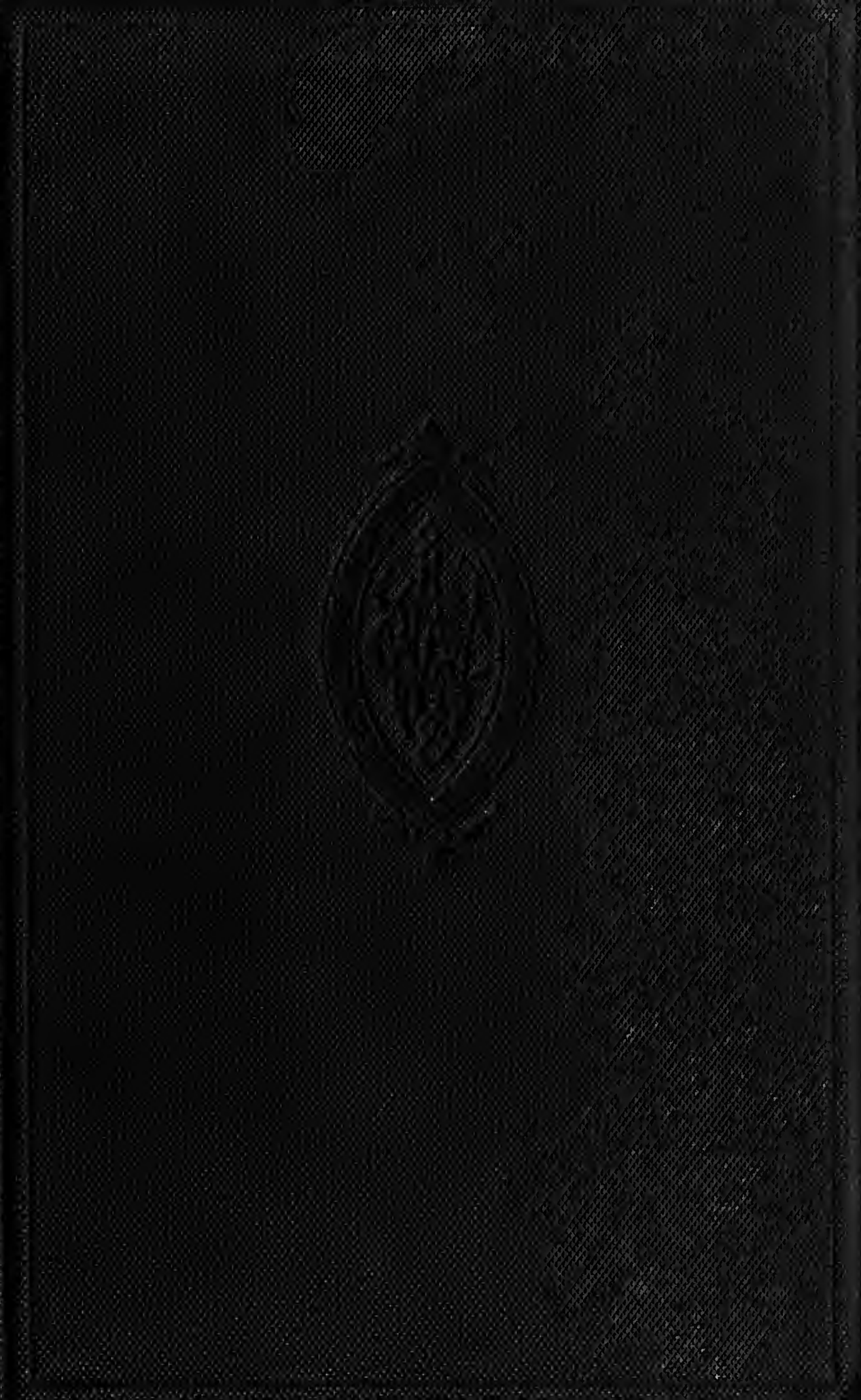




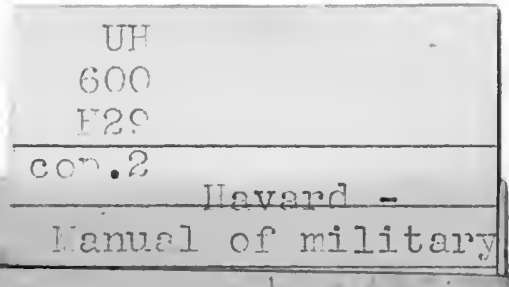

Southern Branch of the

University of California

Los Angeles

$$
\begin{aligned}
& \text { For L } \\
& \text { UH } \\
& 600 \\
& H 29 \\
& \text { cop. }
\end{aligned}
$$


This book is DUE on the last date stamped below

Auid ? 9 re

I Re

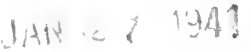

Form L-9-15m-8,'26

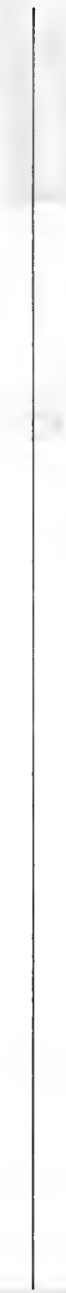


Digitized by the Internet Archive in 2007 with funding from Microsoft Corporation 


\section{MANUAL}

OF

\section{MILITARY HYGIENE}

FOR

\section{THE MILITARY SERVICES OF THE UNITED STATES}

BY

VALERY HAVARD, M. D.

COLONEL, MEDICAL CORPS, UNITED STATES ARMY, RETIRED;

FORMER PRESIDENT, ARMY MEDICAL SCHOOL

Published Under the Authority and With the Approval of the Surgeon General U. S. Army

THIRD, REVISED EDITION

Illustrated with Sez'en Plates and

Two Hundred and Forty-five Engravings

NEW YOR K

WILLIAM WOOD AND COMPANY

MDCCCCXVII

38606 
Copyright, 1917

Br WILLIAM WOOD \& COMPANY

Printed by

Hamilton Printing Company

Albany, N. $Y$. 


\section{UH \\ 600 \\ H29 \\ cob.2}

\section{PREFACE TO THE THIRD EDITION}

The second edition of this work having been exhausted shortly after our country became involved in the present European war, and a third edition called for with as little delay as possible, advantage was taken of the very limited time allowed the author for such revision and addition as seemed most desirable. No material changes have been made and, it is believed, none were necessary.

The aim of the author, in preparing this edition, has been chiefly to present the sanitary devices and methods evolved, as well as the result of the more important investigations pursued, in military hygiene, during the last two or three years, so as to bring it as nearly up to date as stress of time and occupation would permit. The prominent hygienic features of the Great War now being waged have not been overlooked and are duly noted. Some of the subjects, already obsolescent, have been omitted or shortened so that the number of pages is but slightly increased.

It is hoped this new edition will prove worthy of the complimentary reception accorded its predecessors, and commend itself to the favorable consideration of all medical officers.

VALERy Havard.

Fairfield, Conn., August, igi 7. 



\section{PREFACE TO THE FIRST EDITION}

The aim of this Manual is to present in a clear and concise manner the art and science of military hygiene in its latest advances, especially as evolved in this country during the last few years.

While believing that there is a real need for such a book at this time, the writer fully recognizes the gret amerits of the works on military hygiene already published in this country and which have so much contributed to our progress. To them all, but especially to the classic work of Munson, he acknowledges his great indebtedness.

Within the limited compass of this Manual, it was not deemed desirable to include many subjects more or less connected with hygiene, but pertaining, more particularly, to physiology, chemistry and bacteriology, and fully treated in special text-books.

Although primarily intended for medical officers the hope is entertained that it will also be found useful and acceptable by all line and staff officers in command of troops, as well as by the student officers of our service schools. To that end, all unnecessary technical expressions have bee navoided, but without any sacrifice of scientific accuracy.

In order to elucidate important subjects and render long descriptions needless, as many illustrations as was possible have been used, with the trust that they will greatly add to the practical value of the book.

To Dr. W. M. Gray, of the Army Medical Museum, my acknowledgments are due for the photographic work of the plates and engravings.

VALERY Havard.

Washington, D. C., February, I909. 


\section{TABLE OF CONTENTS}

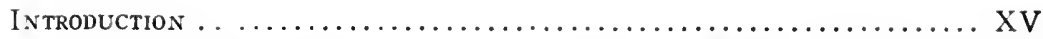

Nature, object and scope of hygiene. What it has already accomplished. The medical officer; his status, duties and responsibilities. Conditions necessary for the successful operation of military hygiene.

\section{CHAPTER I.}

MoRbidity and MORTAlity in the MILITARY SERVice................... Morbidity and mortality in time of peace. Morbidity and mortality in war. Character of wounds in the present European War. Morbidity and mortality in tropical countries. Influence of race. Influence of age and length of service. Influence of climate and station.

\section{CHAPTER II.}

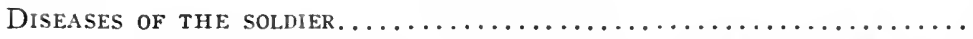

Infectious diseases. Typhoid fever. Paratyphoid fever.

CHAPTER III.

INFECTIOUS DISEASES (CONTINUED)

Diarrhea and dyssentery. Sprue (Psilosis). Cholera.

CHAPTER IV.

INFECTIOUS DiSEASES (CONTINUED)

Tuberculosis. Pneumonia.

CHAPTER V.

INFECTIOUS DISEASES (CONTINUED).

Malaria. Blackwater fever. Yellow fever. Dengue.

CHAPTER VI.

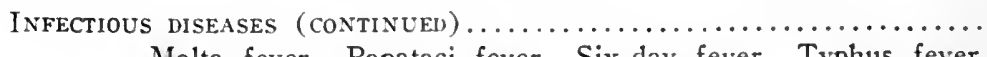

Malta fever. Papataci fever. Six-day fever. Typhus fever.

Relapsing fever. Rocky Mountain spotted fever. Plague. 
CHAPTER VII.

INFECTIOUS DISEASES (CONTINUED) ........................ 90

Cerebrospinal meningitis. Diphtheria. Smallpox. Scarlet fever. Measles. Mumps. Influenza.

\section{CHAPTER VIII.}

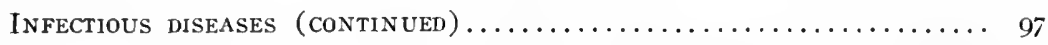

Tetanus. Rabies. Anthrax. Glanders. Leprosy. Yaws.

Climatic bubo. Leishmaniasis. Trypanosomiasis.

\section{CHAPTER IX.}

Parasitic diseases 105

Parasites of the circulatory and lymphatic systems. Intestinal parasites. Skin parasites.

\section{CHAPTER X.}

Nutritional and miscellaneous diseases.................. II 4

Pellagra. Beriberi. Scurvy. Heart diseases. Trench foot.

Asphyxiating gases.

\section{CHAPTER XI.}

Diseases CAUSEd By im MORAL OR INTEMPERATE habits............ I 22

Venereal diseases. Alcoholism.

$$
\text { CHAPTER XII. }
$$

The tobacCo AND DRUG HABITS.......................

Tobacco. Morphine. Cocaine.

CHAPTER XIII.

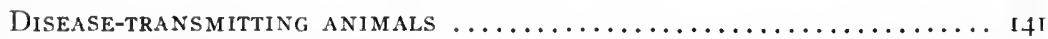

Mites and ticks. Insects proper: bedbug, louse, fleas, cockroaches, flies.

\section{CHAPTER XIV.}

The Mosquito as disease transmitter..................... 159

Description. Classification. Destruction.

\section{CHAPTER XV.}

Pyrethrum. Datura. Mimm's culicide. Pyrofume. Sulphur dioxid. Hydrocyanic acid. Formaldehyde. Mercuric chloride. Liquid insecticides. 
CHAPTER XVI.

ReCRUiting . . .................................... 182

General considerations. Age. Height, weight and chest measurement. Vision and hearing. Mode of examining, physically and mentally. Special disqualifications. Identification record.

CHAPTER XVII.

EXERCISE

Physiology of exercise. Fatigue. Effects of exercise on all the organs of the body.

CHAPTER XVIII.

EXeRCISE (CONTINUED) 218

Regulation of exercise. Overtraining. Gymnastics and physical training. Special exercises. Athletic games and races.

CHAPTER XIX.

THE MARCH

General considerations. Regulation. Care of feet. Irritable heart. Work done in marching.

CHAPTER XX.

Personal hygiene

Daily toilet. Baths. Laundering. Care of bowels.

\section{CHAPTER XXI.}

WATER .

Quantity required. Water in nature. Wells. Examination of: chemical, miscroscopic and bacteriological.

\section{CHAPTER XXII.}

Water purification 273

By heat. By chemical means. The water bag sterilizer.

\section{CHAPTER XXIII.}

WATER PURIFication (CONTINUED)

Filtration. Domestic filters. Sand filtration. Mechanical filtration. The Darnall filter. Improvised filters. 
CHAPTER XXIV.

FOOD . 307

Classification. Proximate principles and their nutritive functions.

$$
\text { CHAPTER XXV. }
$$

ANiMAL FoODs

Meat. Characteristics of good meat. Meat parasites. Meat poisoning. Cooking. Broth and extracts. Preservation.

CHAPTER XXVI.

Animal foods (CONTINUED).

Fish. Mollusks. Crustaceans. Milk. Eggs.

CHAPTER XXVII.

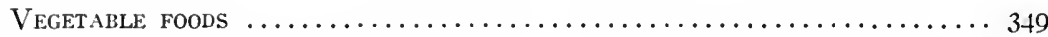

Classification. Composition. Description. Flour and bread.

\section{CHAPTER XXVIII.}

THE NUTRITIVE VALUE OF FOODS. 363

Amount necessary. Chittenden's experiments.

\section{CHAPTER XXIX.}

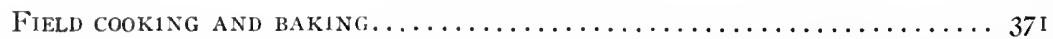

Field range. Fireless cooker. Baking ovens. Field cooking in foreign armies.

$$
\text { CHAPTER XXX. }
$$

THE RATION $3^{8} 3$

U. S. Army ration; its several kinds. Composition and value. Navy ration. Ration in foreign armies.

\section{CHAPTER XXXI.}

Conclusions REgARDING THE COMPOSITION AND FOOD VALUE OF THE MILITARY RATION

\section{CHAPTER XXXII.}

ThE RATION IN TIIE TROPICS......................... 400

\section{CHAPTER XXXIII.}

RULES TO BE OBSERVED IN EATING AND DRINKING. 
CHAPTER XXXIV.

Beverages

Non-alcoholic. Alcoholic.

\section{CHAPTER XXXV.}

Clothing and uniform................................ 419

Properties of textile fabrics. Underclothing. Non-actinic colors. The uniform. Coats. Trousers. Head-gear. Gloves.

\section{CHAPTER XXXVI.}

UNIFORM (CONTINUED)

Foot-wear. Requisites of the military shoe. Leggins.

\section{CHAPTER XXXVII.}

EQUIPMENT . 453

Requirements. Distribution of weight. Knapsack. Equipment in foreign armies. Equipment in U. S. Army.

\section{CHAPTER XXXVIII.}

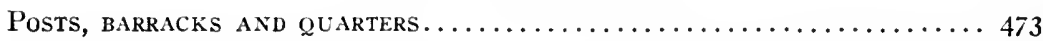

General considerations. Construction and materials. U. S. barracks. Furniture and bedding.

\section{CHAPTER XXXIX.}

POSTS, BARRACKS AND QUARTERS (CONTINLED) .................. 492

Care of barracks and guard-house. Insects and vermin. Care of kitchen and mess-room.

CHAPTER XL.

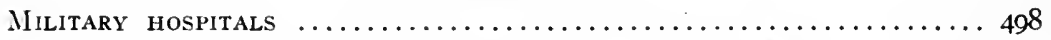

Post hospitals. General hospitals. Base hospitals.

\section{CHAPTER XLI.}

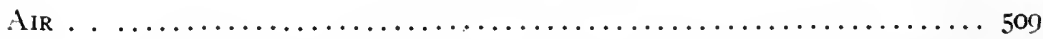

Composition. Humidity. Influence of ocean, lakes and forests. Effects of altitude. Atmospseric dust.

\section{CHAPTER XLII.}

Air contamination. Overheating. High humidity. Air stagnation. Tests for carbon dioxid and carbon monoxid. Nature of rentilation. Amount of air neressary. 
CHAPTER XLIII.

Ventilation (CONTINUED)

Natural ventilation. Artificial ventilation. Draft.

CHAPTER XLIV.

Heating .

Heat transmission. Suitable indoor temperature. Open fire. Stove. Hot-air furnace. Hot water. Steam. Heat and air dust.

GHAPTER XLV.

LightiNG .

Effects of light. Location of lights. Illuminants used.

\section{CHAPTER XLVI.}

EXCRETa, garbage And wastes........................... 564

Definition. Sewerage and plumbing. Fixtures and pipes. Traps. Water-closets. Urinals. Hygiene of toilet rooms. Sewers.

\section{CHAPTER XLVII.}

Excreta, garbage AND Wastes (CONTINUEd) 578

Nature of sewage. Final disposal. Discharge into the sea, a lake or river. Chemical treatment. Irrigation. Septic tank. Imhoff tank. Filtration. Contact filter. Sprinkling filter. Disinfection of affluent. Disposal in garrison and camp. Subsurface irrigation. Disposal of garbage.

\section{CHAPTER XLVIII.}

Soll . . . .

Properties. Ground air. Ground moisture. Ground temperature. Influence of vegetation. Soil bacteria.

CHAPTER XLIX.

CAMPS . . ............................................6 603

Selection of site. Poisonous plants. Venomous animals.

\section{CHAPTER L.}

CAMPS (CONTINUED)

Tents; advantages and objections. Shelter tent. Wall tent.

Conical tent. Pyramidal tent. Hospital tent. Hospital ward tent. Tortoise tent. 


\section{CHAPTER LI.}

Camps (CONTINUed) 624

Improvised and portable barracks. Log hut. Falk's method. Dugouts. Knock-down pavilions. Form of camp. Field hospital. Establishment of camp.

CHAPTER LII.

Latrines . . ....................................... 636

Trenches. Pits or sinks. Latrine box. Post holes. Sanitary trough latrine. Earth-closet latrine. Knock-down sheds.

\section{CHAPTER LIII.}

Disposal of EXCRETA BX INCINERATION....................... 658

The McCall incinerator. The Harris incinerator. The Q. M.

C. incinerator. The Conley incinerator.

\section{CHAPTER LIV.}

Lavatory. Disposal of wastes, garbage and Refuse in CAMp........ 666 Kitchen pit. Company incinerator. Caldwell incinerator. Guthrie incinerator. Rock pile crematory. Disposal of manure.

CHAPTER LV.

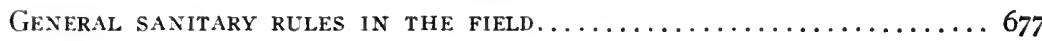

CHAPTER LVI.

Administration of THE SANitARy SERvice in THE FIFLD...........668

CHAPTER LVII.

SERVtCE IN WARM Climates................................6 686

Characteristics of tropical climates. Its effects upon the body. Food. Beverages. Solar rays. Actinic rays. Sunstroke. Clothing. Shelter. General directions. Tropical diseases.

CHAPTER LVIII.

Service in cold climates............................... 7 I 2

CHAPTER LIX.

Disinfection and disinfectants......................... 719

Air and light. Heat. Steam. Sulphur dioxid. Formaldehyde; methods of generation and use. Disinfectants in solution. Lime. Room disinfection. Treatment of hands, feces and sputum. 


\section{CHAPTER LX.}

Hygiene of the battlefield ........................... 744

Precautions to be observed. Sanitary features of modern warfare. Trench hygiene. Disposal of the dead.

CHAPTER LXI.

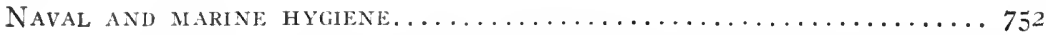

Effects of life aboard on health. Recruiting. Ventilation.

Heat and humidity. Personal hygiene. Disinfection of ships. The sick bay. Hospital ship.

\section{CHAPTER LXII.}

Quarantine

Quarantinable diseases. Special U. S. regulations for each.

Interstate quarantine. Municipal quarantine. Articles of the second international sanitary convention of American republics ( 1905 ).

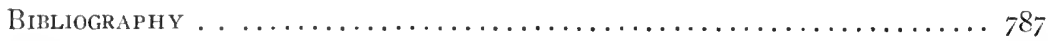

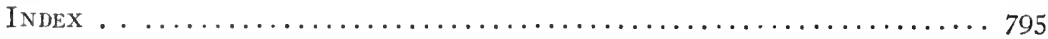




\section{INTRODUCTION}

Hygiene is the science of preserving and promoting health. It seeks out and determines the causes of disease and formulates rules for their prevention and removal. It may then also be defined the science of preventing disease. No science can be more important or beneficial to humanity so long as we consider health the most precious of all our earthly blessings. Prevention is better than cure; therefore, to the world at large, hygiene is of more value than medicine.

But the two sciences are so inextricably blended and mutually beneficial that it would be futile to atiempt to differentiate them. Hygienists, to be successful, must possess a well-grounded knowledge of medicine, while physicians must avail themselves of the researches and discoveries of hygienists if they wish to do justice to their patients.

Hygiene is usually described as individual and public. Under individual or personal hygiene are grouped the subjects of body cleanliness, diet, exercise and habits, while public hygiene considers all those measures which, under the name of sanitation, are instituted for the welfare of communities, such as water-supply, disinfection, sewerage and disposal of wastes. Sanitation deals with the removal of all conditions favoring the growth and propagation of pathogenic, or disease-bearing, germs; to it may be ascribed the chief share in the beneficient effect of hygiene in saving human lives and extending longevity.

All diseases may be classed under two heads: I, those arising within the body (autogenetic diseases), due to disturbances of nutrition, assimilation and elimination, the result of a want of harmony between waste and repair, such as rheumatism, gout, diabetes, arterio-sclerosis, tumors, nervous diseases, etc.; 2, those due to causes from without and depending upon the invasion and multiplication of special germs in the body, such as the infectious or zymotic diseases.

Hygiene is concerned in the prevention of both classes. By determining the relations which should exist between diet, work and 
exercise, it secures a sufficient assimilation of suitable food and a free eliminaton of wastes, without overstrain of any of the organs; thus are the diseases of the first class guarded against. However, it is especially in preventing those of the second class, among which are the so-called camp diseases, that military hygiene is most efficacious.

For the production of infectious diseases two factors are necessary: the germs must be absorbed in the body through the digestive or respiratory tracts, or by inoculation through the skin; and, being absorbed, they must find a suitable soil in which to grow and breed, that is to say, the body must be in a susceptible or receptive state. The hygienist, therefore, should have a sufficient knowledge of bacteriology, but the description and reactions of the pathogenic micro-organisms are of less consequence to him than their mode of transmission and introduction into the human body. Thus we know how yellow fever is conveyed, and this knowledge has enabled us to stamp it out from Cuba and other places, although the causal micro-organism has never been seen. On the other hand, we know the bacillus of leprosy, but on account of our ignorance of the manner in which it effects an entrance into the body, under natural conditions, very little has as yet been accomplished for the prevention of that terrible disease.

As a country develops its material resources under the favorable conditions of peace and acquires wealth, its hygiene, as a rule, progresses correspondingly; the people eat better food, are better clothed and sheltered and make more frequent use of the bath, while the cities and State construct public works of sanitation. The result has been a remarkable fall in the death rate of all civilized countries during the past quarter of a century, as shown by the following figures from the reports of the Census Office:

In the United Kingdom (England, Scotland and Ireland) the yearly mortality per I,000 inhabitants, which was about 22 in I870, has diminished steadily until the present time, being 18.2 in 1900 and 15.5 in 1905 . This means that, since 1870 , for each 1,000 inhabitants there is a saving of 6.5 lives, or, for a population of $40,000,000$, of 260,000 lives a year. In England and Wales, the rate for I9Io was only 13.5 and for $1913,13.7$.

In France the mortality fell from 26 in 1820 to $2 I .5$ in I900, 19.6 in 1005 and 17.7 in 1913 ; in Germany, from 26.8 in 1850 to 
22.2 in 1900, 19.9 in 1905 and 15.6 in 1912 ; in Austria, from 32.2 in I 850 to 29.5 in I890, 26.6 in I900, 24. I in 1905 and 20.5 in I9I2; in Italy, from 27.1 in 1890 to 24.2 in $1900,21.8$ in 1905 and 18.2 in I9I2; in Spain, from 30 in 1900 to 26. I in 1905 and 21.8 in I9I2.

In the United States the death rate for the registration area was I9.6 in I890, I7.8 in I900, I6.2 in I905, I5.0 in I9I0, and 13.6 in I9I4. The registration area (consisting of 67 per cent. of total population in 19I4) includes most of the large cities, where the death rate is higher than in rural districts. Thus the rate for the rural part of the registration States in I9I4 was only 12.3. As the nonregistration States include a much larger rural part than the registration area, it is reasonable to conclude that for the total population the death rate for I9I4 is well under I3. If, furthermore, we take into account the fact that the colored people, which form such an important element of our population, have a mortality at least 60 per cent. greater than that of the whites, it follows that the death rate of the white population of the United States, considered by itself, must be among the lowest in the world.

The decrease of mortality in some of our principal cities has been as follows:

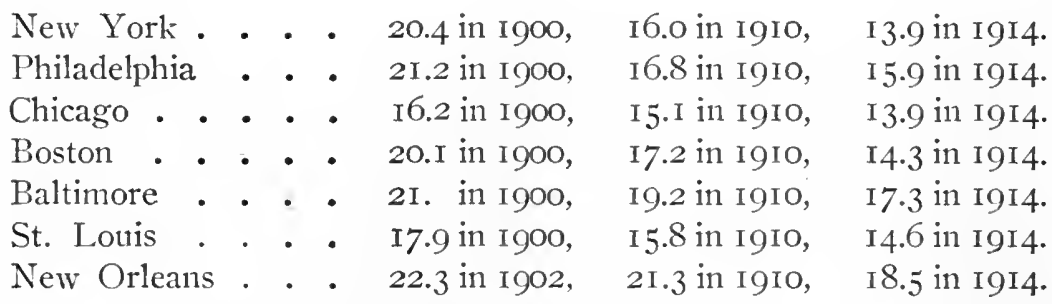

The progress of military hygiene has fully kept pace with that of general hygiene. The death rate in our Army was about I $_{5}$ in I870, II in I880, and had gradually fallen to 5.I I in I897, before the Spanish WVar. As the result of this war, of the subsequent insurrections in the Philippine Islands and of the unusual and ardous conditions of tropical service, the mortality remained for several years above its normal rate, but is again steadily lowering, being $5.8 \mathrm{I}$ in $1907,4.46$ in I9I0, 4.40 in 1914 and 4.45 in 1915 , for the whole Army at home and abroad.

The same improvement is noted in all European armies. Thus in the British Army the death rate for troops at home was 7.20 for 
the decade $1875-84$ and 4.68 for the decade $1887-96$, rising to $5.6 \mathrm{r}$ in 1905 and falling to 2.92 in 1909 and 2.34 in 1912 . In the Prussian Army the rate fell from 4.82 in 1880 to 3.30 in $1890,1.80$ in 1907 and 2.0 in I9II; and in the French Army, from about 8 in 1880 to 5.81 in $1890,3.92$ in 1907 and 3.01 in 1910.

Military hygiene differs from general hygiene only in its application; its laws and principles are necessarily the same. The soldier is subject to the same diseases as the civilian, but in a different degree on account of the special conditions under which he lives. $\mathrm{He}$ is to a large extent bereft of his personal freedom; his food and shelter are provided for him, while his work and exercise are strictly regulated. In war time, his food and surroundings are those which military necessity imposes, the preservation of his health becoming a matter of secondary consideration. The crowding of troops in barracks or camps facilitates the transmission of disease and is an element of danger much more serious than in civil life, but, on the other hand, in a well-administered army the soldier is the object of sanitary measures which, if properly enforced will, to a large extent, safeguard him against the dissemination of infectious germs.

The vital importance of hygiene, in garrison and the field, has been more fully realized of late years, especially since the SpanishAmerican War. The investigations carried on during and after this war have shown that the diseases which caused its terrible mortality were mostly preventable, that they resulted from the lack of efficient sanitary measures or their indifferent application. It is the earnest belief of the Medical Corps that such unnecessary sacrifice of life cannot again occur in our Army, except through unpardonable ignorance or criminal carelessness.

It is now recognized that the sanitary service of an army is not an impedimentum or a necessary evil to be tolerated, but that, on the contrary, it is an essential and integral part of its organization, materially contributing to its fighting efficiency by preventing the depletion, from disease, of the combatants' ranks, and by such treatment of the sick and wounded as will permit their prompt return to the firing line.

On this subject, Field Marshal Evelyn Wood, of the British Army, expresses himself as follows: "I am convinced from my experience of thirty years as a general that the arny doctors should 
be regarded not merely as healers of the sick and wounded, but as trusted staff officers to advise their chiefs how to guard the troops against the originating and spreading of disease and thus maintain the number of effectives in a campaign. This will result not only in the increasing of fire effect, but will raise immensely the fighting value of the troops and will incidentally enable us to reduce the costly and cumbersome hospital establishment and transport."

Hygienic measures are expensive, but they will prove comparatively cheap if they prevent disease, for nothing is more costly than a sick soldier; not only is he useless in war but requires the services of able-bodied comrades, and later burdens the pension list. A trained soldier is a valuable product as an efficient fighting machine; the chief duty of the medical officer is to maintain him in his best physical condition and, in case of sickness or wound, to return him to the ranks in the shortest possible time.

With a fuller appreciation of the importance of hygiene has come, as a natural consequence, a remarkable change in the functions and duties of medical officers. Formerly they were primarily surgeons and so designated in official parlance, but now they are chiefly sanitarians; their principal work is no longer the treatment of the wounded, important as that is, but the prophylaxis of disease. If camp diseases can be controlled and prevented, it is evident that, during a campaign, good sanitation will save more lives than the most perfect surgery ; therefore it follows that most medical officers must be good sanitarians at all times, while only comparatively few need be skilled surgeons. The unfitness of the title of "surgeons" applied to them is especially obvious when we reflect that war is an unusual condition, that modern armies live generally in a state of peace, when wounds only result from accident and are comparatively rare, and that the duties of medical officers are chiefly those of physicians and sanitarians.

The sphere of activity of the medical officer is becoming so extended and comprehensive that the question of specializing his duties is now demanding consideration. The need is becoming more and more felt in the Army for experts, not only in surgery and special diseases, and in general hygiene, but also in sanitary engineering, men capable of installing and directing water-supply and sewer systems, filtering and disinfecting plants, etc. 
The wonderfully low ratio of mortality from disease during the Russo-Japanese War had the result of compelling attention to the organization of the medical department of both belligerents; this, although far from perfect, was seen to be much more complete and on a more liberal scale than had ever been the case in any previous war of great magnitude. The effect, in all civilized countries, was the accentuation of the importance of military hygiene in war and an increased interest in its study and development.

As the happy culmination of the application of modern preventive medicine to the conditions of armies in the field, we are now amazed to see the greatest war that was ever waged (I9I7) nearly absolutely free from any of the dreadful camo diseases so prevalent in former wars.

In our service, garrison and comp sanitation is now recognized as specialized knowledge requiring the direction of experts; therefore no part of it is any longer intrusted to non-professional officers, however intelligent and zealous they may be, but only to such medical officers who have acquired this expert knowledge. To them should be turned over the entire work of sanitation, initiation and execution. For that purpose, increased authority has been given them and a correspondingly greater responsibility laid upon them.

It is obvious that such authority and responsibility must have limitations; in this respect, the position of medical officers is very different from that of civilian health officers whose work is exclusively of a professional character and whose will is supreme as regards the manner of executing it. In war, conditions are often encountered when the demands of hygiene must be more or less sacrified to military exigencies. An Army is organized for a definite purpose, which is war, and all its elements must be shaped and trained so as best to subserve that purpose. The first duty of the medical officer is to contribute as much as possible to the fighting efficiency of the army, bearing in mind that the best way to insure proper treatment to the sick and wounded is by first winning the victory. While unremitting in the use of every available means to prevent the reduction, by disease, of effective combatants, he will acquiesce in and make the most of situations where sanitary considerations must be overlooked for the sake of possible military advantages. Under such circumstances the responsibility for the consequences are assumed by the commanding officer. 
All authority must necessarily be centered in the commanding officer. To divide it would lead to military chaos ; complete autonomy is no more possible or desirable in the medical than in any other staff department. Under existing regulations, officers of the Medical Corps cannot exercise command except in their own department. Their duties concerning sanitary matters, at post or in the field, are limited to investigation, report and recommendation. It is an admitted fact, however, that the best results are obtained by endowing them with as much executive authority as conditions permit, and that it can be delegated to them in the same manner that authority is delegated to adjutants and inspectors. Thus the Field Service Regulations provide that sanitary inspectors may be authorized to direct, in the name of the commanding officer and within prescribed limitations, the prompt abolition of conditions prejudicial to the health of troops. This power was given to sanitary inspectors during our interventions in Cuba, and in most of our recent camps of instruction, with excellent results. It is also given by some post commanders to their post surgeons (see page 683).

Inasmuch as sanitary recommendations may involve important work and large expenditure, it is obviously necessary that a medical officer should possess not only professional knowledge but also good judgment and sound common sense. His reconmendations mutst be reasonable and practicable, and made only after careful consideration of all factors involved; their disapproval by his professional superior would discredit him and impair the usefulness of the Medical Corps.

The above considerations show the wisdom of Congress in investing medical officers with military rank and military title, so that their authority may be commensurate with their responsiblity. for experience has proved that military rank is necessary to confer the power and prestige of authority - and no officer from staff or line, in the field, needs this authority more than the medical officer for the successful handling of the large personnel under his command and the efficient discharge of his manifold duties. Although classed with non-combatant officers, he is none the less exposed to all the hazards of the fire zone. The statistics of the Civil War show that more medical officers were killed in battle than officers of all the other staff corps together, and the same is true of the present European War. 
Although it is a well-established policy that all sanitary appliances, such as sterilizers, filters, incinerators, etc., as well as ambulances, must be under the direct control of the Medical Department, when in use, there is still some difference of opinion as to whether this department should not also manufacture and store them in time of peace so that they may always be in readiness on taking the field. But whichever way this is decided, it is certain that the Medical Department will always be more or less dependent upon the Q. M. Corps for much of its material and hired labor and must therefore receive its thorough co-operation to secure the best results.

In the event of war, the bulk of our armies would necessarily consist of volunteer or drafted forces and be under the sanitary care of volunteer or reserve medical officers. These officers may be assumed to have all the needful qualifications as physicians and surgeons, but their knowledge of practical field sanitation will always be a variable and uncertain quantity. This is a weak point in our system requiring serious consideration. The remedy, as already successfully applied in several States, consists in theoretical instruction during the winter months under the supervision of the Surgeon General of each State and in practical instruction during summer encampments. The sanitary work of the mixed commands of regular and militia troops at our summer camps has been of the highest value as a school of instruction not only to medical officers, but as well to all line officers and enlisted men. The use of sanitary appliances has been clearly exhibited and their beneficial effects upon the health of troops demonstrated in such a manner as to produce a marked impression upon all concerned. It is especially by means of such camps that medical officers will obtain the practical knowledge which they require and that line officers will be made to understand the part which devolves upon them in the work of sanitation.

In the application of the precepts of hygiene, the medical officer, in spite of his efforts, can accomplish but little if unaided. His labors, to be fruitful, must have the hearty and intelligent cooperation of staff and line. Therefore he must instruct, by word and object-lesson, and carry on a sanitary propaganda which will reach down to every enlisted man. He should particularly endeavor to interest the company officers, for it is through them that the men can be most readily influenced and the enforcement of sanitary measures most easily accomplished. The intelligent soldier, in a 
disciplined regiment, complies readily with regulations intended for his welfare as soon as he understands their importance to himself and the service.

The War Department has realized the great importance of hygiene in the Army by adding it to the curriculum of the Military Academy and of all our service schools, and it is most gratifying to note how efficiently our young officers, at the very outset of their career, apply the knowledge thus acquired, to the great advantage of the enlisted man.

If we bear in mind that the great majority of our soldiers remain but a short time in the Arny and then return to civil life, we must realize the highly beneficial effect of this instruction upon the health conditions of the country at large. The thousands of men yearly discharged carry with them to their homes more or less practical knowledge of the prevention of disease, as well as formed sanitary habits, which must be useful to them and the people with whom they associate, so that military hygiene, as taught every soldier in the Army, becomes one of the most valuable educational agencies under the control of the Government.

Military hygiene, to be efficacious and successful, must deal with able-bodied soldiers. The best possible care can never make strong, resistant and useful soldiers of immature, undergrown or otherwise defective youths. In any army composed of men voluntarily enlisted it would seem that only physically perfect individuals should be accepted and enlisted. But this is far from being the fact. It is not always the strong and stout who present themselves to the recruiting officer, nor is it always possible to detect chronic or latent diseases or a predisposition to them, while it also happens that examiners are not as careful or experienced as their duties require. The result is that there is always a certain ratio of sickness due to pre-existing conditions - for which the military service is not responsible. Therefore, great care and diligence are enjoined by the War Department upon all recruiting officers in order to reduce this rat1o to a negligible minimum.

Military hygiene is indissolubly bound up with discipline. It is not enough to be familiar with all its precepts, they must be fully and strictly applied, and this cannot be done successfully except through discipline. Laxity of discipline in an army is the source of many evils, not the least of which is its baneful effect upon the 
dissemination of disease. Assuming that medical officers are equal to their duties, experience shows that the health condition of the command will be directly proportional to its discipline and, therefore, that to preserve the health of troops the action of regimental and company officers is quite as necessary as that of medical officers. This has been exemplified in all modern wars, but especially in the Franco-German and the Russo-Japanese Wars. The difference in the sanitary condition of the French and German Armies in 1870 and $187 \mathrm{I}$ was not less marked than the difference in their fighting efficiency. The Japanese in Manchuria did not discover any new system of sanitation, but they excelled in discipline, prompt obedience to orders and administrative abilities, and this was not the least of the causes which reduced their ratio of sickness to a minimum never before reached by any large army in the field.

The successful application of military hygiene under the best conditions - that is to say, by an efficient medical department to a body of men carefully chosen and under strict discipline-was strikingly illustrated in the German Army which, since the FrancoPrussian War of 1870 , until the present European War, always had the lowest mortality of any army in the world. The military service being compulsory in Germany and the number of conscripts much larger than the annual contingent required, it was possible to make a careful selection of recruits and reject all the physically imperfect. The strict discipline prevailing in its army was also notorious. Thus was its remarkably low death rate readily and satisfactorily explained.

In the present war, in Europe, the very low mortality from disease is chiefly due to our knowledge of how to prevent and successfully combat typhoid fever, typhus, cholera and dysentery. 


\section{MILITARY HYGIENE}

CHAPTER I.

\section{MORBIDITY AND MORTALITY IN THE MILITARY SERVICE.}

As late as the middle of the Igth Century, the crowding of soldiers in ill-ventilated barracks and the neglect of all hygienic rules resulted in the free dissemination of infectious germs and in enormous rates of disease and death in garrisons, several times higher than in civil life. But since the Crimean War in Europe and the Civil War in America, with the progress of scientific medicine, it has become more and more evident that most of the military morbidity and mortality can be prevented by giving soldiers sufficient air space and the benefit of intelligent sanitary regulations. In time of peace, soldiers are presumed to be comfortably quartered, properly fed and clothed, subject to wise hygienic rules and, to a large extent, guarded from excesses and dangerous exposure by military discipline. They are, besides, chosen men, physically sound when enlisted or mustered in. Under such conditions one would expect them to enjoy at least as good health as males of the same age in civil life. It is true that the danger of conveying disease by contact, direct or indirect, in barracks, even when sufficiently roomy, is ever present and cannot be overlooked. The question is complicated by several interacting factors which add to the difficulty of reaching a clear conclusion.

According to the United States census of I900, the male population of military age, namely between 20 and 39 years inclusive, was $12,466,309$; during the year this population suffered a mortality of 95,070 , or at the rate of 7.62 per thousand. For the period of life from 20 to 29 , the rate was 7.II. So far as it is possible to ascertain, England, France and Germany have about the same ratio of civil mortality, being a littie less in Germany and a little more in France. According to the latest American life insurance tables, 
7.80 per thousand of young men 20 years old die annually in this country, and 8.43 of men 30 years old. According to the United States Life Tables for I9IO (probably nearer to the truth), the mean rate of mortality in the registration States for the ten years 20 to 30 , was 5.50 .

How then does military mortality compare with the above? By consulting the official reports of the last few years, years of peace, the figures appear quite favorable to military life. Thus we find that for the $U$. S. Army (exclusive of colonies) the total mortality (from disease, accident and injury) for 1909, I9IO and I9I I was $4.84,4.42$ and 4.72 respectively, or an average of 4.66 . Great Britain reports 2.42 for I9IO, France 3.75 for I909, Prussia I.78 for 1909 and Russia 4.07 for I909. These figures show that the rates for the U. S. Army are higher than those of the leading European countries, but that they are all under those prevaiiing in civil life. A further study of the subject, however, somewhat modifies this first view. It is found that, in all armies, when soldiers contract incapacitating diseases, not likely to be cured within a short time, they are discharged and returned to civil life, so that their death, instead of being charged to army statistics, go to increase the civil rates. Thus in I904, the proportion of discharges per thousand of strength was 48.40 in the French Army, 46.00 in the Prussian Army and 22.00 in the U. S. Army. In the British Army this proportion was $\mathbf{1}_{5} .05$ for 1905 and only 8.65 for 1909. In the U. S. Army it increased to 26 for 1906 and fell to I 5.28 in 1910.

The disease which, in the absence of epidemics, makes the greatest number of victims, is tuberculosis. In the French and German Armics most of the discharges are for that disease; as soon as a case of it is clearly diagnosed the patient is invalided and sent home where his death ultimately swells the civil rates. In our service, such a patient is generally sent to the Army sanatorium at Fort Bayard, N. M., and his death, should it occur there before his discharge, credited to the Army rates.

According to Viry and Marvaud, the ratio of mortality in the French Army should be increased by 4 to give a near estimate of the number of deaths fairly attributable to military life. The same correction should doubtless be made for the German Army. These 4 deaths are to be deducted from the rates of civil life, but owing 
to the large population of military age in France and still more so in Germany, this deduction would only amount to a fraction of one per thousand, a negligible quantity. It is presumed that an increase of 2 would fully correct the rates of the U. S. Army and the British Army.

However, if, instead of taking the mortality rrom all causes, we simply compare the death rates from disease only, the result is still more clearly in favor of military life, for it is a fact that, in the service, in time of peace, more men die of injuries and violent death than in civil life. Thus, in I900, the proportion of deaths from accidents and injuries, to the total mortality, in the male population of military age in the United States, was only I7 per cent., while in the U. S. Army (exclusive of colonies) this proportion, for the three years $1904-6$, is 43 per cent.; the rates of deaths from disease, for those years, being only $3.94,3.55$ and 2.84 per thousand respectively. The death rate in the Army, for 1910, was 2.44 from disease and 2.02 from external causes, or in the proportion of 55 to 45 per cent., whereas for the entire population of the United States this proportion (suicides excluded) was 94 to 6 . The number of accidental deaths in European armies is not as high as in our service, but yet decidedly higher than in the civil male population.

It is plain, therefore, that we are justified in stating that, in time of peace, modern armies suffer less from disease than the corresponding male civil population. This is due largely to the fact that soldiers are subjected to a careful sifting, whereby all applicants below a suitable physical standard are rejected; but it is also an indubitable proof of the efficacy of scientific sanitary measures whell enforced by military regulations and discipline.

\section{MORBIDITY AND MORTALITY IN TIME OF PEACE.}

The diseases of the soldier in time of peace differ from those of war time in their degree of prevalence and rates of mortality. 'In th U. S. Army (exclusive of colonies), for the three years I908I9ro, the following diseases have been those most prevalent, in the order named:

Venereal diseases, tonsillitis, bronchitis (acute and chronic), diarrhea (including enteritis), influenza, alcoholism, malarial fevers, rheumatism, measles, mumps, tuberculosis. 
For 19 I 5 the five most prevalent were exactly the same as above and in about the same order.

In the U. S. Navy and Marine Corps, for the years I9o9 and I9IO, the order of prevalence was: venereal diseases, tonsillitis, skin diseases (suppurative), influenza, bronchitis, rheumatism, diarrhea, malaria. For I9I5 the order was: venereal diseases, tuberculosis, tonsillitis, mumps, appendicitis, influenza, bronchitis.

In the British Army, at home, the order of prevalence, tor I909, was: venereal diseases, minor septic disorders (boils, abscesses, etc.), tonsillitis, digestive diseases, influenza, bronchitis, scabies, rheumatism.

In the French Army, for I907: bronchitis, venereal diseases, mumps, rheumatism, influenza and measles.

In the Prussian Army, for 1907: bronchitis, venereal diseases, influenza, rheumatism and pneumonia.

The preeminence of venereal diseases in America and England, as compared with continental European countries, is largely due to the lack of the safeguards used, for instance, in France and Germany, where prostitution is subject to police regulations. This natter will be discussed in its proper place.

Tonsillitis, in our Army and Navy, comes second in the order of admissions, while bronchitis is not far below it. The high prevalence of tonsillitis is remarkable, showing the sensitiveness of the tonsils to external influences, as well as some serious defect in the ventilation of our dormitories. The rate begins to rise as soon as winter sets in; then the barracks, often crowded, are tightly shut and the air becomes overheated, close, ill-smelling; conditions most favorable to the spread of tonsillitis by air infection, and favorable also to the incidence of nasal, pharyngeal and bronchial affections mostly included under the general heading of bronchitis. The remedy is more air space and better ventilation.

Deaths result chiefly from the following diseases, in the order named:

In the U. S. Army, for the years 1909-19I I : tuberculosis, pneu-. monia, heart disease, typhoid fever, nephritis, measles, alcoholism, appendicitis. For 19I5: pneumonia, measles, heart disease, tuberculosis, appendicitis.

In the U. S. Navy and Marine Corps, for the years 1909 and I910: tuberculosis, pneumonia, heart disease, nephritis, typhoid 
fever. For the year 1915: tuberculosis, pneumonia, nephritis, heart disease, appendicitis.

For the entire population of the United States, the five leading causes of death, in I9IO, were, in the order named: tuberculosis, heart disease, diarrhea and enteritis, pneumonia, nephritis. In I9I4 they were tuberculosis, heart disease, nephritis, diarrhea and enteritis, cancer.

In the British Army, for 1909: pneumonia, tuberculosis, heart disease, nephritis, typhoid fever, rheumatism.

In the French Army, for 1907: tuberculosis, typhoid fever, pneumonia, influenza, scarlet fever.

In the Prussian Army, for 1907: pneumonia, tuberculosis, appendicitis, typhoid fever.

It is admitted that in military medical statistics, the rate of the constantly non-effective, that is, of men constantly on the sick report, is the true measure of the loss in efficiency of an army from sickness and injury. The non-effective rates per thousand in our Army, for 1909, 1910, I9I I and 1915, were 42, 36, 33, and 25 respectively. They show a steady and much-needed reduction during the past decade. The rate of 42 , for 1909 , corresponded to a loss of 14.5 days from sickness or injury for each soldier, or to $3,08_{3}$ men constantly excused from duty. During the same year, the average number of days lost by each British workman was 7 . It was about the same in France and, presumably, in this country. It has also been computed that, in the United States, for each death in the Army, there is a reported period of sickness several times longer than for each death in civil life. In the U. S. Navy, for 1909 and 1910, the non-effective rates averaged 26.59, corresponding to 9.70 days of sickness for each man. In the British Army, for 1909 , these rates were 28.86 , the average sick time for each soldier being 10.39 days.

It is evident that soldiers in most armies, but especially ours, àre often excused from duty for reasons which would not appear sufficient to keep workmen or mechanics away from their daily task. To some extent this is inevitable, but it is an indulgence that, in the interest of the service and of the men themselves, should be guarded with discretion and judgment and never permitted to go beyond reasonable limits. It is certain that our soldiers, as a general rule, have been too easily admitted to hospital and quarters 
and kept there longer than necessary, and that a little more strictness in that regard on the part of medical officers would work neither injury nor hardship to any one. It is a matter of common observation that those posts where the medical department is most efficiently conducted, nearly always show the lowest sick report. It is also remarkable that in summer camps, where the troops are subject to many discomforts and often destitute of suitable sanitary appliances, the sick report is seldom more than half that of troops in garrison. This is becattse the men are liept busy and interested in their work, temptations to dissipation are fewer, and the sanitary service is under the direction of selected medical officers.

Inasmuch as venereal diseases make up one-fifth of all admissions, it is hoped that the systematic measures of prophylaxis now becoming more generally employed, together with the careful application of the recent law stopping the pay of officers and men under treatment for disease resulting from misconduct, will materially reduce the rates of these affections.

The stricter rules now enforced to get rid of incurables and of cases not likely to recover within a reasonable time also tend to greater efficiency.

In comparing our death rates with those of other armies, it should be remembered that our Army contains a certain proportion of negro troops, amounting to 4 or 5 per cent., and that their mortality from disease is nearly double that of white soldiers.

\section{MORBIDITY AND MIORTALITY IN WAR.}

In time of war, sanitary conditions are necessarily very different from those which should prevail in time of peace. Then everything is ruthlessly sacrificed to strategic exigencies, that is to say, the necessity of confronting the enemy as quickly as possible, in the best position and with the greatest number of men. But, even then, on the march or the battlefield, sanitary considerations should never be overlooked or neglected, for hygiene is a source of strength to an army at all times. During a campaign, soldiers are likely to suffer bodily from inclement weather, heat, cold or rain, lack of shelter, improper or badly cooked food, overstrain and, mentally, from excitement and anxiety. Hence, digestive and intestinal disorders, rheumatic, respiratory, cardiac and nervous affections. Furthermore, the soldier is greatly exposed to infectious diseases and, intil hardened by rough service, more susceptible to them. 


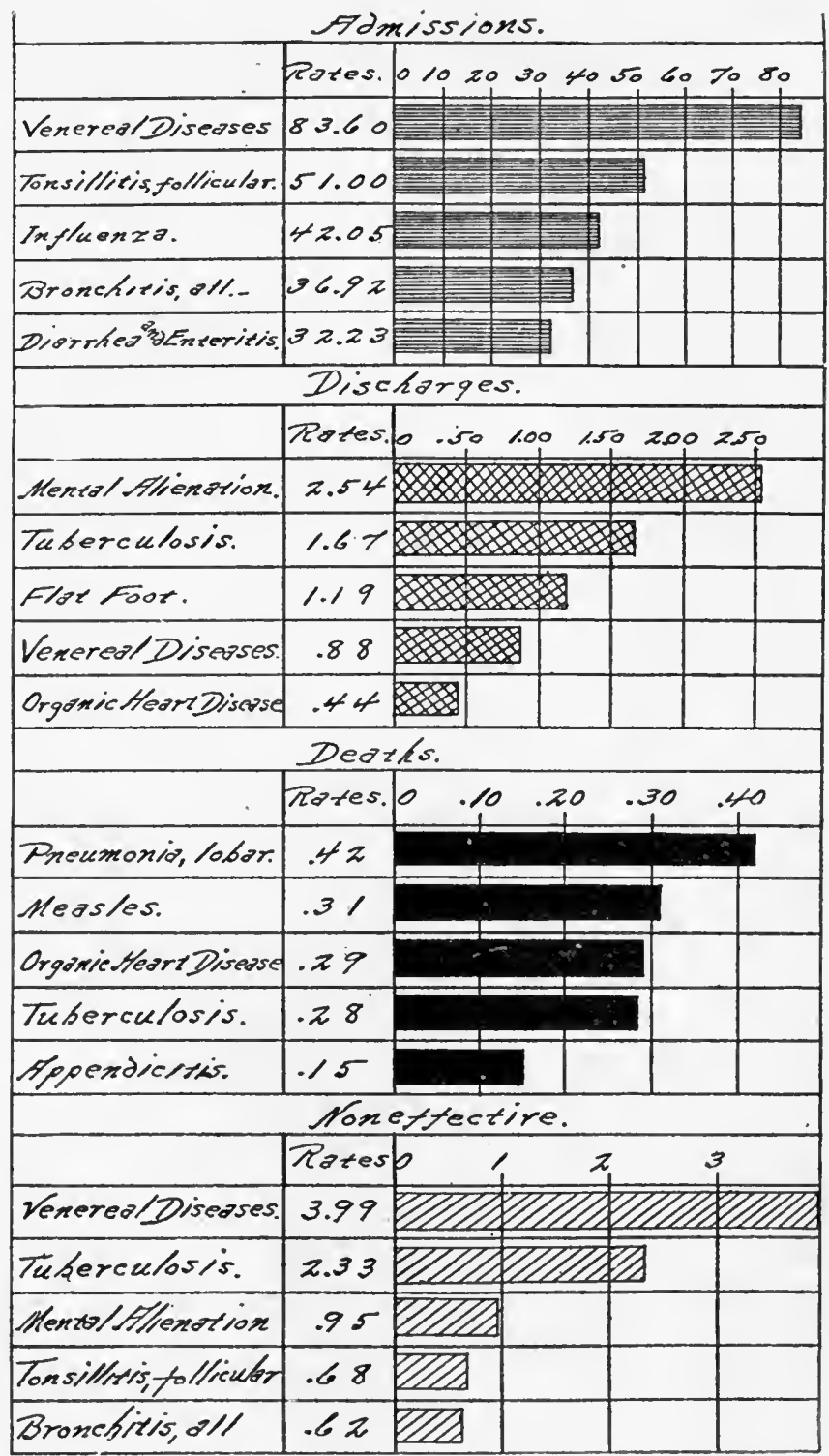

Fig. 1.- Chart showing the relative admission, death, discharge, and noneffective ratios per thousand of mean strength for the five diseases causing the highest rates in the United States proper, for the year 19I5. 
The concentration of regiments in one camp or position cannot always be avoided, while sanitary measures can only be imperfectly applied. The result has been, almost invariably, that until the beginning of this century, the morbidity and mortality from disease, in war, rapidly increased and soon exceeded the rates of the wounded and killed in battle. A few modern historical instances will impress this melancholy fact upon the mind.

During the Mexican War of $1846-47,22$ per cent. of the American regulars died and $\mathrm{I}_{4}$ per cent. were invalided from disease, while only 5 per cent. were killed in battle or died from wounds.

During the Crimean War of $1854-56$, the French lost from disease, in round numbers, 70,000 out of 3 Io,000 men, or nearly 23 per cent., while 65,000 were invalided home. Only 7,500 fell in battle. In the same war the British lost 2I,000 men from disease out of III,oOo.

For the Civil War, I86r-65, the record is as follows:

$\begin{array}{lr}\text { Killed in battle, } & 44,238 \\ \text { Died of wounds, } & 49,73 \mathrm{I} \\ \text { Died of disease, } & \text { I 86,2 I6 } \\ \text { Died, cause unknown, } & 24.184\end{array}$

Leaving out of reckoning those who died from unknown cause, it is seen that for each man killed or dead from wounds, two died from disease.

In the Franco-Prussian War, 1870-7x, the French mortality from disease vastly exceeded that from battle, but reliable statistics are not available. The Germans lost I7,255 in killed and 14,904 from disease, the first conspicuous exception to the rule that, in war, more men die from disease than from gunshot.

For the Spanish-American War, during the year I898, the record is as follows:

Cuba,

From battle. From disease.

Camps in United States, .... deaths. 2,649 deaths.

Philippine Islands,

Porto Rico,

I7 deaths. 203 deaths.

3 deaths. 262 deaths.

Total,

293 deaths. 3,68I deaths.

or I death from battle to 12.5 deaths from disease.

In the war between China and Japan, I894-95, out of 227,600 Japanese brought into the field, I5,850 died of disease and I,3 I I from wounds, a proportion of $\mathrm{I} 2$ to $\mathrm{I}$. 
In the Boer War, I899-190I, out of a total British force of 448,000 men, 7,534 died from wounds and 14,382 from disease (Report of Insurance Actuaries) ; in addition, 63,644 sick and 8,22I wounded were invalided home.

It would be irrational, however, to attach much importance to this comparison of ratios between the sick and the wounded, or estimate from it the sanitary condition of an army. These ratios are not necessarily correlated and remain more or less independent of each other. Thus although the sanitary status of troops may be excellent, the ratio of sick will greatly exceed that of the wounded if there be no severe fighting. The bad showing made by the statistics of the Spanish-American War, in this regard, is not so much the result of a high proportion of sick as it is that of an unusually low ratio of wounded. As a general rule it can be stated that a bloody and decisive campaign, of short duration, will be attended with much less sickness than one consisting of ineffective engagements covering a long period of time. Nothing is more detrimental to the health, as well as to the morale of an army, than a long-dragged out, indecisive campaign, under a pusillanimous commander.

The Russo-Japanese War, 1904-5. marks a new epoch in military hygiene by demonstrating the possibility, even in wars of great magnitude, to keep the rates of disease much below what had always been considered as the irreducible minimum. In a war of nearly two years' duration, the dead from disease were only about one-half the number of killed; often the percentage of sickness among both belligerents was lower than that of garrisons at home. Although the usual diseases were present, none prevailed in an epidemic form, except beriberi, which appears to have been nearly as widespread among the Japanese as during their previous wars, in spite of persistent efforts to eradicate it.

The following is the official Japanese statement for the entire war (including Port Arthur):

Total killed and wounded

220,812

Total sick

236,223

Killed in battle and died of wounds, 58,887

Died of sickness,

If we estimate the total strength of the Japanese in the zone of operations at 700,000 , we obtain a percentage of $8.4 \mathrm{I}$ for the killed and of 3.88 for the dead from sickness. 
The official statistics of the Russian Army (exclusive of Port Arthur) are as follows:

Total killed and wounded, 140,953

Total sick, 237,472

Killed in battlc or died from wounds, 23,008

Died of disease,

Missing,

No satisfactory official explanation has becn offered to account for this exceedingly large number of missing. The number of deserters to the hostile Chinese cannot have been very great. It seems more probable that a large proportion of them, perhaps a majority, should be counted as among the killed. Both Col. Fioff and Dr. Follenfant, medical attachés with the Russian Army, estimate at about 14,600 the missing which should be added to the number of killed, thus increasing the latter to 37.608 . But it seems logical that, of the remaining missing, should be also deducted a certain proportion of men taken sick along the extended lines of positions and left to die in Chinese villages unaccounted for. It is believed that 3,000 or 4,000 might thus be properly added to the dead from disease.

Regarding Port Arthur, it is known that the Russian garrison did not exceed 55,000, and that the number surrendered was $4 \mathrm{I}, 000$, of whom 800 subsequently died (Seaman). It is estimated by Kuhn that at least 10,000 were lilled, so that less than 5,000 must have died of disease.

In thus endeavoring to account for a certain proportion of the missing, and by the inclusion of the Port Arthur statistics, the following corrected estimates are obtained:

Killed in battle, as officially reported,

Added from the missing,

Killed at Port Arthur,

23,008

I 4,600

I0,000

Total killed,

Died of disease, as officially reported,

47,608

Added from the missing.

18,830

Died of disease at Port Arthur,

4,000

5,000

Total dead from disease,

27,830

Although the Russians brought over a million men into Manchuria, it may safely be estimated that the number present in the 
zone of active operations did not exceed 650,000 ; on such basis we obtain a percentage of 7.32 for the killed and 4.28 for the dead from disease.

Were we to take into consideration the fact that the large contingents of Japanese and Russians, outside the zone of active operations, were also subjected to those field conditions which favor the spread of camp diseases, the rates for disease as above stated could be still further reduced.

The common diseases of war. - The two diseases which formerly caused most of the frightful mortality of military camps were: typhus and cholera. Typhus, the febris bellica of older writers, was the scourge of armies during the wars of the I8th century and of the Napoleonic era, as late as the Crimean War, where it made more victims than all other diseases combined. Under the influence of better hygienic habits it has practically disappeared from the United States where it is rarely found under the mild form of Brill's disease. It is common in Mexico under the name of tabardillo. Sporadic cases are still seen in many parts of Europe and Asia; thus during the Russo-Japanese War it was seldom absent from the Russian base hospitals. It is endemic in Galicia and the Balkan States, and became epidemic in Servia in the early part of the European War (see page 8I).

Cholera has not been an important factor in the mortality of late wars, having last prevailed in an epidemic form in the Sino-Japanese War of 1894, but it has so many endemic foci in various parts of Asia and Eastern Europe that it continues to be a serious menace, especially to our troops serving in the Philippine Islands. Serious outbreaks of it were reported from the armies operating in Galicia and parts of Poland in 1915 (see page $5 \mathrm{I}$ ).

Scurvy, the result of defective alinentation, was also common formerly in armies, especially when besieged and cut off from fresh supplies. Being an easily preventable disease it is now rarely seen.

In the Mexican WVar, the principal disease is reported to have been diarrhea of an aggravated type; probably this covered many cases of typhoid fever.

In the Crimean War, the most fatal diseases were typhus, cholera and typhoid fever.

In the Civil War, the prevalent diseases, in order of their admissions, were dysentery and diarrhea, malaria, respiratory diseases, 
rheumatism, venereal diseases and typhoid fever. The most fatal were typhoid fever, dysentery and diarrhea, which have continued to be the chief causes of death in military camps ever since.

During the year of the Franco-Prussian War, the German Army had over 73,000 cases of typhoid fever, equivalent to a rate of admission of 9.3I per cent. of all sickness.

During the Spanish-American War, the prevalent diseases, in order of their admissions, were malaria, dysentery and diarrhea, typhoid fever, respiratory diseases and venereal diseases. The most fatal were typhoid fever for the volunteer camps in the United States, malaria and dysentery for the Philippine expedition, and malaria and yellow fever for the Santiago expedition.

In the Sino-Japanese war, the mortality resulted chiefly from cholera, dysentery and beriberi.

In the Boer War, about one-half of the disease mortality was from typhoid fever, while dysentery and diarrhea made the next: greatest number of victims.

In the Russo-Japanese War, the Japanese suffered chiefly from beriberi, or kakki, a nutritional disease to which the Nippon race is particularly susceptible, more than one-third of the total sickness being due to it ( 84,545 cases according to Seaman), with mortality of 5 or 6 per cent. Dysentery and typhoid fever were the only other notable infectious diseases, but the number of cases of either, as officially reported, never exceeded I per cent. of all sickness. The case mortality, however, was very high in both diseases.

In the Russian Army, the infectious diseases which caused most of the mortality were typhoid fever and dysentery; then, as secondary factors may be mentioned, in order of their importance, typhus, variola, relapsing fever and scurvy. From the beginning of the war to July I4, I905, there were 10,449 cases of typhoid fever with I,O4I deaths; on September I, the number had increased to I7,033 cases with 2,077 deaths. The number of cases of dysentery was approximately one-half that of typhoid fever, with a mortality of 5 to 6 per cent.

In the great European War still raging (I9I7), we find a notable increase in the number and gravity of wounds (page 14), but no tendency to the spread of infectious diseases, thanks to the general use of the sanitary weapons with which the modern military surgeon is armed. In spite of the very insanitary condition of the trenches 
and dugouts in which much of their time is spent, the health of the troops of all the belligerents has been surprisingly good, rather improving than deteriorating with length of service. Outbreaks of typhoid fever, typhus and cholera occurred during the first year of the war, but were soon overcome. Since 1915, the armies of all belligerents have been practically free not only from epidemics, but from marked prevalence of any serious disease, and claim to enjoy better health than while in garrison, before the war.

This remarkable control of preventive medicine over those infectious diseases which formerly decimated troops in campaign, often a greater menace than the enemy, is, from the view-point of the humanitarian, the great wonder of our day and, for the military surgeon, an incentive and stimulus to continued exertions and the attainment of still more perfect results.

Also worthy of notice is the expedition of 10,000 American troops which, in March, 1916, crossed the Rio Grande and entered northern Mexico in pursuit of raiding insurgents. During several months of arduous service under the most trying climatic conditions, these troops maintained a remarkable record of health and efficiency, their non-effective rates comparing favorably, with the best of the home troops under garrison conditions.

\section{CHARACTER OF WOUNDS IN THE PRESENT EUROPEAN WAR (1917).}

The number and nature of the wounds received on battlefields do not directly pertain to the domain of hygiene, but a brief consideration of the subject may be useful, especially to show the decided influence upon them of environment, and the necessity of freeing this environment of at least its most harmful features.

Until the present world war, wounds by rifle (or machine gun) fire had always formed a large majority of casualties. Thus their percentages were 9I in our Civil War, 89 in the Franco-Prussian War, 84 in the Russo-Japanese War, while in the present war it is only about 55 . This decreasing rate shows that the part played by artillery (shrapnel, shell fragments, etc.) and grenades has been steadily growing in importance, causing from less than one-tenth of total wounds in the Civil War, to about one-half in the present world conflict. It is to be observed, however, that for the Crimean War, Chenu reports a percentage of 54 for rifle fire and 46 for 
artillery. Inasmuch as the Crimean War was also mostly waged from trenches, it would seem that this great increase of the deadly power of artillery is largely due to the present mode of warfare from field intrenchments.

This greater proportion of artillery casualties also accounts for the higher ratio of killed. Thus, before the present war, the ratio of I killed to 4 wounded was the generally accepted average. But, according to British reports, the ratio was I killed to 3.4 wounded in the first year of the war, and $I$ to 3 in I9I7, while in trench warfare it is estimated to be $I$ to 2 .

The remarkable effect of preparedness, training and experience on the part of the medical department is clearly shown in this war. Thus the Germans reported, in November, I9I4, that, of the wounded in hospitals, 2 .I per cent. died and 87 per cent. were returned to duty, and in April, I9I5, that 9I per cent. were returned to duty and only I.4 died.

In the French hospitals, the mortality for disease was 2.9 per cent. during the first year of the war, and I.4 during the succeeding 8 months; while for wounds, the mortality was 3.4 during the first year and 1.5 during the succeeding 8 months. In December, I9I5, 86 per cent. of the wounded were returned to duty.

A larger proportion of wounds than formerly, especially of those received in trenches, are severe and extensive, while nearly all are infected. A small proportion of rifle wounds heal by first intention, or nearly so, but most of them, as well as all artillery wounds, suffer from septic infection, often of a severe type. This surprising state of affairs is due to the two factors of insanitary environment and of the extensive lacerations and contusions of the wounds. When on duty in the trenches the soldier's clothing is begrimed with mud and filth, and the skin smeared with dirt, so that myriads of bacteria are carried into the wounds with the projectiles.

Of these bacteria the most dangerous are the bacillus of tetanus and the bacillus aërogenes capsulatus of Welch. "The pyogenic organisms come next in importance and, among them, streptococcus fecalis is the most frequently found. This is ascribed to its universal presence in the terrain which comprises the intensive farming district of the western front. Staphylococci, bacillus pvocyaneus and the colon bacillus are next in order of frequency" (La Garde).

The bacillus aërogenes capsulatus (b. perfringes of the English 
and Frencli) is generally considered the cause of gas gangrene which was such a frequent and terrible complication of lacerated wounds during the first months of the war. "The following signs are very suspicious of infection by the gas bacillus: The wound presents a dirty, sloughing appearance; a dirty serous exudate escapes from it, as well as bubbles of gas; crepitation under the skin is felt, and a fecal-like odor emanates from the discharge; the adjacent tissues are reddened as in cellulitis, edematous and tender" (La Garde). The abundant gas production causes rapidly spreading emphysema, and the mechanical pressure of the effusion and gas obstructs the circulation, with resulting gangrene.

The treatment now generally successful in all septic wounds consists in very free incisions reaching all pockets and sinuses likely to be infected, and irrigation with Dakin's preparation, or saline solutions, by means of rubber tribes with branches extending to all the remote parts of the wound (Carrel method).

\section{MORBIDITY AND MORTALITY IN TROPICAL COUNTRIES.}

Nortality has been in the past, and is still now, much higher in tropical than in temperate climates. This is due chiefly to the ignorance of hygienic laws and neglect of public and personal sanitation, whereby favorable conditions are created for the propagation of pathogenic organisms, such as those of malaria, tuberculosis, dysentery, cholera, plague, yellow fever, dengue and leprosy. It cannot be denied, however, that these organisms are more or less influenced in their rate of. growth and virulence by climatic conditions.

The cliseases above named, and others from which the natives of hot countries suffer, such as beriberi and various intestinal parasitic disorders, are all preventable, in the usual sense of the word, by the application of well-known sanitary measures. On the other hand, the several acute diseases which, in temperate and cold countries, produce a large proportion of the mortality, such as pneumonia, acute and chronic nephritis, cerebrospinal meningitis, cancer, bronchiis, diphtheria, etc., are comparatively rare in the tropics, where, furthermore, all the specific exanthemata (small-pox, measles, scarlet fever, etc.) are very mild.. It follows, therefore, that the mortality of tropical lands need not be necessarily higher than in other countries and that, as anywhere else, it is mostly a matter of care- 
ful and intelligent hygiene. Thus the rate of deaths in Havana during the decade preceding the Spanish War was 36 per I,000; under American administration and sanitation this rate fell speedily to $2 \mathrm{I}$. For the entire island of Cuba it was 17.35 in I90o, gradually falling to 13.96 in I9Ir. During the year 1902, our Army was about equally divided between the United States and the Pacific Islands; while at home the mortality from disease was only 5.66 per I,O0O, it was 20.85 in the Islands; but, in 1903, after our medical officers had learned how to contend against the diseases that beset them in their new surroundings, the mortality fell to II.I4 in the Philippines and has steadily continued to decrease. In the same year, the ratio of deaths in Cuba and Porto Rico was only 3.36 , notably lower than in the United States.

The mortality of Manilla, P. I., is still very high for the natives, among whom sanitary laws are difficult of enforcement, but for the American population it was only 9.05, and for the Spanish population 15.45 per thousand, in I904, a rate considerably below that of New York City.

For the three years I908-19I0, the death rate from disease among our troops in the United States was 3.10, 2.97 and 2.30 respectively, and, in the Philippines, 5.03, 4.09 and 3.08, showing a notable decrease from year to year, with closer approximation of rates, in both countries. For I9I5, the principal causes of admission, death and non-effectiveness in the Philippines, with rates for each, are indicated in Fig. 2.

For I915, the non-effective rate in the Philippines, for disease alone, was 21.42 as against 20.90 in the United States. On the other hand, the death rate for disease was only $\mathrm{r} .6 \mathrm{r}$ as against 2.53 in the United States.

Hawaii, as would be expected from its equable and salubrious climate, has lower rates for admission, non-effective, discharge and death than the United States. Thus, for IgI4 and 19I5, its death rates were 1.63 and 3.04 respectively. as compared with 4.82 and 5.42 in the United States.

In the Isthmian Canal Zone, the total population, including Panama, Colon and all employes, for the year ending June 30, 1907, was 87,2 I 5 with a death rate of 42.08 . For the white employes $(7,727)$ the rate was 15.93 , and for negro employes $(25,360$, mostly from Jamaica and the Barbadoes), 45.34. That the death 


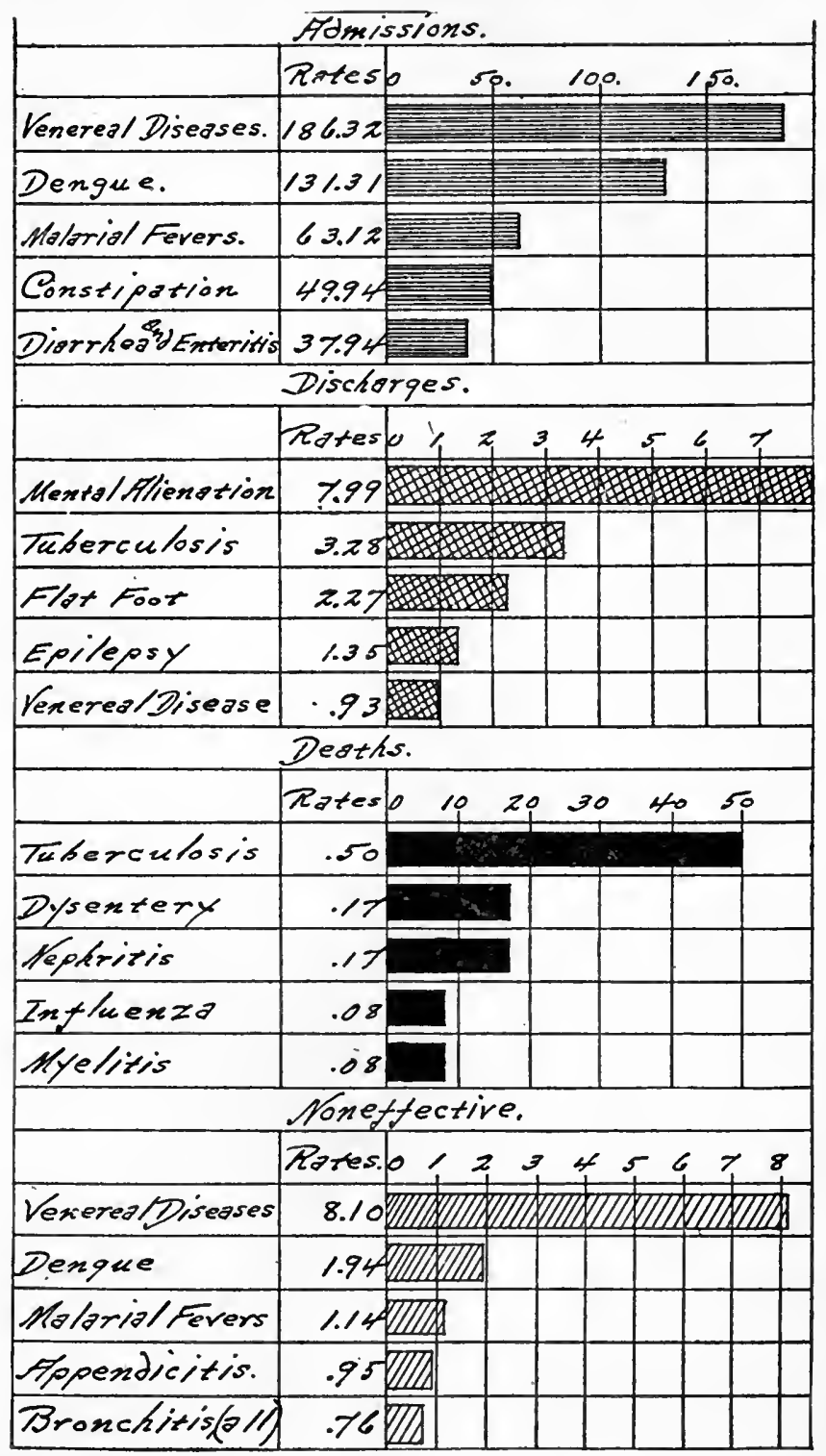

FIG. 2.-Chart showing (for American troops) the relative admission, discharge, death, and noneffective rates per thousand of mean strength for the diseases causing the highest rates in the Philippine Islands, for 1915. 
rate of negroes should be three times that of whites, in a tropical climate, is most remarkable. It would be interesting to know how much of this discrepancy is due to the higher power of adaptation and resistance of the white race; it seems more probable that it should be mostly attributed to the great difficulty of enforcing sanitary regulations among ignorant negroes. Among all the employes, white and black, the most fatal diseases, in the order of their mortality, were pneumonia (especially among the colored), malarial fever, typhoid fever, tuberculosis, acute and chronic nephritis, dysentery, septicemia, heart disease and meningitis.

In I9IO, the general mortality of all employes was 2I.I8 per I,000. For disease only, it was 4.92 for whites and 8.39 for negroes; the chief causes of death for whites being, in the order named: malaria, pneumonia, nephritis, tuberculosis, heart disease; and for negroes, tuberculosis, pnetumonia, malaria, nephritis and heart disease. In I9I $\mathrm{I}$, the death rate for the Zone, including the cities of Panama and Colon, was 2I, while that of the American whites (men, women and children) was only 4.48 .

Among the 6,000 American troops serving in the Zone in 1915, the non-effective and death rates, for disease alone, were 2r.66 and I.3 I respectively, as compared with 20.90 and 2.99 among troops in the United States.

\section{Influence of Race.}

From tables prepared by Chamberlain* for the ten years I905I9I4 for American troops serving in the United States, it appears that, for disease only, the colored soldiers had slightly smaller admission, discharge and non-effective rates than white soldiers, but a much larger death rate, in the ratio of 5.1 to 2.7 per 1,000 .

For the same period of ten years among American troops in the Philippines, the tables show that the colored soldiers had smaller non-effective and discharge rates but higher admission and death rates, the latter being 5.5 and 3.2 respectively per thousand.

For I9I 5 , in the Philippines, the rates of colored troops for admission, non-effectiveness and death exceed those of white troops, the death rates being 5.22 and 1.04 respectively.

In 1914 and 1915 the white troops, in the entire Army, had the higher admission rate, while the colored troops had the higher death, discharge and non-effective rates.

* The Military Surgeon, December, 1916. 
Comparing the four races making up our military strength, we find that in 1915 their rates are as follows:

Constantly non-effective: Colored, 28.46: white, 25.70; Porto Ricans, 22.78; and Philippine Scouts, 15.68 per $1,000$.

Discharge: Colored, I 5.85 : white, I4.I6; Philippine Scouts, I 1.44 : and Porto Ricans, 7.34 per 1,000 .

Death: Colored, 5.5I ; white, 4.54; Porto Ricans, 4.4I ; and Philippine Scouts, 2.54 per I,000.

The average number of days lost for each case treated was: Porto Ricans, I4.98; colored, I4.I2 ; white, I2.59; and Philippine Scouts, 9.97.

From the above statistics and those relative to the Isthmus of Panama, we may conclude that the negro shows about the same degree of resistance that the white does to the exposure and hardships incident to military service, as shown by admission and noneffective rates, but that, as in civil life, when attacked by a serious illness, he is more easily overcome and liable to die, especially from tuberculosis and pneumonia.

The greater vitality of the white, as shown by his lower mortality. is apparent not only in temperate but also in tropical zones, and this notwithstanding the fact that white troops, in the last decade, had three times as many admissions for alcoholism as colored troops, and fifty times as many as Filipinos. It is generally conceded that the colored race was developed with special adaptation to warm climates, but.such adaptation, as compared with the white race, only becomes evident after a long period of years. Were troops of both races to serve together ten or fifteen years within the tropics, there is hardly any doubt that the negro would in the end manifest a higher degree of resistance and vitality than the white, provided they performed similar duties and were protected by about the same hygienic conditions.

Concerning Filipino troops, we find that their rates of admission, non-effectiveness and death, from disease, are much lower than for American troops serving in the Philippines or in the United States. The difference became particularly marked in I9IO, after the eradication of beriberi, the disease previously most prevalent among them.

In Porto Rico, the admission and non-effective rates for native troops, in I9II, were lower than for white troops in the United 
States, but the death rate was higher. In I9I5 the non-effective, discharge and death rates were smaller than for white troops at home.

\section{Influence of Age and Length of Service.}

The experience of the U. S. Army and of all other armies is that the admission rate to sick report is highest for young soldiers under 20 years of age. This is especially true of typhoid fever. Then the admission rate, as well as the discharge and non-effective rates, fall rapidly up to the age of about 45 . The death rate is highest in young soldiers, I9 years and under, but declines after 20 and reaches it minimum between 25 and 30 years, rising again slowly up to 40 years and rapidly thereafter.

From the point of view of length of service all the rates are highest in the first year, and gradually diminish thereafter, to rise again aften ten years' service. The death rate is lowest in the third or fourth year.

The influence of age is about the same in the tropics as in the United States, except that young soldiers are still more liable to swell the admission rates. The lowest rates of admission, discharge and non-effectiveness are among soldiers past 40 , although it is this class which furnishes the highest death rate. In the Philippines, the admission and non-effective rates are highest in the first and second years of service, and then gradually diminish until ten years or more or service. The death rate is high during the first year, then falls and reaches its minimum the third or fourth year, thereafter rising and reaching its maximum after ten years of service or 45 years of age. The steady fall of the non-effective rate after the second year of service, concurrently with the steady rise of the death rate after the third or fourth year, which may seem puzzling, means that while the great majority of the men become hardier and more or less acclimatized, a small proportion (probably less than 3 per cent.) gradually lose their power of resistance and succumb year after year in increasing number.

\section{Influence of Climate and Station.}

Of the various parts of the United States and its colonies, Alaska is the most salubrious. Thus for the three years 1908-1910, its mean rate for the constantly non-effective from disease was only 


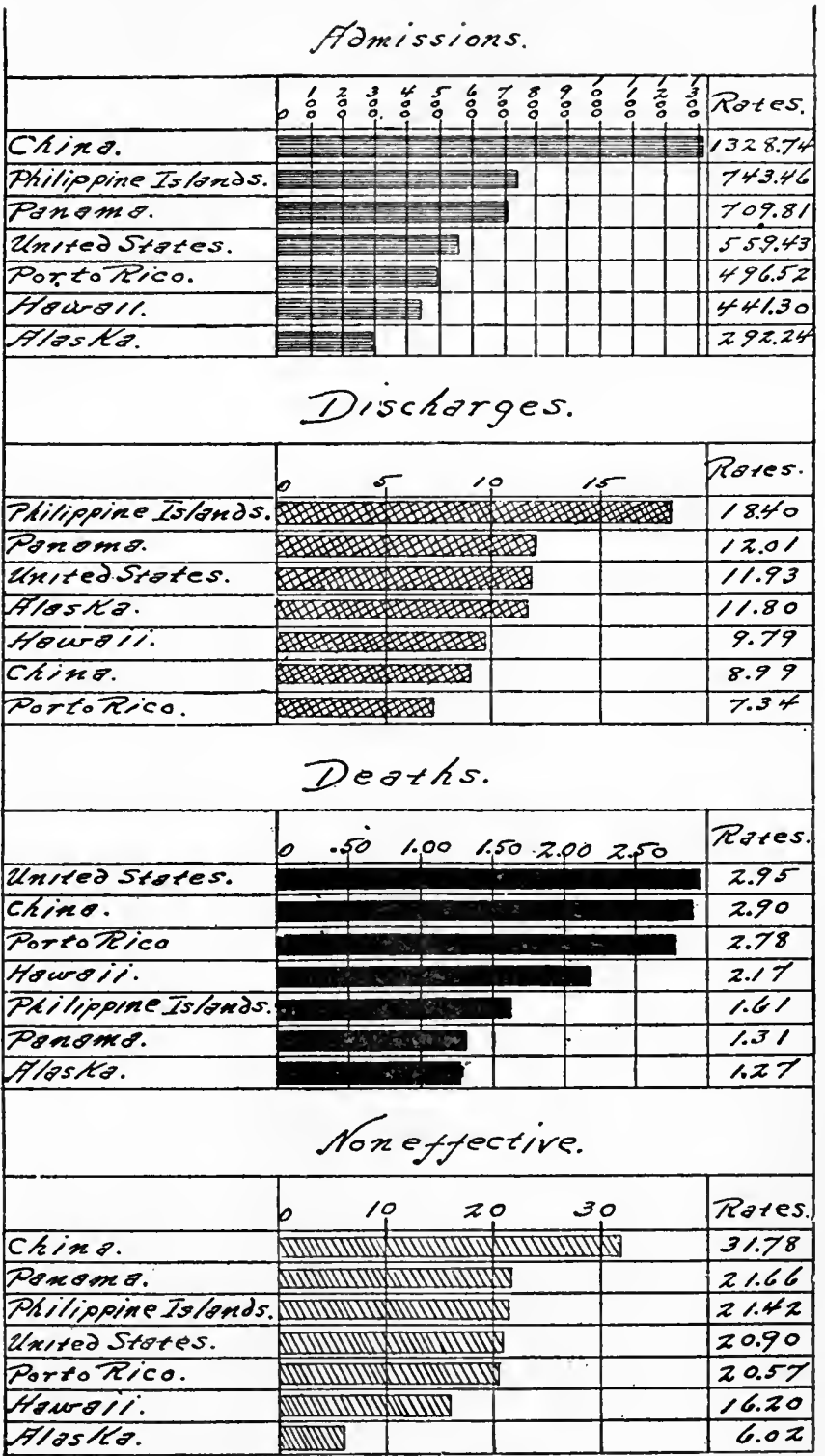

FIG. 3.- Chart showing admission, discharge, death and non-effective rates for disease, by countries, for American and native troops. Year 1915. 
10.7 per thousand strength, while it was 32.4 in the United States, 39.4 in Hawaii and 45 in the Philippines.

Fig. 3, from the Surgeon General's Report for I9I5, shows the effect of climate and country upon American and native troops; from it it appears that Alaska, Hawaii and Porto Rico are the healthiest of our colonies, and that all three have better rates than the United States.

Within the United States there is a notable difference between the health records of the nine military departments into which its territory was formerly divided. For the above three years, the Departments of the Columbia and Texas have the best records, closely followed by the Department of California, then more distantly by the Departments of Missouri, the Colorado, the East, the Gulf, Dakota and the Lakes, in the order named. Thus the admission rate per thousand strength, in I9IO, was 588 for the Department of Columbia and 979 for that of the Lakes. Such disparity is the more remarkable that there has been no epidemic or serious endemic outbreak during the period under consideration, at any of our posts, and that malarial fever, formerly so prevalent in many States, has ceased to be an important factor except at a very few posts, none of which are in the two Departments reporting the highest morbidity. Whatever may be the local influences which, added to the peculiarities of climate, bring about this result can only be a matter of conjecture. 


\section{DISEASES OF THE SOLDIER.}

Soldiers may have any of the diseases that male civilians of the same age suffer from; they possess no special susceptibility to, nor immunity against them, but the conditions in which they are placed often favor the occurrence and rapid spread of certain classes of affections.

Those which mostly impair the efficiency of the soldier are chiefly the large and all-important group of infectious diseases; then, secondarily, those caused by parasites, exposure to inclement weather and extremes of temperature, immoral or intemperate habits, and improper diet.

\section{INFECTIOUS DISEASES.}

The lowest class (Tallophyta) of the great world of cryptogamic plants is made up of algæ, lichens and fungi. The fungi, including the molds, yeasts and bacteria, are of most interest medically. Molds and yeasts are of minor importance as compared with bacteria; they cause relatively few diseases and these principally of the skin. Molds are frequent on meats and other foodstuffs, while cheeses depend upon them for their flavors.

Bacteria are very minute unicellular organisms, which may occur free and separate or else in groups or colonies. They are divided into three main classes: the cocci or spheres, the bacilli or straight rods and the spirilla or curved forms. The bacilli are by far the most numerous; they are elongated cells from two to ten times longer than wide, with ends squarely cut off as in B. anthracis, or gently rounded as in $B$. typhosus. Many bacilli and spirilla are actively motile through the wavy or screw-like motion of long tenuous filaments or flagella.

Bacteria multiply by cell division or fission. The cells may divide so as to form chains (streptococci), packets or cubes (sarcince) or irregularly in various directions (micrococci). The virulence of streptococci is exceedingly variable; in long chains they are commonly the cause of diffuse phlegmonous inflammation; in short chains they are often the normal and harmless inhabitants of the mouth, nose and intestines. Micrococci dividing so as to form masses like clusters of grapes are called staphylococci, of which the 
most important is S. pyogenes aureus, the ordinary cause of boils and abscesses. Micrococci, after division, may remain coherent in twos, or diplo-forms, as in the Pneumococcus, Diplococcus meningitidis and Gonococcus.

The spirilla include Spirillum proper, with rigid cells and polar tufts of flagella (S. of cholera); Spirochota, with sinuous, flexible cells, an undulating membrane and no flagella (S. of relapsing fever); and Treponema which differs from spirochæta in having a flagellum at each end and no undulating membrane ( $T$. of syphilis and yaws).

Many bacteria possess the power of sporulation, that is, to develop into an encysted stage (spore) for the purpose of resisting unfavorable environment. Of this class, the best known are the bacilli of anthrax and of tetanus.

All bacteria need oxygen for their growth and multiplication. Those requiring it in the free state are called aërobes; those for which free oxygen is injurious, and which obtain it by the splitting of carbohydrates and from easily deoxidized bodies, are called anaërobes. Between these two classes are many which thrive well under either condition: these "facultative" bacteria include most of the parasitic and saprophytic kinds.

According to their action upon the higher animals, bacteria are divided into parasites or those capable of living and multiplying within the human or other animal bodies, and saprophytes or those which are unable to maintain themselves in tissues of the higher animals but swarm everywhere in air, soil and water, being concerned in many biological processes beneficial to. animals and plants. The separation between these two classes is not sharply drawn; many bacteria are capable of developing under both conditions, although seldom equally well; some of the pathogenic varieties belong to these so-called facultative parasites.

Diseases caused by the introduction of virulent microorganisms into the tissues of the body are called infectious. Among them are the most common and dreaded disorders from which troops suffer in peace and war, and nearly all the mortality results. The epithet pathogenic (disease producing) is commonly applied to all organisms which thus transmit infections. Pathogenic microorganisms are of two kinds, the microbes or bacteria above described, and Protozoa, the lowest unicellular forms of animal life. Most of 
them are visible under the microscope; others are ultramicroscopic and therefore invisible, their existence, as in yellow fever, smallpox, etc., being postulated from analogy with other diseases in which the causative organisms are well known. Both kinds, bacteria and protozoa, may produce infectious diseases, that is, diseases communicable from man to man. The mode of conveyance is not always clear. The former faith in fomites infection (the transmission of long-persistent germs through clothing and bedding) or in aerial infection (the transmission of more or less desiccated germs considerable distances through the air), is now mostly discredited and replaced by belief in actual contact, direct or indirect, with comparatively fresh organisms. Flïgge and his pupils have laid great stress upon the spray thrown out from the mouth in coughing, sneezing and lour speaking as a possible factor in the spread of tuberculosis and other diseases whose germs inhabit the naso-pharyngeal cavities. From the result of careful experiments, Winslow and Robinson* were unable to confirm Flügge's teaching, and sum up their conclusions as follows: "It appears from these investigations that spray is discharged from the mouth during loud speaking in such amount as to cause a considerable pollution of exposed surfaces upon which it falls. True aerial pollution by particles suspended in the air and liable to be inhaled with it, is on the other hand so slight tinat it can hardly play a relatively important part in the causation of disease."

This contact of infectious organisms is obvious and admitted in typhoid fever and other fecal-borne diseases such as cholera, dysentery and diarrhea. It is still more evident and direct in those diseases in which the infective agent is contained in the secretions of the mouth and nose, as in diphtheria, pneumonia, whooping-cough, tuberculosis, influenza and cerebrospinal meningitis. According to Chapin, there is every reason to believe that even small-pox, scarlet fever and measles are not contracted otherwise than by contact.

Germs may be conveyed in ways that are obscure and difficult of detection, for instance by the patient himself when, being only mildly sick and unaware of the nature of his illness, he continues to attend to his usual avocations. Another common factor of conveyance is by carriers. There are, of course, men who through their soiled hands and clothing and uncleanly habits carry germs

\footnotetext{
* Am. J. Pub. Hyg., 1910.
} 
from the sick to the well or infect the food and drink of healthy people; but the term carrier is here specifically applied to the men and women (common carriers) who after recovering from an infectious disease continue to discharge pathogenic germs for some weeks or months afterwards, or to men and women (contact carriers) who become infected by contact with patients and carry germs in their secretions or excretions, but remain in normal health and show no clinical symptoms of the disease so that they may be active propagators of it for indefinite periods before they are suspected.

From what has been said it follows that the two terms, contagious and infectious, have lost their former distinctive significance and are practically synonymous.

The production of infection, in man, is a complex process, requiring other factors than the mere contact of pathogenic organisms. These must have the required degree of virulence, gain entrance to the tissues of the body in sufficient number and possess the ability to live and multiply in them. It is well known, that most pathogenic bacteria may be found in the body in a quiescent or latent state and without ill effects; thus staphylococcus, streptococcus, pneumococcus and various spirilla are always, and the germs of diphtheria and cerebrospinal meningitis often, present in the mouth, nose and throat; the colon organisms are invariably found in the normal bowel, while the tubercle bacillus is a frequent inhabitant of the lymphatic glands of healthy subjects. The influences which may suddenly light these parasites into toxic activity are still conjectural. In this connection the doctrine of autogenesis suggested by Pasteur and developed by Kelsh and other French epidemiologists, possesses plausibility. It claims that the virulence of germs is a variable quantity, absent or dormant for indefinite periods of time, but capable of manifesting itself under the influence of cosmic or other extrinsic unknown factors. It does not deny the possibility and frequency of contagion, but limits its range of action. Thus would be explained, for instance, the presence of typhoid cases in isolated places, away from sources of infection, or the apparently spontaneous breaking out of diseases such as cerebrospinal meningitis, influenza, cholera, in endemic or epidemic forms, sometimes simultaneously in various disconnected foci.

The effect of weakening or depressing influences on the development of infectious diseases is well known; it explains the paroxism 
of intermittent fever which follows a cold bath, or the attack of dysentery which results from exposure to wet and cold. Calmette, Granjux anc other sanitary authorities in the French Army assert positively that maneuvers or campaigning involving great fatigue, whether at home or in African deserts, are always followed by an outbreak of typhoid fever, independently of any infective cause other than the latent germs carried in the system.

Against the invasion of microorganisms the body is guarded externally by the skin and mucous membranes. They may pass readily into the system through any cut, break or ulceration of the cuticle (as in plague and tetanus), while others are directly injected by stinging insects (as in yellow fever and malaria). Many are absorbed through the naso-pharynx or perhaps the respiratory tract (as in influenza, diphtheria, tuberculosis, pneumonia, etc.), and through the alimentary canal (as in typhoid fever, cholera and dysentery).

Bacteria may do harm to the tissues by mechanical interference, but their chief injurious action is through the poisons or torins which they produce. These poisons may be true toxins, that is real secretions of the bacterial cells, soluble and readily diffused in the blood (as in diphtheria, tetanus and botulism), or they may remain within the bacterial body, separating from it only after its death and disintegration; these are the endotorins and are produced by the greater number of pathogenic bacteria (in typhoid fever, cholera, plague, etc.).

The presence of toxins in the blood determines the prompt formation of antidotes or antito.rins, a reaction taking place between the two, analogous to the chemical neutralization of an acid by a base. The system is also protected by various agents or antibodies making up its defensive or resistance power; they normally exist in the tissues and are increased through the stimulation of the invading organisms and their products, reaching their full development when immunity is acquired. Some of them (ly'sins) disintegrate and dissolve the bacteria; others (agglutinins) cause the latter to lose motility and clump' together; others again (opsonins) facilitate their destruction by the leucocytes.

Another and very important protective agency is phagocytosis. the power which certain cells possess of ingesting and destroying bacteria. These phagocytic cells are blood leucocytes and certain 
large endothelial cells lining serous cavities and blood vessels. The leucocytes most concerned are the polymorphonuclear cells (microphages) and the large mononuclear cells (macrophages). The degree of resistance to any infection seems to be proportionate to the activity of these devouring cells, and Metchnikoff believes that phagocytosis is the cardinal factor which determines immunity; other investigators, however, contend that the struggle takes place in the fluids of the body and that phagocytosis is merely a means of removing dead or inactive bacteria. It has been established that phagocytosis is very much increased by certain special substances contained in immune serum. These substances, the opsonins of Wright, act directly upon the bacteria, facilitating their ingestion and destruction by the leucocytes. They appear to be identical with the opsonins existing in normal blood. The effect of phagocytosis upon protozoa, although not yet thoroughly understood, is probably also well marked.

IMMUNITY. - Immunity against infectious asseases may be natural or acquired; natural when it is the normal attribute of a race, species or individual; acquired when obtained either accidentally or artificially. A first attack of most infectious diseases so strengthens the resistance of the individual that he is no longer susceptible to the same infections. This is natural acquired immunity. The protection conferred by the inoculation of gradually increasing doses of toxin, or of dead bacteria with their liberated endotoxins, inasmuch as it depends upon the reactions of the tissues of the subject inoculated, is spoken of as active immunity. After animals have been actively immunized against toxins, their sera, rich in antitoxins, are capable of protecting man against the corresponding infections. The resistance thus acquired, as for instance by the inoculation of diphtheria antitoxin, is a purely passive process and is known as passive immunity.

The study of immunity against diseases caused by pathogenic protozoa has as yet produced no practical result. From present indications it is probable that it will be found easier to abolish the etiological factors, especially in the case of infections known to be transmitted through the sting or bite of insects. A certain degree of individual immunity is naturally acquired against malaria by a long residence in malarial regions, but such immunity is probably never inherited. Inasmuch as the natives of countries in which 
yellow fever (an assumed protozoal infection) is endemic rarely suffer from this disease, it was formerly supposed that they enjoyed a natural immunity against it, but Guiteras has shown that it was an acquired immunity conferred by a mild attack in infancy. Marchaux and Simond claim to have produced relative immunity against yellow fever by injections of the serum of convalescents.

\section{TYPHOID FEVER.}

Writing in 1905 , Kean could truthfully say that "Typhoid fever is to-day, on account of its wide dissemination, the persistent vitality of the infecting organism, the duration and severity of its attack and its large death rate the most formidable infectious disease with which we have to contend in military life." Since then antityphoid vaccination has been extensively practised and found to be a wonderful prophylactic weapon in the hands of medical officers. Nevertheless, for the reasons stated, as well as on account of its ready transmission from man to man by direct or indirect contact, and the marked susceptibility of the American people to its infection, typhoid fever will doubtless continue to be one of the most dangerous diseases of military life.

In garrisons, typhoid fever is easily controlled, but in large camps, where troops assemble for instruction or mobilization, or in the field during active service, it is a very different matter because of the difficulty of disposing of the excretions from the human body. During the Civil War the rate of admissions was 62 per 1,000 of strength, a rate which has since been exceeded in several wars. Typhoid fever being endemic throughout the United States, it is hardly possible for any volunteer regiment, often made up of units from different localities, to come to such camps without bringing one or more cases in the period of incubation, or already developed. Such primary cases are inevitable; every new batch of recruits may contain some; they should be expected and watched for. But secondary cases, that is, cases infected by the primary ones, are prerentable and their occurrence will justify the assumption of imperfect or careless prophylaxis.

Typhoid fever, as seen in camps, assumes many types, some of which vary widely from the classic form and have only been identified by the improved diagnostic methods of recent years. The 
elaborate report of the Reed Board* appointed to investigate the typhoid fever epidemic of the military camps in the United States, in 1898 , threw much instructive light upon the subject. The members of this board by studying the medical histories, company by company, in to6 regiments, found that, besides the cases clearly typhoid, there were many others of a few days duration only, diagnosed malarial, which were no longer susceptible to the infection of typhoid. They also found that previous attacks of diarrhea and gastro-enteritis were likewise in a large measure protective. Hence their conclusion that, during the prevalence of an epidenic of typhoid fever, many of the cases heretofore diagnosed malarial fever or diarrhea are really mild and atypical forms of typhoid, insuring protection against that disease and quite as capable of conveying its infection as the gravest forms. According to this expert Board, hardly one-half of the cases of typhoid were diagnosed as such, and the rate of admissions which was reported as 88.55 per thousand strength should have been 192.65. Long before this it had been known that the so-called typho-malarial fever of the Civil War was simply typhoid with or without malarial complication. The observations of the Board "appear to indicate that when an individual, the subject of malaria, is subsequently infected by the typhoid bacillus, the manifestations of the malarial parasite remain, as a rule, in abeyance during the active stage of the typhoid infection, to appear, in a certain proportion of cases, during the stage of debility attendant upon the convalescence." The term typho-malarial, denoting a hybrid form of disease, is therefore misleading and should be dropped from the nomenclature.

Typhoid Bacillus.- The specific organism, cause of typhoid fever, is the Bacillus typhosus, a short, flagellated, sporeless bacterium closely resembling the widespread Bacillus coli communis and often hard to distinguish from it. It flourishes best at the temperature of the body and ceases to grow below $48^{\circ}$ or above $108^{\circ} \mathrm{F}$. It is quickly killed in boiling water, or when exposed for 15 minutes to a temperature of $140^{\circ}$. The most intense cold yet produced does not destroy it, and it may remain frozen in ice for months without appreciable loss in virulence. It may retain its vitality for a while

* Consisting of Major Walter Reed, Surgeon, U. S. Army; Major Victor C. Vaughan, Division Surgeon, U. S. V.; and Major Edward O. Shakespeare, Brigade Surgeon, U. S. V. 


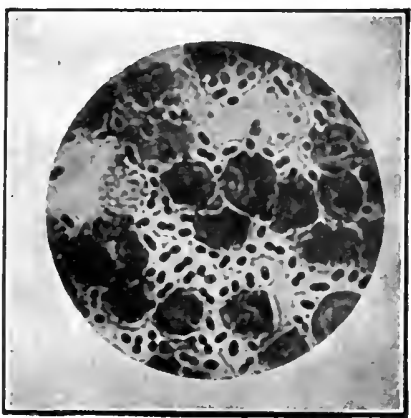

1

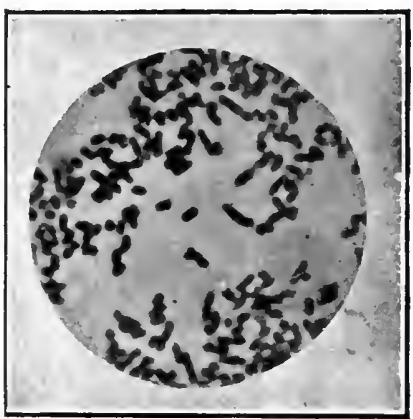

3

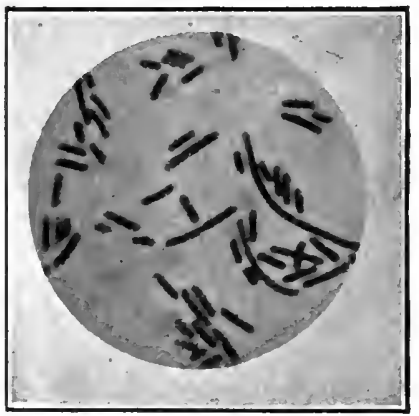

5

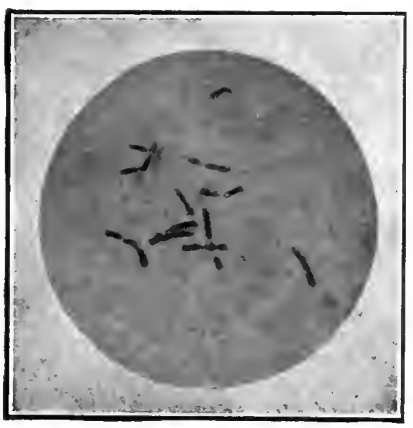

2

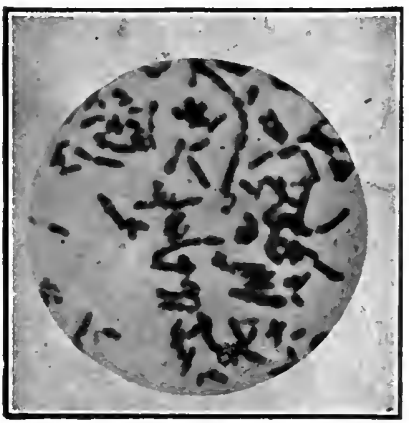

4

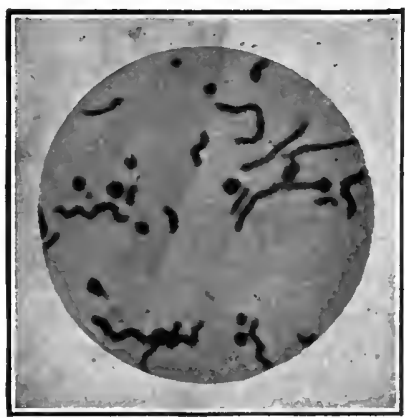

6

I. Diplococcus lanceolatus (pneumonia) in blood of rabbit.

2. Bacillus of tuberculosis in sputum.

3. Bacillus of influenza in pure culture.

4. Pacillus of diphtheria in pure culture.

5. Bacillus of tiphoid fever in pure culture.

6. Bacillus of Asiatic cholera in pure culture. 

in very dry material but complete desiccation soon kills it. It may live and breed for a long time in earth contaminated with organic matter, but soon dies out in clean soil well exposed to the sun. In distilled water it may live for two or three months, but in ordinary water its life is generally shortened to one or two weeks and often to three days (E. O. Jordan) by competition with saprophytic bacteria. In sewage, it tends rather to decrease than to multiply, and seldom survives 48 hours in fecal matter. Experiments with pure cultures seem to show that, in the soil, it may survive one or two months and thus might, during this period, infect the streams draining the contaminated area. But Morgan and Harvey have demonstrated that the results are very different under natural conditions, in which this bacillus is associated with and vastly outnumbered by antagonistic organisms; then its life duration is very short, seldom exceeding two or three days. In clothing and tentage, this organism may survive several weeks and be carried long distances, thus producing fresh outbreaks in unexpected places.

The average incubation period is $8-12$ days, but the interval between exposure and the outbreak of the fever may be much greater. One may carry the bacilli under his nails, in his hair or on his clothing for some days, possibly for weeks, before they find their way into the alimentary canal.

The typhoid bacillus is passed in vast numbers with the feces of the patients from the beginning of the disease, sometimes from the stage of incubation, and often continues to be discharged long after convalescence has set in. It is also passed in the urine in about one-fourth of all cases, often in enormous numbers, as if in pure culture, so that a drop may contain millions. "Because of the frequency with which it is voided, its comparative inoffensiveness, its easy dissemination and the relatively great number of organisms present, infected urine is the most dangerous excretion of the typhoid patient" (Vaughan). Other secretions, such as sweat, saliva and milk, may also contain the organism.

A large proportion of patients continue to excrete bacilli in their feces after the disappearance of all febrile symptoms. This proportion falls to about 4 per cent. ten weeks from the beginning of the fever (Lentz), and to 2-3 per cent. a year afterward. These are the common typhoid carriers; most of them discharge virulent bacilli for years and some throughout their lives. Another class of 
carriers, designated contact carriers, consist of healthy individuals who give no history of ever having had typhoid fever; they amount to about 3 per I,Ooo of the adult population. It is generally assumed, however, that these contact carriers have at some time suffered from a mild attack, overlooked or forgotten. In all carriers the germs are generated chiefly in the gall-bladder and often become the cause of cholecystitis and gall-stones. In the relatively rare urinary carriers, the germs are generated in the bladder, pelvis or kidney, producing a chronic inflammation of these organs. It should be noted that carriers discharge germs more or less intermittently, the excreta, at times, being free from them for several days; therefore suspected persons should be subjected to at least two examinations, a few days apart, before being declared free from infection.

Safety then requires that, in camp, all excreta should be considered infected. The soldier may be readily convinced of the danger of fecal matter, but it will require special efforts on the part of his officers to make him realize that urine is equally dangerous and must be disposed of with the same care.

Dissemination.- Typhoid fever, as a general rule, is spread only by the introduction of the specific organism into the alimentary canal with food or drink; in other words, by the ingesting of infected food or the drinking of polluted liquid. Fecal matter and urine being the infectious media, it is important to know how they are transmitted and disseminated. Man himself is the most active agent in this dissemination, for he may carry them in his alimentary canal and bladder, on his person or clothing, hundreds and thousands of miles. As stated before, at least one man in every regiment of volunteers can be assumed to be infected before he reaches the camp of mobilization, and, as he usually discharges virulent germs during the stage of incubation and before showing any visible clinical signs of infection, he may plant the typhoid bacillus in every latrine in his regiment before being suspected and removed. In each regiment there will be also one or more chronic carriers, unconsciously discharging highly infective stools. Finally, it is not uncommon to find men with such a mild, atypical form of the fever (ambulant cases) that they remain on duty, a constant menace to their unsuspecting comrades. It follows that, in a military camp, all excreta must be considered infected. This infection is not a 
mere possible contingency, but a very probable if not certain condition.

The most common and widespread mode of transmission in camp is that which takes place by contact. Dried fecal matter and urine may be carried on the hands, shoes, clothing and skin of patients in the incubative stage, of chronic carriers and anbulant cases, and, in the close intercourse of tent life, propagated from man to man and from man to food, before the responsible individuals are detected and isolated. Should typhoid patients be taken care of in a regimental infirmary instead of being promptly removed to a field hospital, there would be the additional danger of propagation by nurses and visiting comrades.

It is mostly by contact that the disease is spread through a family after its introduction, and it is doubtless one of the chief modes of its propagation in the field. This was well demonstrated in some of our camps during the Spanish-American War, in I898, where the fever was characterized by a series of company epidemics, with a traceable connection in each company between two-thirds of the cases; thus certain tents were badly infected and the majority of their inmates developed the disease, while adjoining tents wholly escaped. The influence of contact was also clearly shown in a camp of Boer prisoners in the hills of Ceylon, in I9OI, where over 5,000 men were collected. The site was high and the water supply good, "The camp was free of disease until typhoid fever was introduced by some newly arrived prisoners. Within three months there were over 700 cases among the prisoners. The troops guarding them, who were under practically identical conditions as regards food and water, escaped entirely" (Thomas McCrae). It is very probable that the typhoid fever epidemic among the British troops at Bloemfontein (1900), attributed to an infected water supply, was also mostly due to contact infection.

A careful investigation of the causes of the high rates of typhoid fever in Washington, D. C., for the period I906-I9II, led to the conclusions that "contacts and carriers" were the predominant factors in the spread of the infection.

Contact infection may be more or less indirect, as, for instance, when effected through tentage, bedding or clothing. Probably in this way was typhoid fever carried from Fort Snelling, Minn., to Camp Columbia, Cuba, in 1906, and thence to several posts on the 
Island. Therefore it is important that canvas and all other fabrics which have been exposed should be thoroughly fumigated before being packed and shipped away; when unpacked it will be a wise precaution to spread them out in the sun. Another illustration of indirect contact is that furnished by company pets, mostly dogs and cats, with retentive and absorbing furs, caressed alike by hands that infect and hands that become infected.

In civil life, the great epidemics of typhoid fever are generally caused by the specific contamination of drinking water. In garrison or permanent camp the water supply is mostly obtained from deep wells, piped from wholesome sources, or else purified so as to be above suspicion. In the military camps of 1898 , infected water was not an important factor in the spread of typhoid fever. It is very important that the men should be required to use only water and other beverages from authorized sources; there is real danger, for instance, from the unwholesome preparations of the numerous vendors of soft drinks always found in the vicinity of camps and garrisons.

Milk is a common vehicle of bacteria and responsible for a considerable proportion of typhoid fever cases. It is infected indirectly through the water used to dilute it or wash the dairy utensils, or else directly through the unclean hands of the milkers and bottlers. Milk products retain most of the bacilli present in the milk and may also be more or less infected. Ice-cream is the most dangerous.

Of articles of food known to be occasional vehicles of the typhoid bacillus should be mentioned oysters and clams in the raw state (sce Mollusks), as well as fruits and vegetables uncooked, especially when much handled and long exposed to fly pollution. Wherever the soil is fertilized with sewage, there is danger of the plants growing in it becoming infected; if not cooked (lettuce, celery, etc.), they should be carefully washed before consumption.

Bathing in infected water, fresh or salt, is not without danger and should be avoided, for the organisms may enter the system through the mouth, nose and eyes.

Flies.-Flies are known to be one of the chief factors in the dissemination of typhoid fever. In several of our encampments, in 1898 , they were the most active agents in the spread of this disease. The reasons given by the Reed Board for coming to this conclusion were as follows: 
I. The latrines contained infected fecal matter.

2. Flies alternately visited and fed upon this infected fecal matter and the food in the mess-tents.

3. Typhoid fever was much less frequent among members of messes who had their mess-tents screened than among those who took no such precaution.

4. The fever gradually died out, in the fall of 1898 , with the disappearance of flies, and this at a time when in civil life it is generally on the increase.

Fecal matter adheres readily to the feet and proboscis of flies and may be carried, not only from latrines but also from soiled clothing, bedding or bed pans, to kitchens and mess-tents where it 1s deposited on food or in drink; or else it may be carried to the men's tents where it is deposited upon their persons or clothing. It is also known that the excrements of flies that have fed on infected feces contain the typhoid bacillus. (See Flies, page 152.)

It has been reported that in some of the typhoid outbreaks in English garrisons, the cavalry suffered more than the infantry, on account, it is presumed, of the greater number of flies in the cavalry quarters.

Grave as is the danger of fly transmission in camp and under rural conditions, it does not seem to play an important part in the production of typhoid fever in cities. According to C. $T$. Chapin "there is no evidence that in the average city the house-fly is a factor of great moment in the dissemination of disease. In a reasonably clean and well-sewered city, the cases of typhoid due to fly transmission are relatively very few compared with the numbers due to water, milk and contact."

As already stated, the soil becomes readily infected with the typhoid germ when fouled with feces or urine and may therefore be a means of transmitting the disease. This takes place when it dries up and, with the dust, is blown in drinking water, on food, clothing or any object handled by the men. Fortunately, it is likely that the specific organism, when thus mixed with dust, soon becomes desiccated and devitalized.

As before mentioned (page 26), French military hygienists lay some stress on the alleged effect of prolonged physical strain in bringing about outbreaks of typhoid fever. Thus they contend that a large increase of cases is always noticed right after the fall manewvers in the French and German armies, as well as during 
arduous campaigns. For believers in autogenesis this is a natural corollary of the doctrine. Others may argue that inasmuch as the maneuvers usually take place during the season when typhoid fever is most prevalent, an increase of cases in the operating troops might be reasonably expected.

Statistics.-Typhoid fever in the U. S. Army has steadily declined since the Spanish-American War. (Fig. 4.) In I899, the rates per $I, 000$ of strength, for the United States proper, were Io. 85 of admissions and I.O7 of deaths. During the years I904I906, they were 4.95 and 0.28 respectively. In I9I0, they had fallen

\begin{tabular}{|c|c|c|c|c|c|c|c|c|}
\hline 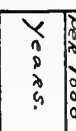 & 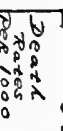 & & 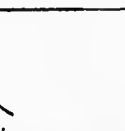 & 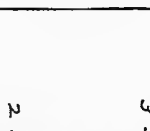 & $\omega$ & $x$ & 9 & 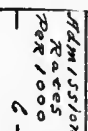 \\
\hline 1903. & .28 & E & $2=0$ & 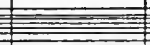 & $=5$ & $=6$ & 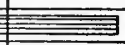 & 5.8 \\
\hline 1904 . & .27 & E & 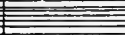 & 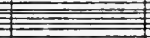 & 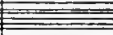 & 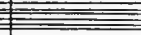 & 事学 & $\frac{1.6}{6}$ \\
\hline 1905. & .30 & 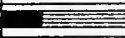 & 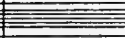 & 量高 & 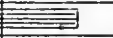 & & & \\
\hline 1906 . & .28 & & & 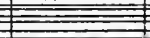 & & 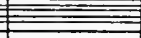 & 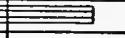 & 568 \\
\hline 1907. & .19 & 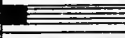 & =a & 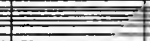 & 焉 & & & 8.5 \\
\hline 1908. & .23 & 5 & an & 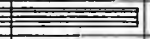 & & & & 2.94 \\
\hline 1909. & .28 & E= & & 戰 & & & & 3.03 \\
\hline 1910. & 16 & 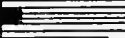 & 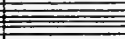 & 帚要 & & & & 2.3 \\
\hline 1911 & .11 & 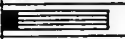 & & & & & & .8 \\
\hline $19 / 2$ & .03 & $\underline{\underline{z}}$ & & & & & & .5 \\
\hline
\end{tabular}

FIG. 4.- Chart showing admission and death rates for typhoid and paratyphoid fevers in U. S. Army. (From A. G. Love, U. S. Army.)

to 2.32 and 0.16 ; and in I9I 1 , to 0.76 and o.Io. In I9I2, the rate of mortality was only 0.03 , almost a negligible quantity. During the four years I909-I9I2 no death occurred among vaccinated soldiers in the United States. This remarkable decline in the curves of morbidity and mortality is the normal result of our better knowl. edge of the principles of phophylaxis and of greater care in applying them, as well as the effect of the process of immunization by vaccination. In the $\mathrm{U}$. S. Navy and Marine Corps, the rates for the years 1909 and I9Io were 3.30 for admission and 0.23 for deaths. Since then prophylaxis by vaccination has also been made compulsory so that for the two years I9I4 and I9I5 there were only $3^{1}$ admissions and one death.

Until three or four years ago, our Army rates for typhoid fever were higher than in most European armies, but it is gratifying to 
note that we have recently progressed at a more rapid pace and that our rates (for I9II and 1912) have fallen below those of foreign armies.

The report of the United States Census Office and that of the Registrar General of England and Wales, for the years I9OI-I905, show that the mortality rate of typhoid fever in the United States is far in excess of that of most European countries. Thus while this rate was 32.2 per IOO,OOO of population in the United States, it was only II.2 in England and Wales, 7.5 in Germany, II.4 in Japan and 5.7 in Norway. The rate for France is not available. During about the same period, it was I6.2 in New York, 24.7 in Albany, 27.8 in Boston, 29.8 in Chicago and 44.2 in Washington, but only 3.8 in Berlin, 4.3 in Vienna, 5.6 in Hamburg and 12.3 in London (E. O. Jordan). Since that time, however, a steady improvement has taken place in the United States, the rate falling to 23.5 per IOo,000 in 1910 and to 15.4 in I9I4. In our large cities, the rates for I9I4 ranged from 6.3 for New York to 22.6 for Baltimore, while the average rate of the four largest cities in Europe (London, Paris, Berlin and Vienna) was only 4.75 for the approximate period I9IO-I9I2.

It is difficult to account for this difference. We may assume that Americans are as well fed and lodged as Europeans, that although our towns leave very much to be desired in the way of sanitation, more of them are provided with adequate water-supply and sewerage than in England or Germany. Typhoid fever is not a disease of the poor but rather of the well-to-do, being most prevalent among the well-nourished and robust, and making most victims in early adult life, at the time of greatest vitality. It is not improbable that this higher susceptibility in the United States is due, at least in part, to intemperance in eating, especially the eating of meat, in which we surpass every other country. This hypothesis is strengthened by the comparative freedom from typhoid fever of countries (outside of cities) whose people are too poor to indulge, more than very sparingly, in a nitrogenous diet. Investigations in many parts of the world indicate that no climatic or racial influences confer immunity against typhoid fever, but that it is much less prevalent among primitive or semi-civilized peoples than in more highly civilized and better-fed countries. There is enough evidence on this subject (see under Food) to make it seem unwise to allow such 
liberal rations as to practically put no limitation to the amount of meat that soldiers and sailors may consume in camp or on board ship, as the tendency has been of late years by those who still disregard the advantages of physiological economy in nutrition.

Prophylaxis. - Troops immunized by vaccination do not require the same strict watch as those that are not thus protected, but, for obvious reasons, they should continue to receive the benefit of all the well-known measures of prevention.

Every case of typhoid fever must be regarded as highly contagious and treated accordingly. The prompt removal of the patient from his company and regiment to post or field hospital, and the disinfection of his bedding, clothing and effects, will be the first indications. One of the chief duties of medical officers is to watch for the mild, obscure, atypical cases, sometines mere gastric malaise or bilious attack in men who do not feel ill enough to report at sick call, or dislike to take the risk of being separated from their comrades. All these so-called ambulant cases are very dangerous and every measure should be taken to detect them in their inception. Any man with febrile temperature not easily accounted for, especially if accompanied with gastro-intestinal disturbance, should be isolated until the nature of his ailment is determined. Blood cultures will generally permit an earlier and surer diagnosis (from the 3 d to the 8 th day in 94 per cent. of cases) than the Widal test. The prompt segregation of initial cases (as in all infectious diseases) is the first and one of the most important measures of phophylaxis.

As it is in camps of instruction and mobilization that outbreaks of typhoid fever are most likely to occur and spread, every effort must be made to guard them against infection. All troop units under orders to proceed thereto should be carefully inspected before leaving their first rendezvous, and suspicious cases left behind until they can safely rejoin their organizations. This inspection gives the special sanitary officers appointed for the purpose the opportunity to detect men who have not yet been vaccinated against typhoid fever, as well as against small-pox, to isolate those in the incipient stage of the disease or who have been dangerously exposed to it, as well as those who, although entirely recovered from an attack of it, are found still discharging bacilli in their excreta.

Transmission by contact is the more insidious and dangerous that it is connected with personal cleanliness and habits, therefore diffi- 
cult of detection and control. The lands are the usual means of transmission and require particular care (see under Personal Hygiene). The proper and careful use of latrines, particularly of pit privies, in camp, so as not to infect hands, shoes or clothing, should be insisted upon. Urine tubs must be provided for the night and their use enforced. The fouling of camp grounds is a capital offense against hygiene, to be visited by swift and severe punishment. In camp, the men must be required, if not supplied with cots, to improvise bedsteads which will lift their blankets and clotling above the dust of a possibly infected soil. (See also under Dissemination, page 32 , and Latrines, page 648.)

Antityphoid Vaccination.- Owing to the impossibility to exclude all typhoid cases from camps, the lax discipline generally existing among volunteer troops, and the lack of sanitary experience of many of their medical officers, it is plain to see that the prevention of serious outbreaks, in case of mobilization on a large scale, would always be a very difficult problem so long as our soldiers remain particularly susceptible to typhoid infection. How to render them immune to it is a sanitary question of capital importance, but one which, happily, has been successfully solved. The institution of antityphoid inoculation is another epoch-making step in the science of medical prophylaxis, one that saves the soldier from his most dangerous enemy and robs war of one of its terrors. It was first elaborated and applied in England by Wright and Leishman, and in Germany by Pfeiffer and Kolle. In this country we owe its introduction to the brilliant work and persistent efforts of Major F. F. Russell, M. C., U. S. Army, under the auspices of Surgeon Generals R. M. O'Reilly and G. H. Torney. Typhoid vaccination in our Army was begun in March, 1909, on officers and men (chiefly of the Medical Department) who volunteered for the purpose. The results were so satisfactory and promising that, in March, I9II, it was made compulsory by the War Department for all troops ordered into active service and, in June of the same year, for all recruits. All men enlisting or drafted, unless they are over 35 years of age or have had a recent attack of the disease, are vaccinated against typhoid fever at the time that they are vaccinated against small-pox.

The preparation of the vaccin is comparatively simple. A nonvirulent strain of the typhoid bacillus is grown on agar in Kolle 
flasks for I 8 hours; the growth is washed off in normal salt solu. tion and standardized so as to contain $I, 000,000,000$ bacilli to the cubic centimeter. It is then subjected to a temperature of 54 to $55^{\circ} \mathrm{C}$. in a water bath for one hour, a temperature which kills all the baccilli without injuring the toxin; 0.25 per cent. of tricresol is added as a perservative, and the finished prophylactic is put up in hermetically-sealed glass ampuls. Only a single strain of the bacillus has been used in this country, as in England, with the idea that the method of preparation should be as simple as possible consistent with good results, so that an adequate supply could always be promptly procured in time of emergency. This rule, however, is liable to modifications, and whenever paratyphoid fever, for instance, is recognized or suspected, it may become necessary to use a mixed vaccin of typhoid and paratyphoid.

Vaccins are also prepared from live cultures; two have been shown to possess practical value, namely, Vincent's polyvalent autolysate, and the attenuated live culture of Metchnikoff and Besredka. Vincent grows several strains (including the paratyphoid) on agar 24 to 48 hours; the growth is taken up in salt solution and allowed to macerate for several days at a temperature of $37^{\circ} \mathrm{C}$. This bacillary emulsion is centrifuged and the supernatant fluid sterilized by shaking it with ether which is then allowed to evaporate. This autolysate has been used in France and among French troops with marked success. In the preparation of Metchnikoff and Besredka, the live culture is "sensitized" by contact with the serum of a horse highly immunized, so that, after vaccination, the bacilli are all speedily destroyed by phagocytes and antibodies. It is claimed for this vaccin, besides absolute safety, an extremely slight reaction and more lasting immunity.

A complete vaccination consists of three inoculations at Io-day intervals, the first of 0.5 c. c. and the others of $\mathrm{r}$ c. c. each. The injection is generally given in the arm at the insertion of the deltoid muscle. It should be subcutaneous, not muscular, and care taken that the needle does not enter a vein, for fear of a possible excessive reaction. With proper system, two medical officers and four assistants can easily vaccinate several hundred men a day and strictly observe all antiseptic precautions. Nolson and Hall paint the skin with iodine, without any previous cleaning, and seal the puncture with collodion; with this method they claim to be able to vaccinate 
300 men per hour. Following the vaccination, within 6 or 8 hours, there is a local reaction consisting of a red and tender area, about the size of the palm of the hand, at the point of puncture. There may also be a general reaction, with pyrexia, headache, malaise, occasional chills and, quite rarely, nausea, vomiting or diarrhea. These symptoms usually subside within 24 hours, or 48 hours in the severest reactions. In the last series of vaccinations published (27,720 men inoculated from January I to June 30, I9II), the systemic reaction was absent or mild in 99 per cent. of the cases, and severe in only 3 per thousand of those receiving the first dose. In no case has there been any untoward result, not even an abscess at the site of inoculation.

It was believed, until recently, that the first dose of vaccin, by temporarily decreasing the antibodies in the blood, produced a "negative phase" during which there was an increased susceptibility to typhoid infection. Experience has shown that, practically, there is no such phase, but that, on the contrary, increased resistance begins almost immediately. There might be danger, however, in vaccinating subjects during the incubation or incipient stage of the disease: therefore in the case of men showing symptoms of fever, or illness, the operation should be postponed.

Laboratory researches demonstrate a marked increase of antibodies (agglutinin, opsonin and bacteriolysin) within 8 or ro days after vaccination, an increase said to be greater than that which follows an attack of typhoid fever. This and other reasons justify the belief that the immunity conferred by vaccination will last at least two or three years, but that the present regulation requiring revaccination upon reenlistment is wise and should be adhered to.

The total number of men vaccinated since March, I909, to July, I9I I, in the U. S. Army, was 45,680 , but only 38,902 received three doses. Of this total number I I have had typhoid fever and none died, a rate of admission of 0.24 per thousand. Of these I I cases, at least 2 had only received two doses, while in 4 the diagnosis was doubtful. The almost perfect efficacy of typhoid vaccination was particularly demonstrated by the troops sent to Texas in March and April, I9I I. By order of the Secretary of War those not already immunized were all vaccinated upon their arrival in camp. The mean strength of the command at San Antonio was 12,800, and of the command in Galveston between 3,000 and 4,000 men, a 
total of at least 16,000 men. So far as known not a single case occurred in the Galveston troops, although 192 cases were reported in the city during the same period. Only two cases, without death, occurred in the San Antonio command. It should be noted that these troops were not confined to the limits of their cantonments, but that when not on scouting duty (which was unusually arduous) they were permitted free intercourse with the populations of neighboring towns.

For the year I9I 5, out of a mean strength of I04,000 men, 8 cases of typhoid fever were reported, of which 7 had been vaccinated at previous periods ranging from 8 months to 4 years. No deaths occurred.

Very striking results have likewise peen obtained in the English, German and Japanese Armies. Of the English troops serving in India, in I9I $I$, the rates of admissions and deaths for the noninoculated were 5.9 and I.I5, and for the inoculated, I.7 and 0.I7 respectively.

It should be added that typhoid vaccin does not immunize against paratyphoid fever, and that it is highly probable that of the cases occurring among the vaccinated in our Army, a few have been of that disease. The observations of English medical officers in India tend to show that paratyphoid fever is more common than generally believed, and that it may be deemed advantageous, in certain circumstances, to use a polyvalent (or rather a bivalent) vaccin to protect against both diseases.

\section{PARATYPHOID FEVER.}

This is a disease resembling mild atypical forms of typhoid fever, with case mortality seldom exceeding 3 per cent. It seldom occurs in epidemic form, but often coexists with typhoid fever in endemic foci. In India, I 5 per cent. of cases reported clinically as typhoid fever were found to be paratyphoid infections (mostly type A). Thousands of cases have been reported from the belligerent armies in western Europe (mostly type B), and believed to be one of the principal causes of " trench diarrhea."

Paratyphoid fever is caused by bacilli intermediate between the typhoid and colon organisms. Of these bacilli two types are recognized, $A$ and $B$. Clinically they are hardly distinguishable, and extremely variable in their manifestations. 
The diarrheal form of paratyphoid, often seen in the French and British armies, closely resembles food poisoning clinically, the patient, within a few hours after the ingestion of meat, being seized with headache, fever, vomiting and severe diarrhea.

The Widal reaction being of no value in inmunized persons, and the differential diagnosis by agglutination always difficult, the best method of distinguishing typhoid from paratyphoid and other continued fevers is by blood cultures, between the $2 \mathrm{~d}$ and 5 th days of the disease.

The modes of infection seem to be exactly those of typhoid fever. Prophylaxis requires vaccination with a mixed vaccin of paratyphoid $A$ and $B$, or as practised in the Frencl and British armies, of typhoid and the two paratyphoid types (the so-called T. A. B. mixture). Special attention should be given to the detection and isolation of carriers.

A few cases are generally reported every year from our troops in the United States and colonies. Thus, in 1915,9 cases occurred in the entire Army, 3 in the Philippines, $I$ in the Hawaiian Islands and 5 in the United States. Of them, 6 were caused by Bacillus A. In August, 1916, an outbreak of paratyphoid occurred among our troops on the Rio Grande, in Texas, in all $\mathrm{I} 20$ cases. This was promptly and satisfactorily cliecked by vaccination with mixed vaccin of both types $\mathrm{A}$ and $\mathrm{B}$, a vigorous search for possible "carriers" and a change of camp sites.

It is to be noted that as the number of paratyphoid cases identified in our Army increases, there is a corresponding decrease of cases of simple continued fever, indicating a greater skill and accuracy in diagnosis. 


\section{INFECTIOUS DISEASES (CONTINUED).}

\section{DIARRHEA AND DYSENTERY.}

Diarrhea is quite prevalent among troops serving at home and in our insular possessions, being always one of the five diseases having the highest rates of admissions. These rates (mean annual), for the period I908-1910, were 34.90 per thousand in the United States and 73.29 in the Philippines, with mortality of 0.02 and 0.05 respectively. They show that diarrhea, in normal times, although one of the principal causes of non-effectiveness, is, so far as death is concerned, a negligible factor. Dysentery, a disease of little importance at home, is much more prevalent and serious in the tropics. Thus the rates (mean annual) for the United States and the Philippines, during the same period I908-I9I0, were, for admissions, 2.12 and 29.56 respectively, and, for deaths, o.or and 0.57. Dysentery is nearly always among the five diseases causing the highest mortality in the Philippines.

For I9I5, a marked decrease in the incidence of dysentery is noted in the Philippines, with an admission rate of 5.32 per $\mathrm{I}, \mathrm{OOO}$, as compared with 12.97 in I9I4; the death rates for those two years being 0.17 and 0.09 respectively.

In war, dysentery and diarrhea assume an importance and produce a mortality which may equal or even exceed that of typhoid fever. In the Medical History of the Rebellion they are thus described:

"These disorders occurred with more frequency and produced more sickness and mortality than any other form of disease. They made their appearance at the very beginning of the war, not infrequently prevailing in new regiments before their organization was complete, and although, as a rule, comparatively mild at first, were not long in acquiring a formidable character. Soon no army could move without leaving behind it a host of the victims. They crowded the ambulance trains, the railroad cars, the steamboats. In the general hospitals they were often more numerous than the sick from all other diseases, and rivalled the wounded in multitude."

They caused 57,265 deaths in the federal armies, corresponding to 


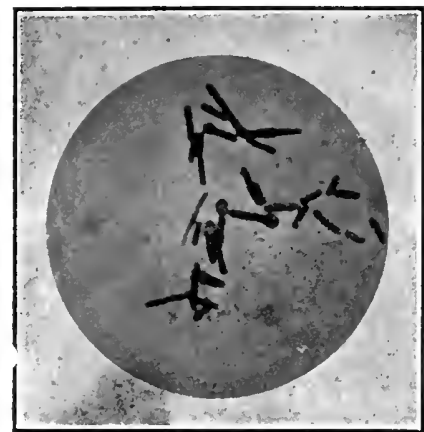

1

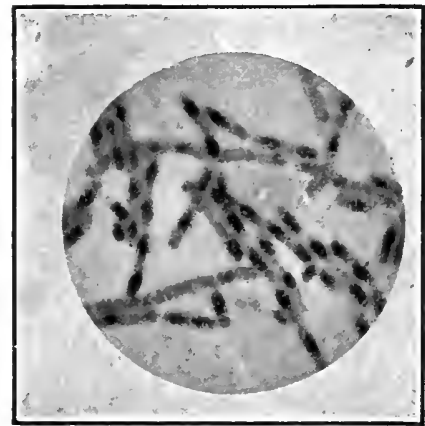

3

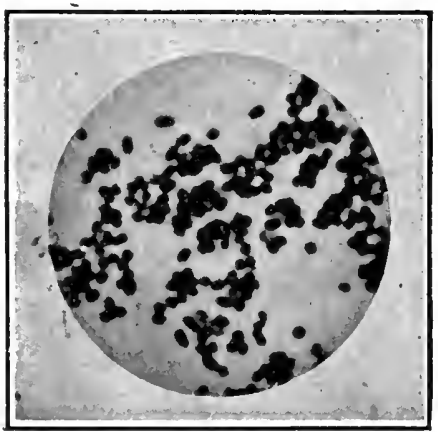

5

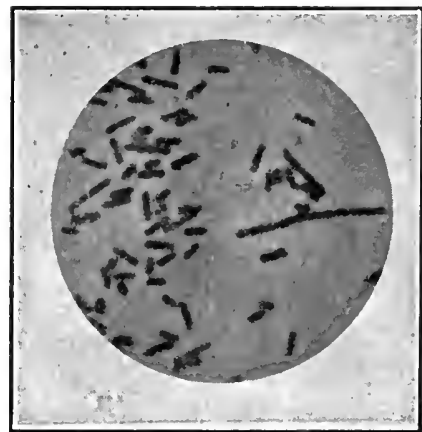

2

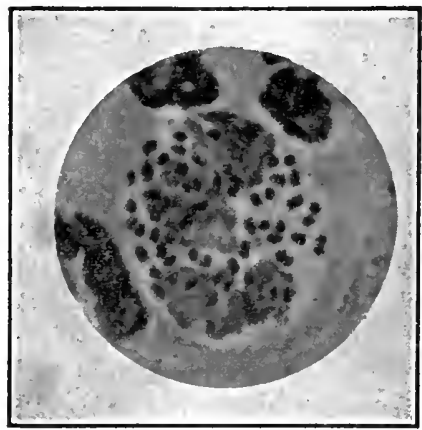

4

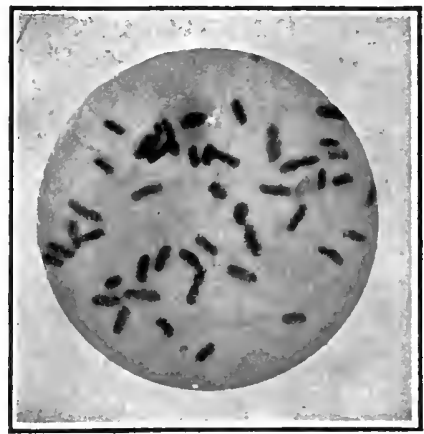

6

I. Bacillus of tetanus in pure culture.

2. Bacillus of dysentery (Shiga) in pure culture.

3. Bacillus of anthrax in pure culture, spores stained.

4. Gonococcus in pus cell.

5. Meningococcus in pure culture.

6. Baccillus of plague in smear from spleen. 

a yearly mortality of $I 7$ per I,OoO, or one death out of every 3.5 deaths from all sickness. The case mortality was I death to 395 cases of acute diarrhea, 57 of acute dysentery, 6 of chronic diarrhea and 8 of chronic dysentery. They, besides, caused over 18,000 discharges. It is probable that many deaths from typhoid fever, wrongfully diagnosed dysentery, should be deducted from the above total. Even with this correction the victims from dysentery and diarrhea greatly outnumbered those from typhoid fever. The great predominance of these diseases during the Civil War is surprising, and has not been equalled in any other modern war, except perhaps the Sino-Japanese War of 1894-5. During the year of the Spanish. American War, I898, their death rate was only 2.I4. In the Boer War, the relation of the mortality from typhoid fever to that of dysentery was as 6 to $\mathrm{I}$; in the Franco-German War, this relation was as 4 to I; in the Spanish-American War as 8 to I. On the other hand, in the Sino-Japanese War dysentery caused more deaths than all other diseases combined.

Simple diarrhea may result from the ingestion of excessive, indigestible or unwholesome food, or impure water, or from exposure to inclement weather, damp soil or foul air. That it is mostly caused by fermentative or putrefactive organisms is proved by the striking effect of heat upon its rate which, in the United States, is lowest in January and February and highest in August. In camps it is often contagious and may become epidemic, indicating the presence in the stools of pathogenic organisms, perhaps some of the ordinary parasitic and generally harmless bacilli of the intestinal canal which assume a certain degree of virulence under special conditions. The younger the soldier the more liable he is to this disease.

The etiology of dysentery has not yet been satisfactorily cleared up; the true causes of its various forms and the symptoms pertaining to each will require further investigation before we possess a complete knowledge of the subject. Our present information leads us to the conclusion, as stated by Manson, that three factors are concerned in the production of dysentery: (I) weakening or irritating influences such as bad food, purgatives, indigestion, diarrhea, intemperance, etc., which prepare the ground for (2) the specific germs which, subsequently, have their action supplemented by (3) the ordinary bacteria of suppuration. The irritating influences re- 
ferred to may produce a catarrhal condition which later develops into severe dysenteric symptoms without the operation of any known specific germs; this is the so-called catarrhal dysentery.

Two chicf types of dysentery are caused by parasites, bacillary dy'sentery and cutamebic dysentery. They are entirely distinct, but occasionally one may complicate the other. Bacillary dysentery is found in all parts of the world and under many different climatic conditions. Its causative organisms are several forms of closely related bacilli, divided by Hiss into four types: the Shiga, HissRussell, Flexner-Strong and the Harris. They are differentiated by fermentation and agglutination tests, but all possess an identical morphology, the same staining characteristics and lack of motility, with the inability to liquefy gelatin and produce gas. The best known and most common is the Bacillus dysenterice of Shiga, a short rod with rounded ends and very similar to the typhoid bacillus. They are all easily destroyed by heat $\left(60^{\circ} \mathrm{C}\right.$.) and the ordinary disinfectants, but show considerable resistance to cold, surviving freezing for several weeks.

Bacillary dysentery, whether caused by one or the other of these organisms, does not differ in its clinical course, pathology, gravity and treatment, so that the identification of the bacillus type is not of practical importance. It is characterized by acnte onset, often with chill, the presence of the specific bacillus in the stools, nonliability to liver abscess and non-tendency to relapse. This is the epidemic dysentery of armies, especially in temperate climates, and susceptible of becoming highly infectious, although probably never to the same extent as typhoid fever. It is the form that prevailed in the Civil War, in European wars, in the Boer War and in the Russo-Japanese War. It is also possible that the bacilli of dysentery may, under less virulent forms, be concerned in the etiology of infections and epirlemic diarrheas, such as sometimes prevail in armies and other human agglomerations. The contention that they are only pathogenic forms of the common colon bacillus which, under special influences, acquires virulent properties, has not been proved, although it is generally admitted that the colon bacillus and other normally harmless intestinal bacteria may become more or less pathogenic and produce severe diarrheal and digestive disturbances.

The incidence of bacillus carriers in dysentery is as great as in Iyphoid fever, if not greater. Therefore the danger of contact and 

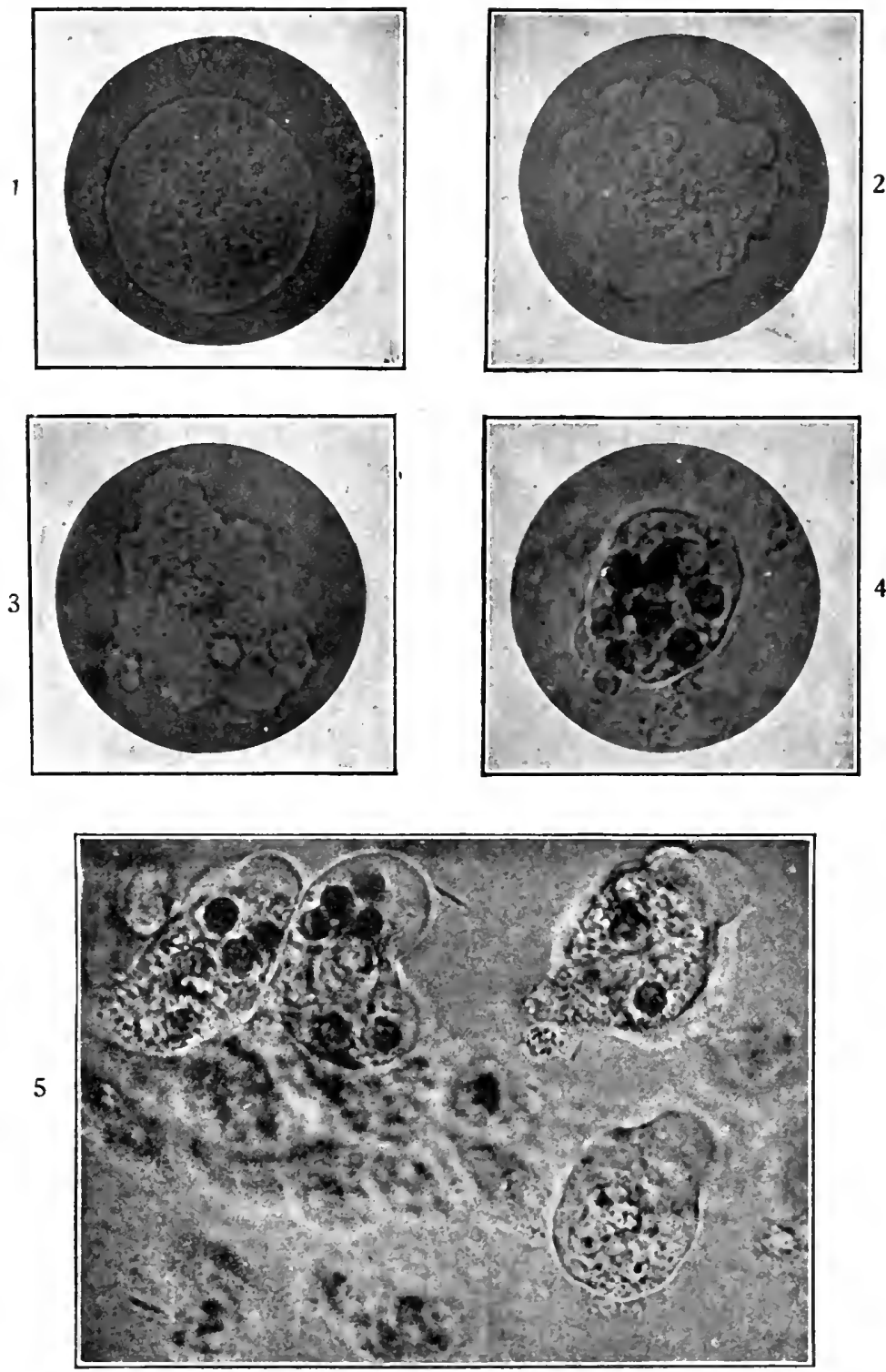

I, 2, 3. Entamaba coli in fresh dysenteric stool; the same parasite photographed at $30-$ second intervals.
4. Entamaba histolytica in fresh dysenteric stool; parasite filled with blood.

5. Entamaba tetragena; living parasites containing red blood cells. 
. 
of infecting latrines is as threatening; convalescents, in the military service, should be returned to duty only under the same restrictions that are applied to typhoid convalescents.

Entamebic dysentery is characteized by the insidious onset, marked tendency to chronicity and frequent relapses, the presence of amœbæe in the stools (mostly in the bloody mucus) and great liability to liver abscess. It is mostly a disease of warm climates and is less contagious than the former, prevailing mostly in a sporadic or endemic form.

Amoba are microscopic animalcules in the shape of rounded masses of 'protoplasm, with nucleus, nucleolus and vacuoles, and often finger-like projections. They are common in all parts of the world and abundant in the tropics. As shown by Musgrave and Clegg they are almost ubiquitous in the Philippine Islands, being found in most surface waters under various forms. From the investigations of Walker, Craig, Whitmore and others, it appears that the amoboid organisms found in the intestinal tract of man belong to a distinct genus, Entamaba, and that they are strict parasites, that is to say, incapable of multiplication outside the body of their host. Three species, distinguishable under the microscope, have been described: $E$. coli, with distinct nucleus near the center of the organism, thick nuclear membrane and abundant chromatin; its movements are sluggish and does not ingest red blood corpuscles; it develops cysts containing 8 nuclei. E. histolytica, with indistinct or hardly visible nucleus, eccentric in position, without limiting membrane and very poor in chromatin; its movements are active and it frequently ingests red blood corpuscles; it develops cysts containing 4 nuclei. E. tetragena, differing from the preceding in having a distinct nucleus with thick membrane, and being rich in chromatin. The latter species has been shown by Walker and Craig to be only the cystic stage of the life-cycle of histolytica into which it merges through various intermediate forms. E. coli is now considered a harmless parasite, while E. histolytica has been proved to be pathogenic and the specific cause of entamebic dysentery. Craig has shown that the latter, whether fed in milk or injected through the rectum, produces in kittens the typical lesions of entamebic dysentery as observed in man, while the E. coli when thus used is absolutely harmless. Ashburn and Craig (Military Surgeon, September, 1907) found that, of 100 healthy American soldiers examined in 
Manila, 72 showed the presence of $E$. coli in their stools; that in many of them the organisms disappeared, but that, in the larger number of cases, they were still found after the lapse of 9 months, during which the men remained perfectly free from dysentery and diarrhea. Other observers have reached the same conclusion, and it may be considered established that a large proportion, sometimes a majority, of healthy people in temperate and tropical countries carry amœbre in their intestines.

Entamebic dysentery is mostly found in tropical and subtropical countries, but is not rare even in the northern United States in a less virulent form. It is the more common type in all our Island possessions.

An outbreak of it, with total of $5_{5} 6$ cases, among the troops serving in the El Paso district and the Punitive Expedition in Mexico, between July and November, I916, was critically investigated by Craig,* who reached the inevitable conclusion that the disease had been carried and disseminated by flies from infected latrines.

It is now generally accepted that the cysts of Entamaba histolytica, as passed by carriers, are the infective agents in the vast majority of instances, infection through the motile forms rarely occurring in man owing to the destructive action of the gastric juice upon them. Therefore the greatest source of danger is not the patient sick with acute symptoms, but the "carrier" of cysts, namely, the healthy individual who discharges cysts although never having had symptoms of dysentery (contact carriers), and the patient who has recovered from the acute attack but develops cysts during convalescence (convalescent carrier). These cysts retain their vitality a number of days in water or when kept moist, but promptly dry and shrivel up when exposed to sunlight. They can be demonstrated in the intestine and excreta of flies as long as eighteen hours after these insects have been fed upon material containing them.

Another form of dysentery, rather rare, distinct from but simulating the entamebic type, is that caused by the Balantidium coli, an infusorial parasite of the intestine, specially studied by Strong and Nusgrave. It is a widely distributed disease, occurring in Europe, Africa, China, North America and the Philippine Islands. Its mode

* The Military Surgeon, March and April, 1917. By Major C. F. Craig, U. S. Army. 
of transmission is still undetermined. Beginning with mild intermittent diarrhea, it gradually becomes dysenteric in character. The symptoms may suddenly disappear for a few days, but always return with increased severity. The prognosis is bad.

Abscess of the Liver.- One of the most serious sequels, or concurrent diseases, of the entamebic dysentery of the tropics is abscess of the liver. There is an intimate relationship between the two diseases, dysentery having been found to be a factor in nearly every case of tropical liver abscess. This disease is rare among the natives, but common among Europeans and Americans, especially the men, its exciting cause being generally intemperate habits and exposure. Women and children seldom suffer from it.

Prophyla.ris.- Dysentery, like typhoid fever and cholera, results from the ingestion into the stomach and intestinal canal, with food or drink, of fecal matter containing the infective organism. Like those diseases, it is often water-borne, many of the epidemics of dysentery being fairly attributable to infected water-supply. There is no doubt, however, that, in military camps, most cases are propagated by flies and direct or indirect contact with patient or carrier, so that, practically, the same preventive measures are required as in typhoid fever. It is quite probable that so long as the mucous membrane of the intestinal canal is sound and normal, it can protect itself against the pathogenic organisms of dysentery, but any cause which produces an irritation and congestion of the bowels, with consequent diarrhea, such as exposure to cold and wet in combination with unwholesome diet, may break this barrier and bring about an infection of the system. Hence the importance, in the presence of an outbreak of dysentery or of a suspected water-supply, to avoid excesses in eating and drinking, unnecessary exposure to inclement weather and sudden changes of temperature. In the tropics there is usually a great fall of temperature during the night, and persons going to bed without enough covering are liable to wake up chilled. It is to be noted that if the body is uncovered while perspiration is free and evaporation active, the abdomen will be first to be chilled; in this way the incipient diarrhea of dysentery may be started. This sensitiveness of the abdomen is not rare in the tropics, and men who suffer from it should wear a flannel abdominal band at night. Such protection, however, is seldom necessary or advisable during the day. 
When diarrheal diseases become prevalent in camp, special care must be observed in the use of vegetables and fruits. No uncooked vegetable should be eaten, unless carefully washed with sterilized water or scalded with boiling water. Fruits should be washed in sterilized water and then skinned or peeled, and only the interior pulp consumed.

Whenever a case of entamebic dysentery is discovered in any organization, the following measures should be strictly carried out to prevent the spread of the infection. A microscopic examination of the stools of all soldiers whose duties are connected with the preparation or serving of food or drink, should be made for cysts of E. histolytica, and all carriers removed at once to the hospital for treatment. If they prove resistant to treatment they should be discharged from the service. The excreta of all carriers must be disinfected immediately after passage whenever practicable. The prevention of the breeding of flies and their destruction, as well as the screening of kitchen and mess tents, are measures of the greatest importance, for these insects are undoubtedly the greatest factor in the spread of entamebic dysentery in temporary or semi-permanent camps (Craig).

\section{SPRUE (PSILOSIS).*}

A very dangerous form of chronic catarrhal inflammation of the mucous membrane of parts of the alimentary canal, and characterized "by an inflamed, bare and eroded condition of the mucous membrane of the tongue and mouth; by flatulent dyspepsia; by pale, phenomenally copious and generally loose, frothy, fermenting stools; by wasting and anemia; and by a tendency to relapse" -(Manson). A tropical disease common in parts of the Philippine Islands, China, India, Africa and the West Indies. Nothing definite is known as to its cause. It may be infectious, but it is not directly contagious. Prolonged residence in an endemic area seems necessary to contract it. All depressing influences predispose to it: exhausting diseases, particularly those involving the alimentary canal, such as dysentery, are apt to terminate in it. Sprue is rare among the natives. To what extent its incidence may be ascribed to any excess in eating or drinking, or unhygienic conditions, has not been determined.

* Not to be confounded with the ordinary thrush or stomatitis of temperate climates, also frequently called sprue. 


\section{CHOLERA.}

Cholera, endemic in India and other parts of Asia, has from time to time spread over Europe and America in an acute epidemic form with case mortality of nearly 50 per cent. Its last visitation to the Lnited States was in 1870-73, when it entered by way of Jamaica and New Orleans. In this country cholera has ceased to be a serious menace for it is believed that, even should some individual cases elude the quarantine, the infection could soon be controlled and prevented from assuming epidemic proportions. In the tropics, however, cholera still persists in many endemic zones and breaks out now and then, showing the necessity for constant vigilance. In the Philippine Islands an epidemic began in March, 1902, raged among the natives and was not suppressed until April, I904. The last case among soldiers during that period was in October, 1903, and no other case occurred until 1905, when there were 9 admissions with 2 deaths; during 1906 there were 7 admissions with 3 deaths. Cholera became again particularly prevalent throughout the Islands in 1909 and 19I0. In the latter year 2 cases occurred among American soldiers, both of whom recovered. In I9I the epidemic had greatly subsided. "When it is remembered that cholera occurs in epidemic form in so many native towns adjacent to our military posts, it must be recognized that the almost entire immunity of the troops is due to the excellent sanitary measures adopted for its prevention."

It has been reported from various parts of southeastern Europe and of Asia, in 1915 and 19I6, but was kept out of the belligerent armies or promptly checked when introduced.

Cholera is carried by man from country to country, following the routes of commercial intercourse, and is often spread by polluted water-supply. But, like typhoid fever, it is also propagated by flies, infected food, clothing and utensils, and by personal contact. In the Philippines, contaminated water seems to have but little to do with the outbreaks of this disease. On being ingested, the germ multiplies in the alimentary canal and abounds in the characteristic rice-water discharges of patients. It is not found in the urine.

The causative organism of cholera is an actively motile spirillum, with single polar flagellum. It multiplies with great rapidity, breaking into comma-shaped fragments, small curved rods, short and thick, to which the name of "comma bacillus" has been given. 
This spirillum is not easily differentiated from other similar organisms; cultural characteristics, like morphology, often yield uncertain results; a positive diagnosis, in many cases, can only be established upon the agglutination reaction ( $\mathrm{I}$ to $\mathrm{I}, \mathrm{O}) \mathrm{O}$ ) with culture from fecal material, and Pfeiffer's phenomenon. Otherwise it matters not whether the organism is straight, curved or spiral. Its growth is arrested below $59^{\circ}$ or above $107^{\circ} \mathrm{F}$. Freezing does not immediately destroy it, but a temperature of $122^{\circ}$ kills it. In the soil, or when dried, it soon dies, but may retain its vitality for a number of days if kept moist, as, for instance, in damp soiled linen and foodstuffs.

It is an interesting fact that it can be injected beneath the skin with impunity, while it may cause death if taken into the bowel.

Prophylaxis. - Cholera is one of the quarantinable diseases in this country, but its incubative period is so variable, ranging from a few hours to ten days, that efficient quarantine is difficult. The official period of detention is five days.

As in typhoid fever, the germs may be conveyed by carriers, that is, individuals harboring it in their intestines without exhibiting any signs of the disease. During an epidemic these carriers are numerous, sometimes amounting to 6 or 8 per cent. of the healthy individuals living in infected neighborhoods. Generally the spirillum disappears from the stools of convalescents in 4 or 5 days, and is seldom seen after 20 days, but, through the infection of the biliary apparatus, may persist several months. Inasmuch as the ordinary quarantine examination would fail to detect carriers, the Public Health Service imposes a bacterial examination of the stools of all steerage passengers coming from infected ports and places.

The quality of the water-supply should first receive attention. No water that is not clearly above suspicion should be used for drinking, cooking or bathing. A sound mucous membrane is much more likely to resist the invasion of the comma bacillus than if abraded; therefore any cause of digestive disturbance and intestinal catarrh must be carefully avoided. The measures of prevention against flies already mentioned under typhoid fever and dysentery must be likewise strictly applied. Raw vegetables and fruits, especially from native markets, are dangerous. All patients must be kept in strict isolation, and their bodies, discharges, clothing and bedding thoroughly disinfected. 
The following practical rules enforced among American troops in the Philippine Islands during an epidenic of cholera are well worth remembering:

Do not visit native houses and shops or partake of native food or drink of any description.

Do not drink, or brush teeth with, any other than distilled or sterilized water.

Do not eat cold or uncooked food, except bread and crackers; all food must be freshly cooked and served hot.

Wash all crockery, glass, knives, forks, spoons, etc., immediately after meals with water that has been raised to a boil.

Boil dish cloths frequently.

Protect all food from flies by gauze or wire netting and do not place it upon the table in advance of the meal hour.

Keep the kitchen and mess-room scrupulously clean, carefully removing scraps of food and particles of refuse likely to draw flies.

Wash your hands before entering the mess-room.

Keep all slops and garbage in closed garbage cans.

Take special care of sinks if sewerage or incinerators are not used.

The prophylactic inoculation of a live culture of the comma bacil. lus, or cholera vaccination, first introduced by Haffkine, of late years practised on a large scale in India, and more recently in some of the belligerent armies in Europe, has given highly favorable results and proved an efficient weapon against the spread of this dreadful scourge. Strong's cholera autolysate is also efficient. 


\section{INFECTIOUS DISEASES (CONTINUED).}

\section{TUBERCULOSIS.}

Tuberculosis, in time of peace, in armies as in civil life, causes more deaths than any other disease. During the three years I904-6, its average annual mortality among American troops, in the United States and colonies, was 0.67 per thousand. During the years I909, I9IO and I9II, the death rate was $0.57,0.28$ and 0.44 respectively. For these years the rates of admission averaged 4.04 in the United States and 4.76 in the Philippines. For the two years, I9I4 and I915, the death rate was 0.47 and 0.33 respectively. Inasmuch as all cases originating in our tropical possessions are promptly returned to the United States, it is difficult to ascertain what difference, if any, there might be in the death rate. From the experience of our Medical Corps it seems fair to conclude that, with good hygienic surroundings, the number of cases is not materially greater in our tropical colonies than at home, but that they progress more rapidly to a fatal termination.

In the U. S. Navy and Marine Corps the death rate averaged 0.58 for the years I905 and 1906; for the years I9ro and I9II it was 0.33 and 0.59 , respectively.

The death rates for tuberculosis in foreign armies, as given by the last available reports, are: Great Britain (I909), 0.3I ; France (1907), 0.76; Prussia and Bavaria (1907), 0.20; Austria (1909), 0.45; Russia (I908), 0.58; Japan.(I909), 0.34. In comparing these rates with our own, it is necessary to remember: first, that whereas in European armies soldiers found suffering from tuberculosis are at once discharged, in our Army and Navy they are kept a variable period of time under treatment in sanatoria, during which they continue to be credited to the Army and Navy rates; second, that our colored troops, which form 5 to 6 per cent. of the Army, always furnish a tuberculosis death rate much greater than that of our white soldiers.

For the 3 years roog-rgrr, tuberculosis, in our Army, caused annually i6 per cent. of all deaths from disease. For all adults from 20 to 29 (males and females) in the United States, during 
I9Io, the nearest class corresponding to the military age, tuberculosis caused 35 per cent. of all deaths.

If we compare our census reports with those of the Registrar General of England and Wales for the period I9OI-5, it appears that the mortality rate of the United States for tuberculosis averages jo per 100,000 of population, which is greater than that of England, Scotland, Italy, Spain, Belgium and Japan, but smaller than that of Germany, France and Austria. In this connection, we must also bear in mind the great susceptibility of the colored race to tuberculosis, furnishing a rate three times as high as that of the white, so that, were the white population alone considered, the tuberculosis mortality in the United States would compare very favorably with that of most civilized countries.

Not only is tuberculosis the most deadly disease in the Army, but also that which, for the decade 1901-1910, caused the highest rate of discharges; the next highest rate being caused by venereal diseases.

At first thought it would seem that in our Army, where all the men are carefully examined and selected, tuberculous recruits could be entirely excluded. But this has been shown to be impracticable. Men may carry the bacillus in a latent or passive form (in the lymphatic nodes, for instance), only awaiting such determining causes as are encountered in the military service to light up an active process; or else the infection is in such incipient stage as not to be recognized by the ordinary methods of examination. It is well known that a majority of cases, in all armies, are first taken sick in the first six months of service, too soon after leaving their homes to ascribe their infection to military conditions. A certain proportion of recruits, therefore, whether conscripted or voluntarily enlisted, although carrying the organisms of tuberculosis, will be accepted. Cornet estimates this unavoidable percentage for the German Army at 0.42 per cent. From the records of the Fort Bayard sanatorium, it seems quite likely that a large proportion of the soldiers who die there of tuberculosis, or are discharged on account of it, had contracted it before enlistment. Thus Bushnell found that, of the 89 patients discharged for pulmonary tuberculosis from January I to August 31, I906, much more than one-half had been examined for enlistment or reenlistment, and passed as sound. only about one year before their admission to the sanatorium, too short a period to dispel a strong presumption that they were already 
infected at that time. In his report for I9I I, the Surgeon General states that of all cases of tuberculosis treated during the year, 74 per cent. were below the standard proportion of weight to height, and I 8 per cent. below the minimum allowed for each height.

The conclusions drawn by Bushnell from the recent work of German investigators (Military Surgeon, Jan., I9I3) must need cause a decided change in our views of tuberculosis infection and prophylaxis. According to these conclusions, all or nearly all civilized adults have come in contact with the tubercle bacillus and been more or less infected in childhood, an infection which has the effect of vaccination, producing an immunity to subsequent outside infection. Inasmuch as these adults remain constantly exposed to the bacillus, they are, as it were, continuously revaccinated. The experiments of Franz, of the Austrian Army, bear upon this question. He found that $6 \mathrm{I}$ per cent. of apparently healthy soldiers from a crack regiment reacted distinctly to tuberculin. It is now believed that a much greater proportion would have reacted had he used a larger dose of tuberctlin. This means that a vast majority of adults, therefore of soldiers, harbor live tubercle bacilli in their lymphatic glands, where they are reduced to a latent, innocuous state by the processes of immunity. No further harm from without is to be apprehended; the danger is from within, when, under depressing influences, there is a breaking down of the immunization and an invasion of the lungs or other tissue.

The infectious organism, Bacillus tuberculosis, is a minute, colorless rod, with regular outlines and slightly rounded ends, about five times as long as it is thick. As found in sputum it varies in length from one-fourth to one-half the diameter of a red blood-cell.

It is chiefly excreted in the sputum or spittle of the patient, so that his expectoration for 24 hours contains billions of it. It sometimes escapes in the urine and feces which, in advanced cases, may thus infect clothing and bedding, as well as the hands of the unclean. It is destroyed in water or milk at a temperature of $150^{\circ} \mathrm{F}$., maintained for 20 minutes, but, like that of typhoid fever, not by any degree of cold. In dry sputum, exposed in thin layers to the direct sunlight, bacilli lose their vitality in a few hours, and much more quickly if the sputum is in the shape of floating dust, but may resist several days, even in dust, if only subjected to diffused light. In thick masses of sputum, in which the coagulation of the outer sur- 
face forms a protective covering, the bacillus may remain alive several months.

Mode of infection.- The chief avenue of infection in tuberculosis was, until recently, held to be the respiratory tract, in view of the fact that the lungs are the parts generally most affected. Cornet and his school taught that the dried, pulverized tuberculous sputum, suspended with the dust and inhaled in breathing, was the ordinary mode of infection. This doctrine has now been abandoned on account of the great difficulty, if not impossibility, of pulverizing a tough, sticky substance like sputum into a sufficiently tenuous dust to float in the air and find its way into the finest ramifications of the bronchial tubes. The experiments of Nägel, Flügge and others have shown conclusively that bacilli are not easily detached from the moist surfaces of mucous membranes; that consumptive patients do not expel them in ordinary breathing, but may do so in coughing, sneezing and loud talking, being contained in the spray of fine droplets then ejected from the mouth. But such spray, as observed by Winslow, seldom extends more than a few feet in front of the patient, and soon falls to the ground, where it becomes harmless. The number of organisms thus expelled, as measured per cubic foot of air, is almost infinitesimal, so that the danger is more from actual contact with the spray than from any possible aërial infection. The dust lying on the neglected floors of wards to which tuberculous patients are admitted may contain enough bacilli to be dangerous, but only when kept in circulation so as to be inhaled by the occupants in sufficient quantity, a very unusual condition.

The food may become infected with the tubercle bacillus in many ways, especially through flies and other insects. Not only do flies carry it on their feet, but, as in typhoid fever, they also excrete it. Spillman caught flies in the spittoon of a consumptive and found virulent bacilli in their intestinal contents. Fly specks have been found to contain them. According to F. E. Lord, flies may continue to excrete living bacilli for 15 days after ingesting them. The tuberculous patient himself is a source of great danger, for his hands, face, beard and clothing are readily contaminated from sputum and fecal matter, and he may convey the infection to everything he touches, especially articles of food. In view of the fact that the bacillus of bovine tuberculosis is practically identical with the human bacillus, and capable of producing tuberculosis in man, 
milk and its products may become dangerous vehicles of infection (see Milk).

The contention that tuberculosis very rarely, if ever, occurs through direct inhalation of the bacillus into the smaller bronchi and air-cells was confirmed by the demonstrations of Aufrecht, Calmette and others that the tuberculous processes in the lungs always have their beginning in the finer capillaries, and not in the finer air tubes, as would be expected if the latter were the channels of transmission of the bacillus.

According to our best authorities, man acquires tuberculosis by inhalation and deglutition. By inhalation the bacilli (discharged with the spray from the mouth of patients) lodge in the mouth or naso-pharyngeal cavities; those that survive pass through the mucous membrane into the lympathics and are carried to the nearest cervical and bronchial glands. By deglutition, probably the usual mode of infection, the bacilli are carried with the food into the stomach and intestine; the survivors penetrate through the walls of the intestine and reach the mesenteric glands. In the lymph glands the bacilli may remain latent temporarily or permanently. Should they happen to overcome the resistance of the gland tissue they find their way into the veinules or the thoracic duct, and the right heart, from which they are carried to the lung capillaries, the finest and most complex capillaries of the body. It is also known that they may reach the left heart and thus enter the general circulation. The recent work of German investigators has confirmed the observations of Rosenberger demonstrating the frequent presence of the tubercle bacilli in the blood. They are usually quickly destroyed in the arterial blood, but when abundant or the vitality of the subject is much lowered and the power of resistance lost, they are capable of invading various tissues. Thus there is no doubt that bone and joint tuberculosis is always hematogenic in its origin.

The bovine bacillus, so far as pulmonary tuberculosis is concerned, is of little importance, but, according to Park, it causes Io per cent. of all deaths due to tuberculosis outside the lungs, while in children 25 per cent. of all cases involving glands, joints and bones are of bovine origin and due to the drinking of milk.

Prophylaxis.- If the doctrine of acquired immunity described above is accepted, as Bushnell thinks it should be, place and contact infections, for adults, become practically negligible dangers. There 
is no special need of separating a patient from his home, except to secure better treatment. The prevention of tuberculosis in the Army is practically reduced to the maintenance of good hygiene of the individual and his surroundings, above all an abundant supply of fresh air, so that his immunity may remain effective. But even then, there will always be a certain proportion of individuals, children and adults, unprotected or only imperfectly immunized, and, for their sake, it remains necessary to observe the usual precautions of prophylaxis, especially a reasonable degree of isolation.

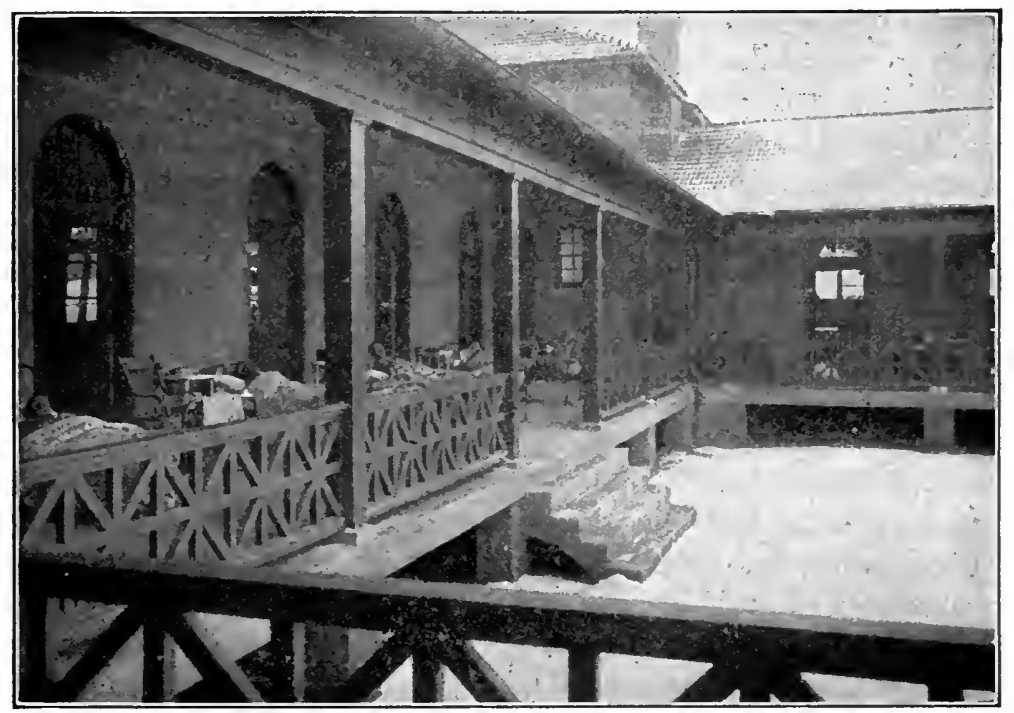

FIG. 5-- U. S. Army General Hospital, Fort Bayard, N. M. Patients sleep on the verandas in all weathers except during snow or rain storms. Note snow on roof and ground.

Nothing has been more satisfactorily proved than the preventive and curative effect of fresh air in tuberculosis; fresh air has accordingly become the prominent factor in the prophylaxis and treatment of this disease. (Fig. 5.) No dormitory should contain more beds than it has room for; this is the first principle in barrack hygiene, but one still insufficiently appreciated and frequently violated by the responsible authorities. Each man is entitled to a 
minimum of 60 square feet of floor space and 720 cubic feet of air space. To give him less is to greatly increase his chances of contracting infectious diseases, and the disastrous effects of overcrowding are shown in no more striking way than in rising rates of tuberculosis. But it is not enough to provide sufficient room; it is also necessary to supply adequate ventilation so that each man will get from 2,000 to 3,000 cubic feet of fresh air every hour, without draft. With plenty of air space and free ventilation, the danger of inhaling enough bacteria, of any disease, to become infected is greatly reduced if not entirely eliminated.

We may fairly assume that, in each company and barrack, there are or may be at any time one or more individuals in the infective stage of tuberculosis. The first care of the responsible officers must be to remove all such cases as soon as recognized. To that end noncommissioned officers should be instructed to regard as suspicious any man, especially a recruit, with an habitual morning cough, and to send him to the surgeon for examination. This precaution is not only on account of danger to his comrades, but also that he may receive the benefit of proper treatment as soon as possible. Under existing regulations, cases of tuberculosis are discharged on certificate of disability or else, if they so desire and their condition permits, sent to the Army Sanatorium at Fort Bayard, N. M., where they may be discharged from the service but kept under treatment as beneficiaries of the Soldiers' Home. The high altitude of the region, its equable temperature, dry air and abundant sunshine are the qualities of climate which make this sanatorium most suitable for the treatment of tuberculosis. Its chief purpose is the cure or arrest of the disease in incipient and moderately advanced cases, but it also cares for advanced cases, the beneficiaries of the Soldiers' Home. The majority of the patients whose disease is not so far advanced on admission as to end in death within the year, have their lives prolonged indefinitely. Too often, however, the disease is only discovered during an exacerbation due to the breaking down of the tubercle, and when already too far advanced to expect the best results. The remoteness of this sanatorium from the majority of posts is its main objection ; should a patient be subjected to the necessary journey of 3 or 4 days while in a high febrile condition, he may lose irrecoverable ground; such patients, before undertaking the journey, should be kept in bed until the fever abates. Compara- 
tively few men, when cured, are returned to duty, for the reason that, by that time, there is generally such a small fraction of his period of enlistment left that it is deemed better to discharge him than subject him to the mortification of being looked upon in his company as an object of suspicion.

The result of treatment in 358 "completed" cases, as reported in 1911 , was as follows: Of 212 incipient and moderately advanced cases, 12 were cured, I6 arrested, I 47 improved, 30 unimproved and 7 died. Of 146 far advanced cases, 1 was arrested, 88 improved, 27 unimproved and 30 died.

A similar sanatorium for the Navy and Marine Corps has also been established at Las Animas, Colo., and another for the Public Health Service at Fort Stanton, N. M.

An altitude of a few thousand feet is favorable to most patients but detrimental to some. The question of climate is not as important as formerly believed, and it is now generally recognized that most of the advantages of a sanatorium can be obtained at home by securing plenty of fresh air, day and night, rest of body and mind, and suitable food.

Since the bacilli are spread through the sputum, strict rules must be enacted and enforced against the habit of promiscuous expectoration, a habit as dangerous as it is loathsome. Spittoons should be provided, partly filled with a disinfectant solution, and anybody found guilty of fouling the floor, walls or furniture of his squadroom or dormitory with his expectoration, mercilessly dealt with. But, as in everything else when dealing with intelligent men, more is accomplished by appealing to the understanding than by threat of punishment. All enlisted men should receive such primary instruction in practical lygiene from their company officers or medical officers, as to know the principal diseases to whicl they are exposed and the measures to take to avoid them. In this way each man is interested in contributing to the general good health of his company and post. All apartments which have been occupied by cases of tuberculosis must be thoroughly disinfected. Clothing and bedding should be preferably steamed or boiled. In the case of a barrack, or other large room, fumigation is of doubtful utility since aerial infection is a negligible factor in the etiology of tuberculosis, but it should be thoroughly ventilated, and the floor, woodwork and furniture wiped with a solution of formalin, phenol or lysol. 


\section{PNEUMONIA.}

Next to tuberculosis, pneumonia is the most deadly of all diseases in the U. S. Army. It occurs in all climates but is much less common in tropical than in temperate or cold countries. For the entire Army, at home and abroad, during the decade of I900-I909, the yearly mean death rates per thousand of strength were 0.70 for tuberculosis and 0.42 for pneumonia; they have steadily decreased these latter years, especially for the former disease, so that for the period $1908-19$ Io they stand 0.44 and 0.36 respectively.

The usual etiological organism of pneumonia, whether of the croupous, catarrhal or septic type, is the Diplococcus pneumonic or Pneumococcus, a rather large, lancet-shaped coccus, occurring in pairs, and surrounded by a definite capsule. It is frequently found in the secretions of the buccal and nasal cavities of healthy persons, producing infection only when acquiring a certain degree of virulence, in a subject with lowered resistance. It may also cause (generally as complications of pneumonia) pleurisy, pericarditis, endocarditis and meningitis. Other organisms are also concerned in the causation of pneumonia, chiefly streptococci and the bacilli of influenza and of Friedlander.

In dry sputum the pneumococcus may remain alive and virulent 2 or 3 weeks when exposed to diffused light. In finely divided sputum, such as ejected in coughing, it survives only one or two hours. Exposed to strong sunlight it dies in a few minutes. It is very sensitive to heat and readily destroyed by the usual germicidal agents.

Pneumonia occurs most frequently in crowded, dark and illventilated dwellings. The habitual breathing of vitiated air renders the lungs especially vulnerable and increases the chances of infection.

This disease is not very contagious, but can doubtless be transmitted by the particles of sputum discharged in coughing or expectorating. It is best to isolate the patient and disinfect the sputum as well as all articles which come in contact with his lips and mouth. 


\section{INFECTIOUS DISEASES (CONTINUED).}

\section{MALARIA.}

Malarial fever, intermittent fever, ague, chills and fever, are different names for the same disease. Formerly ubiquitous in the inhabited parts of the United States, it has of late years lost much of its importance and is still steadily decreasing in prevalence and severity, the rate of admission for I9I I being only 14.68, or less than half that of $1907(30.20)$ shown in Fig. 6. For the years I9I4 and I9I5 the rate was I0.74 and 12.37 respectively. Not a single

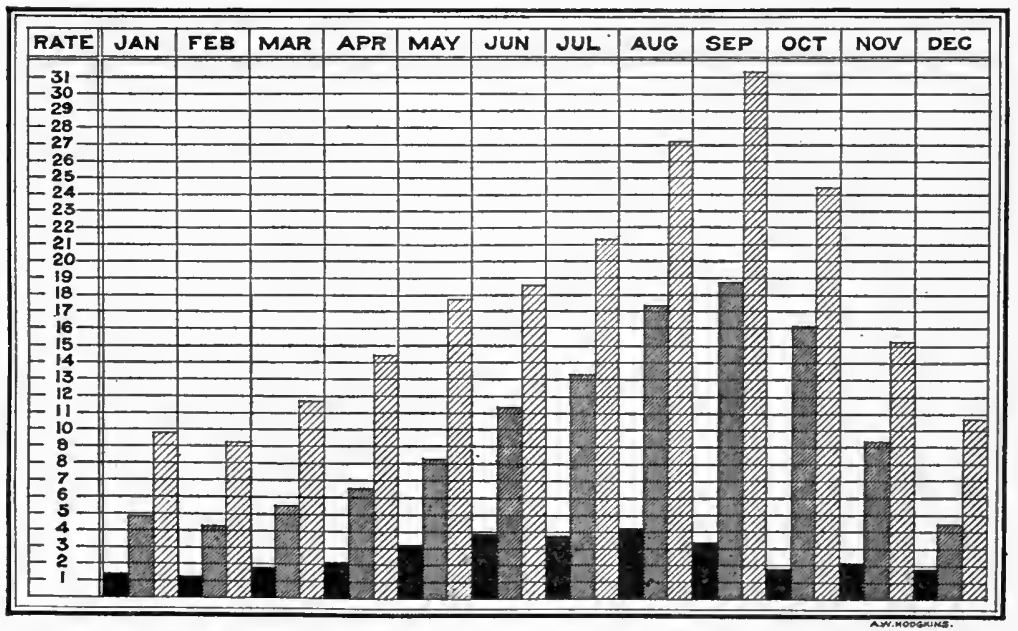

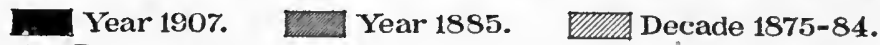

FIG. 6.-Diagram illustrating the distribution of cases of malarial fever by months per thousand strength, in the United States Army at home. Also showing their constant reduction.

death from malarial fever occurred in the Army in the United States from I906 to I9I5 (in which year two deaths were reported). This continuous improvement is due not alone to the active efforts of medical officers but also to the liberal provision for wire-gauze screens to protect doors and windows made by the Q. M. Corps. 
In the Philippines, the rate of admission for American troops has likewise steadily fallen from 167.79 for 1907 to 75.56 for I9I I, but rose again to $I 7 \mathrm{I}$ in $19 \mathrm{I} 2$ and $1 \mathrm{I} 2$ in 1913 , with a marked drop to 45 in 19I4. In I9I 5 malarial fever still stands third among causes of admission and non-effectiveness, that is, next to venereal diseases and dengue. Our troops in the colonies being now mostly comfortably quartered in permanent posts where preventive measures against mosquitoes can be efficiently carried out, it is confidently expected that the rates of malarial fever will continue to show a steady decrease.

Malaria is an Italian word meaning bad or poisonous air, from the belief that the disease was caused by emanations from the soil. It is now generally recognized that it results only from the biting of certain species of mosquitoes, and that soil or water are not directly concerned in its etiology. (See under Mosquitoes, page I59.)

Parasites.- In the act of stinging, while sucking blood, the mosquito injects saliva into the puncture and, if infected, the germs or parasites of malaria are carried along with the saliva. These parasites are microscopic animalcules, of the class sporozoa, genus Plasmodium, found in the red blood-cells. They were described by Laveran, in 1880 , but the first definite mosquito-malaria theory was formulated by Manson in 1894 and demonstrated by Ross in I898.

It is generally accepted that each form of malaria is produced by a distinct variety or species of parasite. The best classification of the malarial plasmodia, according to Craig, is as follows:

I. Quartan parasite (Plasmodium malaric).

2. Tertian parasite (P. vivax).

3. Estivo-autumnal tertian parasite ( $P$. falciparum).

4. Estivo-autumnal quotidian parasite ( $P$. falciparum quotidianum).

They are divided into two groups, the benign and the malignant. The benign parasites are two: the quartan, with cycle of 72 hours in the human body and causing a fever recurring every 3 days, and the tertian, with cycle of 48 hours, causing a fever recurring every 2 days. They are morphologically distinguished by not forming crescents and, clinically, by comparatively mild types of fever, easily controlled by quinine. The malignant parasites are the estivoautumnal tertian, with cycle of approximately 48 hours, and the estiro-autumnal quotidian (a sub-species), with cycle of approxi- 


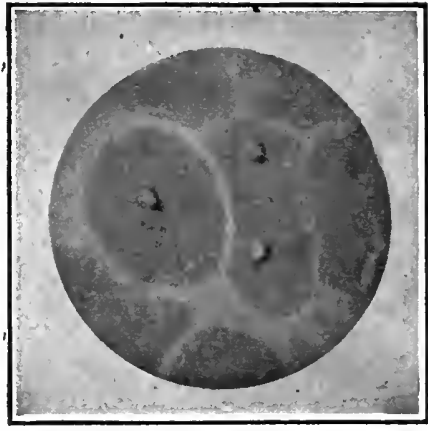

1

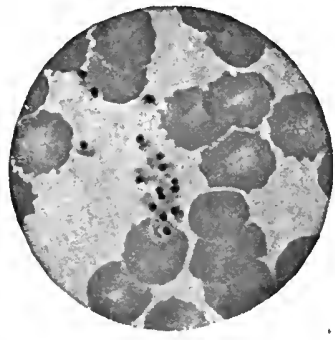

3

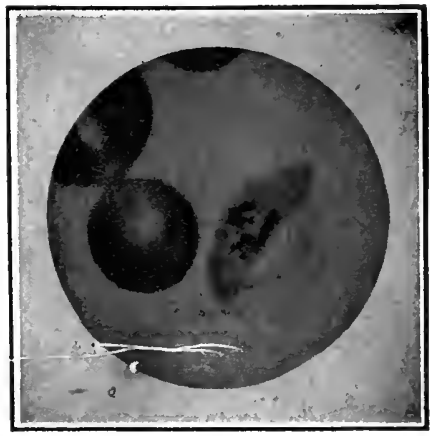

5

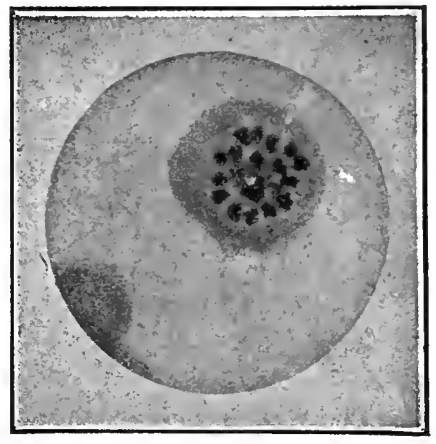

2

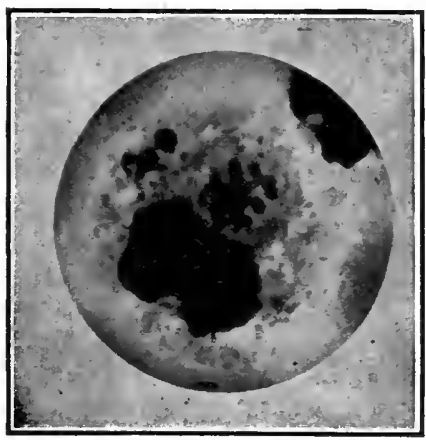

4

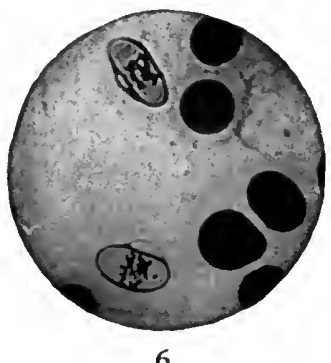

6

I. Estivo - autumnal plasmodium; young or ring forms.

z. Estivo - autumnal plasmodium; sporulating parasite.

3. Estivo - autumnal plasmodium; free spores or merozoites.

4. Estivo - autumnal plasmodium; phagocytosis of sporulating parasite.

5. Estivo - autumnal plasmodium; female gamete or crescent.

6. Estivo - autumnal plasmodium; male gamete or crescent. 
mately 24 hours. Craig has not been able to demonstrate the existence of a pigmented and unpigmented quotidian plasmodium as specifically distinct forms, "for while it is not uncommon to observe only unpigmented quotidian plasmodia in the peripheral blood in estivo-autumnal infections, the spleen invariably shows pigmented organisms." The malignant parasites are very much smaller than the benign ones, frequently causing the invaded blood-cells to become shrivelled, crenated and to assume a dark "brassy" color, but are chiefly distinguished by their assuming the shape of crescents. Clinically, they give rise to fevers of very irregular course and often of pernicious type, over which quinine has but little effect.

Whatever its species, the parasite exhibits two distinct phases or cycles, one inside the human body and one in the body of the mosquito, both cycles being necessary to its full sexual development and perpetuation.

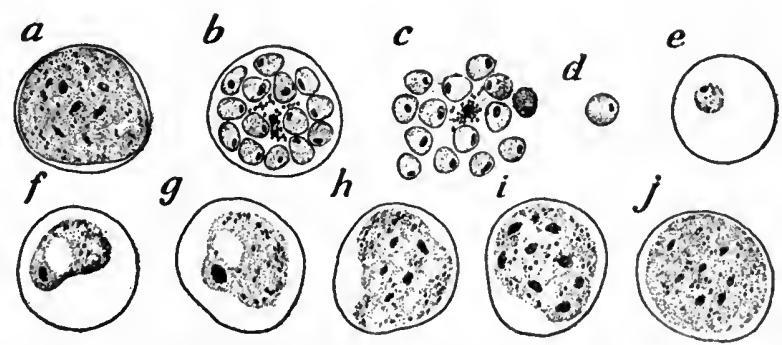

FIG. 7.- Evolution of the tertian parasite, stained. (Manson.)

Human cycle.- If the blood is examined microscopically shortly after a malarial paroxysm (Fig. 7), some cells may be found containing the young parasite, in the form of an ill-defined, pale, roundish body showing active movements; scattered through it are fine granules of reddish-black pigment (f. g.) ; after a while these granules collect into groups or lines $(h, i, j, a)$; still later, the body of the parasite, which fills the cell, divides into segments which become well-defined spherules arranged, rosette-like, around the pigmented center (b). The blood-cell now breaks down and the spherules become free in the liquor sanguinis (c, d). It is at this time that the chill, or first stage of the attack, takes place, due probably not so much to the presence of the spherules as to that of some toxin set free in the circulation. Most of the spherules are absorbed 
and devoured by the white cells (phagocytes). but some escape, attach themselves to other red blood-cells which they contrive to enter (e), and begin another cycle of life. The malarial parasite, thus exposed to the attacks of the white cells of the blood and other enemies. would soon disappear from the human body, and out of existence, did it not have means of propagating itself outside the body and re-entering it afterward.

It has been seen that the parasites, in reaching maturity, develop into rosette-like or segmenting bodies, but not all of them; some

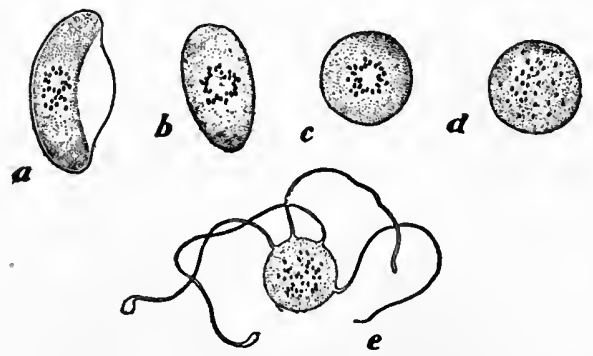

FIG. 8.- Evolution of the flagellated body from the crescent. (Manson.)

develop into the sexual form or gametes. The gametes are spherical in benign fevers, looking like ordinary large intracellular pigmented parasites, and crescent-shaped in the malignant fevers. The latter are of slow development and found only after a week or ten days of malarial infection; they are readily distinguished by their shape, their pigmented center and the convex line bridging over the concavity (Fig. 8, a). In the human body the gametes do not proceed any further in sexual development. The spherical gametes seldom last long, while the crescents may persist for months, sometimes disappearing from the superficial circulation but generally numerous in the deeper viscera.

The pigment elaborated in the parasite is hematin, set free by the decomposition of hemoglobin. At the time of the segmentation of the parasite and breaking down of the blood-cell, this pigment is liberated into the circulation. W. H. Brown has shown that it is an active factor in the production of many if not all of the important changes in the blood that characterize the various forms of malaria, and that to it are directly referable, at least in part, the acute and chronic symptoms of this disease. 


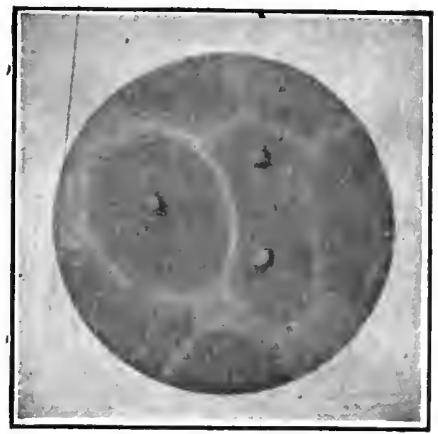

1

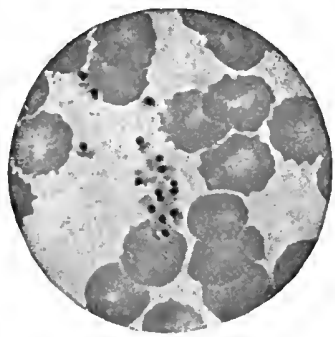

3

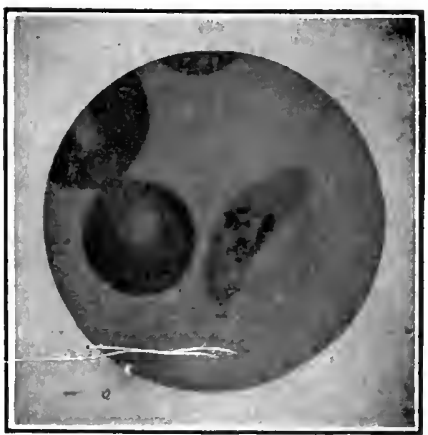

5

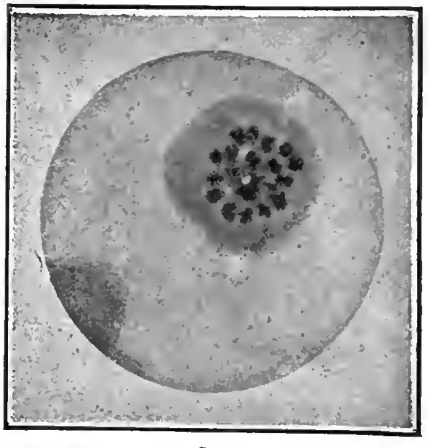

2

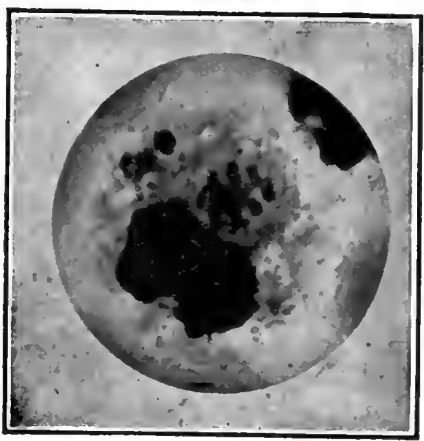

4

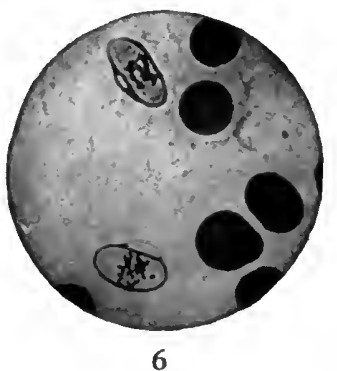

6

I. Estivo - autumnal plasmodium; young or ring forms.

z. Estivo - autumnal plasmodium; sporulating parasite.

3. Estivo - autumnal plasmodium; free spores or merozoites.

4. Estivo - autumnal plasmodium; phagocytosis of sporulating parasite.

5. Estivo - autumnal plasmodium; female gamete or crescent.

6. Estivo - autumnal plasmodium; male gamete or crescent. 

Mosquito cycle.-When blood containing gametes (spheres or crescents) has been sucked by certain species of mosquitoes, further changes soon take place. The crescents become transformed into spheres. The spheres from either source, benign or malignant, can now be differentiated into two kinds, male and female. The former are transparent or hyaline, the latter are filled with granular protoplasm. In the male sphere, one or more filaments or flagella (microgametes) suddenly grow out from the periphery. These flagella execute characteristic waving and lashing movements and often succeed in breaking loose from the sphere. Each flagellum thus set free enters and impregnates a granular or female sphere (macrogamete). The latter soon changes shape, becomes pointed and vermicular and moves about with great vigor. It finally lodges in the walls of the mosquito's stomach where it increases in size. Its nucleus and protoplasm divide into a number of spherular cells around which spindle-shaped, spine-like bodies are formed. These bodies or "sporozoites" become loose in the capsule and are discharged into the stomach. Thence they find their way, through the blood circulation, to the 3 -lobed salivary glands lying one on each side of the forepart of the thorax, and, as stated before, are injected, with the saliva, into the animal bitten by the mosquito.

It appears, from what has been said, that the malarial parasite undergoes important changes when passing from one host to the other, and is not infective until these changes are completed. Thus a man bitten by an infected mosquito does not develop symptoms of malaria until a week or ten days afterward, and probably does not become infectious to another mosquito for several days later. The mosquito which has become infected by sucking the blood of a malarial patient is not dangerous until the lapse of about ten days, or until the sporozoites have matured and been conveyed to the salivary glands

\section{Prophylaxis.}

This may be considered under the following heads:

I. Destruction of mosquitoes.

2. Protection against mosquito bites.

3. Isolation and protection of malarial patients.

4. Medical prophylaxis. 
The first three means of prevention are entirely based on our knowledge of the rôle played by the mosquito in the genesis of malarial fever. Not only the first, but the second and third as well, if carried out singly with completeness for a couple of years, would eliminate malaria. Thus, were all healthy men protected against mosquitoes, no new cases of infection would be possible; likewise, were all malarial patients so protected that no mosquitoes could get at them and become infected, the disease would necessarily come to an end. But it is evident that much better and quicker results can be obtained by a combination of these means.

I. Destruction of mosquitoes. - This is sometimes possible, in one or two seasons, by intelligent concerted action between individuals, or with adequate financial means provided by State or municipality and expended under the supervision of experts. Generally, complete extinction, in civilized countries, is only the result of steady and progressive cultivation and drainage for a period of years. But, fortunately, long before such result is obtained, in temperate climates, malarial fever will have practically disaappeared; that is to say, that the chances of contracting it decrease in a much more rapid ratio than that of the reduction of mosquitoes, on account of the coincident reduction of malarial cases from which the mosquitoes are infected. There is therefore, for each place, what may be called a minimum of safety, depending upon the amount of infective material accessible; for instance, two or three anopheles mosquitoes in a Filipino village, a majority of whose natives carry the germs of malaria, are much more dangerous than swarms of them in a New England town.

Only certain species of mosquitoes transmit pathogenic germs. Therefore it is important to ascertain, in each locality, whether those species are present and, if so, to study their habits and places of breeding. As a rule, however, different species, innocent and guilty, will be found breeding together under the same circumstances so as to make it difficult and often impossible to discriminate between them in our warfare.

2. Protection against mosquito bites. - In a malarial country, or wherever mosquitoes are prevalent, the mosquito-bar is indispensable. To be effective, it must be sufficiently long to rest upon the floor all round the bed, and its meshes sufficiently small to exclude all mosquitoes. The regulation mosquito-bar (model I9I2) now supplied 
to all the men of the U. S. Army, is made from bobinette, with not less than 182 meshes to the square inch ( $14 \times 13$ ). It is 66 inches long on ridge and base, and 61 from ridre to base. The triangles are $591 / 2$ inches long on ridge and 64 on base. It has 40 tapes arranged in pairs, each tape I I inches long and stayed with a piece of muslin. (Fig. 9.) It is intended not only for bedsteads, but also for shelter tents, cots and couches of any kind, and follows the lines previously indicated by Vedder of the Medical Corps.* This excellent bar is open to the objection that its meshes are somewhat too large; it is

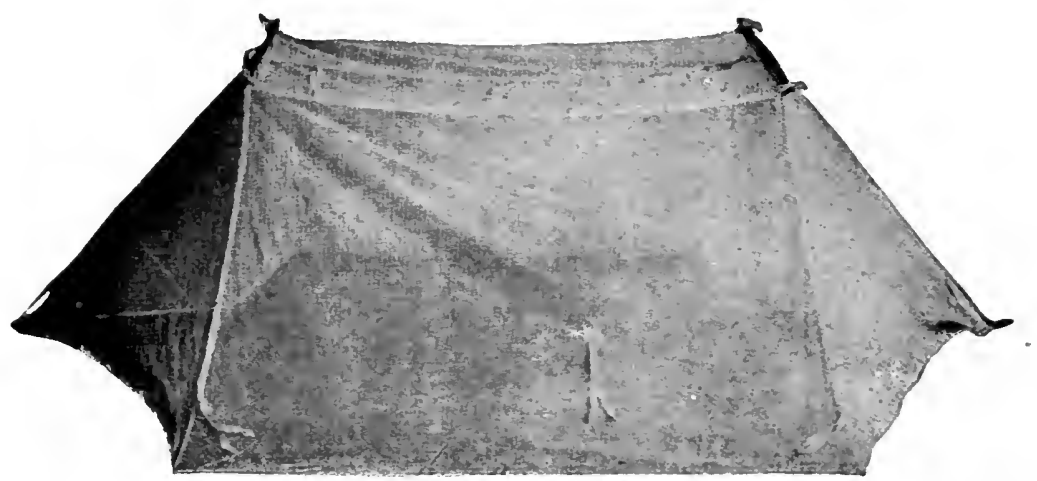

FIG. 9.- Regulation Mosquito Bar (Model 19I2). For single bedsteads and shelter tents.

the opinion of most observers that, in order to exclude all mosquitoes, the meshes should not be less than 16 to the inch. The mosquito-bar requires careful handling: a hole or tear makes it worse than useless, for mosquitoes will get in and be caught as in a trap, unable to escape. Its careful use should be the subject of strict disciplinary measures.

In countries where malarial or other mosquito-borne diseases prevail, or are apprehended, thorough prophylaxis requires also that, so far as practicable, all doors, windows, ventilators, etc., should be screened with wire netting so that mosquitoes may not get inside of buildings. Copper or other rust-proof wire is preferable; iron wire should receive two coats of good paint. There are few barracks

* The same officer has also devised a portable mosquito and fly-proof hut consisting of a knock-down frame and netting structure, 9 feet in all three dimensions. 
or other large inhabited structures, however, especially in the tropics, which can be so completely screened as to be mosquito-tight and render mosquito-bars unneecessary. Such result is obtainable only when the architect plans and builds with this end in view. All the new buildings in the Isthmian Canal Zone, for instance, are so constructed as to admit of complete screening, and it has been shown that this can be accomplished without sacrificing any of the requisites of air space and ventilation. (Fig. Io.)

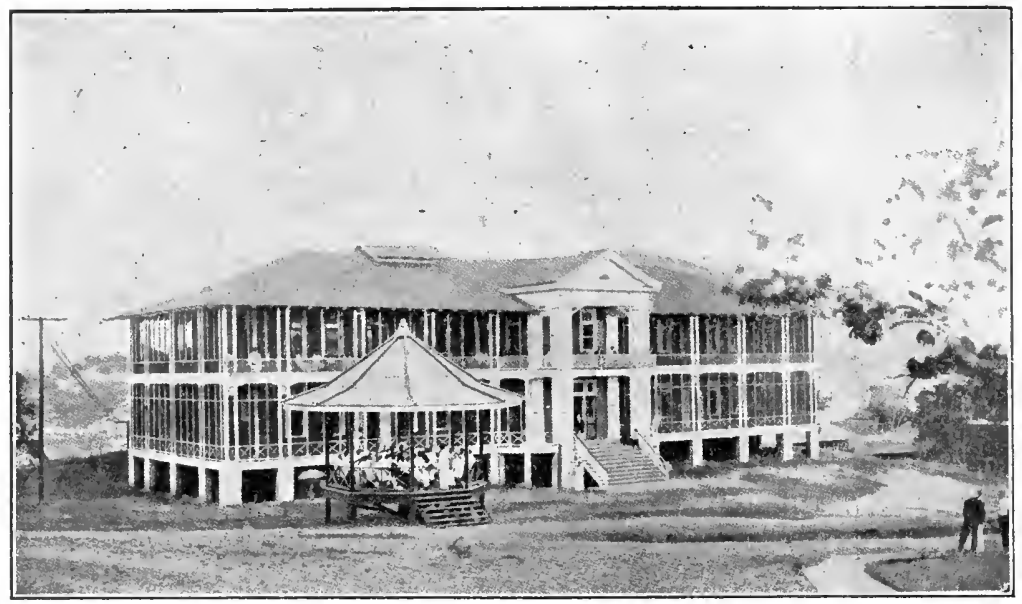

Fig. Io.-Club-house at Culebra, Panama, showing construction for thorough screening against mosquitoes.

In a malarial country and so long as mosquitoes are about, the soldier should be protected not only in garrison, but also in camp and in the field. Under these circumstances he should be supplied with a mosquito-bar, as part of his equipment, just as certainly as he is with a blanket and shelter-tent.

3. Isolation and protection of malarial patients.- As soon as a man suffers from febrile symptoms which may be reasonably ascribed to malaria, he should be isolated and protected, that is to say, placed in a screened ward, under a mosquito-bar, so that no mosquito can have access to him. Should the development of the case prove it to be not malarial, nothing will be lost by these preliminary measures.

Unfortunately, it is well known that the natives of malarial dis- 
tricts, especially in tropical countries, become more or less immune to the disease, and that, although their blood contains the parasite, they enjoy a fair degree of health, and attend to their usual daily occupations. In certain parts of the Philippines, and in the Isthmus Canal Zone, more than half of the natives were found thus infected, although not apparently incapacitated for work. These immune natives are a constant and widespread menace to other people, especially young soldiers. In and about their quarters infected mosquitoes are always found, so that to visit them, especially when these insects are most active, in the evening or during the night, is to court serious danger. Such visiting should be strictly prohibited. For this and other reasons which suggest themselves, a camp, other things being equal, should never be pitched in the immediate vicinity of native villages.

Before establishing a post or cantonment in an unknown country, it is very important to determine whether malarial fevers are present or not, and, if so, to what extent. This is best done by the examination of the blood of natives and of mosquitoes for the development stages of the plasmodia.

From what precedes it may be deduced that the prophylaxis of a post or camp, in a malarious district, involves likewise that of the surrounding communities; much can be done in that direction by education so as to secure the coopperation of all concerned and develop an appreciation, even by the poor and ignorant, of the advantages of screening, mosquito-bars and quinine.

Medical prophylaxis. - Two medicines have been recommended as preventives of malaria, arsenic and quinine. The prophylactic virtue of arsenic is very doubtful, and the drug is now seldom used for the purpose. Quinine is a well-known specific as curative and preventive. Its systematic use when campaigning in malarious districts will always considerably reduce the number of admissions. It should be supplementary to other means of prophylaxis, especially the use of the mosquito-bar, and not take their place. Where only tertian and quartan infections prevail, 2 grains (0.15 gram) a day is sufficient. Where the estivo-autumnal form occurs, quinine should be given in larger doses at longer intervals, no less than 15 grains ( I gram) once a week. Such preventive treatment need not be continued throughout the year, but only during those months when malaria prevails. 
Prompt and active treatment upon the first manifestation of the infection will almost always prevent the development of the malignant forms of plasmodia. Small daily doses should be given for at least three months after the disappearance of acute symptoms.

The researches of Bruin Mitzmain, Public Health Service, appear to have proved that the malaria parasites cannot live through the winter in hibernating mosquitoes, and that man is the sole winter carrier of malarial infection. Therefore it follows that malaria can be practically eradicated in any community in one year, independently of the number of anopheline mosquitoes, by proper quinine treatment.

As it is very probable that the immunity of natives in malarious districts is acquired at the expense of a certain amount of energy and vitality, it would be clearly to their advantage and that of all people having intercourse with them, should they be subjected to a sufficiently prolonged course of quinine to get rid of the infectious parasite and cease to be a menace to their neirhbors.

\section{BLACKWATER FEVER (HEMOGLOBINURIC OR HEMATURIC FEVER).}

An acute disease characterized by an initial chill, fever, bilious vomiting, jaundice and hemoglobinuria. The urine becomes very dark, sometimes almost black, and, as the paroxysm subsides, returns gradually, through shades of red, to its normal color. It is frequently diminished in quantity and sometimes suppressed. The fever may be intermittent, remittent or continued.

It is found in many tropical and subtropical countries, as well as in our southern States, and, as a rule, is most prevalent in malarial regions. The exact nature of it has not yet been determined. Many writers consider it simply a manifestation of malarial intoxication in subjects who have suffered grave or repeated attacks of malaria with marked destruction of blood globules. Christophers and Bentley, in India, have furnished strong evidence in favor of this view. Other investigators, however, maintain that the two diseases are not necessarily connected, that blackwater fever may be absent where pernicious malaria is prevalent, and exhibit itself in patients in whom the plasmodium parasite cannot be found. Sambon, Blanchard and others are inclined to regard it as a specific disease, like the hemoglobinuric fevers of cattle and horses, caused 
by a protozoön (probably Bebesia) which has hitherto escaped the search of the microscope. Quinine has also been incriminated, but on quite insufficient evidence. Even in toxic doses it never produces hemoglobinuria in healthy people, but in patients already suffering from the first stage of blackwater fever it may provoke or increase some of the manifestations of that disease.

Since blackwater fever is mostly observed in individuals who have, or have had, malaria, prophylaxis should consist in avoiding exposure to the causes of malaria. Quinine is indicated if plasmodium parasites are present in the blood, not otherwise.

\section{YELLOW FEVER.}

The home of this dread disease appears to nave always been the central portion of the American Continent, and more especially the West Indies. From such endemic foci as Vera Cruz, Havana, Kingston and Panama it has been carried southward as far as Buenos Ayres, and along the Pacific coast from Mexico to Peru. It has also occasionally visited the western European countries and the coast of Africa. Most of the Gulf and Atlantic seaports of the United States, as far north as Providence, have been repeatedly scourged by it, New Orleans and Philadelphia being the two greatest sufferers. This disease caused fearful loss of life in all the military expeditions to the West Indies during the last two centuries, and compelled the English, after their conquest of Havana in 1763 , to return that city to the Spaniards in exchange for Florida.

Etiology.- The mode of transmission of yellow fever was unknown until the year I900, when a commission of medical officers from the United States Army, consisting of Reed, Carroll, Agramonte and Lazear, appointed to investigate tropical diseases, made one of those brilliant discoveries which mark epochs in the progress of medical science. They proved that yellow fever cannot be contracted by contact with the patient or his effects; that it is not conveyed through the air nor through food or drink, but is always the result of mosquito bites. They supported their doctrine by such convincing demonstrations and arguments that, in spite of strong and prejudiced opposition, it was promptly accepted by the scientific world. So far as now known, yellow fever is transmitted by only one species of mosquito, Stegomyia fasciata (page 166). This mosquito, to be infected, must bite a patient during the 
first three days of the disease, and it cannot transmit the infection until the lapse of at least I 2 days after the bite. The subcutaneous injection of blood, or blood serum, from a yellow-fever patient into a non-immune person will also cause an attack within the usual incubation period.

The nature of the specific virus transmitted from patient to mosquito, and from the latter to non-immunes, remains unknown. Whether it is a soluble toxin, a bacterium or protozoön has not been determined, although the indications strongly favor the protozoön theory. However, if it be an organism, vegetable or animal, it is so tenuous as to be ultra-microscopic, beyond the power of our best microscopes. It has been proved that blood serum from a yellow-fever patient is capable of conveying the disease by subcutaneous injection, therefore contains the specific virus, even after being passed through a Berkefeld filter which excludes all visible microbes.

The propagation of yellow fever obviously requires three conditions: preexisting cases of the disease, Stegomyia mosquitoes and the presence of non-immunes. If there is no yellow fever in the locality, everything else may be disregarded. If in a town with good sanitary service there are but few cases, properly isolated, while, on the other hand, the Stegomyia mosquitoes are not common, the danger is a negligible quantity.

Prophylaxis.-Our full knowledge of the mode of transmission of yellow fever has robbed this disease of its terrors and made its prevention a comparatively simple matter. It has already disappeared from Cuba, Vera Cruz and Panama, and we may reasonably assume that it will be practically stamped out from the continent before many years have elapsed.

The rules to be followed suggest themselves; it is mostly a question of their strict and efficient application. As in the case of malaria, every effort must be made to destroy the mosquitoes or at least reduce their number. The Stegomyia is a domestic insect, therefore tanks and cisterns should be protected against it, and water not allowed to stand in gutters, spouts, tubs, vases and other receptacles in or about dwellings. It is a twilight mosquito, feeding early in the morning, and in the afternoon and evening until ten o'clock, so that non-immunes can go about in infected localities between $9 \mathrm{~A}$. M. and $3 \mathrm{P}$. M. with almost absolute impunity (Carroll). 
Houses should be screened, so far as it is practicable, and all inmates sleep under efficient mosquito-bars. As soon as any person (especially if non-immune) in an infected locality experiences symptoms of fever not readily accounted for, particularly if it begins with a chill and headache, he should at once be isolated and placed behind wire screens and mosquito-bar. Since the patient is only infectious to the mosquitoes during the first three days of the disease, the necessity of discovering and promptly isolating him, in the incipient stage, becomes clearly apparent. As soon as he is removed from his room, and before the mosquitoes have a chance to escape, disinfection must follow. As the only pathogenic germs are in the bodies of infected mosquitoes, the sole object of this disinfection, or rather fumigation, is to destroy all mosquitoes in the patient's bedroom and adjacent rooms and thus remove the only cause of further danger. For this purpose, the fumes of pyrethrum powder, campho-phenol or sulphur are used, as described under Insecticides. The disinfection of the patient's person, bedding, clothing and effects is entirely unnecessary.

The natives of zones where yellow fever is endemic are immune to it. This is attributed to the effect of a mild form of the infection which they are supposed to have had in infancy and childhood, the so-called borras fever of the Cubans. All non-immunes, in an infected locality, should be identified, brought together as much as possible, regularly inspected and the object of special sanitary measures.

From the day a man is taken sick, no secondary case can result before the lapse of at least I4 days, namely, I day or more before the secondary mosquito becomes infected, 12 days for the elaboration of the germ in its body, and I or more days of incubation after biting the secondary patient. Therefore if, in an outbreak, after a lull of 2 or 3 weeks, a new crop of cases appears, the presumption is very great that the mosquitoes infected by the primary crop, or some of them, are still at large.

The incubation of yellow fever ranges usually from 3 to 4 days, so that a quarantine period of at least 5 days is necessary. Any person suspected of having been exposed to the bite of infected mosquitoes should be kept under observation until 5 days have elapsed. Ships visiting infected ports should keep away from other vessels and anchor at least half a mile from shore. See Quarantine. 
Historical APPENDix. - The mosquito-malarial theory passed from the region of conjecture to that of fact in 1900 . The experiments of Ross, Grassi, Sambon and others had been closely followed by the medical officers of the U. S. Army, and suggested to Reed the possible agency of the mosquito in the transmission of yellow fever; nor were the medical officers, then serving in Cuba, slow in assuming the responsibility of recommending the general application of the conclusions reached concerning the conveyance of malarial and yellow fevers. As early as Oct. 15, I900, Col. J. R. Kean, then Chief Surgeon of the Department of Western Cuba, made the following statement in a communication to the Adjutant General of the Department:

"The role of the mosquito in the transmission of certain diseases is now well established. The evidence is now perfect and conclusive that malaria, as well as filarial infections, are carried by this insect, and there are reasons to suspect that it may be connected with the transmission of yellow fever also."

This was written before the Reed Board had published the results of its experiments; as soon as these were completed, upon the recommendation of Col. Kean, then acting Chief Surgeon of the Department of Cuba, the following order was issued:

\section{Genfral Orders; \\ No. 6 . \\ The Chief Sur} The Chief Surgeon of the Department having reported that it is now well established that malaria, yellow fever and filarial infection are transmitted by the bites of mosquitoes, the following precautions will, upon his recommendation, be taken for the protection of the troops against the bites of these insects :

I. The universal use of mosquito-bars in all barracks and especially in all hospitals, and also in field service when practicable.

2. The destruction of the larvæ or young mosquitoes, commonly known as "wiggletails" or "wigglers," by the use of petrolenm on the water where they breed.

The mosquito does not fly far and seeks shelter when the wind blows; so it is usually the case that each community breeds its own supply of mosquitoes in water barrels, fire buckets, post holes, old cans, cesspools, or undrained puddles.

An application of one ounce of kerosene to each fifteen square feet of water, twice a month, will destroy not only all the young, but the adult females who come to lay their eggs. The water in cisterns or tanks is not affected for drinking or washing purposes by this application if only it is drawn from below and not dipped out.

For pools or puddles of a somewhat permanent character, draining or filling up is the best remedy.

The Medical Department will furnish oil for the purpose above mentioned.

Post commanders will carefully carry ont these precautions. By Command of Major General Wood:

H. L. SCOTT, Adjutant General. 
The doctrine that it is un11ecessary to isolate yellow-fever patients, beyond screening them against mosquitoes, was so novel and startling that there was considerable hesitation in the official recognition and application of that great and far-reaching discovery. However, the irresistible conclusions of the Reed Board having soon removed all doubts, it became advisable to lay greater emphasis upon the new measures of prophylaxis required, and the Governor General ordered the publication of the following circular prepared by the writer, then Chief Surgeon of Cuba:

\section{C1RCULAR, \\ No. 5. 1 \\ Headquarters Departanent of Cuba, Havana, April 27, 1901.}

Upon the recommendation of the Chief Surgeon of the Department, the following instructions are published and will be strictly enforced at all military posts in this department:

The recent experiments made in Havana by the Medical Department of the Army having proved that yellow fever, like malarial fever, is conveyed chiefly, and probably exclusively, by the bite of infected mosquitoes, important changes in the measures used for the prevention and treatment of this disease have become necessary.

I. In order to prevent the breeding of mosquitoes and protect officers and men against their bites, the provisions of General Orders No. 6, Department of Cuba, December 2I, I900, shall be carefully carried out, especially during the summer and fall.

2. So far as yellow fever is concerned, infection of a room or building simply means that it contains infected mosquitoes, that is, mosquitoes which have fed on yellow fever patients. Disinfection, therefore, means the employment of measures aimed at the destruction of these mosquitoes. The most effective of these measures is fumigation, either with sulphur, formaldehyde* or insect powder. The fumes of sulphur are the quickest and most effective insecticide, but are otherwise objectionable. Formaldehyde gas is quite effective if the infected rooms are kept closed and sealed for two or three hours. The smoke of insect powder has also been proved very useful; it readily stupefies mosquitoes, which drop to the floor and can then be easily destroyed.

The washing of walls, floors, ceilings and furniture with disinfectants is unnecessary.

3. As it has been demonstrated that yellow fever cannot be conveyed by fomites, such as bedding, clothing, effects and baggage, they need not be subjected to any special disinfection. Care should be taken, however, not to remove them from the infected rooms until after formaldehyde fumigation, so that they may not harbor infected mosquitoes.

Medical officers taking care of yellow fever patients need not be isolated; they can attend other patients and associate with non-immunes with perfect safety to the garrison. Nurses and attendants taking care of yellow fever

* It was soon after this that formaldehyde was shown to be unreliable as an insecticide. 
patients shall remain isolated, so as to avoid any possible danger of their conveying mosquitoes from patients to non-immunes.

4. The infection of mosquitoes is most likely to occur during the first two or three days of the disease. Ambulant cases, that is, patients not ill enough to take to their beds and remaining unsuspected and unprotected, are probably those most responsible for the spread of the disease. It is therefore essential that all fever cases should be at once isolated and so protected that no mosquitoes can possiblv get access to them until the nature of the fever is positively determined.

Each post shall have a "reception ward" for the admission of all fever cases and an "isolation ward" for the treatment of cases which prove to be yellow fever. Each ward shall be made mosquito-proof by wire netting over doors and windows, a ceiling of wire netting at a height of seven feet above the floor, and mosquito-bars over the beds. There should be no place in it where mosquitoes can seek refuge not readily accessible to the nurse. Both wards can be in the same building, provided they are separated by a mosquitotight partition.

5. All persons coming from an infected locality to a post shall be kept under careful observation until the completion of five days from the time of possible infection, either in a special detention camp or in their own quarters; in either case, their temperature should be taken twice a day during this period of observation so that those who develop yellow fever may be placed under treatment at the very inception of the disease.

6. Malarial fever, like yellow fever, is communicated by mosquito bites, and therefore is just as much of an infectious disease and requires the same measures of protection against mosquitoes. On the assumption that mosquitoes remain in the vicinity of their breeding places, or never travel far, the prevalence of malarial fever at a post would indicate want of proper care and diligence on the part of the surgeon and commanding officer in complying with General Orders No. 6, Department of Cuba, I900.

7. Surgeons are again reminded of the absolute necessity, in all fever cases, to keep, from the very beginning, a complete chart of pulse and temperature, since such a chart is their best guide to a correct diagnosis and the proper treatment.

By Command of Major General Wood:

H. L. SCOTT, Adjutant General.

\section{DENGUE.}

Dengue, or break-bone fever, is an acute infectious epidemic disease of tropical and subtropical countries. It has frequently prevailed in the Southern States, as far north as Charleston, and occasionally reached Philadelphia and New York. Its mortality is very small, in uncomplicated cases practically nil.

There has been no case of dengue among troops in the United 
States during the last few years, but it is more or less endemic and of considerable military importance in the Philippine Islands on account of the rapidity of its spread and the large number of people infected during an outbreak. It is there one of the important causes of admission, the annual rate for the period 1904- 6 having been about 60 per thousand of strength, and, for the period 1909-1911, about IIo. As it is hardly ever fatal (only I death during the period 1909-19II) and has a very small non-effective rate, it constitutes rather an inconvenience than a sanitary danger.

Dengue presents much analogy to yellow fever in some of its peculiarities; it only prevails in warm weather, always ceasing with the advent of winter in the Southern States, and spreads most readily along the coast line, seldom extending far inland, or upward to a high altitude.

Nothing definite is known regarding the virus of this disease, but its mode of propagation seems to have been finally settled by the experiments of Ashburn and Craig (The Military Surgeon, September, 1907), whose conclusions are as follows: No organism, either bacterium or protozoön, can be demonstrated in either fresh or stained specimens of dengue blood under the microscope; the intravenous inoculation of unfiltered or filtered blood into healthy men is followed by a typical attack of the disease; the disease can be transmitted by the mosquito Culex fatigans and this is probably the more common method of transmission; the period of incubation in experimental cases averaged 3 days and 14 hours.

Bancroft, in Australia, also concluded that the germ is ultramicroscopic, but that it was conveyed by Stegomyia fasciata. 


\title{
INFECTIOUS DISEASES (CONTINUED).
}

\author{
MALTA FEVER. \\ (Mediterranean Fever.)
}

A continued fever of complex and irregular symptomatology, prolonged and indefinite duration, with frequent relapses, undulating thermal curve, sweats, rheumatic and neuralgic pains, constipation and frequently orchitis. Mortality low, seldom exceeding 2 per cent.

Its home is in Malta and other islands of the Mediterranean, as well as in all countries (of Europe, Asia and Africa) bordering on that sea, but has extended, in sporadic or endemic form, to most tropical and subtropical countries, including the southern United States and the Philippines. It was doubtless spread by the exportation of goats from Malta and Asia Minor to India, South Africa and America. Gentry and Ferenbaugh* have demonstrated its endemic existence in certain goat-raising sections of Texas where goats were first imported from the Mediterranean. They found that about 20 per cent. of the animals examined gave a positive agglutination reaction, although showing no manifest symptom of illness, and that several herders had suffered from this disease. It is probable that, in those regions, many cases are overlooked, being mistaken for atypical typhoid fever.

Malta fever is caused by the presence in the blood of a very small coccus (Micrococcus melitensis) found in all the organs, especially the spleen, and often in secretions and the urine, but not in the feces. It enters the body by the drinking of infected milk, or by the contact of the urine or milk with mucous membranes or the abraded skin. The urine may act indirectly by polluting the food or contaminating the soil. This organism possesses great power of resistance, having been recovered from khaki cloth, blankets and serge on the 8oth day.

This disease may affect all domestic animals. In Malta it was

*J. Am. Med. Ass., Sept. 30, I9II. 
found to be propagated mostly through the milk of goats. When cows become infected their milk is likewise a source of danger. The diagnosis can only be positively settled by hemoculture at the hands of an expert, or by the agglutination test. The Bureau of Animal Industry found that serum from healthy goats may give an agglutination of $I$ to 40; hence an agglutination of at least $I$ to 70 , within one and a half hours, is required for positive diagnosis.

Prophylaxis consists chiefly in the bacteriological examination of goat herds and the isolation and destruction of all diseased animals. The flesh of infected animals can be consumed with impunity if properly cooked. Their milk could also be utilized after boiling.

\section{PAPATACI FEVER. \\ (Three-day Fever, Sandfly Fever.)}

An infectious fever of short duration, never fatal but attended with marked debility and causing great loss of time in the military scrvice of Austria and Italy. It prevails in the basin of the Adriatic, often epidemically, and is occasionally found in all other countries bordering on the Mediterranean Sea. It is also reported from North India. It is caused by the bite of a very small fly (Phlebotomus papatasii). (See page 158 .) The infective organism has not yet been identified, being apparently invisible under the microscope and passing through the Berkefeld filter.

\section{SIX-DAY FEVER.}

Under this name, Dr. W. E. Deeks* has described a febrile disease peculiar to the Panama Canal Zone. It has an acute onset with chills, headache and backache, the temperature reaching as high as $104^{\circ}$ and falling to normal on the 6th day. The spleen is enlarged. Leucopenia, with high percentage of polynuclears, is the most important blood change. Albuminuria is almost always present. A rash, especially on the extremities, is frequently seen. The cause of this fever has not been determined.

\section{TYPHUS FEVER.}

An acute, very infectious epidemic disease, characterized by a profuse roseola and petechial eruption, formerly causing a fearful

*J. Am. Med. Ass., Oct. 26, I9I2. 
mortality in many parts of Europe, especially in camps and along trails of armies. Outbreaks have been rare in the United States and easily controlled, always resulting from imported cases. The disease is still endemic in several countries, especially Austria-Hungary (more particularly in Galicia), Russia, the Balkan countries, Great Britain and Ireland. The last epidemics of it were in the TurcoRussian War of 1878 , and in Servia in 1915. Many sporadic cases were found in most of the Russian base hospitals during the RussoJapanese War, and outbreaks of it were frequent in the belligerent armics during the first year of the European War.

It has long been observed that typhus fever is not spread by fomites or simple direct contact. The fact that it usually breaks out in crowded tenements, jails, emigrant ships and insanitary camps has long pointed to the probability of vermin as the propagating factor. Nicolle, in Tunis, $\uparrow$ transmitted this disease to monkeys by the injection of blood from human patients, and then from monkey to monkey by means of the ordinary body louse. Similar experiments were carried on by Ricketts and Wilder, + who transmitted the typhus fever of Mexico (tabardillo) to man and monkey through the same insect. It is now well established that the body louse and, to a lesser extent, the head louse, are the usual communicating agents of typhus infection. The above investigators also believed that the infectivity of the louse may be transmitted from one generation to the next by heredity, thus facilitating the spread of the infection in space and time, but this has not been confirmed by the later researches of Anderson and Goldberger.* The louse is infective until the $5^{\text {th }}$ day after feeding and perhaps later, but probably not after the 7 th. The bedbug and flea appear to be incapable of propagating this disease. The virus is present in the blood throughout the febrile period and for a day or two after the return of the temperature to normal. There is no evidence that it is capable of passing through a Berkefeld filter.

Anderson and Goldberger were unable to produce typhus in monkeys by the injection of the buccal and pharyngeal secretions from human cases, and therefore express the opinion that it is not transmittable by droplets ejected from mouth or nose.

$\dagger$ J. Am. Med. Ass., Feb. 5, I9Io.

† J. Am. Med. Ass., July 23, I9io.

* Collected Studies on Typhus. Hyg. Lab. Bull. 86, I912. 
A peculiar continued fever, known as Brill's disease, not uncommon in the lodging and tenement houses of our large cities, has recently been identified as a mild form of typhus fever. Thus an attack of it immunizes against typhus fever, as well as against tabardillo, a fact which also proves the identity of these two diseases. Therefore there is danger that, through increased virulence of the causative germ in Brill's disease, serious outbreaks of typhus may occur in this country, should proper sanitary measures be neglected.

The cause of the disease, whether bacterial or protozoal, still remains unknown.

Our knowledge of the mode of transmission in typhus fever suggests the necessary prophylactic measures, namely, prevention of crowding, house sanitation, personal hygiene and destruction of vermin.

"The fact should be kept in mind that the louse is as necessary for the spread of typhus as the mosquito is for malaria. Where lice are present typhus is possible; where typhus is present lice will be found." (Bull. 10, War Dept., 1916.)

Where typhus is present, daily individual inspection for vermin of the person and clothing of those caring for the patients, or otherwise exposed, should be strictly required.

\section{RELAPSING FEVER (RECURRENT FEVER).}

An infectious disease usually occurring in epidemics, characterized by second and third attacks after periods of remission. Fcrmerly common all over Europe and Asia, but there have been few epidemics of it in this country, where it never showed any tendency to spread. It is still endemic in the Philippines although no cases have so far been reported among our troops. Like typhus, it is a disease of poverty, overcrowding and insanitary conditions, but its mortality is small.

The specific cause is a spirillum (Spirochaeta recurrentis).

Nicolle (in Tunis) has shown that the louse becomes infected with it, although incapable of conveying it by biting; but when this insect is crushed on the skin of its host, the organism is released and may then be inoculated by scratching, probably the usual mode of transmission. This infection of the louse may also be inherited by its young. 
The relapsing fever of South Africa (African Tick Fever) is caused by another and larger spirochete (S. duttoni) which is transmitted by a tick (Ornithodoros monbata).

\section{ROCKY MOUNTAIN SPOTTED FEVER.}

(See Ticks, page 142.)

As defined by Rucker,* an acute endemic febrile disease, occurring chiefly during the summer months, and characterized by a continuous moderately high fever, severe arthritic and muscular pains, and a profuse petechial or purpuric eruption, appearing first on the ankles, wrists and forehead, but rapidly spreading to all parts of the body. The incubation period is usually 7 days.

This disease has been reported from nearly all the States of the northern Rocky Mountain region, but is especially prevalent in Montana and Idaho, having been first observed and still existing in its most virulent form in the Bitter Root Valley of Montana.

The mortality varies greatly, according to localities, indicating different strains of the infective organism; thus it is only 4 per cent. in Idaho, while often exceeding 70 per cent. in parts of Montana.

Although the causal germ has not yet been discovered, careful investigations and experiments have demonstrated that it is transmitted by ticks, but chiefly if not exclusively by the wood-tick, Dermacentor andersoni (D. venustus), which attaches itself not only to man but to various other mammalian hosts, infecting them and, in turn, being infected by them. It is evident that man himself is only an occasional and accidental host and that, when sick with this fever, ticks have seldom any chance of becoming infected by feeding upon him.

From the experiments of Ricketts and others, it appears that the following animals, which are also the most common hosts of the tick in its immature stages, are susceptible to spotted fever through tick bites and can infect other ticks: The Columbian ground squirrel (Citcllus columbianus), woodchuck (Marmota flaviventer), sidestriped ground squirrel (Callospermophilus), yellow-bellied chipmunk (Eutamias). Whether all of these, or more, act as the usual intermediary hosts for the causative parasite, thus perpetuating the discase, has not been determined. The Columbian ground squirrel,

* The problem of the Rocky Mountain spotted fever, by W. C. Rucker, The Military Surgeon, Dec., I91 1 . 
on account of its great prevalence in the infected zone, should be regarded as the most dangerous.

The measures of prevention suggest themselves, namely, the destruction of ticks, especially of adults while feeding on domestic animals, and personal prophylaxis, that is to say, the wearing of tick-proof clothing by persons entering the infested zone, and the careful daily search of the body.

It is known that in cattle having Texas fever (likewise conveyed by ticks) the causative organism may remain in the blood for years afterwards and that ticks feeding upon these apparently healthy animals may become infected and transmit the disease. Should it be likewise with persons having spotted fever, they could act as carriers and spread the infection to new regions should suitable ticks be found there to act as transmitters.

\section{PLAGUE.}

Plague is an acute infectious disease with rapid and severe course and great mortality. Formerly one of the worst scourges of the Old World, it is now rarely seen in epidemic form, but remains e11demic in many parts of America and Asia. For us there is danger chicfly from South and Central America, Mexico and the West Indies. In 1912, spots of infection still existed all along the western and eastern coast lines of South America, and small outbreaks were reported from Porto Rico and Cuba.

In this country it first manifested itself in 1900 , in the Chinese quarter of San Francisco, probably imported in rats from Hawaii. and was not eradicated until 1904, after having caused the death of 225 people. It reappeared in 1907 , but was promptly suppressed.

Three types of plague are recognized: bubonic, pneumonic and septicemic. In the bubonic form, which is the most common, the infection occurs throngh the skin and is first localized in the nearest lymphatic glands, which swell into characteristic buboes. In the pneumonic form, the respiratory tract is the portal of entrance and the disease takes primarily the form of a bronchopnemmonia. In the septicemic, there is a general blood infection from the beginning. The last two, almost invariably fatal, were the "black death" of the Middle Ages.

Bubonic plague does not resist a temperature higher than $85^{\circ} \mathrm{F}$. or lower than $50^{\circ}$. The pneumonic form is favored by cold, being restricted between the isothermal lines of $40^{\circ}$ and $50^{\circ} \mathrm{F}$. 
The causal organism is the Bacillus pestis of Kitasato, also pathogenic for nearly all animals. It is a short, thick bacillus with wellrounded ends, showing characteristic bipolar staining with intermediate unstained area. It is frequently found in the urine of patients, but very rarely in the feces. Outside of the animal body it may retain its viability for months in a dark and moist environment. In pus or sputum from patients it may live one to two weeks. Direct sunlight destroys it within four or five hours. Steam or boiling water is effectual in a few minutes.

In pneumonic plague the bacilli ejected in coughing are soon destroyed in a dry atmosphere, but retain their vitality much longer in humid air. As pointed out by Teague and Barber, inasmuch as the atmospheric relative humidity increases with the degree of cold, it follows that pneumonic plague is more commonly found and spreads more rapidly in cold than in warm countries. The only great epidemic of this infection in modern times occurred in Manchuria and northern China during the winter of I9IO-I9II.

The incubation of the several types of plague, in man, is from 3 to 5 days, while the duration of the disease seldom exceeds 5 or 6 days.

Investigations in India have definitely determined that bubonic plague is primarily a disease of rats, and that the flea is the intermediary in the transmission of the infection from rat to rat and from rat to man. Two species of rats, found nearly in all parts of the world, are chiefly concerned: the brown or Norway rat, also called ship or sewer rat (Mus decumanus), by far the most common, and the black or house rat (Mus rattus), including a white-bellied variety (Mus Alcrandrinus). An epizootic of these rats always precedes a human epidemic. The common house mouse (Mus musculus) may also become infected, but its flea (Ctenopsylla musculi) hardly erer bites man.

The several species of fleas concerned (see page 145) live indifferently on the brown and black rats. It is only three days after the death of these hosts that they seek others and are likely to bite man; therefore, in plague-stricken countries all rats should be burned up within 48 hours after their death so as to insure the destruction of their fleas. The two most dangerous, because readily attacking man when hungry, are the Xenopsylla cheopsis in tropical and subtropical climates, and Ceratophyllus fasciatus in cooler 
regions. The dog, cat and human neas seldom play any part in plague transmission.

The plague bacillus is found in the digestive tube and excrements of the flea from 4 to 6 days after sucking infected blood, and thereafter rapidly disappears. The flea infects directly while biting, through the germs adhering to the mouth parts, or oftener indirectly through its habit of defecating while sucking blood, so that germs are deposited on the skin close to the bite; if the latter is rubbed or scratched, the germs get into it or in any other minute wound of the skin

Certain authors, notably Le Dantec, hold that, since rats are promptly destroyed by plague there must be other animals of the same or allied families, like marmots, gophers and woodchucks, which by acquiring a certain degree of immunity can act as permanent hosts and natural reservoirs for the plague bacillus, until opportunities happen for rat infection and a human outbreak. Such a permanent host was discovered in California. In I907, bubonic plague having reappeared in San Francisco, it was ascertained that the ground squirrel or gopher of the Pacific Coast States (Citellus beecheyi), a rodent quite common east and south of that city, was the remote cause of it, many of these animals being found infected and harboring fleas capable of transmitting the bacillus to rats by which it was conveyed to man. They are the more dangerous that they acquire a relative immunity and may continue indefinitely as hosts. Two other species of gophers ( $C$. franklini and $C$. tridecemliveatus) are numerous enough east of the Rocky Mountains to be troublesome in cultivated districts and a source of danger in case of plague outbreak. The plague bacillus has likewise been found in the wood rat (Neotoma fuscipes) of the Western, Middle and Southern States.

The mode of transmission of the pneumonic plague has not yet been completely cleared up. The rat and flea do not appear to be important factors. In the great epidemic which prevailed in Manchuria in I9II, only the pneumonic type, or a combination of this with the septicemic, was seen. It is supposed to have originated among the trappers of tarbagan (Arctomy's bobac*) in handling the skins; whether through direct inoculation, the biting of fleas or the

* A marmot related to the common American woodchuck or ground hog (.1. monax) which is abundant in the United States and Canada. 
eating of the flesh has not been ascertained. A severe epizootic had been prevailing among these animals and destroyed many. Neither rats nor other animals were found to be infected. The disease was introduced into every new center by the arrival of persons actually suffering from plague or incubating it. It was shown that the human host and rather close contact were the two most prominent factors in the spread of the infection. Transmission by clothing, merchandise or vermin was not proved. In pnetumonic plague the bacilli are not usually expelled in quiet breathing, but are abundantly disseminated through the surrounding air by coughing, sneezing and speaking. The sputum is loaded with them, but they may be ejected in large numbers even when there is no visible particle of it. From the studies of Strong, Crowell and Teague, it would appear that plague pneumonia results from inhalation, the infection first involving the bronchi and thence extending to the air-cells and the blood, and that the bacilli are not primarily absorbed into the general circulation and secondarily carried to the lungs. To what extent the bacilli may be absorbed indirectly through soiled hands or contaminated food is not known.

Diagnosis. - The material for smears and cultures in bubonic plague should be drawn from a bubo with a syringe; in the pnet1monic type, the sputum must be used. Animal inoculation, especially of the guinea pig, is very useful; smearing the material into the intact, shaven skin of this animal with a glass spattula will produce the infection. In pneumonic plague the pulmonary plysical signs are obscure in the early stages and render the diagnosis difficult.

Prophylaxis.-Consists essentially in good sanitation, the destruction of intermediary hosts, especially rats, and the use of protective vaccination. Plague thrives in filthy places, while it seldom attacks people living in clean surroundings. The greatest risk always threatens bare-footed and bare-legged people. In an exposed community, a rigorous quarantine of at least five days should be maintained against all stuspected ships and persons, even at the risk of serious interference with business, for when plague has secured a foothold it is exceedingly difficult of eradication. Every effort should be made to recognize and isolate the first cases.

Since man, in bubonic plague, is almost always infected from rats, the first preventive measures should be directed against them, 
by rat-proof buildings and sewers, cleaning out and rendering inaccessible their usual haunts, removing their food supplies, especially ill-kept garbage cans and, finally, by sulphur or hydrocyanic acid fumigation and the use of traps and poisons. Of the latter, phosphorus, in the proportion of 4 per cent. in a glucose base ( $W . C$. Rucker), is among the best; rats like it; it is cheap and certain in its action. The rat is the most abundant, useless, destructive and dangerous of the predatory animals infesting human communities, and a continuous warfare should be waged against it until its complete extinction. Should any other rodent be found infected and a possible reserve source of bacilli for rats, their systematic extermination should also be undertaken. For the destruction of fleas, see page 146.

In pneumonic plague, attention should be mostly directed to measures of isolation and disinfection, the destruction of rodents being only a secondary matter. The use of masks or respirators, by the attendants, is the most important measure of direct presonal phophylaxis. The mask should protect the mouth, nose and eyes, as well as any abrasion of the face and neck.

Patients should be promptly removed to a special hospital, and their houses, with all contents, thoroughly disinfected, special attention being paid to rats, mice, fleas and flies.

Haffkine's method of prophylaxis by the inoculation of cultures of the bacillus killed at a temperature of $65^{\circ} \mathrm{C}$., reduces the mortality, it is claimed, to about one-fourth. The immunity thus conferred only lasts a few months. Yersin's serum from immunized horses has also prophylactic value. 


\section{CHAPTER VII.}

\section{INFECTIOUS DISEASES (CONTINUED).}

\section{EPIDEMIC CEREBROSPINAI MENINGITIS.}

An acute infectious disease with special lesions of the meninges of the brain and cord. Common in Europe and America, but chiefly at home in this country, where it causes a notable proportion of the yearly mortality in crowded and unsanitary districts. Among our troops, in the United States, small outbreaks occur every year, with a mortality of over 50 per cent. During the 5 years I907-I9II, the number of admissions was 65, with 49 deaths. During I9I5, 23 cases were reported, with 9 deaths. Very few cases occur in our colonies.

The accepted canse, in the epidemic and sporadic forms, is a double coccus, Diplococcus intracellularis meningitidis of Weichselbaum, or Meningococcus, found not only in the involved tissues, but also in the secretions from the mouth, nose and pharynx. It possesses a low degree of vitality and quickly perishes when removed from the body and exposed to air and sunlight, or to heat and cold, so that it is impossible to demonstrate it in air, dust or fomites. It is most probable that transmission takes place directly, from person to person, by inhalation of droplets ejected by patients or carriers in coughing, sneezing or speaking. The mode of access to the cranial cavity is a matter of some doubt, probably by absorption into the circulation through the mucosa of the nose and pharynx.

It is also quite likely that infection may result from the use of common eating or drinking utensils, contamination of food by soiled fingers, etc.

Very few persons of those who acquire the organism are susceptible to it and become infected, but they may transmit it to stisceptible persons, so that the disease is mostly disseminated by carriers. 'The germs, in the naso-pharyngeal cavities of carriers, seldom last longer than 30 days, but may remain alive several months; some persons carry them permanently.

Whenever the diagnosis is doubtful it can be cleared up by a lumbar puncture and the examination of the spinal fuid for the 
meningococcus. But as this is not always easily differentiated, resort may be had to the high agglutinating value of immune-horse serum.

The prophylaxis consists in the isolation of patients, with sufficient air space and ventilation, and all other measures tending to prevent contamination with the oral and nasal secretions. The attendants should wear gowns and, besides, a cap and a mask of gauze.

Wheraver this disease prevails, carriers are so many that it is generally impracticable to quarantine them, but, as far as possible, they should be detected and isolated, or at least kept under observation and carefully instructed as to what to do and what to avoid.

The treatment consists chiefly in the injection of the antimeningitic serum of Flexner and Jobling in the spinal canal after a certain quantity of spinal fluid has been withdrawn. Generally from 20 to 30 c. c. are injected daily until 3 or 4 injections are made. This serum has reduced the mortality from 73 to 25 per cent. in adults, and to less than 20 per cent. in children.

Excellent results have also been reported from vaccination with meningo-bacterin, the reaction being very much like that observed after the use of typhoid vaccin.

\section{DIPHTHERIA.}

A disease of minor importance in the Army. During the 3 years I908-I9IO, I56 cases were reported, with 7 deaths, corresponding to yearly rates of 0.75 per thousand of strength for admission and 0.03 for deaths. Diphtheria is rare in tropical countries; during the decade I900-I9IO, only 82 cases were reported in the population of Manila. Since the introduction of a specific antitoxin in its treatment, the mortality has fallen in New York City from 14 per I0,000 of population (before I895) to 2.17 in I9II.

Diphtheria is caused by the bacillus of Klebs and Loeffler, which induces a specific reaction in mucous membranes, especially of the throat, larynx and nose, resulting in the formation of psendomembranes. The symptoms depend partly upon the mechanical disturbances and local inflammation caused by these membranes, but chiefly upon the systemic poisoning from the toxin secreted by the bacilli.

Active and virulent bacilli may linger for weeks in the mouths, throats and noses of persons who have entirely recovered from the 
disease. It has also been found that mild colds and sore throats may be due to the diphtheria germ, although the subjects are not sick and present no characteristic symptoms. Thus carriers are not uncommon during outbreaks of this disease.

The infection is directly transmitted by the minute particles of psettomembranes ejected in coughing and sneezing. Therefore no patient should be released from isolation until the absence of bacilli from the throat and nose has been demonstrated by 2 or 3 successive negative cultures at 48 -hour intervals.

With the exception of the clothing and bedding, no terminal disinfection is now deemed necessary.

\section{SMALIPOX (VARIOLA).}

Smallpox, although the most preventable of infectious diseases, by vaccination, continues to be widespread in the United States. The number of cases reported from 27 States, for I9IO, was 30,352, with $4 \mathrm{I} 5$ deaths. The very low case mortality (r.4 per cent.) shows the type of the infection to be very mild, as it had been during the previous I2 years, probably an indirect result of generalized vaccination. During the 3 years I908-1910, 32 cases occurred in the U. S. Army, with I death, all sporadic cases contracted outside of military reservations. During 1915 , the same widespread prevalence continued in the United States, but among our troops only 3 cases occurred (all in United States), without death.

The period of incubation in smallpox is about 12 days, and that of invasion 3 or 4 days. It is a highly contagious disease from the earliest symptoms until the completion of desquamation, about six weeks. Its causative organism or virus has not yet been determined; it exists chiefly in the contents of the pustules, as well as in their crusts and scales. It is certain that this disease is transmitted with extreme ease, being one of the so-called air-borne infections. Most probable, however, as in measles, it is only transmitted by contact, direct or indirect.

Vaccination, properly done, confers absolute immunity against smallpox for a number of years. Calf lymph is almost exclusively employed at the present day. There is strong evidence, however, that the matter obtained directly from the pustules of variolous patients confers more complete and lasting immunity, and is therefore preferable whenever the subject furnishing it is known to be 
free from syphilis and other inoculable infections. The immunity given by calf lymph lasts 8 or Io years in youth, but much longer in the adult period of life. It is necessary that a person vaccinated in infancy be revaccinated when II or I2, and again when about 22 or 23 years old. Many careful observers contend that an adult with good scars showing two successful vaccinations should be considered immune for life and excused from further operation. As the degree of protection greatly depends upon the amount of virus inserted, not less than three spots, an inch apart, and each about half an inch in diameter, should be scarified and inoculated. The scar left by vaccination to be perfect, that is to say, an evidence of successful operation, should be uniformly pitted and free from smooth cicatrix tissue, the result of sloughing.

It is doubtful whether there are any persons, not having had smallpox, immune to primary vaccination. Failure, as a rule, is the result of inert lymph, careless method or removal of virus by rubbing or washing. It is therefore expedient to vaccinate scarless recruits, having no history of smallpox, repeatedly until success follows or absolute immunity is satisfactorily established.

At the first enlistment all recruits must be vaccinated, except those who are pock-marked, and those who have good scar evidence of two successful vaccinations, the last not to be more than six years old, or of one successful vaccination performed within the three previous years. At every reenlistment a soldier should be revaccinated who does not bear the evidence of two successful vaccinations, or of one successful vaccination performed within the three previous years. Before scarifying the skin, it should be carefully disinfected and the disinfecting material washed off with sterile water; after the operation a protecting shield or dressing should be applied.

The usual measures of prophylaxis where smallpox breaks out consist in the strict isolation of patients and the thorough disinfection of their bedding and clothing. Their rooms may also be fumigated, although this is of doubtful utility. All unprotected persons who have been or may be exposed to the contagion should be promptly vaccinated.

Varioloid is a mild form of smallpox occurring in persons who have been imperfectly vaccinated or whose immunity has been partly lost. It is dangerous only in its capability of transmitting true smallpox to unprotected persons. 
Chicken-pox (Varicella), a mild, harmless eruptive fever, lasting but a few days, is entirely distinct from smallpox and therefore does not protect against it.

\section{SCARLET FEVER (SCARLATINA).}

Scarlet fever is of secondary importance in the Army. During the three years 1909-I9II, only 43 cases, with one death, were reported. It is not as highly infectious as measles, nor is there the same susceptibility to it on the part of soldiers, but, on the other hand, the vitality of its causative agent or virus is very remarkable, often persisting for several months. The nature of this specific cause is not known. The streptococci nearly always present in the throat of patients are only a secondary growth, responsible for some of the complications of the disease. Certain inclusion bodies are always found in the polymorphonuclear leucocytes of the blood during the first week, but they are also secondary invaders, likewise present in diphtheria and other infections; their only value is in differentiating scarlet fever from measles and rötheln.

Scarlet fever is transmitted by the secretions from the nose and mouth, and probably by the desquamating skin and the urine. There is danger of contagion from the first appearance of the symptoms until the cessation of discharges and completion of desquamation.

On account of the peculiar resistance of the virus, careful disinfection is more essential than in measles and most other infectious diseases. It should include not only all fomites, but also the patient's room. It is advisable to segregate cases with secondary infections from simple uncomplicated cases.

\section{MEASLES (RUBEOLA OR MORBILLI).}

Measles is a highly contagious disease from which few children, in cities, find it possible to escape. Although usually a mild form of infection, with low mortality, its prevalence is such that, according to the official census of I9IO, measles stands next to diphtheria, of all infectious diseases, as a cause of death among children. In the Army it is responsible for more deaths than all the other eruptive fevers together. For the three years I908-i9ı, the yearly ratio of admissions per thousand of strength was 9.62, and of deaths 0.24. During the year 19I0, 3I7 cases occurred in the Army with only one death, but in I9I I, a severe epidemic prevailed at Columbus 
Barracks, O., with 126 cases and 16 deaths, the principal complications being broncho-pneumonia, pleurisy and otitis media.

During 1915, 555 cases occurred in the Army (chiefly at the recruit depots) with 20 deaths and 8 discharges, being the disease causing the greatest number of deaths, next to pneumonia. In camps, cases of measles should always be expected, watched for and promptly isolated.

The incubation period is about 12 days, and that of invasion 3 or 4 days. The causative agent is unknown, but Herkoen (I905) first demonstrated its presence in the blood. Goldberger and Anderson of the Public Health Service, by experimenting with mon. keys, have lately shown that it is present in the blood at least a few hours before the eruption appears and for about 36 hours after. They have also demonstrated that it is contained in the nasal and buccal secretions during the first 48 hours of the eruptive period, and that it is absent from the desquamating epidermis. From these discoveries several inferences may be drawn, namely: that measles is not an air-borne disease as generally understood; that most, if not all, cases are contracted in the early part of the eruptive stage, often before a diagnosis is made and the patient isolated; that its virus is short-lived; and that the quarantine after desquamation, usually imposed on patients, may be considerably reduced.

No evidence exists that there are carriers of measles or that healthy persons have anything to do with its propagation; the patient alone is the source of danger. It is therefore an unnecessary hardship, when a case occurs in a family, to quarantine the entire household.

The measures to prevent the spread of this disease consist chiefly in an early diagnosis and the effective isolation of patients. It is desirable, when possible, to separate cases with secondary infections from simple uncomplicated cases. In an outbreak at a post or camp, every man who has not had the disease should be required to report daily to a medical officer to be examined, especially for Koplick's spots, rise of temperature, coriza and cough. The discharges from the eyes, nose and mouth should be received on pieces of cloth and burned or otherwise disinfected. The hands of the nurses must be carefully and frequently washed. It is best to disinfect the patient's clothing and bedding, but unnecessary to disinfect his room.

Rötheln or German measles (rubella) is a contagious eruptive dis- 
order bearing some resemblance to measles and scarlet fever, but showing its distinct specific character in the fact that it protects from neither of these diseases. It is very mild, seldom attended with any febrile movement and always ending in perfect recovery.

\section{MUMPS (PAROTITIS EPIDEMICA).}

A very infectious disease, of little gravity and no mortality, but sufficiently serious to keep soldiers, when attacked with it, off duty for several weeks. In the Army it is nearly as common as measles, the yearly ratio of admissions per thousand of strength, for the period igo8-igro, being $8.5^{2}$.

The causative virus (not yet determined) is contained in the discharges from the throat and may transmit the infection from the earliest symptoms until about Io days after the swelling disappears, a period of about three weeks.

The parotitis may be accompanied or followed by orchitis which, occasionally, proves a tedious complication.

Petrilli, of Rome, believes that mumps generally begins as a pharyngitis and that the infection can be arrested before it reaches and invades the duct of the parotid gland, by the application of iodin.

\section{INFLUENZA (GRIPPE).}

A very infective disease characterized by the rapidity of its spread over vast areas. Its causative bacillus is an extremely small organism, very sensitive to heat, desiccation and disinfectants, dying in dry sputum within one or two hours. During epidemics, it is found with much regularity in the nasal passages and bronchial secretions of patients, most frequently causing a form of broncho-pneumonia; it may also be found in various parts of the nervous system causing corresponding morbid symptoms; nor is it rare in the secretions of normal and healthy individuals. In local outbreaks or sporadic cases the agency of this bacillus is uncertain and puzzling, being generally associated with other organisms so that the course of the disease is irregular and atypical. It is also a common invader of the air-passages in other diseases, notably whooping-cough, bronchitis and tuberculosis.

The spread of influenza is particularly favored by overcrowding and defective ventilation. Patients should be isolated, so far as possible. The secretions from the mouth and nose being the means of transmitting the disease should be carefully disinfected. 


\section{INFECTIOUS DISEASES (CONTINUED).}

\section{TETANUS OR LOCKJAW.}

Tetanus is usually, if not always, of traumatic origin, and results from an infection of the nervous system, generally through a neglected punctured, lacerated or gunshot wound of the extremities.

The Bacillus tetani is the most important organism of the anaërobic spore bearers; the spore, being strictly terminal, gives it a characteristic drumstick appearance. It occurs commonly in the superficial layers of the soil, especially in cultivated and manured fields. The spores, if protected from sunlight and other deleterious influences, may remain viable for many years. It is probable that, in many cases of wotnd infection, they fall a prey to phagocytosis before toxin is formed, and are therefore harmless. This may explain the comparative rarity of tetanus, although its germs are widely distributed in nature.

The tetanus bacillus seems to grow better in symbiosis with aërobes; hence a dirty, suppurating wound, contaminated with various cocci, offers the most favorable soil. According to A. P. C. Ashlurst, tetanus will never result from any wound properly treated from the beginning.

The pathogenicity of the bacillus depends entirely upon the soluble toxin which it produces and which is one of the most powerful poisons known. It is conducted to the nerve centers along the path of the motor nerves, but is also absorbed by the lymph vessels and conveyed into the general circulation. The period of incubation, that is to say, the time required for the toxin to reach the nervous centers, ranges from I to 4 weeks and averages about I5 days.

An antitoxin, obtained from highly immunized horses, in the same manner as diphtheria antitoxin, is successfully used as a prophylactic as well as in the treatment of the disease.

Tetanus is more common in warm than in cold and temperate countries, and in some tropical regions may result from a lärge proportion of all wounds fouled by carth or dirt. During the Russo-Japanese War, in Manchuria, a few cases of it were always 
under treatment in the Russian hospitals. In the U. S. Army, during the period of II years I898-1908, only I7 cases were reported, with 12 deaths. Since then the number of cases has been steadily decreasing.

In the present European War, owing to the great soil pollution, the severity of the wounds and the almost insuperable difficulty of transporting patients to field or base hospitals for suitable treatment within a day or two, tetanus was at first alarmingly frequent in shrapnel and other artillery wounds. But as the prophylactic value of anti-tetanic serum became better understood it was administered subcutaneously in all cases of septic wounds, and a great improvement at once took place. The primary injection, consisting of 500 units, is given at the dressing station or field hospital as soon as the wounded soldier reaches it. It should be inserted near the principal nerve supplying the region of the wound. A second injection of the same strength is generally given 7 days later. A third and a fourth may be indicated. Tetanus is now rarely seen in patients who have received, betimes, a sufficiency of serum and whose wounds are freely incised and disinfected.

In the curative treatment of tetanus the element of time is allimportant, and active intervention should begin as soon as the diagnosis is made. The first manifestations of the infection are rigidity, twitchings or local increased reflex to gentle tapping in the muscles round the wound. It is therefore desirable that these muscles be examined whenever the dressings are removed. An early bacteriological examination of the secretions from any suspicious wound is also important. Clinical experience has shown that the best results are obtained by injections into the spinal canal; as only 20 c. c. can thus be injected, the serum should be of high potency. At the same time, 5,000 to 10,000 units are injected intra-muscularly around the wound. Intraneural and intravenous injections have also been found useful.

The symptomatic treatment consists chiefly in the exhibition of sedative drugs.

The local treatment recommended by Ashhurst is to incise the wound freely, clean it carefully and then swab it with a 3 per cent. alcoholic solution of iodin. This solution is also painted on the surrounding skin. The wound is daily irrigated with hydrogen peroxid and dressed with gauze soaked in the iodin solution. 
The Carrel treatment with Dakin's solution is now generally preferred.

\section{RABIES.}

\section{(Hydrophobia, Lyssa.)}

Primarily a disease of dogs, cats, wolves and skunks, but communicable to man through the saliva of the rabid animal gaining entrance to the wounds made in biting and scratching. According to the investigations of the Public Health Service, there were in the United States, in I9I I, I,38 I reported cases of rabies, with 98 deaths. For 1914 the Census Bureau reports 65 deaths in the registration area of the United States. In I9I5 the dissemination of the disease among coyotes and sheep dogs in California and other Pacific States had resulted in heavy losses to stock owners from bites inflicted upon cattle and sheep. In spite of this, little if any increase in the incidence of the disease has occurred in man.

The time of incubation in man varies from 14 to 90 days, and in the $\operatorname{dog}$ and cat from 14 to 60 days. This slow and irregular incubation is explained by the fact that the virus is conveyed to the central nervous system chiefly by the nerve trunks and not by the blood-ressels; therefore it is shortest and the disease is most severe when the wounds are about the head, neck and upper extremities.

Rabies in man begins with headache and nervous depression, followed by difficulty in swallowing, especially water, spasms of the respiratory muscles, perhaps maniacal attacks and, finally, paralysis and death.

The causative agent is not known. Peculiar round bodies, "Negri bodies," always form in the cells of the central nervous system of rabid dogs, especially in the cornu ammonis, and their determination is an extremely important method of diagnosis. They are considered protozoa by some and the parasitic cause of rabies, but the existing evidence does not warrant such assertion. Most pathologists believe them to be simply products of cellular reactions.

The prophylactic treatment (Pasteur's) applied to persons bitten by rabid animals and suspected of being infected, consists in an active immunization with an emulsion of the spinal cord of a rabid rabbit. in doses of increasing virulence. By this treatment, which lasts about 20 days, the mortality is reduced to one per cent. It can be carried out at the home of the patient, or at any post hospital, 
by the use of glycerinated virus which is sufficiently stable for shipment by mail or express. It is estimated that 16 per cent. of persons bitten develop rabies (if untreated) and that all die.

As soon as possible after the bite, the wound should be cauterized with nitric acid (followed by salt solution) or phenol (followed by alcohol). Better still, according to J. G. Cumming, is a 5 per cent. formalin solution applied for 12 hours.

In diagnosing rabies in dogs, it is best to keep the animal alive, securely tied, so that the symptoms may be noted. A rabid dog is restless and irritable, easily startled and scared; it has difficulty in chewing and swallowing, but not in drinking; there is a change in its bark or a loss of it; later it may snap at imaginary things and perhaps develop a furious stage. Paralysis is always present and begins early, at first shown by difficulty in walking (from paralysis of left hind leg) and drooping of the lower jaw, and finally causing death in from 4 to 7 days after the appearance of the first symptoms.

In case a rabid dog has been killed, it is generally possible to make a diagnosis by the demonstration of the Negri bodies, or the inoculation of a rabbit (into the brain substance or under the dura) with a salt-solution emulsion of the medulla of the dead dog.

\section{ANTHRAX.}

Usually an infectious disease of herbivora, especially cattle and sheep, but occasionally occurring in horses, hogs and goats, as well as in man. Its causal organism (Bacillus anthracis) is an aërobic, spore-bearing, straight rod with square-cut ends, often forming chains. The large oval spore is located in the middle of the bacillus. Because of this spore-forming property the anthrax bacillus is extremely resistant to chemical and physical agents; it may be kept in a dry state for many years without losing its viability.

Infection in animals takes place mostly by way of the alimentary canal, also cutaneously through abrasions or punctures about the mouth, and is quickly fatal. In man the disease is usually acquired by inoculation, but may also be contracted by inhalation and more rarely, by deglutition. Inoculation occurs through small cuts or scratches on hands or arms of men who handle live-stock, of butchers and tanners, or on the neck, face and back of men wearing infected sheepskin coats or hats. The primary lesion (" malignant pustule") resembles at first an ordinary furuncle, but 
soon its center is filled with sero-sanguineous and purulent fluid, while it becomes surrounded with a ring of vesicles and an angry, red, edematous areola. Generally, especially if prompt excision is practised, the patient recovers. The pulnonary infection known as "wool-sorter's disease," and which manifests itself as a violent, irregular pneumonia, occurs in persons handling infected wool, hair or hides, by the inlualation of spores which, from the mucosa of the upper respiratory tract where they are arrested, are carried to the lungs and pleura by the lymphatics.

Spores do not form in the body of an animal dead from anthrax until free access of air is made possible by a post-mortem examination or natural disintegration. For this reason, it is better to bury such animal without opening the body, and to make the diagnosis by cutting off an ear.

The general prophylaxis consists chiefly in the killing and burial or cremation of infected animals, and in disinfection with corrosive sublimate. Pasteur has shown that earthworms will bring anthrax spores to the surface from a depth of at least five feet; hence the necessity of cremation, or of deep burial after disinfection.

Anthrax should not be confounded with Symptomatic Anthrax or "blackleg," which is caused by an entirely different organism and has never been observed in man.

\section{GLANDERS.}

An infectious disease chiefly prevalent among horses, mules and asses, but occasionally transmitted to other domestic animals and to man. The causal organism, Bacillus mallei, is a small, nonmotile rod with rounded ends. Heating to $75^{\circ} \mathrm{C}$. kills it within an hour, and is easily destroyed by the ordinary chemical disinfectants. In water it may remain alive several months.

Infection takes place through the broken skin or mucosa, and not infrequently through the digestive tract. In horses, glanders occurs in an acute or chronic form: the former is usually limited to the nasal mucosa and upper respiratory tract from which an abundant sero-purulent secretion is discharged; the latter, usually known as "farcy," is characterized by multiple swellings of the skin and general lymphatic enlargement, some of the swellings showing a tendency to break down and ulcerate.

Glanders in man is usually contracted from contact with diseased 
liorses, and is almost always fatal. The initial point is more frequently some part of the skin (where scratched or broken) than the nasal mucosa; there a nodule appears, with swelling and lymphangitis. In the acute form the onset is usually violent, with high fever and grave systemic symptoms. A general papular eruption may occur, soon becoming pustular and simulating variola. The chronic form is much like that of the horse, with the same foul discharging ulcerations, but is more frequently fatal.

To diagnose glanders in horses, mallein, the endotoxin of $\mathrm{B}$. mal$l e i$, is frequently and successfully used, its injection into an infected animal being followed within a few hours by a sharp rise of temperature.

The general prophylaxis consists in the killing and burial of infected animals, and disinfection. Great care should be taken by all persons handling diseased horses or mules to prevent any discharge from the nose or sores of these animals coming in contact with wounds or scratches on their bodies, or being blown in their faces.

\section{LEPROSY.}

A widespread chronic infectious disease, most common in China, India and Japan, but found in nearly all countries, including the United States and its colonies. The Bacillus lepre is accepted as the cause, but the manner of its introduction into the body and the conditions favoring it are still uncertain. It seems probable that the infection is by inhalation of the bacillus, and absorption through the mucosa of the nasopharynx, as the result of prolonged and intimate association with patients. . Prophylaxis consists in segregation and isolation of patients.

According to the Public Health Service, there were, in I9I5, over 200 lepers (of whom IO2 were in Louisiana) in the United States, 670 in Hawaii, 4,472 in the Philippines and 37 in Porto Rico. In Cuba there are at least 200 . Too many lepers have complete freedom of movement and action in this country, and are a menace to their families and people they associate with. A suitable home, under federal authorities, should be provided for them.

Since the occupation of the Philippines, to I9Io, there have only been three cases of leprosy among Americans in the Islands, all of whom gave a history of excessive sexual indulgence with native women. 


\section{YAWS (FRAMBOESIA).}

Yaws is a highly contagious, inoculable chronic disease characterized by mild constitutional disturbance and eruptions of fungoid incrusted tubercles. Common in Africa, the West Indies and parts of India. Not rare in some of the Philippine Islands among the very poor and ignorant, but seldom seen in Manila. The causative agent is a minute, spirally-twisted organism or spirochete, the Treponema pertenue of Castellani, which so closely resembles the $T$. pallidum of syphilis that few bacteriologists claim to differentiate them. The disease itself suggests syphilis and may prove to be a form of it, but is clinically distinct.

For its production, simple skin contact is not sufficient; a break of surface, previous sore or ulcer is necessary. Probably the virus can be conveyed by insects.

\section{CLIMATIC BUBO.}

A form of non-specific adenitis found in all warm countries, and always causing a few admissions to the sick report anong our troops in the colonies. The inguinal and crural glands are those generally affected; they may swell to the size of a hen's egg or even larger, and occasionally suppurate. The cause of this affection has not yet been determined, but it is probably of an infectious nature and transmitted by some biting insect.

\section{LEISHMANIASIS.}

Under this heading are included three distinct diseases caused by different varieties of Leishmania, an intracellular roundish protozoön containing two nuclei, one oval, the other smaller and rodshaped. I, Kala-Azar, a highly fatal infection of India and other parts of Asia, attacking both sexes at all ages; caused by L. donovani. 2, Infantile Kala-Azar, prevailing in all countries bordering the Mediterranean, but restricted to children between 2 and 5 years; caused by L. infantum. 3, Tropical or Oriental Sore, a benign ulcerating affection of the skin common in the tropical regions of the Old and New Worlds; caused by L. tropica. For the 3 years 1909-I9II, 26 cases of tropical sore were reported from the American troops in the Philippines.

These three varieties of the parasite are much alike, and Leishman was unable to detect any constant differential features between 
them. Kala-Azar is probably transmitted by bedbugs. The infantile form is transmitted from the $\operatorname{dog}$ by fleas. The mode of transmission of the tropical sore is not yet known.

\section{TRYPANOSOMIASIS (SLEEPING SICKNESS).}

An almost always fatal African disease caused by a spindleshaped blood protozoön, Trypanosoma gambicnse, transmitted by several "Tsetse flies," small brownish biting flies of the genus Glossina, chiefly G. Palpalis and G. morsitans. (See page I $^{6}$.) The latter species also transmits $T$. brucci, cause of the widespread cattle disease, nagana, in Africa. Another trypanosome ( $T$. evansi), apparently transmitted by several kinds of biting flies, causes the "surra" of horses and mules in India and the Philippines. 
1

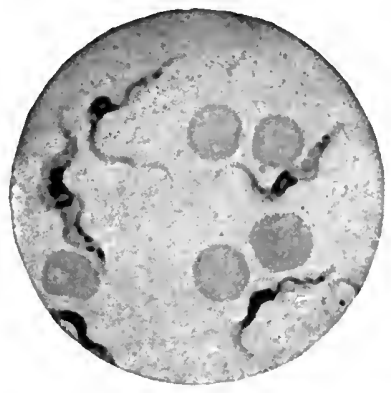

3

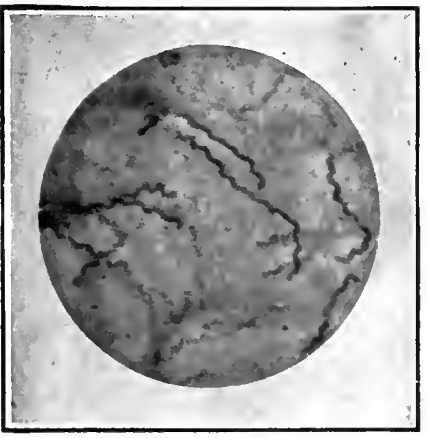

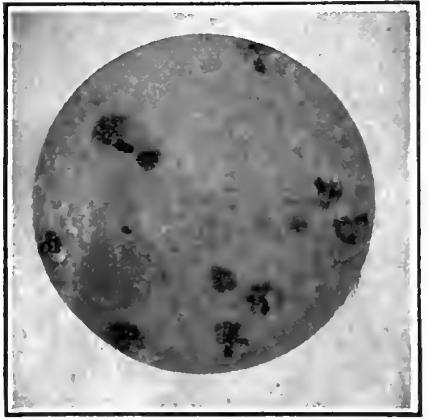

2

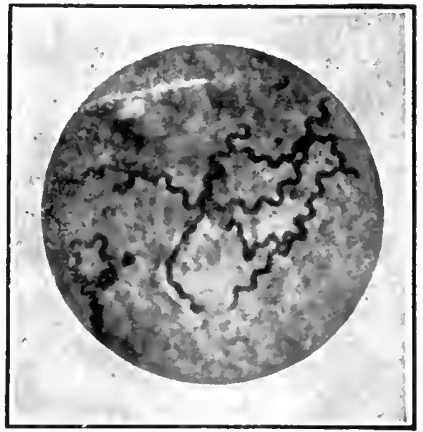

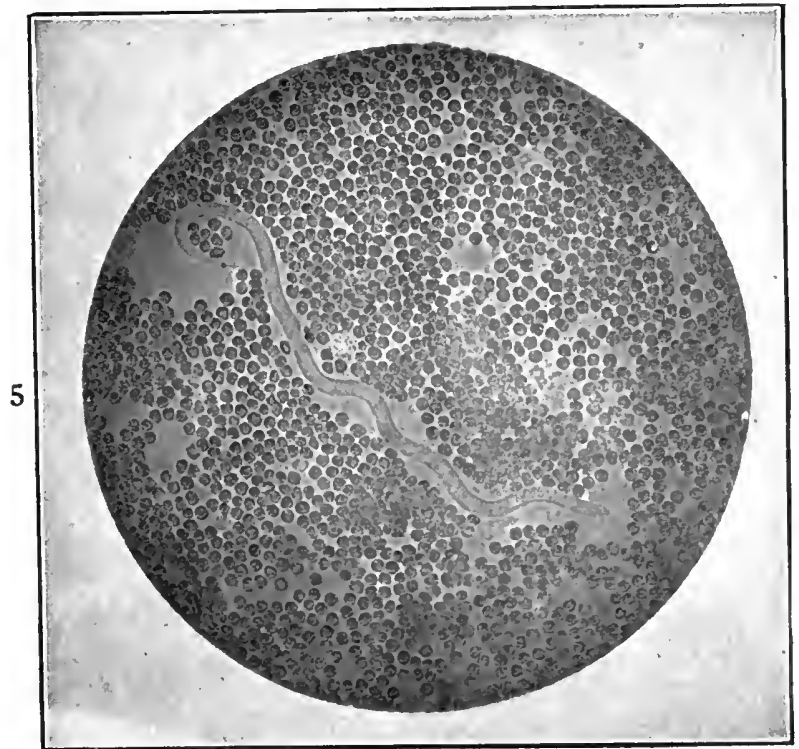

I. Trypanosoma gambiense; trypanosome of sleeping sickness.

2. Lcishmania donozani; splenic smear from case of kala-azar.

3. Treponema pallidum of syphilis in chancre.

4. Treponema pertenue of yaws in section of skin.

5. Embrio of Filaria bancrofti in fresh human blood. 


\section{CHAPTER IX.}

\section{PARASITIC DISEASES.}

Under this heading are included diseases caused by various parasites other than bacteria and protozoa.

\section{PARASITES OF THE CIRCULATORY AND LYMPHATIC SYSTEMS.}

Filariasis is the condition resulting from the presence in bloodvessels and lymphatics of minute, filiform nematode worms belonging to several species of Filaria. Only one, $F$. bancrofti, appears to have important pathological bearings. It is the most common and widespread. In the adult stage it inhabits the lymphatics of man; there fecundation occurs, soon followed by the pouring out of new generations (microfilaria) which find their way into the circulation. In most cases of filariasis the parasite scems to exercise no injurious influence, except mechanically by obstructing the lymphatics. In this way are produced various complications such as chyluria, lymph varix, lymph scrotum and probably elephantiasis. $F$. bancrofti exhibits a remarkable day and night periodicity, being absent from the peripheral circulation during the day and usually abundant at night.

This disease, with all its complications, has a very extensive distribution throughout all tropical and subtropical countries where a notable proportion of the poorer natives are often infected with it, and is therefore always a menace to our soldiers in the colonies, several of whom have already suffered from it. According to Phalen and Nichols, an average of 2 per cent. of the population of the Philippine Islands is infested with filaria. These observers record that among 250 American soldiers stationed 18 montlis in one of the most highly infested filarial districts of the Islands, not a single case occurred, indicating how easily white people can be protected from this parasite.

Filariasis is a mosquito-borne disease, being transmitted to man by Cule.r fatigans and probably other insects. Man is the definite host of this blood-worm and the mosquito only the intermediary host (Plate VI).

Ashburn and Craig have discovered another species (F. Philip- 
pincnsis) in the blood of Filipinos, likewise transmitted by Culex fatigans.

The prophylaxis of filariasis is that of all mosquito-borne diseases, namely, destruction of mosquitoes and protection from their bites.

Bilharziasis (Bilharz's disease) is caused by several species of Schistosomum (Bilharsia), trematode or fluke worm, in the blood and lymph. S. hcmatobium, of Africa, lodges mostly in the walls of the bladder, causing chronic hematuria; S. mansoni, of the West Indies, inhabits chiefly the mesenteric veins and gives rise to dysenteric symptoms; S. japonicum, of Japan and China, is not very rare in the Philippine Islands; it attacks specially the liver, spleen and intestines.

Paragonomiasis, not uncommon in the Philippines, is a disease caused by the Paragonimus westermani, an oval reddish-brown fluke, half-inch long, inhabiting chiefly the lungs but more rarely also the liver and brain. It is readily diagnosed by a chronic cough, hemoptysis, and the characteristic ova in the rusty-brown sputum.

Clonorchis endemicus ( $C$. sinensis), a narrow oblong parasite, ro to $20 \mathrm{~mm}$. in length, inhabits the bile ducts and gall-bladder. Very common in Japan and China and not rare in the Philippines.

\section{INTESTINAL PARASITES.}

In the United States, the most common intestinal parasites, according to Stiles and Garrison (I906), are the following:

Per cent. of population in which found.

Trichuris trichiura (Trichocephalus trichiurus) Whipworm,

Oryuris vermicularis, Pinworm,

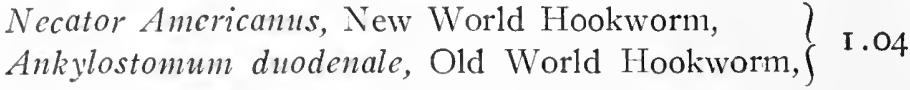

Ascaris lumbricoides, Eelworm, Roundworm,

0.49

Hymenolepis nana, Dwarf Tapeworm,

Strongyloides stercoralis, Cochin-China Worm,

0.23

Tcriia saginata, Fat Tapeworm,

From an examination of the stools of 1,280 patients in the Philadelphia General Hospital, Rosenberg found I 70 harboring parasites, or 13 per cent. 


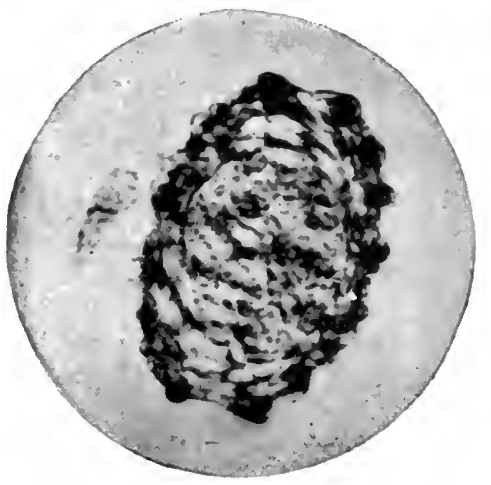

1

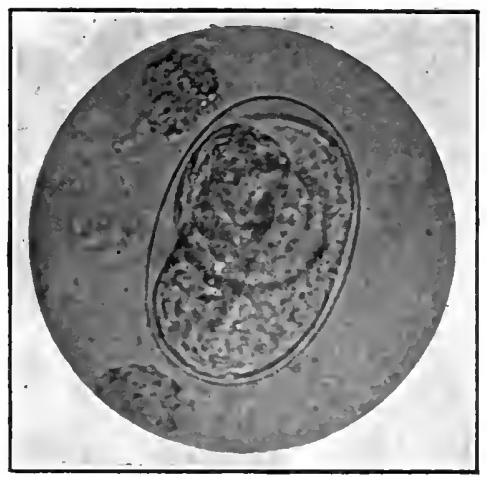

3

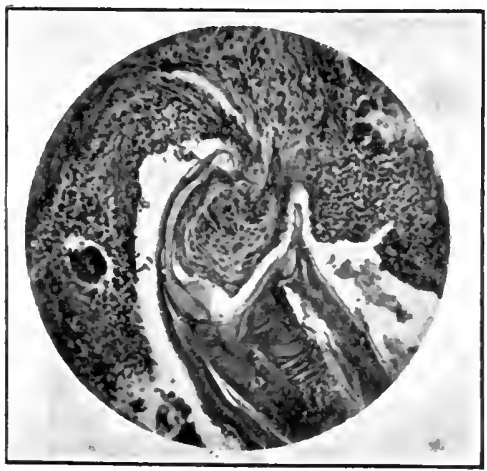

5

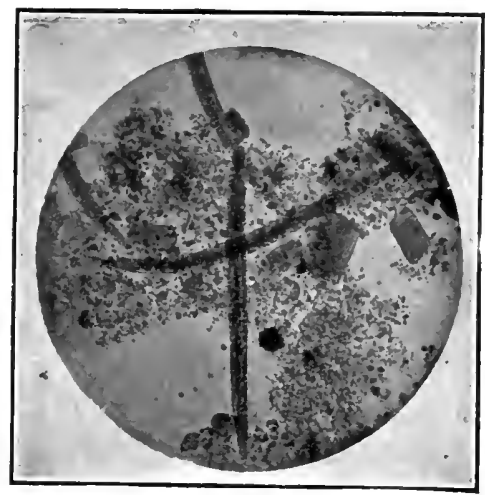

2

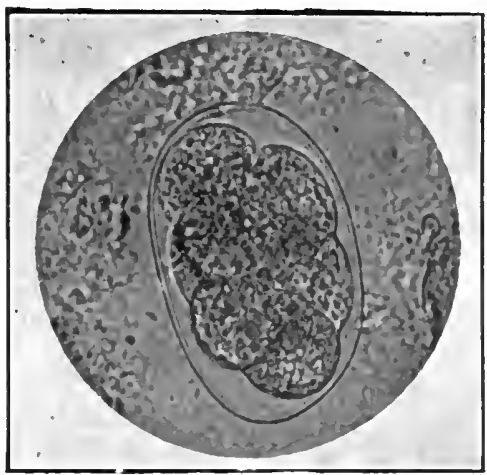

4

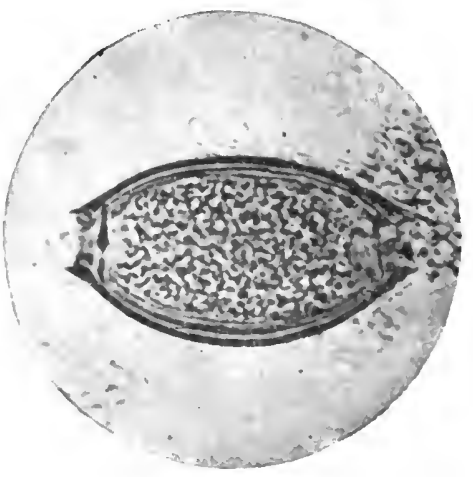

6

1. Ovum of Ascaris lumbricoides.

2. Larve of Strongyloides stercoralis in fresh stool.

3. Ovum of Necator americanus in fresh stool.

4. Unhatched larva of Necator americanus in fresh stool.

5. Adult Necator americanus, showing head of worm attached to the mucous membrane of the intestine.

6. Orum of Tricocephalus trichiurus. 
• 
These figures appy mainly to the middle and northern States. In most of the southern States it is well known that the percentage of individuals affected with hookworm far exceeds that of individuals larboring all other parasites combined.

From an examination of the stools of applicants for enlistment from the southern States, at Columbus Barracks, O., in I9IO, Love ascertained that 37.50 per cent. were infested with one or more kinds of parasites, Necator being found in 24.62 per cent., Ascaris in 14.77 , Strongyloides in 4.93 and Trichuris in $3.4 \mathrm{I}$.

In Porto Rico, the prevalence of intestinal parasites was as follows in 1904:

Necator Americanus, Per cent. of population. Ascaris lumbricoides, 90 to 100

Trichuris trichiura, $3 \mathrm{I} \cdot 4 \mathrm{I}$

Strongyloides stercoralis, 7.27

Oxyuris vermicularis,

0.80

0.07

Since I904 the above proportion of Necator has been greatly reduced.

In the Philippine Islands, not less than 80 per cent. of the population is infested with one of more kinds of intestinal parasites, so that if the different species are separately considered there is an average of about 200 infections to each 100 of population (Garrison). The most common species, in order of prevalence, are Ascaris, Trichuris and the hookworms. There, as in many other tropical countries, ideal conditions often exist for the propagation and dissemination of parasites, namely: crowding of dwellings, absence of privies, scattering of excrements or use as fertilizer, questionable water-supply, disregard of personal cleanliness, practice of eating off common dishes with dirty fingers, lack of footwear.

As a remarkable indication of the detrimental effect of intestinal parasites in the tropics, even in the absence of obvious symptoms, may be noted the fact that the mortality of the Bilibid prison (P. I.), in spite of satisfactory sanitary conditions, remained at 75 per 1,000 until the prisoners were systematically treated for parasites, after which the death rate dropped to about $\mathrm{I} 3$ per I,000 (Garrison).

Outside of the hookworms and the tapeworms in certain regions, the large roundworm (Ascaris) seems to be the most prevalent of intestinal parasites in all parts of the world. The eggs are readily recognized by their rough, mamillated shell; they are endowed 
with remarkable vitality, lasting at least four months in moist feces after being passed from the intestines, as shown by Stiles. This worm is also found in cattle and hogs, and the eggs may be conveyed with the excrements of these animals to the vegetable garden or washed into the water supply.

The reports of the Surgeon General for I9Io and I9I I show that next to the hookworms, the most common intestinal parasites among troops serving in the United States are, in order of prevalence, Tania saginata, T. solium, Ascaris, Bothriocephalus and Oryuris. In the Philippines, Ascaris is more prevalent than Tonia.

Ankylostomiasis. - The hookworms, both Old World and New World species, are not only the most prevalent of intestinal parasites in many regions, but also the most dangerous, producing a form of anemia known as ankylostomiasis.

The Old World Hookworm (Ankylostomum duodenale or Uncinaria duodenalis) is about half-inch in length (the male often less, but the female generally more), the thread-like body tapering to a narrow neck ending in a mouth cavity armed with 4 hooks and 2

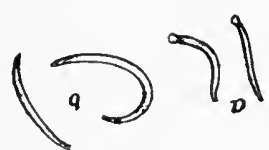

Fig. II.-Ankylostomum duodenale (natural size). (Dubini.)

$a$, Male; $b$, female. conical teeth. (Fig. II.) It inlabits the upper part of the small intestine, where it attaches itself to the mucous membrane by means of its strong buccal armature, sucking the blood out of it (Plate VII). The male, besides being smaller than the female, is recognized by the caudal umbrella-like expansion (copulatory bursa). The female produces a prodigious number of eggs which pass out in the feces, thus betraying the presence of the parasite. (Plate VII.) These ova, under the microscope, are clear and transparent, with regular oval form and delicate shell through which can easily be seen 4 to 6 grayish yolk segments. They generally have to be differentiated under the microscope from those of the two most common intestinal parasites, the roundworm and the whipworm; these are both bile-stained, the former having a tuberculated shell, the latter looking like an oval platter with handles. (Plate VII.) Outside of the intestine the hookworm ova undergo rapid changes, and the embryo is born within one or two days. After reaching a certain development, the minute larva, about $0.5 \mathrm{~mm}$. long, whether in water, mud or sand, remains in a torpid condition waiting for a 
chance, should it survive, to be transferred to the human alimentary canal, where it acquires sexual characters and permanent adult form. Thus it appears that, although the eggs are produced in the intestine, there is no hatching in the human body, and therefore no reinfection, except from the outside.

The ova in feces, as when passed in latrines, hatch in due course, but the embryos soon die unless supplied with air and earth.

The larva find their way to the human intestine chiefly by boring through the skin; then, entering the blood-vessels and lymphatics, they are carried to the lungs and ascend the trachea until the esopliagus is reached, whence they pass to the stomach and small intestine. This mode of entrance is the usual one in the case of farm laborers walking on infected soil with bare feet. The result is the condition known as "ground itch." Its symptoms are a stinging sensation in the feet and legs, followed by intolerable itching for several days, then vesiculation and incrustation. Within two months the ova may be found in the stools. Infestation is also possible by ingestion of contaminated water or food, but this is thought to be comparatively rare.

This parasite occurs nearly in all but the most northern countries, being abundant in the south of Europe as well as in the tropical and subtropical regions of Asia and Africa, and common in America. It is most prevalent in southern China, Japan, India and northern Africa, where at least 75 per cent. of all natives are infested. It is found in most of the mines of the L'nited States and Mexico, having been implanted therein by foreign workmen.

The hookworm disease (as caused by both Ankylostomum and Necator) belts the earth with a zone about 66 degrees in width, namely, from $36^{\circ}$ lat. north to $30^{\circ}$ lat. south. Both species are common in China and Japan, but only the former is found in India. In Panama both species are found, but seldom in sufficient number to produce noticeable symptoms.

The New World Hookworm (Necator Americanus), differentiated and described by Stiles in I902, is abundant in the Southern States, Porto Rico, Cuba and very likely in Central America ; also a common parasite in the Philippine Islands, and likewise found in certain parts of Africa. In the United States it occurs in a large proportion of the population, white and colored, from New York and Illinois sotthward and westward to Florida, Louisiana and Texas, 
especially among the rural farming classes of sandy zones, being most abundant in central Tennessee and the sandy coastal plains of Virginia, North and South Carolina, Georgia, Alabama and Mississippi. It is estimated that, in Georgia, one-fourth of the total white population is infested. The negro is much less susceptible than the white. The period of life most affected is from 6 to 16 years, so that in the schools an incidence of 75 to 80 per cent. is not unusual. Chamberlain found that at least 60 per cent. of southern-bred recruits were infested. In four North Carolina militia regiments, Strosnider found the percentage of infestation to range from 30 to 58 .

The Necator is distinguished from the Ankylostomum by a smaller mouth armed with semilunar plates instead of hooks. The female has the same length (II-I2 mm.) in both species. The ovum is larger. (Fig. I2.)

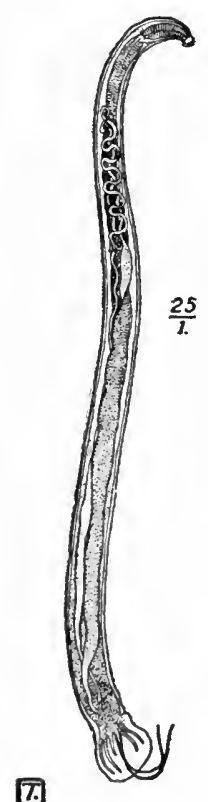

团

Male.

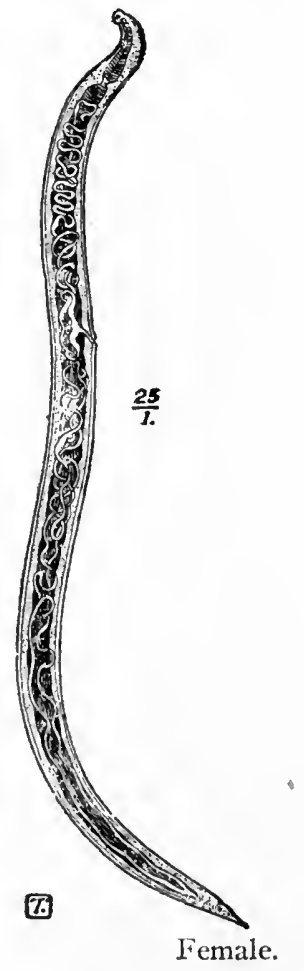

Female.

FIG. I2,- Necator Americanus. (Manson.) 
Often, hookworms in the intestine, especially if few in number, give rise to no appreciable symptoms. But if numerous they may, in poorly nourished subjects, especially those already suifering from some chronic exhausting disease, give rise to a severe form of anemia leading to permanent degeneration of important organs and death. The chief symptoms are: depraved appetite, with gastric and intestinal disturbances; marked retardation of general development, with weak, flabby muscles, serous effusions into the subcutaneous tissues, retinal hemorrhage, night blindness, etc. The mind is slow and torpid. The Ankylostomum sucks blood from the mucous membrane (as shown in fig. 5, plate VII); not so with Necator which feeds only on epithelial cells. Both, however, produce a constant drain on the system, and, through the minute wounds, a greater susceptibility to the attacks of in fectious bacteria. It is also believed that additional harm is done by a poisonous substance secreted by the worm. In the southern Atlantic States, ankylostomiasis must be considered one of the most common and widespread diseases, especially among the lower classes of white, being even more prevalent, in rural sand districts, than tuberculosis and malaria. It accounts for the fact that a large proportion of the recruits for the Army from the Southern States are under-developed and physically inferior to northern-bred men of the same age. In Porto Rico, before 1904, from 5,000 to 7,000 natives died from it annually, while 70 per cent. of the worm-carriers were more or less incapacitated in consequence of it (Ashford). In the Philippine Islands both kinds of worms are found, but the Necator predominates. Among the general population of Luzon the percentage of infestation was found to range from i i to 16 , and to reach 29 among the natives of the northern mountains; it has exceeded 50 in the scout companies and among the inmates of the Bilibid Prison. It is remarkable that, in these Islands, severe clinical manifestations of ankylostomiasis are comparatively rare, and that there is no prevalence of anemia nor of any other symptoms among the natives attributable to the hookworm. This is probably due to the fact that, although many individuals are infested, the number of parasites in each is generally small.

Prophyla.ris.- Since the parasite is spread through the stools of patients, fecal contamination of soil and water must be carefully prevented by compelling all people concerned to use privies. Cleanly 
habits and the wearing of shoes should be insisted upon. The systematic administration of thymol or betanaphthol to all persons affected or suspected, to expel the worm, has produced excellent effects in Porto Rico. Permanent practical results can only be expected after a patient and persistent campaign of education.

Thymol is recognized as the best all-round remedy against hookworm, and betanaphthol the next best. After a fast of I 2 to 24 hours and a purge, thymol is given in capsules, the quantity (from 0.5 to 4 grams) being divided into two doses administered one hour apart.

\section{SKIN PARASITES.}

Skin diseases are now much less common than formerly in the Army and Navy. True itch, or scabies (see page I41), is becoming rare. It has been found, in the Navy, to involve oftener the gluteal folds and dorsum of penis than the hands, indicating its transmission by the seats of water-closets. In the tropics, the so-called "dhobie itch" is not infrequently seen among our soldiers. Although the name is applied to various itching, ringworm-like affections, it usually refers to some form of bacterial intertrigo of the crotch and axillæ. In hot and damp weather the germs proliferate actively, causing excessive irritation and scratching. "Camp itch" is an indefinite term applied to various itching skin affections, generally beginning with papillary inflammation which, when complicated with filth and scratching, may become eczematous; its treatment should always begin with water and soap.

The following parasitic skin diseases are also mentioned on account of their comparative frequency:

Tinea circinata or Ringworm, caused by the fungus Trichophyton tonsurans, appears upon both the hairy portions of the body as well as upon free skin, forming circular scaly patches within which the hair falls out. Occurs mostly in children.

Tinea imbricata or Tropical Ringworm, is prevalent in most warm countries, " characterized by a concentric arrangement of closely-set rings of scaling epidermis" (Manson), from one-eighth to half-inch apart, and without dermatitis. All parts of the body may be involved except the scalp, crotch and axilla. Each scale, under the microscope, shows an enormous profusion of a trichophyton fungus, the causal organism. 
Pemphigus contagiosus, a non-febrile, highly contagious affection of tropical countries, beginning with minute papules which rapidly mature into vesicles, bullæ or even large blebs. These are round, with well defined margins and no areola of congestion. After rupture of the blisters a process of desquamation sets in, extending to the surrounding skin. Any part of the body may be affected but, in adults, the disease is mostly confined to axillæ and crotch. There is an entire absence of constitutional symptoms. The causal organism has not yet been determined.

Common in most countries is Favus, calised by several forms of the fungus Achorion. It attacks chiefly the scalp of ill-nourished children, forming small sulphur-yellow cupped crusts at the base of the hair. It is rarely seen among our soldiers. 


\section{CHAPTER $\mathrm{X}$.}

\section{NUTRITIONAL DISEASES.}

\section{PELIAGRA.}

An endemic, non-contagious disease of slow evolution, characterized by various cutaneous, gastric and nervous symptoms, most marked in the spring and early summer months.

It is of comparatively modern origin, having been first observed in Italy and Spain in the middle of the XVIIIth century, then gradually spreading to all countries of southern Europe and northern Africa. Although first discovered in the United States only a few years ago, it has now become so prevalent in some of the Southern States as to constitute one of the most important health problems of the day. Thousands of cases have been reported from more than thirty States, especially Ky. Tenn., N. Car., S. Car. and $\mathrm{Ga}$.

The skin symptoms are characteristic. An erythema, like a severe sunburn, in irregular patches, appears suddenly, first on the back of hand and feet, then on forearms, legs and other parts usually exposed to the sun. It lasts about two weeks, leaving the skin rough and thickened (pellagra meaning rough skin). The tongue and other parts of the mouth become inflamed and denuded. Gastro-intestinal disturbances are usually present. There is also great weakness and emaciation. The patient becomes irritable, morose, and suffers from attacks of giddiness. All these symptoms may greatly improve or almost disappear before winter, but always recur the following spring in a more severe form. His depression of spirits deepens into melancholia and, in time, he becomes paralytic and completely demented. It is estimated that, in this country, 50 per cent. of the cases die during the first two years:

The etiology of pellagra has been the object of numerous investigations, for until this was discovered no decided progress in prophylaxis and treatment was possible. The Italian theory, until recently widely accepted, is that pellagra is caused by the toxins of mouldy maize. According to Sambon, who first advanced this view, it is produced by a protozoal parasite probably transmitted, in southern Europe, by a blood-sucking fly, Simulium reptans, and 
in this country by other species of the same genus, such as $S$. pecuarum (buffalo gnat), S. pictipes and others (see page 157). Fortunately the persistent observations and experiments of the Public Health Service, during a number of years and completed in 1916, have finally settled this most important and troublesome question. It is now established that pellagra is a deprivation disease resulting from a faulty diet containing an excess of carbo-hydrates and deficient in certain food elements (vitamines) generally supplied in milk, eggs, fresh meat, beans and peas. It was found that corn bread prepared by means of baking soda, without the addition of buttermilk or sour milk, is deficient in vitamines owing to the destructive action of the alkali upon them.

The prevention of pellagra, therefore, consists simply in a wellbalanced diet, with a sufficiency of animal protein foods and legumes, and free from any excess of carbo-hydrate components.

\section{BERIBERI OR KAKKE.}

A specific, non-infectious, acute or chronic form of multiple peripheral neuritis, endemic in Japan and China and, to a lesser extent, prevalent in most tropical and subtropical countries. In Manila alone, I,002 cases were reported during the year 19I0. It was the disease which most disabled the Japanese troops in the Russo-Japanese War.

A disease exceedingly variable in the nature and severity of its symptoms, generally assuming one of two forms, the paralyticatrophic (dry beriberi) or the dropsical (wet beriberi), or various combinations of them (mixed beriberi); death, when it occurs, being usually the result of heart paresis.

Cause.- The cause of beriberi has now been sufficiently elucidated to enable us to control this disease and prevent its development. Until recently, owing to its peculiar endemic and localized character, beriberi was generally believed to be a place disease produced by a germ breeding in certain soils or buildings under the influence of dirt and other unhygienic conditions. To-day the germ theory has but few supporters. The persistent researches carried on in the Philippines, chiefly by boards of Army medical officers, as well as in the Malay Peninsula by English physicians, have established the fact that beriberi is a disease of nutrition, the result of the lack in the dietary, of certain essential elements. It is not improb- 
able, however, that camps or buildings in which beriberi prevails may become contaminated by secondary germs or toxins which, although non-specific as regards the transmission of the disease, render exposed persons more susceptible to it. Thus might be explained its apparent epidemic character under certain circumstances.

Experiments by Strong and Crowel, in Manila, upon prisoners under sentence of death, who volunteered for the purpose, furnish definite evidence that beriberi is due to the prolonged consumption of a diet lacking certain substances (vitamines) necessary for the normal physiological needs of the body, while there is none suggesting that it is an infectious disease.

The fact that beriberi is generally found in races which use rice as a more or less exclusive article of diet, long since called attention to this food as the possible cause of it. Some years ago, when beriberi was disabling at least one-fourth of the Japanese Navy, a change was made in the ration at the suggestion of Takaki, consisting in the reduction of the rice component and the increase of other vegetables richer in nitrogen. The result was the almost complete disappearance of this disease; but as other hygienic reforms were instituted at about the same time, the chief cause of this great improvement in the health of the crews was not made manifest.

A few remarks about the structure and composition of the rice grain will be useful for the better comprehension of the subject. It consists of the small embryo, the endosperm, the episperm or seed coat and the skin or pericarp, all of which parts are closely coalescing, so that the episperm is not distinguishable. Outside are the glumes (husk) which adhere to the grain. Rice with the husk still on is called " padi " by the English, and " palay" in the Philippines. Under the microscope can be seen the flattened and dried cells of the husk, then the elongated cells of the pericarp in one or two layers, and within these the large polygonal cells of the endosperm filled with very numerous and minute angular starch grains. The framework of the cells itself consists largely of protein. The thin outer portion of the endosperm, called the aleurone layer, contains albuminous material and most of the fat. The pericarp contains also albuminous material, as well as phosphorus and potassium in notable proportion. The color of the pericarp varies from white to yellow, red and nearly black. The common varieties grown in the 
Philippines have the pericarp either brick-red or yellowish-white. Rice, when prepared for consumption, is polished or milled, that is to say, not only husked but its pericàrp ground off; in this process more or less of the aleurone layer is also removed. As a result of milling, a certain proportion of nitrogenous matter is lost; thas while raw rice often contains Io per cent. of protein, polished rice seldom contains more than 7 . The loss, however, is especially great in phosphorus and potassium, rice only superficially polished, or undermilled, containing at least twice as much of these substances as the regularly polished grain.

Experiments on a large scale conducted during the last few years in the Philippines and the Malay Peninsula, under varying conditions, have conclusively proved that a dietary consisting chiefly of polished rice will almost certainly result in a certain proportion of cases of beriberi, and that as soon as the polished rice is replaced by unpolished or undermilled grain beriberi will cease. In accordance with this finding, the Tropical Disease Board recommended a change in the ration of the Philippine scouts; the rice was changed from the polished to the undermilled kind and reduced from 20 to I6 ounces; r. 6 ounces of beans was added; the meat allowance of 12 ounces and other components were left unchanged. From the time this new ration went into effect, in March, I9Io, beriberi rapidly declined, and before September had entirely disappeared.

A certain amount of milling is probably necessary in order to remove the husk and give the grain a more acceptable appearance, but it should be as slight as possible. A simple test to ascertain the proportion of pericarp left is to stain the grain with Gram's iodine solution, then wash and dry; the pericarp (if originally white) remains grayish-white, while the endosperm, or parts denuded of pericarp, will become dark blue, almost black. Unpolished rice contains about 0.55 per cent. of phosphorus (as phosphorus pentoxid, $\mathrm{P}_{2} \mathrm{O}_{5}$ ). It is considered that a rice containing at least 0.4 per cent. of phosphorus will not cause beriberi, and that one with less is unsafe when it forms the principal article of diet. It is not claimed that lack of phosphorus is the cause of the disease, but that its percentage is a reliable index of the proportion of pericarp left, and therefore of the beriberi-producing power of a given rice.

The "cured rice" of India and other places is a rice that has 
been parboiled and then dried before milling, with the result that the pericarp and aleurone layer are less easily and completely removed from the grain. Beriberi is rare where cured rice is commonly used.

Although enough progress has been made in the etiology of this disease to enable us to prevent it, and eradicate it where it already exists, we do not know, as yet, the substance in rice whose loss gives rise to beriberi. To begin with, it is not a starvation disease, for it may coexist with a fair sufficiency of food among well-to-do classes. When the effect of a polished rice dietary was clearly understood it was generally believed that beriberi was the result of a lack of proteid substance; but inasmuch as it may develop with a sufficient amount of nitrogenous food this view must be set aside. Thus it prevailed among the Filipino scouts in I908 and I909 (I2 and 10 per thounsand of admissions) when they were receiving a comparatively generous ration, including 12 ounces of meat which, if only two-thirds of it were consumed, should have furnished all necessary nitrogen. But they also drew 20 ounces of rice, while much of the meat may have been sold or otherwise disposed of. However, it should be borne in mind that the milling process removes less than one-third of the total nitrogen of the grain. As to the phosphorus and potassium removed with the pericarp, most investigators are of opinion that, although useful constituents, they cannot be considered the chief causative factors.

Fowls readily develop multiple neuritis when fed exclusively on polished rice; although this polyneuritis gallinarum and beriberi are not identical, it has been ascertained that the capacity of a rice to produce polyneuritis in fowls is an accurate indicator of its beriberiproducing quality. By experimenting on fowls Chamberlain and Vedder found that the constituent of rice polishings which will prevent neuritis is soluble in both cold water and cold alcohol. These solvents take up only I.34 per cent. of the polishings, namely, sucrose, nitrogen, mineral matters and traces of phosphorus. The solution being dialized, it was found that the diffusate was neuritispreventing, while the substances which did not pass through the membranes were not. Deducting all substances known to be inactive, leaves only 0.4 per cent. in which must be sought the neuritispreventing principle. From his experiments Vedder concludes that this principle is not a fat, proteid, inorganic salt or alkaloid, but probably an organic base as claimed by Funk. 
In conclusion, it is established that a dietary consisting chiefly of rice will produce beriberi if the grain is polished; that it will not do so if the grain be simply undermilled; that rice in a varied, wellbalanced ration is entirely harmless, even if polished, provided the quantity be moderate; but that on account of the marked fondness shown for rice by Orientals and the danger of their consuming it in large amounts to the exclusion of other foodstuffs, the use of undermilled grain should be encouraged and whenever possible enforced wherever beriberi prevails or has been endemic.

It seems probable that other cereals are like rice in this respect, and that when making 11p the bulk of the diet they must be used whole, cortical layers as well as starch cells, to prevent some form of beriberi. Thus Dr. Little found that the people of Newfoundland, when obliged to live for long periods of time on white bread and tea, often suffer from peripheral neuritis. This disorder disappears when the white bread, made of fine white flour, is replaced by bread made from whole-wheat flour.

\section{SCURVY.}

Of diseases caused by scant and improper diet among soldiers, scurvy has been the most common and widespread. It is a disorder characterized by great debility, a spongy condition of the gums and a tendency to hemorrhages.

It results from the absence or insufficiency of vegetables and fresh meats in the food, causing a reduction of the alkalinity of the blood. (See page 349.) That vegetables are not essential, at least for a period of several months, is shown by the remarkable experience of the polar explorers Nansen and Johansen, who subsisted on meat and fat alone during their memorable winter in Franz-Josef Land, and remained in good health

This disease was formerly very prevalent among soldiers and sailors but, with more varied and wholesome food, it has long ago ceased to play an important part in the norbidity of camps. It is still liable to occur wherever troops are besieged and cut off from fresh supplies; thus, during the Russo-Japanese War, the Russians in Port Arthur suffered severely from it. At the time of the surrender of that fortress there were 9,093 cases of scurvy out of a total number of I I, 105 sick.

The horrible ravages of scurvy in former times, even when men were fairly well fed, lead to the belief that, under conditions of 
crowding and bad ventilation, an infectious element becomes developed whereby its virulence is greatly increased. Thus reliable witnesses have described epidemics of scurvy which could not be controlled on board ship, but ceased as soon as crews and passengers were put ashore, although given the same diet and treatment, showing the presence of some secondary infectious agent clinging to the ship.

The proper treatment consists in a diet of fresh vegetables (especially raw potatoes, tomatoes and onions), milk and fresh meats, together with plenty of pure air.

\section{MISCELLANEOUS DISEASES.}

\section{HEART DISEASES.}

These affections have always been among the four or five diseases causing the highest rates of mortality and discharge among soldiers. For the period I909-I9II, they caused a mean death rate, in the United States, of 0.28 per $\mathrm{I}, 000$, ranging from $0.3 \mathrm{I}$ in 1909 to 0.27 in I9II; and in the Philippines, of 0.39 per 1,000 , ranging from 0.61 in 1909 to 0.16 in 1911.

The death rates for the United States and British armies, for troops at home, are about the same, but several times greater than for the French and German armies. This marked difference is difficult to account for, inasmuch as, in the latter armies, the drill, training and hardships to which the men are subjected are probably more arduous than in the former. For lack of a more convincing reason it may be conjectured that these higher rates in cardiac diseases are attributable to the corresponding higher rates for venereal diseases and alcoholism.

It is natural to suppose that soldiers would be particularly liable to heart disease, from tight fitting coat, pressure of belts and straps, weight of equipment, drill, marching and hardships, to say nothing of cigarette smoking and alcoholic and venereal excesses. But this supposition is not confirmed by statistics, and any comparison between military and civil rates is rather to the advantage of the former. The exceedingly low death rate from cardiac disease in the Prussian Army (0.02 per I, 000 ) tends to prove this. The prevalence of heart affections among young men in civil life is well shown in the reports from our recruiting depots. De Loffre found that 
exactly Ioo out of I,O00 applicants for enlistment at Columbus Barracks suffered from some abnormal cardiac condition, requiring the rejection of 19. (See page 198.) Of the total number of applicants rejected for the 3 years $1909-1911$, 10.6 per cent. were for heart disease, a ratio much higher than for any other class of diseases except venereal diseases (II.I per cent.). The ratio of rejections for heart disease is likewise the highest in the British Army.

See also under Recruiting, and the effects upon the heart of training, marching and athletics, under Exercise. For Irritable Heart, see page 244 .

\section{TRENCH FOOT.}

This condition is one of the results of the trench warfare so characteristic of the present European conflict. Owing to the difficulty and often impossibility of draining trenches, the men are frequently compelled to stand for hours in cold water or mud, up to or above the ankles. Stagnant water becomes warmed to some extent, but in running water the suffering and danger are much greater. The factors of wetness, cold and tight-fitting foot and leg wear render the circulation sluggish and the skin liable to infection, conditions which tend to the production of extensive chilblains and a type of frostbite which may terminate in gangrene.

The preventive measures consist in keeping the feet dry and warm. Rubber boots over woolen socks will do, but are expensive and seldom available. A simple practical device is to thoroughly grease the feet and socks. Still much better is to wear socks made of thin, soft oil-silk, between two ordinary socks, the outer one well greased.

\section{ASPHYXIATING GASES.}

Notwithstanding the clause of the Convention of Geneva forbidding belligerents " To employ poison or poisoned weapons," asphyxiating gases, one of the new and most terrible developments of warfare, are used on an extensive scale. We are, therefore, in presence of a trauma rather than a disease. Chlorine seems to be the most used, but carbon monoxid, sulphurous acid, formaldehyde, bromin and hydrocyanic acid are alleged to have been utilized. The symptoms are most distressing: gasping respiration with marked "air hunger," sharp pain in the air passages and extreme cyanosis.

The only preventive measure so far found effective is the wearing of a mask or respirator, whereby the inspired air is made to pass through a preparation which neutralizes the poisonous gas. 


\section{DISEASES CAUSED BY IMMORAL OR INTEMPERATE HABITS.}

\section{VENEREAL DISEASES.}

These diseases belong to the group of infectious disorders, but it is deemed best to consider them by themselves under the above caption.

They include three distinct affections, all infectious and contagious, namely :

I. Gonorrhea, caused by the gonococcus, a micro-organism (diplococcus) which not only produces the primary and direct lesions of the genital tract, but may also reach many other parts of the body, resulting in gonorrheal septicemia, endocarditis, arthritis, myositis, ocular complications, neuritis, etc.

2. Soft chancre, caused by the bacillus of Ducrey; this organism is arrested by the inguinal lymph glands, so that the sore always remains localized.

3. Syphilis, caused by a sprrally-curved organism or spirillum (Treponema pallidum) which, at first localized in a small, inactive. chancre, soon invades the entire system, so that there is no organ in the body nor any tissue in the organ which may not become involved. Frequently associated with this organism is another spirillum (Spirochata refringens), larger and more darkly stained, but bearing no causal relation to syphilis.

Venereal diseases, which always have the highest rate of admissions in peace time, may become less prevalent under the strenuous and exciting conditions of war. During the Civil War and the Spanish-American War they caused fewer admissions ( 83 and 82 per $\mathrm{I}, \mathrm{OOO}$, respectively) than several of the other common camp diseases, passing from the first to the fifth place. This, however, was due, not so much to an actual reduction of venereal diseases as to the greatly increased rates of the other diseases.

In the war ficlds of Europe (I9I5 and 1916), a decided increase has been noted (from Io to I6 per celli. according to Gaucher) not only in the belligerent armies but also in the civilian population.

They are, by far, the most important factor affecting the efficiency 
of modern armies in peace. Their rates in the U. S. Army at home, per I,000 of mean strength, are given in the following statement (Report of Surg. Gen., I916) :

\begin{tabular}{|c|c|c|c|c|c|c|c|c|c|c|c|c|}
\hline & \multicolumn{3}{|c|}{ Admitted. } & \multicolumn{3}{|c|}{$\begin{array}{l}\text { Discharged on } \\
\text { certificate of } \\
\text { disability. }\end{array}$} & \multicolumn{3}{|c|}{ Died. } & \multicolumn{3}{|c|}{ Noneffective. } \\
\hline & $\underset{\sim}{\stackrel{5}{0}}$ & 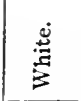 & 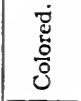 & 蒄 & 莒 & $\begin{array}{l}\stackrel{0}{0} \\
\stackrel{0}{0} \\
0\end{array}$ & 焉 & 莣 & $\begin{array}{l}\overrightarrow{8} \\
\stackrel{0}{0} \\
\dot{0}\end{array}$ & 焉 & 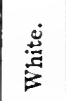 & $\frac{\pi}{0}$ \\
\hline \multicolumn{13}{|l|}{$\begin{array}{l}\text { Gonorrhea and re- } \\
\text { sults: }\end{array}$} \\
\hline 1915. & 57.56 & 50.91 & $4 \mathrm{I} .82$ & $0.3^{T}$ & 0.32 & & 0.02 & 0.02 & & 2.05 & 2.04 & 2.19 \\
\hline 19 & $\begin{array}{r}5 \mathrm{I} \cdot 35 \\
40.66\end{array}$ & $\begin{array}{r}52.04 \\
0 .\end{array}$ & $\begin{array}{l}28.99 \\
60\end{array}$ & .32 & $\begin{array}{r}.33 \\
68\end{array}$ & & & $\ldots$ & & $\begin{array}{r}1.80 \\
1.81\end{array}$ & 1.8I & 1.44 \\
\hline $\begin{array}{l}191 \\
191\end{array}$ & 49.46 & 49.14 & 60.14 & .66 & .68 & & & 0 & & $\begin{array}{l}1.84 \\
3\end{array}$ & 1.84 & 1.65 \\
\hline $\begin{array}{l}1912 \\
1911\end{array}$ & 70.07 & 69.87 & 74.36 & .60 & $.6 \mathrm{I}$ & 0.40 & .02 & .02 & & 3.26 & 3.27 & 2.94 \\
\hline 19 I I & 94.52 & 94.48 & 45.12 & .79 & .84 & . & & & & 4.68 & 4.72 & 3.93 \\
\hline \multicolumn{13}{|l|}{ Chancroid: } \\
\hline I915. & 15.80 & 15.73 & 17.63 & .03 & .02 & $.4 \mathrm{I}$ & & & & .72 & $.7 \mathrm{I}$ & .85 \\
\hline & 19.96 & 20.09 & 18.56 & & & & & & & .80 & .79 & I. $1 \overline{8}$ \\
\hline & 15.38 & 15.11 & 24.76 & & & & & & & .57 & .56 & .98 \\
\hline & 20.09 & 20.52 & 10.45 & .03 & .03 & & & & & I.OI & 1.03 & .50 \\
\hline $19 \mathrm{I}$ & 25.04 & 25.22 & 21.82 & & 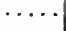 & $\cdots$ & & & $\cdots$ & 1.32 & 1.34 & 1.05 \\
\hline \multicolumn{13}{|l|}{ Syphilis: } \\
\hline 1915. & 17.24 & 17.44 & 12.30 & .54 & $5 \mathrm{I}$ & 1.23 & .05 & .03 & 0.41 & 1.22 & I.23 & I. 18 \\
\hline IOI & 18.53 & 18.86 & 7.66 & .55 & .43 & 4.38 & .05 & .05 & & I.15 & I.II & 2.46 \\
\hline 191 & 20.99 & 20.98 & 21.23 & .35 & .29 & 2.36 & .07 & .05 & .59 & I.I7 & I.15 & 1.90 \\
\hline 19 & 25.58 & 25.84 & 19.69 & .62 & .61 & $.8 n$ & .03 & .0 .3 & & 1.70 & I.68 & $2.1 \mathrm{I}$ \\
\hline I9I I & 44.30 & $45 \cdot 33$ & $25.9 \mathrm{I}$ & .52 & .48 & 1.36 & .11 & . II & & 2.82 & 2.85 & 2.30 \\
\hline \multicolumn{13}{|l|}{ Total venereal: } \\
\hline 1915 & 83.60 & 84.08 & 71.75 & .88 & .85 & I. 64 & .06 & .05 & $.4 \mathrm{I}$ & 3.99 & 3.98 & 4.22 \\
\hline & 89.84 & 90.99 & 52.52 & .87 & $.7 \pi$ & 4.38 & .05 & .05 & & I.15 & I.II & 2.46 \\
\hline & 85.83 & 85.23 & 106.13 & I.01 & .97 & 2.36 & .07 & .05 & .59 & 3.58 & 3.55 & 4.53 \\
\hline & 115.74 & 116.24 & 104.50 & I. 26 & I.26 & I. 2 I & .05 & .05 & & 5.96 & 5.98 & 5.55 \\
\hline 191 & 163.85 & 165.03 & 142.85 & I. $3 \mathrm{I}$ & $1.3 \mathrm{I}$ & I. 36 & .11 & $.1 \mathrm{I}$ & & 8.82 & 8.91 & 7.29 \\
\hline
\end{tabular}

It is seen from the above table that, as the natural effect of the prophylactic measures urged and imposed upon enlisted men, a decided improvement in all rates has taken place since I9I I.

In the Philippines, for $191 \mathrm{I}$, the admission rate was 305.12 and for non-effectiveness 16.63 . For 1915 the admission rate was reduced to 186.32 .

For the entire Army, at home and in the colonies, for I9II, the total admission rate was 185.13 , and non-effective rate IO.I4, corresponding to 738 men constantly sick and excused from duty by reason of venereal diseases. For I9I4 and I9I 5 the non-effective rate was reduced to 4.73 and 4.78 respectively or less than one-half.

In the U. S. Navy, the rates do not materially differ from those of the Army. In 1907, the Surgeon General reported that their pro- 
gressive increase "is alarming and calls for the most serious consideration." The rates of admissions for 1909-I9I I were 199, I96 and 177 , respectively, but have, since then, also been substantially reduced.

Comparing our rates with those of foreign armies, a great difference is apparent. The latter, as found in the latest obtainable data, are as follows for home troops: British, 66; Austro-Hungarian, 54.2; French, 27.8; Prussian, I8.7; Bavarian, 15.2. In trying to account for this discrepancy it must be remembered that the methods of reporting disease vary in different armies; in some, only patients treated in hospital are accounted for, while in our service all patients excused from any duty, even while in barracks, must be entered on the sick report. As regards venereal diseases, our soldiers, for hygienic reasons, are advised to report to the surgeon as soon as possible, without fear of punishment, and probably fewer fail to do so than in foreign armies.

But after making due allowance for all those causes which render venereal statistics so proverbially unreliable, we must accept the fact that, in this class of diseases, our army occupies an unenviable preeminence. Two general causes will mostly account for this, and they likewise apply mutatis mutandis to the preeminence of the British rates among European armies before 1915. I. The American soldiers, as volunteers, receive a liberal pay, and financially are much better off than those of other countries under compulsory military service; furthermore, they are not subjected to the same stren110us work and irksome restraints; hence they enjoy greater opportunities and facilities for dissipation. Let the average soldier, from any race or nation, be given money and leisure, and abundant pleasure resorts within easy reach, and the inevitable result, as expressed in terms of venery and alcoholism, can be readily foretold. 2. In continental Europe, the State realizing the gravity of the ravages of venereal infection endeavors to restrain prostitution, or mitigate its effects, by police regulations whereby prostitutes are subjected to systematic registration and examination so that all found diseased may be segregated and cured. There can be no doubt that such a system is justifiable on the part of the State on the ground of self-defense against the most widespread and loathsome of contagious diseases, nor of its great efficacy in checking them, especially among soldiers. But a strong public sentiment has 
opposed this recognition and regulation of prostitution by the State authorities in England and America, with the result that venereal rates in both countries greatly exceed those of other civilized nations where the system is still more or less in vigor.

It may be argued that volunteer soldiers, that is to say, men who freely enlist in time of peace, do not ustally come from the more industrious and sober classes, that they are mostly men of unformed and unstable character and habits, of neglected education, uncultivated moral sense, and who take but little thought of the consequences of their acts. But, on the other hand, one must remember that they are sifted men, who have passed a careful physical and mental examination, and whose habits have been inquired into and found at least good. It is doubtful, therefore, whether, if placed. under the same conditions, the proportion of our volunteers violating the moral laws would be greater than among the compulsorily enrolled soldiers of European armies.*

Diagnosis. - The discovery of the causative organism of syphilis and the elaboration of the Wasserman reaction have rendered the diagnosis of this disease easier, more reliable and possible at a much earlier date, so that treatment can be started before any damage is done. On the appearance of the initial lesion a positive diagnosis is made by finding the treponema. The demonstration is still more complete if a positive WVasserman reaction can be obtained. This reaction is positive in more than one-fourth of the cases dirring the first week, and nearly in all cases by the end of the fourth week. It may also be positive in a few other infections, but in none whose diagnosis can be confused with syphilis, except perhaps yaws. The chief difficulty lies in the correct interpretation of negative results. Thus it is absent in syphilitic cases undergoing a course of mercurial or arsenical treatment, or immediately after such a course. The Wasserman reaction also enables the physician to recognize latent syphilis in the absence of symptoms, and to determine with exactness the time when a patient may be considered cured. By means of it, Reasoner, U. S. Army, found that 5 per cent. of the enlisted personnel at his post gave evidence of syphilitic lesions. The medical examiners at Fort Slocum (Mil. Surg., June,

* Note.- Inasmuch as a large American drafted army is soon to be mobilized in training camps, and later proceed to the battlefields of Europe, we shall probably obtain data that will throw further light upon this subject. 
I9I I) found that 46.1 per cent. of all men discharged on certificate of disability gave a positive reaction.

Thus has also been ascertained that gangosa, a disease of the Pacific Islands causing serious disfigurement of the nose and adjoining regious, is a form of tertiary syphilis.

The luetin test is of most value in the latent and tertiary stages, when the IVasserman reaction becomes less reliable. It sometimes happens that in cases in which the Wasserman reaction has been negative for one or two years, the lnetin test or the provocative Wasserman test will still demonstrate the presence of the disease.

The standard of cure in the Army is thus formulated by Nichols: "One year without treatment, without any suspicious clinical signs, with several negative Wasserman reactions and no positive ones, and with a negative provocative Wasserman reaction and luetin test at the end of the year."

Prophylaxis.-Outside of purely moral teaching, which must ever remain the fundamental basis of all sound home and school education, the most fruitful prophylactic measures may be considered, seriatim, as follows :

I. Soldiers should be taught all knowledge deemed necessary and useful on the subject. For instance, that sexual intercourse is not necessary for the attainment of the best physical and mental health, and that a strong, manly character is developed only by self-control and continence. They should have a proper appreciation of the prevalence and gravity of venereal diseases. Thus it is estimated that fully one-third of all prostitutes are suffering from some form of them in an infectious stage. According to Leser, 33 per cent. of all syphilitics eventually die of tuberculosis, paralysis or aortic aneurism. The sinister predilection of tertiary syphilis for the nervous system is well known. All deaths from general paralysis and locomotor ataxia, as well as half of those from paraplegia and softening of the brain, are attributable to syphilis.

Many men expose themselves thoughtlessly, with the impression that, at the worst. a few days in hospital will suffice to get rid of the consequences. This is foolish and dangerous ignorance which officers, especially medical officers, should endeavor to dispel by a few plain talks, demonstrating to them the many complications and sequels of gonorrhea as well as the ravages of syphilis which affect not only the incontinent but, through matrimony, many innocent 
women and children. Let them know that, in the opinion of gynecologists, a majority of the hazardous surgical operations performed upon married women are made necessary in consequence of gonorrheal infection by the husband; and another terrifying fact, that syphilis, so far as absolutely known, is not only directly transmitted from father to children, but the sole disease thus transmitted, as it were a special curse upon the human race.

All this lnowledge should be particularly imparted to recruits, for it is a matter of record that they are much more prone to expose themselves and contract venereal infections than trained soldiers.

2. All efforts to abolish prostitution have been futile; universal experience has shown that it is an inevitable social evil; but that if it cannot be prevented, its effects can certainly be mitigated and reduced to a minimum by suitable regulations. It has been found that at a certain number of posts, at home and in our colonies, the civil authorities have coöperated with commanding officers in exercising sanitary supervision over prostitutes. It is believed that such coöperation, wherever it can be secured, will always result in a marked decrease of venereal rates.

In war the conditions are different, and the strong hand of military power can be exerted to protect camps and garrisoned cities from the worst evils of prostitution. It is even reasonable to suppose that, under such circumstances, the civil authorities will willingly lend their assistance. The registration and inspection of prostitutes, as a measure of military necessity, was done with great success at Nashville and Memphis, Tenn., during the Civil War, so that there are excellent precedents for it.

3. The life of the soldier in garrison and canip should be made as attractive as possible, so that he may not so readily succumb to the allurements of the outside dens that lay their snares for him. The post exchange should have all its recreation features fully developed, such as reading-room, card and billiard rooms, lunch room. gymnasium and bowling-alley. The restoration of the canteen feature, with the regulated sale of beer and other mild spirituous refreshments, would probably do more to keep the men at home and away from the haunts of vice than any other preventive measure. The establishment of soldiers' ciubs, independently of the post exchange, has also been strongly recommended.

4. The systematic physical inspection of the men is of undoubted 
value, not only in discovering neglected and unreported cases, but also in prompting them to submit to treatment as soon as the disease manifests itself. Under G. O. No. I7, I912: "Commanding officers will require a medical officer, accompanied by the company or detachment commander, to make a thorough physical inspection twice in each month of all enlisted men (except married men of good character) of each organization belonging to or attached to the command. These inspections will be made at times not known beforehand to the men and preferably immediately after a formation. The dates on which the physical inspections of the various organizations are made will be noted on the monthly sanitary reports."

"At these inspections a careful examination of the feet and footwear, and of the condition of personal cleanliness of the men will be made, as well as a careful observation for the detection of venereảl diseases."

Men found suffering from venereal disease are to be subjected to treatment and strictly confined to the limits of the post.

5. It is essential that men contracting any form of disease after sexual intercourse should report at the hospital as soon as the first symptoms manifest themselves, for examination and treatment, so that the infection may be attacked when most curable and before complications arise. As part of the treatment, each man should be furnished with a printed leaflet telling him clearly what to do and what to avoid to cure himself and prevent the contamination of his comrades. While under treatment, or at least as long as the disease remains in an infective stage, he should be confined to the limits of the hospital.

6. The venereal register kept at all post hospitals, as prescribed by the Surgeon General, is an excellent means of control whereby a man, once admitted to treatment, is kept under continuous observation until cured. Should he be transferred while under treatment, his venereal history is transmitted, together with other pertinent details, to the medical officer who is to have future charge of the case.

7. The question of personal prophylaxis, that is to say, of the employment of disinfectant applications to prevent infection after sexual intercourse, has been much discussed from the moral and sanitary points of view. There is now a general consensus of pro- 
fessional opinion, in this country and Europe, that such prophylaxis is not only justifiable but desirable. There is no good reason why a man who lacks the necessary moral stamina to control his sexual appetite should not, at least, try to prevent the possible danger of a physical disability which "unfits him for the performance of his duty as a soldier and seriously impairs his usefulness as a citizen." (Rep.Surg. Gen.) The contention that the application of a disinfectant removes the fear of contracting venereal infection, and is an incitement to licentiousness by making it safe, has been disproved by actual observation. It is notorious that the influence of fear is a deterrent factor of slight importance. On the contrary, it has been remarked that the prominence given to venereal diseases by instruction and the application of personal prophylaxis, brings the better class of men to a greater realization of the enormity of the evils likely to result from exposure

Medical officers are unanimous in their belief that at least 90 per cent. of venereal cases can be prevented by the proper application of a suitable disinfectant within a few hours after exposure.

G. O. I7, I912, directs that "Commanding officers will require that men who expose themselves to the danger of contracting venereal disease shall at once, upon their return to camp or garrison, report to the hospital or dispensary for the application of such cleansing and prophylaxis as may be prescribed by the Surgeon General. Any soldier who fails to comply with these instructions, if found to be suffering from a venereal affection, shall be brought to trial by court-martial for neglect of duty."

The regulations prescribed by the Surgeon General, under this order, are briefly as follows:

A suitable, easily accessible room is set apart for the purpose and placed in charge of a properly instructed hospital-corps man, who is on duty or within call at all times, and to whom the men report for treatment.

The genital organs are thoroughly washed with soap and warm water. An injection is made into the urethra of $4 \mathrm{c}$. c. of the standard solution of 2 per cent. protargol dissolved in glycerine (I5 parts) and water ( 85 parts). This should be retained in the urethra for three minutes. In individual cases, when the protargol solution is found to produce an irritating effect, a 20 per cent. sclution of argyrol may be used. The entire penis is rubbed with calomel 
ointment ( 30 per cent. in benzoated lard), care being taken that the folds of the prepuce and about the frenum are thoroughly covered. The parts are then wrapped in a napkin of soft paper furnished for the purpose in order to protect the clothing. Medical officers who deem it necessary to use other preparations than those above specified are required to report the reasons for their departure from the usual treatment.

In view of the fact that the hospital method described above is not applicable to soldiers on pass or furlough of more than 24 hours, it is recommended that protective packets be kept on hand for sale at post exchanges. Men should be advised to provide themselves with these packets before leaving camp or garrison, for self-treatment as soon as possible after exposure, if they do not intend to return within a few hours thereafter. In such cases the medical officers, if satisfied that the packet has been properly applied, may omit the hospital treatment.

Several types of prophylactic packets or tubes are being successfully used. The Slee tube is a two-compartment tube, with a 33 per cent. calomel ointment in one compartment and a 20 per cent. argyrol (or 2 per cent. portargol) solution in the other; the solution is injected into the wrethra and the ointment rubbed on the outside.

Later investigations have demonstrated that calomel ointment will not only prevent chancre and chancroid, but that, when injected into the urethra, it will also kill gonococci and prevent gonorrhea, so that argyrol and protargol can be dispensed with and the process simplified." The so-called "A and N packet" has been found very efficient in a series of laboratory experiments; it consists of :

$\begin{array}{lrcc}\text { Camphor, } & 3 & \text { per } & \text { cent. } \\ \text { Phenol, } & 3 & \text { " } & \text { " } \\ \text { Calomel, } & 25 & \text { “ } & \\ \text { Lanoline, } & 34 & \text { " } & \text { " } \\ \text { Bcnzoated lard, } & 35 & \text { " }\end{array}$

The same constituents, but without camphor and lard, were found much less efficient against gonococci, showing the essential part played by these latter substances in securing a diffusible mixture and close contact of the active ingredients with the mucous surfaces.

*The Military Surgeon, Sept. and Dec., 1910. Col. L. M. Maus, U. S. Army. 
Of the contents of this packet a small quantity is injected into the urethra and the remainder rubbed on the glans and prepuce.

8. Stoppage of pay whilc on sick report for venereal disease, as provided by law, $\uparrow$ is just and logical punishment for a soldier who. in spite of frequent admonitions, deliberately exposes himself to venereal contagion and then neglects the use of the prophylactic measure which is almost certain to save him from the physical consequence of his act. Not only does the government lose the benefit of his services, but is put to greater expense on his account, while his comrades are obliged to perform his duties in addition to their own. It has also been observed that some men of indolent and vicious nature expose themselves purposely with women known to be infected in order to secure hospital treatment and get released from irksome duties. Many men turn a deaf ear to the promptings of their moral sense, but few are insensible to a loss of pay, and no form of punishment could have a greater deterrent effect. It will make offenders particularly careful to have recourse to prophylactic treatment and thus greatly reduce their chances of infection.

\section{ALCOHOLISM.}

\section{(See Alcoholic Beverages, page 4I4.)}

Alcoholism and its immediate results, in the U. S. Army, increased steadily from 1900 to 1907 . From the latter date it has constantly diminished, so that the admission rate of I9II was only 20.31 and for $1915,13.87$ per I,O00 of strength, the latter rate being the lowest for any year since I870. In the Philippines, the admission rate for $19 \mathrm{I}$ I was 28.50 and the non-effective 0.43 , practically the same as for I9Io. For 1915, the admission rate is only 12.0 and the non-effective o.I4.

For alcoholism, even more than for venereal diseases, statistics of admissions are quite valueless for purposes of comparison. In foreign armies drunken men are kept in the guard-house until again fit for duty, and seldom made a matter of record, as in our service. Statistics of mortality, although not entirely reliable, are more useful

$\dagger$ The following clause of an act making appropriation for the support of the Army for the year ending June 30, 1913:

"Provided that no officer or enlisted man in active service, who shall be absent from duty on account of disease resulting from his own intemperate use of drugs, or alcoholic liquors, or other misconduct, shall receive pay for the period of such absence." 
as a basis for comparison. For " acute alcoholism" the death rate for our Army, at home, was 0.II in I9I0, 0.04 in I9I I and 0.06 in I9I 5 ; for the British Army, o.03 in I9Io; and for the French, o.oor in I909. There is no doubt that, in alcoholic intemperance, our Army still occupies an unenviable high standing.

The reasons already given for the prevalence of venereal diseases in our army will also explain this high standing in alcoholism. Both forms of vice, alcoholic intemperance and sexual debauchery, are always closely associated, one leading to the other, so that the soldier who frequents the saloon falls an easy prey to the prostitute. It is the conviction of most officers that the abolition of the canteen, that is to say, the prohibition to sell any kind of alcoholic beverage, however mild, on military reservations, has maintained the rates of alcoholism and venereal diseases at an unnecessarily high level. Most soldiers, at the time they enlist, have formed the habit to indulge moderately, whenever so inclined, in beer or other spirituous refreshment. The fact of becoming soldiers cannot be expected to render them totally abstinent. Formerly they could get beer and other mild beverages at the canteen to any reasonable extent and always under official supervision. This feature of the canteen being abolished, the men now seek their alcoholic refreshments outside, away from supervision and restraint, where, for the weak, there is every inducement to drunkenness. Its restoration has been strongly urged by the War Department and recommended to Congress in Presidential messages. 


\section{CHAPTER XII.}

\section{THE TOBACCO AND DRUG HABITS.}

\section{TOBACCO.}

Tobacco is the dried leaves of Nicotiana tabacum, indigenous to tropical America and now cultivated in all warm and temperate climates.

Its principal constituents are: nicotine, an alkaloid; nicotianin, a solid, camphor-like body to which its odor is chiefly due; traces of hydrocyanic acid; various irritant aldehydes, particularly furfural; a minute quantity of essential oil which causes its flavor; about 25 per cent. of albuminoids; a high proportion of malic, citric and other organic acids; a little sugar, and a large amount of salts, yielding I 5 to 20 per cent. of ash.

Tobacco smoke contains: vapor of water, free carbon (in minute particles), ammonia compounds, carbon monoxid and dioxid, nicotine, prussic acid and furfural.

Nicotine, generally considered the active principle of tobacco, is a volatile liquid alkaloid, very soluble in water and virulently poisonous, standing next to prussic acid in the rapidity and energy of its toxic action. Tobacco may yield as much as 7 or 8 per cent. of this alkaloid, but seldom contains more than 3 , the average (as consumed) being less than 2 per cent.; thus Havana cigars seldom exceed I per cent. Nicotine is colorless in its pure state, but rapidly turns brown on exposure, when part of it is transformed into an allied substance, pyridine, having practically the same properties. The amount of hydrocyanic acid in tobacco smoke, when present at all, is too minute to have any importance in this connection. More suspicious is the aldehyde furfural, a highly poisonous volatile oil which, according to the investigations of the London Lancet, * may prove to be the most injurious factor in smoking. It is commonly present in the smoke of cheap Virginia cigarettes. To it, by many, are also attributed the toxic effects of cheap liquors.

The primary effects of tobacco in those not yet habituated to its use are well known: giddiness, nausea, vomiting, diarrhea, faintness and extreme. prostration with complete muscular relaxation.

* The toxic factor in tobacco, 1912. I, 994 and II, 547. 
If used in increasing quantities it produces cold perspiration, dilated pupils, very slow and intermittent pulse, spasms, laborious and oppressed breathing, great numbness as well as impaired power of the limbs, and sometimes heart failure, although death, when it occurs, usually results from paralysis of the respiratory muscles. These symptoms show that nicotine acts primarily upon the spinal and sympathetic nervous centers, and only secondarily upon the brain.

As in the case of other narcotics, a stage of comparative toleration is reached by most people, after which tobacco may be used with apparent immunity. The degree of susceptibility varies greatly; a certain percentage of men, although in perfect health, have such an idiosyncrasy in regard to tobacco that, in spite of repeated efforts, they never acquire toleration, and therefore never develop the smoking or chewing habit; others are never able to exceed a very moderate indulgence without suffering from gastric disturbance and nervousness.

The advantages claimed for tobacco, when used in moderation, are that it gives one something to do when bored, that it has a calming and soothing effect on the worried mind, aids digestion after meal, conserves strength when food is scant, and blunts the edge of hardship. It has been observed that the first effect of tobacco is to produce a slight vaso-motor constriction of blood-vessels with attenclant increase of blood pressure; in other words, to stimulate the circtlation. This explains the cheering effect of a smoke upon the exhausted athlete after a hard contest, or upon the hardmarching soldier after reaching camp. In view of the universal use of tobacco and from the result of daily observation, it seems safe to say that in the case of men who, having reached their full physical development, readily acquire the stage of toleration, and indulge in smoking or chewing with discretion and moderation, the injurious effect of tobacco is probably a negligible quantity.

It is impossible to believe, however, that the habitual and constant use of such a virulent poison as nicotine can ever be of any real benefit to the system. All that can be claimed for tobacco is that, under the circumstances just mentioned, it may be practically harmless. It should be stated that most authorities on the subject are inclined to believe that the habit of smoking, persistently indulged in, is a form of slow, insidious chronic poisoning, detrimental to 
health at all times, and that although a period of years may elapse during which the smoker enjoys apparent immunity, a day of reckoning is almost sure to come after middle life when the deteriorated nerve-cells are unable to furnish sufficient energy for the proper functioning of the bodily organs. It is true that sometimes hard smokers are also men of strong physique, great energy and high mental attainments, but it must be remembered that only men of. naturally strong constitution are capable of acquiring the tobacco habit with apparent immunity, and that their physical and mental output is more likely to be in spite, than on account of the habit, and would probably have been still greater and of finer quality had they been entirely free from the effects of chronic nicotine poisoning.

It may seem surprising that in view of the camparatively large amount of nicotine present in tobacco, smoking and chewing should not be more immediately injurious. This is explained by the fact that only a very small proportion is absorbed, most of it being burned in smoking, stored in the bowl of the pipe or the stump of the cigar, or ejected with the expectoration in chewing.

Tobacco, in a general way, but particularly in youth, interferes with metabolism, therefore with the normal growth and repair of tissues, thus lowering physical and mental vigor, endurance to fatigue and resistance to disease. That, in young men, it checks the height and weight of the body and chest development has been well established. That it also checks their mental development is the consensus of opinion of hygienists, thus fairly stated by Dr. G. L. Meylan of Columbia University: "It is generally conceded that the use of tobacco by college students is closely associated with idleness, lack of ambition, lack of application and low scholarship, though these may not be due entirely to the tobacco." It is also notorious that the use of tobacco predisposes to worse habits. A young man always starts smoking before he starts drinking. Tobacco by its sedative effect makes him feel a craving for alcoholic stimulation; thus the two habits are nearly constantly associated.

The tobacco habit, like the opium habit, once developed becomes tyrannical and demands to be satisfied. It produces a craving which unfits its victims for work or enjoyment until gratified, and this gratification often leads them to sacrifice many of the proprieties and amenities of life.

Chewing is less deleterious than smoking. the tobacco juice com- 
ing in contact only with the mucous membrane of the mouth, whereas smoke reaches a much larger area of absorbent surface; furthermore it is probable that nicotine volatilized in the smoke is more readily absorbed than in liquid form. Tobacco chewing, however, considered simply from the esthetic point of view, is a most repugnant habit which no well-bred man should cultivate and inflict on his associates.

From what has been said it is rational to conclude that the use of tobacco in any form by students in all educational establishments, including the Military and Naval Academies, should be absolutely prohibited. The development and strengthening of the degree of manly self-control necessary to comply with such prohibition would not be the least of the benefits conferred by such establishments.

Local and special effects.- Smoking and chewing often cause irritation and congestion of the mucous membrane of the mouth and pharynx. The catarrhal condition of the throat and hoarseness of the smoker is commonly observed. The saliva secretion is excessive and its digestive quality more or less impaired.

The heart is readily affected by tobacco, especially in young men. The so-called tobacco heart, with rapid, irregular or intermittent beat, is one of the usual manifestations of hard smoking. Many candidates for the Military and Naval Academies, as well as for enlistment in the Army and Navy, are rejected on account of it. Every athlete knows that smoking "hurts the wind," that is, the ability of the heart to respond quickly to extra work.

The optic nerve is also specially liable to the injurious influence of nicotine; the vision becomes weak and blurred, with floating spots, and complete amaurosis may follow.

One of the most common effects of smoking is degeneration of the motor nerves, with consequent impairment of muscular control; the result is tremulousness of the hands and inability to perform delicate movements requiring nice coördination. Students addicted to smoking are recognized by their unsteady hands and inability to draw a clean, straight line. This tremulousness is of course much aggravated when the habit of drinking is superadded. Inasmuch as the chief requirement of a soldier is that he should be a good shot, it follows that tobacco which affects the precision of eye and hand must necessarily greatly impair his efficiency.

Pipe, cigar and cigarette.- Tobacco smoke, as above mentioned, 
contains several deadly poisons, nicotine which is volatilized by the heat, carbon monoxid and furfural. Carbon monoxid, the dangerous constituent of coal gas probably plays only a minor part in the toxic effect of tobacco smoke; furfural is still under investigation and may be shown to be a much more serious factor; as matters stand, nicotine must still be considered the chief offender.

Most of the nicotine is destroyed in the burning of the tobacco, and only a relatively small proportion is drawn in with the smoke; the greater the access of air to the tobacco the more complete is its combustion and the nore nicotine is destroyed.

According to careful experiments recorded by the London Lancet, Virginian cigarettes, with a nicotine value of $\mathrm{I} .40$ per cent., gave up only i 2 per cent. (of the total nicotine) to the smoke. When, however, the tobacco of these cigarettes was smoked in a pipe, 53 per cent. of the nicotine came out with the smoke. Havana cigars, with nicotine value of 0.64 per cent., gave up to the smoke 3I per cent., while with a British cigar containing I.24 per cent. of nicotine the proportion in the smoke was 83 per cent. From these observations it follows that the smoke of cigarettes contains much less nicotine than that of pipe or cigar. This is due to the fact that, in cigarettes, the tobacco is generally drier and better aërated, so that its combustion is more complete than in pipe or cigar. For the same reason, cigarette smoke contains less of carbon monoxid and other products of combustion, and is less irritating.

Notwithstanding all this, it is very doubtful whether cigarettes should be commended and preferred to other forms of smoking. On account of their cheapness, convenience and quick combustion, the smoker is but too prone to develop a peculiar craving and muscular unrest only satisfied by a constant succession of them, so that, in the end, more tobacco is consumed. However, the chief objection to the cigarette is that the smoke, being less irritating than that of the pipe or cigar, is almost always inhaled, that is, drawn into the larynx and the bronchial tubes, and therefore exposed to a much greater area of mucous membrane for the absorption of toxic substances.

Between the pipe and cigar there is but little choice. In both, the smoke is more or less charged with various irritant products of combustion. The pipe is said to yield more nicotine than the cigar and, to that extent, to be more objectionable. The cigar is much 
less harmful if a holder is used, and if thrown away when but little more than half consumed, that is, before the products of combustion, through which the smoke must pass, accumulate in the stump.

\section{THE MORPHINE AND COCAINE HABITS.}

Morphine is the principal alkaloid of opium, the exhudation obtained by incising the unripe capsules of Papaver somiferum.

In medicinal doses it produces a soothing calm of body and mind, with pleasant fancies and a disposition to sleep; the pulse becomes slower, the pupils contract slightly and the slin is moist. In larger doses, it causes redness of the face, heat and fullness of the head and marked contraction of the pupils; the mind is excited even to delirium, the pulse full and frequent and the skin hot and dry. Depression follows, with slow pulse, pale and damp skin, nausea, torpid mind and uncertain gait. The digestive functions are impaired and the bowels constipated.

From official documents it appears that over 400,000 pounds of opium are annually imported and consumed in the United States; 300,000 are manufactured into morphine, and it is estimated that 80 per cent. of this alkaloid is used by victims of the habit. To show the appalling extent of this evil in the United States, it is enough to note that Germany, with $65,000,000$ inhabitants, consumes only about 17,000 pounds of opium annually.

Morphine, by those addicted to its use, is usually self-administered by hypodermic injections, so that, in the habitué, the scars of numerous punctures are found on the arms and other parts of the body. As with alcohol and tobacco, the primary stimulation is followed by debility and depression which nothing but a fresh resort to the drug relieves; the longer it is used the larger the dose required and the more deep-rooted the habit. In spite of the terrible sufferings morphinism entails, including throbbing pains, spasms, neuralgia, insomnia, fearful anxiety, hallucinations, etc., such is its powerful hold on its victims that they are very seldom completely cured.

The habitual morphine consumer may be recognized by great emaciation, flabby muscles, inability to execute quick and complex movements, pale skin, contracted pupils, general nervousness, tremulousness of the hands, disturbed mentality ranging from abnormal vivacity and excitement to sluggishness and torpidity. His moral sense is blunted and distorted. 
Opiun smoking is a vice seldom if ever indulged in among soldiers and needs no further mention here.

The synthetic alkaloid heroin (diacetyl-morphine) is an allied narcotic, the addiction to which is likewise readily formed and leads to the most deplorable results. It is taken hypodermically or else as a snuff; in the latter case the patient generally suffers from chronic rhinitis. Otherwise the symptoms closely resemble those of the morphine habit.

Cocaine is an alkaloid obtained from the leaves of Erythroxylon coca, a small South American shrub.

In its native country the leaves are used as a gentle stimulant, diaphoretic and an aid to digestion. Medicinally, cocaine is mostly. used as a local anresthetic. When absorbed in sufficient quantity, it produces a pleasurable glow throughout the body, a general sense of cheerfulness and elation, relief from worries and an increase of bodily and mental vigor. These symptoms are associated with a remarkable endurance of fatigue and insensibility to hunger. After larger doses, the pulse is quick, feeble and thready, the skin pale and cold, the respiration shallow, slow and labored, the pupils dilated and insensible and the vision blurred. Formication has been noted. Hallucinations, convulsions and even maniac violence may also occur.

Most persons addicted to the cocaine habit take this narcotic by way of the nostrils, in snuff, and seldom use the hypodermic syringe. Therefore, in suspected cases, the membrane over the cartilaginous septum should be inspected for signs of inflammation and ulceration. The intoxicating effects of cocaine are described as being much more enticing than those of morphine and the habit has become alarmingly widlespread in this country. As in the case of morphinism, it is most difficult of eradication.

The many manifestations of cocainism are varied and confusing. At first are noticed anorexia, dyspepsia, constipation, restlessness, insomnia, inabilify to concentrate the mind. Later, the body is emaciated; the face pale and cadaveric, with sunken, wandering eyes, dilated and insensible pupils, twitching muscles and lack of co-ordination. There is impairment or loss of memory and, at times, emotional excitement with voluble talk, hallucinations and delusions of persecution. Epistaxis from nasal ulcerations is not rare. 
Careful experments have shown that cocaine, after exhausting work or forced marching, will temporarily restore physical and mental vigor, and blunt the sensation of hunger. This, however, is at the expense of the reserve energy stored in the system and which must be made up by sufficient rest later. The use of cocaine for this purpose may be permissible in individual cases, by persons of discretion, under medical supervision; but for a body of men, there are no conceivable circumstances which would make its use desirable and justifiable, and where coffee or tea would not be more serviceable.

In the Army and Navy, the attempt to cure men addicted to drug habits is futile and dangerous; the only logical course to pursue is to promptly discharge them from the service. 


\section{CHAPTER XIII.}

\section{DISEASE-TRANSMITTING ANIMALS.}

Man is the chief transmitter of the diseases from which he suffers. With few exceptions, it is through his agency, direct or indirect, that they are disseminated. But the germs of some of them are also conveyed, sometimes exclusively so, from man to man by insects and vermin with which man is more or less in contact. A few diseases special to domestic animals may be likewise directly transmitted to man; for instance, glanders by the horse and mule, anthrax by cattle and sheep, hydrophobia by the dog and cat.

Animal carriers or transmitters of disease may be conveniently classified and described as follows:

Class Arachnida. Animals with wingless body consisting of only two regions, the cephalo-thorax and abdomen, distinctly divided in spiders and scorpions, but merged together in mites and ticks. Also distinguished from insects in having four pairs of legs.

Order Acarina. Includes Mites and Ticks.

The mites are mostly very minute animals. The most notable is Sarcoptes scabiei, the cause of true itch or scabies. The egg-bearing female tunnels in the skin, chiefly between the fingers and on flexor surfaces, and lodges at the blind end of the tunnel, along which are scattered feces, eggs and larvæ. Another troublesome mite is the "red bug" or "harvest mite" (Trombidium), which attacks both man and animals in autumn. Other kinds are found in sugar (causing "grocers' itch"), cheese, flour and dry fruits.

Family Ixodidae. Ticks Animals with ovate, oval or rounded bodies without articulation or division into segments, the head, thorax and abdomen being merged together into one piece. Generally the rostrum (mouth parts) is visible at the anterior end, forming what is commonly regarded as the head, but in the suborder Argasince the rostrum is concealed by the overlapping cephalo-thorax so that the animal appears headless.

Recent researches have shown that ticks play an important part in the transmission of disease. They are widely distributed, and almost every mammal is liable to attacks from one or more kinds. 
The species of particular interest to the sanitarian are Ornithodoros moubata, the transmitter of the spirochete of African relapsing fever; Argas persicus, the transmitter of the germ of the Miana disease of Russia and Asia; Margaropus anmulatus, which conveys the protozoön (Babesia bigemina) of Texas fever; and Dermacentor andersoni of North America, the usual transmitter of the germ of the Rocky Mountain spotted fever (see page 84). Doubtless other species will be found capable of conveying disease to man.

Dermacentor andersoni Stiles (D. venustus Banks). Adult male and female about the same size, namely, 4 by $2.5 \mathrm{~mm}$., but the female, when gorged with blood, becomes at least three times as large. The male has a hard plate or shield covering the entire back and decorated with a somewhat complicated pattern of white and brown stripes. In the female the shield is much smaller, covering only the anterior portion of the body. Like other species, this tick exists under four distinct stages, namely, egg, larva, nymph and adult. The eggs are deposited on the ground in large masses (several thousands). The larvæ (seed ticks) which emerge from the eggs are minute six-legged animals. After feeding upon a suitable host, they drop to the ground and molt, becoming nymphs. In this stage they have eight legs, but no genital organs. The nymph waits until it can attach itself to a host, engorges blood, drops, molts its skin and becomes adult. Although a few of the individual ticks may complete their life cycle during one season, the majority require two years.

A very important peculiarity of this tick is that when the adult becomes infected with spotted fever, the germs of the disease pass through the eggs to the young of the next generation, which, even as larvæ and nymphæ, may thus transmit the infection. This power of transmission has also been observed in the tick of the African relapsing fever, as well as in that of the Texas cattle fever, so that it presumably exists in most species.

The area infested by this tick includes the northern Rocky Mountain region in the United States, and the valleys and plains to the east and west of it, from Montana, Wyoming, Colorado and New Mexico through Idaho and Utah to Washington, Oregon and Nevada. It also occurs in southern British Columbia and in the Rocky Mountains of Canada.

The animals which act as hosts are many, including cattle and 
other domestic animals, and most of the wild animals of the region. The adult tick is found mostly on cattle and horses, while, in the immature stages, it is found only on small wild animals.

Five other species of ticlis have been observed in the Bitter Root Valley of Montana, but their host relations are such as to render them comparatively harmless.

According to the experiments of Maver, spotted fever may be transmitted by various species of ticks, so that it is very important to prevent the spread of the infection to new territory where are found ticks more or less related to Dermacentor andersoni and, like it, known to attack man. These dangerous species are $D$. occidentalis of California and Oregon, Amblyomma americanum (lone-star tick) and $D$. variabilis (American dog-tick) of the Southern and Eastern States, Amblyomma maculatum of the Gulf Coast and $A$. cajennense of Texas and New Mexico.

Inasmuch as those ticks which are dangerous to man also feed on cattle, horses, sheep and other domestic animals, the most practical and effective way to destroy them is to dip these animals as often as may be necessary, during the proper season, in an insecticide bath, such as an arsenical solution or crude oil. Where dipping is not practicable, hand treatment of these animals should be resorted to. The killing of the small wild animals which serve as hosts to the ticks in their immature stages is also useful, as well as the clearing and burning of the brush in the shelter of which the eggs hatch and the larva and nymphxe wait for their prey.

Class Hexapoda. Insects proper. Body with three distinct regions, head, thorax and abdomen; the thorax consisting of three segments, the prothorax in front (sometimes hardly distinguishable), the mesothorax or main segment, and the metathorax or rear segment, each bearing a pair of legs. Generally the mesothorax and sometimes the metathorax bear a pair of wings. Eyes both compound and simple.

Order Hemiptera. Mouth-parts consisting of a horny sheath inclosing three sharp bristle-like organs, the whole forming a sucking proboscis.

Family Cimicidae. Genus Cimex. Bedbug. Wingless insects with brownish-red, flattened body and penetrating odor. The eggs are deposited in cracks, and in Io days hatch out into larvæ which develop into adults by a series of moltings. 
Two species are of interest as disease bearers: C. lectularius, the common cosmopolitan species, and $C$. rotundatus, of tropical and semi-tropical regions, with shorter, rounded abdomen.

A striking peculiarity of the common species is its ability to survive for long periods without food, specimens having remained alive for a year in a sealed vial with absolutely no means of sustenance (Marlatt). It is the carrier of European relapsing fever. Dutton by experiments, and Riggs, U. S. Navy, by observations, have shown that it transmits typhoid fever, the virulency of the typhoid bacillus remaining undiminished in its body for several weeks. According to Howard and Clark, it can absorb the virus from the blood of a poliomyelitis patient and maintain it active within its body for at least seven days. Also probably capable of transmitting plague. The rotundatus is believed to be connected with the transmission of kala-azar and probably leprosy. Kerr; U. S. Navy. found large numbers of acid-fast bacilli in bedbugs fed on the infected areas of leprosy patients.

According to Ricker, this animal conveys pathogenic germs with its saliva into the wound, like the mosquito.

For destruction of bedbugs, see page 495 .

To an allied family of the same order belongs the Texas or Mexican bedbug (Conorhinus), a winged insect with dark-brown body nearly an inch long, and a projecting, narrow head. Its bite is much more severe than that of the common bedbug.

Family Pediculidae. Genus Pediculus. Louse. Wingless insects hatched from acorn-shaped eggs or nits, and without metamorphosis. Three species are parasitic on man:

$P$. capitis. Head-louse. Thorax as broad as the abdomen. Eggs deposited on the hairs of the head.

$P$. vestimenti. Body or Clothes-louse. Larger than the preceding the abdomen broader than the thorax. Eggs deposited in the clothing.

P. pubis. Crab-louse. With almost square body, and large claws. Preferably inlabiting the pubic region, but may also be found in other hairy parts of the body.

The body-louse and head-louse have been proved to be the ordinary carriers and agents of trasnmission of typhus fever and recurrent fever (which see). They are also suspected of transmitting relapsing fever and possibly leprosy. Lice feeding on typhoid-fever patients have been found to contain the bacillus of this infection. 
Of insect parasites, lice are the most loathsome, but also the most easily gotten rid of. Any soldier infested with either kind should be promptly isolated and subjected to vigorous treatment so as to destroy both insect and egg. In case of head infection, or of other hairy parts of the body, the hair is cut very short, shampooed and washed with kerosene oil or a solution of formalin $(4 / 100)$. Kerosene is a powerful destroyer of lice and eggs; it can be used by spray or directly with a cloth. The body-louse is destroyed by disinfecting the clothing in steam, boiling it in water or immersing it for at least one minute in gasoline. Dry heat is also very effective. For blankets and woolen clothing there is nothing better than a hot flatiron well pressed down. Insect powder may be used on outside garments. Great attention must be given to the eggs or nits found attached to the hairs and the fibres of the clothing, especially along the seams.

Order Siphonaptera. Family Pulicidae. Subfamily Pulicinae. Fleas. Wingless insects, with two simple eyes and no compound eyes. Antennæe inconspicuous. Edges of head and prothorax armed with stout spines directed backward. Puncturing apparatus consisting of long, serrate mandibles and epipharynx, together forming a tube throngh which the blood is sucked up. Body compressed laterally and hind legs.specially adapted for leaping. The eggs of fleas are laid in the dust of crevices and hatch in about a week. The larva forms a cocoon, molts into a nymph and emerges as adult about three weeks later.

Fleas are the transmitters of bubonic plague from infected rats to man (see page 86 ).

The most important species in this connection are Xenopsylla cheopsis, Ceratophyllus fasciatus, C. anisus, Ctcnopsylla musculi, Cteriophthalmus agyrtes. $X$. cheopsis is the most prevalent in the tropics and subtropical regions; it is also common during summer and autumn in port towns of temperate zones having maritime intercourse with the tropics (Marseilles, London, San Francisco, etc.). In cooler regions, $C$. fasciatus is the most universally distributed, being associated more or less with $C t$. musculi and $C t$. agyrtes. The latter species seems to be the most common rat flea in England. but very seldom bites man. In Japan, $C$. fasciatus is replaced by $C$. anisus, a closely allied species. $X$. cheopsis and $C$. fasciatus are the 
species, which, when hungry, readily bite man, and therefore the most dangerous.*

The dog and cat flea (Ct. canis) and the human flea (Pulex irritans) may also be found accidentally on rats, but too rarely to play any part in plague transmission. In this country, the dog and cat flea is much more likely to become a nuisance in houses than the human flea which seldom occurs in great numbers. According to the experiments of W. F. Dutoon, it is capable of inoculating the germs of typhoid fever.

To get rid of fleas all rugs and, if necessary, mattings and carpets must be removed, beaten and then dusted with pyrethrum powder or sprinkled with benzine or gasoline. The floor is then scrubbed, especially the cracks and crevices, with hot soapsuds or a kerosene emulsion, or (which is much more effective) a 5 per cent. emulsion of creolin. The house dogs and cats must be washed with soapsuds and then freely dusted with pyrethrum or sprayed with benzine; but still more effective is a good washing with a 3 per cent. solution of creolin. Another plan to rid a room of fleas is to turn guinea pigs loose into it; after the fleas have collected on these animals they are stupefied with chloroform spray, combed out and burned. The most reliable method is doubtless sulphur fumigation wherever its destructive action upon fabrics and metals is not to be feared.

To the subfamily Sarcopsyllince belongs Sarcopsylla penetrans, the chigo, jigger or sand flea of warm countries, not unlike a reddish flea in size and appearance, but distinguished by the larger head, which is distinctly pointed, somewhat like that of a fish. The female, when impregnated, bores its way into the skin of man, especially the soles of the feet and about the toes, and then distends enormously, even to the size of a small pea. The treatment consists in the enucleation of the entire insect with a sharp needle.

Order Orthoptera. Mostly winged insects, with wings lying straight along the back, the under ones larger and thinner and folded in plaits.

Family Blattidae. Cockroaches. Four species are described by

\footnotetext{
* In an investigation of the rats of Providence, R. I., and their parasites, in I9I2, Geo. H. Robinson found that, of $34 \mathrm{I}$ rats captured, 333 were the brown or Norway rat: that 195 were infested with fleas, and that, of these fleas (as determined by Nathan Banks), 75 per cent. were Xenopsylla cheopsis and 22 per cent. Ceratophyllus fasciatus. These surprising results show the necessity of further investigations to ascertain the climatic distribution of fleas.
} 
Marlatt as the most common in this country: the American roach (Periplaneta americana) (Fig. I3), Australian roach (P. australasia), Oriental roach or black-beetle of Europe (Blatta orientalis), and German roach (Blatella germanica). In the Oriental roach the

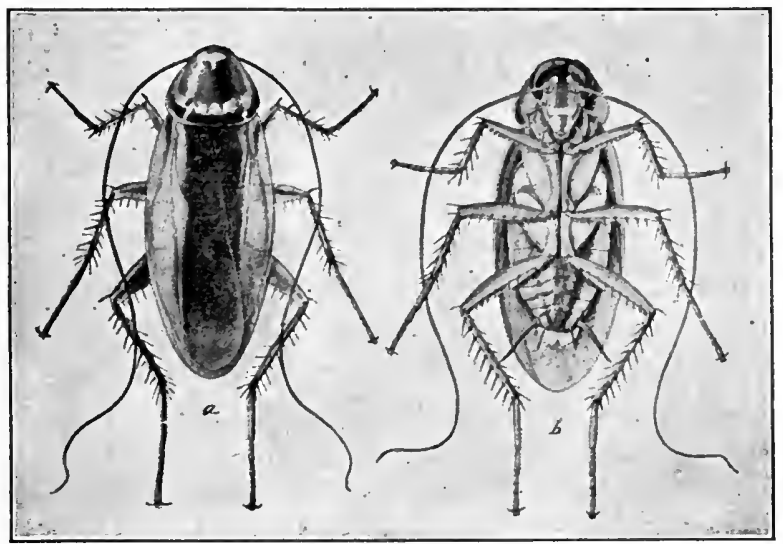

Fig. I3.- The American roach (Periplaneta americana) : $a$, View from above; $b$, from beneath. (From C. L. Marlatt.)

wings are only rudimentary in the female, and rather short in the male. In the other three species the wings are well developed in both sexes. The German roach or "Croton bug," the smallest of our domestic species, and easily recognized by the two converging dark stripes on the thorax, has become the commonest in the eastern United States. (Fig. I4.)

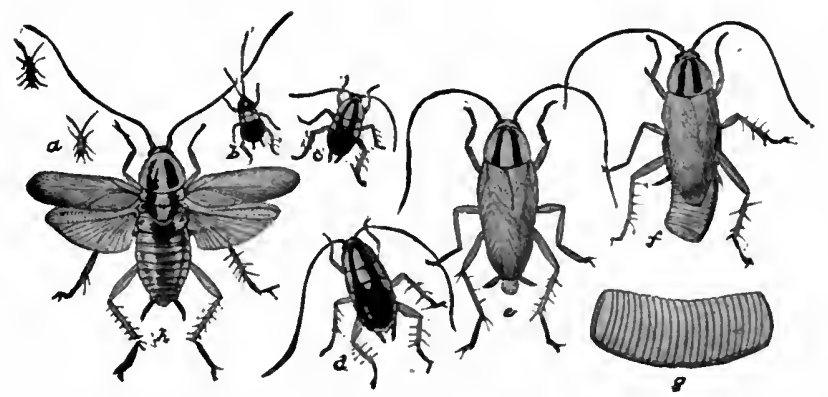

FIG. I4.-The German roach (Blattella germanica) : $a$, First stage; $b$, second stage; $c$, third stage; $d$, fourth stage; $e$, adult ; $f$, adult female with eggcase; $g$. egg-case, enlarged; $h$, adult with wings spread. All natural size except g. (From Riley:) 
Nocturnal animals, omnivorous and destructive, infesting kitchens, pantries and storerooms; also objectionable from the nauseous and persistent odor of their secretions. They are accused of infecting food and milk with intestinal bacilli, and of distributing destructive molds and pyogenic organisms. Also suspected of disseminating the germs of tuberculosis and typhoid fever. Gales, from long experience with epidemics in the U. S. Navy, believes that roaches are often responsible for the spread and continuance of typhoid fever, diphtheria, tonsillitis and tuberculosis. Riggs, U. S. Navy, traced an epidemic of paratyphoid fever on board ship to these animals. Longfellow (Toledo Clinical Laboratories) claims to have proven that roaches can and do carry, on their feet and in their viscera, all of the common bacteria of the acute infectious diseases, as well as any others met in their travels.

The most effective means for their destruction is fumigation with hydrocyanic acid or sulphurous acid. A phosphorous paste made of sweetened flour paste containing I or 2 per cent. of phosphorus, is effective. Pulverized sodium fluoride used as an insect powder is highly spoken of in the Navy. Suitable traps are also very useful.

Order Diptera. Insects with only two wings, borne on the mesothorax, and a pair of knobbed threads (the halteres or balancers) borne on the metathorax. Mouth formed for sucking or lapping. Includes the insects most concerned in the transmission of disease. The principal families are readily distinguished by the wing venation.*

Family Muscidae. True Flies.

Musca domestica. Common, Domestic or House-fly. (Fig. I5.) By far the most abundant of flies, forming, according to Howard, nearly 99 per cent. of all flies caught in houses. $\dagger$ Following man into all but the coldest climates, it is found in practically all parts of the world as a household insect. Wherever troops form camp in this country, or any of our colonies, however desert and sterile the place, flies will be present. It is true that in many situations the domestic fly is associated with or entirely replaced by other species undistinguishable from it to the average observer.

It is differentiated from similar species by the following char-

* Simple methods of differentiating disease-bearing insects. C S. Ludlow. The Military Surgeon, July, I912.

† The House Fly. Disease carrier. L. O. Howard. 
acteristics: Four black lines on the back of the thorax; vein ending near the tip of the wing distinctly elbowed a short distance from the tip; absence of bristles on the abdomen except at the end. The head consists chiefly of the large compound eyes, made up of thousands of facets; between them, above, are three simple eyes; thus
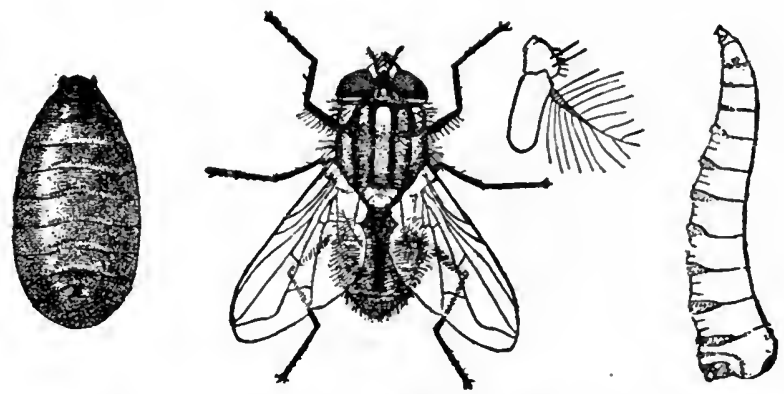

FIG. 15.- The common house-fly (Musca domestica): Puparium at left; adult next, then antenna (with feathery bristle) and larva. All enlarged. (From L. O. Howard.)

can a fly see in all directions. Below, in front, are two large pendant organs, the antennae or feelers, each with a large feathery bristle. Still further down are the mouth parts or proboscis, a curious jointed appendage with another pair of feelers (maxillary palpi) at the base; at the tip it expands into two lobes covered with transverse hard ridges; between the lobes is the oral orifice. Fluid substances are readily sucked up. With soluble solids the process is more complex; the oral lobes, widely opened, are closely applied to the solid surface; saliva (or fluid from the crop) is freely ejected upon it and strong sucking movements made, with rasping frictions of the hard ridges of the lobes, until some of the substance is sufficiently dissolved to be absorbed.

Each of the six feet consists chiefly of two sticky pads and two claws. (Fig. 16.) The pads are covered with numerous minute hairs, viscous at the end, making them stick to everything they touch. On smooth surfaces the fly adheres by the combined action of pads and claws, while on rough surfaces it clings mostly by its claws. This stickiness of the feet is one of the reasons which render the house-fly dangerous; they constantly become clogged with adhering substances, mostly microscopic particles, which are thus carried from one place to another. Considering the wonderfu] 
activity and strength of the house-fly, much surpassing those of any bird in comparison to size, we can readily realize the amount of matter thus carried about. As this clogging interferes with the free action of its feet, the fly is frequently seen preening itself and lapping off the collected dirt which thus enters the stomach and passes out with the excreta.

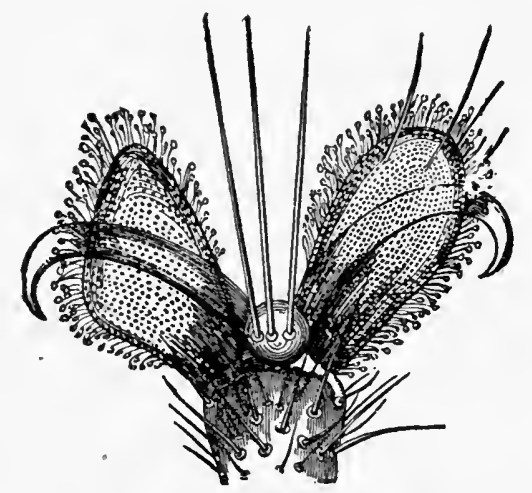

FiG. I6.- Foot of the house-fly. (From Elements of Zoology by Sanborn Tenney. Copyright, 1875 and 1903. Reproduced by permission of the American Book Company.)

The house-fly lays its eggs preferably upon horse manure, which seems to be the favorite food of its larvæ; but it also oviposits on human excrement and all kinds of manure; in the absence of better material it will breed in decaying vegetable and animal substances, as well as in fermenting garbage and rubbish.

The female lays about I 20 eggs, in small irregular clusters. They are glistening white in color, cylindrical-ovoid in shape, one-twentieth of an inch, or little more, in length. (Fig. I5. ) They hatch in about 8 hours. The white, conical larva undergoes several moltings, often turning yellowish, and reaches the pupal stage in about five days, being then about $6 \mathrm{~mm}$. long. From the moment of hatching it is very active, burrowing into the substance upon which the egg was laid and rapidly disappearing from sight. The pupa is nearly cylindrical, with both ends evenly rounded, and rapidly changes in color from yellowish to red and finally to a dark chestnut. Its duration is also five days, making an average period of ten days from the time of oviposition to the emergence of the adult fly.

The great majority of flies oviposit only once or twice, but, excep- 
tionally, females have been observed to lay three or four times. As it requires Io days for the evolution of the full-fledged insect and about Io days more for the young fly to attain sexual maturity, a new generation may therefore be expected every 20 days, that is to say, six or seven generations during the summer from any one female which, after hibernating, begins to lay about April i5. A simple calculation will show that, from this female ancestor, numerous billions of flies may be bred before autumn. It is not unusual to find I,O0O maggots to the pound of manure; as many as 200 have been counted in less than one cubic inch of it.

The eggs, larvæ, most of the pupæ, as well as all unprotected flies, are killed when the frosts come, the species being perpetuated by the impregnated females that find suitable shelters in which to hibernate, and the comparatively few pupæ sufficiently protected under manure heaps.

Levy and Tuck record the very important observation* that the larvæ of the house-fly remain in the manure heap only until they have attained their full growth, and that they then leave it and enter the earth wherever it is possible for them to do so, the full-grown maggots being mostly found on the outer edges of the heap, whence they rapidly burrow into the ground. We may therefore accept the fact that the house-fly does not pupate in manure if the full-grown maggots can find any means of reaching and entering the earth; in other words, that the soil is the normal place for its final transformations. Of course, when the manure is placed in closed containers, the larvæ, unable to escape, will enter the pupal stage within it. In due time, if left undisturbed, the adult fly finds its way to the surface, generally through the hole made by its larva. Stiles has shown that a certain proportion of flies, hatched from eggs buried under four feet of sand, will find their way out. Doubtless the larvæ had crawled up as high as possible before pupating. The maggots scattered with the manure upon the surface of the ground are much more likely to be destroyed by dryness than by any thickness of earth ploughed upon them. It is likewise probable that most of the flies bred in the contents of earth-closets or open privies will, in the absence of special preventive measures, find their way out of them.

* Am. J. of Public Health. July. rigr3. 
The transmission of disease by flies is effected in three ways: I, by direct conveyance of the germs on varions parts of their bodies; 2 , through the liquid vomited from the crop ; 3 , through the intestinal discharges or fly-specks.

It has already been seen that the feet are well adapted to collect and transport microorganisms, but other parts of the body cain also act as carriers, especially the large proboscis and the hairy legs. Knowing the predilection of the fly for filth, including human excrement, and the ceaseless activity which leads it from latrines, manure piles and dumps to kitchens and mess-rooms, we realize how pernicious it may be. The investigations of Esten and Mason, who studied the bacterial population of several hundred flies, show that the average for each was over a million organisms. One fly falling into a pan of milk may therefore be responsible for the millions of bacteria to the c. c. found in it a few hours later. Fortunately, the experiments of Graham-Smith have proved that the pathogenic bacteria collected on the proboscis, legs and wings do not usually survive more than a few hours. Much more dangerous are those which come from within the body.

In the alimentary canal of the house-fly is found a diverticulum or crop which is a temporary storehouse for food; here the food remains practically unchanged. The contents of the crop are drawn into the stomach, or they may be regurgitated through the proboscis, as in feeding on solid food. According to Graham-Smith, it is these regurgitated drops which make the larger stains upon a window covered with fly-specks. Pathogenic bacteria frequently survive several days within the crop, and still longer in the intestine, but there is no evidence that they multiply in either of these situations. The digestive fluids appear to have but little effect upon the organisms contained in the food, and most of these can be recovered in the excreta.

J. C. Torrey has investigated the numbers and types of bacteria carried by city flies (J.Inf. Dis., March, X, No.2). Fecal bacteria of the colon-type were first found abundantly in the early part of July. The bacteria in the intestine were 8.6 times as numerous as those on the surface; the colon-group bacteria constituted 37.5 per cent. of the total within the intestine, and only r3. I per cent. of those on the surface. Of the streptococci cultures none were of the 
pyogenes type. Several cultures of $B$. paratyphosus, type A, were obtained.

Some English experiments seem to indicate that flies which, as larvæ, fed on infected fecal matter, will continuc to carry typhoid germs in their intestine for a number of days although having ingested none since their emergence from the pupal state.

The house-fly is an enormous feeder, swallowing in the course of a day, under favorable conditions, nearly its own weight of food. While feeding upon the discharges of typhoid, cholera, dysenteric or diarrheal patients, it swallows millions of pathogenic bacteria. When well fed it defecates every few minutes during the day. As a natural consequence the number of fly-specks, wherever flies are at all numerous, is often amazing; a proportion of 1,000 per square foot on window panes is not uncommon, and on neutral-tinted surfaces, less often cleaned, the proportion may easily rise to millions $(N . A . C o b b)$. It thus clearly appears that the chief danger from the house-fly is through the contamination of foodstuffs by flyspecks.

It must be considered, next to the mosquito, the most dangerous of insects. It is a common disseminator of typhoid fever, and, on that account, often called typhoid $\mathrm{fly}$. It is also known to transmit cholera, dysentery, infantile diarrhea and tuberculosis. Howard and Clark found that it can carry the virus of poliomyelitis in an active state for several days. There is likewise strong circumstantial evidence that it conveys the germs of plague, smallpox, leprosy, anthrax, yaws, trachoma, ophthalmia, erysipelas, tropical sore, and the eggs of parasitic worms.

Yet, in spite of the above formidable list of infectious diseases, one should guard against being led to exaggerate the pernicious activities of the house-fly, bad as they may be. Most of the experiments which have proved its guilt were made under the favorable conditions of the laboratory and with pure pathogenic cultures. It is certain that such conditions seldon prevail in nature, and that diseases from infected flies are not as common and widespread as one might believe from the foregoing statements. Thus GrahamSmith, a judicious and careful investigator, is of opinion that our knowledge of the subject does not afford conclusive evidence that naturally infected flies " are a frequent source of disease in man by infecting food materials." Other trustworthy authorities, however, 
consider such estimate too conservative and liable to encourage dangerous neglect. There is no doubt that under such conditions as often prevail in military camps, on many a country farm and wherever the primitive open privy is used, the house-fly is a really dangerous agent in the transmission of infections.

Prevention and Destruction of House-flies.-Bearing in mind the comparatively few flies which survive the winter, it is evident that the campaign should begin by the destruction of these hibernating females wherever found in basements, barns, stables and other sheltered places. Hibernating mosquitoes should be destroyed at the same time. Thus will the problem be wonderfully simplified, since each female killed then represents millions of offspring which would be bred during the summer.

Since flies breed in excrement, manure, garbage and filth, all such material should be frequently removed from the vicinity of houses, and, until so removed, carefully screened. Garbage cans must be kept covered with a well-fitting lid. Wherever domestic animals are kept, there should be in connection therewith a bin, closet or pit for the reception of manure, properly screened against flies. The insecticides so far found of most practical value for the destruction of fly maggọts in stables are borax and hellebore.* Borax finely powdered is used in the proportion of 0.62 to I pound per 8 bushels ( Io cubic feet) of manure, applied particularly around the outer edges of the pile with a fine sieve, and followed by a sprinkling of 2 or 3 gallons of water. If used carelessly in large quantity, borax is liable to injure vegetation, and for this reason, hellebore, which is harmless in any proportion, should be preferred when treating manure on a large scale. It is used at the rate of one-half pound of the powdered root to Io gallons of water, for 8 bushels of manure. Since the average time elapsing between the laying of eggs and the escape of the larva into the ground is three or four days, it is advisable to have manure accumulations removed at least twice a week. Daily removal and spreading out in a thin layer is better; thus spread and broken up, it dries quickly and ceases to be attractive to flies.

The observations of Levy and Tuck, above referred to, have suggested an effective means of fighting the fly. If the manure is placed

* Bull. No. 245, U. S. Depart. of Agriculture. 
in a barrel or other container, in the bottom of which several large holes have been bored, and said container is set in a large tub, from the bottom of which it is elevated by several bricks, it will be found that all the maggots escape from the container and drop into the tub in their efforts to reach the ground. Surer results are secured by replacing the bottom of the container with a piece of stout wire gauze of about a quarter-inch mesh. The tub should contain a few inches of water to drown the maggots.

The preference shown by flies for horse droppings seems to be due to the film of mucous covering them; if they are broken up and scattered as soon as possible they quickly dry and do not become a nuisance. In camps, manure not needed for fertilizing purposes is best disposed of by burning. The sprinkling of kerosene on polluted soil will keep flies away. The attraction of dirt for fies is a strong argument for the strict policing of camp grounds, especially in the vicinity of kitchens, mess-tents and lavatories. Latrines require great care in this connection, as described under the proper heading.

Under ordinary conditions the house-fly does not travel far from its breeding place, seldom more than 500 yards, which simplifies measures of control.

The exclusion of flies from houses presents no serious difficulties. If the careful screening of doors and windows will not accomplish it, we must, besides, have recourse to fumigation (see Insecticides), fly papers and traps; of the latter, those devised by Prof. Hodge, of Clark University, are particularly efficient. Formalin is also very useful in solution of one part to seven parts of milk or sweetened water, placed in shallow vessels.

Of the natural enemies of flies the most common and effective is a parasitic fungus, Empusa musca, which carries off great numbers of them in the early autumn. It has also been found that ants often feed voraciously upon the eggs and larva of flies in manure, although they cannot always be relied upon for the purpose. P. L. Jones, U. S. Army, attributes the comparative scarcity of flies in the Philippine Islands to the prevalence of ants, and advises to favor the multiplication of these predatory little insects wherever flies breed abundantly.

Other common flies of this family, likewise liable to communicate disease, are : 
Muscina stabulans. Large House-fly. Often mistaken for the domestic fly, but somewhat larger and lacking the lines on the thorax and the elbowed vein near the apex of the wing. Its larva feeds upon rotten vegetables and human excreta, as well as dung, and there is no reason to consider it a special inmate of stables, as the name would indicate.

Stomoxy's calcitrans. Biting Stable-fly. (Fig. 17.) Recognized by its beak or proboscis formed for blood sucking. Farm yards and stables are its favorite haunts, being more common in country than in city districts, and feeding voraciously on the blood of horses and cattle. Its puncture is not poisonous, and is less painful than that of the mosquito. Rosenau and others believe that this fly can convey and inoculate the virus of poliomyelitis. According to Austen and Osborn it is probably also capable of transmitting trypanosomes, glanders and anthrax from infected to healthy animals.
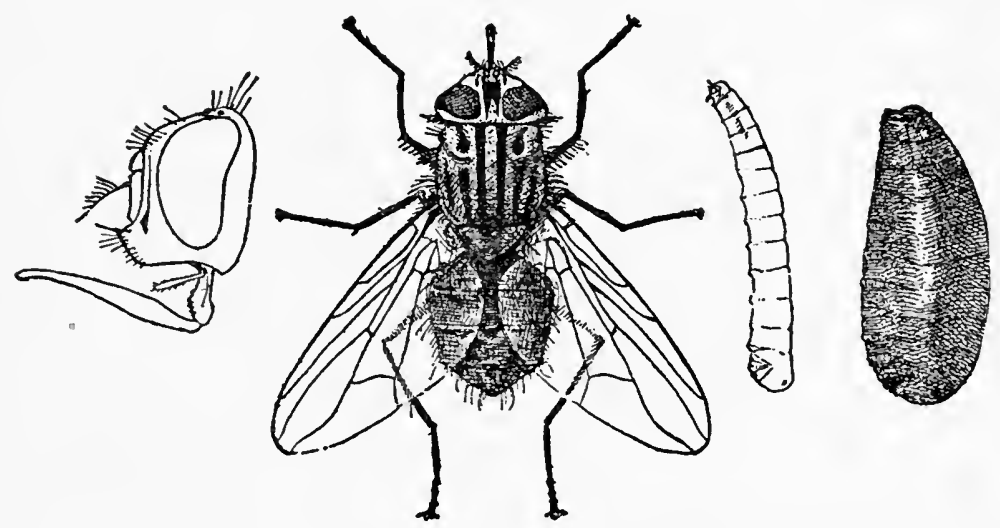

FIG. I7.- The Biting Stable-fly (Stomoxys calcitrans). Adult, larva, puparium, and details, all enlarged. (From L. O. Howard.)

Pollenia rudis. Cluster fly. A little larger than the domestic fly. with dark-colored smooth abdomen and a sprinkling of yellowish hairs. A sluggish fly, sometimes abundant in spring and fall.

Homalomyia canicularis. Little House-fly, with conical, pointed body and, on account of its small size, often mistaken for the young of the common fly. Rather frequently seen upon window panes in houses.

To this family also belongs the genus Glossina, the Tse-tse flies 
of Africa, with dark-colored body, a centimeter long, tnick, projecting proboscis formed for blood sucking, and a wing characterized by the cleaver-shaped central (or discal) cell. When at rest the wings overlap, crossing each other like the blades of a pair of scissors. $G$. palpalis is the common transmitter of the organism of sleeping sickness in Africa, but G. morsitans, G. fusca and other species are also believed to be concerned in disseminating this disease.

Family Sarcophagidae. Flesh or Blow Flies, with shining metalcolored abdomen, laying their eggs on meat and dead animals or even in wounds and open cavities of living animals.

Calliphora erythrocebhala. Large Bluebottle Fly, with black spines on the striped thorax. Common in Europe and America.

Phormia terranova. Small Bluebottle Fly. Widespread in the United States. Displays a conspicuous dark median band on thorax and abdomen.

Chrysomyia macellaria. Screw-worm Fly. Another quite common bluebottle fly, with striped thorax. Sometimes lays its eggs in the nostrils of man, whence the maggots may gain access to the frontal sinus or other cavity and do serious harm.

Lucilia casar. Greenbottle Fly. Abundant in Europe and America, and commonly found about dead animals. Displays the same band on thorax and abdomen as the small bluebottle fly.

Sarcophaga carnaria, the Flesh Fly of Europe, a great flesh eater and general scavenger, does not occur in the United States, but is replaced by similar species with the same habits.

Family Simulidae. Genus Simulium. Wing characterized by having only 2 or 3 veins. Small humpback, biting. gnats with comparatively stout legs and short, curved, hairless antennæ.

$S$. reptans has been accused by Sambon of being the transmitter of the germ of pellagra in Europe. The conmon species in the United States are S. venustum, or "black fly" of the northern woods, and S. pecuarum, the southern "buffalo-gnat." Other southern species are $S$. rittatum and $S$. pictipes. The part played, if any. by these native species in propagating disease has not yet been determined.

Family Tabanidae. Horse-flies. Wing venation strongly marked. characterized by the wide-open mouth of the apical cell. Known to carry disease to mammals, and believed by Austen to be also capable of infecting man, especially in tropical countrics. 
Family Psychodidae. Moth-winged Flies. Wings with straight, nearly parallel veins covered with hair-like scales.

Genus Phlebotomus. P. papatasii, the propagator of papataci fever in the Mediterranean region and in India, is a very small, stinging insect, passing easily through the usual mosquito net.

Family Culicidae. Mosquitoes. Wings with characteristic venation, their apical and dorsal margins fringed, and the veins bearing small scales; antennæ long, bearing whorls of hairs; mouth parts forming a long suctorial beak or proboscis. 


\section{CHAPTER XIV.}

\section{THE MOSQUITO AS DISEASE TRANSMITTER.}

Of all insects, the mosquito is preëminently the worst enemy of man, for not only does it disturb and irritate him with its sting, but also infects him with the germs of several diseases. It is known that the mosquito is the chief and probably exclusive factor in the transmission of malaria, yellow fever, dengue and filariasis, and surmised that it may also play a part in the dissemination of other infectious disorders.

The mosquito, like all insects, consists of a head, thorax and abdomen, with a pair of articulated legs attached to each of the three segments of the thorax. Each leg comprises femur, tibia and tarsus, the latter piece consisting of five joints, with claws on the last one. Between the middle or main segment of the thorax and the posterior segment is a little band or plate, the scutellum, whose shape and scales are useful in classification. The head consists chiefly of the large compound eyes, the feelers or antennx, and the mouth parts, namely, the palpi and proboscis. The sexes are readily differentiated by the antennx which, in the male, bear long plumelike, silky hairs, and, in the female, short, scant hairs. (Fig. 23.) The palpi (mouth feelers) vary in length according to sex and species. The proboscis (sting or beak) consists of six distinct piercing elements, or stilets, enclosed and protected in a sheath called labium. This sheath does not penetrate but bends backward while the stabbing elements are thrust into the animal or vegetable from which the mosquito seeks food. These elements form a tiny channel along which the blood or other food is sucked into the mouth cavity.

The wing venation is especially important. (Fig. I8.) The comparative length and width of the first submarginal cell (between forks of second vein) and the second posterior cell (between forks of fourth vein), as well as the position of the cross veins which determines the length of the petiole or stalk of the cells, are of value in differentiating genera and species.

Mosquitoes feed mostly on vegetable juices, the male exclusively so, but the females of most species, when the opportunity offers, also suck the blood of mammals, birds and other animals. The male 
mosquito, not being a blood sucker, takes no part in the propagation of disease; the female only bites and infects animals. This infection takes place through the salivary glands, in which are found the malarial germs in great numbers. While the female sucks blood,

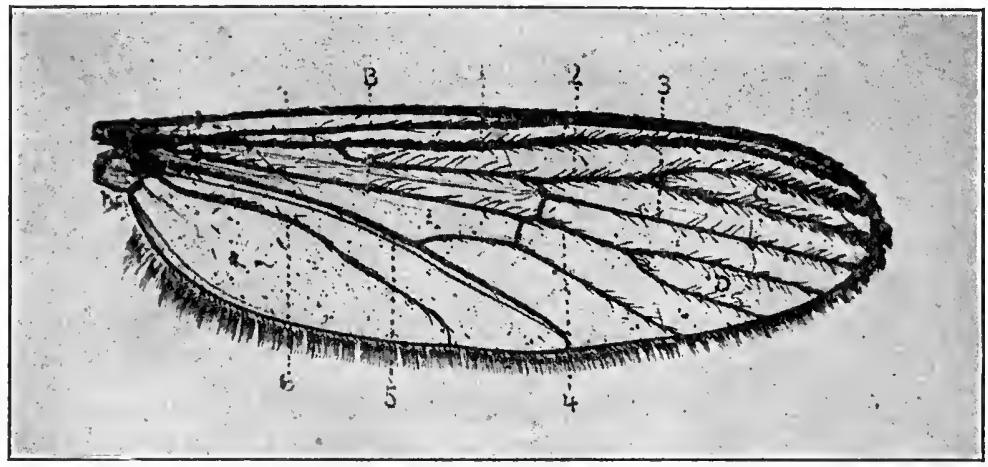

Fig. I8. - Wing of Culex mosquito. A, costa or marginal vein; $\mathrm{B}$, subcosta or auxiliary vein; C, first submarginal cell; D, second posterior cell; I, first long vein; 2, second long vein; 3 , third long vein; 4, fourth long vein; 5 , fifth long vein; 6 , sixth long vein.

the secretion of these glands is ejected into the tissues of the bitten animal through the salivary duct and down a minute canal which opens at the tip of one of the stabbing elements of the proboscis. This secretion contains also an irritant poison which causes the smart and swelling of the sting. The function of this poison appears to be to determine a flow of blood to the bitten part and prevent its coagulation.

The mosquito lays its eggs, as a general rule, on the surface of water, but this water must be still or stagnant; any movement from current, tide or pumping will prevent oviposition. Occasionally, as in the case of salt-marsh species, the eggs may be laid on mud or even the dry bottom of holes and depressions likely to become submerged. The eggs are glued together in boat-shaped masses of several hundreds, a quarter-inch or less long (Culex), or may lie more or less loosely in groups of a few scores (Anopheles). See Fig. 20. They hatch in a couple of days, giving birth to the larve or wigglers which at once exhibit great activity in feeding themselves. Being air breathers, much of their time is passed at the surface of the water with the breathing tube (near the tail) protrud- 
ing into the air. (Figs. $2 \mathrm{I}$ and 22.) After undergoing several moultings, the larva, in eight, ten or more days, becomes transformed into the pupa which, unless disturbed, quietly floats on the surface of the water. In about two days the pupa case bursts and the perfect insect emerges from it. The entire period elapsing from
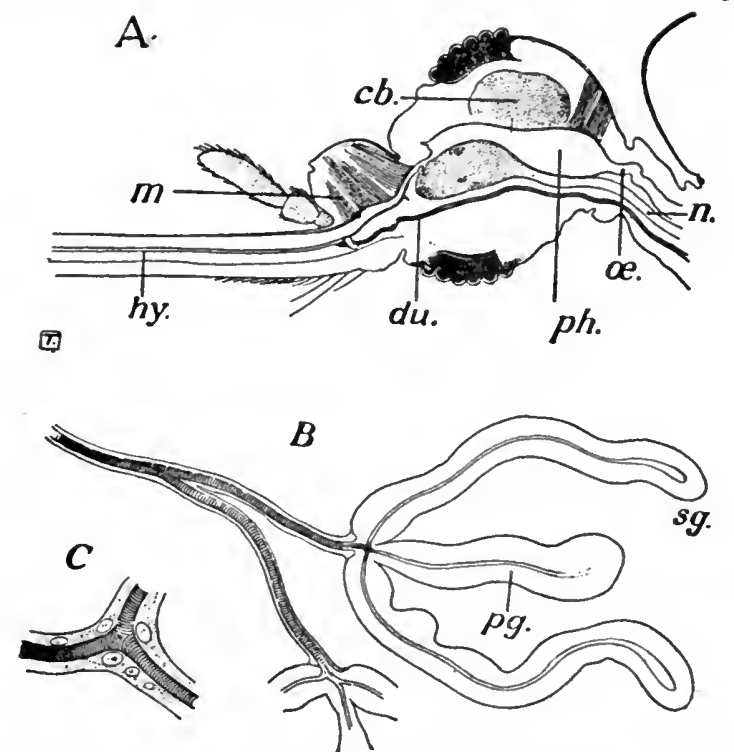

FIG. I9.- Dissection of head of mosquito: A, Median section of head, showing $d u$, the veneno-salivary duct, with its insertion in hy, the hypopharynx; $c b$, cerebrum; below this are the cerebellum and the pumping enlargement of $\alpha$, the cesophagus; $m$, muscle; $n$, nerve commissure. The other parts have been removed. $B$, the veneno-salivary duct, showing its bifurcation and the three glands on one of its branches; $p g$, poison gland; $s g$, marks the upper of the two salivary glands. C, the bifurcation of the duct with its nucleated hypodermis. (Manson.)

the laying of eggs to the birth of the full-fledged mosquito occupies from 8 or ro days to 3 or 4 weeks, according to species and also to clinate and temperature, the process being particularly active in hot, damp weather. The same female may lay eggs several times and become the ancestor of many successive generations during the summer. Few males outlive the summer. In a few species, notably the house mosquitoes (Culer pipiens and $C$. quinquefasciatus). the malarial mosquito (Anopheles maculipennis) and the yellow fever mosquito, the impregnated females hibernate in sheltered places, ready to lay eggs as soon as the temperature permits in 
the spring. But in most species, such as the salt marsh, woodland and prairie mosquitoes, males and females are killed by the first frost of winter. These species hibernate mostly in the egg stage. The egg is known to be endowed with astonishing vitality, remaining unaffected by the intense winter cold of the northern States. Thus, as observed by Dr. J. B. Smith, the eggs of the saltmarsh species may remain in dried water holes for at least two years without deteriorating, and will promptly hatch if covered with water during the third year. Larvæ may also pass the winter in ice without being killed, reviving as the ice melts and then developing into perfect insects. But they require water for their development; if the pool in which they are hatched dries up within a week, or before their transformation is completed, they soon perish, especially if exposed to the sun.

In tropical countries, mosquitoes breed throughout the year, except during the dry season, when the female remains inactive, waiting for a rainy spell. It has been noted that eggs laid in water
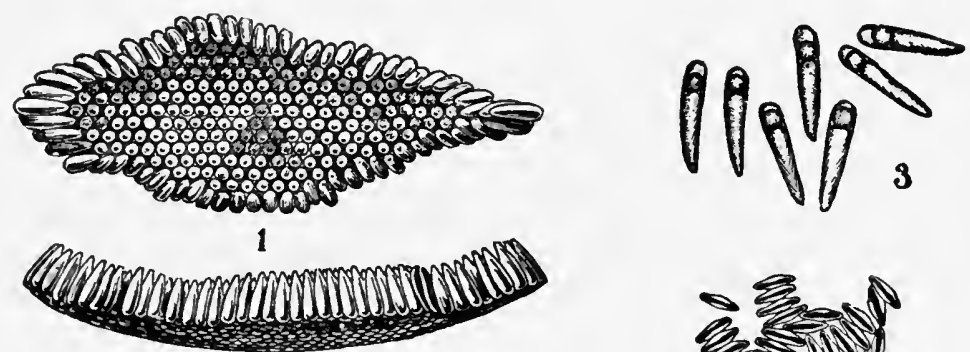

2
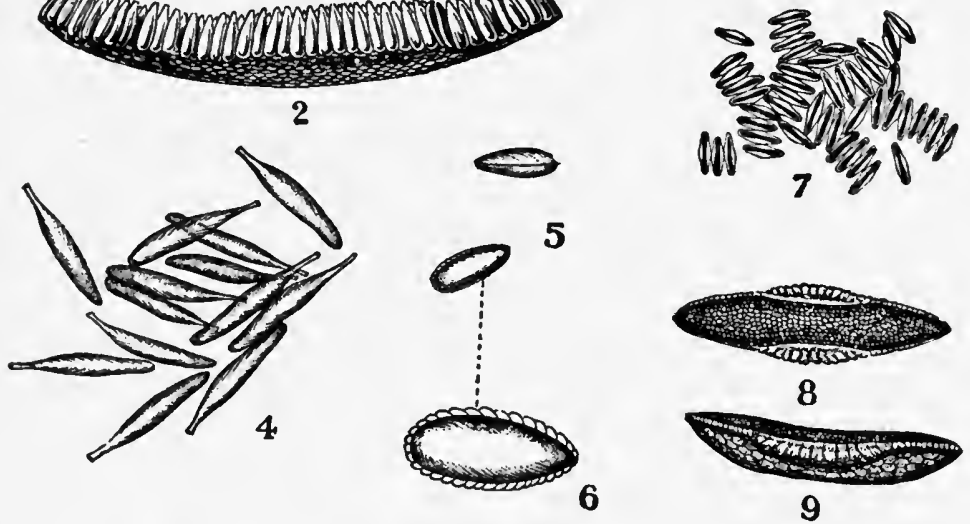

Fig. 20.- Mosquito eggs. (1), Egg-boat of Culex seen from above; (2), the same, side view; (3), separate Culex eggs; (4), eggs of Panoplites; (5), eggs of Stegomyia; (6), the same, more magnified; (7), group of Anopheles eggs; (8) and (9), eggs of Anopheles maculipennis, showing lateral floats. 
which dries up before they hatch may retain their vitality for a number of weeks.

The female sucks blood in order to obtain suitable nutriment for the ripening of her eggs, and it is after such a meal that she oviposits. As the blood ingested considerably increases her weight and bulk, making her flight slow and laborious, she lights on any suitable water nearest at hand.

Although, when scenting a prey, it is persistent in pursuing it, the mosquito does not usually take long flights, seldom straying far from its breeding place. In a town, each square or street is generally responsible for the mosquitoes that plague it. In the absence of water near by, mosquitoes will fly short distances in search of suitable places to lay their eggs; or where they breed in crowding swarms, the instinct of self-preservation leads them to allow themselves to be carried away by the breeze. The salt-marsh mosquitoes, for instance, (C.cantator, C. sollicitans, etc.) have decidedly unpleasant migratory habits, although seldom breeding outside of salt or brackish water. Governor's Island, in the bay of New York, is periodically infested with swarms of mosquitoes in summer; careful inspection has failed to discover any breeding place on the island, and as they appear with easterly winds the inference is justified that

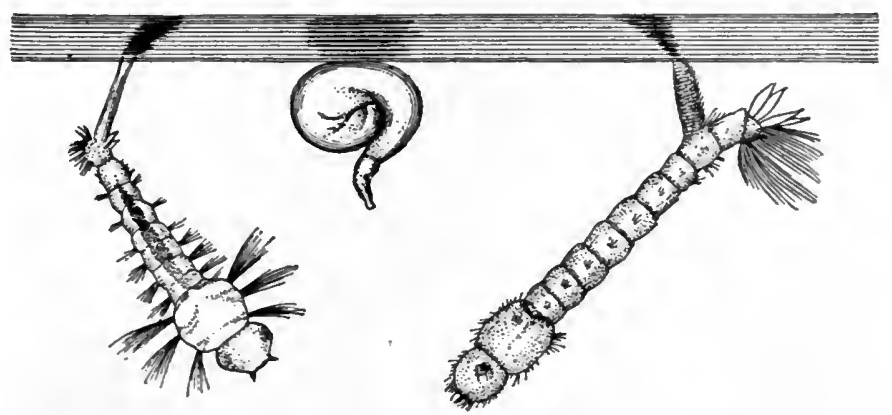

FIG. 2I.-Larvæ and pupa of Culex. Note the breathing tube.

they are blown from Long Island, probably a distance of several miles. As a general rule, however, as soon as the wind begins to blow, mosquitoes seek shelter in the grass and in the lee of shrubs and trees. It must be borne in mind that they are readily transported to distant places by ordinary vehicles of comnumication, such 
as ships, trains, carriages etc., so that they may suddenly appear where they have never been known before, or reappear where they have not been seen for many years.

Isolated mosquitoes may exceptionally appear, one or few, in unexpected places and soon again disappear, but, as a general rule,

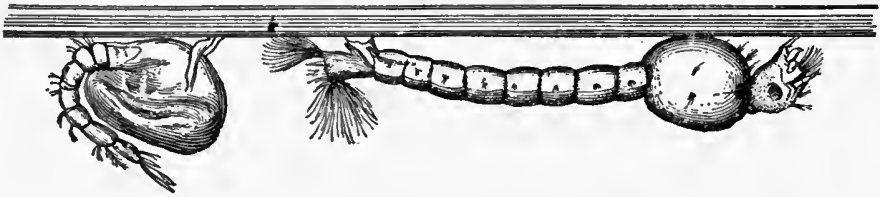

FIG, 22.- Larva and pupa of Anopheles. Note shortness of breathing tube, compelling the larva to hold its body parallel with the surface.

their constant presence anywhere indicates that they are bred in some neighboring marsh or pond on a large scale, or else in holes, cisterns, barrels or gutters, freely exposed, for unless they have favorable recruiting grounds they soon become extinct.

\section{CLASSIFICATION AND DESCRIPTION OF DISEASE-BEARING MIOSQUITOES.}

The following subfamilies, genera and species include all mosquitoes known, or reasonably suspected, to transmit disease.

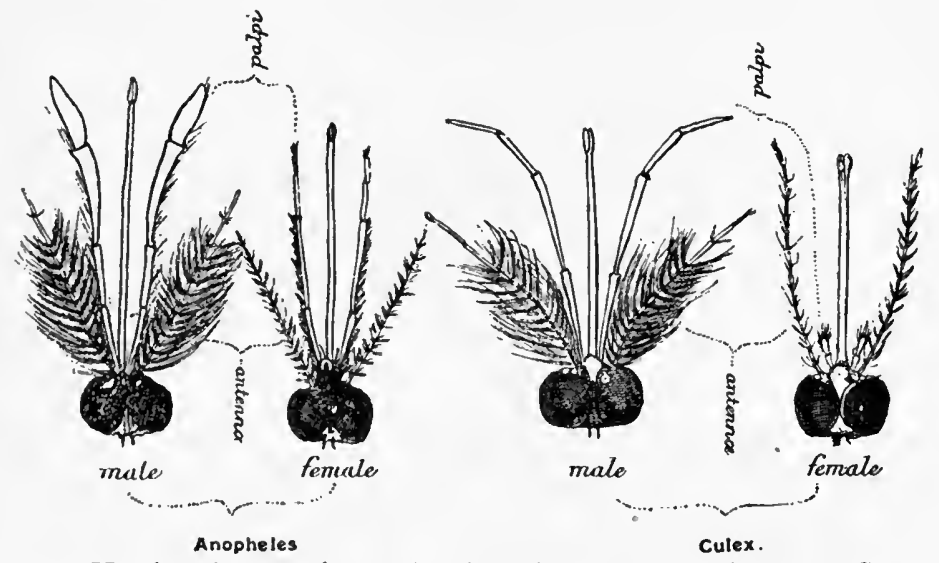

Fig. 23.- Heads of mosquitoes showing the difference between Culex and Anopheles in both sexes (Giles).

Apparent similarity of structure between allied species may not necessarily imply the same pathogenic capability, but with our'still 
very incomplete knowledge of the subject it seems logical and safer to consider such similarity a reason for suspicion.*

$A$. Subfamily Culicinae. $\dagger$ - Scutellum trilobed. Palpi short in female, long in male; first submarginal cell as long as or longer than the second posterior cell.

I. Culex. - " Head ornamented with narrow curved scales over the occiput, and upright forked scales, especially thick on the back

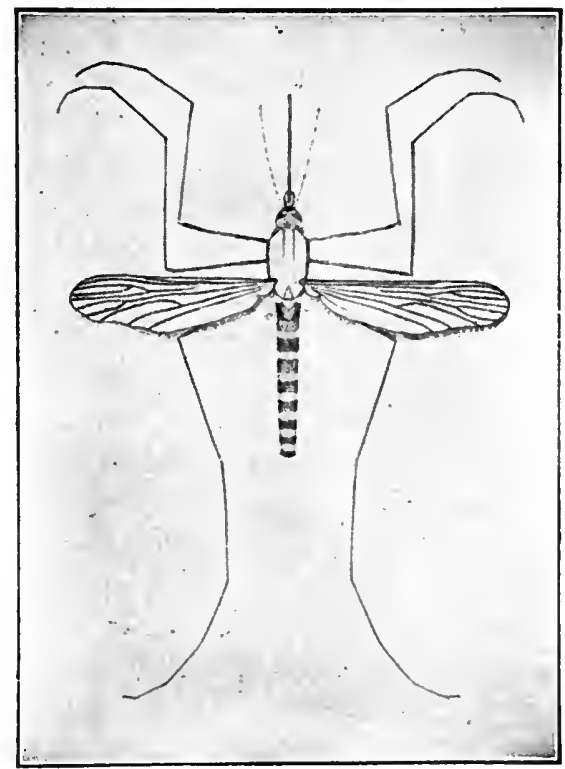

FIG. 24.-Culex fatigans, female. (Ludlozi.)

of the head; flat scales on the sides." Wings with small median scales, and thin linear lateral ones, to the veins.- Large and unwieldly genus of which $C$. pipiens, the common European mosquito, is the type. It is remarkable that of the numerous species of Culex, only one is positively known to be disease-bearing.

C. fatigans (C. pungens, C. quinquefasciatus).- Thorax clothed

* For much of this information, I am indebted to Bull. No. 4, Surgeon General's office, by C. S. Ludlow, Ph. D.

t The nomenclature of North American mosquitoes being still in course of revision and elaboration, it was deemed best, in this edition, to retain the previous familiar names rather than adopt recent changes which may need further modifications when the classification has been finally settled. 
with golden scales and three lines of black bristles; petiole of first submarginal cell $1 / 4$ or more the length of the cell; abdominal segments nearly black, with straw-colored bands; legs generally dark brown. (Fig. 24.) Found throughout all the warmer parts of the world, as a purely domestic species, with the same general distribution as Stegomyia fasciata. This Culex is the intermediary host of Filaria bancrofti and probably of $F$. philippinensis which it transmits to man. It seems to be also the chief factor in the transmission of dengute.

North of Washington, $C$. fatigans is replaced by the closely allied $C$. pipicns, introduced from Europe, and the common "house mosquito" of the northern Atlantic States and Canada. Like fatigans, it is nearly uniformly dark-yellowish, with light-colored bands at base of the abdominal segments, but characterized by the very short petiole of the first submarginal cell, not more than one-sixth the length of the cell. It winters, by preference, in cellars or other damp and dark shelters in or near houses. The female lays her eggs (about 200), glued together in the shape of a raft, on any kind of water, however foul, from rain barrels to catch basins and cesspoois. This mosquito persistently strives to get into dwellings, not only by open doors and windows, but also by forcing its way through the meshes of nettings. It does not usually travel much, seldom more than a few hundred yards from the place of its birth.

2. Stegomyia. - Head scales all flat and broad. Abdomen completely covered with flat scales. Wings with small scales, both spatulate and linear. Mostly jet-black insects with ornamental liries of silvery scales on thorax and legs. - A genus of some I9 species, all with a smooth satiny appearance.

$S$. fasciata ( $S$. calopus.). - Thorax velvety-black to goldenbrown, marked with two median straight and lateral curved lines, in the form of a lyre (or Jew's harp). Abdominal segments with snowy-white bands and lateral tufts. Proboscis pure black. Legs prettily banded. (Fig. 25.)

This is the most widely distributed mosquito of the family, being found throughout the entire tropical and subtropical zones, from the Hawaiian Islands to the Philippines, India, Egypt, Italy, Spain, the West Indies, Central and South America, and our southern States as far north as Virginia. Although of domestic habits and breeding freely in cisterns and tanks, it is also a good traveller on 
steamers, and may be expected to establish itself in any moist tropical zone, a fact which should be borne in mind when much of our Asiatic trade passes through the Isthmus of Panama. It is the only known agent of transmission of yellow fever. That it is the only one cannot be asserted and does not seem probable, but it is a practical fact that campaigns against yellow fever based on that assumption have always been successful.

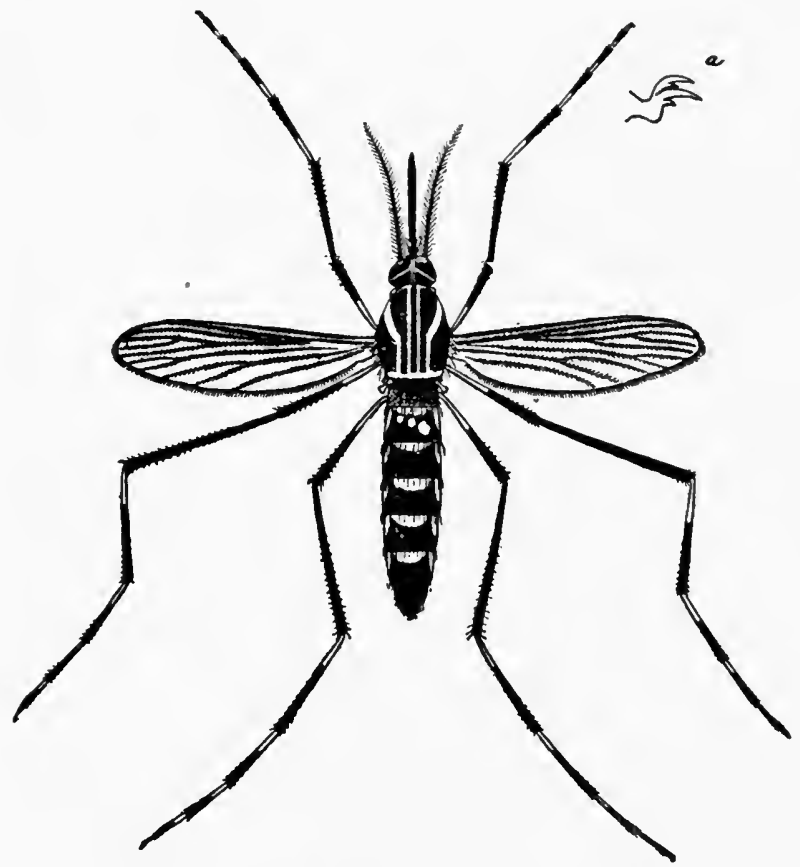

FIG. 25.- Stegomyia fasciata (S. calopus).

S. scutellaris of India, Siam and the Philippine Islands is very much like the preceding, but with only one median line and no curved outer line on the thorax.

The larvæ of Filaria are said to occur in these two species of: mosquitoes, but unable to reach maturity.

S. persistans Banks, from the Philippines, is only a common form of fasciata.

3. Mansonia. - Wings with large, broad and asymmetrical scales. M. uniformis. - Thorax chestnut-brown. Wings brindled but 
unspotted. Legs mottled and banded. Proboscis with broad yellowish band. - India, Africa and the Philippine Islands. This, and M. pseudotitillans of Brazil, are hosts of Filaria.

$B$. Subfamily Anophelinae. - Soberly tinted insects, with palpi of about the same length as the proboscis in both sexes, usually clubbed in the male. Scutellum simple, never trilobed. In this subfamily are found all the mosquitoes concerned in the transmission of malaria. Anopheles is the only genus in this country, but the other genera here described occur in our colonies. Of the I $_{5}$ or more species of Anophelinae found in the Philippine Islands, 4 have been proved to be hosts of the malarial parasite and responsible for most of the malarial fever there, while at least three other species are open to suspicion.

I. Anopheles. - "Thorax and abdomen hairy. Palpi of females thin, generally umbanded. Wing veins with long lanceolate scales which may or may not form tufts, but rarely showing much colorspotting. Mostly large species from temperate or mountain climates." Feet wholly black. - Represented in the United States by 9 or more species. Of the malarial mosquitoes of the Philippines and West Indies none belong to this genus.

The Anopheles is readily distinguished from Culex by the long palpi of the female which produce the appearance of three probosces. Its attitude when at rest is also characteristic, with body in a straight line but at an angle with the resting surface, head down and tail up, while the Culex, although more humpbacked, holds its body nearly parallel with the resting surface. The Anopheles lays only 40 to 100 eggs, more or less separated and not attached together like those of Culex. The eggs of the maculipennis are characterized by fine reticulate hexagonal sculpturing. The larva (wigglers) of both genera are also readily distinguished; that of Culex projects its long breathing tube through the water surface and holds its body downward at an angle, while that of Anopheles has short breathing tube and holds its body parallel with the surface. The entire period covered by the metamorphosis of the maculipennis, from the laying of the egg to the escape of the insect, ranges between ' 12 to 20 days, being somewhat longer than that of our common Culex pipiens and fatigans. The Anopheles is more particular as to breeding places than Culex, generally preferring clear water containing green algæ and other aquatic plants. 
The following are our noteworthy species:

A. maculipennis (Fig. 26). - Rather large but inconspicuous; easily recognized by the four small black spots on the wings, and the wholly black margin and veins. In the female the palpi are yellowish-brown, with dense dark scales at the swollen base, and shorter than the proboscis. Found almost everywhere in North America and Europe, and the common malarial mosquito of both continents. Also found to be a host of the Leishmania parasite in all stages of development by Franchini. Shy and difficult to secure, even when not rare.

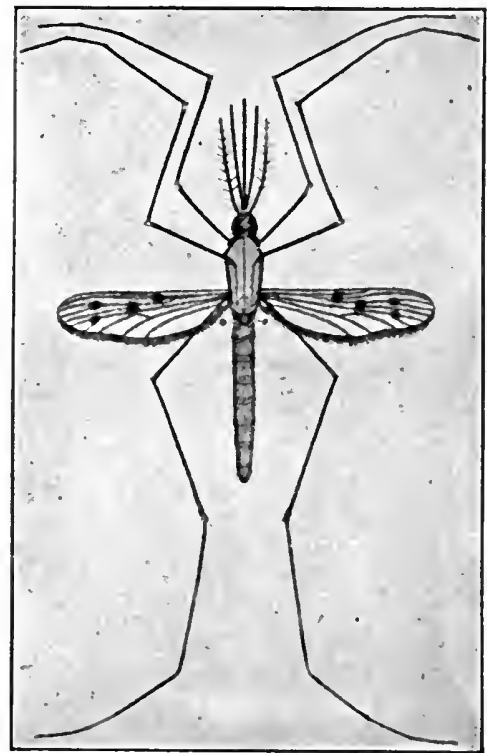

FIG. 26.

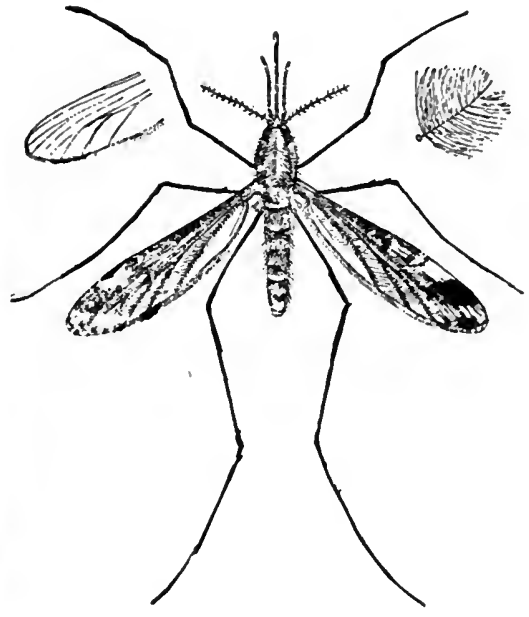

FIG. 27.

FIG. 26.-Anopheles maculipennis, female. (Ludlox.)

Fig. 27.- Anopheles punctipennis, female, with male antenna at right and wing tip showing venation at left; much enlarged. (How'ard.)

A. punctipcnnis (Fig. 27). - Handsome insect with two brilliant yellow spots on margin of the dark wing, one near the apex, the other larger, above the middle. More than five black spots on the veins. Base and tip of last vein black, remainder white. Very common in this country and Canada. 
The experiments of Duprée* and Mitzmain, P. H. S., have shown that it may become infected with the parasite of tertian malaria and transmit it. It is infrequently found in human habitations and therefore probably of little importance in the spread of malaria. Said to breed along rapidly moving water-courses.

A. pseudopunctipennis. - Characterized by having the 3 d, $4^{\text {th }}$ and last veins white, patched with black. Said by Darling to be "only slightly concerned in the transmission of malarial fever" on the Isthmus of Panama.

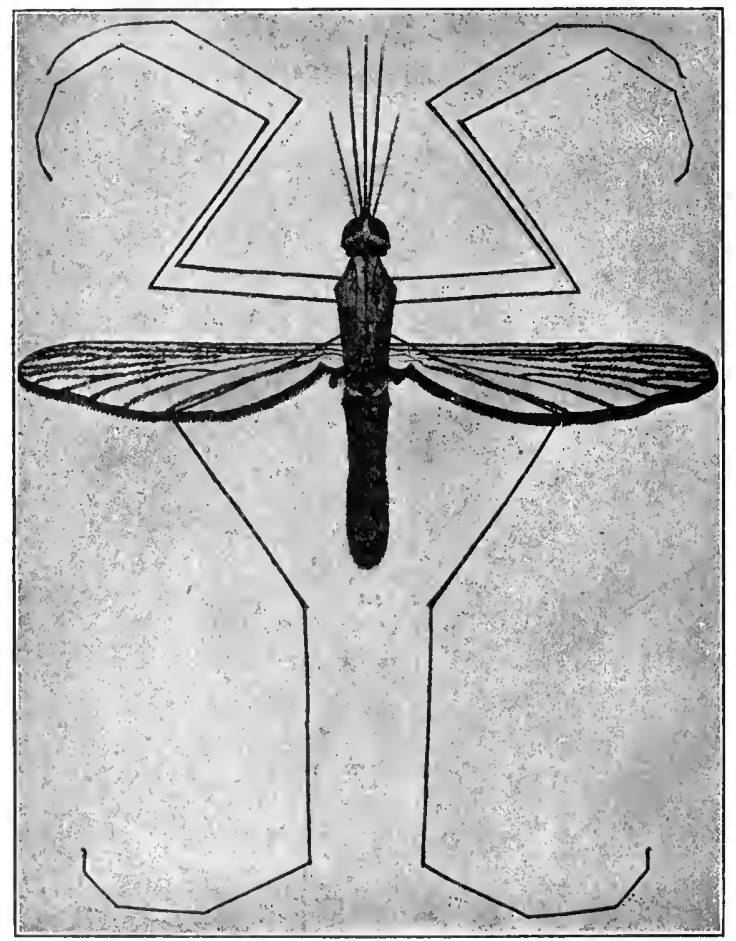

IIIG. 28.-Anopheles crucians, female. (Ludlow.)

A. franciscanus. - Much like pseudopunctipennis and believed by Knab to be identical with it; the dark costa (edge) of the wing with two nearly equal yellow spots. Basal half of last vein white,

* The New Orleans Med. and Surg. Journal. January, 1902, and July, 1905. 
remainder black. Found from California to Texas and Louisiana, and strongly suspected of conveying the malarial parasite.

A. crucians (Fig. 28). - Front margin of the wing wholly black. The last vein white, with three black spots. Tawny, the thorax with three deeper tinted lines. Often seen in tidal waters. Southern States to California. Claimed by Pothier and Beyer to become infected with the parasite of estivo-autumnal fever, and by Mitzmain by the parasites of both tertian and estivo-autumnal malaria. Like punctipennis, it is seldom found in human habitations.

2. Cycloleppteron. - Wing with lanceolate scales, and patches of large inflated scales, densely pigmented, of almost circular outline.

C. grabhamii. - Small dark mosquito with black dot on each side of thorax, yellow patches on wings and brindled legs; palpi very hirsute, almost to the tips. West Indian Islands, where it has been found to convey malaria.

3. Myzomyia. - Thorax and abdomen hairy. Wings with long narrow scales projecting far apart; more or less spotted in contrasted colors. Feet with white bands.

M. funesta (Anopheles funestus) (Fig. 29). - Small dark mos. quito, with three yellow spots on the intensely black costa (edge of wing), and spotted wing fringe; palpi with white tip and two snowy bands. - A host of the malarial parasite in Africa, and always taken in the Philippines wherever malaria is prevalent, so as to leave no doubt of its guilt.

11. rossii (A. rossii). - A common species of India, where it transmits filaria; also considered the malarial carrier in Ceylon; but too rare in the Philippines to be of importance.

M. ludlowii. - Small, with banded palpi and white-tipped proboscis; legs mottled and spotted with yellow; wings with 4 large costal spots and one or two small basal ones. - One of the few mosquitoes breeding indifferently in fresh and salt water, being mostly found in tidal back waters. Widely distributed in the Philippines where it appears coincidently with malaria and, according to Banks and other observers, positively connected with its transmission.

$M$. inde finita. - Very closely related to the preceding two species, but without the leg spots of ludlowii. Also quite common in the Philippines, but its connection with malaria still doubtful.

Several other species of Myzomyia have been found carriers of the malarial parasite in India, and one in Brazil. 


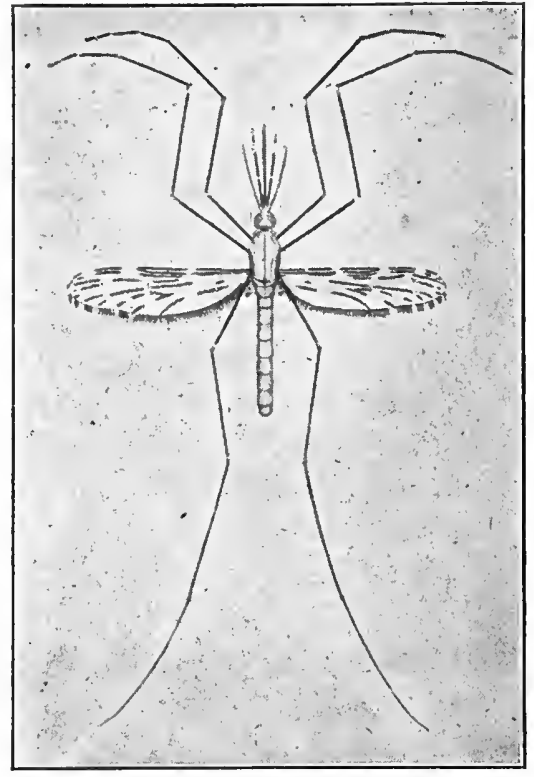

FIG. 29.

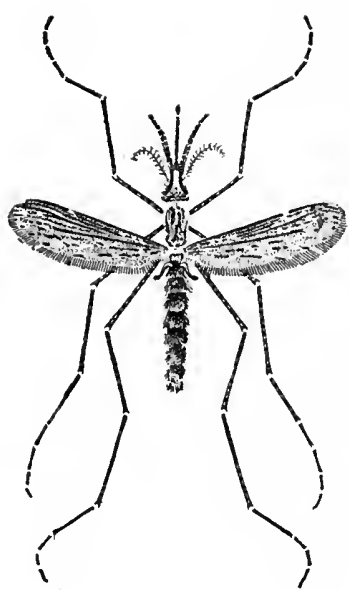

FIG. 30 .

FIG. 29.- Myzomyia funesta (Anopheles funestus) female. (Ludlow.)

FIG. 30.- Myzorhynchus barbirostris (Anopheles barbirostris).

4. Myzorhynchus. - Abdomen without lateral scale tufts; wing scales broadly lanceolate, sometimes short and broad; palpi and proboscis bushy with densely packed scales.

M. barbirostris (Anopheles barbirostris) (Fig. 30). - Dark insect; wing intensely black, with many small spots; head and appendages entirely black except a white frontal tuft. - A host of the malarial parasite in India, and found in the Philippines coincidently with malarial fever.

$M$. pscudobarbirostris, an allied species, may also be suspected.

M. sinensis, a host of the malarial and filarial parasites in Japan and China, has been found at some of the stations in the Philippine Islands, but its connection with malaria there is still doubtful.

Several other African and Indian species are also known agents of malarial and filarial infection.

5. Nyssorhynchus. - Thorax with narrow, curved and fusiform scales. Abdomen with lateral tufts and dorsal patches of flat scales. Palpi densely scaly. Legs banded and spotted white. 
N. fuliginosus (Anopheles fuliginosus). - Small, very dark mosquito, with three yellowish spots on the black costa and numerous black dots on the veins, the last three tarsal joints of hind legs pure white. - Always appears in the Plilippines in connection with malarial outbreaks and may be regarded as one of the transmitters of the parasite.

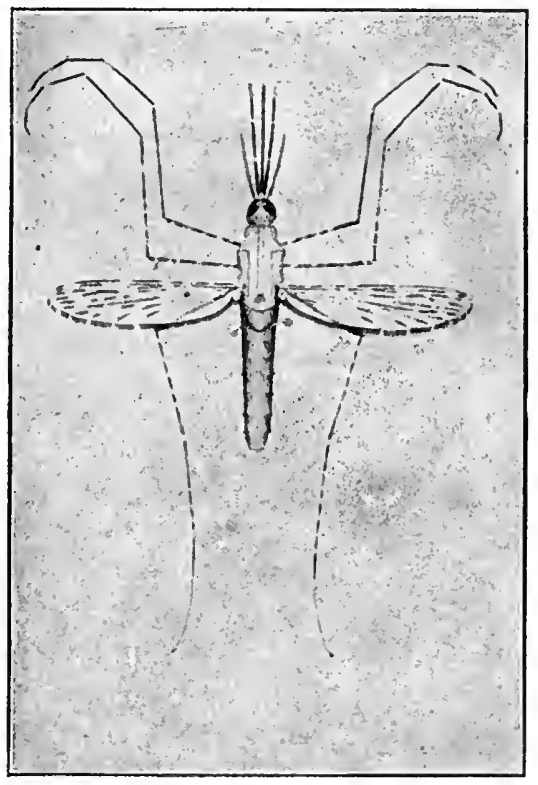

FIG. 3I.-Cellia albimana, female. (Ludlow.)

6. Cellia. - Abdomen almost completely but irregularly densely scaly, with large lateral tufts. - Handsome insects, among which are the best-known malarial carriers of the West Indies and Panama.

C. albimana (Anopheles albipes, $A$. cubensis). - Dark mosquito with the last three hind tarsal joints, and nearly half the second, pure white, save for a black band at base of the terminal joint. (Fig. 3I.) - "Certainly much the commonest Central American and West Indian anopheline, said to breed in canals, puddles, and in the most varied situations, even in brackish water" (Giles). It bites severely at any time of day and night. One of the usual hosts for the parasite of malignant malaria, and of Filaria bancrofti. It 
is inefficient for Filaria demarquaii. According to Darling, by far the most abundant mosquito on the Isthmus of Panama, a host for the estivo-autumnal as well as the tertian and quartan parasites.

C. argyrotarsis (Anopheles argyrotarsis). - Much like the preceding but not so dark, and without the black band at base of terminal tarsal joint. Also common in all the West Indies and further south, and likewise an efficient host and transmitter of malarial parasites.

Another species is a common agent of malarial infection in Africa.

\section{DESTRUCTION OF MOSQUITOES.}

As with flies, much can be done during the winter when many of the surviving mosquitoes, including house species as well as the malarial and yellow-fever species, can be found in cellars, basements, stables, barns, outhouses, etc., in sheltered places. These hibernating mosquitoes are the most dangerous from the fact that they are all females, most of them impregnated and ready to oviposit in the spring. Each one killed is equivalent to the suppression of thousands in the following summer. Many can be destroyed by cautiously holding under them a small vessel containing a little ammonia, petroleum, spirits of turpentine or cotton moistened with chloroform. A small tin can nailed through the bottom to the end of a stick forms a convenient receptacle for the purpose. It is held a moment against the ceiling so as to prevent the escape of the insects.

Whenever a building or room has become invaded by mosquitoes, especially if there is reason to believe that it contains infected ones, the most effective way to get rid of them is by fumigation, as described under Insecticides.

Hand-catching with a chloroform bottle, the use of "slappers" made of wire gauze, and of traps, are all useful measures to get rid of indoor mosquitoes, especially in the tropics. Anopheles are mostly found in early morning and in the dusk of the evening on the inside and outside of the screens, seldom more than six feet from the floor, and in that situation are easily killed by slappers.

Mosquitoes cannot breed unless there is water wherein the eggs may hatch and the larva develop. Hence the accepted axiom: no water, no mosquitoes. This water must be relatively still; no running or ruffled water will do. The first measure then that im- 
poses itself is the drainage or filling of all surrounding swamps and stagnant pools. In marshy lands the breeding places are mostly near the edges, along the uplands, and such places should preferably be filled up. In tidal marshes, narrow ditches 20 to 30 inches deep, dug at intervals of 100 to 150 feet, will drain all intervening pools, with the addition of an occasional spur ditch. Creeks, ditches and pools in which the water rises and falls with the tides will not breed mosquitoes; therefore if a pool cannot be emptied, it should, if possible, be connected with a tidal creek. Another method to prevent breeding in a marshy area which cannot be drained is to dig a pit at its lowest point so as to form a catch basin into which all surrounding stagnant waters will gravitate, and then stock it with fish.

In the tropics, open ditclies soon become obstructed with vegetation and are often breeding places; there, buried tile drains are effective and econonical; where they cannot be used, open concreted ditches are best.

In conjunction with drainage and filling, another important measure is the removal of shelters, such as thick underbrush, long grass and thrifty weeds, especially along streams and on border of ponds, which afford protection to mosquitoes and prevent the drying of water holes.

Within the boundaries of a post or camp, swarms of mosquitoes may be produced from sewers, cisterns, tanks, pits and cesspools, water barrels, neglected buckets and tin cans. Therefore, before blaming the neighboring grounds it is always judicious to make a careful investigation of the military reservation and exclude therefrom all possible breeding places. Pits and holes should be filled up. All receptacles likely to hold rain-water should be broken up or turned bottom up. Barrels and cans in which water must be kept should be covered with a well-fitting lid. Tanks and cisterns, if not roofed over and mosquito-tight, should be protected with wire screens. Pools and ponds which cannot be drained or filled up should have a thin film of kerosene or petroleum poured upon the surface, or sprayed with a nozzle, at the rate of one ounce to each I5 square feet, once every two weeks, or whenever necessary; if washed away by heavy rains or blown against the shore by high winds, more frequent applications should be made. This "petrolization" is easy, cheap and quite effective; not only does the film 
of oil prevent the female mosquito from laying eggs, but all the larvæ already in the water, unable to breathe through it, promptly perish. The best oil for the purpose is one which spreads readily but does not evaporate too rapidly, the crude oil or fuel oil of trade. The addition of crude carbolic acid renders it more diffusible and efficient.

There are situations in which petroleum is not satisfactory, especially in the tropics where the vegetation is rank and pouring rains frequent. It must also be borne in mind that if it kills larvæ it is likewise dangerous to fish and other useful aquatic animals. Therefore efforts have been made to discover a more suitable larvicide, that is to say, a cheap substance which will thoroughly diffuse in the water, kill the larvæ promptly as well as green algæ, and remain efficient for some days without being poisonous to man, domestic animals and fish. Of the several proprietary mixtures found in the market none seems entirely satisfactory. Certain aniline products have been recommended by Celli. "Phinetas oil" has been more or less used on the Isthmus of Panama, especiaily along borders of swamps and streams. It forms a milky compound with water which destroys not only larva but all other animal life. It varies in quality and loses its efficiency very soon after application. The department of sanitation of the Canal Zone has devised a cheap larvicide which gives excellent results, although not supplanting petroleum in suitable places. It is prepared as follows: I 50 gallons of crude carbolic acid are heated in a tank to a temperature of $212^{\circ} \mathrm{F}$.; 200 pounds of powdered and sifted resin are then poured in, followed by 30 pounds of caustic soda previously dissolved in 6 gallons of water, the mixture being kept at $212^{\circ}$ and thoroughly stirred until a perfectly dark emulsion, without sediment, is obtained. One part to 5,000 parts of water will kill larvæ in 5 to Io minutes. It has also high value as a germicide and algicide, thus destroying the food and hiding places of the larva. It is used by spraying it, diluted with water ( $\mathrm{I}$ to 5 ), over the surface and along the margins of mosquito-breeding places.

Ponds and lakes will be sufficiently protected if well stocked with fish. The best kinds for this purpose, in the United States (according to W. P. Seal, a recognized authority on the subject), are a combination of goldfish, roach (Leuciscus) and top-minnows (Gambusia affinis and Heterandria formosa) which feed near the 
surface and almost entirely upon insect larvæ. Also very useful are several species of Fundulus, the killies, in their natural habitat in tidal streams and salt marshes. On account of their predaceous habits it is doubtful whether they would be desirable in land-locked waters. Two small species of sunfish (Enneacanthus) are also recommended. In Cuba, the guayacones, species of Gambusia, especially $G$. punctata which is the most common, have been successfully used to get rid of mosquito larva. The sticklebacks have been mentioned in this connection but, being bottom feeders, are undoubtedly useless for the purpose.

Certain water plants grow rapidly upon the surface of water, forming a thin floating mantle which interferes with the breeding of mosquitoes. Species of Azolla have been recommended; the common American form does not spread much and is of little value; a Pacific form, often covering extensive stretches of quiet water with a dense purple-red mantle, is more promising for mild climates; the European form which covers many of the canals in Holland is also worthy of trial in fresh water. Our duckweeds (Lemma) may be useful in certain situations, although they do not begin to expand much until summer.

Various trees and shrubs have been extolled as effective agents for repelling and killing mosquitoes through their peculiar emanations, especially certain species of Eucalyptus, the castor-oil plant, the china-berry tree and the papaw; but more trustworthy observations have demonstrated their absolute worthlessness for the purpose. 


\section{CHAPTER XV.}

\section{INSECTICIDES.}

Substances generally used, by combustion or in gaseous form, for the destruction of flies, mosquitoes, fleas, lice, bedbugs, roaches and other obnoxious insects. Some are also effective against rats and mice. All possess more or less germicidal properties, as described under Disinfectants. In using them, the rooms should be made reasonably tight by pasting strips over the big cracks, which also prevent the escape of the insects. Closets, cases, drawers, etc., likely to harbor the latter, should be opened to allow free access of the gas or fumes. Anything that will make a dense smoke in burning will kill or drive away mosquitoes and flies, but the following substances are those most commonly used as insecticides:

Pyrethrum insect poreder. - Made from the dried flower-heads of several species of Pyrethrum and owes its power to a volatile oil that disappears with age and exposure. If burned in an open room, mosquitoes and flies are temporarily driven out. In a closed room, in the proportion of 4 to 6 ounces of the powder to each I,OOO cubic feet of space, it will kill some and stupefy the others, causing them to fall on the floor, where they can be swept and burned. It is well to pull down all window shades except one; the insects will then congregate on or near this excepted window, where they can be more easily swept up. This powder is liable to many adulterations. A bright yellow color is often imparted to it by chromate of lead, a poisonous substance generally disguising an article of inferior quality. Pyrethrum is not a disinfectant, but simply an insecticide of variable efficiency. As its fumes are harmless to man, as well as to contents of houses, it affords a convenient, every-day means of getting rid of mosquitoes in spite of its comparatively high cost. In rooms suspected of harboring mosquitoes infected with malarial or yellow fever germs, more effective fumigants must be employed.

This powder is also largely used, as such, against vermin, freely sprinkled wherever needful.

Datura stramonium, the common Jimson weed, powdered, is a good insecticide if comparatively fresh and mixed with one-fourth 
of saltpeter to make it burn brightly. But it is of variable strength and does not keep well.

Mimm's culicide (Campho-phenol). - Made of equal parts, by weight, of carbolic acid crystals and gum camphor. The crystals are melted over a gentle heat and the solution poured slowly over the camphor, the result being a clear, somewhat volatile liquid of rather agreeable odor. It is permanent and may be kept in tight jars. It is inflammable, burning with an intensely smoky flame, but not explosive. It volatilizes rapidly if placed over an ordinary alcohol lamp. As it may take fire spontaneously if overheated, it should be kept at a certain distance from the flame, and not more than 8 ounces put in any one pan. In the proportion of 4 ounces per 1,000 cubic feet of air, volatilized in a closed room (with all large cracks pasted up) for two hours, it kills all the mosquitoes and temporarily stupefies the flies and other insects. For its use a simple apparatus can be readily improvised with a section of stove pipe, 8 inches long, cut below so as to rest on 3 legs, and with a row of quarter-inch holes punched around near the top for the outlet of draft (Fig. 32); an alcohol lamp is placed beneath and the shallow evaporating basin on top. Eight ounces of the liquid will require two ounces of alcohol for its volatilization. Properly used, this preparation ranks next to sulphur as an insecticide in practical fumigation, but costs nearly twice as much. Unlike sulphur, it does not affect metals nor delicate fabrics.

Pyrofume. - A clear, straw-colored liquid derived from pine wood by fractional distillation. In a Public Health report (I906), it is said to be as efficient as sulphur, as an insecticide, acting more quickly and without its disadvantages. For its proper application a special device is necessary.

Sulphur dioxid. - Probably the best of known insecticides. Being equally efficacious in dry or moist air no moisture need be artificially produced, as the drier the air the less the injury to furnishings, colors, etc. With all cracks and crevices closed, the burning of one pound of sulphur per 1,Ooo cubic feet of space will kill flies and mosquitoes within two hours with very little damage to the furniture. Two pounds will kill rats within four hours, and five pounds will destroy most bedbugs, roaches, lice, within six hours, although some of these insects usually escape by running into crevices. (Public Health Bull. No. 42.) The pot method de- 
scribed on page 727 may be used, but without water. Giles recommends a mixture of one pound each of niter and charcoal to eight of sulphur, which burns much more briskly and completely than pure sulphur.

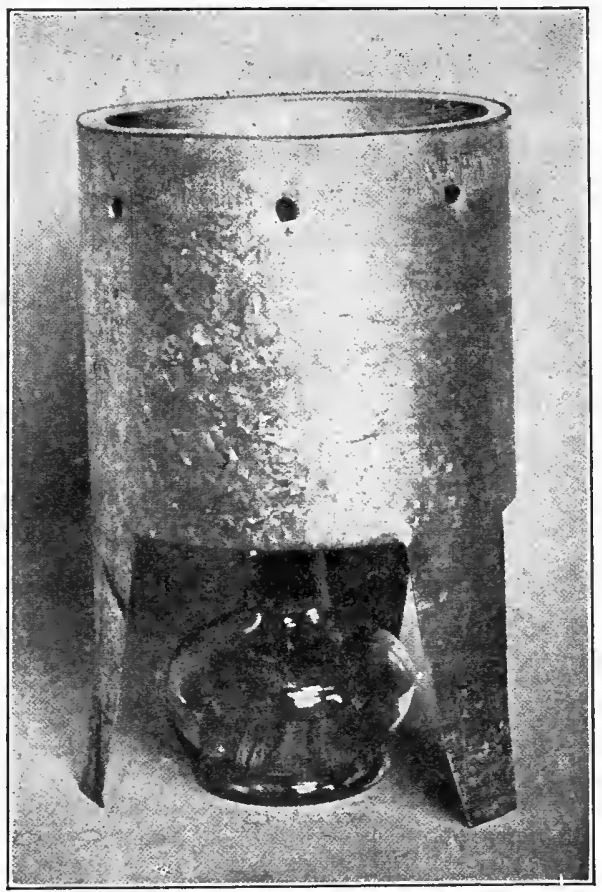

FIG. 32.- A simple apparatus for fumigation, from piece of stove pipe.

Hydrocyanic acid gas. - This gas is deadly to all forms of animal life; it kills surely and promptly not only insects and vermin of all kinds, but also rats and mice. But it is a dangerous agent in the hands of the inexperienced and should seldom be used in dwellings. In careful hands, however, it is very useful for destroying rodents and all forms of vermin in granaries, barns, stables, etc. The proportions for each $\mathrm{I}, \mathrm{OOO}$ cubic feet of space are: potassium cyanid I pound, sulphuric acid I.5 pint, water 2.25 pints. The acid and water are first mixed in an earthen vessel and, when the mixture has cooled down, the cyanid (previously placed in a ganze bag) dropped into it. On account of the rapid evolution of the gas the 
operator must quickly leave the room. The time of exposure is only two or three hours, and no one should enter the building until it has been aired 8 to io hours (Pub. Health Bull. No. 42).

Formaldehyde is a feeble insecticide and not to be relied upon for the destruction of mosquitoes and flies unless a very large volume of gas is liberated quickly so that it may diffuse to all portions of the space in sufficient concentration. When thus generated, as in Munson's method, it has been proved to be a convenient and effective insecticide (see page 732).

Mercuric chloride (corrosive sublimate) evaporated in a porcelain dish over an alcohol lamp, in the proportion of one ounce per I,O0O cubic feet of space is, according to Surgeon G. M. Guiteras, P. H. S., as effective as sulphur against flies and mosquitoes, and much cheaper. If metal surfaces are wiped off immediately after fumigation they are not tarnished, nor are fabrics injured.

Of liquid insecticides may be mentioned lime bisulphide, carbon bisulphide, gasoline and benzine. Lime bisulphide is prepared by boiling together for an hour or two, in water, equal parts of flowers of sulphur and quicklime, and diluting with more water. It is used by pouring into the cracks and crevices supposed to harbor vermin. Gasoline and benzine are used in the same manner. Carbon bisulphide evaporates rapidly at ordinary temperature, and one pound to $\mathrm{I}, \mathrm{OOO}$ cubic feet of air-space (in sealed room) will generate enough fumes to destroy all insects, including roaches. All these liquids, except lime bisulphide, are highly inflammable and must be used with the utmost caution against fire. 


\section{CHAPTER XVI.}

\section{RECRUITING.*}

Next to hygiene, the most important factor in securing the physical efficiency of the soldier is the proper selection of the recruit. None but able-bodied men must be enlisted or mustered in. They alone can stand the exposure and hardships to which troops are exposed and acquire the endurance to fatigue and resistance to disease which make up the reliable and effective soldier. Delicate, undeveloped and immature young men and those who suffer from chronic ailments are easily exhausted by hard work, privations and the inclemencies of the weather, and an easy prey to infectious diseases, weakening the ranks they are intended to strengthen and falling by the wayside when most needed, so that they crowd the ambulances and hospitals especially needed for the wounded, and later become pensioners of the Government which they never have usefully served. The best hygienic conditions will never make hardy men or good soldiers of them. Not only are they ineffective but, still worse, they often require the care of able-bodied men thus withdrawn from the effective forces.

There is therefore no more important duty devolving upon the medical officer than the examination of applicants for enlistment, none that is productive of better and more important results. This is shown, in our past wars, by the difference between the morbidity and mortality of regulars and those of volunteers, the medical officers of volunteer troops having examined their applicants without the necessary strictness or, rather, having been compelled by the exigencies of the situation to accept, against their better judgment, men below the required standards. Thus, in the Civil War, the annual mortality from disease and discharges for disability were respectively 55 and $9 \mathrm{I}$ per I,O00 for volunteers and only 32 and 68 for regulars. In the year 1900 , the admissions for disease per 1,000 of strength were I,82I for regulars and 2,762 for volunteers, while the mortality was 12 and 25 respectively.

This matter of examining applicants for enlistment is deemed so

* See Tripler's Manual on the Examination of Recruits, by Chas. R. Greenleaf, Col., Asst. Surg. Gen., U. S. A. 
important that, under existing United States Army Regulations, medical officers are required to perform the duties of recruiting offcers at recruit depots and other large posts where they are available for the purpose, and are alone responsible for the acceptance or rejection of applicants.

As a general rule, the higher the social class to which the recruit belongs, the better he will be physically as well as mentally, for it is notorious that in European armies, where military service is obligatory, the officers are usually taller and heavier than the enlisted men. Vision is an exception to this rule, for it grows more imperfect as we ascend the social scale, so that perfect eyesight is oftener found in the middle and lower than in the higher and better educated classes. The physical and mental aptitudes of recruits are, to a great extent, the resultant of their previous environment, occupation and education; these factors therefore must be duly considered. Youths from the country are stronger, heavier, healthier and more enduring than city-bred boys, generally also of better habits and character, but, on the other hand, they are hard to break in, slow to accommodate themselves to their new conditions and to acquire the smart step and martial bearing of the trained soldier. The city recruit is more supple and adaptable, and sooner trained for an emergency; he is also more likely to be immune to infectious diseases and to have had some training in mechanical trades, the latter being valuable accomplishments in modern war; but he is lacking in vigor, endurance and stability of character.

AGE. - "Applicants for first enlistment must be between the ages of 18 and 35 years, of good character and temperate habits, able-bodied, free from disease, and must be able to speak, read and write the English language.

"No person under I8 years of age will be enlisted, reenlisted, or accepted with a view to enlistment, and minors between the ages of I8 and 2I years must not be enlisted, or accepted with a view to enlistment, without the written consent of the father, only surviving parent, or legally appointed guardian, to the minor's enlistment.

"Original enlistments will be confined to persons who are citizens of the United States or of Porto Rico, or who have made legal declaration of their intention to become citizens of the United States."

It is a well-known fact that the human body grows and develops until at least the age of 25 . Before this the bones are not fully 
formed; the epiphyses are not all ossified and united; the sacrum and sternum are not yet consolidated; nor has the body reached its full height, weight and chest capacity. We enjoy our best physical development and greatest strength from 25 to 30 , the lungs not gaining their maximum capacity until 30 or 35 . Therefore, in theory, the older the recruit, if under 30 , the better he is physically and mentally. But, in practice, there are other aspects of the question to be considered. After 25, most of the desirable young men have chosen their trade or profession, therefore are more or less settled in life and no longer think of enlistnig. Furthermore, at that age their habits are formed and they could not so easily be broken into the new and very different ways of military life. Were it possible to secure enough recruits 22 years old, it would be in the interest of the service to do so; but as this is generally impossible, the minimum age must remain at $2 \mathrm{I}$.

In European countries, where the military service is obligatory for all, the age is 20 ( $2 \mathrm{I}$ in Russia), although many of the young conscripts do not really get under the colors until they are $2 \mathrm{I}$. There the age has to be that which follows the period of education and precedes that of the selection of a profession or trade.

Experience has shown that, under 20, soldiers are incapable to stand the fatigue and hardships of a campaign, and military history contains many complaints of commanders whose hospitals were full of young, immature soldiers. It is stated that the French Army which won the glorious battle of Austerlitz, in I805, after a forced march of more than $\mathrm{I}, \mathrm{OOO}$ miles, leaving but few sick en route, contained no men below the age of 22 ; while the army which won the bloody and indecisive battle of Wagram, in I809, after scattering many sick and wounded on the way, was mainly composed of much younger soldiers.

In our service, more men enlist in their twenty-first year than at any other age.

In the British Army the age of endistment is between 18 and 25, more than half of the recruits being under 20 years, an age, according to Melville, "of greater moral and physical receptivity." Such age would certainly be dangerously low, were it not that the enlistment is for I 2 years, 7 with the colors and 5 in the reserve, so that, in reality, the Army is composed of a majority of welltrained soldiers from $2 \mathrm{I}$ to 25 . A convincing proof that this sys- 
tem is not detrimental to health is that, notwithstanding the worldwide colonies of the empire, the mortality of the British Army is less than that of the U. S. Army.

In France and Germany, where the term of military service is only three and two years, respectively, the proportion of men under 2I years and imperfectly trained is necessarily much greater than in the English Army.*

At what age ceases the aptitude for military service? It will seldom be in the interest of the service to enlist men over 30 years old, and the legal limit of 35 is only intended to be used in times of emergency. "It has been observed that for acceptable colored recruits the age of 25 or 26 is practically the maximum, because after that they are liable to be physically stiffened and mentally dulled" (Greenleaf). There is no age limit for reenlistment in our Army. but it is recognized that the soldier grows old quickly in barracks and in the field. After 20 years in the ranks, when past 40 years of age, he soon becomes unequal to the arduous duties of field service. The Army Regulations provide that when an enlisted man shall have served as such for 30 years he may apply for retirement; but

* Note.-All that is stated in this chapter, as may readily be seen, was written before the present European War had been begun or expected.

Physiological facts cannot be changed, but their adaptation to new war conditions now demands consideration. This is a war between armed nations, in which all available resources in men and supplies must be utilized. The problem is no longer how to mobilize a certain number of ablebodied men, but how to obtain the greatest output from universal service. In Europe, all men under 45 , not clearly disqualified, are already under arms. Men of 50 may soon be called, where they are not already mobilized.

There is no doubt that men suffering from minor disabilities, or who are past 40 and therefore beyond the period of highest physical efficiency, are still capable of rendering valuable military service, provided they are assigned to the duty for which they are fitted, and it is the medical officer who should determine the nature of this duty.

Notwithstanding our deep-rooted hostility to conscription, the irresistible logic of events has compelled Congress to enact the Universal Training and Service law, calling to the colors all able-bodied men from $2 \mathrm{I}$ to $3 \mathrm{I}$. The principle that, in the event of war, it is the duty of all citizens to take up arms in defense of their country being thus established, we may assume that our wars, hereafter, will be fought not by costly and inefficient volunteers but by drafted and well-trained citizens.

In order to mobolize large numbers of men in as short a period of time as possible, it may become necessary to relax the strictness of some of the requirements of our physical examination, at least for some of the branches of the service. In such case, we need hardly say, the physical defects of the recruit should be carefully recorded in order to guard the national treasury against subsequent claims which, with our loose and extravagant pension system, might become an intolerable burden upon the country. 
there are few enlisted men who serve usefully and efficiently until the age of $5^{1}$. The so-called veterans of Napoleon were men of 26 to 28 years, and his "old guard" consisted of men from 28 to 29; at Austerlitz the oldest soldiers were 33, and at Friedland 36 years old (Rouget et Dopter).

Youths under 2I often present the appearance of the physical signs of maturity and may attempt to deceive the recruiting officer. The latter therefore should be very particular to ascertain the correct age of applicants, the more so that a minor who enlists by deceit may, upon application of his parent or guardian, be discharged from the Army, thus causing the loss to the Government not only of his service but of his pay and equipment. According to Greenleaf, there are certain evidences of maturity which usually accompany the period of legal majority and with which the recruiting officer should familiarize himself.

"At twenty-one years of age the wisdom teeth are usually cut, and on each side of both jaws there should be found five grinders, viz., three large double or molar teeth and two smaller double or biscuspid teeth. In case of the loss of teeth the spaces originally occupied by them may be seen.

"Under twenty-one years of age the wisdom teeth are seldom to be found, and there will, therefore, be but four grinders on each side of both jaws, viz., two molars and two bicuspids.

"At maturity there should be some beard upon the face, and hair under the arms, a full growth around and above the genital organs, and some scattered hairs in the neighborhood of the anus. The hair of the body is generally fine and silky.

"After maturity, the hair is thick and coarse in the various places mentioned.

"After maturity the skin of the scrotum is somewhat darker in color than the surrounding parts, is opaque, and is marked in various directions by wrinkles or folds:

"Before maturity this skin retains the soft, velvety condition of youth, its pink or fresh flesh color, and is more or less translucent, while the wrinkles or folds are not well pronounced, or are entirely absent, particularly at the sides. This condition of the scrotum is the most valuable of the signs of maturity."

In hot countries, where man has to contend against depressing climatic conditions and endemic infectious diseases, very young sol- 
diers are particularly exposed to grave dangers and soon become non-effective. Only matured, well-trained men, of at least 22, should be sent to the tropics for active duty.

\section{HEIGHT, WEIGHT AND CHEST MEASUREMENT.}

To determine the physical condition of applicants for enlistment it is necessary to ascertain whether the height, weight and chest measurements come up to established standards and bear certain definite proportions to one another.

Height. - Our Army is recruited not only from native Americans, but also from the naturalized emigrants of many countries, differing more or less in physical characteristics. According to Kilbourne, the North American Indians are taller than any other race on this continent, the adult males averaging 5 feet 7.93 inches. Next in size are the native white Americans, whose average height. in the Civil War, was 5 feet 7.67 inches. Among our naturalized citizens, the Norwegians, Scotcl Canadians, Swedes and Irish come next, in the order named. The American negro follows, with an average of 5 feet 6.62 inches. Lower in the scale, but still about 5 feet 5 inches, are the English, Hungarians, Germans, Swiss, French and Poles. The Italians and Austrians are 5 feet 5 inches or under.

In this country, "the average stature of a youth of 18 years of age, a 'growing lad,' is a little over 5 feet 4 inches, and this increases gradually until he reaches the age of 25 years - about the stage of physical maturity or manhood - when his average height is between 5 feet 7 inches and 5 feet 8 inches" (Greenleaf).

For the years I9Io and I9II, in our Army, the average height of white recruits was 5 feet 7.56 inches. Of white recruits 49 per cent., and of colored recruits 47 per cent., were 5 feet 8 inches or over. In the British Army, the average height of recruits for Igog was 5 feet $6 \frac{T}{2}$ inches.

Our Army Regulations provide that: For infantry, coast artillery and engineers the height must not be less than 5 feet 4 inches; for cavalry and field artillery (except mountain batteries) not less than 5 feet 4 inches and not more than 5 feet Io inches; for mountain batteries, not less than 5 feet 8 inches and not more than 6 feet. A variation not exceeding a fraction of an inch above the maximum height given for cavalry and artillery, is permissible if the applicant is in good health and in other respects desirable as a recruit. 
In foreign. countries, the ninimum height is 64 inches for the English, 62 for the Germans and less than 62 for other armies. In the French Army, the minimum limit, reduced to 60.5 inches in 1872 , was entirely discarded in I90I, on the ground (for us inadmissible) that physical aptitude is independent of height.

It must be admitted, however, that height has not the va!ne of former days when a certain spectacular effect was expected from the uniformed soldier, and that relatively small men are quicker, more active, enduring and resistant than tall men; thus the French troops which are the best marchers in Europe average at least two inches less than the English and American troops. There is no doubt that, in our service, the minimum height for enlisted men could be safely lowered to 63 inches without loss of efficiency.

In a full-grown and well-proportioned subject, his strength and staying power increase with his height, but a maximum limit is soon reached beyond which it is not advisable to go. Tall men, that is, men exceeding 6 feet, are objectionable, for in them, as a rule, the lungs and heart are not developed nor their muscular energy increased in proportion to their height, so that they are less able to stand hard marching and endure hardships than much smaller men. Furthermore, tall men require more food, more clothing, and - not a negligible consideration - offer a larger target to the enemy.

For admission to the United States Military Academy, candidates I7 years old must be at least 64 inches tall, and those 18 years and upward at least 65 inches.

Weight. - In our Army the minimum weight for all arms of the service is 128 pounds, subject to slight variations as explained on page 192 ; but in no case will an applicant whose weight falls below I 20 pounds be accepted without special authority from the Adjutant General of the Army. The maximum must not exceed I9o pounds for infantry, coast artillery and engineers, nor 165 pounds for cavalry and field artillery.

Weight is of the greatest importance in the examination of recruits, giving, in connection with the height, a valuable indication of the development of organs as well as of the firmness and compactness of tissues, but it is necessary to see that the weight is chiefly that of the organs and tissues most concerned in the production of physical strength, namely, bones and muscles, and that there is no excess of fat or protuberance of abdomen. 
In our service, the proportion which should exist between height and weight is formulated by Greenleaf as follows:

"For each inch of height from 5 feet 4 inches to 5 feet 7 inches, inclusive, there should be calculated 2 pounds of weight. When the height exceeds 5 feet 7 inches, calculate 2 pounds of weight for the whole number of inches of height; add to this product 5 pounds of weight for each inch of difference between 5 feet 7 inches and the actual height ; the sum will be the normal weight in pounds."

For the years 1910 and I9II, in the U. S. Army, the average weight of white recruits was 145 pounds. Of white recruits 58.07 per cent., and of colored recruits 73.6 per cent. weighed I 40 pounds or over.

In Europe, the minimum weight admissible ranges from i Io to 120 pounds. The weight requirement, in proportion to size, is also somewhat less than with us; thus in France and Belgium, the recruit is accepted if his weight is 7 or 8 kilos below the number represented by the decimals of his height expressed in the metric system; for instance, a man I meter 63 centimeters high ( 64 inches) need not weigh more than $63-7=56$ kilos, or 123 pounds.

In the British Army, for I909, the average weight of recruits was I 30. I pounds, the minimum required being $\mathbf{I} 20$.

Chest Measurement. - The chest contains the heart and lungs, the most important organs of the body; therefore its development is of particular significance as indicating the vital power and endurance of the recruit. As the body increases in size and weight, a proportional increase of the chest must take place in order to ftirnish the greater amount of nervous and muscular energy required. A short, narrow or thin chest is never desirable in an applicant for enlistment, particularly if long-legged.

A still more useful indication than the mere size of the thorax is the respiratory capacity of the lungs. This capacity is best determined by the spirometer which shows the actual amount of expired air after a forced inspiration. But the use of this apparatus has not yet become generalized and the respiratory capacity is usually determined by measuring the chest mobility.

This measurement of the chest mobility, however, is open to objections. It makes no allowance for diaphragmatic breathing, or the fact that narrowness of chest may be compensated by length: nor does it take account of abnormal contraction of muscles and 
obesity of chest walls. Errors are also frequent in the handling of the tape so that different examiners of the same subject seldom obtain the same figures. From careful experiments, Owen (Mil. Surg., May, I90o) concludes that mistakes with the tape are much more liable to occur than with the spirometer; that the use of the latter gives more accurate and valuable information, aiding in excluding men in the pre-tubercular stage, men perhaps with large muscular development but small pulmonary capacity. The two systems could be usefully combined, applying the tape to robust and normally developed men, and reserving the spirometer for thinchested or very stout men.

According to the regulations governing recruiting in our Army, "The chest mobility, $i$. e., the difference between the measurement at inspiration and expiration, should be at least 2 inches in men below 5 feet 7 inches in height, and $2 \frac{1}{2}$ inches in those above that height." A large proportion of men have a chest expansion of 3 to 4 inches.

It may be formulated, in a general way, that the measurement at expiration should be at least equal to one-half of the height; thus a man 66 inches high should not measure less than 33 inches. In our service, the rule is that a man whose chest measures less than 32 inches at expiration, whatever his height may be, should be rejected, unless otherwise specially desirable.

For the years I9IO and I9II, in the Army, 48.3I per cent. of white recruits, and $52.4 \mathrm{I}$ of colored recruits had a chest measurement of 34 inches and over at expiration.

Antony found that in French soldiers affected with respiratory diseases, especially tuberculosis, 43 per cent. present a chest perimeter smaller than half the height.

"The circumference of the chest is measured by passing the tape around it immediately at the point of the shoulder blade, the arms hanging down. Generally the tape will then be found to fall below the nipple.

"In taking this measure contortions of the body, such as bending backward to 'throw out' the chest or bending forward to 'draw it in,' should be avoided.

"The applicant should stand erect without muscular strain or rigidity. After the tape is placed in position and lies snugly and evenly upon the skin, and while the loose ends are held between 
the fingers of the examiner so that when the chest is expanded the tape will run readily through them, the applicant should be directed to draw in slowly and steadily a long breath until his chest is inflated to its utmost capacity, when the record of chest circumference at inspiration should be read from the tape. He is then to expel the air by counting slowly and steadily from one onward until he can no longer resist the urgent demand for inspiration, when the record of chest circumference at expiration should be read. This should be repeated several times to insure accuracy. Many men are extremely awkward in developing their chest capacity on demand and great care and patience are necessary in getting the true measurements" (Greenleaf).

The French regulations prescribe to place the tape along the lower pectoral line, which is 2 or 3 centimeters below the nipple.

Pignet's "index" to estimate the physical condition and efficiency of recruits, and now much used in Europe, is obtained by adding the chest measurement (in centimeters) at expiration, to the weight (in kilos), and then subtracting the sum from the height (in centimeters); thus: index $=\mathrm{H}-(\mathrm{W}+\mathrm{C})$. The smaller the index the stronger the man, and any recruit may be accepted whose index does not exceed 20 or 25 . This method rests on correct principles so long as the height does not fall below the accepted standard.

The following table is given in our recruiting regulations for convenience of reference:

Table of physical proportions for height, weight, and chest measurement.

\begin{tabular}{|c|c|c|c|}
\hline \multirow{2}{*}{ Height } & \multirow{2}{*}{ Weight } & \multicolumn{2}{|c|}{ Chest measurement } \\
\hline & & At expiration & Mobility \\
\hline $\begin{array}{c}\text { Inches } \\
64 \\
65 \\
66 \\
67 \\
68 \\
69 \\
70 \\
71 \\
72 \\
73\end{array}$ & $\begin{array}{c}\text { Pounds } \\
\text { I28 } \\
\text { I30 } \\
\text { I32 } \\
\text { I34 } \\
\text { I4I } \\
\text { I48 } \\
\text { I55 } \\
\text { I62 } \\
\text { I69 } \\
\text { I76 }\end{array}$ & $\begin{array}{c}\text { Inches } \\
32 \\
32 \\
32^{\frac{1}{2}} \\
33 \\
33^{\frac{1}{4}} \\
33^{\frac{1}{2}} \\
34 \\
34^{\frac{1}{4}} \\
34^{\frac{3}{4}} \\
35^{\frac{1}{4}}\end{array}$ & $\begin{array}{c}\text { Inches } \\
2 \\
2 \\
2 \\
2 \\
2 \\
2 \frac{1}{2} \\
2 \frac{1}{2} \\
2 \frac{1}{2} \\
2 \frac{1}{2} \\
3 \\
3\end{array}$ \\
\hline
\end{tabular}


It is not necessary that the applicant should conform exactly to the figures indicated in the foregoing table. The following variations below the standard given in the table are permissible when the applicant for enlistment is active, has firm muscles, and is evidently vigorous and healthy:

\begin{tabular}{|c|c|c|}
\hline Height & Chest at expiration & Weight \\
\hline Inches & Inches & Pounds \\
\hline 64 and under $68 \ldots$ & 2 & 8 \\
\hline 68 and under $69 . .$. & 2 & 12 \\
\hline 69 and under $70 \ldots \ldots \ldots \ldots \ldots \ldots$ & 2 & 15 \\
\hline go and upward $\ldots \ldots \ldots \ldots \ldots \ldots \ldots$ & 2 & 20 \\
\hline
\end{tabular}

In 1897 , there were 6,062 native whites accepted for the United States Army. Of these, 3,243 ranged in age from 2I to 24; they averaged in height 67.79 inches, and in chest measurement 33.92 inches at expiration and 36.99 inches at inspiration. The remaining 2,8 I 9 ranged in age from 25 to 39 , with practically the same average height but about a half-inch increase of chest measurement, at both expiration and inspiration, the expansion being the same, almost exactly 3 inches.

During the Civil War, Baxter found that the native-born whites had an average expansion of 2.80 inches.

Causes of Rejection and Discharge. - For the years I9Io and I9I I, in the U. S. Army, the principal causes of rejection, by recruiting and examining officers, in order of frequency, were venereal diseases, heart diseases, affections of the eye and defects of vision. affections of the ear and deafness, flat feet, malformation or defects of development, alcoholism.

The principal causes of discharge during the same years, in order of frequency, were tuberculosis, venereal diseases, insanity, heart diseases, flat feet, congenital malformation or defects of development, epilepsy. It thus appears that, with the possible exception of vencreal diseases, all the other conditions mentioned probably existed previous to enlistment and that many could have been detected by a stricter scrutiny on the part of the examining officers. 


\section{MODE OF EXAMINING AN APPLICANT FOR ENLISTMENT.}

This examination is physical, mental and moral.

Physical. - The recruit is to be examined stripped, in a large, well-lighted room, after he has taken a bath. The examining officer proceeds in the following order:

I. General physique, skin, scalp and cranium, ears, eyes, nose, mouth, face, neck and chest; chest measurements.

2. The arms being extended above the head, backs of hands together, the applicant is required to cough vigorously; any form of rupture may now be discovered by the hand and eye, but still better by the index finger passed up to the external ring.

3. The arms remaining extended above the head, the man is required to take a long step forward with the right foot and bend the right knee; the genital organs are now conveniently exposed and varicocele and other defects in the scrotum may be recognized.

4. Arms down and the man required to separate the buttocks with his hands, at the same time bending forward; this exposes the anus.

5. Heart and lungs; rate of pulse and respiration.

6. Upper extremities, making sure that all joints are free and supple, from the phalanges to the shoulder.

7. Lower extremities; the applicant is required to leap directly up, striking the buttocks with the heels, to hop the length of the room on the ball of first one foot and then the other, to make a standing jump as far as possible and repeat it several times, to run the length of the room in double-time several times; after which his heart and lungs are reexamined.

Mental and Moral. - No standard is prescribed for the mental examination, but the man should be able to read and write, and give evidence of primary education and of normal, sound understanding. The advances in the art of war and the use of modern weapons require a much higher degree of intelligence on the part of recruits than formerly, and no future war can be waged successfully except by soldiers who think for themselves and have a clear conception of their duties.

Mental diseases form one of the chief causes of discharge from the Army. During the 5 years 1905-1910, 520 mental cases were reported, or about 1.40 per thousand. In the German Army, the ratio was I.Io in I906. There is no doubt that most of these cases exist at the time of enlistment and could be eliminated by a trained 
psychiatrist. The most frequent mental abnormalities in military life are weak-mindedness, dementia precox, degenerative psychoses and hysteria. About 50 per cent. of the cases are more or less complicated with alcoholism.

In view of the many and serious troubles caused by defectives and dements in military organizations, careful attention should be given to the mental state of applicants for enlistment by medical officers specially instructed in psychiatry. The life history of applicants, whenever obtainable, will furnish most useful information in this regard: an aimless and wandering life, frequent changes of occupation, lack of primary education, confinement in reform school, etc., are all suspicious indications. Much of the mental and moral states of applicants can be revealed by suitable questions. Defectives, dements and degenerates exhibit defects of memory and an inability to do connected thinking; they have little power of attention and concentration, and tire very easily; or else give evidence of irritability, boastfulness, sometimes of brilliancy, with more or less excentricity of manner or speech. Some information may also be obtained from the so-called stigmata of degeneration, such as asymmetry of face and head, low and narrow retreating forehead with projecting eyebrows, deformed nose or ear lobes, high-arched or cleft palate.

The moral character should be scrutinized with care in order that enlistments from the vagrant, vicious and criminal classes may be avoided. The evils of intemperance in the use of stimulants or narcotics are so great that men whose habits in this respect are under suspicion should be rigidly excluded. It may sometimes be difficult to form a correct opinion of the character of the applicant, but "long indulgence in habits of intemperance will almost surely be indicated by persistent redness of the eyes, tremulousness of the hands, attenuation of the muscles - particularly of the lower extremities - sluggishness of the intellect, an eruption upon the face and purple blotches upon the legs." In the drunkard, the face and nose are often congested, with dilatation of superficial capillaries, producing a bloated appearance ; there may be a prominent dropsical abdomen; the pulse is soft and quick, and the skin hot. 


\section{VISION AND HEARING.}

Vision. - To determine the acuity of vision, place the applicant with back to the window at a distance of 20 feet from the test types. Examine each eye separately, covering the other eye with a card (not with the hand). The applicant is directed to read the test types from the top of the chart down as far as he can see, and his acuity of vision recorded for each eye, with the distance of 20 feet as the numerator of a fraction, and the size of the type of the lowest line he can read correctly as the denominator. If he reads the $20-$ feet type correctly, his vision is normal and recorded $20 / 20$; if he does not read below the 30 -feet type, the vision is imperfect and recorded $20 / 30$; if he reads the 15 -feet type, the vision is unusually acute and recorded $20 / 5_{5}$, etc.

Until I908, normal vision was required of all recruits, with few exceptions, but, in that year, Banister and Shaw, U. S. Army, conducted a series of experiments which tended to prove:

That a perfectly sharp image of the target or bull's-eye is not necessary for good shooting.

That a visual acuity of $20 / 40$, or even $20 / 70$, in the aiming eye is consistent with good shooting, provided the soldier is able to accurately focus both sights of his rifle.

That as rifle shooting is an act of monocular vision, a comparatively high standard of vision is necessary for one eye only.

In accordance with these conclusions, the prescribed minimum visual requirements in the U. S. Army are now as follows:

"I. For the line of the Army and for the Signal Corps: 20/40 for the better eye, and 20/100 for the poorer eye, provided that no organic disease exists in either eye.

"Recruits may be accepted for the line of the Army when unable with the better eye to correctly read all of the letters on the $20 / 40$ line, provided they are able to read some of the letters on the $20 / 30$ line.

"2. For the Ordnance Department and for the Hospital Corps: $20 / 70$ in each eye, correctible to $20 / 40$ with glasses, provided that no organic disease exists in either eye."

The conclusions of Banister and Shaw have been strongly contested by Major R. P. O'Connor (J.M.S. I., Jan.-Feb., I9I I), who maintains that the first requirement for accurate and rapid shooting is the ability to see the bull's-eye clearly and hold the sights upon it 
while the trigger is pulled. To do this the bull's-eye must be focused clearly by direct vision, the sights being seen by indirect vision, the front one appearing but slightly blurred in the center of a much blurred rear-sight notch. The necessary sighting corrections cannot be made intelligently if the target is blurred, and the bull's-eye cannot be used as a unit of measure. The great majority of sharpshooters and expert shots, according to O'Connor, have normal vision or had it when they learned to shoot.

The ability to correctly estimate distances, under all circumstances, forms an important element in the education of the soldier, and is indispensable to become a good shot. It implies the power, as one changes position, to perceive differences in the clearness, color and shape of topographical features, details of uniform, moving bodies, etc. This is impossible for anyone with a $20 / 40$ vision in the right eye and a possibly poorer one in the left eye. Shooting may be an act of monocular vision, but in all situations where a quick and accurate estimate of distance, speed and direction is necessary, binocular vision is indispensable.

There are two special reasons in modern warfare calling for acute normal vision: the distant and hidden enemy, often with only the head showing, if anything; and the inconspicuous color of his uniform, blending with the background.

To sum up, it should be said that, although it may be necessary. at times, to enlist applicants with vision below $20 / 20$ in order to obtain a sufficient number of recruits, the normal binocular vision should remain the standard, and be required whenever possible.

In the examination of candidates for admission to the United States Military Academy and of candidates for commission, whether from the ranks or from civil life, the vision, as determined by the official test types, must not fall below 20/40 in either eye, and not below 20/20 unless the defect is a simple refractive error, not hyperopia, is not due to ocular disease, and is entirely corrected by proper glasses. Hyperopia requiring any spherical correction, anisometropia, squint or muscular insufficiency, if marked, are causes for rejection.

For admission to the United States Military Academy, as well as for candidates for commission, color blindness, red, green, or violet, is cause for rejection. For enlistment it is only a bar for applicants for the Signal Corps. Total color blindness is exceedingly rare, but 
partial blindness, that is, blindness in one of the fundamental colors, may be expected, according to Seydel, in 6 or 7 per cent. of soldiers examined. Violet blindness is very rare; the green is the most frequent, being about twice as common as the red.

Hearing. - To determine the acuity of hearing, place the appiicant facing away from an assistant who is 20 feet distant, and direct him to repeat promptly the words spoken by the assistant. If he cannot hear the words at 20 feet, the assistant should approach foot by foot, using the same voice, until the words are repeated correctly. Examine each ear separately, closing the other ear by pressing the tragus firmly against the meatus. The examiner, whose hearing should be normal, faces in the same direction as the condidate and closes one of his own ears in the same way as a control. The assistant should use a low conversational voice (not a whisper), just plainly audible to the examiner, and should use figures, names of places, or other words or sentences until the condition of the applicant's hearing is evident. The acuity of hearing is expressed in a fraction, the numerator of which is the distance at which the words are heard by the candidate, and the denominator the distance. in feet, at which the words are heard by the normal ear; thus $20 / 20$ records normal hearing, 10/20 imperfect hearing, etc.

Deafness of either ear constitutes an absolute cause of rejection. A slight degree of deafness in only one ear may be overlooked.

Candidates for aviation must have perfect vision and perfect hearing. The ear, particularly, requires to be examined by a series of special tests to ascertain the functional powers of the vestibule and semi-circular canals, and the state of the sense of equilibrium.

\section{SPECIAL DISQUAIIFICATIONS.}

The following defects and conditions are the most frequent causes of rejection:

Skin. - Chronic, contagious and parasitic diseases, vermin, chronic ulcers.

Head. - Abrupt depression in skull, the consequence of old fracture; marked baldness.

Spine. - Curvatures, caries, abscess. Lateral curvature is cause for rejection when it exceeds one inch to either side of the line of spinous processes, especially when it throws the shoulders out of symmetry. 
Ears. - Deafness of one or both ears; all catarrhal and purulent forms of otitis media; perforation of tympanum.

Eyes. - Defective vision in either eye; conjunctival affections, including trachoma and entropion; strabismus, disease of the lachrymal apparatus, exophthalmos, ptosis, asthenopia, nystagmus.

Mouth, Nose and Fances. - Deformities interfering with mastication or speech, chronic ulcerations, fissures or perforations of the hard palate, hypertrophy of the tonsils sufficient to interfere with respiration or phonation, loss of voice or manifest alteration of it. The applicant must have " at least six serviceable molar teeth, two above and two below on one side and one above and one below on the other side, and so opposed as to serve the purpose of mastication."

Obstruction of nostrils, or foul discharges indicative of ozena. Simple atrophic rhinitis is readily curable. Nasal polypi often mean chronic sinusitis, but are not a bar to enlistment if, after removal, the sinuses appear free. Sunken or scarred nose is often indicative of syphilis, while a red, bulbous nose suggests alcoholism or indigestion.

Men with marked hypertrophy of tonsils should be accepted only after excision. According to Le Wald, 2 out of 3 cases with hypertrophied tonsils will also have adenoids, and every 2 out of 3 recruits with adenoids have visible changes in the middle ear. All large adenoids should be excised before acceptance, as well as smaller masses if associated with pathological changes in the middle ear; refusal to be operated should, at least, disqualify the applicant for the artillery.

Neck. - Goiter, great enlargement or ulcerations of the cervical glands.

Chest. - Diseases of lungs and heart, especially in flat or narrow or malformed chest. In examining the heart, care must be taken not to ascribe to disease the hurried, sharply accentuated action sometimes due to nervousness, fright or embarrassment, or the irregular action caused by the excessive use of tobacco. Nor should the examiner attach undue importance to the soft systolic murmurs often heard in growing athletic youths, functional and temporary in their nature.

De Loffre, in his examination of applicants for enlistment at Columbus Barracks (Mil. Surg., Sept., I910), found that, out of 
1,000 , just 100 suffered from affections of the heart, namely, 18.64 with organic cases (rejected) and 8I.37 with functional cases (accepted). Of the organic cases, the ratios of valvular disease were: mitral regurgitation 12.28, aortic regurgitation 2.09, mitral stenosis 1.23, and tricuspid regurgitation 0.57 . Of the functional cases, the valvular exhibited murmurs, in order of frequency, at the pulmonary, aortic and mitral valves, while the neurotic were marked by arrhythmia, tachycardia and irritability. A simple murmur at a valve was called functional unless hypertrophy, dilatation or other physical signs were present. Osler considers that " with an apex beat in the normal situation and regular in rhythm, the auscultatory phenomena may be practically disregarded." Simple murmurs without the presence of other physical signs; especially those referable to the mitral valve, may often be overlooked, but not until the effect of sharp exercise has been observed.

Abdomen. - Chronic inflammations of the gastro-intestinal tract, including diarrhea and dysentery, and other diseases of contained organs; hernia in all situations.

The experience of the medical examiners at Fort Slocum has led them to believe that indirect inguinal hernia among enlisted men is, with rare exceptions, always congenital.

Anus. - Hemorroids, prolapsus, fistula and fissures.

Genito-urinary organs. - Syphilis in all its stages; venereal sores (both. chancre and chancroid) and gonorrhea, whether acute or chronic; urethral stricture, balanitis, phimosis, undescended testicle, orchitis, hydrocele, incontinence of urine; all diseases of the bladder and kidneys. Varicocele " does not constitute a cause of rejection unless it is either painful or so large as to interfere with locomotion;" it frequently occurs among the most robust men and often without their being aware of its existence.

Latent syphilis has been shown, by the Wasserman test, to be so much more prevalent than was formerly believed that the examiners of applicants for enlistment at Fort Slocum (Mil. Surg., June. I9II) consider as syphilitic a large proportion of applicants with perforated nasal septum, slight general glandular enlargement, thickening of radial arteries, recurrent superficial ulcerative keratitis, or marked follicular conjunctivitis (trachoma).

Affections common to both e.tremities. - Chronic rheumatism, diseases of joints, irreducible dislocations or false joints, old dislo- 
cations if attended with impairment of motion or distortion of the joint, severe sprains, synovitis, badly united fractures, caries, necrosis, atrophy or paralysis, extensive or adherent scars, permanent contraction of muscles.

Hands. - Webbed fingers, permanent flexion, extension or loss of motion of one or more fingers; loss or serious mutilation of either thumb, total loss of index finger of the right hand, total loss of any two fingers of the same hand, or loss of the second and third phalanges of all the fingers of either hand.

Lower E.rtremities. - Varicose veins, especially when attended with edema or marks of ulceration, knock-knees, club feet, flat feet, webbed toes, bunions, overriding or marked displacement or deformity of any of the toes, hammer toes, ingrowing nail, corns on the soles of the feet, or soft corns between the toes.

The shin-bone, if rough, nodulated and tender, suggests syphilis.

A broad, flat sole is common in laboring classes, particularly among negroes, and is in no way disabling. In the flat foot which renders a man unfit for service, the arch is so far gone that the entire inner border rests upon the ground, with the inner ankle lowered and very prominent, and the foot apparently pushed outward. Flat feet are not infrequently the result of a tuberculous process.

\section{IDENTIFICATION RECORD.}

A personal identification record consisting of all body marks indicated on an outline figure on his identification card, of finger prints and photographs, forms also part of the examination of the recruit, under orders issued from time to time by the War Department.

This record is very important as it affords sure means of identifying him should he at any time during his subsequent service be found dead or unconscions. It is also a perfect method to identify deserters or men fraudulently reenlisting under assumed names.

\section{Vaccination Against Small-pox and Typhoid Fever.}

After enlistment the recruit is vaccinated on the left arm and given his first typhoid prophylactic on the right. 


\section{CHAPTER XVII.}

\section{EXERCISE.}

"We march with our muscles, run with our lungs, gallop with our heart, resist with our stomach and succeed zeith our brain." (Dr. Phillippe Tissié.)

Exercise should not be regarded merely as an amusement or recreation, but also as an obligation, inasmuch as it is indispensable for the maintenance of body and mind in a healthy condition and the proper performance of all bodily functions. Correctly applied, it not only maintains health, but corrects physical defects, supplies deficiencies by strengthening and developing our organs and rendering them capable of greater and more persistent effort. It is therefore especially needed by the recruit in order to train him into a strong, agile and enduring soldier and obtain from him a maximum of useful work.

\section{PHYSIOLOGY OF EXERCISE.}

Exercise is generally understood to mean the more or less active contraction of voluntary muscles.

We know that our nervous energy and consequent capacity for mental and physical work depend upon active cell metabolism, that is, constant and rapid renovation of tissues. The most vital factor in this work of nutrition, assimilation, dissimilation and elimination, whereby heat and energy are evolved, is oxygen. Unless the blood be thoroughly oxygenated, all functions suffer. An abundant supply of oxygen to the tissues is the great end of exercise.

When voluntary muscles are set in motion, they require more blood, especially more oxygen. If this motion be active and continued, or becomes violent, the need of oxygen is so much greater that the heart is stimulated to quicker action in order to furnish a more rapid flow of blood to the contracting fibres; hence the increased frequency of pulse in exercise. But since the blood obtains its oxygen from the lungs, it follows that, simultaneously with increased pulse, there must be increased respiration, increased first in depth and then also in frequency. Heart and lungs, then, are func- 
tionally, as well as structurally, very intimately connected, and any stimulation or disturbance of the one is necessarily felt by the other.

At the beginning of active exercise more venous blood is returned to the heart; the right ventricle labors to empty this increased amount into the resistant lungs. There is, in this first stage, a rise of blood pressure, indicated by the labored heart-beats and more or less breathlessness. But soon, with the increase of body temperature, the resistance lessens; the lungs expand, the peripheral vessels dilate, sweat breaks out and the blood pressure falls; the heart pulls itself together, adjusts itself to the respiration and the so-called "second wind" is established.

The muscles are the main furnace of the body and the principal source of our body heat. When at work they are supplied with 4 or 5 times as much blood as when at rest, consume 20 times as much oxygen and discharge 25 times as much carbonic acid (Cheveau \& Arloing). Their contractions, therefore, must exert a profound influence on all the organs and functions of the body. As a general rule, the absorption of oxygen and discharge of $\mathrm{CO}_{2}$ proceed pari passu, so that the latter gas, as stated by Macfie, may be considered a measure of the intensity of the fire of life. The greater the muscular activity the greater the respiratory exchange, that is, the intake of oxygen and discharge of $\mathrm{CO}_{2}$. Thus E. Smith found that a man discharged 162 c. c. of $\mathrm{CO}_{2}$ per minute when asleep, and 84I when walking three miles an hour, or more than 5 times as much. This respiratory exchange is the true measure of vital capacity, a term which should be applied not to the capacity of the lungs, but to the respiratory capacity of the tissues. The heat generated in the muscles is so efficiently distributed by the rapid circulation of the blood that the temperature of the body is seldom raised nore than a degree or two. Through this increase of temperature, the hemoglobin of the blood yields oxygen more abundantly so that a more active metabolism of the tissues is rendered possible. Physiologists consider a temperature of $102^{\circ} \mathrm{F}$. (in rectum) as the highest that can be produced by exercise without abnormal effects; above that point, an irregular, inefficient pulse is likely to result.

The main factor regulating respiratory movements, as well as the cardiac beats, has been shown by Yandell Henderson to be the pressure of the $\mathrm{CO}_{2}$ in the blood, as gauged by its pressure in the pul- 
monary alveoli. During exercise more $\mathrm{CO}_{2}$ is produced, the greater its pressure in the blood and it $\dot{s}$ stimulating effect upon the respiratory center, causing increased breathing in rate and depth. This results in greater ventilation of the alveoli and the prevention of a dangerous accumulation of $\mathrm{CO}_{2}$ in the blood. Thus rapid, deep breathing, for about half a minute, produces a condition of apncea, in which there is no inclination to breathe, owing to the washing out of the $\mathrm{CO}_{2}$ from lungs and blood. In this condition, the composition of the alveolar air will show only 2 or 3 volumes per cent. of $\mathrm{CO}_{2}$ and 19 or 20 of oxygen, instead of the usual 5 to 6 volumes of $\mathrm{CO}_{2}$ and $\mathrm{I}_{3}$ or $\mathrm{I}_{4}$ of oxygen. Henderson found that even a slight reduction of $\mathrm{CO}_{2}$ in the arterial blood causes a marked quickening of pulse, and that a further reduction may cause extreme tachycardia. Increased tension of $\mathrm{CO}_{2}$, on the contrary, slows the action of the heart and increases the ventricular output.

The dyspncea of violent muscular exercise is doubtless the result of the accumulation of $\mathrm{CO}_{2}$ in the blood when not adequately relieved by the ventilation of the lungs. Contributory causes are the presence of other waste products, especially lactic acid, and the inability of the heart to respond to the higher demand made upon it. When, from strenuous muscular effort, the pulse and respiration have doubled in frequency, it will be noticed that, after a rest of a few minutes, when the dyspnca is all over and respiration has returned to its ordinary rhythm, the pulse rate continues much above the normal; whence we may conclude that, since the heart does not recover its normal action until long after the disappearance of dyspnoa, it cannot be the main agent in its production.

The principal non-nitrogenous extractives in muscles are glycogen, dextrin, sugars and lactic acid. The amount of glycogen varies in different muscles, its rate descreasing in proportion to their activity. It is the form in which carbohydrate is stored up. By muscular work it becomes transformed into dextrin, maltose and glucose before being oxidized. After prolonged activity glycogen may disappear from the muscles, but not until it has disappeared from the liver, which, therefore, must be a supplying organ.

The lactic acid of muscle (often called sarcolactic acid) differs from that obtained by the fermentation of lactose, by rotating the plane of polarized light to the right, and is probably derived from the glucose of muscle. It is always present in the urine and appears 
to be a normal constituent of the blood. Violent exercise causes it to be present in muscles and to be actively excreted by the kidneys; it, likewise, increases the amount of it in the blood, the alkalinity of which is thus reduced. Moderate exercise, such as walking, even if continued for hours, does not increase lactic acid in the urine, while a marked increase will follow a run of 2 or 3 minutes, if sufficiently vigorous to produce dyspnœa. It appears to be the result of imperfect oxidation; the oxygen, although increased during hard muscular work, not being supplied in sufficient quantity to prevent its formation. As soon as the period of violent activity ceases, lactic acid is quickly oxidized, so that, in less than an hour afterward, the tissues have returned to their normal state. A slight increase would paralyze the nerve-endings and poison the muscles of the cardio-vascular system were it not thus promptly destroyed before accumulating in sufficient quantity to produce its toxic effects. Burridge suggests that each muscle contains within itself enough creatin to neutralize, by its change to creatinin, the amount of lactic acid it may produce.

This increased formation of lactic acid in strenuons exercise is said to serve a very useful purpose in rendering the blood more acid and thus stimulating the respiratory center and the heart. Inasmuch as the alveolar carbon dioxid soon falls to normal or even below normal after violent exercise, it is held that increased respiration cannot be due to carbon dioxid alone (J.H. Ryffel).

According to Bohr, lactic acid has, like $\mathrm{CO}_{2}$, the remarkable effect of reducing the affinity of the blood for oxygen, thus becoming a valuable accessory in tissue respiration; it is especially when the oxygen reaches the capillaries at a low tension, under stress of exhausting exercise, that it exerts this beneficial power of turning out the oxygen from the hemoglobin of the blood.

The gentle or moderate exercise of a few muscles, for instance, those of the upper extremity, may not produce any appreciable fatigue, or, if prolonged. may produce only local fatigue without respiratory difficulty; but in all violent muscular effort there is always a corresponding disturbance of heart and lungs, a disturbance which is in direct ratio to the sum total of work done, and therefore not necessarily proportional to the degree of fatigue felt; thus, as mentioned above, a man may be tired with hardly any increase of pulse or breathing, while, on the other hand, he may run up-stairs and get out of breath without muscular fatigue. 
It is readily understood that, whenever the heart is spurred on to more vigorous effort, not only the working muscles are benefited but, through the greater velocity of the more highly oxygenated blood, all the organs and tissues of the body receive an increased share of oxygen and other nutritive principles; therefore the man who walks not only exercises his lower extremities but his brain and liver as well.

There is no doubt that thinking and mental work are good exercise for the brain and necessary to a normal and complete development of the mind; it is also common experience that muscular exercise, by insuring an active metabolism of the brain tissue, is necessary for our best mental work; but body and mind can seldom be vigorously employed at the same time or in quick succession successfully. Mosso has proved conclusively that mental exertion produces not only psychical weariness but also a distinct loss of muscle power. The onset of fatigue is much more rapid after severe mental strain; conversely, mental application is much more difficult after severe muscular exercise. It is a great mistake to imagine that the brain does not share the fatigue of the body and that a physically exhausted man can think as clearly and successfully as when in a state of rest.

It has not yet been conclusively shown that mental effort affects in any appreciable manner the metabolism of nitrogen, phosphorus and silphur, so far as determined from the urine. The pulse rate, body temperature and production of $\mathrm{CO}_{2}$ may be increased, but such increase is so slight and so often absent that one cannot assert that mental effort has a positive influence on metabolic activity (Benedict and Carpenter). Mosso's belief that more blood flows to the brain in mental work so that the head becomes heavier, has not been confirmed. On the contrary, the experiments of E. Weber (endorsed by Leonard Hill) show that mental work causes a determination of blood not to the brain but to the abdomen; that pleasurable ideas and feelings send the blood from the abdomen into the peripheral parts, and that during muscular exercise the venous cistern in the abdomen supplies more blood to the limbs.

The influence of exercise upon metabolism and excretions is obvious. As already stated, the intake of oxygen and output of $\mathrm{CO}_{2}$ are immediately raised and increased in proportion to the work done, the necessary energy resulting chiefly from the combustion of 
carbohydrates and fats. The nitrogenous excretion is not sensibly affected, being dependent upon the intake of nitrogenous food. The phosphates in the urine are increased. Albuminuria is found in all cases of vigorous exercise, even after short runs, and increases in proportion to the extent and duration of subnormal blood pressure. It has been observed that the best athletes, that is, those capable of greatest exertion, always pass albumen in their urine after contests in sports, such as rowing, running and football. This may be due to the coincident feeble blood pressure, and the clogging of the kidneys from an unusual increase of waste products.

Forms of exercise. - Exercises have been divided into exercises of strength, exercises of speed and exercises of endurance. We might add a fourth category, namely, exercises of skill. In all games and sports the characteristics of these several classes of exercises are more or less combined.

Exercises of strength, such as wrestling, lifting weights, tug of war, etc., demand the simultaneous, sustained action and whole force of many muscles. In order that these muscles may take a very firm attachment, it is necessary that the chest be filled with air and all the bones of the trunk strongly fixed, with glottis closed. This fixation of the trunk requires will-power, a special effort. The highest blood pressure is produced by a prolonged straining effort, as in lifting, the contraction of the muscles partly closing the lumen of blood-vessels and increasing the peripheral resistance; but this is soon overcome and followed by an abundant and continuous flow of blood into the muscles, producing all the conditions necessary for energetic tissue repair. Exercises of strength need very little work of co-ordination, or repetition of movement, occasion but little nervous disturbance and do not demand great brain work; in other words, "they increase energetically, and even violently, the working" of all the organs of the body, while leaving in relative repose the nerve centers and psychical faculties." *

Exercises of speed are those which require frequent repetition of movement; the muscles are not called on to act with their utmost energy, but to contwast and relax a great many times and at very short intervals, the result being the same amount of mechanical work performed and the same increased activity of the respiratory and cardiac functions as in exercises of strength; but experiments have

* Physiology of Bodily Exercises. Lagrange. 
shown that muscles subjected to small, frequently repeated contractions, receive less blood than during one long-sustained contraction; therefore the nutrition and development of muscles is much less marked in exercises of speed than in exercises of strength; it is noted that professional runners have trim legs and comparatively small calves. Furthermore, Lagrange also calls attention to the excessive expenditure of nervous energy and certain phenomena of exhaustion produced by exercises of speed, out of proportion to the quantity of mechanical work performed; a state of nervous excitability which prevents repose and sleep, defective nutrition and repair, and, sometimes, great loss of weight.

Exercises of endurance are those in which the muscular effort is moderate and the movements not too rapid, but in which the work is continued for a long time. The duration is subordinate to the power of the lungs and heart, and the intensity of the nervous energy which actuates them. Walking is the type of exercises of endurance, but when performed up a steep slope may become an exercise of strength. Rowing over a short course is mostly a work of speed, but, in a long race, becomes also a work of endurance. In these exercises all the functions are stimulated but in a milder way and without danger of violence to any organ. A serious objection is that they do not excite the respiratory movements with sufficient strength to expand the air-cells and increase the capacity of the chest. They are also rather tedious and irksome from the monotony of the same movements long continued. For the soldier, marching is the most important of requirements and he should be diligently exercised in it until the proper degree of endurance has been attained.

In exercises of skill, the psychical faculties are more severely taxed than in any other kind; they require speed, repetition and accuracy of movement, and special training of certain sets of muscles. Their effects are mainly those of exercises of speed, but as moments of rest are more frequent and the strain is less continuous, they seldom give rise to the utter prostration which occurs, for instance, in running and rowing races.

Fatigue may result from the active use and consequent exhaustion of the nerve centers, or from muscular exertion, or from a combination of both causes. The part played by the psychic factor in producing fatigue should not be overlooked; thus great mental 
worry is often more exhausting than strenuous physical exercise, and the exercise which requires thought and attention is much more fatiguing than mere mechanical work. But if nervous impressions may greatly aggravate physical fatigue, they are also capable of greatly alleviating it, as, for instance, the stimulating effect of a lively tune upon tired troops, and of the sight of camp after a weary march.

On the other hand, muscular fatigue has well marked depressing effects upon the various faculties of the mind: memory fails; names of things and places are temporarily forgotten; the will power weakens and may be unable to control unreasonable impulses whereby men, for instance, straggle away from marching columns or are seized with panicky fear; discipline is relaxed and the morale loosened. Hence the danger of engaging in battle with exhausted troops.

Fatigue acts as a sedative upon sexual instincts, but creates a desire for alcoholic drinks.

In physical fatigue, physiologists tell us, the stimuli sent out from the central nervous system are blocked in the nerve-endings. The causes of this blocking are not yet clearly known. It is generally attributed to lack of oxygen and the accumulation of waste products such as carbon dioxid and lactic acid in the blood. Masso has demonstrated that the blood of a fatigued animal produces fatigue in another animal into which it is injected. It has also been shown that during hard work the sweat becomes toxic, and that the toxicity of the urine is increased. According to Burridge ( $J$. of Physiol., I910), the conditions present in a muscle working at a fairly high rate, are the continued liberation of potassium and production of lactic acid, together with lack of oxygen and increased tension of carbon dioxid. In his opinion, the early stage of general fatigue is due to the increase of potassium salts in the circulation; this corresponds to the normal physiological fatigue from which the system rapidly recovers without after effect. The excessive fatigue of overworked muscles corresponds to the action of lactic acid; should this acid be present in the free state beyond the merest traces, stiffness of muscle results; in this stage, the condition of the muscle is more an inability to relax than to contract, being then particularly liable to contracture and cramp.

Experiments made to determine the effect of oxygen inhalations 
on muscular exertion seem to show that this gas, thus used, is beneficial; that if inhaled before strenuous exercise, it facilitates the performance of the latter, and that if inhaled after exercise it diminishes the existing fatigue and distress. Hill and Flack,found that it allows a man to stand a much lower, as well as a higher tension of $\mathrm{CO}_{2}$ than normal ; that quiet breathing of oxygen, before exertion, gives the best results, causing the atlulete to excel by enabling him to stand a higher tension of $\mathrm{CO}_{2}$ The sprinter who would otherwise suffer from a temporary asphyxia benefits from a full initial supply of oxygen, and the long distance runner in whom oxygenation does not keep pace with the demand of the muscles is likewise benefited by oxygen inhalation. It is also possible that oxygen thus introduced in the blood may play a part in the rapid elimination of lactic acid.

On the other hand, Cook and Pembrey contend that the amount of oxygen in the alveolar air is so little affected by muscular exercise, that its administration is of value only when the conditions are pathologic.

\section{EFFECT OF EXERCISE ON MUSCLES.}

The voluntary muscles make up the bulk of the body. They consist of fibers which, through the nerves, contract and relax in obedience to the will, thus acting upon the bones and producing the various movements of the body. Exercise, that is, the frequent contraction and extension of the muscle, especially against resistance, at first produces (especially in fleshy and stout men) a reduction in the size of it, as well as in the total weight of the body, due to the melting away of all superfluous fat: but after two or three weeks, the muscles begin to increase in size, hardness, power and endurance. The increase in size is due to the more active growth of the individual fibers and not to any multiplication of their number. Systematic exercise also develops a quicker command and more perfect control of all our muscles so that complex movements and delicate manipulations requiring the simultaneous play of many muscles become easier and less fatiguing. Hence the alertness of the trained soldier, that is, his power to respond quickly to the perceptions of the senses and execute promptly the commands of his officers.

It is obvious that soldiers do not require great strength and therefore large muscles. What they need is suppleness, skill and rapidity 
of movements, as well as great resistance to fatigue and disease. To that end they must have sound lungs and heart, and well trained, sufficiently developed but not excessive muscles. It is very likely that the process whereby a muscle becomes physiologically hypertrophied, and afterward (when the training is discontinued) again reduced to its normal size, is accompanied by a loss of tonicity. The endurance of a muscular fiber depends much more upon this tonicity than upon its size, and the soldier with medium but tense and firm muscles, quickly responding to the stimuli of the nervous centers, is the one who will march furthest, carry his equipment with least fatigue and handle his rifle most efficiently. The nimble, hardtrained medium-sized soldier will always (other things being equal) circumvent and overcome a much heavier and stronger but slower adversary. To obtain the optimum tonicity of muscular fiber without material hypertrophy is the problem, and this is solved by a proper combination of systematic gymnastic training with exercises of speed and endurance, and sane athletic sports.

Involuntary muscles are also strongly affected by exercise, their health and the activity of their physiological processes depending greatly upon the work of the voluntary muscles.

\section{EFFECT OF EXERCISE ON THE HEART.}

As stated before, the heart is concerned in any form of exercise. Nore blood has to be sent to the working muscles and the heart must supply it by increased contractions, so that its fibers, like those of other muscles, undergo a more rapid oxidation and renovation. The result is increase in size and power, as well as in frequency of beat. The ventricular walls become thicker and stronger, especially on the left side, and the whole organ enlarged and strengthened. This strengthening, with or without enlargement, is a normal physiological process and must be one of the most important aims of exercise, for physical power necessarily depends upon vigor of heart.

Another ordinary and normal effect of strenuous athletics is temporary dilatation of the right side of the heart, due to the passive congestion of the lungs and increased intracardiac pressure existing in the primary stage of muscular effort. To this dilatation are chiefly due the increased size of the heart during and immediately after a hard contested game, and various murmurs often heard at that time over the cardiac region. 
The accommodating power of the heart is enormous, and so long as the strain upon it is not excessive and prolonged, it recovers itself without ill effect. Thus the pulse may rise to 160 , I75, even to 200 , without permanent injury, provided the heart be given time to recuperate. Even a dicrotic pulse following strentous exertion is not rare and need not always be considered a dangerous sign. But, unless the training has been carefully graded and the muscular effort is always kept within physiological limits, there is constant risk, in violent exercise, of incurring the dangers of overstrain.

In young healthy soldiers and students, even when not engaged in active athletics, functional and temporary murmurs over the precordial region are common and their significance must not be exaggerated. They are often the result of training, worry and excitcment, and disappear as the subject grows stronger or returns to quieter conditions. These murmurs are almost always systolic (with first sound or immediately after), soft, blowing in character and heard with greater frequency at the base of the heart, although likewise common at the apex. Perhaps the greatest number are heard over the pulmonary area, in the second left interspace, close to the sternum. They have been ascribed to dilatation of the pulmonary artery (conus arteriosus), and to leakage of the mitral and aortic orifices due to temporary dilatation of the heart and unequal tension of the valve leaflets. Unless these murmurs are accompanied by cardiac enlargement, venous engorgement, dyspnoea or disease of some other organ, they cannot be regarded as indicative of organic heart disease.

Dr. R. Tait McKenzie, professor of physical education in the University of Pennsylvania, found, after exercise, 74 murmurs out of 266 young men in apparently good health, and therefore contends that this presence of murmurs in 28 per cent. of normal young men, even on slight exertion, should lead to caution in determining their significance after severe strain or fatigue.

Oswald S. Lowsley (Am. J. of Plyisiol., I9I I), has shown the effects of all forms of exercise upon blood pressure. Any exercise sufficiently active to cause an increase of pulse rate also causes a rise in systolic and diastolic pressures, the former being greater than the latter, so that it is evident that the heart beats are strengthened as well as accelerated. As soon as fatigue sets in, the pressure falls to normal and, if the exercise be continued, invariably falls below 
normal, the systolic more rapidly than the diastolic, and remains in this stubnormal stage a period of time proportionate to the strenuous nature of the exercise. The pulse, at first strong, becomes weaker as the pressure falls; its rate which always increases during exercise, decreases rapidly after it but seldom falls below normal. The subnormal stage of blood pressure which follows short but violent and exhausting exercise, lasts much longer than the same stage following prolonged but moderate exercise, an indication of more serious strain. Speed exercises such as basket ball, football and rumning races are particularly liable to overstrain. There is less strain put upon the circulatory system by walking a number of miles at a moderate rate than by sprinting a hundred yards at top speed; in the former case the depressed blood pressure returns to normal in about 30 minutes, while in the latter case it may take three or four times as long. Lowsley proposes to determine the beneficial or injurious effect of exercise by the extent and duration of the subnormal phase following it. When the pressure returns to normal within 60 minutes, the exercise may be considered as lying well within hygienic limits, while a return to normal delayed beyond 2 hours may be regarded as exceeding these limits. In speed or other strenuous exercises, the systolic pressure rises about $40 \mathrm{~mm}$., and the diastoilc $25 \mathrm{~mm}$., above normal, while the pulse rate is increased from 65 to So beats per minute; this is followed by a systolic drop of $\mathrm{I}_{5}$ to $20 \mathrm{~mm}$. below normal, the pressure returning to normal in from two to three hours, but sometimes not until four to six hours or even longer. It is noteworthy that even moderately active exercise, such as is indulged in for recreation, is invariably followed by a decided drop to subnormal.

\section{EFFECT OF EXERCISE ON THE LUNGS.}

The end of exercise being a free supply of oxygen to the tissues, it follows that the organs which supply this oxygen must be adequately developed and equal to the demands made upon them. Therefore it is impossible to expect stout arms, vigorous legs and strong heart except with an ample chest capable of large expansion. During exercise there is a largely increased absorption of oxygen and a correspondingly larger production and elimination of carbon dioxid, aqueous vapor and other waste matters; therefore the respiratory function is greatly stimulated. Thus a man marching fast 
will inhale five times as mucl air as if reclining, at rest. This means that instead of breathing 16 times a minute and inhaling 30 cubic inches of air at each inspiration, or a total of 480 inches a minute, he will breathe so mucl more frequently and deeply as to inhale 2,400 cubic inches. The amount of oxygen absorbed during an average working day is about three times greater than during a day of inaction. During severe labor, the consumption of oxygen and excretion of carbon dioxid and aqueous vapor are frequently in. creased from 7 to 10 times. Nothing therefore should interfere with the free play of the lungs during work or exercise, neither tight clothing nor badly suspended or ill-distributed equipment.

It is very important that, in all exercises, so far as possible, the breathing be through the nose. The nostrils are cliefly for the purpose of warming, moistening and purifying the air. Thus air at $14^{\circ} \mathrm{F}$., in passing through them, is warmed to $77^{\circ}$ by the time it reaches the throat. Their devious, convoluted course and extensive mucous surfaces, to which most of the organisms and foreign particles in the inspired air adhere, render the access of germs and dust to the lungs very difficult. But in severe exercise, as in games and races, it is impossible to inhale enough air through the nose, and the mouth must be kept open. Under such conditions, mouth-breathing can be done with impunity or even with advantage, for the body temperature is then more or less above normal, and colder air taken into the lungs tends to cool the body and restore the equilibrium.

Few soldiers know how to expand their chests properly so as to fill up all the air vesicles and take the fullest advantage of their respiratory capacity. They must be taught the proper use of all the respiratory muscles, including the diaphragm. The usual tendency is to expand chiefly the upper part of the lungs, raising the clavicles and shoulders, but complete respiration must be abdominal as well as thoracic. While the ribs and sternum are drawn outward, the diaphragm should be correspondingly depressed, so that the lower lobe be as fully expanded as the upper lobe. The shoulders must not be perceptibly raised. The Drill Regulations prescribe that the lungs should be inflated to full capacity by short successive inhalations through the nose, and emptied by a continuous exhalation through the mouth. The rhythm should be as nearly normal as possible, that is, the inspiration followed withont delay by the expiration; holding the breath with a fully expanded chest is harm$\mathrm{ful}$ to heart, lungs and abdominal muscles and should be avoided. 
In taking a long breath the downward movement of the diaphragm is facilitated by contracting the abdominal muscles and depressing the shoulders. According to Thooris, the strong contraction of the abdominal muscles not only permits a greater lowering of the diaphragm, but also increases the amplitude of the chest at all its levels, while preventing too rapid an inflation of the lungs. The broad belt often worn by gymnasts is very useful in this connection as an abdominal support. Mobility, more than the size of the chest, is the test of efficient respiration, but it must be entirely due to the movements of the thoracic walls and not, in part, to the forced contraction and relaxation of the muscles of the chest and shoulders. A true mobility of 3 or 4 inches is abundantly capable of supplying all the oxygen the blood can possibly absorb, and there is more to lose than to gain in attempting to increase it. Furthermore, as shown by IIutchinson, there is no accurate relationship between the expansion of the chest and the amount of air inspired, for the thorax may be uselessly expanded beyond the extensibility of the lungs, in which case the abdominal viscera are drawn upward to occupy the vacant thoracic space.

The expansion of the lungs is best promoted by exercise, especially marching and athletic sports, that is, all muscular efforts which increase the rate and depth of breathing. The mere practice of deep breathing does not affect the metabolism of tissues, and therefore does not generate heat nor energy, but, nevertheless, is useful in maintaining and increasing the mobility of the chest wall and the power of the respiratory muscles, so that when the tissues themselves require extra oxygen, the lungs may be fully capable to obtain it. Deep breathing, besides, assists the circulation of the blood and lymph through the thorax and, by the play of the diaphragm, massages the liver and other abdominal organs.

Under proper military training an increase of chest measurement and mobility are soon noticed. The average increase in size, at the end of the first year, for French recruits, is about an inch (Abel, Chassagne and Dally'), and that in mobility, according to Fetzer, very little less (2I $\mathrm{mm}$.). The vital capacity, in the experiments of Fetzer, rose from 3 lit. 800 to 4 lit. 500; Dettling has seen it reach 5 and even 6 liters at the school of Joinville.

The classes which graduated at the United States Military Academy in 1900, I90I, I903, I904 (346 cadets in all), gave a measure- 
ment of 35.48 inches at expiration and 37.78 inches at inspiration, with mobility of 2.30 inches. At the time of entrance, four years before, the measurements had been 33.72 and 35.90 , respectively, with 2.18 mobility. Therefore the increase during their stay at the Academy, as the result of natural growth, drill, gymnastics and athletics, was 1.76 inches at expiration and 1.88 at inspiration, the mobility meanwhile having only increased o.12.

The class which graduated in 1910 gave measurements of 33.7 and 37 , respectively; at the time of entrance, in 1906 , they had been 31.75 and 34 , and, in $1907,32.60$ and 36 ; therefore the total increase at expiration was 2 inches, at inspiration 3 inches, and in mobility I inch.

For the period 1906-1910, the average increase of the fourth classmen, from October to June, was I.32 at expiration, I.52 at inspiration and 0.20 in mobility, corresponding to an increase in lung capacity of 16.34 cubic inches.

The variations in the above figures show the difficulty of comparing lung measurements with a view to formulating definite conclusions, unless made by the same person and method.

It has also been observed that the effect of exercise is to reduce the number of respirations and increase their depth. Thus Marey has seen the number fall from 20 to 12 a minute while the amplitude was almost quadrupled.

\section{EFFECT OF EXERCISE ON THE SKIN.}

Exercise causes an increased supply of blood to the surface of the body, dilating the peripheral capillaries and reddening the skin; at the same time the sweat glands are stimulated and pour out an abundant excretion. From the more active oxidation and combustion of the tissues produced by exertion, the body temperature is increased, and this increase would soon cause serious injury to the system were not means provided to check it. These are the direct radiation of heat from the congested skin and the evaporation of the sweat, so that an equilibrium is maintained and the body temperature seldom rises more than $\mathrm{I}^{\circ} \mathrm{F}$. above normal. As sweat continues to be excreted and evaporated after the exercise ceases, the temperature soon falls to normal, or even below, and a chilly sensation results if the skin is not properly protected; hence the need of a sweater or woolen garment after active work. Under the effect of 
strenuous and sustained exertion, during a game or race, it is not unusual to see the temperature fall one degree or even more below normal, without subsequent ill effects, provided the young athlete is at once wrapped up in a blanket and allowed to recuperate.

\section{EFFECT OF EXERCISE ON HEIGHT AND WEIGHT.}

Height is sensibly increased by exercise in young men under 20 , especially under $\mathrm{I} 8$, but it is doubtful whether that of young soldiers (2I years old or over) is perceptibly affected.

The average increase of height of the four classes which graduated at the United States Military Academy in I900, I9OI, I9O3 and 1904, during the four or more years of their stay at the Academy, was 0.97 inch (from 67.85 inches to 68.82 inches). Their average age at entrance was slightly over 19. For the class of I9I0 the increase was only 0.70 (from 68.66 to 69.36 ). The greatest rate of increase is during the first year, the period of compulsory gymnastics, amounting to 0.40 inch as the average of the fourth classmen for the five years I906-igIo. The rate of increase thereafter, to the time of graduation, is much less marked; thus, for the I9ro class ( 83 members), the height was 68.66 in $1906,68.89$ in 1907 and 69.36 in 19Io. After graduation (at average age of 23 years), the increase in height, if any at all, is a negligible quantity.

Weight is quickly and markedly affected by exercise. In the first stage there is almost always a temporary loss ranging from a few ounces to a few pounds, but this is readily made up in a few days by a more active digestion and greater absorption of food. This loss is due not only to the oxidation of fat and glycogen (or its product, alcohol), but also to an increase of all the excretions, especially sweat; it may continue or increase if violent exercise is persisted in, without sufficient recuperation, and then becomes one of the signs of overtraining. The permanent gain in weight, the net result of exercise, varies according to the physical condition of the stubject, being much greater in small and immature than in large, well-developed men. This probably explains the striking results obtained in the training of recruits in certain European countries, as compared with our own results. Dettling found that after three months' training at the gymnasiun, the young soldiers of the Joinville School had gained 2.425 kilos, or 5.33 pounds. Similar figures are recorded by other German and French military gymnastic 
schools. According to Onslow the average increase in British soldiers who completed the full course of training, in 1887 , was $2 \frac{1}{2}$ pounds. In this country, Butts determined that after a course of instruction at Columbus Barracks extending over a period of $3 \frac{1 / 2}{2}$ months, the average gain was $2.8 \mathrm{I}$ pounds. At Harvard, the increase of weight, based upon a class of 200 men averaging 18.3 years, after 6 months of moderate work in the gymnasium, was 2 pounds.

At the United States Military Academy, for the class of 1910, the total average increase in weight, as the result of natural growth, drill, gymnastics and athletics, during the four or more years of their stay at the Academy, was Io pounds (from I39 to I49). The greatest rate of increase is during the first year (October to June), the time of active gymnastics, amounting to 6.29 pounds ( I 41.60 to I47.89) as the average of the fourth classmen for the period 1906 to I9Io. Thereafter the increase, to the time of graduation, is much less; thus, for the I9ro class, the weight was I 39 pounds at entrance (in 1906), I46.25 the following June, and only I 49 in I9Io.

The increase in weight produced by exercise is almost exclusively in the muscles, which, in consequence, become harder and larger, so that there is a gain in the size of all parts of the body, including chest, shoulders, hips, upper and lower extremities. Thus, at the U. S. Military Academy, the muscular development of the arms during the first year, as averaged for the period I906-1910, was as follows: the right and left arms measured 9.68 and 9.54 inches, respectively, in October, and 10.59 and 10.16 in June. The only exception is in the waist and abdomen which become reduced in size, much of the fat stored in these situations being oxidized. Thus Butts found that the average loss in the waist of recruits was about one inch. 


\section{EXERCISE (CONTINUED).}

\section{REGULATION OF EXERCISE.}

Young soldiers not accustomed to systematic training have but little endurance and are soon tired out. Therefore the exercises should be easy and short at first, gradually increasing in duration and intensity, but ceasing as soon as great fatigue is noticed. As the training of the recruit progresses, his muscles become stronger and his joints more supple, his movements are better coördinated, almost automatic; he feels fatigue less and less and becomes capable of stremuous and long-sustained efforts without losing breath and without exhaustion. It is only when thus perfectly trained that he is equal to the physical demands which are made upon him in camps of maneuvers, in the field, on the march or on the battlefield.

The rules which should guide instructors in drilling recruits may be formulated as follows:

I. The exercises should be progressive, each day a little harder than the previous day, but without fatigue, avoiding violent, sudden efforts for which the recruit is not yet prepared.

2. Each man should be examined with a view to his special needs and deficiencies, and placed in the section or squad where he may receive the training most appropriate to his condition.

3. Agility for the soldier is as important as strength and should be carefully developed, particularly in awkward recruits with big, strong muscles. This is done by light but continued exercises especially directed to the suppling of joints, and calling for short, quick movements.

4. Drills should take place in the open air whenever possible, or else in large, well-ventilated rooms. The maximum time devoted to them should not exceed 5 hours a day, namely, 3 hours in the morning and 2 in the afternoon, with a sufficient number of halts, during which the men should be allowed to stand at ease, sit or lie down.

5. As a rule, the soldier should not be exercised before breakfast, or such exercise should be short, not exceeding half an hour. The 
best time for drill is between meals, that is, beginning about an hour after meal and stopping soon enough to allow at least half an hour's rest before the next meal. When exercise immediately follows eating, the blood which is indispensable to the proper work of the stomach is diverted to the muscles and severe indigestion may result. It is necessary to watch over the digestion and to bear in mind that any system of training which impairs the appetite is harmful. The use of alcolnolic drinks, or other stimulants or tonics, before or during exercise, should be strictly prohibited.

6. The action of the heart and lungs must be closely watched, and any exercise likely to bring about pulmonary congestion or difficulty in breathing avoided. No exercise should be so strenuous as to produce severe dyspnœa, while the pulse, however quick it may be, should remain regular and of fairly good strength.

7. Drills must be made as varied and interesting as possible. A long continued mechanical repetition of the same movements soon tires the recruit and renders him listless. He profits but little by exercises in which he ceases to be interested and which he performs in an imperfect and perfunctory way. Therefore the monotonous work of the "School of the soldier" and the "School of the company" should alternate with gymnastic exercises, marching, wrestling, boxing, fencing and athletic games.

\section{Overtraining.}

When, as the result of muscular exercise, carbon dioxid and other waste products accumulate in the blood, we experience a sense of fatigue. The power of the brain to generate impulses, as well as the nerve force which transmits them, are weakened, and the contractile power of the muscles is impaired so that the heart beats more feebly and the arterial tension falls. If, as the result of violent exercise without sufficient rest, these waste products continue to accumulate faster than they can be eliminated, and the oxidized tissues are not properly renovated, the symptoms of overtraining show themselves.

The most common form of overtraining is that which results from continued, hard muscular work without sufficient rest or sleep, and is not rare among soldiers during the period of instruction, maneuvers, practice marches, and especially during a campaign. Its symp- 
toms are constant lassitude, with drawn, haggard face, impaired appetite and disturbed digestion, loss of weight and strength, fall of temperature; the heart is somewhat dilated and the pulse soft and quick, while the cyanosed skin and mucous membrances indicate a sluggish circulation. In this so-called "stale" state, soldiers become an easy prey to infectious diseases, especially typhoid fever and tuberculosis.

In another form, the result of a more strenuous but less prolonged exertion, the overtrained men suffer from myalgia, lumbago, general stiffness and well-marked febrile symptoms, with coated tongue, gastric disturbance, headache and insomnia. These symptoms usually last a few days and disappear, but sometimes, in more aggravated cases, the patient falls into a typhoid state, the so-called overtraining fever (fièvre de surmenage) which imitates true typhoid, being distinguished chiefly by the normal condition of the respiratory organs, the weak, dilated heart, cardiac dyspncea, and the small, soft, irregular pulse.

In the so-called overstrain, occurring mostly among young men taking part in athletic races and games, the symptoms are chiefly those of dyspnœa, caused by the rapid accumulation of waste products in the tissues, and cardiac failure. If the athlete heeds the first warning and stops in time he soon recovers his normal condition, but if he prolongs the effort, determined to win at any risk, he is liable to the worst effects of overstrain - a hypertrophied and dilated, flabby heart, with irregular, dicrotic pulse - a condition from which he may never fully recover. In other words, the normal physiological hypertrophy of the heart becomes pathological. In such heart the persistent increase of the tension to which the segments of the aortic valve are subject during diastole induces a slow, progressive sclerosis of these segments, and eventually aortic insufficiency, causing the so-called "athlete's heart."

There is also very good reason to believe that the hypertrophied heart of the athlete, physiologically adjusted to the demands of severe training, may give him trouble in after years, when he settles down to a quieter life, especially if he fails to reduce the generous diet to which he is accustomed. As a rule, the enlarged muscular fibre of an athletic heart degenerates gradually to its normal size and gives no trouble, but there are exceptions in which the heart retains its abnormal strength and vigor; in stch men, according to Hare, 
the condition is comparable to that of a steamer whose engines are too strong for her hull. As noted long ago by Clifford Allbutt, the liypertrophied left ventricle slowly causes stretching and dilatation of the aorta with subsequent insufficiency of the valves, the patient often not seeking medical aid until after middle life.

One of the most frequent evil effects of violent athletics is renal congestion and overstrain. Albumen is often, if not always, found in the urine after severe and protracted muscular effort, and it would be well if this symptom were taken as a warning that the kidneys are taxed dangerously near to the pathological point (see page 205). Those young men who, in their normal state, before training, present traces of albumen, should refrain from all athletic contests.

\section{Gymnastics and Physical Training.*}

Physical training, that is, the system of exercises which enables us to use our special senses and motor organs to the best advantage, is of greatest value to the soldier. Its effects may be summed up as follows: The superfluous fat and water are eliminated. There is increased size and tonicity of the muscular fiber, and increased muscular power. There is also a distinct economy of the nervemuscular mechanisms of respiration and circulation. New movement complexes are established with the use of fewer muscles by the correction of the needless tension of antagonists. This economy of muscular effort prevents excessive metabolism and results in a decreased output of carbon dioxid. The exercise which at first raised the pulse to $\mathrm{I} 2 \mathrm{O}$ and generated a temperature of $\mathrm{IO}^{\circ}$, can, after suitable training, be performed with a pulse of 90 and a temperature of $100^{\circ}$. The trained soldier can march at least a third further than the untrained recruit, without any more fatigue.

The exercises of the gymnasium are susceptible of great variation and therein lies one of their chief advantages. It is possible so to order and combine them as to contract, to any desired degree, every muscle, tendon and ligament, thus securing suppleness of joints as well as development of tissues, for it must always be borne in mind that agility is as desirable as strength. Simple callisthenics, without

* The authorized manuals and guides for the physical training of recruits are Butts" "Manual of Physical Drill," for outdoor and parade ground instruction, and Koehler's "Manual of Gymnastic Exercises," for indoor or purely gymnastic work. 
apparatus, can be made exceedingly useful and should never be overlooked. An objectionable tendency in the gymnasium is to give a preponderant share to the exercises of the upper extremities. The arms are vigorously trained as in suspending or supporting the body, and often made to usurp the office of the legs; they soon become greatly developed, often out of proportion to the rest of the body, this development, in professional gymnasts, often amounting to deformity, such as protuberant shoulder-blades and round back. The legs, served by powerful muscular masses, are capable of much work with little fatigue. A man who runs quickly up-stairs, or up a steep slope, performs a sum of work which far exceeds any muscular effort he is able to do in any other way during the same time.

The vital importance of chest development need not. be divelt upon; on the size and shape of the chest depend, to a great extent, the capacity and vigor of lungs and heart. The best way to increase the expansion of the chest is to strengthen the so-called respiratory muscles, those concerned in elevating the ribs and sternum and depressing the diaphragm. If is an error to believe that this is best achieved by exercising the upper extremities; experience shows that it is best attained by the exercise which compels the deepest inspiration and insures the most complete inflation of all the pulmonary vesicles; we must therefore seek to increase the amplitude and frequency of the respiratory movements. These movements depend upon the intensity of the respiratory need, while the intensity of this need depends on the quantity of mechanical work performed in a given time. The sum of work performed by a muscular group is according to the strength of this group; the legs possessing three times as much muscle as the arms, can perform three times the amount of work before being exhausted. Therefore, it is chiefly by the use of the legs, as in running or ascending slopes, that the chest is to be developed.

Another advantage of gymnastic exercises is that they are easily watched, regulated and controlled, consequently free from injury or accident. Thus it is a very remarkable record that during the last twenty or more years there has not been a single serious injury among the cadets in the IVest Point gymnasium. Furthermore, gymnastics can be carefully graded and adapted to individual wants so that overtraining is impossible. There is no question, then, that they can be made to answer fully and successfully all the physical 
needs of a growing youth or young recruit. They are performed at the word of command, requiring attention and prompt obedience; this, however, is not an ummixed advantage, for exercises under coercion are not always performed with alacrity and thoroughness; they are more like work than play, may become irksome and often fail to bring out will-power and nervous energy. This is why athletic games, with their greater freedom, variety and excitement, will always be a pleasant, useful and necessary addition to mere gymnastics.

For the soldier, gymnastics are not only for the purpose of developing his strength, agility and endurance, but also a means to the end of overcoming the many obstacles which, in warfare, will stand in his way. Therefore, in a complete system of physical training, they should be supplemented by those special exercises requiring knowledge, skill and self-confidence, which the soldier would be called upon to perform under the actual conditions of a campaign. These applied gymnastics have the advantage of requiring but few appliances, only such as can be improvised in a post or camp. They comprise various kinds of races, jumping ditches, vaulting over fences, scaling walls, climbing poles and trees, walking on narrow boards placed over ditches, etc.

Circular 33, War. Department, of May 6, 1908, is a compilation of purely military exercises which have been conducted throughout the Army from time to time in athletic meets, and was published as a convenient guide for the government of competitions. It consists of 9I "events" covering most of the field duties of soldiers on foot and horseback, conducted in competition, and therefore in an attractive and stimulating form.

In our Army, the question of physical culture, outside of the Military Academy, has not yet received the systematic treatment which it deserves. Gymnasiums have been established at some of our largest posts, in connection with the exchanges, but exercise therein is entirely voluntary or left to the discretion of post commanders. Existing War Department orders provide for garrison training and field training. Garrison training includes gymnastics, outdoor athletics and all other military exercises practicable in garrison. Field training includes practice marches, camping and all other field work. The details of this training and the time devoted to it are prescribed by Department Commanders. 
It seems to be the opinion of officers who have given special attention to the subject, that gymnastic drill should be made compulsory for foot troops and discretionary with the post commander for mounted troops, but never exceed 30 minutes each day.

Physical training for officers.- G. O. No. I48, W. D., I9Io, enjoins upon all officers on the active list to keep themselves in fit physical condition to perform active duty with troops under war conditions. Regular and systematic exercise will be required of them by their immediate commanders under the supervision of the Department Commanders.

All officers on the active list (general officers excepted) will be examined physically once a year. As soon as possible after this examination, all officers above the grade of captain, not physically found disqualified, will take riding tests of thirty miles each day for three consecutive days. "One of the rides will be concluded within six hours and thirty minutes, and two within seven hours and thirty minutes each, after starting, all to include proper rests." The annual riding test for officers serving in the tropics will cover, in the same number of days, two-thirds of the distance, and in two-thirds of the time each day.

Field officers of the Coast Artillery Corps will take a marching test in lieu of the riding test, consisting of a march of fifty miles to be made in three consecutive days and in a total of twenty hours, including rests. The marching test for officers serving in the tropics will cover, in the same number of days, two-thirds of the distance and in two-thirds of the time each.

Field officers of the permanent staff corps and departments who have reached an age and rank which render it highly improbable that they will ever be called upon to participate in active field operations may, upon their own application, be excused from the physical tests above described.

This riding or marching test, as the term indicates, is not intended as an exercise, but rather to show whether field officers are physically fit for any active duty they may be called upon to perform. From the medical point of view it is open to some objections. In the first place, it does not seem rational that senior colonels and junior majors, with a difference of 20 or more years in age, should be subjected to the same test. All men above 50, but particularly above 60 , suffer more or less from organic degenerative changes and, 
for some of them, the test is a dangerous strain. They may not know the nature and extent of those changes, and a medical examination cannot always reveal them. The test may thus, by throwing strenuous work upon enfeebled organs, aggravate their condition and seriously impair the efficiency of officers who, in actual war, would have successfully discharged all the duties normally devolving upon them. It may be noted, in this connection, that elderly officers with ripe judgment and accumulated experience, and whose physical condition is normal for their age, are too valuable an asset of a wellconstituted army to subject them to the risks of a test much beyond anything likely to befall them in real warfare.

In the second place, there is no doubt that field officers can be induced to keep themselves in proper physical condition by less strenuous and more efficient means, namely, by regular and systematic exercisc covering a certain number of hours weekly or monthly, varying according to age and rank.

In the Navy and Marine Corps, the physical test is based on different principles; it partakes more of the nature of exercise and is apparently free from objections. All officers on the active list are required to take, once a month, a walk of Io miles, to be completed in not more than 4 and not less than $3^{\mathrm{T} / 2}$ consecutive hours. In the tropics or " any place where the temperature is correspondingly high at the time of the exercise, the distance walked and the times required shall be two-thirds those above stated." Before taking the first monthly walk, in each year, each officer is examined by a medical board to determine whether it can be taken without danger to the officer, and again within 3 hours after completion thereof. All officers are excused from the test during the calendar year prior to their retirement for age.

\section{SPECIAL EXERCISES.}

Marching. - Marching being the most important of military exercises will be the subject of a special chapter.

Manual of Arms.- The manual of arms exercises all the muscles of the body, but very unevenly; those of the shoulders, arms and chest are much more affected than those of the lower extremities. and the right side more than the left. It is an imperfect exercise and develops quickness of movement more than strength.

Setting-up Exercises.- The six setting-up exercises prescribed in 
the Drill Regulations are simple, easily performed and, if all practised successively, affect all the muscles of the body pretty evenly, correcting the tendency to muscular asymmetry and defective attitudes produced by the manual of arms, bayonet and saber drills, and fencing. Each exercise should be short, as it brings into play only few groups of muscles and soon produces great fatigue.

Fencing.- This is one of the very best and most complete of military exercises; bringing into vigorous play all the muscles of the body, expanding the lungs, cultivating agility, quicliness of decision, rapid coördination and accuracy of movements. It is a violent exercise, only to be indulged in by the physically sound and which should be carefully graded. A serious drawback to fencing is that the side of the hand which handles the foil is liable to become somewhat hypertrophied, the muscles of the shoulder, arm and leg on that side growing larger and stronger; as a consequence there may also be some degree of lateral curvature, with concavity toward the foil hand. This danger is prevented or corrected by fencing with the left as well as the right hand.

Sword and saber exercises are similar in their character and effects to fencing. In bayonet exercise, both arms being employed, the muscular exertion is more evenly distributed.

Ruming Drill.- This drill, like double-time, is prescribed by infantry regulations and forms part of the soldier's training, but it is a violent exercise requiring careful supervision. At first, the soldier should run in light garb and without encumbrance; later, his arms, canteen and equipment may be added. The distance is gradually increased until it reaches a maximum of 200 or 300 yards. To avoid the danger of overstrain, men running, especially in competitive races, should be allowed to fall out at will; men with signs of cardiac weakness should not be allowed to run at all.

Equitation.-Equitation brings into play the muscles of the basin and the lower extremities, while it has but very little effect upon the upper extremities and chest. Therefore it is an imperfect exercise and must be supplemented by gymnastics. The continuous shaking and jolting to which are subjected the abdominal viscera, if not excessive, have a beneficial effect and tend to improve digestion and nutrition. This exercise should be practised in open air whenever the weather permits so as to avoid the breathing of the more or less infected tan-bark dust of the riding-hall. 
The dangers of equitation are the production of hernia, ptosis or displacement of various abdominal organs, and hemorrhoids. It has been accused, but without proof, of causing varicocele and varicose veins. The mounted soldier is particularly liable to furuncles and ecthyma in the region of the buttocks, the result of local infection; daily washing and clean linen will prevent their occurrence. Chafing and excoriations of the thighs and buttocks are more or less inevitable in recruits; they should be washed with boiled water and dressed with antiseptic gauze.

Srvimming.- Swimming is a useful accomplishment as well as a capital exercise, and wherever possible should be practised by the recruit. It produces contraction of all the muscles of the body, as well as free expansion of the lungs, under the best possible conditions. It has, furthermore, the added advantage of the marked tonic effect of cold water, while the skin is thoronghly cleansed. It is somewhat violent and should be carefully graded to the cardiac strength and power of endurance of each man. A non-commissioned officer, or Hospital Corps man, well instructed in the care to be given cases of asphyxia by drowning, including artificial respiration, should always be on hand with the necessary ontfit.

To secure the best effects and prevent the dangers of swimming, the following indications will be found useful:

Salt water is best, but if a river or pond be used the water should be reasonably clear and free from organic filth, and the temperature not below $65^{\circ}$ or $70^{\circ} \mathrm{F}$.

Do not enter the water while perspiring very much or when clilled, or too soon after eating; the best time is a couple of hours after meal.

On entering the water, submerge the whole body, including the head, and begin swimming at once to prevent chilling.

Diving and swimming under water may injure the ear-drum or otherwise affect the hearing, and therefore should be avoided by men whose ears are not perfectly sound.

Leave the water before feeling exhausted or very cold, and before the legs and feet show signs of numbness.

After the swim, dry the skin thoroughly to restore circulation and prevent chilling.

Hygiene of the gymnasium and the saimming pool.- In the gymnasium, exercise more or less violent is indulged in ; brcathing 
is increased in frequency and depth, and takes place through the mouth as well as through the nose. It is therefore necessary that the air should be as pure as possible. Such purity, however, is rendered difficult by the conditions generally prevailing therein, especially when frequented by many people. Dust is freely introduced in various ways and kept in perpetual motion, so that even with proper care it cannot be excluded to the extent possible in other buildings. It has been noticed that in badly kept gymnasiums wounds frequently become infected. All surfaces should be hard and smooth so as to be easily cleaned. Only wet mops or vacuum cleaners should be used. Mats and mattresses must be frequently shaken or beaten outside, and renewed as often as necessary. Smoking should not be allowed under any circumstances, and spitting only permitted in specified places where suitably disinfected cuspidors are provided. A common cause of danger is sudden chilling during perspiration from strenuous exercise; this is guarded against by avoiding drafts and by the use of sweaters. The temperature of the gymnasium should never be above $50^{\circ}$ or $55^{\circ} \mathrm{F}$. An abundant fresh-air ventilation is absolutely necessary. Besides the ventilators which always remain open, a free wind perflation through the windows is highly desirable during recesses.

The swimming pool is a necessary adjunct of a well-equipped gymnasium. It affords a form of exercise which should not be neglected. By reason of the large capacity of such pool the water can seldom be renewed more than once a week, so that, if used by many persons, it is liable to more or less pollution and may become a medium of transmission for infectious disease. However pure the water when admitted, the number of microbes increases rapidly from day to day, so that a bacterial count of 100 per c. c. the first day is very likely to be 100,000 the sixth day, even when the swimmers are required to take a preliminary shower bath. If a sufficient supply is available, a certain amount of water may be drawn out every day from the bottom so as to carry off some of the sediment, and afterward replaced by fresh water. It is also a good plan to remove, with small hand pumps, any accumulation of hair or other debris observable on the bottom. Once a week the tank is emptied, scrubbed and refilled.

In order that swimmers may have a place to spit out swallowed water, and also to prevent drippings from running back into the tank, it is advisable to provide a shallow trough along the edges. 
The best way to prevent the transmission of infection in the swimming pool is to sterilize the water. It has been found that the application of calcium hypochlorite, in the proportion of one or two parts per million, is entirely satisfactory, offering a cheap, convenient and efficient method of insuring a hygienic pool. In the above proportion there is no noticeable odor or taste. The lime, finely pulverized, may be put in a cheese-cloth bag and dragged about the pool, or else dissolved in 3 or 4 buckets of water and the supernatant solution siphoned off into the tank. As the effects of this purification last at least four days, it is enough to practise it once a week. No swimmer should be allowed to enter the tank except after taking a soap and water shower bath.

Some investigators contend that complete purification of a pool used by many bathers requires a proportion of 2 or 3 parts of hypochlorite per million and that, in such quantity, it becomes objectionable on account of its odor and its irritating effect on the eyes and mucous membranes. They recommend, as preferable, sulphate of copper in the proportion of one-half part per million. It is not irritating, is free from odor and cheaper.*

\section{Athletic Games and Races.}

Games and races, such as football, baseball, basket ball, la crosse, cricket, tennis, running and rowing, provide the natural and most pleasant forms of exercise. They should therefore always form part of a well-regulated system of physical training and, when kept within proper bounds and intelligently supervised, receive every encouragement. There is no doubt that the best effects of exercise can be obtained from them, provided the contestants are physically sound and propcrly trained.

Athletic games give useful results in the Army only in so far as all recruits are permitted and encouraged to indulge in them, that is, when the games are practised in a general systematic way, for the benefit of all. To pick out a team in a garrison from a few exceptionally strong men and train them for a contest with a similar team from another garrison may lead to pleasant and exciting diversions, and to that extent is unobjectionable, but such training and contest add practically nothing to the physical efficiency of the company or regiment concerned. This tendency to make spectacular displays

* Jour. A. M. A., Sept. 25, I9I5. 
of athletic games is one of the most serious objections urged against them, for instead of an average gain among all the men, the great majority of them are allowed to be simple idle spectators, while a small minority are strenuously trained, even to the point of danger. It is doubtful whether those who participate in these contests derive from them any physical benefit which they would not more fully obtain from ordinary gymnastics. Thus it was found that at the United States Military Academy, the football players of the gradnating classes of I9O0, I9OI, I9O3 and I9O4 only increased 4.36 pounds in weight during their stay at the Academy, while the average increase for the four entire classes was 5.62 pounds.

For these reasons the War Department has wisely decided that athletic " meets" between posts should not be encouraged, although they may be permitted once a quarter between the units of the post.

Surgeon General Stokes, U. S. Navy (Annual Reports for I9II and 1912), believes that competitive and spectacular athletics are undesirable for midshipmen. In his opinion, the prolonged rigorous course of training necessary to excel in games and races is particularly dangerous in its after effects in the Navy, under the conditions of service at sea where active exercise is impossible and the body more liable to the degenerative changes of age. An examination of the medical records of 625 former athletes at the Naval Academy, from the classes of I89I to I9I I, showed that 9 had died and I 2 had retired. Of these 2I casualties, 6 were due to tuberculosis, 8 to mental or nervous diseases, 2 to alcoholism, 2 to heart disease (directly attributable to track and crew racing) and I to injury received in football contest. Of the remaining 604 in service, I 98 had disabilities of sufficient moment to be of official record; 48 being referable to the heart or blood-vessels, I6 to the joints and I I to the kidneys. About I7 were tuberculous or had had symptoms of tuberculosis, I6 were cases of neurasthenia, 25 of appendicitis and $\mathbf{5}_{5}$ of hernia. The last two classes of disabilities were particularly associated with football.

The above rates of casualties and disabilities are not any greater than those occurring among non-athletic midshipmen. They are practically the same. But it is contended that the athletes being all exceptional men, especially selected on account of their size, strength and endurance, should show a much better record, a more striking superiority in their freedom from disabilities. In analyzing the 
character of these disabilities it becomes apparent that a large percentage of them should be ascribed to the overtraining and overstraining undergone during the years of their academic life.

As stated by Gulick, of Harvard, no evidence has been presented showing that the selected men taking part in spectactular intercollegiate athletics are benefited in health, strength and vitality. On the ground of benefit to the individual there can be no defense of the present system of competitive athletics.

Football.- Of football, as it affects body and mind, there is a great diversity of views. That it is a dangerous game, attended with many injuries of all degrees of severity, is generally admitted. From the records of the members of football teams who graduated at the Military Academy from 1892 to 1903 ( 159 in all), it appears that, in 1905, 5 had died from disease (typhoid fever 2, cancer I, malarial fever I, and acute mania I), evidently too small and imperfect data from which to draw useful conclusions. At that date, 7 had not yet filly recovered from injuries received while playing football, although not incapacitated for duty. That, like all violent athletics, football may cause a breakdown in middle life is maintained by competent observers. Not enough years had elapsed when the records of the above teams were examined, to judge of any possible after effects upon the heart and lungs.

As regards mere physical development, better results can be obtained by graded gymnastics and less strentous games. At the United States Military and Naval Academies, as well as all other educational institutions, it is detrimental to intellectual culture for the folowing simple reasons

I. It absorbs time which the athlete should devote to his books. His leisure hours are no longer his own; he must train and practise as bidden, whatever may be his class standing and need of study.

2. It produces intense fatigue. An exhausted body means a tired mind, one incapable of useful study. Each game involves the expenditure of an enormous quantity of nervous energy, and time is required to recuperate.

3. It causes many injuries, from which, indeed, the athlete generally recovers but for which he must be treated in hospital for days, weeks or months, valuable time irrevocably lost to him.

The effect of football on character is noteworthy. It is obvious that the qualities which it demands and develops: attention, sub- 
ordination, self-restraint, clear judgment, prompt decision, pluck, etc., are precisely those most needful to the officer on the battlefield. Therefore it seems a logical conclusion that this game, although somewhat dangerous to the body and rather seriously interfering with the curriculum, may be tolerated, if not encouraged, at the Military and Naval Academies, not indeed as a means of physical culture but rather as a means to develop useful military traits of character.

From what precedes it does not follow that football is suited to soldiers and sailors. Most of them have not received the thorough training which is indispensable, and, at their age, such training is hard and often impossible. Furthermore, they are less capable of the self-control and subordination absolutely necessary for a clean game. The result is that when soldiers play football the casualties are likely to be many and serious.

In colleges and other institutions, not military, the serious objections existing against football, as now played, are not off set by compensatory advantages and the game should be radically changed or else completely banished from them 


\section{CHAPTER XIX.}

\section{THE MARCH.}

Maurice de Saxe taught long ago that it was much more important to exercise the legs of the soldier than his arms, that in his legs was the secret of the success of a campaign, and that ability to march was far better than knowledge of the manual of arms. Napoleon is credited with the saying that battles are won with legs rather than with arms. In modern warfare, everything tends to indicate that victory will continue to be with the commander who gets first in position with the largest number of men.

Marching is the normal exercise of infantry. It is the simplest, easiest and most important of all military exercises, but also the most exhausting on account of the enormous amount of muscular work performed, especially when the soldier carries his equipment, arms and anmunition. The infantry man, therefore, should be constantly and persistently trained in marching, with and without his load, until he is able to walk 15 to 20 miles a day, with arms and equipment, without much fatigue, almost automatically.

The effect of practice on marching is well shown by the experiments of Dr. Pembrey. At first, marching a certain distance caused a rise in pulse of 72 beats, in temperature of $2.2^{\circ} \mathrm{F}$.. and a loss of weight of $23 / 4$ pounds. After 3 weeks of training, the same march only increased the pulse 28 beats, the temperature $0.6^{\circ}$, while the loss in weight was under 2 pounds. A moderate rise of temperature. in marching, as during any form of exercise, is physiological and beneficial, favoring chemical changes and enabling the muscles to do their work more economically. According to Melville, the optimum temperature for marching is about $100.6^{\circ}$, and may rise higher without ill effects.

It is the general experience that soldiers on the march, even when subjected to many discomforts and hardships, are in better physical condition and suffer less from infectious diseases than when in standing camps. Thus in Sherman's march to the sea, which lasted about six months, the sick were less than 2 per cent. in the Georgia colımn, and between 3 and 4 per cent. in the Carolina column which was operating in swampy country and harassed by the enemy. 
Any march exceeding 20 miles in 24 hours is a forced march, but soldiers in good training should always be able, when circumstances require, to make a forced march of 25 or more miles. "The maximum for a day's march of infantry and trains may be assumed at 28 to 30 miles; a repetition of this performance on the next day cannot be counted upon unless conditions are quite favorable" (Field Reg.) One of the most remarkable forced marches on record is that of Friant's division which covered 78 miles in 46 hours, and the next day (December 2) fought in the battle of Austerlitz, where it lost 40 per cent. of its strength in killed and wounded. Equally remarkable, on a larger scale, is the march of the Grand Army, in I802, from Boulogne to the Rhine, 400 miles in 25 days, or at the rate of 16 miles a day.

During the period of the year assigned especially to practical instruction, as determined by Department Commanders, existing orders direct that there will be "one march in each month of not less than three nor, ordinarily, more than six days, and during such period commands will take the field for not less than twenty-one consecutive days."

According to our Regulations, the length of the full step, in quick-time, is 30 inches measured from heel to heel, and the cadence at the rate of 120 steps per minute. The foot is moved smartly, but without jerk, straight forward, sole near the ground, the knee straightened and slightly turned ont; at the same time the weight of the body is thrown forward and the foot planted without shock. The arms hang naturally, the hands moving about six inches to the front and three inches to the rear of the seam of the trousers. In quick-time the soldier marches IOO yards a minute and $32-5$ miles an hour.

In double-time, the length of the full step is 36 inches and the cadence at the rate of 180 steps per minute. The hands are raised until the forearms are horizontal, fingers closed and elbows to the rear. The knees are slightly bent and the arms allowed to swing naturally. This is not a marching step but a run, and is too exhausting for more than spurts of a few moments, although careful training may enable most soldiers to keep it up I 5 to 20 minutes.

In the route-step, the men are not required to preserve silence nor keep step, but the ranks must cover and preserve their distance.

In European armies, the quick-time step is the same as in our 
service, except that in Germany it is $3 \mathrm{I}^{\mathrm{J} / 2}$ inches with cadence of II 5 steps per minute, and in Russia only 28 inches. According to Marey, the length of the step increases with the cadence up to I5O steps per minute and then diminishes. It is generally recognized that better results are obtained by lengthening the step than by accelerating the cadence; this is done by inclining the body forward, completely extending the rear leg and flexing the front one.

In the double-time step there should be no hopping movement, the center of gravity being maintained as much as possible along a horizontal line; the leg should be flexed as the foot strikes and the foot set flat upon the ground.

The military step is more fatiguing than the ordinary walking step; the movements are more rigid and constrained, ease of carriage being sacrificed to precision and uniformity. The body is erect and the legs extended or only slightly flexed, the heel touching ground first, followed after an appreciable interval by the toes. The progression of the body is attended with a succession of vertical and lateral oscillations very conspicuous in a regiment of marching troops, the body rising as each foot is lifted from the ground, and swaying to right and left as the corresponding foot is set down. The vertical oscillation often reaches an amplitude of two inches, a useless waste of effort.

The 'military step, therefore, however desirable on the parade ground and on occasions of ceremony, is not suited to marching in the field. To render it less irksome and fatiguing, the body should be slightly inclined forward, but with head erect and chest thrown out to favor breathing; the foot moves parallel with the ground and nearly straight forward, being raised only just enough to clear obstacles; the muscles of the knee are relaxed as the foot strikes ground (heel and toes almost simultaneously) to prevent shock. The feet should be slightly turned out to increase the base of support, but this eversion must not exceed an angle of Io or I 2 degrees so that the foot may rest in its normal position, namely, on heel, ball and outer edge; beyond this, there is a waste of muscular effort and the foot is made to rest too much on its inner edge, with danger of straining and flattening the arch. Under instruction, the vertical and lateral oscillations of the body can also be reduced to a minimum.

Flexion step.- Carrying these indications still a little further, we obtain the "flexion step" of the French as developed by De Raoul, 
said to be the most natural route-step, and the best to cover long distances with the least fatigue. It is that generally taken by tired laborers, messengers and mail-carriers. In this step the body is bent; the hips, knees and ankles are flexed; the feet just clear the ground and move parallel with it, being planted flat, heel and toes together. By leaning the body forward, the center of gravity is
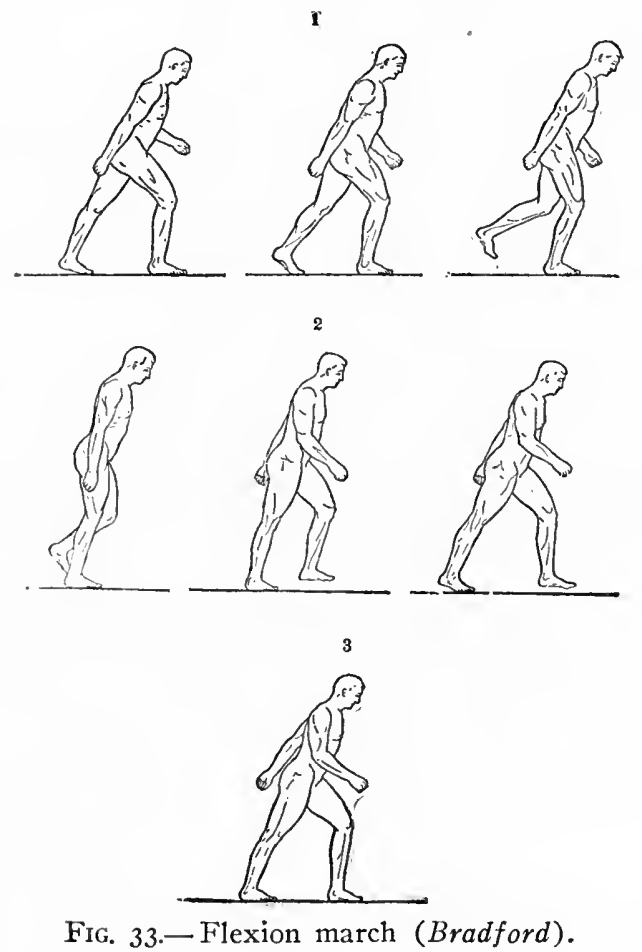

displaced and the legs follow with least muscular effort; the greater the inclination forward the greater is the speed. This step, it is claimed, enables trained soldiers to cover a distance of to miles in about I hour and 45 minutes, with less fatigue than in double the time with the ordinary step. Against it the ob; ection has been made that the continuous extension of the quadriceps femoris (fleshy mass in front of the thigh) will ultimately cause a larger expenditure of muscular work than in the ordinary walk. A useful modification, suggested by Demeny, consists in fully extending the rear leg which 
thus helps materially in increasing the length of the step and propelling the body forward. (Fig. 33.) This step is not as much used as formerly in the French Army.

\section{REGULATION OF THE MARCH.}

The length of the average march for infantry and for mixed commands consisting partly of foot troops, is ${ }_{5} 5$ miles per day, with a day of rest at least once a week. Small commands of seasoned infantry marching on good roads in cool weather can cover 20 miles per day, but in extensive operations involving large bodies of troops, the average rate of progress will not exceed 12 miles per day. Field artillery marches $I_{5}$ to 20 miles a day; cavalry, after men and animals are hardened, 25 miles a day: wagon trains about the same as infantry. (Field Reg.)

It is of great importance that an even, uniform rate be maintained throughout the column, so as to avoid the alternate checking and hurrying produced by an irregular pace, so fatiguing for the body and trying to the temper. In the field the maximum rate to be counted on, while marching, is 3 miles per hour, or, including halts, $2 \mathrm{I} / 2$ miles per hour. "Sandy, muddy or slippery roads, great heat and dust, strong head winds and storms, or broken country reduce the rate of progress." Under average conditions the rate for infantry columns may be assumed at $2 \frac{1}{4}$ to $2 \frac{T}{2}$ miles; thus, for a march of 15 miles, a period of 6 to 7 hours is necessary.

After marching half to three-quarters of an hour, the column is halted for 15 minutes to allow the men to relieve thenselves and readjust their clothing and accoutrements. After this first rest there should be a halt of 10 minutes every hour, that is, the troops march 50 minutes and then halt 10 . This is not a rigid rule and may be modified according to circumstances.

Men on the march should not breathe hurriedly, but regulate the number of inspirations to the cadence of the step, taking a deep breath every few respirations in order to fill all the air-cells and secure thorough oxygenation of the blood. The air should be inhaled through the nose as far as possible, but, in hard marching. most men are obliged to breathe through the mouth as well.

It is advisable to start slowly so as to gradually supple the joints without fatigue and get the second wind established without breathlessness. It is likewise well to slow the pace at the end of the march 
so as not to get into camp in a heated and perspiring state. Halting places should always preferably be on clean, dry ground, sheltered from the sun in summer and from the wind in cold weather. The men should be, encouraged to lie down so as to fully relax the muscles and place the body in the best position for prompt recuperation. The practice of massage at the halts is often advisable; it consists in the forcible flexion and extension of each leg three or four times, followed by the kneading of legs and buttocks ( C. H. Melville). Such massage frees the muscles from the waste products accumulated in them and prevents cramp and stiffness. If the ground is wet the men should not sit or lie down, unless they can do so on their ponchos or other water-proof material. In diffcult or mountainous country, halts should be more frequent. If the march be unusulally long, a rest of about one hour is necessary after covering two-thirds or three-fourths of the entire journey, during which the men may eat a light meal.

At halts the company commander calls out the side or direction where the men may attend to calls of nature; a non-commissioned officer designates the place and sees that the feces are properly covered with earth. The equipment shovel may be used for the purpose. In the vicinity of dwellings, a small trench should be dug.

"When the roads are soft with mud or deep with sand, it may be advisable to divide the column longitudinally, thus permitting men and animals to pick their way with better footing and leaving the middle of the road clear. The suffering from heat and dust may also be materially reduced by this method. But, whatever the widening of the column thus produced, increase of length of the road space occupied by any unit should not be permitted" (Field Serv. Reg.).

The moisture evaporated from the men in front, in a marching column, on a warm, still day, saturates the air so that the men in rear may suffer much discomfort. This is relieved by the division of the column as above described, as well as by changing the order of march from day to day.

The composition and weight of the equipment and clothing on the march should be carefully determined by the commanding officer according to circumstances. The men may find their burden heavy and irksome, especially in hot weather, but should be made to realize that each one of the articles they are required to carry 
is practically as essential to their efficiency as rifle and ammunition. The blanket and poncho are the articles most likely to be discarded, but precisely those that may be most needed in the cold and muddy bivouac. If the march be through a malarial country, or one in which dangerous insects or poisonous animals are known to be common, each man should carry his mosquito-net and use it in connection with the shelter-tent or (in the absence of the latter) stretched over his couch. It will protect him not only from mosquitoes and flies, but likewise from other obnoxious insects, ticks, scorpions and reptiles.

In hot weather the men should be allowed to remove their coats or blouses, or at least to unbutton them so as to promote free evaporation of perspiration.

The march should begin at as early an hour as possible so that the men may arrive at their destination by noon or as soon thereafter as practicable. In hot weather or in a tropical climate, an early start is still more necessary; then the march may even begin before daylight, being intermitted at 9 or ro oclock and the troops allowed to rest until 4 or 5 o'clock, when it is resumed. Night marching is very exhausting and justifiable only in emergencies.

The men should breakfast before starting, see that their canteens are full, and carry their luncheon in the haversack. Any purification which the water may require should have been attended to the previous evening, especially boiling, so that it may have time to cool before morning. It is always advisable to fill the canteens in the evening and leave them exposed to the night air. All forms of alcoholic drinks are pernicious and must be strictly prohibited. Smoking and chewing are injurious during the march and should be postponed until the camp is reached.

Concerning water drinking, thirst is not an entirely reliable guide. It is often a mere expression of a dry condition of the mucous membrane of the mouth and pharynx, or sometimes only an artificial craving cultivated by indulgence into a habit. This thirst of habit should be distinguished from true or physiological thirst, the expression of a real water hunger of the tissues. It should also be noted that the sensation of thirst, like that of hunger, does not disappear as soon as the needs of the econony are supplied but lingers for some time afterward. The trained marcher drinks moderately and stops promptly as soon as his thirst is relieved (not quenched), 
thus economizing water and strength. Any one who continues to ingurgitate water so long as the process affords him gratification, runs the risk of becoming more or less water-logged. Such unrestrained water drinking, especially in hot weather, produces profuse perspiration, gastric disturbance, marked debility and predisposes to heat-stroke and heat-exhaustion. It is a good rule not to use the canteen until the half-way halt is reached. The careless soldier who drinks early in the day creates an artificial thirst that must be frequently gratified, and is quite likely to become exhausted and fall out before the march is completed. Free drinking is often the result of alcoliolic indulgence, injudicious eating or excessive smoking, conditions which, it is true, call for plenty of water but which may also be easily aggravated by an excess of it. Outside of such abnormal conditions, free drinking is physiologically required only when the air temperature is so high that the body does not lose any of its heat by radiation, but depends altogether upon evaporation of the perspiration to maintain its temperature down to normal; then, water may be drunk at shorter intervals, in sufficient amount. Whenever there is uncertainty as to the quantity and quality of the next water-supply, the thoughtful soldier will always keep a reserve in his canteen.

According to Col. Melville, R. A. M. C., even the untrained man in England is able to march 7 miles without drinking. Thereafter he should have a pint of water every hour. Every soldier, in his opinion, should be in condition to cover an ordinary day's march of 14 miles, on a fair road, without having recourse to his water bottle, but needs about a pint every hour after his limit of endurance is reached.

To relieve thirst, especially the thirst of habit, the chewing of a twig of some bitter shrub or of a piece of bark is recommended, as it excites the salivary secretion and keeps the mouth and throat moist. Weak tea or coffee in the canteen is often better than water; it is a sterilized, gently stimulating drink and less of it is necessary to quench thirst.

Sugar is a special tonic and restorative of the muscular system, and may be used freely either in coffee or tea, or in the form of chocolate tablets. Coca and kola have been praised as useful muscular tonics on the march, but without sufficient reason; coca is too dangerous a drug for soldiers, and inasmuch as the active principle 
of kola is caffein, the same result can be more easily obtained with tea or coffee (see page 4I3).

The sources of water along the route must be carefully investigated by medical officers marching with the vanguard and, so far as possible, their quality (whether potable and safe, or otherwise) indicated on conspicuous posters. The character of any water, under such canditions, can only be determined hurriedly and approximately by taste, color, smell, surroundings, depth of well,

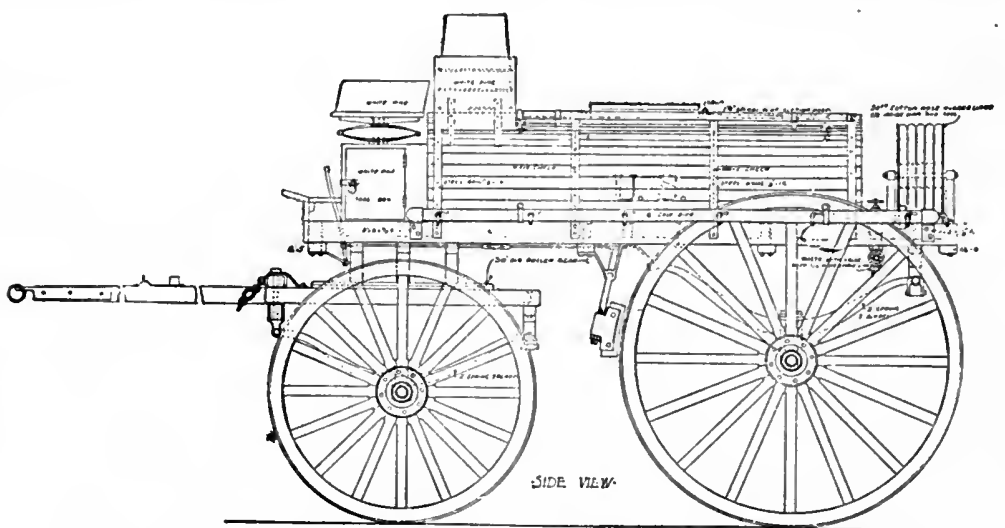

Fig. 34- - Army water wagon, with pump, hose and distributing pipe.

opinion of inhabitants, etc. The men shotld be forbidden to drink from unauthorized supplies. To insure entire compliance with such prohibition, it may be well to throw a few grains of methylene blue in contaminated springs or wells; the deeply colored water will not be touched.

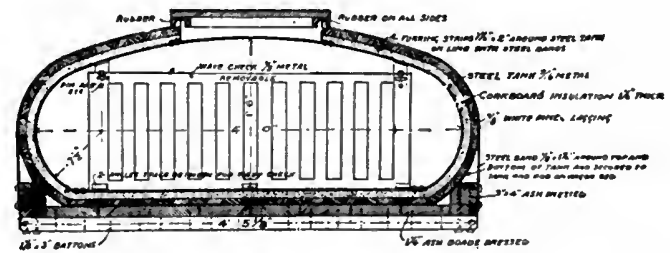

FIG. 35.- Section through tank of Army water wagon.

Before crossing desert tracts where water is scant and of bad quality, a sufficient quantity of good water should be taken in bar- 
rels or in specially constructed carts. The army water wagon recently adopted by the Q. M. Department, of a eapacity of 225 gallons (Figs. 34, 35) fills a long-felt want and will doubtless prove very useful under such circumstances.

On rnaking camp, it is refreshing and comforting to take a bath whenever practicable. Even where water is scant a satisfactory degree of cleanliness is possible by means of a sponge or washcloth. On daily marches, it is not advisable to wash the face and neck with soap, as the partial removal of the natural oil of the skin renders it more susceptible to the dust and heat of the march; it is enough, morning and night, to rub those parts with a damp towel.

CARE OF FEet.- As the result of marching, the feet are liable to various injuries, such as painful arch, corns, blisters, ingrown nails, excoriations or abrasions, synovitis of tendons, tarsalgia, etc., which may be followed by lymphangitis and adenitis. When walking over rough ground, with heavy equipment, there is also a liability to sprain or even fracture of metatarsal bones. These injuries are prevented or mitigated by the use of well-fitting, comfortable shoes, rendered soft with grease or oil, and by cleanliness. The feet should be washed every day by immersion in cold water, but only long enough to remove the perspiration and dirt and relieve the sensation of heat and fatigue; long soaking softens the skin and does more harm than good. It is a good plan to change shoes every day or two; stockings which are soiled, wet or wrinkled should be replaced by a clean pair as soon as possible or else dried, cleansed of all dust and dirt, and kneaded until again soft and pliable. Careful greasing of exposed or painful parts with fresh tallow, lanoline or vaseline, is advisable. It is also recommended to soap the feet or dust them with talcum powder, before starting, to diminish friction. Parts exposed to friction may be protected by wide strips of adhesive plasters. Taleum powder (the foot powder supplied by the Medical Department) consists of talcum 87 parts, starch Io parts, salicylic acid 3 parts; it is a useful lubricant and antiseptic and generally preferable to grease or soap on foot or in stocking. Tender feet can be hardened by bathing them in alttm, lead water, or diluted alcohol.

The pied forcé of the French, or Fussgeschu'ulst of the Germans. is the condition produced by the fracture, partial or complete, of the second or third metatarsal bone, at the junction of the anterior and middle thirds. The symptoms are sharp, localized pain and 
swelling, rarely with ecchymosis or lymphangitis. The nature of the lesion, unless diagnosed by the $\mathrm{X}$-ray, may only be recognized by the presence of a callus, 2 or 3 weeks after the injury. It occurs in marching with heavy load, especially in double time, or in jumping A predisposing cause is the use of shoes with comparatively high heels and thin soles, as in the German and French armies, tilting the foot forward and transmitting the shock of the step directly to the metatarsals.

Bunion is an enlarged and more or less inflamed bursal sack over a joint, most commonly the metatarso-phalangeal joint of the big toe, in connection with hallux valgus, and results from the wearing of narrow and pointed shoes. If small it is cured by the use of a rational shoe; if large and swollen the man should be discharged. Corns should be pared down and protected with corn plasters; more radical treatment must be reserved for the hospital. Blisters are pricked with a needle, gently squeezed, then protected with adhesive plaster or bandage. Care must be taken not to tear off the raised cuticle. Abrasions, if large and painful, may be painted with a 5 per cent. solution of chromic or picric acid ; tincture of iodine has also been recommended. Toe nails are not so likely to become ingrown if cut square across or only slightly rounded, and not too close.

Ingrowing nails are often very painful, incapacitating soldiers for marching. They are promptly relieved by a wider shoe, but, for a few days, may require treatment, consisting in trimming the nail and inserting a pledget of cotton under its offending edge. In old cases it may be necessary for the surgeon to remove part of the nail with its matrix.

A painful arch may be due to flat feet, or to weak muscles and ligaments, or perhaps to teno-synovitis. It may require to be supported on the march with felt pads, adhesive plaster and bandages. It is also present (says Munson) in probably a majority of soldiers exercised in hard marching under field equipment soon after being fitted with the broad army shoe, owing to the stretching of muscles and ligaments in adapting themselves to the new conditions of greater foot expansion. Such pain wears away in a very few days.

Teno-synovitis is a painful inflammation of muscle tendons, usually due to pressure or friction. In the foot, those most liable to injury lie over the instep, especially the extensors of the toes. 
The tendo achillis also frequently suffers from blister, abrasion or inflammation from shoes improperly fitted to the heel of the foot.

Irritable Heart.- This condition, a form of heart strain, is seen in young, immature and untrained soldiers, and appears to be the result of temporary dilatation and failure of the right heart, under severe and steady marching, or prolonged nervous and mental strain. There is more or less exhaustion and shortness of breath; the action of the heart is short, irregular and "irritable," that is, greatly quickened by the least exertion, and is often attended with a murmur; the pulse is weak, intermittent, quite variable in frequency, sometimes dicrotic. The treatment is complete rest, under which the patient ultimately recovers, but although there is no indication of valvular disease the irritable cardiac action usually persists for a long time.

This affection is reported to be frequently observed on the battlefields of Europe (I9I7).

For sunstroke, heatstroke and heat-exhaustion, see under Service in Warm Clinates.

Work Done in MARChing.- - It has been ascertained that an ordinary day's work for an adult is equivalent to about 300 foot-tons, a hard day's work to 400 , and a very hard day's work to 500 foottons. From the investigations of Haughton, Rubner and others, it is also possible to estimate the amount of labor performed by men in marching. According to Haughton, as quoted by Harrington. this labor, in walking over a level surface, is determined by the following formula:

$$
\begin{aligned}
\frac{\left(\mathrm{W}+\mathrm{W}^{\prime}\right) \times \mathrm{D}}{2240} \times \mathrm{C} & =\text { number of foot-tons. } \\
\mathrm{W} & =\text { weight of the person. } \\
\mathrm{W}^{\prime} & =\text { weight carried. } \\
\mathrm{D} & =\text { distance in feet. } \\
2240 & =\text { number of pounds in a long ton. } \\
\mathrm{C} & =\text { coefficient of traction. }
\end{aligned}
$$

The coefficient of traction varies for different rates of speed. For two, three, four and five miles per hour, it is approximately $x / 26$, $\mathrm{I} / 20, \mathrm{I} / \mathrm{I} 6$ and $\mathrm{I} / \mathrm{I} 4$, respectively. Thus a man weighing $\mathrm{I} 6 \mathrm{o}$ pounds, carrying 40 pounds and walking $\mathrm{I}_{5}$ miles at the rate of 3 miles per hour, will perform an amount of work equivalent to 353.57 foot-tons : 


$$
\frac{(160+40) \times 79200}{2240} \times \frac{1}{20}=353.57
$$

or, if at the rate of 2.5 miles per hour, 307.45 foot-tons.

The nature of the terrain has naturally very much to do with the amount of exertion required; a very muddy, sandy or dusty road enormously increasing the work of the soldier. He may even be obliged to give up the road to the wagon trains and pick his way alongside of it. A gently rolling country is better for him than a flat surface, as all the muscles concerned in locomotion are alternately brought into action and rested. The expenditure of energy is generally greatest when marching over rough and ascending ground. If, furthermore, we consider that, in the field, marching is only part of the day's work of the soldier, that he must also pitch and strike camp, load and unload wagons, do guard duty, etc., we realize that an average daily march of 15 miles, with full equipment, cannot be long exceeded without danger of overstrain and of seriously impairing his efthciency. 


\section{CHAPTER XX.}

\section{PERSONAL HYGIENE.}

The mode of life of the soldier who, in camp or in garrison, is constantly thrown in contact with his comrades, renders personal cleanliness and good sanitary habits especially necessary.

We bathe and wash the skin:

I. To promote and stimulate its physiological functions. These functions are of great importance. The skin, besides being the seat of the sense of touch, is an excretory organ, pouring out from one and a half to over four pints of sweat daily, and the regulator of the body temperature. It also contains numerous sebaceous glands yielding a semi-fluid, greasy substance which forms a tenuous lubricating film over many parts of the body. The sweat holds only one or two per cent. of solids, consisting of sodium chloride, fatty acids and, in case of disease of the kidneys or liver, some of the waste products normally eliminated by these organs. The evaporation of the sweat, that is, its conversion into vapor, can only take place by the absorption of heat, chiefly from the skin, so that the more rapid the evaporation the more active the cooling of the skin and the greater the reduction of the body temperature. Washing the skin, therefore, opens the pores or mouths of the sweat glands and promotes free perspiration, especially during muscular exercise when the temperature tends to rise. It also stimulates the sebaceous glands; but, on the other hand, repeated bathing, when accompanied by hard rubbing, may remove their secretion more or less completely and cause dryness of the skin; this, however, is seldom to be apprehended.

2. To remove dirt and prevent the breeding of germs on its surface. The dead superficial layers of the outer skin or epidermis are constantly thrown off and, togther with dried sweat, sebaceous secretion, dust and other outside impurities, form, if not washed, a foul coating which affords an excellent soil for the growth of microbes. Remlinger has counted 40,000 on a square centimeter of skin. Not infrequently they cause prickly heat, furuncles, ecthyma, cellulitis, whitlow, etc.. in persons careless of their personal habits. 
Any wound of a dirty skin is also much more likely to become seriously infected. It is well known that several of our most prevalent and dangerous infectious diseases, such as typhoid fever, cholera and dysentery, are aften propagated by personal contact, that is, through the conveyance of infected excretions on skin and clothing, but especially on the hands; hence the increased necessity, in the presence of one of these infectious diseases, to bathe and wash frequently.

3. To prevent fouling of the air by emanations resulting from the accumulation and decomposition of excretions and filth.

4. For the tonic and stimulating effects which cold water produces on the terminal filaments of nerves and, through them, on the whole nervous system.

DAILy ToILET. - Every man should wash his face, head, neck and hands every morning, in cold water. Tepid water is better for cleansing purposes and, if available, may be used, but should always be followed by cold water. Although it is well for the scalp to bc washed every day, soap should not be used upon it more than once or twice a week for fear of removing too completely the oil which nature provides, and making the hair dry and brittle. The hands should be washed with soap whenever soiled, before each meal, and the nails frequently cleaned and brushed.

The soap used on the human skin should preferably be neutral in reaction, with potash base and a slight excess of fat ready to combine with the alkali set free as the soap is dissolved (Unna). The water should be soft, that is, as free from lime and magnesia as possible; the addition of a little lime-water, soda, glycerine, bran or starch renders hard water less objectionable. Soap does not only act as a detergent in removing dirt, but has also considerable bactericidal power and is therefore a useful disinfectant for the skin as well as for soiled linen. This action does not appear to be due to either the alkali or the fatty acid of the soap, but to the combination of the two.

The mouth and throat, particularly when the seat of catarrh, ulcerations, abscess, etc., should be frequently sprayed, rinsed or gargled with solutions of boric acid, borax, chloride of sodium or peroxid of hydrogen; Dobell solution is a popular and very efficient preparation for the purpose.

The teeth demand especial attention. As soon as they give indi- 
cations of decay the care of the dentist must be sought so that they may be saved if possible. The preservaion of teeth means better health and probally increased longevity. Tooth-picks should never be of metal and must be used gently, lest the gums be wounded and become infected. The tooth-brush is necessary, but should be comparatively soft. It is easily contaminated, not only from the mouth of the person using it, but also from exposure to extraneous dirt, so that each bristle may become an inoculating needle. It is well; therefore, to wash it thoroughly after using, rinse it if convenient in a mild antiseptic solution, such as boracic acid, and then keep it in a bottle closed with a rubber cork into which the end of the handle is permanently stuck. Such bottle is particularly desirable while marching or traveling. Hygienic tooth-powder should contain as little hard and insoluble matter as possible in order not to bruise the mucous membrane, or lodge between the teeth; ordinary alkaline soap is preferable.

The nostrils require some care; they can be sprayed daily with Dobell solution, or else liquid vaseline or a tepid solution of common salt may be snuffed up. The frequent snuffing of cold water is not advisable.

The ears collect much dirt and should receive their share of the daily toilet. Considerable wax is often secreted at the mouth of the auditory canal and should be removed at least once a week; this is best effected with a little absorbent cotton or gauze wrapped around the point of a little wooden stick or tooth-pick and dipped in tepid water or diluted alcohol; the parts must be wiped dry afterward. To push the stick deep into the canal is unnecessary and dangerous.

The beard, if worn at all, should be neatly trimmed, brushed every day and frequently washed. It is best for the soldier, at least when in barracks, to shave the whole face, although the wearing of the mustache, if properly clipped, is unobjectionable.

The hair should be kept short, less so in the tropics, and for its ordinary daily care needs nothing more than comb and brush. Let the brushing be free but not so hard as to scratch or irritate the scalp. Once or twice a week it may be shampooed with soap or an alcoholic lotion.

It is well known that contagious diseases, such as syphills and various skin affections (tinea, favus, acne, impetigo, forms of eczema, etc.) can be conveyed by the hands, instruments and imple- 
ments of the barber. Every soldier, therefore, should endeavor to shave himself. But as this cannot be enforced, post barbers should be required to keep their shops and their persons scrupulously clean and take stch sanitary measures as will prevent the transmission of disease. For the disinfection of shaving mugs, scissors, razors and brushes, boiling water is efficient but somewhat difficult of employment. Alcohol followed by a solution of formalin (4 per cent.) or tricresol ( $\mathrm{I}$ per cent.) is preferable. Powder puffs should be replaced by wads of gauze which are thrown away after being used.

It is imperative that each soldier should have his own toilet articles, such as soap, brushes and towels; if he does not shave himself he must have his own 'mug, shaving-brush and razor in the barber shop. Roller-towels should be banished from the lavatory. The use of toilet articles in common is very repugnant to cleanly men and insanitary; it should be severely forbidden. As a rule, only careless men, those most likely to transmit infective disease, try to borrow such articles. Pipes and cigar holders, likewise, must be strictly reserved for their owners.

The feet get soiled very quickly, not only from outside dirt but also from their own secretions, the product of numerous sweat and sebaceous glands. These secretions, especially between the toes, unable to escape, collect and soon form a culture medium for many micro-organisms, some of which produce the repulsive smell so noticeable in certain individuals. Furthermore, the retained sweat soaks the epidermis, softens it and renders it more liable to rapid abrasion.

The feet should be washed whenever the body is bathed, that is, at least twice a week, but whenever convenient facilities exist it is a good practice to wash them in cold water every morning. Soldiers who do much walking or marching, especially in the field, should bathe them every afternoon, after getting into camp, as already noted. In case of great tenderness of the epidermis, very little soap should be used, and only cold water, to which alcohol or an astringent can be added. It may even be better to use simply a wet cloth. In that condition of the feet known as bromidrosis, or stinking feet, which occurs in one or two per cent. of soldiers, the feet sweat profusely and give off a fetid, repulsive odor. The skin is soft, white and easily rubbed off ; in old cases it may become congested and eczematous. The usual treatment consists in painting the affected 
area with a 10\% solution of formalin, taking care to avoid fissures and abrasions. Applications are generally made every other day, and half a dozen may be necessary for a cure. In the German and French armies, a ro\% solution of chromic acid is used, and repeated in about a month.

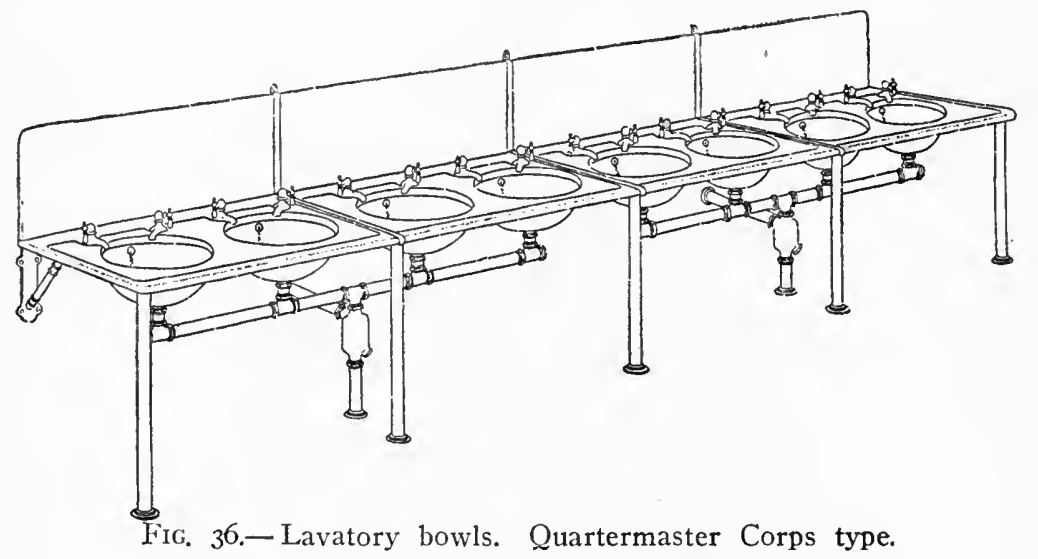

Each company, in our posts, has it own lavatory which, besides water-closets and urinals, contains wash-bowls, shower-baths, laundry tubs and sinks, with all necessary plumbing fixtures. (Fig. 129.) The bowls are of porcelain and supplied with hot and cold water. (Fig. 36.) There should be at least one for each five men. They may be thus safely used in common if kept scrupulously clean ; it is always desirable, however, that one or two in each company be reserved for men suffering from contagious affections. Any man who so desires can use a removable individual basin. A very useful addition to the lavatory would be a special porcelain or zinc-lined trough set up about a foot above the ground to enable the men to wash their feet at any time.

Soap is furnished by the Quartermaster Corps, therefore each man should be required to have and use his own piece. An excellent sanitary and economic device is that illustrated in Fig. 37, in which as much of the soap as necessary is grated off by turning a crank, without any handling of the cake. Another efficient and excellent device, commonly seen in hotels and other public places, and likewise well adapted to garrison lavatories, consists in the use of liquid soap from small receptacles conveniently placed over the faucets. 
Drinking cup.- It is well known that there is danger in drinking from a cup or glass used in common by a number of persons without washing or disinfection, danger not only from mouth and lip affections but also from various infectious germs lurking in the saliva of carriers. For this reason the common cup is gradually disappearing from all public drinking tanks and replaced by the hubble fountain which enables the drinker to absorb as much water

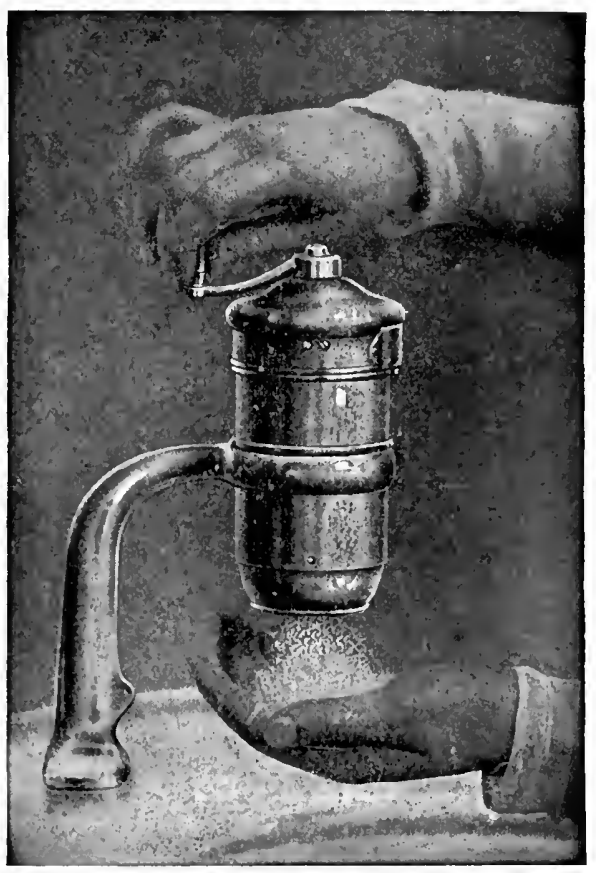

Fig. 37.- Soap gravulator in use. (Hygienic Soap Granulator Co.)

as he wishes without touching the fixtures with his lips, a device as economical as it is sanitary. (Fig. 38.) This bubble system should be installed in all company barracks, as well as in standing camps. In its absence, the soldier must use his individual canteen cup.

BATHS.- The short morning bath is the rule for everybody who can do so. The soldier can seldom enjoy this luxury, nor is it necessary for cleanliness, but whenever the facilities are adequate he should be required to bathe the entire body twice a week. 
A bath can be taken at various temperatures. It is cold when ranging from $40^{\circ}$ to $80^{\circ} \mathrm{F}$; t tepid, from $80^{\circ}$ to $90^{\circ}$; warm, from $90^{\circ}$ to $100^{\circ}$, and hot when above $100^{\circ}$. The water has also different effects according to its temperature; thus for cleansing purposes warm water should be used, while if a bracing, tonic effect is desired the cold bath is indicated. The benefit of both cleansing and tonic effects can easily be obtained by following the warm bath by a cold sponging or shower, or a plunge into cold water.

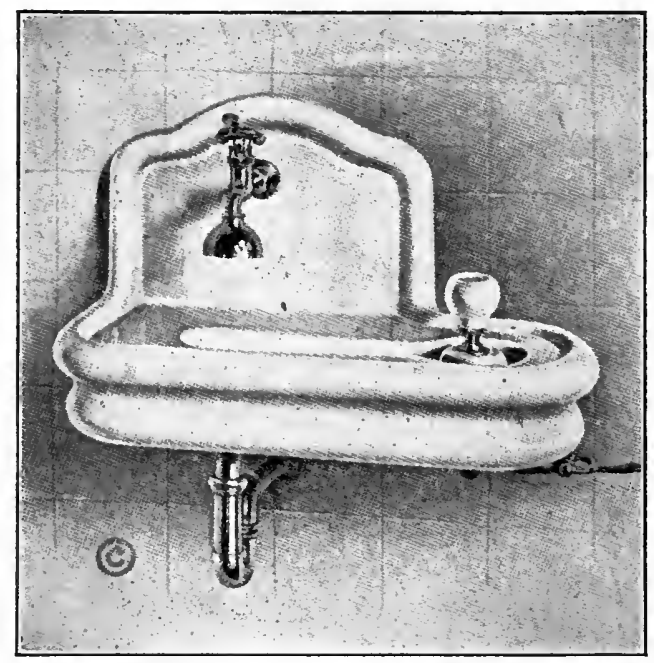

FIG. 38. - Wash-hand basin, with sanitary bubbling cup at the right, which flows when the lever below is pressed down. (American Medical Association Building.)

That the cold bath, either with sponge, in tub or as shower, has a decided strengthening effect is undoubted and therefore should be preferred by those who can safely take it. Not only is it a general tonic, but it also hardens the peripheral nerves and enables them to stand exposure with greater impunity, so that the cold bather seldom contracts affections of the nose, throat and bronchial tubes. The shock of the cold bath is severe and cannot be borne by every one; it is positively dangerous for men with cardiac weakness or degenerate arteries. The test of its usefulness is in the immediate after-effect which should be a pleasurable sensation of warmth and 
increased energy. Should the bather, on the contrary, remain chilly and depressed, he should abstain. Cold sponging can often be enjoyed by delicate persons if standing with feet in tepid water.

The warm bath dilates the slin capillaries and causes free perspiration. It has a general soothing, relaxing and sedative effect, removing the soreness of tired muscles and restoring sleep in insomnia.

The Turkish bath and the Russian bath are taken in air heated to very high temperature $\left(120^{\circ}\right.$ to $150^{\circ} \mathrm{F}$.), dry in the former, moist in the latter; the body is vigorously rubbed and massaged, then bathed in warm water, and the process ends with a cold douche or plunge, and thorough drying. Either Turkish or Russian bath is somewhat severe and should be carefully tried and adjusted to the resistance of the individual. It produces a perfect cleansing of the surface, abundant elimination of waste matter through the profuse sweat, and relieves internal congestion. It is especially useful to well-fed men of sedentary habits and therefore seldom needed by soldiers.

In the Army, the ordinary tub bath is inconvenient, expensive and insanitary, and should seldom be seen outside of post hospitals. It is being entirely superseded by the shower bath which has superior advantages and none of its objections. The shower bath requires a simpler, more easily handled apparatus, and consumes much less water and time. With it the transmission of disease, not unlikely in a tub, is made impossible. Moreover, the percussion of the water upon thie body intensifies the tonic effects of the cold water. To get the full benefit of this percussion the nozzle should be movable so that the shower may" be projected at any angle. Although both warm and cold water are provided, the men should gradually accustom themselves to cold water, at least in summer; but warm water, whenever used, must always be followed by cold water, or as cold as can be borne.

While bathing, the soldier should give special attention to the hairy parts of the body, under the arms and about the genitals and anus. The secretions from the mucous membrane of the prepuce, particularly in men with long foreskin, soon become acrid and irritating and should be regularly washed out. In such cases circumcision is often advisable. The skin around the anus, as well as 
the anus itself, especially in case of hemorrhoids, must also be thoroughly cleansed.

LAUNDERING. - But little benefit will be derived from washing the body if the linen in contact with it and constantly absorbing its secretions is not frequently changed. Underclothing wet with perspiration should be removed and dried at the first opportunity, before being worn again. No man, while in barracks, should be permitted to go to bed in the underclothing worn during the day. Each soldier is expected to provide himself with two or more sets of pajamas for night use.

In the process of laundering, the inen is first soaked in cold or tepid water, then washed in hot or boiling water, with soap, and finally rinsed out. The water in which it is first soaked becomes charged with a large amount of organic filth, including many more germs, according to Miguel, than are contained in ordinary sewage. This polluted water is therefore particularly dangerous and must be disposed of in such manner that it will not contaminate the soil nor the water-supply. Clothing which is boiled is thereby disinfected, that is, freed from all ordinary pathogenic germs. Boiling, however, does not remove the ill-smelling organic dirt, especially from woolen articles, imparted by an unclean skin; this must be done by subsequent rinsing in running water or pure water frequently changed. Properly laundered clothing, when tested by the nose, should have no other than a clean, sweet smell.

In our garrisons there is no general regulation governing the washing of clothing. A few laundry tubs are provided in the lavatory of each company for the washing of such articles as men may find necessary to do. (Fig. 39.) Each soldier is expected to have his own laundry done as best he can, sometimes at much inconvenience and expense. The weekly wash thus received from various outside places is always a source of danger since it may introduce vermin and disease germs into the garrison. It is therefore highly desirable, as a sanitary measure as well as for the convenience of the men, that the laundry work should be done under official supervision, either in connection with post exchanges or by means of properly equipped post steam laundries. The Act making appropriation for the support of the Army for the fiscal year I908, provides "for the construction, operation and maintenance of 
laundries at military posts in the United States and its island possessions" by the Quartermaster Corps. This is a step in the right direction and may lead to a complete solution of this vexed problem.

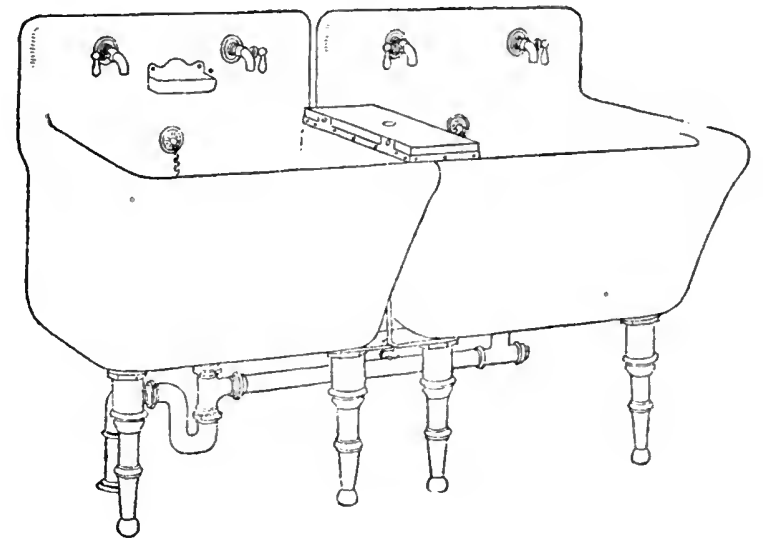

Fig. 39.- Laundry tubs. Quartermaster Corps type.

In the field, the cleanliness of clothing must not be neglected whatever may be the difficulties in the way. It is well known that some of our most dreaded camp diseases result from the conveyance of infectious matter from man to man by the skin and clothing. Therefore, in the absence of special provision for the purpose, each man must be required to wash and boil his own linen in camp, the only material needed being water, kettles to boil it in. and soap. Every precaution, however, must be taken to secure the proper disposal of the waste water. When a command is camped on a stream, the part used for washing should be the lowest, or the farthest removed from that used for drinking purposes.

Care of boi'cls.-An important feature of personal hygiene is that which relates to the evacuation of the bowels. This is a bodily function which must be attended to with great regularity, that is to say, daily and at the same hour. The proper time for most men is as soon as convenient or possible after breakfast. The habit of so doing is very easily formed so that the desire for evacuation is always felt at the right moment and should be promptly complied with. Carelessness in heeding this call of nature leads to bodily 
discomfort, digestive disturbance, constipation and the various ills which, soon or late, follow in its train. Such neglect is a distinct violation of one of the primary laws of nature. The young soldier who enjoys the advantages of regular meals, wholesome food and active exercise, should never have occasion to take cathartic medicines. Any sluggishness of bowels should be overcome by a change in the diet, that is, less meat and more vegetables and fruits, massage of the abdomen and more exercise. In the field, where the regular habits of garrison life are more or less disturbed, regularity in bowel evacuation is not so easily maintained and requires greater care, but is also quite possible and fully as necessary.

The washing of the hands after defecation is one of the cardinal precepts of modern hygiene. Inasmuch as many people are the unconscious carriers of the germs of various infectious diseases in their intestinal canal, the contamination of their fingers with those germs in using toilet paper, or otherwise, is always possible, especially by men of unclean habits. Infection may thus be conveyed to the squad-room or tent, mess-room and kitchen. Therefore, washing of the hands with soap and water, preferably running water, must be insisted upon and necessary conveniences provided for it. (See pages 495, 648, 742.)

Skin parasites, see page $\mathrm{I} 12$.

Bedbugs, lice and fleas, see pages 495, I44, I45.

Swimming and swimming pool, see pages 227, 228.

Use of tobacco, see page I33.

Addiction to narcotics, see page $\mathbf{I}^{8} 8$.

Venereal diseases, see page I22.

Alcoholic beverages, see page 4I4.

Rules in eating and drinking, see page 406. 


\section{CHAPTER XXI.}

\section{WATER.}

Water is necessary to all animal and vegetable life. In man it forms over 60 per cent. of the weight of the body and is indispensable to the ingestion and absorption of food and the maintenance of the normal composition of all our tissues. While a mammal can use up almost the whole of the body fat and 50 per cent. of its protein before dying, it becomes moribund when it has lost little more than Io per cent. of its body water $(L$. Hill $)$. The water of the body being constantly excreted through the skin, kidneys and intestines, and exhaled through the lungs, must be constantly replaced. To supply this loss, from 70 to Ioo ounces are consumed daily, about one-third of which is contained in our food.

The quantity of water required for all the ordinary needs of the body may be liberally computed in gallons as follows: For drinking, I ; cooking, 2 ; ablutions, 2 ; laundry, 8; water-closet, 6 ; shower bath, 5 ; total, 24 gallons. Where strict economy is necessary, onehalf this quantity, or 12 gallons, may be made to answer the purpose. So much water, however, is wanted for needs not directly connected with the human body, namely, for animals, street watering, fountains, factories, etc., that the minimum daily supply per head in our cities should never be less than 100 gallons. In fact, sanitary engineers, in determining the capacity of waterworks, generally base their calculations upon a minimum of 150 gallons per capita. Much of this amount, however, is recklessly wasted. In our garrisons, a supply of from 50 to 75 gallons is amply sufficient for foot troops, but twice that amount must be provided for mounted troops.

\section{WATER IN NATURE.}

All water used by man comes from the condensation of the aqueous vapor of the atmosphere in the form of rain, snow, fog and dew. The larger proportion of this water remains on the surface as brooks, rivers, ponds, lakes and ocean. The rest percolates through the porous earth 'until stopped by impervious strata, at various depths. 
Rain-aiater is the purest of natural waters. It would be more correct to say that it is the least polluted, for it is never absolutely pure, containing many of the constituents and impurities of the atmosphere. The air which it dissolves is particularly rich in oxygen (22 to 30 per cent.) and in carbon dioxid ( 2 to Io per cent.). Besides coal, sand and clay dust, and various other mineral substances such as sodium chloride, calcareous salts, etc., in almost infinitesimal proportion, rain-water contains also as constant constituents, especially in cities, ammonia (mostly as carbonate) and nitric acid, as well as a minute quantity of organic matter and a few bacteria ( 4 to I9 per cubic centimeter according to Miguel).

Rain-water is highly aërated, wholesome and palatable. The absence of earthly salts makes it very soft, an excellent solvent of soap, and admirably suited for washing and cooking purposes; but, for drinking water, this absence of salts is rather a defect than a quality.

Cisterns for the collection of rain-water are now seldom used at our military posts, but there are times and places when and where they may be necessary. Of whatever material constructed,

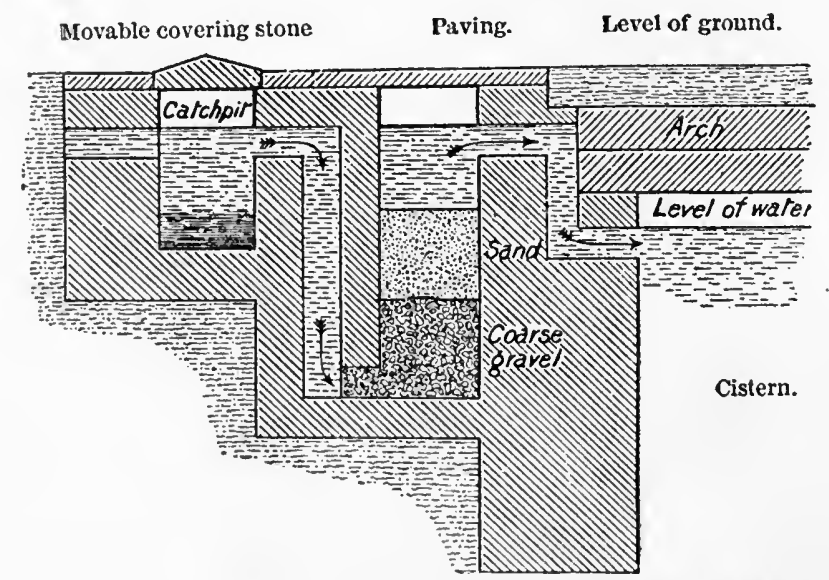

FIg. 40.-A filter for rain-water. (Notter and Firth.)

their first requirement is to be water-tight, so as to preclude leakage inward or outward. If of stone or brick, they should be thoroughly cemented; ordinary mortar will not do for the lime dissolves in water and makes it hard. If of iron, an unpleasant taste and color 
are imparted to the water unless covered with a protective coating. Galvanized iron, unless of the best quality, may be attacked by water charged with nitrates and its component zinc dissolved, a metal disagreeable to the taste and poisonous when continuously absorbed. A cistern must be well ventilated but kept dark to prevent the growth of animal and vegetable life. It is an ideal place for the breeding of mosquitoes and therefore must be carefully screened. The first portion of the rainfall, more or less contaminated by the roof surfaces, should be rejected; this is generally done by some automatic device. Should the water flow over the sloping surface of adjoining grounds, it may be advisable to pass it through a sand filter before letting it into the cistern (Fig. 40).

River-ciater. - River-water is of complex and variable composition, according to the character of the affluents of the river, the geological nature of the water-shed feeding it, the number of towns and factories located on its shores and the degree of cultivation of the adjoining lands. It may be nearly as free from organic matter as rain-water or so polluted by human sewage and wastes from factories as to destroy all fish, batracians and mollusks. The pollution is greatest in spring, when the melting snow washes into it the excrementitious matters scattered on the shores over the frozen ground. In countries having dry and rainy seasons, the water is most polluted at the onset of the rainy season, generally marked by an increase of intestinal disorders. It always contains a large amount of matter in suspension, especially in our western and southern rivers, ranging from about 15 parts per $1,000,000$ in the Hudson River at Albany, to about I,000 parts in the Mississippi at St. Louis, and much more in the Rio Grande and Colorado.

River-water, as it flows, undergoes a very important spontaneous purification, so that at a distance of from 20 to 50 miles below a large city, the water has nearly recovered the degree of organic purity that it had above it. Thus the river Seine, 25 miles below Paris, is hardly any more contaminated than above that capital. The Illinois River, some 24 miles below the point where the Chicago sewage canal empties into it, shows a bacterial content but slightly in excess of that of the local tributary streams (Jordan). This self-purification is the result of the following agencies: I. Dilution by tributary streams, especially when their water is purer or of different temperature and composition.

2. Sedimentation, the natural 
effect of gravitation, whereby all organic and mineral particles tend to fall to the bottom, much more rapidly, however, in the quiet waters of lakes than in rivers; micro-organisms adhere to these particles and are carried down with them, hence the comparative freedom from germs of the water of our western rivers, rich in suspended sand and clay, after it has been allowed to settle. 3. Solar action, which exerts a well-marked oxidizing and destructive effect upon the organic matter and microbes of the surface and down to a depth of a few feet, according to the intensity of light and transparency of the water. 4. Biological action, probably the chief and most powerful purifying factor. Numberless animalcules, as well as aquatic cryptogams (algæ, infusoria, etc.), feed upon organic matter, but their action is slight compared to that of the usual nitrifying bacteria which decompose and mineralize animal and vegetable substances with great energy, as will be explained later. This action of bacteria requires the presence of an abundant supply of oxygen and is therefore heightened by falls and rapids. Along with this oxidation and decrease of organic matter there is a corresponding fall in the number of organisms, large numbers of which are thus starved out.

Despite this self-purification, there is in the streams flowing through well-settled parts of all countries, a residuum of contamination which makes their water unsafe for drinking without undergoing some form of artificial purification. In England, the conclusion of a commission appointed to make a special study of the subject was that there is no river wide or long enough, in that country, to completely purify itself.

Lake-water, compared to river-water, contains less matter in solution and suspension, nor is it so likely to become polluted, except at certain points along the shore where sewage or waste waters are discharged; but this pollution seldom extends very far in large lakes and leaves the water relatively pure half a mile or so inward. In the most contaminated part of Lake Michigan, off the Calumet River, there is a zone of constant pollution extending somewhat over a mile, and a zone of occasional pollution extending one or two miles farther; beyond the 4-mile limit the water is remarkably pure, giving bacterial counts as low as 8 bacteria to the c. c.

Spring-water is water that has percolated through deep strata and which therefore is thoroughly filtered. It is cool, clear, well 
aërated, sparkling and palatable. It is also comparatively free from organic matter and absolutely so from pathogenic germs, but likely to be rich in mineral salts.

WeLls.- Ordinary dug wells are objectionable but sometimes inevitable. The deeper they are the more completely filtered and purer is the water that flows into them. However, provided a well traverses at least one impervious stratum, the depth is less important; thus a well that penetrates through two impervious layers of clay

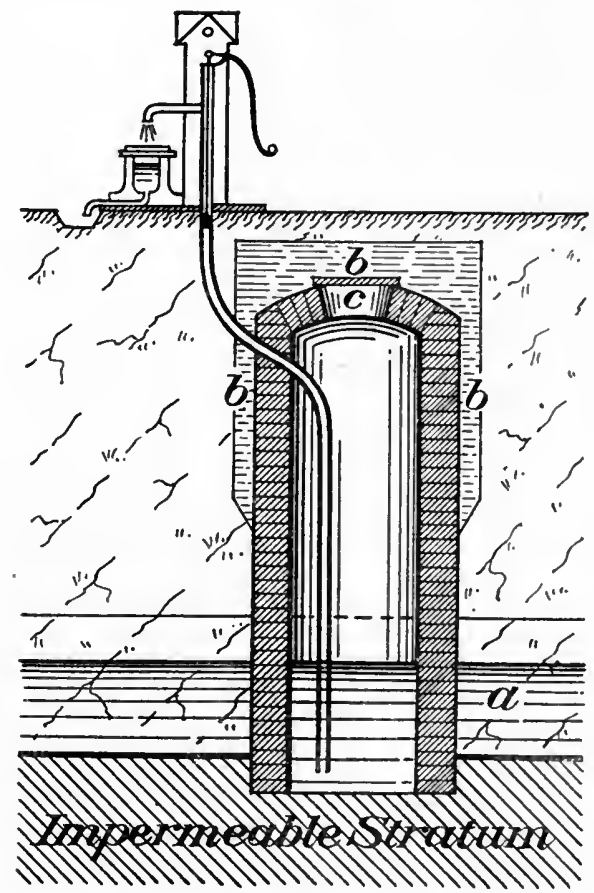

FIG. 41.-A properly constructed well; $a$, water stratum; $b$, layer of clay; $c$, manhole.

or rock, even if only 30 feet deep, possesses all the advantages of a deep well, that is to say, regularity of output and constancy of purity, for the water is obliged to travel a long and circuitous route. Shallow wells are dangerous, being fed by surface waters and liable to be polluted by many impurities. The surface area drained by a well should be kept as clean as possible, especially from human 
excreta. This area is a circle whose radius is about twice the depth of the well, therefore being 200 feet all around a well roo feet deep. It varies according to the nature of the soil and the daily fall of the water produced by pumping, widening in direct proportion to this fall.

A well, to be above suspicion, must comply with the following requirements (Fig. 4I): It should traverse the entire aqueous stratum (a) and extend to the subjacent impervious clay or rock; it should be lined throughout, or at least above the water, with a well-constructed masonry wall vaulted at top, with manhole in center (c) and thoroughly cemented inside, but with enough drain holes in the aqueous stratum. The pump, instead of being placed directly over it, should be by the side of it. If there be especial danger of surface contamination, the top and outside of the wall, to half its depth or more, may be covered with a layer of well-tamped clay $(b)$. Sometimes it is preferable to build up the lining wall two or three feet above the surface of the ground; in such case the well must. be tightly closed at top and the ground made to slope away from it.

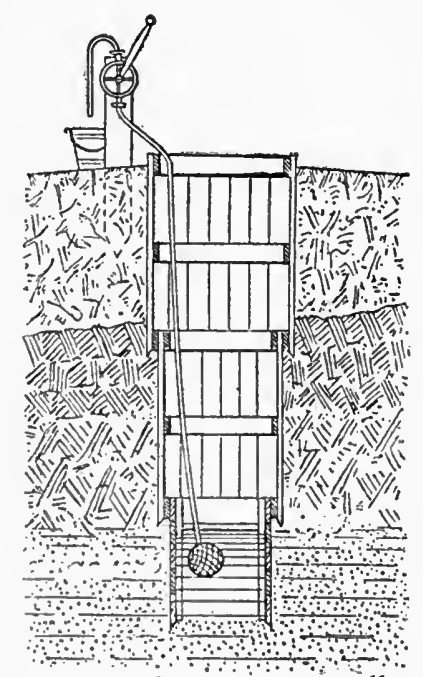

Fig. 42.- Temporary well for camps, with portable pump. (Munson.)

In locating a well it is important to ascertain the trend of the ground water and make sure that it is not likely to be infected. It should be above and sufficiently far away from cesspools and latrines. Even when the movement of the ground water, under a cesspool, is away from the well, there is still danger of pollution if the drainage from the cesspool, as it spreads in all directions, reaches the well before being carried off by the ground water (Fig. 43). The best and easiest test to ascertain the possibility of such infection is by the use of a strong alkaline solution of fluorescine, which, if thrown into the cesspool will, in case of leakage into the well, produce a characteristic red tint in the suspected water, recognizable to the naked eye even in a dilution of $\mathrm{I} / \mathrm{100,000,000}$ and, by 
its strong fluorescence, in a still much higher dilution. Sodium chloride can be used in the same manner, and its leakage into the water ascertained by the usual silver reagent, having, first, ascertained the normal amount of chlorides in said water. For the same purpose, the culture of certain germs has also been recommended, such as the ordinary beer yeast (Miquel) or, still better, the bacillus prodigiosus which is seldom found in any water, harmless and readily identified by its bright red color.

Pipe or tubular wells are greatly superior to dug wells, in all situations. The tube prevents contamination from surface waters and can be sunk until good pure water is reached, at least through the first impermeable layer of clay, so as to be entirely beyond the possibility of infection. Some of our American cities are being supplied entirely from deep tubular wells with water organically pure, and whenever a sufficient amount can thus be obtained, not containing mineral constituents in excess, it should be preferred to any that requires some form of purification.

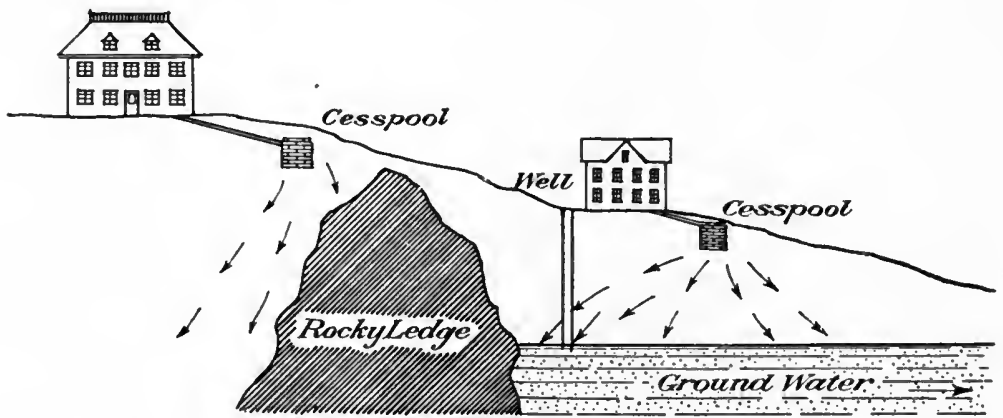

FIG. 43.- Showing how a well may be polluted from cesspoo! below it and protected from one above it.

\section{EXAMINATION OF WATER.}

E.rternal Qualities. - Water may be blue, green, gray, yellow or red according to its depth, matter in suspension and reflected sky, so that color (unless distinctly muddy) is not significant of its quality. It may even be dark reddish-brown as in the Dismal Swamp and many streams, the effect of the peaty beds over which it flows, or sometimes the result of a combination of the tannic acid dissolved from aquatic plants, with iron oxids. This dark-colored 
peaty or inky water used to be preferred by ship captains, when, going on long voyages, on account of its keeping qualities. Several of our western rivers have received the name of Colorado (red) because of the color of the water seen by reflected light, due to a large amount of reddish clay in suspension.

Drinking water should be perfectly clear and transparent, but turbidity caused by mineral matters in suspension is quite compatible with good quality, after sedimentation. A bright, sparkling water may be assumed to contain much oxygen and carbon dioxid in solution, but does not contraindicate the presence of infectious organisms.

But little reliance can be placed on taste in determining the value of drinking water; it may be very palatable and yet contain dangerous bacterial life; while the taste of salt, magnesia, iron, sulphur, etc., although objectionable, does not indicate harmful constituents. Iron is detectable by taste when present to the extent of I/5 grain to the gallon, and salt to the extent of 75 grains. The use of water with distinct taste of sodium and magnesium salts, not uncommon in the western States, is often, at first, accompanied by slight bowel trouble but, as a rule, the system soon accommodates itself to it. A water from which the gases have been more or less expelled is flat and unpalatable, as, for instance, boiled water not sufficiently aërated, but quite safe.

Water should be odorless; any smell is suspicious, especially so if of animal origin, and of a fecal or putrefactive character. Odors are more readily detected if the water is heated and shaken in a half-filled quart bottle. Decaying vegetable matter (mostly algæ and diatoms) often produces a repulsive taste and odor in the water of small, badly-constructed reservoirs, rather intensified by heating. They are mostly noticed in the fall when the chilled water of the surface falls to the bottom, causing vertical currents and a stirring up of the strata. Such water, although decidedly objectionable, is probably harmless.

\section{CHEMICAL EXAMINATION.}

The chemical analysis of water, that is, the determination of its mineral and organic constituents, is essential to estimate its qualities for drinking and other purposes; but it does not give sufficient information; it tells nothing of the presence of micro-organisms, 
especially of the germs of infectious diseases, and must therefore be supplemented by a bacteriological examination. Both are necessary; they mutually help and complete each other.

It is not intended here to describe analytical processes, but only to indicate the mineral and organic substances usually found in water, and show their sanitary significance, that is, to what extent they may be injurious or indicative of the presence of infectious organisms.

Total Solids. - The amount of total solids in solution conveys useful information but is not of much sanitary value. Smart sets the safe maximum limit at 300 parts per million, and Wanklyn at 575 , but many of our waters, particularly those of our western rivers, greatly exceed those figures without being considered harmful.

Hardness. - Water, in nature, always contains mineral salts in variable quantity, and we may assume that these salts supply some of the needs of the body. Water containing them to a moderate extent is therefore much better than distilled water for drinking purposes. The proportion, however, should be small and the water remain "soft." Soft water, that is, water free from an excess of earthy salts, is best for all purposes, drinking, cooking, washing, steam boilers and manufactures. Water is said to be "hard" when it contains so much earthy salts that soap does not readily form a lather with it. The hardness is "temporary" or "permanent." Temporary hardness is caused by calcium and magnesium carbonates held in solution by the free carbon dioxid present in the water. Boiling expels this gas and precipitates the salts, leaving the water soft; the same result is obtained by the addition of fresh lime (Clark's process) which combines with the free $\mathrm{CO}_{2}$ (one gallon of clear lime-water to every ro gallons of water). Permanent hardness is caused by calcium sulphate and other salts of calcium and magnesium, not carbonates. Boiling has but little effect upon such water; it is generally treated with sodium carbonate, which sets up a reaction resulting in the formation of neutral and inert sodium sulphate and insoluble carbonates which are precipitated. When soap is used in hard water, it is at once decomposed with formation of insoluble calcium stearate, and does not begin to have any cleansing or detergent effect until all the calcium has thus been combined; hence the enormous waste of soap when used with such water. 
The degree of hardness of water is readily estimated by Clark's soap test, in which a standardized soap solution is poured into a measured quantity of water until a persistent lather is formed. Permanent hardness is estimated by first boiling the water and getting rid of the carbonates.

The average hardness for American rivers, expressed in so many parts of calcium carbonate per million of water, is 50 for soft, and I 50 for hard water (Leeds' standard).

The metals most commonly found in solution in water are lead, copper, arsenic, manganese and iron, but rarely in sufficient quantity to be obnoxious, except iron which, when exceeding the proportion of I part per million, renders water unsuitable for washing white goods, bleaching and dyeing; when exceeding 3 parts it is no longer fit for drinking. Iron waters are usually treated simply by aëration which causes oxidation; the oxid is removed by sand filtration.

Organic MATter. - The determination of organic matter in water is much more important from the sanitary point of view than that of mineral substances, for it is in it that danger lurks. It is estimated by the combustion process, that is, by evaporating a given quantity of water in a platinum dish and, after weighing, gradually raising the dish and its contents to redness, and ascertaining the "loss on ignition." It is important to observe the intensity of the charring and the presence or absence of fumes; the latter, if present, may indicate the character or source of the organic matter. Water of high organic purity should give no appreciable blackening nor odor. According to Smart: " The blackening during the process is of more interest than the mere loss of weight. No matter how few parts are lost, if the lining of the capsule blackens all over and the carbon is afterward dissipated with difficulty, the water is to be viewed as suspicious. What are called "peaty" waters here constitute the exception."

Another method of estimating organic matter is by determining the amount of " required oxygen" to oxidize it. It consists in the addition of a solution of potassium permanganate of known strength to a measured amount of water acidified with sulphuric acid and heated to the boiling point, until the red color persists. The amount of oxygen required, in good water, should not exceed two or three milligrams to a million milligrams ( 1 kilo) of water.

A pretty constant relation has been noticed between the " required 
oxygen " and the carbon of organic matter, the latter being obtained by multiplying the former by 2.50 or 3 . Again, the proportion of carbon to nitrogen affords evidence as to the origin of the organic matter; a ratio of 3 to I, or less, would indicate animal matter, while if as high as 8 to $\mathrm{I}$, matter chiefly or entirely of vegetable origin.

The percentage of dissolved and available oxygen is of great value in determining the quality of water, for the higher this percentage the greater is the amount of organic matter that can be disposed of without objectionable effects. It is estimated that to maintain aquatic animal life in its normal state, and not produce nuisances, water should contain at least 70 per cent. of dissolved oxygen. A fair index of pollution is furnished by the percentage reduction of dissolved oxygen when the water is kept in tightly closed bottles and exposed to a constant temperature for a definite length of time.

Carbon is a constituent of every living tissue, but, from the sanitary chemist's point of view, of little importance.

It is from nitrogenous organic matter (nearly always from animal sources) that we obtain our most useful information; of its constituents, four are of especial significance, namely: chlorine, ammonia, nitrites and nitrates.

Chlorine is almost invariably found in water, generally as sodium chloride (common salt) washed from the air or soil, or added from cess-pools. Salt, of itself, is harmless, but, when in excess, shows probable sewage contamination. Ordinary sewage contains from I Io to 160 parts per million of chlorine, which comes almost entirely from the urine, the solid excrement containing but a minute proportion. Before estimating the significance of chlorine it is necessary to know the normal amount found in the waters of the region, for this is very much influenced by the proximity of the sea and the existence of local salt strata. Leeds' standard for American rivers is from 3 to IO. Frankland places the permissible limit at 5 .

Ammonia exists in water as free ammonia and albuminoid ammonia. Free ammonia, either really free or as an ammonium salt, passes over unchanged with the steam when the water is distilled. Albuminoid ammonia does not exist ready formed, but is a product of the decomposition of organic nitrogenous substances by the action of potassium permanganate. Rain-water, especially when collected near large cities, often contains a large proportion of free ammonia, while river-water seldom shows more than traces of it. As water 
filters through the porous earth, its ammonia is rapidly converted into nitrites and nitrates, so that the presence of this gas in any considerable quantity in shallow wells indicates probable recent contamination with animal matter. Growing plants have a marked influence in reducing free ammonia; thus a lake which contains as much as 0.57 in January may not show any at all in August (Drown). A high proportion of ammonia, without animal contamination, is likely to be found in waters from ferruginous swampy regions, owing to the action of iron oxids upon organic natter. Likewise, much free ammonia may exist in deep-well waters of excellent quality, due to the reduction of nitrates and nitrites by sulphide of iron or other agent, such nitrogen salts being the result of oxidation in past ages, and in nowise indicative of pollution $(C . B$. Forr). In testing for ammonia, the rate at which it is evolved, according to Smart, is as important as the total amount: "Gradual evolution of albuminoid ammonia indicates the presence of organic matter, whether of vegetable or animal origin, in a fresh or comparatively fresh condition, while rapid evolution indicates that the organic matter is in a putrescent or decomposing condition."

According to Wanklyn, water containing over o.I5 of albuminoid ammonia should be condemned; but Mason thinks that many waters, especially brown, peaty waters, of proved wholesomeness, may far exceed this proportion. The following maximum limits are proposed by Leeds :

Free ammonia

Albuminoid ammonia
.OI to .I2 per million.

.Io to .28 per million.

An excess of free ammonia over albuminoid ammonia, indicating an active process of decomposition, is always a suspicious sign, unless both numbers be low.

Nitrites result from the oxidation of the ammonia of organic matter, the second step in the process of nitrification, or may likewise be due to the reduction of preexisting nitrates. Being transition products, their presence in ground or surface waters is usually evidence of active fermentative changes and, even in the slightest proportion, should always be looked upon with suspicion. In spring and deep-well water, Frankland has shown that they are without significance, being generated by the deoxidation of nitrates "brought about either by the action of reducing mineral substances, such as ferrous oxid, or by that of organic matter which has been imbedded 
for ages, or, if dissolved in water, subjected to exhaustive filtration." The absence of nitrites proves nothing; it may be due to lack of available oxygen and does not show the absence of dangerous organic matter. Leeds' standard for American rivers is 0.003 . Good water, unless from deep well, should not exceed o.or.

Nitrates represent the completed process of the oxidation of nitrogenous organic matter, the process of nitrification; they are the ashes of organic matter and therefore simply indicate contamination at some previous time. This contamination is more likely to be of animal than of vegetable origin, not only because of the greater quantity of nitrogen present in animal matter, but also an account of its more ready decomposition. Nitrates form very slowly in rivers, animal matter being much less actively oxidized in running water, especially if deficient in dissolved oxygen, than in water percolating through the soil, and are, besides, constantly deoxidized by the addition of fresh organic pollution. A marked departure, either by increase or decrease, from the usual proportion of nitrates in any water may be taken as evidence of contamination. There are two natural sources of nitrogen which should be considered in estimating the normal proportion of nitrates in the water of any region: the first is washed from the atmosphere as nitric acid and amounts from 0.15 to 0.40 ; the second is washed from soils in which certain leguminous plants are cultivated (alfalfa, clover, cowpea, etc.) and where it is stored by various kinds of bacteria which abstract it from the air. (See page 5 IO.)

Leeds' standard for American rivers is I.I I to 3.89. Good surface water should not exceed I or 2 parts. Spring or deep-well water may contain a much larger amount without significance, having dissolved it while filtering through various strata, or from old beds of nitrified organic matter entirely free from organisms. When the proportion of potassium or sodium nitrate exceeds 0.30 gram per liter, the water acquires well-marked diuretic properties.

\section{MICROSCOPIC AND BACTERIOLOGICAL EXAMINATION.}

Water contains many forms of low cryptogamic life, such as fungi, algæ, desmids, diatoms, sponges, infusoria, etc., which have but little effect upon its sanitary value, except in occasionally producing very disagreeable odors. Some branching forms of minute fungi (cladothrix, crenothri. and beggiatoa) multiply rapidly and may clog 
pipes and drains, or sometimes become objectionable on account of the large amount of iron which they secrete; they do not grow in the absence of sunlight. Water may also contain the eggs of intestinal parasites such as ascaris, oxyurus, trichocephalus, tænia, ankylostomum, etc. It ordinarily abounds with many forms of bacteria, nearly all of which are saprophytic and probably more beneficial than harmful. Some of them, however, have been accused, by causing putrid fermentation, of increasing the virulence of pathogenic germs.

According to Leon Gérard (of Brussels), a properly conducted microscopic examination may afford much useful information concerning the organic impurities of water. For this purpose, the water is passed successively through four wire screens, each with meshes finer than the preceding (from 500 to 5,300 meshes per square centimeter), the last screen consisting of two thicknesses of wire cloth with white filtering paper between. The process can be much simplified and expedited by the use of large centrifuges. The sediments on each screen are then examined with magnifying lens and microscope. Contamination by man and animals is indicated by four classes of substances: I. Fragments of textile fabrics; fibers of wool, cotton, linen, silk; hairs of man and animals; sections of human hair cut in shaving, in the shape of flat, oval or roundish disks, sometimes extremely thin and remarkably well preserved. 2. Grains of starch (cereals, potatoes, etc.) which reveal pollution from kitchen wastes. 3. Débris of muscular fragments, easily recognized by their structure and certain microchemical reactions; such débris indicating almost certainly fecal pollution. 4. Eggs of intestinal parasites, which also prove contamination by the fecal matter of man or animals.

The usual and principal object of the bacteriological examination is to ascertain whether or not the water contains pathogenic organisms, or the organisms which are usually associated with them and reveal the presence of contamination, whether from sewage, manure, or other source. The total number of bacteria contained in a cubic centimeter of water gives us doubtless more or less knowledge of the nature and extent of the contamination; it is mostly valuable, however, in ascertaining the degree of efficiency of filtration; thus a properly constructed and well-conducted filter should yield water containing less than 100 bacteria to the cubic centimeter, that is to say, water producing less than that number of colonies when sown 
on nutrient gelatine and kept in a temperature of $20^{\circ} \mathrm{C}$. for four days. In unfiltered water, the number varies enormously; but in really good water it should not usually exceed $\mathrm{I}, 000$, while in merely passable water it ranges from 1,000 to 10,000 , and in bad water exceeds 10,000. Spring-water and deep-well water are most free from bacteria ; lake-water comes next in purity, especially at some distance from the shore, while river-water is the worst. The Lake of Geneva was found to contain I50,000 bacteria to the c. c. near the shore and only 38 in the middle (Fol et Dunant); the river Seine, above Paris, from 46,340 in December to 13.710 in August; the Thames (in I886) from 45,000 in January to about 3,000 in July. It is noticed that, in temperate regions, the number falls considerably in summer when rains are few and shorter, while in winter the more continuous rains and many small streams from melting snow carry with them a much larger amount of soil pollution.

The degree to which a river can be infected by the sewage of a large city is illustrated by the Spree, which shows only 8,95 I bacteria above Berlin and 343,000 two miles below it.

The most usual and dangerous pathogenic organisms present in water are those concerned in the etiology of typhoid fever (bacillus typhosus), cholera (comma bacillus or cholera spirillum) and dysentery (bacillus dysenteria of Shiga, and others). Unfortunately they are very difficult of detection in water, on account of their comparative rarity, lack of resistance and shortness of life, often disappearing before the first case of the disease is diagnosed, so that their presence can only be assumed by the recognition of other and more common intestinal organisms, nore or less harmless to man but plainly indicative of fecal pollution. These witness bacteria ("bacterial indicators"), readily found and recognized in water, are always present in the intestinal discharges of man and other mammals, and, therefore, in sewage and manure. They are:

I. Bacillus coli communis, or colon bacillus. The best indicator of dangerous contamination. Common everywhere from the excrements of man, mammals and birds. Under favorable conditions it is capable of multiplying outside the body so that it may become a constituent of road dust, likely to be blown into reservoirs and even cisterns, but this mode of pollution seldom exceeds a few bacteria to the cubic centimeter. There is no evidence that the true type of this bacillus is ever present in any large number in rivers which have not been exposed to fecal contamination. Under ordinary con- 
ditions it does not multiply in water supplies, unless they contain fecal matter. Although its vitality outside the body is not great; it is more resistant that the bacillus typhosus, so that, if absent, it is nearly certain that the latter is also absent.

As it is not possible to differentiate the human colon bacillus from that of animals, a careful study should be made of all the factors involved so as to determine, if possible, the source of contamination; for instance, whether from the excrements of pasturing animals or from latrines.

The colon bacillus includes a number of forms differing in characters and reactions and often hard to separate. The typical form is the only one that has much significance. It is identified by the following characteristics: fermentation of glucose or lactose broth with rapid gas production, most or the whole of it being evolved in twenty-four hours, the total amount approximating about 50 per cent. and consisting of $\mathrm{H}$ and $\mathrm{CO}_{2}$ in the proportion of 2 to $\mathrm{I}$; liquid in bulb of fermentation tube strongly acid; a distinct indol reaction; coagulation of milk in I to 3 days; growth on gelatine in the form of non-liquefying, opaque, whitish expansions with irregular margin.

2. Bacillus lactis aërogenes, closely allied to the colon bacillus and, like it, almost constantly present in the human intestine. Also nearly always found in milk.

3. Streptococci, under several forms, are abundant in human and animal excreta and therefore in sewage. They are naturally parasitic, delicate germs, unable to multiply and rapidly dying outside the animal body, although some persistent forms are met with. They seldom live more than a week or two in water; their detection in any quantity, therefore, shows recent fecal pollution, and therein lies their chief value as bacterial indicator.

4. Bacillus enteritidis sporogenes (Klein), possibly identical with B. aërogenes capsulatus of Welch, is also abundant in human and animal excreta, but its usefulness as an indicator is very much limited by the fact that the spores only can be used for its detection, not the bacillus itself. As these spores are highly resistant and may persist for long periods, their presence simply indicates pollution at some indefinite time in the past (perhaps weeks or months before) and which may have long ceased to exist. Therefore, of themselves, they are without signficance, but when found with the colon bacillus add confirmation to the evidence yielded by that organism. 


\section{CHAPTER XXII.}

\section{WATER PURIFICATION.}

Water, to be pure, or at least potable and safe, must be ridden of its obnoxious constituents, especially its organic matter and microorganisms.

The three general methods of purification are by heat, chemical means and filtration.

For an army in the field, the ideal method of purification is one that can be carried out in all situations, with a small, simple, transportable outfit yielding plenty of good, cool, palatable water within a short time. Thus a regiment of 1,800 men should have at least 400 gallons of purified water within two hours after going into camp. However strict the discipline, it will always be very difficult to prevent men, when impelled by thirst, to drink from condemned sources if good water is not promptly available.

\section{HEAT.}

Heat is the most certain and effective of purifying agents and the surest means of obtaining an absolutely sterile water. Boiling destroys all pathogenic germs; it also removes the temporary hardness of water by precipitating the carbonates. It does not decompose organic matter, nor destroy its odor or color, but renders it less putrescible. An objection to boiling drinking water is that its gases are driven out, leaving it flat and unpalatable. But boiling is not necessary for the practical sterilization of water. A temperature of $165^{\circ} \mathrm{F}$., maintained for ten minutes, is sufficient for the destruction of all ordinary pathogenic bacteria; thus less fuel and time are required; less gas is lost and the more rapidly is the water cooled down. Water purified by boiling should always be thoroughly aërated after cooling, by dipping and pouring from a height, decanting from one kettle to another or blowing air directly into it.

In the field, the camp fire can generally be resorted to, in the absence of special apparatus, provided suitable kettles are available. The water should be sterilized in the evening, properly aërated and the canteens filled directly afterward so that it may be quite cold in the morning. This primitive method of sterilization, although oftert necessary, is seldom satisfactory. 
THE FORBES STERILIZER.

-rilizer (Fig. 44) was officially adopted in the Army after ative tests by a special board in 1898 . Fig. 45, which is . ely diagrammatic, illustrates the principle of its operation.

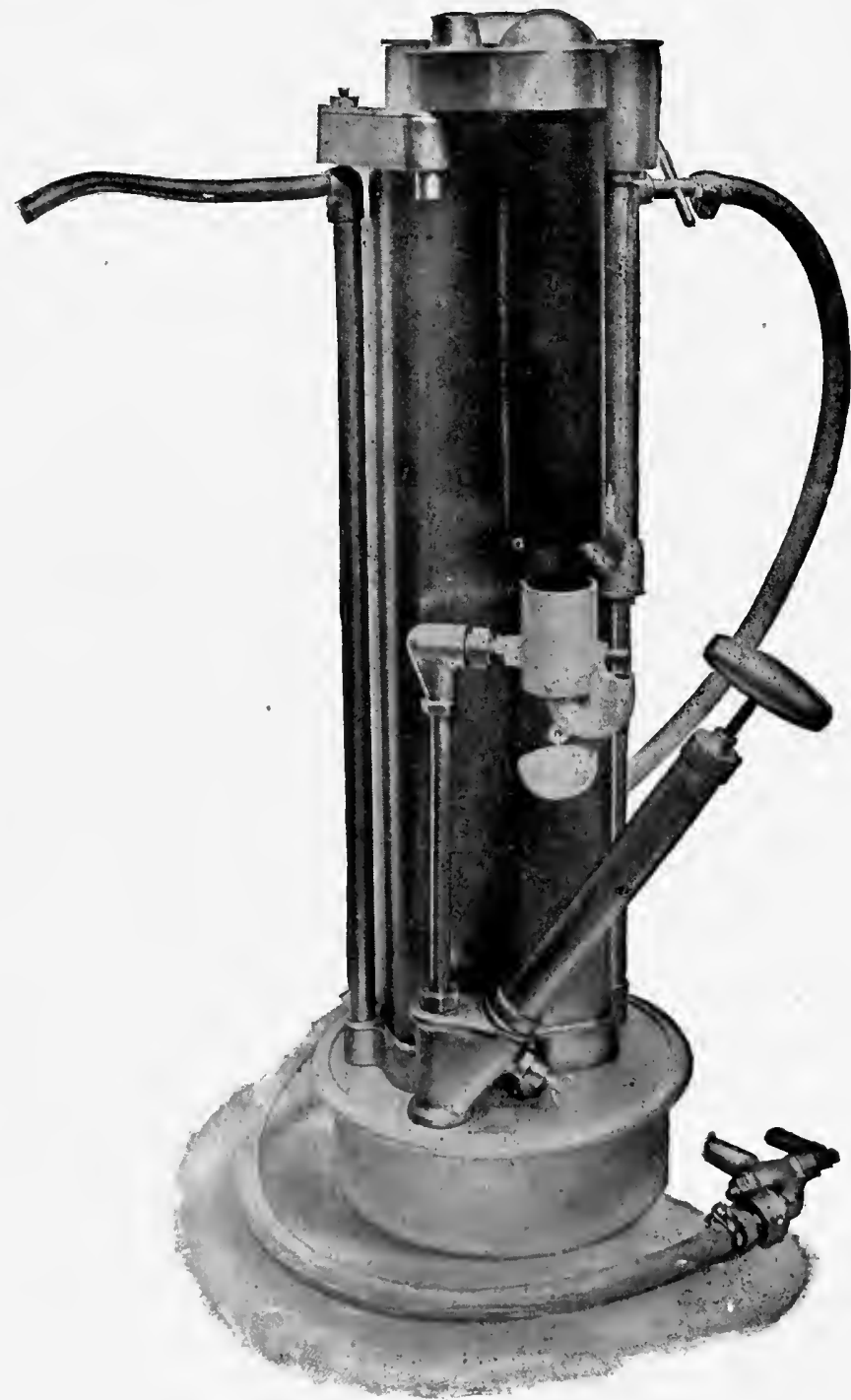

FIG. 44.- Forbes sterilizer ready for operation. 
Raw water enters at I and passing through the open valve 7 flows down through the tube 2 and enters the lower end of the raw water compartment 3 of the "heat exchange." Rising in the compartment 3, the water, which is shown by light shading, fills the compartment and enters the float-box 4. Passing from the float-box 4 , the water enters and fills the heater 5 up to the level $\mathrm{X}$. The water

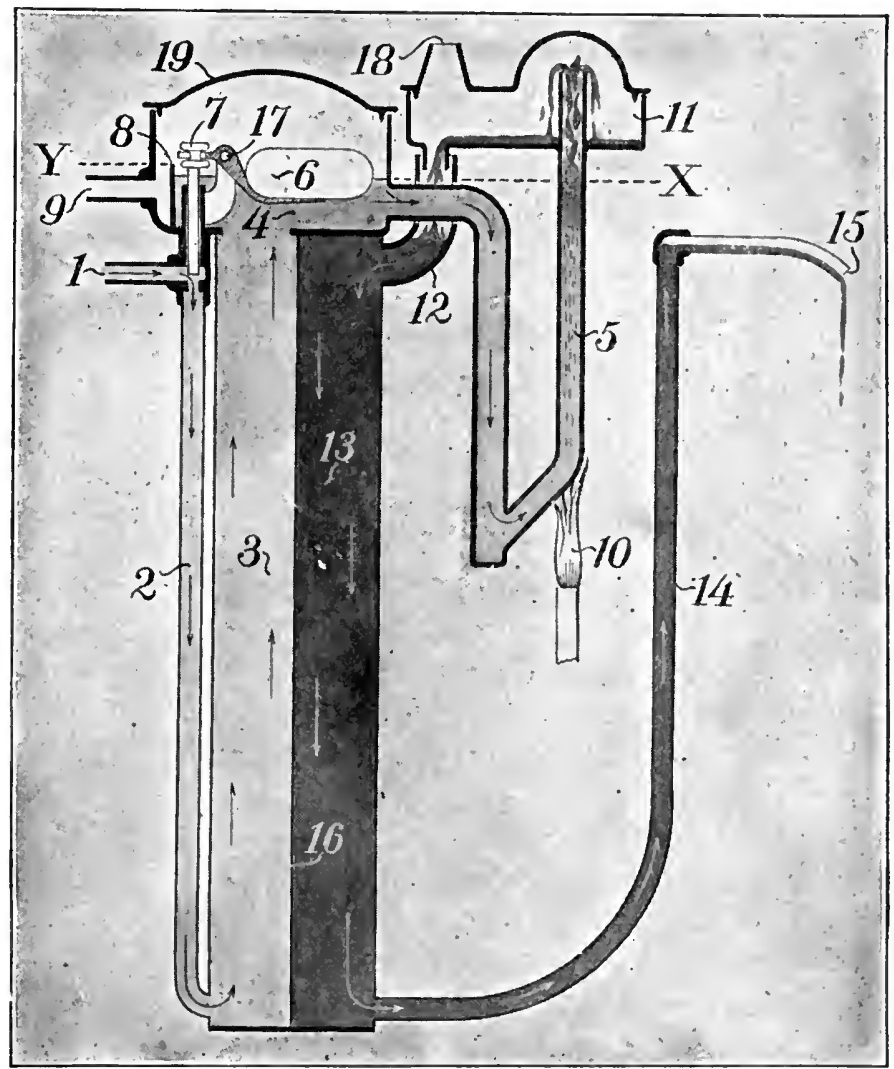

FIG. 45.- Schematic diagram of the Forbes sterilizer.

in the float-box 4 must also be at the same level $\mathrm{X}$ and at this point the water lifts the float 6 and closes the valve 7 , thereby preventing more water entering the apparatus at $\mathbf{I}$.

Should the float or valve stick or fail to work through any cause, the water will continue to rise in the float-box 4 and likewise in the 
heater 5 until it reaches the level $\mathrm{Y}$, where it will overflow at 8 and pass through the pipe 9 to waste. Higher than this level $Y$ the water cannot go.

Heat, in the shape of flame 10, is applied under the heater 5, thereby causing the water in 5 to boil, and in boiling it will rise in the tube and overflow at the top into the catch-all II. From the catchall I t the hot sterile water, represented by dark shading, flows down through $\mathrm{I} 2$ into the sterile water compartment I 3 of the "heat exchange." The pipe 14 conveys the sterile water up to the discharge 15. This outlet I $_{5}$ is brought to the top of the "heat exchange" to insure the sterile water compartment $\mathrm{I} 3$ always being full of water. While passing down through the sterile water compartment I3, the heat of the water is absorbed by the cold water rising through the raw water compartment 3 , and the transfer of the heat from the hot to the cold water takes place through the thin metal diaphragm I6; so that the water leaves the apparatus cold, having given up its heat to the cold water entering while, on the other hand, the cold water entering the apparatus, having absorbed the heat of the hot water leaving the apparatus, enters the float-box 4 and heater 5 very hot and nearly at the boiling point. Only a small amount of heat is, therefore, necessary to keep the sterilizer in continuous operation.

The heating apparatus consists of the oil reservoir, air pump and the burner proper. This is the most important feature of the sterilizer and its construction and management must be thoroughly understood and mastered by the operator if good results are desired.

The Forbes sterilizer, packed in its field case of galvanized steel, is 37 inches high and $\mathrm{II} / 2$ inches in diameter, and weighs 96 pounds. It burns one quart of oil in three hours, and averages an output of 15 to 20 gallons of sterile water an hour.

As in every other form of sterilizer, the water remaining in the apparatus, when not in use, becomes rapidly contaminated with the ordinary saprophytic bacteria, and on that account it is advisable, when again started, to reject the output of the first half hour.

This sterilizer, when properly operated, is entirely reliable and has rendered excellent service in many of our camps and garrisons. Its advantages may be státed as follows:

$x$. The water is not deprived of its natural gases.

2. All living micro-organisms are destroyed, except a few harmless spore-bearing bacteria. 
3. It may be kept in action for 24 hours without renewing the supply of oil in the reservoir.

4. The water flows out of the apparatus only $41^{1} 2^{\circ} \mathrm{F}$. warmer than when it entered it.

5. It is easily taken apart, cleansed and put together again.

6. It is durable, not liable to breakage and very easily transported. Besides the usual form above described, for burning mineral oil, there is another with fire-box attachment for burning wood or coal.

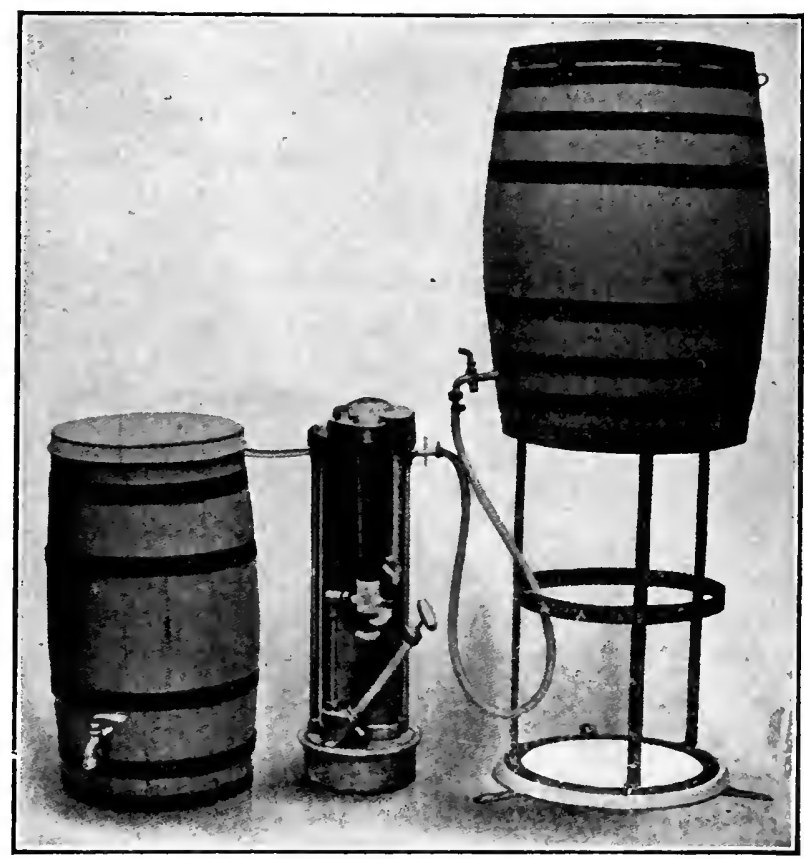

Fig. 46.- Barrel accessory set up, ready for operation in connection witis the Forbes sterilizer.

There is also a "barrel accessory" (Fig. 46) consisting of two barrels, a large one for raw water, elevated on a steel stand, supplying the sterilizer through a rubber tube, and a smaller one into which the sterile water is discharged.

WheEled Sterilizers. - For field purposes, however, a sterilizer to be entirely satisfactory should be mounted on wheels and able to 
follow a moving command, consume either wood or coal, yield a sufficient daily output for at least a regiment and always have a reserve of available cool water in its tank. Such are the German Henneberg Trinkwasser (Fig. 47) and the French Vaillard-Desmaroux outfits, in which the water is raised, under pressure, to a temperature of $240^{\circ} \mathrm{F}$. without boiling, so that it is rendered absolutely sterile without the loss of any air. These types are open to

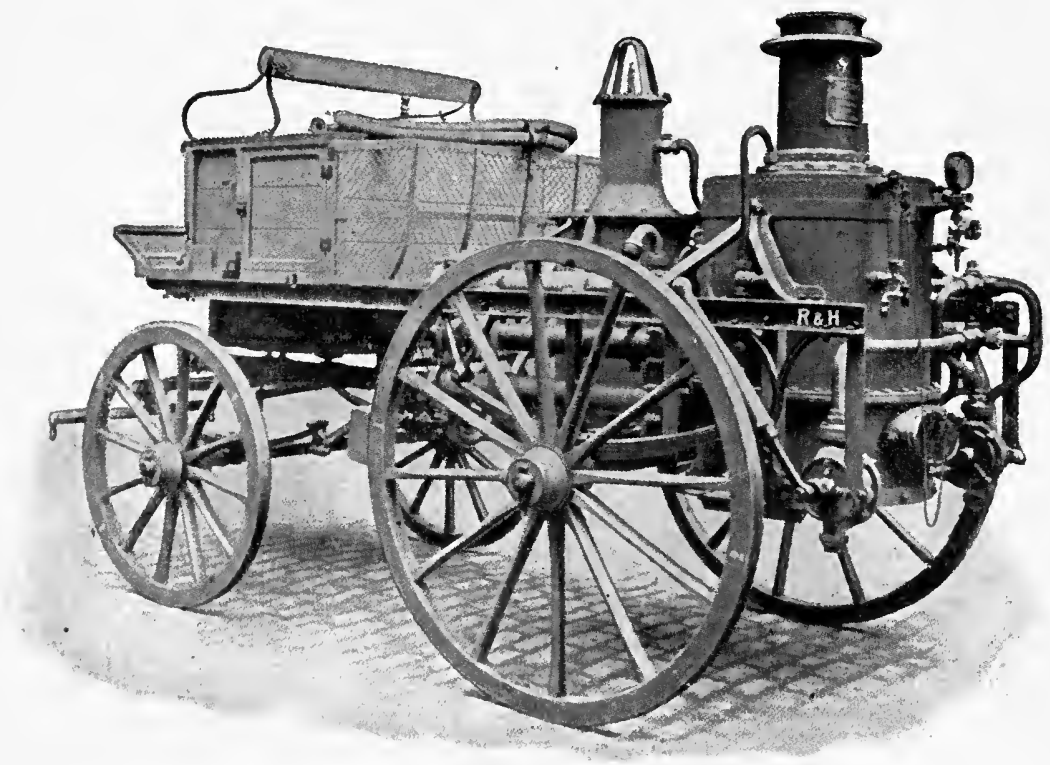

Fig. 47.- The German Henneberg Trinkwasser wagon.

the objection that the absolute sterility which they produce is not required, therefore that they consume more fuel than is necessary and deliver a water relatively hot.

The Forbes Co. also constructs several kinds of sterilizers on wheels, all on the same heat exchange principle, and consisting of boiler, pumps, filter, sterilizer and storage tank. The "Army IVagon Sterilizer," mounted on the regulation Army-wagon wheels, filters and sterilizes 300 gallons of water per hour, and 14 men draw their supply at the same time from its I50-gallon storage tank. The "Forbes Transportable Water Sterilizer" (Fig. 48) is a more recent type, mounted on the British Army limber. It is 
lighter and more mobile. Its capacity is 400 gallons per hour and consumes approximately one gallon per hour of gasoline. Its collapsible canvas storage tank has a capacity of 2000 gallons.

Griffitil Sterilizer. - In England, the Griffith sterilizer is highly spoken of and, apparently, well adapted to military purposes. It consists essentially of a heater and a cooler. The heat is obtained from a lamp burning coal oil under pressure. As soon as the water reaches the temperature of $180^{\circ} \mathrm{F}$., which is more than sufficient to destroy all disease-bearing organisms, a valve opens automatically, by expansion, and allows it to flow into the cooler. A larger type, mounted on wheels, gives 350 gallons of water an hour and has a

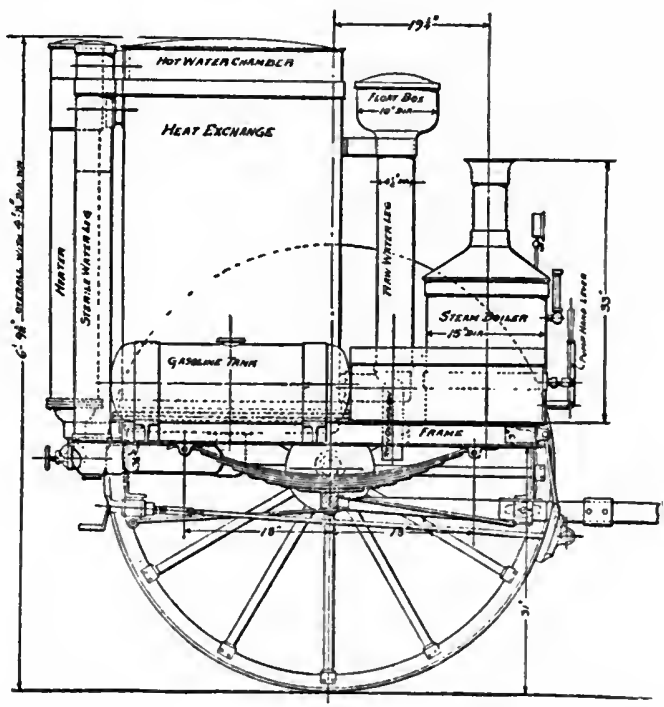

FIG. 48.- The Forbes Transportable Water Sterilizer.

storage tank for 50 gallons. It consumes I gallon of oil to sterilize 480 gallons of water.

Disadvantages of Sterilizers. - Several objections may be raised against heat sterilizers. They are costly and heavy. The fuel, whether it be oil, coal or wood, will often be difficult to procure and transport in sufficient quantity. They can seldom follow troops to the front, and are best adapted to more or less stationary camps. All sterilizers, particularly the large types, are more or less com- 
plicated and require careful and intelligent management. Their efficiency is also materially impaired by water containing much carbonate of lime which soon incrusts the partitions or tubes of the heat exchange, as well as the boiler, and lessens the output.

Distilled W ATER. — Distilled water is necessarily pure and sterile, but the absence of mineral waters and scarcity of gases in solution render it somewhat insipid and less fitted to the needs of the body than good natural water. Through osmosis it absorbs mineral substances from the tissues without contributing any solids in return, a drain to which has been attributed decay of the teeth and various other symptoms of a lack of salts in the system. Distilled water has a noticeable solvent action on metals and care must be taken that it does not come in prolonged contact with lead, zinc or copper. It is also liable to be contaminated with objectionable gaseous substances if distilled from foul water, salt or fresh.

COLD. - The purifying action of cold also deserves to be noted. Water in freezing forces out, into the subjacent layer, most of its matters in solution but only a part of those held in suspension, retaining most of its organic matter and bacteria, especially when much air is imprisoned as in snow or porous ice. Therefore, clear ice is purer than snowy ice, and top ice (if clear) than deep ice. The first effect of congelation, according to C. Frankel, is the death of the greater part of the micro-organisms; four-fifths disappear in two days and nine-tenths in five days. The pathogenic bacteriá, however, are among those most resistant to cold; when subjected to a freezing temperature, the cholera bacillus succumbs in seven days, but the anthrax bacillus may persist for weeks and the typhoid bacillus for several months. It is also of common notoriety that the larvæ of mosquitoes may remain in ice all winter in a quiescent state and, in the following spring, develop into full-fledged insects. We may then conclude that ice is better than the raw water beneath, especially if from a deep, calm pond or lake, but that it is not always safe, that it may contain thousand of microbes and has been the means of transmission of typhoid fever. Therefore, unless it is known to be reasonably pure, it should not be used in water nor placed in contact with articles of food. Artificial ice, if made from distilled water, as it always should be, is entirely above suspicion and should always be preferred.

Snow takes up foreign substances from the air as it falls, absorbs 
them from the soil on which it lies, and is constantly exposed to pollution. After a heavy snowfall the top layer is more or less sterile; but, as a rule, snow water needs purification.

\section{CHEMICAL MEANS.}

Chemical agents purify water by the oxidation of its organic matter and microbes, or by the precipitation of its matters in suspension. The use of these agents is attended with some difficulties and seldom practicable in the field. The proportion of chemicals must be adjusted to the character and impurities of the water, rendering a careful and variable dosage necessary. They often require a partial removal of organic matter before their use, and as they may produce precipitates or turbidity, clarification afterward. Moreover, foreign' substances are introduced into the water, often imparting an unpleasant taste to it, and the effect of which upon the body economy has to be considered.

Ozone. - One of the best methods of purification, so far devised, is undoubtedly that by ozone, based upon the powerful oxidizing effect of ozonized air upon organic matter and micro-organisms. First introduced by Ohlmïller and recently perfected by Siemens and Halske, this method has already given very remarkable results, under favorable conditions, absolutely eliminating both organic matter and bacteria from the water. The ozone is generated by a special apparatus and, after being mixed with a current of dry air, forced up a cylinder containing gravel and down which the raw water percolates. It imparts no unpalatable or harmful element to the water and in no way modifies its chemical and physical properties, dissolved salts or gases. Its action is very rapid and, being eminently unstable, leaves no trace a few moments after its mixture with the water. It first oxidizes the organic matter, and afterward the more resistant bacteria; the amount therefore must vary with the proportion of organic matter and demands a nice adjustment at the hands of a skilled operator. Turbid water generally requires a preliminary filtration.

The entire sterilizing outfit has been mounted on wheels with the object of making it available for troops in the field, but it is not well adapted to that purpose on account of its complexity, the expert manipulation required and the variable composition of the waters to be purified. 
With filtered water, I or 2 grams of ozone to the cubic meter of water is sufficient, but, in the field, dealing with variable waters, larger doses are needed, averaging about 15 grams (3 cubic meters of ozonized air) per cubic meter of water.

Ozone, as water sterilizer, on account of its cost and the difficulty of accurate dosage, is still seldom used.

Ultra-violet ray's. - Water sterilization by means of these rays has been shown to be possible and practical. Mercury-vapor lamps, with quartz walls, are almost an ideal source of ultra-violet rays, emitting the intermediate rays (from 3000 to 2200 Angström units) which are most efficient for sterilization, being highly bactericidal and sufficiently penetrating. These lamps act best when immersed in the water to be purified, their radius of action being at least a foot. Sterilization is complete in less than a minute without any alteration of the physical and chemical properties of the water. Experiments in Manila have shown that typhoid bacilli as well as amœer (motile or encysted) are killed in 5 seconds. These rays are applicable only to clear or clarified water, not heavily polluted. They are useless for liquids containing colloid substances such as milk, broth, wine, etc.

This method of purification promises to become well adapted to the requirements of permanent camps by the simplicity of the apparatus and cheapness of its operation.

The Nogier ultra-violet mercury lamp has been successfully used in France. It is claimed to yield from 400 to 500 liters of absolutely sterile water per hour. It is from 6 to 12 inches long and only requires a continued current of 30 to 35 volts and 5 to 7 amperes.

The other chemicals recommended at various times for the purification of water include bromine, iodine, chlorine, potassium permanganate, sodium bisulphate and copper.

Bromine, according to the Shumburg method, is used in a solution of potassium bromide ( 20 grms. each of bromine and potassium bromide in roo cubic centimeters of water). About two cubic centimeters are sufficient for one quart of ordinary raw water; at the end of $I_{5}$ or 20 minutes the excess of bromine is neutralized with ammonia or sodium hyposulphite. A large proportion of microbes are destroyed but many escape; furthermore, the transportation and handling of bromine and other chemicals in solution are inconvenient and often impracticable in the field. 
Iodine, as recommended by Vaillard, is less objectionable as only tablets are used. Three kinds are required: No. I (blue), containing potassium iodide and sodium iodate; No. 2 (red), of tartaric acid; No. 3 (white), of sodium hyposulphite. If one blue and one red tablets be crushed and dissolved in a little water, a brown fluid containing 0.06 grain of nascent iodine results; this, if added to a liter of water, will destroy its micro-organisms in ten minutes. Tablet No. 3 may then be added to neutralize the excess. This is a neat and efficient process, somewhat complex for general use but well adapted to the needs of officers and small detachments. Nesfield recommends that the iodine be increased to $1 / 2$ grain per gallon for the best results.

Chloride of lime (chlorinated lime or bleaching powder) is one of the most effective agents in water purification and now extensively employed for this purpose in connection with filtration or (where the water is reasonably clear) independently of it. It consists of about equal amounts of calcium chloride and calcium hypochlorite, the proportion of available chlorin averaging about 35 per cent. When added to water, the calcium chloride remains inert; the hypochlorite, acted upon by the $\mathrm{CO}_{2}$ in the water, splits into calcium carbonate and hypochlorous acid. This acid which is exceedingly unstable breaks up, its chlorin combining with the hydrogen of the water to form hydrochloric acid while its oxygen is liberated. The hydrochloric acid decomposes the carbonates of the water and becomes reduced to calcium chloride. It is to the oxygen thus liberated in an atomic or nascent state that the strong sterilizing action of hypochlorites is due. As ordinarily used their effects are: destruction of most non-spore bearing bacteria, oxidation of organic matter proportional to the amount of chemical employed, partial removal of color as well as more or less complete removal of any swampy taste and odor. On the other hand, there is a slight increase in total hardness and total solids. Unless used in excessive doses the changes in physical and chemical characteristics of the water are barely noticeable; free chlorin, as a rule, cannot be detected, and any imparted taste or odor is quickly dissipated by agitation of the water.

Sodium hypochloritc (chlorinated soda) as obtained, through the electrolysis of sea water or of solution of common salt, is slightly more efficient than calcium hypochlorite, forming less precipitate, 
and may supersede it should the process of manufacture become cheaper.

The amount of chloride used is from 5 to I 5 pounds for each million gallons of water, or about one pound to 100,000 gallons. The common practice is to make a solution of $1 / 2$ per cent., that is, one pound to 200 pounds of water, in concrete tanks, and feed it to the raw water, before or after filtration at the proper rate and in such manner as to insure a quick and thorough mixture. The contact period should be at least one hour before the water is delivered for use. For clear water simply needing organic purification, chloride of lime is largely taking the place of filtration. For turbid water a combination of both methods if often practised and highly advantageous. When added to the reservoir before filtration, chloride of lime is said to materially lessen the cost of operating filters by reducing the amount of coagulant needed and (through a decrease of organic matter) lengthening the runs of the beds between washings. On the other hand, by its inhibitive action upon organic life it reduces the bacterial efficiency of the filter. It seems best, therefore, to have recourse to a rough preliminary filtration if any previous treatment is necessary to prevent undue clogging of the sand filter, and use the chloride in the effluent from the latter.

Of all chemical methods of water purification, that by chloride of lime is the cheapest and one of the most efficient; it is also one of the most readily adapted to the needs of troops in the field. For a small party of men, the following simple device is recommended: a teaspoonful of chloride, leveled off by rolling a pencil over it, is rubbed up in a cup of water. This is diluted with three cupfuls of water, and a teaspoonful of this dilution is added to a two-gallon pailful of the raw water, mixing it thoroughly. This will give four or five parts of chlorin in a million parts of water and will destroy all pathogenic bacteria without leaving any taste or odor.

Chlorin gas, as water sterilizer, has been shown by C. R. Darnall, U.S. Army, to possess certain decided advantages over hypochlorites because of its uniform strength and of the ease and accuracy of its dosage. In this method, the purified, dried and liquefied gas is used, as found on the market, in steel drums holding from Ioo to 140 pounds each. Chlorin attacks metals, but only in the presence of water (by liberating oxygen); when dry it can be kept (liquefied) indefinitely in steel drums and may be conducted through pipes and 
valves without undue corrosion. Its sterilizing action in water is due, as with hypochlorites, to nascent oxygen. Owing to the strong attraction of chlorin for hydrogen, water is decomposed with formation of hydrochloric acid and liberation of oxygen. As stated before, this acid is then neutralized by the carbonates of the water.

This method is somewhat more expensive than the use of hypochlorite, but cheaper than the ozone process and much easier to operate. It is not applicable, however, to the needs of troops in the field.

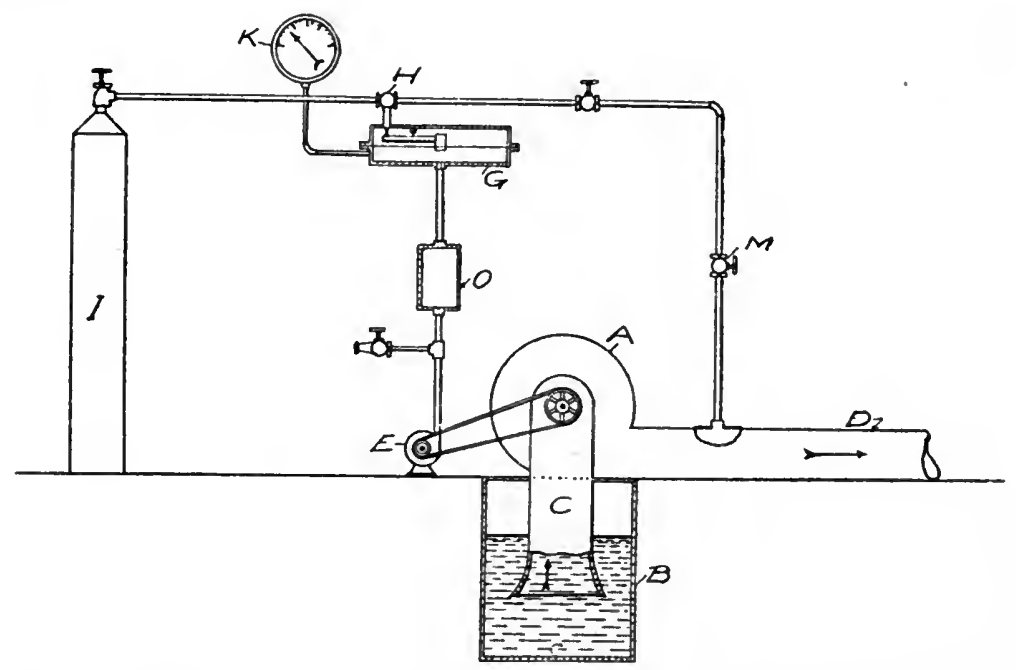

Fig. 49.-Diagram of the Darnall chlorin apparatus for sterilization of water.

The apparatus used by Darnall (Fig. 49) consists essentially of the chlorin container (I) and a pressure regulator (G) actuating the valve $(\mathrm{H})$ which admits the gas to the water to be sterilized (through the conducting pipe $\mathrm{M}$ ) in such manner as to maintain the predetermined ratio between the gas and the water. The regulator is a casing in the form of a disk made up of two horizontal compartments separated by a movable diaphragm. This diaphragm is connected with the valve by a lever, closing it as it is depressed and opening it as it is raised. The chlorin enters the upper compartment, and as its pressure increases the depressed diaphragm closes the valve and decreases the flow of gas. The lower compartment is connected with an air chamber $(O)$ in which the pressure is 
regulated by the velocity of the flow of the raw water; as this velocity increases, the greater is the action of the small auxiliary pump $\mathrm{E}$ (air or water pump), increasing the pressure so as to raise the diaphragm, open the valve and increase the current of chlorin. A centrifugal pump (A) raises the water from a well or tank (B) through a suction pipe (C) into the main (D) where the chlorin is discharged. This apparatus admits of various modifications applicable to most conditions of water-supply.

The official board appointed to investigate this method came to the conclusion that it is as efficient as purification by ozone or hypochlorites and more reliable in operation; that the apparatus is very simple, almost automatic, occupies very little space, and can be installed in connection with any water-supply.

\section{WATER BAG.}

Concerning the easiest, cheapest and most efficient method of water purification for mobile troops in the field and in active service, the preponderance of evidence, at this time, is for the chemical treatment by calcium hypochlorite in a convenient, portable container. This is officially recognized, in our service, in the shape of an appliance* designated on the supply table as "Water Bag. Field, Sterilizing," and described as follows (Fig. 50) :

"Consists of a canvas bag of specially woven flax, 20 inches in diameter and 28 inches in length, sewn to a flat galvanized iron ring, hinged so it folds at one diameter. Spliced at four equidistant points on the ring are two crossed pieces of hemp rope, enabling the bag to be suspended on any convenient support capable of holding the weight of the bag when filled with water, which is about 330 pounds. Five nickel, spring faucets are placed at equal spaces about the bottom edge of the bag. The neck of these faucets is small enough to enter a canteen, which can be filled in Io seconds. The self-closing faucets prevent wastage.

"The purpose of the bag* is not for transporting water, but to provide a stationary receptacle in which water can be held long enough to sterilize and then distribute it. The empty bag weighs from 7 to $7 \frac{\mathrm{T}}{2}$ pounds and folds into a convenient package for carriage in the field.

* Devised by Major Wm. J. L. Lyster, M. C., U. S. A. 
"After the bag is suspended and filled with water, it is sterilized by the addition of a small amount of hypochlorite of calcium. This is carried in measured doses, sealed in glass tubes. A package of 60 of these tubes weighs 10 ounces and measures $7^{\mathrm{T}} / 2$ by $3 \frac{1}{2}$ by $4^{1 / 4}$ inches. Packed in corrugated paper it will stand rough usage.

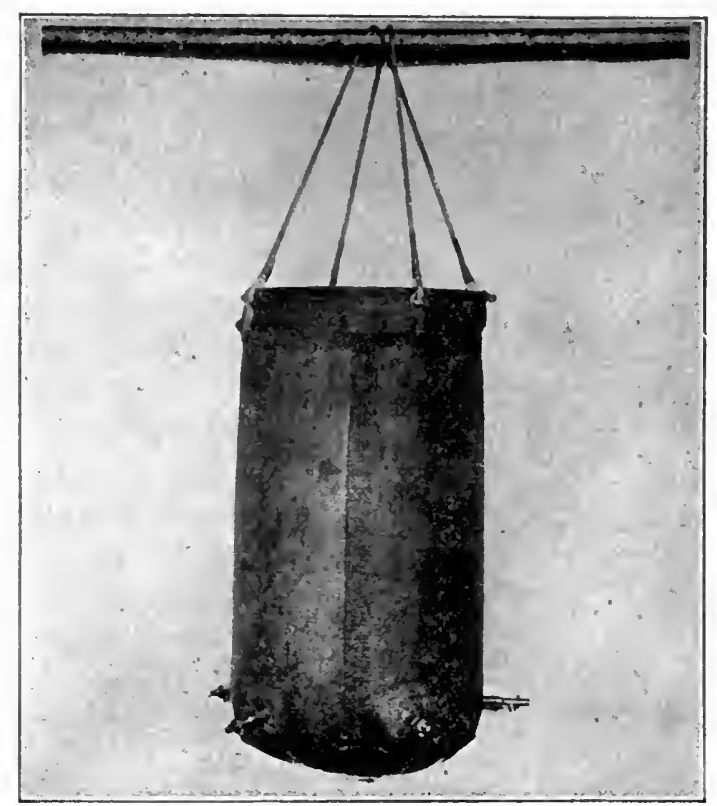

FIG. 50.-Bag for Sterilization of Drinking Water by Calcium Hypochlorite.

"The tubes themselves are 3 inches in length by three-fifths of an inch in diameter and are marked with a file, enabling them to be easily broken in the fingers without fragments. They contain from I4 to 15 grains of calcium hypochlorite. This chemical contains from 30 to 32 per cent. chlorin, which forms in the water hypochlorous acid and results in sterilizing the water. The process is one of oxidation. In the strength used, waters highly infected are rendered safe. Of course, in such strength (I-500,000) grossly polluted water, such as sewage, will not be rendered safe. Water ordinarily used will be entirely safe after being so treated. As the chemical acts more efficiently in clear waters, a filter cloth, to be 
fastened over the opening of the bag and weighing I ounce, is provided, or water may be strained through a blanket. The bag is filled after it is in place. Suspended matter, such as clay, is largely removed and not left to interfere with the action of the chlorin.

"Comprehensive experiments demonstrate the bacteriological efficiency of the appliance. The organisms causing typhoid fever, the dysenteries, including amœic or tropical dysentery, and ciliates are promptly destroyed. Even in the vegetative form amœbæ are killed in 15 minutes; ordinarily 5 to 10 minutes suffice after the addition of the powder to render the water safe to drink. Exposure of 30 minutes has been found to destroy all amœbæ and ciliates under most severe conditions in a test."

Other chemicals worth mentioning are:

Potassium permanganate has long been used as a water purifier and yields fairly good results. A sufficient quantity must be added to the water to impart a faint pink tinge which should persist for half an hour. From 5 to Io centigrams to a liter (about a grain to the quart) is required in average water to oxidize and destroy the organic matter and bacteria, but its action is very slow and somewhat uncertain. Vaillard, however, declares that in water previously clarified, 2 to 4 centigrams to a liter will destroy, in 30 to 40 minutes, all pathogenic and sporeless bacteria. After using permanganate it is well to filter the water to get rid of the brownish precipitate of manganese oxid, although probably innocuous. The water often retains an unpleasant taste.

In the Lambert process, manganese sulphate, aluminum sulphate and an alkaline carbonate are added to the permanganate, so that the water is clarified as well as sterilized.

Sodium bisulphate has been found an efficient destroyer of the ordinary pathogenic bacteria in water. To a quart of water is added a 30-grain tablet of this salt sweetened with saccharin and flavored with oil of lemon (as advised by Firth); the water, which acquires a pleasant acidulous taste, may be drunk in about 20 minutes. It is doubtful whether the use of such water could be long continued on account of its probable laxative effect.

Copper sulphate has been highly recommended as germicide and algicide. In the proportion of I to $1,000,000-10,000,000$, it will destroy most of the algæ which often infest reservoirs and drains. 
If evenly distributed and the water is filtered afterward it can safely be used in the above proportions in drinking water. It cannot be depended upon to destroy all micro-organisms except in dangerous doses (I/50,000 to $\mathrm{I} / \mathrm{100,000)}$.

Citric acid, in the proportion of one ounce to 16 gallons of water, is well spoken of as a corrective of the repulsive fishy taste and odor due to the decay of minute alga found in certain waters, and which remain unaffected by filtration and boiling.

Those chemical agents which act as coagulants and precipitate matters in suspension will be considered under filtration. 


\section{CHAPTER XXIII. \\ WATER PURIFICATION (CONTINUED).}

\section{FILTRATION.}

Filtration is the method of purifying water in which we seek to imitate the natural percolation taking place in nature, through the superficial porous strata of the earth, whereby contaminated surface water is transformed into the pure water of springs and deep wells.

Filtration is chiefly a mechanical process; that is to say, the action of a filter is mainly that of a very fine strainer and therefore limited to the suspended or insoluble matters of the raw water. We may, therefore, expect to see the turbidity, sediment and bacteria largely or entirely disappear, while organic matter and other substances in solution, such as those producing hardness, color and smell, are removed only to a slight extent, if at all. Besides this mechanical action, however, we also often find the evidences of a biological action in filters, varying in degree according to the method pursued. We know that bacteria are more minute than the pores of a Berkefeld or Chamberland filter, or the spaces between grains of fine sand; if they do not pass through with the water, it is because of a molecular attraction whereby they adhere to the grains of sand or the walls of the pores, an adhesion aided and strengthened by the viscous organic matter found in most waters and which lines the pores, or smears the grains. of sand of filters. Thus arrested, the bacteria are subjected to unfavorable conditions which soon bring about their oxidation and destruction. It readily happens, however, that if certain precautions are neglected, the bacteria find suitable conditions for their multiplication and gradually extend, by growth, through the filter into the filtered water which thus may become worse polluted than the raw water. Therefore, although filtration when properly carried out is a very efficient means of purification, it may also, when carelessly conducted, be the source of serious contamination.

Whenever coagulants are used, previous to filtration, there is a chemical action added to the mechanical and biological actions. In quick mechanical filtration, there is no time for any biological action to take place, the organic matter and bacteria being simply removed instead of being oxidized. 


\section{Domestic Filters.}

Of these filters the number is legion. The best-known types and the most effective, like the Pasteur-Chamberland and Berkefeld, consist of unglazed porcelain cylinders made from a mixture of kaolin and other special clays. The Berkefeld filter was also formerly made of infusorial earth (microscopic skeletons of diatoms). Finely powdered asbestos is sometimes added to the clay as in the Mallié or Porcelain-Adamant filter. They are mostly in the form of hollow cylinders called "candles" or " bougies," closed at one end and open at the other or nipple-shaped end (Fig. 5I). Each bougie is inclosed in a glass or metal jacket with an intervening space

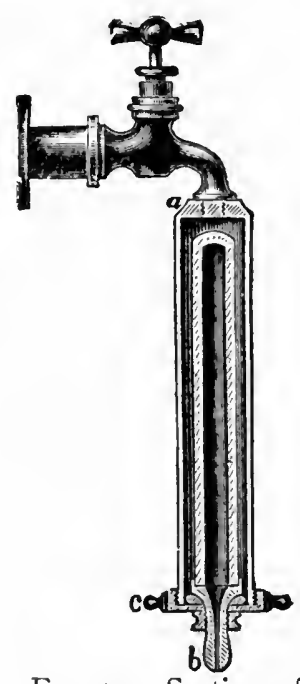

FIG. 51.- Section of Berkefeld filter. (Munson.)

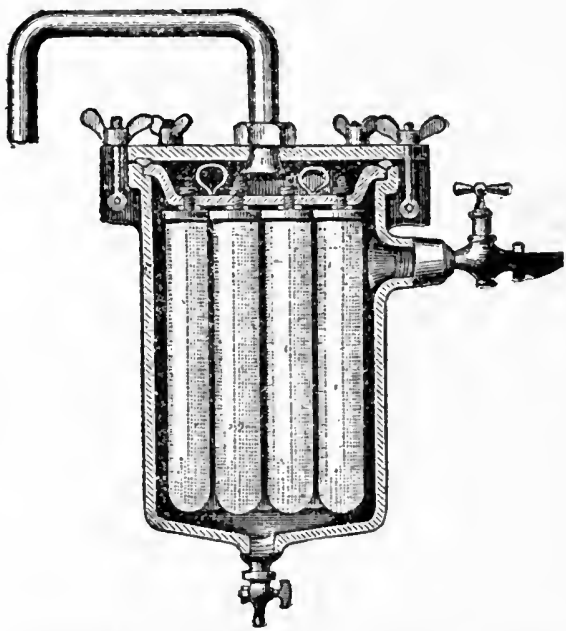

FIc. 52.- Battery of Berkefeld filters. (Munson.)

between. The head of the jacket being screwed to a water tap, the raw water enters into the space surrounding the bougie and, passing through its walls into the hollow interior, discharges at the nipple end. For proper functioning, the pressure should be at least equal to one atmosphere. These cylinders can be set together and operated in batteries (Fig. 52). In the field such batteries are supplied with a hand-pump attachment (Fig. 53). The output is small, seldom exceeding five quarts an hour per cylinder, being somewhat larger with the Berkefeld than with any of the other kinds. 
These filters not only clárify but are also capable of purifying the water. When put in operation, after sterilization, the Chamberland and Berkefeld furnish at first a water absolutely sterile. But it is noticed that the output decreases rapidly, falling from 4 to 5

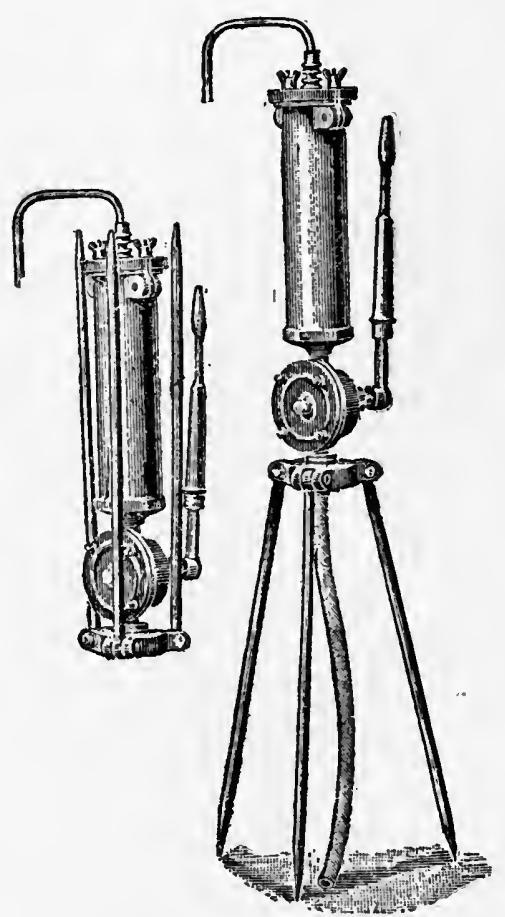

Fig. 53.- Berkefeld filter with pump for field use. (Munson.)

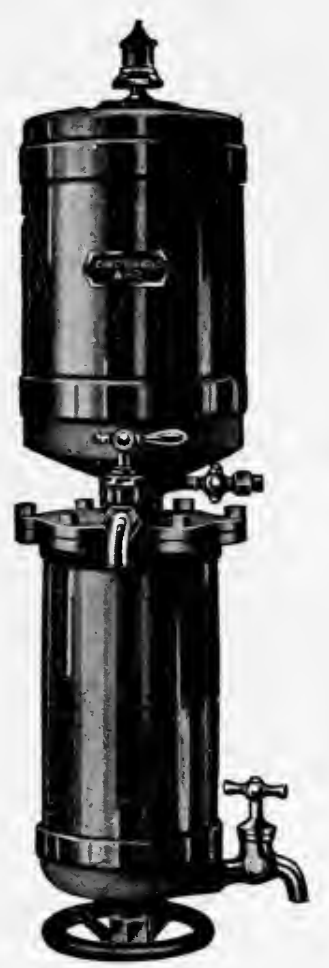

FIG. 54.- Cincinnatus filter with scraping device controlled by wheel at bottom, and reservoir above.

quarts an hour to 2 , I or even less in the course of three or four days; at the same time the bougie becomes covered with a coating of the impurities in suspension, and germs begin to appear in the filtered water. These germs did not pass through with the raw water but result from the growth of those deposited upon the surface, gradually extending along the pores through the walls of the bougie. They are nearly always harmless water saprophytes, the 
conditions being generally unfavorable to the multiplication of pathogenic bacteria. It is quite possible, however, that, under favorable conditions such as suitable temperature of the water and sufficient nutrient medium, the germs of typhoid fever and cholera could also grow through these filters. It is necessary therefore that they should be brushed every day, and sterilized in boiling water at least every 3 or 4 days. This cleansing and sterilization require special care, such as can seldom be given in households or military organizations, so that, outside of laboratories, these filters are seldom of much practical utility.

In the Cincinnatus filter type (Fig. 54), the water when filtered passes into the reservoir above. It is cleaned by opening the flush cock and revolving the wheel; this, by special device, reverses the filtered water and scrapes the mud off the surface of the tube. Such filter can be depended upon for thorough clarification and a fair degree of bacterial efficiency.

\section{SAND FILTRATION.}

Sand filtration is the method of water purification on a large scale used by towns and other large communities. It is applied in two different ways, either according to the original sand-bed or English system, or the more recent mechanical or American system.

In the English system, the plant consists of a certain number of sand beds, each capable of independent operation (Fig. 55). The average sand bed is one acre in area and about four feet deep. It consists, from the bottom upward, of broken stone, gravel and sand; the broken stone and gravel forming a layer one foot thick, and the sand another layer three feet deep. The broken stone is graded from one or two inches in diameter to about $I / 5$ of an inch or less. The sand should be clean, free from lime and clay, the grains ranging from 0.30 to 0.40 millimeter. The finer the sand the better the purification and the lesser the depth of the surface layer becoming soiled, but the smaller the output. The bed is thoroughly underdrained with lateral drains of open-jointed tile emptying into a central conduit. The bottom and sides of the compartment containing the bed are carefully cemented and made water-tight. A roof is necessary wherever the winter is severe and much ice forms; by excluding light it also prevents the growth of algæ and other microscopic plants, sometimes very troublesome in summer. In Eng- 
land and Holland the beds are mostly left open. Unless the water is pretty clear one or more settling basins are necessary; the more complete is this preliminary sedimentation the more rapid and effective will be the functioning of the filter. Turbid water clogs the sand bed and requires frequent and expensive cleaning.

The suspended matters in water are retained on the surface of the sand forming a sediment which becomes a much finer strainer than the sand itself. This sediment (Schmutsdecke of the Germans)

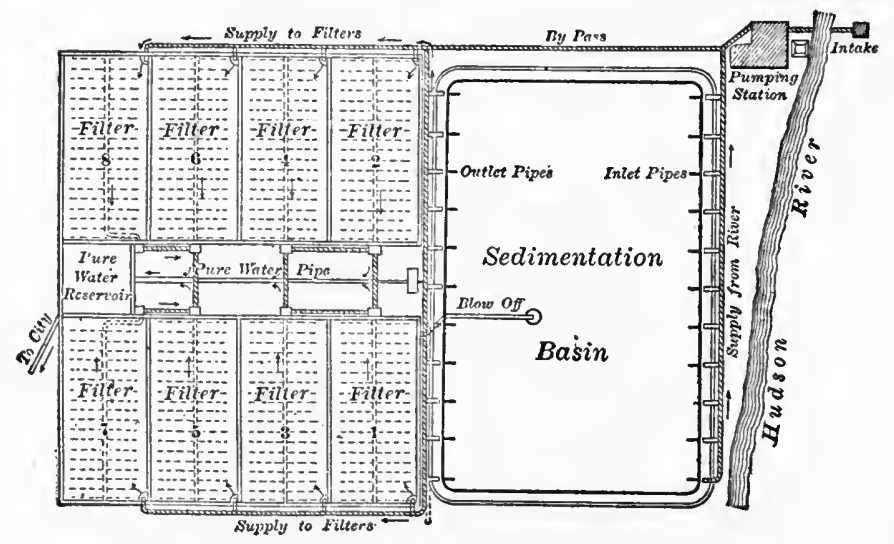

FIG. 55.-Plan of filter beds at Albany, N. Y.

consists of mineral and organic substances in variable proportions according to the character of the water. In water relatively clear, as in most of our eastern rivers, it is mostly made up of organic matter, especially miscroscopic algæ and diatoms, which by the swelling up of their cell membranes form a slimy, gelatinous layer in which nearly all the micro-organisms of the water are caught. This gelatinous substance (bacterial jelly or zooglœa), which also smears each grain of sand in the upper layer, plays an important part in the process of sand filtration, for it is after its formation that the filter reaches its highest degree of efficiency, the bacteria caught in its meshes being promptly oxidized and destroyed. But, after a while, the sediment becomes so thick and impervious that the rate of filtration is very much reduced. It is then necessary to clean the bed, that is, remove the gelatinous sediment and one or two inches of the surface sand. When the water contains much fine silt and the organic gelatinous sediment is scant, several inches of sand may 
become clogged and require removal. Sand filters are generally cleaned every 3,4 or 5 weeks in winter, depending upon the amount of impurities in the water, and much less frequently in summer. In improved plants (as in Washington, D. C.), the process of cleaning is largely mechanical and automatic; the sand is shoveled into movable ejectors, aspirated into washers, then conveyed into bins for storage and distribution. The filters may be scraped several times before new sand is put on, provided the sand bed is never reduced to a thickness of less than two feet. In still more recent plants the bed is cleaned by raking and flushing without removal of sand.

After the cleaning, leveling and smoothing of the surface, the bed is refilled with filtered water, from below, through the underdrains. This is in order not to break or roughen the surface. After cleaning there is a reduction in the bacterial efficiency of the filter; more bacteria will pass through if full pressure is put on at once; it is better, therefore, to start again slowly and gradually increase the rate. The maximum rate of downward flow should seldom exceed four inches an hour.

In most of our southern and western rivers the abundant clay and sand in suspension absorb most of the organic matter so that no gelatinous layer forms. The water of such rivers would clog the filter in a few days and must therefore receive special treatment. It is first run through one or more settling basins, and then a coagulant is added to it, as in mechanical filtration. Simple sedimentation, that is to say, the natural settling down of matters in suspension in quiet waters, produces of itself a marked degree of purification. Not only do the mineral particles subside, thus clarifying the water, but in so doing attract the floating bacteria and carry them down; this is one of the sanitary advantages of large reservoirs admitting of long storage. The addition of a coagulant causes the microscopic atoms of silt (smaller than the pores of the sand) to coalesce in larger and heavier particles which settle down as a flocculent precipitate, forming a sediment which takes the place of the gelatinous layer of clear rivers. This addition of a coagulant, previous to sand filtration, is always desirable in turbid waters. (See page 297.)

Lime and sulphate of iron, in combination, have been used to precipitate sewage for many years. Lately this combination has been adapted to water purification, on account of its cheapness and 
the softening action of the lime when added to water containing an excess of carbonates. The iron hydrate is the active coagulating agent; if magnesium is present, magnesium hydrate also acts as a coagulant. The process is particularly adapted to turbid waters containing calcium and magnesium bicarbonates, and is largely used in the Middle and Western States. At Saint Louis, Mo., it is claimed that it removes 99 per cent. of bacteria and reduces the turbidity from $\mathrm{I}, 200$ to $\mathrm{IO}$.

In Richmond, Va., the result of sedimentation and coagulation is so satisfactory that filtration is dispensed with. The reduction in turbidity is 76 per cent. in the settling basins and reaches a total of 96 per cent. in the coagulating basins, while the bacteria seldom exceed 200 to 300 per c. c., and the colon bacillus is hardly ever found in the effluent.

The experience of recent years in Philadelphia tends to prove that, where a large amount of the coarser suspended matter consists of small organic life which would not be thrown down by precipitation, a preliminary rough upward filtration (through coarse gravel and crushed furnace slag, topped with compressed sponge) doubles the yield of the sand beds, besides insuring a uniformly better quality of effluent. These preliminary filters do in a few hours what it may take weeks to accomplish with sedimentation basins, and much more cheaply.

The changes produced by effective sand filtration are: complete removal of solids in suspension, a reduction of at least 50 per cent. in free ammonia, 35 per cent. in albuminoid ammonia and 25 per cent. in oxidizable organic matter, and an increase of 25 to 30 per cent. in nitrates. It should also remove at least 99 per cent. of the micro-organisms of the raw water, so that the remaining bacteria will not exceed 50 to the cubic centimeter.

If a disinfectant is judged necessary, it is best, as a general rule, to use it after filtration, for if added before, it may destroy or injure the efficiency of the filter by reducing its bacterial life and activity.

The filter plant of Washington, D. C., one of the latest and most complete in this country, consists of 29 sand beds, each one acre in size, in basins of concrete, completely covered. The water is taken from the Potomac River, I4 miles above the city, and allowed to settle in three successive reservoirs. The beds are cleaned every six weeks in winter and only once or twice in summer, the thickness of 
sand removed, owing to the large proportion of fine clay in the raw water, being 6 to 12 inches. This cleaning is mostly automatic, as noted above. The cost of operation, including interest on cost of construction, is 0.9 of a cent per $\mathrm{I}, 000$ gallons. The output exceeds 200 gallons per capita. During the year 1906, the number of bacteria in the filtered water ran from 17 to 39 per cubic centimeter.

\section{MECHANICAL FILTRATION.}

This system is of American origin and extensively used in this country. It is particularly adapted to very turbid waters in which no organic gelatinous sediment forms, as well as to highly colored waters; it is the most practicable system of purification for the water of the lower Mississippi and many other western rivers. It is also the system best adapted to military posts or small communities. It consists in rapid sand filtration, after sedimentation and the addition of a coagulant. Its efficiency depends chiefly upon the use of coagulants; these do not effect purification by destroying or inhibiting the bacteria contained in the water; they simply cause the formation of a precipitate of a more or less flocculent character which entangles and surrounds all suspended particles, including the bacteria. Thus clay, sand, considerable organic and coloring matter, and bacteria, are removed; but the bacteria thus carried down are not killed, for cultures can be made from the precipitate. Water thus treated and allowed to stand until the precipitate has settled to the bottom is clear and brilliant, and nearly sterile (Darnall).

The coagulants most commonly used are alum (aluminum and potassium sulphate), aluminum sulphate and iron sulphate. When either of these chemicals is added to the water, it is decomposed by the action of the carbonates present in the water, with formation of a flocculent precipitate of aluminum hydroxid or ferrum hydroxid, and of another of basic sulphates, the latter to some extent helping the former in carrying down the suspended impurities. Should the water be lacking in natural carbonates it will be necessary to add them.

In the mechanical (gravity or open) filter plant, the raw water is conveyed or pumped into settling tanks, entering near the bottom after having received a graded quantity of coagulant, usually one to two grains per gallon. It rises until it overflows into the filter tanks, leaving behind. in the settling tanks, much of the precipitated sus- 
pended impurities. The filter tank is circular, of wood or steel (Fig. $5^{6}$ ), or rectangular, of concrete. It is filled with sand through which the water passes at a rate 50 to 60 times faster than through

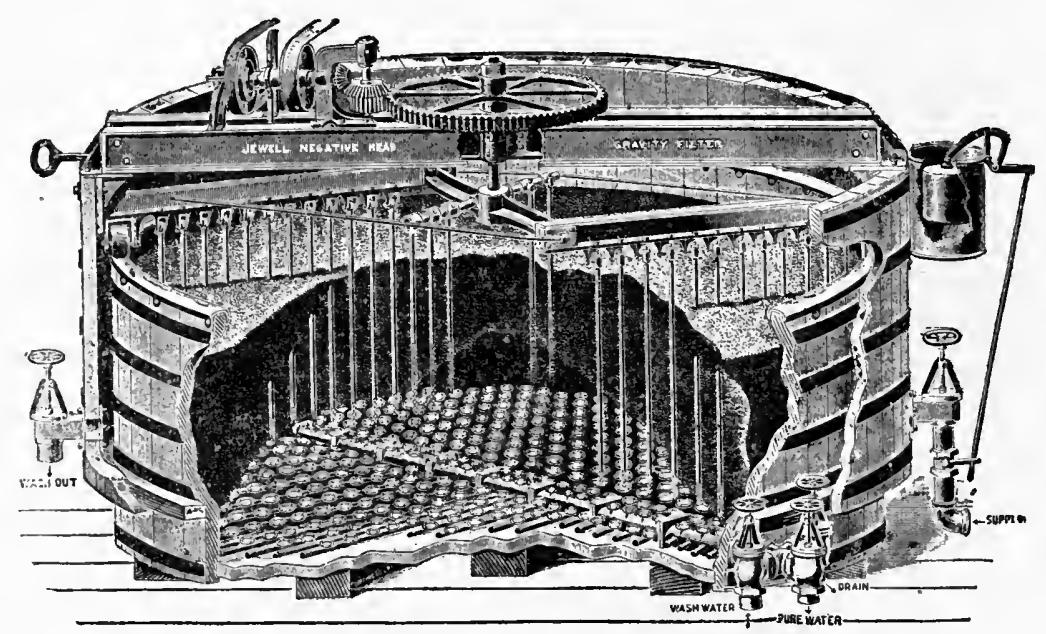

Fig. 56. - Jewell gravity filter with rake. (N. Y. Continental Jewell Filtration Co.)

an ordinary bed filter, the best average rate being IO5 gallons an hour per square foot of area. The sand is somewhat larger than in the ordinary filter, but should be of more uniform size to prevent "breaking" or "channelling" in the process of washing. Whenever the sand becomes clogged, once or twice a day, it is cleaned, in circular tanks, by using a reverse or upward current of filtered water, being meanwhile thoroughly stirred by a revolving rake, the impurities overflowing over the edge of the tank.

In plants using concrete rectangular tanks, the revolving rake is replaced by the "air wash" device, the sand being washed by driving innumerable jets of air and water alternately through it from the bottom, the air for agitation and scrubbing and the water for rinsing. This system is likewise applied to circular tanks. (Fig. 57.)

The so-called pressure mechanical filter is in an entirely closed receptacle through which the water is forced under pressure. (Fig. 58.) When the location is favorable, as when the plant is below the source of supply, this system is indicated and satisfactory. Pressure filters are readily cleansed and operated, and in the case of 


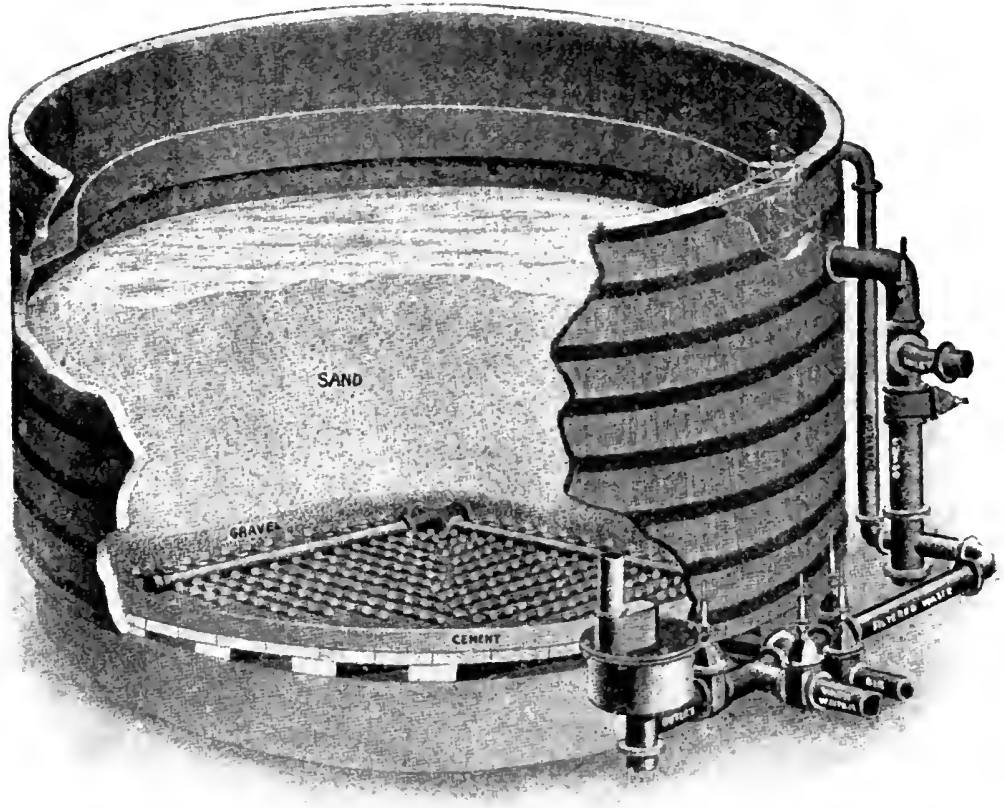

FIG. 57.-Continental " a1r wash" gravity filter.

small communities quite economical. Nevertheless, they are not as reliable as gravity filters and cannot be recommended for drinking water unless supplemented by the use of chloride of lime.

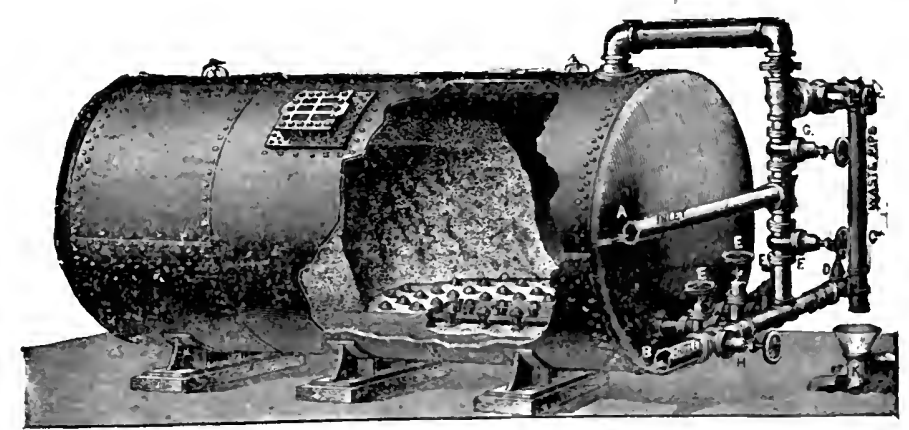

FiG. 58. - New York sectional wash horizontal pressure filter. 
The efficiency of the mechanical filter is not quite as high as that of the best sand-bed filter, seldom removing more than 98 per cent. of the bacteria; but it is 25 per cent. cheaper in total first cost and requires an area of only about one-thirtieth the size.

According to Rudolph Hering: "With good design and construction, and with good operation, these two classes of filters can and do remove about equally well both pathogenic and ordinary bacteria."

THE DARNALI FILTER.*

This apparatus is an ingenious adaptation of the principle of mechanical filtration to the needs of troops in the field. It consists of a galvanized-iron tank, two water cans, a siphon filter and cloth,

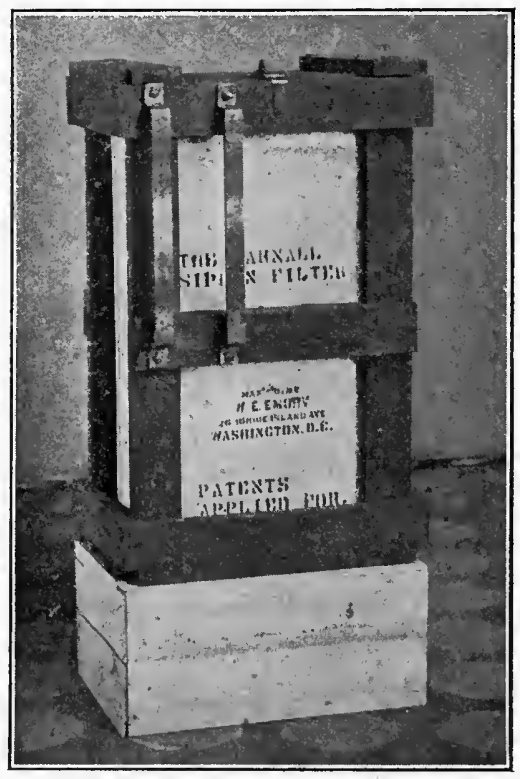

FIG. 59.- The Darnall filter, packed.

a siphon primer (small hand pump to start the flow of water) and a crate (Figs. 59, 60, 6r). The essential part is the cylindrical metal framework of the siphon over which is wrapped the filtering material, a closely woven cotton fabric. This is placed in the tank filled

* Devised by Major Carl R. Darnall, Medical Corps, U. S. Army. 
with raw water to which the precipitant has already been added, and the water, after passing through the filtering cloth into the cylinder, is discharged by siphonage into the water can.

As matters in suspension deposit upon the cloth and the flow of water becomes much diminished, the filter should be taken out and brushed, but when the brushing no longer restores a full flow, the

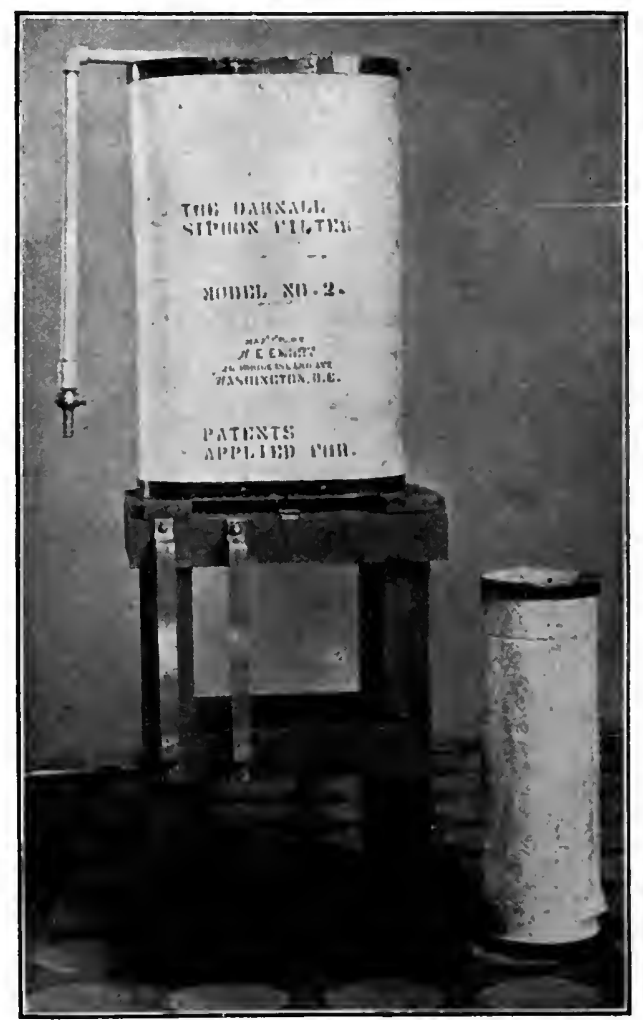

Fig. 60.- The Darnall filter, ready for operation.

cloth must be removed, washed and put aside to dry; a new cloth is put on and sterilized in the can by siphoning boiling water through it.

The precipitant consists of alum and sodium carbonate in such proportions as to neutralize each other. Five grains of alum to the 
gallon is sufficient for the most grossly polluted water. Each gallon of water treated with this amount of alum and its equivalent of soda and then filtered, will contain, in addition to its original chemical constituents, about 2.5 grains of sodium sulphate, 0.93 of potassium sulphate, and 0.7 of carbon dioxid, all of which are harmless if not beneficial. The alum and soda may be used in separate solutions fed automatically, but for use with marching troops and in temporary camps it is more convenient to use them already mixed, in the form of a stable powder, one pound of which is sufficient for 500 gallons of water.

This filter complete (with crate) weighs 52 pounds and will deliver about 200 gallons of water in four hours. It completely clarifies the water. Its bacterial efficiency is about 98 per cent. with

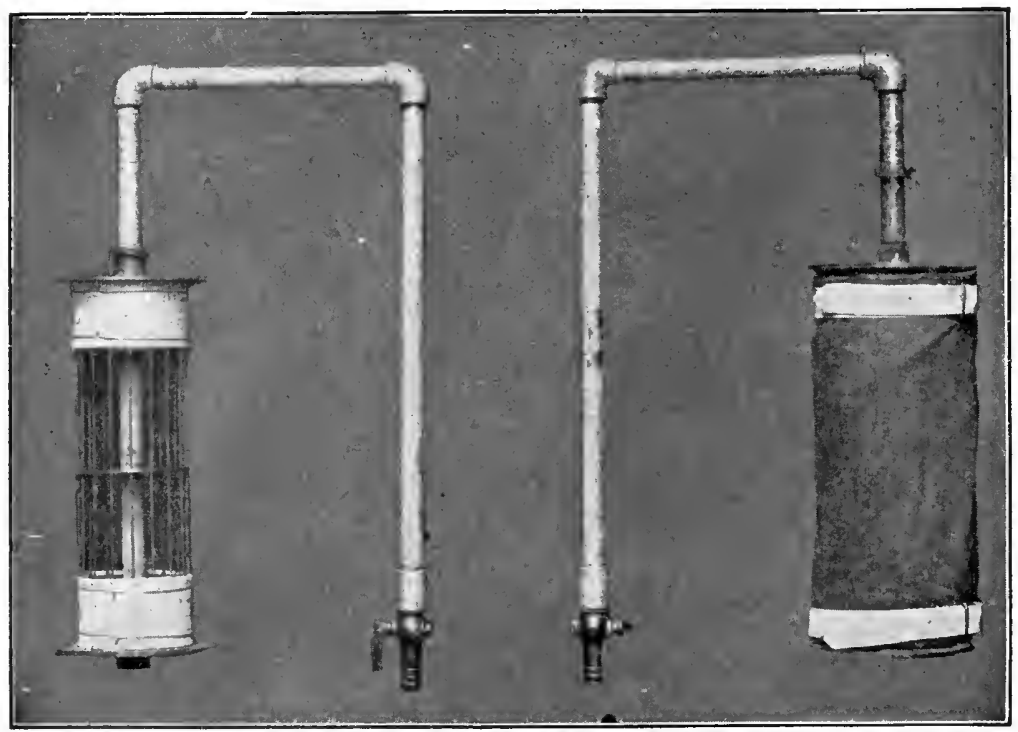

FIG. 6r.-Filtering cylinder of the Darnall filter, with and without filtering cloth.

ordinary waters, and more under favorable conditions, therefore comparing favorably with most municipal filtering plants. This apparatus cannot be depended upon for the certain elimination of all pathogenic bacteria, as with heat sterilizers, but it purifies ordinary waters to a degree that renders them reasonably safe. It does not 
require any fuel except for heating water to sterilize the filter cloths, once daily; it furnishes plenty of clear water, unchanged in temperature and taste, within an hour after getting into camp; the whole outfit is simple, cheap, easily transportable and workable in any situation. It has been carefully investigated by a board of medical officers and its use in the field recommended.

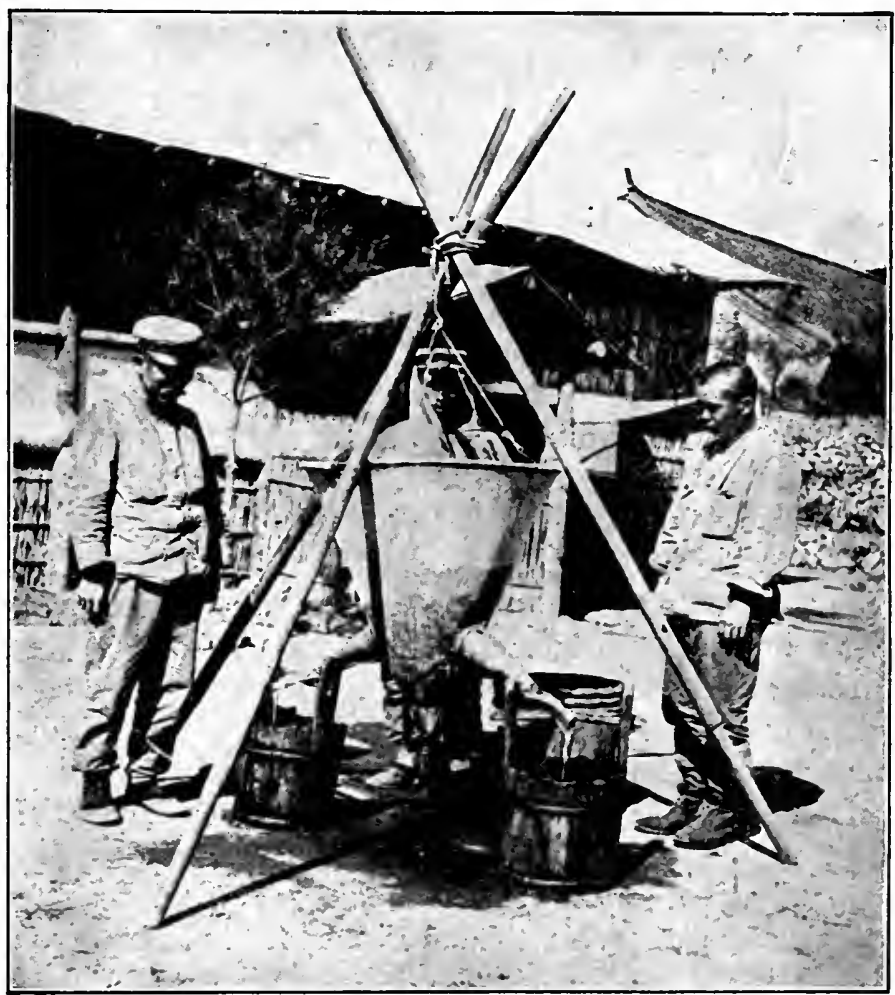

Fig. 62.-The Japanese field filter. (Eshitzi filter.)

\section{THE JAPANESE FIELD FILTER (Eshitzi Filter).}

During the Russo-Japanese War, the Japanese used another simple and practical adaptation of the principle of the mechanical filter. (Fig. 62.) It consists essentially of a conical canvas bag of a capacity of 24 gallons, with two spouts or branches near the point; these spouts are filled with charcoal and sponge disks and constitute the 
filters proper; the point, or apex, receives the sediment. The whole apparatus is suspended between the branches of a tripod.

Two powders are used. The first (A) consists of potassium alum, potassium permanganate and (to give bulk) aluminum silicate; the second (B), chiefly of aluminum silicate and of small amounts of tannic acid and hydrochloric acid. The filter having been filled, a suitable quantity of powder A, enough to plainly discolor the water, is added and stirred up; after a few minutes, about half as much of powder $\mathrm{B}$ is stirred in until the discoloration caused by the first has been removed. Then the water is allowed to stand 15 or 20 minutes for the bactericidal action and subsidence of the precipitate, after which the lateral spouts are untied and the water allowed to pass through. The hydrochloric acid in powder $\mathrm{B}$ facilitates the decomposition of the permanganate, while the tannic acid removes the color imparted to the water.

The result is quite satisfactory with comparatively clear water, but much less so with turbid water. The output is small and the disinfection of the apparatus difficult.

Granular Filters. - This name is given to devices whose filtering material consists of some hard granular substance other than sand, often contained in asbestos cloth. Bone charcoal was formerly commonly used for this purpose; it has the property of removing coloring matters, but on account of its porous nature soon absorbs, and becomes impregnated with, the impurities of the water, when the only way of sterilizing it is by calcination. It also yields nitrogen and phosphates which favor the growth of bacteria in water. Vegetable charcoal, coke and spongy iron are more or less efficient against suspended matters and micro-organisms, but soon deteriorate and, unless constantly watched, are likely to yield more bacteria, saprophytic and pathogenic, than are contained in the raw water.

\section{IMPROVISED FILTERS.}

In camps, filters for the clarification of water can be readily improvised if sand be available, and a certain degree of purification can likewise be obtained. But, on the other hand, it must be remembered that such filters, unless they can be washed or sterilized, may soon become infected and that clarification may be obtained at the expense of purification.

Hard, clean sand is the best material for improvised filtration. 
For reasons stated above, bone charcoal is not suitable. If sand be placed over pebbles and coarse gravel in a barrel, we have the usual sand filter on a small scale; through the perforated bottom the filtered water is discharged into another barrel or can. If the water is turbid it should first be treated with alum, 2 or 3 grains to the gallon. In the absence of sand, this treatment by alum may be sufficient of itself, but with water likely to be contaminated with sewage, it should be followed by boiling.

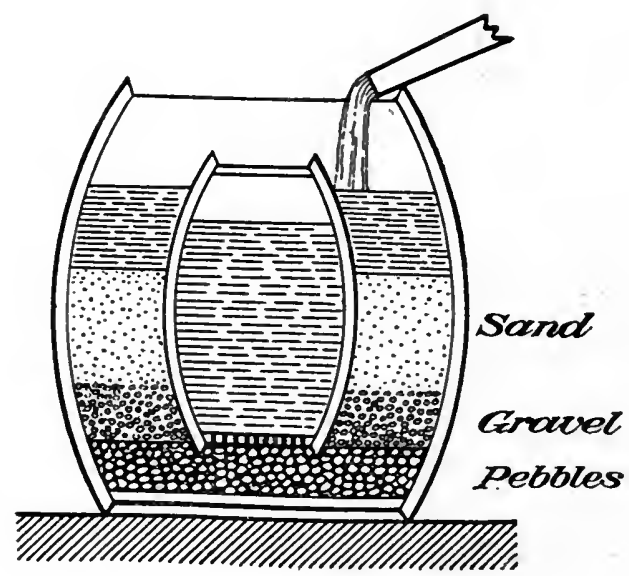

Fig. 63-Double barrel improvised filter.

If a hogshead and a barrel are available, the latter, with perforated bottom, is placed inside the former upon a bed of coarse gravel or pebbles and the interspace filled with gravel and sand; the water is gently poured or sprinkled over the sand and, after filtration, rises up to its level in the barrel. (Fig. 63.) The upper layer of sand should be frequently scraped out and washed or replaced by fresh, clean sand.

Another expedient is to dig a pit at a distance of a few feet from the river's edge, where the bank is sandy, and let the water percolate through the intervening sand into it; or, again, a trench may be dug connecting the river with the pit, then boxed and filled with sand.

The so-called "Venetian cistern" can also be readily improvised in certain situations. It consists (Fig. 64) of a pit with sloping walls of clay (D) lined with cement (C), and of a circular wall of 
clay, likewise cemented, in the center; the interval is filled with clean sand (B) and receives the raw water (A) which, after filtering through, passes into the central well (E).

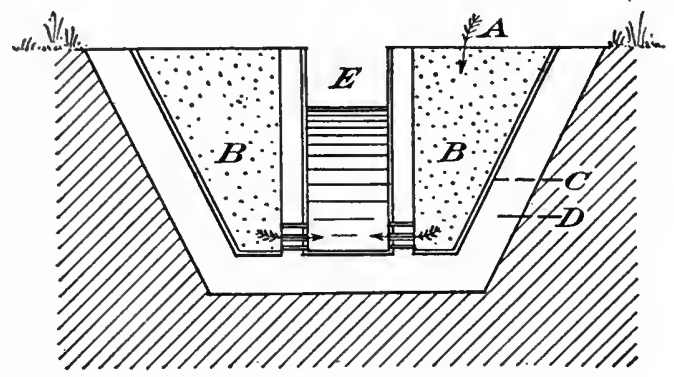

Fig. 64.- Venetian cistern.

In certain arid regions where cactus plants are common, the leaves (or rather joints) of these plants, cut up and crushed, are quite useful to clarify water in the absence of alum. They contain an abundant mucilaginous juice which, like alum, causes the very fine silt suspended in the water to coalesce into larger and heavier particles which precipitate, carrying down with them a large proportion of the micro-organisms. 


\section{CHAPTER XXIV.}

F00D.

Food is any digestible substance introduced into the alimentary canal for the nourishment of the body.

All foodstuffs may be conveniently divided and classified as follows :

Organic
(animal and vegetable). $\begin{cases}\text { Nitrogenous. } & \begin{array}{l}\text { Proteins or proteids. } \\ \text { Non-nitrogenous. }\end{array} \\ \begin{array}{l}\text { Hydrocarbons-fats. } \\ \text { Carbohydrates-sugars } \\ \text { and starches. } \\ \text { Vegetable acids. }\end{array}\end{cases}$

Inorganic

(mineral).

$\left\{\begin{array}{l}\text { Mineral salts. } \\ \text { Water. }\end{array}\right.$

Organic foodstuffs are primarily divided into two great classes, according to the presence or absence of the all-important element nitrogen. They consist of a few constituents, or "proximate principles," existing ready formed in nature. The three important proximate principles entering into the composition of all animal and vegetable foods are the proteids, hydrocarbons and carbohydrates. None of these principles is ever found alone in nature, but they are always associated in variable proportions, as in bread, meat, milk and vegetables, for man is omnivorous and needs them all for his best physical development. '(Fig. 65.)

Nitrogenous constituents of foods. - These constituents, according to Prof. Mallet, may be divided into the following classes:

I. Proteins proper (or albuminoids) and their closely related derivatives, the proteoses and peptones.

2. Gelatinoids or collagens (sometimes also called albuminoids) and allied substances immediately derived from them, such as gela-

3. Amins, amino-acids and allied substances, such as "meat bases" or "extractives" (creatin, creatinin, etc.) of animal origin, and the asparagin, glutamin, etc., of vegetable substances.

4. Alkaloids, or amin-like compounds of well-determined basic character, the active principles of many plants. 
5. Ammonia and its salts.

PROTEIN (proteos, first) is a hypothetical substance formerly believed to be the essential nitrogenous constituent of animal and vegetable foods, but now applied to a number of nitrogenous or "proteid" bodies of definite chemical composition making up the bulk of the soft tissues and blood of animals, and also widely distributed in the vegetable kingdom. Protein and proteid are practically synonymous, although the latter is more generally used adjectively. In analytic work the term protein includes the total nitrogenous substance of animal and vegetable food materials, exclusive of the so-called nitrogenous fats. It is employed, in common usage, to designate the product of the total nitrogen by the factor 6.25 , the per centage of nitrogen in most proteins averaging 16.

Proteins form the chief part of every animal cell, being the principal constituent of muscle, and making up about i 8 per cent. by weight of the body. They are found under many forms in all animal and vegetable foodstuffs. Animal and vegetable proteins are identical in chemical composition and, when equally digestible, have about the same nutritive value. Chemically they consist of carbon, oxygen, nitrogen, hydrogen and sulphur, in the average percentages of $52,23,16,7,0.5$ to 2 , respectively. Phosphorus is also a frequent constituent. Nitrogen is their characteristic element. All proteins are insoluble in alcohol and ether, but differ more or less as to their solubility in water and their decomposition products. Their solutions do not dialyse, that is, do not diffuse through animal membranes or parchment paper. Most of them coagulate when heated. Especially important are the two groups, albumins and globulins; the former, soluble in distilled water, are represented chiefly by egg albumin, serum albumin and lactalbumin (of milk); the latter, insoluble in water, are represented chiefly by egg globulin (vitellin), serum globulin, fibrinogen, and myosinogen (of muscle). Other noteworthy proteins are the glutenin and gliadin of wheat, caseinogen of milk, legumin of peas and beans. Of phosphoproteins, that is, proteins with a large proportion of phosphorus in their molecule, the most important are the caseinogen of milk and the vitellins of egg-yolk. Of compound proteins, the principal is nucleo-protein (protein in combination with nuclein) which forms the main constituent of the nuclei of cells. Nuclein itself is a pro- 
teid compound with an organic acid (nucleic acid). By digestion, proteins are transformed into the more soluble and diffusible proteoses and peptones which, by oxidation and the hydrolytic action of specific ferments (enzimes), break up into amino-acids (such as leucin, tyrosin, etc.) with the final end-products of carbon dioxid, water and urea, and small proportions of uric acid and creatinin.

The quantity of protein ingested is readily ascertained from the proportion contained in foodstuffs. The amount discharged from the body is determined from the amount of nitrogen excreted. It is assumed that each gram of nitrogen corresponds to 6.25 grams of protein. It is further assumed that meat contains an average of 3.4 per cent. of nitrogen so that each gram of nitrogen corresponds to 30 grams of muscle. Nearly the entire quantity of nitrogen excreted (over 90 per cent.) is found in the urine, chiefly as urea, uric acid, creatinin, ammonia and amino-bodies. Creatinin remains practically constant, while creatin, from which it is derived, is only found in urine under abnormal conditions, such as starvation and fevers, indicating destruction of muscular structure. Only about one gram of nitrogen per diem occurs in the feces, made up from unabsorbed nitrogenous bodies and others excreted from the blood. Nitrogen, as urea, is also found in the sweat, but usually in such small amount as to be negligible.

Among the decomposition products of nucleo-protein is the group of purin bodies. They are substances chemically built around a nucleus having the formula, $\mathrm{C}_{5} \mathrm{H}_{4} \mathrm{~N}_{4}$, and to which the name purin has been given. They result from the breaking up of nucleo-protein into adenin and guanin (amino-purins); these by oxidation form xanthin and hypoxanthin which, by further oxidation, yield the terminal product uric acid, the chief form in which the purins are

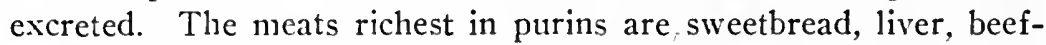
steak, sirloin, chicken, turkey, veal, pork and mutton. There is no difference between red and white meats as to their purin content. This content varies in fish, being low in white fisl, like cod, and as high as in meat in other fish, like halibut and salmon. Milk and eggs, as well as vegetables, are practically purin free.

The daily uric acid excretion, on an ordinary diet, is about one gram, and does not greatly depend upon the amount of nitrogen in food, being only reduced to 0.6 gram on a purin-free diet. A uric acid diathesis, however, is generally promptly improved by a diet 
of rice, bread and butter. In gout, the increase of uric acid in the blood may continue on a purin-free diet, apparently the effect of a change into an insoluble form which the kidneys cannot excrete. Violent muscular exercise, even on a purin-free diet, increases the output of purin bases and uric acid, probably on account of the presence of xanthin and hypoxanthin in muscle.

Nutrinve ingredients, refuse, and fuel value.

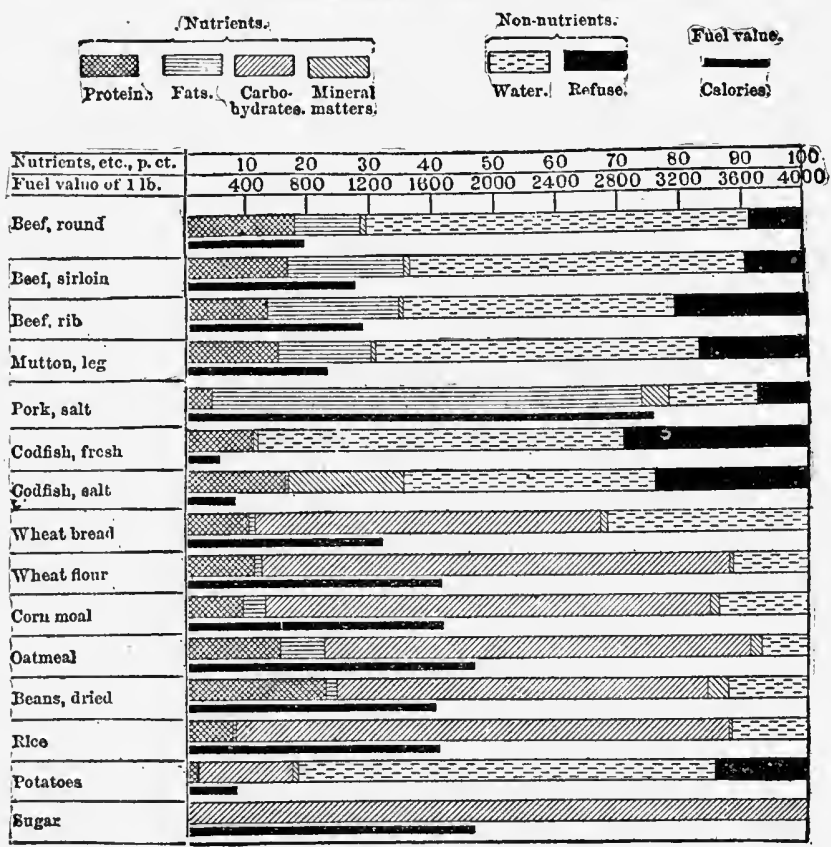

FIG. 65.- Composition of the chief foodstuffs entering into the United States Army ration. (Atwater.)

Gelatinoids. - Include ossein, the organic substance of bone ; collagen, the chief constituent of white fibrous tissue and connective tissue; cartilage and ligament; the air-bladder and various other parts of fish. By boiling, these various tissues are mostly transformed into gelatin and chondrin. Continued boiling produces soluble gelatin, gelatoses and peptones.

Organic extractives. - These are substances extracted from muscular and connective tissues by boiling, such as creatin, creatinin, 
xanthin, hypoxanthin and allied cleavage products of the proteins. They are soluble in water, of simpler constitution than the proteins, and (together with certain mineral salts, chiefly potassium phosphate) the most important constituents of beef-tea and many meat extracts. It is to be noted that flesh extractives contain also nonnitrogenous components, of which the most important are sarcolactic acid and glycogen.

It seems probable that the xanthin and hypoxanthin of muscular tissue are metabolized into uric acid in view of the considerable increase of this acid in the urine by active exercise.

Hydrocarbons or Fats. - Fats, in nature, occur mostly under three forms, stearin, palmitin, and olein, which are compounds of glycerin with stearic, palmitic and oleic acids respectively. These three compounds are always more or less combined in nature. Stearin and palmitin are solid, while olein is liquid at ordinary temperature. They are free from nitrogen, consisting chiefly of hydrogen and carbon, with a proportion of oxygen too small to combine with the hydrogen to form water, their general formula being $\mathrm{C}_{10} \mathrm{H}_{18} \mathrm{O}$. Fat forms about 15 per cent., by weight, of the body of man, being mostly deposited in the abdominal, muscular and subcutaneous tissues. All foodstuffs, animal and vegetable, contain fat in variable proportions; especially rich in it are meats, milk, eggs, nuts and such cereals as corn and oats; while some, like olive oil, cotton-seed oil, butter, bacon, suet and lard consist chiefly of it.

Carbohydrates are so called because, in addition to carbon, they contain hydrogen and oxygen in proportion to form water, that is, twice as many atoms of hydrogen as of oxygen. They occur abundantly in plants, chiefly as sugars and starches, but form less than I per cent. of the body tissues.

Grape sugar, glucose or dextrose $\left(\mathrm{C}_{6} \mathrm{H}_{12} \mathrm{O}_{6}\right)$, the common sugar of fruits and flowers, characterized by its property of reducing solutions of copper sulphate, and manufactured on a large scale by the action of dilute acids on starches. It is found normally in animal tissues, generally from the transformation of other carbohydrates, or as a morbid product excreted by the kidneys, as in diabetes.

Maltose is the sugar produced from starch by the action of ptyalin and amylopsin; also produced by the ferment diastase in germinating barley, acting upon the starch of the grain which becomes "malt." 
Cane sugar or saccharose $\left(\mathrm{C}_{12} \mathrm{H}_{22} \mathrm{O}_{11}\right)$, the crystalline sugar of sugar-cane, beet-root and the sap of maple, birch and other trees. It does not reduce solutions of copper sulphate, but is easily transformed into glucose by fermentation or the action of dilute acids.

Milk sugar or lactose, only found in milk.

Saccharose, maltose and lactose when taken into the alimentary canal are not utilized as such, but promptly converted into forms of glucose. Sugar, as glucose or dextrose, is one of the normal constituents of the blood, its quantity being regulated by the liver so that a definite proportion is maintained in spite of all fluctuations in the supply. This function of the liver consists in transforming the excess into glycogen or animal starch $\left(\mathrm{C}_{6} \mathrm{H}_{10} \mathrm{O}_{5}\right)$; this is stored up as reserve material, chiefly in the liver and the muscles, to be reconverted into sugar when needed by the body.

Saccharin, a complex substance derived from one of the components of coal-tar naphtha, is a white, crystalline powder, odorless but intensely sweet, frequently used as a substitute for sugar. According to an official statement from the Department of Agriculture, "the continued use of saccharin for a long time in quantities over three-tenths of a gram ( $4^{\mathrm{T}} / 2$ grains) per day is liable to impair digestion; and the addition of saccharin as a substitute for cane sugar or other forms of sugar reduces the food value of the sweetened product and hence lowers its quality."

Starches $\left(\mathrm{C}_{6} \mathrm{H}_{10} \mathrm{O}_{5}\right)$ exist in all plants, being the principal constituent of seeds and cereals, as well as many tubers and roots. They are mostly derived from wheat, corn, potatoes, the roots of cassava and arrowroot, and the pith of the sago-palm. They occur in characteristic grantles made up of concentric layers, of variable size and shape according to the plants producing them (Fig. 66). Starches are insoluble but, when heated in water, the granules swell up, burst their membranes and form an easily digestible paste. With tincture of iodine they yield a characteristic intense blue color. In the body they are acted upon by ferments (enzymes) and converted, at first into a gum-like substance (dextrine), then into dextrose and glucose.

Other carbohydrates worth mentioning are: cellulose, which forms the framework of plants and constitutes the fiber of flax, hemp, cotton, the hull of seeds, etc., being quite insoluble and of no dietetic value; pectin, which forms the jelly of various ripe fruits. 
Vegetadle Acids consist of the same clemical elements as carbollydrates, but the oxygen is in quantity more than sufficient to convert the hydrogen into water. They are derived from fruits and other parts of plants. Those most used as food are: tartaric acid, found in grape juice as potassium tartrate; citric acid, found in the orange, lemon and other fruits of the citrus genus, as well as

1

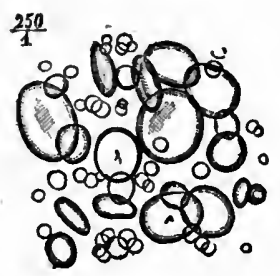

4

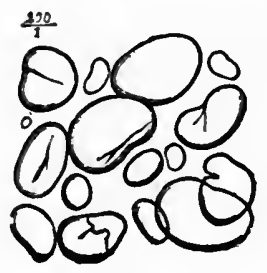

7

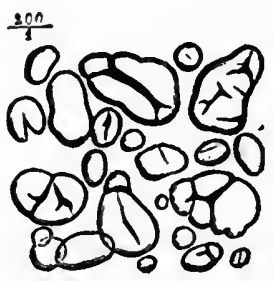

2

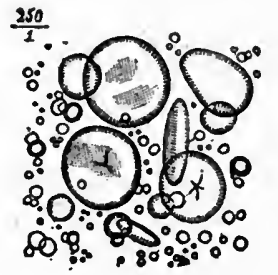

5

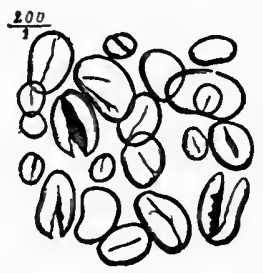

8

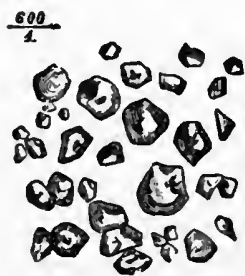

3

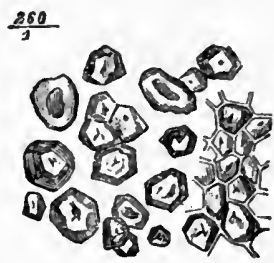

6

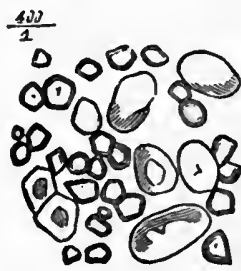

9

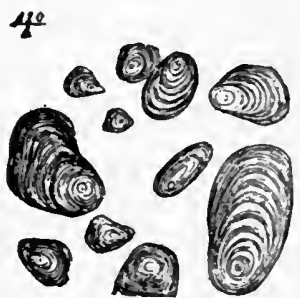

FIG. 66.- Starch grains of various vegetables. I, wheat; 2 , rye; 3 , corn; 4. rice; 5 . horse-bean; 6 , potato; 7 , bean; 8 , pea; 9 , buckwheat.

in gooseberries and potatoes; malic acid, found in the apple and pear; oxalic acid, found in rhubarb, tomatoes, sorrel and other plants with acid foliage; acetic acid, the active constituent of vinegar.

They exist in the free state or, more commonly, in combination as salts. In the body, they are decomposed and become oxidized 
into carbonates which help to maintain the alkalinity of the blood and other fluids. Their absence in foods is soon severely felt, resulting in a lowering of vitality, malassimilation and symptoms of scurvy.

Mineral Salts. - The salts most necessary for the body are sodium and potassium chlorides, iron, and phosphates of lime, potassium, sodium and magnesium. Chlorides are indispensable to keep in solution the globulins and albumin of the blood and other fluids, as well as to supply the hydrochloric acid of the gastric juice. The phosphates are needed for bone growth and repair, while phosphorus is a constituent of nerve tissue. Phosphate of lime is found in all tissues and seems essential to cell growth. Iron is a necessary constituent of the coloring matter of the hemoglobin of the red blood-corpuscles. All these salts, with the exception of sodium chloride, which is used as a condiment, exist generally in sufficient quantity in drinking water and the ordinary articles of food.

\section{NUTRITIVE FUNCTIONS OF FOOD PRINCIPLES.}

The proteins are the tissue formers and repairers. They form the chemical basis of all living cells, whether animal or vegetable, and are absolutely necessary for the building and renewal of tissues and organs. No life is possible without them. The amount used in the repair of tissues bears no relation to the amount of muscular work performed; it is a daily constant, regardless of bodily exertions. Another function of protein, but secondary and unimportant, is to furnish heat by its combustion as fuel. Under exceptional circumstances proteins can be converted into carbohydrates as, for instance, in the case of diabetic patients who although fed on an exclusively proteid diet continue to excrete sugar. As much as 60 per cent. of the protein ingested can thus be converted into sugar.

Physiologically two facts differentiate proteins from other foodstuffs in adults: I, the daily demand for them is fixed and does not vary with the amount of work done as is the case with fats and carbohydrates; 2 , there is no provision for their storage, so that any excess ingested must be catabolized and eliminated.

An adult animal puts itself promptly into equilibrium with its supply of nitrogen; if this supply be equal to the needs of metabolism there is neither loss nor gain of the body proteids; the output of nitrogen is practically equal to the intake; only an evanescent 
gain of body nitrogen can be produced, in the adult, by the most liberal supply of proteid food.

Bacteria play an important part in the digestion and decomposition of proteins. The conditions necessary for their growth are very favorable in the intestines where they live in symbiosis, that is to say, with benefit to themselves and their host. Bacteria decompose foodstuffs and bring about products often identical with those resulting from the action of the digestive juices, but sometimes of a different nature. In the small intestine the carbodydrate bacteria are the most active, whereas in the large intestine they are mostly replaced by those which act upon protein; here their action corresponds to that of trypsin, but they digest further and produce a multitude of simple degradation products (ammonia, fatty volatile acids, carbon dioxid, etc.) as well as ptomains and various substances belonging to the aromatic series. These aromatic bodies (which also result from the trypsin digestion of proteins) are immediately derived from certain amino-bodies, namely tyrosin, tryptophan and phenylamin; from tyrosin come phenol and its derivative kresol, while from tryptophan come indol and its derivative skatol. A considerable proportion of these bodies are absorbed into the blood and reappear in the urine as ethereal sulphates. Their presence in the urine has generally been considered a convenient index of the degree of putrefactive bacterial action going on in the large intestine. To check, this source of "auto-intoxication" which Metchnikoff contends is the chief cause of senility, the ingestion of lactic-acidproducing organisms has been recommended, especially as found in milk inoculated with Bacillus bulgaricus (see page 342). Another organism, the Glycobacter peptolyticus of Metclinikoff and Wo.1man, is reported, when in the presence of potato starch, to be another preventive of the putrefactive production of phenol and indol.

Gelatinoids generally contain somewhat more nitrogen than the proteins proper but, for reasons not well understood, possess little nutritive value, the nitrogen not being in an assimilable form. Of themselves they are incapable of keeping the body in nitrogen equilibrium, but have a rather marked protein-sparing effect when added to proteins in the diet, so that, within certain limits, they may act as a substitute; thus, according to Kauffmann, one-fifth of the protein ration can be replaced by gelatinoids without detriment to the body. 
Fats and carbohydrates are the sources of heat and energy, the natural fuel foodstuffs of the body, by their oxidation maintaining the body temperature and evolving power to run the bodily machinery. The greater the muscular exertions, and the colder the weather, the larger is the amount needed and consumed. They undergo complete combustion in the body to simple gaseous products, namely carbon dioxid and water. Fats containing much more carbon than carbohydrates have a much greater fuel value per pound but are less easily oxidizable. Like proteids they form an essential part of the structure of tissues, the surplus becoming stored in them for the needs of the body economy. In the process of digestion they are mostly split by the steapsin of the pancreatic juice into fatty acids and glycerine, with formation of sodium soaps; only when finely emulsified are they also split in the stomach by the gastric lipase. After absorption of their parts, fats are reconstituted by the action of lipase, a ferment thus shown to be reversible in its power, splitting fats and later bringing about their synthesis, as needed in the tissues. Bacteria in the intestines act upon fats like steapsin, but carry the process a step further down the fat-acid series.

Bile assists materially in the absorption of fat. When its secretion is checked from any cause, there is an excess of fatty acids in the intestinal contents, coating the particles of food and preventing the digestive juices from getting at them, thus favoring the growth of the bacteria of putrefaction.

Fats are specially concerned in the growth and repair of brain and nerve tissues, forming one-fifth of the solid matter of the brain, and are likewise of much value in wasting diseases such as tuberculosis. Much fat may be derived from carbohydrates as seen by the increased plumpness of men employed in sugar mills, as well as by the amount of cream in the milk of cows simply fed on grass, this amount being much in excess of the fat contained in the grass. There is no evidence that fat can be formed from proteins in the animal body, or that it may be converted into sugar in the metabolism of the diabetic. In fatty degeneration there is no change of protein into fat, but simply a wasting of the protein, so that the fat previously existent in the cells runs together and comes prominently into view. When not required in the system, fats are absorbed with difficulty and render many articles of food indigestible; or " too 
rich." The free use of water seems to favor the deposit of fat in the body, while, on the other hand, a diet consisting chiefly of lean meat causes rapid oxidation of fat and reduces obesity.

The acetone bodies, namely oxybutyric acid, diacetic acid and acetone, are largely derived from the fatty acids and make their. appearance in the urine, when fats are metabolized at an unusually rapid rate, as for instance in the urine of diabetes, starvation and low carbohydrate diet. They reduce the alkalies of the body and produce the condition known as acidosis.

Carbohydrates are all absorbed as sugar. From the ready conversion of sugar into fat, it does not follow that these principles are interchangeable in the diet; both have doubtless different functions to perform before their final reduction to carbon dioxid and water. Sugar is of special importance as muscle food, rendering men capable, at least for a few days, of unusual muscular exertion, and alleviating hunger, thirst and fatigue. When soldiers are called upon to do strenuous work, nothing is better than small cups of black coffee strongly sweetened with sugar or glucose.

Experiments have shown that if the supply of carbohydrates be deficient in the organism, creatin appears in the urine, with a rise in the output of total nitrogen, indicating increased catabolism of protein, whereas if the supply of carbohydrates be abundant, not only is there no creatin but the required nitrogen may be lessened and a normal equilibrium obtained with from 8 to Io grams daily.

Fat in the food, even in considerable amount, does not save protein catabolism.

The action of bacteria on carbohydrates in the intestines is even more energetic than on proteins. Besides doing all that ptyalin and amylopsin can do, they also decompose saccharides into simpler bodies such as ethyl alcohol, lactic and butyric acids. Without their aid cellulose cannot be digested so that for herbivora, at least, bacteria are indispensable. 


\section{CHAPTER XXV.}

\section{ANIMAL FOODS.}

For our purpose, animal foods may be classified as follows: I. Meats, including poultry and game. 2. Soups and meat extracts. 3. Fish, mollusks and crustaceans. 4. Milk and its derivatives. 5. Eggs.

\section{MEAT.}

Meat consists of muscular fibers, connective tissue which holds the fibers together, and fat cells in the connective tissue. Each fiber is enveloped and protected by a sheath of sarcolemma, a non-contractile but elastic structureless membrane, and consists of many fibrils or minute, striated, microscopic tubes whose contents continually undergo change and renewal.

The muscular fiber contains proteins, salts and extractives. The chief protein is myosinogen, and the principal salt potassium phosphate. Extractives are nitrogenous substances derived from proteins and "extracted" by boiling water; they have very little nutritive value but give to meats their characteristic flavors, increasing with the age of the animals and varying much in quality according to the food of the latter; thus lamb and veal are less flavored than mutton and beef, while game animals feeding upon wild herbs are more savory than domesticated ones. The younger the animal the more watery is its flesh and the lower its nutritive value. The amount of fat in meat is quite variable, ranging, according to the condition of the animal, from 3 per cent. in young fowl, rabbit or veal in which there is no visible fat, to 25 per cent. in fat steer and 90 per cent. in fat hog; its increase is always at the expense of water, the more fat the less water and conversely.

According to Farrington (Bureau of Animal Industry), fat cattle dress 60 per cent. of live weight, sheep 50 per cent., hogs 80 per cent. Much of the waste, however, could be saved by better methods and more suitable machinery.

The average composition of the meats most used is as follows (from Bulletin 28, revised edition. U. S. Department of Agriculture): 


\begin{tabular}{|c|c|c|c|c|c|c|}
\hline MEATS. & Refuse.* & Water. & Protein. & Fat. & Ash. & $\begin{array}{l}\text { Fuel } \\
\text { value } \\
\text { per } \\
\text { pound. }\end{array}$ \\
\hline risket: $\quad$ BEEF, FRESH. & Per cent. & Per cent. & Per cent. & Per cent. & Per cent. & Cals. \\
\hline $\begin{array}{l}\text { Edible portion**............ } \\
\text { As purchased*..... } \\
\text { Chuck, including shoulder: }\end{array}$ & 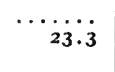 & $\begin{array}{l}54.6 \\
41.6\end{array}$ & $\begin{array}{l}15.8 \\
12.0\end{array}$ & $\begin{array}{l}28.5 \\
22.3\end{array}$ & $\begin{array}{r}0.9 \\
.6\end{array}$ & $\begin{array}{l}1,495 \\
I, 165\end{array}$ \\
\hline $\begin{array}{l}\text { Edible portion } . \ldots \ldots \ldots \ldots \ldots \\
\text { As purchased........ }\end{array}$ & ${ }^{\prime}{ }_{17.3}$ & $\begin{array}{l}65.0 \\
54.0\end{array}$ & $\begin{array}{l}19.2 \\
15.8\end{array}$ & $\begin{array}{l}15.4 \\
12.5\end{array}$ & .9 & $\begin{array}{r}1,005 \\
820\end{array}$ \\
\hline 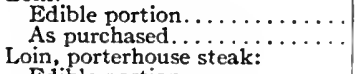 & $\cdots \cdots \cdots$ & $\begin{array}{l}61.3 \\
52.9\end{array}$ & $\begin{array}{l}19.0 \\
16.4\end{array}$ & $\begin{array}{l}19.1 \\
16.9\end{array}$ & $\begin{array}{r}\text { I. } \\
.9\end{array}$ & $\begin{array}{l}1,155 \\
1,020\end{array}$ \\
\hline $\begin{array}{l}\text { Edible portion. . . } \ldots \ldots \ldots \ldots \ldots \\
\text { As purchased. } . \cdots \cdots \cdots \\
\text { oin, sirloin steak: }\end{array}$ & $\cdots \cdots$ & $\begin{array}{l}60.0 \\
52.4\end{array}$ & $\begin{array}{l}21.9 \\
19.1\end{array}$ & $\begin{array}{l}20.4 \\
17.9\end{array}$ & $\begin{array}{r}\mathrm{I} .0 \\
.8\end{array}$ & $\begin{array}{l}\text { I, } 270 \\
\text { I, I IO }\end{array}$ \\
\hline $\begin{array}{l}\text { Edible portion. . } \\
\text { As purchased............... } \\
\text { Ribs: }\end{array}$ & 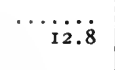 & $\begin{array}{l}61.9 \\
54.0\end{array}$ & $\begin{array}{l}18.9 \\
16.5\end{array}$ & $\begin{array}{l}18.5 \\
16.1\end{array}$ & .0 & $\begin{array}{r}1,130 \\
985\end{array}$ \\
\hline $\begin{array}{l}\text { Edible portion. . . . . . . . . . } \\
\text { As purchased. } \\
\text { Round: }\end{array}$ & $\cdots \cdots$ & $\begin{array}{l}57.0 \\
45.3\end{array}$ & $\begin{array}{l}17.8 \\
14.4\end{array}$ & $\begin{array}{l}24.6 \\
20.0\end{array}$ & .9 & $\begin{array}{l}1,370 \\
1,110\end{array}$ \\
\hline $\begin{array}{l}\text { Edible portion. } \ldots \ldots \ldots \ldots \ldots \\
\text { As purchased....... } \\
\text { Rump: }\end{array}$ & $\cdots \ddot{8.5}$ & $\begin{array}{l}67.8 \\
62.5\end{array}$ & $\begin{array}{l}20.9 \\
19.2\end{array}$ & $\begin{array}{r}10.6 \\
9.2\end{array}$ & $\begin{array}{l}\text { I. I } \\
\text { I.O }\end{array}$ & $\begin{array}{l}835 \\
745\end{array}$ \\
\hline 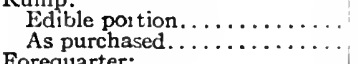 & $\begin{array}{l}\cdots \cdots \\
19.0\end{array}$ & $\begin{array}{l}57.9 \\
45.9\end{array}$ & $\begin{array}{l}18.7 \\
15.2\end{array}$ & $\begin{array}{l}23.1 \\
18.6\end{array}$ & .9 & $\begin{array}{l}1,325 \\
1,065\end{array}$ \\
\hline $\begin{array}{l}\text { Forequarter: } \\
\text { Edible portion..... } \\
\text { As purchased..... }\end{array}$ & $\cdots \cdots$ & $\begin{array}{l}62.5 \\
49.5\end{array}$ & $\begin{array}{l}18.3 \\
14 \cdot 4\end{array}$ & $\begin{array}{l}18.9 \\
15.1\end{array}$ & $\begin{array}{l}9 \\
.7\end{array}$ & $\begin{array}{r}1,135 \\
905\end{array}$ \\
\hline $\begin{array}{l}\text { Hindquarter: } \\
\text { Edible portion..... } \\
\text { As purchased..... } \\
\text { Sides: }\end{array}$ & ${ }^{\prime}{ }_{10.3}$ & $\begin{array}{l}62.2 \\
52.0\end{array}$ & $\begin{array}{l}19.3 \\
16.1\end{array}$ & $\begin{array}{l}18.3 \\
15.4\end{array}$ & .9 & $\begin{array}{r}\text { I, } 130 \\
950\end{array}$ \\
\hline 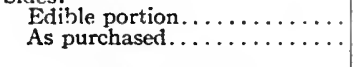 & $\cdots \ddot{8} \ddot{8}$ & $\begin{array}{l}62.2 \\
50.5\end{array}$ & $\begin{array}{l}18.8 \\
15.2\end{array}$ & $\begin{array}{l}18.8 \\
15.5\end{array}$ & .9 & $\begin{array}{r}1,145 \\
935\end{array}$ \\
\hline BEEF ORGANS. & & & & & & \\
\hline $\begin{array}{l}\text { Brain, edible portion........... } \\
\text { Heart: }\end{array}$ & $\cdots$ & 80.6 & 8.8 & 9.3 & I. I & 555 \\
\hline $\begin{array}{l}\text { Edible portion } . \ldots \ldots \ldots \ldots \cdots \cdots \\
\text { As purchased.......... } \\
\text { Kidney: }\end{array}$ & $\begin{array}{l}\cdots \\
\quad 5.9\end{array}$ & $\begin{array}{l}62.6 \\
53.2\end{array}$ & $\begin{array}{l}16.0 \\
14.8\end{array}$ & $\begin{array}{l}20.4 \\
24.7\end{array}$ & $\begin{array}{r}1.0 \\
.9\end{array}$ & $\begin{array}{l}1,160 \\
1,320\end{array}$ \\
\hline $\begin{array}{l}\text { Edible portion } . \ldots \ldots \ldots \ldots \ldots \\
\text { As purchased. } \ldots \ldots \ldots \ldots \ldots \\
\text { Beef liver: }\end{array}$ & ‥9 & $\begin{array}{l}76.7 \\
63.1\end{array}$ & $\begin{array}{l}16.6 \\
13.7\end{array}$ & $\begin{array}{l}4.8 \\
1.9\end{array}$ & $\begin{array}{l}1.2 \\
1.0\end{array}$ & $\begin{array}{l}520 \\
335\end{array}$ \\
\hline $\begin{array}{l}\text { Edible portion.............. } \\
\text { As purchased............... } \\
\text { Sweetbreads, as purchased.... } \\
\text { onque: }\end{array}$ & $\begin{array}{r}\cdots \cdots \\
7 \cdot 3 \\
\cdots \cdots\end{array}$ & $\begin{array}{l}71.2 \\
65.6 \\
70.9\end{array}$ & $\begin{array}{l}20.4 \\
20.2 \\
16.8\end{array}$ & $\begin{array}{r}4.5 \\
3.1 \\
12.1\end{array}$ & $\begin{array}{l}1.6 \\
1.3 \\
1.6\end{array}$ & $\begin{array}{l}605 \\
555 \\
825\end{array}$ \\
\hline $\begin{array}{l}\text { Edible portion } \ldots \ldots \ldots \ldots \ldots \ldots \\
\text { As purchased............... }\end{array}$ & $\cdots 2 \ddot{26.5}$ & $\begin{array}{l}90.8 \\
51.8\end{array}$ & $\begin{array}{l}18.9 \\
\text { I } 4.1\end{array}$ & $\begin{array}{l}9.2 \\
6.7\end{array}$ & $\begin{array}{r}1.0 \\
.8\end{array}$ & $\begin{array}{l}740 \\
545\end{array}$ \\
\hline BEEF, & & & & & & \\
\hline $\begin{array}{l}\text { Roast, as purchased ........... } \\
\text { Round steak, fat removed, as }\end{array}$ & $\cdots$ & 48.2 & 22.3 & 28.6 & I. 3 & \\
\hline 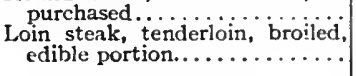 & & $\begin{array}{l}63.0 \\
54.8\end{array}$ & $\begin{array}{l}27.6 \\
23.5\end{array}$ & $\begin{array}{r}7.7 \\
20.4\end{array}$ & $\begin{array}{l}1.8 \\
1.2\end{array}$ & $\begin{array}{r}840 \\
1,300\end{array}$ \\
\hline BEEF, CANNED. & & & & & & \\
\hline 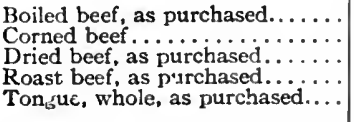 & $\begin{array}{l}\cdots \cdots \\
\cdots \cdots \\
\cdots \cdots \\
\cdots \cdots\end{array}$ & $\begin{array}{l}51.8 \\
51.8 \\
44.8 \\
58.9 \\
51.3\end{array}$ & $\begin{array}{l}25.5 \\
26.3 \\
39.2 \\
25.9 \\
19.5\end{array}$ & $\begin{array}{r}22.5 \\
18.7 \\
5.4 \\
14.8 \\
23.2\end{array}$ & $\begin{array}{r}1.3 \\
4: 0 \\
11.2 \\
1.3 \\
4.0\end{array}$ & $\begin{array}{r}1,425 \\
1,280 \\
960 \\
1,105 \\
1,340\end{array}$ \\
\hline
\end{tabular}

* Meats, as purchased, always contain more or less refuse (bone, gristle, skin, etc.) which lowers the relative proportion of nutrients.

** That is to say, with refuse deducted. 


\begin{tabular}{|c|c|c|c|c|c|c|}
\hline MEATS. & Refuse. & Water. & Protein. & Fat. & Ash. & $\begin{array}{c}\text { Fuel } \\
\text { value } \\
\text { per } \\
\text { pound. }\end{array}$ \\
\hline & Per cent. & Per cent. & Per cent. & Pec rent. & Per cent & Cals. \\
\hline \multicolumn{7}{|l|}{ BEEF, CORNED. } \\
\hline $\begin{array}{l}\text { Corned beef: } \\
\text { Edible portion }\end{array}$ & & & & & & \\
\hline $\begin{array}{l}\text { Edible portion. } \ldots \ldots \ldots \ldots \ldots \\
\text { As purchased. } \ldots \ldots \ldots \ldots \ldots\end{array}$ & $\ldots \ldots \ldots$ & 53.6 & I5.6 & 26.2 & 4.9 & $r, 395$ \\
\hline As purchased. . . . . . . . . . & 8.4 & 49.2 & $14 \cdot 3$ & 23.8 & 4.6 & I, $27 \mathrm{I}$ \\
\hline \multicolumn{7}{|l|}{ VEAI, FRESH. } \\
\hline Leg, hind: & & & & & & \\
\hline 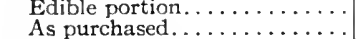 & $\cdots \cdots \cdots$ & 71.7 & 20.7 & 6.7 & I. I & 670 \\
\hline $\begin{array}{l}\text { As purchased.............. } \\
\text { Loin: }\end{array}$ & $\mathrm{Ir} \cdot 7$ & 63.4 & 18.3 & 5.8 & I. 0 & 585 \\
\hline Edible portion. . . . . . . . & & 60.5 & 19.9 & Io. 0 & I. I & 790 \\
\hline As purchased.............. & I 8.0 & 56.3 & I6. I & 8.2 & .9 & 645 \\
\hline \multicolumn{7}{|l|}{ LAMB, FRESH. } \\
\hline Leg, hind: & & & & & & \\
\hline Edible portion. . . . . . . . & $\cdots \ldots$ & 58.6 & 186 & 22.6 & I. 0 & I, 300 \\
\hline $\begin{array}{l}\text { As purchased................ } \\
\text { Loin, without kidney and tallow: }\end{array}$ & 13.8 & 50.3 & 16.0 & I9. 7 & .9 & 1,130 \\
\hline Edible portion................... & . & $53 . I$ & 18.7 & 28.3 & 1.0 & I, 540 \\
\hline $\begin{array}{l}\text { As purchased } \\
\text { Side, without tallow: }\end{array}$ & 14.8 & $45 \cdot 3$ & 16.0 & $24 . I$ & .8 & 1,3 I 5 \\
\hline Edible portion.................... & $\cdots$ & 58.2 & 17.6 & $23 . I$ & I. I & 1,300 \\
\hline As purchased............ & 19.3 & 47.0 & 14.1 & 18.7 & .8 & 1,055 \\
\hline \multicolumn{7}{|l|}{ LAMB, COOKED. } \\
\hline Leg, roast................ & $\ldots \ldots$ & $67 \cdot I$ & 19.7 & 12.7 & .8 & 900 \\
\hline \multicolumn{7}{|l|}{ MUTTON, FRESH. } \\
\hline Leg, hind: & & & & & & \\
\hline $\begin{array}{l}\text { Edible portion. } \ldots \ldots \ldots \ldots \ldots \ldots \\
\text { As purchased. . . } \ldots \ldots \ldots \ldots \ldots\end{array}$ & $\cdots \cdots$ & 6.3 .2 & I 8.7 & $17 \cdot 5$ & 1.0 & $I, 085$ \\
\hline Loin, without kidney or tallow: & I 7.7 & 51.9 & 15.4 & 14.5 & .8 & 900 \\
\hline Edible portion. ........... & 0 & 47.8 & 1.5 .5 & 36.2 & .8 & $I, 8 I 5$ \\
\hline As purchased. $\ldots \ldots \ldots \ldots \ldots \ldots$ & 14.8 & 40.4 & 13.1 & $3 I .5$ & .6 & 1,575 \\
\hline $\begin{array}{l}\text { Shoulder: } \\
\text { Edible portion. ............. }\end{array}$ & $\ldots \ldots$ & $60: 2$ & 17.5 & $2 \mathrm{I} .8$ & .9 & 1,245 \\
\hline 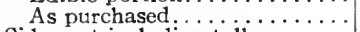 & 22.1 & 46.8 & 13.7 & I 7.1 & .7 & 975 \\
\hline Side, not including tallow: & & & & & & \\
\hline Edible portion. . . . . . . . . & $\cdots \cdots$ & $53 \cdot 6$ & 16.2 & $29: 8$ & .8 & 1,560 \\
\hline As purchased............. & 19.3 & $43 \cdot 3$ & I3.0 & 24.0 & .7 & 1,255 \\
\hline MUTTON, COOKED. & & & & & & \\
\hline Mutton, leg roast, edible portion & $\cdots \cdots$ & 50.9 & 25.0 & 22.6 & I. 2 & $I, 420$ \\
\hline PORK, FRESH. & & & & & & \\
\hline Chuck ribs and shoulder: & & & & & & \\
\hline Edible portion............ & $\cdots \cdots$ & $5 \mathrm{I} \cdot \mathrm{r}$ & 17.3 & $3 I . I$ & .9 & I, 635 \\
\hline Ham, fresh: & I8.I & 41.8 & I4. I & $25 \cdot 5$ & .8 & 1,340 \\
\hline Edible portion. . .......... & $\ldots \ldots$ & $50 . \mathrm{I}$ & 15.7 & $33 \cdot 4$ & .9 & $\mathrm{I}, 700$ \\
\hline As purchased............. & 10.3 & 45.1 & $14 \cdot 3$ & 29.7 & .8 & $I, 520$ \\
\hline $\begin{array}{l}\text { Loin (chops): } \\
\text { Edible portion. }\end{array}$ & $\ldots$ & 50.7 & I6.4 & 32.0 & .9 & 1,655 \\
\hline 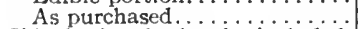 & 19.3 & 40.8 & 13.2 & 26.0 & .98 & $\begin{array}{l}1,055 \\
1,340\end{array}$ \\
\hline Side, lard and other fat included: & & & & & & \\
\hline Edible portion. ........... & $\ldots \ldots$ & 29.4 & 9.4 & $6 \mathrm{r} \cdot 7$ & .4 & 2,780 \\
\hline As purchased.... & I I. 2 & 26 . I & 8.3 & 54.8 & .4 & 2,465 \\
\hline
\end{tabular}




\begin{tabular}{|c|c|c|c|c|c|c|}
\hline MEATS. & Refuse. & Water. & Frotein. & Fat. & Ash. & $\begin{array}{c}\text { Fuel } \\
\text { value } \\
\text { per } \\
\text { pound. }\end{array}$ \\
\hline PORK, SALTED AND SMOKED. & Per cent. & Per cent. & Percent. & Per cent. & Per cent. & Cals. \\
\hline 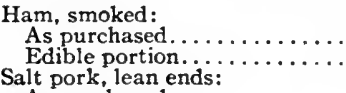 & 12.2 & $\begin{array}{l}35.8 \\
39.8\end{array}$ & $\begin{array}{l}14.5 \\
16.5\end{array}$ & $\begin{array}{l}33.2 \\
38.8\end{array}$ & $\begin{array}{l}4.2 \\
4.7\end{array}$ & $\begin{array}{l}1,670 \\
\mathrm{I}, 945\end{array}$ \\
\hline $\begin{array}{l}\text { As purchased........ } \\
\text { Edible portion..... } \\
\text { Bacon, smoked: }\end{array}$ & $\begin{array}{r}\text { II.2 } \\
\ldots \ldots\end{array}$ & $\begin{array}{l}17.6 \\
19.9\end{array}$ & $\begin{array}{l}7.4 \\
8.4\end{array}$ & $\begin{array}{l}59.6 \\
67.1\end{array}$ & $\begin{array}{l}5.1 \\
5.7\end{array}$ & $\begin{array}{l}2,665 \\
2,985\end{array}$ \\
\hline $\begin{array}{c}\text { As purchased. } \ldots \ldots \ldots \ldots \ldots \\
\text { Edible portion } \ldots \ldots \ldots \ldots \\
\text { chickens. }\end{array}$ & $\begin{array}{r}8.7 \\
\ldots .7\end{array}$ & $\begin{array}{l}18.4 \\
20.2\end{array}$ & $\begin{array}{r}9.5 \\
10.5\end{array}$ & $\begin{array}{l}59.4 \\
64.8\end{array}$ & $\begin{array}{l}4.5 \\
5.1\end{array}$ & $\begin{array}{l}2,685 \\
2,930\end{array}$ \\
\hline $\begin{array}{l}\text { Young: } \\
\quad \text { As purchased................. } \\
\text { Edible portion.......... }\end{array}$ & $\begin{array}{r}18.8 \\
\ldots \ldots\end{array}$ & $\begin{array}{l}55.5 \\
68.4\end{array}$ & $\begin{array}{l}17.8 \\
21.9\end{array}$ & $\begin{array}{l}7.2 \\
8.9\end{array}$ & $\begin{aligned} .9 \\
I .1\end{aligned}$ & $\begin{array}{l}765 \\
945\end{array}$ \\
\hline 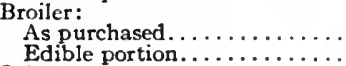 & $\begin{array}{r}29.1 \\
\ldots \ldots .\end{array}$ & $\begin{array}{l}51.2 \\
69.7\end{array}$ & $\begin{array}{l}15.5 \\
20.7\end{array}$ & $\begin{array}{l}3.3 \\
8.3\end{array}$ & $\begin{array}{r}.8 \\
1.1\end{array}$ & $\begin{array}{l}540 \\
890\end{array}$ \\
\hline $\begin{array}{l}\text { Other: } \\
\text { As purchased } \ldots \ldots \ldots \ldots \ldots \ldots \\
\text { Edible portion.............. }\end{array}$ & $\begin{array}{r}25.2 \\
\ldots \ldots\end{array}$ & $\begin{array}{l}47.3 \\
59.5\end{array}$ & $\begin{array}{l}14.4 \\
20.4\end{array}$ & $\begin{array}{r}0.3 \\
12.6 \\
19.2\end{array}$ & $\begin{aligned} .7 \\
I .1\end{aligned}$ & $\begin{array}{r}910 \\
1,350\end{array}$ \\
\hline OTHER FOWL. & & & & & & \\
\hline 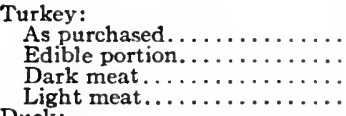 & $\begin{array}{r}14 \cdot 3 \\
\cdots \cdots \cdots \\
\cdots \cdots \cdots\end{array}$ & $\begin{array}{l}49.2 \\
57.4 \\
57.0 \\
63.9\end{array}$ & $\begin{array}{l}19.0 \\
22.2 \\
21.4 \\
25.7\end{array}$ & $\begin{array}{r}16.2 \\
18.9 \\
20.6 \\
9.4\end{array}$ & $\begin{array}{l}1.0 \\
1.2 \\
1.1 \\
1.3\end{array}$ & $\begin{array}{l}\mathrm{I}, 185 \\
\mathrm{I}, 385 \\
\mathrm{I}, 435 \\
\mathrm{I}, 065\end{array}$ \\
\hline $\begin{array}{l}\text { Duck: } \\
\text { As purchased... } \\
\text { Edible portion.. }\end{array}$ & $\begin{array}{l}15.9 \\
15.9\end{array}$ & $\begin{array}{l}5 \mathrm{I} .4 \\
6 \mathrm{I} . \mathrm{I}\end{array}$ & $\begin{array}{l}15.4 \\
18.3\end{array}$ & $\begin{array}{l}16.0 \\
19.0\end{array}$ & $\begin{array}{l}\mathrm{I} . \mathrm{I} \\
\mathrm{I} .3\end{array}$ & $\begin{array}{l}1,085 \\
1,290\end{array}$ \\
\hline $\begin{array}{l}\text { Goose: } \\
\text { As purchased......... } \\
\text { Edible portion....... }\end{array}$ & $\begin{array}{r}\text { II.I } \\
\cdots \cdots\end{array}$ & $\begin{array}{l}48.0 \\
54.0\end{array}$ & $\begin{array}{l}14.8 \\
16.6\end{array}$ & $\begin{array}{l}25.5 \\
28.7\end{array}$ & $\begin{array}{l}I .0 \\
I . I\end{array}$ & $\begin{array}{l}1,475 \\
1,660\end{array}$ \\
\hline $\begin{array}{l}\text { Ageon: } \\
\text { As purchased. } \ldots \ldots \ldots \ldots \ldots \ldots \\
\text { Edible portion. } \ldots \ldots \ldots \ldots \ldots \ldots\end{array}$ & $\begin{array}{r}13.6 \\
\ldots \ldots\end{array}$ & $\begin{array}{l}55.2 \\
64.0\end{array}$ & $\begin{array}{l}19.7 \\
22.8\end{array}$ & $\begin{array}{r}9.5 \\
\text { II } .0\end{array}$ & $\begin{array}{l}1.3 \\
1.5\end{array}$ & $\begin{array}{r}915 \\
1,060\end{array}$ \\
\hline
\end{tabular}

Digestibility. - The nutritive value of food does not only depend upon its constituents but also upon its availability, that is, the extent to which it is digested and absorbed. Thus roast mutton and dried peas contain approximately the same amount of protein, but their nutritive value is very different. As a rule, animal foods are more digestible and completely absorbed than vegetable foods; thus, of animal foods, 97 per cent. of the proteins, 95 of the fats and 98 of the carbohydrates are digested, while, of vegetable foods, only 84 per cent. of the proteins, 90 of the fats and 97 of the carbohydrates are digested (Atwater), the balance being discharged with the intestinal excreta.

Rubner found that all but 3 per cent. of roasted beef was digested by a healthy man; that flesh is more completely digested when 
roasted than when either raw or boiled, and when raw than when boiled.

Of the constituents of meat, the muscular fibers are most digestible and most nutritive. The shorter and thinner they are, the more tender the meat. The older the animal the thicker the walls of the tubes, the denser the connective tissue and the less digestible the meat. Connective tissue is readily dissolved in the stomach but has little nutritive value. A moderate amount of fat facilitates the digestion of meat; more than that hinders the action of the gastric juice upon the fibers; therefore, except when much heat and energy are needed, fat beyond a small quantity is undesirable. The digestibility of meat is also favored by the amount of savory extractives which it contains, and by the acids which naturally form in it as first product of decomposition; therefore it never should be eaten fresh; but always kept until it begins to soften, previous to actual decay. This softening may be produced more rapidly by macerating the meat in weak vinegar, or by pounding it across the cut ends.

Mutton is drier than beef and contains more fat; it is a pleasant substitute for it at times, but its exclusive or steady use is not so well borne. Pork differs from both in having less protein and more fat, being therefore less digestible; somewhat different is bacon in which the fat is drier, more granular and generally accepted by delicate stomachs. Veal contains less protein and more fat than beef and does not agree with everybody, while lamb differs but little from mutton. The white meat of poultry contains less fat than the dark meat and is more delicate and more digestible. Tripe and siveetbreads are easily borne, but liver and kidneys require a vigorous digestion.

Horse meat has a peculiar sweetish flavor and is generally less tender than beef, but, unless from old or emaciated animals, is not unpalatable nor indigestible and is largely consumed in Europe. When well cooked it is an excellent substitute for beef in emergencies. Its fibre is coarser and of a dull reddish-brown color. Horse meat is distinguished from other meats by the fat, which has a lower melting point and a higher iodine absorption figure.

Cinaracteristics of Good Meat.- For Army use the only beef accepted is from steers 2 to 6 years old, and occasionally spayed heifers. The meat from bulls, stags (bulls castrated late in life) and females (except spayed heifers) is rejected. 
The lean of good beef which has been cut at least an hour (freshly cut meat is always dark), should have a bright, lustrous, cherry-red color, with a distinctly marbled appearance due to the lines of fat around the polygonal bundles of muscular fibers; it should be soft and silky to the touch, but, at the same time, firm and elastic, neither pitting nor crackling on pressure, the bright red juice slowly oozing out. Pale, moist muscle marks young sickly animals, while dark, tough, stringy meat probably comes from old ones, or perhaps from bull or horse. A deep, purple tint suggests that the animal has died from disease. The fat should be reasonably abundant, hard and firm; the fatter the meat, the smaller is the proportion of protein. Good meat has a slight but not disagreeable odor. In temperate climates the marrow of the hind legs is still solid 24 hours after killing, and rosy red. The first evidence of decay is detected by thrusting a wooden skewer deep into the flesh, preferably alongside a bone, then withdrawing and smelling it. Veal and lamb are paler than beef and softer to the touch. Mutton is of a dull red color, with very white (occasionally yellowish), hard fat and no marbling. Pork is light red, softer than beef or mutton; its fat should be white and firm.
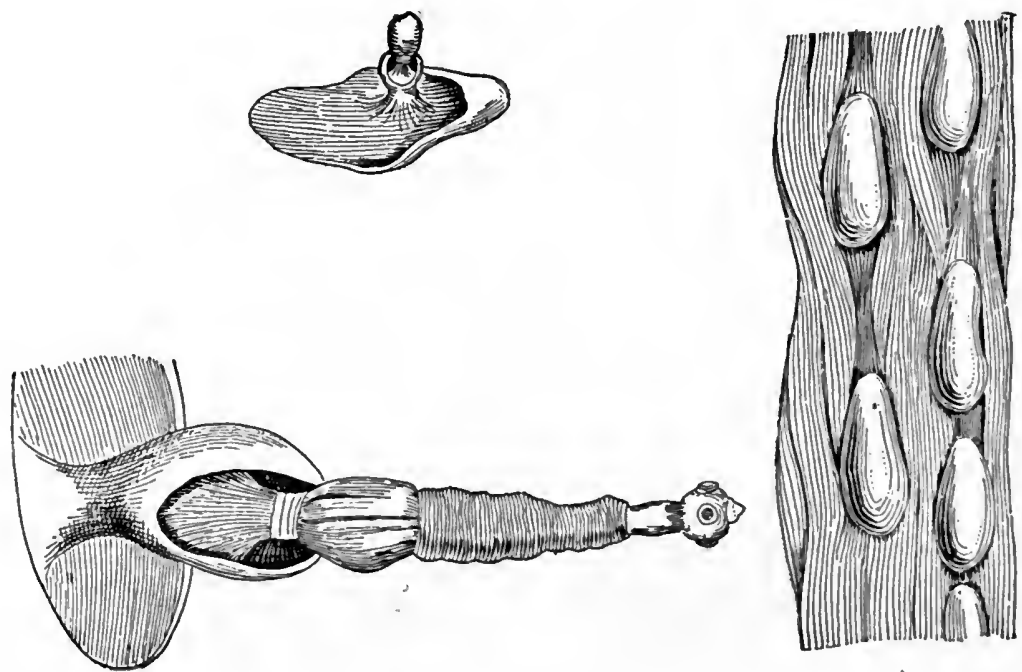

FIG. 67.-Cysticercus of pork; I, cysts in muscle : 2 and 3 , inner coat cut open and parasite extruded. (Davaine.) 
Diseases of Cattle and Hog. - The diseases of cattle which render their flesh more or less unfit for food are chiefly pleuropneumonia, foot-and-mouth disease, Texas fever, rinderpest, anthrax, actinomycosis, tuberculosis, pyemia and septicemia. Those of the hog are anthrax, muco-enteritis, hog cholera and tuberculosis. The flesh of cattle, sheep and hogs may also be infested by various parasites. Some of the diseases and parasites are transmissible to man.

Meat Parasites. - The meat parasites from which man most suffers are the tapeworms and trichina spiralis. Of the several species of tapeworms, only two are known to infect man in this country, namely Tania saginata due to measly beef, and Tania solium due to measly pork. In cattle and swine they only exist in the larval form known as Cysticercus bovis and C. cellulose (Fig. 67 ). Their life history is as follows: From the adult tapeworm, in the intestines of man, a number of eggs are discharged; these are expelled with the feces and some find their way, with water or food, into the digestive tract of cattle or swine; there the embryos

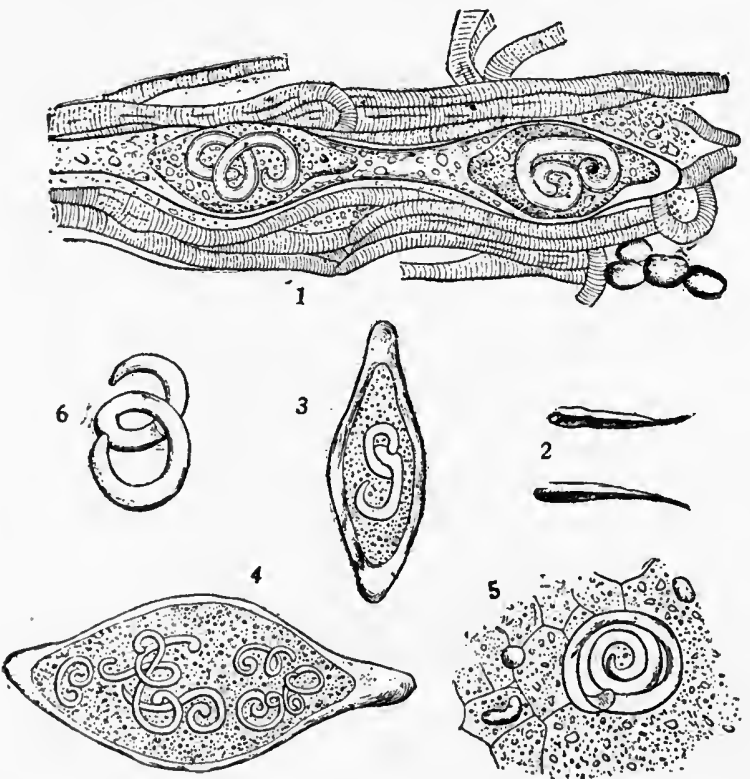

FIG. 68. $\rightarrow \mathrm{I}$, Trichinæ in muscle; 2 , embryos; 3 , encysted trichinæ; 4 , cyst containing seven trichinæ; 5 , trichina in fatty tissue; 6 , trichina removed from cyst. (Laveran.) 
issue from the eggs and piercing the intestinal walls migrate to all parts of the body, where they grow as "bladder-worms." The whitish bladders, oval or elliptic in shape, vary in size from a large bean to a pin's head. The meat thus infested is said to be " measly;" if eaten raw or imperfectly cooked, by man, the cysticercus develops into the adult tapeworm, thus completing the cycle. Bladder-worms are readily killed by ordinary cooking and the meat rendered harmless.

Trichina spiralis, outside of man, is found mostly in pork, occasionally in other mammals, especially the rat. It is a filiform worm, a millimeter long, coiled within a calcareous capsule $0.2-0.3 \mathrm{~mm}$. in diameter (Fig. 68). It infests chiefly the voluntary muscles, being more abundant near their tendinous extremities, and is generally visible to the naked eye as a small white speck, the coiled worm itself being easily revealed under a magnifying glass after treatment in a weak solution of caustic potash. Trichinosis in hogs is but too common all over the world, at least 2 per cent. being thus affected in this country. When trichinous pork is eaten raw or imperfectly cooked, by man, the capsules are dissolved in the stomach and the worms liberated; in a few days they grow to full adult size and the females give birth to numerous embryos which at once migrate to all parts of the body. This migration begins 8 or lo days after the ingestion of the infested meat and lasts about a week; it is marked by fever, puffiness of face, edema and intense muscular pain. The symptoms often suggest typhoid fever, rheumatism or acute tuberculosis. A characteristic feature of the blood is marked eosinophilia which gradually increases to 55 or more per cent. An examination of the feces will reveal the embryos. Death may occur within a few weeks, but generally the disease ends in recovery, the worms becoming encysted and incapable of further injury. Hogs are probably infected by eating meat wastes or excrements from trichinous animals.

The incidence of trichinosis in man varies according to times and places but is seldom less than $\mathrm{I}$ or 2 per cent. Ostler found trichinæ in 5 per cent. of unselected autopsies in Buffalo, N. Y.

The only reliable prophylactic measure is the thorough cooking of pork meat. The process used in smoking and curing hams does not kill the parasite; they should be boiled for several hours. While the worms are in the intestines, before their migration into the 
muscles, they can be expelled by free catharsis and the administration of thymol.

Flukes. - Parasitic flat worms found in the liver of cattle and sheep, producing the condition known as "rot." As this disease is very rarely conveyed to man, the flesh of animals thus affected (liver excepted) need not be condemned.

Actinomycosis, or "lumpy jaw," caused by the presence of a yellow ray-fungus, occurs in cattle and swine. If care be taken to remove the involved parts, the meat may be consumed in safety. It also occasionally occurs in man, apparently not from diseased animals but from the same unknown source that infested them.

Transmission of Disease by Meat. - Of the animal diseases transmissible to man the following are noteworthy:

Tubcrculosis, one of the most common diseases of cattle, affecting also sheep and swine. In Europe, from to to 40 per cent. of cows are tuberculous. In this country, the percentage is not so great, ranging from 5 to 30 . The proportion of steers affected is distinctly less, because they are not so constantly stabled, and are much younger when slaughtered. It is significant that at the abattoirs under federal supervision, in this country, hardly 2 per $\mathrm{I}, \mathrm{OOO}$ of cattle inspected are condemned for tuberculosis. This may be explained by the facts that many cattle reacting to tuberculin show no obvious evidence of disease, and that the majority of tuberculous cows are slaughtered by local butchers without inspection. The number of swine condemned for tuberculosis at the abattoirs is more than twice that of cattle. For diagnostic purposes the use of tuberculin is entirely satisfactory and safe; a clear reaction to it is an unequivocal assurance that the animal is tuberculous. Safety requires that it should be, if not slaughtered, at least segregated and under observation. The identity of human and bovine tuberculosis has been conclusively proved, as well as the positive danger, for children, of using milk from infected cows, especially when the udder is involved. There is also a strong presumption that man may become infected by the meat of tuberculous cattle. The organs most frequently involved are the liver, lungs, kidneys and brain; they should always be rejected in diseased animals. The muscles are very rarely affected, and whatever danger there might be in their consumption is completely removed by cooking. (See under Milk.) 
Anthrax, when occurring in cattle, sheep and hogs. The meat should be condemned and destroyed, not only because of the difficulty of killing all the bacilli and spores by cooking, but also on account of the great danger of infection through cuts and abrasions. The handling of the hides is equally dangerous (see page roo).

Glanders. - There is also danger of infection in the handling of the flesh and hide of glandered horses (see page IOI).

The Foot-and-Mouth Disease of cattle and sheep, characterized by a vesicular eruption about the mouth and feet, may be transmitted to man by direct inoculation, or the use of milk of diseased animals, but there is no evidence that the meat is ever infected.

Meat Poisoning. - Bacteria are dangerous not only by their toxins but also through their proteolytic action upon nitrogenous substances whereby many decomposition products, harmless and toxic, are formed. These products, thus resulting from the bacterial splitting of proteid substances, have therefore nothing in common with toxins. Preeminent among them are the ptomains. These are nitrogenous bodies, basic in character, that is, able to combine with acids to form salts, and therefore analogous to the vegetable alkaloids. A great number of ptomains are known; many are innocuous; some are highly toxic and to them are attributable frequent cases of poisoning from the use of infected meat, cheese, ice-cream, or even vegetables. The meats most liable to be contaminated are pork and veal, and their preparations, especially if used raw or imperfectly cooked, or containing part of the entrails or viscera.

Besides the harmful products of proteid decomposition, meats may be infected by the toxins of various bacteria, especially the following:

Bacillus botulinus, large spore-bearing, slightly motile anaërobe, the cause of botulism, a dangerous infection, chiefly of the nervous system, usually the effect of the ingestion of tainted ham, canned meat or sausage. The poisoning of the meat occurs after the death of the animal; it develops an offensive odor and its unfitness for food is generally apparent to the senses. The symptoms of botulism, due to a potent soluble toxin (like that of diphtheria), are chiefly paralysis of the eye muscles, aphonia, dysphagia, cardiac and respiratory distress. There is no fever and consciousness is preserved. The case mortality may reach 25 per cent. This bacillus, although spore-bearer, is readily destroyed by a temperature of $160^{\circ}$ F. 
Bacillus enteritidis of Gärtner, and allied bacilli (forming the hogcholera or paratyphoid group) intermediate between the colon and typhoid types and only distinguished from them by some special reactions. This bacillus, when ingested with food containing it, produces a high fever and acute gastro-intestinal symptoms. It affects the animal in life but, after death, the flesh containing it does not show any visible sign of unwholesomeness. Its toxin (endotoxin) is extremely resistant to heat and not easily destroyed by boiling, hence the danger of meat contaminated with it.

Bacilli of this group have been isolated in cases resembling mild attacks of typhoid fever and called paratyphoid (see page 42 ).

$B$. proteus vulgaris, type of a group of the bacilli of putrefaction, widely distributed and abundant wherever decomposition takes place. Its pathogenic powers are feeble, although some outbreaks of meat poisoning have been attributed to it.

\section{COOKING.}

Cooking produces in foodstuffs certain important physical and chemical alterations, affecting their quality, digestibility, absorption and nutritiousness. It often improves their appearance and, at the same time, develops new and appetizing flavors. It destroys parasites and pathogenic germs, as well as the germs of putrefaction and those which bring about the production of ptomains. Its effect upon muscular fibres is to render them firmer and more brittle by the coagulation of albumen, and to separate them by the softening or gelatinizing of the connective tissue, thus greatly facilitating mastication. It diminishes the amount of water and removes more or less of the fat, salts and extractives. The digestibility of vegetable foodstuffs (some fruits excepted) is always much increased by cooking, but that of animal food may be decreased. Thus raw beef disappears more rapidly from the stomach than when boiled; experience shows that raw or underdone meat is most suitable for delicate stomachs, provided it be chopped fine or scraped into a pulp and well seasoned.

If meat is placed in cold water, parts of the organic salts, the soluble albumen and the extractives or flavoring matters will be dissolved out. At the same time, a small amount of lactic acid is formed which renders other parts of the meat soluble. The quantity of materials dissolved in water depends upon the surface exposed 
(the smaller the pieces the greater the surface), the temperature to which the water is raised and time of exposure. If the water be heated gradually, more and more of the soluble substances are dissolved. At a temperature of about $\mathrm{I} 34^{\circ} \mathrm{F}$. the soluble albumen will begin to coagulate, and at $160^{\circ}$ will rise as a brownish scum to the top and the liquid become clear. Upon heating still higher, the connective tissues begin to be changed into gelatin and are partly dissolved, while the insoluble albuminoids are coagulated. The longer the action of the hot water lasts the tougher and more tasteless the meat becomes, but the better the broth. Treated in this way, meat may lose over 40 per cent. of its weight; this loss is from the evaporation of water, with the exception of 6 to Io per cent. made up of fat, soluble albumen, gelatin, mineral matters, organic acids, muscle sugar and flavoring materials. The nearly tasteless mass of fibers left undissolved contains about all the protein of the meat; it has practically the same nutritive value and, if properly combined with vegetables and the usual condiments, is almost as completely digested as the same weight of ordinary roast.

All proteins coagulate at $170^{\circ} \mathrm{F}$. or less; therefore, in cooking meat, it is unnecessary to go much beyond this point, except for a short time. The guiding rule in preparing animal and vegetable foods is to cook them slowly and at the lowest temperature that will accomplish the purpose. In cooking a piece of meat by any method (except when stewing or making broth), the first step is to subject it to a temperature high enough to speedily seal up the superficial layer by the coagulation of its albumen and thus prevent the loss of juice and soluble constituents. In boiling, the meat is plunged into boiling water and, after a few minutes, the water brought down to about $180^{\circ}$, at which temperature it is maintained at the rate of $\mathrm{I} 5$ minutes for each pound. If it be desired to prepare broth, a different process must be followed. In broiling, it is exposed to the direct heat of the fire which converts the surface extractives into new, savory substances. In roasting (more properly baking), it is placed in a hot oven, at $400^{\circ} \mathrm{F}$., for a few moments, and then kept at a much lower temperature at the rate of 15 minutes per pound, bearing in mind that the heat should be more moderate with a large joint than with a small one, to prevent burning. Meat being a poor conductor of heat, a large piece exposed to intense heat would become burned outside long before the heat could penetrate to the 
interior. This is the most popular and satisfactory method of cooking. In frying, the meat, cut into small pieces, and if desired rolled in dry crumbs, cracker dust or flour, should be dropped and completely immersed in oil or fat heated to $400^{\circ}$, or until little jets of steam issue from it; if the temperature is not high enough, the pores of the meat not being immediately closed, it becomes impregnated with fat and indigestible. Chops or fat meats are best cooked by sautéing, that is, putting them in the hot pan with just enough fat to prevent sticking, turning frequently and cooking quickly. In stewing, it is cut into small pieces and placed in cold water which is heated slowly to $180^{\circ}$ where it is maintained for several hours. All the extractives and other soluble substances are retained without loss.

Dressed meat, as usually issued to troops, contains about i 5 per cent. of bone. Furthermore, in cooking, it loses from 20 to 30 per cent. of its weight, mostly from the evaporation of water, so that the quantity consumed is reduced in weight to about 60 per cent. of the raw material. The loss varies greatly according to the time of cooking; thus with rare roasts the loss in weight was found to be I9.48 per cent (I3.87 water, 5.5 I fat, 0.07 nitrogenous matters and 0.03 ash), while with well-done roasts it was $3 \mathbf{I} .29$ per cent. (I 7.79 water, I 3.29 fat, 0.1 5 nitrogenous matters and $0.06 \mathrm{ash}$ ). There is greater economy, therefore, as well as improved digestibility, in stopping the cooking before the "well-done" stage is reached. To the meat proper, however, should be added the value of the juices utilized as gravy, broth, etc., and that of the bones which can be used for soup.

Good cooking is deemed so important in our Army that three "training schools for bakers and cooks" have been established by the War Department, one at Washington Barracks, D. C., another at Fort Riley, Kansas, and the third at the Presidio, San Francisco, Cal., under the immediate charge of officers of the Quartermaster Corps. The classes under instruction are composed of specially selected men who manifest a desire to learn the trade of baker or cook and who show aptitude for the work. The course lasts four months and covers the management of messes, baking and cooking in garrison and the field. 


\section{BROTH, SOUP AND MEAT EXTRACTS.}

In preparing soup or broth, the object is to dissolve out as much of the constituents of the meat as possible. The meat should be cut into small pieces and placed in cold water which is very slowly raised to the boiling point. Cracked bones, whose marrow dissolves readily, are a valuable addition. In this way, the broth will contain the extractives and mineral matters, a small proportion of soluble proteids, a little fat and, if the boiling be prolonged, some gelatin; that is to say, all the savory and appetizing elements of the meat but hardly any of its nutritive principles. It stimulates the digestive functions, promotes the flow of gastric juice and prepares the way for more nourishing food; it is therefore a condiment and a stimulant. To make a beef-tea really nutritious, the meat, after boiling, should be dried, pounded fine, screened and then added to the liquid extract.

One pound of beef meat and 7 ounces of bones should make about a pint of strong broth, containing 95.2 per cent. of water, I.2 protein, I.5 fat, I.8 extractives and 0.3 mineral matters.

Good soup, that is broth or "stock" to which are added vegetables, slowly cooked and carefully skimmed, is such valuable and appetizing food that no pains should be spared to cultivate the art of making it and developing a taste for it among soldiers.

Meat extracts are obtained by the action of boiling water upon fresh meat and the concentration of the liquid by evaporation. They are mostly made from the "soup liquor" resulting from the parboiling of meat which is to be canned. They should be entirely soluble in cold water and free from albumen, fat and gelatin.

Meat juices are obtained from raw meats by high pressure or otherwise.

Inasmuch as, in the preparation of both extracts and juices, it is necessary to bring them to a certain temperature and remove the greater part of the coagulable proteins, to prevent spoiling, most of their valuable nutritive principles are lost. They are useful, however, for their stimulating and flavoring qualities. Beef-tea, carefully prepared in the home or hospital, is, as a rule, much superior to commercial extracts and juices.

\section{PRESERVATION OF MEAT.}

As meat must often be transported long distances, it is necessary 
to make it undergo certain preparations whereby it may be kept a variable period of time without decay or material loss of nutritive qualities. The methods of preservation mostly in use are refrigeration, canning, desiccation, salting and smoking.

Refrigeration. - Preservation by cold, whenever applicable, is generally preferred. Cold-storage houses are mostly cooled by the ammonia process, which is based on the property possessed by a fluid of absorbing heat on passing from the liquid to the gaseous form. Liquid ammonia, under pressure,-is allowed to escape into pipes where it vaporizes by absorbing the heat of contiguous surfaces. These pipes being coiled in a tank of brine, this liquid is cooled below $32^{\circ} \mathrm{F}$. and then pumped through the pipes used in refrigerating the cold-storage rooms. The moisture of the air condenses on the pipes and. freezes. in the form of a dense coating of frost. The gaseous ammonia, under the combined effect of pressure and cold, is again liquefied and then released, thus making the process continuous.

Refrigerated meat may simply be chilled by exposure to a minimum temperature of $25^{\circ} \mathrm{F}$. or else frozen throughout at a temperature of $-8^{\circ}$ or $-10^{\circ} \mathrm{F}$. Frozen meat, after being removed from the refrigerator, will remain sound as long as a week if protected from the sun and wrapped in non-conductive material. Meat should be kept frozen only when necessary for transportation and trade. The Q. M. Corps requires that beef and mutton should be frozen solid when delivered so that it may not begin to decompose before it can be used. Otherwise it is best to keep it just above the freezing point, from $32^{\circ}$ to $40^{\circ} \mathrm{F}$. At such temperature the activity of the micro-organisms is checked but the action of the ferments normally present still continues, causing it to "ripen" and improve in flavor without decaying. Refrigerated 'meat is wholesome, digestible and nutritive; it has nearly all the qualities of fresh meat and, when prepared from inspected animals, is often much better than the fresh beef from local markets. Freezing causes rupture of the muscle cells, so that in thawing there is more or less infiltration of the disintegrated tissues and free escape of the liquid contents, with loss of weight and some impairment of quality.

Frozen meat, when removed from storage, is much more sensitive to infection and liable to the rapid development of deleterious substances than fresh meat. Therefore it should be placed in very cold 
water to prevent rapid thawing and further destruction of tissues, and to permit the absorption of the water lost during storage. It should then be consumed as soon as possible. The loss in cooking is also somewhat greater than with fresh meat.

Beef kept two years in cold storage at Manila, P. I., was found "mouldy outside, of dark color, dry, with fat turning yellow." It had lost the aroma of fresh beef but retained a good flavor and was still of excellent quality. The loss in water was 7 per cent.

The extent of decomposition, not appreciable to the senses, may be determined according to Folin's methods, by the amount of ammoniacal nitrogen generated, or still better, by the degree of acidity of the fat.

Poultry. - For cold storage, poultry should be dry picked; scalding (as practised for the easy removal of feathers) impairing the integrity of the skin and its resistance to bacterial infection. It should also be dry chilled (instead of being cooled in water) in a room at $35^{\circ}$ or $40^{\circ} \mathrm{F}$. until most of the animal heat has been removed. Then it is customary to freeze it at a temperature below zero and afterward to carry it hard frozen at $10^{\circ}$ to $15^{\circ} \mathrm{F}$. until used. If birds are carefully drawn (entrails removed), they will keep better and longer, but as this cleaning process is often carelessly done and the flesh liable to be contaminated, most dealers adopt the safer course of keeping them "undrawn." Wild game that has been shot, therefore with intestines probably perforated, should always be drawn.

Poultry is never improved by cold storage, but its deterioration takes place very slowly. It is only after the sixth month that it becomes notably shriveled and that a careful observer can notice a difference in the taste. Up to the ninth month this difference is very slight. Thereafter, although the meat continues wholesome, there is a loss of flavor, increasing with time. After removal from cold storage, it should be thawed slowly in cool air, or in the kitchen refrigerator, rather than in cold water which extracts a good deal of the flavor from the meat.

Canning. - Next to refrigeration, canning is the best method of meat preservation. Canned meat is largely used in our service as well as in foreign armies, on account of its very convenient shape for transportation and distribution.

Canned beef is usually from the forequarter of the carcass. It 
is trimmed so as to remove as much gristle as possible and the larger pieces of fat. It is then parboiled about 20 minutes and packed in the cans, with the addition of a little "soup liquor" (the parboiling liquid), and sometimes a little salt or molasses. The tops are now soldered on and the cans "processed," that is, sterilized by heat, sometimes in vacuum pans, at temperatures ranging from $216^{\circ}$ to $250^{\circ} \mathrm{F}$. according to the process used; the air is driven out through a puncture in the top, afterward closed with a drop of solder. The preliminary parboiling prevents any great alteration in the appearance of the muscular tissue; it also removes a large quantity of water, thereby increasing the relative percentage of the nutritive constituents; thus a 30 -ounce can of roast beef contains the equivalent of 49 ounces of fresh beef, minus the extractives and a little fat.

Canned meat, prepared under proper inspection, is perfectly wholesome; the process destroys all germs and parasites while preserving the nutritive constituents, but melts the fat, gelatinizes the gristle and softens the muscle so that the meat is less attractive to the eye; having lost some of its extractives it is also less savory.

Bulging, in cans, generally indicates decay and formation of gases, the result of a small leak and access of air. It may also be produced by freezing without detriment to the quality of the contents, or by overstuffing the can with meat; in the latter case the swelling is on the sides, near the top, and the contents remain good if the tin is not broken. A leaky can placed in water will show air bubbles. A can may also be concaved or collapsed from atmospheric pressure if insufficiently filled.

All canned meats contain more or less tin from contact with the can; extracts, more than meats on account of their acidity. Lobster and fruits absorb relatively large quantities of it. Schryver estimates that tin is not harmful unless exceeding 20 centigrams to the kilo ( 1,000 grams) of contents, a proportion hardly ever approximated. In meat cans six years old, this observer found only 5 centigrams to the kilo.

The canned meats issued by the Q. M. Corps, all in key-opening lacquered cans, are corned beef, corned-beef hash, roast beef, roastbeef hash, beef and vegetable stew.

Corned beef is from the brisket, chuck and plate of the carcass, in two-pound net can, with not more than I ounce of clear jelly from 
soup stock. Chemically it contains 51.8 per cent. of water, 26.3 of protein and 18.7 of fat. Corned-beef hash consists of equal parts of vegetables (potatoes and onions) and meat, suitably seasoned with salt and pepper. Roast beef is also in two-pound net can, with not more than one-half ounce of salt and I ounce of clear jelly made from soup stock. It contains about 59 per cent. of water. 26 of protein and 15 of fat. Roast-beef hash consists of equal parts of vegetables (potatoes and onions) and meat, suitably seasoned with salt and pepper, in $28 \mathrm{r} / 2$-ounce net can. Beef and vegetable stew consists of $101 / 2$ ounces of meat, 7 ounces of potatoes, 5 ounces of onion and 6 ounces of sauce, in $28 \mathrm{r} / 2$-ounce net can.

Salting. - With rare exceptions, the only salt meats issued in our service are bacon and corned beef. Bacon, when properly cured and smoked, usually consists of I 6.8 per cent. of water, 9.2 of protein and 6r.8 of fat. It is appetizing and digestible when well cooked, and on account of its high calorific value particularly useful in cold countries. In warm countries it should be eaten sparingly, as a relish or for a change of diet, using the lean rather than the very fat and frying it until crisp and crackling.

Corned beef is prepared by pickling in a solution of salt, sugar and saltpeter. It contains about $5^{2}$ per cent. of water, 26 of protein and 19 of fat. As compared with fresh meat, it is more nutritive but less digestible, tougher and much too salty for prolonged use.

Desiccation. - This process was formerly extensively used in the West and Southwest or wherever the dryness of the air permits complete desiccation of thin strips of meat without putrefaction. Meat thus prepared, or " jerked," retains all its nutritive qualities. Pemmican consists of pounded jerked meat, mixed with fat and berries, to which sugar may be added; it is a rich food furnishing the necessary energy for strenuous work on the frozen plains of the Northwest or in the Arctic Circle.

Rapid Processes. - In emergencies, when it is desirable to preserve meats quickly and for a short time, the fumes of sulphur $\left(\mathrm{SO}_{2}\right)$ may be used; they form a firm superficial protective layer of alkaline sulphites on the surface of the meat. In this process the bones should be disarticulated rather than severed. Formaldehyde has also been found useful, as it combines readily with the albuminous matters of the surface, forming likewise a protective envelop. Another simple method recommended is to fire the surface of the meat after spraying it with alcohol. 
Food Preservatives. - The antiseptic substances employed for the preservation of foods, but especially of meats, are boric acid and borax, benzoic acid and benzoate of soda, salicylic acid and salicylates, sodium sulphite and formaldehyde. There is general agreement of opinion that the tendency of these chemicals, in daily doses, even if very small, is to injure the quality of the food with which they are mixed and impair its digestibility, to disturb the metabolic functions of the body and irritate the secretory and excretory organs. It is contended by the U. S. Bureau of Chemistry that there is not a single article of food which cannot be offered to the consumer in a perfectly sound and wholesome state without having recourse to any of these preservatives, and that their use leads to the neglect of the processes necessary to the proper selection, cleaning and preparation of foods. Borax, as well as benzoate of soda, are frequently used in this country and in Europe to conceal a slight smell of decay and neutralize injurious products of decomposition; such use may be justified, under special circumstances, as the lesser of two evils. According to German experts, benzoic acid (or equivalent of benzoate) in small doses amounting to 0.5 gram daily, is harmless to the human body, but this quantity should never be exceeded. It is evident that the presence of any of the above preservatives, in meat or other food, will justify the presumption that the latter was not perfectly sound when prepared, or previous to canning or refrigeration. 


\section{ANIMAL FOODS (CONTINUED).}

\section{FISH, MOLLUSKS AND CRUSTACEANS.}

Fish is a wholesome, nutritious and easily digested food. Its percentage of protein ranges widely, from I 5 or I6 as in Spanish mackerel, salmon, halibut, to only 8 or 9 in haddock, mullet and turbot. Fish flesh contains more gelatin-yielding material and less extractive substances than meat. More of its protein is lost in boiling. It also contains less fat. A few species, such as salmon, turbot, eels, mackerel, lake trout and herring, contain ro per cent. or more of fat, while the majority have less than 5 per cent. As regards digestibility, there is no appreciable difference between fish and meat; in both, 97 per cent. of protein and over 90 of fat are assimilated. There is very little, if any, difference in thoroughness of digestion between fat and lean fish. The popular belief that fish is better brain food than meat is without scientific foundation and not proved by experience.

Fish is never improved by keeping, like meat; it decomposes quickly and should be eaten while fresh. It should be considered unfit for food when it floats in water, when the eyes have lost their sheen and the cornea is somewhat clouded, when the gills are pale red, the scales dry or easily loosened, and the meat dents on pressure of the finger.

In cold storage, fish cannot be preserved at the freezing point, this not being cold enough to hinder the action of ferments and production of bodies of unpleasant flavor, although there is no decay. For fish previously frozen, the proper temperature for keeping is $25^{\circ} \mathrm{F}$. If redipped in water now and then so as to retain a thin coating of ice over the skin, it will keep indefinitely.

Mollusks, such as clams, oysters and mussels, contain a fair percentage of proteids and a notable amount of carbohydrates, but are very poor in fat. They are very digestible, oysters somewhat more so than clams but less nutritious. It is estimated that one quart of oysters (removed from the shell) contains about the same quantity of nutritive substance as one quart of milk, one pound of bread or three-fourths pound of beef. Good oysters and clams, when removed from the water close their shell, move when touched, are of normal size and color, and the fluid inside the shell is clear. Oysters are generally considered unhealthy during the spawning 
season (June I to Oct. I), being then bathed in a milky, viscous liquid containing innumerable ova; during this season they also deteriorate more rapidly than at any other time.

Before being marketed, oysters are generally "freshened" or "floated," that is, placed for 2 or 3 days in fresh or slightly brackish water, where they become plump and improve in flavor. This plumpness is not a real gain in flesh but simply the effect of osmosis, whereby water is absorbed and some of the nutrients of the oyster lost.

Mollusks, but especially oysters, have often been the vehicle of typhoid fever, and may, in the same way, be that of other diseases. This happens when oyster or clam beds are located near sewer outlets or in the way of currents from infected places, or when oysters are "floated" in polluted waters. Chantemesse has found the typhoid bacillus in the intestine of the oyster, but this is exceptional and only the result of great contamination. As a rule the germs of sewage are found only in the water within the shell or on the surface of the tissues. The standard recommended by the U. S. Bureau of Chemistry condemns all oysters in the shell which show the presence of Bacillus coli types in O.I c. c. of the shell liquor, in 3 out of 5 oysters, this examination to be supplemented by an inspection of the beds and a bacterial examination of the water in which the oysters are bedded or floated. All danger is removed by placing the suspected oysters in pure sea water for one or two weeks, by washing them thoroughly before using, or else cooking them.

The liquor of shucked oysters is particularly liable to infection from polluted ice water and unclean methods of handling.

Oysters in cold storage should never be frozen. In the shell, the proper temperature is $40^{\circ} \mathrm{F}$, and out of the shell $35^{\circ}$.

Mussels have been observed to grow and multiply preferably in sewage-polluted waters. Cockles, on the contrary, generally keep out of contaminated zones.

Crustaceans, such as lobster, crab, crawfish and shrimp, are richer in proteids than mollusks, but less digestible and not suited to delicate stomachs. Abalone, the ear-shell of the Pacific Coast, is more nutritious than most other sea products and of excellent flavor. Large quantities are canned.

The following table (from Bull. 85, U. S. Department of Agriculture) exhibits the average composition of the fish, mollusks and crustaceans most used as food material: 


\begin{tabular}{|c|c|c|c|c|c|c|c|}
\hline NAME. & $\begin{array}{l}\text { Refuse } \\
\text { (bone, } \\
\text { skin, } \\
\text { etc.). }\end{array}$ & Water. & Protein. & Fat. & $\begin{array}{c}\text { Carbo- } \\
\text { hy- } \\
\text { drates. }\end{array}$ & $\begin{array}{l}\text { Ash or } \\
\text { mineral } \\
\text { matter. }\end{array}$ & $\begin{array}{c}\text { Fuel } \\
\text { value } \\
\text { per } \\
\text { pound. }\end{array}$ \\
\hline FRESH FISH. & Per ct. & Per ct. & Per c. & Per $a$. & Per ct. & Per at. & Calories. \\
\hline Bass, sea, dressed *. . . . . . . & 46.8 & 42.2 & 10.5 & .2 & $\ldots \ldots$ & .7 & 200 \\
\hline Bass, sea, whole........... & 56.1 & 34.8 & 8.7 & .2 & $\ldots \ldots$ & .6 & I68 \\
\hline Bass, striped, dressed . . . . . . & $5 \mathrm{I} \cdot 2$ & 37.4 & 8.8 & 2.2 & $\ldots \ldots$ & .5 & 249 \\
\hline Bluefish, dressed........... & 48.6 & $40 \cdot 3$ & 10.0 & .6 & $\ldots \ldots$ & .7 & 204 \\
\hline Cod, dressed.............. & 29.9 & 58.5 & I I. I & .2 & $\ldots \ldots$ & .8 & 209 \\
\hline Cod,steaks............. & 9.2 & 72.4 & 17.0 & .5 & $\ldots \ldots$ & I. 0 & 327 \\
\hline Haddock, dressed......... & 51.0 & 40.0 & 8.4 & .2 & $\ldots \ldots$ & .6 & I 59 \\
\hline Halibut, dressed........... & 17.7 & 61.9 & 15.3 & $4 \cdot 4$ & $\ldots \ldots$ & .9 & 454 \\
\hline Herring, whole..... & 42.6 & 41.7 & II. 2 & 3.9 & $\ldots \ldots$ & .9 & 363 \\
\hline Mackerel, dressed........... & 40.7 & 43.7 & 11.6 & $3 \cdot 5$ & $\ldots \ldots$ & .7 & 354 \\
\hline Mackerel, Spanish, dressed... & $24 \cdot 4$ & 51.4 & 16.3 & 7.2 & $\ldots \ldots$ & I. 2 & 585 \\
\hline Mackerel, Spanish, whole.... & 34.6 & 44.5 & I4. I & 6.2 & $\ldots \ldots$ & I.o & 508 \\
\hline Mullet, dressed........... & 49.0 & 38.2 & 9.9 & 2.4 & $\ldots \ldots$ & .6 & 277 \\
\hline Mullet, whole............ & 57.9 & $3 \mathrm{I} .5$ & 8.2 & 2.0 & $\ldots \ldots$ & .5 & $23 \mathrm{I}$ \\
\hline Pickerel, dressed........... & 35.9 & 51.2 & 12.0 & .2 & $\ldots \ldots$ & .7 & 227 \\
\hline Pickerel, whole.......... & 47.1 & 42.2 & 9.9 & .2 & $\ldots \ldots$ & .6 & 186 \\
\hline Pompano, whole........... & $45 \cdot 5$ & 39.5 & 10.3 & 4.3 & $\ldots \ldots$ & .5 & 358 \\
\hline Salmon, California (sections). & 10. 3 & 57.9 & 16.7 & 14.8 & $\ldots \ldots$ & .9 & 903 \\
\hline Salmon, Maine, dressed..... & 23.8 & $5 \mathrm{I} .2$ & 15.0 & 9.5 & $\ldots \ldots$ & .9 & $65^{8}$ \\
\hline Shad, dressed $\ldots \ldots \ldots \ldots \ldots$ & 43.9 & 39.6 & 10.6 & 5.4 & $\ldots \ldots$ & .8 & 408 \\
\hline Shad, whole............. & 5.01 & 35.2 & 9.1 & 4.8 & $\ldots \ldots$ & .7 & 363 \\
\hline Shad, roe $\ldots \ldots \ldots \ldots \ldots \ldots$ & $\ldots \ldots$ & 71.2 & 23.5 & 3.8 & $\ldots \ldots$ & 1.5 & $58 \pi$ \\
\hline Trout, brook, dressed ...... & 37.9 & 48.4 & I I. 9 & I. 3 & $\ldots \ldots$ & .7 & 268 \\
\hline Trout, brook, whole......... & $4^{8} .1$ & 40.4 & 9.9 & I. I & $\therefore \ldots$ & .6 & 222 \\
\hline Trout, lake, dressed......... & $37 \cdot 5$ & 44.4 & I I.O & 6.2 & $\ldots \ldots$ & .7 & 44 \\
\hline Turbot, dressed,.......... & 39.5 & 43.1 & 8.9 & 8.7 & $\ldots \ldots$ & .8 & 513 \\
\hline Turbot, whole........... & 47.7 & 37.3 & 7.7 & 7.5 & $\ldots \ldots$ & .7 & 445 \\
\hline Whitefish, dressed........ & 43.6 & 39.4 & 12.8 & 3.6 & $\ldots \ldots$ & .9 & 376 \\
\hline Whitefish, whole...... & 53.5 & 32.5 & I0.6 & 3.0 & $\cdots \cdots$ & .7 & 313 \\
\hline $\begin{array}{c}\text { General average of fresh fish } \\
\text { as sold } \ldots \ldots \ldots \ldots \ldots \ldots \ldots\end{array}$ & 41.6 & 44.6 & 10.9 & 2.4 & & .7 & 295 \\
\hline \multicolumn{8}{|l|}{ PRESERVED FISH.** } \\
\hline Macleerel, No. I, salted...... & 19.7 & 34.8 & 13.9 & $2 \mathrm{I} .2$ & 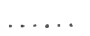 & 2.1 & I, 107 \\
\hline Cod, salted and dried ....... & 24.9 & 40.2 & 19.0 & $\cdot 4$ & $\ldots \ldots$ & I. 2 & 363 \\
\hline $\begin{array}{l}\text { Cod, boneless codfish, salted } \\
\text { and dried................ }\end{array}$ & & $54 \cdot 4$ & 26.3 & .3 & & I. 7 & 490 \\
\hline Herring, salted, smoked and & $\cdots$ & 34.4 & & & & & \\
\hline 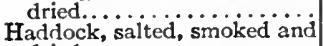 & $44 \cdot 4$ & 19.2 & 20.5 & 8.8 & & .9 & 726 \\
\hline dried............... & 32.2 & 49.2 & 15.8 & I . & & I.0 & 290 \\
\hline 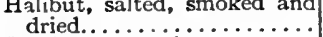 & 7.0 & 46.0 & 19.3 & 14.0 & & 1.9 & 916 \\
\hline Sardines, canned.......... & 5.0 & 53.6 & 23.7 & 12. I & $\ldots \ldots$ & $5 \cdot 3$ & 916 \\
\hline Salmon, canned.. & 14.2 & 56.8 & 19.5 & $7 \cdot 5$ & $\ldots \ldots$ & 2.0 & 658 \\
\hline \multicolumn{8}{|l|}{ MOLLUSKS. } \\
\hline Oysters, removed from shell.. & & 88.3 & 6.0 & I. 3 & 3.3 & I. I & 222 \\
\hline Oysters, in shell. . & $81 \cdot 4$ & $16 . \mathrm{r}$ & I. 2 & .2 & .7 & .4 & 41 \\
\hline 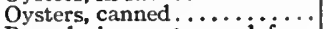 & $\ldots \ldots$ & 83.4 & 8.8 & 2.4 & 3.9 & 1.5 & 327 \\
\hline $\begin{array}{l}\text { Round clams, removed from } \\
\text { shell } \ldots \ldots \ldots \ldots \ldots \ldots \ldots \ldots\end{array}$ & & 80.8 & 10.6 & I. I & 5.2 & 2.3 & 331 \\
\hline Round clams, in shell........ & 675 & 28.0 & 2.1 & $\mathrm{I}$ & 1.4 & .9 & 68 \\
\hline \multicolumn{8}{|l|}{ CRUSTACEANS, ETC. } \\
\hline Lobsters, in s & 61.7 & 30.7 & 5.9 & .7 & .2 & .8 & 141 \\
\hline & $\ldots \ldots$ & 77.8 & I 8.1 & I. I & .5 & 2.5 & $38 t$ \\
\hline Crabs, in shell... & 52.4 & 36.7 & 7.9 & .9 & .6 & I. 5 & I9I \\
\hline Abalone, fresh........ & $\ldots \ldots$ & 72.8 & 22.2 & .3 & 3.3 & 1.4 & $50 t$ \\
\hline Terrapin, in shell. & 75.4 & I 83 & 5.2 & .9 & $\ldots \ldots$ & .2 & 132 \\
\hline Green turtle, in shell.. & 76.0 & 19.2 & $4 \cdot 7$ &. $\mathrm{I}$ & $\ldots \ldots$ & .3 & 91 \\
\hline Frogs' legs.......... & 32.0 & 56.9 & 10.5 &. $\mathrm{I}$ & $\ldots \ldots$ & .7 & 195 \\
\hline
\end{tabular}

* "Dressed" here means prepared for market and, generally, ready for cooking.

** The percentage of salt is left out. 


\section{MILK.}

The composition of cow's milk of average good quality is as follows :

$\begin{array}{ll}\text { Fat (cream), } & \text { 4.00 per cent. } \\ \text { Sugar, } & 5.00 \text { per cent. } \\ \text { Proteins, } & 3.30 \text { per cent. } \\ \text { Mineral matter, } & \text { o.70 per cent. } \\ & - \\ \text { Total solids, } & \text { I3.00 per cent. } \\ \text { Water, } & 87.00 \text { per cent. }\end{array}$

It differs f:rom human milk in containing twice as much protein and salts, thie proportion of fat and sugar being about the same. Therefore to make cow's milk of approximately the same composition as woman's milk, it is usual to dilute it with an equal bulk of water, and to add cream and sugar.

The fat exists in very minute globules which, rising to the surface, form the creamy layer. The last part of the milking ("strippings") is the richest in fat.

The proteins consist chiefly of caseinogen ( 2.6 per cent.) and lactalbumin ( 0.7 per cent.). Caseinogen, the substance from which casein is formed in the presence of digestive ferments, is a phosphoprotein; its chief property is to clot when treated with the enzime rennin (or pepsin) in presence of soluble calcium salts, or to curdle in presence of acids, producing the curd of sour milk. The sugar or lactose is much less soluble than dextrose or cane sugar, and less sweet; through the action of certain bacteria it changes into glucose and then splits with formation of lactic acid, some of the lactic acid being further split into butyric acid. The mineral matter consists of phosphates and chlorides of potassium, sodium, calcium and magnesium. Potassium phosphate, indispensable for the growth and functions of muscles, is the most abundant, while calcium phosphate, so necessary for the growth and repair of bony tissues, comes next in quantity.

Milk is an excellent food medium for bacteria which, under favorable conditions, multiply in it at the most amazing rate. Even when the most careful aseptic precautions are taken, freshly drawn milk always contains bacteria; these are mostly cocci and come from the udder. In a few hours, under ordinary exposure, it will contain thousands of them. Cleanliness and a temperature below 
$50^{\circ} \mathrm{F}$. will keep them from rapidly increasing for a day or two, but under the ordinary conditions obtaining in dairies during the summer, Harrington found them to increase in 24 hours to 48,000 in a cubic centimeter, and to 680,000 in 48 hours. Milk, as sold in city stores, seldom shows less than 100,000 bacteria to the c. c., and often contains several millions. The effect of these swarming bacteria upon the health of the consumer is still a matter of conjecture. That they modify the quality of the milk and that some of them may render it unwholesome or even poisonous is certain. Several highly toxic substances, doubtless due to bacterial action, have been found in milk, cheese and ice cream, such as tirotoxicon, a crystalline body first isolated by Vaughan, and tirotoxin, similar to curare in its action, discovered by Dakkum, substances which are the more dangerous that they develop without any apparent indication of their presence. It is possible that the summer diarrhea of children is mostly caused by certain special pathogenic bacteria and the poisons they elaborate, but the belief is general that the greater the number of bacteria in milk the more likely it is to produce intestinal disturbances. Some cities have established a maximum limit which must not be exceeded in milk offered for sale. Boston's standard is 500,ooo bacteria to the c. c. Other cities have set it as low as 100,000. For infant feeding it should not be over 10,000. It is well to bear in mind that bacteria in milk are carried up to the top layer with the globules of fat, so that their number is much greater, nearly a hundredfold, in the cream than in the milk below it. On the other hand, the bacterial content of separator cream (cream mechanically: separated) is said to be smaller than that of the milk from which it is obtained.

The infectious diseases most commonly transmitted by milk are tuberculosis (page 58) and typhoid fever (page 34), but it may also be the vehicle of cholera, dysentery, diphtheria, scarlet fever and septic sore throat. The bacilli of tuberculosis are derived from the cow itself; those of typhoid fever, cholera and dysentery infect the milk generally through the water with which it is diluted or the dairy utensils are washed. The germs of the other diseases are mostly derived from the infected hands or uncleanly habits of milkers and others handling the milk.

When it is considered that 15 to 20 per cent. of cows in the United States suffer from tuberculosis in its various stages (as detected by 
the tuberculin test), and that at least 8 per cent. (E. C. Schroeder, I909) of all samples of milk in the market contain tubercle bacilli, the danger of human infection is fully realized.

Tuberculous cows expel tubercle bacilli more commonly with their feces than in other ways. Even when only slightly affected, they may thus pass virulent bacilli. As they do not expectorate, the matter raised from the lungs is swallowed and the organisms discharged with the contents of the intestines. Thus it happens that milk is frequently infected through the dust and manure of the stable, especially while milking ungroomed cows whose flanks are incrusted with fecal matter. Tubercle bacilli are also expelled directly in the milk when the udder is affected, as well as in advanced generalized tuberculosis. They are likewise contained in the matters slobbered and coughed from the cow's mouth, and may thus infect healthy cows eating off the same box or manger.

The ingestion of raw milk is doubtless an important cause of primary intestinal tuberculosis during childhood; at that age the bacilli are generally arrested in the mesenteric glands. In adult life they pass through them much more readily, so that pulmonary tuberculosis is more likely to develop without involvement of the lymphatic glands.

But all bacteria in milk are not objectionable; may be regarded beneficial those which by their action upon the sugar of milk produce lactic acid and sour milk. Says Metchnikoff: "Among the useful bacteria, the place of honor should be reserved to the lactic bacilli. They produce lactic acid and thus prevent the development of butyric and putrefactive ferments, which are among our dangerous enemies." Sour milk, in various forms, whether naturally or artificially produced, is healthful and, for many stomachs, a better food than sweet milk. Metchnikoff contends that senility in man is chiefly due to the action of toxic bacteria breeding in the large intestine, and that lactic acid, in the shape of sour milk, is their natural antidote. He, accordingly, recommends the use of certain acid-generating microbes, especially two, the "Bulgarian bacillus," a powerful lactic acid ferment obtained from the Bulgarian fermented milk "yahourth," and the "paralactic bacillus," not so strong but imparting to the beverage a pleasant flavor. Skimmed milk is boiled a few minutes, then rapidly cooled and pure cultures of the above bacilli sown into it. The result is a sour, curdled milk, containing 
about Io grams of lactic acid per litre, palatable, slightly diuretic, and active in preventing intestinal putrefaction.

In the examination of milk, it is necessary first to determine whether water has been added to it, not only on account of the dilution it causes, but because of the pathogenic germs of which it is the ordinary vehicle. Then a bacteriological count should be made and the sediment investigated. Miscroscopic examination may disclose tubercle bacilli; the typhoid bacillus has been very rarely detected, while the colon bacillus is very common in milk. Streptococci are almost constantly found and, if numerous, may be of suspicious origin. An excessive number of leucocytes would indicate disease of the udder. The sediment is easily separated by straining through a fine wire sieve or by centrifugation. It consists of hair, epithelium, fecal matter, fragments of straw, hay, etc.; in dirty milk it is readily seen as a collection of particles of foreign matter at the bottom of the bottle.

Milk fresh from a healthy cow, and kept in clean vessels at a temperature not exceeding $50^{\circ} \mathrm{F}$., is best for all purposes. But the average dairy milk, as supplied to the consumer, is far from being always fresh, and has generally been exposed to many causes of contamination; therefore, to destroy the bacteria it may contain, it should be subjected to the action of heat, that is, sterilized or pasteurized. The complete sterilization of milk requires a temperature of $248^{\circ} \mathrm{F}$., but this process, as well as ordinary boiling, changes the taste of the milk, kills its natural ferments and probably otherwise impairs its nutritive qualities. This impairment, however, has not yet been satisfactorily proved; it is even contended by some investigators that boiling causes the casein to coagulate in finer flakes in the stomach and renders it more digestive. Nevertheless, it is certain that the present tendency of sanitarians is to use no more heat than necessary to accomplish the end in view, which is to kill all the pathogenic bacteria in the milk, a process called pasteurization. According to Rosenau, the heating of milk to $140^{\circ} \mathrm{F}$., for $20 \mathrm{~min}$ utes, kills the micro-organisms of tuberculosis, typhoid fever, Malta fever, diphtheria, cholera, dysentery, etc., as well as most bacterial toxins, including those of tetanus and botulism. It does not injuriously affect its taste, composition, quality or food value. The ferments remain intact, so that the milk is in no way devitalized.

An objection urged against pasteurization is that it kills the spore- 
less lactic acid bacilli which, by souring the milk, tend to preserve it, while the spore-bearing peptonizing bacteria which cause the decomposition and putrifaction of milk are less completely destroyed. In practice, however, it is found that enough acid bacilli are left (or added by subsequent handling) to sour the milk so that the acid development in well-pasteurized milk is about the same as that in clean raw milk, although somewhat slower. Another objection to pasteurization is that the bacteria thereafter increase more rapidly than in raw milk, especially if carelessly handled. This happens, however, only when the process is imperfectly carried out and the product not properly chilled.

There are two methods of pasteurization, the flash or continuous method, in which the milk is subjected to a temperature of $165^{\circ} \mathrm{F}$, for one minute, and the holder process, in which the milk is subjected to a temperature of $140^{\circ}$ to $150^{\circ}$ for 20 to 40 minutes. The latter method is the more reliable and should be preferred. Whichever process is used, the milk must be cooled at once to a temperature of $45^{\circ}$ or less to prevent the rapid multiplicatoin of the surviving bacteria. The standard of reduction should be 99 per cent. of the bacterial content of the raw milk, but the maximum count allowable after pasteurization should never exceed Ioo,000 to the c. c.

For improvised home or hospital pasteurization, the bottles of milk, after being warmed for five minutes, are immersed up to the lip in boiling water. The source of heat being then removed and the receptacle covered with a lid or towel to prevent loss of heat, the bottles are left standing in the gradually cooling water for 25 minutes. They are then taken out, corked and quickly cooled in cold water or ice.

Dried or powdered milk is prepared by passing a thin film of milk over a revolving cylinder heated to about $250^{\circ}$. It is a coarse, granular, cream-colored powder, readily soluble in water. The composition of the solids is but little altered and their relative proportions remain the same. It has the great advantage, when packed in air-tight tins, of keeping indefinitely. Amundsen used it on his journey to the South Pole and speaks highly of it.

Ice-cream. - Standard ice-cream, as defined by law, is a frozen product made from cream and sugar, with or without a natural flavoring, and contains not less than i 4 per cent. of milk fat, or not less than 12 per cent. when fruit and nuts are also used. This defi- 
nition excludes all substances commonly used as thickeners, such as eggs, gelatin, starch, glue, etc. Frozen products differing from the standard are not ice-cream in a legal sense, and should be designated by suitable names descriptive of their composition. A striking peculiarity of commercial ice-creams is their enormous bacterial flora. In 1907, an examination of 263 samples collected in the city of Washington showed an average of $26,000,000$ organisms per c. c. Of these samples, $7 \mathrm{I}$ per cent. showed the presence of gas-producing bacteria. According to Pennington and Walter, so per cent. of commercial ice-creams contain streptococci. The abundance of organisms in ice-cream is accounted for by the fact that the great bulk of milk bacteria are found in the cream (except when mechanically separated), but it is surprising to note the comparatively slight effect of freezing upon their vitality. Generally their number decreases during the first few days when placed in cold storage (at about $6^{\circ}$ F.), but nearly always increases afterward. "Sometimes the killing off of the organisms is very slight, their number remaining almost stationary or making a continuous upward curve" (G. W. Stiles). The number and varieties of bacteria always found in ice-cream, and the great ease with which they multiply under conditions of careless and uncleanly manufacture, explain the many cases of gastro-intestinal disturbances, as well as the occasional cases of acute poisoning attributed to its use. Outbreaks of typhoid fever have been traced quite definitely to infected ice-cream.

Butter is obtained by the churning of milk, and consists chiefly of the fat (cream), with salts, a little casein and lactose. It is one of the most acceptable and digestible of fats, and the usual accompaniment of our daily bread. It is liable to infection, although the presence of many vigorous saprophytes, the washing out of numbers of organisms into the buttermilk, and the salting, somewhat lessen the chances of pathogenic bacterial growth. When made from infected milk, it may retain the typhoid bacillus sixty days (IVashburn), and the tubercle bacillus 4 or 5 months (Bureau of Animal Industry). But inasmuch as butter is sterilized in cooking, and consumed otherwise in comparatively small quantity, the danger from its use may not be great but is sufficiently real to make it advisable to pasteurize it, as well as milk; it is in no way altered by the process and, with suitable apparatus, nearly as easily accomplished. 
Oleomargarin, which is made of fat, sour milk, dairy butter and some vegetable oil, is even more liable to infection than butter, because of its several constituents, any of which may be contaminated, and the more complex process of manufacture, involving more handling and exposure. It is but little inferior to good butter in nutritive qualities.

Buttermilk, or the milk from which butter has been removed, has still decided food value, and its slight acidulous taste renders it refreshing, healthful and palatable to most persons. It shares the bactericidal properties attributed to sour milk by Metchnikoff. There is no doubt, however, that it can also be infected by the pathogenic bacteria which may be abundantly contained in the cream from which it is derived. Therefore it should never be used, as an habitual beverage, unless made from pasteurized milk or milk known to be reasonably pure.

Koumiss, originally made by the alcoholic fermentation of mare's milk, is now made from cow's milk by the addition of cane sugar and yeast, which results in the evolution of alcohol (about $0.76 \%$ ), carbon dioxid and lactic acid. Kephir, the "milk wine" of some Caucasian tribes, is prepared from sheep's and goat's milk with a special ferment.

Cheese consists essentially of the casein of milk. It is made by heating the milk to above $80^{\circ} \mathrm{F}$. and then curdling it by rennet, sour whey, or by the acids formed by the lactic bacteria. After pressure to the proper consistence, the curd is allowed to ripen, when are produced the bacteria, moulds and ferments concerned in the development of special flavors. The composition of cheese is variable according to the milk used, and whether it is whole or skimmed. American cheeses of good quality (under the names of Cheddar and Emmental or Swiss cheeses) contain about 36 parts of fat, 30 of proteins and 30 of water. It is therefore richer food than meat. One pound has nearly the same food value as two pounds of fresh beef or three pounds of fish, although costing only about one-third more than good beef. Properly ripened cheese, with a well-developed flavor, is not only palatable and nutritious but wholesome and of comparatively easy digestion. However, it is not, as a rule, as digestible as meat. For habitual use it is only suitable to laborers or men doing hard physical work. In small quantity, the finer kinds make a delicious dessert on account of their savor and pungency. 
Cheese, like other milk products, may be infected by the ordinary pathogenic organisms. Tubercle bacilli may retain their virulence in it for at least two or three months. But as most cheeses require several months for their ripening, they become practically free from infectious germs before they are consumed. Cottage cheese and other kinds which are eaten within a much shorter time, especially if "full cream," may not be above suspicion in that regard. Toxic bacterial products are sometimes elaborated in cheese, causing acute poisoning, as already mentioned.

The following table (from Bull. 28, revised edition, U. S. Department of Agriculture) shows the average composition of dairy products and of eggs:

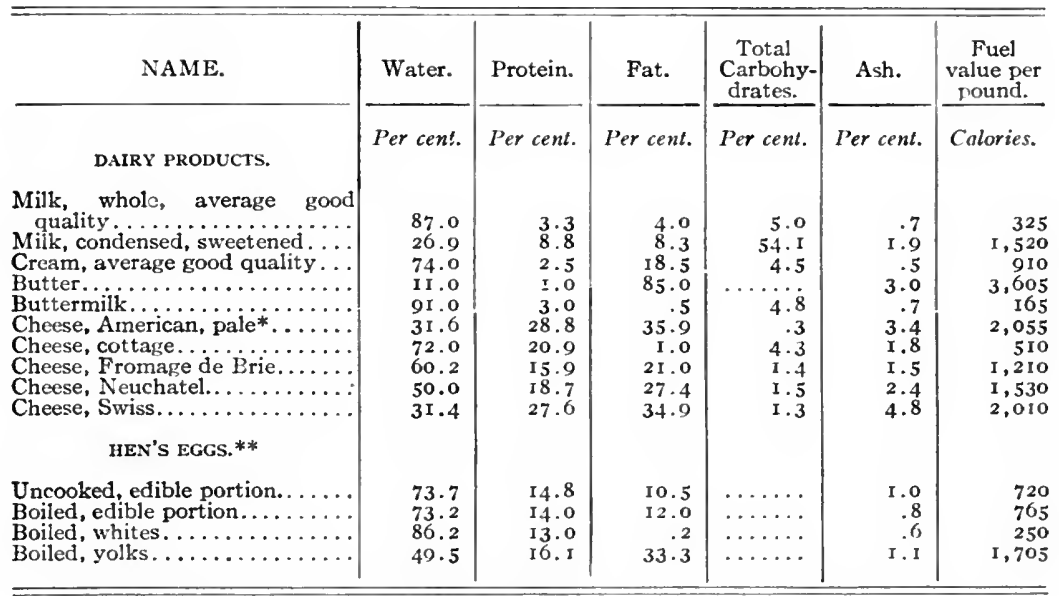

* The carbohydrates are milk surar and lactic acid.

** The refuse, consisting of the shell, amounts to I I.2 per cent. of the whole egg.

Eggs. - The white and the yolk of egg are of very different composition. The yolk contains a number of substances, namely: I5 per cent. of protein (vitellin), combined with nuclein and a phosphorized fat (lecithin) into a body called lecithin-nucleo-vitellin; 20 per cent. of other fat in emulsion; sulphur and salts of calcium, magnesium, potassium and iron. The total phosphorus is equivalent to about I per cent. of phosphoric acid. It is mostly present, as well as the iron, in organic combination which renders their assimilation easier and more complete. The white contains less protein than the yolk, about twice as much water and no fat. 
Eggs are among the most quickly assimilated foods; the yolk, especially, is most digestible and nourishing, either raw or softcooked. They are often better borne than meat and an excellent substitute for it, containing about the same percentage of nutritive matter, that is, less protein but more fat, both in a very digestible form.

Eggs keep very well in cold storage provided the temperature is as low as possible without freezing their contents to the slightest extent, that is, ranging from $28^{\circ}$ to $32^{\circ} \mathrm{F}$. They are as good as when put in at the end of 6 months. The separation of the white from the yolk becomes difficult after the $7^{\text {th }}$ month, but the eggs remain good to the end of the $9^{\text {th }}$ month and sometimes much later. For temporary keeping or transportation, $38^{\circ}$ to $40^{\circ}$ is the proper temperature.

Fresh eggs are generally sterile, but if kept in insanitary surroundings they soon become invaded (through the shell) by microorganisms (bacteria, yeasts and moulds) of the most objectionable character; hence the advisability of putting them promptly in cold storage. 


\section{CHAPTER XXVII.}

\section{VEGETABLE FOODS.}

Vegetable foods are characterized by the large proportion of carbohydrates which they contain, especially starch, but they also contain proteins and fats in variable proportions, thus furnishing, more completely than animal foods, all the principles needed by man for a normal and sufficient diet. In vegetables the nutrients are enclosed in cell walls composed of cellulose or woody fiber which interferes with their digestion, and by irritating the intestinal canal increases peristalsis and hastens the food onward before there is time for absorption. For this reason the digestibility of vegetable foods is lower than that of animal foods; thus, with cereals, it is 85 per cent. for protein, 90 for fat and 98 for carbohydrates, while for other vegetables the percentages seldom exceed 83,90 and 95 respectively. Cooking is necessary for the digestion of most vegetables; it renders not only starch but also more or less cellulose amenable to the action of the digestive juices.

The balance of acids and bases in the body is very important for the maintenance of a healthy physiologic equilibrium. Contrary to popular ideas, vegetables and fruits show a marked predominance of base-forming elements. The acids which they contain being essentially organic in their nature are wholly burned up in metabolism so that, for instance, sodium citrate, to which are attributed the antiscorbutic properties of potatoes, becomes converted into sodium carbonate, and functions as an alkali. Meats and fish, on the contrary, show a decided excess of acid-forming elements; this excess is somewhat less in eggs, and much less in cereals. Milk shows a slight predominance of bases.

Vegetable foods may be classified as follows:

I. Cereals. - Wheat, rye, oats, barley, rice, maize.

2. Pulses. - Beans, peas, lentils.

3. Tubers and roots. - Potatoes, sweet potatoes, carrots, turnips, beets, radishes.

4. Green vegetables. - Cabbage, spinach, lettuce, onion, celery. 
5. Fruits used as vegetables. - Tomato, aguacate, cucumber, squash, pumpkin, egg-plant.

6. Fruits. - Apple, pear, plum, cherry, grapes, peach.

7. Nuts. - Walnut, hickory-nut, chestnut, peanut, cocoa-nut.

The following table shows the composition of the vegetable foods most commonly used (from Bull. 28, revised edition, U. S. Department of Agriculture) :

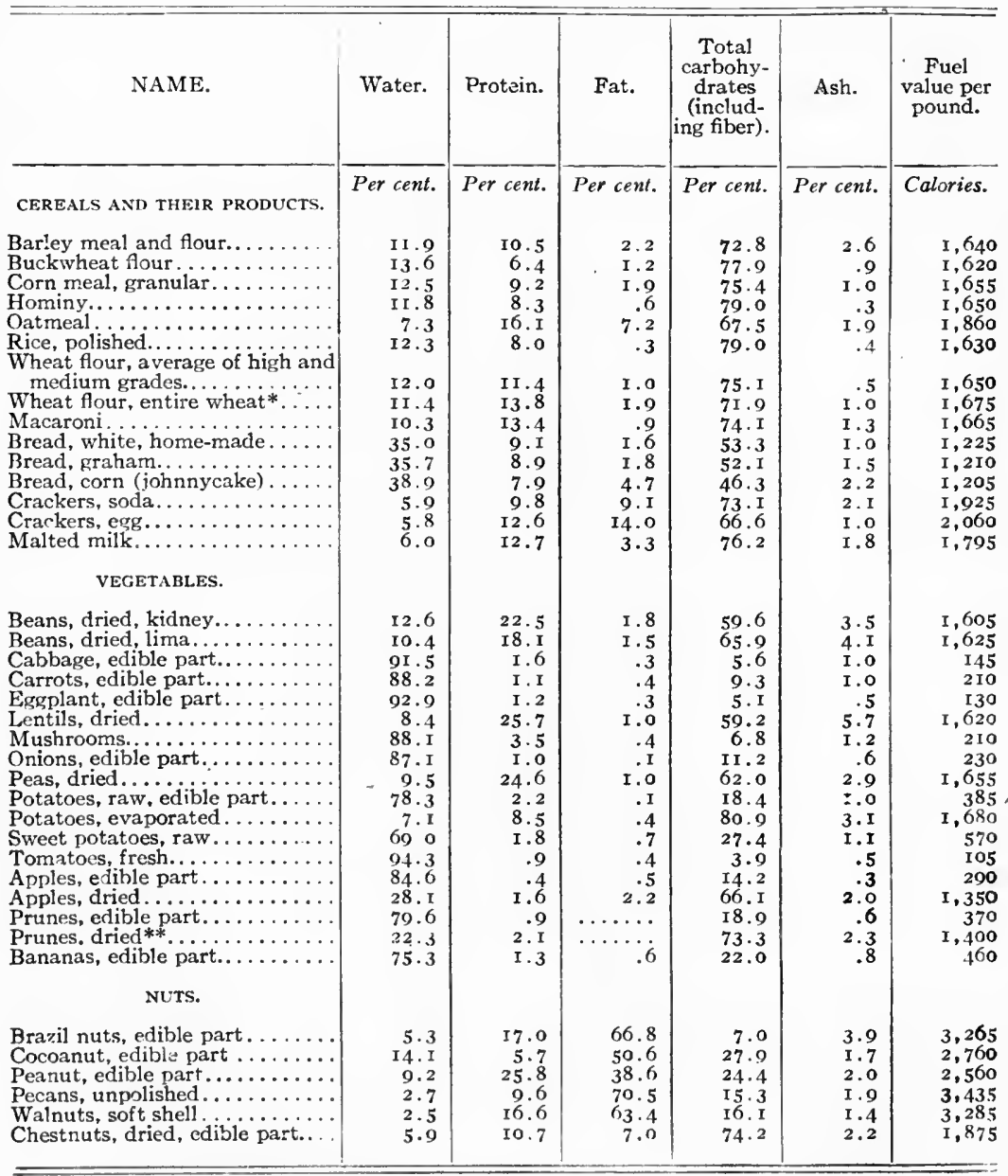

The ash consists almost entirely of potassium phosphate.

** Ar least one third of the carbohydrates is sugar. Ash rich in potassium phosphate. 
Wheat. - The section of a grain of wheat (Fig. 69) shows the following parts: Five layers (A, B, C, D, E) which form the bran, or about 5 per cent. of the entire grain, the 3 outer making up the skin or pericarp and the 2 inner the coats of the seed proper; the outer of these coats is the testa which contains most of the coloring matter of the bran. Inside the seed coats is the endosperm, comprising the aleurone layer ( $F$ ) made up of large rectangular cells, and the starch cells $(G)$. The bran layers are mostly indigestible cellulose. The aleurone cells are filled with nitrogenous material, and form about 8 per cent. of the grain. The starch cells, more or less irregular in shape, consist of a wall of cellulose, within which

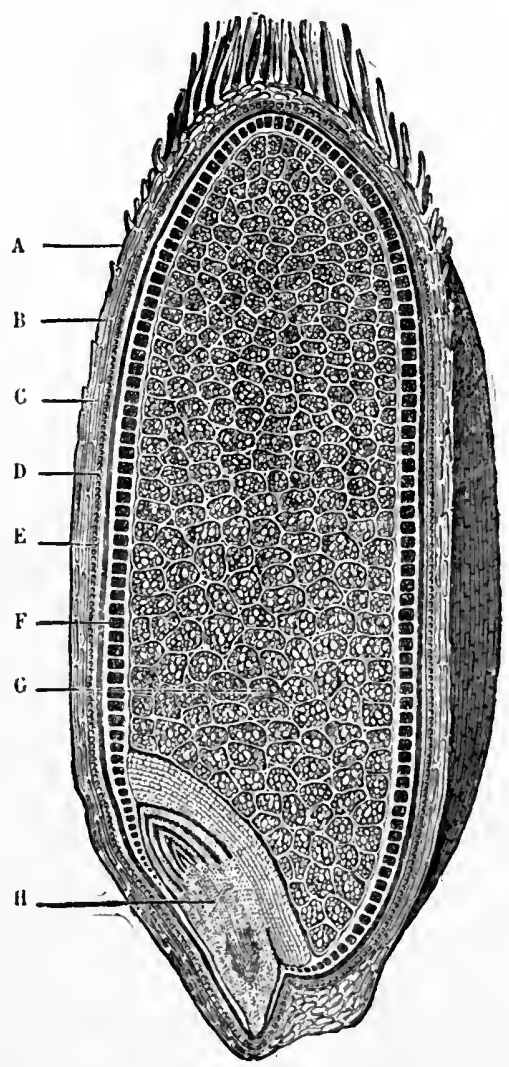

Fic. 69.- Longitudinal section of grain of wheat. A, B, C, layers of skin or pericarp; D, E, coats of the seed proper; $F$, aleurone layer; $G$, starch cells ; $\mathrm{H}$, germ or embryo. 
is a network of nitrogenous protoplasmic material thickening toward the center into a nucleus, and containing proteins, starch granules and fat. The outer starch cells have thicker walls than the inner ones and are generally sifted out in the making of good flour. The germ is rich in fat.

Wheat flour varies, not only according to the quality of the grain from which it is made but also according to the method of grinding the grain itself. The outer part contains more of the proteins, fat and mineral matters than the white interior part. It follows that the whitest, high-grade flours are poorer in proteins, also slightly poorer in fat and salts, but correspondingly richer in starch, than the low-grade, colored flours. What they lose in nitrogen and other constituents, however, is more than made up by increased digestibility.

According to their comparative contents in protein and starch, flours are divided in three grades:

Graham flour, from the grinding of the entire grain, skin and all. Whole-wheat flour, from the grinding of the grain after removal of the skin or outer bran layers.

Standard patent (or family grade) flour, made after removal of the bran, aleurone layer, outer starch cells and the germ. By careful milling, 73 per cent. of the grain can be obtained in flour of this quality.

The amount of protein is greatest in the Graham and smallest in the standard patent (in the proportion of I2.65 to II.99), while the amount of carbohydrates is smallest in the Graham and largest in the standard patent (in the proportion of 74.58 to 75.36 ). Careful experiments by the Department of Agriculture (Farmer's Bull., No. 389) have proved that the digestibility and assimilation of bread made from standard patent flour is so much greater than that of bread made from the other grades, that it actually utilizes a larger amount of both proteins and carbohydrates, the proportion of proteins thus absorbed (for equal weight of milled grain) being 10.62 for the standard patent, I0.05 for the whole-wheat and 9.47 for the Graham.

It clearly follows that to eat Graham bread under the impression that (the cost being the same) it yields more nourishment than good white bread, is a groundless delusion. Graham bread and, to a lesser extent, whole-wheat bread, act as mild irritants to the in- 
testinal canal, increasing peristalsis and producing a laxative effect. This property may sometimes be useful in the field where constipation often prevails.

It should also be noted that wherever bread forms the great bulk of the diet, whole-wheat flour is preferable to the standard patent on account of the presence of certain substances (vitamines) in the outer layers which are useful to the nervous system; thus it is well known that wherever rice constitutes the main article of diet, removal of its pericarp is likely to be followed by symptoms of peripheral neuritis. (See under Beriberi.)

Good flour should not be of a pure white color but of a creamy, yellowish shade. "After being pressed in the hand; flour should fall loosely apart; if it stays in lumps it has too much moisture in it; when rubbed between the fingers it should not feel too smooth and powdery but its individual particles should be vaguely distinguishable; when put between the teeth it should 'crunch' a little; its taste should be sweet and nutty, without a suspicion of acidity." Flour is peculiarly sensitive to the exhalations from other substances and should not be stored in the same room with decaying vegetables or articles that emit unsavory odors. As it deteriorates rapidly when exposed to dampness, it should be kept in dry, cool, well-ventilated storerooms, and in barrels rather than in sacks. Flour which has fermented and become sour can be partly corrected and yield fairly good bread by the use of good lime water.

The proteins of wheat consist chiefly of gliadin and glutenin which, in contact with water, form gluten, the tenacious elastic substance that gives consistency to the dough and enables it to be baked into bread. The quantity and quality of these proteins, especially gliadin, has much to do with the value of the bread.

BREAD. - There are two general types of bread: fermented bread when made with yeast, and unfermented bread when made without yeast. Fermented bread is always preferable. It is generally made, in post bakeries, by the sponge-and-dough process. In this method, one-half the flour to be used in the baking, all of the yeast and a little more than half the water, are thoroughly mixed and set aside in an even temperature of about $80^{\circ} \mathrm{F}$. to rise. This is the sponge. When bubbles begin to break at the surface, lard, and water in which salt and sugar have been dissolved, are added and the sponge made into a thin batter. The remainder of the flour is put in and 
the whole mass mixed and kneaded into a stiff elastic dough. In the straight-dough process all the ingredients are mixed together at the same time. As it saves time and labor it should always be preferred in the field. Equally good results can be obtained by either method.

For each roo pounds of flour, about 7 gallons of water will be required, no matter what process is used. Each gallon will take about 3 ounces each of salt, sugar and lard, and one ounce of compressed yeast.

Compressed yeast, a pure culture of yeast with enough starch to give it body, is the best kind; when not procurable it is generally replaced by a larger quantity of liquid yeast made either with flour or potatoes. Under the influence of the yeast, another ferment, diastase, originally present in the flour, becomes active and hydrolyses some of the starch into sugar. The yeast then acts upon the sugar, splitting it into alcohol and carbon dioxid; the latter, as it forms and expands, causes the dough to "rise" and to become spongy. If the fermentation proceeds too far, lactic and butyric acids may form in sufficient quantity to make the bread sour; if not far enough, the bread will be heavy and soggy. Heavy bread may also result from cheap flour poor in gluten, too much water, too little or poor yeast, insufficient kneading of the dough and imperfect baking. The fermented dough, cut into loaves, is baked in an oven heated to about $450^{\circ} \mathrm{F}$. In this process, the surface of the dough is transformed into crust by desiccation and partial caramelization; the gases expand still more and the little cavities are further enlarged by the evaporation of the moisture; starch is rendered more soluble and some of the proteids converted into peptone-like bodies.

"A loaf of good, well-baked bread should be well raised and have a thin, flinty crust which is neither too dark in color nor too tough, but which cracks when broken. The crumb should be porous, elastic and of uniform texture, without large holes, and should have a good flavor and odor." Bread should not be eaten until Io or I2 hours after its removal from the oven. Warm bread is objectionable because readily compressed into solid masses offering more resistance to the digestive juices. This objection has much less force against rolls or other forms in which the crust is very large in proportion to the crumb. 
In non-fermented bread, the carbon dioxid is generated by "baking powders" consisting of sodium bicarbonate and an acid or acid salt, most commonly potassium bitartate (cream of tartar), with enough starch to prevent these ingredients from reacting upon each other until dissolved in water. Sometimes alum is used instead of sodium bicarbonate, but the opinion is pretty general that it is an undesirable and harmful constituent. Again, the baking powder may be previously mixed.with the flour as in the so-called self-raising flour; or the carbon dioxid may be evolved in the water used to make the dough, or otherwise forced through the latter. But the bread resulting from any of these artificial methods is distinctly inferior in taste and digestibility to fermented bread.

In the process of making dough, the flour absorbs 50 per cent. of its weight of water, one-half of which evaporates in the oven, so that the added weight is about 25 per cent.; in other words, IOO pounds of flour should yield at least 125 pounds of bread.

The typical high-grade American bread, according to Wiley, consists of : water 35 , proteins 8 , carbohydrates 54.45 , fat 0.7 .5 , ash I.5O. Bread, therefore, is somewhat deficient in proteins, much more so in fats and cannot be considered a perfect food; it must be supplemented with butter, cheese, bacon, or rich gravy. Well-made bread is highly nutritious and digestible, the total loss in the intestinal tract being less than 3 per cent.

Crackers, biscuits and hard-bread are made from unleavened or slightly leavened dough, with as little water as possible, and very little salt, slowly and carefully baked and afterward kept for some time in a heated room to complete desiccation. As they contain much less water than ordinary bread they are more nutritious and have greater keeping qualities but are also less palatable and digestible.

The French pain de guerre, the best hard-bread yet baked, can be kept sound and wholesome at least a year. It is made from an excellent quality of flour, bolted to 30 per cent., with yeast and salt, and therefore undergoes a certain degree of fermentation which makes it more appetizing and absorbent.

Fresh soft bread is, under all circumstances, the best of the components of the ration and should be furnished, even in the field, whenever possible; hard-bread is but a poor substitute. Experience has demonstrated that baking bread in camp is not attended with 
great difficulties. Each division in an army should have a baking detachment provided with a sufficient number of portable ovens, which establishes itself at some convenient central point in rear, or divides into brigade sections. Thus the Russian troops in Manchuria (1904 and I905) were most of the time furnished with fresh bread, often baked only a few miles from their lines.

Field bread. - A type of fresh bread recently supplied by the Q. M. Corps, and of which the constituents are issue flour, yeast, sugar and salt. It contains less water than ordinary bread and is baked more slowly and a longer time. It has a thick crust made to withstand handling and long transportation, and keeps comparatively fresh for a period of from to to I4 days, or longer, depending upon weather conditions. After the crumb has become hard and dry, through long keeping, it may be freshened by wrapping with wet cloths and reheating in a very slow oven. That this bread serves a very useful purpose and is greatly appreciated by troops in the field has been demonstrated during maneuvers.

Somewhat similar is the pain biscuite of the French, which has been exposed to prolonged baking and is drier than ordinary bread. It can be kept for 18 to 20 days after issue, but has the defect of breaking up and crumbling in the haversack.

MAIZE OR CORN, next to wheat, is our most important cereal. It is richer in fat than any other cereal excepting oats; much of this fat is in the germ which is often removed to prevent the meal from becoming rancid and mouldy. On account of its deficiency in gluten, corn cannot be made into fermented bread, but is consumed under various other forms and is always nutritious and wholesome.

Parched corn, mixed with the pods of the mezquit tree (the seeds being removed) and ground into a coarse flour, is the "pinole" of the Mexicans, a wholesome and easily transported food on long journeys.

OATS is the richest of cereals in proteins, fat and mineral matters, its ash containing 0.42 per cent. of phosphorus. Oat-meal is mostly consumed in the form of cake and as porridge. It is highly nutritious but liable to produce acidity and disagree with some persons. The large proportion of bran scales it contains gives it distinct laxative qualities. One advantage of oatmeal for soldiers in the field is the ease and quickness with which it is prepared; in the absence of fire it can even be eaten raw, after soaking in water. 
RICE is the poorest of cereals in protein and fat, but one of the richest in very digestible starch. It is the staple food of about one-third of the human race. In the absence of meat, rice should be supplemented with vegetables rich in proteins, such as beans and peas. (See Beriberi.)

Proper cooking is essential to insure its palatability and digestibility. It should be sprinkled into boiling water so slowly that it will not cool the water, for a constant brisk ebullition is necessary to keep the grains separate until cooked.

Pulses or Legumes. - Plants of the family Leguminosc, characterized by butterfly-shaped flowers and 2-valved seed pods. Their seeds are notable for their richness in proteins, which equals or often exceeds that of meat. They also contain considerable potash and lime but are poor in fat and sodium chloride. The chief proteins are legumin and glutenin, forming what is commonly called vegetable casein. They are highly nutritious but of more difficult digestion than cereals or meats; under the most favorable conditions, from 15 to 20 per cent. of the proteins are unabsorbed and lost. It is therefore necessary to cook them thoroughly and with great care.

The ordinary pulses are beans, peas and lentils. The proteins of beans and peas contain sulphur, an ingredient which often gives rise to flatulency by the formation of carbureted and sulphureted hydrogen. Dry beans and peas should be soaked in warm water for I2 hours and then boiled several hours until perfectly done. The water must be soft, for the legumin forms insoluble compounds with lime; if hard, it should be previously boiled to precipitate the carbonates, or else have sodium carbonate added to it. Peas are best used ground, as meal, making palatable and very nourishing soup; they are a good occasional substitute for beans. The pea-sausage of the German Army consists of pea flour, fat pork and salt; it is a good emergency food, being issued cooked, and readily made into soup. Lentils are the best of the pulses and deserve to be better known and more generally used in this country. They contain even more proteins than beans and peas, and are free from sulphur; they are more digestible, more easily cooked and fully as well flavored.

The cow-pea, much cultivated in the Southern States as forage plant and green manure, also belongs to this family. Some varieties 
are as good as beans in regard to nutritive value, digestibility and flavor.

The Soy Bean (Glycina hispida) is the most important pulse of China and Japan, being remarkable for its high percentage of protein (34) and fat ( $I 7$ ). It is manufactured into a number of products, all rich in protein and, when combined with rice (the staple food of those countries), helping to make a well-balanced dietary. The best known are shoyu or soy sauce, a thick brown liquid with pungent, agreeable taste, and several kinds of cheese-like preparations. They contain little or no starch and, on this account, have been recommended for diabetics.

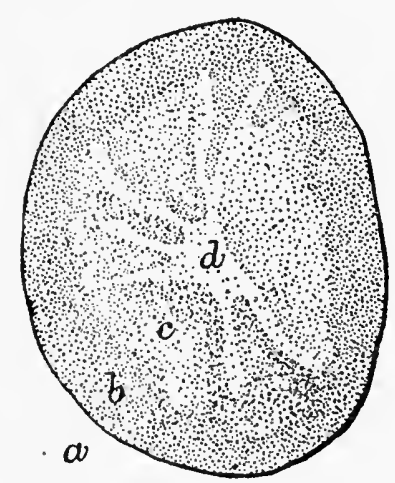

FIG. 70.-Transverse section of the potato; $a$, skin; $b$, cortical layer; $c$, outer medullary layer; $d$, inner medullary area.

Tubers.-Tubers are underground stems thickened into oblong or rounded bodies bearing "eyes" or buds. The only true tubers commonly used as food are the potato and the Jerusalem artichoke.

Potato is a wholesome and easily digested food but, on account of its very small content of proteids and fats, an imperfect one.

If a cross section of a raw potato (Fig. 70) is held up to the light, four distinct zones can be seen: the skin; the cortical layer, slightly colored and denser than the inner parts; the outer and inner medullary areas. The skin and cortical layer make up about II per cent., and the medullary areas 89 per cent. of the tuber. The outer medullary area contains the greater part of the nutrients. The inner area, or somewhat star-shaped core, spreads irregular arms into the outer. When this core is large it makes a soggy mass, full of holes after cooking. In peeling a raw potato, the loss in weight is about 20 per cent., namely the skin, most of the cortical layer, and more or less of the medullary areas; in reality the loss of nutrients is even greater than this would seem to indicate owing to the larger proportion of proteins and mineral matters existing in the outer layers. It is best therefore to remove the skin mechanically, by scraping, rather than by cutting. 
The most important mineral matters in the potato are potash and phosphoric acid compounds; it also contains citric, tartaric and stuccinic acids, forming salts with potash, soda and magnesia to which are due its well-known antiscorbutic properties.

In the process of boiling a potato, more or less protein and mineral matter are lost; comparatively little if boiled with the skin, but a great deal if it has been peeled, amounting to nearly one-half if, besides, it has been soaked in water. If peeled before cooking it should be placed directly in hot water. Potatoes cooked with jackets on are more nutritive and palatable than if peeled; a section of skin should first be removed at each end so that the moisture may escape. They should be slowly boiled for 30 minutes. If cooked rapidly or too long they become soggy. Mealiness is highly desirable in a cooked potato, on account of its increased palatability, but waxiness is probably an indication of greater quality, being due to a higher proportion of protein, as often seen in young tubers.

Normally the potato contains

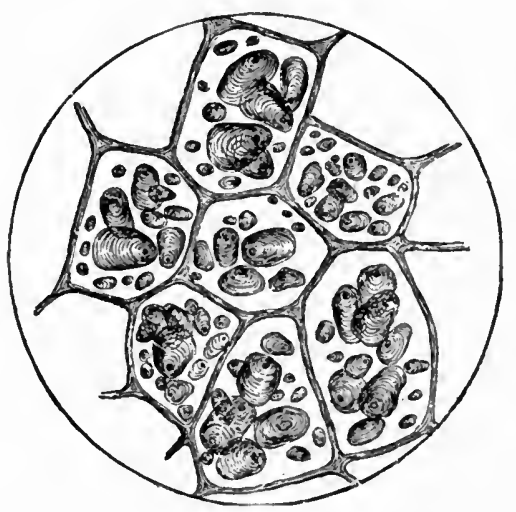

FIG. 7I.-Cells of a raw potato, with starch grains in natural condition. (Munson.)

Jerusalem artichoke, a tuberous perennial sunflower, growing wild in the middle western United States, is more or less cultivated in this country and Europe. The small, knobby tubers contain I5 per cent. of carbohydrates, mostly in the shape of inulin and levulin instead of starch. They are wholesome, digestible, palatable, and often used in salad. An easily grown and very prolific plant. 
Other plants furnishing tuber-like fleshy rhizomes used for food in warm countries, including the southern United States, are:

Yam (Dioscorea), a slender twining vine with often enormous rhizomes, ranking next in value to the sweet potato in the West Indies.

Taro or Eddoes (Colocasia), also frequently cultivated for the beauty of its huge, arrow-shaped leaves ("Elephant's Ears").

Tannier or Yantia (Caladium), with leaves and fleshy rootstocks much like the preceding.

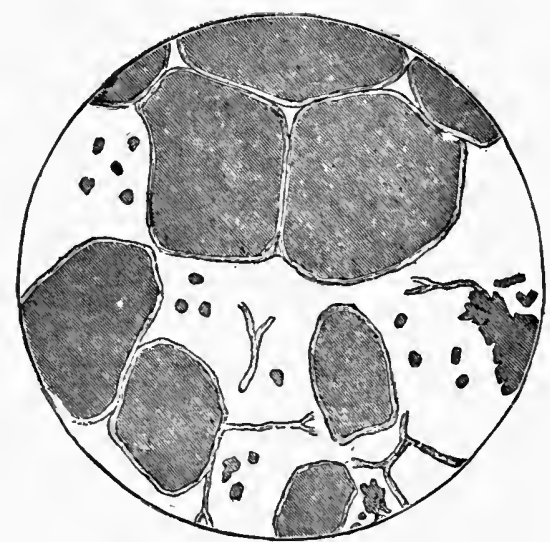

FiG. 72,- Cells of a thoroughly boiled potato. (Munson.)

Roots. - To the true roots belong the following commonly cultivated plants :

Sweet potato (Ipomca), species of morning glory, with long creeping stems, cultivated in this country as far north as New Jersey for its valuable root, which contains as much starch as the potato and about 6 per cent. of sugar. The sweeter and more juicy kinds (often miscalled yam) are preferred in the South, whereas the drier and mealy kinds are in greater demand in northern markets.

Cassava (Manihot), a stout herb with palmate leaves and huge fleshy roots extensively consumed, in tropical and semi-tropical America. From their juice is obtained the starch known as tapioca.

The ordinary garden roots, such as carrot, beet, turnip, parsnip, oyster plant and radish. They are watery and contain little nutri- 
tive substances, but they add variety and pleasant flavors to more substantial foods. Carrot contains 6 per cent. of sugar, and beet

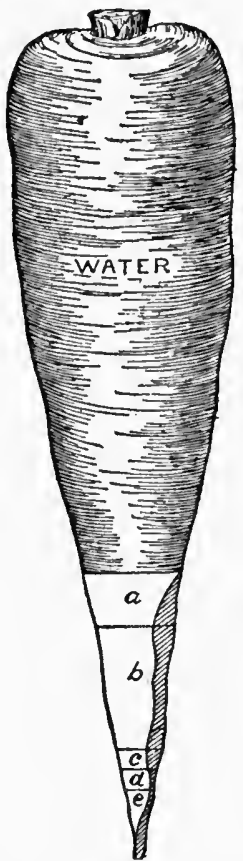

FIG. 73.- The com. position of the carrot and the loss of nutrients when boiled: $a$, fiber, starch, fat, etc.; $b$, sugar; $c$, nonproteid nitrogenous matter; $d$, proteid nitrogenous matter; $e$, mineral matter. The hatched portion represents the loss when medium-sized pieces were boiled. at least twice as much. (Fig. 73.)

Green vegetables consist of the leaves and stems of various plants. As a class they contain somewhat less carbohydrates than roots and tubers, but a little more protein and salts; they are also valuable antiscorbutics; their appetizing flavors make them indispensable adjuvants to a well-ordered dietary, besides giving it bulk and promoting the action of sluggish bowels. Cabbage is not always easily digested; it contains sulphur, which may produce flatulence, and a fair proportion of potassium salts. Lettuce and cresses are healthful and digestible. Celery and asparagus have useful diuretic properties. Onions are savory and wholesome, owing much of their value to a pungent oil containing sulphur. Vegetables such as celery, lettuce and all others eaten in salad and which, therefore, do not undergo the purification of fire, are liable to convey the eggs of parasites and pathogenic germs; they should always be carefully washed before being prepared for the table.

Fruits Used as Vegetables. - They include melon, cucumber, squash, pumpkin, egg-plant, tomato, etc., all very watery, the melon and tomato containing as much as 95 per cent. of water, but, nevertheless, useful, palatable and wholesome. Tomato contains nearly as much organic acids as potato, and as it is mostly consumed raw, more of them are available in the body, giving it valuable antiscorbutic qualities.

Fruits and Nuts. - Fruits, properly so-called, are mostly used in the raw state, as relish and dessert, for their pleasant acidulous taste. They may be divided into pulpous fruits (apples, peaches, cherries, plums, oranges, strawberries, etc.) and farinaceous fruits (chestnut, banana, bread-fruit, etc.). The former are very watery, 
containing sugar, acids, gum and pectin in variable proportions and having but little value as food; the latter contain a notable amount of starch and sugar and have decided nutritive value.

Nuts (see composition, page 350 ) are rich in proteins, carbohydrates and especially in fats; they constitute a highly nutritious food, but greatly tax the digestive powers. As dessert they should be eaten sparingly.

Peanut belongs to the pulses botanically and, like them, is rich in protein; but, as food, is more akin to the true nuts by its high proportion of fat. When roasted and ground into meal it is somewhat oleaginous and marketed as peanut butter. 


\section{CHAPTER XXVIII.}

\section{THE NUTRITIVE VALUE OF FOODS.}

\section{AMOUNT NEEDED.}

We take food to build and repair the bodily tissues, as well as to evolve heat and the necessary energy for work. The body is a machine capable of converting potential into kinetic energy. The potential energy is supplied by the food, and the metabolism of the tissues converts it into the kinetic energy of heat and mechanical power. The potential energy contained in any foodstuff is determined by ascertaining the amount of heat it will yield on complete combustion. It is possible, by laboratory experiment, to measure the exact amount of heat liberated by the burning of a definite weight of food in a calorimetric bomb, and this is assumed to represent the energy evolved by the oxidation of the same quantity in the system. This assumption is correct enough in the case of fats and carbohydrates, which are completely consumed in the body to carbon dioxid and water, but needs qualification in the case of proteins which are only oxidized to urea, their final product of decomposition, so that their physiological value is about 25 per cent. less than their calorimetric value.

The potential energy of food as measured by the amount of heat obtained by combustion, is expressed in units of heat or calories. A calorie is the amount of heat required to raise I kilogram of water $\mathrm{I}^{\circ} \mathrm{C}$. (or one pound $4^{\circ} \mathrm{F}$.). A " small calorie" is the amount required to raise $\mathrm{I}$ gram of water $\mathrm{I}^{\circ} \mathrm{C}$., so that there are $\mathrm{I}, 000$ small calories in the ordinary or large calorie. There are marked differences between the various proteins as regards their calorimetric or fuel value, animal proteins having a greater value than those of vegetable origin; the same difference exists between animal and vegetable fats, also in favor of the former. But, for practical purposes, the following averages of the physiological value of the three proximate principles of foodstuffs, as determined by Rubner, are generally accepted. 

I gram of protein, $=4.1$ calories. $*$
I gram of carbohydrates,
I gram of fat, $=4$. $\mathrm{I}$ calories. $=9.3$ calories. $\dagger$

The unit of mechanical energy of food used in this country is the foot-ton, that is, the energy required to raise 2,240 pounds I foot. One calorie is equal to $\mathrm{I} .53$ foot-tons. Therefore we assume that I gram of fat, oxidized in the body, evolves sufficient heat to warm 9.3 kilos of water $I^{\circ} \mathrm{C}$., or sufficient mechanical energy to raise 14.2 tons one foot. Expressed in metrical terms, I calorie is equivalent to 425.5 kilogram-meters, that is, the energy required to raise 425.5 kilograms I meter.

An adult man at rest, lying down but not sleeping, has been found to require only 2,300 calories (33 per kilo of weight) per day for the normal performance of all the animal functions. For a man at work must be added the calories needed for the greater activity of the body functions and the muscular energy expended, a variable quantity.

Of the calories evolved in the body during a hard's day's work, 300 or more are expended in mechanical energy. The remainder, according to Stewart, are given off as heat in the following proportions :

From the skin by $\left.\left\{\begin{array}{l}\text { radiation } \\ \text { conduction } \\ \text { convection } \\ \text { evaporation of perspiration } \\ \text { evaporation } \\ \text { of water } \\ \text { heating of the } \\ \text { expired air }\end{array}\right\} \begin{array}{c}80 \\ \text { per cent. }\end{array}\right\} \begin{gathered}\mathrm{I} 7 \cdot 5 \\ \text { per cent. }\end{gathered}$

Heating of excreta

2.5 per cent.

According to another authority, the loss of heat in man is 77 percent. by radiation, conduction and convection, and 23 per cent. by water evaporation. (See page 422.)

* Deduction made of the calorimetric value of urea passed with the urine. If a further reduction is made for the escape of some of the products of combustion of the proteins in the feces, the value would be nearly exactly 4 calories.

† According to Atwater, the latest and most reliable researches give the following averages: protein, 4 calories; carbohydrates, 4 calories; fats, 8.9 calories; but they take into account only the material which is digested and oxidized and actually available for the body 


\section{AMOUNT OF FOOD NECESSARY.}

According to the generally accepted standard of Carl Voit, a man of average weight ( 70 kilos or I54 pounds), doing moderate work, needs II 8 grams of protein,* 500 grams of carbohydrate and 56 grams of fat, with total fuel value of 3,055 calories. This was found to be the average food consumption of laboring men in Germany. German soldiers in active service, says Voit, eat 145 grams of protein, 500 of carbohydrate and roo of fat, with fuel value of 3,574 calories. In France, according to Gautier, the ordinary laborer must have I 35 grams of protein, 700 of carbohydrate and 90 of fat, with fuel value of 4,260 calories. In England, weavers were found to take I5I grams of protein, with enough fat and carbohydrates to make a total fuel value of 3,475 . In the United States, according to Atwater, a man doing moderately active muscular work consumes 125 grams of protein, with enough fat and carbohydrates to make a total fuel value of 3,400 calories, while when doing hard muscular work the protein is increased to I 50 grams and the fuel value to 4,150 calories. The dietary of the Yale University crew, at Gales Ferry, averaged I7 I grams of protein, I7 I of fat and 434 of carbohydrates, with fuel value of 4,070 calories; that of Harvard University crew averaged I60 grams of protein, I70 of fat and 448 of carbohydrates, with fuel value of 4,074 calories (Chittenden).

Langworthy, of the United States Department of Agriculture, appears to have reached conclusions somewhat at variance with those of the preceding observers; he says: "In the average of a large number of dietary studies with men at moderately active muscular work, the quantity of protein in the food actually eaten is between IOO and ro5 grams per day.".

For the healthiest condition of the body and the greatest development of energy, the three proximate principles must be present and combined in-suitable proportions; neither proteins nor carbohydrates alone could supply the necessary nutriment; fats, on the other hand, can only be digested and absorbed in relatively small quantity, while neither carbohydrates nor fats can perform the essential functions of proteins; furthermore, the digestive fluids are obviously intended to act upon mixed and varied foods. The relative proportion of these food constituents, in well ordered dietaries, is generally set down, in round numbers, as one part of protein, half a part of fat

\footnotetext{
* One pound of protein is contained in 6.4 pounds of beef sirloin.
} 
and four parts of carbohydrates, the proportion of nitrogen to carbon being $I$ to $I 6$ or I8. The amount of protein should not fall below I.69 grams, according to Voit, or one gram, according to Lapicque, for each kilo of body weight. This amount varies only within narrow limits; when more energy is required for an increase of muscular work, it is supplied by carbohydrates and fats, especially the latter, which possess the highest fuel value. Fat is therefore the element of the dietary which oscillates most, in accordance with the amount of work performed and the temperature of the air.

The amount of food stated above as necessary is based entirely upon the assumption that whatever is habitually consumed by a class of men is a correct basis upon which to determine the actual amount required by such men. But it is obvious that such an assumption does not rest upon scientific grounds and is liable to lead into serious errors.

The ideal diet. - Any excess over what is really necessary to meet the wants of the body is certainly useless and may be harmful. It should always be remembered, says Atwater, that "the ideal diet is that combination of foods which, while imposing the least burden on the body, supplies it with exactly sufficient material to meet its wants." According to Prof. R. H. Chittenden, who has devoted much careful study to this question, the ideal diet is the smallest amount of proteins, fats and carbohydrates: "sufficient to establish and maintain physiological and nitrogen equilibrium, sufficient to keep up that strength of body and mind that is essential to good health, to maintain the highest degree of physical and mental activity with the smallest amount of friction and the least expenditure of energy, and to preserve and heighten, if possible, the ordinary resistance of the body to disease germs." To determine this ideal diet, habits are untrustworthy. The fact that they are shared by many individuals, or a whole nation, does not prove that they are correct and hygienic. The extent to which we indulge our cravings for food is not a measure of the extent to which it is best to carry such indulgence. In many communities, a large proportion of the men drink and smoke immoderately without any apparent harm, yet no one will contend that such habits respond to any actual need of the body economy, subserve any useful purpose or are innocuous. It cannot be denied that, at table, our desire for food, stimulated by the senses of taste, smell and sight, often continues to be gratified after all the 
needs of the system have been supplied. To overeat is one of the habits most easily contracted, often long tolerated by the system without obvious protest, and always most difficult to correct. It is probably the origin of many of the diseases of modern civilized society, and therefore no question, within the whole field of preventive hygiene, seems more worthy of careful attention than the exact determination of suitable dietaries for all conditions.

The food constituent which is most commonly eaten in excess is the protein. Meat, under its many forms, is appetizing and savory, and almost always an important part of the diet of people who can afford it. But, being expensive, we find it used much more abundantly by prosperous and rich nations than among poor ones, the latter being often, from necessity, reduced to an entirely vegetable diet. The assertion, sometimes made, that the most civilized nations have reached their present intellectual standard because of their high consumption of meat is an absurd confusion of cause and effect.

Conclusive experiments.* - In 1902, Prof. Chittenden began a series of experiments to determine, on scientific grounds, the amount of proteins necessary to maintain the best physical condition of the adult body. During the course of several years, he experimented with groups of professional men, athletes and soldiers, gradually and steadily reducing their protein food, and in all cases with the same striking and convincing result, namely, that the protein constituent advocated by Voit and others is much too high, at least twice greater than actually necessary. He found, by multiplied demonstrations, that the so-called nitrogen equilibrium, that is, the condition of the active body in which the ingestion and excretion of nitrogen balance each other, without loss of body weight, can be easily maintained with a daily intake of 0.85 gram of protein per kilo of body weight, and that any quantity in excess of this is wasted, if not harmful. He concluded that the proper diet for a man weighing 70 kilos (I54 pounds) should not exceed 60 grams of protein, with enough fat and carbohydrates to make up a total fuel value not exceeding 2,800 calories.

The I 3 soldiers experimented upon by Prof. Chittenden, during a period of six months, were fed upon this reduced diet, which, however, within the terms stated, admitted of many varied combinations. They led an active life, performing each day a certain amount of

* Physiological economy in nutrition, by Russell H. Chittenden, 1904 . 
prescribed exercise in the gymnasium in addition to their regular drill and ordinary duties. Their weight remained practically the same, several losing one or two pounds during the first few weeks and their weight remaining stationary thereafter. At the end of the experiment, they were in the best of health, having all gained materially in strength and endurance, as ascertained by careful tests in the gymnasium.

It is well known that the amount of nitrogen excreted rises and falls with the amount ingested and is directly proportional to it, which shows that there is no appreciable storing of it even when the intake is very much increased; only a very small proportion becomes transformed into living organized tissue, most of it, while still circulating in the fluids of the body, being metabolized into urea and thrown out as of little or no value. The greater energy needed for hard or violent muscular work is best obtained from an increased quantity of fats and carbohydrates, and so long as these principles are freely supplied there will be no loss of muscular tissue, even though the protein of the food remains unchanged.

Food which contains more proteins than the body requires is not only wasted, but there is every reason to believe that it is positively dangerous. The many decomposition products resulting from the breaking down of the circulating unorganized nitrogenous material crowd the blood, lymph and tissues; it is believed that they impair the phagocytic function of the white blood-corpuscles, that is, their power to ingest and destroy the pathogenic bacteria which invade the system; it is also probable that they exert an inhibiting effect.upon the peripheral endings of the motor nerves or upon the muscle fibres themselves, thus impairing the functional power of the tissues, causing fatigue to be readily felt after exertion and loss of the power of endurance.

We know that gout often follows, or is aggravated by a free meat diet, doubtless the result of a failure of the nitrogenous decomposition products, when in excess, to be properly oxidized and eliminated.

A consumption of protein food much beyond physiological needs means a large amount of urea and uric acid, as well as amino-acids and other products of protein decomposition, which must be passed out through the kidneys, thus throwing a constant strain upon those organs. It is the opinion of many medical authorities that a large proportion of cases of high blood-pressure, leading to arteriosclerosis, are due to an effort of the system to force out these wastes. 
Dr. L. Duncan Bulkeley, of New York, with all the authority of an eminent specialist, has called attention to the relative absence of cancer and psoriasis in the far East, as well as among Chinese and Japanese, and other countries where animal food is sparingly eaten. $\mathrm{He}$ also shows by conclusive statistics that a strictly vegetarian diet is the most important factor in the treatment of psoriasis and various acute inflammatory affections of the skin, affections in which the urine is found to contain abundant uric acid and urates.

Without pretending to find therein any necessary relation of cause and effect, it is pertinent to note that the United States, where the consumption of meat is undoubtedly greater than in any other civilized nation,* is the country which exhibits the highest rates for typhoid fever. It also shows a steady increase of the degenerative diseases of old age, so that the longevity of people past 50 is less now than one or two generations ago.

Again, we know that many symptoms of toxemia, or self-poisoning, result from absorption into the blood of anaërobic putrefactive bacteria and their toxins from the large intestines. In view of the fact that these bacteria, according to Dr. C. A. Herter, are always more abundant in the intestinal tract of carnivorous than of herbivorous animals, it seems quite probable that a free meat diet pro. motes their multiplication.

Kendall, of Harvard, found that the intestinal organisms dominant on a protein diet are the Bacillus subtilis group, $B$. coli and various forms of $B$. proteus, with alkaline reaction and the formation of indol, skatol and other products of protein putrefaction. A change of diet from protein to carbohydrate is associated with the development of acid-forming bacteria. Some bacteria are facultative in. their metabolism, producing an alkaline or acid reaction according to the culture media; $B$. coli, for instance, when grown on dextrose broth, becomes an acid former instead of a toxin producer. Toxic substances from protein decomposition are not found to any extent in media containing a certain amount of utilizable carbohydrate. These conclusions are in accord with the régime recommended by Metchnikoff for the destruction of the so-called microbes of senility, namely, low protein diet, free sugar supply and direct ingestion of

* The consumption of meat per capita in the United States, in I9I5, was 18 I pounds, while England, the greatest meat-eating country in Europe, only consumed 120 pounds in 1913 and 1914. 
lactic-acid bacilli by the mouth. From his observations, Kendall is led to recommend lactose (the most assimilable form of sugar) as an essential element in the treatment of typhoid fever, cholera, dysentery and summer diarrheas.

Conclusions. - From the results reached by Chittenden and other experimenters, and from the above considerations, we are irresistibly led to the conclusion that the standard diet of Voit, and other physiologists, must be modified so as to average about as follows:

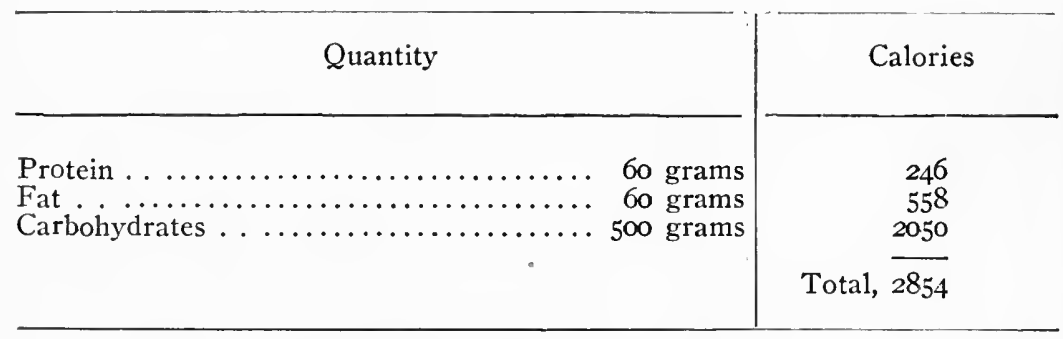

Sixty grams of protein are contained in about Io ounces of fresh lean beef; but as milk and eggs, as well as all vegetable foodstuffs, contain nitrogen, they will necessarily contribute a large part of the required protein, seldom less than one-half, so that the amount to be supplied in the form of flesh (meat, poultry, fish) will seldom exceed 30 grams (one ounce), contained in 4 or 5 ounces of boneless, fresh, lean beef. Thus Prof. Irving Fisher, of Yale, has shown by experiments upon students, that the highest degree of endurance was reached at the close of a period of five months, upon a diet which, beginning with a daily average of $5^{2}$ grams of protein from flesh foods, and total fuel value of 2,830 calories, was reduced to about 8 grams from flesh foods, with total fuel value of 2,220 calories.

This subject is further considered in connection with the chapter on Rations. 


\section{CHAPTER XXIX.}

\section{FIELD COOKING AND BAKING.*}

This very important subject has not yet received the study which it deserves nor reached the development of which it is capable. Under field conditions the cooking outfit should be as light and simple as possible, but experience has shown that, even then, it is generally practicable to provide each company with a few utensils, carried by pack animals or wheeled transport, which will greatly contribute to the comfort of the men and the improved quality of their food.

In the absence of field ranges, the most easily improvised kitchen consists of a trench 4 feet long, dug in the direction of the wind, with chimney at the leeward end, one or two feet high, built of sod, stone or mud; the trench should be a foot deep, well open at the

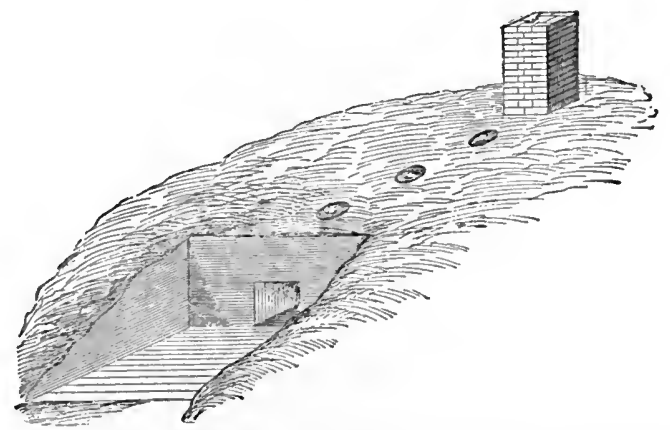

FIG. 74.- Excavated fire-place inside of bank.

windward end, where the fire is made, and shallow up under the chimney, its width being 2 inches less than that of the kettles; if material is at hand, it can be closed above so as to make a regular flue, leaving two or three openings for the kettles. As the wind changes, another trench is dug accordingly, leading under the same chimney. Several trenches may thus radiate from the same chimney, those not in use being temporarily clogged up. If time permits,

* Manual for the Subsistence Department, igio. Manual for Army Cooks, 1910. Handling the straight Army Ration and Baking Bread. T. L. Holbrook. 
a crane can be put up, consisting of a pole supported on two forked uprights, or of an iron crossbar with hooks for hanging the pots.

In clayey soil, an underground horizontal flue, one foot square, is dug in the side of a bank, one foot from the surface of the ground, and its internal end connected with a chimney. Along its course, openings are made in which to place the kettles. (Fig. 74.)

Economy of fuel often demands that company cooking fires should also be utilized to destroy as much of the garbage as possible. The regular kitchen incinerator is described on page 668. Cooking over the incinerator fire is often practicable and desirable. For this purpose the long-legged "spider," supplied to various militia regiments, is the best fixture whereon to set kettles and pots. The regulation field range necessarily requires a separate fire, but even in such a fire much of the wastes can be burned up.

In the field, the individual mess kit of the American soldier consists of canteen with cup, and meat can. (See under Equipment.) The only cooking device now supplied troops in the field is the

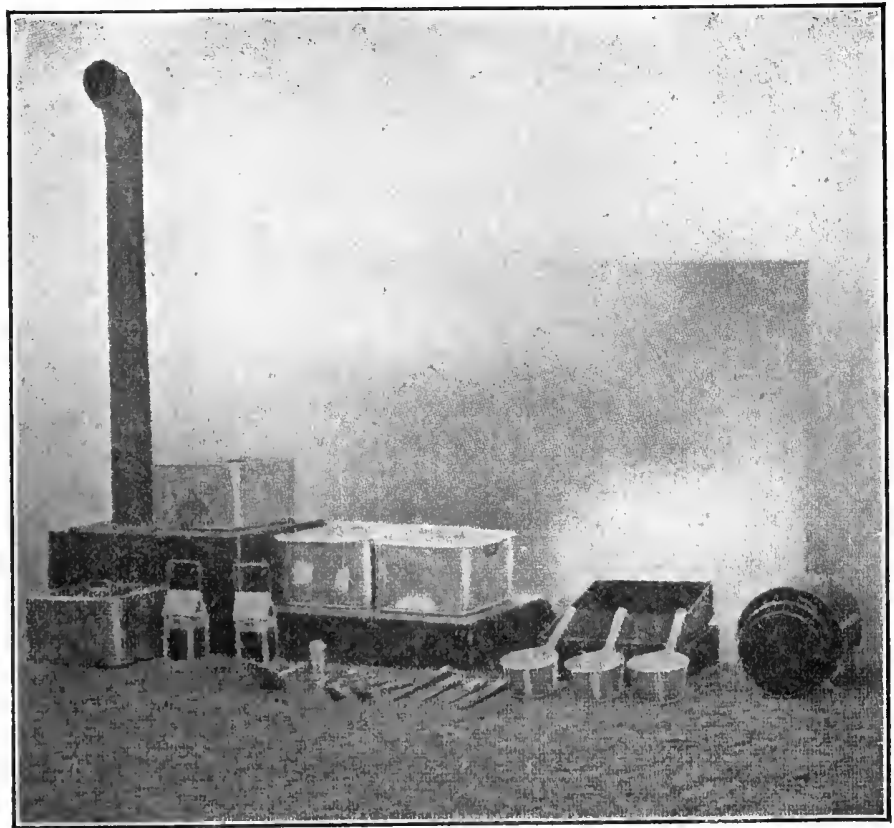

Fig. 75.-New Army field range. 
Army field range, in two sizes, No. I and No. 2; the former weighs approximately 264 pounds, with Alamo attachment and utensils, and is designed to cook for I 50 men; the latter weighs about I 50 pounds, with utensils, and is designed for $55 \mathrm{men}$; it is without the Alamo attachment. (Fig. 75.) Both consist essentially of a sheetiron cover, or boiling plate, with turned down edges, under which the fire is made, and the oven or roaster (4I), which is a sheet-iron box with double walls and top, surmounted by the smoke-stack. In No. I the boiling plate has three sections, $42,42 \mathrm{~A}$ and $42 \mathrm{~B}$, the last two forming the Alamo attachment. (Fig. 76.) The heat is drawn from the fireplace to the space between the walls, thus enveloping the oven and being thoroughly utilized. The range should be set up with the firing end to the wind, and the sides banked just enough to seal cracks. A slight excavation under the boiling plate will make a larger firing place and prove more satisfactory. It can boil, roast, fry and stew any of the components of the ration. If installed upon a pit filled with stones (see Company Incinerator), it is possible to burn much of the garbage in the fire and to evaporate most of the liquid wastes on the heated stones.

When prepared for transportation, with utensils nested inside, No. I makes a package $32 \times 20 \times 16$ inches in size which can be packed on a mule by placing the oven on one side and the boiling plate with utensils on the other side, making a well-balanced load.

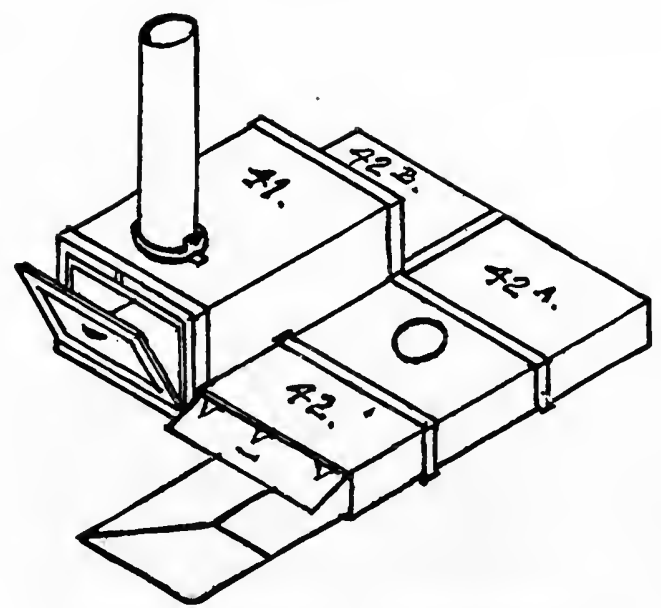

Fig. 76.-Field Range No. I in position. 
When transportation ceases to be available, it is left behind, except the boilers and other utensils, which are carried by the cooks as far as practicable.

Cooking in paper bags, as now frequently practised in many households, seems to be worth trying in the field range. Meats and vegetables can thus be cooked in less time and with less fuel; they require less attention, no basting, for instance, being necessary; there is no shrinkage and hardly any loss of juices or of nutritive properties.

Fireless Cooker. - Any cooking device saving fuel and time is worthy of consideration in the field, and, on that account, much attention has been given, of late years, to the so-called self-cooking stove or fireless cooker. It consists of a strong box closing tightly and containing vessels in which the articles of food, partly cooked, are placed. The vessels fit snugly in the box and are surrounded on all sides by a thick layer of some non-conductive substance. The food prepared in the ordinary way and placed in the vessels is boiled or otherwise cooked for a short time (20 to 30 minutes) in a range or over a fire, and the vessels, securely clamped down, are returned to the box. Usually, heated soapstones, in the shape of disks, are used as radiators, one being placed beneath and the other (when necessary) over the vessel. As the loss of heat is very small, the temperature of the vessels is not sensibly lowered, and the food continues to cook until it is thoroughly and evenly done, requiring about twice the time that would be taken in an ordinary stove. A longer stay in the cooker is said not to be detrimental to the quality of the food inasmuch as there is no loss by evaporation. (Fig. 77.)

This system of cooking possesses advantages for the field; it greatly economizes fuel and gives the men a well-cooked meal on their arrival in camp. Where wood cannot be procured it might be exceedingly valuable. On the other hand, it requires a heavy and rather unwieldly apparatıs which does not take the place of the field range, but only supplements it, thus adding materially to the weight of the company baggage. On this account the general opinion of competent observers is adverse to its use for military purposes. It seems probable, however, that a combination of coal-oil stove and fireless cooker (as already found in trade) could be developed in such manner as to be quite adaptable to permanent and semi-permanent camps. 


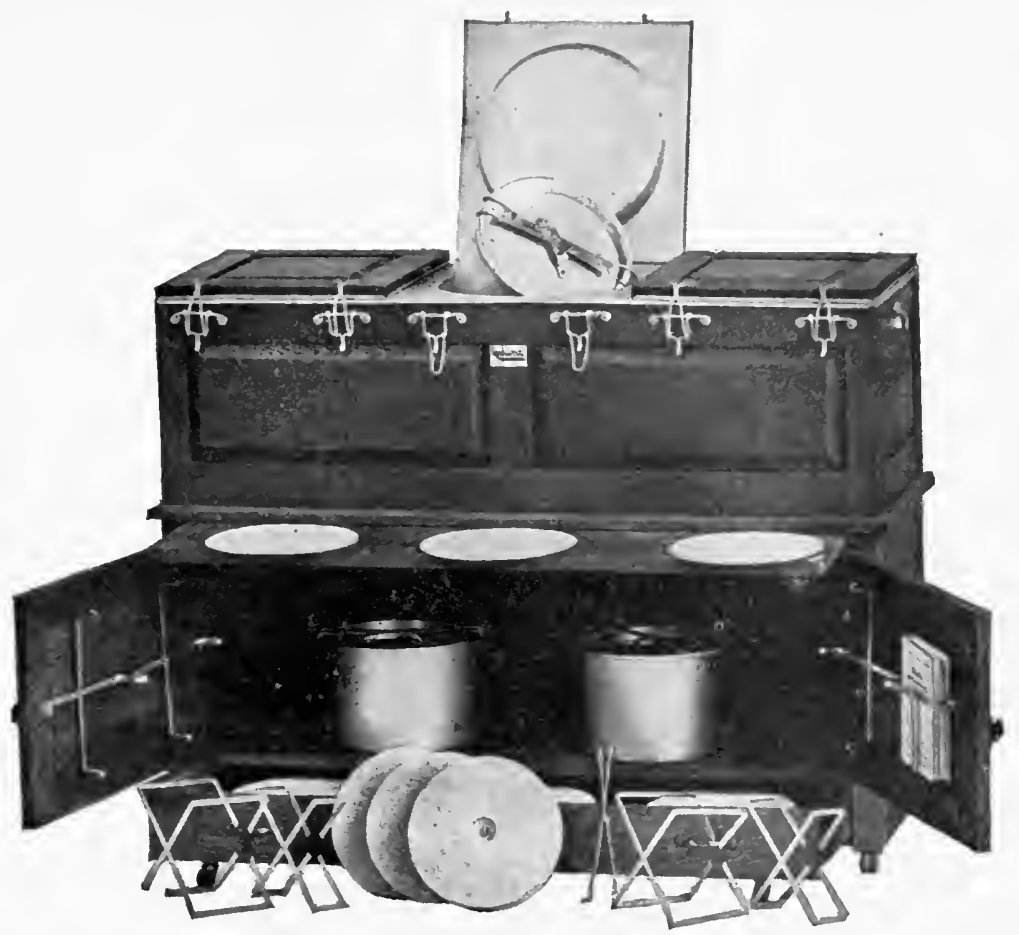

FIG. 77.- "Caloric" Fireless Cookstove, with storage cabinet below. The equipment consists essentially of three kettles with seamless aluminum lining, six radiators, trays, baking racks, radiator racks and tongs. The covers are equipped with valve that allows the excess steam to escape.

Gas Cooker. - For traveling detachments, in trains or wherever gas is obtainable, the Q. M. Corps provides a convenient and portable cooking appliance, the "Gas Cooker," compactly packed in one trunk.

Baking 0vens. - In the field, portable ovens should be provided whenever practicable; in our Army, the old-type field oven is made of two pieces of sheet iron, so curved that when their upper edges are connected and the lower edges fixed in the ground, they form an arch 5 feet long, 3 feet 9 inches wide, and $x$ foot 8 inches high; the front is closed by a two-handled iron door and the rear by a plate; when set up, the whole, excepting the door, is covered with a layer of earth; the door serves both as draft and vent for the smoke. (Fig. 78.) Such an oven, if kept in constant operation for 24 hours, 


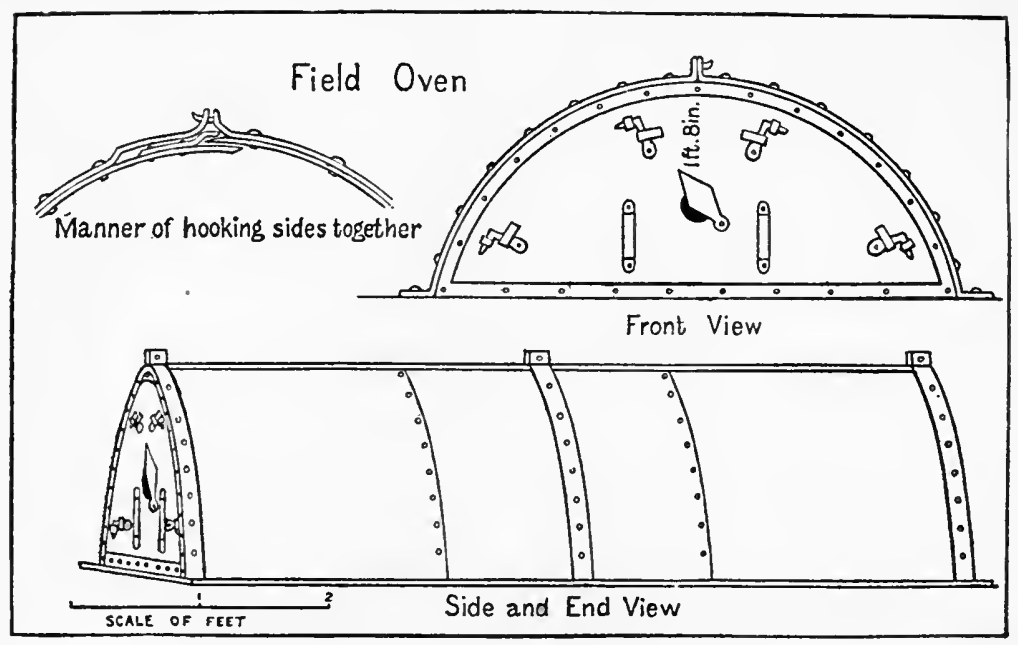

FIG. 78.- Field oven for baking bread.

can bake enough bread for I,O0O men. A larger size is also supplied, 6 feet long and 4 feet wide, formed of three pieces of sheet iron; it is operated in the same manner.

A larger and more efficient field oven is that devised by W. H. Hart of the Q. M. Corps, and known as field oven No. I. (Fig. 79.) It is a knock-down steel structure containing three tiers of pans, and designed for continuous baking. Its base is banked around with earth and the fire made in a pit underneath. The top is covered with sand to prevent loss of heat. The walls are double, and, through the space between them, as well as through the space between the tiers of pans, the flames and smoke freely circulate, so that all parts of the oven are bathed in heat. This oven has the great merit of a large output (from 3,000 to 3,600 rations per day) with comparatively little fuel.

Baking ovens can be readily improvised, as shown in Fig. 8o. On the left is seen a mud oven made by moulding sand or loam over two barrels placed end to end, the moulds thus formed being covered with about 6 inches of clay, into which hay or straw has been chopped. The oven is allowed to dry in the sun for about two days and then baked by slow fire. In the absence of barrels, a framework of flexible twigs closely wattled together will answer the same purpose. 


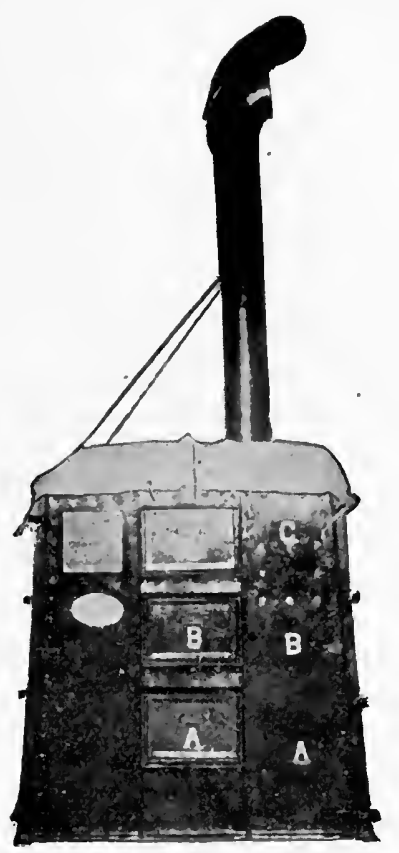

FiG. 79.- Field Oven No. I.

In the bank are seen two simple excavated ovens, one with mouth built out and narrowed.

On the right is seen a mud range constructed in the same manner as the oven first described, except that suitable holes are left in the top for kettles. A mud chimney should be added if time permits.

In all these types of draw-fire ovens the fire is built in the oven 2 or 3 hours before baking; the coals are then withdrawn, the mouth closed (if possible) to equalize the heat, and the baking begun when the temperature is sufficiently reduced, that is to say, when the bare arm can be retained in the oven 12 to 15 seconds. Such ovens are much more efficient if built upon a stone or brick floor.

Dutch ovens are also convenient for baking bread, when at hand.

\section{FIELD COOKING OF FOREIGN ARMIES.}

For cooking in the field, European troops were, until sately, left entirely dependent upon what they carried on their persons. But after the Russo-Japanese War, having recognized the advantages of 


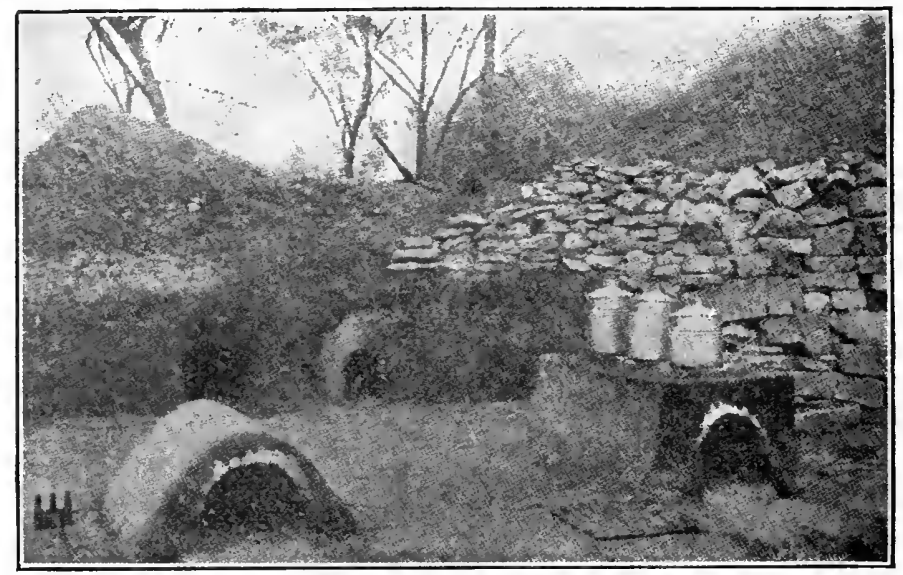

Fig. 8o.- Improvised field ovens.

the Russian ambulant kitchen, Germany, France, England and other countries conducted experiments which have led to its general adoption, with such modifications as were deemed advisable.

The Japanese field cooking outfit consists of a stove or segmented cylinder of sheet iron, 22 inches in diameter, without top or bottom; of a thin cast iron kettle which fits into the stove; of the rice boiler or colander which rests by handle lugs on the rim of the kettle; and of various cans and small utensils. Four cooking outfits are allowed for a company of 235 men. (Fig. 8I.) They are transported on pack animals, each stove knocked down into six segments. (Fig. 82.) Such outfit is specially adapted to a ration consisting chiefly of rice. The individual mess-can of the Japanese soldier contains several compartments for pickles, vegetables and sauces, and is also used to cook rice $(K u h n)$.

The Russian field cooking system, now in actual use by all the belligerent armies in the present European War, is doubtless the best as yet devised, having successfully stood the test of hard campaigns. It is practically an ambulant kitchen mounted on wheels. Two sizes are used, the larger for an infantry company of 240 men, on four wheels drawn by two horses (Fig. 83), and the smaller for a cavalry troop of 120 men, on two wheels drawn by one horse (Fig. 84). The cooking proceeds while on the march, so that, on retting into camp, the men do not have to wait for their meal. 


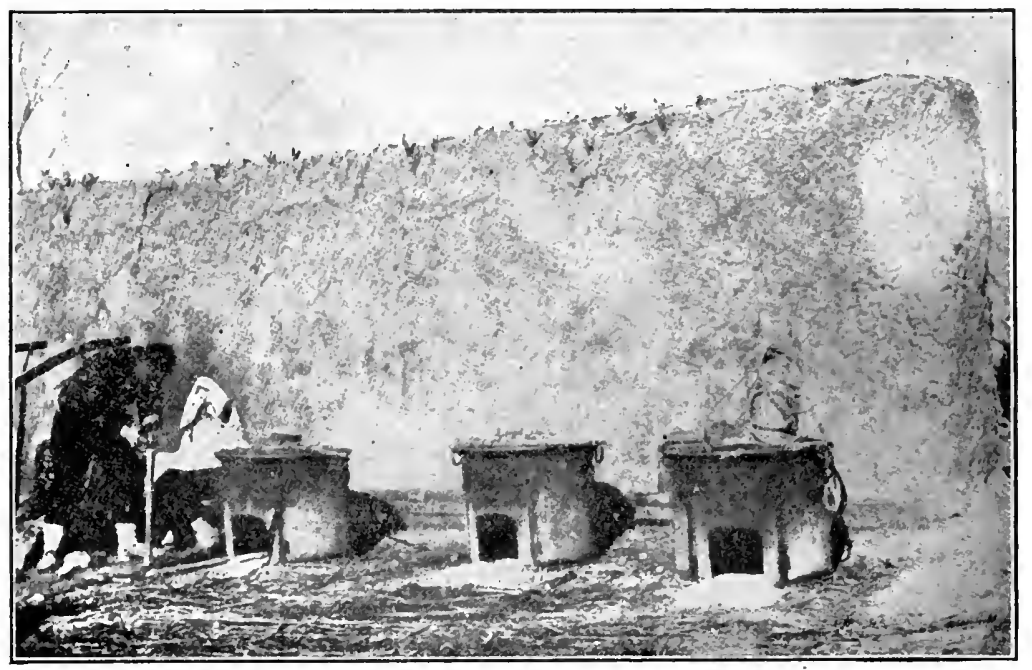

FIG. 81.- Japanese field company kitchen. (Kuhn.)

Two types were used by the Russian troops in Manchuria, one devised by Colonel Braün and the other by Colonel Debronrawoff. The Braün type, which was by far the most common, consists essentially of a boiler, with fire-box and chimney. The boiler is double, the inner wall of copper, tinned inside, the outer of iron lined with asbestos. The lid can be screwed down air-tight so that the contents are cooked under considerable pressure, the danger of explosion being prevented by a safety valve. A perforated aluminum bottom, for cooking grits or cereals, can be placed in the boiler. This type therefore admits only of boiling and steaming, which is not much of an objection in the case of the Russian soldier accustomed to soup and boiled meat. For officers, a special form is constructed which admits of boiling, steaming and roasting. (Fig. 85.) The Debronrawoff type is somewhat more complex, but equally strong and practical, admitting of boiling, roasting and stewing.

The Russian system is ideal in principle and, with a few modifications, could be readily adapted to our service; not only would it supply varied and well-cooked food with least trouble and when most needed, but also sterilized water. The chief objection against it is the additional transportation involved, namely, one horse or 


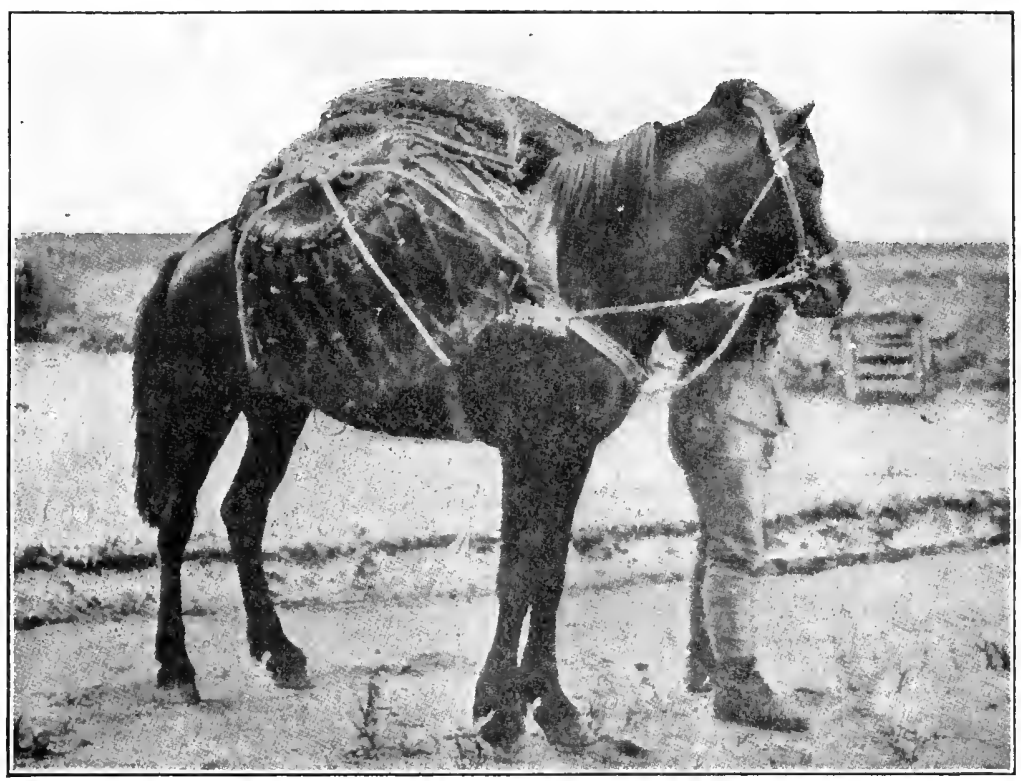

FIG. 82.- Japanese field stoves and kettles packed on regulation saddle. (Lynch.)

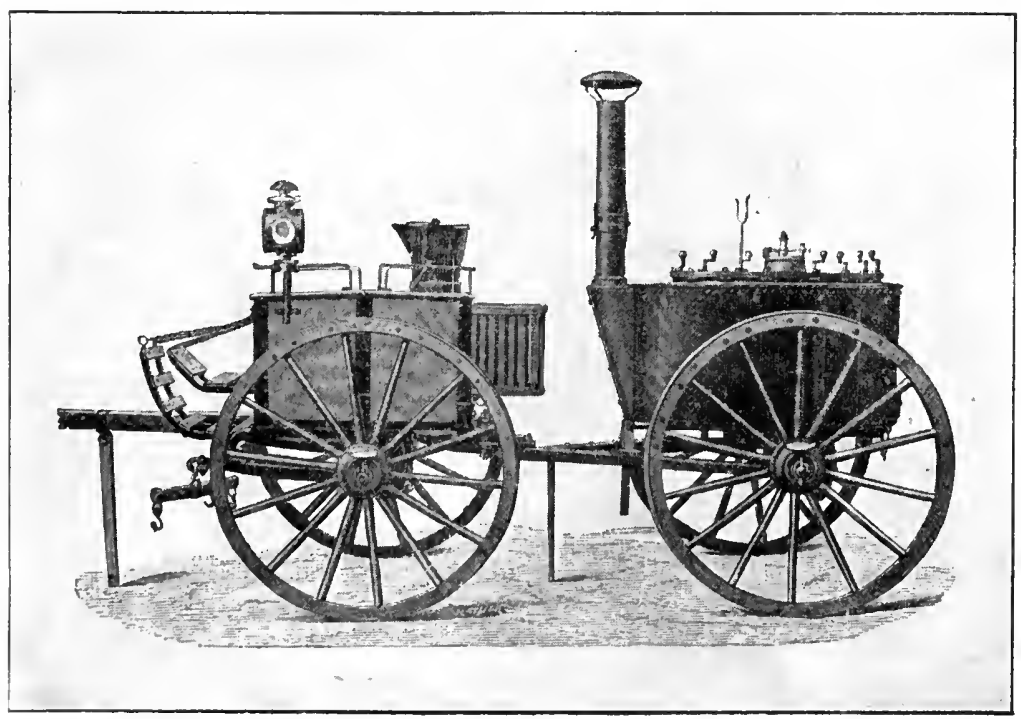

FIG. 83.- Russian wheeled kitchen for a company of infantry in the field. 


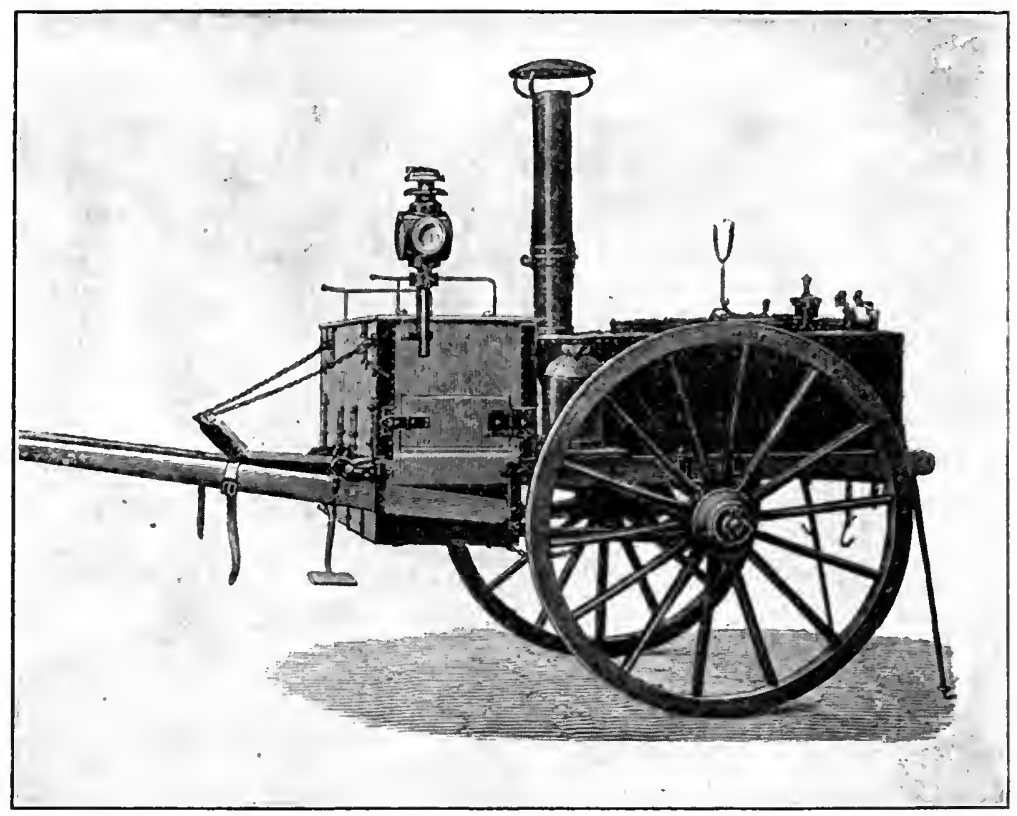

Fig. 84.-Russian wheeled kitchen for a company of cavalry in the field.

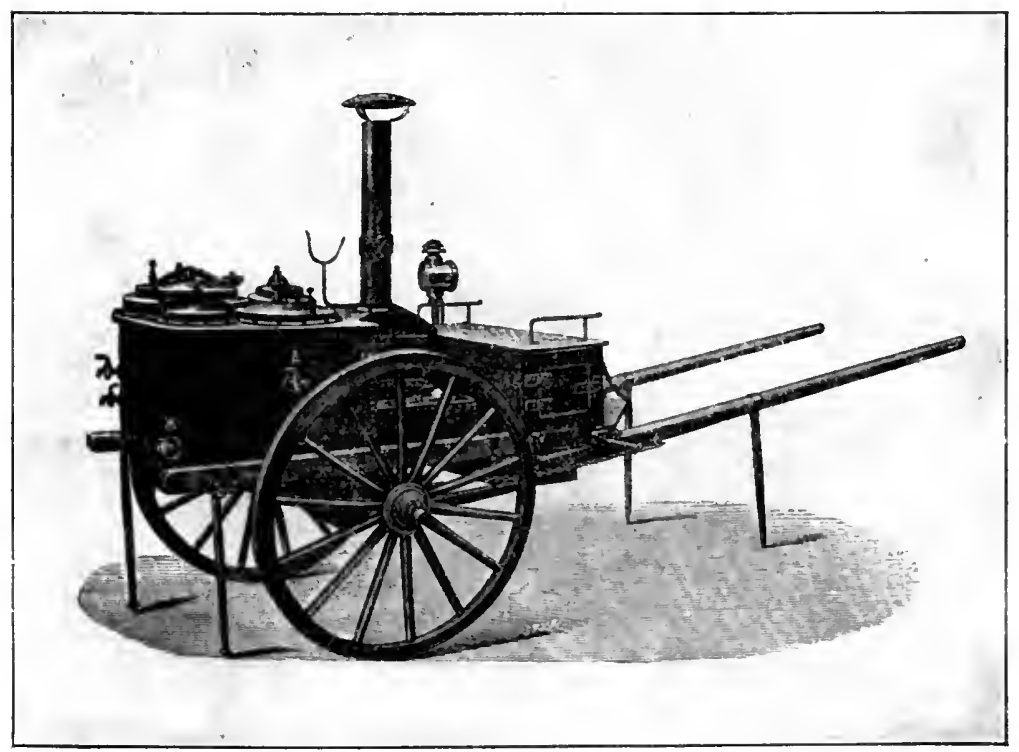

FIG. 85.-Russian wheeled kitchen for officers in the field. 
mule per company. It must be remembered, however, that the one wagon allowed each company to carry all its baggage and supplies will often be overloaded or insufficient, and that the ambulant kitchen would be the easiest and most effective method of relief.

In the Norwegian service, a cooking outfit mounted on wheels has also been tried, but instead of kettle and fire-box, a fireless cooker is used, a system, for reasons already stated, deemed unsatisfactory. 


\section{CHAPTER XXX.}

\section{THE RATION.}

A ration is the allowance for the subsistence of one person for one day, and varies in components according to the station of the troops or the nature of the duty performed, being severally known as the garrison ration, the travel ration, the reserve ration, the field ration, the Filipino ration and the emergency ration.

\section{Garrison Ration.}

The garrison ration is intended for troops not only in garrison, but also in camps and during maneuvers, and wherever practicable in peace and war. It is as follows:

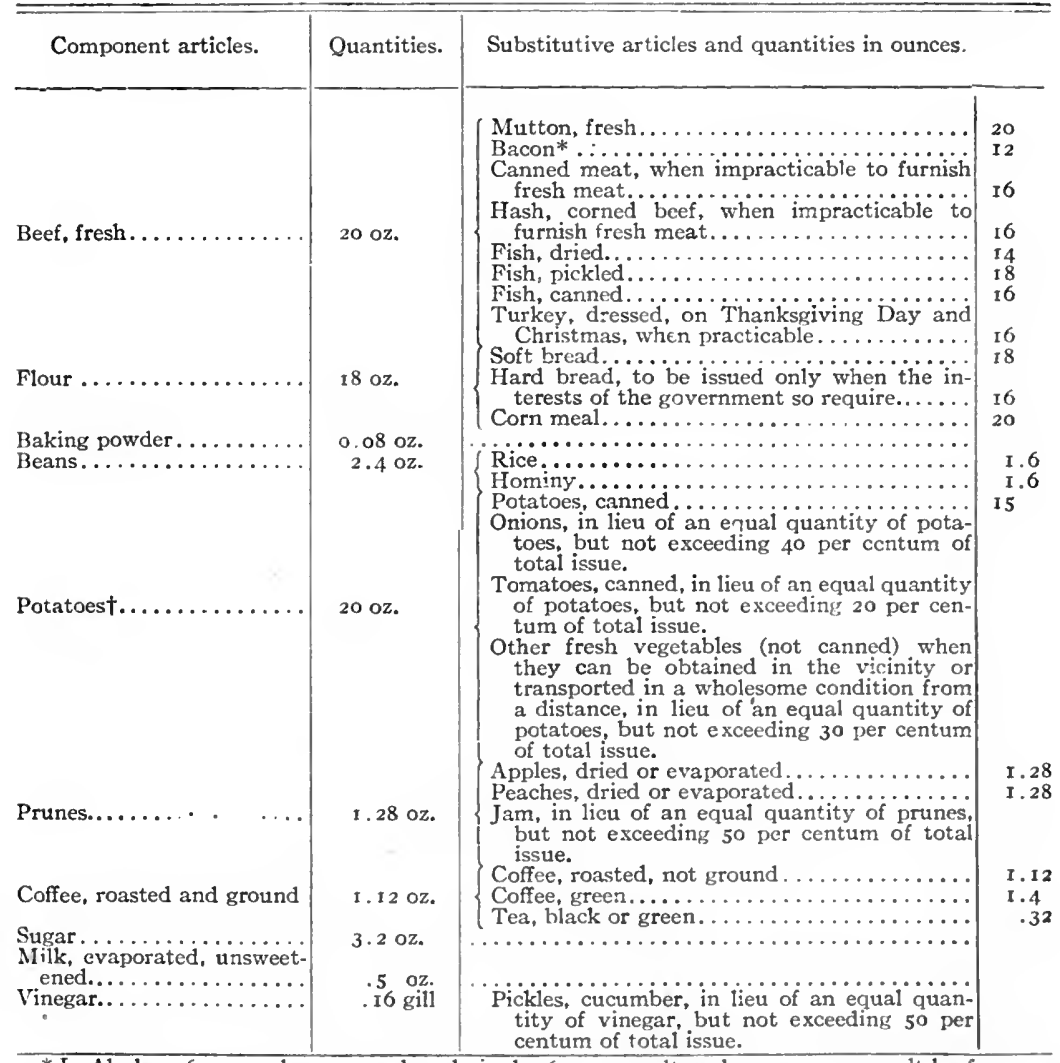

* In Alaska, 16 ounces bacon or, when desired, 16 ounces salt pork, or 22 ounces salt beef.

† In Alaska the allowance of fresh vegetables will be 24 ounces instead of 20 ounces. or canned potatoes, I 8 ounces instead of 15 ounces. 


\begin{tabular}{|c|c|c|c|}
\hline Component articles. & Quantities. & Substitutive articles and quantities in ounces & \\
\hline $\begin{array}{l}\text { Salt. ... } \\
\text { Pepper, black.............. }\end{array}$ & $.64 \mathrm{oz}$. & 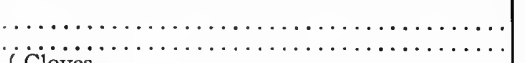 & \\
\hline Cinnamon... & $.014 \mathrm{oz}$ & 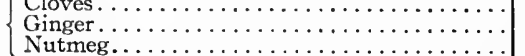 & $\begin{array}{l}.014 \\
.014 \\
.014\end{array}$ \\
\hline 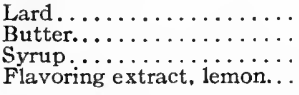 & $\begin{array}{l}.64 \mathrm{oz} . \\
.5 \mathrm{oz} \\
.32 \mathrm{gill} \\
.014 \mathrm{oz}\end{array}$ & 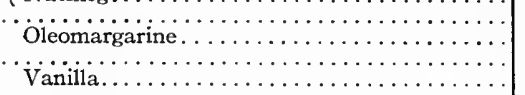 & .014 \\
\hline
\end{tabular}

Nore.-Food for troops traveling on U. S. Army transports will be prepared from the articles of subsistence stores which compose the ration for troops in garrison, varied by the substitution of other articles of authorized subsistence stores, the total cost of the food consumed not to exceed 24 cents per man per day, except on Thanksgiving Day and Christmas, when not excceding 39 cents.

The fuel value, in calories, of the principal components is as follows:

\section{Component articles}

Fresh beef

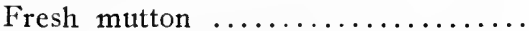

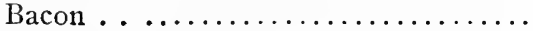

Dried fish $\ldots \ldots \ldots \ldots \ldots \ldots \ldots \ldots$

Pickled fish $\ldots \ldots \ldots \ldots \ldots \ldots \ldots \ldots$

Canned fish $\ldots \ldots \ldots \ldots \ldots \ldots \ldots \ldots$

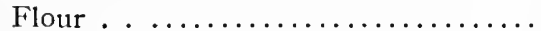

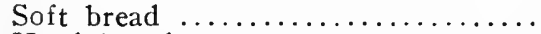

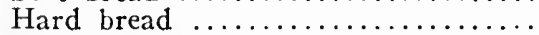

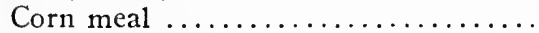

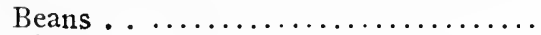

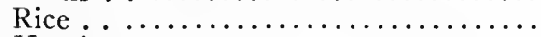

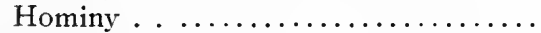

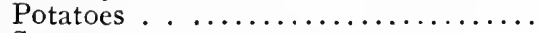

Sugar . . . . . . . . . . . . . . .

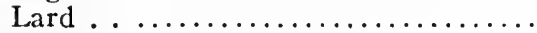

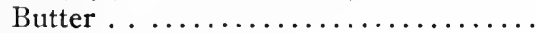

Sirup . . . . . . . . . . . . .

\begin{tabular}{|c|c}
$\begin{array}{c}\text { Quantity } \\
\text { in ounces }\end{array}$ & $\begin{array}{c}\text { Fuel value } \\
\text { in calories }\end{array}$ \\
\hline & \\
20 & 1287 \\
20 & 1440 \\
12 & 2040 \\
14 & 276 \\
18 & 1029 \\
16 & 680 \\
18 & 1828 \\
18 & 1355 \\
16 & 1712 \\
20 & 1986 \\
2.4 & 228 \\
1.6 & 160 \\
1.6 & 172 \\
20 & 368 \\
3.2 & 350 \\
0.64 & 160 \\
0.5 & 106 \\
1.3 & 192 \\
& \\
\hline
\end{tabular}

The garrison ration, as may be seen, admits of many combinations which insure variety. It is comprehensive and elastic and can be adjusted to any climate. By selecting the most nutritive articles, such as bacon, hard bread or cornmeal, beans, potatoes, dried fruit, butter and sirup, we can obtain from it a maximum fuel value of 5,378 calories, according to Langworthy, or 5,674 calories, according to Wiley. On the other hand, by using such articles as dried fish, soft bread, rice, potatoes, canned tomatoes and dried fruit, the fuel value can be reduced to 2,500 calories. The average garrison ration, habitually consisting of fresh beef, soft bread, beans, potatoes and 
onions, dried fruit, butter, sirup and sugar (or their nutritive equivalents), weighs 65 ounces and contains 99 grams of fat, $48 \mathrm{I}$ of carbohydrates and 157 of proteins, with total fuel value of 3,536 calories.

Fresh meats are ordinarily issued seven days in ten, and bacon three days.

The garrison ration is often supplemented by articles obtained from the post garden or purchased from the company fund, and which largely contribute to give it variety and appetizing value.

In order to facilitate the supplying of troops and the keeping of accounts, the former system of issue has been replaced by that of purchase.

"All articles of the garrison, travel or Filipino ration due a company, or other military organization, will be retained by the Q. M. Corps and credit given to the organization for the money value of these articles at the current price of the articles."

The stores required by the organization will be purchased from the Q. M. Corps, and the latter will pay as savings to the organization commander any excess in value of the stores retained over those purchased. At the end of the month, or whenever necessary, the organization commander will settle the account with the $Q$. M. Corps, when the savings to the organization, or the amount due to the Q. M. Corps, as the case may be, will be paid and the account certified as required.

The price of bread, as charged against organizations, is determined by adding together the cost of flour and other ingredients used, the extra-duty pay of the bakery personnel and the cost of the power used in operating the baking machinery, and then dividing by the total number of pounds of bread baked. It follows that the organizations are thus given the benefit of whatever savings accrue from the conversion of flour into bread after deduction of all expenses.

"Money accruing from the 'ration and savings account' of an organization will be spent only for food."

All articles of the ration required for the supply of troops will be obtained from the Q. M. Corps when on hand, but should any organization want more of any article than is allowed by regulation, the excess may be purchased elsewhere; or if any article is not in stock it can likewise be bought elsewhere.

When necessary to renew reserve rations, or to avoid loss of ration articles that have accumulated, the commanding general or 
commanding officer, as the case may be, may order the issue of such supplies to troops, not to exceed the ration allowance and only for such time as the interest of the Government requires.

The value of the garrison ration is estimated at 30 cents; the Filipino ration at 20 cents, and the travel ration at 40 cents.

Under circumstances when enlisted men or nurses cannot be furnished with rations in kind, or it is impracticable to carry them, commutation may be allowed at rates ranging from 25 cents to $\$$ I.5O a day.

The ration of enlisted men sick in hospital, and of female nurses while on duty in hospital, is commuted at the rate of 30 cents per ration, except that at the general hospital at Fort Bayard, N. M., 50 cents per ration, and at other general hospitals, 40 cents per ration, is authorized for enlisted patients therein.

Other issues of stores, not components of rations, may be authorized when necessary for the public service, and made on ration returns, approved by the commanding officer, such as soap, candles, matches, toilet paper, towels and ice.

Ice. - Ice is issued by the Q. M. Corps to organizations of enlisted men as follows: For each ration, 4 pounds, the maximum allowance to any organization or detachment of less than Ioo men to be 100 pounds a day, and to organizations of Ioo men or more to be I pound a day, per man. The full allowance may be issued for the entire year to troops stationed south of the 37 th parallel. To troops stationed north of the 37 th parallel, and where from any cause it is impracticable to cut and store ice for their use, the allowance will be only for the summer months, from April I to October 3I. A special allowance is provided for States on the Pacific Coast.

\section{Travel (or Cooked) Ration.}

Issued to troops traveling otherwise than by marching, and separated from cooking facilities.

Component articles and quantities

in ounces

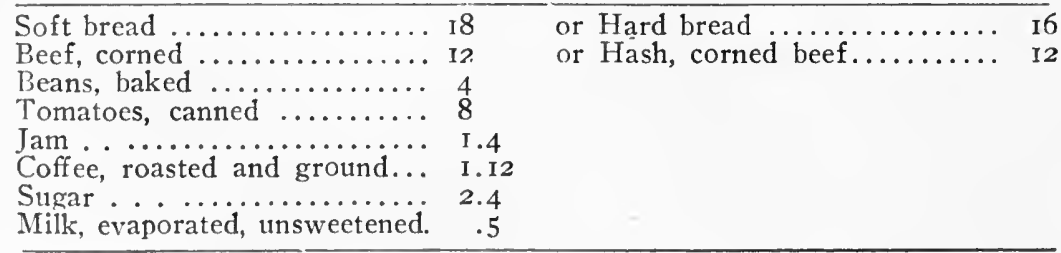


Its fuel value is about 2,735 calories.

Enlisted men supplied with travel rations may, in lieu of the coffee, milk and sugar components thereof, receive funds for the purchase of liquid coffee, at the rate of 21 cents per day for each man.

\section{Reserve (or Haversack) Ration.}

Issued to troops in the field when beyond the advance supply depots. It is carried on the person of the men and in the trains, and constitutes the reserve for field service.

Component articles and quantities in ounces

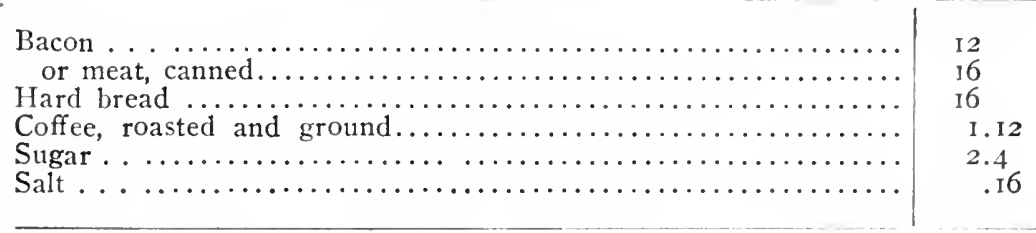

These articles contain about 218 grams of fats, 489 of carbohydrates and II 3 of proteins, with total fuel value of 4,448 calories.

Should it be found practicable to supplement it by local purchase or otherwise, the commanding general may direct the issue in kind of such additional articles of food as are available, at whatever cost, but not in excess of the amounts allowed of corresponding articles in the garrison ration.

The bacon is contained in a rectangular tin can with capacity for two rations. The sugar, coffee and salt are contained in another rectangular tin can, $2 \frac{1}{2}$ inches square, 5 inches long, and with rounded corners; a cross partition divides it in 2 compartments, for 3 days' rations of coffee and sugar; the ends are closed with screw covers. The screw cover on the sugar compartment has a round receptacle 2 inches in diameter, $\mathrm{T} / 2$ inch deep, closed with a compression friction top, for carrying 3 days' rations of salt. (See Haversack, p. 465.)

In the field, bacon, in the absence of fresh meat, becomes an invaluable component of the ration, easily kept and transported, readily digested when well cooked, and furnishing abundant energy for severe muscular work. 
In the field, a quarter ounce of soap per ration is also issued (in ounce cakes.)

Existing orders prescribe that one day in each alternate month of the season of practical instruction, not exceeding three days in each year, the use of the reserve ration, with individual mess-kit, will be required of all troops in the field for purposes of instruction.

\section{Field Ration.}

The field ration is the ration prescribed in orders by the commander of the field forces. It consists of the reserve ration in whole or in part, supplemented by articles of food requisitioned or purchased locally, or shipped from the rear, provided such supplements or substitutes correspond generally with the component articles or substitutive equivalents of the garrison ration.

\section{Filipino Ration.}

Issued to the Philippine scouts (Philippine Islands).

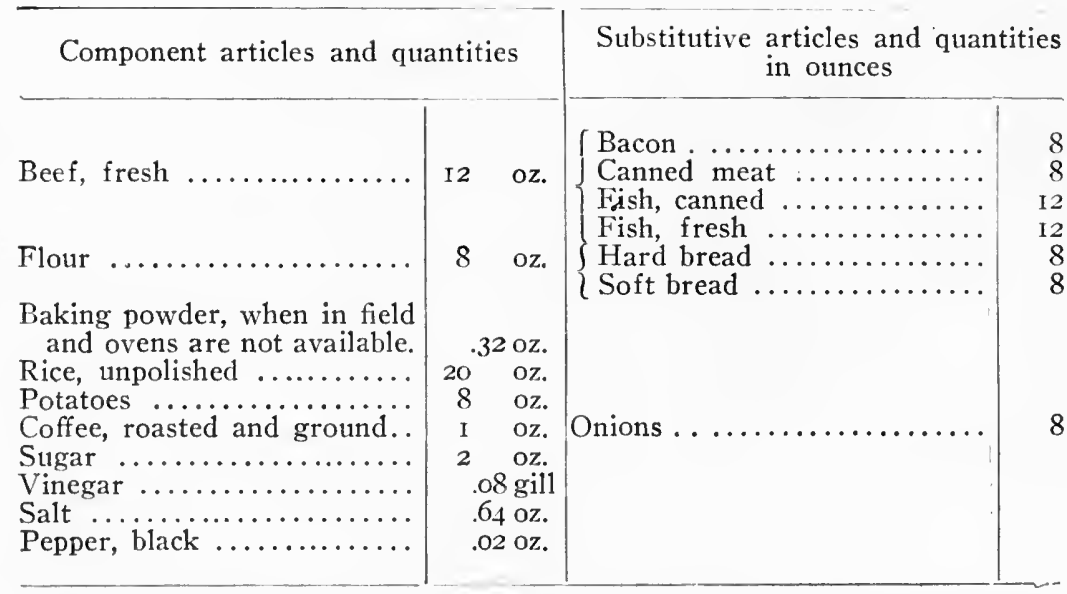

The components of the ration yield a maximum fuel value of 3,980 calories.

As is well known, Filipinos, like other Oriental races, manifest a marked preference for rice, to the exclusion of more nutritious food, thereby rendering themselves liable to beriberi (which see). To guard the scouts against such possibility, it is prescribed that only unpolished rice will be issued to them and that no more than 
I6 ounces per day will be used. They are also required to use the entire meat allowance. For the portion of the rice ration not drawn, I.6 ounces of beans are substituted, while native products, such as camotes, mongos and squash, are utilized to as large an extent as possible.

\section{Emergency Ration.}

"The emergency ration is furnished, in addition to the regular ration, as required for troops on active campaign or in the field for purposes of instruction, and will not be opened except by order of an officer, or in extremity, nor used when regular rations are obtainable."

The emergency ration used in our service, prior to I9Io, weighed I 2 ounces net and consisted of wheat, meat, chocolate and seasoning. Its preparation was so elaborate as to require special plants so that, in case of mobilization on a large scale, the supply would have been inadequate. The components of the present ration are such as to be readily obtained and prepared to any extent needed. They are as follows: .

Chocolate liquor,

Nucleo-casein,

Malted milk,

Egg albumen,

Powdered cane sugar,

Cocoa butter,
45.45 per cent.

\begin{tabular}{|c|c|}
\hline 7.25 & " " " \\
\hline 7.25 & 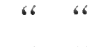 \\
\hline & $\begin{array}{ll} & " \\
، & 6\end{array}$ \\
\hline 3 & " $\quad$ " \\
\hline
\end{tabular}

25.24 per cent. .88 28.05 " "

39.1 I “" "

.17

3.27

Each ration weighs 8 ounces net and is put up in 3 cakes of equal size, each cake wrapped in tinfoil and all three inclosed in a hermetically sealed and lacquered round-cornered tin, with key-opening attachment. Its fuel value is $I, 272$ calories, and its cost 40 cents.

From previous experiments it is believed that this ration can be kept in store, even in the tropics, for several years without loss or deterioration. As a compact and portable food preparation, intended to tide over a day or two until regular supplies are available, 
it is undoubtedly well adapted to its purpose. However, there is serious doubt of its necessity, and the Infantry Equipment Board of I9I 2 recommended that it be abolished and replaced by the reserve ration.

The "Trail Ration," for tuse in Alaska only, is the garrison ration with an increase in the allowance of beans, potatoes, coffee, sugar, sirup and butter.

\section{THE RATION OF THE UNITED STATES NAVY.}

This ration consists of the components embraced under I, 2 and 3 , and of a weekly allowance of other articles as enumerated below.

Component articles and quantities

Salt or smoked meat..... $20 \mathrm{oz}$

I)

Fruit, dried .......... 6 oz. or canned or preserved. $6 \mathrm{oz}$.

Flour .............. I2 oz. or beans or peas...... 3 gills

Preserved meat ........ 16 oz.

Fruit, dried .......... 3 oz. or canned or preserved. $6 \mathrm{oz}$.

Rice............. $8 \mathrm{oz}$. or canned vegetables... I2 oz. or desiccated vegetables. $6 \mathrm{oz}$.

Biscuit . ............. I6 oz.

Butter.............. 2 oz.

3 Sugar $. . \ldots \ldots \ldots \ldots \ldots, 4$ oz.

Coffee or cocoa........ 2 oz.

or tea $\ldots \ldots \ldots \ldots \ldots \ldots$ a $\frac{1}{2} \mathrm{oz}$.

with condensed milk or evaporated cream .... I oz. WEEKLY :

Macaroni ............. 4 oz. Cheese................ 4 oz.

Tomatoes ............. 4 oz.

Vinegar or sauce.......... $\frac{1}{2}$ pint Pickles ................ $\frac{1}{4}$ pint Molasses . ............. $\frac{1}{4}$ pint Salt ................. 4 oz. Pepper ................ $\frac{1}{2}$ oz. Spices ............... $\frac{1}{8}$ oz. Dry mustard ............ $\frac{1}{2} \mathrm{oz}$. Lard or suitable substitute... 7 lbs. Yeast and flavoring extracts as necessary.
Substitutes when deemed necessary by the senior officer present

Fresh meat or fresh fish.. $28 \mathrm{oz}$. or 8 eggs.

\}

Fresh vegetables ...... $28 \mathrm{oz}$.

Fresh meat or fresh fish. . 28 oz. or 8 eggs.

\}

Fresh vegetables ...... 28 oz.

Soft bread ............. zo oz. or flour ........... I8 oz.

for every $100 \mathrm{lbs}$. of flour issued as bread. 
The following substitutes can likewise be issued when authorized by the senior officer present.

$\overline{\text { For }}$

Condensed milk or evaporated cream ............... I $1 \mathrm{~b}$. Dried fruit ............. $3 \mathrm{oz}$. Canned or preserved fruit... $6 \mathrm{oz}$. Flour ................ I2 oz. Rice or other starched food.. $8 \mathrm{oz}$. Canned vegetables ......... I2 oz. Macaroni ............. $4 \mathrm{oz}$. Cheese............... 4 oz. Vinegar or sauce.......... $\frac{1}{2}$ pint Pickles . .............. Molasses . ............. ${ }^{\frac{1}{4}}$ pint Spices . .............. $\frac{1}{8} \mathrm{Oz}$.

\section{Substitute}

Flour .............. $12 \mathrm{oz}$. or rice............ $8 \mathrm{oz}$. or other starched food.... $8 \mathrm{oz}$. or canned vegetables....... I2 oz.

Fresh milk ............ I quart Fresh fruit ............ 9 oz. Fresh fruit ............ $9 \mathrm{oz}$. beans or peas............ 3 gills Beans or peas............ 3 gills

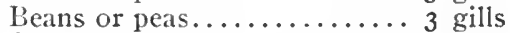
Sugar . ............... 3 lbs. or condensed milk...... I $\frac{1}{2}$ lbs. or coffee ............. I lb. or canned fruit......... I $\frac{1}{2} \mathrm{lbs}$. or canned vegetables..... 4 lbs. or flour ............ 4 lbs.

An extra allowance of one ounce of coffee or cocoa, two ounces of sugar, four ounces of hard bread or its equivalent, and four ounces of preserved meat or its equivalent are allowed to enlisted men of the engineer and dynamo force who stand night watches between 8 o'clock postmeridian and 8 o'clock antemeridian, under steam.

This ration provides a great variety of foodstuffs and condiments and permits almost any substitutes so as to give it perfect adaptability to all climatic conditions. It is only open to the criticism that, in quantity, it is manifestly in excess of requirements, and that its issue may be attended with serious risks of overfeeding and waste.

\section{THE RATION IN FOREIGN ARMIES.}

France. - In France, four types of rations are provided, two for peace (garrison ration and maneuver ration) and two for war (ordinary and large field rations).

Their components are as follows: 


\begin{tabular}{|c|c|c|c|}
\hline Article & Garrison ration & $\begin{array}{l}\text { Ordinary } \\
\text { field ration }\end{array}$ & $\begin{array}{c}\text { Large } \\
\text { field ration }\end{array}$ \\
\hline 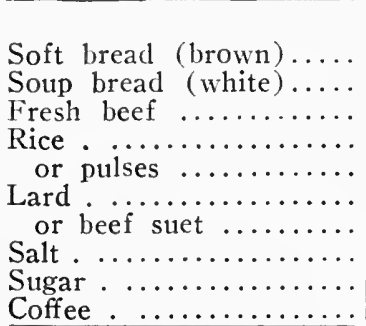 & $\begin{array}{c}\text { Kg. Grams } \\
0.750 \\
0.250 \\
0.320 \\
0.030 \\
0.060 \\
0.030 \\
0.040 \\
0.016 \\
0.021 \\
0.016\end{array}$ & $\begin{array}{c}\text { Kg. Grams } \\
0.750 \\
\ldots . . \\
0.400 \\
0.060 \\
0.060 \\
0.030 \\
0.040 \\
0.020 \\
0.021 \\
0.016\end{array}$ & $\begin{array}{c}\mathrm{Kg} . \text { Grams } \\
0.750 \\
\ldots . \\
0.500 \\
0.100 \\
0.100 \\
0.030 \\
0.040 \\
0.020 \\
0.031 \\
0.024\end{array}$ \\
\hline
\end{tabular}

The soft bread ("pain de munition") is a leaven-made bread from flour bolted to 20 per cent.; it may be replaced by pain biscuité (page $35^{6}$ ) or by hard bread and partly by Italian pastes and flours of cereals; the beef, by any kind of fresh or preserved meat, fish, cheese or milk; the rice and pulses, by any available dry or green vegetables.

In bivouac, or whenever ordered by the commanding general in the field, a liquid ration is issued of either wine ( $1 / 2$ pint), beer ( 1 pint) or brandy $(2 \mathrm{oz})$.

The garrison ration contains (according to Rouget and Dopter):

Proteins

Fats,

Carbohydrates, I25.06 grams, yielding $5 \mathrm{I} 3$ calories. 60.46 grams, yielding 562 calories. 573.52 grams, yielding 2,35 I calories.

\section{Total,}

3,426 calories.

The maneuver ration contains less protein and fat, having a fuel value of $3, \mathrm{I} 64$ calories.

The ordinary field ration contains:

Proteins,

Fats,

Carbohydrates,
1 23.60 grams, yielding 507 calories. 64.74 grams, yielding 602 calories. 476.99 grams, yielding 1,956 calories.
Total,
3,065 calories.

Soup bread may be added if available (which is seldom the case), increasing the total calories to 3,687 .

The fuel value of the large field ration (without soup bread) is 3,383 calories. 
The French soldier takes two meals a day, breakfast at Io A. M. and dinner at 5 P. M., besides black coffee at reveille.

In time of peace, the commissary department supplies only hard bread, sugar and coffee, as well as wine and brandy when authorized. The rest of the ration is purchased by the company messes (" ordinaires") from special money allowances (about io cents per man), under the supervision of a council appointed by the commanding officer ("commission des ordinaires"). Money allowances remaining unexpended, that is, resulting from savings on the ration, cs well as proceeds from fines, sale of wastes, etc., are used in purchasing such additional articles of food as deemed desirable.

In war, the men carry two days' rations in the haversack; two more are carried in the regimental trains and four on the administrative trains.

Germany. - In Germany, four types of rations are also provided, two for peace time and two for the field. The large peace ration, such as issued during maneuvers, consists of $75^{\circ}$ grams of bread, or 500 of field biscuit, or 400 of egg biscuit; $25^{\circ}$ grams of 'fresh meat, with 60 of kidney fat, or 200 of smoked bacon, or 200 of canned meat; and vegetables. According to Bischoff its composition is: protein, 89.9 grams; fats, 80.7 ; carbohydrates, 514.8 ; with fuel value of 3,23 o calories.

The other peace ration is somewhat smaller and, in certain garrisons, has to be supplemented from the private means of the soldier. The small field ration averages $14 \mathrm{I}$ grams of protein, $5^{\mathrm{I}}$ of fat and 458 of carbohydrates, with fuel value of 2,929 calories; the large field ration, I8I grams of protein, 64 of fat and $55^{8}$ of carbohydrates, with fuel value of 3,625 calories.

The commissary furnishes bread and meat, most of the other articles being purchased out of a daily allowance of 4 cents per man.

The German soldier takes a cup of black coffee, or coffee with milk, and bread in the morning, dinner at noon and a light supper in the evening. During maneuvers, or in the field, he may also receive $\mathrm{I}$ quart of beer, $\mathrm{I}$ pint of wine or about 3 ounces of spirits.

Great Britain. - The ration of the British soldier, in garrison, consists of 16 ounces of bread, 12 ounces of meat, and such additional articles (vegetables, groceries) as are purchased out of a daily allowance of 7 cents. During maneuvers the meat is increased to I6 ounces.

In the field, his ration varies according to climate and the char- 
acter of his work; it generally consists of 16 ounces of salted or preserved meat, or 16 to 20 ounces of fresh meat; 20 ounces of fresh bread or 16 ounces of hard bread or flour; I ounce of compressed vegetables, or 8 ounces of potatoes (or other fresh vegetables), or 2 ounces of rice (or split peas), or 4 ounces of onions; coffee, tea or chocolate, sugar and the usual condiments. Two ounces of spirits and some tobacco may also be allowed.

An emergency ration is provided in the British Army, for use only when no other food is procurable and when authorized by the commanding officer. It is contained in a flat can and consists of chocolate, sugar and protein in the form of "plasmon," weighing $6 \mathrm{r} / 2$ ounces net. According to Langworthy, its proximate principles average: protein 59 grams, fat 50 and carbohydrates 65 , with fuel value of 973 calories. This ration, like our own emergency ration, is relatively too rich in protein and deficient in carbohydrates.

Russia. - The Russian soldier, in the field, receives $2^{1 / 4}$ pounds of black bread (from unbolted rye flour), $14 \frac{1}{2} 2$ ounces of fresh meat or I I of preserved or salt meat, 9 ounces of green vegetables or the equivalent in compressed vegetables, I to 2 ounces of suet or butter for cooking, together with 4 to 5 ounces of grits, tea, sugar and condiments.

According to Bischoff its composition is: protein, I20.7 grams; fat, 39.2 ; carbohydrates, 628 ; with fuel value of 3 ,I 47 calories.

Most of the articles of the ration (bread excepted) are boiled together in a large wheeled kettle and served as soup (see page 379).

Japan. - The Japanese ration, in the field, consists of:

Rice, uncooked, 30 ounces.

or steamed and dried, 25 ounces.

or fresh bread, 20 ounces

or hard bread, I3 ounces.

Meat, canned, Io ounces.

or fresh (with bones) I3 ounces (which may be increased to 20 if procurable).

or salt, dry or smoked meat, 8 ounces.

or fish.

Vegetables, dry, 8 ounces.

or green, 32 ounces.

Pickles, sauces and condiments.

Tea and sugar.

Sake (beer made from rice). 
The Japanese soldier does not take readily to bread, either fresh or hard. During the Russo-Japanese War, the meat component was scant and issued irregularly; rice, fish and vegetables formed the staple ration.

The Japanese medical regulations provide that the nutritive value of the ration should never fall below 2,580 calories.

Emergency Ration. - All foreign armies have a so-called emergency ration, but, with the exception of England, it is nothing else but a field ration in a more condensed and portable form, consisting of hard bread, and preserved meat and vegetables in cans. 


\section{CHAPTER XXXI.}

\section{CONCLUSIONS REGARDING THE COMPOSITION AND FOOD VALUE OF THE MILITARY RATION.}

In a previous chapter it was stated, on good grounds, that an adult man weighing I54 pounds is in better physical condition, stronger and capable of greater endurance, with a ration yielding 2,800 calories, especially if the proteins are kept within a maximum of 60 grams, than with one greatly exceeding this value. But we have seen that in our service, as in all other leading countries, the soldier's ration exceeds these limits in the number of calories which range from 3,000 to 5,000 , but especially in the amount of proteins. (Japanese excepted) which hardly ever falls below Ioo and often ranges up to 160 or more grams. There is no indication therefore that, in this country or in Europe, the soldier is underfed, provided he actually receives the ration called for by military regulations.

Much has been written on the necessity of feeding the soldier well, and much attention and study have been devoted to the composition of adequate rations. That he should be sufficiently and properly fed needs no discussion, but the belief generally entertained that the more he eats the greater is his energy and efficiency is groundless and mischievous. The danger of overeating has been too much overlooked, or else esteemed a negligible factor. It is the opinion of many careful observers that the American soldier is much more likely to be overfed than underfed, and that his health and efficiency stand in greater peril from excess than from lack of food. As a general rule, the soldier does not know how to regulate his appetite, nor does he appreciate the necessity of doing so. $\mathrm{He}$ eats what is allowed him hurriedly and often without proper mastication, and, between meals, frequently patronizes the lunch counter of the post exchange or the outside shop where pies and other tempting pastry are displayed. This is characteristic of the soldiers of all countries, particularly of those who receive the most liberal rations. Thus the remarks of Rouget and Dopter, in their "Hygiène militaire," although aimed at their countrymen, are of very general 
application. "Many Frenchmen, especially in the country, have the pernicious habit not to cease eating until they experience a sensation of fullness in the stomach. Little do they care about the nutritive value of the food ingested. Their conviction is that, so long as this abdominal repletion is not distinctly felt, they have not been sufficiently fed. This is particularly observed each year at the time of the incorporation of the new contingent. The amount of bread in the ration, although considerable, is not enough to satiate these young soldiers; they buy more from outside bakeries."

It should be remembered that within an hour or two after taking a full meal, at least a pint of gastric juice is poured into the stomach and added to its contents, so that what was at first mere repletion may become uncomfortable distention. Soldiers should be advised that a sensation of fullness or distention following a meal is a clear admonition that they have eaten too much, and that if such excess is kept up, as a habit, they will surely suffer in health and efficiency. Such advice may do good, but more practical results will follow if the food, especialy the meat, as served in the mess-room, is simply sufficient and not wastefully abundant.

Experience shows that it is while actively engaged in the field, when the rations are reduced and the cooking simple, that the men enjoy the best health and show most endurance, provided, of course, there is no actual lack of food. Thus, during the active part of the Santiago campaign, in 1898 , there was but little sickness in spite of the trying climate and of short commissary supplies, but as soon as the work was over and rations became abundant, the morbidity began to rise and before long exceeded 75 per cent. of the command. It is hard to resist the conviction that injudicious feeding was responsible for many of the cases of " undetermined fever," and prepared the way for much of the malaria which prevailed in our camps near Santiago, as well as of the typhoid fever which decimated our troops in the United States. It is noteworthy that the Spanish soldiers in Santiago, although reduced to very scant rations of poor quality, had a much smaller proportion of sick than the American troops. During the Boer War, in South Africa, the English troops besieged in Ladysmith. were fed for several months, according to Dunlop, on a ration of 73 grams of protein, 69 of fat and I4I of carbohydrates, with fuel value of only I,527 calories, but which, as re- 
marked by Munson, furnished energy enough for a stout and successful resistance. The Russo-Japanese War has taught us an important lesson in dietetics. Both Russians and Japanese had meat rations much smaller than those provided for American, English and French soldiers; the meat ration of the Japanese, in particular, was decidedly meagre and often lacking altogether. Yet we know that no armies, during an arduous war of twenty months, ever suffered so little from infectious diseases and had fewer men incapacitated from sickness. To what extent this immunity is due to the food can only be conjectured. It certainly cannot be attributed exclusively to the sanitary measures taken, for it was the opinion of the American and English attachés with both armies, that had their own troops been placed under similar conditions, but fed with their own rations, typhoid fever would undoubtedly have prevailed among them in an epidemic form. It is true that the Japanese suffered much from beriberi during this war, a disease known to be due to an excess of rice combined with a deficiency of protein, but there is no doubt that it would have been entirely prevented had the Japanese soldier received one-half of the protein component called for by the ration of the United States soldier.

Surgeon Tsui, of the Chinese Army (Mil Surg., Nov., 1909), states that, in Northern China, the country laborers from whom the army is mostly recruited, men of fine physique and most remarkable power of endurance, live almost exclusively on a vegetable diet. The Chinese soldier receives meat only twice a month. The rates of sickness and death in the Chinese Army, according to this trustworthy authority are much lower than in our Army.

It is a well established fact that the troops of the belligerent armies in the European War (1917), after several years of arduous service, on rations often necessarily curtailed and indifferently prepared, are hardier, capable of greater effort and more resistant to disease than at the beginning and during the first year of the war.

Formerly, the price of savings made from the ration and paid by the $\mathrm{Q}$. M. Corps to the organization commander, could be expended by the latter in any way deemed most advantageous to the organization, that is, not only in the purchase of other kinds of food, but of any articles intended for the comfort and enjoyment of the men, such as games, sporting goods, etc. Under existing regulations, 
money received as balance of the ration and savings account" can be spent only for food. It follows that the company commander has no longer any discretion in regulating the amount of food consumed by his men, but is expected to expend the entire money value of the ration in the purchase of foodstuffs. The writer cannot commend this change. He believes that the former system whereby the company commander was allowed" discretion in regulating the quantity as well as the quality of the food was better calculated to safeguard the health and comfort of his men.

As the outcome of the latest study and experience in the physiology of nutrition among adult men, we may state that, in garrison or camp, the fuel value of the ration actually consumed need not be over 2,800 and should never exceed 3,000 calories, and that there must be very few circumstances in the field, except in a very cold climate, when a value of 3,500 calories is desirable or necessary. Half a pound of beef, when freed from bones and other waste, is reduced to about six ounces which, with the proteins of other foodstuffs of the ration, exceeds the 60 grams we have seen to be quite sufficient for the daily needs of the active body. It is therefore unphysiological, wasteful and against the best interest of the soldier and of the service to give him, while in garrison or camp, much more than that amount of beef or its equivalent of other meats, nor is it necessary in the field to exceed one pound. The solicitude of all officers concerned should not be, how to increase a ration already extremely liberal, but to make it comprise as many components as possible, to see that these components are most advantageously combined to secure variety, that they are cooked, seasoned and served in the most digestible and appetizing manner, and that the men be made to understand the advantages of thorough mastication. In the field, or wherever a desirable variety of food cannot be obtained from the Q. M. Corps, every effort should be made to convert part of those components allowed in excess, especially meats and fats, into lighter and healthier articles, according to what local markets afford. 


\section{CHAPTER XXXII.}

\section{THE RATION OF THE UNITED STATES SOLDIER IN THE TROPICS.}

\section{(See Service in Warm Climates.)}

The climatic conditions characteristic of the tropics are a constant high temperature and great relative humidity. The effects of these conditions upon the body, so far as food is concerned, may be stated thus :

I. Increased bodily temperature (half degree or more until acclimation is established) from diminished loss of heat by radiation, convection and evaporation.

2. Loss of flesh, especially of fat, so that the normal weight is reduced several pounds.

3. Decreased oxygenation, from the expansion and rarefaction of the air and lower atmospheric pressure.

4. Greatly increased perspiration, causing a diminution of urine and of the digestive fluids.

5. General depression of the nervous system and loss of vital energy, with weakening of the digestive and assimilative functions, as well as diminished power of mental applicaton and muscular acton.

Let us remember that food is the fuel which generates animal heat, and that it must be constantly supplied in order to maintain the normal temperature of the body and furnish the necessary mental and muscular energy. The body temperature of $98.6^{\circ}$ is a constant term, admitting of only slight variations, but the rate of heat dissipation is variable and depends chiefly upon atmospheric conditions. This dissipation takes place mostly through radiation and evaporation. The smaller it is, the less food will be required to maintain the body at its normal temperature. As the air temperature increases and approximates that of the body, the rate of outward radiation decreases; but, if the warmer air be also drier, the diminished radiation may be offset by increased evaporation, so 
that the loss of heat remains about the same. It is only in climates both warm and damp, such as are found in tropical and subtropical latitudes, that the loss of heat from the body is more or less permanently lessened, . both by radiation and evaporation. It is therefore in such climates that the amount of food should be correspondingly diminished in order to avoid the discomfort and danger of a greater evolution of heat than can be disposed of by the system.

Besides the fuel value of food, as measured by calorimetric combustion, there is another source of heat which should not be overlooked, what has been called the "specific dynamic action" of food. This is the restrlt of the increased metabolism caused by the activity of the digestive organs in the processes of mastication, digestion and absorption. It is well marked within an hour of eating, and steadily increases during the next 2 or 3 hours. It is greatest after the ingestion of proteins, less with carbohydrates and least with fats, in the proportion of 20 , Io and 7 . According to Rubner, the evolution of heat may thus be rapidly increased by rich feeding to the extent of 44 per cent.

The normal physiological amount of food required for the needs of the body is smaller in the tropics than in temperate regions. Man, there, is not capable to absorb and assimilate the same quantity, nor has he the same craving for it. The loss of heat from the body being smaller, less fuel is needed to maintain the temperature required for the performance of all its functions. The decreased oxygenation is an important factor in considering the question of alimentation in the tropics; the constructive and destructive metabolism of tissues depends chiefly on the amount of available oxygen present, and food is useless or detrimental which for want of it cannot be converted into assimilable bodies or else broken up and eliminated. The loss of weight, the weakened power of digestion and assimilation, the diminished physical vigor and muscular exertion, are so many reasons for a corresponding reduction in the diet. Increased perspiration diminishes the amount of turine and output of urea, so that more work is thrown upon the liver and skin as excretory organs. Unless the food is suitably adjusted to the new conditions, there is danger of hepatic congestion, with subsequent inflammation and degeneration, as frequently seen in hot climates.

These theoretical considerations are confirmed by the established 
dietary habits of people living at different latitudes; in other words, we find that the nutritive amount of food consumed by the same class of laborers diminishes as we advance from the northern portion of the temperate zone to the tropics. Thus the Department of Agriculture (Bull. 38 and 71 ) has shown that while negro families in northern Virginia consume 109 grains of protein, 159 of fats and 444 of carbohydrates, with fuel value of 3,745 calories, families of the same class in southern Alabama only consume 62 grains of protein, I 32 of fats and 436 of carbohydrates, with fuel value of 3 ,OI 2 calories. The fuel value of the food consumed by the natives of all tropical countries is estimated to range from 2,000 to 3,000 calories.

Admitting that a reduction of the soldier's ration, as adjusted for temperate climates, is necessary in the tropics, the next question is in which of the proximate principles should it be made; undoubtedly in the fats and proteins.

Fats are only digestible to a limited extent, according to the needs of the body. When taken in excess they are extremely apt to split up in the stomach into irritating acids which, together with hepatic congestion and disturbed bile secretion, produce the condition known as biliousness, often attended with putrefaction of the intestinal contents and catarrhal inflammation of the bowels. As fats contain but little oxygen, they are often unable to obtain the large amount of it required for their complete combustion, thus becoming an incubus in the system; on the other hand, if completely metabolized they evolve much more heat than is desirable. It is not contended that all fats should be banished from the tropical ration; a certain amount is necessary, but this should be very moderate and in the most acceptable form. Vegetable oils have been recommended. The butter accompaniment of the daily bread, if not entirely left out, should be reduced to a minimum. Bacon, when well cooked so as to be dry and granular, is quite acceptable even in hot countries

The reasons before urged for a reduction of proteins in temperate zones apply with still greater force in the tropics, where the digestive functions, oxygenation, metabolism and elimination are more or less impaired. The amount of proteins which is digested and utilized in a temperate zone may here overload the system with 
unoxidized nitrogenous waste, or else, if oxidized and discharged by the kidneys as urea, strain these organs already functionally weakened by a diminished excretion of urine. Proteins, although reduced in quantity, should be as varied and digestible as possible. Beef can be now and then replaced by mutton, often by fresh fish, which is always good and abundant in the tropics, while eggs, cheese, beans (frijoles) and macaroni will also help in furnishing the necessary nitrogen in acceptable forms. Fish is often objected to by the company cook and left out of the men's dietary simply because of the trouble in preparing it, a striking instance of the indifference of some company commanders in the discharge of one of their most important duties.

All authors agree that, in the tropics, carbohydrates should form the best part of the dietary. They are the most easily oxidized of the proximate principles, the most readily metabolized and eliminated, their decomposition products being simply aqueous vapor and carbon dioxid.

The natives of tropical regions obtain their food almost entirely from the vegetable kingdom, consisting chiefly of bread, rice, beans, vegetables, fruit, coffee and sugar, to which poultry, eggs and fish are occasionally added; hence the natural inference that this diet is likewise the best adapted to the needs of white people in the tropics. Such an inference, correct in the main, needs to be qualified ; the natives did not consciously select their diet; as a rule, they are ignorant, indolent and poor, and therefore consume the food procured with the least effort and expense. There is no doubt, however, that this food, which is mostly that provided by nature, is the best for them; its chief defect is a lack of variety. As soon as they can afford it they are but too apt to buy the viands and more appetizing foods of the higher classes, thereby losing their native immunity to various infectious diseases and becoming liable to the ills of those whites who neglect to adjust their habits to climatic conditions.

Munson, in his elaborate study of the subject,* proposes several dietaries for the soldiers in the tropics, in which the proteins vary from 123 grams to 104, the fats from II 4 grams to Io and the carbohydrates from 630 grams to 517 , with fuel value ranging from

* The ideal ration for an army in the tropics, 1900. 
2,947 to 3,825 calories. As already seen from the conclusions drawn above, such liberal allowances cannot be accepted as representing the actual amount of food needed by the body in the tropics, but, to some extent, may be used as the basis of the ration to be actually issued.

With our very elastic system of supplying troops, it is not necessary to make the tropical ration different from the regular garrison ration. The money value of the articles of the ration not needed can be expended in purchasing others better adapted to the climatic requirements. Care, however, should be taken that such liberal policy does not engender wasteful habits. The important point to determine is the amount of proximate principles which, in the performance of the ordinary duties of garrison and camp, is actually needed by the men, to be used as a guide by the officers in command of organizations, so as to provide plenty and at the same time avoid overfeeding. It has been seen that this amount, in temperate zones, does not exceed 60 grams of proteins and about the same quantity of fat, with enough carbohydrates to yield a fuel value of 2,800 calories. This then should be the maximum for the tropics, to be exceeded only in case of unusually arduous work. It is furnished by the following quantities of the ordinary components of the ration :

Articles.

Fresh beef, or fresh mutton, or pork, or bacon, or dried fish, Flour, or fresh fish,

or soft bread, or hard bread,

Beans,

$$
\begin{aligned}
& \text { or peas, } \\
& \text { or frijoles, } \\
& \text { or macaroni } \\
& \text { or rice }
\end{aligned}
$$

Quantity.

IO ounces

Io ounces

6 ounces

6 ounces

Io ounces

I6 ounces

I 5 ounces

I 5 ounces

I2 ounces

2.4 ounces

2.4 ounces

2.4 ounces

2.4 ounces

4 ounces 
Potatoes, or substitutes, Dried fruit, Sugar, Coffee,

or tea.
16 ounces

I6 ounces

Such ration will yield at least 3,000 calories and, after making allowances for wastes, comes up fully to requirements.

The best cereal and one of the best foods for tropical regions is rice; therefore an effort should be made to cook and season it in an appetizing manner, not boiling it into a gelatinous, sticky mass, but so that each grain, when thoroughly cooked, stands unbroken, separate and dry (see page 357 ).

As pointed out by Kean, it is a blunder to make potatoes an indispensable article of the tropical ration and export them, at great trouble and expense, to our colonial possessions where native roots, tubers and fruits, at least equally palatable and nutritious, can be readily obtained. Potatoes rapidly decay in transit or when stored in hot countries, and most of them become unfit for use. before they can be issued to the companies. In the tropics it is generally possible to secure sweet potatoes, various kinds of cucurbits (squash family) and of yam, cassava (manioca), taro (colocasia), tannier (caladium), etc., and any number of savory and palatable fruits. It should also be made one of the important duties of the cook to learn how to prepare them for the table. Tropical fruits, contrary to popular prejudices, if mature and not over ripe, are all perfectly wholesome.

In attempting to change the quantity and quality of the ration in the tropics, it is well to bear in mind the strong influence of habit. The soldier going from the United States to our colonial possessions quickly understands the necessity of a change in his clothing, but is slow in realizing that of a change in his diet, and any attempt to reduce too rapidly the quantity of food he has been accustomed to will be strongly resented. In this, as in other things, he should be properly instructed; the advantages of such a reduction must be pointed out to him so that he may submit to it intelligently and more completely appreciate the benefits of it. 


\section{CHAPTER XXXIII.}

\section{RULES TO BE OBSERVED IN EATING AND DRINKING.}

The general hygienic rules to be observed in eating and drinking, in order to maintain our best health, may be summed up as follows :

I. Let the food be simple but nutritious and varied, containing all the proximate principles in suitable proportion; the digestive organs soon tire from the same articles while they are stimulated by change and new combinations. Highly seasoned food, rich pastry and savory cheese should be the exception, never the rule. Pungent condiments are pernicious; for a while they excite the digestive glands to abnormal action but eventually cause diminished secretion and dyspepsia.

2. Cook it properly so as to bring out its savors, promote a free flow of digestive fluids and cause its prompt disintegration therein.

3. Eat slowly and masticate thoroughly, for unless the food is properly cut and ground by the teeth, lumps of it will escape the action of the saliva and gastric juice, not only remaining undigested but likely to ferment and give trouble.

4. Drink as much water as may be necessary to thoroughly soften and mix the food, but slowly, not too much at a time, and not too cold. The old belief that water should not be drunk at meals on the ground that it hinders digestion and absorption has been proved erroneous. On the contrary, water promotes digestion, especially of fats, and insures more complete utilization of proteins. To quench the thirst, however, it is best to drink between meals, or before beginning to eat. Water does not appear to be absorbed by the stomach but escapes into the intestine, at the rate of at least a pint an hour.

5. Avoid dishes or beverages too hot or too cold, for extremes of temperature check the secretion of the gastric juice, while their alternations may fissure the enamel of the teeth. Water should be cooled with ice, but never ice-cold.

6. Observe regularity in the hours for meals, so that the digestive juices may be poured out more abundantly at the accustomed times. 
7. Avoid over-indulgence or intemperance in eating, for not only does the stomach resent abuse, but the whole system becomes clogged and the congested and strained organs function imperfectly.

8. Do not divert blood from the stomach during active digestion by mental or physical work. Rest of body and mind, or at least abstention from active exercise, is necessary for a few moments before meals and for about an hour afterward. Depressing thoughts and business preoccupations should be banished from the table.

Concerning the interaction of food and work, nothing is more illuminating than the following remarks of Chittenden:

"It is a mistake to assume the necessity for a hearty meal because heavy work is about to be done. It is certainly far more rational from a physiological standpoint to leave the hearty meal until the day's work is accomplished.

"We seemingly forget that the energy of muscular contraction comes not from the foodstuffs present at the time in the stomach and intestinal tract, but rather from the absorbed material stored up in the muscles and which was digested and absorbed a day or two before. Further, it is to be remembered that the very process of digestion draws to the gastro-intestinal tract a large supply of blood, and that a large amount of energy is needed for the process of secretion, digestion, absorption, and peristalsis, which are of necessity incited by the presence of food in the stomach and intestines, thereby actually diminishing the amount of energy available at the place where it is most needed.

"Why, then, draw upon the resources of the body just at a time, or slightly prior to the time, when the work we desire to perform, either muscular or mental, calls for a copious blood supply in muscle or brain, and when all available energy is needed for the task that is to be accomplished.

"We are wont to compare the working body with a machine, the boiler, engine, etc., overlooking the fact that the animal mechanism differs from the machine in at least one important respect. When we desire to set machinery in operation we must get up steam, and so a fire is started under the boiler and steam is generated in proportion as fuel is burned. The source of energy made use of in moving the machinery is the extraneous combustible material introduced into the fire-box, but the energy of muscular contraction, for example, 
comes not from the oxidizable food material in the stomach, but from the material of the muscle itself. In other words, in the animal body it is a part of the tissue framework that is burned up, and the ability to endure continued muscular strain depends upon the nutritive condition of the muscle involved, and not upon the amount of food contained in, or introduced into the stomach." 


\section{CHAṔTER XXXIV.}

\section{BEVERAGES.}

Beverages are conveniently divided into alcoholic and nonalcoholic.

Of the non-alcoholic beverages, those which especially concern the soldier are coffee, tea and chocolate.

Coffee is the name of the seed of the shrub Coffea arabica cultivated in many tropical countries, as well as of the beverage prepared from it. Except in the hands of an expert, it is difficult to judge of the quality of the seed, or berry, from its size, shape or color, as these vary considerably on the same plant and are otherwise easily altered. By roasting the green berries until they acquire an even, dark reddish-brown color, characteristic aromatic and empyreumatic principles are developed; as they are volatile, coffee should be roasted in a closed vessel and only a short time before being used; for the same reason, roasted coffee should be kept in tight vessels and only ground as needed. The taste is often much improved by the addition of a little butter and sugar before roasting. The composition of roasted coffee is about 2 per cent. of water, I of caffeine, 12 of fatty matters, I2 of nitrogenous substances, 4 or 5 of tannic acid, very little sugar, a minute proportion of volatile oil and of aromatic principle, a large amount of cellulose and extractive matters and nearly 5 of mineral salts. The principal active constituent is the alkaloid caffeine, of which a cup of black coffee contains about I grain.

The berry should be ground into coarse powder; if ground too fine, part of the flavor is destroyed. In order to preserve its aromatic principles coffee should be prepared only by infusion. The ground coffee is put in boiling water, two or three heaping tablespoonfuls to the quart, and then the pot removed far enough from the fire to keep it just below the boiling point for Io to 15 minutes.

Caffeine produces a slight increase of blood pressure and slowing of the pulse. It is our best cerebral stimulant, improving all the mental functions, causing clearness and rapidity of thought, alertness and wakefulness. It is also a typical motor stimulant, causing 
a decided increase in speed, as well as in the accuracy and coördination of movements. It enables the muscles to contract more vigorously, and without secondary depression, so that the sum total of muscular work is increased $(H . C$. Wood, Jr.) The real stimulation of caffeine does not begin until about an hour after ingestion and, so long as it is used in moderation, this stimulation is not followed by subsequent depression. Caffeine is also an active diuretic. When taken too freely, as coffee or tea, it is liable to cause general nervousness, irritability, insomnia, functional irregularity of the heart and derangement of all the digestive functions.

Coffee acts as a gentle laxative, whereas tea, owing to its astringent constituents, may have the opposite effect. It is not usually so well borne as tea by dyspeptics.

Tea consists of the leaves of the shrub Thea chinensis cultivated in many tropical and semi-tropical countries, and is likewise the name of the beverage prepared from them. Tea of excellent quality is grown in our Southern States. Green tea and black tea differ only in the method of preparation; the former being immediately dried after picking, and the latter previously allowed to undergo a slight degree of fermentation. Black tea is generally higher in caffeine and much lower in tannin than green tea. According to König, average tea contains $2 \mathrm{I} .22$ of nitrogenous mattcrs, $\mathbf{I} .35$ of theine, 0.67 of volatile oil, I0.75 of fat, resin, gum, etc., 7.76 of tannic acid, 37 of other extractives, cellulose, etc., and 5.I I of mineral matters. Its active principle, theine, is identical with caffeine, and quite variable in quantity, sometimes amounting to 3 per cent. in black teas.

Tea, as beverage, should be made, like coffee, by infusion. Boiling dissipates the aroma and dissolves much of the astringent and bitter principles. As the quality of tea depends largely upon the mode of its preparation, the following rules should be observed: Take fresh water, not water that has become flat by standing on the stove or in the waterback; bring it quickly to a hard boil; fill the teapot and set it on the fire for one minute in order that it may get thoroughly heated; pour off the water and put into the pot one teaspoonful of the leaves for every teacupful of tea that is to be drawn, and one for the pot; then pour on the boiling water and set the covered pot on the stove or near the fire to "draw," but not to boil. It should draw just enough to liberate the essential oil which 
constitutes its aroma, but not long enough to drive it off or to dissolve the tannin which gives the tea a disagreeable taste. The time varies with the different teas, from five to ten minutes. (Manual for Army Cooks.) The most delicate portion of the infusion is that poured off within three or four minutes.

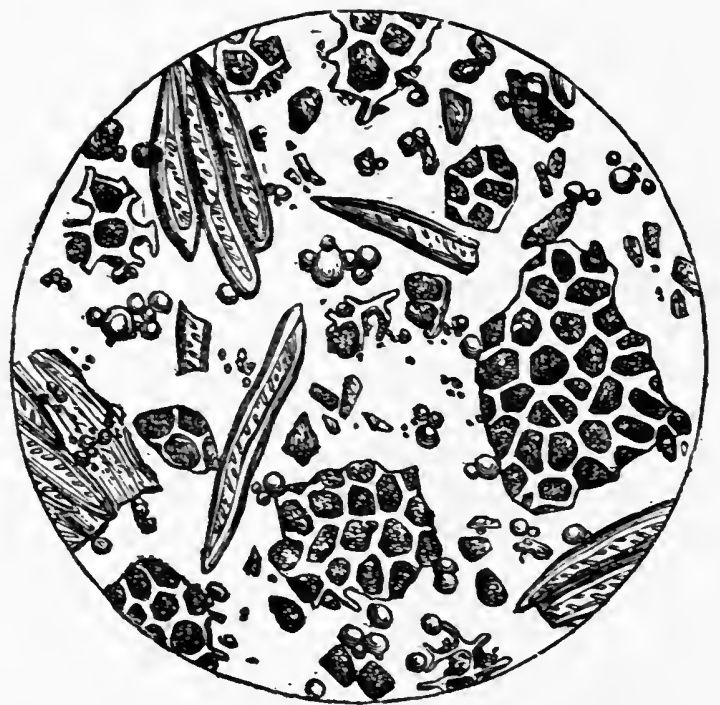

FIg. 86. - Coffee roasted and ground, free from all adulteration. (Hassall.)

Should the soldier use coffee or tea in the field? Both are gentle stimulants which cheer and refresh, relieving fatigue and the sensation of hunger. The addition of sugar and cream gives them decided nutritive value and increases their power to evolve muscular energy. Coffee, among male adults, is more generally used than tea in this country, as well as in France and Germany, while tea is preferred in England, Russia, Japan and China. This appears to be in a large measure a matter of habit and custom. There is good evidence that, to relieve thirst on a hot or cold day, as well as to appease hunger and sustain energy, nothing is quite so satisfying as a glass of tea; the extended experience of English officers on the torrid plains of India, as well as that of Russians in Siberia and Manchuria, appears to be conclusive on that point. The opinion of the English in India is doubtless correctly expressed in the following declaration of Sir Havelock Charles: "Tea is the drink par excel- 
lence from the snows of the Pamirs to the scorching sands of Rajputana; I have tried it and seen its effects." It seems also that more caffeine can be taken with impunity in the form of tea than as coffee.

For the soldier in the field tea possesses some obvious advantages. For equal weight it yields one and a half or frequently twice as much caffeine as coffee, so that less is needed to make the same quantity of infusion. It is much easier to transport, preserve and prepare. The weight and bulk of enough tea, in compressed tablets, to last a soldier a week is a negligible quantity in his haversack. Not only is coffee heavier and more bulky, but its proper roasting and grinding in the field is often a difficult process, or, if issued roasted and ground, great care is required to prevent deterioration." The most practical way to use coffee in the field is in the form of compressed cakes with impervious wrappers, as in the French service, or of a strong and stable liquid extract requiring nothing but the addition of hot water, such as is sometimes used in the Russian service. Tea and coffee being prepared with water that has been boiled are sterilized beverages and of great value wherever water is contaminated; but tea, being more aromatic than coffee, can be diluted to a much greater extent without ceasing to be palatable, therefore can be used practically ad libitum and more readily take the place of water as a safe drink. In the absence of fire, good tea can also be prepared with cold water if time permits. The tannin in tea is objectionable on account of its astringent and constipating effect, but a properly prepared infusion, especially when diluted, never contains enough of it to be harmful. On the other hand, tannin in tea is not without redeeming features; it may render good service in diarrhea and, as an antiseptic, may help to purify water that cannot be boiled.

Cocoa is derived from the seeds of Theobroma cacao, a tree of tropical America, in no way related to the cocoanut palm. The fruit is a rigid, pointed pod, six to ten inches long, containing from 25 to 40 seeds or "beans." These undergo a process of fermentation, then are dried in the sun, roasted and freed from the germs. Mixed with about an equal quantity of sugar and spiced with vanilla beans and cinnamon, they constitute the ordinary sweet chocolate. Cocoa contains I 3 per cent. of proteins, 49 of fat (cocoa butter), I3 of starch and 2 or 3 of potassium phosphate. It contains also an average of 1.50 per cent. of the alkaloid theobromine, closely related to 
caffeine, but somewhat less stimulant. The principal constituents of good chocolate are 57 per cent. of sugar, 22 of cocoa butter, I.33 of theobromine, 4.75 of proteins and $\mathrm{I} .48$ of tartaric acid.

Cocoa, therefore, especially as chocolate, is not only a mild stimulant, but, unlike tea and coffee, a valuable food in condensed form, capable of evolving much muscular energy in time of need. Chocolate is an important component of the emergency ration and an excellent article for the soldier to carry in his haversack whenever rations are scant and the work ardious.

Kola nut, the fruit of an African tree, contains about 2 per cent. of caffeine, some theobromine, starch, sugar and a volatile oil. It is used by mastication or in the form of various fluid preparations. It is a stimulant of the heart and respiration, temporarily increasing muscular energy and mental power, and has been recommended to relieve the fatigue of long marches on scant rations. It is generally believed, however, that its active effects are only those of caffeine and can be more conveniently produced by the use of coffee or tea.

Other noteworthy plants containing caffeine are:

Mate (pronounced matay), species of Ilex which, in Paraguay and other South American countries, takes the place of Chinese tea, containing about the same proportion of alkaloid.

Guarana, a preparation from the seeds of a climbing slirub of Brazil, containing 4 or 5 per cent. of caffeine.

The so-called " soft drinks," consumed in such vast quantities during the summer, are seldom above suspicion, and should be used with discretion. They are often prepared with contaminated water, or water taken from the nearest and most convenient supply. They nearly all contain saccharin, which has been shown to be injurious when habitually consumed. The extract of soap-bark, to produce foaming, is also largely used in their preparation; this bark contains saponin, a toxic principle. Besides chemical flavorings and coloring substances usually added, caffeine is also a common ingredient, particularly dangerous for children and young people who indulge so freely in those drinks.

Concerning water drinking, see under March, p. 239, and also p. 694 . 


\section{ALCOHOLIC BEVERAGES.}

These beverages include: I, fermented drinks, either made from malt, such as beer, ale and porter, or from saccharine liquids and fruit juices, such as wine and cider; 2 , distilled drinks, the product of distillation of fermented saccharine solutions, such as whiskey, brandy and rum.

The active and essential principle of all these beverages is ethylic alcohol in variable quantity.

The food value of alcohol and its effects upon the economy is a subject beset with difficulties, one studied by many physiologists, but often with contradictory results. The following conclusions are those now generally accepted. Atwater and others have demonstrated that if alcohol, in small quantity and for a few days, is made to take the place of its isodynamic equivalent of fat in the diet, the number of calories evolved remains the same; therefore, that within certain limits, alcohol is oxidized and a real food. In small quantity it may also act as a stimulant, that is, increase the blood pressure and the systolic output of the blood. This rise in the blood pressure, however, is transitory, seldom lasting more than a few minutes. It has been found that, even in very moderate doses, alcohol increases the discharge of uric acid in man, a fact leading to the suspicion that, even then, it has a deteriorating effect. Cheveau has shown that when alcohol is substituted for some time to the fats, the muscular energy falls and the body weight decreases. The sensation of warmth experienced after the ingestion of an alcoholic drink is due to the dilatation of the peripheral and pulmonary capillaries; but this is soon followed by a more active radiation of heat and increased evaporation, so that the loss exceeds the gain; thus after an ordinary intoxication the body temperature may fall $2^{\circ}$ or $3^{\circ} \mathrm{F}$. below normal. There is a general agreement that in large doses, namely, in excess of Io to I $_{5}$ c. c., alcohol acts as a depressant and is harmful. The line separating the stimulating from the depressing dose varies according to individuals, and in the same individuals at different times. We may then conclude that, except in small doses, or when the body actually lacks food, the temporary increase of pulse, respiration and muscular energy produced by alcohol is succeeded by a stage of depression and mental dullness which more than offsets the good effects of the stage of stimulation.

As a medicine, alcohol, under many forms, has doubtless a useful 
part to play, and is often advantageously administered in cases of exhaustion or heart failure, but even in such cases other stimulants may be preferable. It should always be given in small quantities and its effects watched, for the toxic dose is soon reached and the second state of the patient may be worse than the first.

In health, physiologists agree that alcoholic beverages are unnecessary, and the statistics of insurance companies tend to show that total abstainers may expect a greater longevity than even those who indulge in moderation. The use of whiskey, wine or beer, formerly pretty general in armies and navies, as part of the ration, is now restricted to exceptional occasions of fatigue and exposure and, in some countries, as in ours, completely suppresseed. There are doubtless circumstances in very cold and very hot regions when a little wine or small amount of diluted whiskey may be beneficial, but the difficulty of correct dosage and the danger of over-indulgence are so great that it is safer, if we wish to obtain the best results from our nervous and muscular energy, as well as from our mental powers, to trust to coffee, tea and chocolate.

Concerning the use of alcoholic beverages, several points must be borne in mind. The most important is that the active toxic principle is alcohol and not any of the impurities which may be associated with it ; the proportion of these, as a very general rule, is much too small to produce any decided effect. The various manifestations of alcoholism, therefore, are due to the quantity, not to the quality of the alcohol. The exception to this rule is in the case of absinthe, vermouth and various bitters, in which poisonous essential oils are used, or again in the case of the crudely distilled spirits found in certain countries, like the "mescal" of Mexico and the "vino" of the Philippines. Another point is that the more diluted the beverage, the less likely is the passage into the blood of enough alcohol at any time to produce toxic symptoms, and the less harmful the result. Thus France is said to consume more alcohol than any other nation (Arnould), but as much of it is in the form of wine, alcoholism does not appear to be worse there than in several other countries. Spain and Italy consume large quantities of alcohol, but also mostly as wine, and they are among the countries which suffer least from alcoholism. All observations tend to show that, for those who desire or need an alcoholic beverage, the most acceptable, that provided by nature from the beginning, is light, unadulterated wine. 
It seems probable that a very moderate use of it, at meals, can be indulged in with comparative impunity.

The proportion of alcohol in the many drinks consumed varies greatly. Any beverage containing less than 2 per cent. may be considered as non-intoxicant. Beers average from 3.5 to 5 per cent.; wines from 6 to 15 ; spirits (whiskey, brandy, rum, etc.), from 40 to 45. Of the latter, pure native whiskey, a few years old, when much of the fusel oil has disappeared and been replaced by aromatic principles, is the least objectionable. The maximum amount of alcohol which anybody can take in health, without immediate apparent harm, is variable; it should never be more than enough to produce a mild degree of temporary stimulation, with no depressing after-effect. This amount for the day should not exceed, as a general average, one ounce of alcohol, or two ounces of whiskey, or ten of light wine, or sixteen of beer. Every prudent man should know what is, for him, a safe maximum dose and never go beyond it, keeping full mastery over this, as well as other dangerous appetites. For many people of nervous, ill-controlled temperament, the only safe rule is complete abstention.

There is a consensus of opinion among experienced field officers, especially among medical officers, that soldiers on active duty do not need alcoholic drinks and are capable of much better and more continued achievements without them. Therefore during the present European War it has been the endeavor of all the belligerent governments to reduce the consumption of alcohol in the field to as low a minimum as circumstances permit, too often a minimum which could be still further reduced with great benefit were it not for the antagonism of uneducated public opinion.

Alcoholism.- Only a few remarks on alcoholism are necessary. (See p. I3I.) The toxicity of alcohol in the blood is mainly due to its dehydrating effect upon cell protoplasm and inhibiting action upon all forms of cell activities, with consequent degeneration of the tissues. The chief effects and symptoms of alcoholism are: congestion of the stomach with increase of connective tissue, degeneration and atrophy of the secreting glands and consequent impaired digestion; congestion and degeneration of the liver, the contracting connective tissue causing atrophy of the portal canals and hepatic cells, a condition known as cirrhosis and generally accompanied by abdominal dropsy; degeneration of the brain substance with motor 
and sensory disturbances, accompanied by gradual dulling and failing of the mental and moral faculties; fatty degeneration of the heart and atheromatous or sclerotic condition of the arteries, leading to apoplexy; decrease of muscular energy and capacity for work; failing of the power of resistance to all diseases, the facility with which free drinkers succumb to pneumonia or contract tuberculosis being notorious. It has also been observed that even moderate drinkers contract disease (outside of alcoholism) more frequently than total abstainers.

From the examination of the bodies of 460 alcoholics, Hultgen (J. Am. Med. Ass., I9IO) found morbid alterations in the central and peripheral nervous system in 93 per cent., thus proving that alcohol is essentially a narcotic. The effects on the gastro-intestinal system were likewise marked and striking, pharyngitis, gastritis and hepatic disorders existing in 83 per cent. Arterio-sclerosis and valvular lesions were not common, indicating that the sclerogenic action of alcohol is perhaps not as great as had been believed. It was surprising to find that those tissues through which alcohol is eliminated, namely, lungs and kidneys, were least affected. As regards the lungs, however, these observations do not accord with those of other observers. Thus Jacques Bertillon, after a thorough investigation of the subject, sums up in the statement that "of all causes which favor the development of tuberculosis, in France, there is none that has the importance of alcohol." Fiis statistics show that alcohol, as brandy or whiskey, is much more deleterious than in the form of wine, the ratio of tuberculosis, in France, increasing proportionally with that of the per capita consumption of spirits. This divergence of views, however, is easily reconciled by the consideration of the fact that alcoholics who develop tuberculosis, lose, to a large extent, their appetite for alcohol in the advanced stage of that disease, so that the true cause of their death is overlooked and recorded simply as tuberculosis.

Methylic alconol, (wood alcohol), although a well-known rank poison, is commonly used as an adulterant of, and substitute for, ethylic alcohol in cheap whiskey and other alcoholic beverages; it is also extensively employed in the arts, as well as in many patent medicines and toilet preparations. The symptoms of acute poisoning are severe gastro-intestinal disturbances, accompanied by abdominal pain, weakness, nausea, vomiting, vertigo, headache, dilated 
pupils and blindness. In fatal cases there is marked cardiac depression, sighing respiration, cold sweats, delirium, convulsions and coma. The silly, irresponsible excitement of ordinary drunkenness is not seen, and even staggering may be absent. Wood alcohol is much more slowly absorbed and eliminated than ethyl alcohol, so that even small doses may have a cumulative effect and do serious damage. Sometimes, after taking a large dose, one or two days may elapse before symptoms of poisoning manifest themselves.

It is estimated that if Io persons drink, each, 4 ounces of wood alcohol ("Columbian spirits") within three hours, all will have marked abdominal distress; four will die, two will be permanently blind and four will recover.*

The treatment consists in getting rid of the poison from the stomach and intestines by means of the stomach pump and rectal injections; and the use of stimulants, especially strychnine and coffee.

The manufacture and sale of wood alcohol are an obvious menace to public health and call urgently for strict Government intervention.

* Death and blindness from wood alcohol. C. A. Wood. J. A. M. A., Nov. 30, 1912. 


\section{CHAPTER XXXV.}

\section{CLOTHING AND UNIFORM.}

The fabrics used to clothe the body are made from animal and vegetable fibers, especially from those of wool, silk, cotton and linen. These fibers are easily distinguished under the microscope. (Fig. 87.) The wool fiber is cylindrical and covered with imbricated scales; these are sharp and numerous in the best material, but worn out in shoddy; they are the cause of the matting together and shrinkage of wet woolen fabrics under pressure. The cotton fiber is flat and twisted, with thickened borders. The silk fiber is a structureless tube without any marking. The linen fiber is a cylinder with cross lines indicating the division of the cells, and occasional filaments at the lines.

The chemical analysis of textile fabrics is difficult and unreliable; an estimate of their relative proportion may be formed by placing them in a Io per cent. solution of potash or soda; the fibers of wool and silk will be dissolved while those of cotton and linen remain intact (Rubner).

The specific weight of the various materials used for clothing depends chiefly upon their structure, that is to say, the mode of weaving or knitting, their compactness and the quantity of air inclosed in their meshes; thus linen goods having all the fibers in close parallel planes, and smooth surfaces, are the heaviest; woolen flannels, with fibers loosely interlaced in several directions, are the lightest, while knit goods are intermediate. As will soon be apparent, the qualities and defects of textile fabrics are all closely related to their specific gravity. The following table, from Arnould,* is instructive:

* Nouveaux élements d'Hygiêne de Jules Arnould. 5th edition, revised by E. Arnould. 


\begin{tabular}{|c|c|c|}
\hline Articles & Specific weight & $\begin{array}{l}\text { Volume of interstices } \\
\text { per cent. }\end{array}$ \\
\hline 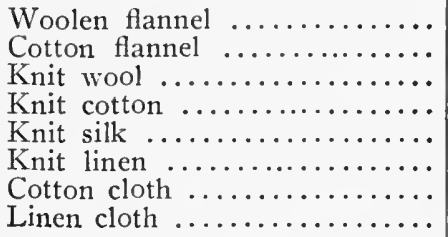 & $\begin{array}{l}0.101 \\
0.146 \\
0.179 \\
0.199 \\
0.219 \\
0.348 \\
0.624 \\
0.665\end{array}$ & $\begin{array}{l}92.3 \\
88.8 \\
86.3 \\
84.7 \\
83.2 \\
73.3 \\
52.0 \\
48.9\end{array}$ \\
\hline
\end{tabular}

Furs weigh only about half as much as flannels, containing 95 or 96 per cent. of air.

The general properties of textile fabrics which concern the hygienist are: permeability to air, hygroscopicity, heat radiation, heat conductivity and heat absorption.

Permeability. - Clothing should be permeable to air and allow a free interchange of gases in its meshes, that is to say, sufficiently porous to permit the external air to penetrate to the surface of the body and allow the free escape of the carbon dioxid $\left(\mathrm{CO}_{2}\right)$ and sweat (as aqueous vapor) constantly excreted by the skin. The

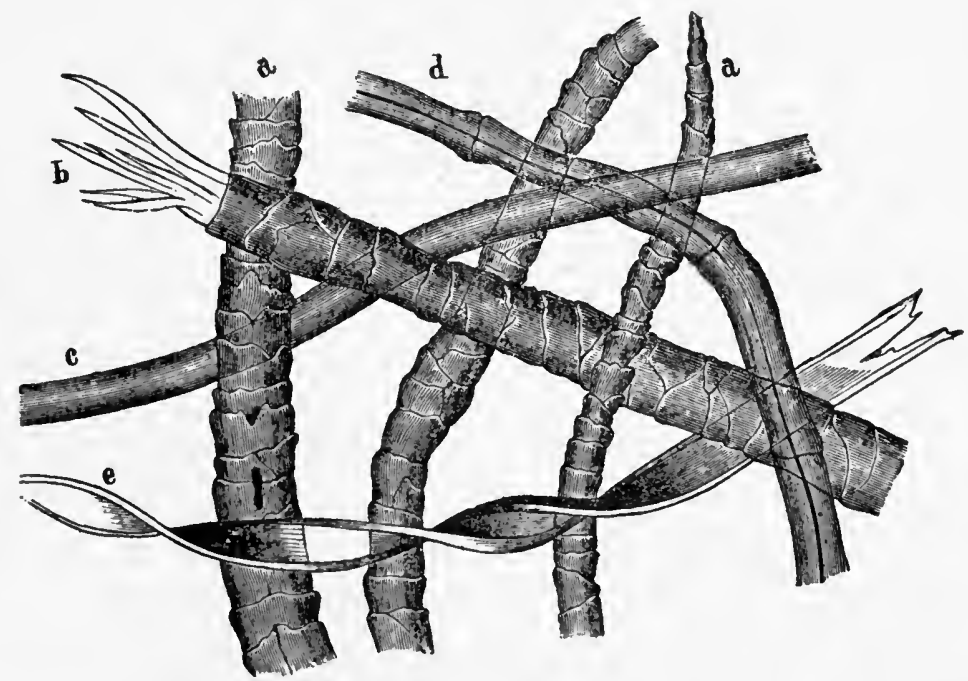

FIG. 87. - Textile fibers: $a$ and $b$, wool fibers; $c$, silk fiber; $d$, linen fiber; $e$, cotton fiber. (Arnould.) 
lighter and more porous the fabric the greater its permeability, but, with the same fabric, permeability is inversely proportional to its thickness. Woolen flannel is the most permeable textile material, and linen cloth the least.

Hygroscopicity. - Fabrics hold moisture in two ways: in the substance of the fiber itself (hygroscopic moisture) and in the interstices or meshes between the fibers (interposition moisture) ; pressure and wringing can remove the latter but not the former. Woolen fabrics absorb the most hygroscopic moisture; then, in order, those of silk, cotton and linen; this moisture swells the fiber to some extent but does not materially impair the permeability of the tissue to air. The capacity of fabrics for interposition moisture depends chiefly upon their texture; thus the maximum capacity of woolen flannel to fill up its interstices by sweat or rain is only $\mathrm{I} 3$ per cent., while that of cotton cloth is 100 ; in other words, those fabrics capable - of holding the largest quantity of hygroscopic water are those that retain interposition water in the smallest ratio and in which, therefore, permeability is least diminished by wetting. Flannel and knit goods absorb moisture, and lose it, much more slowly than cotton and linen cloth. When, after exercise, a woolen garment is put on, the vapor from the body is condensed and absorbed in the fibers; in this process of transformation from gas to liquid, it gives off its latent heat, producing a comfortable feeling of warmtl. At the same time the evaporation proceeds slowly and without any sensation of cold.

Heat Absorption. - The heat absorbing power of garments, in the sunlight, depends entirely upon their color and not on material or texture; white absorbs least; then, in increasing order, khaki, olive-drab, green, red, brown, blue and black, the last-named color absorbing more than twice as much as white.

For this reason black outer garments are best for outdoor use in cold weather (in order to absorb more solar heat), and white garments in hot weather. Color has the same effect upon artificial heat; thus water in a black kettle on the stove will boil more quickly than in a light-colored one.

The heat-absorbing power of garments is noteworthy and, in warm comtries, deserves special attention, as shown by the experiments of the "Board for the Study of Tropical Diseases" in Manila, especially in reference to khaki and olive-drab cotton cloth. 
A thermometer placed beneath olive-drab material exposed to the direct rays of the sun, rose from $93^{\circ}$ (air temperature in sun) to $126^{\circ} \mathrm{F}$. in about an hour, the air temperature meanwhile remaining unchanged, whereas beneath white drilling the temperature usually remained below that of the body. The average temperatures of several observations, in sunlight, were $108.3^{\circ}$ beneath olive-drab, 105. $6^{\circ}$ beneath khaki, and $95.4^{\circ}$ beneath white. These observations were made at a time when the intensity of sunlight was not at its maximum, so that the difference between the three materials would have been still more marked at other seasons. Between helmets covered respectively with khaki and olive-drab material, and exposed to a bright sunlight, the difference between the thermometers placed beneath them wás $8.28^{\circ} \mathrm{F}$. in favor of khaki.

It should be stated that the difference in the temperatures beneath khaki and olive-drab worn on the human body is not as great as when these materials are placed on a table as in the above experiments; this is owing to the power of warm-blooded animals to prevent the body temperature from rising, by perspiration; therefore the warmer the garment, on a warm day, the more active the perspiration and the greater the strain upon the heat-regulating mechanism.

Heat Radiation. - According to Rubner, the body at rest expends, in a day, about 2,700 calories; of these, 95 per cent. are lost through the skin, namely I,I 8 I by radiation, 833 by conduction ard $55^{8}$ by evaporation. (See page 364.)

Heat radiation, such as takes place indoor or in the shade, is, like absorption, affected by color, black radiating most and white least. For this reason, indoor outer garments should be darkcolored in hot weather and light-colored in cold weather. The water which boils more rapidly in a black kettle, also cools off more quickly when removed from the stove. As regards military uniforms, radiation is of little importance because at the time when it would be of value, that is, in barracks, the soldier generally discards his outer garment.

Heat Conductivity. - If the conductive power of air be represented by $\mathrm{I}$, that of wool fiber will be 6 , of silk fiber 19 , and of cotton and linen fibers 30. The heat conductivity of a fabric will therefore depend upon the nature of its material and the amount of air it contains; flannels and knit goods having the least conductive fibers 
and the most porous texture will conserve the body heat much better than cotton and linen cloth.

Permeability to air reduces heat conductivity but, on the other hand, favors the loss of heat by evaporation and convection; therefore it must be properly adjusted to atmospheric conditions by varying the thickness or nature of the clothing.

The conductive power of water being 28 times as great as that of air, that of any tissue is necessarily modified by the amount of moisture it contains, especially interposition moisture; wet cotton and linen fabrics, therefore, lose heat much more rapidly than wet woolen ones; they cling to the skin, expelling the protective layers of air between the several garments, and by their rapid evaporation chill the body.

Evaporation. - The evaporation of the sweat and consequent cooling of the surface are favored by permeable porous clothing. In cold weather, when the sweat is reduced to a minimum, the same degree of permeability is not required and more compact clothing can be worn. Evaporation from wet garments depends chiefly upon the temperature and humidity of the ambiant air, but also increases directly wtih the proportion of water they contain, being greater from linen or cotton cloth than from woolen fabrics.

Having thus briefly summed up the properties and effects of textile fabrics, it is easy to determine those best adapted for underclothing and for outer garments.

\section{UNDERCLOTHING.}

For this are required materials of low conductivity to guard against any sudden or excessive loss of body heat, permeable to air so as to promote the excretion and evaporation of the sweat, and of great hygroscopic power so that the sweat be readily absorbed in the fibers without interfering with the air circulation. The material which best subserves these purposes is woolen flannel. and next to it cotton flannel. Woolen flannel, in contact with the skin, will readily absorb during active exercise in hot weather, $5^{\circ}$ per cent. of its weight of perspiration; but this would only fill ro to I 2 per cent. of its interstices, while, under the same circumstances, a cotton or linen undershirt would have 68 per cent. of its air spaces filled up, causing nearly complete impermeability to air and forming a serious obstacle to the evaporation of the sweat, with resulting 
heating of the body, increase of perspiration and more complete saturation of the undergarments. It is also to be remembered that flannel gives off its moisture by evaporation more slowly than cotton and linen, and cools the skin correspondingly less. Lastly, wool does not retain the organic matter and salts of the perspiration and the sebaceous glands to the same extent as cotton and linen, and therefore is less soiled in the same space of time. Flannel is not only the best fabric for undergarments in winter but also in summer, by varying its thickness. The undershirt must be of ample size when issued and reach half way down the thigh. In cold regions soldiers should be allowed to draw two sizes and, in winter, to wear one over the other when necessary. Fleece-lined undershirts and drawers are now issued for service in Alaska.

The chief objection against woolen garments is their irritation of sensitive skins, and their shrinkage and hardening when laundered. Their washing requires special care: it should be done with soap containing but little alkali as this dissolves the natural oil of the fiber and injures its texture; the garments should be immersed and shaken in lukewarm or cold water, rinsed out without being beaten, and dried after only very moderate wringing.

Pure wool, however, is not necessary for undergarments; cotton or other material may be advantageously mixed with it to a large extent so long as the flannel or knit structure is preserved.

Silk possesses most of the qualities of wool, being hygroscopic and a poor heat conductor but, in warm weather, gets too easily sodden with perspiration and, in that state, is too good a heat conductor to be a suitable material for underclothing. A combination of wool and silk is the ideal material for the purpose in hot countries, the so-called "Anglo-Indian gauze;" it is more lasting and less apt to shrink than cheaper fabrics of cotton and wool.

Cotton, in the shape of flannel or knit fabric, although inferior to wool for underwear, may take its place when cost is to be considered. Certain cotton fabrics, such as "twilled lining," are much used in India for underwear, shirts and pajamas, being absorbent, easily tolerated by delicate skins and poor conductors. Linen is not suitable for the purpose unless especially woven or knit, and then only for warm weather. It becomes readily saturated with perspiration and requires frequent changes.

Outer Garments. - The natıre and thickness of outside garments 
will depend chiefly upon the temperature of the air. In cold weather, dark woolen fabrics will be best. In warm weather, cotton and linen garments which are better conductors of the body heat, and therefore cooler, are preferable; in color they should be white or nearly so in order to reflect the solar heat as much as possible. Linen goods are smooth and lustrous, heavier and more durable than cotton and of high heat conductivity; they make the best outer garments for warm weather and tropical countries. For outdoor use in the tropics, under a blazing sun, the outer garment should not only be white but of sufficient thickness to prevent solar penetration, for, under such conditions, solar heat may readily overcome nature's method of maintaining the body temperature within physiological limits; to prevent this risk, white flannel of suitable thickness is indicated.

\section{SUITABLE GARMENTS AGAINST THE ACTINIC RAYS.}

Of late years much attention has been paid to the actinic or chemical effects of sunlight upon the body, especially in the tropics (see page 695). They are chiefly produced by the ultra-violet, or short rays of the spectrum, and least active in the red and orange. In order to exclude these dangerous actinic rays, it has been proposed to wear red or orange underwear or to line outer garments and head covering with these colors. As red is more conspicuous and a less durable dye, orange is more commonly used; orange silk being preferable on account of its lightness and non-conductivity. To avoid complexity of garments, Dr. Sambon has devised peculiar fabrics ("solaro" fabrics) with white warp or outer surface, and black, red or orange weft or under surface. For soldiers, the warp is made of yellow and blue, producing a khaki or drab effect, and the weft of red.

The application of non-actinic colors to the soldier's uniform presents difficulties. Orange or red are too conspicuous to be used as lining to the blouse, or for undergarments in hot countries where the soldier is strongly inclined to discard his outer clothing.

It has been found, however, that opacity of garment is at least as effective as color, and that a black fabric (preferably silk) is altogether best for the purpose. Therefore a black undershirt suggests itself. For the soldier, the best garment would be a " solaro" flannel overshirt, olive-drab outside and black inside. It 
is especially the head and spine that should be protected against the injurious actinic sunlight of the tropics; hence the necessity of a black lining to the helmet or campaign hat, and of a black band, 6 inches wide, along the middle line of the blouse or overshirt.

Although the foregoing remarks, which appear in the first edition of this work, are left unchanged, the recent experiments with colored underclothing, described elsewhere (page 697), have shown the comparative unimportance of the effects of actinic rays, and that for the great majority of soldiers these effects are negligible.

\section{THE UNIFORM.}

The soldier's uniform must combine various hygienic and military requirements. The proper fabrics having been selected, it should be of such shape and size as to be suitable and comfortable, and enable the wearer to perform his duties to the best advantage. Everything must be sacrificed to these primary requirements. It must also be simple, easily put on and removed, and consist of as few garments and have as few detachable parts as possible. All this being granted, there is no reason why it should not also be neat and possess a certain degree of smartness and ornamentation, such as will help to develop in the soldier a spirit of pride in his calling; it is possible that, in our service, not enough importance has been given to the esthetic aspect of the uniform.

In size, the uniform should fit the body, but not too snugly, so that the free movements of the extremities, and those of the chest in breathing, be in no way impeded; it is especially necessary that the neck be not constricted by a tight collar. The layers of iwarm air interposed between the skin and the clothing should be allowed to escape freely in summer, especially at the neck; but, in cold weather, it may be advisable to restrict this air circulation by tightening the garments at the collar, wrists and ankles. It is important that the trousers and breeches extend well above the navel to protect the abdomen, and be tolerably loose about the seat and crotch to prevent friction and abrasion in marching or riding. In our service, they are held up with suspenders or a webbing waistbelt; the suspenders, which leave the waist and abdomen free from pressure, are always preferable.

The color of the uniform in garrison is regulated simply by esthetic and military considerations. In the field, it is chiefly regu- 
lated by the necessity of being as invisible as possible to the enemy. The visibility of the uniform will depend more or less upon the background against which it is projected, but, under average conditions, white is the most conspicuous color and therefore the most objectionable; then come the following colors in order of conspicuousness; scarlet, dark blue, yellow, green, butternut, dust or khaki, olive-gray drab. At dawn and sunset of a cloudy day, as well as by moonlight, white becomes as invisible as gray and brown, blue and scarlet being then the most conspicuous colors. The khaki
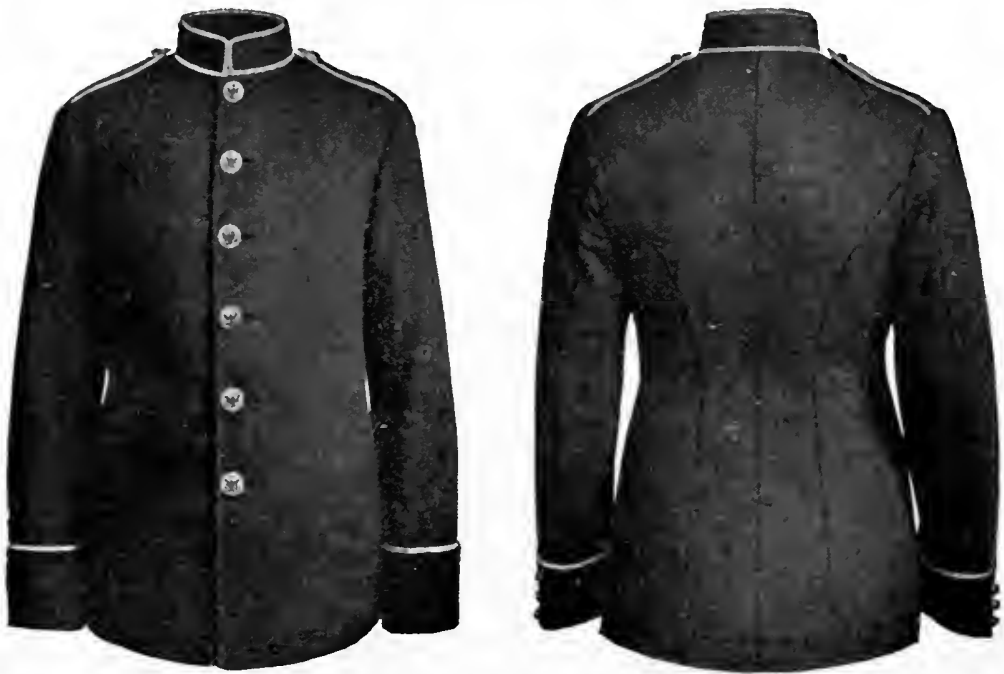

FIG. 88.- Dress coat for all enlisted men.

uniform formerly worn by our men, after being washed became pale yellowish-gray and comparatively conspicuous; under the same conditions the olive-drab uniform is nearly completely invisible. For the same reason, all buttons and metal ornaments, as well as articles of equipment, should be of dull material not liable to reflect sunlight.

It is still an open question, however, whether invisibility is an unmixed advantage, especially when both belligerents wear nearly the same kind of inconspicuous uniform. There may be serious danger of failing to recognize our own men in the distance and mistaking them for the enemy, or vice versa, allowing the enemy to secure important positions before being recognized. 
A service uniform of greenish-gray color has been adopted by nearly all the leading European armies. The French army has only recently reached some definite conclusion in regard to this; meanwhile, its very conspicuous red kepi has been screened under a darkblue cover.

United States Army Uniform. - In all arms of our Army the enlisted man is provided with only two uniforms: the dress and the service uniforms. The dress coat (Fig. 88) is a single-breasted sack coat of dark blue cloth, fastened with six regulation buttons down the front, with standing collar and shoulder loops. On occasions of ceremony, cords and tassels of mohair are fastened across the front of it. The dress trousers are of sky-blue kersey
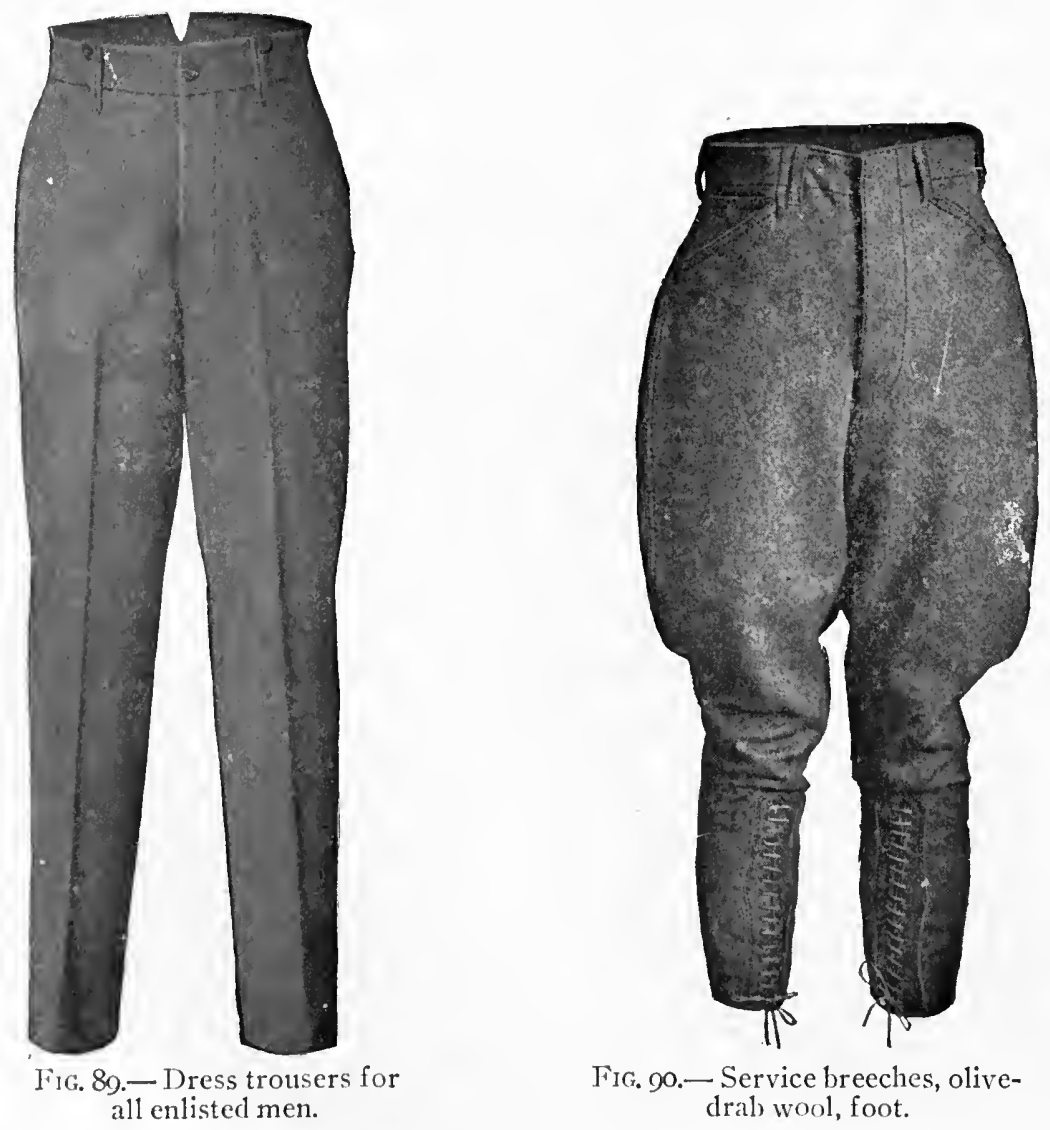

FIG. 90.- Service breeches, olivedrab wool, foot. 
(Fig. 89). The service coat is a single-breasted sack coat of olivedrab woolen material (Fig. 91), or olive-drab washable cotton material (for hot weather), with plain patch pockets, and buttons and collar ornaments of dull-finished bronze metal. The service breeches are of the same materials, made loose above but fitting closely below the knee, extending to the tops of the shoes and fastened with tapes (Fig. 90). They should always allow free and complete flexion of the knee. They are worn with shoes and leggins. For mounted men they are reinforced with a piece of the same material inside the thighs, and the lacing is on the outside instead of in front.

Long service trousers (cotton or woolen), worn without leggins, are only issued to enlisted men on duty in offices.

The service uniform has now been used several years under various conditions of service and climate, and has proved very satisfactory.

The cotton khaki,-formerly used for the service uniform, has been replaced by an olive-drab cotton cloth said to be greatly preferable, being dyed in the raw cotton so that, when woven, its softness and permeability are not impaired. It is also lighter (the uniform weighing about to ounces less) as well as stronger and more serviceable. The dye is absolutely fast so that the cloth can be washed repeatedly without fading. It absorbs more heat than
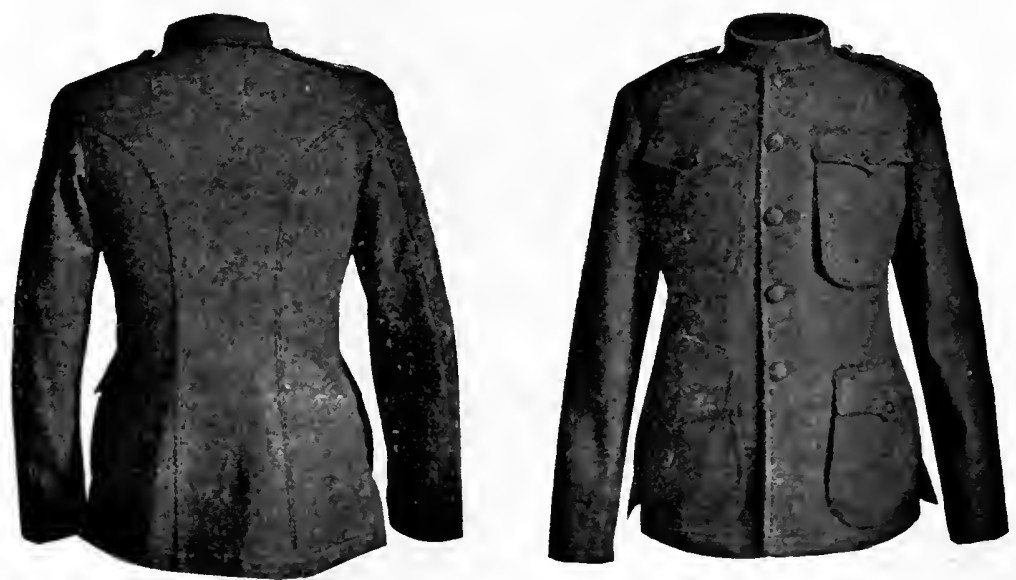

FIG. 91.-Service coat, olive-drab wool, for all enlisted men. 
the lighter-hued khaki and is therefore somewhat warmer, but is much less conspicuous. Furthermore, khaki was of such an unstable color that a company in that cloth presented few uniforms of exactly the same shade or hue.

In laundering the olive-drab cotton cloth, the clothing should be washed in cold or lukewarm water, and no starch used. In ironing, a woolen cloth should be interposed, or the uniform ironed on the reverse side.

The hospital corps when on duty in hospital, the members of the general recruiting service, in summer, as well as cooks and bakers, wear white suits, coat, trousers and cap, of bleached cotton duck, each cap provided with two detachable covers for washing.

A fatigue suit, coat, trousers and hat, of brown cotton duck (denim), is issued to all enlisted men for use when on fatigue work or certain special duties.

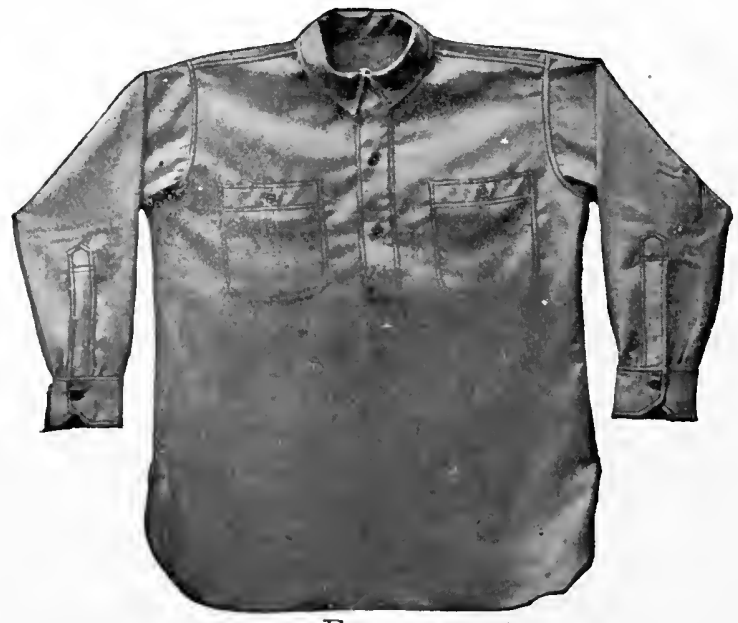

FIG. 92.

The olive-drab flannel shirt (Fig. 92) and the sweater are also parts of the service uniform. The former may be prescribed without the coat and without the sweater as the normal outer garment for field service, recent tests under all weather conditions having shown it to be highly satisfactory; a belt is then worn instead of suspenders. The insignia are worn on both sides of the collar, but the chevrons of non-commissioned officers are on the sleeve. 
The sweater is designed to replace the blouse for field use, being a much more comfortable garment to sleep in when necessary, and when the weather is too cold for the flannel shirt alone, especially on the march. It has a standing collar and two pockets in front knitted into the fabric. It is neat and not unsoldierly in appearance. When worn over the flannel shirt the collar of the latter is made to cover that of the sweater. The insignia of rank of non-commissioned officers are worn on the sleeve. It is carried in the surplus kit bag.

The overcoat for all enlisted men is a double-breasted ulster of olive-drab woolen material, suitably lined, extending down the legs 8 to Io inches below the knee, with standing rolling collar, and closing by means of five large horn buttons (Fig. 93). A hood
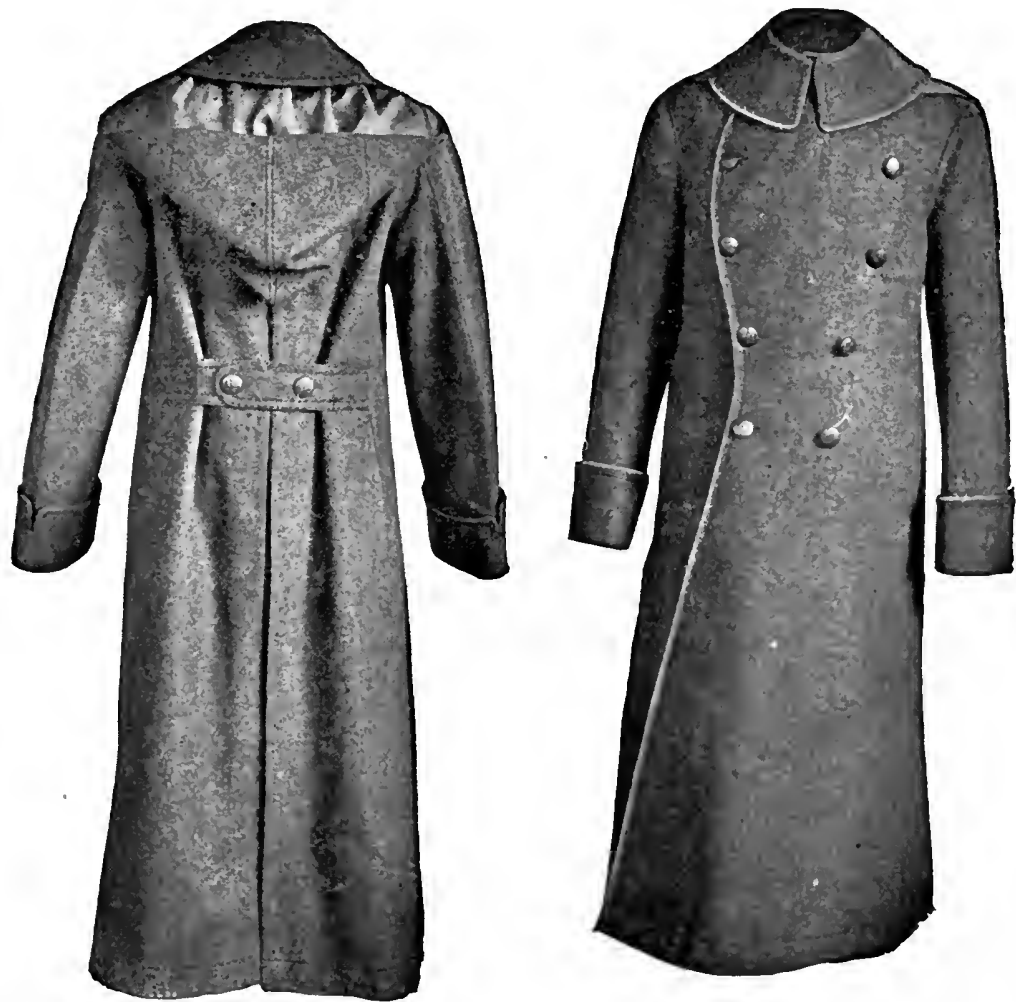

Frg. 93-- Overcoat, olive-drab wool, for all enlisted men. 
of the same material, large enough to cover head and cap, can be buttoned around the neck, under the collar, so that when drawn over the head it raises and holds the collar against the neck; in garrison it is worn only in inclement weather. The front corners of the skirt are provided with buttons or hooks, so that they may be turned back to facilitate marching. This overcoat can be worn over any uniform without change of head-gear.

The Infantry Equipment Board of I9I2, recommended that foot soldiers in the field should wear a short overcoat extending about half way to the knee, on the ground that long skirts are in the way and possess no advantage in providing warmth that cannot be better obtained by warm underclothing. This recommendation has not been approved.

A blanket-lined or fur-lined canvas overcoat is issued to troops stationed in extremely cold regions, but only for use while on guard duty or in field service. Mackinaw coats and trousers, of thick navy-blue mackinaw cloth, lined with dark-blue flannel, are issued for service in Alaska.

Waterproofing. - Garments, when dry, prevent the loss of body temperature but, when wet, increase it, often to a considerable degree. Thus, wet woolen flannel loses $5 / 8$ more of its heat than when dry, and cotton cloth $7 / 8$. This increased loss of body heat is the result of the greater conductivity of the water which has replaced the air in the interstices of the fabric, the expulsion of the warm layers of air from between the articles of clothing and, lastly, of the greatly increased evaporation.

Furthermore, the clinging of the undergarments to the skin stops the circulation of air upon its surface and, therefore, the excretion and evaporation of the sweat. It follows that wet garments are colder in winter and more oppressive in summer. They also become much heavier, sometimes nearly doubling in weight; thus the overcoat alone will take up 5 or 6 pounds of moisture in an ordinary shower. As a consequence, the burden of the soldier is much increased and his movements impeded.

In view of the extent to which rain may impair the efficiency of the soldier it seems desirable that his uniform should be rendered waterproof. This, however, must be done without impairing the permeability of the fabrics to air, in this respect assimilating them to the fleece of sheep or the feathers of birds which exclude 
moisture while retaining a free air circulation. The waterproofing of the uniform in a practical way, and with permanent results is a difficult problem and it is very doubtful whether it can be accom. plished without increase of weight and sacrifice of permeability. It seems preferable to preserve this most important quality of permeability at all times and provide an impervious outer covering for use in rainy weather. In our service the question has been decided, and apparently wisely, by the issue of a "poncho" to the foot soldier and a "slicker" to the mounted man (see page 468). These articles do not add much to the weight of the pack and are otherwise valuable in protecting the soldier from direct contact with damp soil in camps and bivouacs.

Should it be deemed advisable, under special circumstances, to waterproof the outer clothing, at least temporarily, various preparations have been recommended. Among the best is that of Cathoire who combines two parts of paraffin with one part of vaseline and dissolves 25 grams of the mixture in a liter of naphtha or benzin. Another and simpler preparation is a ro per cent. solution of lanolin (freed from water) in benzin, the cloth being soaked in it and then passed through rollers to remove the excess of solution and insure evenness of impregnation. (Munson.)

Underclothing. - According to the necessities of the climate and service, enlisted men are provided with two kinds of undershirts, wool knit (half wool and half cotton) and cotton knit; two kinds of drawers, unbleached canton flannel (Fig. 94), and jean (Fig. 95); three kinds of overshirts, olive-drab flannel (Fig. 92), heavy and light, and white muslin; two kinds of stockings, woolen and cotton. When the coat is not put on, the olive-drab flannel overshirt is worn as part of the service uniform, as noted above. In Alaska, fleecelined undershirts and drawers.are likewise supplied. A blue-andwhite chambray overshirt is also issued, but will be discontinued after the stock on hand has become exhausted. Pajamas have also been removed from the list of clotling.

In emergency, paper is useful, plastrons of one or two thicknesses of newspaper being placed over the chest. Paper soles within the shoes may also prevent much discomfort.

Head Covering. - The covering for the head should be light in weight, well ventilated, and a bad heat conductor; it should also protect the head from heat and cold, shield the eyes in front and 
the back of the neck from sun and rain. It should have a firm, even hold of the head so as not to be easily blown off, but without constriction at any point; in other words, it should have the contour of the average head which is oval or elliptical and not circular. Good ventilation is assured by providing plenty of space between the scalp and the top of the covering and a free circulation of air

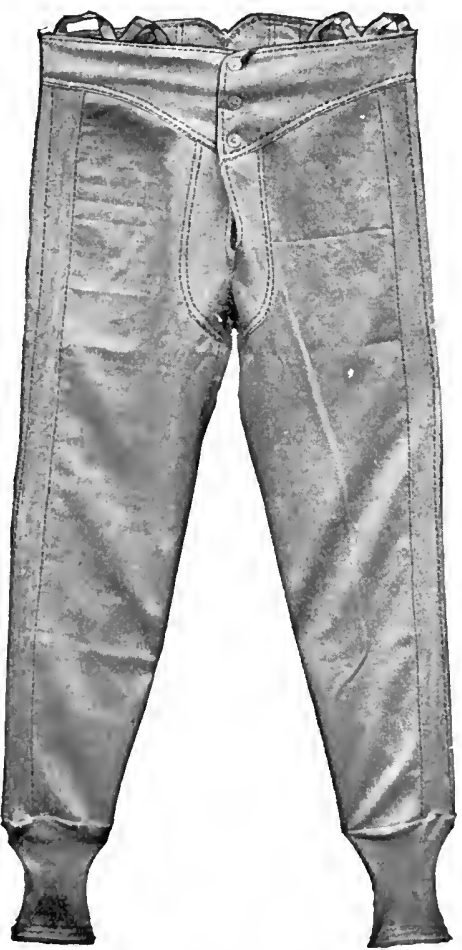

FIG. 94.-Winter drawers of canton flannel.

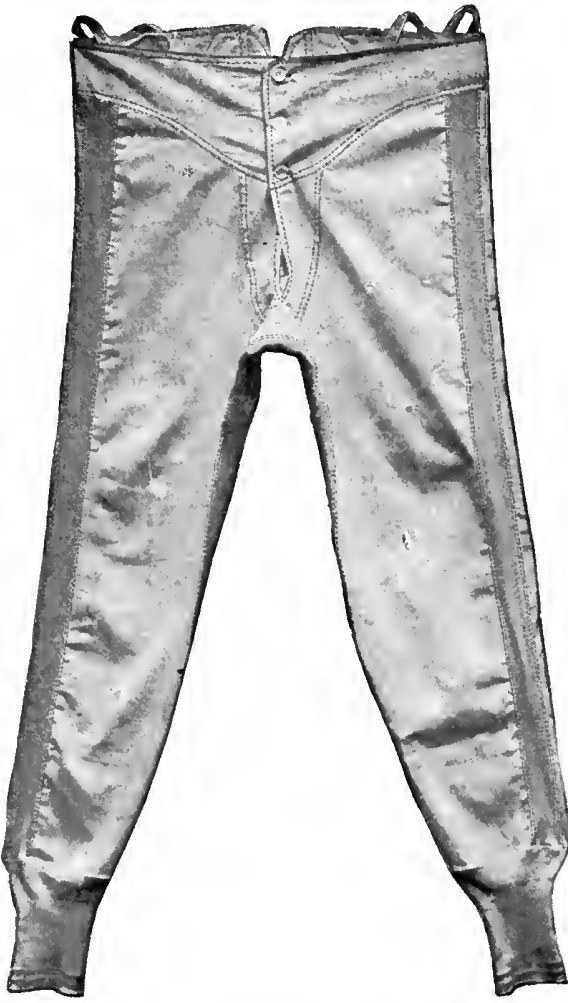

FIG. 95.- Summer drawers of jean.

through it. The ventilating holes should be preferably in front and rear rather than on the sides, so as to set the air in circulation by the motion of the wearer. The head-dress, more than any other part of the clothing, must be non-actinic, that is, exclude the short ultra-violet rays; this is best accomplished by black or orange lining, good ventilation and absence of constriction of the scalp. 
The head-gear which, in a hot country, best combines these requirements, is a properly constructed waterproof pith helmet, of white color, with ample brim in front and rear, and an all-round ventilated sweat band. The lining should be black inside the body and dark blue or green inside the brim.

Experiments made in the Philippines have shown that while the temperature inside a white helmet was $35.36^{\circ} \mathrm{C}$. (or always below the body temperature), it rose to $38.38^{\circ}$ in a khaki helmet, to $40.42^{\circ}$ in an olive-drab helmet and to $46.60^{\circ}$ in the campaign hat.

The ideal tropical helmet is one made up of two shells, one within the other, with complete air space between. The use of aluminum or tin-foil is not to be recommended as metals are good heat conductors and interfere with air permeability. Felt or cork may be used instead of pith, but cork, if thick enough, is too heavy. The objections to the helmet, especially for the use of enlisted men, are its bulk, its stiff, unyielding material, and the ease with which it gets broken or damaged, or, if not waterproof, softened by rain.

The only kind atthorized in our service is the olive-drab, worn by troops in the Philippines when in garrison, the service hat being worn at other times. The superiority of the white helmet, in hot weather, as a comfortable and hygienic head covering, has been so clearly shown that there seems to be no good reason why it should not be used on garrison duty in the tropics and in the Southern States. Even when worn with the olive-drab uniform the contrast in color is not at all unpleasant.

The. "havelock," a piece of muslin hanging loose from the rear border of the helmet, protects the neck from the sun; but this is more effectively done by a sufficiently wide brim.

Caps. - Enlisted men are provided with a dress cap, a service cap, a service hat, and a fatigue hat (denim). The dress cap (Fig. 96) is of dark-blue cloth, with flared top, stiffened in front and projecting all around from three-quarters to an inch beyond the band. The flat crown, slightly oval in outline, is held firm by wire springs and slopes down in rear. Its total height is 4 inches. The visor, of solid patent leather, lined with embossed green leather, is I $5 / 8$ inch wide at its center and slopes at an agle of 45 degrees. It is ventilated by two eyelets on each side and one in front, of $1 / 8$ inch aperture. In the front eyelet are inserted the fastenings of the detachable gilt insignia. It has a detachable band for occasions of ceremony varying in color according to the arm of the service. 
The service cap (Fig. 97) is of olive-drab woolen material, but otherwise like the dress cap in size, form and construction. It takes a firm grasp of the head without irksome pressure and is light and comfortable. From the viewpoint of hygiene, it would be improved by raising the slanting crown to a level so as to increase its air space, and widening the visor to 2 or even $2 \mathrm{~T} / 2$ inches, the better to protect eyes and face. Like all caps and kepis, it has the defect of not protecting, or only imperfectly, the nape of the neck. It is not worn in the Philippines, being unsuitable for warm countries.

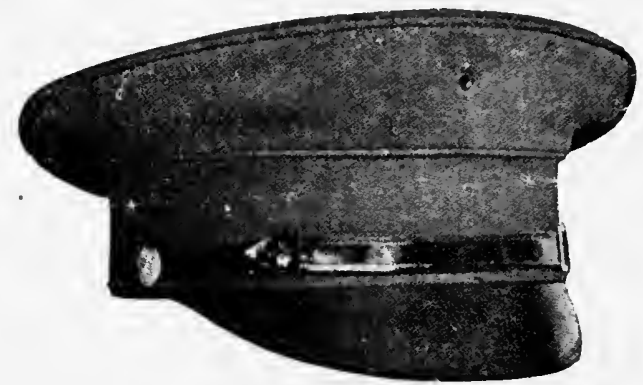

FIG. 96.-Dress cap for all enlisted men.

Service Hat. - The service or campaign hat (Fig. 98) is of drab felt, as near as possible the color of the service uniform, practically waterproof, with stiff brim 3 inches wide, and soft crown $5 \frac{1}{2}$ inches high; to be worn peak fashion by making four slight indentations between the ventilating eyelets. Ventilation is obtained by four eyelets $3 / 16$ inch wide, one in front, rear and on each side. It is

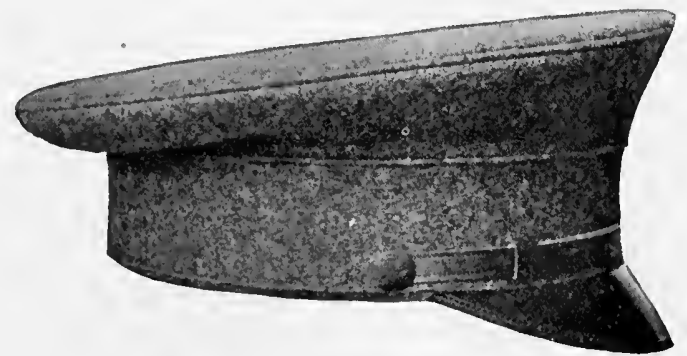

FiIG. 97-- Service cap for all enlisted men, olive-drab serge, 


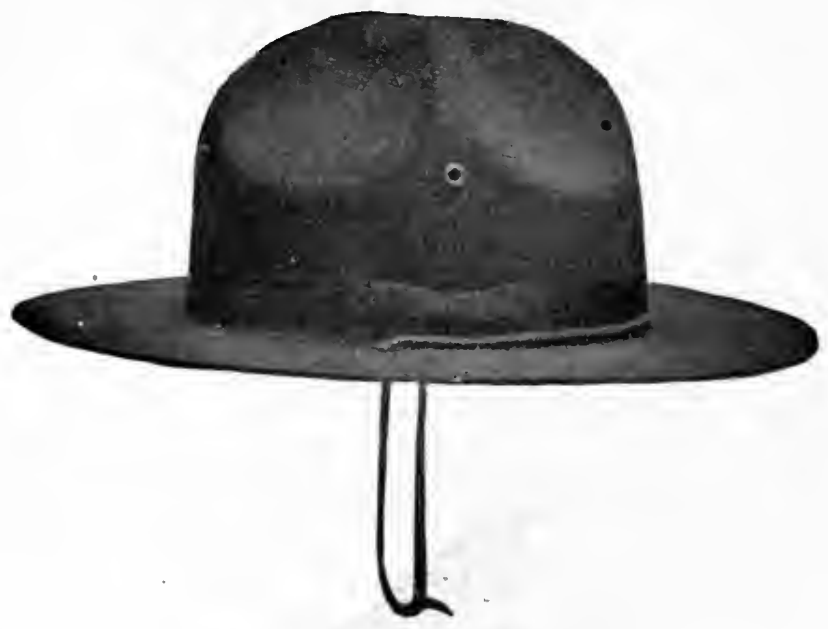

FIG. 98.- Service or campaign hat.
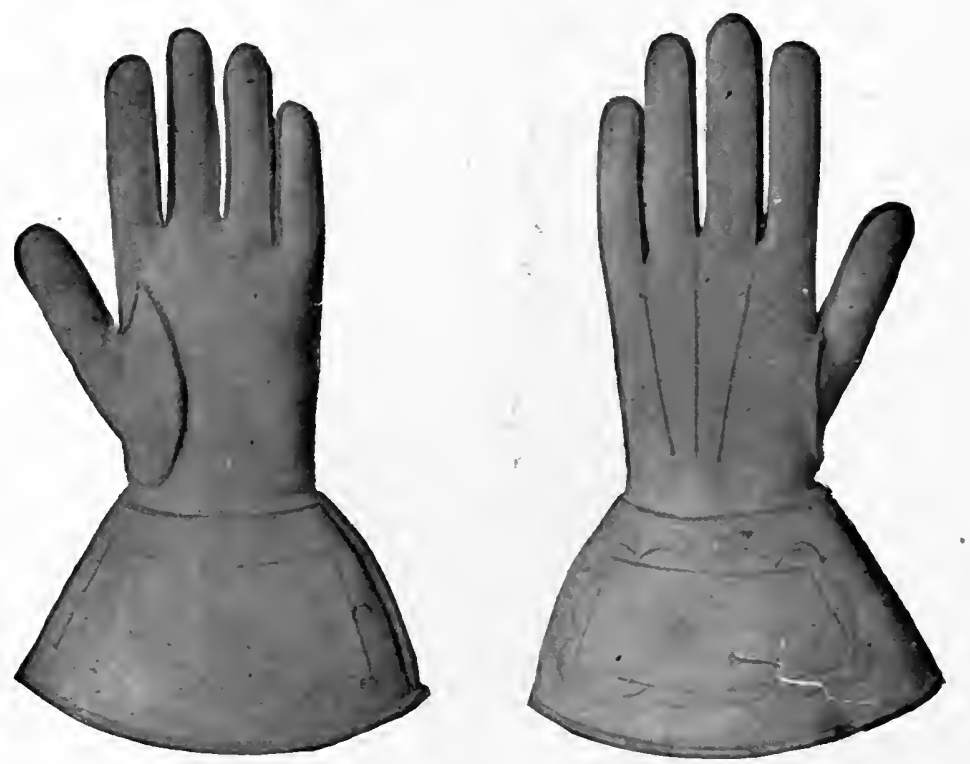

FIG. 99.- Leather gauntlets for mounted men. 
held by a braided cord passed over the back of the head. To the leather sweatband, $\mathrm{I} 3 / 4$ inches wide, is attached a non-rustible reed covered with an oiled muslin backing.

It is a very service ble headgear, protecting also the face, temples and nape of the neck, with larger air space and better ventilation than the cap. Its softness and flexibility allow of rough usage in the field without rendering it unserviceable; in this lies its superiority over the helmet. Creasing the crown reduces the air space and therefore is not advisable. A wet cloth may be placed within it to cool the head.

The recommendations of the Infantry Equipment Board of I9I2, that the brim be $3 \frac{1}{2}$ inches wide, and that a ventilating cork pad be placed under the sweatband in front, are judicious. Some such de-

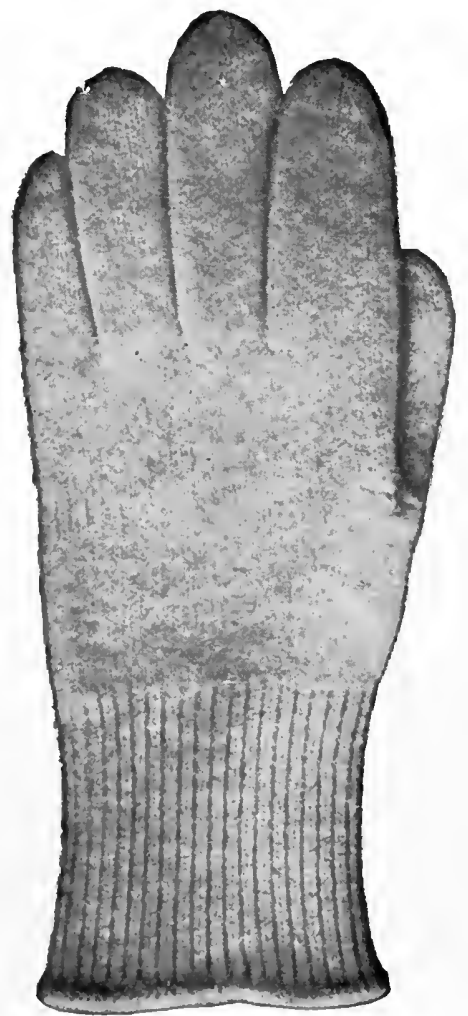

Fig. 100.- Olive-drab woolen glove. 
vice to permit the air to circulate freely around the head is highly desirable, at least in warm regions. The cord, when used around the back of the head, is uncomfortable and not effective in keeping the hat on. A recent regulation directs that "the hat strings provided on service hats will be used, tied either under the chin or as chin straps on the point of the chin."

In very cold regions, canvas caps lined with olive-drab kersey, which cover the head and neck, may be issued to enlisted men; this takes the place of the former fur cap now only allowed in Alaska.

A worsted knitted cap, light weight, has also been recommended by the Infantry Equipment Board of 1912, for use in climates requiring it, to be worn at night, or over the ears under the hat on the march. It is to be carried, normally, in the pocket of the overcoat or sweater.

Gloves. - The gloves issued are: leather gauntlets for mounted duty (Fig. 99); leather gloves for men of the coast artillery and ordnance department, to protect their hands when handling guns; white cotton or olive-drab woolen gloves (Fig. IOo), for dismounted duty; thick buckskin mittens (admitting woolen gloves inside) or fur mittens (Fig. IOI) for very cold regions.
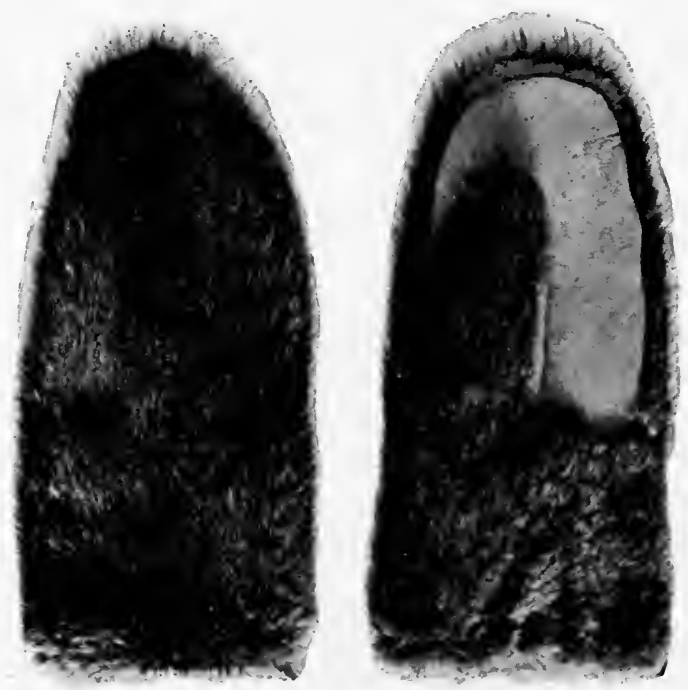

FIG. Ior.-Fur mittens, fleece-lined. 
Abdominal Band. - The woolen abdominal band, formerly highly recommended in the tropics, may be occasionally useful in the case of men predisposed to gastric or intestinal disturbances, but should only be worn at night and when the abdomen is not otherwise suitably protected. It is no longer isstued. When the bowels need special protection, the triangtular apron advocated by Woodhull is preferable to the band. It consists of two thicknesses of flannel sewn together, and is suspended from a tape tied around the waist see page 704). 


\section{CHAPTER XXXVI.}

\section{UNIFORM (CONTINUED).}

\section{FOOT-WEAR.}

In warfare, the chief aim of the commanding general is to occupy advantageous positions with the greatest possible number of men in the shortest possible time. This is largely governed by the marching capacity of the men which, in its turn, depends to a great extent upon their foot-wear, for this, according to Marshal Niel, is to the infantry what horses are to the cavalry. It has been observed in the French maneuvers that, in the first few days, 15 per cent. of the infantry have their feet more or less injured by ill-fitting shoes. In I883, Von Lindau estimated that, in Germany, there were, each year, 60,000 conscripts exempted from service on account of disability produced by foot-wear. It has been estimated that an average loss of Io per cent. must be expected from this cause in European armies, among unseasoned troops on taking the field. Therefore the importance of well-fitting, comfortable and serviceable shoes cannot be overestimated. Each man should watch his shoes and do such precautionary mending as he is able, to save them from becoming unserviceable, or else take them to the company shoemaker who, on the march, should have his tools and materials carried in the company wagon or advance train.

Before determining the most suitable covering for the foot it is necessary, first, to know something about its anatomical lines: The foot is remarkable for its lack of synmetry from whichever side it is looked at. The plantar surface is distinctly arched lengthwise and also, but more slightly, from side to side, a structure which combines strength with elasticity. The arching is greatest on the everted internal border and disappears on the external border which touches the ground. In the standing postitre, the foot rests on the heel, the head of the first metatarsal bone (ball of the big toe) and the outside border. In a normal, well-shaped foot, the internal border is practically straight, that is to say, if placed side by side, the feet will touch each other from heel to tip of big toe, the shallow lateral curve of the arch excepted. (Fig. I02.) According to Meyer: "The great 
toe must lie in such a position that its axis, when carried backward, shall pass through the center of the heel." This "Meyer line" is now generally accepted as practically correct. (Fig. IO3.) The external border, on the contrary, describes a marked curve, from the

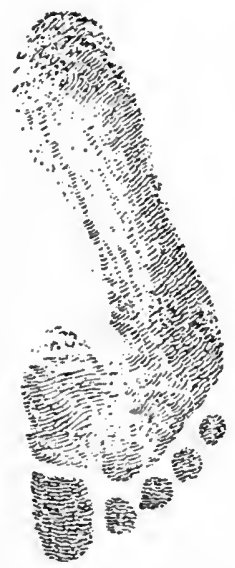

FIG, 102. - The normal foot and footprint. (Munson.)

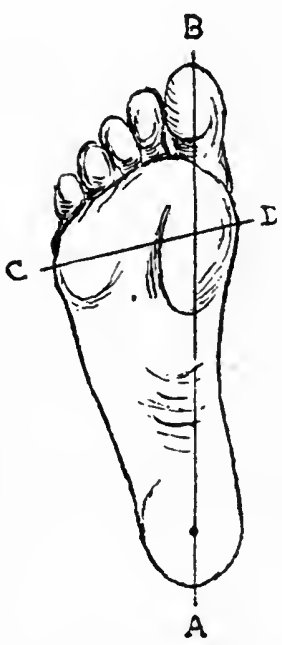

FIG. IO3.- The normal foot. A-B, Meyer's line; $\mathrm{C}-\mathrm{D}$, ball line.

tip of the big toe (the longest of the toes) outward along the receding toes, the summit of the convexity being opposite the base of the little toe. The widest part of the foot is an oblique line drawn from the base of the big toe to that of the little toe (ball line). It is to be noted that the ridge of the instep does not run along the middle line of the foot but inside of it and toward the big toe.

A badly-shaped shoe, or one too short or too narrow, produces a misshapen foot and various lesions which seriously impair the efficiency of the soldier, such as corns, callosities, jamming and clubbing of toes, ingrowing nails; some of the distal phalanges become bent down (hammer toes). Most commonly the toes are crowded toward the middle line, the second and third overriding the others, while the big toe, not infrequently, is partly dislocated at the metatarso-phalangeal articulation, which may swell into a bunion. This displacement and deformity of the toes deprive the foot of much of 
its propulsive power in walking. It must also be borne in mind that any interference with the full play of the arch by tight shoes not only impairs the efficiency of the foot but greatly increases the fatigue of the march by the more direct transmission of the jar of the step to the body. Soreness of feet from any cause will also make the

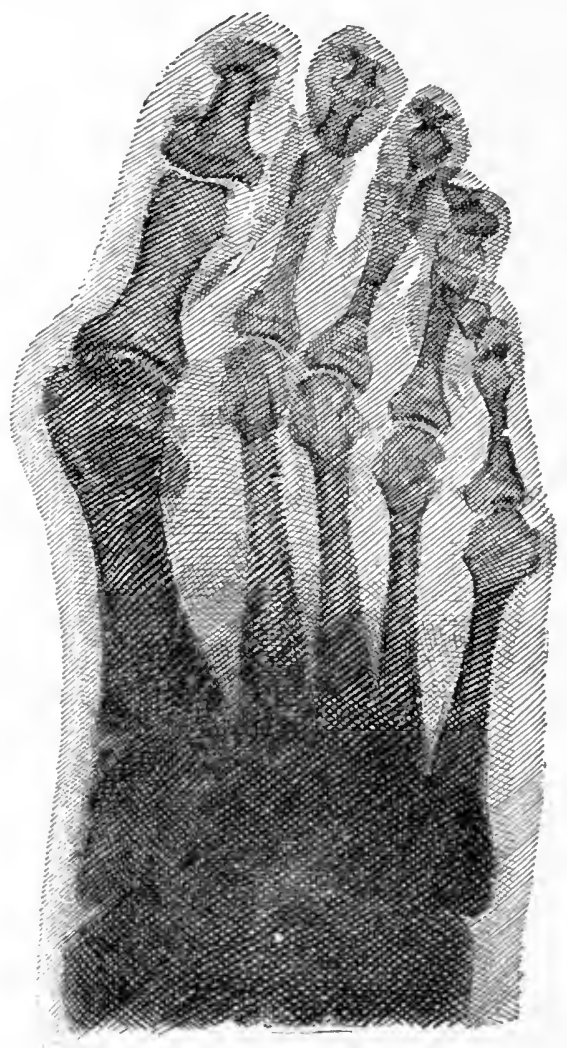

FIg. 104.- Radiograph of foot of soldier, showing hallux valgus and clubbed toes due to bad shoes. (Munson.)

sufferer bring into action unusual muscles in order to diminish the movement or pressure which produces pain, thus adding another cause of muscular effort and fatigue.

Hallu.x valgus is the name given to the very common condition described above, in which the great toe is pushed away from its 
proper, straight inner line (Fig. IO4), its two phalanges making a marked angle with the first metatarsal. The big toe is such an important part of the foot that its displacement, if more than slight, has a very appreciable effect upon the soldier's ability to stand and walk, while, if considerable, it may constitute a cause for rejection in applicants for enlistment.

The Meyer line is only seen in the perfect foot, that is, in the foot of children and of adults who have never worn shoes. In all civilized, shoe-wearing people, the big toe is more or less pushed out of line, so that a certain degree of hallux valgus has come to be regarded as practically normal and must be given due consideration in the construction of the shoe last, so that there be no loss of space or excess of material in the shoe.

Munson has called attention to the fact that there is a military foot type, distinct from the average civilian foot, that is to say, a better-shaped, stronger, more stocky and muscular foot; a difference due, in the first place, to the selection of the recruit, and to the active marching exercise to which he is subjected. The military shoe, therefore, requires a special last, one that follows more strictly the anatomical lines of a normally and strongly developed foot.

The foot should be measured while standing, for the mere weight of the body causes it to spread I/3 inch in length and I-5 inch in width, while, under a heavy weight, such as the soldier may carry in the field, the elongation may reach at least half an inch and the widening at least a quarter inch. After a long march the whole foot becomes still further temporarily enlarged. In the tropics, according to Giles, the feet (as well as the hands) become a full size larger than at home.

But little benefit would result from a well-constructed shoe unless carefully fitted to each individual foot. This fitting is considered so important that the Q. M. Corps is required to provide 15 lengths of shoes, running from 5 to 12 by half sizes, each length with 6 widths; in all, 90 sizes.

Experience has shown that very few soldiers know how to select suitable shoes, such as will enable them to march for a number of days with comfort and without injury to any part of the foot. Their chief aim, like most young men in civil life, is to obtain the smallest size that will permit them to walk. Therefore, under existing regulations, the company commander is required personally to 
measure the feet and fit the shoes of men of his company. All measurements are taken with the soldier standing in bare feet and with a 40-pound burden on his back, bearing the entire weight upon the foot to be measured. To the actual length of the foot thus obtained, two sizes are added (2/3 of an inch), to provide space for the socks and for the free play and congestion of the foot. The circumference of the foot is taken with the measuring tape around the ball. To verify the fitness of shoes, the soldier having put them on, over light woolen socks, and laced them tightly, is made to stand squarely on each foot, supporting himself and his load so as to maintain easy equilibrium. Fitness in length is ascertained by pressing down the leather in front of the great toe, where there should be no less than half an inch of vacant space. "Fitness in width is verified by grasping the vamp of the shoe at its widest part and bringing the thumb and fingers slowly together; the leather should not be so loose as to wrinkle, nor should it feel hard; tense and bulging.

A suitable fit, especially in width, may require several trials before entirely satisfactory shoes are secured. The guiding principle in this fitting is that shoes larger than necessary seldom do any harm, whereas it is short and narrow shoes which cause the vast majority of injuries. Shoes of the proper length and width, but which are not wholly comfortable, are eased by the application of the shoe stretcher provided by the $Q$. M. Corps. A free use of this stretcher in the shoes just selected is nearly always advisable. By means of adjustable bulbs, stretching of such local areas as are especially liable to cause friction, blisters or corns is easily accomplished.

With the few men who, on account of anatomical peculiarities, are obliged to wear somewhat too loose shoes in marching, the Coindreau strap, as used in the French army, is recommended. It is $3 \mathrm{~mm}$. thick, $\mathrm{I} 5 \mathrm{~mm}$. wide, and 75 centimeters long. It is passed under the foot, crossed over the instep, passed around above the heel and buckled on the outside of the foot, above and in front of the ankle, thus describing a figure of eight. This strap keeps the shoe from slipping and prevents chafing.

According to Munson, an excellent method of moulding the shoes to the feet, after careful fitting, consists in making the man, with shoes on, stand in about three inches of water for about five minutes, or until the leather is thoroughly wet and pliable. He then walks on 
a level surface for about an hour, or until the shoes have dried on his feet. This method is particularly valuable where troops are issued new shoes which there is no time to break in slowly, before they must be used for marching.

It has been observed that the foot of a recruit put in the Army shoe tends to broaden, thicken and strengthen, so that another fitting

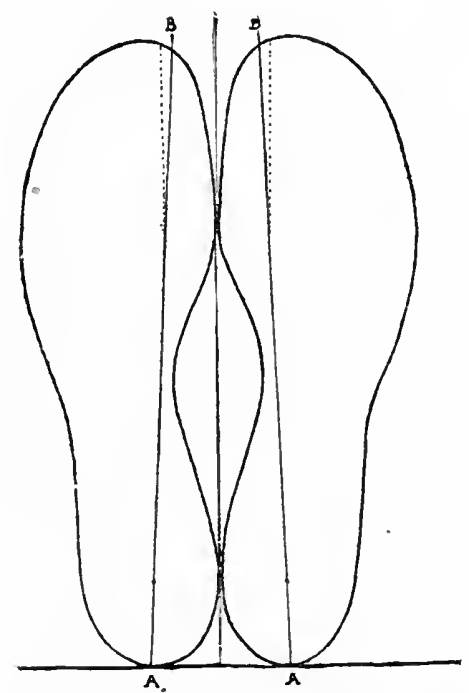

FIG. I05. - Shape of new military shoe. Insoles placed side by side. A-B, Meyer's line as it would be in perfect foot with straight inner border, but slightly deviated outward, as shown by dotted line, in the military shoe so as to fit the average sound foot.

may be needed six months later. Thereafter the soldier's size remains a constant quantity for future requisitions.

Inasmuch as the rational shoe must follow the lines and contours of the foot it follows that instead of having a symmetrical rounded tip, with apex in the middle line, it should be broadly triangular, with the apex of the triangle opposite the big toe, the internal side slightly slanting outward from the ball of the big toe, while the external side is well curved around the toes. In theory, as already explained, the inner edge of the big toes should be straight, that is, 
parallel with the Meyer line, but as the adult foot is never perfectly normal in this respect, the big toe being always deflected more or less outward, the shape adopted in the new military shoe and illustrated in Fig. I05 is practically anatomical. In the narrow part (shank) of the sole, the concavity of the inside border is more marked than on the outside, corresponding to the everted form of the arch. Laced shoes which permit to vary the degree of tightness are preferable.

The requisites of a good military shoe may be summed up as follows :*

It should be strong and substantial, yet sufficiently flexible, the upper and quarter being as soft and yielding as compatible with hard service. It must be comfortable, by proper shape, careful fitting and suitable material. It must be durable by making the soles as thick and strong as the leather permits, and having half soles at hand to be attached as required. In sandy and rocky regions brassscrewed or hobnailed soles are preferable. Box toes have been left off our military shoe on account of the serious defect, when wet and then drying off the feet, of warping and curving down so as to press on the toes; they are replaced by a double thickness of leather forming a toe cap; this must be high so as to avoid pressure on the toes from above, and must rise abruptly from the front of the shoe; as it is soft and pliable it expands where space is most needed.

The shoe must be as light as is compatible with durability. Viry is responsible for the statement that every additional ounce carried on the shoe is equivalent, as regards effect on the wearer, to 100 ounces carried on the back. This additional weight has not only to be carried, but lifted a height of 340 feet in every mile. Our army shoe weighs only 2 pounds 5 ounces, without nails, while the thickly hob-nailed French shoe weighs $33 / 4$ pounds. According to Corbusier it would seem advisable, under certain conditions, "to use a medium-sized hob-nail on the marching shoe, as the roughened surface prevents wear and slipping, both in muddy ground and on hard roads."

The shoe must be easily put on and taken off; to this end the new shoe has a wide half-bellows tongue and only six eyelets on each side, or seven when the size exceeds No. 8. Eyelets and not hooks are used for the laces. The heel should be broad, flat and

* The Soldier's Foot and the Military Shoe: E. L. Munson, U. S. Army. 
long; as the outer edge tends to wear more rapidly than the inner part, it should be heavily reinforced with nails. The stability of the foot is secured by a snug fit over the instep and around the ankles and heel; at these points the contact with the foot must be firm to prevent slipping and wabbling, the posterior wall curving and narrowing so as to embrace the natural curvature of the heel. The sole is flat across but has a slight upward curve at its front end to prevent the toe from striking stones or sinking in the mud, and also to facilitate the heel-and-toe movement of the marching step.

To form a more complete idea of the requirements of foot-wear, we must also bear in mind a physiological peculiarity of the foot, namely, its abundant excretion of sweat, the amount poured out by both feet being estimated at one-fourth of the total quantity excreted by the skin surface covered with clothing. The permeability of leather, permitting the evaporation of the sweat, is therefore a desirable quality, especially in hot weather, but this quality only exists to a limited extent and is further diminished by the use of grease. The result is that the perspiration is liable to accumulate inside the shoe and cause soaking and softening of the skin. These drawbacks are reduced to a minimum by the use of laced shoes which leave openings for the escape of the sweat, and of appropriate socks or stockings. Russet-leather shoes, prepared with vegetable tan, without coloring and filling, and only lightly oiled, are more porous than black shoes and therefore preferable in summer, or at any time for marching.

The chief object of the shoe is to protect the foot against outside cold, moisture and mud, as well as from injury. Moisture in leather, as in textile fabrics, increases the heat conductivity and, in cold weather, may chill the feet. Waterproofing, therefore, may be occasionally advantageous in certain conditions of climate and soil. It is readily done by the soldier himself by rubbing the dry, clean leather, slightly warmed, with neatsfood oil until the proper degree of saturation is reached. Such waterproofing is not perfect nor permanent, but can be renewed when necessary and answers the soldier's purpose. It is better to let the feet get wet now and then than to keep them constantly hot and sweaty by interference with the evaporation of perspiration.

Shoe blacking contains acids which harden and crack the leather. In the field, and in garrison when not required to be polished, the 
soldier's footgear should be cleaned, then lightly oiled or greased. Neatsfoot oil is now issued to enlisted men for the purpose. It is the natural oil of the animal and free from substances deleterious to leather.

Various devices have been recommenaed to increase the elasticity of the shoe and facilitate marching by the use of rubber in the heel and sole, but none has as yet been found sufficiently practical and durable for military purposes.
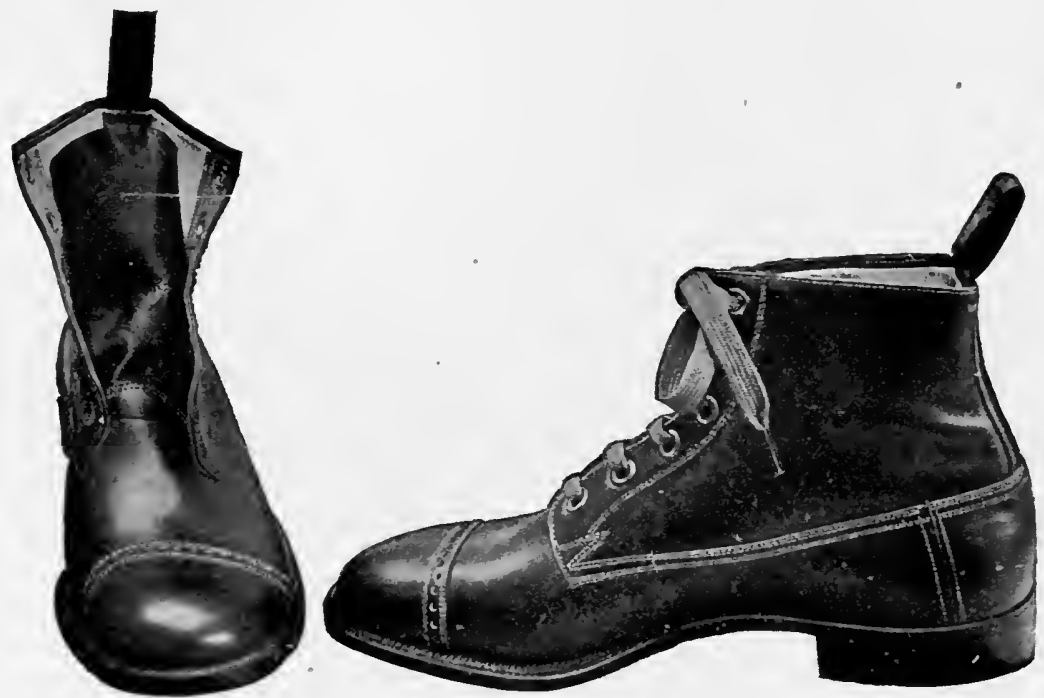

FiG. 106.- Russet shoes.

In our service, the foot-wear issued to enlisted men of all arms is reduced to two forms, the russet or tan-leather shoe and the gymnasium shoe.
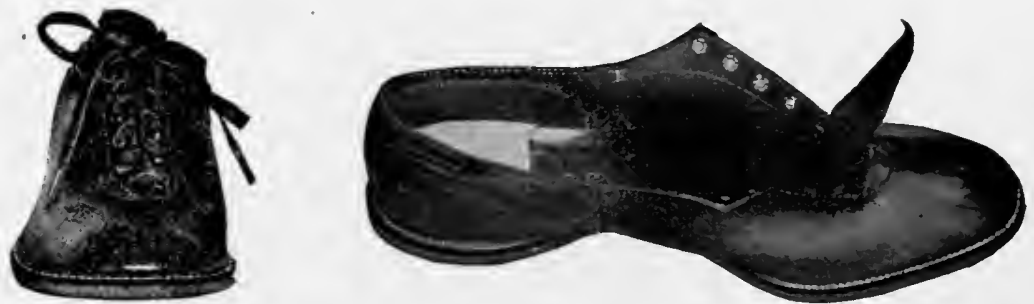

FIG, I07.- Gymnasium shoes. 
The russet shoe (Fig. I06) is a laced shoe, with half-bellows tongue, $6 \frac{1}{2}$ inches high from bottom of heel. It is worn "for all occasions" in garrison and on the march. It is polished with the usual russet dressing in garrison, or oil-tanned with neatsfoot oil in the field.

The gymnasium shoe (Fig. IO7) is a low shoe of soft black
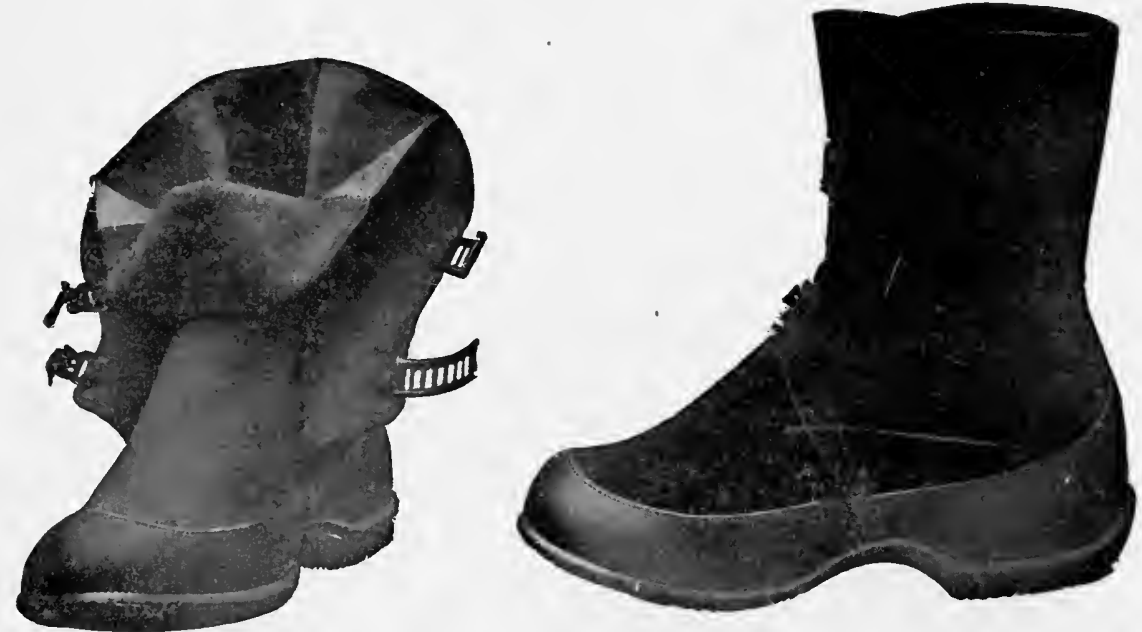

FIG. 108.-Arctic overshoes.

"vici" kid. It is worn in gymnasium work. It may be worn in barracks and may, when prescribed by the commanding officer, be carried in the surplus kit and used as a camp shoe.

Overshoes. - In extremely cold regions, when the necessity therefor is certified by the post commander, arctic overshoes (Fig. Io8) or lined moccasins may be issued to the men.

Leggins. - Canvas leggins are worn by all infantry men with the service uniform. (Fig. Io9.) They are of cotton duck, dyed through in the fiber to the proper olive-drab shade, and fastened with a round braided cord partly concealed. Made in five sizes. A piece of tape stitched on the inside of the back holds a piece of lacing cord which is fastened to the back of the shoe to keep the leggin from slipping up. According to a recent decision, mounted troops are to be supplied with leather leggins fastened with outside strap and buckle. 
The spiral "puttee" has been recommended for our Army by the Infantry Equipment Board of I9I2. It consists of a long woven flannel or worsted bandage which is wound around and supports the leg from ankle to knee, requiring neither strap nor buckle and adapting itself perfectly to the shape of the limb. It is lighter and more easily cleaned than leggins. It also excludes insects and other animals more completely. In spite of these advantages, a properly made and well-fitted leggin is generally considered preferable.

The abolition of the service trousers, and the wearing of breeches
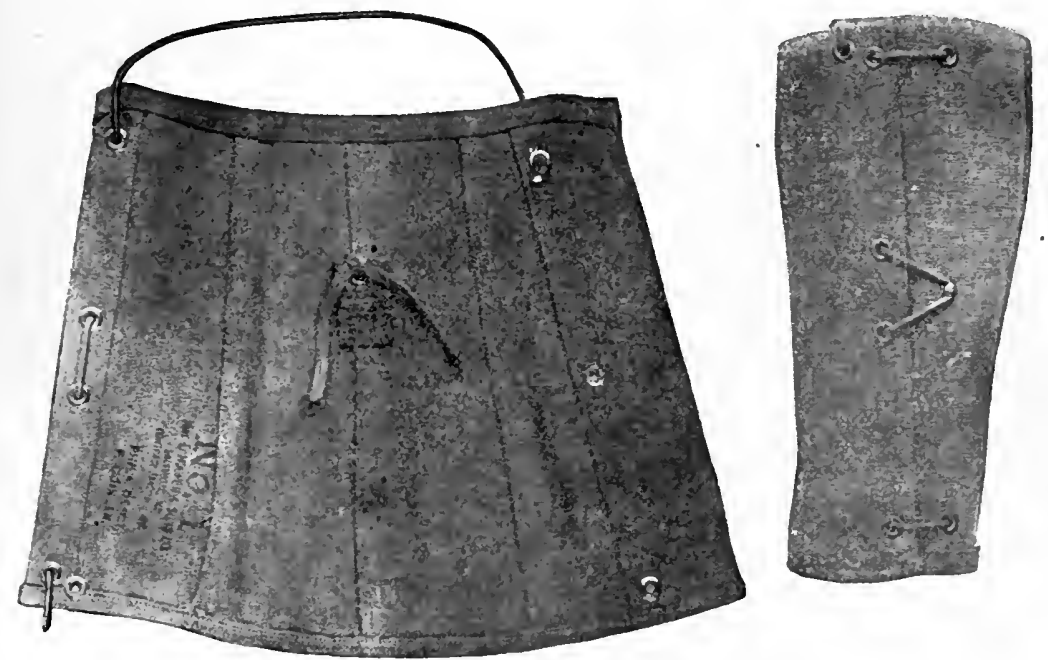

FIg. ro9.- Canvas Leggin, for infantry men.

and leggins by all enlisted men and on all duties (except for the few wearing the white duck uniform), as at first required, was a sacrifice of comfort to appearance, without any compensating gain in efficiency. Leggins thus constantly worn are liable to cause, especially in warm climates, increased perspiration of legs, a predisposition to skin disease and more or less interference with the circulation. Upon the recommendation of the Inspector General, the issue of service trousers (cotton or woolen), to be worn without leggins, is now authorized for the men of the Q. M. Corps when 
not under arms. This desirable privilege was further extended by the following order:

"Trousers, cotton or woolen service, may be issued to and worn without leggins by such enlisted men as are on duty in offices, in the buildings in which offices are situated, to be worn only during the time actually engaged on such office duty."

Socks. - Two kinds of socks are issued, woolen (heavy and light) and cotton. Woolen are in many ways superior to cotton socks, having greater compressibility, elasticity and absorbent power, and are preferable for the soldier in the field, provided their thickness can be varied according to circumstances. Cotton socks may be worn in hot weather or when wool provokes abundant perspiration, but need frequent changing. Each man should be allowed to wear the kind most comfortable to him.

Socks, like shoes, should fit the wearer. If too small they are not only painfully uncomfortable, but wear out more quickly and help to produce displacement and deformity of the toes. Allowance must also be made for the shrinking of woolen socks in washing. As a rule, the soldier should wear socks one size larger than the length of the foot. 


\section{CHAPTER XXXVII.}

\section{EQUIPMENT.}

In the field, the soldier, besides the clothing he wears, carries arms and accouterments, ammunition, extra clothing and toilet articles, intrenching tools and rations, all of which constitute his equipment.

The general requirements of the equipment are the following:

I. Reduction of weight to the minimum consistent with strength and durability.

2. Ease and convenience of carriage by the proper suspension and distribution of the load.

3. Facility of putting on and removal.

4. Easy segregation and removal of unnecessary parts for marching and fighting.

5. Simplicity of construction and ease of reparr.

6. Sanitary construction, so as to prevent fouling irom contents, and permit cleansing and renovation.

To reduce the weight of the equipment to the minimum necessary and distribute it over the body so as to be most conveniently carried and least impede the marching capacity of the soldier are important questions to which much attention has been given. From the experiments of Zuntz and Shumburg the weight carried by the soldier, when exceeding certain limits, reduces his vital (respiratory) capacity to a serious extent, namely, 9 per cent. with a load of 48 pounds, and about i 1 per cent. with a load of 59 pounds. This embarrassment of respiratory movements is often followed by engorgement at the bases of the lungs and an increase in cardiac and hepatic dulness. These disabling effects become less marked after well-regulated practice. From other experiments carried out in Germany it results that the weight (including overcoat) should seldom exceed 55 pounds, and that a load of 65 pounds is oppressive and exhausting, and can only be carried a short distance. The weight of the full equipment of various European countries ranges from 57 pounds in France and 58 in England, to 59 in Germany, 6r in Austria, 64 in Russia and 70 in Japan. The Japanese soldier carries at least onehalf his own weight in winter. A notable diminuation of the weight 
and bulk of the equipment could be obtained in several countries by making a freer use of aluminum.

The weight of our own equipment, as shown further on, compares favorably with that of any other country.

It is highly desirable that the equipment be divisible as in the United States, British and Japanese armies, so that when transpor-

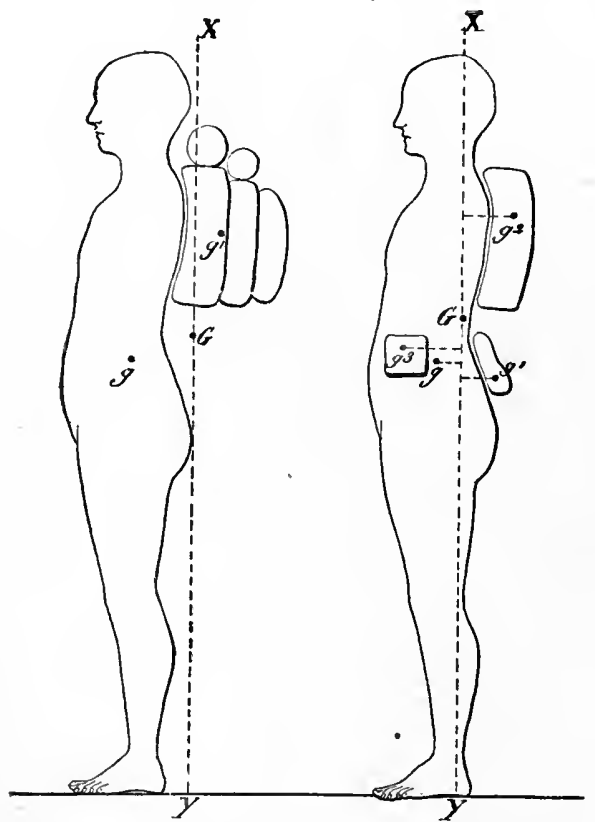

FIG. IIO-CImproper and proper methods of distributing the equipment. (Laveran.) $G$, center of gravity of body and load; $g$, center of gravity of body.

tation is available the men may be relieved from part of it. The load of the British soldier can thus be reduced to 50 pounds.

It is important, however, to bear in mind that there is a point beyond which the equipment cannot be reduced without greatly impairing the efficiency of the soldier. Troops in bivouac, exposed to cold and rain, without necessary food, clothing or covering, suffer much more acutely and lose more of their military efficiency than could possibly have resulted from the addition of a few more pounds to their pack. It must be admitted that the more equipment the 
soldier is able to carry without materially interfering with his marching and fighting capacity, the better will his physical and mental conditions be maintained. Thẹse considerations warrant us to state that it is much safer and wiser to train the soldier, by vigorous marching exercise, to carry as much as possible of his equipment on the battlefield, than to depend upon baggage trains which, in all probability, will fail to turn up when most needed.
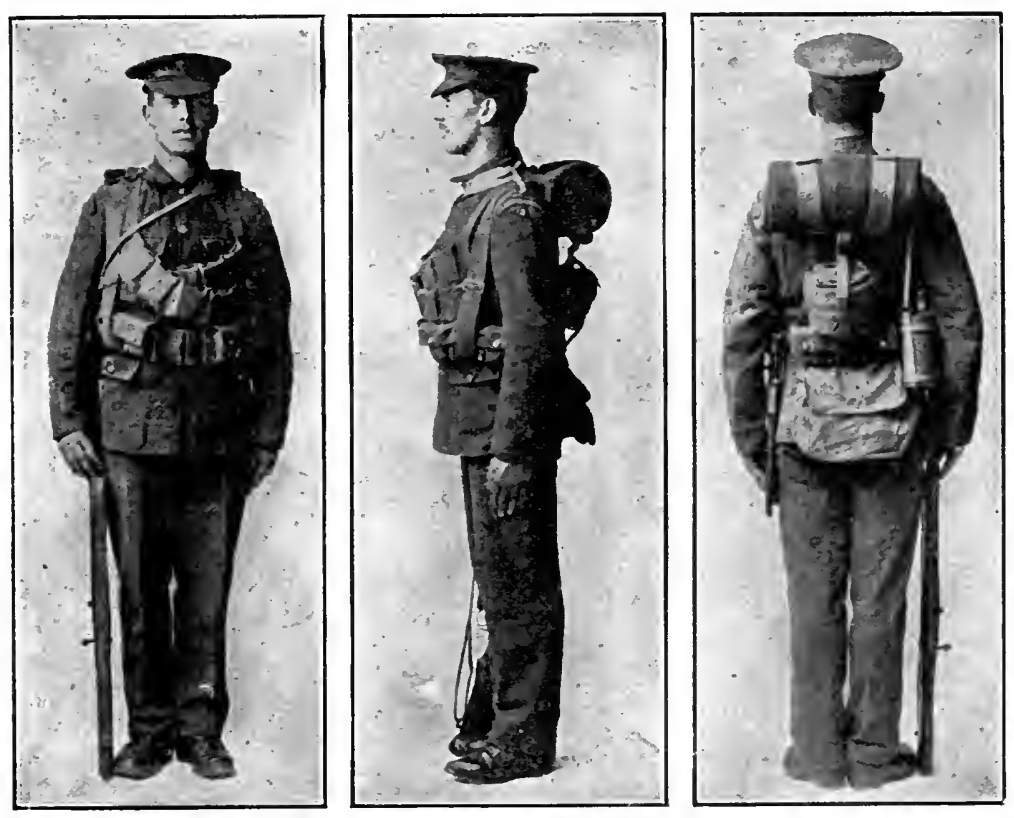

FIG. II I.- English field equipment.

Distribution of Equipment. - The center of gravity of the body is opposite the second lumbar vertebra and 2 or 3 inches in front of it. The line of gravity is a vertical line passing through this point. It strikes the ground on a line connecting the summits of the arches of the feet and midway between them. The weight of the equipment, to be properly balanced, should be as near the line of gravity as possible, and the center of gravity of the load and that of the body should correspond as closely as practicable. (Fig. I Io.) This is best accomplished by distributing the weight around the body over 
as many points as possible; such distribution has the further advantage to bring all available muscles into play without overexerting any special set. The bony parts which practically bear the entire weight are the shoulders, back and pelvis brims. They should all be utilized to the full extent. Pressure over the blood-vessels and nerves of the inner part of the arm, in the axillary region, must be carefully.

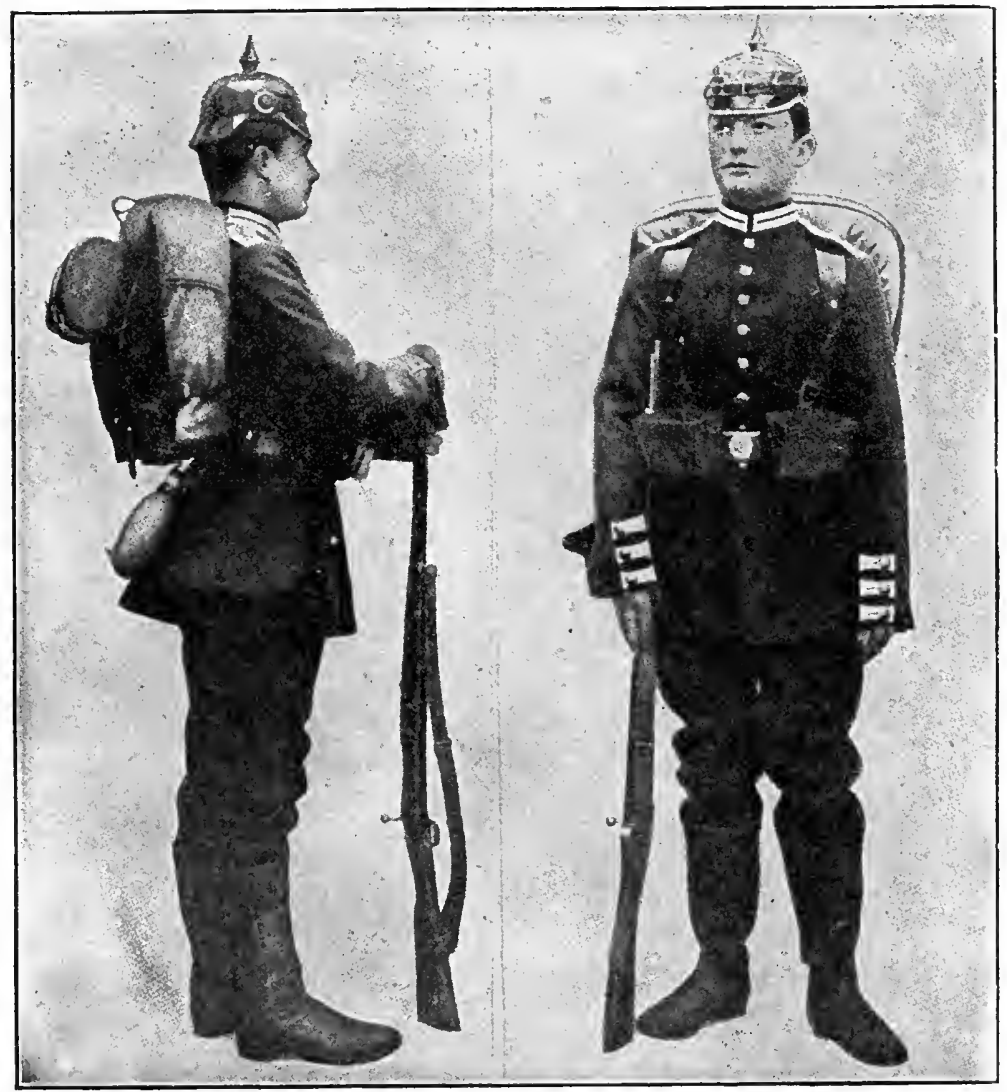

FIG. I 12.-German field equipment. (Lavisse.)

avoided; therefore straps and slings passing under the arm should lie close to the body and run in a direction more vertical than transverse. The free play of the chest is most important and nothing should seriously interfere with it. It is also obvious that nothing 
must impede the free movements of the arms, especially in the handling of the rifle.

Knapsack. - The knapsack is an essential part of the equipment of all European armies: it contains spare clothing. toilet and per-

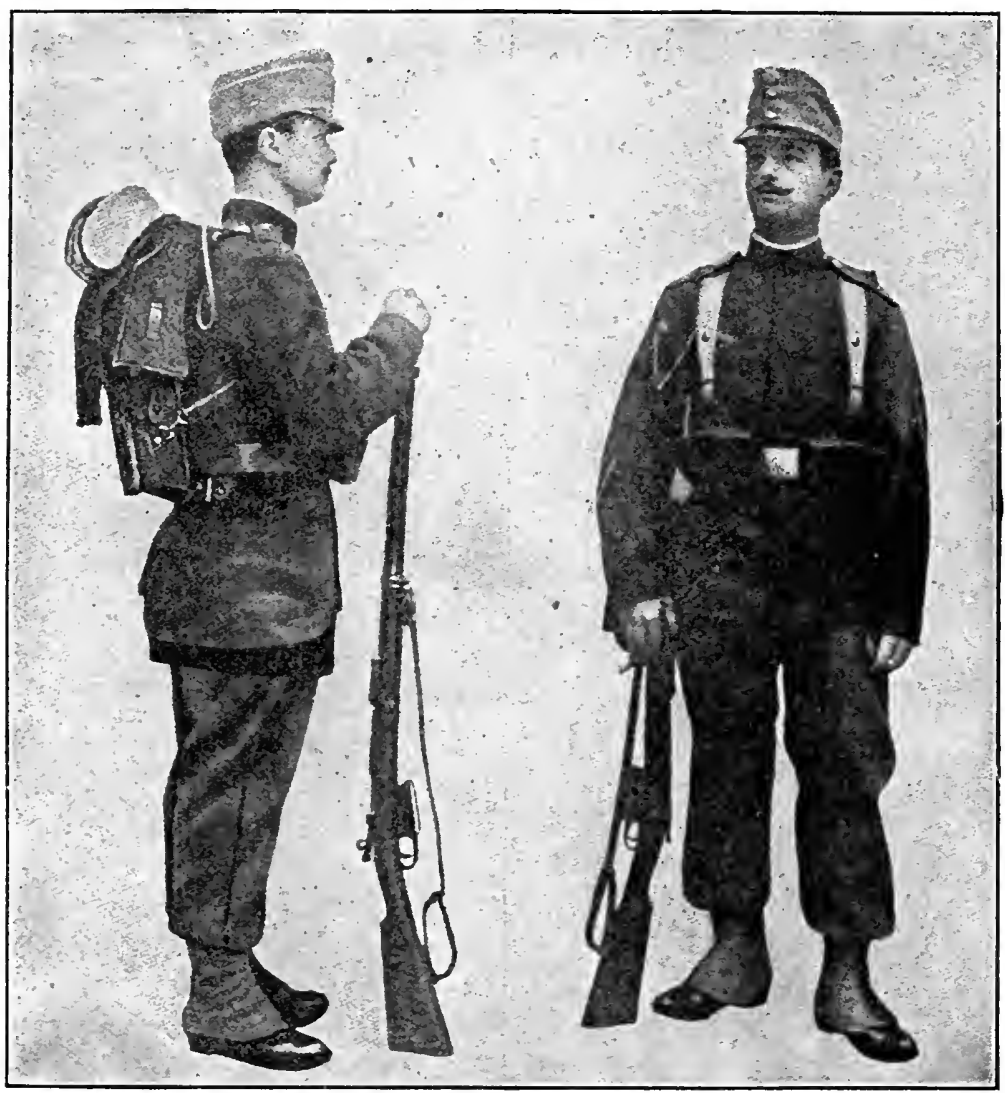

FIG. I13.- Austrian field equipment. (Lavisse.)

sonal articles, sometimes cartridges and rations. According to Lavisse, it should be made of hide covered with its hair, except on the inner face, which should be of pliable leather to avoid heating the back; this inner face should be slightly concave and made to fit the back, thus bringing it nearer to the line of gravity; to secure 
this fit, 2 or 3 sizes of knapsacks should be provided, as in the German army. Lavisse also calls attention to the advantages of giving sufficient length to the knapsack, thus enlarging its capacity, causing it to be supported by more points, and removing the straps

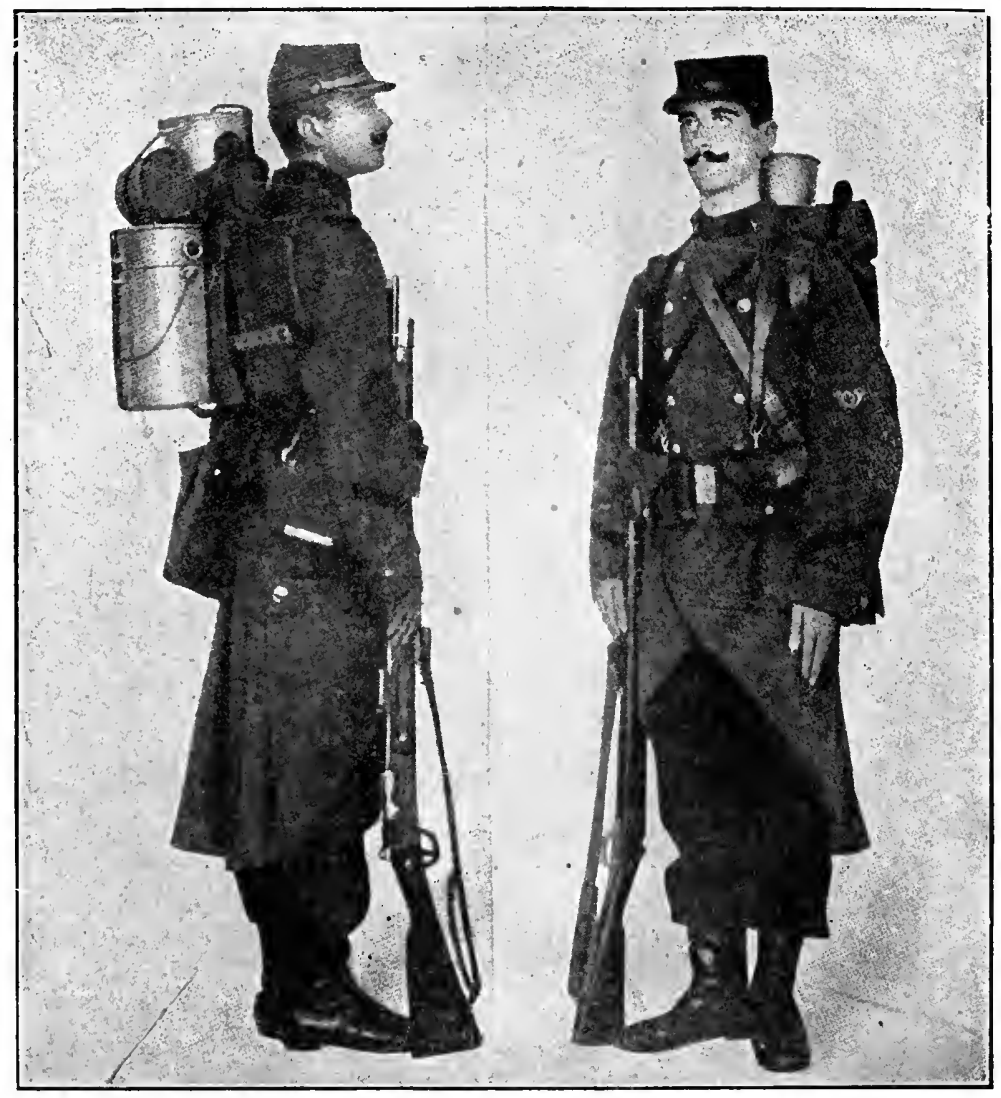

Fig. II4.-French field equipment. (Lavisse.)

attached to its lower end from the axillary blood-vessels and nerves. The French knapsack is particularly defective in this regard, as shown in Fig. I I4. The contact of the sack against the back can be softened by two pads (as in the Swiss army) which have, besides, the advantage of separating slightly the sack from the back, thus 
facilitating the circulation of air between them. This separation is also secured in Norway by means of a wooden framework fitting the back.

The knapsack is suspended by slings from the shoulders according to various methods, and generally connected by straps with the

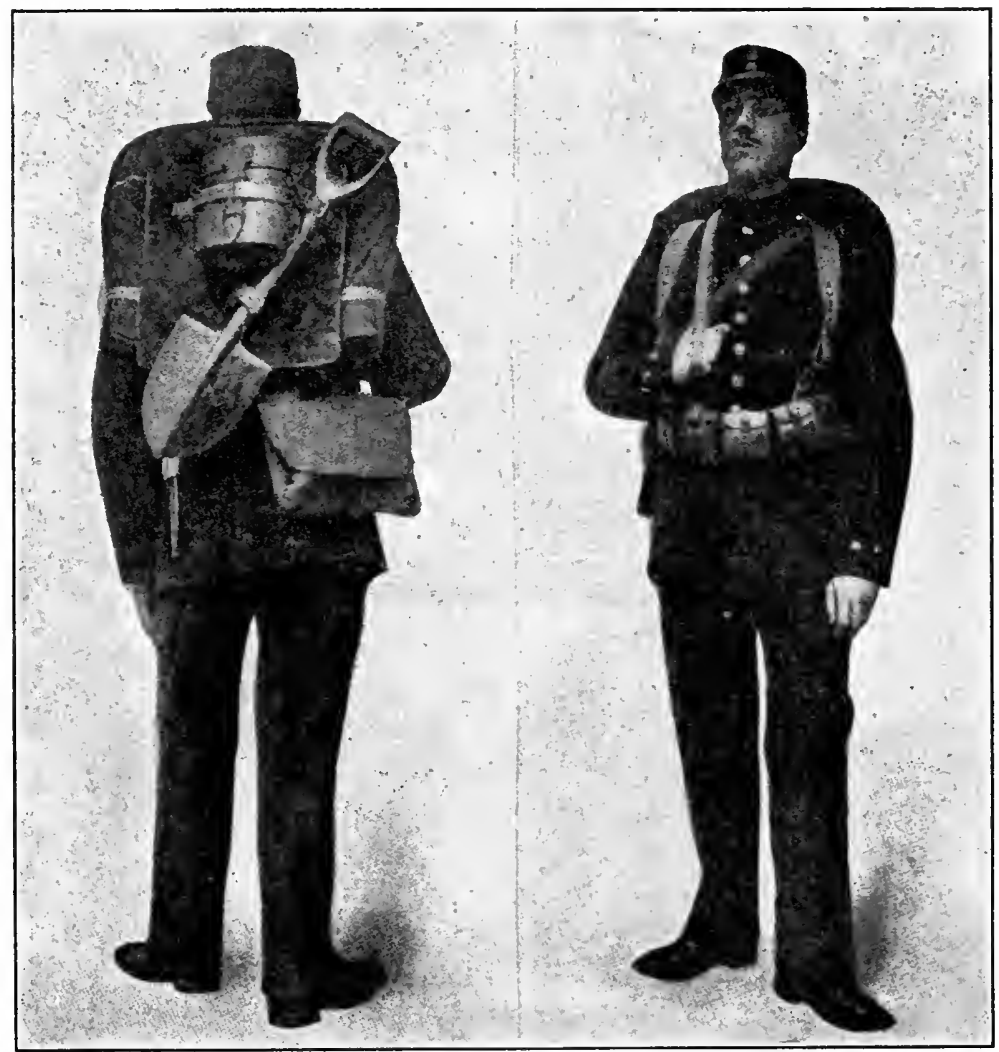

Fig. II5.- Swedish field equipment. (Lavisse.)

cartridge boxes carried on the belt in front, so as to balance the weight.

Ingenious and practical devices have been recommended to support part of its weight upon the hips or lumbar region. Of them, the Merriam, pack (first suggested by Dr. E. A. Parkes, of Eng- 
land) is probably the best. (Fig. I I6.) By means of stiff wooden side braces, the main part of the soldier's load is transferred from the shoulders directly to the large bones of the hips. The advantages claimed for this method are the greatly reduced pressure from the shoulders, entire freedom of motion of the arms and unobstructed ventilation; the abolition of every form of cross belt so that the nerves and vessels of the armpits are wholly relieved from pressure; the strapping of the blanket, shelter-half and poncho around the knapsack out of the way of the rifle; the suppression of the haversack.

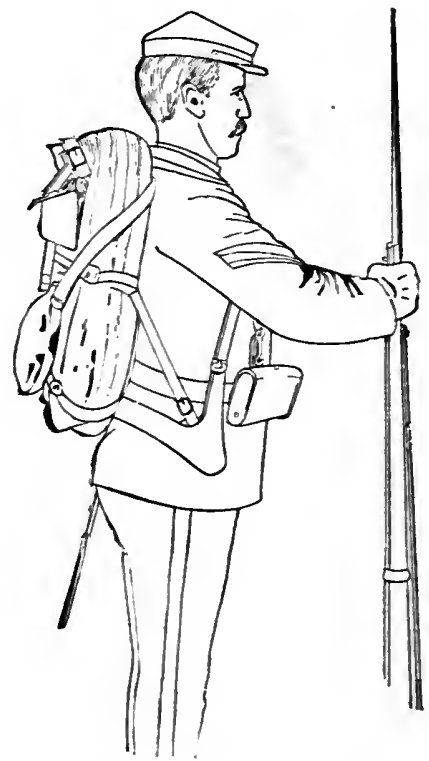

Fig. Ir6.- Merriam pack.

In Europe, this principle of support is only applied in Denmark, where the knapsack rests on a strap lifted by a rod which is secured to the belt. Barthelemy, of the French Army, has proposed the use of a cartridge box, moulded to the curve of the lumbar region and firmly resting upon it, as a support for the sack, a method which, besides, brings the weight of the latter much nearer the center of gravity of the body. 


\section{U. S. ARIY EQUIPMENT.}

In our service the knapsack, never popular, was discarded in Igo8 and replaced by the blanket-roll which, bent into a horseshoe shape. was slung across the chest over one or the other shoulder. (Fig. I I7.) The advantages of the roll were soon found to be more than.
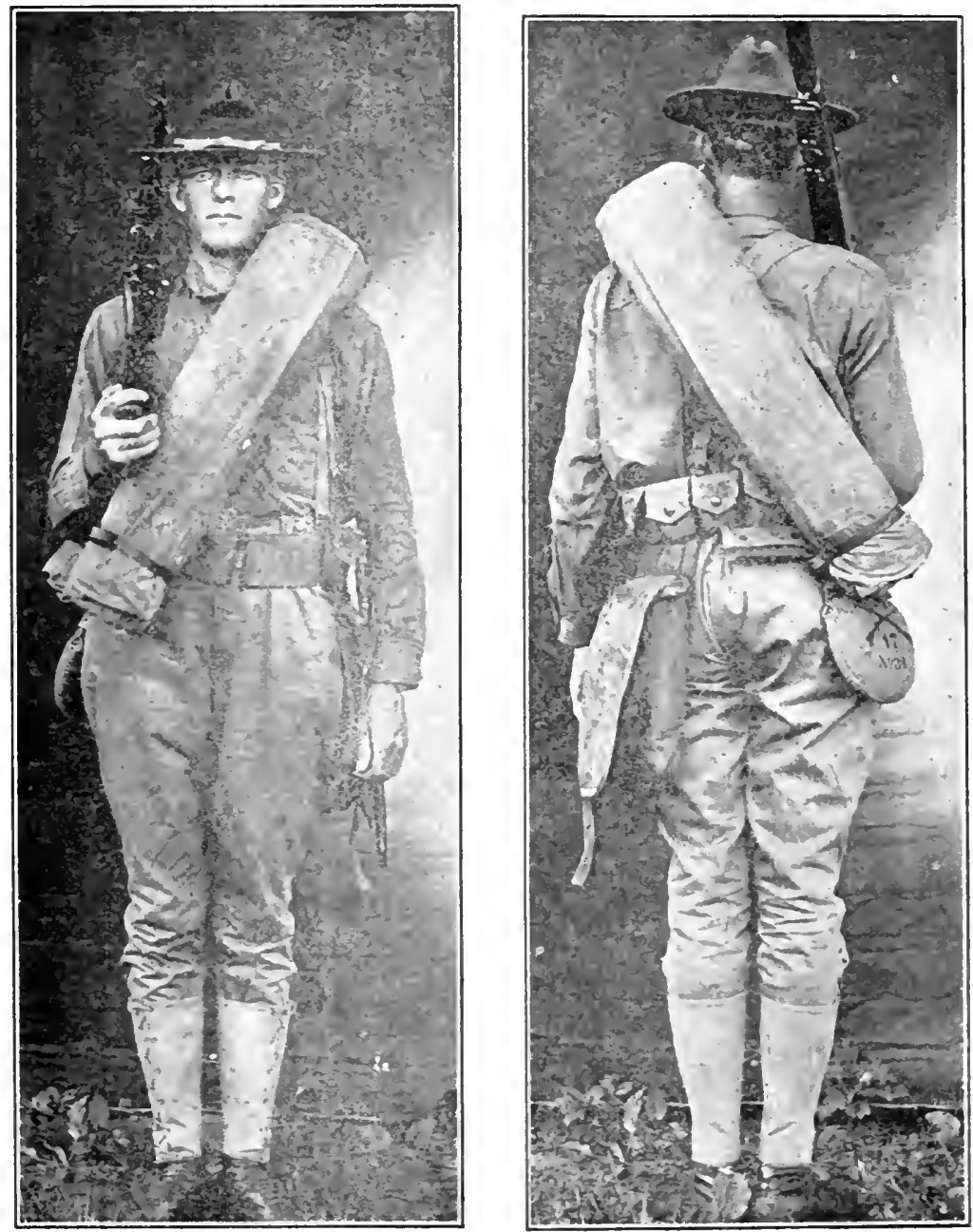

FIG. II7- The blanket roll. Equipment of the U. S. foot soldier, from Igos to 19 TO. 
counterbalanced by its defects. It heats the neck and chest to an uncomfortable degree, restrains the expansion of the lungs and interferes with the free use of the arms and handling of the rifle. In I9IO, it was replaced by the present equipment, which was constructed on entirely new and original lines.

Three forms of it are used:

The full equipment (Fig. II8),

The marching equipment,

The fighting equipment.

The full equipment consists of :

Identification tag,

Rifle and sling,

Bayonet and scabbard,

Cartridge belt,

Ammunition ( Ioo rounds),

Intrenching tool and carrier ( 6 tools carried by every squad: 3 shovels; I pick, I hand ax and I wire cutter).

First-aid packet and pouch,

Canteen, cup and cover,

Haversack, containing:

Mess-kit (meat-can, fork, spoon and knife),

Rations ( 2 reserve rations or I reserve and I emergency),

Socks, one pair,

Toilet articles (towel, comb, tooth-brush, soap),

Pack, consisting of carrier, blanket, poncho or slicker, clothing, shelter-tent half, pole and pins.

The marching equipment is the same as the full equipment, minus the pack which is normally carried in the company wagon.

The fighting equipment is the same as the marching equipment with the addition of two bandoleers of 60 rounds each, thus giving the soldier 220 rounds of cartridges.

The weight of the full equipment is $40 \mathrm{lbs} .5 \mathrm{oz}$; of the marching equipment $3 \mathrm{I}$ lbs. $4 \mathrm{oz}$, and of the fighting equipment $39 \mathrm{lbs} . \mathrm{r} \mathrm{oz}$. To this should be added the weight of the clothing carried on the person (exclusive of coat, sweater and overcoat), namely $7 \mathrm{lbs}$. I I oz. This clothing, for infantry in the field, consists of : service hat, neckerchief, olive-drab shirt, olive-drab breeches, undershirt. drawers, woolen socks, shoes, leggins, waist belt (optional), gauntlets (only when mounted). 
Thus it appears that the weight of the marching equipment, plus the ordinary clothing worn, is $38 \mathrm{lbs}$. I5 oz. When weather conditions require it, the sweater is worn over the shirt, and the weight

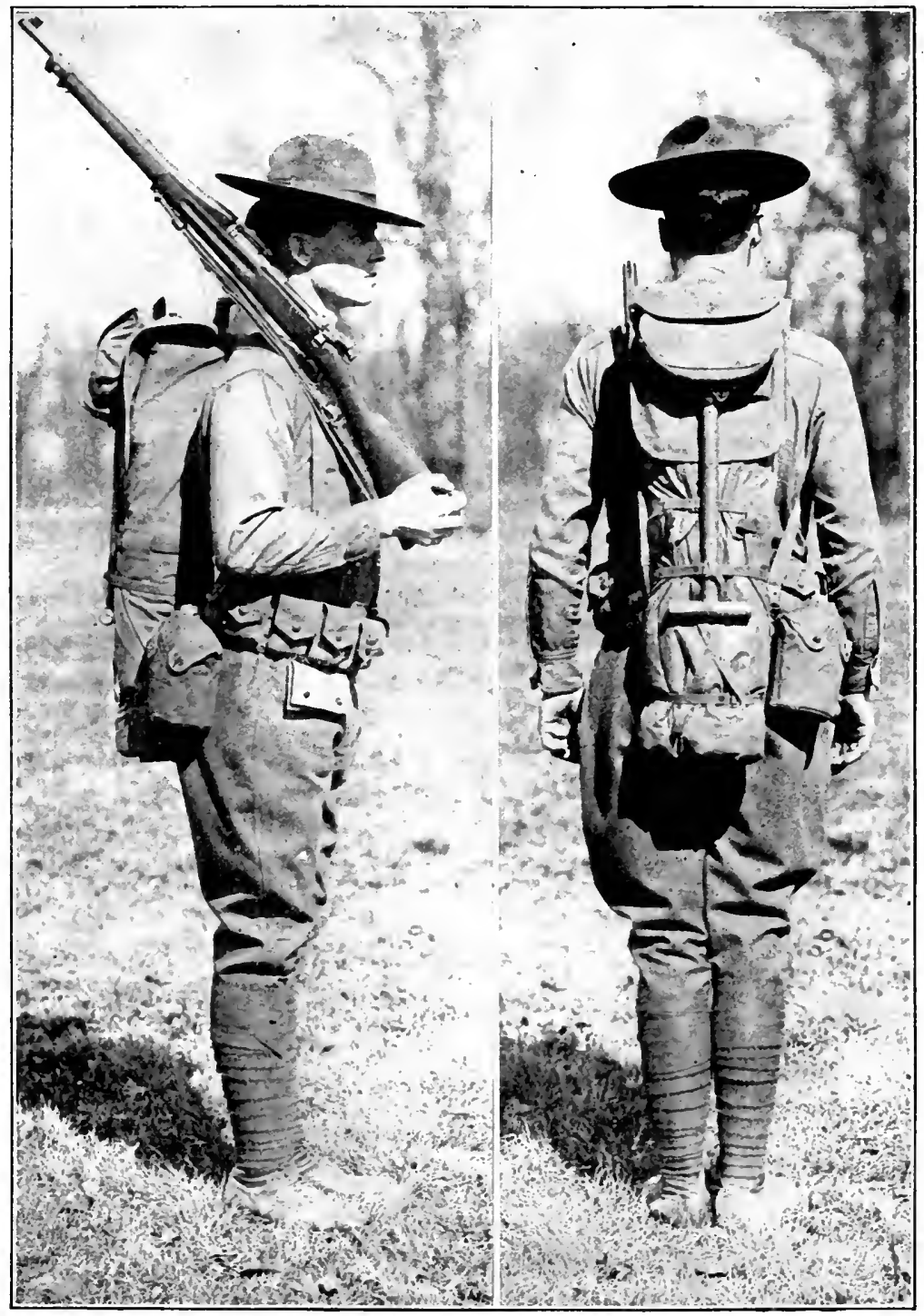

FIG. I I8.- Full equipment, U. S. Infantry. The puttees should be replaced by leggins. 


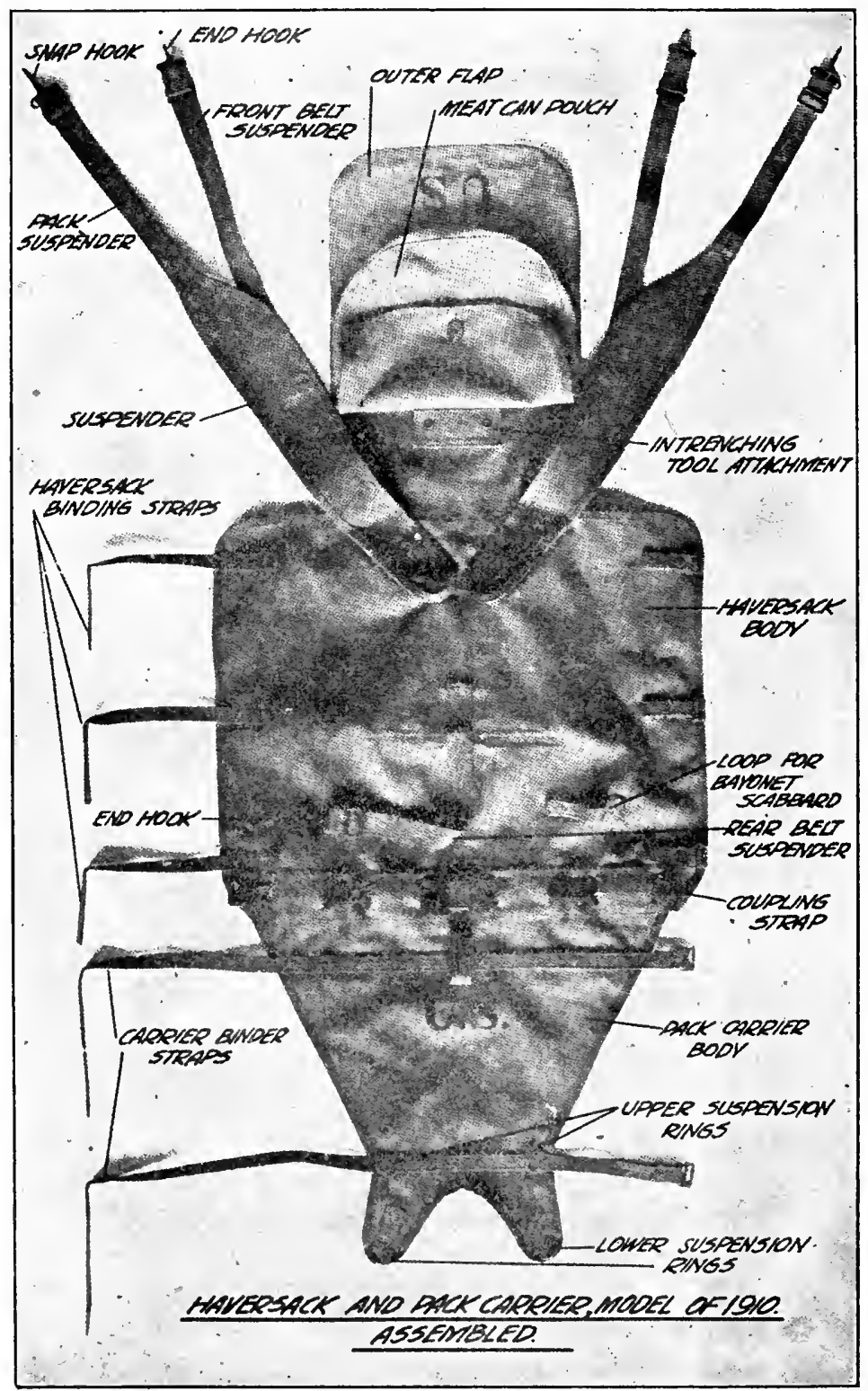

Fig. I19.- The haversack and pack-carrier, assembled and spread out. 
(about 2 lbs.) must be added, making a total of $40 \mathrm{lbs}$. and $15 \mathrm{oz}$. If overcoats and sweaters are not needed they are packed in boxes and left at the nearest convenient depot. If the overcoat is worn, the weight ( 9 lbs.) must be added, making a total of 49 lbs. $15 \mathrm{oz}$. It is doubtful whether this weight can be reduced (except possibly in the overcoat) without sacrificing something of strength and durability.

The "field kit" which is carried on the person by dismounted and on the saddle by mounted men, is supplemented by the "surplus kit," carried in the train, the two together making up the clothing component of the service kit.

The field kit clothing component, in addition to the clothing worn on the person, is composed of the following articles:

I blanket; I poncho or slicker; I drawers, pair; I undershirt; 2 stockings, pairs; I towel; I housewife (for one man of each squad).

The surplus kit consists of :

I breeches, pair; I drawers, pair; I shirt, olive drab; I shoes, russet leather, pair; 2 stockings, pair; one shoe laces, extra, pair; I undershirt.

The haversack and pack-carrier are made of olive-drab cotton duck, bound with olive-drab cotton webbing (Fig. II9). The haversack consists essentially of body, meat-can pouch, flaps and suspenders (Fig. I2O). By means of the inside flap, the body can be converted into a bag of varying dimensions capable of carrying anything up to 4 days' rations. It holds two small tin cans, one for bacon, the other for sugar, coffee and salt (see reserve ration). It can be washed and scrubbed when necessary. The meat-can consists of body (or dish) and cover (or plate), both of. aluminum (Fig. I2I). To the body is attached a steel handle (aluminized) by a hinge of German silver, so that it can be used to cook food on the fire. The cover fits accurately over the body and is held firmly in place by the handle folded over it and caught in the rim of the body. The knife, fork and spoon are carried in the meat-can.

The haversack and pack are fastened together by a coupling strap, forming a compact and roughly cylindrical bundle, 6-8 inches in diameter and, when in position, extending from the level of the 


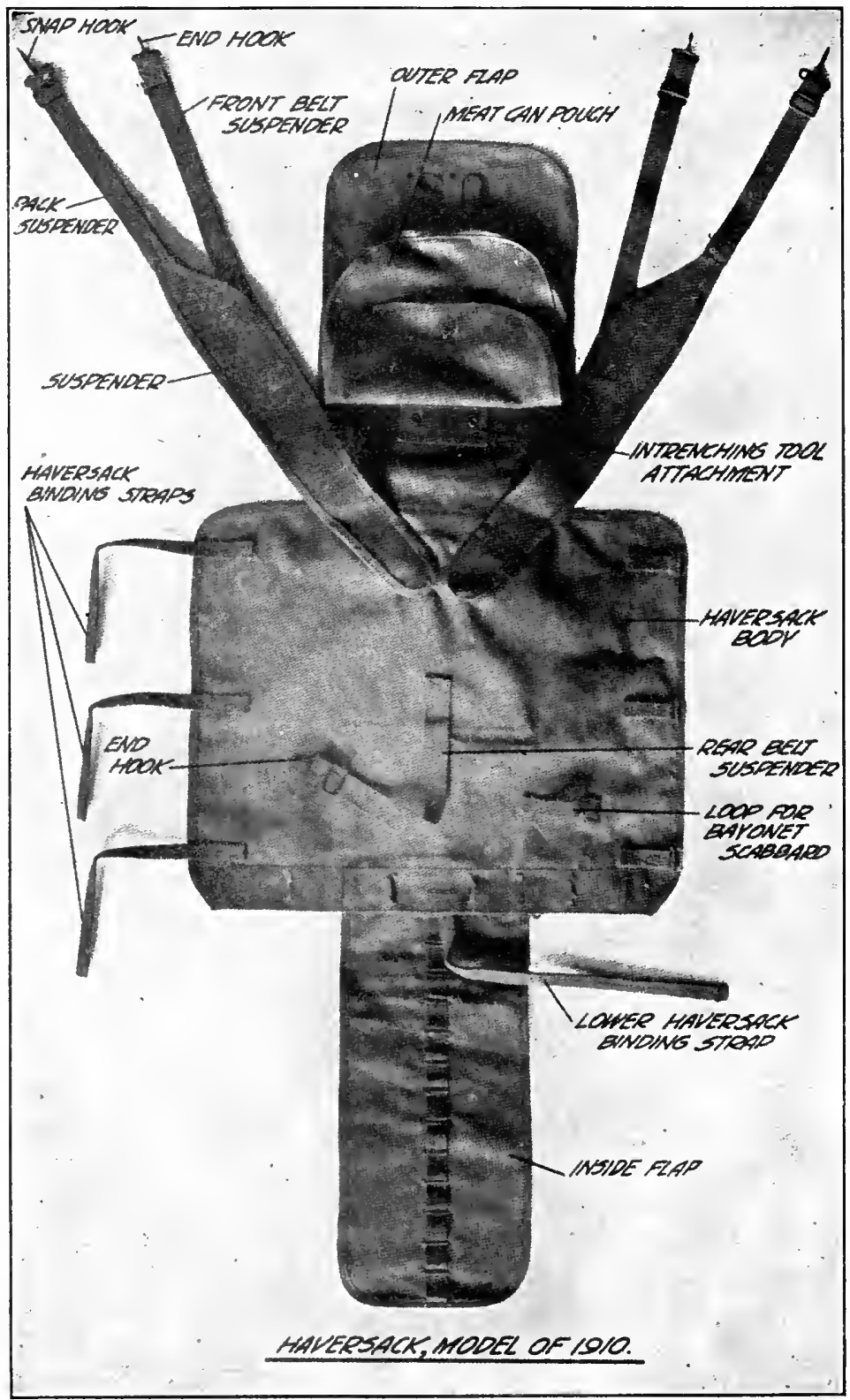

FIG. I20.- Haversack, spread out, the carrier detached. 
shoulders to a point $3-5$ inches below the waist line (Fig. 118). It is held in a vertical position on the back by means of wide suspenders attached to the top of the haversack, carried over the shoulders, beneath the arms and secured to the pack carrier near the bottom of the bundle. A branch is given off from each suspender and attached to the beit in front, thus utilizing the weight of the ammunition to balance the load on the back and bring its center of gravity as near as possible to that of the body. A short back suspender hangs from the haversack and is fastened to the belt in rear to further steady the load and serve as third point of support for the loosely worn cartridge belt.

At halts, during the march, the bearer can quickly remove or replace the bundle, or if he sits down without removing it, the bottom rests upon the ground, instantly releasing the pressure and weight from the body.
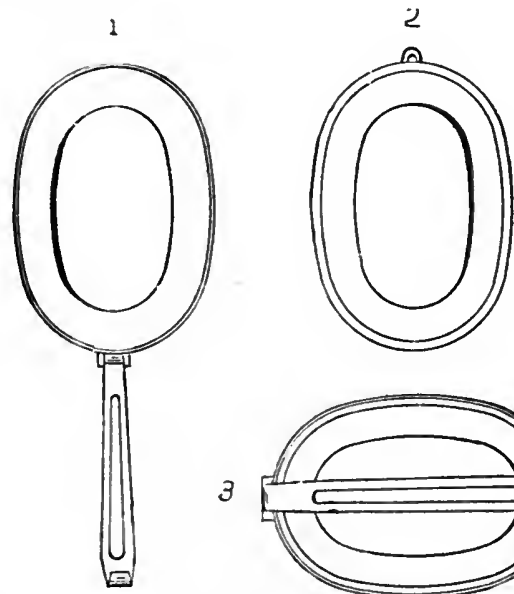

3

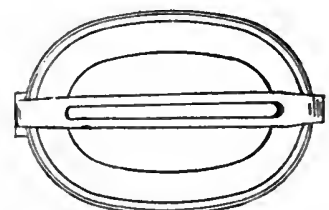

FIG. I2I.-Meat-can. I, Body with handle; 2, cover; 3, meat-can closed.

By withdrawing the coupling strap, the pack is separated from the haversack, in compact shape easily transportable and identified, while the haversack remains in position.

This equipment, as a whole, fulfills all necessary desiderata. The bundle is in its proper place anatomically, supported by the shoulders and spine. Its cylindrical shape presents a minimum surface of contact with the back, interfering but slightly with ventilation. Its center of gravity, in connection with the cartridge 
belt, is brought reasonably near that of the body. It hangs comfortably, with but little pressure or constriction of muscles, bloodvessels and nerves. It places no restriction upon the full expansion of the chest and free play of lungs and heart, there being no strap of any kind between the suspenders in front; nor is there any interference with the full use of arms and legs or the free handling of the rifle from any position. It might be objected that the suspenders, as they pass under the arms, are liable to compress the axillary vessels and nerves, but inasmuch as their direction is obliquely downward and inward, such compression can seldom have any injurious effect.

The pack, as already stated, consists of a detachable carrier containing the blanket, poncho and shelter-tent half.

Only one type of blanket is issued; it is an olive-drab woolen blanket 7 feet long and $5 \% \frac{1}{2}$ feet wide, with tapes along the sides and bottom, weighing 3 pounds. It has been ascertained that, with the sweater and poncho to supplement it, this blanket will afford ample protection against the ordinary weather of temperate zones. For winter, or service in cold climates, a second or even a third blanket may be issued.

The poncho, for the use of foot troops (Fig. I22), is made of waterproof cotton sheeting, to weigh no less than 2 pounds ro ounces, nor more than 3 pounds. It consists of body, fly and extension. It is 75 inches long, exclusive of extension, and 59 inches wide exclusive of fly, and is passed over the head by a crosswise opening 13 inches long in center seam. The slicker, for the use of mounted troops, is made of the same material, and so shaped as to protect not only the rider but also his saddle equipment.

The poncho and blanket are of stitable size and shape to form a comfortable sleeping bag, the blanket being folded and tied together by means of its tapes, and the poncho buttoned together over the blanket. A double sleeping bag can also be made by means of two ponchos with two blankets between, the latter being tied together along the edges and the foot end.

Cartridge belt. - (Fig. I23). This is a woven webbing belt, 3 inches wide, consisting of two pocket sections, right and left, connected by an adjustment strap at the back, 2 inches wide. Each section has 5 pockets of the required size for holding two clips of five cartridges, each pocket with flap secured by a glove fastener. 
In the belt for mounted men, the front pocket, on each side of the fastener, is replaced by two smaller pockets, one above the other, and of suitable size for holding five revolver cartridges. Thus it is seen that the ammunition is always in front, and the adjustment entirely in rear. The belt is adjusted so as to fit loosely about the

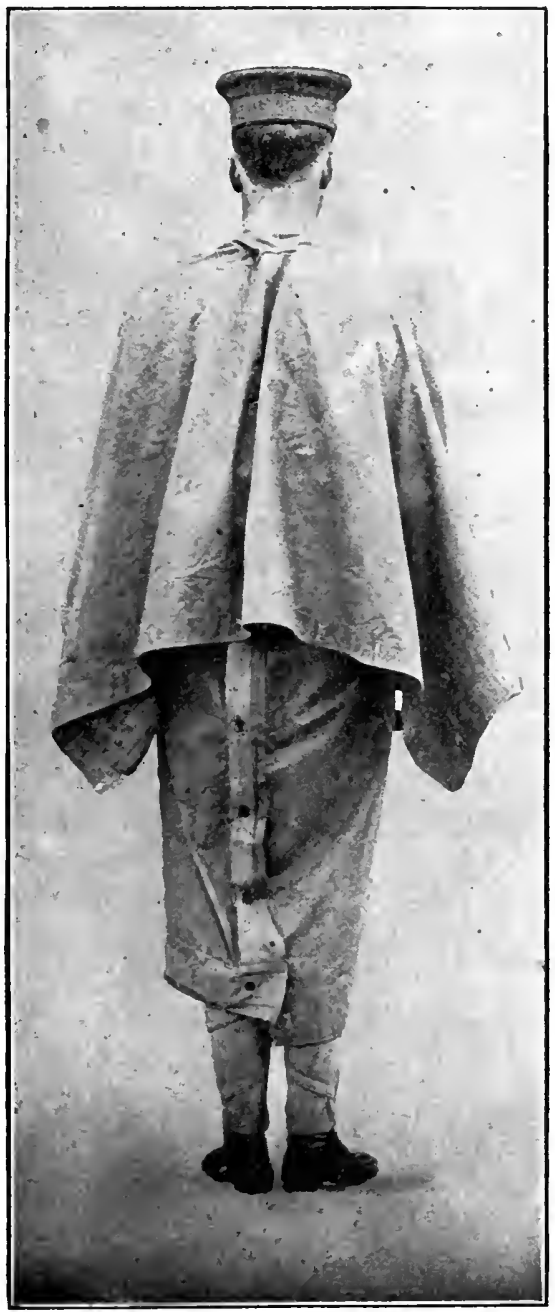

FIG. 122.- Poncho, for U. S. Infantry. 
waist and rest well down over the hip bones, and below the pit of the abdomen in front. It is held up by three suspenders from the haversack, one in rear and two in front, all hooked into corresponding eyelets in the upper edge. Therefore it is supported, as it should be, from the shoulders and by the hips. It also balances the weight of the bundle on the back and tends to bring the center of gravity of the equipment towards the front. To the belt, on the right side, are suspended the first-aid pouch and the canteen cover by double-hook attachments in the eyelets between the pockets, on the lower edge, the former under the second pocket and the latter under the rear pocket.

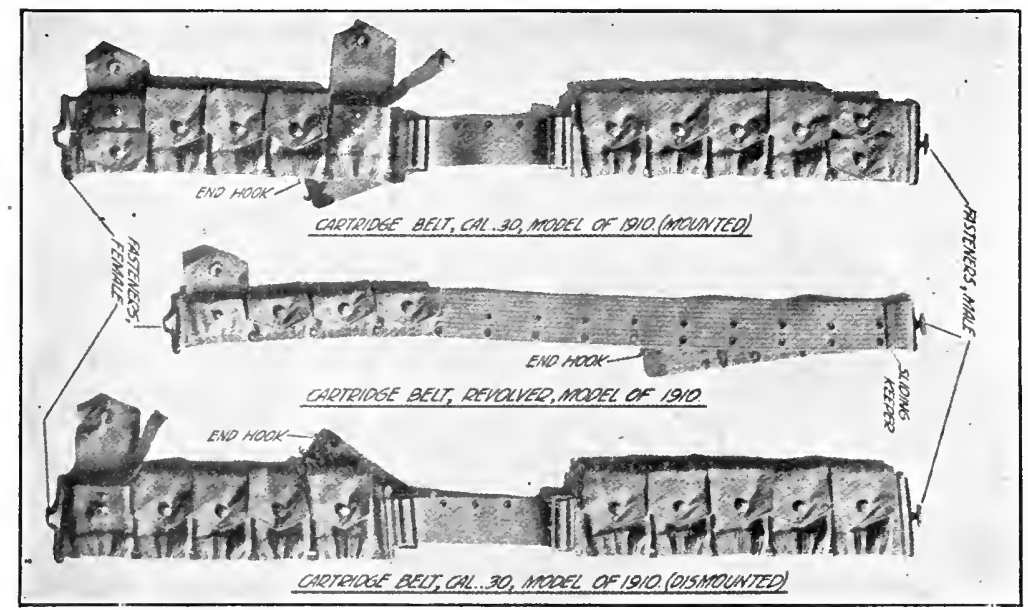

FIG. I23-Cartridge belts, for infantry and cavalry.

Canteen and cup. - The canteen is flask-shaped with a flat bottom, slightly concave on the side next to the body, and is nested in the cup for transportation. The body is of one piece of seamless aluminum and has a capacity of two pints. The neck is threaded to receive the aluminum screw cap which is held by chain and sliding ring also of altminum. The canteen cover is of olive-drab cotton duck, lined with gray felt, both materials stitched together; the top of that portion forming the back terminates in two flaps which, with the canteen and cup in position, fold over and are secured to the front part by glove fasteners. A double-hook attach- 
ment is stitched to the back for suspension to the belt under the rear pocket on the right. The canteen can be readily withdrawn from its cover in order to heat or sterilize its contents, and as readily replaced.

The cup is of one-piece seamless aluminum drawn and shaped to correspond to the lower half of the canteen. The folding handle, when open and fixed in position makes an additional support for the cup when placed on the ground. When the cup is not in use the handle is sprung over the cup body.

The aluminum canteen has been objected to on the ground that it imparts a metallic taste to tea and coffee kept in it; but this is due to the metals with which aluminum is alloyed, principally iron and copper. Pure aluminum, or aluminum alloyed with magnesium is not open to this objection.

The pouch for first-aid packet is of olive-drab cotton duck, with flap secured by two glove fasteners, and a double-hook attachment for suspension to the lower edge of the belt under the second pocket on the right.

\section{CAVALRY EQUIPMENT.}

In the cavalry, the equipment is distributed on the saddle as shown in Fig. I24 (Model of I912), the trooper carrying only the cartridge belt and bandoleer on his person. The cantle roll is formed of the shelter tent containing tent pins, underclothing and toilet articles. It is about 30 inches long, its ends resting on top of the ration bags. The ration bags will carry, together, two reserve rations and one emergency ration. They are so constructed as to permit of their being assembled into a haversack for use on dismounted duty; it is carried on the trooper's back and secured by suspenders and belt. When on such duty, a roll about 42 inches long, consisting of saddle blanket, or shelter tent, containing necessary clothing and toilet articles is placed symmetrically on top of the knapsack, the ends bent down. and secured to the top and sides of the knapsack by the thongs provided for this purpose.

\section{MEDICAL DEPARTMENT EQUIPMENT.}

The Medical Department has recently discarded the large, unwieldy pouch carried by the members of the Hospital Corps, and replaced it by a belt carrying Io even-sized pockets. From this belt depend an instrument case, the canteen and a hand axe (which 


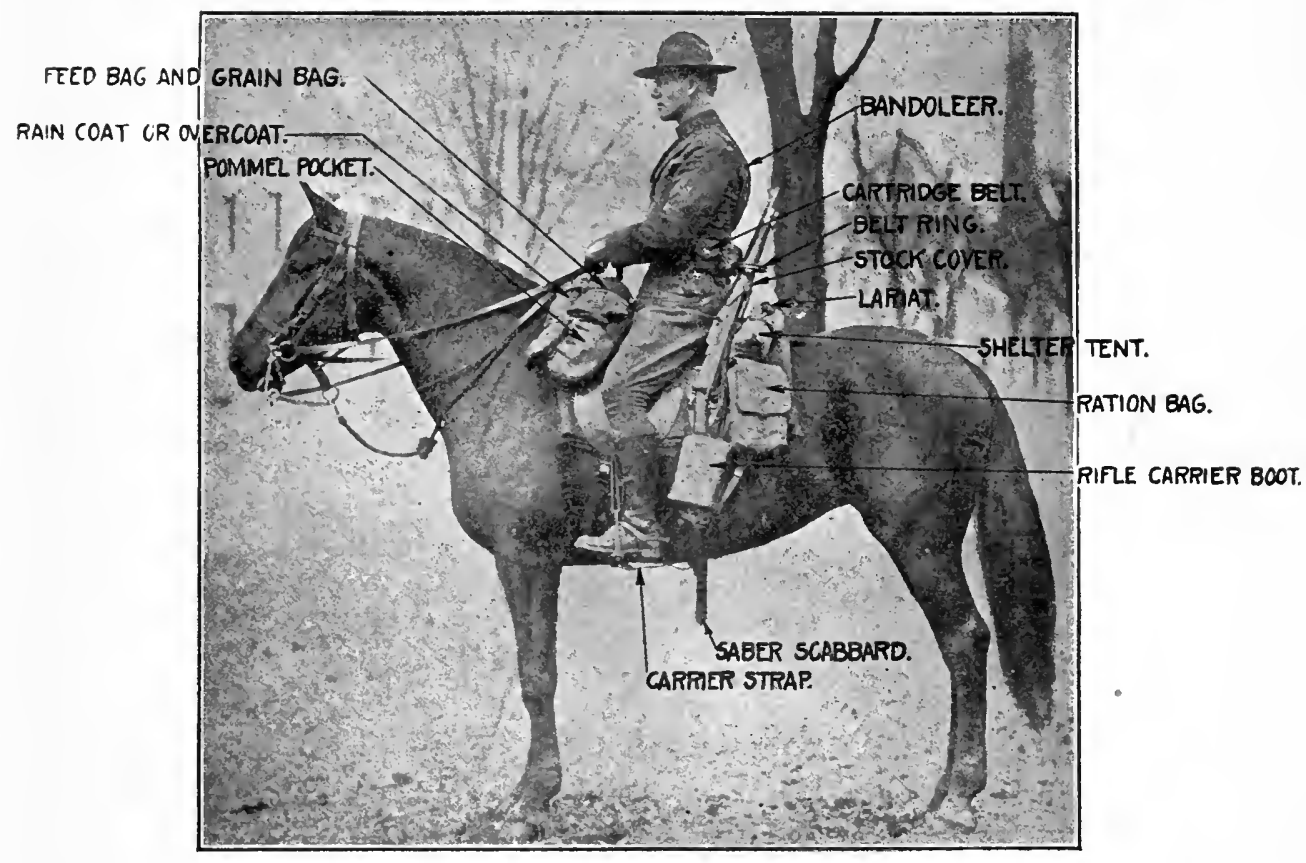

takes the place of the Hospital Corps knife). The articles in the belt are distributed uniformly around the waist line and so placed that they are all easy of access.

The Medical Department has also adopted so much of the new model cavalry equipment as is suitable for the use of Hospital Corps men. This equipment, as noted above, is so constructed as to be readily adaptable to either mounted or dismounted service, a feature especially desirable for the Hospital Corps. 


\section{CHAPTER XXXVIII.}

\section{POSTS, BARRACKS AND QUARTERS.}

The site for a military post should be selected with the greatest care, so as to meet not only military but also economic and hygienic requirements. A post should be a model and an object-lesson to the surrounding region in regard to the most approved methods of caring for a large aggregation of men. Therefore no selection of site should be made and no plans decided upon without consulting a competent medical officer. Ample grounds are necessary to prevent crowding of buildings and have plenty of space for formations and drills. For this reason it will seldom be possible to establish a post in or very near a city; such location would, besides, be open to other objections: the noise, smoke and dust of railroads and factories, the allurements of dissipation and the danger of contracting infectious diseases. It is best, therefore, that a post should be well outside of a town, where a sufficiently large reservation can be secured, but yet not so far as to remove it from the many conveniences and advantages which such neighborhood offers. Not only does the vicinity of a town afford to officers and men facilities for general information tending to their greater efficiency, but it is also in the interest of the country that the civilian should become familiar with the military uniform.

From the hygienic point of view, the site of a post should be high and dry, away from marshes, neither on a wind-swept summit nor in a contracted valley where surface waters collect, but rather on gently sloping grounds with good natural drainage, not liable to contamination from any neighboring town, and with sandy or gravelly, porous soil. The ground-water should not be less than 8 or Io feet deep. Should it be necessary to occupy a low site, with ground-water within a few feet of the surface, thorough underground drainage would be necessary, for there is no medical doctrine better established than that of the detrimental effects of constant humidity upon health. The question of water-supply will require careful consideration. As there are now few places in the inhabited parts of the United States and our colonies where good drinking water in sufficient quantity can be obtained from uncontaminated 
streams or lakes, it will generally be necessary to provide a purification plant, or else connect the post with the system of a town having such a plant.

The buildings of a post must be so constructed and exposed as to get as much air and sunlight as possible; hence the rule that the interval between them will be at least equal to one and a half times their height. In cold and temperate climates they should face east and west so that they may receive the full benefit of the warm afternoon sun, while in warm countries they should face south and north; but this question of exposure will necessarily be influenced by the direction of prevailing winds, local conditions and architectural exigencies (Fig. 125).

The size, shape and internal arrangements of barracks have been quite variable and, until recently, with but little regard to hygienic requirements. In Europe, where economy of space imposes itself, they are mostly large, several-storied, monumental structures, each accommodating a battalion or even a regiment; they generally suffer from serious hygienic defects and have often been unduly crowded, with consequent higher mortality. During the last thirty years a strong reaction has manifested itself in favor of smaller barracks, especially of separate I-story pavilions, and the health of troops has much improved in consequence. In France, for instance, the Tollet pavilion has been introduced in several garrison towns. It is a simple structure of brick and iron, 27 feet wide and 25 high, unceiled, with ogival roof, and ventilated through the ridge. Its claims are: that it separates men and organizations and reduces the chances of communicating disease, diminishes as much as possible all infectible and putrescible material, renders all surfaces impervious to germs and vermin, suppresses angles and furnishes a maximum of enclosed air with a minimum of enclosing surface, favors natural ventilation, and relegates all services likely to compromise health (kitchens, laundries, latrines, etc.) to outside places. It has become obvious, however, that such a system demands too large areas and scatters the command to an inconvenient extent. It has been given up in Europe, where the present tendency is to return to the principle of moderate concentration. Experience has demonstrated that in temperate as well as in tropical climates, barracks of two stories are fully as hygienic as I-story pavilions, besides being less expensive of space and money, and more easily administered. 
Each company, troop or battery should preferably have its own separate barrack, kitchen and mess. In order that they may receive as much light and air as possible, barracks should be parallel to one another and, if the terrain permits, in echelon; that is to say, each one projecting more or less beyond the last. The disposition in squares or quadrangles is very objectionable, as one or more sides, as well as the enclosed court, will be shut off from the sunlight and prevailing breezes; were such an arrangement rendered imperative by local conditions, a wide interval between adjoining buildings should be left at the four corners.

In England, according to Melville, the latest plans show a disposition to concentrate an entire battalion in a block of one or two buildings. Thus the Windsor Barracks, recently erected to accommodate 5 companies, consist of two parallel buildings, two and three-story high, separated by an open space of 75 feet covered by a flat roof with large ventilating lantern lights. The greater part of this space is used for mess-rooms.

A striking innovation in these barracks is the introduction of the individual cubicle system, the cubicles or cells opening on each side of a long corridor, with partition walls 7 feet 5 inches high, leaving a space of 2 feet 7 inches above to the ceiling, as well as a space 6 inches below to the floor. Each cell is 7 feet 6 inches long and 6 feet wide, and has its own window, 2 feet 6 inches broad, the upper half of which is a fanlight, opening inward and controlled by a screw rod operated from the N. C. O.'s room. Large recreation rooms are provided, so that there is seldom occasion for the men to go to their cubicles during the day except collectively, before and after formations. The floor space of the cubicle ( 45 square feet), although comparing favorably with that of most European barracks, is certainly smaller than that deemed necessary by all hygienists. The window in each cubicle, however, must be taken into consideration as a means of ventilation.

The above system is excellent in principle. There is no reason why soldiers should not be given a reasonable degree of privacy in dormitories, so as to foster a sense of self-respect and decency. A room to each man, however, is hardly to be commended, for such arrangement seriously interferes with supervision, ventilation and cleanliness. A desirable compromise would be to divide the dormitory into squad-rooms, each for a squad of $8 \mathrm{men}$, including the N. C. O. in charge of it, and with partitions open above and below. 


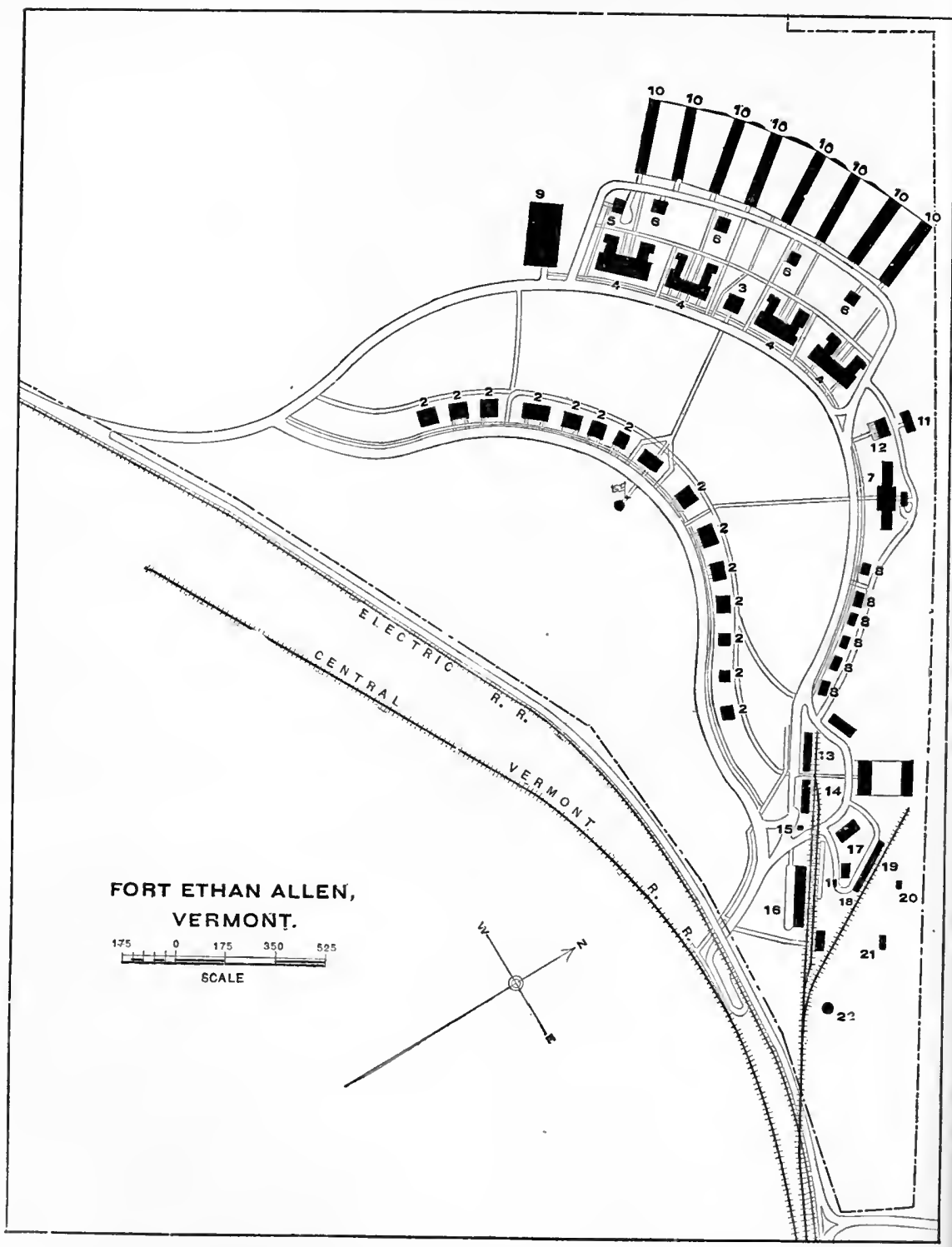

Fig. 125-Fort Ethan Allen, Vermont. Post of modern construction. showing arrangement of buildings. I, Commanding offlcer's quarters; 2, officers' quarters; 3, guard house; 4, barracks; 5, band quarters; 6, saddler shops; 7 , hospital; 8 , non-commissioned staff quarters; 9 , cavalry drill-hall; Io, cavalry stables; II, post exchange; I2, bakery; I3, store-house; I4, storehouse; 15, scale-house; 16 , forage-house; 17 , shops; 18 , ordnance; I9, fuelshed; 20, oil-house; 21, magazine; 22, water tower. (From Munson.) 
Construction and Materials. - The property of building materials which most concerns the hygienist is porosity, that is to say, the amount of air they contain. From this depend their heat conductivity, moisture capacity, air and water permeability. Since air is a worse conductor than any liquid or solid substance, the more porous a material the lower is its heat conductivity and capacity; in other words, the more easily are its surfaces warmed, but the more slowly does heat penetrate through it. Walls of such material will absorb but little sunheat and lose but little of the inside artificial heat, therefore will keep a building cool in summer and warm in winter. Another property they possess is bad conductivity to sounds.

The above qualities of porous materials are necessarily modified by moisture, according to the quantity of air which is replaced by water; they become better heat conductors and lose more or less of their permeability; the larger the pores the less is the increase of conductivity and loss of permeability, and the quicker do they dry after a rain.

The permeability to air of building materials has sometimes been considered a valuable factor in the ventilation of buildings. But the researches of Lang and Recknagel have demonstrated that the amount of air capable of passing through the most porous of them is practically insignificant and a negligible quantity with regard to the renewal of air and ventilation of rooms, especialiy when the walls are plastered, painted, or paper-covered.

Bricks are light, cheap, durable and porous, and, for these qualities, very generally used. Their marked porosity makes them a bad heat conductor. Thus a brick wall Io inches thick will protect as efficiently against external variation of temperature, in a moderate climate, as one of limestone 20 inches thick (Arnould). It must be borne in mind, however, that to prevent the transmission of solar heat into a building, a wall, of whatever material, must have suffcient thickness; thus, a brick wall only 12 to 15 inches thick may become so heated in the day that it only partially cools off at night, and its temperature will continue to rise from day to day until its internal surface is reached. The use of perforated brick in walls, at least for the inside course, adds very much to their non-conductivity and is therefore highly desirable in all climates. In tropical countries, it is a further advantage to build double walls with an air space of 2 to 4 inches between them; this space should be provided with screened outlets for the free escape and renewal of air. 
The porosity of brick renders them quite permeable to air, especially under the pressure of a strong wind. They are also very absorbent, taking up to to 20 per cent. of their volume of water. For these reasons, brick walls should always be plastered, at least inside, and unless otherwise protected from rain it is often advisable to paint them outside; nor should they ever be in direct contact with the soil.

Stone, especially marble, hard sandstone, granite, etc., is less porous than brick and a better conductor of heat; it absorbs less water but retains it longer; on the other hand, it is more durable and sightly.

Wood is a very poor conductor of heat and affords efficient protection against the sun, but is very hygroscopic, moisture causing it to expand irregularly, with disjunction of parts, cracks and fissures leading to decay and favoring the collection of dirt and parasites. It is, besides, always in danger of fire.

Iron, now so largely used for the framework of buildings, is an excellent conductor of heat and sound; but this defect is mitigated by a sufficient covering of stone or brick and mortar. It has the advantage of being impenetrable to humidity.

Ordinary mortar, consisting of lime, sand and water, is very porous, especially when made with coarse sand. Plaster is still a poorer conductor of heat than mortar, although less permeable; it is the most hygroscopic of materials. Cement and concrete are waterproof and have little porosity and permeability, therefore are good conductors of heat.

A new building contains a large proportion of water, from i 30 to 230 liters in a cubic meter of brick masonry, according to Flügge. Such excessive humidity must be gotten rid of before it is habitable.

In a damp building the cutaneous evaporation is checked, while the loss of body heat by radiation and conduction is increased; it follows that the occupants are unduly overheated when the temperature rises, and chilled when it falls. Furthermore, damp walls being better heat conductors, the building is difficult to warm in cold weather, or becomes overheated by the sun in hot weather. For these reasons, confined dampness has an unfavorable influence upon human health, leading to catarrhal and rheumatic affections as well as to tuberculosis. No plastering or coating of any kind should be put 
on the walls until they are quite dry, that is, until the mortar, which in the fresh state contains 15 per cent. of free water, has dried down to 2 per cent. or less. Plaster contains nearly twice as much water as mortar and dries very slowly. It is readily seen, therefore, why a newly completed house should be thoroughly ventilated and gradually heated, at least for a week or two, according to the weather, before it is fit for occupancy.

Cellars and Basenents. - Cellars and basements improve the healthfulness of buildings, provided they are properly constructed. Unless they are impervious to ground-air and water they do more

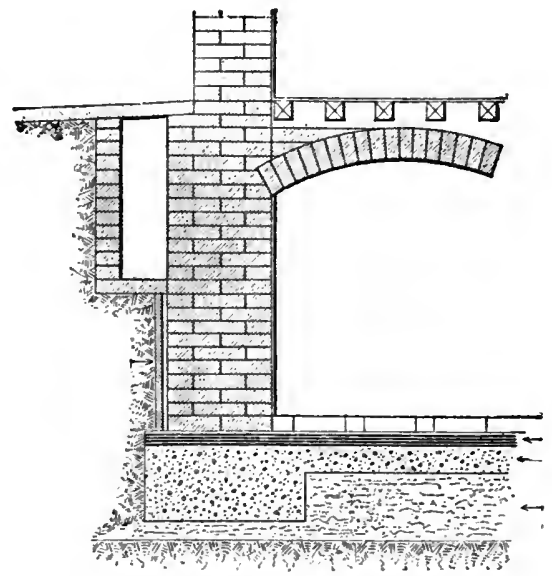

FIG. I26. - Method of flooring cellars to prevent dampness. $a$, Disconnected air-space lined with cement: $b$, cement; $c$, concrete; $d$, clay. (Munson.)

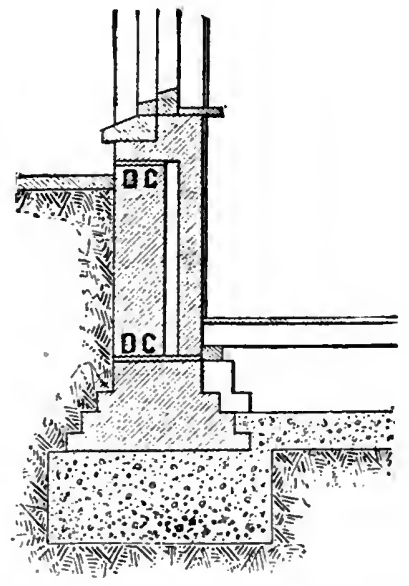

FIG. 127.- Method of preventing dampness of walls. Letters DC denote location of dampproof courses. (Munson.)

harm than good. Ground-water may rise into them or keep the floor and walls constantly damp; ground-air, more or less polluted, readily escapes into them and, as it becomes warm, ascends into the building carrying more or less humidity with it. Even under the best conditions, air moisture readily condenses on the cool walls of a cellar, and unless well ventilated or artificially heated it will seldom be perfectly dry. It is necessary therefore that cellars and all other substructures should be made impervious. Damp grounds must be underdrained. The floor should be covered with a layer of concrete coated over with cement or asphaltum. A more perfect 
result is attained by placing the concrete over a stratum of welltamped clay.

Foundations. - The best material for foundations is stone. Their most important hygienic requirement is that they be waterproof. Therefore, besides being drained, they should rest on a bed of impervious concrete (concrete footing) and be protected from outside moisture by layers of tar and cement (Fig. I26), or by a trench extending down to the footing and filled with broken stone. A double foundation wall, with air space of 2 or 3 inches, is also very useful in preventing much of the outside moisture from reaching the internal surface (Fig. I27). The space may be loosely filled with a non-hygroscopic material, such as cinders. In order to preclude the rising of moisture into the walls by capillary attraction, it is also necessary to interpose, at the level of the soil, a " damp-proof course," consisting of slates, vitrified bricks or waterproof felt imbedded in cement or asphaltum.

Barracks and other buildings without basement or cellar should be raised above the ground, leaving a clear air space beneath, large enough, if possible, for inspection and cleaning. A further improvement would consist in cementing the floor of this space.

Plastering. - Walls may be furred, lathed and plastered, with an air space of about two inches between the brick and lathing, materially contributing to their dryness and non-conductivity. The use of perforated brick for the inside course renders lathing unnecessary; such brick furnish a sufficient air space and are of equal hygienic value, without providing a harbor for rodents and vermin. The use of metallic woven-wire lathing, now becoming general, is likewise entirely satisfactory. Plaster may also be applied upon a wall of solid brick by the intervention of a thin coating of adhesive material, but this is clearly undesirable.

Partition walls should preferably consist of large hollow tile, of light, incombustible material, non-conductor of heat and sound; such walls are readily plastered without the intervention of any other material.

Ordinary plaster is porous and absorbent, and therefore a poor material for the inner finishing of the walls of barracks or any building occupied by a number of men. The vapor of respiration condenses upon it and all the impurities floating in the air readily adhere to it. It should be whitewashed or kalsomined at least once 
a year. Water paints, however, are always unsatisfactory. They are not durable and soon scale, nor can they be washed down and disinfected, while the binding material (glue, dextrin, casein, etc.) is liable to develop musty and foul odors. Much preferable is the use of a harder, less porous material, one that can be readily washed, such as adamant plaster, soapstone finish; good oil or enamel paint, etc. Where a certain degree of ornamentation, together with more complete asepsis are desired, as in bath-rooms, operating rooms, etc., enameled or vitrified tiles of various types are used.

PAPERING. - The papering of walls and ceilings is unlyygienic and not permissible in barracks, hospitals and other buildings occupied by a number of men. In private houses it is still more or less used for economic and esthetic reasons. Paper presents an absorbent surface and, together with the paste used, furnishes not only a resting place for the germ-laden dust but also a culture medium for the growth of bacteria. Bright green and red papers should be avoided for they probably contain arsenic and, when detached from the wall, may give off arsenical vapor. In repapering a wall, the old paper must always be removed and the surface scraped before the new paper is laid on.

Floors. - The ideal floor, in temperate and warm countries, is a hard, smooth, impenetrable mineral floor, free from open joints and crevices, one that can be washed and scrubbed without danger of dampness, decay and shrinking, and that affords no harbor of refuge to vermin and dust. But the expense and weight of such floor render it too often prohibitive; it is also open to the objection of being uncomfortable in cold weather.

The material most used for flooring is wood. It should be laid in two layers, with a thickness of flooring felt between, the lower, of boards laid diagonally, close together, and the upper of tongued and grooved boards. For lavatories, latrines and other places whose floors need frequent washing, an impervious material is necessary, such as cement, asphaltum, "terrazo" (made of small pieces of marble laid in cement and smoothed down), or various types of mineralized wood pulp for which are claimed the combined advantages of wood and stone. In tropical countries, tiles or marble slabs laid in cement make perfect floors.

Between the floor above and the ceiling below there is often an empty space. This is an advantage, as it deadens sounds and pre- 
vents heat conduction. It is essential, however, that it should not become a receptable for organic matter passing through the cracks and defective joints of the flooring, and liable to be stirred up by air currents and contaminate the air of the room above. This is rendered impossible with a tiled floor properly laid in cement, or a wooden floor resting on a ceiling of hollow tile or reinforced cuncrete, especially if imbedded in asphaltum.

But the organic dust, full of micro-organisms, which constantly accumulates on the wooden floor of inhabited rooms, especially barracks, finds lodgment in the joints, cracks and all interstices, however minute; it is constantly raised by air currents, as well as by sweeping and dusting, and a menace to the occupants. Furthermore, any moisture applied to an unprotected wooden floor will cause it to expand, crack and decay. Hence the necessity of filling and closing up all places capable of harboring dust and vermin, and to render the wood impervious to water. Coal tar, diluted with heavy coal oil, has long been used for these purposes in French barracks, but lately was replaced by carbonyle (coal-tar product) which, besides rendering the wood impermeable, possesses also bactericidal and parasiticidal properties; it has the objection of imparting a dull brownish tint to the floor. In German barracks, boiling linseed oil is used once or twice a year, but although undoubtedly useful fails to fill up the joints and cracks. The use of paraffin and wax gives excellent results, but is too expensive for general application. Certain coal-oil products, cheap and easily applied (such as the Standard Floor Dressing) are reported to be very satisfactory.

In our barracks, all finished wood floors and stair treads are simply given one coat of raw linseed oil, well rubbed in. More complete treatment is certainly desirable.

Windows. - Windows should extend up within a short distance of the ceiling, and down to about three feet of the floor. In cold and temperate climates it is best that the two sashes slide vertically one over the other, ventilation being thus more readily graded; in warm countries, French windows descending nearly to the floor, with lateral sashes hinged at the sides and opening their whole length, are preferable. An excellent combination is to have an independent transom over the French window, to be used for ventilation when the weather does not admit of the opening of the window itself. Doors and windows must be protected by suitable 
screens against flies and mosquitoes whenever these insects are troublesome or the diseases which they convey are prevalent. In cold countries, double windows are used so as to save heat and prevent drafts; in such case, the outside window should always have a ventilating panel which can be opened or closed to the desired extent. In northern European countries, the lower part of the 'space between the two windows is often filled with wool, a device to be commended only when the temperature renains long below zero.

Roofs. - For most countries, slate laid on heavy builder's paper is the best roofing material. Tiles are less conductive, therefore cooler and better adapted to hot climates. Metal roofs become very hot and are therefore objectionable unless they rest upon a layer of coarse cement. When an emergency requires the use of corrugated iron or other metal, it should be laid in two layers separated by an interval of six inches. In tropical countries, it is well to provide an air space, or attic, between the top floor and the roof, and, between this attic and the tiles, to interpose a layer of porous cement laid between and over the rafters, consisting chiefly of some nonconductive substance (cinders, infusorial earth, pozzuolana, etc.).

\section{General Specifications for United States Barracks.}

In accordance with the specifications of the Chief Quartermaster Corps, our barracks are two-story and basement brick buildings; the exterior basement and area walls and piers, of stone; the footings under all walls, piers, chimneys and iron columns, of concrete; the superstructure, interior basement walls and all chimneys, of brick; the roof of building to be covered with slate, and the roofs of porches, of tin.

A subsoil drain of 6-inch vitrified, hub-joint, terra-cotta pipe is laid outside of the footings, the lower third of joint cemented and the remainder left open. Through 2-inch cast-iron pipes, it receives the drip from refrigerators and drainage from area-ways. It has no connection with the sewer.

The downspouts are of corrugated galvanized steel, provided, below the water table, with cast-iron, hub-joint leaders which connect with a 4 -inch vitrified terra-cotta drain emptying into the main drain or wherever desired, but not connecting with the sewer.

The entire basement floor (including toilet rooms, barber and tailor shops) is properly filled, graded and rolled to a firm surface, 
then covered with $4^{\mathrm{T}} / 2$ inches of concrete; before this has set, a finishing coat, half-inch thick, of one part cement and two parts sand, is put over it. The floors of toilet rooms and shower stalls are graded from walls to floor traps. The steps, coping and floor of each areaway likewise receive a layer of concrete and finishing coat. •

The stone work must.be of sound, durable local stone, of good quality of rubble work, faced full height on inside, and below grade

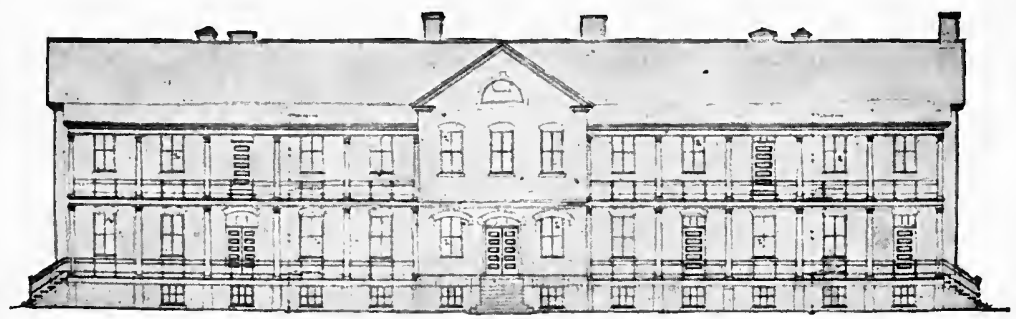

FIG. 128.- Standard barrack, U. S. Army. Front elevation.

on outside, the mortar used consisting of one part cement and three parts sand. After basement walls are well set and dry, the outside, from bottom of footings to surface of ground, is to be plastered halfinch thick with mortar composed of equal parts of cement and sand.

No damp-proof courses are used except in exceptional cases.

The brick must be sound, hard, well-burned and dark-red common brick, laid with flushed, solid joints; dry if laid in frosty weather, and well wetted if laid in dry, hot weather.

The floors consist of two layers: the under layer of boards not over 8 inches wide, dressed on upper side and laid diagonally, close together, upon the wood joists; the upper layer, of maple or yellow pine boards; tongued and grooved, $3 \frac{1}{4} 4$ inches wide, tightly driven up and blind nailed; with one thickness of flooring felt between them (no tarred paper used).

The hand rails are of oak throughout; the stair treads of same wood as upper floors; all other inside finish of best quality of white pine or yellow poplar.

The entire halls of basement, first and second stories are wainscoted four feet high, with baseboard and cap moulding. All rooms in first and second stories have baseboards, with moulding at bottom. 
All walls and ceilings (except ceilings of first story and soffits of all stairs) are furred, lathed and plastered; the plastering consisting of three coats, the first two of quick-setting cement plaster, the third, or finishing coat, of hard white cement-finish troweled to a smooth surface. The stone walls in toilet rooms are given a coat of plaster made of one part of Portland cement and three parts of sand applied directly to the stone and floated smooth and even.

All ceilings of first story and soffits of all stairs, on account of the great jarring to which exposed, are covered with one thickness of heavy flooring felt and then cross-furred with strips properly spaced to receive steel ceiling plates. These plates, about 20 inches square, have lock slips or countersunk joints with no ornament of any sort except in extreme corners to conceal wrinkles.

All roofs of building (except where tin is specified) and the walls of dormers are covered with the best quality of unfading black slate. Before laying the slate, the roof is covered with a thickness of best red-rope, waterproof sheathing paper, free from wood pulp and rosin. The roof of all porches is covered with tin, in locked and soldered plates, laid on one thickness of flooring felt.

All outside wood and metal work (except copper and tin) are painted three coats of paint made of white lead mixed with linseed oil, the last coat to be one-third zinc white. Tinwork receives one coat of mineral paint on under side and two on upper side. All interior woodwork and exposed iron columns are painted three coats of paint made of equal parts of white lead and zinc white mixed in linseed oil. The walls of each toilet room receive two coats of lead and oil paint and a third coat of white enamẹl paint.

Distribetion of Rooms. - The basement and two stories of barracks are generally assigned to the following uses, as shown in Figs. I29, I30, I3I. In the basement is the lavatory, containing waterclosets, urinals, wash-bowls, laundry tubs, 4 to 6 showers and one bath-tub. On the first floor is the kitchen, with pantry, store-room and cook's room; mess-room ; company office, with adjoining storeroom and clerk's room; a large recreation room; first sergeant's room; one dormitory. On the second floor are only dormitories, with separate rooms for non-commissioned officers.

This arrangement is entirely satisfactory. The location of the lavatory in the basement, assuming that the plumbing is perfect, is quite unobjectionable; it is of convenient access and is heated in the 
easiest and most economical way possible. The kitchen and messroom are properly placed on the first floor, the kitchen in a wing and the mess-room in the main building; a large hood over the hearth carries off all odors so that none are detected in any other part of the barrack. It is a recognized principle in hygiene that dormitories should be as high as possible above the soil; it is therefore one of the advantages of a 2-story barrack that the upper floor can be exclusively used for dormitories.

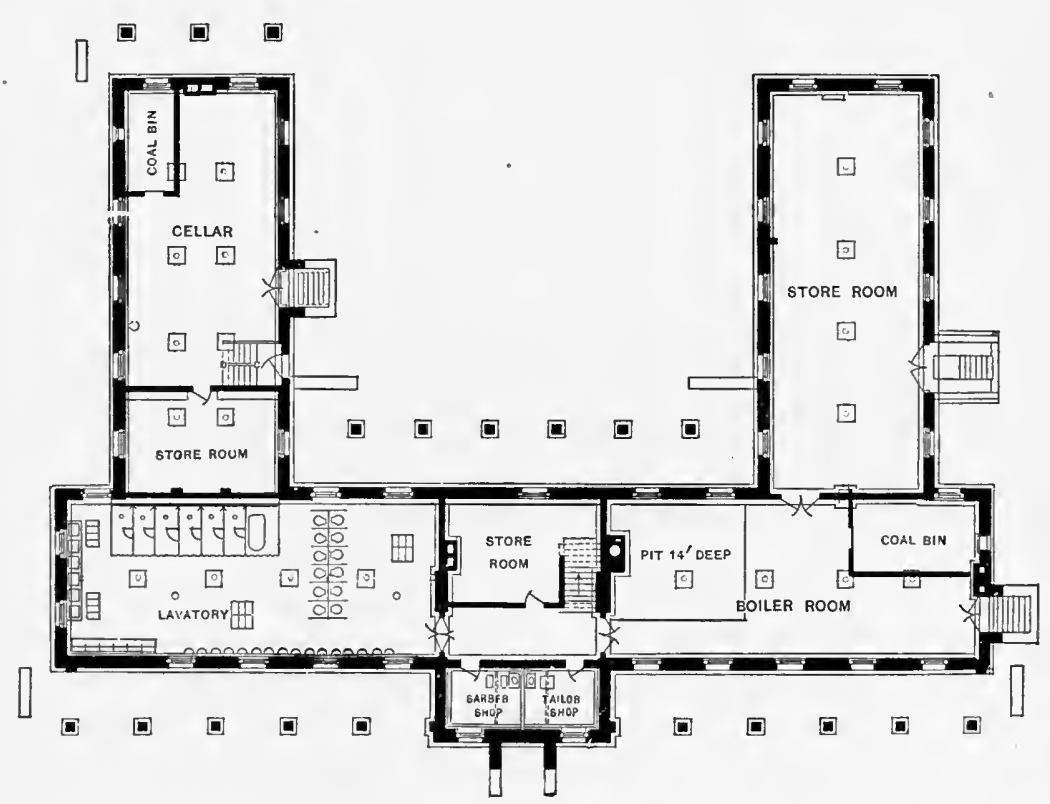

FIG. 129.-Standard barrack, U. S. Army. Basement plan.

Sometimes lavatories are detached (Fig. I32), each being placed in rear of its corresponding barrack. This may be rendered necessary by the difficulty of getting a suitable outlet for the excreta and wastes at a lower level ; but, all things considered, it is less desirable than a properly installed basement lavatory. When thus detached it generally contains the barber shop and tailor shop.

Several of our large posts are provided with general messes, that is to say, with one common kitchen and mess-room for the several organizations of the garrison. Such messes have decided advan- 


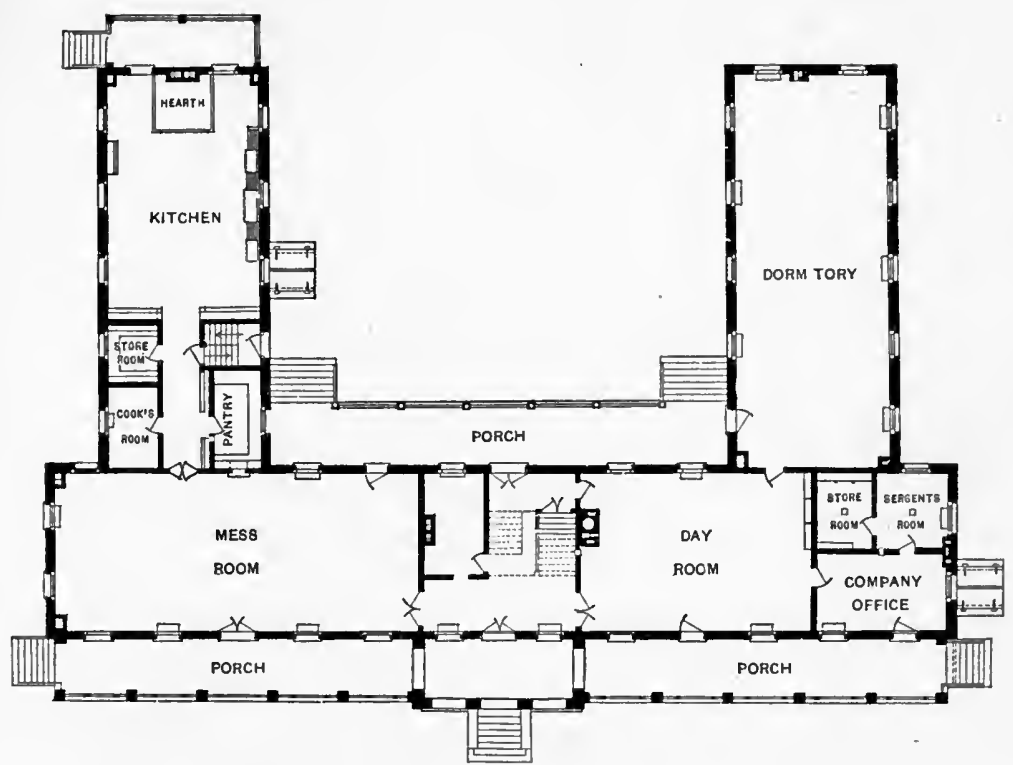

FIG, I30.- Standard barrack, U. S. Army. First floor plan.

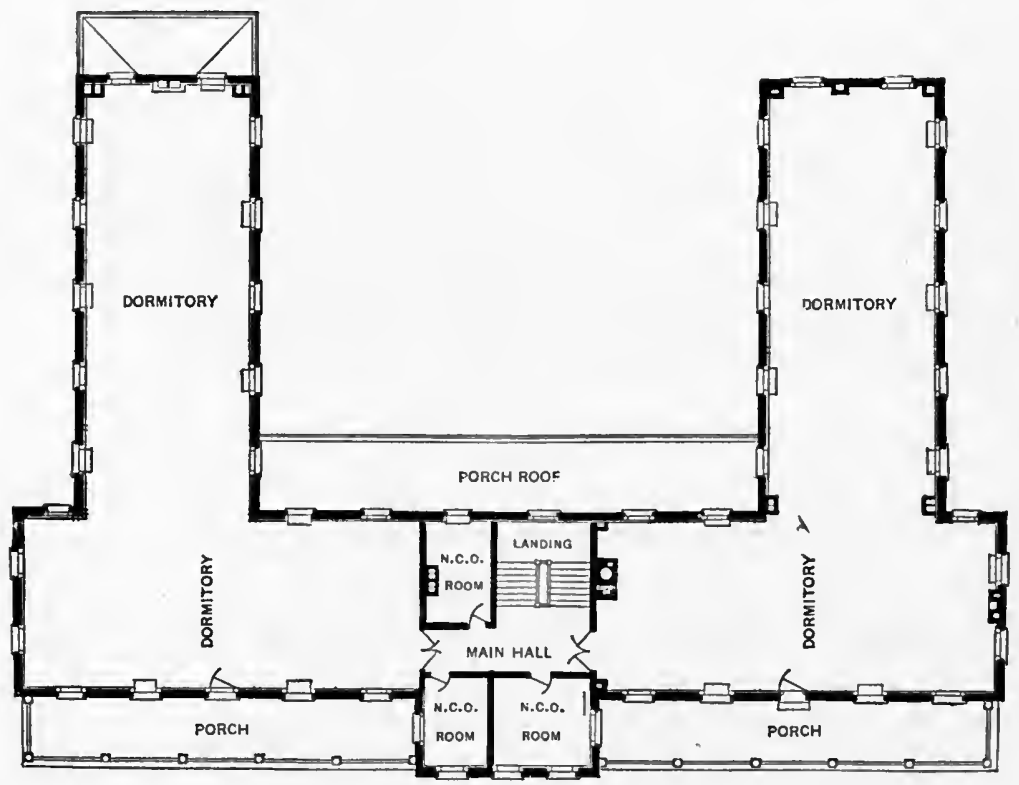

FIG. I3I.- Standard barrack, U. S. Army. Second floor plan. 
tages, provided they are managed by competent and interested officers; they are more economical, admit of a higher grade of cooking, are more easily supplied and their wastes more readily disposed of. Notwithstanding all this, there is a general opinion that separate company messes are preferable, inasmuch as each company trains its own cooks and is always ready to take the field independently.

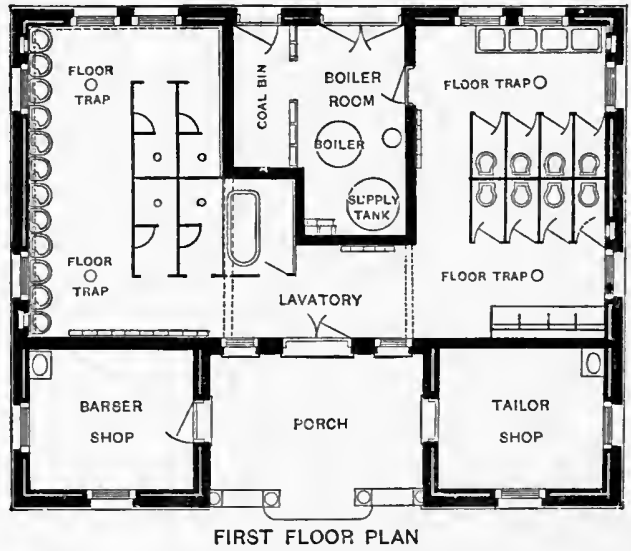

FIG. 132.- Detached company lavatory.

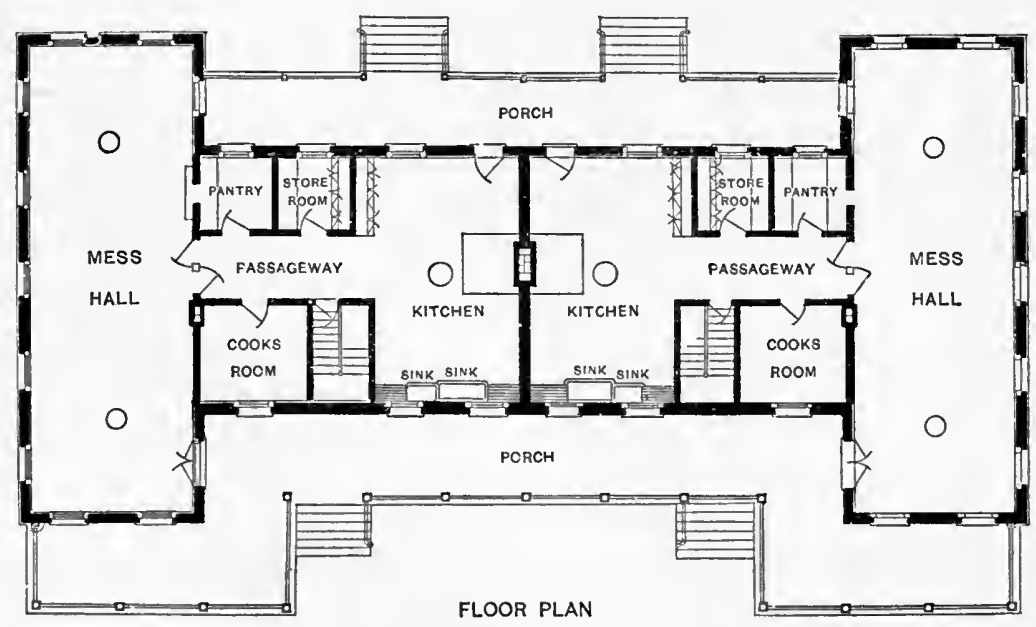

FIG. I33.- Double company kitchen and mess hall. 
Besides, companies may thus benefit from the careful and thrifty management of their officers and indulge in such delicacies as their resources permit.

When barracks are so planned that kitchens and mess-rooms have to be detached, a very convenient and economical arrangement is that illustrated in Fig. I33, in which two sets are contiguous and under the same roof. This is particularly suitable for barracks disposed in double sets.

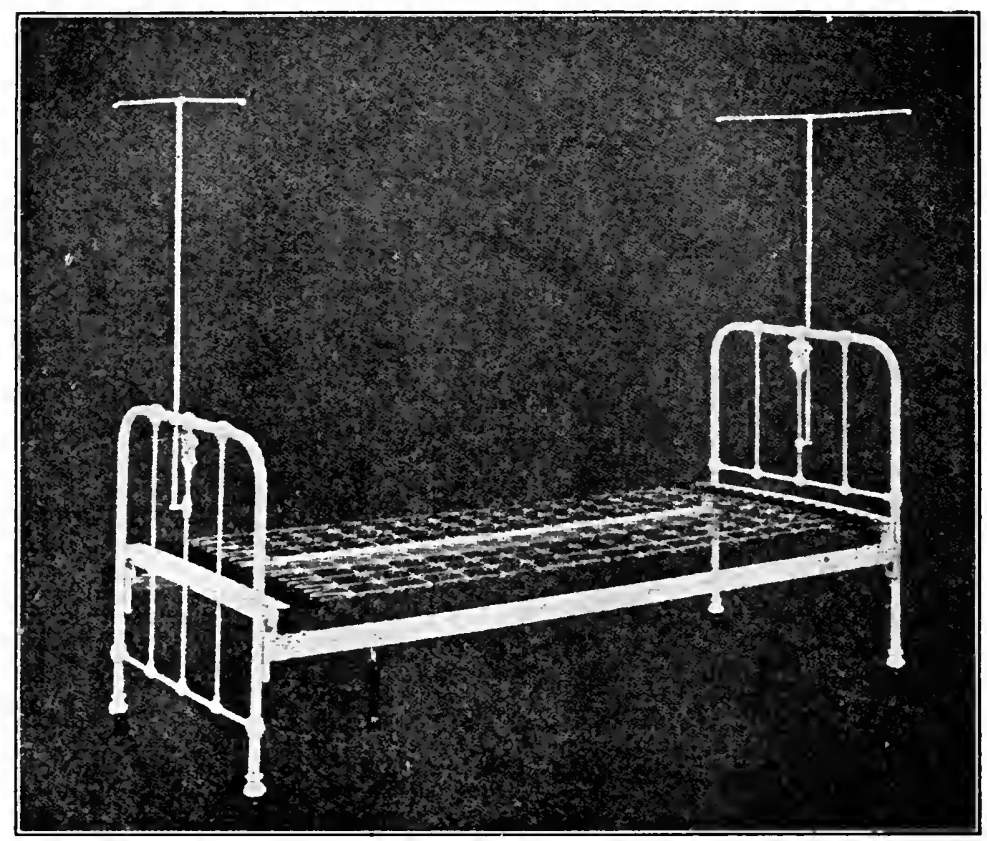

FiG. 134.- Barrack bedstead.

The features which should characterize barracks in the tropics are described in the chapter on Service in Warm Climates.

\section{Furniture and Bedding.}

All woodwork and furniture in barracks should be of the simplest kind and easily accessible, with as few recesses, angles, mouldings, projections and open shelving as possible to avoid the accumulation and dissemination of dirt and dust. 
Wainscoting is superfluous and harmful in barracks, affording refuge to vermin and rodents.

The bedstead now provided for enlisted men is above criticism. It is entirely of metal, the link-meshed spring bottom, or " fabric," being 9 meshes wide by 16 meshes long, in all 65 inches long and $3 \mathrm{I} 3 / 8$ wide, and connected to rail at each end by 19 spiral springs. Head and foot pieces have mosquito-bar rods. (Fig. I34.)

The bedding consists of mattress, mattress cover, pillow, pillow cases, bed sacks, pillow sacks, mosquito-bars, sheets and blankets. The mattress consists of narrow-striped blue and white ticking filled with one unbroken sheet of interlaced carded cotton felt. (Fig. I35.) For protection it is enclosed in a cover of unbleached cotton duck. The pillow is made of the same material, and enclosed in cases of unbleached muslin. The bed sacks, of unbleached cotton drilling, and pillow sacks, of unbleached cotton duck, are intended

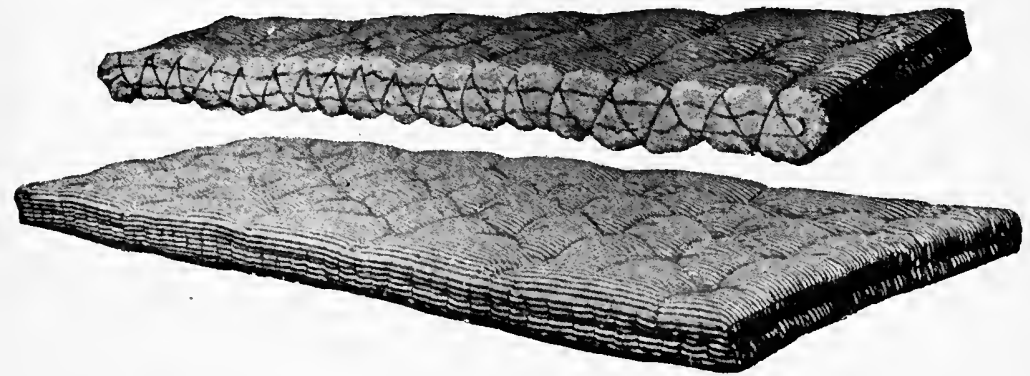

Fig. I35.- Mattress of felted cotton, for use with barrack bedstead.

to take the place of mattresses and pillows in the field, stuffed with hay or grass. Mosquito-bars are indispensable in garrison and field, wherever malaria and yellow fever prevail, and stringent orders should be issued to insure their proper use. The sheets are of unbleached sheeting, 90 inches long and 48 wide. The blanket, of olive-drab woolen material, is 7 feet long, $5^{\mathrm{T} / 2}$ feet wide, and weighs 3 pounds. It is provided with tapes along sides and bottom to permit its being folded into a sleeping bag (see under Equipment).

All soiled clothing is placed in a "barrack bag" of brown cotton duck, 32 inches deep and 15 in diameter.

Lockers. - Each enlisted man is supplied with two lockers, a " metallic wall locker" attached to the wall and a permanent fixture 
of the squad-room (Fig. 136), and a "trunk locker" which he carries with him whenever changing station.

The wall locker is con-

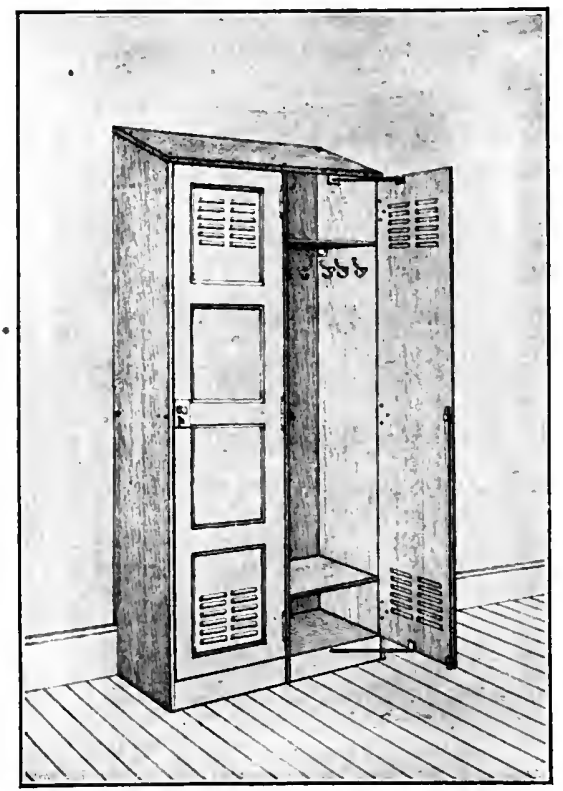

FIG. 136.- Metallic wall locker, double. structed of sheet steel, varnished and finished in oak or olive green; 7 feet 4 inches high in rear, but only 6 feet 8 inches in front on account of sloping top; 19 inches deep and 20 inches wide (when single). The door is perforated for ventilation by 12 slots, with hood to prevent the settling dust from getting to the contents. Eleven hooks are provided, 3 in each wall and 2 double ceiling hooks, all triple brass plated. These lockers may be single, but are generally double, treble or quadruple.

The trunk locker is a rectangular box 30 inches long, I6 inches wide and 12 inches deep, inside measurement. It has a tray $23 / 4$ inches deep, divided into 3 equal compartments. It is made of wood in 3 -ply veneer, with outside covering of vulcanized hard rubber bound on the corner edges with vulcanized fiber and clamped at the corners. 


\section{CHAPTER XXXIX.}

\section{POSTS, BARRACKS AND QUARTERS (CONTINUED).}

\section{CARE OF BARRACKS AND GUARD-HOUSE.}

Barracks, kitchens and mess-rooms should be as neat and clean at all times as conditions permit. This is one of the practical tests of the competency of company commanders. The officer who knows how to look after the comfort and health of his men in garrison can generally be depended upon to give a good account of himself in the field.

The temperature of barracks and guard-house must never exceed $70^{\circ}$ in winter. It need not be absolutely uniform; on the contrary, a certain range of variations (say from $60^{\circ}$ to $70^{\circ}$ ) is desirable to produce the cutaneous stimulation most conducive to active metabolism (see under Heating). Ventilation by perflation, through doors and windows, is the most satisfactory; in cold weather this should be done mostly when the men go out for mess, drill or other duty.

Most of the dirt and dust in barracks, guard-house and other buildings is carried in on the feet. This should be reduced to a minimum by the use of scrapers and metallic mats at the doors. The tendency of soldiers to accumulate property and fill their lockers and other available spaces should be restrained; all superfluous objects diminish the air space, favor the collection of dust and germs, and interfere with sweeping and policing. A space-sufficient for cleaning and inspecting should be left between the bedsteads and the wall.

The bedding soon gathers dirt and dust from the body, clothing and surrounding objects; it should be taken out, well shaken and sunned a few hours once a week whenever the weather permits. Sheets and pillow-cases must be changed weekly or, at least, before they become visibly soiled. It is well to make irregular inspections of the beds for unclean clothing and forbidden articles stowed beneath the mattress or under the pillow. All soiled linen must be sent to the laundry every Monday, or other specified day, and not be allowed to accumulate in bags or other receptacles. 
The guard-house is more exposed to dirt and vermin pollution than any other part of a garrison, and requires correspondingly strict supervision and policing. The prison room, with occupants and contents, must be visible from end to end, as provided by existing plans, so that accumulation of soiled clothing, foodstuffs or other unauthorized or superfluous articles be easily guarded against. The heat must be kept down in winter and plenty of fresh air constantly provided. Concrete floors and well-plastered walls are necessary to prevent the harboring and breeding of vermin. Unless the sanitary condition of the guard-house be very satisfactory, the bedding and clothing of prisoners should be subjected to some form of fumigation before being returned to barracks.

Floors. - In addition to the dust which settles upon them, the floors of barracks are constantly contaminated by mud and dirt brought from the outside, by sputum, fragments of food and various organic débris shaken from the clothing and bedding. All these impurities as they dry and become ground into a fine dust by the tramping of shoes, are raised by air currents and made part of the atmosphere breathed by the occupants. Therefore to clean the floors, walls and furniture without raising and scattering the dust and contaminating the air is a vital hygienic desideratum. It is evident that the ordinary method of dry sweeping and dusting is inefficient and may be worse than useless since much of the dust, if not the whole of it, is simply scattered through the room to again settle over the same or other parts of it; meanwhile it pollutes the air and is much more dangerous than if it had been left undisturbed. Floors should not be swept but cleaned with damp mops. The process consists in dipping the mop in a bucket of water, wringing out, rubbing the floor, then washing and rinsing it in another bucket of water. Dust should be removed by catching it on damp cloths rubbed on the woodwork and furniture, especially all ledges, mouldings and shelving. The feather duster has become obsolete. The soldier's former way of cleaning floors by pouring streams of water upon them, preparatory to sweeping and scrubbing, is pernicious and should never be permitted. It fills the joints, fissures and holes with moisture, thus promoting the breeding of micro-organisms, besides causing the wood to warp, crack and decay. Scrubbing is the best method of cleaning mineral floors (tile, marble, terrazo, etc.); it may also be necessary for wooden floors when very much soiled, 
but it must be done with as little water as possible; in such case the better plan would be to detach and disintegrate muddy or incrusted spots with a wet stiff brush, previous to mopping the floor.

An excellent system, to prevent the raising of dust, consists in coating the floor with an agglutinative dressing* to which the dust adheres, but from which it is easily removed by a stiff broom, in little particles too heavy to rise and float in the air. In barracks or other buildings where the wear is unusually heavy, an application every 2 or 3 months is necessary. Properly put on, such dressing catches and holds every atom of dust coming in contact with it, thus saving much labor in sweeping and scrubbing; it is also an excellent preservative of wood and a repellant of insects and vermin.

In this connection may also be mentioned the vacuum cleaning system whereby all dust is completely removed by suction from carpets, hangings, upholstery and decorations without the least air pollution. Wood and tile floors are cleaned in the same manner, previous to mopping. An obvious advantage of this system is the pumping out and removal, together with the dust, of much of the musty and germ-laden air which too frequently stagnates in obscure corners and clings to upholstered furniture.

Spittoons. - The sputum is frequently contaminated with the germs of tuberculosis, diphtheria, pneumonia and other diseases, generally without the knowledge of the man ejecting it; therefore it is a source of great danger and every precaution'must be taken to prevent its dissemination in the air, especially in rooms occupied by a number of people. Spittoons or cuspidors should be provided in sufficient number, and punishment swiftly inflicted upon any one guilty of spitting on the floor. Cuspidors must be emptied and washed daily, preferably in boiling water, and then partly filled with a disinfecting solution. They must be so shaped as not to easily upset, and to permit easy cleaning. They should be set upon a square piece of linoleum, rubber matting or other washable material, so as to save the surrounding floor from possible pollution.

Insects and Vermin. - The various insects liable to infest barracks and other buildings need careful attention. Flies and mosquitoes are best guarded against by screening the doors and windows, and the use of mosquito bars. Their prevention and destruction are described in other chapters.

* Such as the Standard Oil Dressing, one of the best known and most extensively used. 
Bedbugs are not only the most noisome and troublesome of the insects infesting barracks, but are also capable of conveying disease (see page 143). Therefore every effort should be made to exclude them, or to exterminate them after their invasion. The frequent examination of the bedding and clothing is necessary, as well as of the linen returned from the wash. They are destroyed by subjecting all infested clothing and bedding to the action of steam under pressure in. a large disinfecting chamber, or boiling them in water. With iron beds, fire can be used, alcohol being poured into all the crevices and immediately ignited. An emulsion of petroleum ( $\mathrm{I}$ to 3 or 4 of water) may be applied with a stout brush, or else a spray of benzine or gasoline, in the cracks of the woodwork and bedding. Such cracks should then be filled with putty, or covered with strips, and painted over. A saturated solution of corrosive sublimate is efficacious but cannot be applied to metals. Insect powder is useless; it has only a stupefying action upon the insects and no effect upon the eggs. If a whole barrack be infested, thorough sulphur fumigation is best; formaldehyde gas cannot be relied upon for this purpose. Hydrocyanic acid, generated from potassium cyanide by the addition of sulphuric acid, is very efficient against bugs and all kinds of vermin, but is such a deadly gas that only experts should handle it.

For destruction of fleas, see page 146.

\section{CARE OF KITCHEN AND MESS-ROOM.}

The personnel required for duty in the company mess consists of a mess sergeant, cook, assistant cook, mess-room orderly and 2 or 3 cooks' police, according to the size of the company. Such a personnel permits a suitable division of labor and an orderly and systematic management of the mess. The first requirement from these men is cleanliness, in their persons and in all parts of the kitchen and mess-room for which they are responsible. The cook and assistant cook wear white clothing, aprons and caps. The others, if not provided with white clothing, should at least wear a high white apron. The hair and beard or mustaches must be kept trimmed and well brushed. The hands demand special attention, since it is mostly through them that food is liable to be infected. The nails must be kept short and clean. The rule that all men should wash their hands after visiting the toilet-room applies 
with particular force to cooks and others detailed for duty in the kitchen. The latter must also wash their hands whenever they are soiled and always before serving the meals. It is therefore necessary to provide a place for the purpose where soap, towels and nailbrushes are kept.

Before detailing a man in the kitchen or mess-room, the possibility of his being a carrier of typhoid fever or other infectious disease prevailing in the vicinity, such as dysentery or cholera, must be considered; in case of doubt he should be rejected or else submitted to a bacteriological examination. Likewise unfit for such duty is any man with venereal disease of any kind, tuberculosis or conspicuous skin eruptions.

Cats, dogs and other pets, as well as all men not on duty therein, must be excluded from kitchen and mess-room.

The kitchen floor is normally a hard, smooth mineral floor; it should be scrubbed every morning with soap and lye, and, besides, swept and mopped after dinner and supper. The kitchen table is scrubbed after preparing each meal. Rubbing off the range with a greasy cloth keeps. it in far better condition than stove polish applied once a week. Metallic surfaces and tinware are cleaned with soap, sapolio, lye, or with a cloth sprinkled with kerosene.

The ice-chest deserves special attention. The tendency of cooks is to overcrowd it. Articles with decided odors, like cheese, bacon, etc., and others which do not readily spoil, had better be kept out of it, in a properly screened box. Milk and butter are especially absorbent of smells and, if possible, should be kept in a separate compartment.

Meats from cold storage should be consumed without delay. Freshly slaughtered meats, on the contrary, improve in flavor and digestibility if kept a suitable time, according to the weather. After being cooked and seasoned, all foods should be consumed as soon as possible. Likewise, fruit and meat cans, once opened, should have their contents used up within a day or two. Foods fermented, sour or decayed, are unfit for use; but a short, recent growth of mold on meat, cheese, jam and preserves is not a necessary sign of decay, and, after being scraped out, such article can usually be safely eaten.

All wastes and scrapings from plates are thrown into a pail which is frequently emptied into the garbage can (kept outside the kitchen) and washed. 
Cockroaches are a common pest of kitchens and pantries, especially when carelessly kept. They are most numerous where grease and food fragments are left in sinks, scattered on the floor, or in garbage pails. For their destruction see page 148 .

The mess-room should not only be a model of cleanliness, but must also be made cheerful and attractive. The old practice of covering the tables with oilcloth is unhygienic. They should be scrubbed once a day with soap and hot water, and the stools also wiped daily with a clean, damp cloth. The floor is mopped every morning and swept after each meal. Nothing is more attractive and cheering in the mess-room than scrupulously clean crockeryware, forks and spoons. The forks need special care to wipe off the grease and food particles lodging between the prongs. The dishes should be served neatly, in an appetizing way; good service has a decided favorable effect upon digestion, not only by the contentment it promotes but also by its undoubted psychic influence upon the secretions of the digestive organs.

All the men should come to the mess-room with clean hands and face, and neatly dressed in the uniform prescribed. 


\section{CHAPTER XL.}

\section{MILITARY HOSPITALS.}

The typical post hospital consists of a main or administration building, with a wing on one side or both sides of it according to the number of patients to be accommodated, the main building and wings consisting of basement, two stories, and attic. In the main building are the various administrative services, while the wings are exclusively used for wards and their annexes. When, on account of an increased garrison, such hospital is no longer adequate, it is usually enlarged by an addition in rear of the administration building and parallel with it so that the central halls of both structures are in line and connected by a covered way. In this addition are transferred the kitchen and mess-room, as well as the isolation and prison wards, dormitories, etc. At all of our large posts a separate annex, entirely detached from the hospital building and supplied with all necessary disinfecting appliances, is erected for the treatment of infectious diseases. (See chapter on Disinfection.)

The administration building of the post hospital is mostly without verandas, but 11sually has a front porch over the main entrance, corresponding to a balcony on the second floor. The wings are provided with verandas on each floor and on both sides, the upper and lower verandas being connected by stairways.

In the administration building the arrangement is as follows: In the basement are the boiler room with water or steam heating apparatus, heater room to supply hot water throughout the building, fuel room, store-rooms, dead room, lavatory, etc. On the first floor (Fig. I37) is an ample vestibule, with dispensary and room for pharmacist on one side and, on the other side, offices for the surgeon and his assistant; further back are the kitchen, pantry and messroom. On the second floor (Fig. ${ }_{3} 8$ ) are the operating room, sterilizing room, instrument room, surgical ward, surgeon's toilet, laboratory, recreation room, linen room and n.-c. o. room. In the attic are the isolation ward with toilet, prison ward with toilet, and dormitories for hospital corps men, also with toilet.

The wing is 73 feet 7 inclfes long, with both stories exactly alike. 
On entering it from the main building, there is a lavatory on one side and a bath-room on the other, each in feet 9 inches long. The rest of the wing, on each floor is occupied by the ward; this is 60 feet 9 inches long and 23 feet wide, inside measurement. It is lighted by 4 windows on each side and 2 at the end, and contains 16 beds in two rows, namely, two beds between the windows and one near each corner. The heating is by the direct-indirect system, the

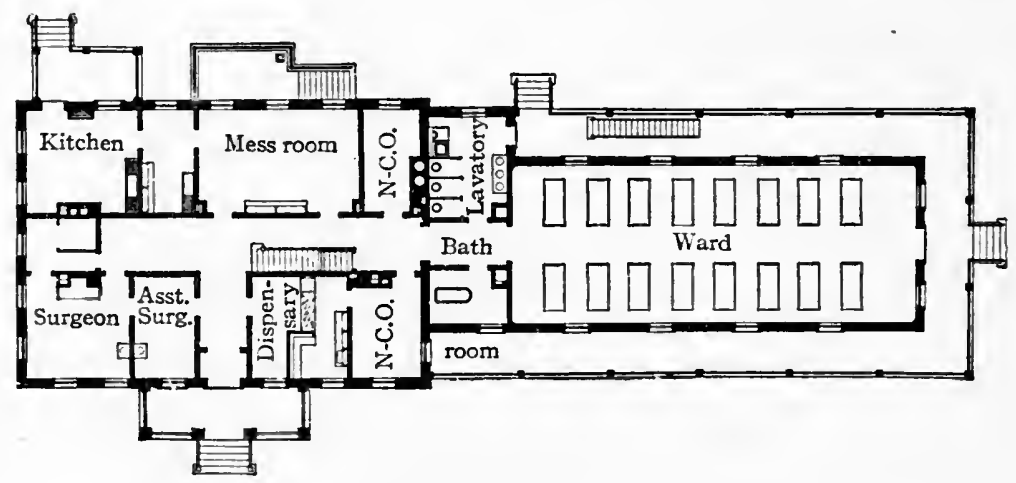

FIG. 137.-Standard post hospital for 36 beds. First floor.

hot water or steam radiators being placed under the windows. The ventilation is effected by two aspirating shafts, $20 \times 22$ inches, placed in the inner wall; two outlets (lower and upper) open in each shaft.

The general specifications governing the construction are mostly the same as those already described for barracks. The entire structure, with exception of the concrete footings and stone foundations,

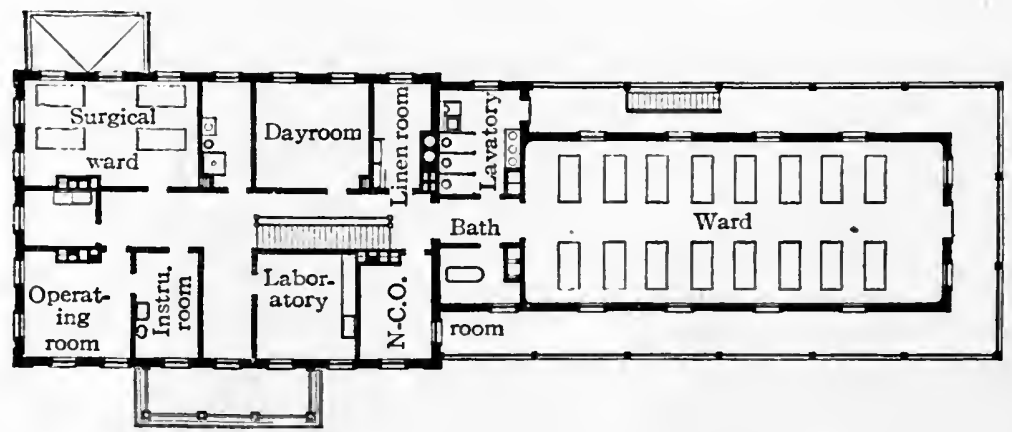

Fig. 138. - Standard post hospital for 36 beds. Second floor. 
is of brick. All exterior walls are built hollow, the "shells" being tied together with galvanized steel ties every sixth course. The walls and ceilings are lathed, plastered and hard finished. The basement floor is of concrete; the floors of first and second stories, except as noted below, are of wood, rarely of reinforced concrete finished with cement. The exterior walls in contact with earth,

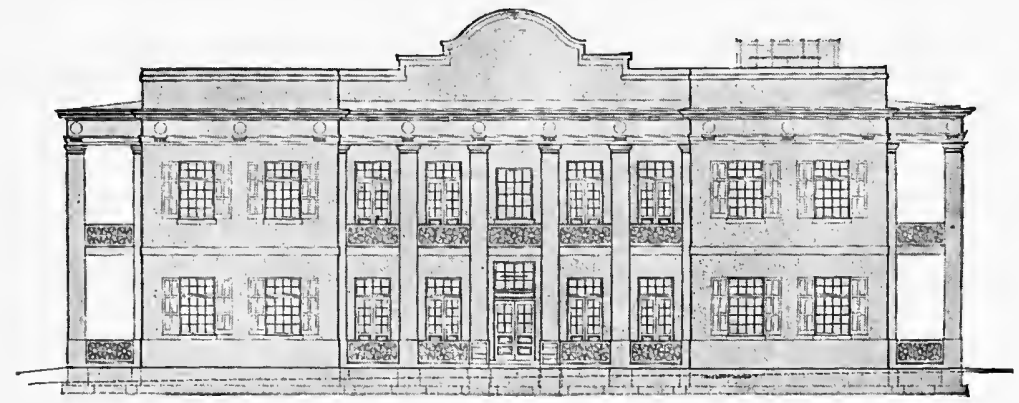

FIG. I39.- Post hospital in the Tropics.

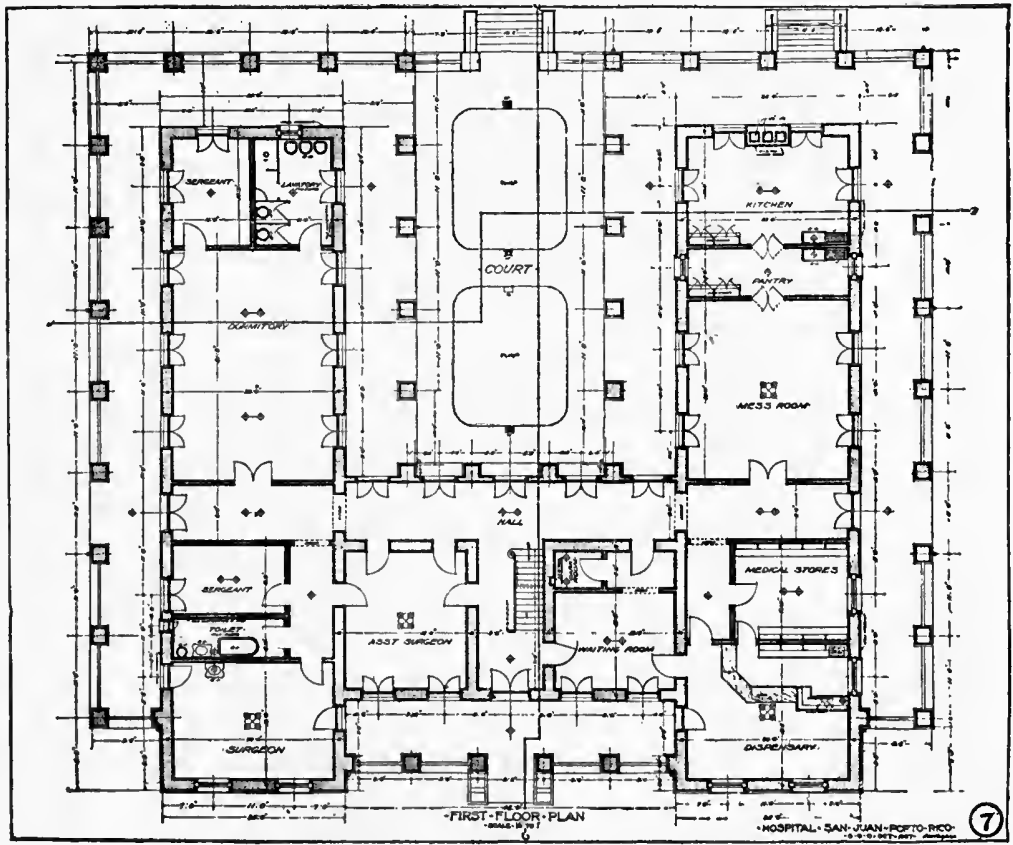

Fig. I40.- Post hospital in the Tropics. First floor plan. 
from bottom of footings to the grade line, are given a heavy coat of boiling-hot asphaltum, or similar waterproofing compound, and plastered over with cement mortar. All interior walls receive a dampproof course a few inches below the floor level, composed of two layers of waterproofing felt cemented with hot asphaltic cement, or of slates imbedded in cement.

The floors of all lavatories, toilet rooms, operating room and its annexes are of 2-inch hexagonal, vitreous, white tiles, with border of one row of $3 \times 6$ inches glazed white tiles. All rooms having tile floors are likewise tile wainscoted, 5 feet high in operating room and annexes, and 4 feet high in the other rooms, with $3 \times 6$ inches glazed white tiles having concave base and rounded top, curved angles at

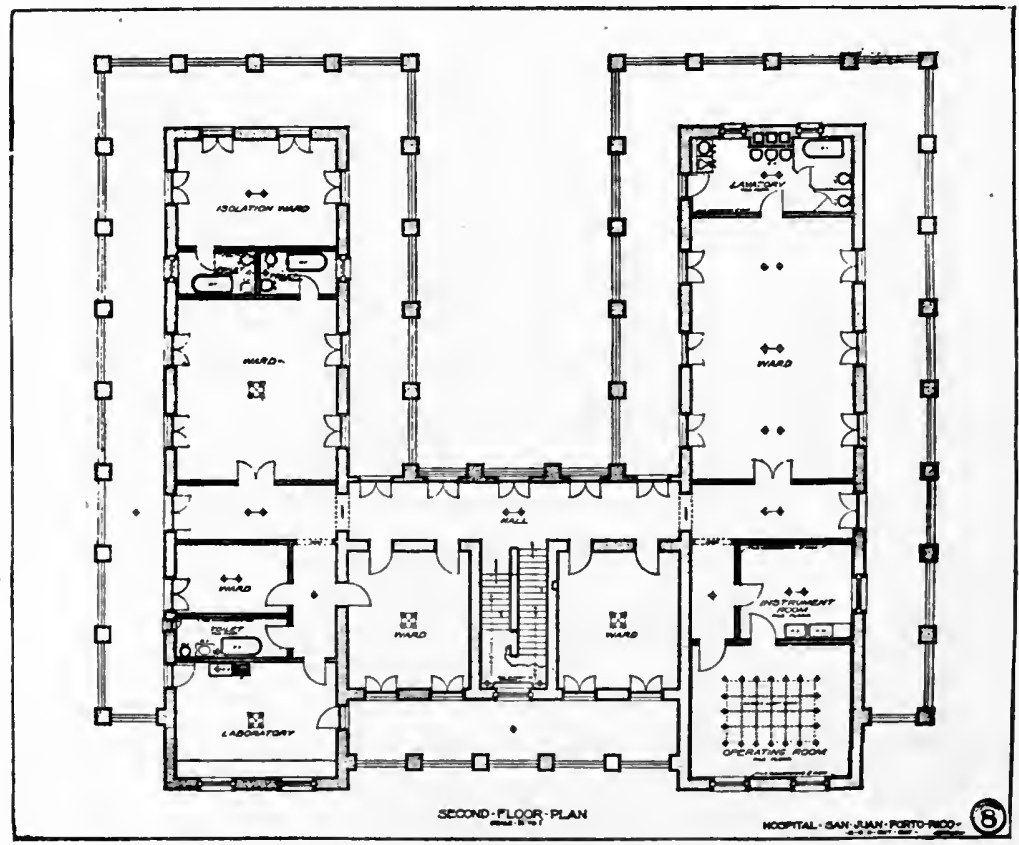

FIG, I41.- Post hospital in the Tropics. Second floor plan.

corners and rounded return at openings. In all lavatories, above basement, the partitions are usually of Italian marble around bathtubs, water-closets, showers, urinals, etc., while all metal parts are of nickel-plated brass. 
All the walls of a hospital should be painted, and the paint such as to form a hard, smooth and impervious surface that may be washed with antiseptic solutions and withstand disinfectant fumes. In the wards the walls should receive a dull non-reflective finish, restful to the patients' eyes. Otherwise, for the general trim of the hospital, satin or glass-finish enamels are recommended, except where tiling may be preferred. The highest grades of enamel finish are said to be obtained with American zinc oxid, ground in suitable varnish.

All finished wood floors and stair treads are given, by the contractor, two coats of floor polish, the last coat to be well rubbed down with flannel cloth or floor brush. This polish consists of paraffin dissolved in linseed oil, to which liquid drier and turpentine are added. To keep these floors in perfect condition, a suitable dressing must be applied and well rubbed in at least once a month. In

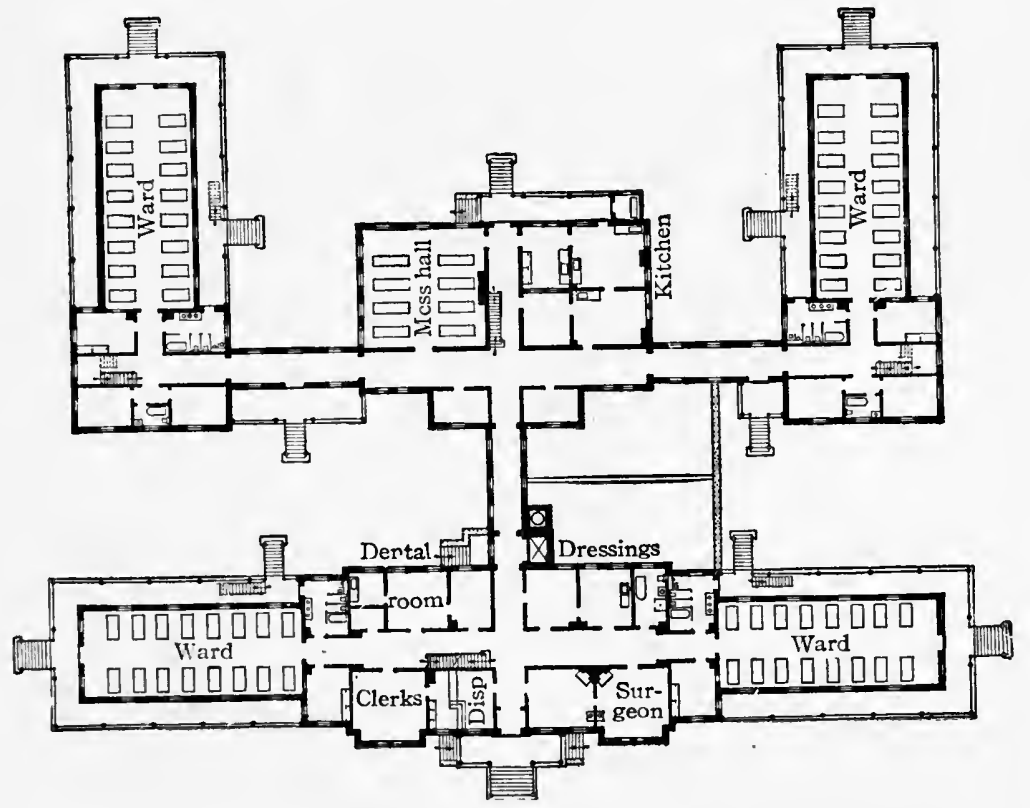

Fig, 142.- Brigade post hospital for $\mathrm{I} 32$ beds. First floor.

our hospitals, this dressing consists of a solution of $1 / 2$ pound of paraffin and 2 pounds of beeswax in I gallon of turpentine. Munson recommends I ounce each of paraffin and wax in a quart of turpentine. 
In the tropics, where skilled labor is hard to procure and the woodwork often attacked by boring ants, it has been found advantagreous to build hospitals and other public buildings of iron and concrete. The specifications for the construction of a military hospital at San Juan, Porto Rico, call for a 2 -story reinforced concrete and cement structure, with high ceilings, wide porches and electric lighting. (Figs. I39, I40, I4I.)

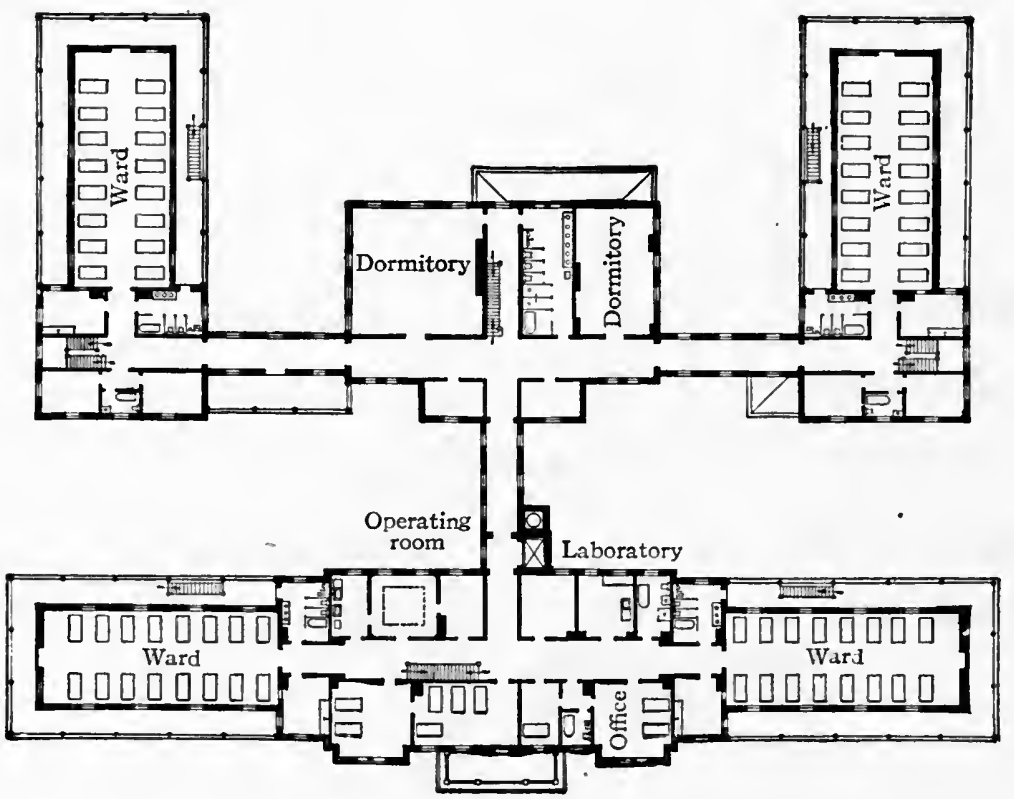

FIG. 143- - Brigade post hospital for 132 beds. Second floor.

In the Philippines, large, flat, translucid shells are extensively used in windows instead of glass panes. They have the advantage of toning down the intensity of light and reflecting the heat rays.

At large posts, the hospital assumes various shapes according to local conditions, or as the result of several successive extensions, but seldom departs materially from the above structural specifications. (Figs. 142, I43.)

Goneral Hospitals. - The plans of large permanent hospitals, such as base or general hospitals, while remaining within the generally accepted principles of hygiene and administration, are susceptible of 
wide variation, according to the views of medical officers and architects, available means and peculiarities of site. The pavilion system is the one that most commends itself, the pavilions being conveniently grouped and so connected with all the other buildings as to secure efficient administration. Each pavilion should be of 2 or 3 stories, with a ward and annexes on each floor, each ward to accommodate about 30 patients. One-story pavilions, in a permanent

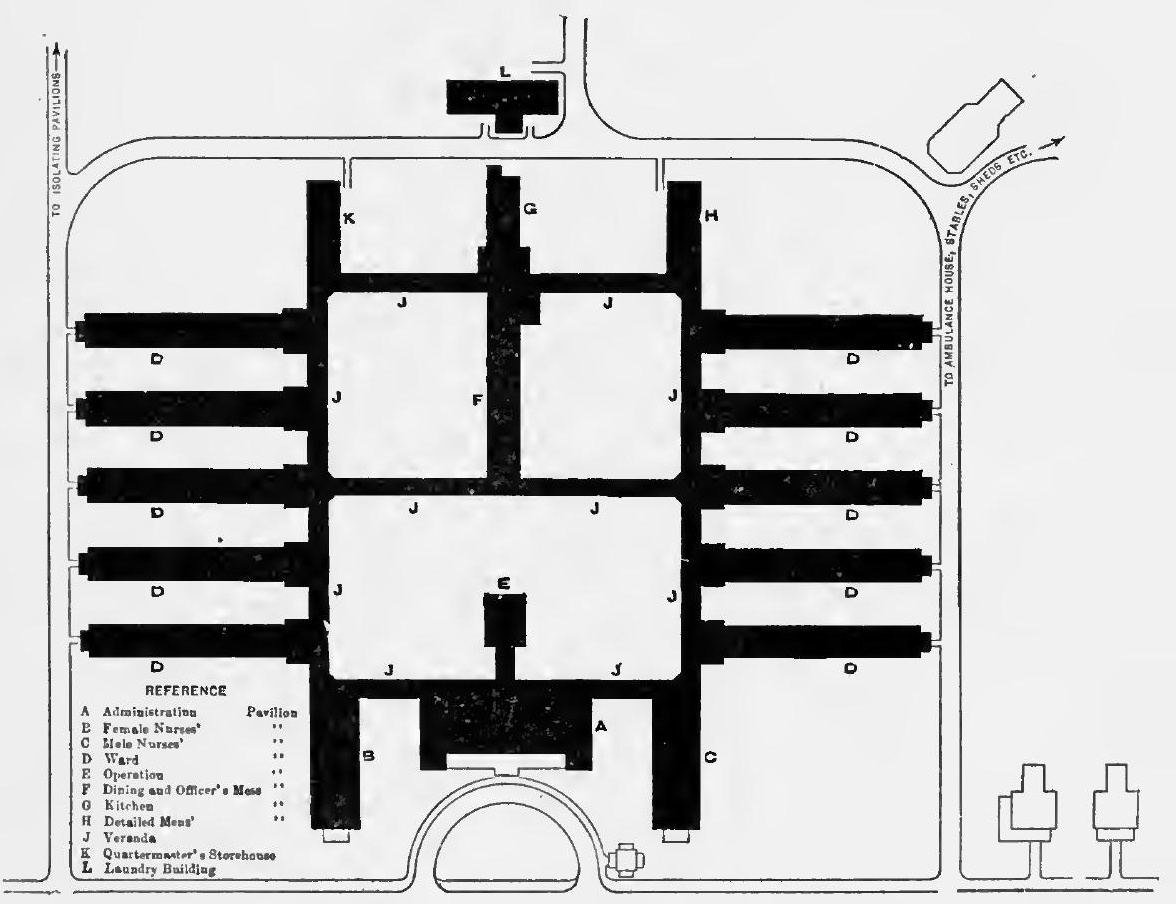

Frg. 144.-Plan of the U. S. Army general hospital, at the Presidio, San Francisco, Cal.

hospital, involve an unnecessary waste of space and material, and cause a very inconvenient scattering of wards. The general hospital at Presidio, San Francisco (Fig. I44), is excellently planned and one of the best types of that class, but open to the objection that its pavilions are I-storied; the ward, excluding lavatory and service rooms, is I 53 feet long and accommodates at least 40 patients, a larger number than should be placed in one room. 
Temporary General Hospital. - In the event of war, when preparations must be made quickly for large numbers of sick and wounded, the pavilion system necessarily imposes itself. The plans and specifications for temporary base and general hospitals, on this

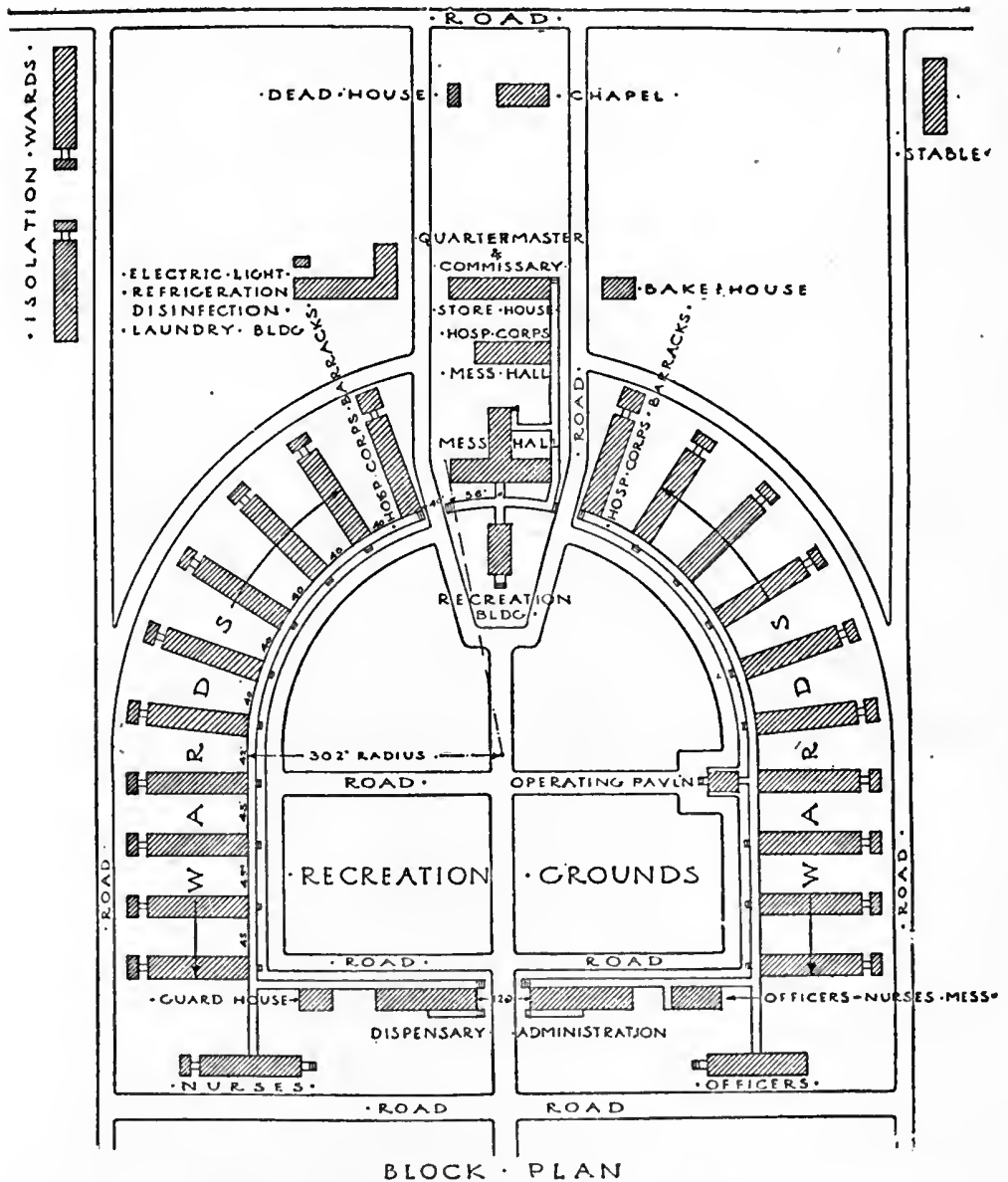

FIG. I45.- Plan for temporary base hospital, with pavilion wards.

system, are on file in the Surgeon General's Office so that they can quickly be put into execution.

The buildings are of simple construction and can be erected by ordinary builders with material that can be purchased in any market. 
In the interest of simplicity a single type has been adopted for all purposes, and the various buildings differ only in their interior arrangement.. They are all I-story substantial frame structures, with walls and ceilings finished with tongued and grooved, beaded boards; heated by stoves and roofed with corrugated iron. One-story pavilions are preferred because of the greater simplicity of construction, the greater facility of heating and ventilating and, in case of fire, the lesser risk to the patients.

Each ward (Fig. I46) consists of a building I 20 feet long and 26 feet wide, with ceiling I 2 feet high, lighted by Io windows on each. side. It is connected, by a passage ro feet long, with an annex in which are the lavatory on one side and the water heater on the other. In the end nearest the annex is a room on either side, one for special diet and the other for office and linen. The ward proper is 106

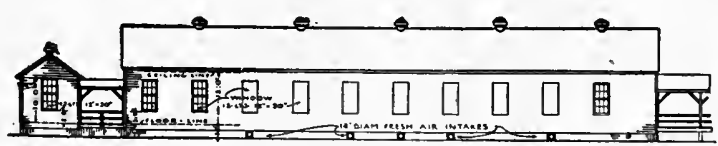

LSIDE ELEVATION

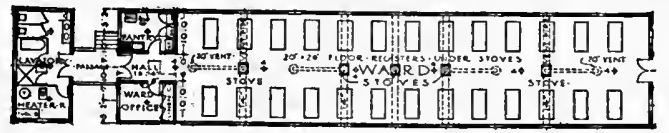

- ERST: HLOR PLAN

WARD AND IAVATORY.
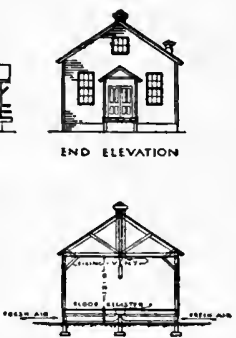

-SICTION. W. WARD

FIG. I46.- Elevation and floor plan of a pavilion. Temporary general hospital.

feet 8 inches long and accommodates 26 beds, each patient having Io6 square feet of floor area and I,272 feet of cubic space which, under the circumstances, is quite sufficient. It is heated by 5 jacketed stoves with bent pipes running up into ventilation shafts. The ventilation is effected by means of galvanized pipes conducting fresh air under the floor to the stoves, and of 5 ventilation shafts which receive the smoke pipes and are topped with star ventilators.

The general arrangement and grouping of the pavilions varies greatly. The wards may be set, like divergent rays, on the outer side of a covered way shaped like a horseshoe (Fig. I45) or an inverted letter $\mathrm{V}$, with all or most of the other buildings inside: they may also be located on each side of a covered way, or on two 
sides of a square or rectangle. The chief objects to be attained, in determining this general plan, are to give each ward a maximum of space, light and sunshine, secure accessibility to all parts and convenience of service, and provide open, attractive grounds for the use of convalescents.

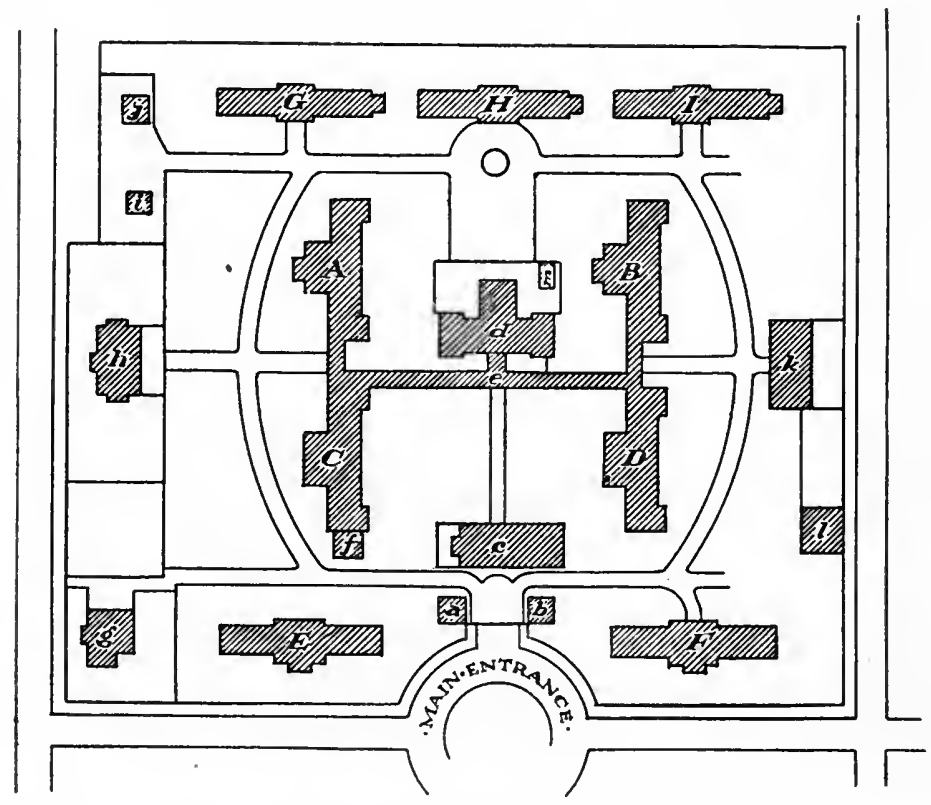

FIG. 147.- Garrison hospital, Tempelhof, Berlin. A, B, C, D, two-story buildings for wards with corridors on north side; $\mathrm{E}, \mathrm{F}$, convalescent wards; $\mathrm{G}, \mathrm{H}, \mathrm{I}$, wards for contagious diseases; $a$, guard and telegraph; $b$, pharmacy and laboratory; $c$, administration ( 3 stories), reception room, quarters for officers and nurses; $d$, laundry, kitchen, distilling and steam plants; $e$, covered passage connecting buildings $\mathrm{A}$ and $\mathrm{B} ; f$, operating room; $g$, chief surgeon's quarters; $h$, hospital corps quarters; $i$, ice house; $j$, chapel, deadhouse, and vivisection room; $k$, store house; $l$, detached ward; $m$, disinfection house.

One of the best German types of military hospitals is illustrated in Fig. 147, which represents the Tempelhof garrison hospital in Berlin, with a capacity for 500 beds, but susceptible of expansion to 600 . It comprises 14 wards with 16 beds each, 27 rooms with 6 beds, 22 rooms with 3 beds, 16 rooms with 2 beds, and single rooms for officers. Each bed has a cubic air space of about 37 meters 
( 1,3 I 5 cubic feet). It is under the direction of a chief medical officer, with personnel of 17 medical officers and 125 enlisted men. Some of its features are a gymnasium for the special exercise and development of any part of the body, an experimental biological laboratory, a model hospital train all fitted out (including ward, kitchen, pharmacy, etc.), and 6 distillation wagons for the field, each capable of distilling and cooling 500 liters per hour. 


\section{CHAPTER XLI.}

\section{AIR.}

Composition. - Air is a mixture of several gases, chiefly oxygen, nitrogen and argon, in the proportion, by volume, of about $2 \mathrm{I}, 78.15$ and 0.85 , respectively. The other normal constituents of air, all in very small or minute quantities, are carbon dioxid, ozone, aqueous vapor, ammonia, nitrous, nitric and sulphuric acids, and dust. The proportion of all these constituents varies only within narrow limits, and when temporarily disturbed becomes again quickly readjusted under the operation of natural laws, that is to say, through the ceaseless convection currents due to the sun's heat and, to a lesser extent, the diffusion of gases. Wide fluctuations in the oxygen tension of the air may occur without notable disturbances of the body functions, until the proportion falls to about 13 per cent. or rises to 50, when adjustment to the change becomes difficult.

At sea level and a temperature of $0^{\circ} \mathrm{C}$. the normal pressure of the atmosphere is sufficient to support a column of mercury $760 \mathrm{~mm}$. ( 30 inches) high, and amounts to 15 pounds on every square inch of surface. This pressure, as shown by the barometer, varies within certain limits, being higher when the air is cold, dry and compressed by upper downward currents, and lower when the air is hot, damp and lifted by upward cuirrents. Damp air is lighter than dry air because the density of aqueous vapor is less than the air, in the ratio of 0.62 to $\mathrm{I}$.

Oxygen is indispensable to the maintenance of all life, animal and vegetable. Without it there can be no growth or repair of tissues, nor combustion and destruction of organic and inorganic matter. In man and animals, the oxygen of the air is absorbed in the lungs by the red blood-corpuscles, uniting with the hemoglobin, or coloring matter, and carried by the arterial current to all parts of the body; it is taken up by the tissue cells, where it combines with the metabolized carbon and hydrogen, the resulting carbon dioxid and water being conveyed by the venous blood to the lungs and eliminated. According to Prof. Foster, an average adult inhales a little more than 7 pounds of oxygen daily, about one-fourth of which, or nearly 2 pounds, is absorbed by the lungs. 
Plants breathe like animals by absorbing oxygen and giving off carbon dioxid, a function especially active during germination and blossoming; oxygen is therefore essential to their growth and life.

Nitrogen is an inert gas, chiefly serving to dilute the oxygen; it takes no part in the respiration of animals, but contributes to the nutrition of plants. It is the most important constituent of animal tissues. Animals obtain it exclusively from the vegetable world, while plants derive it from the soil and the air; from the soil out of ammonia salts, nitrites, nitrates and proteid compounds from dead plants and animals; from the air through the agency of certain "nitrifying" bacteria which stand in symbiotic relationship to the plant and enable it to obtain nitrogenous compounds at the cost of the atmospheric nitrogen. These bacteria develop nodules, ranging in size from a pin's head to a small pea, upon the roots of many plants, particularly species of the leguminous family (peas, beans, clovers) which, in consequence, often thrive in poor soil and enrich it. Cultures of them are now commercially prepared and made available for sowing in sterile soil in connection with stitable plants. It is worth noticing that nitrogen is also one of the principal ingredients of explosive powders, so that if it is indispensable to the life of animals it is also one of the chief agents in destroying it.

Argon, so far as known, is an inert, indifferent gas.

Carbon dioxid or carbonic acid $\left(\mathrm{CO}_{2}\right)$ is a constant but variable constituent of air, averaging from 3 to 4 parts in 10,000 , or 0.03 to 0.04 per cent. In a dense fog it may rise to $\mathrm{I} 2$ or $\mathrm{I} 4$ parts in the open air of large cities. It is derived from the respiration of animals, the combustion of all substances used for heating or illuminating purposes, the oxidation, fermentation and decomposition of all organic matters, the eruption of volcanoes, etc. The ground-air is much richer in carbon dioxid than the open air above, sometimes reaching $\mathrm{IOO}$ or more parts in. $\mathrm{IO}, 000$; by diffusion it contaminates the layers next to the ground which on this account contain much more of $\mathrm{CO}_{2}$ than the normal average.

An adult man exhales about I 5 cubic feet, or about 2 pounds of $\mathrm{CO}_{2}$ a day. Illuminating gas gives off twice its volume of it. One ton of coal, by its combustion, produces three tons of $\mathrm{CO}_{2}$. The burning of coal, therefore, is the chief source of carbon dioxid in the atmosphere, the yearly production of this gas being estimated at I,200 millions of tons. Huxley calculated that the atmosphere super- 
incumbent upon any area of the earth's surface contains enough carbon to cover this area with a dense forest.

How is this great excess of $\mathrm{CO}_{2}$ in the atmosphere disposed of and the normal ratio of 0.03 per cent. maintained? Much of it is absorbed by the ocean, since water takes up its own volume of the gas, but a larger proportion is removed by plants which, through the action of their green pigment, or chlorophyl, and under the influence of light, decompose it, retaining the carbon and discharging the oxygen. Thus it is estimated that one acre of woodland withdraws, in one season, $4 \frac{1}{2}$ tons of $\mathrm{CO}_{2}$ from the air, appropriating $\mathrm{I} / 4$ tons of carbon and discharging $3 \frac{\mathrm{T} / 4}{4}$ tons of oxygen. This function of plants, carried on through their foliage, is a purely nutritive one and not concerned in their respiration, the oxygen thus returned to the air far exceeding the comparatively small amount absorbed in the performance of the latter function, and maintaining the normal equilibrium of atmospheric gases. Since the decomposition of carbon dioxid by plants ceases in darkness, while their respiration continues, an increase of that gas may be expected at night in rooms in which plants are kept, especially when blossoming, if not properly ventilated.

Ozone, an allotropic form of oxygen, is a normal but not constant constituent of the air, being only found in the country and at sea, and the amount increasing with the altitude. It has very strong oxidizing properties, eagerly combining with decaying animal or vegetable matter, so that it quickly disappears wherever such matter is abundant. Any locality, therefore, where ozone is present may be assumed to be free from putrefying organic substances. Very little is known of the effects of ozone on the system beyond the fact that, except in largely diluted form; it is dangerously irritating (see p. 736).

Ammonia, as well as nitrous and nitric acids, are always present, at least in traces in the air, and benefit the soil through the rain which dissolves more or less of them.

Coal contains about 8 pounds of sulphur per I,ooo, which by combustion becomes oxidized into sulphuric acid; thousands of tons of this acid are every year showered upon the eastern United States by the rain.

Aqueous vapor is a constant and very important constituent of air, although variable in amount and unequally diffused. It is derived 
from many sources: the evaporation of water and soil, the evaporation from the skin and lungs of animals, from combustion and from the transpiration of plants. An adult man, under average conditions, gives off from 3 to 4 pounds of watery vapor from his skin and lungs, namely, $2 \mathrm{~T} / 2$ pounds from the skin and the remainder from the lungs. The amount given off by animals, however, is very small compared to that contributed by plants which absorb water in large quantity from the soil, through their roots, and exhale it as vapor through the pores (stomata) of their leaves. According to Hellriegel, the amount of water thus exhaled by plants is from 250 to 400 times the weight of the dry wood formed during the same time.

The amount of invisible vapor which the air can hold depends on the temperature; thus the amount which at $70^{\circ} \mathrm{F}$. would condense into cloud, fog or rain, will completely disappear at $90^{\circ}$ and leave the sky perfectly clear. The proportion averages about I per cent., varying from less than o.I to 4 per cent. The air expired in breathing is saturated, containing about 6 per cent. of moisture. The capacity of the air to hold moisture is doubled for every temperature increase of $27^{\circ} \mathrm{F}$. As already stated, moist air is lighter than dry air, and upon this largely depends the motive power of the atmosphere. The air lightened by heat and vapor ascends and, on cooling, parts with vapor which condenses as cloud; this condensation sets free the latent heat of vaporization and this heat serves to carry the neighboring air to still loftier altitudes (Leonard Hill). Water vapor and dust modify the transparency and diathermancy of the air; they both soften the scorching power of the sun and prevent the rapid scattering into space of the heat gained by the earth, a parasol at noon and a blanket at night. Water vapor acts like the glass panes of a greenhouse in absorbing and retaining heat, therefore as a temperature regulator; it is also a great reservoir and carrier of latent heat which becomes actual heat when the vapor condenses.

The degree of temperature to which the air must be cooled to cause condensation of its vapor is the "dew point," while the air which is so charged with vapor that any lowering of temperature would produce precipitation is said to be "saturated." When the air is saturated, it is unable to absorb more vapor and therefore evaporation from the skin ceases, but perspiration being a necessary 
function of life continues, although very much diminished; this is a very uncomfortable weather condition popularly described as "sticky" in summer and "raw" in winter.

Relative Humidity. - The "relative humidity" of the air is the percentage of the total amount it can take up to become saturated at any given temperature. It is obtained by dividing the weight of vapor actually existing in the air by the weight of vapor which would be present if the air were saturated. Thus the relative humidity of the Eastern States ranges from 60 to 75 , that is to say, is from 60 to 75 per cent. of saturation, and diminishes as we advance into the drier interior.States, reaching its mininum in Arizona (Fort Yuma), where it averages only 35. It is 42 at Denver and 66 at Los Angeles. In California it is exceedingly variable, often dropping from nearly 100 at dawn to 22 at noon. In England it averages about 75 , being highest at 9 А. м. The temperature we actually experience, in summer, that is, our subjective sensation of heat and cold, is chiefly determined by the amount of our perspiration and consequent evaporation, and this depends upon the dryness of the air more than its temperature, or, in other words, upon its relative humidity. The drier the air the more freely we perspire and the more active is the evaporation of the sweat and cooling of the skin. Our skin surface, always excreting sweat, may be compared to a wet bulb thermometer, so that the reading of the latter is an index to our sensation of heat. Thus, while the mean July reading of the wet bulb thermometer in Boston is $65^{\circ} \mathrm{F}$., and $75^{\circ}$ in Savannah, it may be only $60^{\circ}$ at Yuma, Arizona, in spite of its much higher, torrid summer heat; Arizona, therefore, is cooler and more comfortable in hot weather than the Eastern States, provided one is protected from the direct sun-rays. Dryness of the air is also expressed by the difference between the wet and dry bulb thermometers; this difference is about $5^{\circ}$ or $6^{\circ} \mathrm{F}$. in the Eastern and Southern States, increases in the interior and reaches its maximum of about $20^{\circ}$ in Arizona. There the skin is dry and harsh, the hair crisp, the furniture shrinks and falls apart, newspapers are brittle and pencils make no marks.

To determine atmospheric humidity the easiest method is that of the dry and wet bulb thermometers; from their readings and by means of hygrometric tables, can be deduced the dew-point, the vapor tension and the humidity, absolute and relative. 
Physiologically, the essential point is not the amount of humidity present, but the amount that can still be taken up, that is, the shortage from saturation or "saturation deficit." This deficit expresses the evaporative or drying power of the air from which the cooling effect depends, and is indicated not only by the difference between the dry and wet bulbs, but chiefly by the temperature. Thus the evaporative force of the air with readings of $45^{\circ}$ and $50^{\circ}$ is much greater than with readings of $30^{\circ}$ and $35^{\circ}$, and will permit the evaporation of much more moisture. This means that, as the temperature rises, there will be an increase of evaporative force, a wider separation between the dry and wet bulbs and correspondingly greater cooling effect in the shade. This effect, as in arid regions, will often more than offset increased solar radiation. As noted by Macfie, saturated air at $89^{\circ} \mathrm{F}$. will not relieve the human system of its heat as fast as produced and a febrile condition will develop. The same air at $13 \mathrm{I}^{\circ}$, containing the same amount of vapor, will readily dispose of the heat production by virtue of the brisk evaporation which it permits.

A high degree of humidity in hot weather not only checks perspiration but also hinders radiation from the body. On the other hand, since moist air is a better conductor of heat than dry air, it will increase the loss of heat from the body by conduction. This loss, however, is small compared to what it would be from radiation and evaporation of the perspiration in drier air, so that much humidity renders hot weather decidedly more oppressive. In cold weather the blood is driven from the skin so that radiation of body heat is not active; if the air be damp, the loss of heat by conduction, especially. if the wind be blowing, is greater than the loss which would result from radiation in drier air. It follows, therefore, that humidity produces opposite effects, intensifying both heat and cold, and that dry air is preferable in either extreme of temperature.

High humidity, in a cold or temperate climate, increases the urine and intestinal secretions, causing great dilution of the blood, slower circulation and other conditions tending to develop the phlegmatic temperament. Dry air, on the contrary, decreases the liquids of the body, quickens the pulse and stimulates the nervous system, producing a more restless and excitable temperament. Dry air has also a marked tonic influence and is beneficial in most chronic diseases. This is attributable, in part, to its desiccating and inhibitory effect upon many of the micro-organisms which prey upon the body. 
Three main factors influence the temperature of the air: latitude, altitude and the proximity of large bodies of water.

OCEAN AND LAKES. - The influence of large bodies of water on the temperature of the air and climate is. worth noticing. Water having much greater specific heat than land is much more slowly heated by solar radiation, but the heat penetrates deeper and is not so easily parted with. Is follows that the diurnal and annual changes are nuch smaller on the ocean or near the coast than in the interior; the range of temperature is smaller, the winter milder, the summer cooler, and the clinate therefore more equable. This influence is also exerted by great lakes; thus a difference of 10 to $15^{\circ} \mathrm{F}$. has been noticed between the northern and southern shores of Lake Ontario in winter, the southern shore being protected by the water against the northern wind.

Forests. - The influence of forests upon the climate is only local and not marked. They mitigate extremes of temperature, the summer heat much more than the winter cold, and check the violence of the winds. Their cooling effect in summer is due to the shading of the ground, the increased surface of heat radiation, the active radiating power of leaves and the free evaporation of the moisture which they discharge. There is no proof that the air of forests is richer in oxygen or ozone than outside air, but it is often stinulating from the exhaled essential oils it contains.

EFfect of Altitude. - As we rise above sea-level, air pressure diminishes; at an altitude of 6,000 or 7,000 feet it has decreased onefourth and the first symptoms of "mountain sickness" may be noticed. At an altitude of 18,000 feet it has decreased one-half and these symptoms become much aggravated; they consist in shortness of breath, weakness, headache, dizziness, nausea, increased pulse, palpitations, quick irregular respiration, nose-bleed and fainting spells. Mountain sickness is due to lack of oxygen, produced by diminished air pressure. As this pressure decreases, the force of the heart beat remaining about the same, there is a flux of blood to the skin and mucous membranes, sometimes causing the latter to bleed; the pulmonary arterioles become congested and dilated, thus to some extent reducing the respiratory surface. Such mechanical impediment, however, is probably only temporary and not of much moment. The illness is mostly due to the failure of the red bloodcorpuscles to carry enough oxygen to the tissues, so that the organs 
are unable to function properly, the heart and brain being especially sensitive to this deprivation.

Even in the rarefied air of the highest mountains there would always be enough oxygen to supply the blood cells and the needs of the tissues were it not for the fact that the chemical affinity of oxygen for hemoglobin is influenced by pressure, diminishing as soon as the tension of this gas in the alveolar air and the blood falls below a certain point, causing a dissociation between hemoglobin and oxygen, which increases with the fall of pressure.

Nature endeavors to supply the deficient oxygen in various ways so that by suitable training there is hardly any altitude on the earth that a man with sound heart and lungs may not be able to overcome. These compensatory provisions are:

I. Deep breathing, whereby the alveolar oxygen tension can generally be maintained near the normal point. A superficial breather, normally inhaling $270 \mathrm{c}$. c. of air 20 times a minute, only absorbs 5,400 c. c., while another, inhaling 440 c. c. I 4 times a minute, takes in $6,160 \mathrm{c}$. c., and will be able to reach a much higher altitude than the former.

2. Slight increase of pulse rate.

3. Increased activity of the alveolar epithelium in secreting oxygen inward into the arterioles; so that the tension of this gas is soon much higher in the blood than in the alveolar air.

4. Increase of the hemoglobin and red cells of the blood. This increase has long been noted. Even at the comparatively small altitude of 5,500 feet, Jaquet and Suter found that the blood of rabbits, within two weeks, became greater in quantity (I 5 per cent.) and richer in quality, containing more red cells ( 13 per cent.) and more hemoglobin ( 9 per cent.). This increase appears to be permanent and is found in most highlanders.

5. Increased amplitude of chest and capacity of lungs. The relatively large chest of the natives of the elevated plateaus of Mexico and Peru is a well known fact.

Such are nature's adjustments for life in a rarefied atmosphere, which render possible the growth and prosperity of cities like Mexico at 7,524 feet, Quito at 9,520, Leadville (Colo.) at 10,200, and Fotosi (Bolivia) at 13,600 feet above sea-level.

If the change of altitude is not too great, too sudden, or of too long duration, the symptoms are slight and ephemeral, lungs and 
heart soon recovering their normal condition. But if the change is permanent, some time is required for the system to accommodate itself to it. Thus people going from the Eastern States to reside at Cheyenne, Wyo., or the neighboring post of Fort Russell, about 6,000 feet high, may not be aware of any difference in their sensations, but it will be several weeks before they regain their full capacity for active physical and mental work. There is always danger for people with unsound heart and lungs to ascend to the summit of high mountains by steam or electric cars, their power of accommodation being unequal to the great and sudden fall of air pressure. The same risk is incurred by aeronauts and aviators who rapidly reach high altitudes.

As one rises above sea-level the temperature falls, at the approximate rate of $\mathrm{I}^{\circ} \mathrm{F}$. for each 300 feet. Owing to the absence of dust and water particles at high altitudes, as well as to the greater diathermancy of the thinner air, the radiant heat of the sun is much greater; its rays are more scorching, although the air itself may be like that of an arctic climate. The heat radiation from the earth is also greater, rendering the nights colder. The air is drier and purer; dead animals often mummify without decaying; meat can be dried (jerked) by exposure to the sun, and the grass is self-cured on the plains. Giles* observed that at an altitude of 16.500 feet. while the temperature at noon, in May, stood at $-20^{\circ} \mathrm{F}$., the sun thermometer registered $165^{\circ}$; one side of the hand turned to the sun would be scorched while the other was chilled, as if in contact with cold water.

The therapeutic value of mountain air lies in its purity from dust and bacteria, its bracing cold, intense insolation, greater radioactivity and in the increased formation of hemoglobin, resulting in more active metabolisn and the strengthening of the heart and respiratory mechanism.

Atmospheric Dust. - Dust is a normal and very important constittent of the air, being derived from the soil by the action of the winds. from combustion, volcanoes and meteorites, while, at sea, much salt dust is shaken off from the spray of the waves. It is of organic and inorganic origin, a certain proportion of it consisting of micro-organisms which are either free or, more commonly, adherent

* Climate and Health in hot countries. 
to mineral particles. Dust is abundant everywhere but especially in the vicinity of towns and manufacturing centers. Smoke consists chiefly of fine carbon dust; it has been estimated that a puff from the cigarette smoker contains 4,000,000,000 particles of it. The haze of summer days is chiefly the result of smoke. Much of the atmospheric dust is readily visible to the naked eye, but more is microscopic and even ultra-microscopic, that is to say, so extremely tenuous as to be beyond the power of the microscope.

The chief function of atmospheric dust is to condense aqueous vapor and cause precipitation, each particle being a nucleus upon which a minute droplet forms, these droplets coalescing into drops. Were it not for this dust, there would be no cloud, no mist and no rain, the aqueous vapor condensing directly upon the surface of the earth, wherever the air penetrates. "The inside of our houses would become wet; the walls and every object in the room would run with moisture" (John Aitken).

The great importance of atmospheric dust in scattering and diffusing sunlight, and thus rendering objects visible, should also be mentioned.

For dust in connection with respiration and heating, see pages 520 and 554 . 


\section{CHAPTER XLII.}

\section{VENTILATION.}

"There is nothing so priceless and yet so costless as air. There is no financial investment which does or can yield so sure and so large returns as money wisely expended for pure air." (Prof. S. H. Woodbridge.)

Pure air is as necessary as pure water and wholesome food for the maintenance of health. Its vivifying qualities are best manifested when unconfined, in the open; as soon as its free movement is interfered with, these qualities are altered. Thus the comparatively pure air of well-ventilated rooms is different from the fresh outside air; the latter possesses tonic and stimulating properties which are lacking in the former. This is shown by the great success of the open-air treatment not only of tuberculosis but of all infectious diseases, in winter as well as in summer. It is also shown by the excellent results obtained in open-air schools. In what consists this vivifying principle of the free air still remains one of the secrets of nature.

Ventilation may be defined as the continuous and systematic renewal of air, so as to keep it as fresh and pure as possible.

Causes of Air Contanination.- The air of inhabited buildings is fouled by many causes, but especially by respiration, excretions, dust, combustion and decomposition.

In the process of respiration, a certain amount of oxygen is absorbed by the blood, so that expired air contains only 16.40 parts of this gas; the proportion of nitrogen remains unchanged, whereas carbon dioxid is increased from 0.03 to 4.40 per cent., the lungs of the average adult at rest eliminating about $\mathrm{I} 6 \mathrm{cubic}$ feet of it in the twenty-four hours, or 0.66 of a cubic foot per hour. Expired air has nearly the temperature of the body and is therefore generally lighter than outside air; it is, furthermore, saturated with moisture, the amount given off per man in a day being about a pound. Whether expired air contains any special organic matter, some volatile alkaloid, as described by Brown-Séquard, seems quite doubtful, the weight of evidence being against such assumption. This ques- 
tion, however, was recently reopened by the experiments of Rosenau tending to show that there is a substance in the condensed moisture from the expired breath capable of sensitizing guinea-pigs so that they will react to anaphylaxis, that is, to a subsequent injection of normal human serum. Further investigation will be required to determine the practical significance of this phenomenon. There is general agreement that expired air received directly from the trachea of a normal animal is odorless, as well as the liquid obtained from its condensation by cold. "In ordinary quiet respiration, no bacteria, epithelial scales, or particles of dead tissue are contained in the expired air. The cause of unpleasant, musty odors in rooms may in part be due to volatile products of decomposition from decayed teeth, foul mouths, or disorders of the digestive apparatus, and in part to volatile fatty acids given off with or produced from the excretion of the skin, and from clothing soiled with such excretions" (Drs. Mitchell, Billings and Bergey). Straus found, in one experiment, that although the inspired air contained over 20,000 bacteria, only 40 were present in the expired air, and concluded that the nasal mucus possesses marked bactericidal properties. Many germs, however, may be contained in the particles of saliva or mucous secretions ejected in coughing, sneezing or even speaking, sometimes of a dangerous character, as in diseased conditions of the throat, tonsils or lungs.

The skin contributes materially to air pollution. It excretes two or three times more water than the lungs; this water, or sweat, contains fatty acids, ammonia and soda salts; mixed with the sebaceous secretion, epidermic débris and dust, it soon gives rise to ill-smelling products. The $\mathrm{CO}_{2}$ excreted by the skin is a negligible quantity.

From these several sources are derived the offensive organic and nitrogenous matters, either gaseous or in the form of invisible dust, which contaminate crowded rooms, being slowly oxidized and long retained by hygroscopic surfaces and clothing.

The proportion of oxygen is but slightly affected by bad ventilation, even under the worst conditions being seldom more than I or 2 per cent. below normal.

Dust. - The dust which contaminates the lower air strata and is commonly breathed by man is a compound of many ingredients, mineral and organic, namely: house and street sweepings; mud and dirt dragged into houses, dried and pulverized; powdered earth, 
plaster, asphalt, ashes, iron dust ; soot from chimneys; garbage frequently strewn in streets; excrements of horses, dogs and other animals; human excrements in congested tenement districts; desiccated sputum; microbes of all kinds, etc. Of mineral constituents, in cities, iron and steel dust deserves special attention; it is produced by elevated railroads, subways, trolleys, automobiles, horse shoes and the nails on shoes of pedestrians, and may amount to many tons a month. According to Soper, the dust collected in the air of New York, 300 to 400 feet above street level, contains Io to I 5 per cent. of metallic dust; in the streets, 35 per cent., and in the subways, 63 per cent.

The dust which gathers inside houses is largely made up of organic matter, consisting of vegetable and animal fibers, hairs, epithelial cells, foodstuffs, pollen, spores, etc. Micro-organisms, pathogenic and others, are also abundant in air, in and out of habitations, forming the so-called "microbial dust." They generally adhere to mineral or other particles, and their viability depends much upon the hygroscopic moisture of the latter. In a bed-room, Miguel found 4,500 to the cubic meter, and 40,000 in the ward of a hospital; in a barrack, at reveille, Kiener and Aldiber counted 220,000. The number found in any room varies enormously according to the occupation of the inmates, the agitation of the air and the currents formed through it; for dust settles rapidly on walls, woodwork and furniture in a quiet atmosphere, but is again quickly raised and floated by air disturbances.

Micro-organisms are much more abundant in towns than in the country, in the proportion of nearly Io to I ; thus while the average number in Paris is 3,910 to the cubic meter, that at Montsouris (just outside the city) is only 455 (Miguel). The number decreases with the altitude and they soon disappear above 6,000 feet. Thus none are found on the summit of Mount Blanc. Sea air, Ioo miles from the coast, is also entirely free from them, as well as the high altitudes of the Arctic Circle.

Mineral dust, when abundant, may produce irritation and possibly inflammation of the respiratory passages. By maintaining a congested condition of the mucosa it favors the implantation of pathogenic germs, and becomes a predisposing cause of asthma, hay fever and bronchitis. It is also a frequent cause of conjunctivitis. The inhalation of metallic dust may, in time, seriously damage the pul- 
monary tissue. Workers in dust-producing industries are notoriously liable to tuberculosis. Ordinary atmospheric dust, stich as is produced by natural agencies, causes wonderfully little irritation of the healthy respiratory mucosa, being arrested and disposed of by the mucous secretion of nostrils and tonsils and the ciliated epithelium of the bronchi. Even when loaded with dust, air can often be breathed for many days with surprising immunity. This is explained by Prof. K. B. Lehmann, who demonstrated that the great bulk of the inspired dust is caught by the nasal, oral and pharyngeal mucosa and swallowed, so that, at best, less than a quarter of it enters the bronchi. The soluble dust may be absorbed along the gastro-intestinal path and cause chronic intoxications.

The serious danger from dust, however, is supposed to be from the more or less desiccated micro-organisms it carries. From their elaborate experiments in connection with aërial infection, Winslow and Kligler arrived at the following findings and conclusions:*

City-street dust (as determined by plating on lactose agar at $20^{\circ}$ C.) averages about 50,000,000 micro-organisms per gram, and indoor dust between 3 and 5 millions. Spore-forming aërobes make up only I/IO or less of the total. All street dust, and nearly all indoor dust, show the colon bacillus in the ratio to total count of $I$ in 963 for street dust and $I$ in 3,582 for indoor dust, indicating that the origin of this bacillus is mainly the horse droppings. The ratio of acid-forming streptococci (of type characteristic of human mouth and intestine) is five times greater for indoor than for outdoor dust. Actual isolations of non-spore-forming bacilli from dust were confined almost wholly to a few $B$. diphtherice and many of the tubercle bacilli, as well as of streptococci and diplococci more or less closely resembling the pneumococcus. All bacilli suffer a more or less rapid reduction in drying. Thus 99 per cent. of the colon bacillus when dried in sand perish in 24 hours. Bacilli which are completely eliminated in Io to 15 days are probably 90 per cent. gone in a few hours, therefore making the danger of infection negligible long before the last have disappeared. The spread of cholera, plague, influenza and gonorrhea by dust is practically impossible, that of typhoid fever and diphtheria is possible though not probable, while the tubercle bacillus and the pus cocci are the only organisms likely to be transmitted in that way. The tubercle bacillus may sometimes be

* Dust. Am. J. of Public Health, 1912. 
found in 5 to Io per cent. of samples of indoor dusts, even when not especially exposed to tubercular infection, but such dust lies inert on floors and other surfaces and can only be distributed by more or less violent air agitation. In conclusion, these observers feel warranted to state that, with the exception of anthrax, the evidence of infection by air or dust is practically nil.

According to Anders, $\dagger$ pus-producing germs are especially abundant in dust and liable to cause boils, carbuncles and purulent catarrh ; this author calls attention to the outbreaks of tonsillitis, influenza and bronchitis often associated with long periods of dry, gusty weather.

In cleaning streets care must be observed not to raise dust. The primitive method of sweeping them in the dry state, with hand brooms, is an evidence of careless ignorance now fortunately seldom seen. The best method of cleaning city streets is undoubtedly by automobile vacuum or pneumatic cleaners, which work much more rapidly and efficiently than horse-drawn sweepers. Flushing with water so as to wash all the dirt and dust into the gutters is also very satisfactory where the sewerage is adequate. Oiling gives excellent results on macadam and earth roads.

Combustion, like respiration, absorbs oxygen and gives off $\mathrm{CO}_{2}$, and aqueous vapor. Besides raising the temperature of the room, it also generates various gases and volatile substances according to the fuel or illuminant used, namely: carbon monoxid, nitrous and nitric acid, compounds of ammonia and of sulphur, marsh gas and fatty acids. An ordinary burner consumes 5 cubic feet of gas per hour; as each foot of gas needs 5.33 cubic feet of air'for its combustion, the burner will therefore require 26 feet per hour or 624 per day. Each cubic foot of gas produces, in burning, half a cubic foot of $\mathrm{CO}_{2}$, therefore the burner, in one hour, will evolve $2 \frac{1}{2} \mathrm{feet}$, or about 4 times as much as an adult man. Each pound of coal requires 300 cubic feet of air for its combustion, but most of the products pass up the chimney and do not contaminate the breathed air.

The atmosphere of manufacturing cities is greatly vitiated by smoke. It is estimated that London is thus deprived of about onehalf of its possible sunlight and daylight in winter, and that 20 per cent. of its notorious fogs are due to the same cause. According to

† J. Am. Med. Ass., Nov. 4, I9II. 
Prof. C. Roberts, the air of that city is daily tainted with 250 tons of the gases, acids and volatile substances enumerated above, as well as with 50 tons of soot. In Indianapolis, Ind., Barnard and McAbee have shown that at least 1,200 tons per annum of soot and ash fall over an area of a square mile near the center of the town. Besides its direct baleful effects upon health, smoke is also indirectly injurious in causing housekeepers to keep the doors and windows closed to exclude the all-pervading soot, thus preventing normal ventilation.

When many people are crowded in a badly ventilated room, the sense of smell first takes cognizance of the fouling of the air, which becomes close and musty. The first impression on entering the room should be noted, for the effect upon the olfactic nerve wears off in a minute or two. Then certain symptoms manifest themselves; first, discomfort and oppression, followed by headache, fall of temperature, perspiration, exhaustion and, in extreme cases, delirium and death. These symptoms, although partly due to the products of respiration and combustion, and to fetid odors, must be chiefly ascribed to overheating, high humidity and air stagnation which operate by interfering with the chief function of the skin, namely, the regulation of the delicate mechanism of temperature equilibrium upon which normal metabolism depends. Thus the recent experiments of Leonard Hill, Benedict, Milner and others prove that, in confined air, although the oxygen pressure is reduced to 16 per cent. and the $\mathrm{CO}_{2}$ increased to 2 or 3 per cent. (so as to make it impossible to light a match), the condition of extreme discomfort thus produced can be immediately relieved by stirring and cooling the stagnant air with electric fans. Provided the humidity be absorbed and the temperature kept down, a man can remain in a small air-tight chamber, of a capacity of less than 2,000 feet, without ventilation for several hours; and, with partial ventilation (one-half or less the amount of air considered necessary), for several days without discomfort or notable change in body metabolism. Hough notes that in such confined air, although the odor is almost overpowering, the subject is unconscious of it. Apparently there is nothing to prove that chemical changes in respired air, whether lack of oxygen, increase of $\mathrm{CO}_{2}$, or accumulation of organic effluvia, are immediately injurious to the human organism under any tolerable condition of bad ventilation.

From their experiments, Hill and others conclude that the high 
death rate from tuberculosis and other diseases found among persons living in crowded and unventilated rooms, must be attributed to air stagnation under improper conditions of temperature and humidity, the effects of such conditions being to lower nervous stimulation and check metabolism, and, in the second place, to favor the growth of disease-producing organisms.

This new view of air contamination rests so clearly upon experiments that it must be accepted as a general proposition. It is not proved, however, that chemical changes and the presence of impurities in the air are entirely free from ultimate deleterious effects upon the system in general and the lungs in particular. Such conclusion would be unwarranted. Nature teaches us the efficacy and necessity of pure air in our instinctive repugnance for air contaminated by human respiration or the smells of organic decomposition. The fact that we can get used to the vilest stenches shows a wonderful power of accommodation but not their innocuousness, although immediate effects may not be noticed. It is indisputable that the natural and normal proportion of the air constituents is best for man and should always be approximated as near as possible, but we may admit that wide fluctuations for short periods of time are apparently harmless.

When death occurs from crowding and lack of ventilation, as in the historic instances of the Black Hole of Calcutta, ${ }^{*}$ the prisoners of Austerlitz, the passengers on the steamer Londonderry, etc., it is chiefly the result, according to Leonard Hill, not of asphyxia but of heat stroke, causing an acute febrile condition and heart failure.

The accumulation of carbon dioxid seems to have very little part in the symptoms produced by ill ventilation. It is to be remembered that although the proportion of this gas in normal air is only 0.03 per cent., in ordinary respiration at rest, it averages 5.57 , and oxygen only I 4.89 per cent., of the alveolar air; directly after muscular exercise the percentages are 6.52 and 14.33 , respectively (Cook and Pembrey). Not only is $\mathrm{CO}_{2}$ devoid of toxicity but it possesses an importance not generally appreciated. Boycott and Haldane attribute the subjective sense of exhilaration produced by cold weather to the high tension of $\mathrm{CO}_{2}$ in the alveolar air. Henderson has shown that the cessation of respiration in shock results from a

* In June, 1756, 146 Europeans were shut up in a room hardly 20 feet square, with only two very small windows; the next morning only 23 were taken out alive. 
diminished amount of $\mathrm{CO}_{2}$ in the blood and tissues of the body. As a strong stimulant of the respiratory center its administration is advised in asphyxia and all other cases of suspended animation, to be immediately followed by oxygen (if deemed necessary) as soon as breathing returns.

Carbon dioxid being a necessary product of respiration and combustion, and its presence and amount easily ascertained, has been chosen as the index of air pollution, it being assumed that the proportion of other contaminating matters rise and fall with it, an assumption, of course, only approximately correct. The value and significance of such an index vary according to the source of the $\mathrm{CO}_{2}$. If it comes chiefly from respiration, it will indicate the proportion of accompanying excretory substances and micro-organisms, but if mainly from combustion, it will rather indicate the ratio of toxic gases. As thus understood, the permissible amount of $\mathrm{CO}_{2}$ in air, that is to say, the amount which, with the corresponding accompaniment of deleterious substances, has no appreciable effect upon health, is 6 or 7 parts per Io,ooo, or an increase of 3 or 4 parts over the normal quantity. Such amount is not detectible by smell. Air containing ro parts begins to be "close;" with I 5 to 20 parts it is "stagnant," while with more than 25 parts it becomes distinctly musty and oppressive. There are few schools, factories, barracks, etc., in which the amount of $\mathrm{CO}_{2}$ does not reach Io to I 5 parts. In crowded halls and theatres it may range from 50 to 75 parts, and, if only for a few hours, without any harm. In the cars of trains it often amounts to 20 or 25 parts, the air of smoking cars being particularly foul, frequently containing 4 to 5 times as much ammonia as the outside air and a notable amount of carbon monoxid. It is prolonged exposure to impure air, rather than the degree of impurity, which is detrimental to health; thus a constant ratio of 12 to 15 parts in bed-rooms, school-rooms or shops will impoverish the blood, stunt the growth and impair the power of resistance to disease much more certainly than occasional exposures to air containing 40 or 50 parts.

Tests for Carbon Dioxid. - The standard method of determining $\mathrm{CO}_{2}$ for sanitary purposes recommended by the Committee of the Laboratory Section of the American Public Health Association, is the time method of Cohen and Appleyard, which combines practicality and reasonable accuracy. 
"Standard Method. - If a dilute solution of lime water, slightly colored with phenolphthalein, is brought in contact with air containing more than enough $\mathrm{CO}_{2}$ to combine with all the lime present, the solution will be gradually decolorized, the length of time required depending upon the amount of $\mathrm{CO}_{2}$ present. The quantity of lime water and volume of air remaining the same, the rate of decolorization varies inversely with amount of carbon dioxid. The method is scientific in principle because it recognizes the fact that the absorption of $\mathrm{CO}_{2}$ by $\mathrm{Ca}$ or $\mathrm{Ba}$ hydroxid solution is a time reaction.

" Collect samples of air in one-half liter glass-stoppered bottles by any of the methods of collection. Run in ro c. c. standard lime water, replace stopper, and note time. Shake bottle vigorously with both hands until color disappears. Note time required, and ascertain corresponding amount of $\mathrm{CO}_{2}$ from table."

TABLE.

\begin{tabular}{|c|c|c|c|}
\hline $\begin{array}{c}\text { Time in Minutes } \\
\text { to Decolorize } \\
\text { Solution }\end{array}$ & $\begin{array}{c}\mathrm{CO}_{2} \text { per } \\
10,000\end{array}$ & $\begin{array}{l}\text { Time in Minutes } \\
\text { to Decolorize } \\
\text { Solution }\end{array}$ & $\begin{array}{c}\mathrm{CO}_{2} \text { per } \\
\mathrm{IO}, 000\end{array}$ \\
\hline $\begin{array}{l}1 \frac{1}{4} \\
1 \frac{1}{3} \\
1 \frac{1}{2} \\
2 \\
2 \frac{1}{4} \\
2 \frac{3}{4} \\
3 \frac{1}{4}\end{array}$ & $\begin{array}{r}16.0 \\
13.8 \\
12.8 \\
12.0 \\
11.5 \\
8.6 \\
7.7\end{array}$ & $\begin{array}{l}3 \frac{1}{2} \\
4 \\
4 \frac{1}{4} \\
5 \\
5 \frac{1}{4} \\
6 \frac{1}{4} \\
7 \frac{1}{2}\end{array}$ & $\begin{array}{l}6.0 \\
5.3 \\
5.1 \\
4.6 \\
4.4 \\
4.2 \\
3.5\end{array}$ \\
\hline
\end{tabular}

For rough work, may also be recommended the "shaker method" of Wolpert and of Fitz, in which the volume of air that must be brought into contact with a definite quantity of lime water, in order to neutralize all the lime, is taken as a measure of the $\mathrm{CO}_{2}$ in the air.

The standard lime water used in all these tests is prepared as follows :

"Standard Lime Water. - To a litre of distilled water add 2.5 c. c. of phenolphthalein (made by dissolving 0.7 grams of phenolphthalein in 50 c. c. of alcohol and adding an equal volume of water). Stand the bottle of water on a piece of white paper and add drop by drop saturated lime water till a faint color persists for a full minute. 
Now add 6.3 c. c. of saturated lime water and quickly cork the bottle, or connect the pipette."

The simplest of the rough tests, with plain lime water, is that of A. Wolpert, as modified by Prof. Boom. "Make a mark on any test-tube, say one inch from the bottom. Fix the bulb of an atomizer to a small glass capillary tube, sufficiently long to reach to the bottom of the test-tube, and in such a manner that a definite quantity of air is forced from the bulb through the tube at each compression. To use: Fill the test-tube exactly to the mark with a saturated solution of lime-water, take the apparatus into the outdoor air and find out how many compressions of the bulb are needed, driving the air slowly through the lime-water each time, to make the lime-water just turbid enough to obscure a pencil-mark on white paper placed beneath the test-tube and viewed from above."* Repeat this operation in the room to be tested and note the number of compressions required. Assuming that the outdoor air contains the normal amount of $\mathrm{CO}_{2}$, namely, 0.03 per cent., the amount contained in the air of the room will be to this normal amount as the number of compressions in the outdoor air is to the number in the room; for instance, if the air of the room requires only one-third the number of compressions needed in the outside air, the amount of $\mathrm{CO}_{2}$ will be three times greater, or 0.09 per cent.

Carbon Monorid (CO).-By far the most toxic of the gases liable to contaminate the air of inhabited rooms is carbon monoxid, the more dangerous from being odorless. It is chiefly derived from the leakage of illuminating gas and from imperfect combustion. Gas prepared from bituminous coal contains only 6 or 7 parts of $\mathrm{CO}$, while water gas, now largely superseding it, contains about 30 parts and is therefore much more poisonous. It is also freely evolved from burning charcoal, a fact taken advantage of in some countries to commit painless suicide. The smoke of tobacco contains a rather large proportion of $\mathrm{CO}$, two puffs of it upon blood being sufficient to demonstrate the absorption of the gas, by the spectroscope. The leakage of coal gas into the ground is enormous, amounting to at least one-fifth of the total output, and much of it may be aspirated into houses by the higher temperature of indoor air. Less than I per cent. of carbon monoxid in the air of inhabited rooms may cause the death of their occupants, owing to its great affinity for the hemo-

\footnotetext{
* A Manual of Hygiene and Sanitation. Seneca Egbert.
} 
globin of the blood which thus loses its property of carrying oxygen to the tissues and eliminating carbon dioxid. Such blood acquires a bright-red color (carmin or cherry-red) easily recognized if a drop or two, diluted with distilled water, are examined in a test tube; it is more pink, less yellow than normal diluted blood. If boiled it yields a brick-red mass, while normal blood becomes brown-black.

A good indicator of the presence of a poisonous amount of $\mathrm{CO}$ in any confined space is a small warm-blooded animal, such as a mouse or bird, which is affected by this gas much sooner than man.

The spectroscopic test is the most reliable. If normal blood, properly diluted, is examined with the spectroscope, the two characteristic absorption bands of oxyhemoglobin appear (both on the blue side of the $\mathrm{D}$ line). If blood which has been exposed to air contaminated with $\mathrm{CO}$ is thus examined, the same, or almost identical bands are present. But if a reducing agent, like ammonium sulphide, be added, the normal blood, from scarlet becomes purplish and its two bands coalesce into one broad band; the contaminated blood, on the contrary, retains its cherry-red color and its two distinct bands.

The symptoms of poisoning by $\mathrm{CO}$ are vomiting, headache, great debility, loss of muscular power, unconsciousness, shallow stertorous breathing, rapid pulse and possibly convulsions. The face and hands, at first cyanotic, become pink in color. In certain industries in which workers are exposed to the breathing of $\mathrm{CO}$, there may be chronic poisoning indicated by general physical failing with deficient nuuscular power. In such cases nature endeavors to correct the evil by a large increase of blood cells.

Coal gas combustion also evolves sulphurous and sulphuric acids, sometimes enough to produce an unpleasantly irritating and acrid smell. It should never contain more than 20 grains of sulphur in IOO cubic feet, corresponding to 0.032 of a cubic foot of $\mathrm{SO}_{2}$.

Air Purification. - The means employed by nature for the purification of air are winds and storms, oxidation, bacterial action, rain and vegetation.

The winds keep the constituents of the air in constant agitation, mixing them thoroughly and maintaining them in their normal relative proportions; they disperse noxious gases and organisms and subject them more completely to the destructive action of sunlight and oxygen. By oxidation, all organic matters are decomposed and 
transformed into simpler innocuous elements; these changes are materially assisted by the agency of bacteria. Rain washes out the air and carries its impurities into the soil. Vegetation, as already seen, removes the excess of carbon dioxid from the air and absorbs from the soil much moisture and organic matter.

Nature of Ventilation. - Ventilation is not an occasional or intermittent renewal of air; it is a continuous process, as is the pollution; it therefore implies a constant movement of air in two directions, inward and outward, the entrance of fresh air and the escape of foul air. The fresh air, however, does not drive out the foul air; it diffuses itself into the room and dilutes the air already used so as to render it innocuous. Perfect ventilation would be that in which foul air is at once removed and replaced by pure outside air, so that none of the occupants of a room would breathe the same air twice; but this is not practicable; the incoming air cannot be kept separate from the air already used, but rapidly diffuses with it. A certain proportion of air will be inhaled more than once, and all that can be done is to so dilute it with fresh air that it will be harmless. The movement of air in ventilation should be imperceptible and never amount to a draft; that is to say, it must not usually exceed 3 feet a second. Cold and damp air is much more readily felt and likely to produce unpleasant currents than if at a temperature of about $50^{\circ}$ or $55^{\circ}$; therefore it is advisable, in cold weather, to warm it moderately, thus combining ventilation and heating. Much heating of the incoming air, however, is highly objectionable as it robs it of some of its purifying and invigorating properties.

The experiments above referred to (page 524) show that the object of ventilation is not only the removal of noxious chemical substances but also the maintenance of comfortable atmospheric conditions in regard to temperature and humidity. Nothing so far said should be understood as tending to lessen the importance of fresh air. The beneficial influence of pure air on oxygen absorption, pulmonary exchange, and metabolism cannot be questioned or exaggerated, but it is evident that to produce its optimum effect it must be cool, in motion, and not too humid, that is to say, in such condition as to favor the chief function of the skin, namely the regulation of body temperature upon which metabolism and vitality largely depend. The necessity of admitting an abundance of pure outside 
air into dwellings remains unchanged, but greater attention should be given to indoor temperature. In this country, the chief defect of ventilation, in winter, is excessive heat and the abnormal dryness which results therefrom.

Amount of Air Necessiry. - One of the chief practical points in ventilation is to determine the amount of fresh air necessary to keep the carbon dioxid down to the permissible limit of 6 parts per Io,000, namely 3 parts as normal constituent of the air and 3 parts as the result of contamination. The amount discharged per hour by an adult male, during ordinary activity, is practically one cubic foot; during repose but while awake, it is 0.72 of a cubic foot, 0.60 by an adult female and 0.40 by children. During sleep, adult males discharge only 0.56 cubic foot. It follows that 0.60 represents the maximum amount exhaled by individuals in a mixed community, or by soldiers asleep in dormitories. To this respiratory average should be added the proportional amount resulting from combustion, as the index of contamination by the various gases and substances of combustion. This is a widely variable quantity depending upon the illuminants used and mode of heating. It is estimated that this additional amount should be about one-half that of respiration, probably a liberal allowance, making in all o.90 of a cubic foot (or 25 liters). The question then is how much fresh air is required to dilute 0.90 cubic foot of $\mathrm{CO}_{2}$ so that there will be no more than 3 parts of it (the permissible ratio of contamination) in ro,ooo parts of air. It reduces itself to a simple rule of three:-

$$
0.90: x:: 3: 10,000 ; \text { hence } \frac{10,000 \times .90}{3}=3.000 \text { feet. In termis }
$$

of the metric system the equation would be $\frac{10,000 \times 25}{2}=83 \mathrm{cubic}$ meters.

It thus appears that each person should receive. per hour, about 3.000 cubic feet of fresh air, in a steady, even, continuous manner, without draft or perceptible change of temperature. The larger the room the fewer renewals of air will be required in the same space of time and the slower the interior air movement; in other words, the larger the cubic space allowed to each occupant the more easily can the necessary amount of fresh air be furnished without draft. Experience shows that when the amount of air, per hour, introduced into a room, is more than three or four times its cubic 
capacity, a current becomes perceptible. It follows, then, that the capacity of said room, or cubic space allowed to each man, should be at least $\frac{3000}{4}=750$ cubic feet. In large buildings, such as public

halls and theaters, where the occupants have only 300 cubic feet per head, the air can be changed ten or more times an hour and sufficient ventilation furnished without draft. This result, however, is only obtained by means of special systems of artificial ventilation neither practicable nor desirable for barracks.

It is obvious that the above calculations as to the amount of air necessary rest upon a somewhat arbitrary basis and can only give approximate figures. Thus it has been contended by reputable authorities that the permissible limit of carbon dioxid should be 9 parts per I0,000, that is, 6 parts from contamination instead of 3 as assumed above. This would only require an hourly supply of $I, 500$ cubic feet of fresh air. The consensus of opinion of most investigators, however, is that the supply should never be less than 2,000 cubic feet.

The floor space allowed each soldier in barracks varies too frequently in accordance with the strength of companies and the area of available quarters. All medical officers agree that it should never be less than io by 6 feet, or 60 square feet; with a ceiling 12 feet high the soldier will thus have a minimum cubic space of 720 feet. It is highly desirable, however, to make the minimum floor area 80 square feet and the cubic space 960 feet. More space should be provided for cavalry than for infantry, the better to dissipate the unavoidable stable odors carried by the men. The workable basis upon which to determine the size of dormitories should be a space of $\mathrm{r}, 000$ cubic feet per man. The number of men that can be accommodated in any dormitory having been determined by proper authority, should be conspicuously painted over the door and, except in emergencies, never exceeded.

In hospitals, to prevent the spread of disease, not only is thorough ventilation necessary, but as much room as possible should be provided so that the patients may be properly separated. The cubic space allowed each patient in wards should never be less than $x, 600$ feet and should reach 2,000 feet whenever practicable.

The ceilings of barracks, in cold and temperate climates, must never be less than io nor more than i2 feet high. If higher than 
this, the upper part of the room is difficult to aërate and to clean; the added space is of but little use for ventilation; much heat is lost, and the cost of construction is much increased without corresponding gain. This, however, does not apply to hospitals, schools, or public halls, etc., in which special means of ventilation are provided. In the tropics, also, where artificial heat is not necessary and an active air movement is desirable, high ceilings, together with high doors and windows, are advantageous. 


\section{CHAPTER XLIII.}

\section{VENTILATION (CONTINUED).}

\section{NATURAI VENTILATION.}

Ventilation is conveniently described as natural and artificial, but both systems are often more or less combined.

Natural ventilation is the resultant of several forces, namely, diffusion of gases, action of the wind and thermal circulation. These forces are constant but quite variable in degree. All gases diffuse so that when two or more come in contact a homogeneous mixture is obtained in spite of the action of gravity, the force of the diffusion being inversely as the square roots of the density of the gases. The wind acts in two ways, by perflation and aspiration; by perflation when blowing through confined spaces, and aspiration when blowing more or less horizontally over the top of chimneys and ventilating shafts; in the latter action of the wind a partial vacuum is produced and the air sucked up.

The movement of the air may be a hardly perceptible breeze or the blast of a .storm, but is seldom if ever entirely absent, often having a velocity of 2 or 3 feet a second in apparently perfectly still atmosphere and being seldom less than 5 or 6 feet. Free perflation, through doors, windows and ventilators, affords the best kind of ventilation and should be used whenever possible, if only for a few moments every day. Even on calm days the air is thus renewed many times an hour. In barracks or other buildings, with many occupants, the windows should be opposite each other so that the air may be swept through the rooms. Air thus naturally blown in possesses a freshness and produces a stimulating and purifying effect not equalled in any system of artificial ventilation.

The chief acting force, however, in natural ventilation, is thermal circulation, or the air movement resulting from the difference in density between masses of air of different temperatures. Heated air expands and becomes lighter; consequently it rises until reaching a layer of the same density, being replaced by colder and heavier air rushing in from below. 'The heating of buildings necessarily produces a difference of density between the outside and inside air ; 
it is therefore more or less involved in ventilation and one of its necessary factors. As air gets warmer it tends to rise and escape through the openings in the upper part of the room or the ventilating shafts; to fill the vacuum thus made, the colder air pours in from the outside through the lower openings. If a vertical slit be cut in the wall of a room, or one of the lateral sashes of a French window opened, it will be noticed that the direction of the current through the lower part of the opening is inward while that through the upper part is outward. Between the two currents

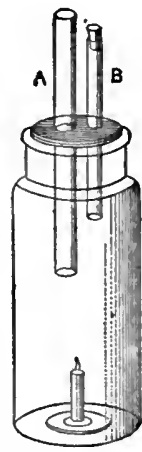

FIG. I48.Sh owing the necessity" of two currents to sustain com bustion.

(Munson. is a neutral zone where the air is not displaced. The further above this zone is an opening made, the greater will be the difference of density and the more active the outward current of air through it. It follows that, for the proper ventilation of a room, at least two openings are needed, preferably at different levels, one for the incoming and the other for the outgoing air. With only one opening, unless it be reasonably large, the two currents will oppose each other, with much friction and loss of efficiency. Thus, if a lighted candle be placed in an open 2-quart bottle, it will speedily die out, whereas if the stopper be pierced with two holes and glass tubes of unequal length placed in them, although their combined areas are less than the area of the open mouth, the candle will continue to burn, the inward and outward currents passing through the separate tubes without interference (Fig. 148).

In an inhabited room, the air in contact with the bodies of the occupants becomes warmer; it mixes with the expired air which has a temperature of about $97^{\circ} \mathrm{F}$. and together rise in slow but steady waves toward the ceiling. Although expired air contains a large proportion of $\mathrm{CO}_{2}$, it is so rarefied that it weighs only I.I2 grams per liter, whereas ordinary air at $62^{\circ}$ weighs 1.22 grams. It is a law that, within a limited space, gases which have no chemical action upon one another are uniformly diffused, independently of their respective density. The $\mathrm{CO}_{2}$, therefore, although heavier than air, remains diffused mostly in the upper layers of the air and, with good ventilation, should escape before it can vitiate the lower layers. This ascensional movement of air which has been used and polluted, 
is nature's way to remove it as soon and as completely as possible. The incoming fresh air which replaces it should follow the same upward direction. Therefore, air inlets, in natural ventilation, are near the floor, or at least lower than the outlets, and the outlets near the ceilings. Downward currents are prevented by insuring a sufficient velocity to the streams of vitiated air escaping into the atmosphere and by protecting the tops of ventilating shafts from the perturbing action of winds.

It is a general rule that the air movement upon which natural ventilation depends is upward, but conditions may exist, especially in hot countries, when the movement is temporarily reversed. Thus with an external temperature in the shade of $90^{\circ}$ or above, it may happen that the interior of a house will be a few degrees lower, according to the amount of solar radiation excluded by the roofs and walls. The colder and denser interior air settles downward and escapes through the lower openings, being replaced by the outside air rushing in from above, through outlets, chimneys, ventilating pipes and shafts. This reversed circulation may have no unpleasant result, the inflowing and diffusion of air being sufficiently active for the needs of the inmates, but, as the difference in temperature is seldom marked, the air movement is correspondingly sluggish; it is promoted by opening the lower sash of windows. Its effect upon the main vent pipe of the house plumbing will depend chiefly upon the nature and location of its intercepting trap (Fig. 164). If this opens outside the building no harm will result. In warm countries it is best to have the fresh-air pipe of the intercepting trap not only opening outside, but at least 7 or 8 feet from the ground, that is, above man's breathing line.

The forces of diffusion and perflation act not only through the openings espeoially provided. but also through all joints and interstices, and even through the walls and floors, their action depending much upon the porosity of the materials used and mode of construction.

This filtering in of air, however, as shown by Recknagel, is too small to be of much value as a ventilating factor, although often sufficient for the production of unpleasant cold drafts. Good sanitary construction requires that a house should be as impermeable to air as possible.

For the admission of fresh air, inlets through the walls are used; 
they will be further considered under artificial ventilation. Various devices are also resorted to. The simplest and commonest is to open the lower and upper sashes of a window, or either of them. To prevent draft the lower sash may be raised and a board fitted in the opening; then the air inlet is at the broken junction of the two sashes. Another device consists in setting a glass plate, or glass frame, vertically or slightly slanting inward, on the window sill, a couple of inches inside the lower sash, so that when the latter is raised, the stream of air, striking the plate, is diverted upward.

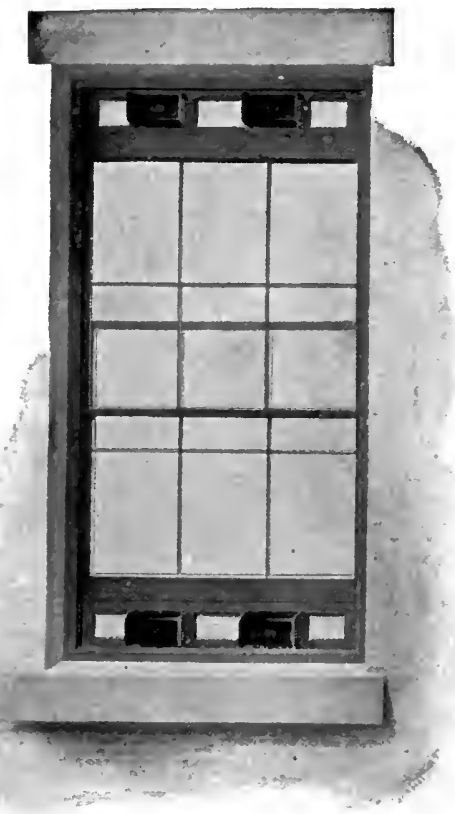

OUTSIDE VIEW.

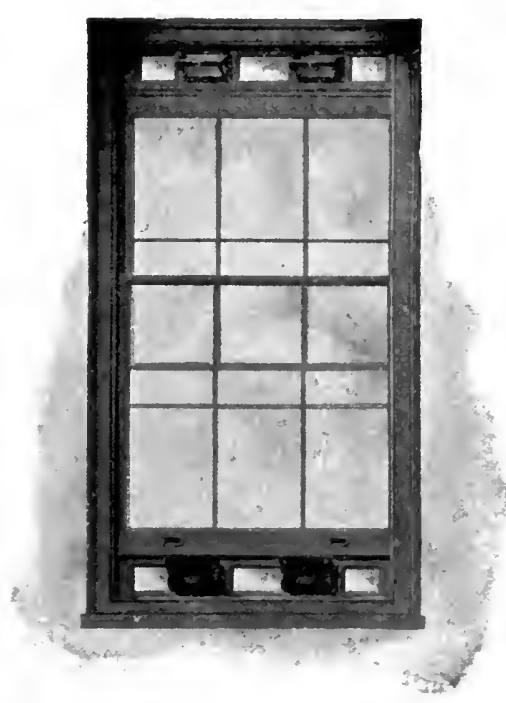

INSIDE VIEW.

F1G. I49.-Ventilating panels. (Mobile and Victor sy'stem.)

A method of natural ventilation now coming into pretty general use is the insertion of ventilating panels in windows, below and above, the sashes being raised or lowered for the purpose. The lower panel contains one or two boxed inlets through which the admission of air is regulated by a valve and the air itself filtered and diffused upward; the upper panel contains the outlets (Fig. I49). This method is specially valuable in winter. French windows are 
frequently constructed with a transom above, hinged outward for the escape of air, thus permitting a certain degree of ventilation without opening the sashes. The outlets may also be simple valvi1lar openings through the walls, or they may be connected with regular ventilating shafts; these shafts are often heated above, or contiguous to heating or smoke flues so as to accelerate their ascending current.

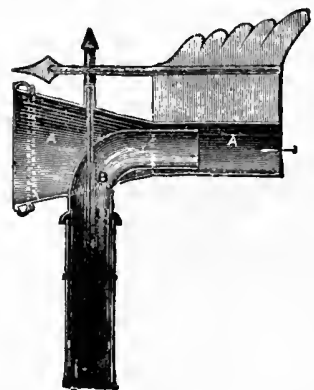

A method of natural ventilation formerly common in our barracks and still advantageously used in the tropics, is ridge ventilation, the outlet consisting of an opening extending along the apex of the roof, protected by a screened hood against rain, flies and mosquitoes (Figs. 24I, 242). With this system, I-story barracks may be left unceiled for more perfect ventilation, but when 2 -storied, ventilating shafts are used and made to open Fig. I50.-Rotating . exhaust ventilator. in the ridge under the hood. (Munson.)

In temporary barracks, or other buildings heated by stoves, ventilation is rendered easy by bringing fresh air in conduits to the jacketed stoves and proriding for the escape of foul air by ventilating shafts through which pass the smoke-stacks (Fig. I54).

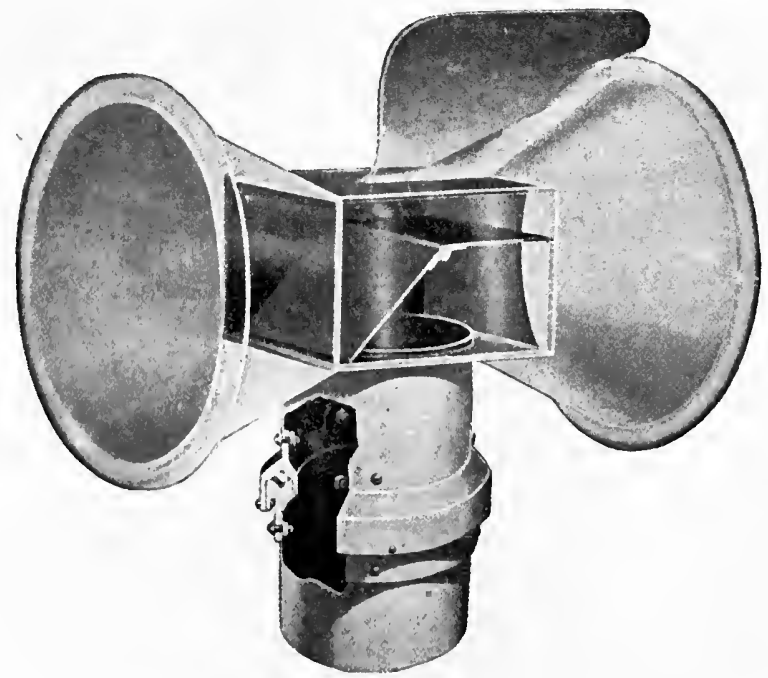

FIG. 151.- Rotating ball-bearing Ariel ventilator. 
Chimneys, with or without fire, have a marked effect upon ventilation, causing an upward draft which increases proportionately with the difference between the temperature of the inside and outside air. Thus Morin found that even without fire, but with a difference of temperature of $2 \mathrm{I}^{\circ} \mathrm{F}$., the chimney of his study evacuated 400 cubic meters of air per hour. This strong draft of chimneys is far from being always beneficial; it may produce objectionable currents along the floor and draw down the foul air from above. Chimneys should preferably have a separate supply of air from the outside, to be regulated as desired (Fig. I53), while the up-draft created by the fire can be utilized by placing a mica flap-valve in the flue just below the ceiling as an outlet for foul air.

The wind blowing horizontally upon or at right angle to the mouth of a chimney or ventilating shaft causes a marked movement of aspiration up the flue or shaft, thus increasing its draft. The wind, however, is subject to so many disturbances in force and direction that it may also drive the air down and reverse the current. This is prevented by various cowls and ventilators so constructed that from whatever direction the wind blows and at whatever angle, it passes over the opening nearly horizontally, thus assisting the upward draft; besides, they protect the flue against rain and snow.

Fig I50 shows the ordinary funnel-shaped

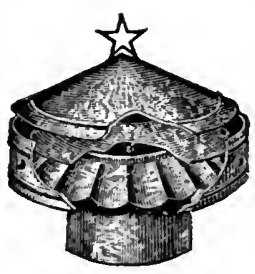

FIG. I52.- Star fixed ventilator. ventilator revolving so as to always present the mouth of the funnel to the wind. Likewise revolving is the Ariel ventilator (Fig. I5I) in which the wind is deflected over the mouth of the chimney so as to be always transformed into a horizontal draft. Fig. I 52 shows a fixed ventilator largely used in the construction of barracks and hospitals. In this type the wind is given an upward direction by numerous slanting pipes. The "Anchor" ventilator is of the same fixed and efficient type.

\section{ARTIFICIAL VENTILATION.}

Artificial or mechanical ventilation is obtained either by the direct propulsion of fresh air. through blowers or fans, into the building to be ventilated, the vitiated air being forced out by displacement (plenum system), or else by the aspiration of the vitiated air out 
of it (vacuum system), both systems being generally combined. The plenum system is often used in large public buildings, including hospitals. The process, briefly, is as follows: The air taken from a pure source is drawn, through filtering bags, to the fan in the basement, then forced into the heating chamber, where it comes in contact with steam coils and acquires the degree of heat desired, and thence distributed throughout the building. From the individual rooms, in which it diffuses itself, the air passes through the outlets into ducts which convey it to a central exhaust or extracting flue. In the vacuum or extraction system a strong up-draft is produced in the central flue by means of steam coils (acceleration coils) or an exhaust fan, or both. This system is rarely used alone as it causes fresh air to rush in from all sides below, sometimes from undesirable sources, and numerous drafts.

Artificial ventilation has decided advantages: It is more or less under control in all weathers; the air can always be drawn from a pure source, filtered and freed from dust, heated, cooled and moistened as desired. Against it may be objected the difficulty of regulating its mechanism so as to obtain satisfactory results under all conditions, and the deterioration of the air as it passes from the fan, through the heating chamber and the extended ducts, to all parts of the building. These objections are serious and have caused the abandonment of this system of ventilation in many buildings where it had been established at great cost. The necessity of preserving to the air the invaluable qualities of freshness and sweetness, to which its invigorating and vivifying properties are due, having become more fully realized, the present tendency of sanitary engineers is to rely more than formerly upon natural ventilation and to utilize it as far as possible.

For purposes of ventilation and heating, two kinds of openings are provided, inlets for fresh air and outlets for foul air. Since the air entering through the inlets mostly escapes through the outlets, it follows that, from the position of these openings will depend the direction of the movement of air, whether it will ascend, as in natural ventilation, or descend before escaping. Both upward and downward systems have their advocates; their relative merits may be thus summed up. Downward, or inverted, ventilation prevents the dust on the floor from rising and being breathed by the occupants; it produces a more thorough diffusion of the air with less 
danger of drafts. But, on the other hand, having to overcome the ascensional currents from the human bodies, as well as from all gas flames used for lighting, the quantity of air needed, and the power to extract it, are enormously greater than in the upward system, with proportionately increased cost. Furthermore, in large halls with galleries and amphitheaters, it is impossible to draw the air downward without exposing the people in the lower seats to breathe the foul air descending from the upper tiers. It is also necessary for the successful operation of this system to keep the windows carefully closed, so that no supplemental fresh air can be directly admitted. As a general rule, therefore, and unless there is some special indication to the contrary, the natural upward system is to be preferred. The objections against it, namely dust and drafts, are readily obviated by the use of rugs frequently shaken or of some adhesive floor dressing, and by the proper location and size of inlets. It is the system used in the Capitol, Washington, and the Houses of Parliament, London.

The position and size of inlets and outlets will be determined by the following considerations. The inlet registers should admit the requisite amount of air without currents of such velocity as would cause discomfort to the occupants. The larger the area of register openings, per person, the slower will be the movement of air passing through them. This area will therefore vary according to the location of the registers : for instance, if, in an assembly room, the fresh air is introduced through the floor, the inlet area for each occupant should be at least Ioo square inches, to prevent drafts, while it need not be more than 30 square inches if the registers are placed near the ceiling and a fan is used to ensure the requisite increased velocity. A good practical rule is to give each person no less than 30 square inches; this, with a velocity of 2 feet a second, will deliver I,500 cubic feet per hour. In most buildings, however, and always in our military barracks and hospitals, artificial ventilation is never exclusively relied upon but is largely supplemented by natural ventilation. A better distribution of air is obtained by providing smaller but more numerous inlets rather than larger and fewer openings.

Cold, raw air is liable to produce unpleasant drafts and it is always advisable, in artificial ventilation, to warm it before admission to occupied rooms. This warming, however, must be moderate. never exceeding $55^{\circ}$ or $60^{\circ} \mathrm{F}$, so as not to deprive it of the fresh- 
ness and invigorating qualities which are so readily affected by a higher temperature. Therefore, air admitted for ventilating purposes should not be depended upon for warming the building as, for instance, in that most objectionable method of heating, the hotair furnace.

The incoming fresh air, to be most available, must diffuse itself thoroughly in the room so that it may be breathed by the occupants before it escapes. Inlets and outlets should therefore be as far apart as possible, or at least so situated that the entering air does not pass out through the outlets before it has served its purpose. Thus when the fresh air is warmed, the inlet registers should never be placed directly below the foul-air registers, for direct currents would easily be established between them, with little effect upon the mass of air in the room. The inlets may be near the base of one wall and the outlets near the base of the opposite wall, the air becoming diffused while passing from one side to the other. If both inlets and outlets are in the same wall, the latter should be below the former; in such case a circulation is established from the inlets upward along the ceiling and across the room to the opposite wall, thence down to and along the floor to the outlets.

In our barracks and hospital wards the fresh air is generally introduced by valvular openings through the walls directly under the radiators (direct-indirect system), while the foul air escapes through outlets in the inside wall.

Whenever the air is warmed, inlet registers, especially if sufficiently large, may be near the floor without fear of draft; but, with cold air, they should be above the heads of the occupants (winter or summer), that is to say, about six feet high and so shaped as to deiiver the air in an upward direction. Very often two outlets are placed on the same shaft in a room, one below for winter use and the other above, near the ceiling, for the natural ventilation of sum. mer. Fresh-air registers, if placed on the floor should not be flush with its surface, for dust and dirt will fall into the flues and be more or less returned with the ascending air. It is also necessary that each room should have its own fresh-air flue separate from all other flues; thus two or more rooms, one above the other, should never be supplied from one common flue.

Conclusions. - The opinion is growing among sanitarians that the plenum system, alone or in connection with the vacuum system, 
is not satisfactory, especially for schools and hospitals. It is becoming generally admitted that ventilation and heating should be made as independent of each other as practicable, so that the invigorating qualities of the air may not be weakened by exposure to a high degree of heat. This is made possible by the direct system of heating which permits the use of windows and transoms for natural perflation, or the use of a fan to drive air through registers at a temperature not exceeding $50^{\circ} \mathrm{F}$., while a proper circulation is maintained by a sufficient number of outlets and shafts.

DRAFT. - What is generally called a draft in a house is an air current colder than the air surrounding the body; therefore it is not felt in the open, where the temperature of the air, whether still or in movement, is everywhere practically the same. A draft is most likely to be felt when sitting indoor and remaining for some time in the same position, the current striking only a limited part of the body. When the wind blows over the entire body the vascular system of the skin contracts to the extent necessary to prevent too much loss of heat by radiation. But when a draft impinges upon a small area of skin, the vascular system is unable to adjust itself to such fractional requirement, and much heat may be lost from the part affected; it becomes cooler than the rest of the body, with depression of its nerve supply and, perhaps, chemical changes, a condition which may react upon some of the sensitive organs. With the body heated and perspiring, a strong draft may cause rapid and energetic contraction of the superficial blood-vessels and dangerous congestion of internal organs. Windows being generally colder than the walls of a room chill the air in contact with them, with production of a cold downward current; therefore the near vicinity of windows is to be avoided by persons sensitive to drafts, especially in winter. 


\section{CHAPTER XLIV.}

\section{HEATING.}

Heat, whether evolved from the combustion of fuel or from any other source, is transmitted by conduction, radiation and convection:

By conduction when it passes from one particle of matter to another in contact with it as, for instance, in a poker one end of which is in the fire. Substances vary much in their conductive power; metals are much better conductors than wood, stone or-glass. Liquids and gases are poor conductors but heat passes readily from them to solids, and conversely. All textile fabrics containing much air, such as furs and flannels, have likewise a low degree of conductivity. The following common substances are placed in order of their conductive power: copper, iron, lead, slate, glass, water, brick, asphalt, wood, wool, air, asbestos. Conduction plays a part, more or less important, in all methods of heating.

$B y$ radiation when it reaches bodies directly exposed to it through the intervening air, like the solar heat or that emanating from an open fire. It is propagated in straight lines in all directions with equal intensity, the effect lessening according to the square of the distance. The air, especially if dry, is but little affected by these radiant rays; the objects which they reach absorb and reflect them and, in their turn, disseminate the heat by conduction and convection.

By convection when, in liquids and gases, it propagates itself through the mobility of their molecules, those in direct contact with the source of heat becoming expanded, therefore lighter and rising up, while others descend to take their place, so that ascending and descending currents continue until the whole mass of liquid or gas is evenly heated. Convection plays the chief part in the distribution and equalization of heat in an apartment. All sources of heat as well as heated objects in it, warm the air in immediate contact with them and thus establish convection currents, the air remaining in active movement until complete diffusion and an even temperature are obtained. In an inhabited room, each person is the source of a convection current, the heated vitiated air rising from the body, to be replaced by cooler and purer air. 
SUITABLE INDOOR TEMPERATURE AND HUMIDITY.

In winter, the temperature most suitable for living rooms is $68^{\circ}$ to $70^{\circ} \mathrm{F}$, and for bed-rooms not over $65^{\circ}$. In barracks, factories, schools, churches, or wherever many people assemble, it should range from $6_{5}$ to $68^{\circ}$, while in gymnasiums, drill halls, etc., where active exercise is taken, it must not exceed $55^{\circ}$. Whenever, in winter, any part of a barrack is vacated for meal, drill or any other purpose, the windows should be opened so that it may be "blown out" and its temperature lowered to 50 or $55^{\circ}$; upon the return of the men the closing of the windows will soon raise it again to the proper degree. In summer, a natural temperature of $65^{\circ}$ is quite comfortable. In England, the winter temperature recommended for houses is from $62^{\circ}$ to $65^{\circ}$, a difference mostly due to the greater amount of moisture in the British Isles.

The thermometer used to determine the temperature of a room should be suspended at a height of about 5 feet on the inside wall, but not in contact with it, a space of 3 or 4 inches being left between so that the temperature recorded will be really that of the ambiant air and not of the wall. It should also be as far as possible from the source of heat.

As the air of a room becomes heated, its capacity to hold moisture is greatly increased and its relative humidity accordingly diminished; in other words, it becomes drier. Thus if saturated air at zero be heated to $80^{\circ}$, its humidity is reduced to less than 5 per cent., and such air becomes intolerably dry. If no moisture is added by artificial means it will absorb it from the persons, furniture and all objects in the room; from the skin, which becomes hard and rough; from the mucous membranes of the mouth, nose and bronchi, causing irritation and cough; and from plants which may wither and die. The drying up of the mucous membrane of the respiratory passages predisposes to infectious diseases by weakening its bactericidal properties. The necessary humidity, therefore, must be furnished so that it may not fall too far below that of the outside air. In the eastern and middle States the relative humidity of the air is from 65 to 70 per cent. In England it is about 75 ; in Germany, from 50 to 60 , and in our arid western territories, 40 to 50 . The relative humidity of rooms artificially heated, in this country, is seldom more than 30 to 40 . This should be brought up to at least 50 for the requirements of the healthy body. The nearer it is to the degree 
of moisture of the outside air the more pleasant it will feel and the lower the degree of heat which will be necessary; thus Dr. Barnes, of Boston, while keeping his office at 53 per cent. relative humidity (by the use of a cotton wicking "humidifier"), found a temperature of $65^{\circ} \mathrm{F}$. to be perfectly comfortable instead of $70^{\circ}$ as before required (Harrington). This is due to the fact that dried air excites more active evaporation of the perspiration and causes greater loss of body heat which must be made up by a higher air temperature. It is therefore quite evident that a proper degree of humidity in heated rooms is economical of fuel as well as beneficial to health.

The temperature of the room being at $70^{\circ}$, a wet-bulb temperature of $58^{\circ}$ (a difference of $12^{\circ}$ ) would correspond to 50 per cent. of relative humidity; while a wet-bulb temperature of $62^{\circ}$ (a difference of $8^{\circ}$ ) would indicate a relative humidity of 60 per cent. As the proper degree of humidity, with artificial heat, is about $55^{\circ}$, it follows that in a room heated to $70^{\circ}$ the difference between dry and wet-bulb thermometers should never be much over $8^{\circ}$ (see chapter on Air).

The humidifiers used to impart moisture to the air are classed into the spray and evaporative types. In the so-called indirect evaporative humidifier, generally preferred, the air, taken from the outside, is humidified and conditioned before being introduced into the rooms. Humidifiers have also a distinct value in their possession of a marked cooling effect in warm weather. On a smaller scale, moisture is imparted to the air by placing pans of water, cotton wicking dipping in water, or moist towels, in heated currents or over stoves or radiators. Various special appliances, more or less satisfactory, have been devised for the purpose. The use of steam is not to be recommended as a rule; it raises the temperature of the air, often imparts a noticeable odor and is difficult to regulate.

Houses, barracks and hospitals, as well as all other buildings, may be heated by open fire or grate, stove, furnace. hot water and steam.

Open Fire or Grate. - The heat is transmitted chiefly by radiation so that a person facing the fire may be quite cold in the back. Most of it passes up the smoke flue and is lost. This method, therefore, although pleasant and cheerful, is quite wasteful. Much air is drawn into the room by the aspiration of the chimney; according tc Notter and Firth, the best type of open fireplace causes some 2,600 
cubic feet of air to pass up the flue per pound of coal consumed, or about I 8,00o cubic feet per hour, but this air is drafty and does not diffuse itself. There are ways, however, of utilizing open fires and grates for better heating and ventilation as, for instance, that shown in Fig. I53, in which fresh air, warmed by the fireplace and smoke flue, enters above and, after diffusing itself in the room, is drawn up through the hearth.

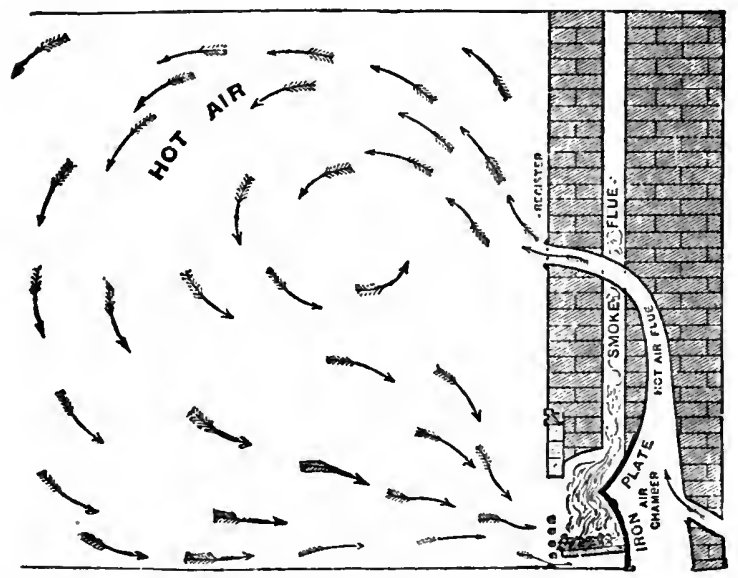

FIG. 153.- Method of heating and ventilating by open fire.

Stoves. - Stoves heat by radiation and convection. They are the most economical and efficient mode of heating, utilizing from 80 to 90 per cent. of available caloric. On the other hand, they are noisy and dusty; they dry the air to a trying degree and scorch the organic particles floating in it, injuring its respirable qualities and causing unpleasant odors. A red-hot cast-iron stove may also permit the highly poisonous carbonic monoxid to pass through it. There is likewise danger, if the damper is closed or the draft otherwise imperfect, of gases escaping from it. These objections, however, can to a large extent be remedied by careful management. The ease with which stoves can be utilized for ventilation is one of their advantages. Thus Fig. I54 shows how barracks can be heated and ventilated by the simple device of surrounding the stoves with a sheet-iron jacket which keeps the cold incoming air (brought under the floor from the outside) long enough in contact with the metal to 
warm it to a suitable temperature. The stovepipes run into and out of a ventilating shaft; but if the latter is capped with a star ventilator (a preferable method) the pipes terminate under it. The shaft must have two outlets, one below, near the floor, the other above, near the ceiling. Whenever the stoves are used, the upper outlet is closed and the lower open so that the heated fresh air, after ascending, is compelled to diffuse itself downward to escape through the shaft. The upper outlet is used in summer to promote natural ventilation, and, occasionally, in winter when the foul air accumulates faster than can escape through the lower vent. Fig. I 55 shows an excellent type of the so-called ventilating stoves in which the fresh air is brought in contact with the fire-box before diffusing itself through the room.

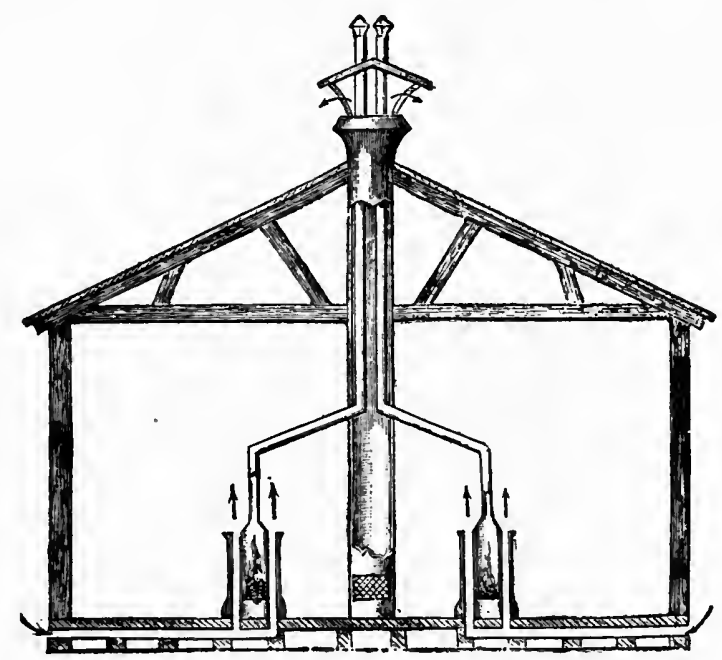

FIG. 154--Diagram showing the use of stoves to heat and ventilate barracks.

As an improvisation, for winter quarters, especially where fuel is scant, the "Russian stove" deserves to be better known. In the field it consists simply of a substantial brick, stone or clay structure through which the flue is made to describe several vertical curves so as to impart its heat to the whole mass. Once heated, very little fuel is required to maintain its temperature. Such stove is never very hot, therefore does not unduly dry or char the air and gives off a pleasant, even, steady, moderate heat. 
Another economical improvisation applicable to tents and huts is the Chinese method in which the fire is built in a pit in front (inside or outside), and the flue runs, under the floor, into an improvised chimney in rear.

With all kinds of stoves it is very important to see that a sufficient degree of humidity in the air is maintained; most of them are provided with receptacles for water and these should always be kept full; but they, are generally insufficient and additional water-pans must be used.

Hot-air Furnace. - In this method, the air is heated in a furnace in the basement and conducted by means of large galvanized iron pipes to all parts of the building. It has but little to recommend it except cheapness of installation, which may have to be considered in private dwellings. It should be used only in buildings in which a very moderate degree of heat is needed, and should always include suitable filters to exclude dust. It both heats and ventilates. The

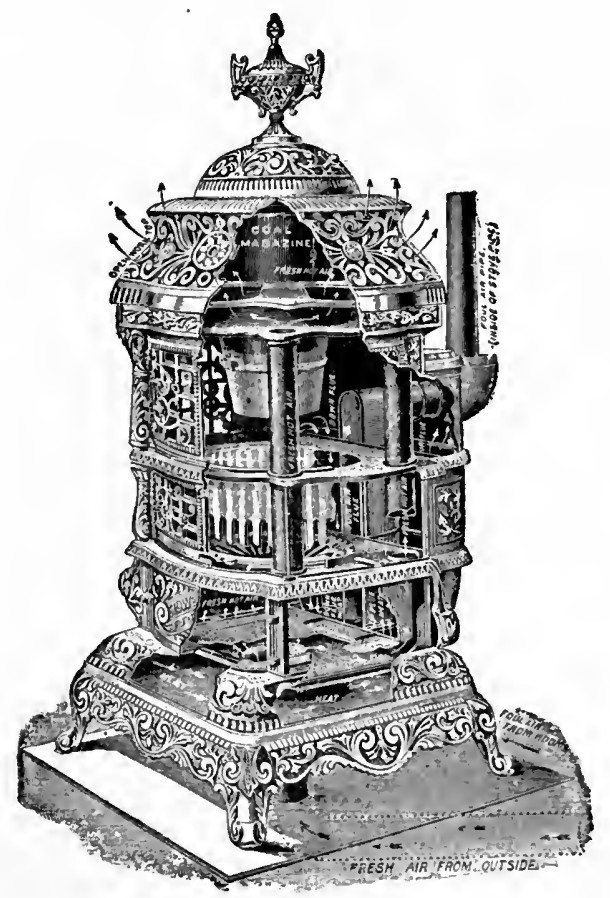

FIG. I55-Cortland Howe ventilating stove. 
ventilation is promoted by providing enough outlets for a proper air movement; this movement being outward, drafts from the outside air are prevented. The disadvantages of the method are serious: The heat is unevenly distributed, its diffusion depending upon the direction of the wind so that the windward side of the house may be cold and the other side too warm, and depending also upon the length, slant and angles of the pipes, the heated air moving readily up vertical pipes but sluggishly along nearly horizontal and crooked ones. The air often carries dust with it which, from contact with the hot furnace, is scorched, rendering it irritating to the throat and nasal passages; in case of any crack or leak in the furnace, it becomes dangerously contaminated with coal gas.

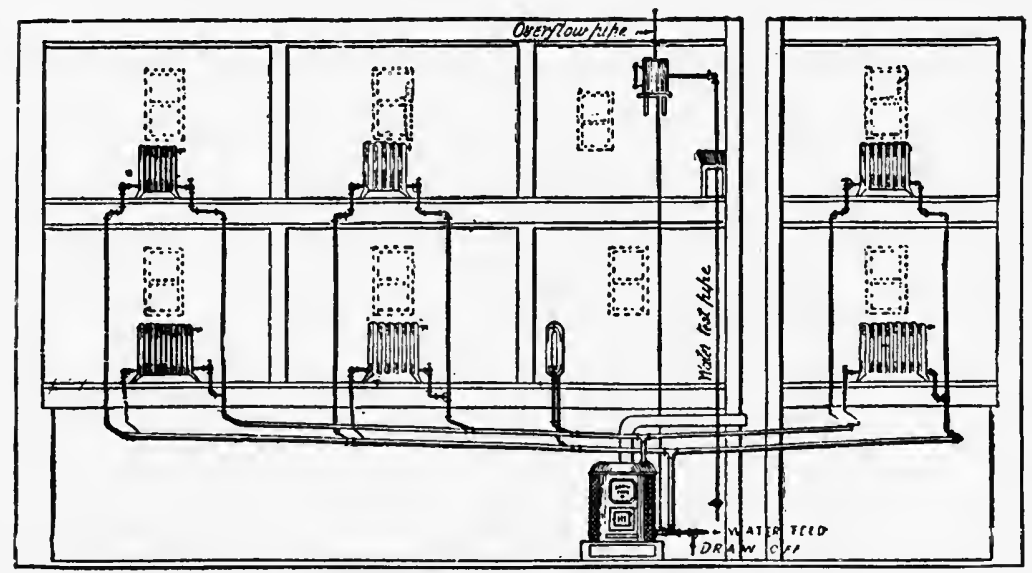

FIG. 156.- System of piping for hot-water heating - Main flow and return pipes. ("Heating and ventilating buildings."-Rolla C. Carpenter.)

Hot WATER. - This method of heating, whenever applicable, is the most satisfactory for houses, barracks, hospitals and public buildings. The high specific heat of water makes it an excellent medium for the storage, transportation and distribution of heat. The quantity which can be stored in equal weights of water and air is in the ratio of $42 \mathrm{I}$ to 100 , which means that the heat set free by one pound of water, cooling down one degree, will raise $4.2 \mathrm{I}$ pounds of air (or 55 cubic feet) one degree. In practice, it is estimated that one cubic foot of water, cooling one degree, will raise to that extent the temperature of 3,000 cubic feet of air. 
The low-pressure system is practically always used, being safer and more easily controlled than the high-pressure one. From the boiler, in the basement, a set of pipes convey the hot water to all the radiators in the building and a second set return the cooled water to the bottom of the boiler. (Fig. I 56.) The system is open above, at its highest point (expansion tank), so as to allow for the expansion of the water, and a vent is provided on each radiator for the escape of dissolved air liberated from the water. The temperature of the water averages $180^{\circ}$ and seldom reaches $212^{\circ}$. The

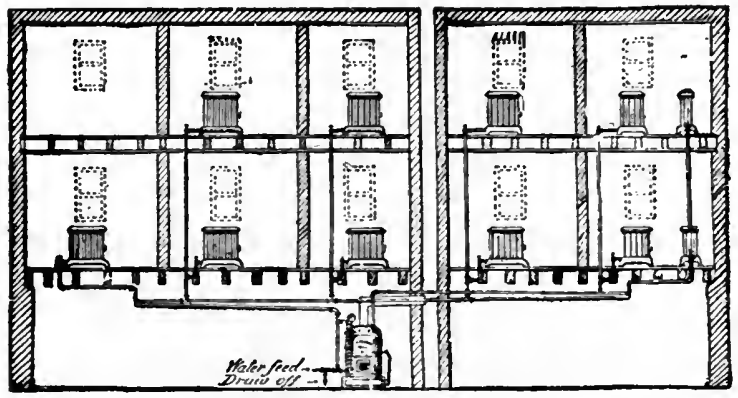

FIG. 157.- System of piping for steam heating. (Baldwin.)

heat, as transmitted from the coils, by conduction and convection, is pleasant, equable and constant, not liable to rapid changes. The system is noiseless, very easily regulated, devoid of danger and, after the first installation, cheaper than steam heating.

Stean. - Steam is still more efficient than water as a medium for the absorption, storage and transmission of heat on account of the enormous quantity of latent caloric which it sets free on condensing. Thus one pound of steam condensing to boiling water gives off enough heat to raise the temperature of 22.5 pounds of air, or 5.36 pounds of water, to $212^{\circ}$. Steam has been more generally used than hot water in this country because of the cheaper and easier installation. The coils are smaller, more compact and no return pipes are required. (Fig. 157.) The heat can be turned on or off much more rapidly, and by dividing the coil in several sections, as many sections are used as needed to obtain the exact degree desired. As the temperature in the pipes may reach and even exceed $225^{\circ}$ there is some danger of scorching the air. An advantage of steam over hot water is that it travels great distances with rapidity and 
without condensation provided the mains are sufficiently large and well insulated. It is also much more efficient for the heating of the upper stories of tall buildings. It is likewise preferable in very cold countries, being much less affected by a temperature below zero. The chief defect of steam heating is the so-called "water-hammer," a loud percussion noise produced whenever condensation water forms in the coils, and the steam, forcing its way, projects it against the sides of the pipes.

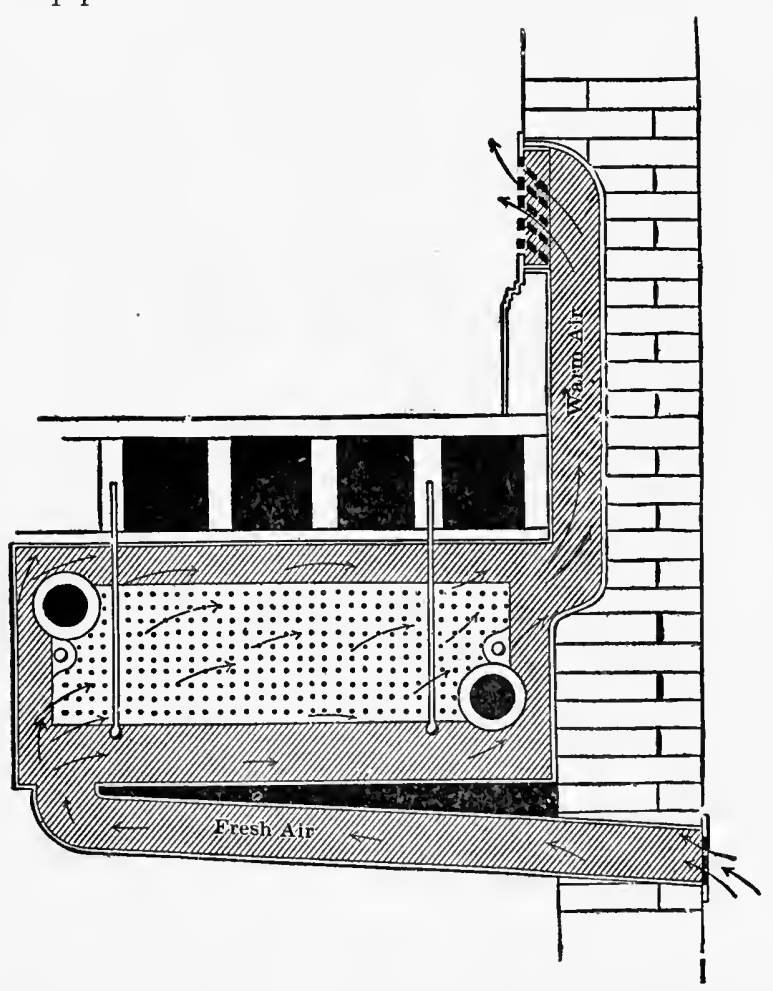

FIG. 158.-Indirect system of heating. (Bashore's "Outlines of practical sanitation.")

Location of Coils. - With either hot water or steam, the location of the coils, within the room, does not matter much, depending upon its shape and the disposition of the furniture. Generally they are best placed under the windows so as to be out of the way and, at the same time, warm the cold air leaking through or flowing down from 
the chilling panes. It is also the best place to receive the cold air from ventilating inlets as is commonly done in our barracks and hospitals. If not placed under the windows, it is best, in dormitories or wards, to set them along the median line so as to be nearer the feet than the heads of the sleeping men.

It should be remarked that the designation of radiators, given to heating coils, is a misnomer inasmuch as they heat mostly by conduction and convection, and least by radiation.

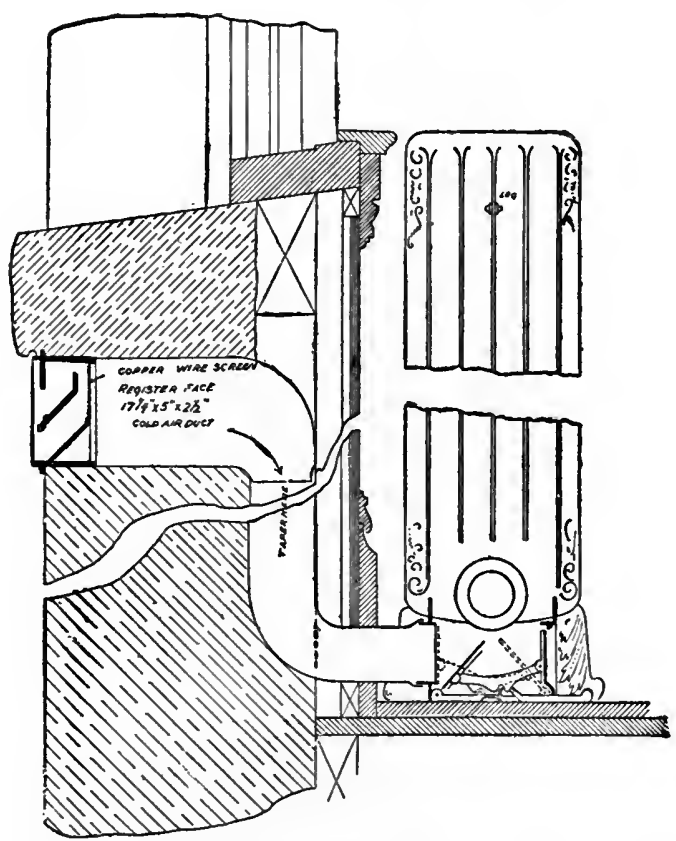

FIG. 159-- Direct-indirect system of heating.

Systems of Heating. - It has been seen that the source of heat can be in the room itself, and not directly connected with ventilation, as with common stove, open fire or grate, steam or hot water radiator; this is the dircet system. When the air is heated before entering the room, either through a hot-air furnace or by passing over steam coils, and is used for heating and ventilation, the system is said to be indirect. (Fig. I58.) In the direct-indirect system, the source of heat is in the room and the air supply is brought in contact 
with it, as in the case of jacketed or "ventilating" stoves, and of hot-water or steam coils with cold-air inlets introduced under them. (Fig. I59.)

As has already been stated, it is desirable that the incoming fresh air, in winter, should be moderately warmed before entering an occupied room. When the building is heated by hot water or steam, the air may be admitted through the walls directly to the coils (direct-indirect system), in which case it abstracts some of their heat so that more coils are required; or else the air is passed over steam coils in the basement and introduced into the room through a sufficient number of registers (indirect system), in which case it brings additional heat to that furnished by the coils.

Heat and Air Dust. - It has been seen that when subjected to artificial heat, the air loses its freshness and becomes more or less "dead" and "stuffy." The exact nature of the change is not known, but there is no doubt that, to a large extent, it may be attributed to the effect of heat upon the air dust. Prof. Esmarch has demonstrated that dust, especially when of organic nature, will begin to decompose on heating surfaces at a temperature of about $160^{\circ} \mathrm{F}$. Of the gases produced, ammonia can be detected in all parts of the room. Even when the heating surfaces were carefully cleaned, before the test, the effect on the air was still noticable, showing that the dust suspended in air will also decompose when passing over heating surfaces and without contact with them. Prof. Nussbaum found that moist organic dust will begin to decompose even at $140^{\circ}$, while dry dust must be heated to about $200^{\circ}$ before gases become detectible. In this respect, however, moist dust does not play an important part. Whatever of it there may be in inhabited rooms will usually lie on the floor and, by reason of its weight, show no tendency to rise and come in contact with radiators until it has lost most of its moisture. It is not believed that the degree of relative humidity necessary for the best condition of the air, can have any appreciable effect in favoring this decomposition of organic dust. On the contrary, it has been seen that a suitable degree of humidity renders possible a lower degree of heat. It is evident however, that where circumstances require the combination of a relatively high temperature and high degree of humidity the careful filtering or washing of the air is very important.

The effect of heat on ozone is also noteworthy. The tendency of 
ozone to combine with organic matter increases with the temperature, and a point is soon reached at which no more ozone is found in the air ; thus it is safe to assume that no ozone can get past a radiator or stack. It is probable that a certain amount of oxygen is also sacrificed by the process of heating. This absorbtion of ozone would account, in a measure, for the lifeless quality of air flowing from a register or ascending from a radiator.

The dust is carried up and kept in motion by the currents of warm air, thus adding certain obnoxious elements to the air we breathe. This movement of the dust is well demonstrated by the black streaks which it often leaves on white walls above radiators. These streaks show also that it is sufficiently scorched to discolor the walls. But it is not likely that live organisms are destroyed to any extent by the ordinary forms of heating apparatus; these merely dry and char the dust and keep it in motion. "Considering this, we are forced to admit that heating increases the bacterial contents of the air" (Konrad Meier). The effect of this air contamination is shown by the increase of nose, throat and bronchial ailments in winter, especially when many people work in the same shops or sleep in the same dormitories. The great increase of tonsillitis among soldiers in barracks, during the winter months, has already been referred to (page 4). In accounting for this, another contributory element comes into action, namely, the usually low relative humidity of the air, causing an abnormal dryness of mucous membranes and the partial loss of their bactericidal qualities. In a word, we have an increase of air pollution, together with a decrease of resistance against it.

Experience shows that in spite of whatever devices used to that end, dust cannot be entirely excluded from buildings and that we must contend with it.

The best way to prevent the chemical adulteration of air through the decomposition of organic matter is by a moderate temperature of dust-free surfaces. With clean surfaces and air reasonably dry there will be no vitiation with a temperature (of heating surfaces) under $160^{\circ} \mathrm{F}$, letting it rise only occasionally to $180^{\circ}$ during unusually cold spells. For this moderate degree of heat, hot water is more suitable than steam.

If, as Flïgge contends, the convection of heat from the body is a necessary function of the system, it is promoted by heating at low 
tension, which means by extended surfaces at relatively low temperature, giving off heat mostly by radiation and least by convection, sufficient to warm the occupants of the room without greatly affecting the ambiant air. The devices which give the best radiant heat and the least air currents are also the easiest to clean. Enamel finish in black and white will help in radiating efficiently and, by showing the dust, will insure clean surfaces. It is insanitary to place heating surfaces overhead or in any place out of reach and sight where the dust will accumulate. Floor registers nearly always become receptacles for dust and are likewise objectionable. 


\section{CHAPTER XLV.}

\section{LIGHTING.}

Light is necessary to the growth and development of animals and plants. Upon animals it favors metabolism, improves the quality of the blood and promotes the performance of all functions. With the exception of the lower cryptogams, plants thrive only under the infutence of light; it is only under such influence that they perform the all-important nutritive function, through their green foliage, of decomposing the carbon dioxid of the air, appropriating the carbon and giving off the oxygen. But light, on the other hand, is inimical to low forms of fungi, including all microbes and bacteria, inhibiting their growth or killing them outright. Therefore it appears that while strengthening man, sunlight destroys some of his silent foes, the pathogenic micro-organisms. Hence the necessity of a sufficiency of window surface in all buildings; this surface should be equal to at least one-fifth of the floor area, or one square yard for each 30 cubic yards of space. In cold or temperate climates, the windows should be disposed on at least two sides of the buildings so that the sun-rays, if possible, may reach all parts of the floor. This is especially desirable in buildings occupied by many people, such as barracks, schools, factories, etc.

In thus providing light and sunshine it is very important to consider the climate and season. The calorific and luminous properties of the solar rays are stimulating and, to a certain point, necessary for the proper work of the bodily functions, but, beyond that point, they become depressing and lharmful; for instance, the sunlight which is cheering and invigorating in winter may be intolerable and dangerous in summer. In tropical countries, the sun, during the hot season, is often the worst eneny that the white man has to enconnter and measures must be taken, not to exclude it altogether, but to mitigate its unpleasant effects. Therefore the houses should face nortl and be protected, at least on the south and west sides, by broad verandas. Not only the sun-rays should be excluded, but the diffused light itself, when very bright, must be stıbdued. Dazzling white surfaces are particularly objectionable and, in warm climates, the 
law should require that all outside walls be lightly tinted. In rooms lighted with electricity the walls should likewise be colored with a soft neutral tint.

According to J. H. Parsons and other authorities, ultra-violet rays are deleterious to the eye and, in men exposed to bright electric light for instance, liable to produce various effects on lens and retina, but especially intense congestion and inflammation of the conjunctiva. Snow blindness is also believed to be due to the same rays. Good protection is afforded by plane, clear glass spectacles which cut off all 11ltra-violet rays of shorter wave length than 300 microns. Blue glasses or smoked glasses are better, but still more effective are amber glasses or glasses tinted greenish-yellow, which are said to absorb all rays below 400 microns. Suitable protective glasses, such as reduce the intensity of light and cut off its actinic rays, are especially needed by men exposed to search-lights.

Location of Lights. - Dazzling lights should be removed from the field of vision or else softened by shades or globes. The socalled indirect method of illumination is a valuable innovation in which the light (from invisible sources) is thrown onto the ceiling and reflected in a soft diffuse form throughout the room. This method prevents glare, gives an illumination free from distinct shadows and is the nearest approach to daylight that it is possible to secure by artificial means. In the semi-indirect method the reflector is made of translucent glass so that it is mildly luminous itself.

In the study or class-room, the reading or writing desk should be placed so as to be clearly lighted, free from shadow and glare. The glare likely to be caused by reflection of the light upon the paper, especially when flat desks are used, may be very trying; it should be prevented by changing the angle of incidence of the rays. Since reading and writing are from left to right, it is best to have the window on the left so that no shadow be projected on the book or paper where the sight is focused. It must not be in front, for every time the eyes look up through it, at any outside object, there is a change of focusing accommodations, followed by another as soon as the reading or writing is resumed, causing fatiguing eyestrain. Artificial lights should be white, bright and steady; they may be placed on the left or in front, so that no shadow shall be thrown upon the field of vision, and must always be shaded in order to concentrate their power and protect the eye. 
Use of Prismatic Glass. -- When a room is lighted only by front windows, its rear part can be rendered much brighter by the use, in these windows, of prismatic or ribbed-glass panes which refract the luminous rays in a horizontal direction and light up all parts of the room, if not too deep.

\section{ILLUMINANTS.}

The illuminants most generally used are candles, petroleum, coal gas, acetylene and electricity. With the exception of electricity, they all absorb oxygen while burning, and give off carbon dioxid, vapor of water and other products of combusion which pollute the air.

In all illuminants, luminosity, or lighting power, is to be distinguished from their heating power. During their combustion, the hydro-carbons, of which they are largely composed, break down and set free the hydrogen and multitudes of carbon particles. In an ordinary flame, the oxygen of the air is not sufficient to combine at once with all these constituents. As the carbon particles pass outward through the flame of the burning hydrogen, they are not oxidized but heated to such a point that they become incandescent, thus causing the luminosity of the flame; as they reach the edge of the light they are converted into carbon dioxid. Whenever it is possible to furnish enough air to the flame, as in the Bunsen burner, to combine with all its constituents at once, the carbon particles are converted into carbon dioxid as soon as set free, producing an intense heat but no luminosity.

Candles. - When necessary for the public service, the $Q$. M. Corps may issue candles in the proportion of 24 ounces to each 100 rations, or 32 ounces in Alaska. Lantern candles are issued in such quantities as the commanding officer deems necessary.

Petroleum, when properly used, is an excellent illuminant, giving a soft bright light, without objectionable odor or poisonous properties. It is still largely burned at many of our military posts, although being gradually replaced by gas or electricity.

GAs. - The ordinary coal gas, made by heating bituminous coal in fire-clay retorts, consists chiefly of about 50 parts of hydrogen, 35 of carbureted hydrogen (marsh-gas) and 6 or 7 of carbon monoxid. Water-gas, now extensively used, is made by the action of steam upon coke or anthracite coal heated to a very high temperature; the 
steam is decomposed and its oxygen combines with the carbon to form carbon monoxid. This gas is usually carbureted, that is, passed through hot chambers charged with petroleum where it absorbs a supplement of carbon to increase the luminosity of its flame. Water-gas contains about 35 parts of hydrogen, 30 of carbon monoxid and 20 of marsh-gas. In whichever way prepared, gas is very poisonous owing to the carbon monoxid it contains; water-gas containing four or five times more of it than ordinary coal gas is proportionately more dangerous; its odor is also much less pronounced so that its presence, from leakage or open burners, is not so readily detected (see page 528). As its use has become more general during the last fifteen years, the number of deaths caused by it, accidental and suicidal, has increased so rapidly that the enactment of legislative measures to restrict the amount of the deadly carbon monoxid to a safe proportion (less than 20 per cent.) would seem to be clearly in the interest of every community.

The usual products of combustion of gas, besides carbon dioxid and vapor, are small amounts of carbon monoxid, ammonia and sulphur compounds. Furthermore, each cubic foot of gas, in burning, generates enough heat to raise the temperature of $\mathrm{I}, \mathrm{I} 6 \mathrm{O}$ cubic yards of air $I^{\circ}$.

Burners have been devised to increase the luminous intensity of the flame with smaller consumption of gas, therefore with less vitiation and heating of air. Such are the so-called " regenerative burners" in which the gas is mixed with hot air, that is, air heated by the flame itself. The most satisfactory, however, are the incandescent burners and, of these, the Welsbach is the best known and most used. It consits of the ordinary Bunsen burner, over the flame of which is hung a mantle of incombustible material. This mantle is usually made by saturating a delicate network of cotton in a strong solution of earthy oxids. When heated by the Bunsen flame it becomes incandescent and intensely luminous, a remarkable instance of heat transformed into light. The Welsbach burner consumes nearly one-half less gas than the ordinary burners, producing therefore only one-half the heat, carbon dioxid and other products of combustion, while its illuminating power it at least three times that of the incandescent electric light.

ACE'TYLENE. - This gas is obtained by the action of water upon calcium carbide. It gives a very brilliant white light, with an illum- 
inating power about equal to that of the Welsbach incandescent burner. It requires a special burner consisting of two tips, each with a minute orifice or slit, the two opposed jets forming a small thin flame able to secure enough oxygen for the complete combustion of the gas. Carbonization, however, is liable to occur on the tips and obstruct the orifices. Acetylene, notwithstanding its very unpleasant odor of garlic, is not as poisonous as coal gas. When

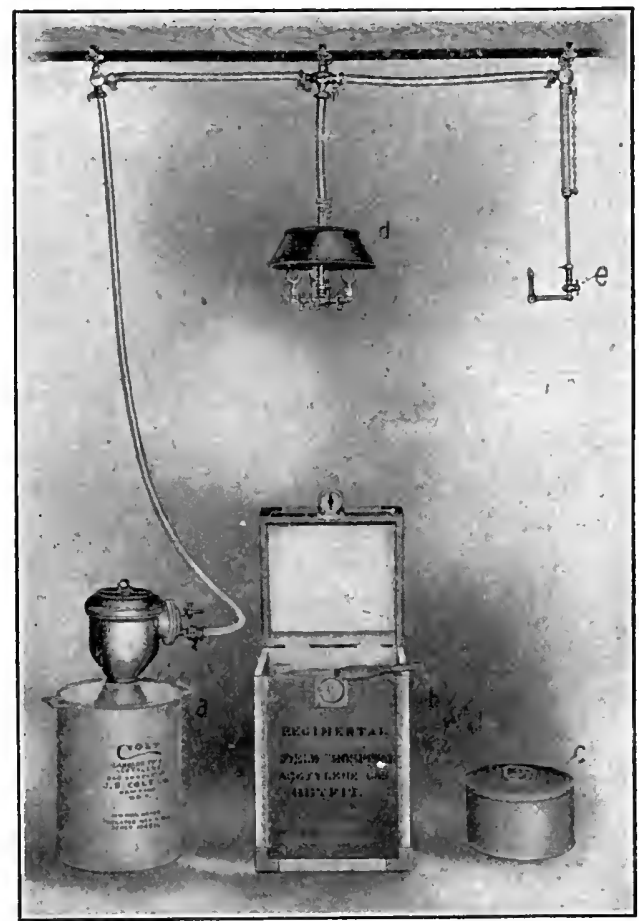

Firg. I6o.-Acetylene outfit for field hospital. (Colt sy'stem.)

mixed with air there is danger of serious explosion in contact with light, so that care must be taken that the piping and fittings are tight and sound:

The advantages of acetylene, besides its intense illuminating power, are its cheapness, being the least expensive of all illuminants, and the facility with which it can be produced in any place. It is espe- 
cially valuable for military purposes on account of the portability, simplicity and ease of operation of the generator. In our service, a small but complete acetylene outfit, consisting of generator, rubber piping, burners, reflectors, etc., weighing, with chest, 74 pounds, is supplied to each field hospital for the lighting of the operating and dispensing tents. (Fig. I60.) Two such outfits are allowed a stationary hospital and three to a base hospital. The generator, of about the size of a water pail, contains two pounds of carbide and can maintain four 25-candle-power burners for four hours, before being resupplied. Fig. I6I shows the application of the system to a

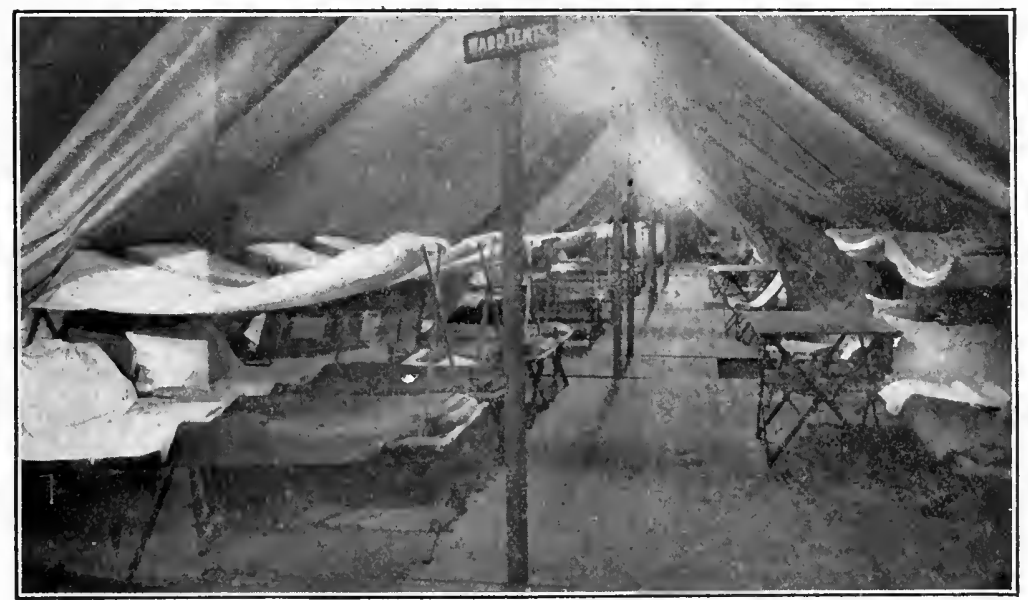

FIG. 161.- Hospital tents lighted with acetylene gas. (Colt systcm.)

hospital ward under canvas. Small acetylene lamps, secured in front of the cap by an elastic band, to be used at night by hospital corps men in searching for the wounded and other purposes, have also been experimented with and found very satisfactory.

Prof. Jacquemet, of Grenoble, has devised an acetylene apparatus whereby light or heat, or both, in any desired proportion, can be instantly generated. It is said to be transportable, easily operated and safe.

Electricity is the ideal illuminant and should always be preferred when available. Used in the form of the incandescent lamp, it gives a white, steady, brilliant light of at least i6-candle power. The 
tungsten lamp, as lately perfected, yields a still more brilliant light and at less cost. As this light is not the result of combustion and does not absorb oxygen from the air, no contaminating product is given off and the composition of the air is in no way affected. Its feeble heating power renders it particularly valuable for tropical climates, but less desirable than gas where lights are depended upon to create an upward ventilating current.

The respective cost of illuminants, for each candle power, per hour, is estimated as follows: Tallow candle, 4 cents; gas, bat'swing burner, 0.76 cent ; electric incandescent lamp, 0.60 ; petroleum, 0.54 ; Welsbach incandescent gas lamp, 0.32; acetylene, 0.20. 


\section{EXCRETA, GARBAGE AND WASTES.}

By excreta must be understood all waste matters, solid and liquid, eliminated from the human body, such as feces, urine, sweat, as well as discharges from the mouth, nose and lungs. Garbage refers chiefly to kitchen refuse, including much animal and vegetable matters in the shape of food débris. The waste waters which hygienists are most concerned with are those from kitchen sinks, lavatories, baths and laundries.

The average daily discharge of fresh fecal matter, per male adult, is 5 ounces, and of urine 45 ounces. In a mixed community, including men, women and children, the average per head may be estimated at 3 ounces of feces and 32 ounces ( 1 quart) of urine. Fecal matter contains 75 per cent. of water and 22 per cent. of organic substance of which 2.2 is nitrogen. Urine contains 95 per cent. of water and I.4 of nitrogen. Feces are quickly putrescible, their decomposition often beginning before being discharged, owing to the agency of the saprophytic microbes which swarm in them. These microbes bring about their transformation into simple harmless substances and therefore play' a useful part in the economy of nature; but excreta may also contain pathogenic bacteria of various kinds and, on that account, are always open to suspicion.

\section{SEWERAGE AND PLUMBING.}

The general system whereby excreta and wastes are removed by water through fixtures, pipes and drains into sewers or other place of final disposal, is called sewerage; while sewage is the name given to the contents of drains and sewers.

A good sewerage system requires an abundant water-supply for the flushing of fixtures and drains, with sufficient outfall and effective means of final disposal; it must be well and substantially constructed, of excellent material, perfectly water-tight and thoroughly ventilated.

The marked effect of sewerage upon human health has long since been conclusively demonstrated. The excreta likely to contain the 
germs of typhoid fever, dysentery and cholera are carried to a safe place of final disposal instead of lurking in pits, vaults and cesspools; furthermore, many of the dangerous waste waters that would otherwise be thrown into yards or leaky receptacles also find their way to the sewers, with resulting improvement in the cleanliness and dryness of grounds. It is a well-known fact that the construction of a sewerage system in a city is immediately followed by a decrease in its mortality and that, next to the introduction of a pure water-supply, it is the most important factor in reducing the death-rate of infectious diseases, especially of typhoid fever which, of them all, is the one that most readily responds to sanitary improvements.

The popular fear of sewer air or emanations has been shown to be groundless. This air contains more $\mathrm{CO}_{2}$ and organic matter than pure outside air, but fewer micro-organisms, in fact much fewer than are found in dwellings. Bacteria abound in sewage but hardly ever find their way into the air above it, so that sewer air is incapable of conveying typhoid fever or, so far as known, any other infectious disease. It is a matter of record that men working in sewers are strong and healthy, and have a low death-rate. Plumbing fixtures properly trapped completely exclude sewer air, so that if foul smells are noticed they are almost always due to the decomposition of excreta or other organic matter within the fixtures, pipes or traps. Such smells may produce headache and affect the appetite and digestion, and therefore are to be avoided, but seldom have serious or dangerous effects.

The plumbing system of a dwelling consists of fixtures (closets, lavatories, sinks, tubs, etc.), waste pipes, traps, soil pipe and drain (Fig. I62). The waste pipes connect the fixtures with the soil pipe. They are generally of lead on account of its malleability, but when too many bends are not required iron is preferable. They must always be readily accessible so that leakage be easily discovered and repairs made. Their diameter should seldom exceed 2 to 3 inches in order to secure a rapid flow and thorough scouring. They are as short and direct as possible and gradually slope to the soil pipe which they join at an acute angle by Y-joints. The soil pipe is a cast-iron vertical pipe, 4 inches in diameter, into which the waste pipes empty the contents of the fixtures. Its segments, 5 feet long, each with spigot and hub. ends, are carefully and strongly jointed 


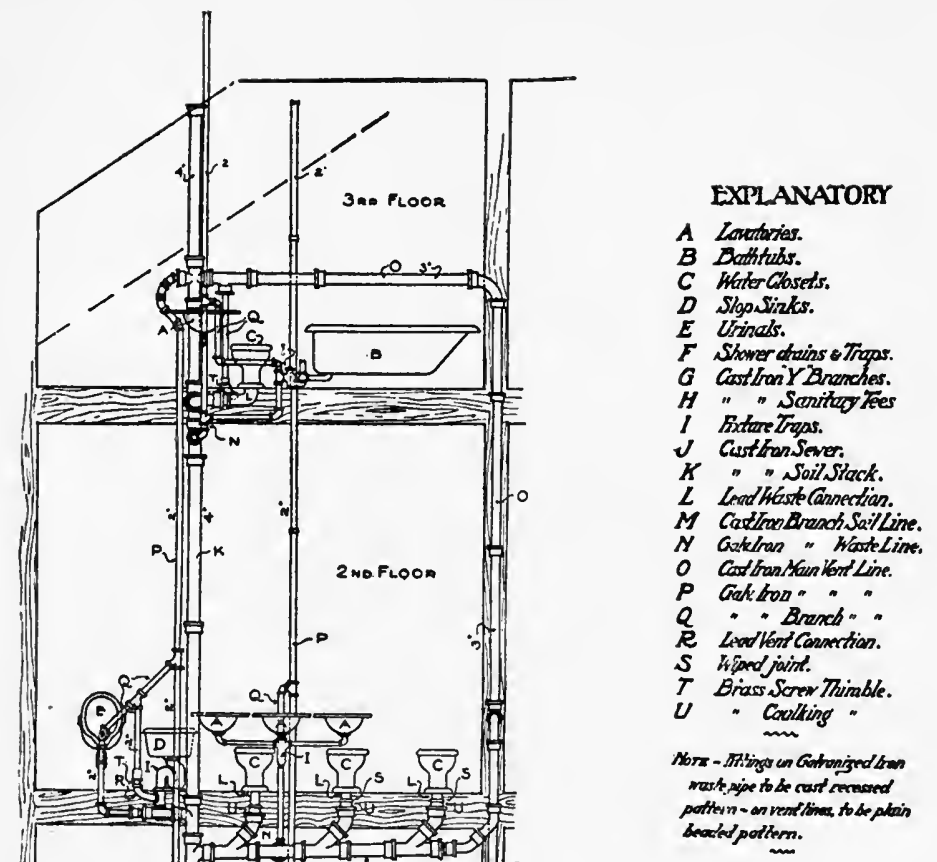

A Louturies.

$B$ Dathitubs.

C Water Cobsets

Shopisizes.

Costron $Y$ Bnances.

" "Sanitugteres

Extme Trass.

$K$ " "Soil Siack.

$L$ Land hesterannectian.

Castran Ananos Sailline.

N Gatlran "Wosteline.

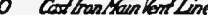

Q c. Bnoto

R Ladlent Conntion.

$s$ ripandjoint.

$T$ Brass Strea Thimble.

Coulting

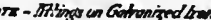

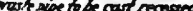

saodispolkm.

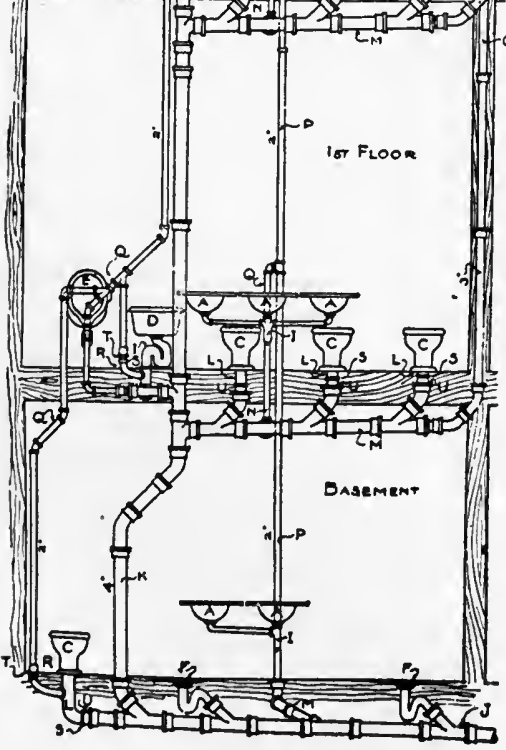

FIG. 162.- Plans and elevation of typical main. soil stack with fixtures and connections, for standard 36-bed post hospital building. 
by gasket of oakum and well-caulked molten lead. It should extend clear through the roof and project a few feet above it, rising if possible a little above the ridge to secure the advantage of wind aspiration. It should not open over a chimney or near a window for fear of unpleasant odors. At the bottom it must be firmly supported to prevent sagging, and make a wide bend at its junction with the drain. The drain forms the continuation of the soil pipe; it is also mostly of cast-iron, its diameter depending upon the number of soil pipes emptying into it. It should have as much slope as possible, and, before passing out of the house or just outside of it (within a manhole), be cut off from the sewer by an "intercepting" or "disconnecting" trap. The soil pipe and drain are thus thoroughly ventilated from above and below.

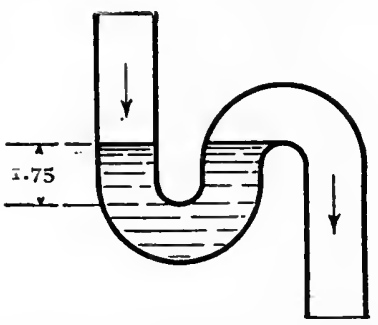

Fig. 163.- Common S-shaped round-pipe trap.

When the system is completed, its tightness is tested, after filling the traps, by pouring a few ounces of oil of peppermint, followed by a few gallons of hot water, into the top of the soil pipe. Any leak will be at once discovered by a distinct odor of peppermint in the room where it occurs.

Traps. - It is a rule that each plumbing fixture must be guarded by a trap, that is to say, a device which while permitting the free passage forward of liquid and suspended matter to the soil pipe, prevents the passage backward of all air, gas or odor. The trap should be as near the fixture as possible and admit of easy inspection and cleaning. The common form consists of an S-shaped bend, filled with water, in the fixture or waste pipe. (Fig. I63.) As the fixture is flushed, the excreta are forced through the trap, with enough water to clean and scour it and afterward refill it to the proper level. The depth between the level of the water and the lowest point of the upper bend is called the seal. (Fig. 163.) This is generally $\mathrm{I} 3 / 4$ inches. The greater the seal the more complete its efficiency but the less perfect is the scouring of the trap.

The round-pipe trap is readily flushed and, being perfectly smooth, keeps itself clean, but loses its seal easily. The flask-trap (Fig. I64). commonly used in barracks and hospitals, is simple, durable, not easily siphoned out and easily cleaned. The bottle or pot-traps 
contain much water and seldom lose their seal but require frequent cleaning. In the ball-trap, which is one of several obsolete mechanical types, the water displaces a ball which, by its weight, fails back upon the outlet. The bell-trap is a form often used in kitchen sinks, areas and gutters, consisting of a reservoir containing water to

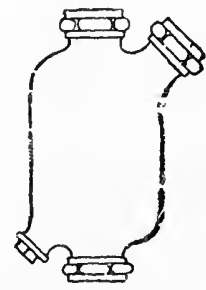

Outside view.

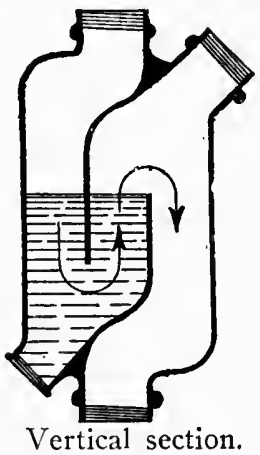

FIG. I64.-Flask trap. Quartermaster Corps type.

the level of the outlet pipe; a bell covers the mouth of the pipe and dips into the water (Fig. 165); it is fastened to the strainer and removed with it to clean the reservoir. The waste, after passing

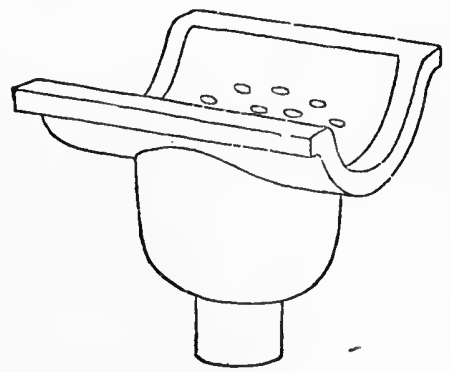

Outside view.

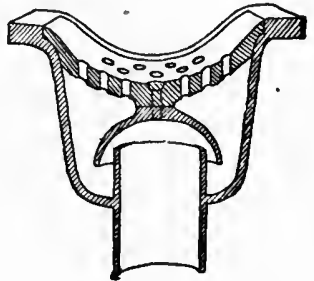

Vertical section.

FIG. 165.- Bell trap. Quartermaster Corps type.

through the strainer, reaches the reservoir and overflows into the outlet. The floor-trap (Fig. 166) used in mineral floors requiring washing, as in bath-rooms, operating-rooms, etc., is fitted with a strainer and sunk flush with the floor; it contains a bent partition with plug for clean-out. The disconnecting or intercepting trap is 
used to separate the outside drain or sewer from the house system. It has a fresh-air vent, as well as inspection and clean-out holes. Fig. I67 shows the one advocated by Harrington, in which the outlet (S) is lower than the inlet in order to secure freer outflow, and the fresh-air pipe $(F)$ is placed far enough from the trap not to become soiled by the splashing filth.

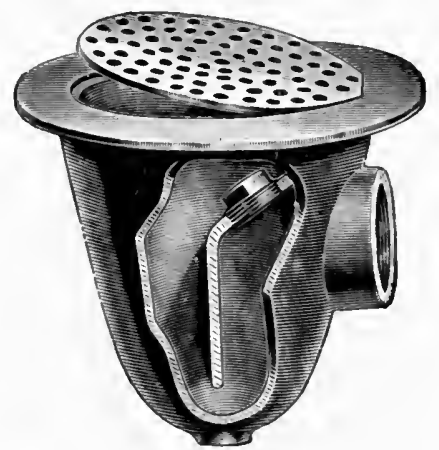

FiG. I66.- Floor trap.

Grease-traps are intended to congeal and collect the liquid fat contained in the warm waste waters of large kitchens and which, were it allowed to escape, would solidify on cooling and eventually choke the pipes. One of the most efficient is the Tucker improved. (Fig. I68.) Its essential feature consists in being lined with a chilling chamber which also projects upward across it. The entire cold water-supply passes through this chamber on its way to the boiler and sink, so that the trap is always kept cool. The flow of greasy water is deflected upwardly by the hollow partition, the grease remaining at the surface while the water passes freely to the waste outlet on the other side. The congealed grease forms a scum easily removed from the top. Grease-traps should always be in a cool place, generally ontside the kitchen. In the absence of such a trap, a strong solution of lye should often be thrown into the sink so as to saponify and dissolve the incrusting fat.

Loss of SEAL. - Traps are exposed to lose their water seal and thus become useless, by siphonage, back-pressure, evaporation, capillary attraction, leakage and accumulation of sediment. The most frequent and important of these causes is siphonage; it results from the rapid discharge, with great momentum, of the mass of water 
from the trap without enough after-flush to refill it, or from the suction produced by a heavy column of water falling down the suil pipe. In loss of seal by back-pressure, which is uncommon, the process is reversed; the column of water falling down the soil pipe

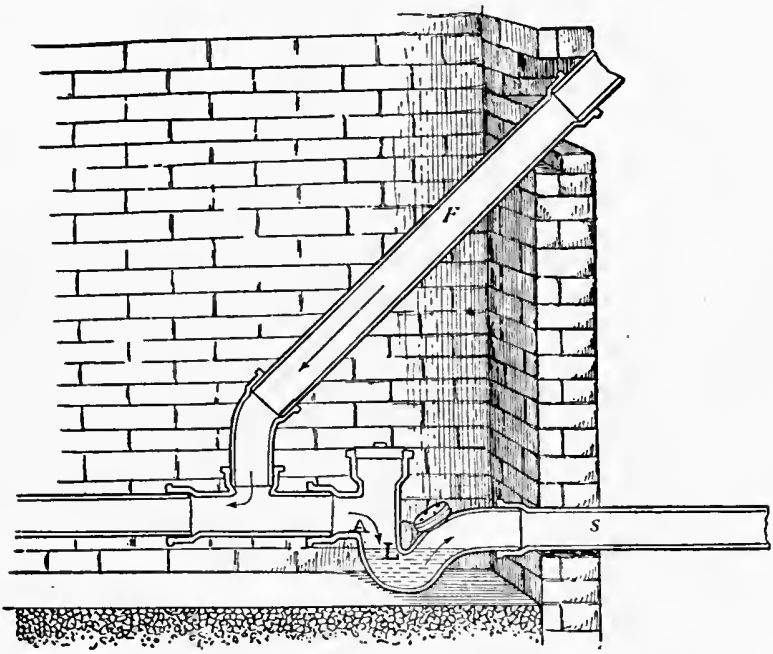

FIG. 167.- Improved intercepting trap. (Harrington.)

and meeting with an obstruction, the compressed air is forced up the waste pipe and breaks the seal. The water of traps, especially vented traps, evaporates more or less rapidly according to the temperature and the movement of the air, so that when a fixture remains unused the seal is soon lost from this cause. It is therefore necessary to flush the fixtures of a vacant house once a week or, preferably, pour oil or glycerine into them. Capillary attraction may empty a trap by means of a rag or string lying partly in the water and partly over the bend into the outlet.

Siphonage, as well as back-pressure, are prevented in either of two ways: by a vent connected with the upcast branch of the trap, on the distant side of the seal, or by the use of a non-siphoning trap. The vent supplies air to the waste pipe on the distant side of the trap and thus prevents the formation of any vacuum which would cause the suction and destruction of the seal; in case of back-pressure it affords an exit to the compressed air. The vent pipe should preferably be placed, not on the summit of the curve but a little below, so 
that the sewage may not be projected into it. (Fig. 169.) Each vent pipe, from the various fixtures of a system of plumbing, connects with a main ventilating pipe which, usually, runs alongside the soil pipe, both projecting above the roof; there is no objection to their being connected, provided such connection be made above the highest fixture. (Fig. I62.)

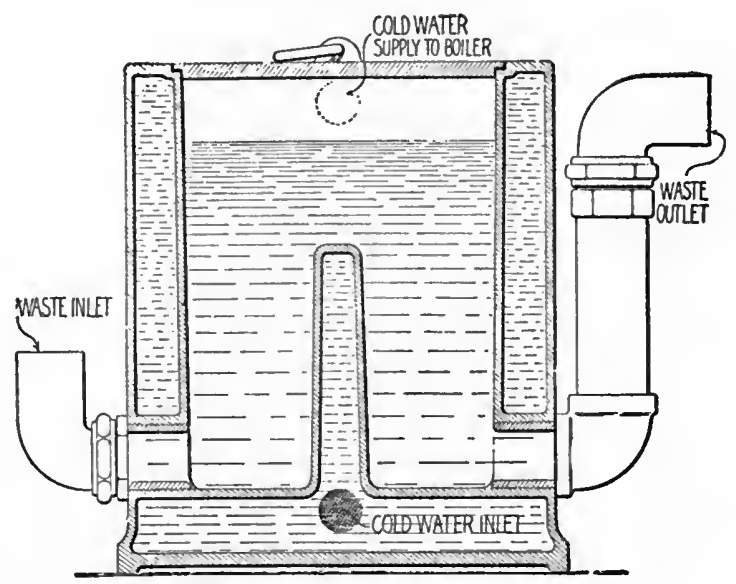

FIG. I68.- Tucker improved grease trap.

Non-siphoning Traps. - These have been devised to obviate the necessity of back-venting which is expensive and sometimes causes rapid evaporation of the seal. They are all so constructed that air may be drawn through them by suction without loss of much water. a sufficient volume of it remaining to reform the seal. Among the best-known traps of this character are the Sanitas (Fig. I67), the

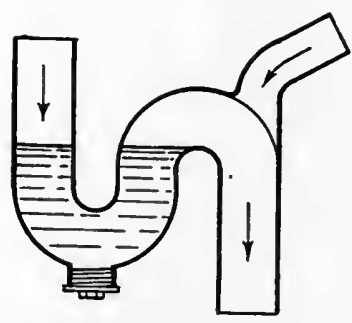

FIG. I69.-Vent pipe properly placed.

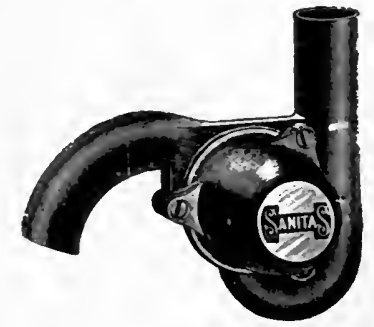

FIG. 170.- Sanitas trap. 
Hydric and Anti-siphon (Fig. I7I). The last-named depends for its efficiency on the fluid contents being given a rotary motion in a vertical plane in such manner as to break the siphonic action before the contents of the trap are withdrawn, leaving sufficient water to form a perfect seal. These non-siphoning traps are greatly more

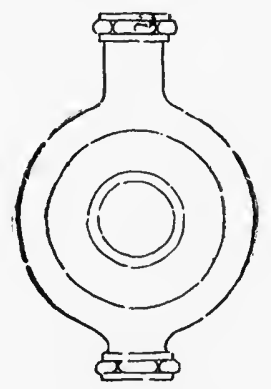

FIG. I71.-Anti-siphon trap.

Quartermaster Corps type.

economical than vent pipe and otherwise entirely satisfactory; there is no good reason why they should not be more extensively used wherever applicable, especially under sinks and lavatories.

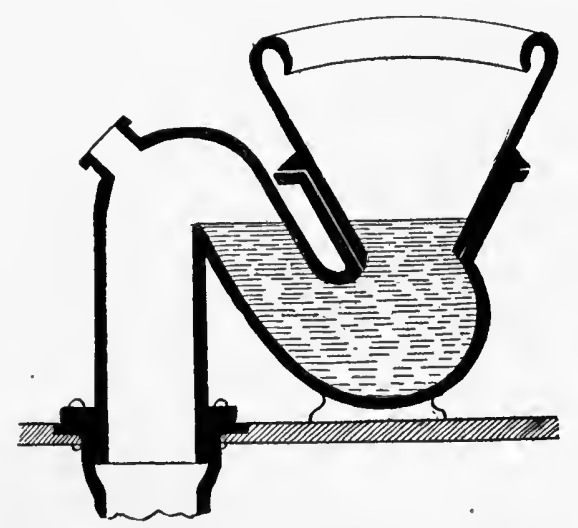

FIG. 172.-Short-hopper closet. (Munson.)

Water-closet Fixtures. - They should be simple, strong and easily operated, with sufficient flush to wash out the bowl, sweep the excreta beyond the trap and leave enough clean water to fill the 
trap and bowl to the proper level. The bowl should be so shaped that its sides are not exposed to fouling, and the water of such depth as to cover the discharges.

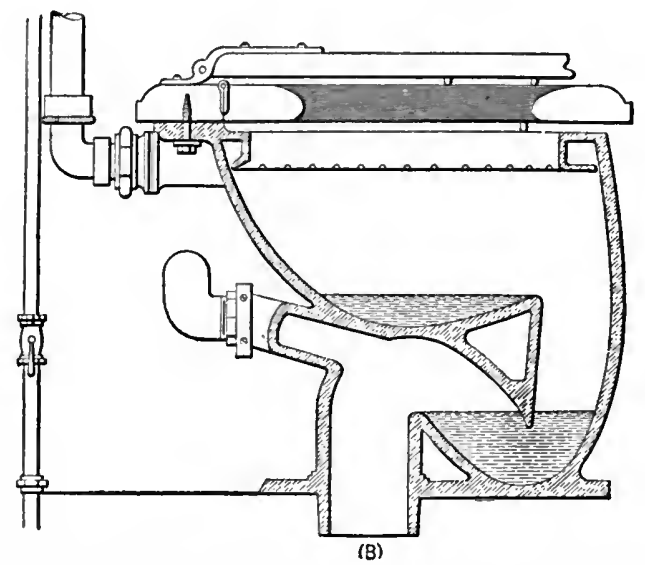

FIG. 173.- Wash-out closet. (Bashore's "Outlines of practical sanitation.")

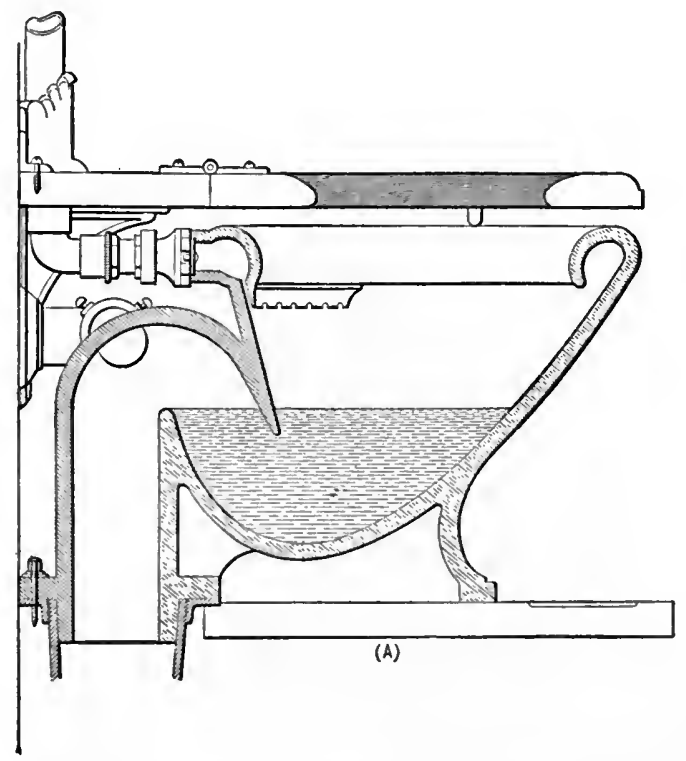

FIG. 174.-Wash-down closet. (Bashore.) 
The specifications of the Quartermaster Corps provide that the bowls for all water-closets in the army will be of the best hard-fired sanitary vitreous china, with flawless white glaze, the bowl and trap moulded in one piece, that a fractured piece of the material must not absorb red aniline ink after being immersed in it for one hour; that bowls of all types (except one) are to be oval, with siphon-jet, top supply and heavy roll-flushing rim with ample perforations properly distributed; that the connection between each closet and soil pipe will be made with drawn lead pipe 4 inches inside diameter.

Water-closets have undergone a marked evolution during the past quarter of a century, keeping pace with the more liberal watersupply now considered necessary for the needs of communities. The former types, such as the pan, valve and plunger closets, still seen in old buildings and which are operated through a complex internal mechanism, have the merit of consuming little water, but

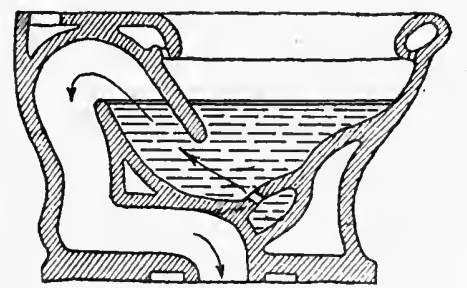

FIG. I75.-Siphon-jet closet with visible jet. Quartermaster Corps type.

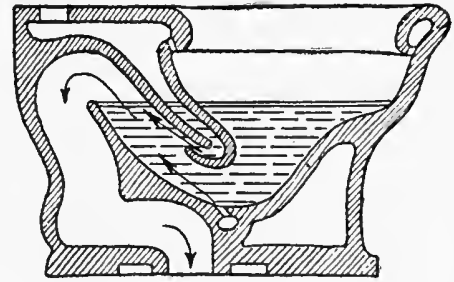

FIG. I76.- Siphon-jet closet with both visible and invisible jets. Quartermaster Corps type.

are always foul, malodorous and thoroughly unsanitary. The shorthopper closet (Fig. I72), consisting simply of bowl and trap, with rim flush, is a distinct improvement. In the wash-out closet (Fig. I73) the excreta are received in a shallow basin and swept out by the flush into the trap below; they often adhere to the basin, fouling: it, as well as the surface against which they are projected, and often partly remain in the trap. This closet, although popular at one time, has but little to recommend it. The so-called wash-down closet (Fig. I74) is an excellent development of the hopper, in which the bowl forms part of the trap and contains a good depth of water. But the best modern type of water-closet, that which is now generally preferred and most used, is the siphon-jet closet, of which there 
are many forms. (Figs. 175, I76.) The bowl and trap are moulded in one piece, the bowl itself forming the inlet of the trap. The flushing operates in two directions, washing the bowl from the rim, while, at the same time, a separate jet drives the water from the outlet of the trap into the waste pipe; the latter forms the long leg of a siphon which, when filled, sucks out the contents of the bowl, the trap and basin being then refilled to the proper level by the afterflush. The jet may be visible in the bottom of the bowl (Fig. I75) or invisible under the partition (Fig. I76). The bowl with visible jet is somewhat more efficient and durable, but, on the other hand, the hole may become soiled and unsightly or clogged. In the siphonjet closet the trap vent, in order not to interfere with the action of the siphon, is placed below the long leg of the siphon, generally under the floor.

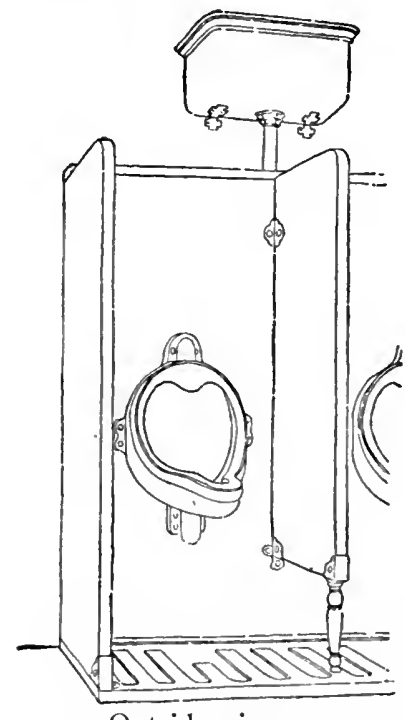

Outside view.

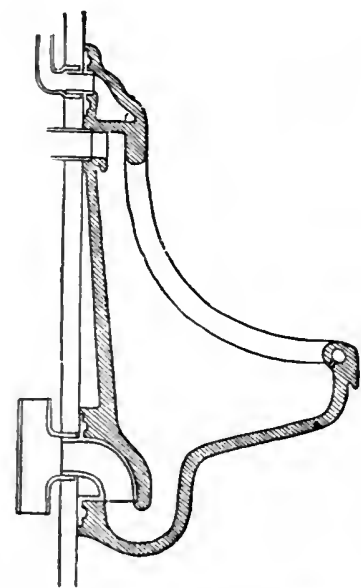

Section through bowl.

Fig. 177.-Urinal for barracks. Quartermaster Corps type.

In order to secure more perfect ventilation in toilet rooms and prevent odors from the water-closet, a vent (Boston or local vent) is sometimes provided in the upper part of the bowl, just above the water level. Such vent, of course, is entirely independent of the trap vent and cannot take its place. 
URINALS. - Modern urinals are constructed of hard vitreous porcelain, with or without bowl. In the latter case, the urine is projected against the walls of the urinal which are constantly washed by running water. The use of bowls is more economical of water, but it is necessary that they be large enough to prevent the dripping of urine upon the bottom slab.

\section{Hygiene of Toilet Rooms.}

These rooms, containing the water-closets, urinals, bath-tubs and showers, wash-bowls and troughs, and sometimes laundry tubs, should be scrupulously clean, thoroughly ventilated and well lighted. The lighting is facilitated and cleanliness promoted by having as many of the surfaces as possible painted white. The floor should be cemented or tiled and free from cracks and crevices so as to make its flushing and mopping easy and effective. All tubs, bowls and troughs should be daily scrubbed and their outlets kept clear from soap, hair, paper, cigar or cigarette stumps, and other refuse which tend to clog them. Gratings must be lifted up and the floor beneath flushed and scrubbed. Metallic surfaces are quite liable to become oxidized unless carefully rubbed and kept dry. Water-closets require frequent inspection to insure cleanliness, efficient functioning and adequate trapping. When out of order they should be closed until repaired. A bad-smelling closet or urinal may be due to defective plumbing but more generally results from lack of care in cleaning the bowls and seats. When urinals with bowls are used, the men must be instructed to exercise care and avoid dripping in urination, but such dripping is almost inevitable and will require strict policing. In addition to the usual flushing, it is well to rub the bowls, the bottom slab and, when necessary, the side slabs with a moist rag sprinkled with kerosene, otherwise a crust of urinary salts will form and bad odors arise. The outlet should be screened with wire gauze to prevent clogging.

As a general rule no disinfectant need be used in the toilet rooms beyond a light sprinkling of kerosene on the damp mop or cloth used to wipe fouled surfaces. As already insisted upon, the hands must always be washed after defecation. A printed poster should remind all visitors of this duty. As no roller-towels are permitted, it is necessary that every man coming to the toilet rooms should bring his own towel and, if needful, his own soap. 
SEWERS.

Sewers are constructed according to the "separate" or "combined" systems. In the former, they receive only the excreta and waste waters of dwellings; in the latter, they carry off not only sewage but also wastes from factories, street washings and rainwater. The separate system has much to recommend it and is often the only one possible in small conmunities; the volume of sewage is pretty constant and can be readily calculated from the daily water consumption; it is more concentrated and uniform in composition than in the combined system and therefore can be better utilized or disposed of ; the sewers being smaller can also be more frequently and effectually flushed. When sewage is to be treated by septic tanks and filter beds, better results are obtained by the separate. system, with matters more homogeneous and not too highly diluted. Thus, for instance, whenever a city, in order not to pollute a bay or river, determines to treat its sewage by irrigation or filtration, it will be in the interest of economy, as well as of efficiency, to build large superficial conduits for storm water along the shortest lines directly to the bay or stream, and separate, deeper and smaller sewers to convey the excreta and wastes to the disposal plant which may be several miles away. In this separate system, in order to insure a rapid flow and good ventilation, the pipes must not be any larger than necessary; the rule is that a sewer should be at least half-full at the time of greatest flow, and that the velocity of the flow should not be less than two feet per second.

In the combined system the size of the sewers must be such as to accommodate all wastes and rain-water. They may be nearly full or, in the dry season, contain only a small sluggish stream, offering conditions most favorable for the settling of solids and putrefaction of nitrogenous matters. It must be remembered, however, that in large cities, where this system is generally used, the waste waters alone are always sufficient to keep the excreta in a state of very free dilution. When this is not likely to be the case, the smaller sewers should be given an ovoid shape with the smaller end downward. 


\section{EXCRETA, GARBAGE AND WASTES (CONTINUED).}

\section{NATURE OF SEWAGE AND ITS FINAL DISPOSAI.}

Sewage in this country is largely diluted, seldom containing more than one part of solids (mineral and organic) per thousand, and generally containing less (Winslow and Phelps). The sewage of English cities may average about twice as much solids, while few European cities exceed two parts per thousand. It is therefore, in this country, nothing but water more or less polluted, and its amount can be readily estimated from the consumption of the water-supply. Of the total solids, one-half is mineral and one-half organic; of the latter, the proportion of nitrogenous matter to carbohydrates is as 3 to 5 . From 60 to 75 per cent. are in solution, the remainder being insoluble matter in suspension. The mineral matter in solution consists largely of sodium chloride. The amount of insoluble mineral matter is normally about Io per cent. of total solids but, with combined sewers, may so increase from storm water as to interfere with the proper operation of filters.

The contents of American domestic sewage in nitrogen (parts per million) are about 24, I 5 as free ammonia and 9 as albuminoid ammonia; in nitrates 0.2 and in nitrites o.o. The bacterial content ranges from I to 2 millions per c. c., of which 20 per cent. are acid formers and 8 per cent. liquefiers.

The final disposal of sewage is according to the following methods:

I. Discharge into the sea or a lake or river.

2. Chemical treatment.

3. Biological treatment.

4. Local or domestic treatment.

Discharge into the sea, wherever possible, is the simplest and most satisfactory method, but open to the charge of unjustifiable waste of our national resources, involving the loss of millions of tons of fertilizing elements which should be returned to the soil. In 1898 , Sir Crichton Browne estimated that in the United Kingdom, fixed nitrogen to the value of no less than $80,000,000$ dollars was thus thrown into the sea. It is hoped that, through improved biological treat- 
ment, such amazing waste will become unnecessary and that the useful constituents of sewage may be recovered and made available.

In the application of this method, measures must be taken that the sewage be entirely carried away and does not float back. It is often difficult, however, to prevent the pollution of neighboring beaches and shores by returning tides. Great care must be exercised that the sewage be only discharged from such points and at such stages of the tide as will insure its permanent removal. Thus, at Fort Monroe, Va., it was found necessary to collect the sewage into a large tank from which it is pumped out to deep water, twice a day, at the setting out of ebb tide.

Sewage can also be safely discharged into any salt, tide-water stream, so long as the tide and current can be depended on to carry it to the sea. The emptying of sewers into fresh-water lakes or streams is never advisable and should be tolerated only when the latter are not used as sources of water-supply. The excreta of a large city may readily contaminate the waters of a lake for a distance of $x$ or 2 miles from the shore. It is a fact that there are now few rivers in the inlabited districts of the United States whose water can be safely consumed without previous purification. This prevalent custom of discharging raw sewage into water-courses, in wellsettled communities, is repugnant as well as dangerous, and sanitary engineers are turning their thoughts to less objectionable methods of disposal. It is highly probable, for instance, that the carriage of the sewage of Chicago to the Illinois River would not be approved at this day. The self-purification of streams has its rather narrow limits; the amount of sewage which they can oxidize and dispose of is roughly stated as I part to 50 parts of water. When that limit is exceeded there is usually a failure of available oxygen; the conditions of putrefaction are set up and an objectionable deposit of decomposing sludge takes place. The condition of our rivers would be vastly improved if the sewage should be made to undergo some form of preliminary treatment, so as to be rendered non-putrescible before being discharged into them.

Chemical Treatment consists in the addition of chemicals. whereby more or less of the putrescible substances in solution are precipitated and subsequently separated, together with all matters in suspension. The solids, or "sludge," are removed, subjected to hydraulic pressure and utilized as fertilizer, while the liquids may" 
be discharged into the sea or a stream. The chemicals most used as precipitants are alum, lime and iron sulphate, either alone or in combination. This system is seldom satisfactory and never adapted to garrisons or camps. The sludge is always difficult to dispose of, while the.liquid effluent contains much putrescible organic matter and can only be turned into a water-course of many times its volume, and not used as water-supply.

BIOLOGICAL TREATMENT.

Fecal matter, as well as all forms of dead organic matter, animal or vegetable, when left exposed to the air, undergo decomposition and disintegration, and eventually become reduced to their primitive elements. This is nature's way to get rid of offensive decaying matter and to transform complex substances, become useless, into simple bodies which can again be utilized by growing animals and plants. In other words, nature operates a conversion of organic into mineral substances. This is effected through the agency of bacteria and, therefore, is a biological process. This process is the result of oxidation and is often designated as the mineralization or nitrification of organic matter. Oxidizing or nitrifying bacteria are found in great abundance not only in excreta but also in the upper layers of the soil, especially in the first 3 or 4 feet, becoming rare beyond a depth of 6 feet. This explains why excreta covered in a shallow trench will be much more quickly distintegrated than if deeply buried. These bacteria are non-pathogenic, and broadly divided into aërobic, or those unable to grow without oxygen, and anaërobic, or those which grow best with little or no oxygen. The great majority of them belong to the first division but both kinds take an active partin the decomposition and reduction of organic matter. With free access of air, dead animal and vegetable substances are decomposed by aërobic bacteria without appreciable odor. But as it is seldom that they are penetrated throughout with a sufficiency of air, anaërobic bacteria, which are the nicro-organisms of putrefaction, are also brought into action and offensive smells generated.

In the biological treatment of sewage disposal, our aim is to create conditions as favorable as possible to the growth and multiplication of nitrifying organisms. This is often a difficult problem for it involves the supply to the organic matter of one to three times its weight of oxygen under the special conditions which will permit 
them to unite. The first step in the process is the conversion of the nitrogenous elements into ammonia $\left(\mathrm{NH}_{3}\right)$ by the direct combination of nitrogen with hydrogen. Ammonia by absorbing oxygen becomes oxidized into nitrous acid $\left(\mathrm{HNO}_{2}\right)$ which, by further oxidation, is in its turn quickly changed into nitric acid $\left(\mathrm{HNO}_{3}\right)$, both acids forming salts by combining with calcium, sodium and potassium. The presence of these alkalies therefore promotes nitrification and renders it more complete. Meanwhile the carbohydrates and fats are also attacked by anaërobic bacteria, but much more slowly decomposed than nitrogenous matters. The outcome of the entire process is the transformation of the putrescible excreta into ammonia and its salts, nitrites and nitrates, carbon dioxid and water. Thus there is rarely as much as o.oI per cent. of nitrogen as nitrite or nitrate in sewage, while 5 or 6 per cent. may be found in the effluent, after filtration.

The two methods by which the biological treatment is applied are irrigation and filtration.

Irrigation. - In this method the sewage is conveyed upon farm land which has been plowed into hills and furrows and properly drained. The irrigation is intermittent, that is, suspended for a few hours every day in order that the air may freely penetrate into the soil and promote the multiplication of aërobic bacteria. Thus are fine crops of vegetables grown near Paris and Berlin, and there is no conclusive evidence that, even when consumed raw, they have ever transmitted disease; simple prudence, however, requires that they should be carefully washed before consumption. One acre of land will dispose of the sewage of from 200 to 400 persons by this method.

Filtration. - Before using any system of filtration it is desirable, if not necessary, to apply to the sewage some kind of preparatory treatment. By means of screening and settling tanks much of the coarser matter is removed, while the sludge itself can be materially reduced by the operation of a septic tank.

Septic Tank. - In a septic tank the sewage is placed under conditions which favor the septic process, that is to say, the propagation and action of anaërobic bacteria, with resulting putrefactive decomposition. The sludge is broken up, liquefied and, to some extent, transformed into gases. About one-third of the suspended solids and at least one-fourth of the organic matters in solution disappear, 
while much of the inorganic matter settles to the bottom or forms a scum or mat on the surface. The escaping gases are mostly hydrogen, nitrogen, carbon dioxid, sulphureted hydrogen, mercaptan, marsh gas and ammonia, some of them highly inflammable and others with offensive odors.

The septic tank is rectangular in shape, five to eight feet deep. After passing through a settling compartment the sewage enters the tank near the bottom so as not to disturb the surface layer nor introduce much air, and flows continuously but very slowly in order to allow the action of bacteria, as well as the rising to the surface or falling to the bottom of the decomposing suspended matters. The capacity of the tank should be at least equal to one-third of the daily amount of sewage to be treated. It is not absolutely necessary to exclude air and light, but desirable that it should be covered so as to exclude rain and prevent smells. A septic tank can be operated several years without any great accumulation of scum or sediment.

The experience acquired in the operation of the septic tank, in this country, tends to show that it has but little value in connection with the use of filter beds, although still advised under certain local conditions to prevent the choking of the beds by a high content of mineral and indigestible fibrous matters. The conclusion has been reached that, on the whole, the septic tank has little to recommend it, that there is no stubstantial gain from retaining sewage in tanks until decomposition has set in, but that, on the contrary, the practice is often distinctly disadvantageous, the slightly increased digestion of the sludge being largely counterbalanced by the greater difficulty of treating the septic effluent. Simple mechanical sedimentation for 3 or 4 hours is preferable.

Imhoff Tank. - A new form of septic tank has been developed by Imhoff, in Germany, in which the sludge appears to be successfully disposed of without altering the character of the liquid sewage, and is being operated in this country with great satisfaction. (Fig. I78.) It consists of two superposed chambers. Sedimentation takes place in the upper, and digestion of the sludge in the lower. The sloping surfaces of the upper chamber project over each other below, leaving a slot through which the settled suspended matters enter the lower chamber, but through which the bubbles of gas generated in the sludge cannot rise into the upper chamber, so that the effluent remains practically the same as raw sewage, less a large part of its 
suspended solids. At the bottom of the lower chamber, under a depth of 20 to 30 feet, the sludge undergoes changes and, when withdrawn through the discharge pipe, appears as a non-odorous, friable, porous mass, resembling vegetable mold. The liquid draining from the sludge is perfectly clear and can be discharged into any watercourse.

Sewage Settling Tank (radialdommend and upwardflow)

4.000 Inhabitants (separate syshem)

580.000 U.S.gallons daily

Capacity of settling basin 8,600 cb.f7.

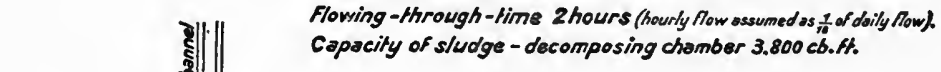

capacity of sludge - decomposing chamber 3.800 cb. At.

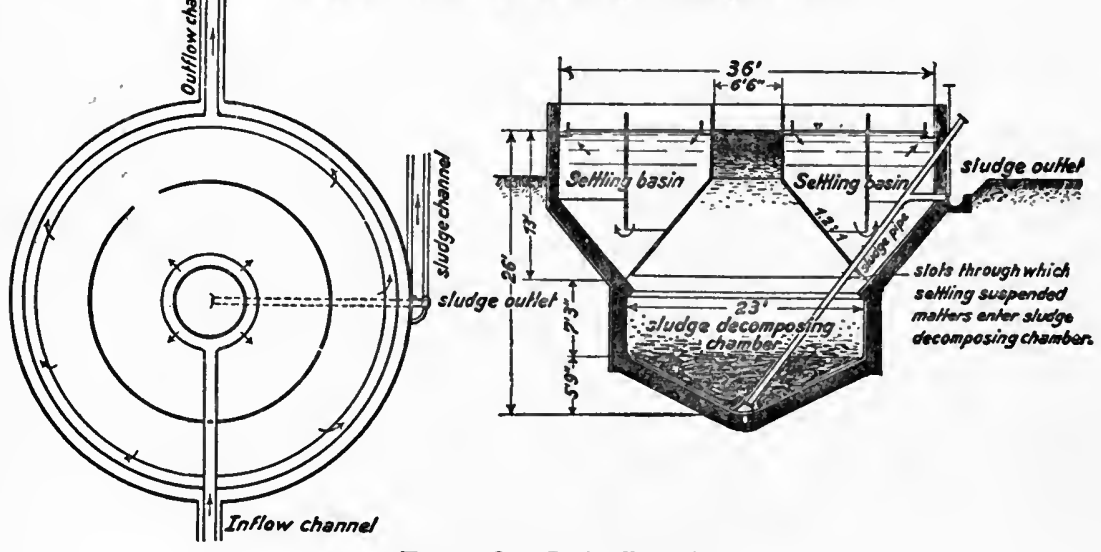

FIG. 178.-Imhoff tank.

Whether the sewage receives preliminary treatment in septic tanks or in simple sedimentation tanks, its final purification must be effected by submitting it to the action of aërobic bacteria by filtration. The filters used are sand filters, contact filters and sprinkling filters.

Sand Filtration. - In this method the sewage is concentrated over small areas of specially chosen and prepared ground, with porous, thoroughly underdrained soil. The filter is generally divided into four equal parts, each in turn receiving sewage during six hours and remaining unused during 18 hours to insure the aëration of the bed and the multiplication of bacteria. Sand filtration, largely used in America, is the most satisfactory and economical method of sewage disposal where the nature of the soil permits its use; it generally 
yields a clear, non-putrescible effluent which may be turned into any stream. One acre of sand filter will dispose of the sewage of $\mathrm{I}, 000$ people.

Contact Filter. - This filter consists of a water-tight compartment, generally of concrete, filled with coarse material such as coke, clinker or broken granite with relatively large interspaces. The sewage is applied at regular intervals, left in contact with the bed for a few hours and then slowly drained out. After each application the bed is allowed a short rest. Contact beds are generally disposed in 2 or 3 sets, the second set containing smaller filtering material than the first, and the third than the second, the effluent passing automatically from one set to the next. One acre of contact beds will treat the sewage of 5,000 people.

Sprinkling Filter. - This filter, also called "trickling" or "continuous" filter; represents the most important advance of the last few years in the treatment of sewage. It consists of the same compartment and material as the contact filter, but is operated differently. Instead of the fill and draw, or intermittent, principle, the sewage is applied continuously either as a spray or in very thin sheets sprinkled over the surface, then trickles down and passes out through the underdrains which are comparatively large in order to insure thorough aëration of the entire bed. The more subdivided and broken up the sewage, the greater the oxidation and the better the result. Therefore it must be discharged slowly and evenly over the whole surface of the bed in a rain of fine drops. The sprinklers may rotate on an axis, in which case circular beds are used, as in England, or they may be fixed, in rectangular beds, as in this country. Fixed sprinklers are simpler, cheaper, more easily operated and less affected by frost. Highly recommended is the use of splashing disks or concave disks into which the sewage is discharged from the bottom of a trough and from which it is projected in a fine circular spray. Sprinkling filters may run io to 5 years before the material becomes clogged and requires renewal. They yield a higher degree of purification than contact filters and their rate of treatment is about four times as great, one acre of sprinkling beds being able to dispose of the sewage of 20,000 people. The effluent is not quite as good as that obtained from intermittent sand filtration; it still contains more than one-half of the soluble organic matter of the sewage and is more or less turbid, but it is non-putrescible, 
stable and fit to be discharged into almost any stream not used as water-supply.

Although the organic matter in the effluent of the contact or sprinkling filter is not completely nitrified, its chemical composition has been so altered in the process that, as a rule, it is no longer putrescible.

Combined Method. - The ideal method of sewage disposal, such as is used at Lawrence, Mass., consists in preliminary sedimentation followed by sprinkling beds and sand filtration. The effluent thus obtained is practically pure and may be safely allowed to run into any water-supply.

Stability of Effluent. - An effuent is said to be stable when its organic matter has been so transformed during filtration that it is incapable of undergoing offensive putrefactive decomposition; it is that condition in which the available oxygen (in solution or as nitrites and nitrates) equals or exceeds the oxygen required to allow nitrification to proceed on to completion.

Immediately after the complete exhaustion of the oxygen in sewage, anaërobic fermentation (putrefactive decomposition) begins, with production of hydrogen sulphide. The property of this gas to discolor methylene blue furnishes a simple test to determine the stability of an effluent. The sample to be tested is collected in a 200 c. c. glass-stoppered bottle. One-half c. c. of a one-tenth per cent. of methylene blue is added to the sample which is then incubated at $20^{\circ} \mathrm{C}$. for four days. If it remains blue at the end of that time, it contains enough available oxygen to prevent its becoming putrescible and a nuisance. When an effluent is discharged into a bay or large river where it is highly diluted and freely oxygenated, the same degree of stability is not necessary. The least that can be required of waters not used for drinking purposes is that the sewage discharged into them should not interfere with fish life. Such interference occurs whenever the dissolved oxygen falls below 70 per cent. of its original amount. Many of our bays and rivers do not come up to this standard.

\section{DISINFECTION OF SEWAGE EFFLUENTS.}

A considerable proportion of sewage bacteria is removed by the operation of the septic tank and of the contact and sprinkling fil. ters, but this removal is not sufficiently complete to have much sani- 
tary significance. Enough micro-organisms remain in effluents to render them highly suspicious, frequently containing nearly as many colon bacilli, spores of Bacillus enteritidis sporogenes and Streptococci as the crude sewage. For this reason untreated effluents should not be discharged into streams used for water-supply, or bays in which are oyster beds or other shell-fish. Slow sand filtration almost totally eliminates the organic matter and yields an effluent practically free from pathogenic bacteria, but is a costly method, seldom applicable. Simple sedimentation improves a very turbid effluent but does not make it bacterially safe. Chemical disinfection offers a simple means to produce a sufficient and satisfactory bacterial purification, even without the complete oxidation of the organic matter. As a finishing process it can be conducted at far less cost than the cheapest form of supplementary sand filtration. Chlorine is the most efficient agent for the purpose, in the form of calcium hypochlorite or the commercial bleaching powder, in the amount of 3 to 5 parts of available chlorine per million parts of effluent. The removal of bacteria averages over 95 per cent., corresponding to 98 or 99 per cent. in the crude sewage, at a cost of I to 1.50 dollars per million gallons. The pathogenic bacteria are all destroyed; the organisms that survive are chiefly of the nitrifying and spore-bearing kinds. The bacterial purification of the crude sewage itself can be accomplished by increasing the dosage of available chlorine to from 5 to Io parts per million of sewage. Gaseous chlorine, found more efficient than any of its compounds in water purification, can probably be also successfully applied to sewage.

\section{DISPOSAL IN GARRISON AND CAMP.}

The disposal of human excreta is one of the most important problems that confront the military hygienist, one that should be the object of the constant solicitude of the medical officer and require his most careful attention. All our military posts, with few exceptions, are provided with modern water-closets and sewerage, and their excreta and wastes, after or without previous treatment, carried away to the sea, neighboring lakes or streams. Other systems more or less applicable to permanent stations are described under Camps. The use of the primitive pit-privy at a post, cantonment or permanent camp could only be justified by very exceptional conditions. 
The method adopted for some of our largest interior posts is in conformity with the highest modern standards, except that contact beds are used instead of the more expensive sprinkling filters. The sewage plant at Fort Benjamin Harrison, Ind., (Fig. I79) has a maximum capacity of 200,000 gallons in 24 hours. It does not receive surface or storm water. The degree of purification required by the $Q$. M. Corps is that all solid matters will be arrested, and 80 per cent. destroyed; that the effluent shall not contain more that $I .50$ parts of albuminoid ammonia in $I, 000,000$ parts, and that I,000,000 parts shall consume less than 15 parts of oxygen in 4 hours; that the effluent shall be clear, colorless, odorless, non-putrescible, and free from typhoid and other pathogenic germs.

The plant consists of five tanks, two sets of contact beds and one sand filter, all built of concrete and lined with a coat of cement plastering one-half inch thick. The tanks are a grit tank or sediment chamber, three septic tanks and a dosing tank, all covered over, with roof properly ventilated and a sufficient number of manholes. The grit chamber receives the raw sewage and retains all heavy insoluble matter; it has submerged openings, guarded with slide gates, one into each septic tank, and a drain-pipe connected with the by-pass drain. The three septic tanks are each of sufficient size to give treatment to 67,000 gallons of sewage in 24 hours. A baffle wall, in each tank, serves to scatter the sewage as it enters from the grit chamber. From the septic tanks the effluent is discharged into the dosing tank by a submerged weir so placed as not to disturb the mat or scum formed on the surface. Each tank is provided with a drain so that the sludge may be removed into the by-pass drain without disturbing the surface mat. The dosing tank collects the effluent until it has enough to charge one contact bed, and then discharges it onto the bed by an automatic alternating device.

Each set of contact beds consists of three beds, each bed of such capacity that the time consumed between doses, that is to say, in filling, resting full, emptying and aërating, shall be no less than 6 nor more than 8 hours. The effluent is distributed over the surface of the beds by open-joint vitrified pipes, and collected at the bottom by horseshoe-tile pipes, being discharged from the beds of the first set to those of the second by an automatic-timed device, and finally upon the sand filter. 


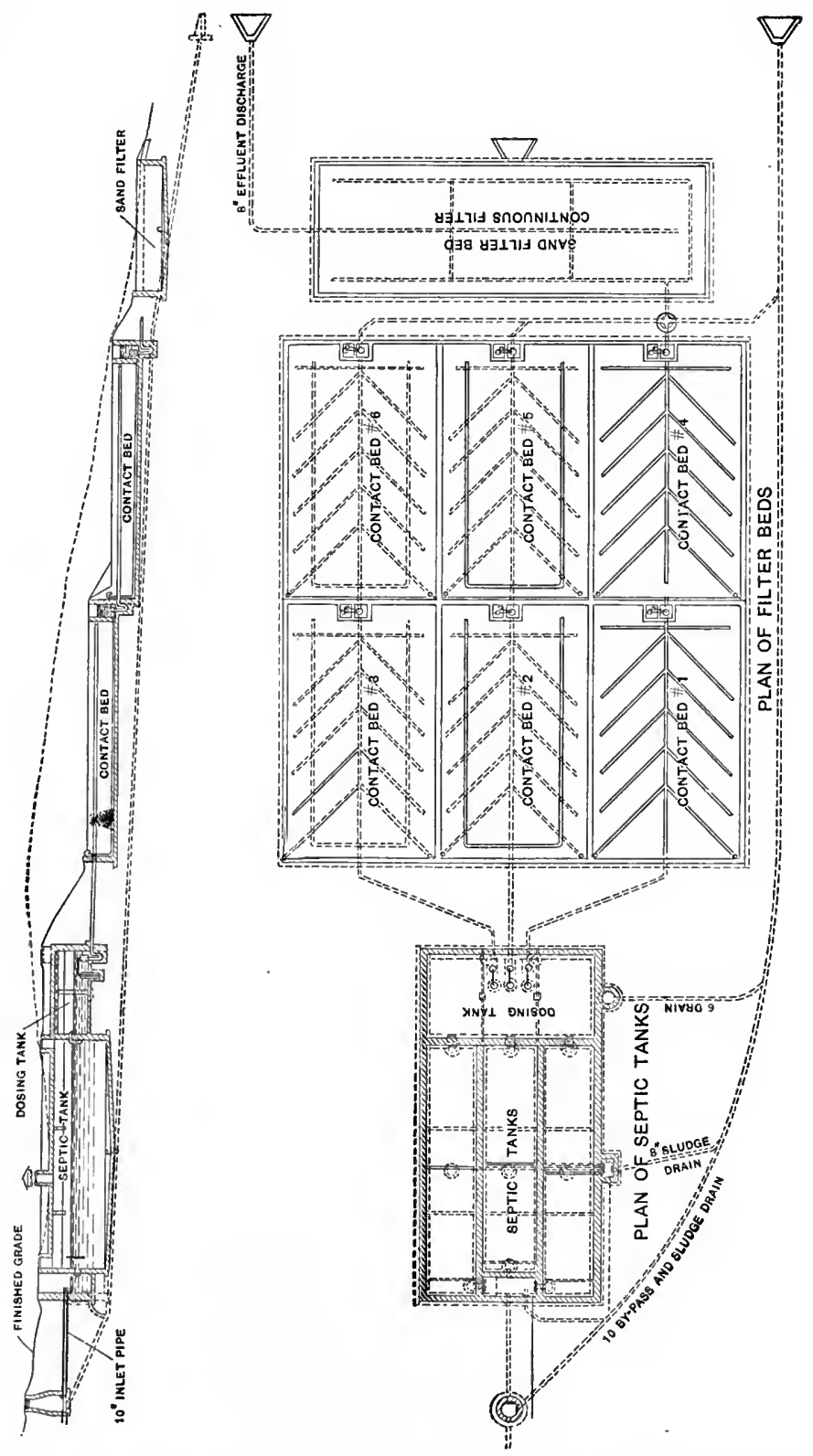

FIG. 179.- Sewage purification plant. Fort Benjamin Harrison, Indiana. 
The filling material of the beds, 4 feet deep, is of good, hard, broken stones or pebbles, capable of resisting the alternate action of water, air and frost without dissolving or disintegrating. In size it ranges from $I$ to 2 inches in diameter at the botton; decreasing to $1 / 2$ inch at top in the first set, and to $1 / 8$ inch in the second set.

\section{LOCAL OR DOMESTIC TREATMENT.}

In the absence of a general or central system of sewerage, various methods may be resorted to for the disposal of excreta and wastes. Ordinary vaults are still used in many communities although rarely at military posts. They should be lined with stone or brick and cemented so as to be water-tight and not endanger neighboring water-supplies. The water-closets may empty directly into them, or else by means of soil pipes and drains. Inasmuch as these vaults have to be periodically emptied, it is evident that they are only a

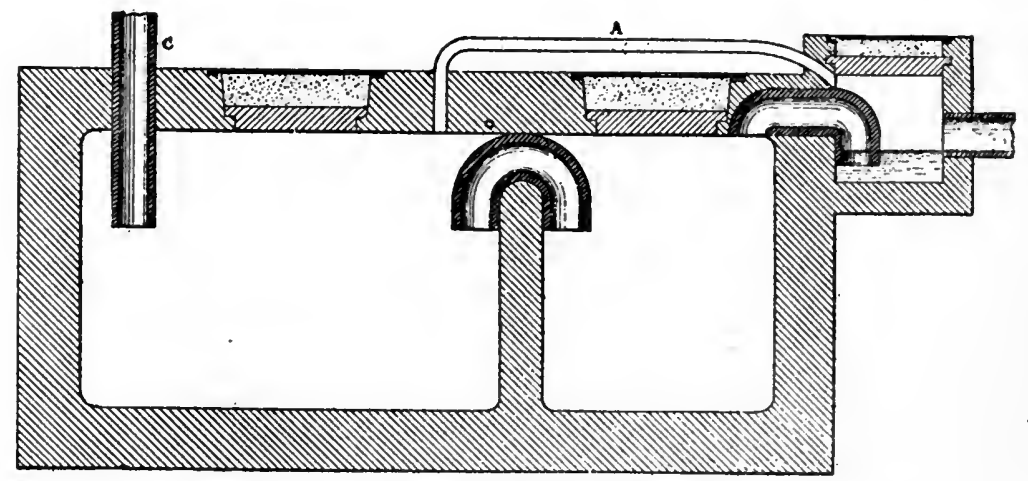

FIG. I80.- Mouras vault.

poor makeshift and not a final means of disposal. The contents can be spread on and plowed into tillable land with safety, and benefit to the soil. The addition of lime is useful, acting as deodorant and disinfectant and generally increasing the fertility of the soil; it is especially advisable when the contents are thrown over the frozen ground.

An improvement upon the ordinary vault is the Mouras vault used with various degrees of success in many countries. In this system a notable amount of septic decomposition takes place, perhaps also a certain degree of nitrification, so that the overflow may 
be allowed to run into a large stream or upon a field for irrigation purposes. As seen in Fig. I80, this vault consists of two unequal compartments connected by means of a curved pipe; the soil-pipe $\mathrm{C}$ dips in the liquid contents of the larger compartment; as these contents rise they pass into the smaller compartment and thence

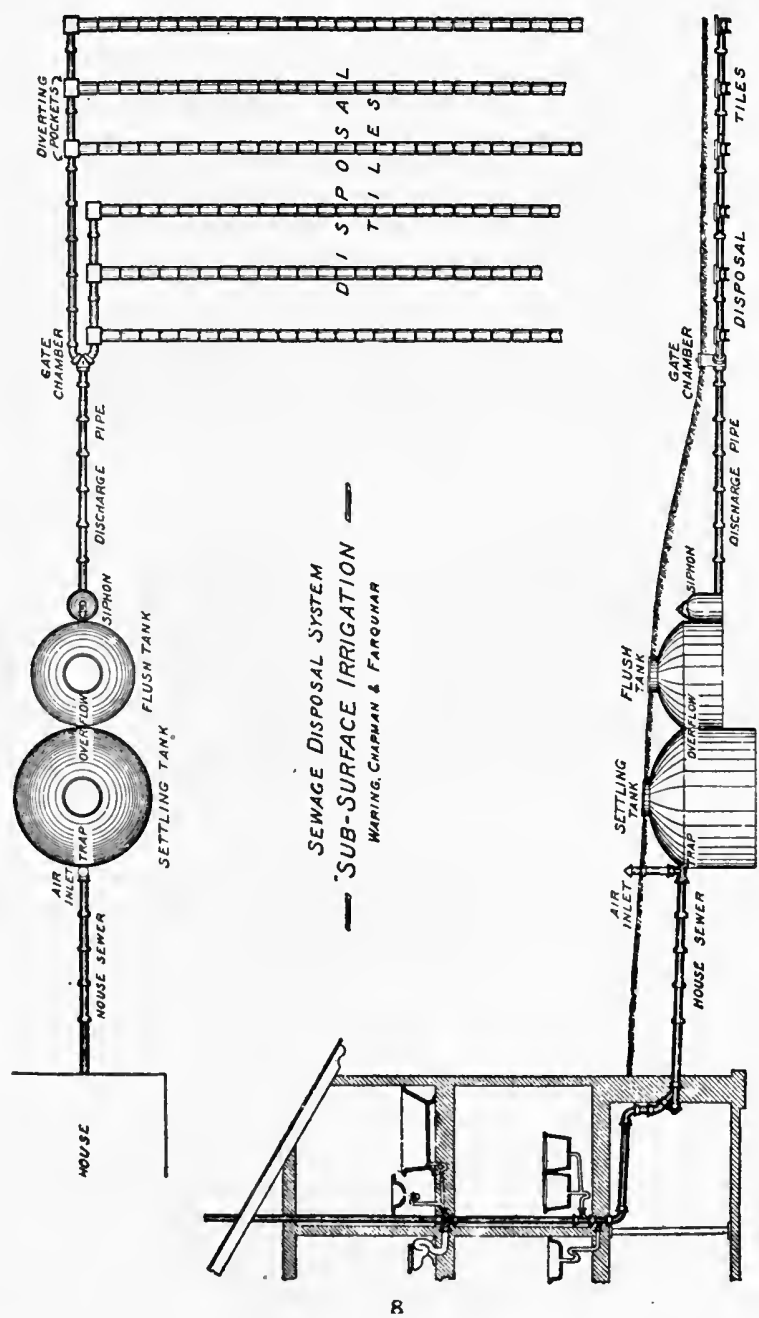

FIG, 181.-Sub-surface irrigation. Waring system. 
siphon into the outlet. The small pipe $A$, intended to relieve the pressure of gases, is of doubtful utility.

Sub-Surface Irrigation. - For small institutions, country houses, military posts or camps, not exceeding roo persons, the Waring system of sub-surface irrigation is available (Fig. 181). It consists of : I, two masonry tanks, under ground, either circular or rectangular, arched over and fitted with manhole covers, the larger for the settling and liquefying of the organic solids, the smaller or flush-tank, to receive and hold the liquefied sewage until the required quantity has accumulated, and then discharge it by means of an automatic siphon; 2, a gate chamber to permit diverting the discharged sewage from one place of disposal to another; 3 , lines of open-jointed disposal tiles to distribute the sewage into the soil surrounding them, below the surface of the ground.

The sewer is trapped where it enters the tank and ventilated by a pipe extending above ground. The tank itself is not ventilated. The discharge by the siphon being intermittent and uniform, the soil never receives more sewage than it is capable of absorbing and oxidizing. The disposal tiles are as near the surface as protection from breakage will permit, so as to favor the bacterial work of nitrification; experience having proved that even the severe winter weather of New England has no appreciable injurious effects upon their efficiency.

Without departing from the principles upon which this system is based, simple modifications applicable to various conditions will suggest themselves. For instance, where the amount of sewage is relatively small, one tank will probably answer the purpose. A simple and cheap improvisation would consist of a barrel as settling tank, a half barrel as flush tank with pipe curved into a siphon for the intermittent discharge of the sewage into a line of sub-surface tiles. The siphon is not essential and, where the tiles are ample, can be dispensed with.

the luMSDEN, Roberts AND stiles SANitary Privy. (Public Health Service.)

This type of the "wet system" admits of ready improvisation and is principally intended for rural households (Fig. I82). It consists of : a water-tight tank or barrel as liquefier; a smaller barrel, can or kettle to receive the effluent; a connecting pipe of which 
the end dipping in the tank is covered with wire gauze; a tight box set on the tank, with opening on top for the seat, and self-closing lid; an anti-splashing device consisting of a small board placed horizontally about an inch below the surface of the water, and which can be raised and lowered by means of a rod.

The liquefying tank is filled with water up to the point where it begins to trickle into the effluent receptacle, through the connecting pipe. The fecal matter falls into the water but the board prevents splashing and thus meets one of the greatest objections raised against the wet system. After defecation the board is depressed to let the fecal matter float free in the water where it undergoes a certain degree of fermentation and liquefaction. The effluent can be used as fertilizer, but if suspected of infection by pathogenic germs, should previously be boiled or mixed with chloride of lime,

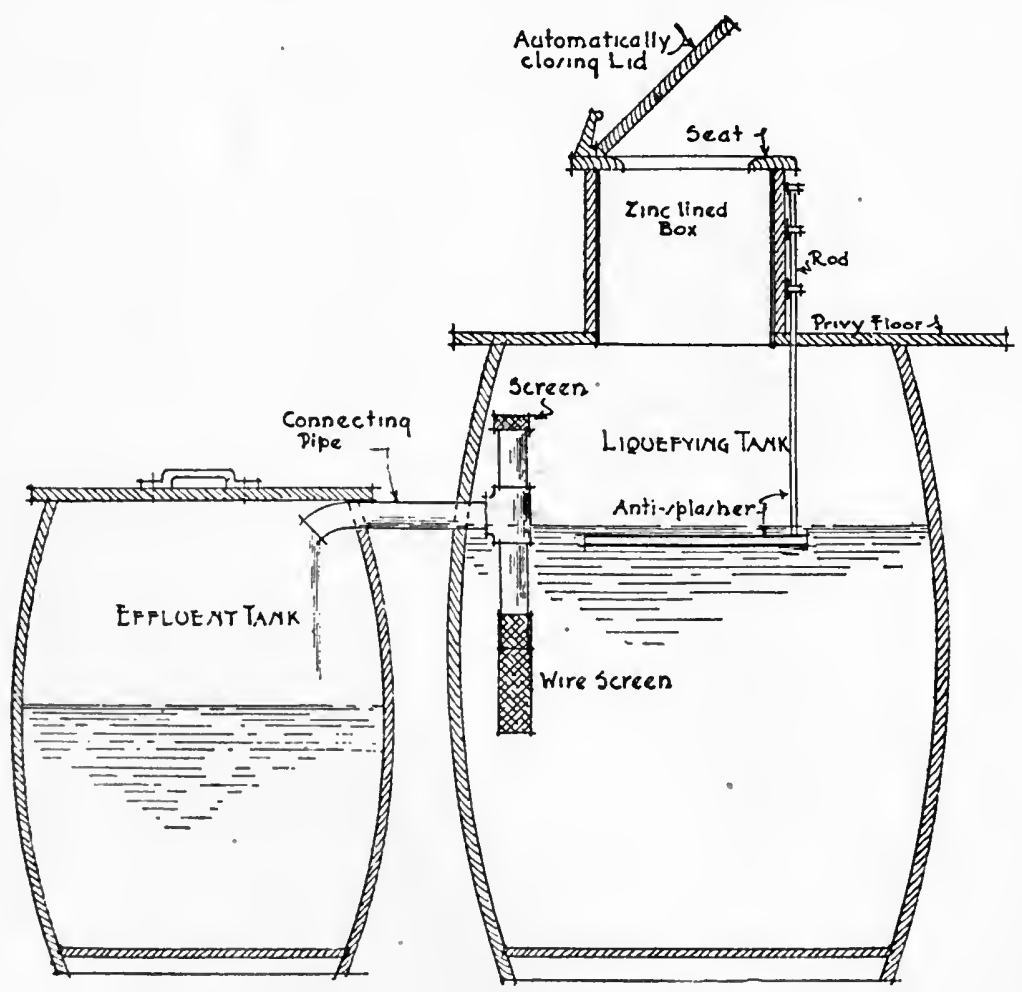

FIG. I82,- Improved L. R. S. privy. Public Health Service. 
one-fourth of a pound to the gallon of liquid, or again may be cairied away by an underground drain as in the preceding system.

The tank may be constructed of sufficient length to accommodate two or more seats, each seat with its corresponding splashing board.

This privy should be in a fly-proof and well-ventilated shed, and, when properly operated, is practically odorless.

Whenever a satisfactory system of sewerage is impracticable at a post or cantonment, for instance where the weather is too cold for water-closets, one of the methods of disposal described under Camps may be used, especially the earth-closet, milk-of-lime closet or, still better, one of the incinerators.

\section{DISPOSAL OF GARBAGE.}

Garbage and all waste material, to be properly disposed of, at posts as well as in cities, must be sorted and divided according to their composition. Kitchen wastes, for instance, which contdin a large proportion of putrescible and dangerous organic matter, should be separated from dry refuse and ashes. At least two cans are required, but three are still better, one for kitchen and liquid wastes, a second for ashes and other dry non-combustible matter, and a third for combustible refuse. The receptacles should be of galvanized iron, air-tight and fitted with lids to exclude flies and prevent odors. Moisture is the immediate cause of the fermenting and smell of garbage, therefore the drier the latter is the longer it can be kept without being a nuisance. An excellent device where kitchen garbage cannot be emptied every day is to drain it and then wrap it up in paper before putting it in the can. In this state it does not smell bad in hot weather, nor freeze and stick to the can in cold weather; the can remains clean and need not be emptied more than once or twice a week.

In cities, at least three-fourtlis of the dry refuse has a salable value. From the kitchen garbage a notable proportion of oil can be obtained, while the residue is utilized as fuel. At military posts, such economic disposal is impracticable. The rule has been, in the absence of crematories, to feed the kitchen garbage to pigs or pour it into pits, and to throw all other refuse and wastes on the dump. The latter is incinerated so far as it is practicable, generally very imperfectly. 
Such primitive method should no longer be countenanced at any of our garrisons or camps. The only proper way to dispose of garbage and all other combustible refuse is by fire, and it is always possible, in all places, to improvise an efficient " rock pile crematory" (see page 672). Most of our large posts are now supplied with regular grate crematories. These are of various kinds. It is generally stipulated that they shall be of a capacity of not less than two tons per hour, or sixteen tons per day of eight hours, and so constructed as to consume manure, garbage and liquids; in other words, all wastes. It is especially necessary to fire empty tin cans; they always contain organic matter adhering to their walls and are favorable media for the multiplication of all sorts of bacteria until thoroughly burned out.

One of the latest and best types of crematories is illustrated in Figs. 183 and 184 . The building is of wood, covered on sides and roof with corrugated sheet steel, and resting on brick or stone walls and piers with concrete footings; the floor is of concrete. The doors, of corrugated sheet steel, are of the rolling pattern. When this building is erected upon a side-hill the runway and platform are omitted, but a longitudinal stone retaining wall is necessary.

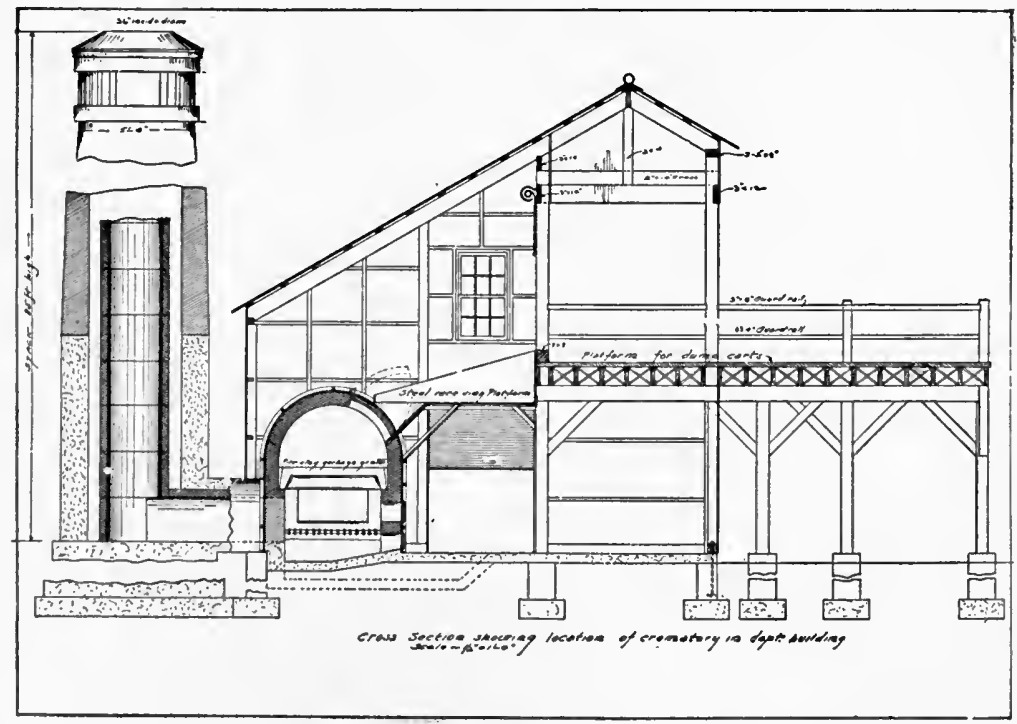

FIG. 183.- Crematory building - Fort D. A. Russell, Wyo. 
The apparatus (Fig. I84) consists essentially of a fire-clay garbage platform above, and of five cast-iron fire grates below, namely, two on each side for incineration and a central one to strengthen the draft and complete the combustion of gases. The garbage beng spread upon the platform, fires are started in the grates A A and C. and the damper $\mathrm{D}$ closed. The heat and smoke current passes through and over the garbage and then under the platform on its way to the smokestack. The garbage, being sufficiently dried on the left half of the platform, is thrown upon the grates A A to serve as fuel. When the garbage on that end of the platform has been disposed of, the damper $\mathrm{D}$ is opened and damper $\mathrm{E}$ closed, and fires started in the grates $B$, thus reversing the heat current and repeating the same process on the right end of the platform; fresh garbage being dumped on meanwhile so as to keep the platform always supplied. 


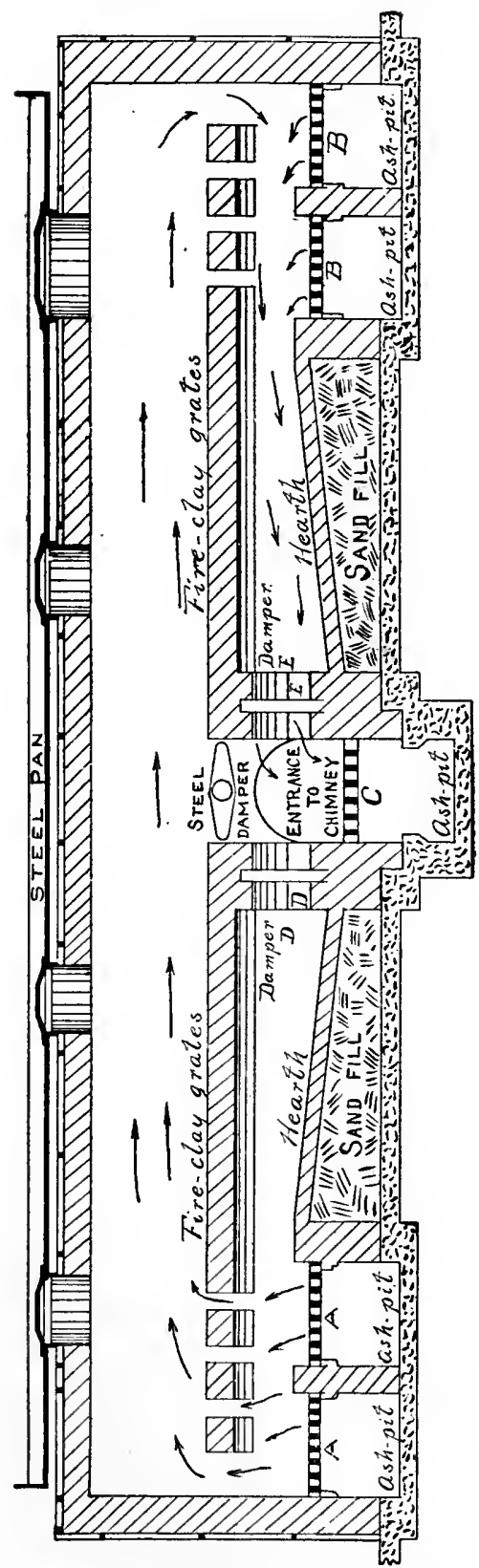

Fig. 184.-Crematory grates-Fort D. A. Russell, Wyo. 


\section{CHAPTER XLVIII.}

\section{SOIL.}

Soil is a mixture of mineral substances, chiefly sand, clay and limestone, with more or less organic matter. Its principal mineral constituents are: silica (quartz, sand, sandstone, etc.) which forms more than half of the earth's crust; aluminum, mostly as clay (silicate of aluminum); lime as carbonate (limestone), phosphate and sulphate; and magnesia as carbonate and sulphate. Other constant but secondary constituents are; iron and manganese, the former playing a very important part in the growth of plants; chlorine as chloride of sodium and potassium; sulphur as sulphides and sulphates; phosphorus as phosphates.

The vegetable mould generally present in the soil and forming the bulk of its organic matter, is called humus, a very complex substance particularly rich in nitrogen and therefore characteristic of fertile land. Peat and muck result from the incomplete decay of vegetable matter under water, the former being more compact and fibrous than the latter. Nitrogen exists in the organic matter not yet decomposed, as well as in the products of its decomposition, namely ammonia and its salts, nitrites and nitrates.

\section{PHYSICAL PROPERTIES OF SOILS.}

All soils are porous to air. This porosity does not depend so much upon the size of the individual particles of earth as upon their shape and arrangement. The total volume of the interstices or pores between the grains, amounts to about 35 per cent. of the entire mass in ordinary soils; even the most compact rocks have more or less porosity although impervious to water. The permeability of soils to air is not necessarily proportional to their porosity, but depends chiefly upon the size of the pores; as these grow larger, permeability increases, although the volume of porosity tends to decrease; thus the permeability of medium gravel is more than Ioo times greater than that of medium sand although its total porosity is less. Clay, consisting of exceedingly fine adhesive particles, possesses but little permeability, unless fissured or crumbly 
or mixed with sand or chalk. The degree of permeability of any soil to air is greatly influenced by the amount of contained moisture ; water drives out the air and may render the soil, especially if largely composed of clay or peat, completely impervious to it.

Ground Air. - Ground air differs from atmospheric air in its much greater proportion of carbon dioxid; it is also somewhat richer in ammonia and occasionally contains sulphureted and carbureted hydrogen. All these gases result from the oxidation of the organic matter in the soil where, therefore, the air suffers a corresponding loss of oxygen. The amount of carbon dioxid varies much according to the nature of the ground and the depth from the surface. A soil rich in organic matter evolves a great deal more of it than if mainly composed of sand or chalk; likewise, more is generated in summer, when decomposition is active, than in cold weather. At a depth of one meter the amount of carbon dioxid is seldom less than 5 parts per 1,000 of air and may reach 15 or 20 parts, while at a depth of 4 meters it is two or three times as much. Ground air is not immobilized in the soil; on the contrary, owing to differences of temperature between it and the atmospheric air, winds, rise and fall of subsoil water, etc., it is always in motion, constantly displaced, renewed and mixed with outside air, so that the atmospheric layers nearest the ground are always more or less contaminated with it. The teaching of Pettenkofer that the amount of carbon dioxid in any part of the soil is an index of the amount of organic matter present is obviously erroneous, since, on account of the permeability and ventilation of the soil, the gas found in one place may easily have been generated at some other place.

When the surface is impermeable, as by freezing, or when cemented, tiled or macadamized, the ground gases may traverse long lateral distances; in this way air from cesspools, broken drains, gas, buried garbage, etc., may be drawn into cellars and escape, by upward draft of air, into heated dwellings.

Ground Moisture. - Water is retained in the ground by two forces: molecular adhesion, that is, the attraction of the surfaces of soil grains for liquids, and capillary attraction. The latter force is active only when the pores or interstices are sufficiently small (less than a millimeter). This capillary water forms the great bulk of the retained moisture; it is the more abundant that the texture of the ground is closer and more compact; thus a soil with grains 
half a millimeter in diameter will hold nearly three times as much water as one with grains $2 \mathrm{~mm}$. in diameter. Sand, with its relatively large pores, absorbs but little water, chalks absorbs a good deal more, while clay retains from 70 to 80 per cent. of its weight. Organic matter is very absorbent of water, humus being capable of retaining several times its weight of it. Permeability of the ground to water, as to air, depends upon the size of the pores, and therefore is generally inversely proportional to capillarity or the capacity of water retention. Thus sand is freely permeable, that is, permits a rapid percolation of water; chalk is less so, while clay may be totally impermeable. Even marls (mixtures of chalk and clay) not containing more than 40 or 50 per cent. of clay, are sometimes quite impervious. Rocks vary greatly in this respect, those of volcanic origin being absolutely impervious, while sandstones and limestones are quite porous and generally allow more or less filtration.

Rain-water percolates through the soil and subsoil until it reaches an impermeable stratum, either clay or rock, upon which it collects to a variable depth. This ground water furnishes more or less moisture to the subsoil through capillary attraction and much of it is evaporated through the soil. It is in constant motion, the direction of the flow depending necessarily upon the slope of the impermeable stratum below, but this slope cannot always be inferred from that of the soil surface. The trend of this ground water may generally be assumed to be toward the nearest watercourse below it, but often the only way to ascertain it, as well as the depth of the water level, will be by the digging of a series of wells. The rise and fall of the ground water depends chiefly upon the rainfall, but it is materially influenced by the extent of vegetation, especially woodlands, the rate of evaporation, and the amount drawn for the supply of neighboring communities.

The effects of constant humidity upon the human organism are decidedly pernicious, probably through its interference with the functions of the skin and the evaporation from the lungs. Long ago, Dr. H. I. Bowditch stated that " A residence on or near a damp soil is one of the primal causes of consumption in Massachusetts, probably in New England."

Ground Temperature. - The soil being a bad conductor of heat, changes in the temperature of the air are less, and more slowly, felt 
a few feet, or even a few inches, beneath its surface; thus a cave, cellar or dugout is cooler in summer and warmer in winter than the outside air. The power of the soil to absorb solar heat depends mostly upon color, black absorbing much more than lighter shades, and white sand least. The power of emission or radiation is proportional to that of absorption, thus dark soil loses more heat at night than chalk or sand. Snow, being also a poor heat conductor, protects winter crops from extreme cold. A hole dug in a snow drift, or shelter built of snow, may save soldiers or travelers from death by congelation. The specific heat of water being about four times that of air and of most mineral substances, it follows that water is not so readily affected by heat and maintains a much more equable temperature than air or soil; moist soil, therefore, absorbs heat more slowly than dry soil and its temperature does not rise to the same degree. For instance, the heat that would raise the temperature of sand $6.67^{\circ} \mathrm{F}$, and of weathered granite $3.33^{\circ}$, will only raise that of water one degree. It is chiefly by its evaporation, however, that moisture lowers the ground temperature, for this evaporation is always at the expense of the heat of contiguous solid substances.

In practice, the color of soils does not vary sufficiently to make much difference in heat absorption. The warming of the soil, as above noted, depends chiefly upon the amount of moisture it retains. Thus a sandy, well-drained soil, although light in color will warm much faster than a wet and heavy soil, although dark in color. This is to be remembered in the selection of camping grounds.

Influence of Vegetation on Soil. - Plants, but especially trees whose enormous system of roots extends many yards in all directions, absorb large amounts of moisture and tend to dry a damp soil; thus most trees evaporate through their foliage a total of moisture several times greater than the amount of rain and snow falling upon the area they cover; hence the wisdom of planting quick-growing trees with abundant foliage in marshy lands, and to keep these clear of underbrush. On the other hand, woodlands have a marked effect in retaining rain-water and regulating its penetration of the soil. The leaves become so many receptacles from which the raindrops, forming tiny streamlets, flow down the stalks and branches to the trunk, and continute to reach the ground long after the rain has ceased, thus preventing any sudden flooding. But 
the water is also entangled and arrested by the compost of vegetable matter, together with the litter of foliage and twigs which cover the forest soil, and therefore more of it penetrates the soil and is utilized. The remainder runs out in brooklets which keep the outflowing streams evenly and constantly fed. It has not been proved that the removal of forests materially decreases the rainfall in any region, but it is certain that it brings about the loss of a large proportion of it; the water, not being arrested by the foliage nor retained by the soil compost or litter, rushes off into the nearest water-courses, causing sudden freshets and destructive torrents. Vegetation tends to equalize the temperature of the soil, absorbing less heat during the day and retaining more during the night. "Herbage acts as a protection against excessive heating in hot climates, and as a blanket to prevent loss of heat in cold ones" (Harrington).

POLLUTION AND SOIL BACTERIA.

The soil is the great receptacle in which all the organic filth not directly carried to the ocean is deposited, and therefore would soon become hopelessly polluted were it not for the provisions made by nature to maintain a safe equilibrium between dead organic matter and living animals and plants. Nature's scavengers are the microorganisms or bacteria of the soil. The ground is full of them, from several thousands to several millions per cubic centimeter. As they feed and multiply on organic matter, the more there is of it, that is the richer is the soil, the more abundant they are. Other favorable conditions to their growth are a certain degree of heat and some moisture. These bacteria, as already stated, are found mostly in the upper layers, especially in the first four feet, below which they diminish rapidly so that few are found beyond a depth of Io or 12 feet. These soil microbes consist mainly of beneficent aërobic saprophytes. Their chief and most important function is the decomposition of all dead organic matter, animal and vegetable, and its transformation into simple, assimilable elements which are again available for growing animals and plants. Among them may be noted the "nitrifying" and "denitrifying" bacteria, the former bringing about an oxidation of ammonia into nitrites and nitrates, while the latter decompose nitrogenous matter into its constituent elements. Another useful office which they perform, through their 
competition and greater power of resistance, is the prevention of the growth and multiplication of pathogenic bacteria. The latter are more delicate organisms; only four are commonly found in the earth and able to perpetuate themselves, the bacilli of tetanus, of anthrax, of malignant edema, and of gas gangrene; the others are generally incapable of enduring long the unfavorable conditions of the soil and the antagonism of the saprophytes. Thus Klein found that very few of the bacteria of cholera, typhoid fever, diphtheria, plague and tuberculosis are found in the bodies of animals, dead from these diseases, a month after their burial. It has been observed that pathogenic bacteria are very quickly destroyed in peaty soils, doubtless on account of the organic acids which the latter contain.

With plenty of air the aërobic saprophytes cause the prompt decomposition of organic matter with little or no offensive odor; but in compact, wet or impermeable soil, anaërobic bacteria, the organisms of putrefaction, come into play and ill-smelling gases are evolved. This, however, is mitigated or entirely neutralized by the remarkable absorbent power of earth for gases and vapors, the gaseotts atoms penetrating into the intra-molecular spaces of the soil elements and condensing therein. This power varies according to the composition of soils; sand has but a feeble attraction for gases, while humus or any soil rich in organic matter, if not too wet, is very absorbent. This affinity is particularly marked for odorous gases; thus it has been observed that ordinary illuminating gas, in passing through a layer of earth, may lose its characteristic odor but not its poisonous constituents which thus become the more dangerous that their presence is unrevealed to the sense of smell. 


\section{CHAPTER XLIX.}

\section{CAMPS.}

Camps vary greatly in their composition, size, form, location and purpose, and, although the principles underlying their sanitation are always the same, the sanitary measures called for will vary correspondingly. There are the summer camps of peace time for field instruction and maneuvers, with many of the comforts of garrison life; camps of mobilization, at the beginning of war, where troops are mustered and organized, after being stripped of superfluities. and the camps of actual warfare, necessarily reduced to the indispensable. In presence of the enemy, before or after a battle, while marching and maneuvering for position, the troops may have to camp many nights on ground which is objectionable from the sanitary point of view. They remain in deployed formation and will generally bivouac on or near the positions they occupy. In this formation they are drawn out into a thin line and therefore not exposed to the dangers attendant upon crowding. Bivouacs, especially if shelter-tents are not available or cannot be put up, and the weather is inclement, expose the troops to severe and mostly unavoidable hardships. In the absence of tents, protection from the wind may be obtained by throwing up an earth wall, three feet high, the earth being taken from the outside to secure exterior drainage and an undisturbed surface to rest upon (Woodhull). When a choice is possible. the principles which guide in the selection of camp sites will apply to the selection of places for bivouacs. According to Lord Wolseley, sanitary reasons should always be considered in the choice of a camp when contact with the enemy is not expected within 48 hours. Every shelter should be ntilized. Woodlands are always desirable on account of the shelter, fuel and material they afford. When troops are likely to remain days and weeks along the line of fire, confronting the enemy, they should make themselves as comfortable as conditions permit, in trenches and dugouts, or in tents, and carefully observe all sanitary measures applicable to their situation.

Selection of Site. - The Field Service Regulations provide that 
"when practicable, a medical officer assists in the selection of camp sites." When there are no tactical questions involved and the camp is to be occupied for some time, sanitary considerations should prevail and great responsibility will rest upon the medical officer concerned in the recommendation of a suitable site.

When in the enemy's territory, careful information must be obtained from the inhabitants regarding the prevailing diseases of man and animals in the zone to be occupied, especially in the proximity of the camp grounds, as well as the quality of the water-supply and the character of the food to be procured from local sources.

The grounds should be large enough to accommodate the command without crowding. The spread of infectious diseases in camp is always to be apprehended, being favored by the concentration of large numbers of men, most of them at a very susceptible age, under hygienic conditions seldom entirely satisfactory. To guard against it, individual men must be given as much space as possible within their respective organizations, and the organizations placed as far apart as topographical conditions and military exigencies permit. A brigade of 4.000 or 5.000 men is as large a command as should be placed in one camp. The site should be high enough to secure dry soil and good natural drainage; the summit of a low ridge with gentle slopes, a high plateau with slight declivity, or the high bank of a river, are very desirable. It is quite important that the location of a camp be such that its drainage shall not pollute the grounds of the camp next below it; if such location cannot be avoided, a large ditch should be dug between them. In cold weather, a slope to the south, with woods to break the force of the wind is an advantage. In hot weather, high grounds, swept by the breeze and, if possible, shaded by trees, should be preferred. Grounds near the foot of a hill are exposed to flooding in rainy weather and remain muddy and wet long afterwards; unless protected by a deep ravine, they should be avoided.

The vicinity of marshes and stagnant waters is objectionable on account of the annoyance and danger of mosquitoes; it is also undesirable on account of the damp atmosphere and possible air pollution from $\mathrm{CO}_{2}$, sulphureted hydrogen, marsh-gas, ammonia and other products of organic decomposition. Although marsh water may be very foul, the vapor rising from it by evaporation is always entirely free from micro-organisms and therefore incapable of conveying any infectious disease. 
An old camp site should not again be used by a command until sufficient time has elapsed for the disinfecting action of the air, sun and rain; this period of time will vary according to the extent of the pollution, especially of the amount of fecal matter left in the soil or subsoil. As a rule, in dry weather, a period of two or three months will remove all danger of soil infection.

A camp site must be dry. A humid atmosphere makes the heat more oppressive and the cold more penetrating. Moisture renders the soil cold and chilly, depresses the body vitality and reduces resistance to disease, predisposing to rheumatism, neuralgia, diarrhea, tuberculosis and malarial fever. The degree of dryness of a site can be estimated from the general topography of the surrounding country, its elevation above the nearest ponds and streams, the character of the vegetation and, more surely, from the depth of the ground water as determined by digging. The ground water, as a rule, should not be nearer than Io or 12 feet, but a depth of 6 to 8 feet is compatible with a dry surface when the drainage is good and the subsoil porous.

Soil. - The soil and subsoil of a camp must be porous and permeable, that is, allow rain-water to sink readily through them. Gravel and sand are excellent for the purpose. Loams (mixtures of sand, clay and organic matter), although not so good are generally satisfactory. The most unhealthful constituent of the soil is clay, for although it absorbs and holds much moisture it is totally impermeable; whether forming the soil or subsoil it retains the surface water which only partially disappears by slow evaporation. Chalk, limestone and sandstone are porous and health ful, but chalk mixed with clay in about equal proportion (marl) becomes impermeable. Volcanic rocks, such as granite, trap, gneiss, etc., except when seamed and fissured, are impervious; but as they absorb little water and dry quickly they make excellent sites, provided there is enough slope for good drainage.

As already stated under Soil, sand allows water to pass through it while retaining very little of it. If white or light colored, it does not absorb much heat during the day and loses but little at night; in other words, the power of heat absorption and radiation of white surfaces is small. As a rule, sand does not contain much organic matter and therefore does not favor the development of pathogenic microbes. These are all good reasons for preferring sand as camp 
soil; but, if nearly pure, it is likely to be soft, yielding and heavy to the foot of man and beast; while, if white, the glare of its surface is trying to the eye; it is best that it should be mixed with a certain proportion of gravel to give it consistency and firmness as well as a neutral color.

Vegetation. - Vegetation on a camp site is highly desirable, but thick undergrowth which excludes the sun and keeps the ground moist must be cleared out. Trees and shrubs should be saved as far as possible so long as they do not seriously interfere with drills and formations. They afford protection from sun and wind and modify the extremes of temperature, cooling the summer heat and tempering the winter cold. Grass is most useful on camp grounds and should be preserved and protected. It mitigates the heating of the soil by day and its chilling by night; does not reflect light and heat, prevents mud and dust, as well as the washing and guttering of the soil by rain.

The effect of vegetation upon mosquitoes and other insects is variable. It has no special attraction for them nor, in the absence of stagnant water, does it afford them breeding places, for it is a well-known fact that mosquito eggs are only laid in water or muddy places. But vegetation affords them shelter during their flights in search of water, or when blown by the breeze, and may thus retain them in the vicinity of habitations. Therefore, in a malarial country it is well, in camps and near dwellings, to have the trees thinned out, no undergrowth, and the grass closely cut. On the other hand, a screen of wood between mosquito-breeding waters and the camp grounds will stop many of these insects and be distinctly advantageous.

The location of a camp will be especially determined by the watersupply which should be abundant, of good quality and conveniently accessible. Grass or hay, as well as wood, are also essential, but can be brought from more distant points.

Poisonous Plants. - There are but few poisonous plants in this country likely to trouble soldiers in camp or on the march. The worst belong to the genus Rhus or Sumach. Rhus toxicodcndron (Poison Ivy or Poison Oak), is found from Canada to Mexico and west to Arizona, Oregon and Pritish Columbia, under various forms ranging from a small upright shrub to a high thrifty climber; easily recognized by its trifoliate leaves and ovate pointed leaflets, one 
of the very few shrubs or woody vines with three leaflets. In California it is replaced by Rhus diversiloba which differs only by the obtuse or rounded apex of the leaflets. Both species are very poisonous to susceptible people and should, so far as practicable, be eradicated from camp grounds. Rhus vernix or Poison Sumach, of the wooded swamps of the Eastern and Middle States, a large upright shrub or small tree, having long pinnate leaves with 7 to 13 entire leaflets, although likewise very toxic, is not common enough to be much of a menace to our camps.

In the West Indies the closely allied genus Comocladia takes the place of Rhus. The species are all shrubs or small trees with compound leaves and stiff leaflets armed with spiny teeth. The best known and perhaps most poisonous is $C$. dentata, the Guao of Cuba, and Carrasco of Porto Rico, a slender upright shrub bearing a whorl of shiny leaves near the top.

The poisoning caused by these species of Rhus and Comocladia, on susceptible persons, is in the form of a more or less severe dermatitis. It has been shown that the toxic principle is an ambercolored, non-volatile liquid resin, so that actual contact,. direct or indirect, with some part of the plant is necessary to produce it. The sticky sap, exhuding from all parts of an injured plant, comes readily in contact with hands or other exposed portions of the body, or may be carried on clothing, furs of animals or various objects. From the hands it is usually conveyed to the face. Poisoning may also result from exposure to smoke from burning stems. The pollen and hairs of the plant do not contain the toxic substance and are therefore harmless. Insects may carry it but probably in too small quantity to do much harm.

Certain peculiarities distinguish dermatitis venenata from eczema and herpes. It frequently begins between the fingers, is acute in character, with swelling and often large vesicles and blebs; occurs in sharply defined patches, elongated streaks and other irregular shapes corresponding with the original area of contact. It seldom attacks the scalp or the inside of the hands.

Since the poison is soluble in alcohol and in alkalies, the rational treatment consists in removing as much of the toxic resin as possible by scrubbing the parts with soapsuds and washing them freely with alcohol. In the absence of alcohol, gasoline may be used. Afterwards, a 2 per cent. solution of potassium permanganate (if 
temporary discoloration of skin is not objectionable), or a 5 per cent. solution of bromin in glycerin, is recommended.

Nearly all the plants of the Spurge Family (Eupliorbiacex) contain a milky juice more or less acrid and irritating to the skin. Some produce skin eruptions through their stinging hairs. One of the worst members of the family is the Manchineel or Manzanillo (Hippomane mancinclla) of the keys of Florida, West Indies and Central America, a medium tree with smooth bark and ovate, finely serrate leaves, growing on seacoast and along river banks. It secretes an abundant white juice extremely caustic. A few drops internally cause drastic discharges, and, in the eye, may produce blindness. The fruit is a small, attractive, pleasant-smelling apple, but so acrid that no one is ever tempted to take a second bite.

Another notable euphorb of the West Indies is the Pinion Botija of Cuba, and Tartago of Porto Rico (Jatropha curcas), a small tree with palmately-lobed leaves, pale yellow flowers and greenish plum-like fruit drying black and containing three large bluish seeds. These have a sweet, pleasant taste and exactly the effect of castor oil; they are an excellent cathartic in the dose of one or two.

Many of the fruits and seeds of the plants of the Nightshade Family are more or less dangerous. Careless persons are sometimes poisoned by eating the seeds of Datura (Jimson weed), of which we have several kinds, or the berries of various species of Solanum.

The Poison. Hemlock of Europe (Conium maculatum) has also become common in this country. Cases of poisoning arise from mistaking the seeds for those of anise, the leaves for parsley and the roots for parsnip. The danger from Water Hemlock (Cicuta maculata and others) is from the roots, often washed out of the soil, exposed to view, and very poisonous.

Mushrooms. - Regarding mushrooms, a good rule for soldiers is never to eat them, except after inspection by an authorized expert.

The common edible mushroom (Agaricus campcstris) has a convex, expanded cap, smooth or oftener silky-hairy, usually white but varying to light brown. The gills are first white but very soon become pink and later purple-brown or dark brown from the numerons colored spores on their surface; they are free from the stem and rounded at the inner ends. The veil (or membrane covering 
the gills in the young plant) is thin, silky and very frail; as the cap expands it is torn irregularly and clings as a thin, ragged collar around the stem, while fragments remain dangling from the margin of the cap. The stem is white, nearly cylindrical or slightly tapering at the lower end. This mushroom grows mostly in open places, such as lawns and pastures.

Very much like it, is another highly esteemed edible species (Lepiota naucina), found at the same time and in the same places. It is chiefly distinguished by its gills which remain white, only becoming pinkish when very old. The cap is very smooth and becomes strongly convex. The veil separates cleanly from the stem as well as from the margin of the cap. The stem is slightly enlarged or bulbous below.

Many other edible species will be found described in special treatises on the subject.

In collecting mushrooms for the table, W. H. Gibson's advice is excellent: "Avoid every mushroom having a cup, or suggestion of such, at its base; the distinctly fatal poisonous kinds are thus excluded. Reject those having an unpleasant odor, a peppery, bitter or other unpalatable flavor, or tough consistency."

The two most common of the deadly mushrooms are the Amanitas, both characterized by having the stem resting in a cup-like bulbous base.

A. phalloides is probably responsible for a majority of deaths from eating mushrooms. It is white in all its parts, with smooth, viscid cap. Generally occurs in woods, but rarely also in open fields.

A. muscaria is distinguished by its bright orange or red cap covered with soft, whitish warts, and its white gills and stem.

Of the poisonous principles of mushrooms, the alkaloid muscarine is probably the most potent. They do not act until several hours after the ingestion of the plant. The treatment consists in producing emesis and then catharsis, and in the administration of atropine as a physiological antidote.

Venomous Animals. - Since soldiers, in camping and marching, are particularly exposed to the bites of poisonous animals, a brief description of them may be useful.

Snakes are the best known and most dangerous. Poisonous snakes are characterized by a movable poison fang on each side of the anterior end of the upper jaw, and a pit between the eye and 
the nostril. The coral snake differs from the other poisonous kinds in having the fangs permanently erect, and no pit. As the snake bites, the pressure of the temporal muscle upon the poison gland forces its contents, along a fine canal, through the fang into the wound. If a bite consists of two isolated punctures (sometimes only one visible), it is almost certain to be from a poisonous snake, and the distance between the punctures gives an indication of the size of the animal and the probable amount of poison injected.

In the United States, the poisonous snakes belong to four distinct genera, as follows:

Coral snake or Harlequin Snake (Elaps fulvius), with small, cylindrical body marked by bright red, black and yellow rings, indistinct head and short tail. Inhabits the Southern and Southwestern States. Feebly poisonous. Another species is found in northern Mexican deserts and probably in Arizona.

Copperhead (Agkistrodon contortrix), of light chestnut color, with Y-shaped, darker blotches on sides, seldom exceeding 3 feet in length, is widely distributed from Mass. to Fla., Ind., I1l., Tex. and Ind. Ty. A more aggressive animal than rattlesnakes, but its poison much less virulent. According to Prentiss Wilson, at least one-fifth of all cases of snake bites in this country are from the Copperhead. Out of 99 cases, 5 fatalities were recorded, but only one was properly attributable to the venom. Therefore severe treatment is seldom called for.

Water Mocassin or Cottonmouth (Agkistrodon piscivorus), of dark chestnut-brown color with darker markings, and white mouth, ranges from N. Ca. to Fla., Tex., Mexico, the Mississippi River and its lower tributaries. Viciously aggressive but its poison even less virulent than that of the Copperhead. Being much heavier than the latter, often exceeding 4 feet in length, it is capable of injecting a larger dose of poison. Records of its bite are rare.

Rattlesnakes, characterized by the horny rings which form a rattle at the tail end. They have been divided into 2 genera: Sistrurus, with top of head covered by a few regular shields, and Crotalus, with top of head covered by numerous scales. The Sistrurus or Ground Rattlesnakes, of which 2 or 3 species are known, are but feebly poisonous.

Of the I7 species of Crotalus, not less than Io are found in the United States, mosî of them extending into Mexico. The other 
species are found in Mexico, Central and South America. None occur in the West Indies. In southern Arizona alone, 7 species are found, probably all extending into Mexico.

Our best known and most dangerous species are:

Dog-faced Rattlesnake (C. molossis), of a general sulphur color, occurs in the States bordering upon the Mexican frontier.

Banded R. (C. horridus), of sulphur color with series of subrhomboidal markings, formerly common all over the Eastern States and still found in New England, but oftener in the Alleghany Mountains.

Diamond R. ( $C$. adamanteus), of general yellowish-gray color. with rhomboidal black blotches, ranges from N. Ca. to Fla. and west to the Mississippi. Often found in water, hence its common name of "water rattler." Our largest species, sometimes 8 feet long, and perhaps the most dangerous.

Texas R. (C. atrox), of general yellowish-gray color, with series of subhexagonal rings from head to tail, inhabits the arid regions of the Southwest.

Prairie R. (C. confluentus), of general yellowish-brown color, with subquadrate to oval dark blotches, ranges between the Missouri River and the Rocky Mountains.

Horned R. (C. cerastes), with projection over the upper jaw, is found in Ariz., Cal. and Sonora.

Pacific R. (C. lucifer), the common rattler of the Pacific coast.

Two other species of the pit-viper or rattlesnake family, with tail ending into a long horny spine instead of a rattle, deserve mention: the Bushmaster (Lachesis mutus) of tropical South America, sometimes 12 feet long, and the most dangerous snake of the New World; the Fer-de-Lance, Lance-headed or Yellow Viper (Bothrops lanceolatus), about 6 feet long, which infests the sugar plantations of the Martinique and other West India Islands.

Cuba and Porto Rico are free from poisonous snakes. The Philippines contain several of them including the deadly cobra ( $N a j a)$, but are nowhere common nor aggressive, and records of deaths from their bites are rare. The best known is the Rice Snake (Dehen Palay') greatly feared of the natives.

The danger of a snake bite depends not only upon the species and the virulence of its venom, but also upon the size of the animal, the more or less successful introduction of one or both fangs, the part struck. whether a vein was entered or not, and the power of resist- 
ance of the person struck. The poison gland may also be emptied, or partly so, from a previous bite. It follows that the effects of bites are quite variable, sometimes hardly perceptible, at other times fulminant.

Dr. Charles B. Penrose, after careful investigations and experiments at the Philadelphia Zoological Garden, recommends the following treatment:

"Ligate the part above the bite with rubber ligature. If the bite is on the finger, use the small ligature on the finger, and put the large ligature on the forearm, or above the elbow.

"Make one or two deep incisions through both fang punctures. The incisions should be $1 / 2$ inch long and $1 / 4$ to $1 / 2$ inch deep, and should bleed freely.

"Suck the wounds. It is best to spit out the blood, though it is not dangerous for adults to swallow snake venom.

"Wash the wound with a freshly prepared solution of chloride of lime, I part of chloride of lime to 60 parts of water. Inject with a hypodermic syringe the chloride of lime solution in the tissues around the wound-chiefly above the wound."

Hypodermic injections of strychnine, repeated as necessary, may be indicated, as well as whisky in ounce doses carefully watched.

In localities where venomous snakes are common, medical officers will do wisely to procure a tube of the anti-venom prepared by the Pasteur laboratories, for use in case of need.

Next to snakes, scorpions deserve mention. These animals do not poison by biting, but by stinging with the sharp-pointed last segment of the body, through which the poison is injected into the puncture. In our species, as found in the Southern and Southwestern States, the sting is hardly ever more serious than that of a bee. In Mexico, dangerous kinds are found, especially the Alacran (Centrulus gracilis), yellowish in color and averaging 2 inches in length. Several deaths are reported from its sting every year in the State of Durango. It is said to occur occasionally in southern Arizona and California. The scorpions found in Cuba and other West India Islands are more troublesome than dangerous.

Of lizards, two species of the genus Heloderma are more or less poisonous; one is the sluggish "Hila Monster" of Arizona and northern Mexico, about a foot long, with stout, ungainly body and short, thick tail. "The poisonous glands are situated at the point 
of the lower jaw and the venom is taken up by the wound while the animal hangs on to its victim with the tenacity of a bulldog." The second species, several feet long, known as Crust Lizard, occurs in Mexico.

In the arid regions of the Southwest, as well as in Mexico, are several kinds of poisonous spiders, injecting venom through movable fangs after the manner of snakes. The tarantula is especially dangerous, its bite causing acute pain, swelling and sloughing, nervous disturbances and sometimes death. The large centipedes of hot and dry regions are capable of inflicting painful excoriations by their numerous claws, but to what extent they are venomous, if at all, has not been clearly determined.

May also be mentioned, the Tropical Toad (Bufo agua) of South and Central America and southern Mexico, some 8 inches long and 4 or 5 broad, with large oval glands behind each ear, which, under irritation, exhude a large amount of a creamy secretion having a pungent aromatic odor, and a deadly arrow poison. It is said to contain epinephrin and a substance akin to digitalin (Drs. Abel and Macht). 


\section{CHAPTER L.}

\section{CAMPS (CONTINUED).}

\section{TENTS.}

Troops in camps are usually under canvas. Tents give shelter against sun and rain and, to some extent, against cold, but do not afford the protection and comfort derived from ordinary buildings; for hospitals and other special purposes they should be resorted to only when suitable buildings are not available. Flies, or outer covers, add much to their comfort and must always be used when on hand, being adjusted so as to leave an air space between them and the tent roofs. This space is important, but need not be so great in cold as in hot weather; the air thus enclosed being a bad heat conductor excludes the sun's heat in summer and prevents loss of interior heat in winter.

Tents are an excellent shelter against nocturnal radiation but, even with flies, afford an imperfect protection against heat and cold, being easily affected by weather changes. In sunlight they absorb and retain heat so that their temperature is higher than that of the shaded open air. Their ventilation is generally satisfactory in summer when the walls can be lifted and the doors left open; it is aided by the free exchange of gases taking place through the canvas in all seasons. But in winter, when it is necessary at times to pin down the walls and close the openings, the ventilation is often imperfect, although, on account of the porous walls, seldom very bad. In rainy weather, however, when the pores are closed by the contracting canvas, the air may become quite foul and diseases of the respiratory tract are likely to spread. Too often the men prefer warmth to fresh air, and, in winter, will generally keep the hood of the pyramidal tent closed tight. For this reason, it was found advantageous in some of the Texas camps (19I6) to replace it by a fixed metal hood, raised 4 inches above the canvas and secured in that position, thus insuring a certain amount of ventilation at all times.

In cold weather, tents are made much more comfortable by lining the walls with blankets or thick flannel; even paper is useful for the 
purpose. Except on very cold days this expedient will prove a good substitute for a stove, especially when fuel is scant.

An economical way of heating tents, in the absence of stoves, is to make a fire in a pit in front, and convey the heat and smoke in a zigzag, underground flue through the tent, to an outside sod chimney.

In permanent camps, the comfort of the soldier is much increased and his health promoted by the use of board flooring in tents, each floor consisting of two or more sections so as to be easily carried. removed and replaced, thus permitting of ready inspection and cleaning of the soil area beneath. In the absence of wood flooring, the earth floor should be made hard and as impervious as possible to subsoil moisture. About 3 inches of fine gravel well rammed down will make a good floor. A better result is obtained by a layer of stone (to break capillary attraction) covered with well-stamped earth, and top-dressed with mortar 3 or 4 inches thick, well stamped down as it begins to dry. This mortar may be simply made of finely: screened soil, but will be more compact and solid if lime is added. A floor thus constructed will be raised, as it should, a few inches above the surrounding level.

The tents used for the shelter of troops in our service are of various sizes and forms, and designated as shelter tent, wall tents, storage tent, pyramidal tent, hospital tent and hospital ward tent.

Shelter Tcht. - The field equipment of all European armies, excepting the French and English armies, comprises a shelter tent. In France, the issue of the tente d'abri is only occasionally authorized for troops operating in certain inhospitable regions. In Great Britain, the shelter tent is still looked upon as an unnecessary luxury. It was not used during the Franco-Prussian War but, since then, has been adopted by both Germans and Austrians.

In our service, each man on the march carries, as part of his equipment, one-half of a shelter tent, weighing 3 pounds, one pole and five pins of cast aluminum. The tent half is made of waterproof olive-drab cotton duck and serves as the wrapper of his pack. Two halves buttoned together at the ridge form the complete tent. It is pitched on two 3 -jointed poles 47 inches long, and occupies a space 64 inches long and 76 inches wide. An additional ground space is enclosed when the two triangular flaps are pinned down. A double tent is formed by buttoning toget::er the open ends of two single tents (Fig. I85). 


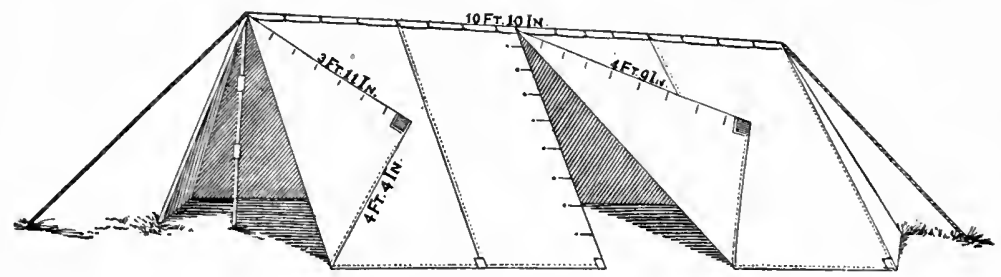

FIc. 185.-Double shelter tent.

In the absence of poles the tent can be supported by two rifles, or by a rifle in front, and by a bayonet in its scabbard, or the intrenching tool, in rear (Fig. I86).

Common Tent. - The common tent (or A-tent) of former days has been discarded in our service.

Wall Tent. - Wall tents are generally intended for the accommodation of officers. Two types are provided, the large and the small, both with flies.

The wall tent, large, is the new name given the former " regulation hospital tent." It is I I feet high, I4 feet 3 inches long and I4 feet 6 inches wide, with wall 4 feet 6 inches high. It requires one ridge, two upright and four eaves poles, i8 large double-notched

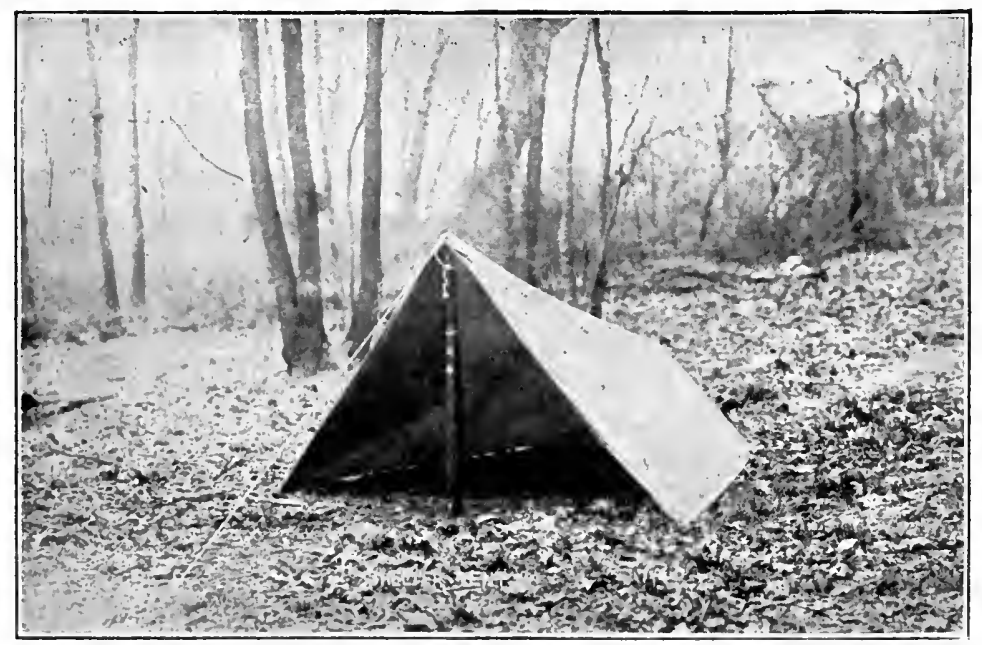

Fig. I86.- Shelter tent, pitched on rifle and bayonet. 
pins and 26 smaller pins. In each end there is a door, and above and to the right is a "ventilator and stovepipe" opening, 6 by 8 inches, to serve as a ventilator in summer and stovepipe hole in winter, with inside flap to close it when necessary (Fig. 187).

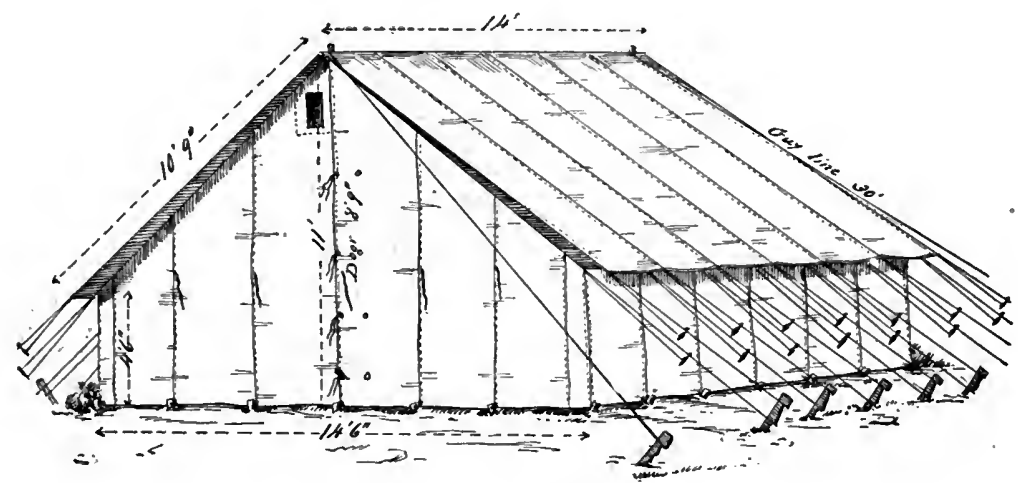

FIG. I87.-Wall tent, large. (Former hospital tent.)

The wall tent, small (or tropical wall tent), is 8 feet 6 inches high, 9 feet 2 inches long, 8 feet I I inches wide, with wall 3 feet 9 inches high (Fig. I88). The sides of the roof, $13^{1 / 2}$ inches from the apex, are drawn in, the whole length of the tent, by a horizontal band of canvas, one foot wide, which forms the floor of an attic or "pocket." This floor is perforated by 6 holes, each 6 inches in diameter, for ventilation, and by 2 smaller holes (one at each end) for the upright poles to go through. Each end of the pocket can be closed by a flap in inclement weather. Besides the ventilation produced by the holes in the floor of the attic, the drawing in of the sides of the roof separates it from the fly and increases the layer of air between them, so that this tent is well adapted to warm climates.

Conical Tent. - The conical tent has been discarded and is no longer used in our service. It is a round tent with conical roof, supported by one upright pole. This pole may be replaced by a stout stovepipe resting upon a tripod and connected with a Sibley stove placed within the tripod. It is a very useful tent in cold climates where stoves are necessary, and the absence of flat surfaces enables it to withstand storms better than other types. On the other hand, it is not adapted to warm climates on account of its easily heated interior, resulting from the low walls and absence of 


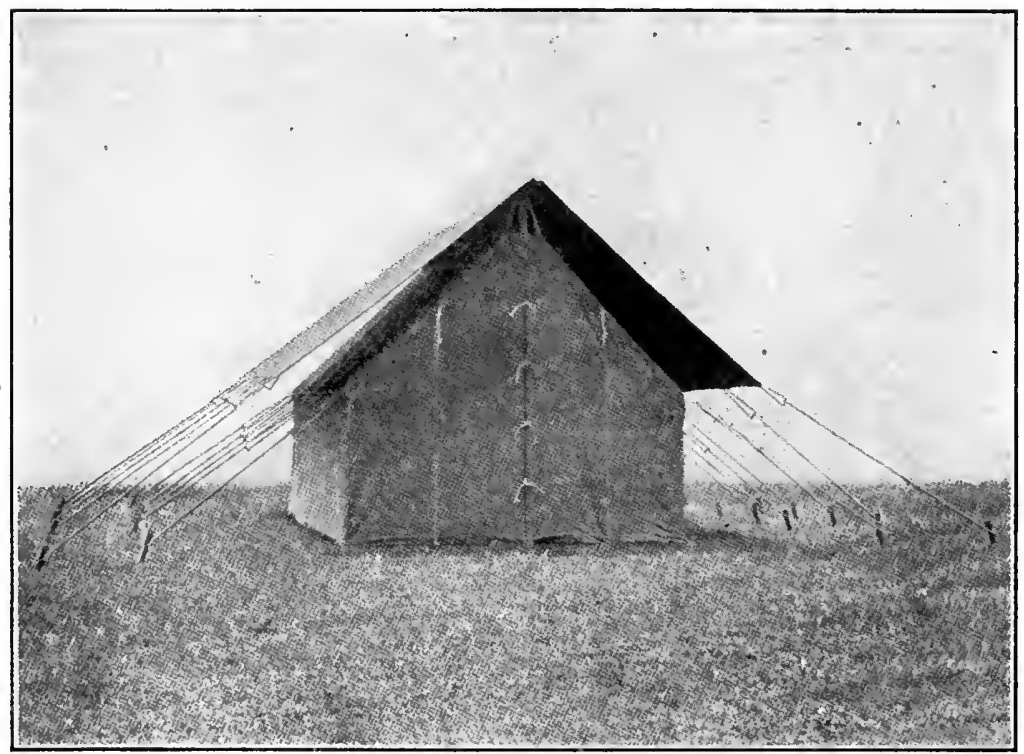

FIG. I88.- Wall tent, small, with fly. (Tropical wall tent.)

fly, its temperature being from 4 to 8 degrees higher than that of a hospital tent under similar circumstances (Fig. I89).

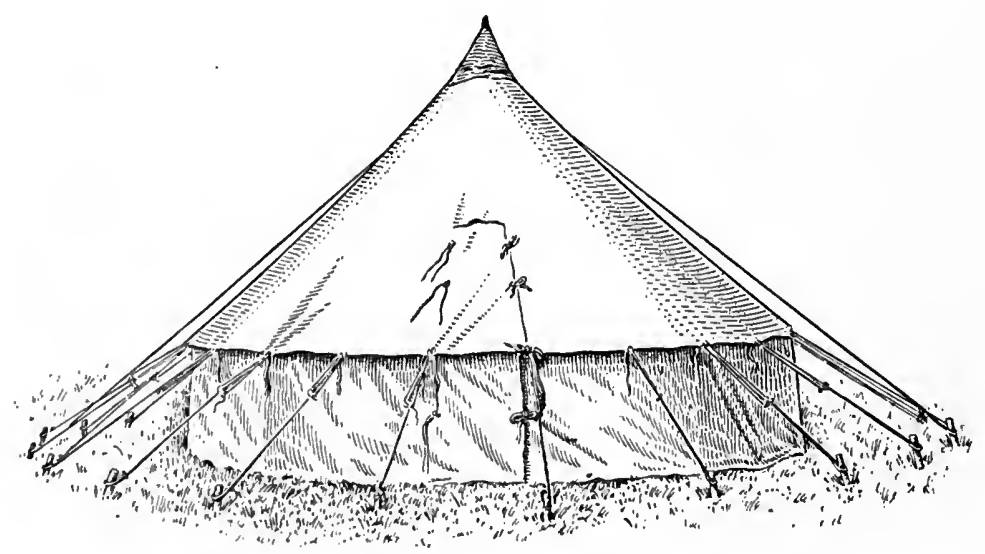

Fig. I89.-Conical wall tent, showing exterior. 


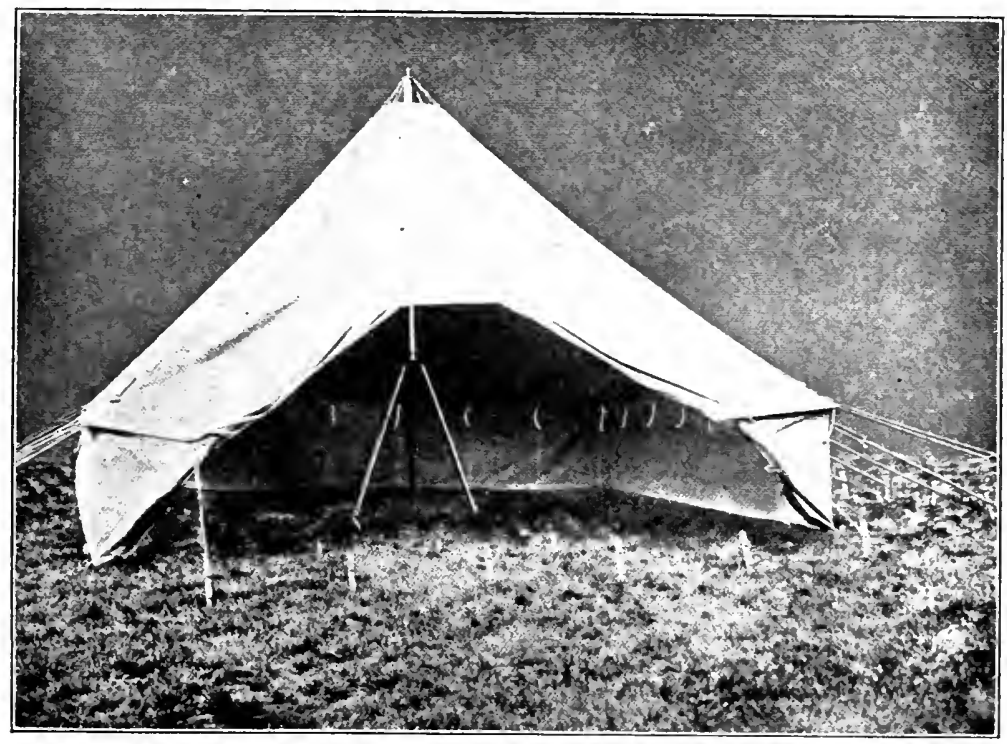

FIG. 190.- Pyramidal tent, open.

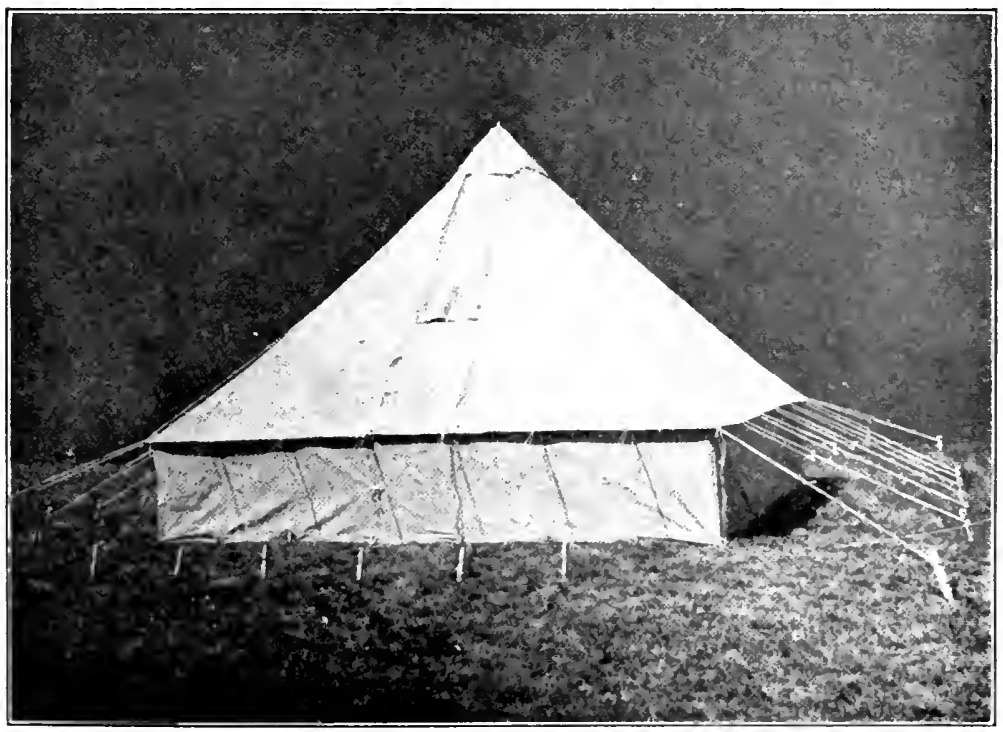

FIg. 'I9I.- Pyramidal tent, closed. 
Pyramidal Tent. - This type has superseded the conical tent. It is 16 feet square, with walls 3 feet high, and I feet high to top of roof, weighing, complete, 164 pounds (Figs. I90 and I9I). Like the conical tent, it is supported by a central pole resting upon a strong folding tripod. It is held above by four chains hanging from a plate passed over the spindle of the pole. The top opening, I 8 inches square, is closed with a movable hood. The method of heating by stove and pipe, used in the conical tent, is also applicable to this tent.

Hospital Tent. - Only one type is provided, the hospital tent, tropical, designed for warm weather. (Figs. I92, I93, I94.)

The tropical hospital tent (model I9O7) is I 2 feet high, Io feet 6 inches to base of pocket, I4 feet 3 inches long, 15 feet 7 inches wide, with wall 4 feet 7 inches high and doors ro feet 3 inches high. The sides of the roof, $20 \mathrm{O} / 2$ inches from the apex, are drawn in, the whole length of the tent, by a horizontal band of canvas, 22 inches wide, which forms the floor of an attic or "pocket." This floor is perforated by 4 holes one foot in diameter, for ventilation, and by' 2 smaller holes (one near each end) for the upright poles to go through. The pocket has 4 holes on each side, 6 inches in diameter, the center of holes being 6 inches from the floor. These side holes can be covered by a small fly 6 feet long by 14 inches wide, with lines to brail it up and haul down. This small fly is especially intended for use in the absence of the regular large fly. The pocket can be closed at each end by a triangular flap 18 inches wide at base and $\mathrm{I}_{4}$ inches long. This tent requires one ridge, two upright and four eaves poles, 36 large double-notched pins and 26 smaller pins. The ridge pole is 18 feet long, spliced in center with a 2 -inch thimble joint protected by a sleeve 30 inches long, and with hole for the spindle of upright pole 2 feet from each" end.

The ridge pole projects 2 feet beyond the upright at each end. The fly is sufficiently long to cover this extension and be secured to a stud at the end of the contiguous ridge pole, so that a completely covered space, 4 feet long, is provided between the tents.

Besides the ventilation produced by the holes in the floor and sides of the attic, the drawing in of the roof separates it from the fly and increases the insulating layer of air between them. 


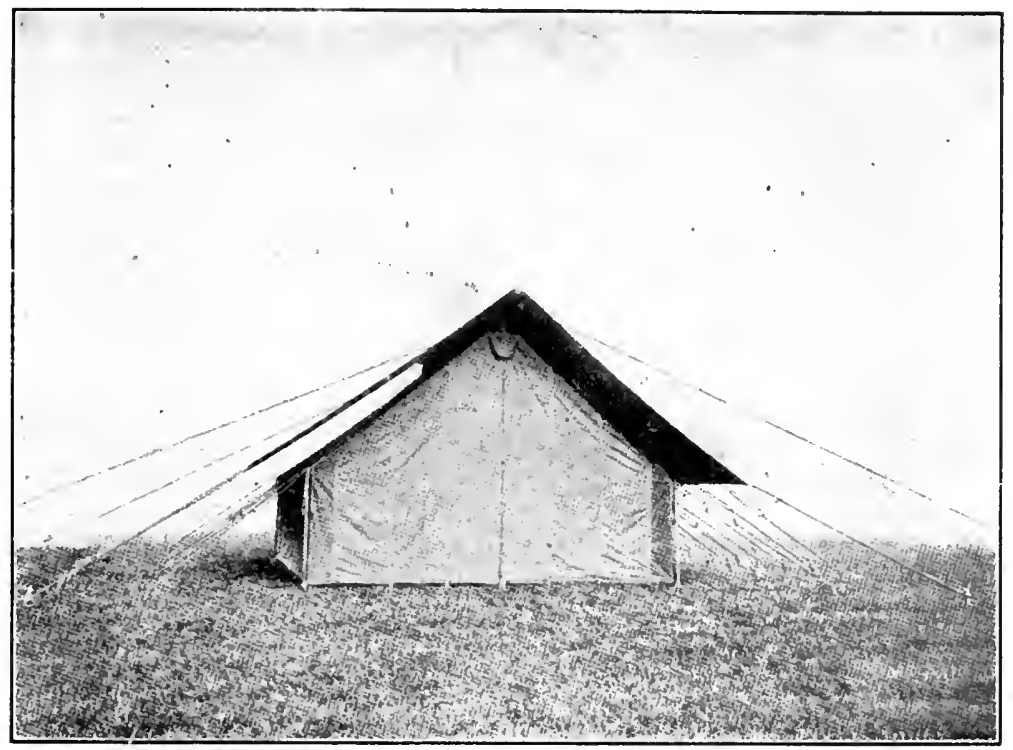

Fig. I92-- Hospital tent, tropical, with fly:

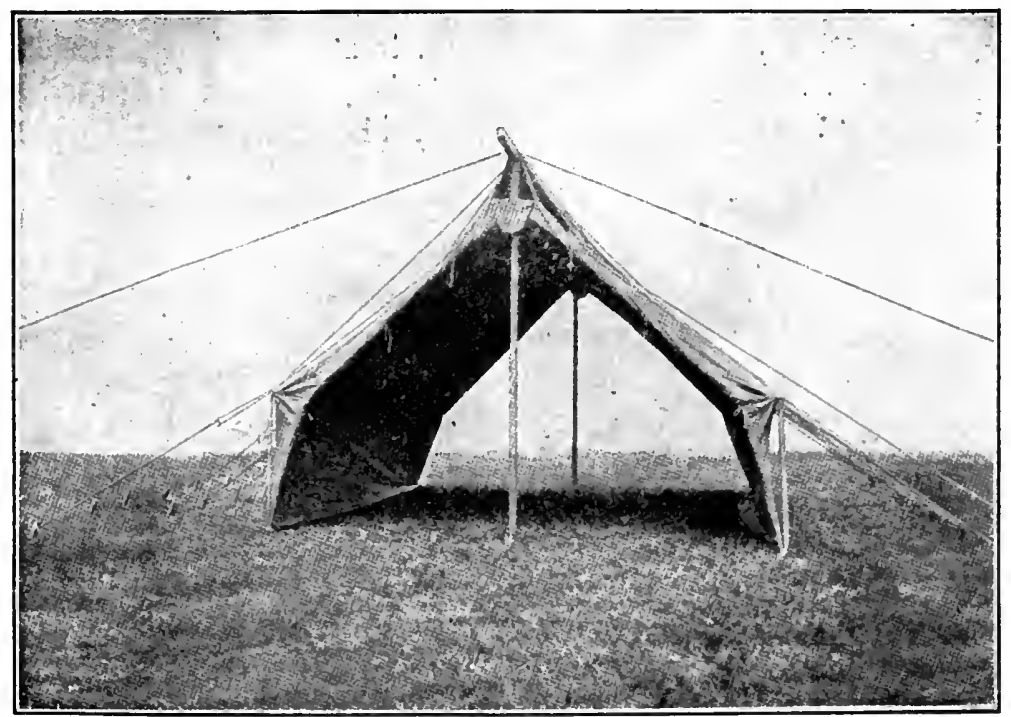

FIG. 193.- Hospital tent, tropical, showing interior. 


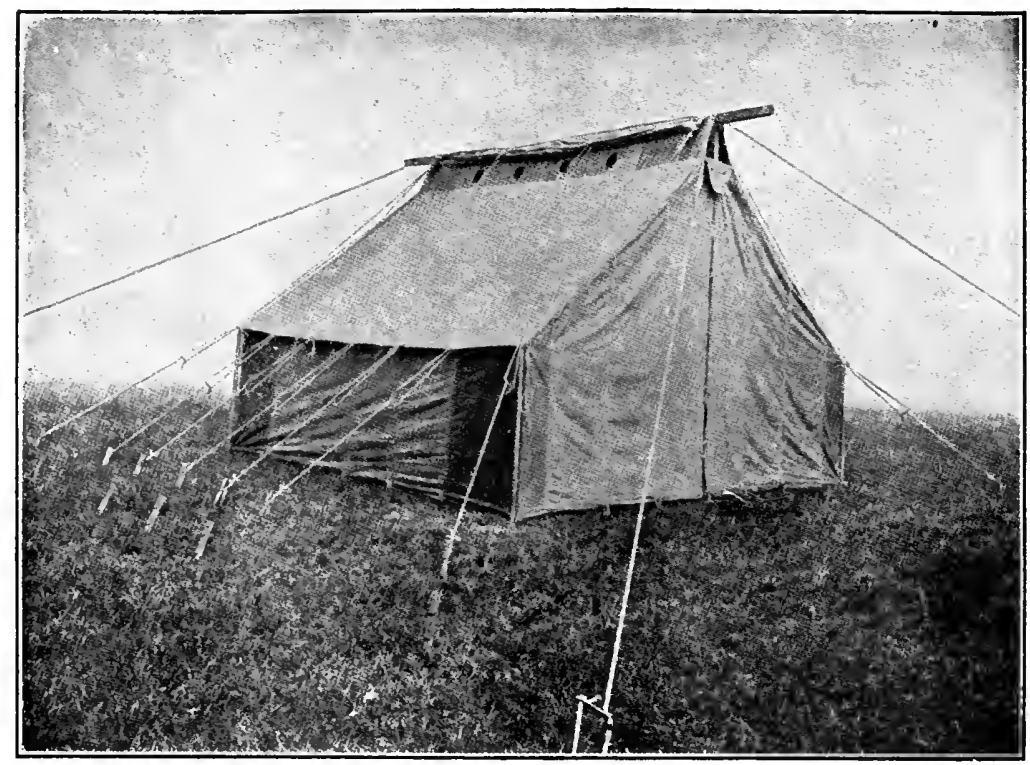

FIG. 194-- Hospital tent, tropical, showing ventilation of attic.

Hospital IV ard Tont. - This tent, as its name indicates, is specially constructed for the admission and treatment of patients, and only used for that purpose, having materially reduced both the cost and weight of the tentage required by field hospitals. (Fig. 195.)

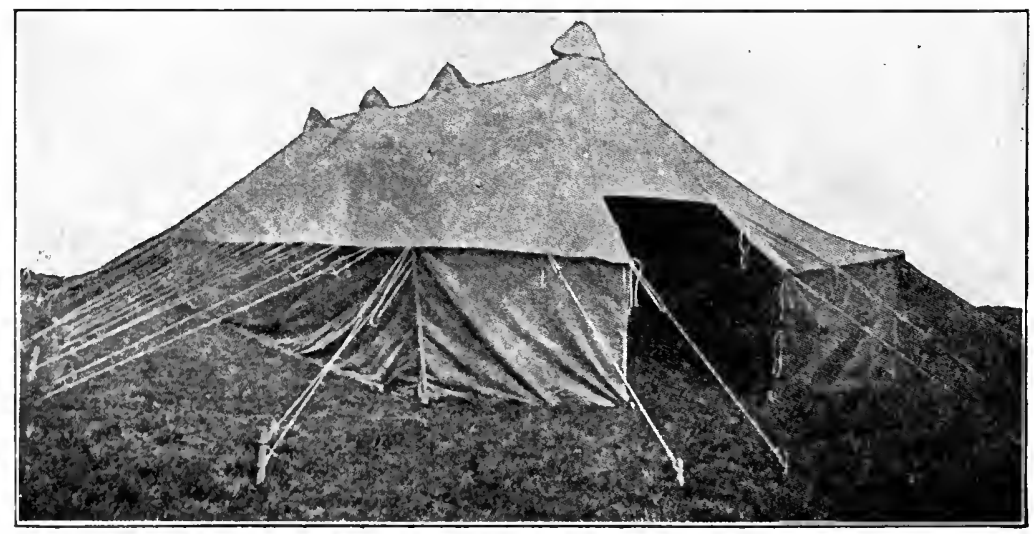

FIG. I95.- Hospital Ward Tent. 
It consists of tent, 4 uprights, 4 corner poles, 72 large and 56 small pins. Its dimensions are: length, 50 feet; width, 16 feet; height, 10 feet; height of wall, 4 feet 6 inches. Its total weight is 555 pounds and packs in 20 cubic feet. Through the four vents on ridge the tent is supported on the poles by plates and chains, the vents being covered by hoods. It accommodates 24 patients.

Storage Tent. - Its dimensions are: length, 20 feet 5 inches; width, I7 feet Io inches; height, 13 feet; height of wall, 5 feet 2 inches. Requires I ridge and 3 upright poles.

Of tents used in foreign armies none appear especially suitable to our service or to possess advantages over those above described, with the exception of the wagon or tortoise tent officially adopted by the English and French and well worthy of special mention. It is rectangular in sliape, 23 feet long by 2 I feet wide and weighs, complete, about 200 pounds. It is carried on top of an ordinary army wagon, forming its roof, the sides being rolled up and supported on brackets. For use, it is unrolled all around the wagon, which takes the place of a ridge pole, and held up by 16 light poles and a sufficient number of pins. It is lighted by windows made of an oiled, translucid fabric. All necessary furniture and supplies are carried in the wagon. This tent can accommodate 16 cots or about 30 patients lying on the ground. The facility with which it is pitched, furnished and supplied, independently of the baggage train, gives it great value. In the colonies or wherever vehicles cannot go, the wagon is replaced by a ridge pole and the whole outfit carried by pack animals.

Two other tents are also officially recognized in the French Army. the Tollet and the Herbet tents, with heavy metallic framework and rather complicated structure, therefore not adapted to our service, although (the Herbet tent especially) offering great sanitary advantages and comforts. 


\section{CHAPTER LI.}

\section{CAMPS (CONTINUED).}

\section{IMPROVISED AND PORTABLE BARRACKS.}

Where timber is abundant and the camp is to be of some duration, $\log$ huts with well-plastered joints, canvas roof and fly, afford

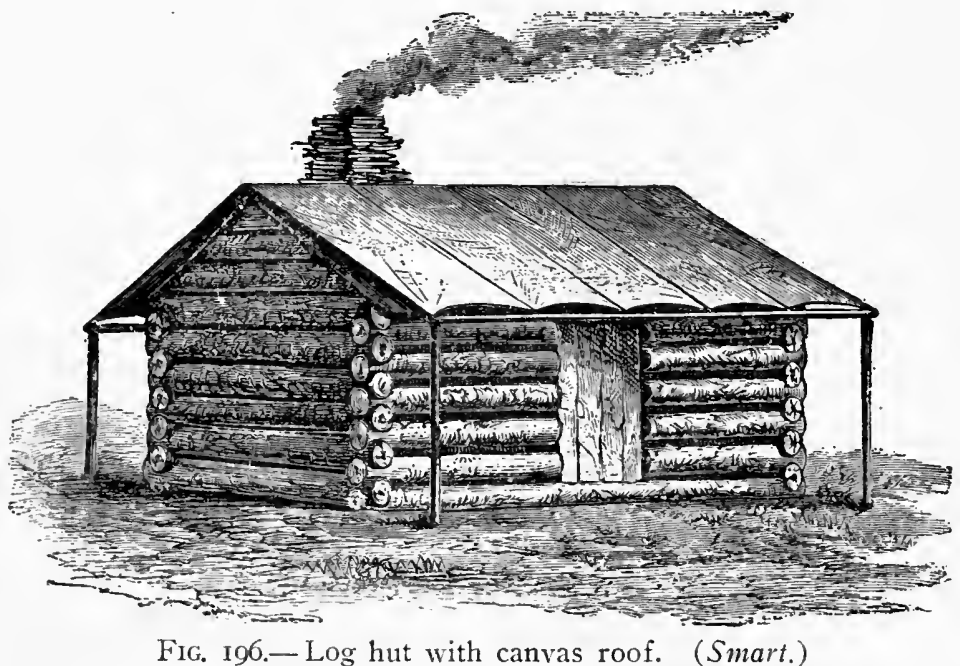

excellent shelter and are preferable to tents. Smart's hut (Fig. 196) is 13 by 7 feet, and 6 feet to the eaves, and accommodates 4 men on two double bunks, one on each side of the doorway. Light and ventilation are obtained through the roof, door and chimney. More light can be obtained by setting a sash or translucid fabric in the upper part of the door.

A better arrangement is that suggested by Woodhull (Fig. 197), in which two huts, 8 by I I feet, stand end to end, 6 feet apart, with doors opening upon a connecting porch. In a mild climate one chimney between the huts would be sufficient.

For winter camps, an excellent arrangement is that devised by Falk, in which 3 wall tents or common tents are placed against three sicles of a small, square, frame cabin, each tent connecting with it by 
a door. (Fig. 198.) A stove or fire in the cabin warms the tents; around it the men gather to smoke and lounge. Instead of glass panes the door should admit light through a translucid tissue.

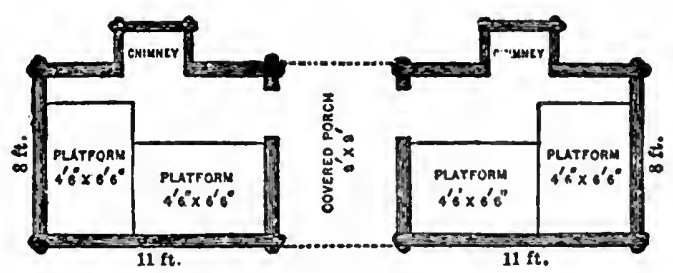

FIG. 197.- Ground plan of huts, for four men each, with covered porch.

When campaigning in very cold countries it may be advisable to construct dugouts, that is to say, huts partly excavated in the ground and covered with mud roofs, as was done on a very exten-

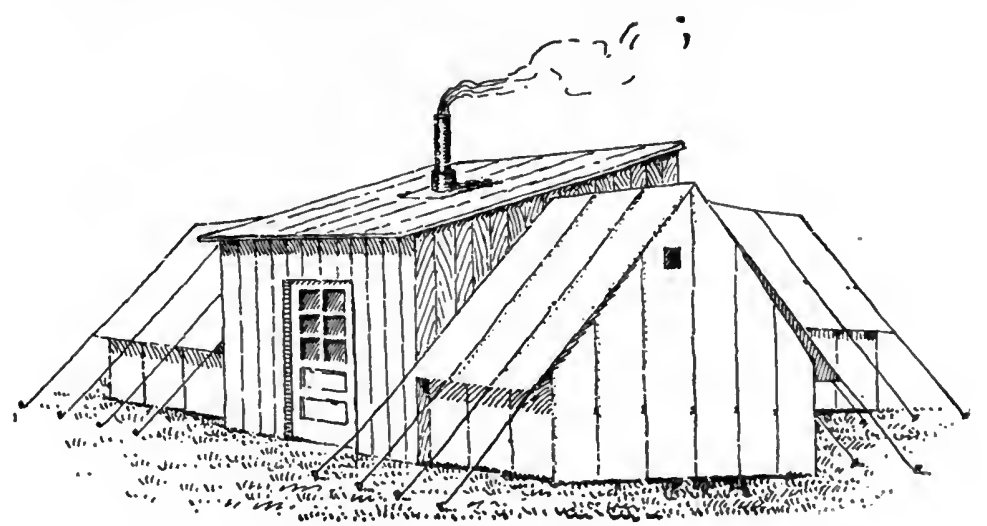

Fic. 198.-Falk's arrangement of tents in winter quarters.

sive scale by the Russian and Japanese soldiers in Manchuria. Such dugouts have the advantages of being quickly and cheaply constructed, easily heated and not conspicuous. On the other hand, they are difficult to police and ventilate, and likely to be insanitary. It should be noted, however, that the health of the Russians and Japanese in Manchuria did not appear to be in any way impaired by their prolonged stay in such underground structures during the winter of $1904-5$. The main reception hospital of the Russians, at 
Mukden, near the railroad station, was of this type. (Fig. I99.)* In sandy soil, the sides of the excavation should be retained by boards and stakes as in the German type. (Fig. 200.)

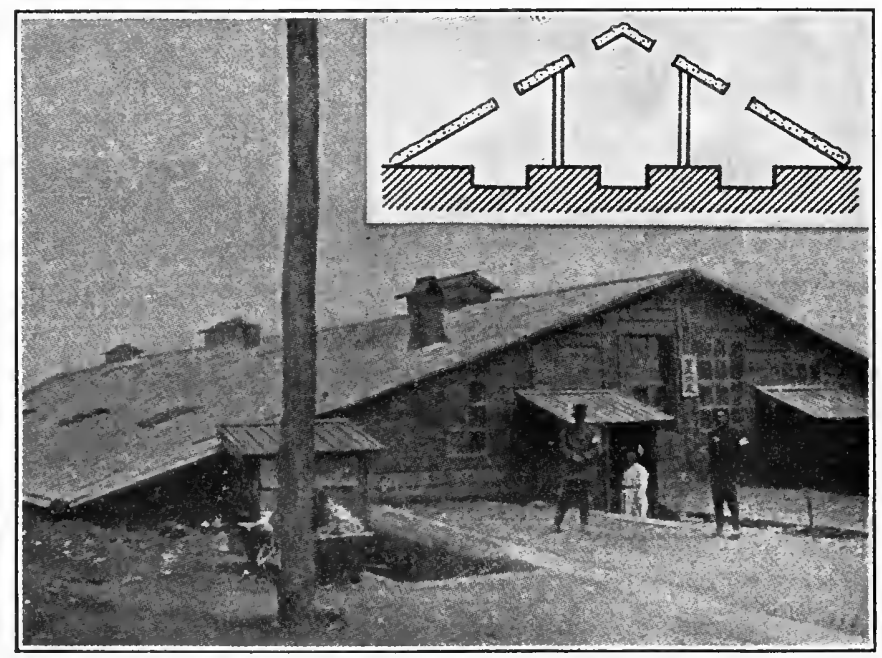

Fig. 199.-Underground Russian hospital at Mukden, Manchuria.

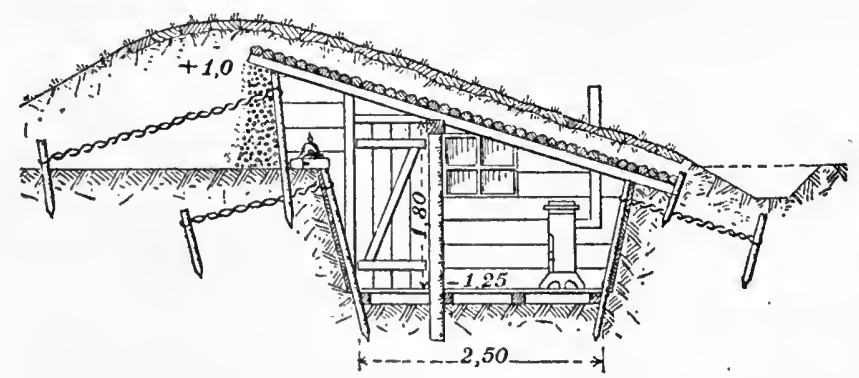

FIG. 200.-Dugout used by the German Army. (Munson.)

For cantonments, light frame barracks of various models have been devised. In the United States they are generally heated by stoves, their ventilation being chiefly by natural perflation, but also, in winter, by ducts which bring fresh air under the floor to the

* Concerning the effect of trench and underground life on the health of helligerent troops in France (1917), see page 747. 
stoves, the foul air escaping through shafts enclosing the stovepipes. An excellent workable type of portable buildings, on the knockdown system, for our cantonments and permanent camps, has been devised by the Quartermaster Corps. They are especially intended to shelter latrines, lavatories, incinerators, kitchens and messes, and will be found described under their proper headings.

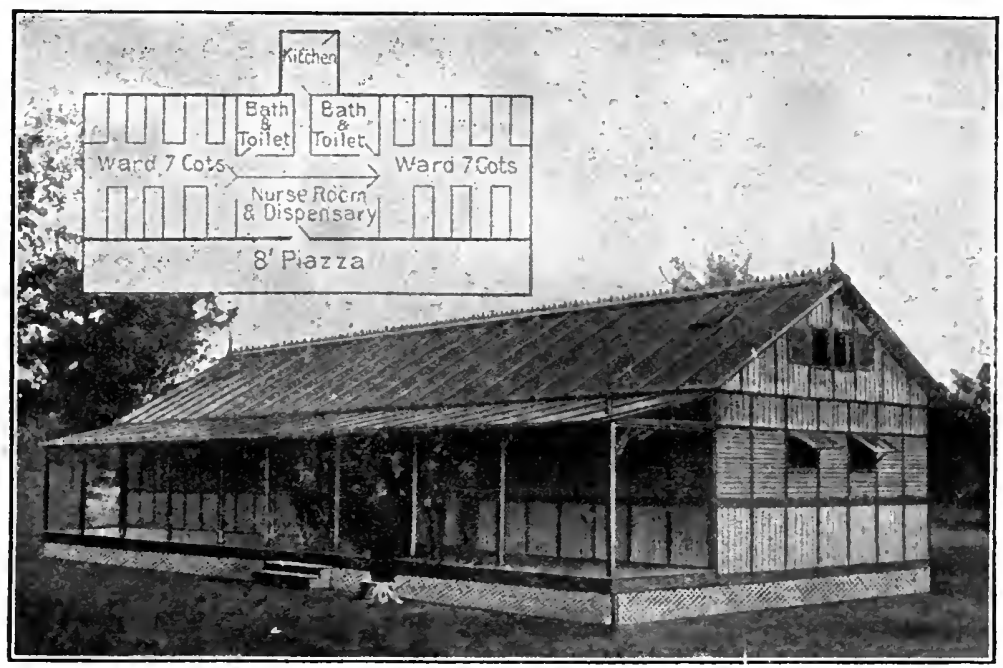

F1G. 20I.- Portable hospital pavilion, system Ducker.

Portable barracks are more or less used in all countries for the shelter of troops as well as for hospital purposes. Two of the bestknown types are the Ducker system manufactured by the Ducker Co., of New York, and the Doecker system manufactured by Christoph \& Lnmack, of Niesky, Germany. The Ducker buildings consist of sections 2 feet 9 inches wide, easily set up and secured, without nail or screw, and have all the advantages of permanent structures, while capable of being speedily knocked down and reconstructed. By the addition or removal of sections they can be increased or reduced to any desired extent. (Fig. 201.) Another model is shown in Fig. 202, a tuberculosis-hospital pavilion at Bellevue Hospital, New York, These portable buildings seem to be particularly well adapted to the needs of permanent summer camps at home and in our colonies. 


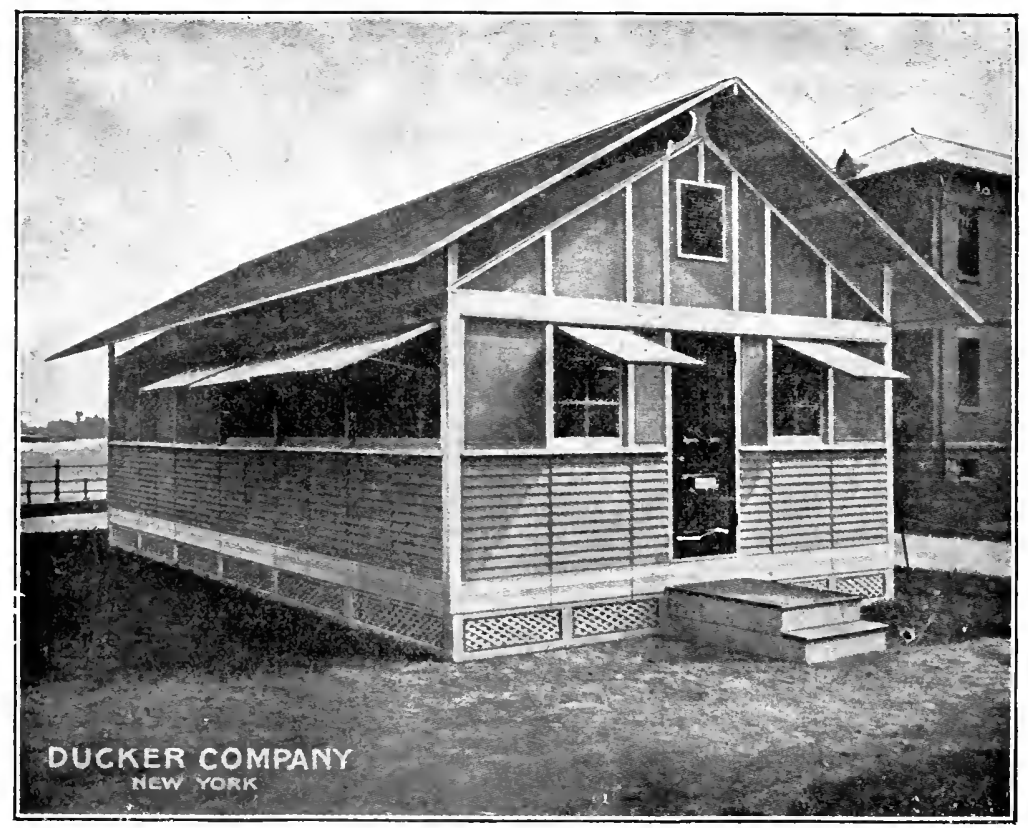

FIG. 202.- Portable hospital pavilion with double roof and double floor, system Ducker.

The Doecker portable hospital was often seen in Manchuria during the Russo-Japanese War, being much used by Red Cross societies with great satisfaction. (Fig. 203.)

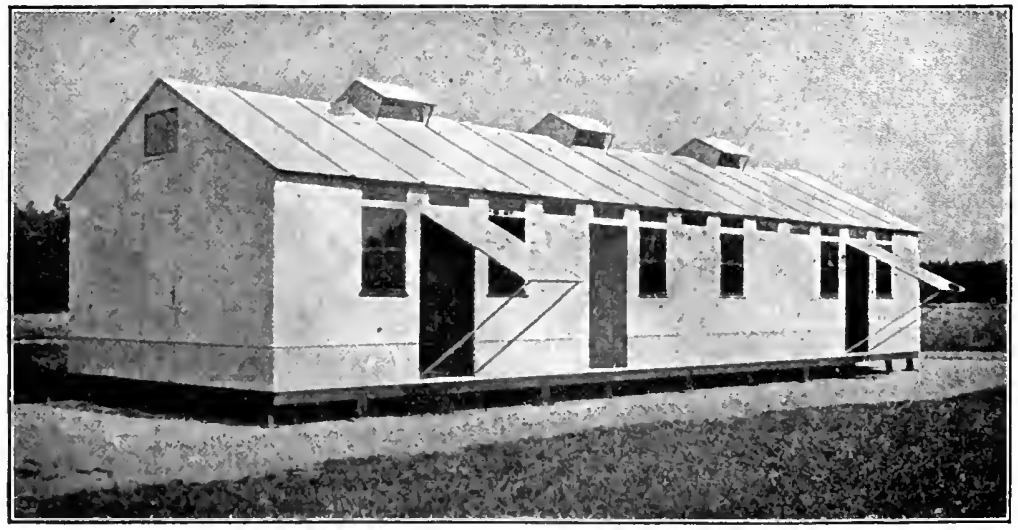

FIG. 203.- Portable hospital, system Doecker. 


\section{FORM OF THE CAMP.}

When not in presence of the enemy, battalions and squadrons normally camp in column of companies or troops at convenient distances.

With shelter tents the arrangement for each company is usually in two lines, facing each other, with a distance of 15 yards between the lines; this space forms the company street. A company of in-

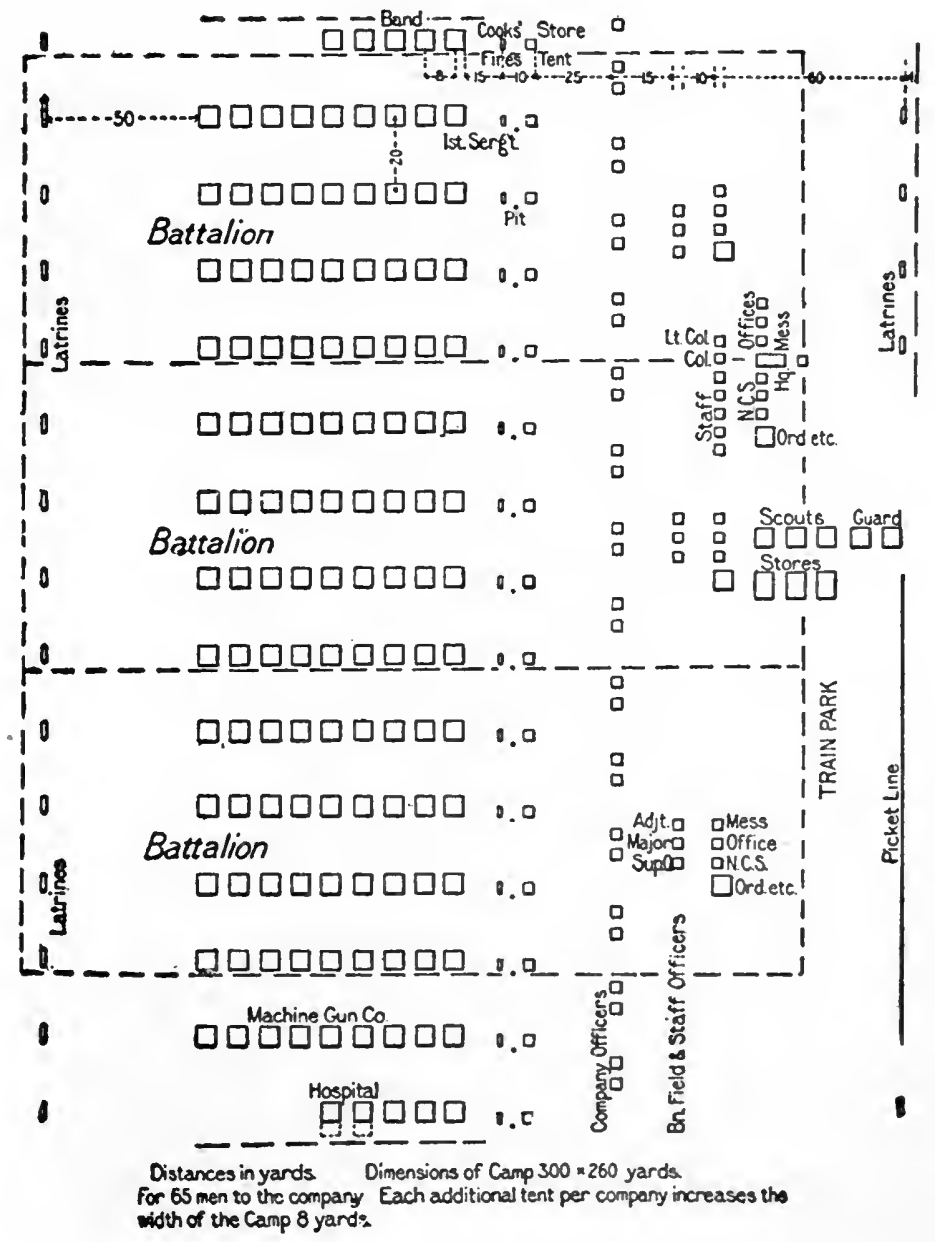

FIG. 204.- Camp of a regiment of infantry. 
fantry thus occupies a space of 20 yards in depth. If pitched in a single line, the tents face the head of the column.

With pyramidal tents each company forms a single row, the distance between the tents, from center to center, being 8 yards.

Between adjacent company rows, picket lines, and gun or carriage parks, center to center the distance is 20 yards.

Latrine seats are provided at the rate of one seat to every Io men, and shower heads at I to every 50 to 100 men.

In a camp of cavalry the picket lines are 15 yards in rear of the latrines.

In pitching tents, the wall or bottom pins are so driven as to slope slightly away from the tent; guy pins, so as to slope slightly toward the tent. For this purpose a maul is better than an axe, the handle not being so liable to break nor the pins to split. It is well to remember that the tent cords contract when wet and expand when dry. Therefore they should be loosened when it rains so that their

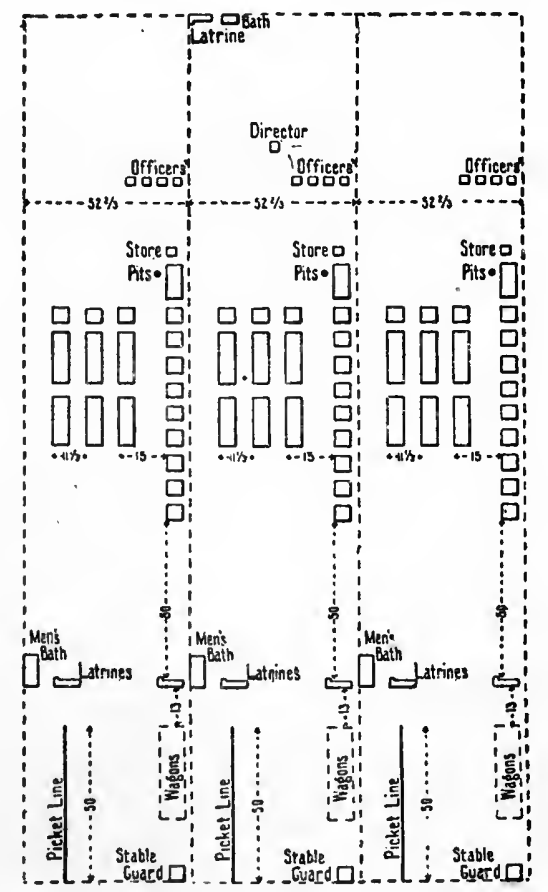

FIG. 205.-Plan of the three field hospitals of a division. Covering an area of 9.2 acres ( $160 \times 280$ yards). 
contraction may not pull out the pins, and tightened again as they dry. In case of very heavy dew this may have to be done night and morning.

From the sanitary point of view, the great guiding principle, in forming camp, is to separate the kitchens and messes as far as possible from the latrines. Therefore, the rule is to place the kitchen on one flank of the company and the latrine on the other. The distance from latrines to the nearest occupied tent is at least 50 yards, but when a smaller camp is desired this space may be used to park carriages and animals.

When shelters or other conveniences for company messes are provided, they are placed in line with the company tents, between the first sergeant's tent and the cook's fire, this interval being increased for the purpose. Near the cook's fire is the store tent, and a pit for liquid wastes when no better provision is made for them. Next to the line of kitchens is the line of officer's tents, an unobjectionable arrangement which facilitates the inspection of the former, thus ensuring greater cleanliness and more efficient management.

When a regiment is camping separately, or operating independcntly, it should be accompanied by a complete regimental hospital, or as complete as transportation permits. When it is brigaded, no regimental hospital is necessary; only an "infirmary" is put up, consisting of two or three wall tents, all hospital patients being sent to the field hospital. This infirmary is placed in the best available site, as free from dust, noise and flies as possible. As it keeps only trivial cases, there is no objection to its location near the men's or officers' rows.

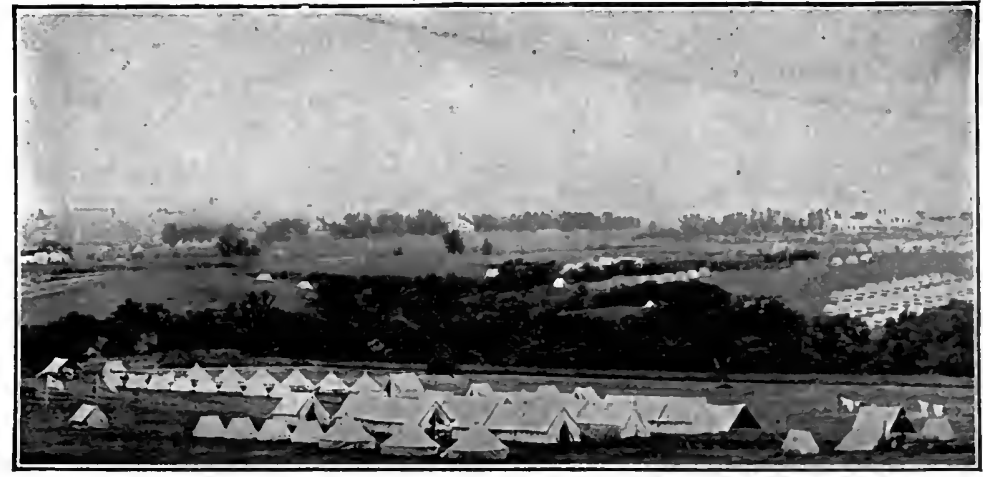

Fig. 206.- Perspective of field hospital. Fort Riley maneuvers, I902. 
One field hospital is usually assigned to each brigade. It should be in an easily accessible site, near a good water-supply and without too much regard to its distance from the regiments for which it is intended. It generally consists of six hospital ward tents, and such

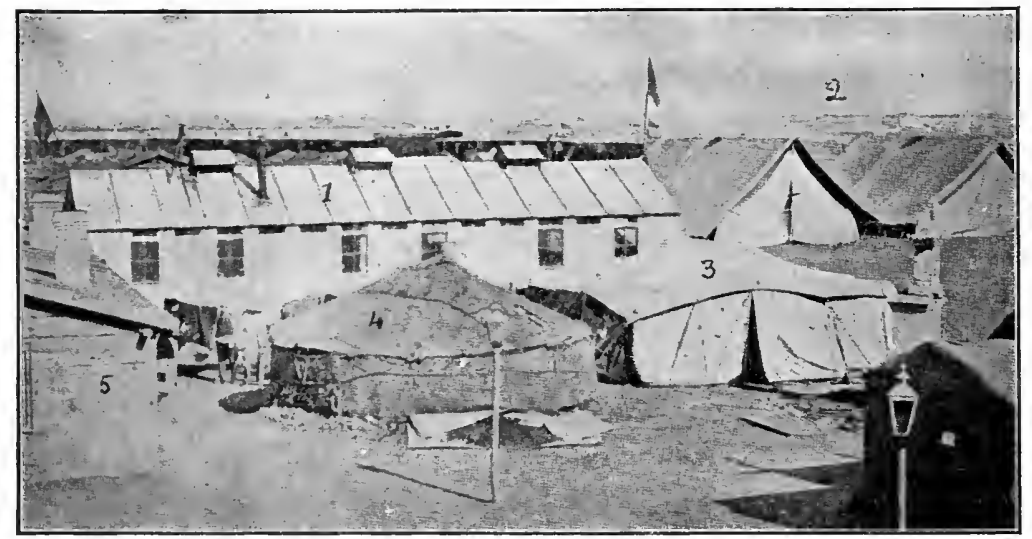

Fig. 207.- Livonian field hospital, Mukden, Manchuria. I, portable Doecker pavilion; 2, large Russian hospital tents ; 3 , tortoise tent or ridge pole (system Lefebvre); 4 , Mongolian felt tent; 5 , small frame barrack for personnel.

other tents as are required for administration, service and quarters. (Fig. 205.) Of the 3 hospital tents in front of the ward tents, the middle one is used as office and dispensary, another as operating room and the third for stores. A perspective view of a field hospital with former types of tents is given in Fig. 206. In the course of a campaign, in the absence of suitable buildings, it may be necessary to utilize all available shelters; this is well illustrated in Fig. 207, representing a field hospital at Mukden, Manchuria, during the Russo-Japanese War.

\section{ESTABLISHMENT OF CAMP.}

Upon arriving at the selected site, the first duty which concerns the medical officer, if the matter has not previously been attended to, is an examination of the water-supply and the determination of the place or places where drinking and cooking water should be drawn. Bclow this, if the supply is from a stream, other places should be likewise set apart for watering animals, for bathing and washing clothing, in the order named. 
If the stream be small it will be of advantage to construct reservoirs by building dams.

Small springs may be dug out and lined with stones, brick, or empty barrels. Surface drainage is kept out by a curb of clay. Animals will in such cases generally have to be watered from troughs or buckets.

If the water be of doubtful quality, measures must be at once taken to filter or sterilize it.

In a permanent camp or cantonment, each company should be provided, for drinking water, with two barrels or large cans with lids, and fitted with fawcets near the bottom, so that the use of dippers may be dispensed with. These barrels should be on a convenient platform, and a soakage pit filled with stones, dug under - the drip.

The next thing requiring the attention of the medical officer is the digging of straddle trenches and sinks. This should be begun as soon as the company lines are laid out, suitable places designated and tools available. Trenches are always necessary. They are the only form of latrines used if the camp is not to last more than 2 or 3 days (see page 637). When sinks are required, as it will take several hours for their preparation, it is expedient that a suitable spot, for each regiment or battalion, be selected and a dozen trenches dug, 3 or 4 feet long, of about the width and depth of the spade. These trenches must be ready by the time the camp is pitched, or even sooner if practicable; otherwise, men will stray beyond their lines and almost inevitably ease themselves wherever they have a chance to do so unseen by their officers, thus violating the first and most important law of camp sanitation. Trenches, like sinks, must be carefully filled up before leaving camp, and all fecal matter properly covered.

"Tents, company streets and picket lines will be ditched if there be time available." The earth from the ditch is usually thrown away from the tent; it should never be thrown upon the sod cloth and against the bottom of the tent wall, unless absolutely necessary to prevent extreme cold or a dangerous irruption of rain-water; in such position the earth interferes with ventilation, prevents the lifting of the walls and, by favoring dampness, impairs the strength of the canvas.

This digging of a small gutter, 4 to 6 inches deep, around tents is 


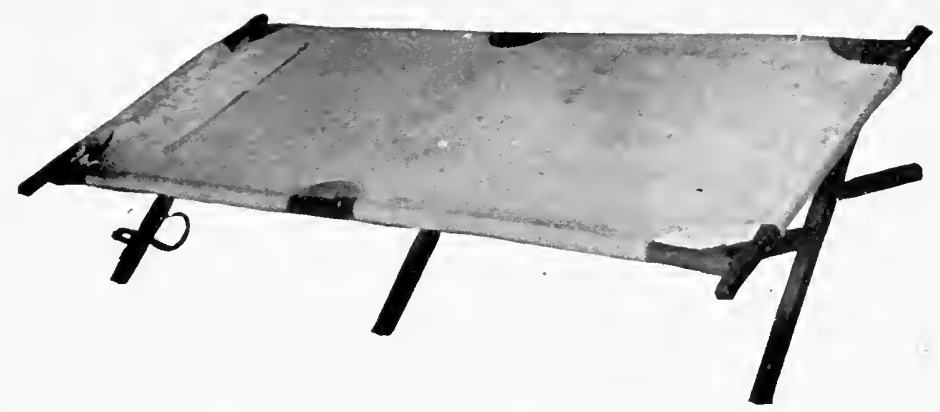

Top View

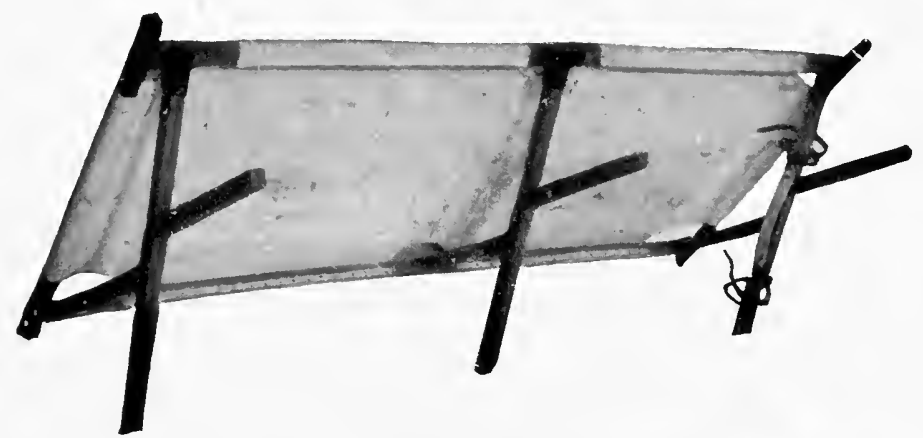

Bottom View

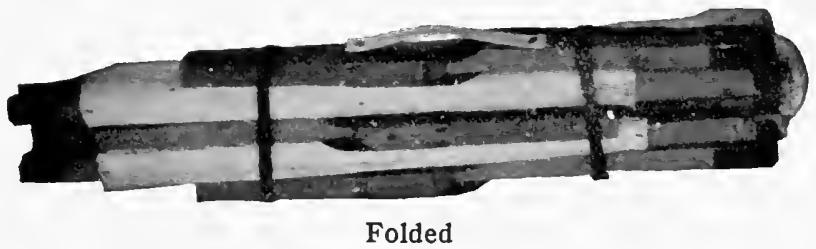

FIG. 208.- Regulation folding cot.

simply for the purpose of keeping their floors dry; the inner edge of the gutter should come just inside the skirt wall of the tent to catch the water running down the wall and thus protect the interior. When the camp is only for a day or two and there is a reasonable certainty that no rain will fall, such digging is unnecessary; but under ordinary circumstances it should not be neglected. These gutters should lead into the trenches of the company street and these into larger ditches so that the whole camp may be properly drained. 
The installation of a camp, so far as the comforts of the men are concerned, will depend chiefly upon the length of time it is to be occupied; thus if it is to last a week or more, the grounds should be cleared of all underbrush, paths and roads ditched, bedded and gravelled; rough chairs, benches and tables may be constructed, as well as shades over mess tables, latrines, bathing and washing places, etc. These simple improvements are easily made and add greatly to the contentment of the men; but so far as the sanitation of the camp and the health of the men are concerned, it matters little what the stay is to be, whether short or long, the sanitary measures are practically the same and to be applied with equal strictness, especially in everything that concerns the correct disposal of excreta and wastes.

Soldiers, so far as possible, should not sleep on or too near the ground: I, because the soil is generally damp relatively to the body, even when apparently dry, moisture rising up very readily from the wet subsoil by capillarity; 2 , because the soil and vegetation being liable to be infected by disease germs, or to harbor poisonous insects. blankets and clothing should be lifted above them; 3 , because the atmosphere near the soil is always more or less contaminated by ground air which, as stated before, contains a large proportion of carbon dioxid and other obnoxious gases resulting from the decomposition of organic matter, especially in hot climates.

In cantonments, or permanent camps, the tents may be pitched in frames and floored, and bunks constructed or cots used. In winter, stoves may be supplied and placed upon brick or stone foundations. The regulation "folding cot" which has been used in our Army for a number of years has been found entirely satisfactory. (Fig. 208.) In temporary camps, the Q. M. Corps supplies bedsacks which the men stuff with straw, grass, leaves or brush. In the absence of cot and bedsack, the soldier can generally make himself a couch of the same material or improvise a bedstead with a few stakes and cross sticks. If none of these things be available he should spread his poncho or slicker upon the ground, under his blanket. 


\section{CHAPTER LII.}

\section{LATRINES.}

Fecal matter is not only repugnant to sight and smell but, in camp, constitutes the chief source of danger to the health of the soldier. It may contain various infectious germs and from it are spread the camp diseases most to be apprehended, namely cholera, typhoid fever, dysentery and diarrhea. As it is always difficult and often impossible to detect cases of these diseases in their early prodromal stage, or before patients have a chance of polluting the latrines, the first sanitary axiom to promulgate in a camp is that all excreta (fecal matter and urine) are to be considered as infected and treated accordingly. A firm mental grasp of this axiom helps to a clearer understanding of the peril to which the men are exposed and suggests the measures best calculated to guard against it.

Fecal matter and urine convey the germs of disease through food and drink, that is to say, by being directly ingested into the stomach. There is also a probability that these germs, as they float in the air dust, are absorbed by inhalation.

Water contaminated by sewage or the seepage from latrines has been a widespread cause of typhoid fever and cholera. But this danger is so obvious and now so well understood that, it can be assumed, few officers will allow their men to drink water, not declared safe by the sanitary officer, without previous sterilization or filtration. Flies are also an active agent of infection, carrying thousands of germs in the minute particles of excreta adhering to their feet. Another prolific cause of the spread of typhoid fever, and probably cholera and dysentery, in camps, is personal contact, that is, the dissemination of fecal matter from man to man, through the clothing, shoes and hands. Human excrement, therefore, must always be looked upon with great suspicion, as a menace to the health of the camp, and be the object of the strictest sanitary measures.

The methods of excreta disposal in camp, wherever water carriage is not provided for, are: trenches, pits or sinks, post holes, sanitary troughs and incineration.

The ideal disposal of excreta in camp is incineration. When this is not possible, the next best method is disinfection and removal (by 
sanitary trough and excavator). But in temporary camps, where troops remain only for a night or a few days, or whenever the necessary sanitary appliances are not at hand, no better system has yet been devised, and probably ever will be, than the primitive trench or pit. Whenever troops go into camp, even if only for a few hours, latrines are necessary, for, if not provided, the surrounding grounds will surely be polluted with fecal deposits.

StraddLe Trexches.- For a day or two, a mere trench, such as used by European armies, the width of the spade or shovel (not to exceed one foot) and a depth of one or two feet, is generally sufficient. The men squat astride of it, in complete security. No pole and supports are necessary, and there is very little danger of fouling the edges. Furthermore, everybody admits that the squatting posture is conducive to a better physiological performance of the act of defecation.

Shallow trenches possess advantages over deep pits. They are more easily dug and taken care of, give off less odor and admit of greater privacy. The excreta being more exposed to air and bacteria are more promptly nitrified and disintegrated; they are less likely to contaminate the subsoil and the water-supply. Trenches can often be dug where pits would be impossible, as in shallow rocky ground, in sandy soil liable to cave in, or in low places where the ground water is near the surface. The objections against them are: the larger area of land required and the necessity of digging new ones once or twice a week; flies readily breed in them; pollution of the shoes is more likely; toilet paper is more liable to be blown about; and the contents are more exposed to be washed out by a heavy rain.

In the English Army, where this system has been best studied and applied, the trenches are 3 feet long, I foot wide and 2 or 3 feet deep, each trench being intended to be used by one man at a time. The earth and sod are heaped up at one end, not at the side. The interval between the parallel trenches, edge to edge, is 3 feet, thus leaving sufficient room for a second set when the'first is filled up. In this way there is always an interval of 3 feet between the trenches in actual use. The new rows are dug on the side nearer to camp so that the men need not walk over the old trenches. A distance of one foot is left between the ends, as between the sides, so that in determining the area required for a given number of trenches, 2 feet 
in width and 4 in length must be allowed for each. As a rule, 5 trenches are provided for every 100 men, but 3 or 4 are enough, according to Melville, for commands exceeding 500 men. With a depth of 2 feet and good management a trench can be used 3 or 4 days. With a depth of 3 or 4 feet it can be used a week. In Europe, however, trenches seldom exceed a depth of 2 feet, as it is considered better to increase their number than their depth.

In this country, trenches, although never depended upon as a permanent system of disposal, are frequently used as a temporary device; for instance, after a command gets into camp and before suitable pits are constructed (see page 637).

Whenever a camp is not to last more than 3 or 4 days, trenches will answer every purpose and pits are unnecessary. Their number, size and arrangement should be as in the English system, except that inasmuch as only one set will be required, the interval between them admits of greater variation; as a general rule it should not be less than one foot nor more than two feet.

Each man must immediately cover his fecal discharges with earth, suitable shovels being provided for the purpose. The trenches are fired with crude petroleum in the same manner as pit latrines, or at least sprinkled with it to keep flies away. When the contents are within 4 or 5 inches of the surface they are filled with earth and the sod carefully replaced and pressed down. It is a good practice, be-

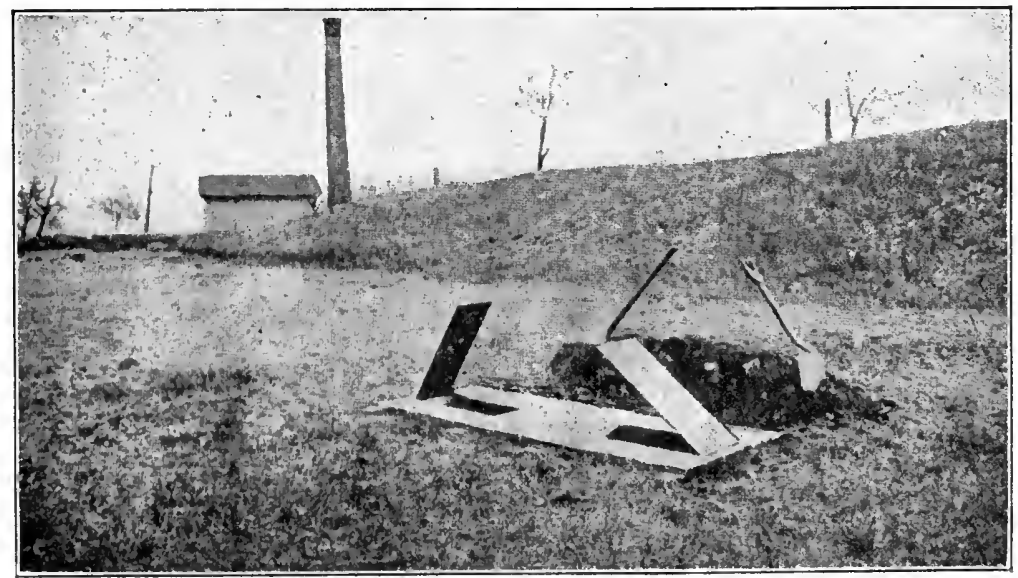

Fic. 209.-The Patterson trench cover. 
fore leaving camp, to scorch the area used for trenches with petroleum, so that all exposed infectious matter may be destroyed and the area itself clearly marked for the information of other commands.

Patterson, M. C., U. S. A., recommends a "straddle-trench cover" (Mil Surg., June, I9I3). Two planks, 8 inches wide and 6 feet 8 inches long, are nailed, Io inches apart, on 4 cross-pieces of 2 by 4 timber. The space between them is closed in the middle, but forms an opening 2 feet long, at each end, for defecation. Each cover is intended for two men and placed over a trench six feet long. The lids are hinged at the terminal end of the opening, so that if two men use the trench at the same time they face each other (Fig. 209). It wottld be preferable to have one of the lids hinged at the central end of the opening so as to be interposed between the two men. Two such covers will usually be ample for a company.

This cover being light and easily handled will practically do for marching commands what is accomplished by the latrine box for maneuver or semi-permanent camps, and obviate most of the objections mentioned above.

Major Lucas, N. G. N. Y., has developed this type of straddle
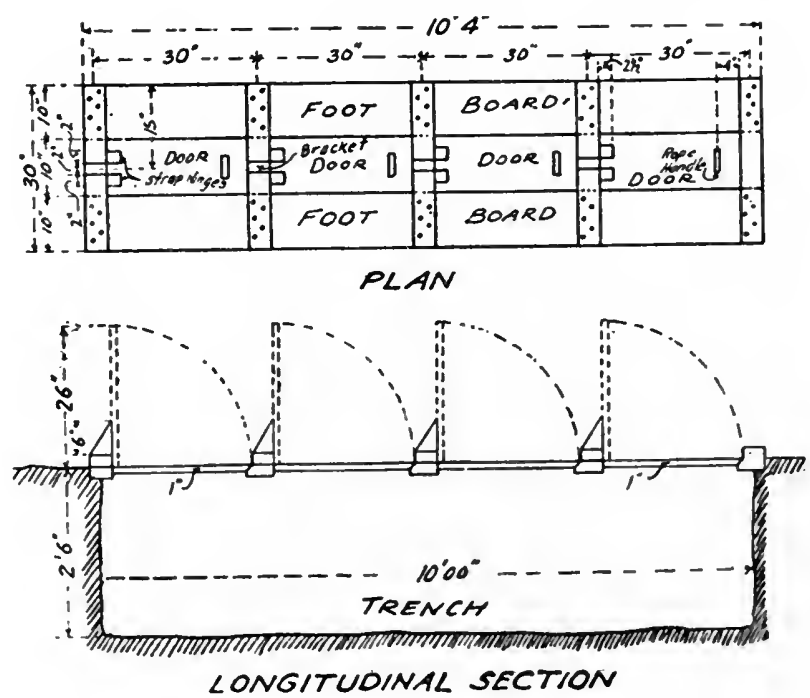

Fig. 210.- The Lucas trench cover. 
trench cover so as to make it more practically useful in camps ( Mil. Surg., Dec., I9I6). Fig. 210 shows plan and section of a trench Io feet long, with 4 lids. Each man squats with back against his lid, and is thus screened from the other men. The lid is prevented from opening to a greater angle than 85 degrees to insure its automatic closure after use.

Pit Latrine or Sink.- If the camp is to last more than a few days, pit latrines (in the absence of incinerators) are indicated. If they are not to be used more than a week, a depth of 4 or 5 feet is sufficient. If needed for a longer period, the pit must be dug full depth; this will vary according to the nature and composition of the subsoil and underlying strata, and also the depth of the ground water. A shallow pit.may be imposed by a rocky stratum, loose sandy soil with tendency to cave in, or by the ground water rising near the surface.

It should be borne in mind that the deeper the pit the greater is the danger of contaminating the ground water and, therefore, any source of water-supply in the near vicinity, and the further fact that the nitrifying bacteria, through whose agency fecal matter is disintegrated and mineralized, are mostly in the upper 3 or 4 feet and that their action is very feeble below a depth of 6 feet. Hiowever, considerations of convenience and economy of space and labor make it generally advisable that, whenever possible, the depth of the pit should be at least 7 or 8 feet. A convenient average width, for open pits, is 3 feet at the mouth and 2 feet at the bottom (Fig. 2rI). For boxed pits, the width does not usually exceed 2 feet at the mouth. In order to increase the capacity of the pit, the walls should be as nearly perpendicular, and the bottom as wide, as the nature of the soil safely permits.

Sinks should never be located in the lower part of the camp where they are liable to contaminate ground water, and exposed to be flooded in rainy weather; as a rule they must be on as high grounds and as far away from the water-supply as possible.

The length of the sink must be such as to afford accommodation for from 5 to 8 per cent. of the command. Under normal conditions the smaller of these percentages will suffice, but as outbreaks of bowel complaints may occur at any time it is better to make more liberal provision if conditions permit. By allowing two feet to each man, a trench 20 feet long will seat io men, or about 8 per cent. of 


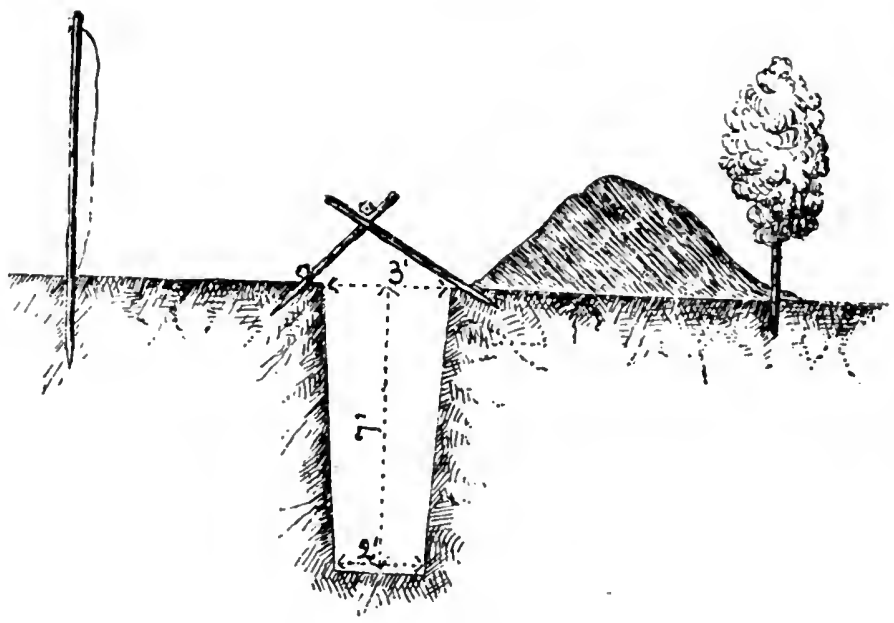

FIG. 2II.-Construction of pit latrine.

an infantry company at its maximum strength of 128 men. But even with a larger company, seldom will more than 4 or 5 men, as a rule, visit the latrine simultaneously for defecation; at this rate, at least 40 an hour can be accommodated, or more than 200 before noon.

To the above length of 20 feet should be added 4 feet to provide for 2 urinals, one at each end, as explained further on.

The question suggests itself whether each company should have its separate sink or if it is not preferable to have only one longer trench for the battalion. The latter system is certainly economical of time, labor and space; it is easier to dig one trench 80 feet long than four separate ones 20 feet long; but, on the other hand, the shorter pits can be placed nearer their respective organizations and maintained in a more perfect state of police. The question will be largely determined by the local conditions of terrain, soil, ground water and by the form of the camp. A very satisfactory compromise is to have one trench for each two companies, with brush partition across the middle.

In digging the pit, the excavated material is usually piled along the edge of the rear side. This material, or as much of it as is good for the purpose, will be used as disinfectant; it is desirable, therefore, if a notable proportion of it should consist of coarse gravel, 
chalk or stone, to throw it further back and pile the loam and other available earth along the edge of the pit.

The usual latrine seat is made up of a smooth, straight pole or sapling, with bark shaved off if possible, resting upon crossed logs. These supports are placed at intervals of 7 or 8 feet, each set consisting of two logs planted opposite each other, one in each edge, and crossing at such a slant that the apex of the upper angle which they form is about $I_{5}$ inches above the mouth of the pit, on a vertical line falling about 8 inches in the rear of the front edge; in this position they are securely lashed or otherwise fastened together. Besides the seat pole placed in the angle of the supports, another horizontal pole must be secured to the front logs, as foot rest and protection to the front edge, just high enough to clear the ground. The front logs (planted in front edge), must be at least 4 , and the rear ones (planted in rear edge), 5 feet long. (Fig. 2I I.)

Whenever the pit need not be more than 5 or 6 feet long, the seat pole is made to rest on the forks of two upright poles, one planted at each end.

One part of the pit must be reserved for urination, preferably one end or both ends of it. For this purpose the crossed poles holding the seat should be set in a couple of feet from the ends; the space thus set apart as urinal may be still further isolated by a brush partition thrown across the trench. The passing of urine which accompanies defecation necessarily takes place in the latrine but, at any other time, urination in any places but those specially prepared and designated must be strictly prohibited. Thus only can the edges and surrounding of the latrine be guarded against urine pollution.

The last requisite to make the sink complete is a draining ditch around it, or at least on the sides from which drainage water might be expected. This is very important; otherwise a heavy rainfall may flood the trench, scatter its contents over surrounding grounds and do irreparable harm.

Latrines should be given as much privacy as possible by building a wall of canvas or of brush, 6 feet high, around them. As to overhead protection against sun and rain, experience at the San Antonio camps, in I9I I, has shown that, contrary to the ideas then prevailing, the latrines left unprotected are more distinctly free from odors and flies than those which are covered over; in other words, that a bright light tends to inhibit putrefaction and acts as an insectifuge. 
Therefore only in case of persistent rain would the use of a tent fly or other shelter be advisable.

LATRINE Box. - Whenever a camp is to assume a permanent or semi-permanent status, the Regulations provide special systems of disposal as described below. But in war time, during a campaign, particuarly in a foreign country, owing to the great difficulty of transportation, the use of such cumbrous appliances will generally be impracticable and sinks made necessary for indefinite periods of time. The question is then how to render these sinks as little objectionable and harmful as possible. There is a consensus of opinion that they should be "boxed" so as to convert them into close vaults more easily policed and from which flies can be excluded.

Boxed latrines have now been used in our service for several years in many camps, and there is unanimous agreement as to their great superiority over the open sink in regard to convenience, comfort and disease prevention. The cost of their construction and operation is so very much less than that of incinerators ( $\mathrm{I}$ to 30 ), while their simplicity, mobility and quickness of installation are so much greater that, although theoretically inferior to them as a means of excreta disposal, they are to a large extent superseding them, experience having shown that they can be safely and successfully used for long periods of time in permanent camps.

Latrine boxes should be designed according to uniform standards, so that they may be readily constructed wherever needed. They should be light and portable, yet strong and tight-jointed so as to endure rough handling and exclude insects. The seat holes must be provided with self-closing lids so that they may never be left open, and also to prevent men from squatting on the seat instead of sitting down during defecation. The principal reason for not leaving the holes uncovered is that flies and other insects may not enter. Therefore if flies are absent, as in winter and early spring, and the latrine is sheltered from rain, lids may be dispensed with.

Several types have been advocated. The simplest is a row of seats with lids, the sides and ends consisting of cotton sheeting extending to the ground and secured by tent pins. This skeleton box can be made folding or knock-down for transportation.

The knock-down latrine box devised by the writer is io feet 6 inches long, I6 inches high and 3 feet 8 inches wide at bottom so 
as to safely cover a pit 3 feet wide (Fig. 212). The sides or walls have an outward slant of 4 inches and are locked together by the end pieces and two traverses. The top consists of two longitudinal halves, simply laid on and kept from slipping by blocks; each $2 \mathrm{I}$ inches wide, projecting 2 inches beyond the side, and perforated by 3 holes which alternate with those of the other half. Each hole, I I inches long, is covered with a strong hinged lid which can only be raised to an angle of $60^{\circ}$, so that it is self-closing and prevents standing on the edge of the box.

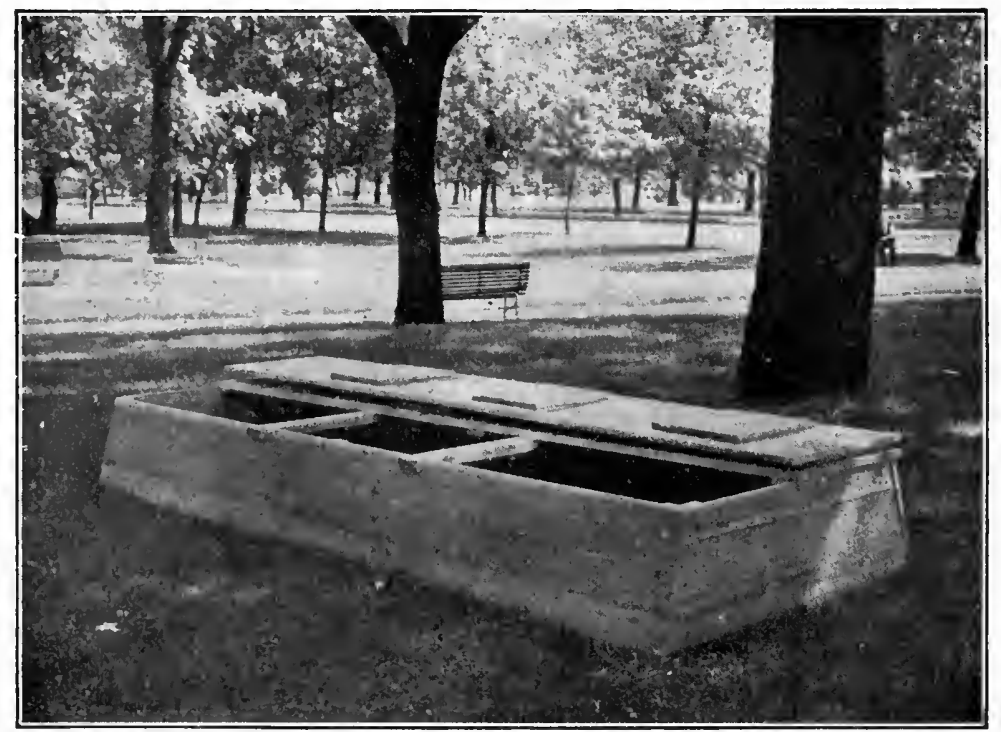

FIG. 212.-The Havard knock-down box, with one-half of the top shifted over the other half for inspection and disinfection.

Each box consists of 8 pieces perfectly interchangeable with those of any other box, and weighs I 75 pounds. It is put together without screw, bolt or hook, or taken apart, by one or two men in a few moments. 'To disinfect the pit, the attendant lifts the half of the top nearest to the earth pile and shifts it over the other half, thus uncovering the pit and obtaining a good view of the contents. One pit Io feet long covered with this box will do for a company for a week or two; if the stay is to be longer, the pit should be of the standard length of 20 feet and 2 boxes used, end to end. 
This knock-down type of box has never been tested on a sufficient scale to determine what its real merits in the field may be, or how it could be perfected. The present tendency of our military hygienists is in favor of a solid, nailed-together box, with a single row of holes. One of this type, devised by Major P. F. Straub (Mil. Surg., Dec., 1911), was used in the San Antonio camps, in 1911, with very satisfactory results. It is Io feet long, with 5 holes, made of tongue-and-groove lumber and designed to cover a pit 2 feet wide. The vertical front is 18 inches high, and the top 20 inches wide; the back slants outward so as to increase the bottom width to 30 inches. The circular holes are 9 inches in diameter, and covered by a solid lid 12 inches wide, reinforced by two battens ( $I^{\prime \prime} \times 2$ ") to prevent warping and to which the hinges are fastened. Longitudinal strips ( $I^{\prime \prime} \times 2^{\prime \prime}$ ) on the rear edge prevent the lid from being raised to a right angle, so that it falls back automatically.

Major Wm. Lyster (Mil. Surg., May, 1912) proposes a similar box but differing in several particulars (Fig. 213). It is only 8 feet long, with 4 holes, and is provided with a grip at each end for convenience of handling. The top is 18 inches wide, with slope of $\mathrm{I} / 2$ inch to the rear to drain rain and wash water. The circular holes are II inches in diameter. The lid is extended forward, flush with the top edge so as to keep the seat dry, and has a block nailed on the upper side to prevent its opening to a right angle. A block $\left(2^{\prime \prime} \times 3^{\prime \prime}\right)$ is nailed at each end of the upper edge in front, so that when the box is turned over, this edge may not be soiled or scratched. A piece of tin, 8 feet by Io inches, is fastened by its upper edge to the inside of the front wall, opposite each seat, and set at an angle that causes the urine projected against it to fall clear into the pit. An important adjunct to the Lyster box is a frame upon which it is set, so as to make the contact with the ground closer. To hold the box on the frame, a strip of board, 4 inches wide, is nailed one inch inside the lower edge of the box, thus projecting 3 inches clear, and snugly fitting inside the frame. It is certain that such a frame renders the box more completely fly-proof and protects the edges of the pit from wear and tear; on the other hand, it adds materially to its weight and complexity and may interfere with the proper aëration of the pit.

The Q. M. Corps, availing itself of previous investigations by medical officers and others, has published the plans of its standard 

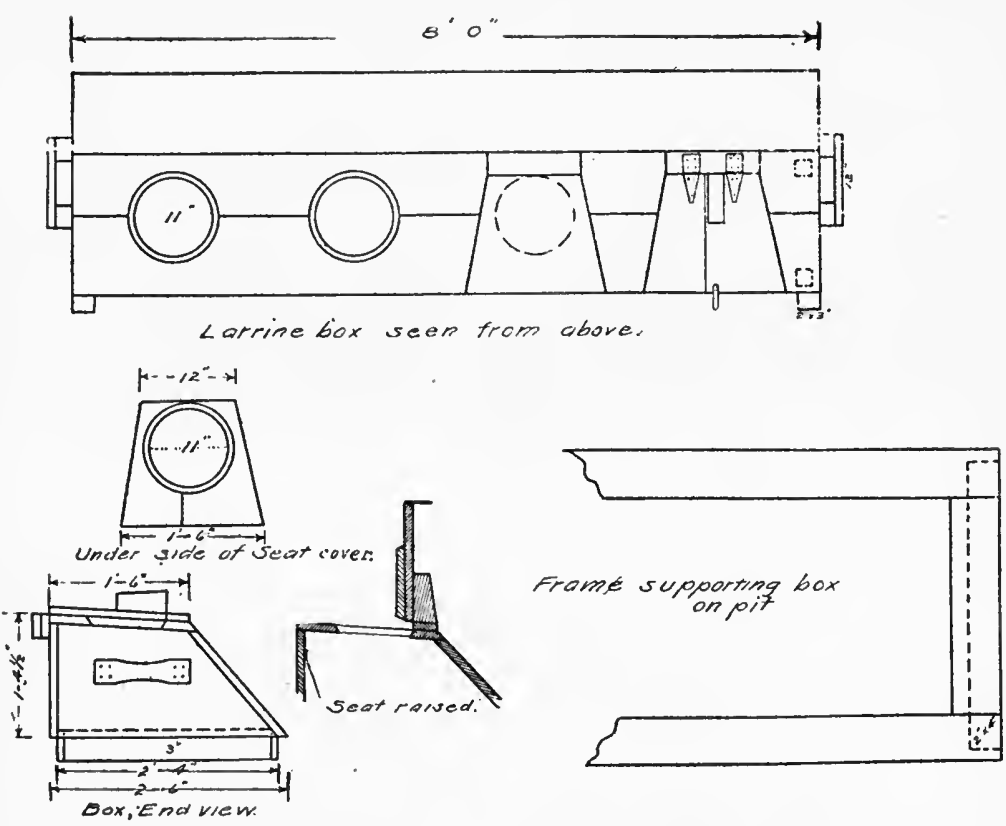

FIG. 2I3.-The Lyster latrine box.

latrine box (Man. for the Q. M. Corps, I9I7), combining most of the desirable features hitherto recommended (Fig. 2I4). This box rests on a frame, and has 4 seats. It is 8 feet long, I 7 inches high and 22 wide on top. The holes are oval in shape, Io by 13 inches. The lid is 14 inches wide, bearing an oval piece beneath made to fit the hole, and prevented from opening to a right angle by a block nailed to the upper side. A piece of tin, 7 feet by ro inches, is fastened to the front wall as advised by Lyster. (Fig. 2I5.)

Some general desiderata may be thus stated: All boards used should be well seasoned, tongued and grooved, and of a uniform width of 6 inches: the timbers to be 2 by 4 inches. The best shape for the seat hole is not circular but ovoid, 8 by I I inches in size. The lid should be 12 inches wide, of one solid piece or of two pieces, and stiffened by one or two battens to prevent warping. To make it self-closing, the easiest way is by the use of a stop block, as in the standard box. Finally, it should be possible to secure the lid so that when the box is turned over for disinfection of the pit, or 


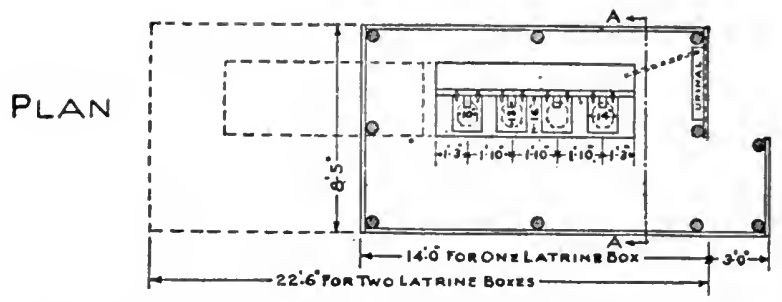

FIG, 214.- Standard latrine box. Quartermaster Corps.

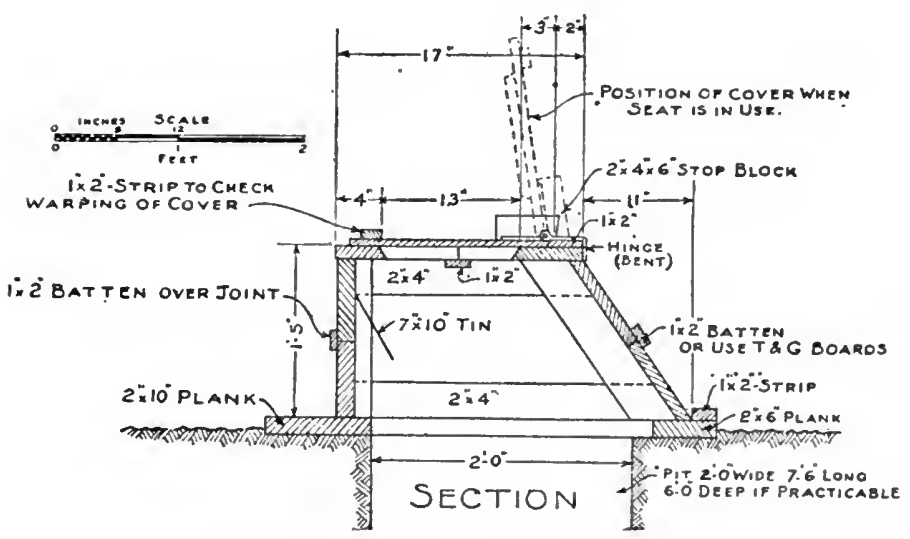

FIG. 215.- Section of standard box.

carried about, it may not swing open. This, with lid flush with the upper edge, as it should be, is easily done by means of a hook (on box) and small screw-eye (on lid).

The ventilation of the boxed latrine also deserves attention. Good ventilation is necessary to favor the work of the aerobic bacteria and thus prevent the production of putrefactive odors. It also helps the evaporation of moisture, preventing its condensation on the under surface of the lid and the edges of the holes. Therefore it is not desirable to make the box, or its contact with the ground, impervious to air, but simply tight enough to exclude flies. With efficient disinfection, special ventilation is seldom called for; when this becomes desirable on account of unpleasant odors, a good method is to cut a round hole, 4 inches in diameter, at or near the center of the top, guarded (when not used) by a sliding lid, and admitting a tin pipe 5 or 6 feet high. This pipe should be screened 
at the top and bear a flange about 6 inches from the lower end to keep it from sliding further down. A pipe of tar paper may also be improvised, with a piece of loose-meshed muslin tied at top. A simpler method of ventilation, and perhaps as efficient, is to provide a screened opening, 6 inches wide and long, in each end of the box.

Inasmuch as a latrine box does not permit direct urination into the pit (outside of defecation), some special urinal must be provided outside of it. The best device is a galvanized-iron trough 6 or 8 feet long, supported by cross sticks. At one end of it is a projecting collar upon which fits the outlet tin pipe. This pipe should run down, under the edge of the box, into the pit, rather than through a hole cut in the end of the box. Tar paper or painted muslin are convenient materials with which to improvise urinals and pipes, the urinals in the shape of troughs supported on forked sticks or of funnels suspended from tripods. Wooden boxes and gutters may also be available. If none of these materials be at hand, a shallow trench, partly filled with stones or gravel must be used.

Urine or water in latrines interfere more or less with the action of nitrifying bacteria and favor putrefactive decomposition; this is particularly the case when earth is mostly relied upon for disinfection. It is therefore preferable, whenever convenient, to separate the urine from the feces. This is readily accomplished by running the outlet pipe of the urinal into a hole dug with the post-hole auger, the lower end of the pipe fitting through an opening in a board covering the hole. A pit urinal (see page 652) may also be used.

Police and Disinfection.- In order to promote personal cleanliness and prevent as much as possible the transmission of infection by personal contact, the men should be enjoined to use paper in latrines, preferably the ordinary thin toilet paper. The Regulations provide for the issue of toilet paper in camps whenever practicable. The use of paper, however, especially light tissue paper, requires great care; the least breeze will often prevent its falling into the sink, while a stronger wind may blow it up and scatter it over the camp grounds. This is much less likely in boxed than in open sinks.

Another important measure to prevent fecal matter dissemination is to require the men to wash their hands, after defecation, before leaving the latrine shed. For this purpose, the attendant (whenever practicable) should have a bucket of clean water, a couple of basins 
and soap always in readiness, each man after washing emptying his waste into the urinal. When an infectious disease is present in camp or vicinity, it will be wise to dip the hands, before washing them, into an antiseptic solution of formalin, cresol, corrosive sublimate, etc. During one of their wars with the natives in South Africa, German troops were required, after defecation, to soak their hands in an antiseptic solution and, after washing, to wipe them with dry sand. This care of the hands will be greatly facilitated by locating the lavatory as close to the latrine as possible.

Strict cleanliness and efficient disinfection will prevent much of the danger lurking in the open sink. In the first place, it should be in charge of a civilian scavenger, or enlisted attendant, permanently detailed if possible and held strictly responsible for its sanitary condition. Every man using it must be required to cover his discharges with a shovelful of earth. Furthermore, the attendant twice a day, or oftener if deemed necessary, should make an examination of the contents and throw earth on any part of the surface not properly covered, not neglecting the fecal matter adhering to the walls. When earth is not available, lime may be used, but it should be one or the other, not a mixture of both. Earth is a deodorant and disinfectant; it absorbs offensive gases to a remarkable extent and brings about their oxidation and transformation; it promotes the multiplication of saprophytic bacteria and nitrifying organisms which feed actively upon all available organic matter and destroy it, with the result that they themselves speedily perish from lack of nourishment. Lime acts by destroying these organisms or preventing their propagation; therefore, if mixed, these agents would partly neutralize each other and lose much of their efficiency.

The attendant should daily scrub the seat with soap and water, and carefully police the edges of the pit and urinals, sprinkling lime wherever fouling has taken place. One of his chief duties will be to see that toilet paper escaped from the latrine is promptly returned to it or otherwise safely disposed of. The easiest solution of this troublesome difficulty is, occasionally, to light a small open fire in which all loose paper, caught with a pointed stick, is readily consumed.

Firing of Pits.- Of late years, disinfection by fire has been found so satisfactory as to be always preferred whenever applicable. The method is as follows: At about 9 o'clock A. M., after a majority 
of the men have visited the latrine, the box being lifted to one side, a layer of straw, grass or hay (Io or 15 pounds for a pit ro feet long) is evenly spread over the contents, sprinkled with a gallon of crude petroleum and set on fire. The hot blaze destroys all the germs lying near the surface of the excreta as well as on the sides of the pit, and completely removes all odors. It tends to keep the flies away, and prevents their laying eggs on the scorched and hardened feces. All loose toilet paper is also burned up. Straub believes that still better results would be obtained by using oil alone, in increased quantity (by an additional quart), as the residue from burned hay or straw fills the pit uselessly. The effect of the firing decreases in the afternoon, and odors, especially on warm days, are likely to be again noticeable; then a liberal coating of lime is recommended, or of fine dry earth. Crude petroleum or diluted formalin sprinkled into the pit is useful at any time as disinfectant and insectifuge.

The box itself demands attention. Before being replaced upon the pit the interior is whitewashed with milk of lime so far as may be needful. The seats must be daily scrubbed and kept scrupulously clean.

Spraying of Pits.- With the recent great increase in the size and number of our camps there has been a decided tendency to reduce the complexity and cost of our sanitary methods. The firing of pits requires a notable expenditure of time, work and material, and efforts have been made to obtain the same results in a simpler and cheaper way. Recent experiments have shown that this is possible.

Major Montgomery, N. G. N. Y., was one of the first medical officers to show that spraying the pit with a mixture of kerosene and lamp-black, without burning, is all that is required. Boneblack is cheaper than lamp-black and can be substituted. It is well known that black is a deterrent to flies and that they will not light upon a surface so treated.

Major Miller, M. C., U. S. A., describes this new method as follows (The Mil. Surgeon, May, 1917):

"In the El Paso district the practice of burning out latrine pits has been entirely discontinued. The routine now carried out is as follows: When the latrine is first established the inside of the box and the sides of the pit are thoroughly blackened with a mixture of I pound of bone black in 3 gallons of crude oil For this purpose 
a spray pump is issued to each regiment. This application to the sides of the pit and to the inside of the latrine box is repeated once in ten days. In addition to the above the contents of the pit are thoroughly covered each day with $1 \mathrm{r} / 2$ gallons of the boneblack-oil mixture, using an ordinary sprinkling pot for the purpose. With a reasonably tight box, flies will not enter a pit so treated. Provided the pit is not used as a receptacle for waste paper and other rubbish it will not be so quickly filled under this treatment as when burning is resorted to. Another advantage of this method of latrine management is that it is applicable to pits that are shored up with lumber to prevent caving and to those containing water either from seepage or flooding. Some experiments have been conducted to determine whether bone black is necessary in the oil used for the daily sprinkling. Definite conclusions have not been arrived at in regard to this point."

It is quite probable that further experience will show that the amount of oil used in the daily sprinkling can be materially reduced. Thus Major Montgomery obtained excellent results with half a gallon or less.

It is also believed that incineration destroys the nitrifying bacteria to a much greater extent than simple spraying, so that the generation of ill-smelling gases is more likely in the former method than in the latter.

Urinals can be disinfected with lime, but scrubbing with crude petroleum is much more effective.

When a sink is filled to within I8 inches of the top, it should be discontinued and filled up with earth; this should be piled a few inches above the surface to allow for sinking.

The fire or spray disinfection above described requires large quantities of oil and is therefore only applicable to more or less permanent camps. With moving commands, latrine boxes will generally have to be abandoned; such dry fuel as locally available may be used, without oil, but, as a rule, no other or better disinfectant will be at hand than the earth dug out of the pits.

Objections to the pit latrine.-These are obvious. We can assume that pathogenic germs may be present at any time in its contents, with resulting pollution of the soil and subsoil, and contamination of neighboring water-courses. The germs of typhoid fever in contact with soil and fecal matter may, under favorable 
conditions, multiply or at least remain alive for a period of several weeks. The pole used as seat is much exposed to fouling, and so are the edges and immediate surroundings of the trench, often wetted by rain and offering favorable conditions for the rapid growth of various pathogenic germs. One of the chief dangers of the open pit is its free accessibility to flies which may thus easily transmit infective matter to the kitchen and mess tents. Toilet paper blown out of it is also a constant menace.

Most of these objections are practically eliminated by the use of a properly constructed box, and careful disinfection. In the absence of incinerators, latrines, therefore, must always be boxed, if possible, and open sinks used only as a necessary evil.

URINALS.- Whatever form of latrine is used, it is essential that a special night urinal be provided. During the day there is no great danger of any one urinating outside the latrines, for fear of discovery, but at night, careless men are strongly tempted to relieve themselves as near their tents as is convenient. An iron can should be placed in each company street as urinal, with a red lantern over it, so that no one may have an excuse for polluting any part of the company grounds. This can should be emptied and cleaned out at reveille. During the day it may be kept near the latrine and used to collect refuse.

Whenever urinals are deemed necessary, apart from latrines, as, for instance, when the latter consist of narrow and shallow trenches, the post-hole system as described above is generally available and sanitary. A urinal can always be quickly improvised by digging a circular pit, 3 feet deep, and filling it a little more than half full with stones, clinkers, ashes, gravel or loose earth.

The urinal recommended in the English Army, for a battalion, consists of a pit and trenches. The pit, 3 or 4 feet in diameter and depth, is loosely refilled with the excavated earth or, much better, half filled with large irregular stones, tin cans or burned rubbish, covered over with coarse gravel and then earth well rammed down. Into this pit, run two trenches (one on each side) filled with small stones, each 12 feet long and 2 feet wide, with gradient of one inch to the foot. All the urine is passed into the trenches, the pit being protected, if needful, by a fence or screen. As soon as the trenches become offensive they are filled up and others dug. The urine is led from the trench to the bottom of the pit by a loosely jointed pipe 
of tin cans running diagonally down to the further end. The covering of earth on the pit keeps flies away and prevents odors. The weak points of this system are the extent of the trench exposed to flies and liable to give off smells, and the splashing of the urine with pollution of the ground on both sides of it. These objections can be mitigated by a daily firing with crude petroleum, or removed by the use of tin or wooden troughs, easily disinfected.

Post Holes. - This method of disposal, first advocated by Major William Jepson, I. N. G., has never been extensively used in our camps but, wherever resorted to, has given satisfactory results. It consists in digging a series of holes, about 3 feet apart, 8 or 9 inches in diameter and 7 to 8 feet deep, with an ordinary post-hole auger. A top, 18 inches wide, with suitable seat openings is set over them at the proper height, each opening corresponding to a hole and bearing a circular guard of tar paper, extending nearly down to the edge of the hole, to prevent scattering of urine and feces. These guards should be frequently renewed, and, when removed, burned. On each side of the top, extending to the ground, is a strip of muslin. In the absence of flies neither muslin nor lids are necessary.

This latrine, even without the use of earth or disinfectant, is said to be remarkably free from offensive smell. It also permits a greater degree of privacy, owing to the distance between the holes and the facility of isolating them by screens. Its use may be impracticable in rocky or damp ground, but in most soils it is a cheap, cleanly and safe method of disposal.

\section{SANITARY TROUGH LATRINE AND OTHER SYSTEMS.}

Several improved systems of disposal for permanent or semipermanent camps are or have been in use in our service.

The sanitary trough latrine was one of the remarkable results of the investigation of the medical board appointed to determine the causes of the typhoid fever epidemics which decimated our camps in 1898. This board, consisting of Major Walter Reed, Major Victor C. Vaughan and Major E. O. Shakespeare, soon realized that the open sinks were largely responsible for the prevailing infection and recommended a system whereby all excreta are disinfected and removed from camp.

The trough latrine (as described in specifications from the office 
of the Chief Quartermaster Corps, January, I908) consists of a small, well-ventilated frame building with doorless opening at each end, containing the trough and urinal. The trough, of galvanized iron, is 14 feet long and 22 inches wide at top, with sloping bottom 15 inches deep at upper end and 18 inches at lower end; it is para-

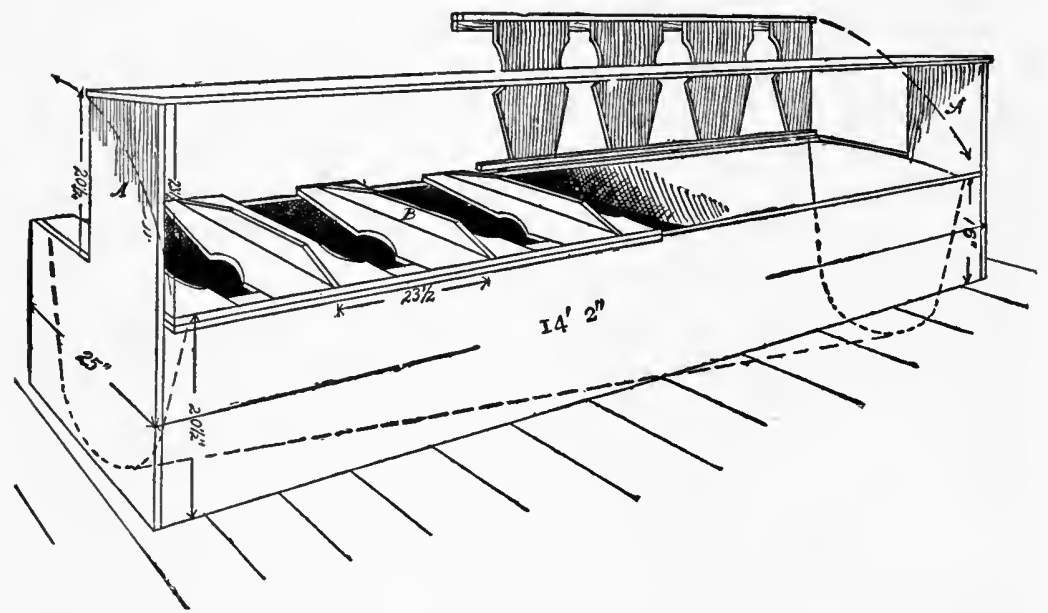

Fig. 2I6.- Perspective view of latrine trough; section of lid open.

bolic in cross section so as to present a curved surface throughout, thus avoiding corners in which the contents could collect and facilitating emptying and cleaning. (Fig. 216.) The seat has 7 openings and is made of 8 separate pieces hinged to a board in rear. The openings are $8 \frac{1}{2}$ inches in diameter, properly bevelled on edges, with cuts in front and rear to prevent fouling, the rear cut widening to I4 inches at back. At each end of the seat, and flush with it, is set up an upright $32 \mathrm{~T} / 2$ inches high; on these uprights is nailed a board to prevent standing on the seat. (Figs. 216, 217.)

The urinal is a galvanized rounded steel trough placed against the rear end of the building in line with the latrine trough, 2 feet 6 inches above the floor. It is 8 feet long, 8 inches wide and 4 inches deep, the rear side extending I 8 inches upward to protect the wall. It has a fall of 5 inches and is connected to the latrine trough by a 2 -inch galvanized steel pipe.

When ready for use, water should be poured into the latrine until it has a depth of at least 2 inches at the upper end. To this is then 


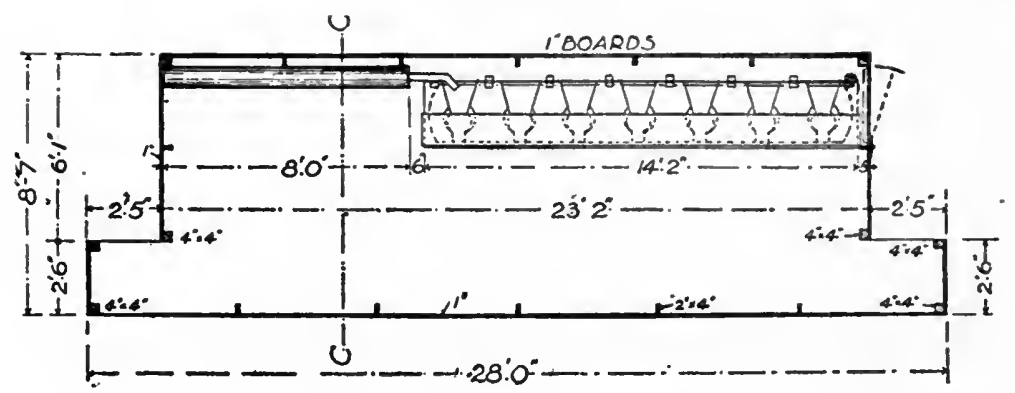

FIG. 217.- Ground plan of trough latrine showing seats and urinal.

added one-sixth barrel of lime and the two well mixed with a wooden paddle. Lime should also be freely sprinkled in the urinal. By this means not only do the excreta immediately fall into a disinfectant solution, but the urine is also mixed with lime prior to falling in the latrine. To better provide for the disinfection of the excreta, the contents of the latrine should be stirred with a wooden paddle two or three times a day.

I.ime is liable to clog the hose and has no deterrent effect upon flies; it is now generally replaced by crude petroleum, one quart in the vater.

Once or twice a day the trough is emptied by the "orderless excavator." This consists of a wagon bed supporting a water-tight tank having a capacity of 500 gallons, and a pumping apparatus mounted on a pair of wheels which track with the wheels of the wagon. For use, two lengths of hose are connected with the pump, one at each extremity; one free end is placed in the lower part of the trough through a hinged door in the wall of the building and the other free end attached to the pipe on top of the tank. The pump is rapidly worked by two men. As soon as the trough is emptied, the hose are disconnected from pump and tank, great care being taken that the ends of the hose and the openings of the pump are securely closed by the couplings or valves provided for the purpose, so that none of the contents be spilled on the ground. In camp or on march the pump truck is hitched to the rear axle of the wagon. Each excavator requires the services of 3 men and disposes of the excresta of 2 or 3 regiments.

The sanitary trough latrine possesses decided advantages. It is very convenient for the men. It need not be more than 20 or 25 
yards from the tents and may be placed wherever desired without regard to the water-supply, character of soil or depth of ground water. The general rule governing the location of latrines, that they should be as far as possible from the kitchen, is not as inflexible with this system as in the case of pit latrines.. From the moment the excreta reach the trough they cease to be dangerous. Flies are less attracted than by open sinks and, if lids are used, can be entirely excluded. The floor, kept dry and hard, is much less likely to become polluted, while toilet paper does not readily escape from the trough. On the other hand, this system requires a heavy and costly material and is only applicable to cantonments and permanent camps. It would also be useless in very cold weather with the temperature below freezing-point. Water and plenty of lime or oil are necessary. Furthermore, after the trough has been emptied by the excavator, there still remains the question of final disposal which may be a troublesome one. It sometimes happens that this very heavy vehicle becomes useless in sandy or miry roads.

Everything considered, it is now the general opinion that wherever the trough latrine can be used, incinerators will be found equally practicable and more efficient.

\section{EARTH-CLOSET LATRINE.}

In camps where the trough latrine is not applicable or available, the earth-closet latrine has been provided. It consists of the same building, row of seats and urinal, but, instead of a trough, there is under each seat a galvanized steel box, I 8 inches wide, I4 inches deep, 2 inches long from front to back at top and 18 inches at bottom, the rear end being vertical and the bottom corners rounded. Each box slides out through a corresponding door upon a platform of joists extending 2 feet in rear of the wall. The contents of the boxes are disinfected with earth or lime. If lime is available, the boxes, before being used, are filled about one-fourth of their depth with milk of lime. The addition of a small quantity of crude carbolic acid forms a very effective disinfecting mixture. The boxes are taken out as often as necessary, emptied, washed and replaced. In cold countries or wherever there is danger of freezing, dry earth or lime is used.

The earth used should be the sweepings of roads, properly 
screened, or the top soil, sun-dried and pulverized. If kiln-dried, the nitrifying bacteria upon which its action depends would be destroyed. Sand, ashes and sawdust are more or less sterile and should only be used when nothing better is at hand.

Figs. 218 and 219 show an earth-closet latrine as above described. but on the knock-down system, that is, consisting of separable sections of siding, floor and roof. Instead of one urinal 8 feet long, there is one 4 feet 8 inches long at each end of the seats, a more suitable arrangement.

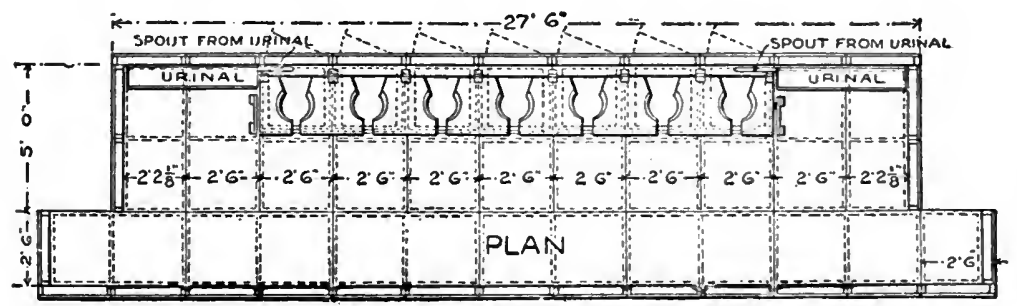

FIG. 218.- Ground plan of earth-closet latrine. Knock-down system.

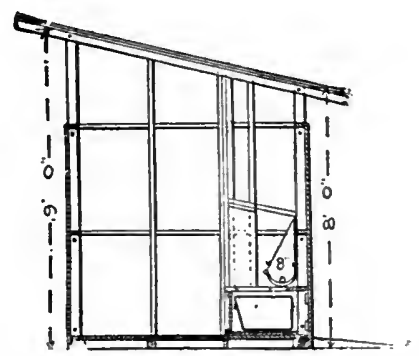

FIG. 219.-Cross section of Fig. 218. 


\section{CHAPTER LIII.}

\section{DISPOSAL OF EXCRETA BY INCINERATION.}

The ideal system of disposal is by incineration, that is, the destruction by fire of all excreta in the very pans in which they are received. With this system no water or disinfectant is required, and as the residuum of combustion is a negligible quantity no further or final disposal need be provided for. It is also applicable to any climate and extreme of weather.

This system, at one time highly praised and extensively used, is not now often seen in our camps. Its cost, difficulty of transportation and operation, and the amount of fuel required are serious objections which are often found to overbalance its advantages.

Of the several types of incinerators tested in our camps, the following appear to possess, in a higher degree, the qualities which best adapt them to military requirements.

The McCall Incinerator. - This is the first type of excreta incinerator used in our Army and has rendered invaluable services in trying situations. (Figs. 220-222.) It is installed in a knockdown building 22 feet 6 inches long in front and 5 feet wide. It comprises two sections at right angle to each other. Each section consists of a combustion chamber in a pit 24 inches deep, lined with brick (2), and of a steel box placed over the pit. In the combustion chamber are the two incinerator pans (25) in which the fecal matter is received. In the steel box, forming the top of the combustion chamber, are two hinged, wide plates (IO) and three narrow, fixed plates (9). Each box is covered with a wooden lid or seat (I3) in which 4 holes are cut, with covers closing automatically. Within the angle formed by the intersection of the two pits is a brick flue common to both. Upon this rests the smokestack base and upon the latter is placed the vertical smokestack (20). In the flue a grate is mounted (2I), forming a second or auxiliary combustion chamber (I8). A' urinal (22), with hinged lid, corresponds to each section. From it a pipe (I I), with valve (26), conveys the urine into the pan. The valve is only opened when the pans are fired. Another pipe $\left(22^{1}\right)$ connects the urinal with the smokestack for ventilation. 
For use, the two plates (10) are lifted up and the wooden seat is placed over the steel box. When the pans are full, the seat is removed and a fire of coke or coal made in the grate (2I); the plates (I0) are then lowered, thus forming a tight cover for the combustion chamber, and the pans are incinerated by burning wood in the pit underneath. This fire, however, is not to be started until the coke in the grate is thoroughly ignited and the auxiliary combustion chamber strongly heated; in this way all obnoxious gases are completely destroyed before they can escape from the smokestack.

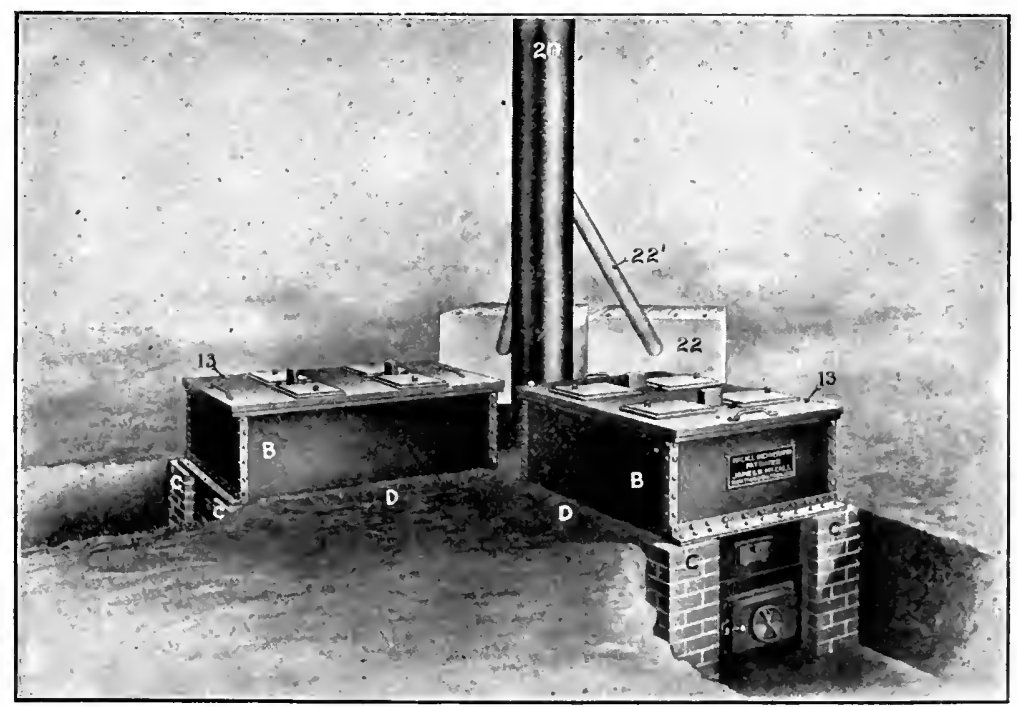

Fig. 220.- Perspective view of the two sections of the McCall incinerator.

After the solid matter in the pans is incinerated, the valve under the urinal is opened and the urine gradually discharged into the pan and evaporated. From 3 to 5 hours, according to size and quality of the fuel, are necessary to complete the incineration of excreta in one pit. Dry, hard wood, 4 feet long, is generally used as fuel. Each section is burned out once a day, or once every other day according to the strength of the company, the most convenient time being in the evening or at night, so that both sections may always be available during the morning hours. 
It is evident that the McCall incinerator, as above described, is only available in camps of some permanency. In order to dispense with the brick work which requires heavy material, skilled labor and time, and that it may be set up, ready for use, as soon as possible after reaching camp, the inventor, in his I9I I model, has replaced the brick pit by a steel fire-box lined with asbestos boards. (Fig. 222.) There is also an independent opening into the coke grate to stoke the fire without interfering with the main combustion chamber, and the urinals have been slightly modified so as to prevent dripping. Thus improved this incinerator, complete, weighs about

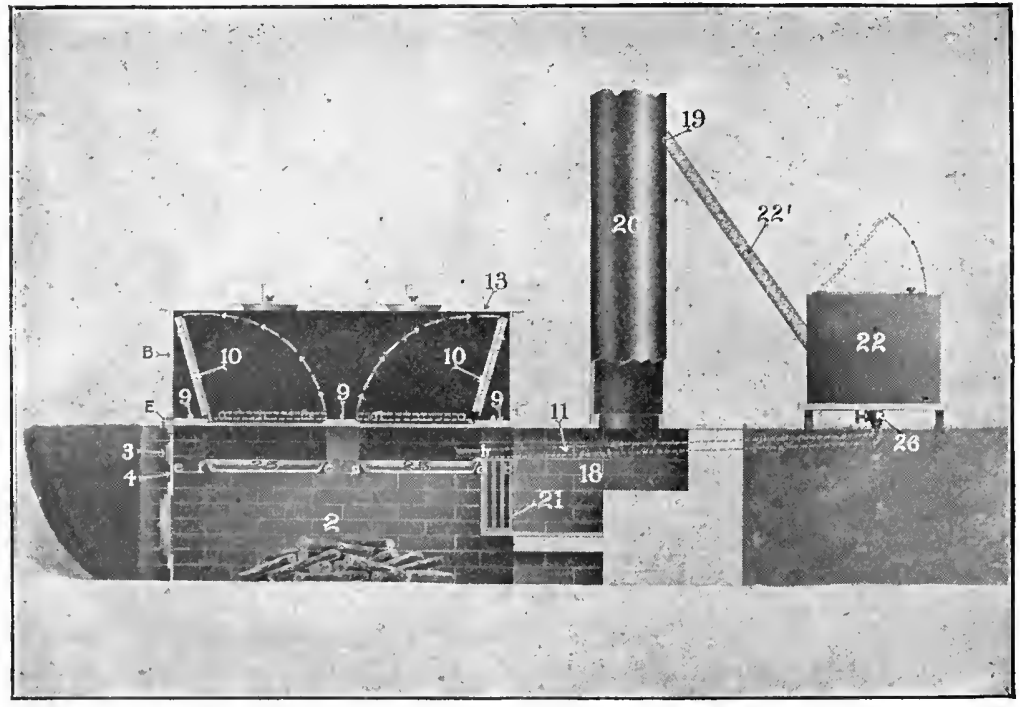

FIG. 221.- Longitudinal sectional elevation of the McCall incinerator.

$r, 800$ pounds; it can be knocked down in conveniently handled pieces, easily transported, and set up, ready for use, within half an hour after reaching camp. For permanent camps, however, the brick pit is still considered preferable.

A garbage crematory constructed on the same plan is also in use.

The Jones Incinerator. - Capt. Percy L. Jones, Medical Corps, drawing his inspiration from the McCall apparatus, has shown how an incinerator can be extemporized almost anywhere. A hole is dug in a side-hill, $2^{1 / 2}$ feet deep, 2 feet wide and $4^{1 / 2}$ feet long, with back 
and floor of brick or stone laid without mortar. If the soil has sufficient consistency or if the camp is only for a day or two this lining of stone is not necessary. It is best, however, not to dispense with the stone floor, always very useful for the evaporation of urine. One foot below the top, the sides have projecting shoulders upon which rest two of the McCall excreta pans. Over all is the boxed seat with 4 holes; this can be hinged for convenience of transportation. Brush or canvas screens may be placed around so as to make the pit fly-proof. This incinerator is likely to produce unpleasant

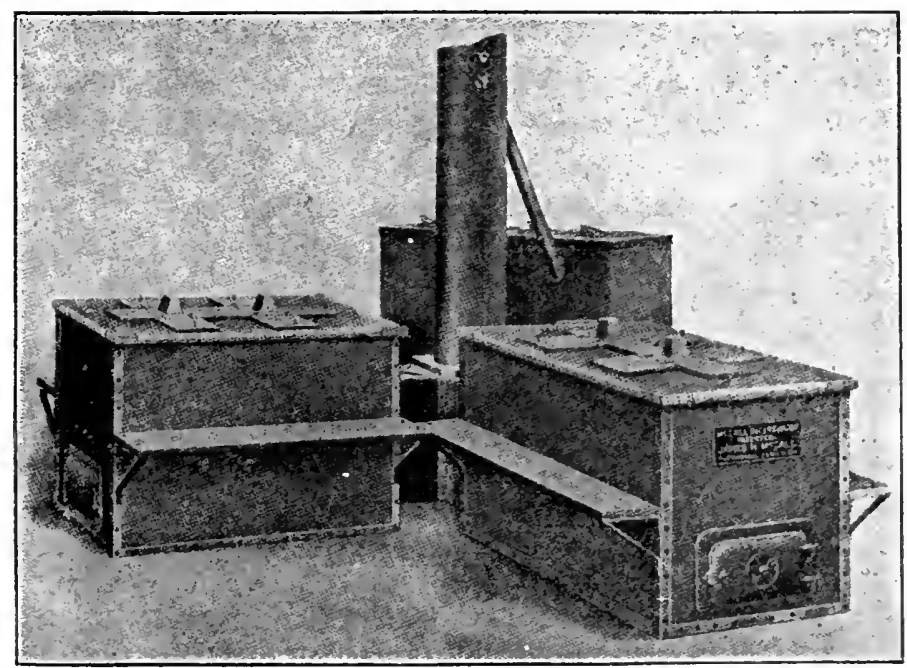

FIG. 222.- McCall Incinerator, Improved 19r I Model, No. 8 (portable).

odors when fired and should therefore be placed to the leeward of the camp. A detached can should be kept near by to be used as urinal, and occasionally emptied upon the heated stones. If this incinerator is only for one night, the iron pans can be dispensed with and replaced by wooden pans or boards which are consumed with the excreta.

Tine Harris Incinerator. - This incinerator (model igi I) consists essentially of a rectangular box and smoke stack. (Fig. 223.) The box is 7 feet 3 inches long, 21 inches high, 36 inches wide, and provides 8 seats. The entire outfit weighs 1,000 pounds. It is 
assembled in one piece, without other detachable parts than the stack, seats and covers, and rests directly upon the ground. The fire chamber extends the entire length of the box, the flame passing from it by means of a hood or flue (forming a projection in rear end) and returning over the excreta on the floor to the stack. The excreta fall directly on a slightly convex floor which is nothing but the roof of the fire chamber; it is depressed on each side into a gutter covered with a perforated guard; into this gutter the liquids drain. The urinal consists of a trough 3 feet long and extending 8 inches from the rear end of the box. The cover is raised for urination, the stream splashing against it, and closes automatically. The trough drains continuously into the gutters of the floor and is sterilized by the heat of each burning. From the above arrangement it is seen that the evaporation of urine and combustion of the solid excreta are simultaneous.

This incinerator has a capacity of 15 men per seat. It is usually

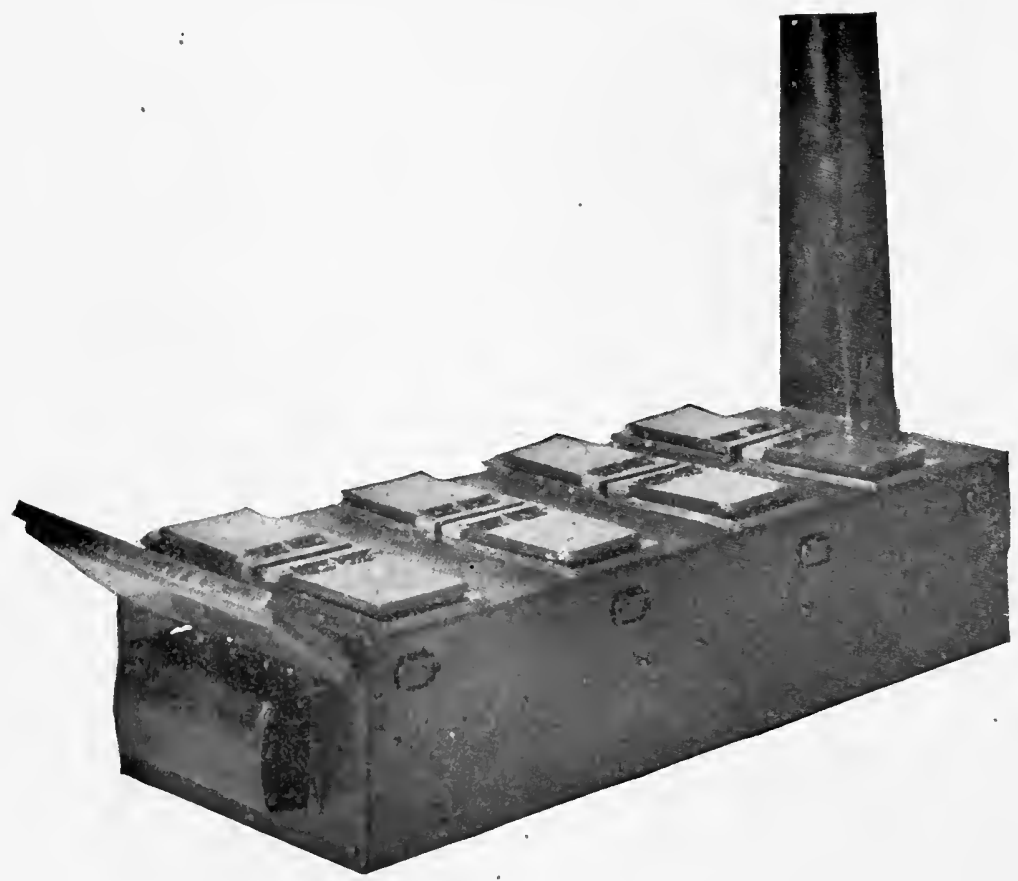

FIG. 223.- The Harris incinerator. Model I9I I. 
burned once a day and requires only $1 / 20$ of a cord of wood for each complete disposal of solids and liquids. Although without secondary combustion chamber, it is claimed that all offensive odors are as thoroughly consumed as in types of incinerators provided with it.

It has been very favorably commented on by official boards, especially for (I) its simplicity of construction and ease of operation, (2) its small weight and bulk, being lighter than any other incinerator with equal number of seats, (3) its economy of fuel, requiring less than any other type, and (4) its general efficiency.

A garbage crematory has also been constructed on the same principles.

The Q. M. C. Incinerator No. I, Model igi . - This excellent incinerator, devised by the $Q$. M. Corps, is 4 feet long, $30 \mathrm{~T} / 2$ inches wide and 26 inches high. (Fig. 224.) It is fitted with 4 seats, 2 on each side, and weighs complete about I,O0O pounds. The shell itself, without linings or trimmings, weighs 3 Io pounds. The top consists of 4 cast-iron panels, each with an oval opening in center closed with cast-iron lid. Over these are fitted 4 wooden seats, their holes corresponding to the openings in the cast-iron panels, and covered with perforated lids, the perforations screened with wire gauze. Inside are found, from above downward, segmented grate, drip pan and charcoal basket-burner. The pan is $461 / 2$ inches long, 25 inches wide and $2 \frac{1}{2}$ inches deep. The basket-burner is 6 inches deep, and has perforated sides, ends and bottom for draft. A urinal, with self-closing lid, is attached on each side of the smoke pipe without screw or bolt, and a galvanized pipe conveys the urine to the drip pan. Another pipe runs from the top edge to the smoke pipe, as vent.

To operate this incinerator, it is set on the ground, having no bottom. The cast-iron panels, without lids, are covered with the wooden seats. The large drip pan is slid in on lugs; being just under the heavy cast-iron grate bars it catches the urine and such fecal matter as passes through the bars. If pulled to the front as far as possible, the odors will escape freely to the smoke pipe. All drafts should be closed so that there will be a downward suction through the screened perforations in the lids, thus preventing heat and odors from escaping. When the pan is in position, the burner, in which a charcoal or coke fire has been started. is placed under it 
and furnishes ample heat to evaporate the urine discharged in the pan. This heat should be so maintained as never to make it uncomfortable for the men using the seats.

At night, or whenever desired, the wooden seats, drip pan and basket-burner are removed, the lids placed in the cast-iron panels,

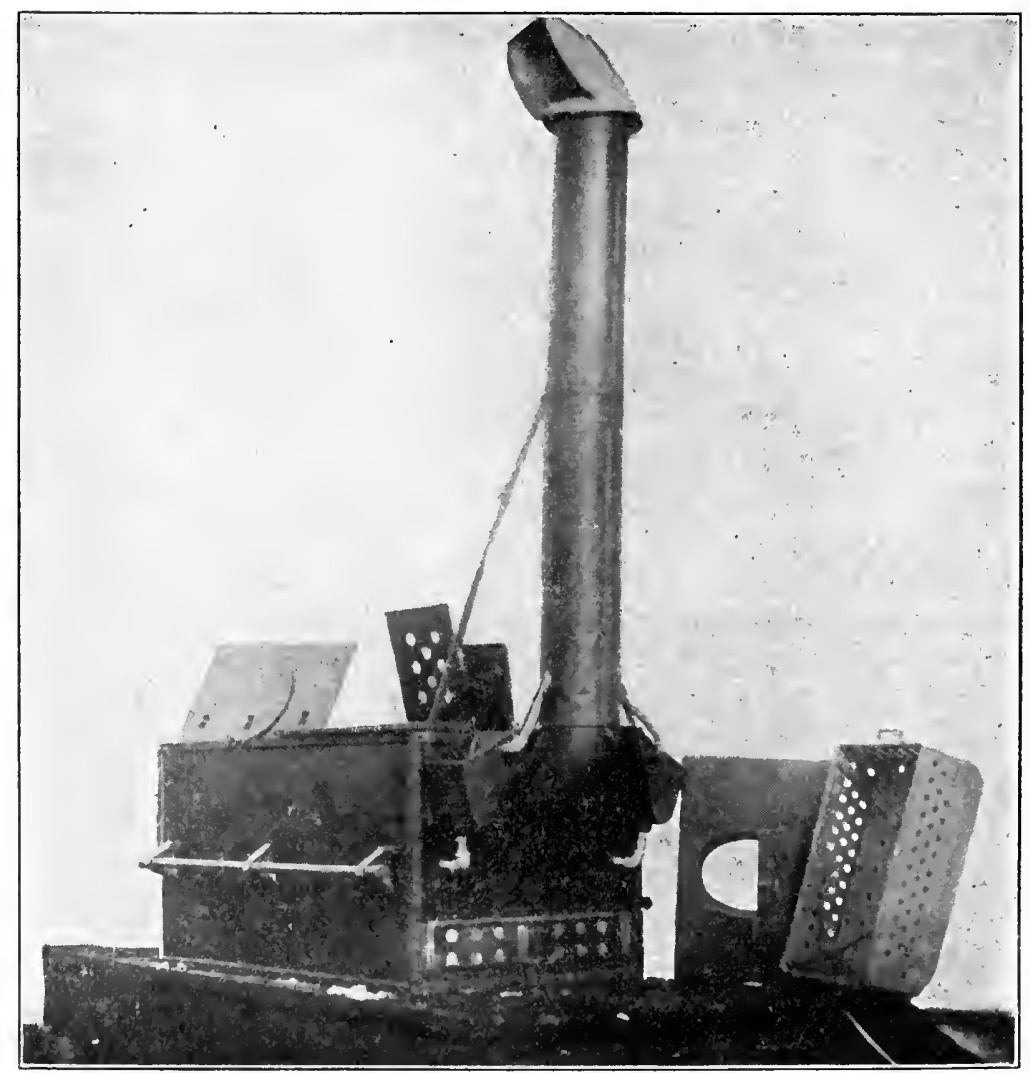

FIG. 224.-The Q. M. C. incinerator, No. I, Model I9II. Showing urinals, cast-iron lid, wooden seat and charcoal burner.

and a wood fire made under the grate bars. All excreta are thus thoroughly incinerated. Should the pan contain matters needing incineration it can be left in until this is accomplished.

This incinerator can also be used as garbage crematory by remov- 
ing any one of the cast-iron panels to dump the garbage on the grates, taking care that the liquid slops do not overflow the pan.

The Conley incinerator, as well as the Learis and Kitchin incinerator, have also been tried in some of our camps with more or less strccess, but have long since been discarded.

Remarks. - The incinerators described are also capable of disposing of a large proportion of the garbage of the camp, and there has been a tendency to develop them in that direction, that is to say, make them burn up garbage and liquid-wastes as well as excreta.

It is quite doubtful, however, whether such combination is desirable and yields the best results. The incinerator sheds, necessarily visited by all the men and always kept scrupulously clean, should not also become garbage pens. Excreta and garbage require somewhat different treatment and can be more conveniently disposed of separately. It is not believed that such separation would require more material or the expenditure of much more fuel, for appliances constructed for one special object can accomplish it more perfectly and economically than if designed for several purposes. It is also to be borne in mind that there are camp sites where only kitchen incinerators are required, the excreta being already provided for by sewerage; or vice versa, where kitchen incinerators already exist and only excreta incinerators are needed.

Ambulant Incinerators. - A system of ambulant incinerators that could follow moving troops and always be available as soon as camp was reached would be ideally perfect, but is obviously impracticable: from 150 to 200 such incinerators, including 600 to 800 mules, would be required for a division, an enormous addition to the train which would seriously hamper the mobility of the command. Furthermore, as they could seldom find their respective organizations at the end of a march, until much too late to prevent the contamination of the camp, they would fail of their purpose. There are doubtless circumstances when they might be serviceable. for instance in connection with summer camps where they would enable organizations to change sites readily and safely, or for small commands marching over good roads. 


\section{CHAPTER LIV.}

\section{LAVATORY.}

\section{DISPOSAT OF WASTES, GARBAGE, REFUSE AND MANURE IN CAMP.}

Conveniences for washing body and clothing must be provided as soon as practicable, especially water barrels or cans, wash tubs and hand basins. If the camp is to last more than a day or two, a place should be set apart in each company, not too near the kitchen, as a lavatory. It is not desirable to have the washing of linen and clothing done on the company grounds. One laundry shed for each battalion is sufficient and this may be located where most convenient with respect to the water-supply. Waste waters from lavatory and laundry should not be allowed to run on the ground and soak into it; they are not only unsightly and ill-smelling, but the most dangerous of all liquid wastes, being always the vehicle of numerous germs (see page 254). They should be emptied into suitable receptacles and carted to the rock-pile crematory or any special evaporator or incinerator used for the purpose. They may also be piped, or conveyed by a disinfected trench to a water-course, pit or distant gully.

The regulation field lavatory for one company consists of a knockdown building 25 feet long in front and 8 feet 6 inches wide, containing 5 shower baths and I cast-iron wash sink ro feet long (in 2 sections), enamelled inside, with 4 nickel-plated compression faucets. When intended for the use of a battalion it is extended in length so as to accommodate 4 two-section sinks and I 5 showers.

This lavatory affords no facilities for the washing of clothing or linen and is so far defective. It should contain 4 wash tubs, or else provision should be made for a battalion or regimental laundry.

A simple sanitary arrangement is the following: Make a hard and impermeable floor, if not naturally so, in the manner described for tents (page 6I5). This floor should preferably have a slight slope. Upon it place a wooden grating, and provide benches for basins as well as for washing clothes. On the lower side, a trench four inches deep and a foot wide is ditg and filled with stones, lead- 
ing to a soakage pit filled with stones, as for urine disposal (see page $\left.65^{2}\right)$.

Another arrangement, sometimes seen in our camps, consists of two wide boards set on trestles, Io inches apart and slightly slanting towards each other so that the water may drip down the space between them. The trench should be directly below this space and have a sufficient gradient to insure good drainage.

The proper treatment of garbage in any place is facilitated by the separation of solids and liquids. This is readily accomplished in camp by means of a boxed screen placed in the mouth of the barrel intended for liquid slops, and only allowing liquids to drain through. This screen should have a projecting rim or flange so as to be supported by the edges of the barrel and easily removed.

All solid garbage should be burned; no other disposition is to be considered whenever fuel is available. Destruction by fire gives absolute security. A garbage pit is highly objectionable and to be tolerated only when incineration is impossible. In such case it should be fired daily with crude petroleum, like the latrines. Weather permitting, solid garbage may also be spread on the ground to dry, then mixed up with inflammable refuse, like horse manure, and burned. For a few days there is no objection to its being thrown in latrine pits.

Kitchen Pit. - Liquid wastes from the company kitchen are best disposed of as described under Company Incinerator. If no method of incineration is available, a pit, dug near the kitchen fire, may be used as provided in the Field Regulations. A convenient size for it is about four feet in length and depth, but not over three in width, so that it may be easily roofed over with cross sticks, grass and earth. At the end nearest the kitchen should be a boxed opening, preferably funnel-shaped, with screened bottom, into which all liquid wastes are thrown, the screen retaining all particles of solid garbage. When not in use, the opening should be kept covered with a lid to prevent odors and exclude flies. A ventilating shaft or pipe may be placed at the other end, but is not essential. The contents will usually seep into the subsoil and seldom overflow; if necessary they can be pumped out by the excavator wagon.

This kitchen pit when properly constructed, in porous soil, is generally satisfactory: but as it is liable to generate offensive smells it is better to dispense with it whenever a better method is available. 
A more satisfactory means of disposal, in porous ground, is an ordinary hole, 8 or 9 inches in diameter, such as is readily dug with a post-hole auger.

One or two barrels, or galvanized-iron cans, with lids, should be supplied each company for its dry garbage, if not burned in the kitchen fire, and an additional one for liquid wastes if a pit is not used. The iron can which serves as night urinal is kept near the latrine and utilized to collect dry refuse during the day. Two more cans may be necessary for the lavatory and laundry wastes if these are not disposed of in some other way.

Sanitary Cart. - A special sanitary iron cart is sometimes supplied by the Q. M. Corps for the removal of garbage, liquid and solid. But it is difficult to empty slop barrels into such a cart without spilling, while leakage is also liable to occur as it jolts on the way, so that the vicinity of kitchens as well as company streets and camp roads are exposed to fouling from these causes. It would be much better to use it exclusively for dry or nearly dry garbage, and to remove all liquid wastes by the usual excavator wagon.

Company or Kitchen Incinerator. - The company kitchen fire, if properly prepared and managed, can be made to burn much of the garbage, liquid and solid, utilizing the latter as fuel. The advice given in the "Manual for Army Cooks" is excellent: "Burn everything [in camp kitchen fire] - coffee grounds, parings, bones, meat wastes; even old tin cans, for if thrown out anywhere, even buried. they attract flies; tin cans are fly traps; burned and cleaned out by fire daily they are harmless. Fires should be cleaned of burnt refuse once a day."

With an ordinary open kitchen fire it is quite practicable thus to dispose of much of the company garbage, but when the fireplace is constructed with a view to its efficiency as garbage incinerator, cooking over it becomes more difficult and requires special fixtures. The fire used in the field range can be utilized but only to a small extent for the burning of wastes.

The company incinerator consists essentially of a pit filled in with loose stones, as first recommended by H. A. Arnold, N. G. Pa. In its construction, care should be taken that the layer of rocks lining the floor is not more than a foot deep, inasmuch as this is about the depth to which the heat penetrates. Liquids gravitating below this level are liable to ferment and become foul when the ground is not 
sufficiently porous, especially should the fire be discontinued for a few days during the temporary absence of the company; in this case the pit may also become a breeding place for flies. Economy of time and material demands that the excavation be not any deeper than necessary.

The type finally developed at the maneuver camps of San Antonio, Texas, in I91 I, by Major P. E. Straub, and since then generally used (Fig. 225), consists of a bed of rocks, level with the ground, I foot deep, 3 feet wide and $4^{1} / 2$ feet long, surrounded by a sloping stone wall, I 8 inches high, except at one end which is left open for draft, fuel and access to the fire. The stone wall absorbs much of the heat which would otherwise be dissipated into space, and increases enormously the evaporating capacity of the incinerator.

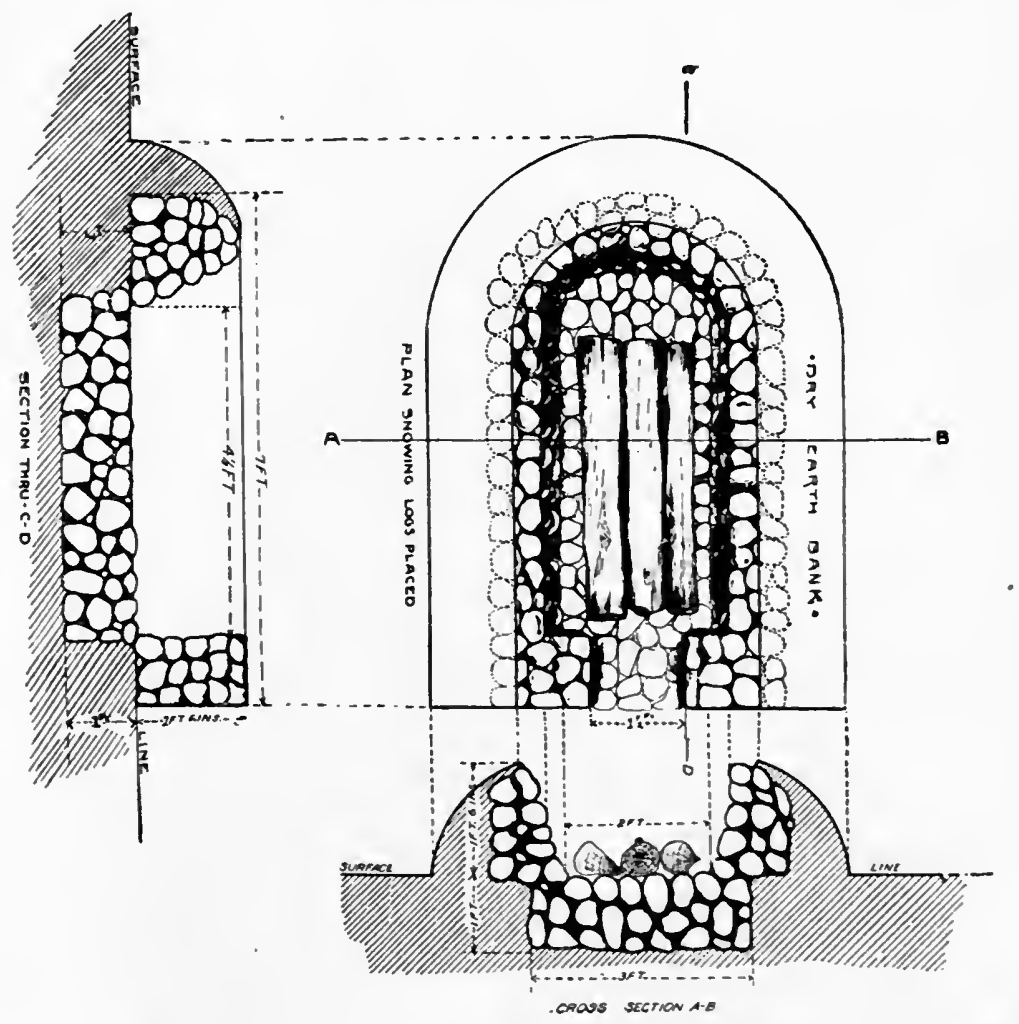

FIG. 225.- The Straub garbage incinerator. Model I9II. 
Large stones (cocoa-nut size) are much better than small ones; as to composition, any kind will do, but limestone which disintegrates and flint which explodes are not as good as sandstone. During a rainy season it would be advantageous to raise the rock bed a few inches above the ground and bank it around with earth to prevent flooding.

Straub found that, with ordinary care and attention, about $\mathrm{I} / 6$ of a cord of wood per day per company was more than sufficient for the destruction of all slops and garbage. Careful experimentation showed that by using this amount of wood, a skilled attendant could destroy Ioo gallons of liquids and 23 cubic feet of solid garbage in about I 2 hours. The liquid slops are evaporated by being poured slowly along the sloping walls of the incinerator, frequently but only a few dipperfuls at a time, while the solid garbage is placed over the firebed, on top of the fuel. When the liquids are excessive it may be expedient to partly evaporate them in a tin boiler. Tin cans and other incombustible material are raked out as often as necessary. It is a good plan to remove the stones making up the floor about once a week so as to clear the pit of ashes and débris. Cooking on such a rock-lined pit is made relatively easy by means of a long-legged spider, such as used by the militia of several States. Where field cooking ranges are used and the companies are small, it will often be sufficient to operate one incinerator for each battalion.

The Straub model is often simplified without much loss of efficiency. Thus, when stones are scarce, the banks of the pit can be built up of the excavated earth, well packed and tamped, without stones. Where the incinerator is only to be used for a few days and the soil is porous, the depth of the stone bed may be reduced to 8 or 6 inches.

Even when stones are lacking, a fire made in the same manner between two walls of earth, in a shallow pit, will generally be capable of disposing of much of the company garbage, solid and liquid. In such case it is well to fill the bottom of the pit with tin cans, if at hand, as a substitute for stones.

Some experience is required before troops can successfully operate the kitchen incinerator, and sanitary inspectors will often need patient persistency to convince officers and men that this method of disposing of kitchen and company wastes in the field is the most practical, economical and efficient. 
The Guthrie incinerator (Fig. 226), adopted by the Qmr. Corps, consists essentially of a fire chamber and an evaporating pan. Its walls are of brick or stone, and a draft is provided by a Sibley stove and pipe. It is very effective for solid and liquid wastes, but requires special material not always on hand. Unless the evaporating pan is thoroughly scraped and cleaned daily a thick crust forms on the bottom, resulting in an increased consumption of fuel and in the destruction of the pan. An overflow hole in the end of the pan is recommended to prevent its contents from boiling over and polluting the surrounding grounds.

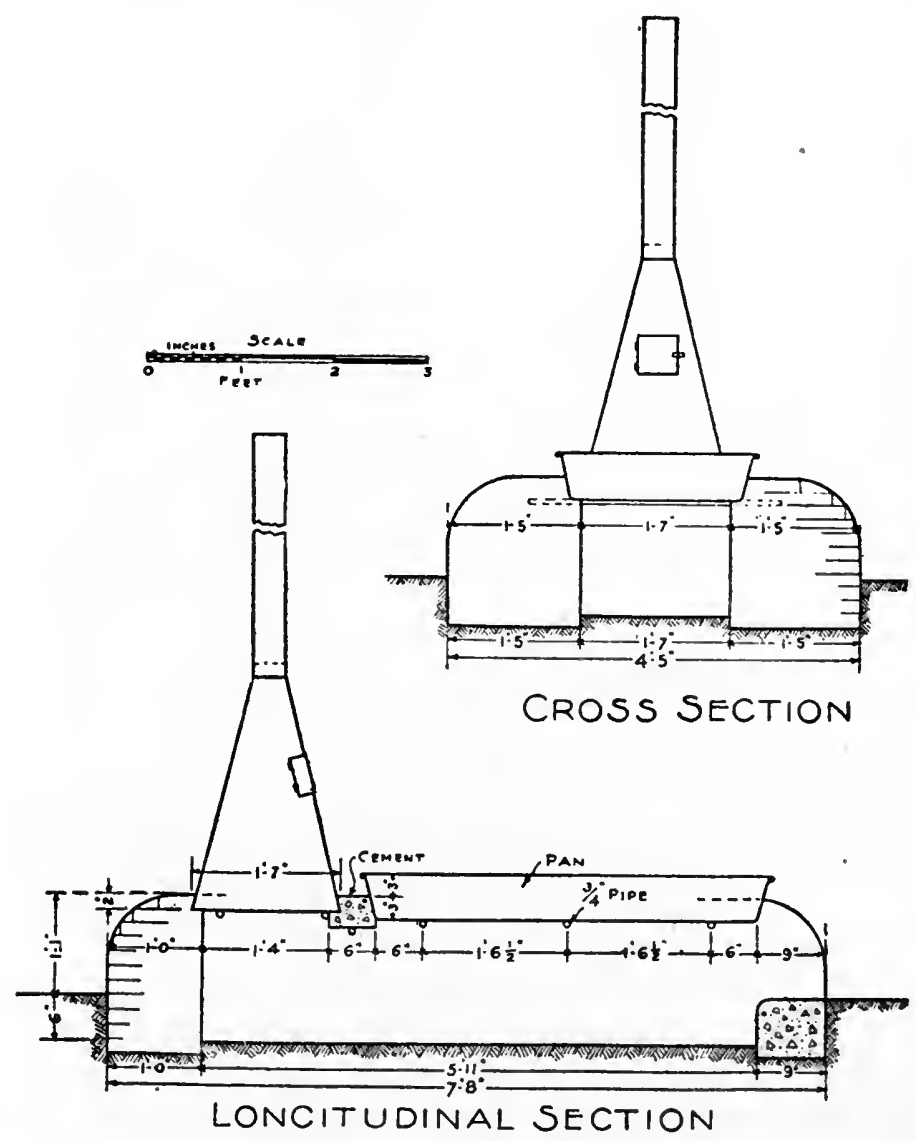

FIG. 226.- Guthrie incinerator. Quartermaster Corps model. 
Caldwell (or English) Incinerator. - Whenever fuel is scarce and stones few, probably no device for the destruction of garbage and refuse is more readily improvised and efficient than this type of incinerator. It consists of a trench Io feet long and I foot wide, about 15 inches deep at the middle and thence gradually shallowing up at each end to the surface level. Over the deep part a barrel is placed and, around it, is constructed a chimney 5 feet high, of clay, earth or sod, sprinkled with water and packed tightly. A fire is made in the interior and the barrel burned out, after which there remains a solid cone of earth. Fuel and garbage are dropped down the chimney. Of the two openings, the one to leeward is closed. A bed of tin cans in the fireplace, through which the air passes freely. is a fair substitute for a grate. Every morning, or as often as necessary, the ashes and cans are raked out and a fresh fire started on a new bed of cans. If the soil is porous, a large quantity of liquids can be evaporated by pouring them slowly into the trench.

This incinerator will burn the solid garbage and refuse of a battalion. With proper material and more skilled labor it can be extended and perfected so as to easily meet the needs of a regiment. Thus, instead of one trench, two trenches may be dug, bisecting each other at right angle. The chimney is built over the point of intersection; it should have an inside diameter of 3 or 4 feet at the base, a little less at the top, and a height of at least 6 feet; across the trenches, boards, flat stones or pieces of sheet iron are required for its support. If iron bars are available they can be placed in so as to form a grate. The great advantage of a four-opening incinerator is that one of the trenches is always in the direction of the wind, and a good draft can always be secured by plugging the throats of the other trenches.

If a few pieces of corrugated roof-iron are at hand they can be easily shaped into a serviceable chimney, thus saving time and labor. Sometimes it is practicable to erect a more permanent structure of stone and brick. Rubble stones plastered with mud will make a very satisfactory chimney.

Rock-PILE CREMatory. - For the general use of a regimental or brigade camp where fuel and stones are plentiful, and in the absence of special appliances, there is nothing better than this type of crematory for the incineration of garbage and refuse, solid and liquid. (Fig. 227.) It is thus described in specifications from the office of the chief Q. M. Corps, January, I908: 
"At some convenient location selected by the military authorities a circular pit is dug, three feet in depth and fifteen feet in diameter, the bottom to be covered with loose stones to the depth of fourteen or sixteen inches. On this is built a circular wall to the height of one foot above the original ground line, and the excavated earth is packed against it clear to the top so as to provide a sloping approach and thereby prevent surface water gaining access to the pit. A pyramid of large stones, four or five feet high, occupies the center. This feature is essential to provide central draft and steady fire.

"The bottom stones receive the liquid portions of the garbage without affecting the fire, and soon evaporate and dissipate them. The solid portions are soon desiccated and become fuel. Care should be exercised to empty the garbage into. and not around the crematory."

Where the supply of stones is scant, tin cans may be substituted to a certain extent, and used repeatedly.

It is desirable to place a few heavy stones along the edge of the pit to serve as bumpers or guard to the rear wheels of the cart or excavator.

This crematory has been used repeatedly in our camps and given general satisfaction. Only one man is required for its service. At camp Captain John Smith, Jamestown Exposition, it was found that one cord of wood consumed about 4,500 pounds of refuse and garbage, including all kitchen wastes and slops. It will likewise incinerate manure and dead animals.

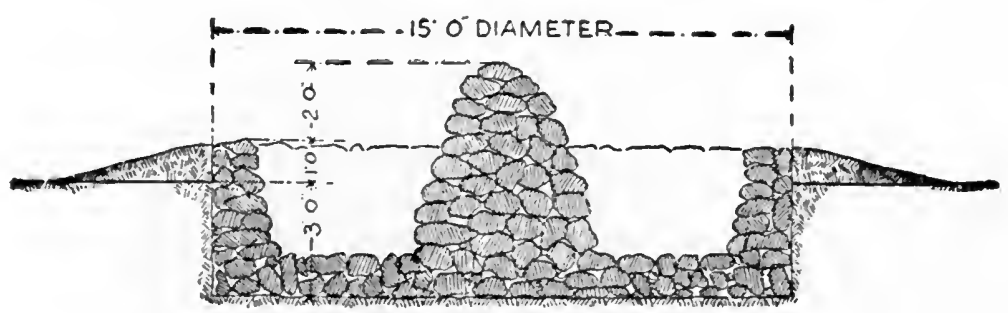

FIc. 227.- Rock pile crematory.

The above crematory has been superseded by a more recent type (Fig. 228), of smaller dimensions and simpler construction, shallower and without central cone. This new model is more practical, requires fewer stones and is more easily cleaned out. 


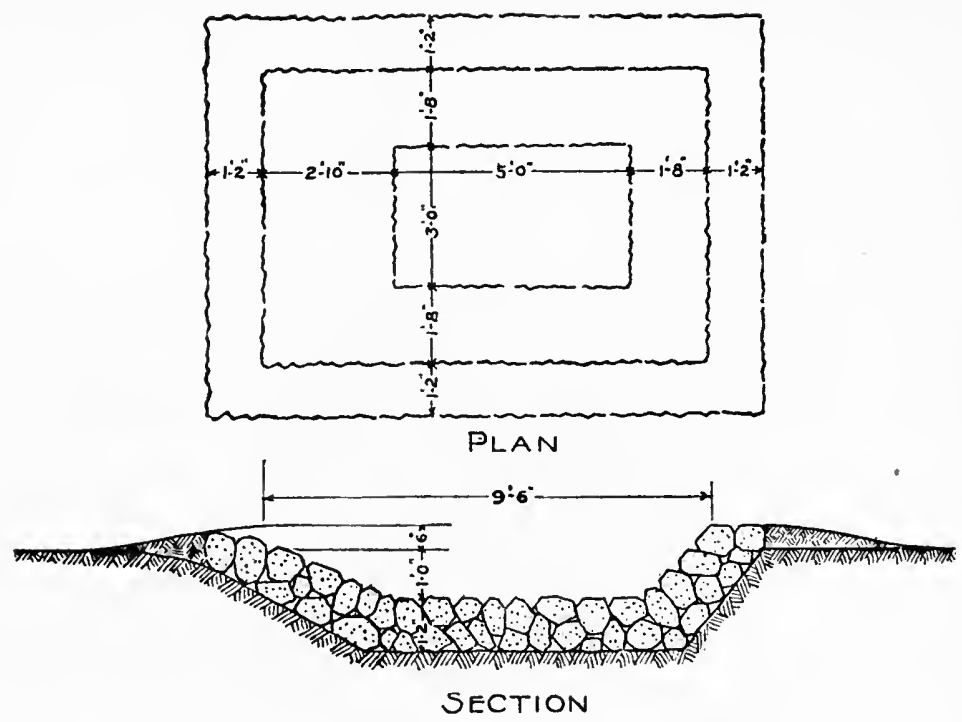

FIG. 228.- Rock pile crematory. New model.

The present tendency in our camps, strongly approved by good hygiene, is not only to burn all excreta and solid garbage, but also to dispose of all liquid wastes by evaporation and incineration whenever practicable. Special appliances have been devised for the purpose and, as stated before, efforts made, more or less successfully, to utilize excreta incinerators to the same end. The effect of the complete burning of excreta and garbage and of strict ground police was strikingly illustrated in our latest camps of instruction. Even with the use of pit latrines, it has been demonstrated that the careful burning of all garbage and refuse, together with the strict observance of all other sanitary measures tending to prevent the breeding of flies and dissemination of infectious germs, will generally keep the ratio of sickness, in camps, below the usual garrison rates.

Disposal of Manure. - Manure breeds flies, renders the soil a better medium for the growth of micro-organisms and is otherwise objectionable. Therefore stable-sheds and picket-lines must receive special attention. They should preferably be located on porous, absorbent soil so that the urine may quickly disappear. As little hay and straw should be allowed to litter the ground as possible 
so as to reduce the amount of manure to a minimum. If the manure is not utilized by farmers, it is best to haul it away and burn it. The picket-lines and stable-sheds should be carefully raked, and all dung scraped up and cremated. As often as may be desirable, they are covered with a thin layer of clean fresh soil, sprinkled with lime, or burned up with crude petroleum.

A general order from the War Department directs that:

"All manure will be hauled to the camp dump. Picket lines will be kept broom swept, and all manure and straw hauled off daily. I weekly incineration of the picket lines will be accomplished with crude oil at the rate of Io gallons to each line."

A simple method of manure disposal consists in building it into a stack and setting fire to it; if dry and containing a good deal of bedding, such stack will continue burning until entirely consumed.

A more effective method is the so-called "Panama plan" in which the manure is piled on a grating of railroad iron raised a few feet above the ground and surrounded by a railing (Fig 229). The rails forming the floor should be laid at right angle to the long axis of the incinerator, and it is an advantage to have them parallel to the direction of the prevailing wind. The fly larva are quickly destroyed so that fewer escape than by the windrow method. No oil or other fuel is used except during wet weather. This incinerator is especially adapted to cantonments and permanent camps.

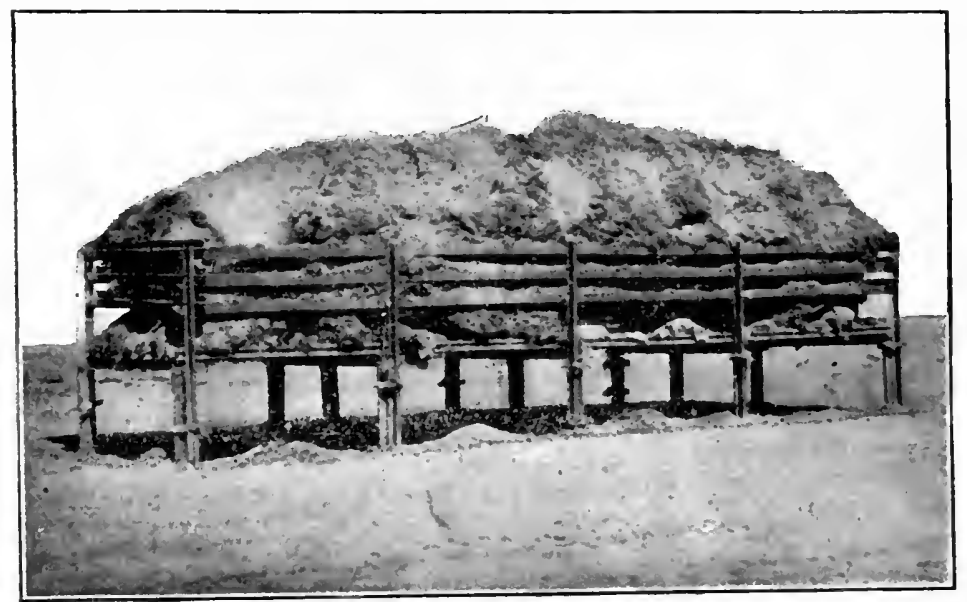

FIG. 229.- Burning manure on grating of iron rails. 
For most camps the windrow method, as recommended by Major R. B. Miller, is the most practical and efficient. The manure is dumped from the tail of the wagons in narrow windrows about 8 feet apart. After drying for a few hours each windrow is spotted with oil at intervals of six feet on the windward side and fired. The next day the wagons straddle the same windrows and dump the manure on the hot, smoldering ashes of the previous day's burning. In order to prevent injury to the animals' feet it is necessary to keep the windrows raked from each side so that they do not exceed two and one-half feet in width. Tin cans, fragments of bottles, etc., should be excluded on account of the danger to the animals' fcat. Waste paper should be separately burned to prevent its scattering by the wind. When the manure is wet, the wagons are required to drive between the windrows where their loads are spread in a thin layer. When dry the manure is raked into the windrows and fired as usual.

It has been observed that a fly never lays its eggs in a dry place, nor will the eggs hatch without moisture. This explains why the great majority of the eggs are deposited on the manure before it leaves the picket lines. Therefore, if it is intended to destroy all the larvæ, the manure should be burned as soon as possible, or not later than Io days after its removal from the picket lines. 


\section{CHAPTER LV.}

\section{GENERAL SANITARY RULES IN THE FIELD.}

Experience has shown that in the course of a campaign, while on active duty involving much marching and fighting. soldiers are seldom sick; that scant rations, worn-out clothing, exposure to inclement weather and hardships are seldom predisposing causes of infectious diseases: that, on the contrary, hard work and plain fare are good preservatives against disease, rendering the men more resistant to germ infection. The danger comes as soon as soldiers get into permanent or semi-permanent camps and begin to make themselves comfortable, that is to say, to devote much of their time to eating, sleeping and diverting themselves, as was illustrated at Santiago de Cuba, in 1898 , after the surrender of the enemy, and by the English during the Boer War, at Bloemfontein where, after an exhausting campaign quite free from sickness, they encamped to rest and recuperate.

In camps, owing to the large aggregations of men thrown into close contact, the liability to water pollution, the imperfection of sanitary conveniences and difficulty of protecting food against infection. the facilities of germ transmission are very much increased, while the effects of such transmission are momentous. The general indications, therefore, to preserve health in camp, are: aroidance of crowding and unnecessary concentrations, care in eating and drinking, adequate sanitary measures (including personal hygiene) and active exercise. These subjects have been treated in their proper chapters, but on account of their importance may now again be briefly considered in their bearing upon camp life.

I. The food should be sufficient, always wholesome, varied and well cooked. As already dwelt upon, there is more danger to be apprehended from excess than from scarcity, especially when the men are at liberty to leave the camp and patronize restaurants not under military control. The sale of foods and drinks in camps by peddlers and hucksters should be strictly prohibited, or at least limited to authorized exchanges, under the supervision of the regimental sanitary officers. During the prevalence of epidemics of typhoid fever, cholera or dysentery in the vicinity of camps, it is 
absolutely necessary not only that the men be prevented from eating or drinking beyond camp linits, but that all supplies from local markets be carefully inspected and their origin inquired into before being allowed in.

The men of each company should be required to eat in the messshelter or at some designated point, and not permitted to carry their food to their tents or eat it at random in the company street, as this always results in pollution of the soil. A receptacle should be provided for wastes and the scrapings of the kit meat-can, as well as a pail of hot water from which to wash the meat-cans, knives and forks.

For permanent camps, the Q. M. Corps has devised a field kitchen and mess-hall combined, 54 feet long, 20 feet wide and roofed with rubberoid (Fig. 230). These dimensions can be reduced and the building covered with one or two paulins.

2. Every effort must be made to prevent contamination of the water-supply. As soon as there is a reasonable doubt of its purity it must be sterilized or filtered. A company will require two barrels of water daily for drinking purposes. The necessary appliances and vessels for boiling or filtering having been provided, a man should be specially detailed to attend to the supply of the company. The Forbes sterilizer. Darnall filter and sterilizing water bag being easily transportable, there will be few situations when one or the other of these approved appliances cannot be made available. A reserve of pure water in the Army water-wagon, the Forbes wagon sterilizer, or other wheeled receptacle should always be on hand during the march and in camp.

As an army advances into the interior of an unknown or hostile country, it is not practicable to make a complete examination of all drinking water. A rapid chemical analysis, however, such as can be made in an hour or two, may give useful indications and is often possible. Therefore, eacl field hospital in our service should be provided with a case containing the necessary reagents and apparatus to that end. A bacteriological examination, to be of any value, must be made in a well-equipped laboratory not liable to frequent and sudden changes, and therefore is not possible in a field hospital; but each base hospital should be furnished with such a Iaboratory, made detachable and portable so that it may take station outside the hospital, wherever most needed. 

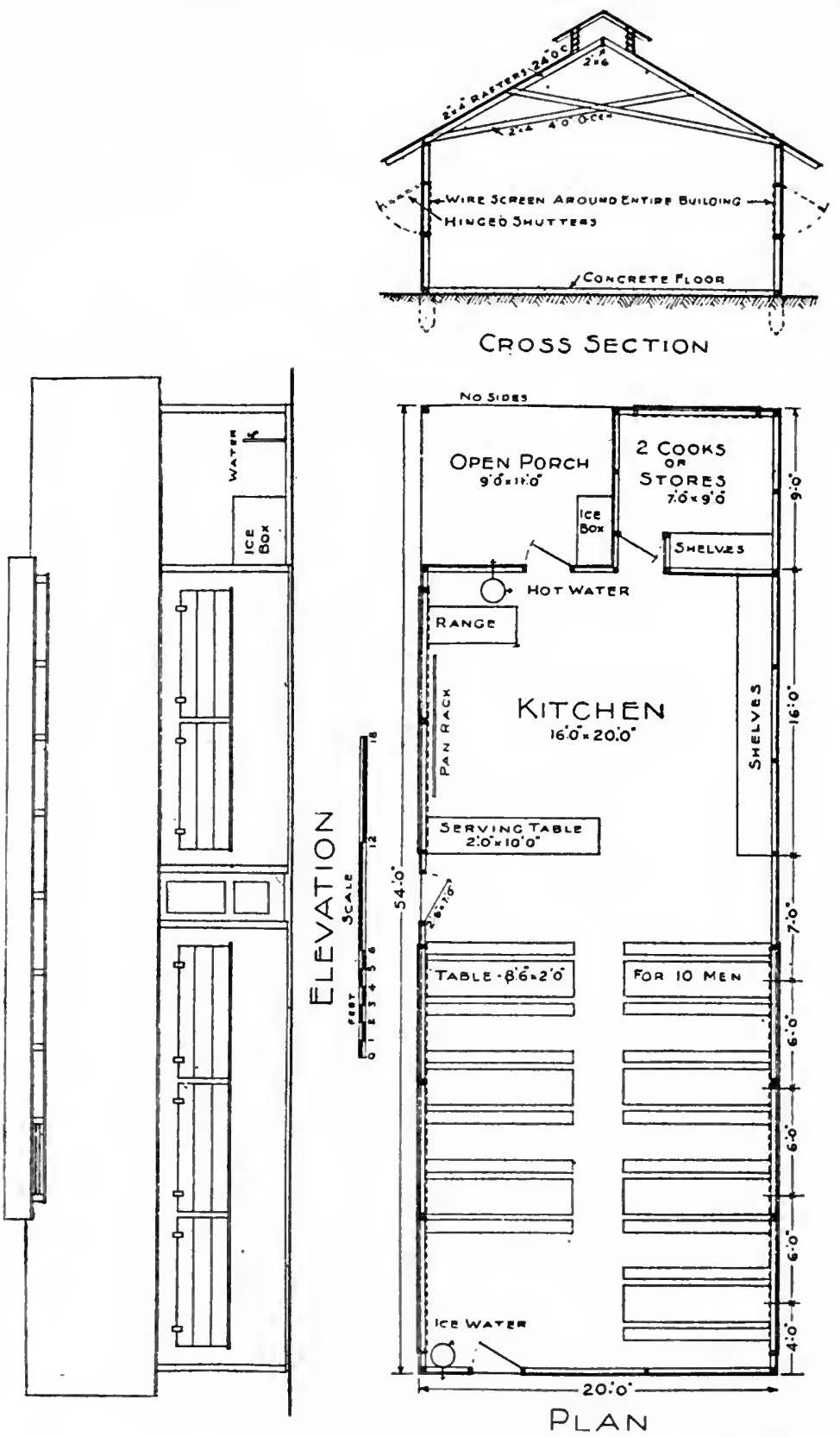

FIG. 230.- Plan for kitchen and dining-room in permanent camp. Quariermaster Corps. 
3. Great concentrations of troops should be avoided and camps made as small as possible, scattering brigades and divisions as much as the topography of the country and the exigencies of the situation will permit. In the presence of the enemy, troops are necessarily strung out in thin lines, making large camps impossible. One of the secrets of the good health of the Russian and Japanese soldiers in Manchuria, as well as. of the belligerent troops now in Europe, is the thinness of their extended lines, seldom more than a battalion or regiment being camped together.

Wherever crowding and promiscuity cannot be prevented, greater care must be given to the early detection and segregation of infectious diseases; even cases of common colds, cough, sorethroat and other apparently trivial ailments had better be removed from their tents and treated in hospital.

4. Personal hygiene, that is to say, cleanliness of body and clothing, is very desirable in the field although often difficult of attainment. The men should not only bathe themselves, but also wash their linen whenever the opportunity offers so that it may always be as fresh and clean as conditions permit. Then not only will they save themselves and comrades from vermin, especially the noisome louse, transmitter of typhus fever, but also from possible contamination by germs of typhoid fever, cholera or dysentery; furthermore, in case of a battle, they will be much less likely to suffer from wound infection, for this dangerous complication is nearly always the result of dirty skin and clothing. It is not amiss to add, in this connection, that soldiers should always go into a fight with empty bowels and bladder so that, if shot through the abdomen, the danger of extravasation and peritonitis be reduced to a minimum. The hands are the most dangerous agent of disease transmission in camp and therefore must be frequently washed.

5. As fully explained under Latrines, the greatest danger to which troops in camps are exposed is from infected human excreta; their proper disposal must always be one of the chief preoccupations of sanitary officers. Whenever a case of infectious disease is detected in a company or regiment, the most effective course to pursue is to remove the patient promptly to a field or base hospital, thus precluding, in a large measure, the possibility of transmission to other men. By vigilantly attending to this prompt segregation of first cases and, at the same time, keeping under observation the men who 
have been in contact with them, no epidemic or serious outbreak of any camp disease is likely to occur.

6. Bearing in mind the agency of flies and mosquitoes in the conveyance of infection, it will be well, so far as means permit, to screen with wire netting all the buildings, sheds and tents which need most protection, namely, mess-sheds, kitchens, latrines and lavatories. It is especially necessary that the food, while being prepared and consumed, be suitably guarded. By the use of the mosquito-bar, malarial fever, formerly the most prevalent of camp diseases, is now the most preventable and the least dangerous; the mosquito-bar, therefore, has become part of the equipment of the soldier, as much so as his blanket and first-aid packet, and its persistent use in camp, wherever mosquitoes are present, must be insisted upon. When these insects are troublesome, a head-net also becomes absolutely necessary, especially for men on guard. In the Russo-Japanese War such a net was issued to each Japanese soldier with his summer uniform; it was collapsible and carried tied to the belt.

7. The cleaning, policing and disinfection of the camp grounds should be the first daily duty of the troops after breakfast. Every part should be carefully inspected and receive the treatment it requires. All organic dust, but especially decaying animal and vegetable matter, must be swept or scraped up and, with all other garbage and refuse, including tin cans, carted to the incinerator. It is upon such decaying matter that fleas and other vermin grow and multiply. Wherever the soil has been fouled by urine or slops, it should be sprinkled over with lime or clean earth. Ditches must be cleaned and all stagnant-water puddles drained or filled up. Disease germs on or in soil which is pounded into dust by the tramping of men and animals are, of course, easily blown about so that a neglected camp is a menace not only to itself, but to all its neighbors. A camp should be kept as scrupulously clean as military exigencies permit. but the custom, much too common, to sweep the entire grounds every morning and raise clouds of dust is distinctly objectionable and insanitary; many of the germs are simply scattered over a larger area, not removed. Dirt can be picked up, raked or scraped, or swept in places, after sprinkling, but indiscriminate sweeping should not be tolerated in camps any more than in city parks.

The use of tar oil on roadways and drill grounds, within and around the camp, is highly commended. 
8. How often should a camp be moved; that is to say, when does a camp, from mere occupancy, become so fouled and infected as to require its abandonment? This depends largely upon the manner and extent of its sanitation. A camp may be so well provided with conveniences, so well policed and safeguarded against the invasion and propagation of infectious diseases, as to be fully as healthy as a properly governed post or cantonment; in such case there is no reason for change of site. Thus during our occupation of Cuba, after the Spanish War of 1898 , camps were kept on the same sites for more than a year with marked benefit to the health of the troops, notwithstanding the depressing effect of a tropical climate. The crowded camp site at San Antonio, in 1910, when vacated after four months of continuous occupation, presented nothing offensive to sight or smell (J.R. Kean). The rule is that, with a good site and good sanitation, a camp can be occupied indefinitely.

There is a change, however, within the camp, which is always practicable and beneficial. Even with good police the soil areas covered by the tents are likely to remain damp and to become contaminated by various kinds of organic matter. Therefore it is well, now and then, to shift the tents to the immediately adjoining grounds, while preserving the relative arrangement of the company street, so that the uncovered soil may be exposed to air and sun; at the end of a week or two they are replaced upon the original sites.

The sanitary reasons requiring the removal of permanent camps are the following: When, through bad sanitation, especially bad management of latrines, the soil has become polluted with fecal matter and urine, or impregnated with decayed organic matter, and therefore a dangerous medium for the multiplication of disease germs; when the water-supply is infected and means of purifying it are inadequate; when the site is so situated that it receives the drainage of camps on higher grounds; when, through irremediable causes, flies, mosquitoes, or other insects are very troublesome; finally, when, another good site being available, it is deemed desirable to give the troops a change of surroundings and active occupation for awhile. 


\section{CHAPTER LVI.}

\section{ADMINISTRATION OF THE SANITARY SERVICE IN THE FIELD.}

This subject is governed by the Field Service Regulations (sections 329-353, 1914).

The senior medical officer of an army or smaller command is charged with the general control of the sanitary troops serving therewith, and commands the independent sanitary units. He may be authorized by the commander to make assignments of the personnel, and in emergencies the entire sanitary service of the command may be placed at his disposition.

Before troops are sent to camps of mobilization they must be carefully examined by medical officers of the regular army when practicable, otherwise by militia or volunteer medical officers especially detailed for this duty, to detect and remove cases of infectious diseases, especially typhoid fever. The spread of such diseases, in camps, during a campaign, might seriously impair the strength and mobility of a command. This medical inspection is made after the assembling of the regiment at some convenient point (usually a general concentration or mustering rendezvous), and within 2 or 3 days of starting for the camp. At the same time, every man not already protected is vaccinated against small-pox and typhoid fever. A certified record of this inspection and vaccination is kept by the regimental commander for the information of the camp commander.

One sanitary inspector, on the recommendation of the Surgeon General, is assigned to each division. (Sect. 33I, F. S. R.)

It is the duty of commanders to remedy sanitary defects reported to them by sanitary inspectors. To facilitate the attainment of proper results in sanitary emergency, a sanitary inspector may be authorized by the commander of the unit to which he is assigned, to direct, in the name of the latter, and within such limitation as the commander may prescribe, the prompt abolition of conditions prejudicial to the health of troops.

It is now recognized, in our service, that military hygiene is specialized knowledge and that only medical officers are competent 
to direct and supervise its application. The former method of turning over much of the sanitary work to the Q. M. Corps caused a divided responsibility which bred indifference and prevented the attainment of the best results. The Medical Department possesses the autonomy which is essential for the successful discharge of its manifold duties. It exercises complete control not only over its own personnel and material, but also upon the civilian laborers and means of transportation temporarily transferred to it, when necessary, by the Q. M. Corps.

Experience has shown that, in the field, the best sanitary service is obtained by the organization of regimental sanitary squads, each consisting of one Hospital Corps sergeant, one Hospital Corps private from each company, and as many hired laborers as may be necessary, under the control of the regimental sanitary officer. Where laborers cannot be procured, special details must be made from the companies. The squad performs all the sanitary work of the regiment.

The Hospital Corps non-commissioned officers and privates of the squad are specially instructed in the installation and operation of filters, the construction and operation of company incinerators, the digging, policing and disinfection of latrines and urinals, the treatment of wastes, care of grounds, picket lines, etc. They give practical demonstrations in all these subjects and supervise the work of police and fatigue parties so far as may be necessary.

In addition to the regimental squads, a general sanitary squad is necessary for the proper care of camp areas beyond the supervision of regimental sanitary officers.

As model of a divisional sanitary order, brief but comprehensive. showing the general scheme of camp sanitation and the duties and responsibilities of the Medical Department under the circumstances, the following is quoted:

Generat. Orners, ?

Headquarters Maneuver Division,

No. 2. $\quad j$

San Antonio, Texas,

The following regulations for camp sanitation are published for the information and guidance of all concerned:

I. CHIEF SURGEON. The Chief Surgeon is charged with the general conduct and supervision of the Medical Department in the performance of its duties, and will make such assignment of personnel as may be required.

2. THE SANITARY INSPECTOR. The Sanitary Inspector is charged 
especially with the supervision of the sanitation of the camp. It is the duty of commanders to remedy defects reported to them by the inspector, in conformity with Section 33I, F. S. R.

3. REGIMENTAL SURGEONS. The senio, medical officer of each command will make at least one inspection daily of the command to which attached, and report any sanitary defects, with proper recommendations to remedy the same, to his commanding officer, who will immediately take the necessary steps for their correction, if within his authority. If beyond his facilities, or if considered impracticable, he will immediately forward the report, with full remarks, for action of higher authority.

4. WATER SUPPLY. The camp water supply is pure and wholesome and no sterilization of drinking water is necessary. Precaution must be taken, however, to prevent subsequent contamination by keeping all containers scrupulously clean and protected from dust and other sources of infection.

5. DISPOSAL OF EXCRETA AND WASTES. Organization commanders will be held responsible for the police of their respective camps. Each company or similar organization will construct incinerators for the disposal of all solid and liquid garbage of the organization, and no other disposition will be made of such wastes. Human excreta will be disposed of in pits provided with latrine boxes unless other provision has been made. Two urinal cans will be placed in each company street at night. Latrine pits will be burned out, and seats scrubbed daily; crude oil and straw or other material will be used. The urinal cans will be burned out daily and bottoms covered with milk of lime before put in use. Defilement of the ground in or about the camp is absolutely prohibited. Manure will be hauled to a designated dumping ground, and there burned in the manner prescribed. Rock pit crematories will be used for disposal of general wastes of camp areas not under the jurisdiction of commanding officers.

6. FOOD AND DRINKS. No food, drinks, or like commodities will be sold in camp except in the authorized exchanges.

7. SANITARY SQUADS. Sanitary squads composed of medical officers, non-conmissioned officers, and privates of the Hospital Corps' will be organized by the Chief Surgeon. The Sanitary Squad will supervise and assist in the disposal of camp wastes. Civilians employed as sanitary laborers will be employed by the Quartermaster Corps and turned over to the Medical Department. The Sanitary Police Officer will make requisition on the Chief Quartermaster for such transportation, labor, or material as may be needed. When assigned to this service they will not be diverted to other uses except by order from these headquarters.

By Command of Mlajor General C......

A. B. C.......,

Colonel, General Staff, Chief of Staff. 


\section{CHAPTER LVII.}

\section{SERVICE IN WARM CLIMATES.}

The territory under the flag of the United States extends from northern Alaska, near the $70^{\circ}$ latitude, to the southern Philippine Islands, only some five degrees north of the equator. Within these limits is the greatest variation of climatic conditions, from a mean annual temperature of about $83^{\circ} \mathrm{F}$. down to one below the freezing point, and from vapor-saturated sea islands to deserts as arid as those of Africa. It must be borne in mind that extremes of temperature are not only found in the Philippine Islands and Alaska but exist as well within the United States. Thus, Manila has a mean annual temperature of $80^{\circ} \mathrm{F}$., while the mean of its hottest month, May, is only $83.5^{\circ}$, and the maximum of any day during this month never reaches $100^{\circ}$. With this may be compared the summer means (May to September inclusive) of Fort Yuma, Ariz., $86.26^{\circ}$, and Laredo, Texas, $84.10^{\circ}$. Furthermore, a maximum temperature exceeding $100^{\circ} \mathrm{F}$. may be expected, with few exceptions, in any city of the United States south of New York and Chicago; while, in the southwest, a maximum of $105^{\circ}$ to $115^{\circ}$ is not rare. However, the combination of high temperature and great humidity only exists in our South Atlantic and Gulf States, and these may be truly said to have a tropical summer climate, a climate indeed often more trying, from the absence of air movement, than that of Manila, Havana or Porto Rico always fanned by the trade-winds. On the other hand, parts of the United States suffer from extreme cold during the winter, the thermometer falling to $-40^{\circ}$, or even lower, in some of the northern States. This extreme variation of temperature, from torrid heat to Arctic cold is the most striking feature of the continental climate of the United States. The records of the Weather Btreau show that, for the decade I898-I908, the lowest annual mean of any station in the United States, namely, $35.7^{\circ}$, was at Pembina, N. D. ( $49^{\circ}$ lat.). During that period, the highest temperature recorded at that place was $103^{\circ}$ in June, and the lowest, $-43^{\circ}$ in January, a range of $146^{\circ}$. But even those States with the highest summer temperature have a comparatively cold winter, often down 
to frost, which partly neutralizes the effects of the summer heat and prevents the deteriorating influence on the human system often seen in true tropical regions.

The climatic conditions characteristic of the tropics are constant higl temperature and relative humidity, actinic action of solar rays, bright luminosity and great electric tension.

High temperature alone does not render a climate unhealthy. Among the most salubrious parts of the United States are the arid regions of the Southwest where the thermometer ranges up to $110^{\circ}$ and above in the shade. The factor which plays the most important part in the deteriorating influence of tropical climates, and is impossible to guard against, is humidity, that is, a constant, high relative humidity. Furthermore, these two elements, heat and humidity, must be present, within limited variations, throughout the year. Thus the annual mean relative humidity of Manila, P. I., is 80.2 (ranging from $7 \mathrm{I}$ in April to 85 in September). The nearest approach to it, in the United States, is that of our Gulf Coast in summer ( 72.3 in New Orleans, in July).

To prevent the temperature of the body from rising above its normal point, nature provides for the dissipation of surplus heat in two ways: by transfer (through radiation, conduction and convection) and by evaporation of the sweat. The loss by transfer varies with the difference of temperature between the body and surrounding air, being greatest in cold weather. With a temperature of $70^{\circ}$, or under, the loss takes place chiefly by radiation and conduction. As the temperature rises above this degree and approximates that of the body, evaporation assumes the more important part. - Through the delicate nervous mechanism whereby the temperature is regulated, more blood flows to the surface and more sweat is excreted, and it is on the evaporation of this sweat that, in hot weather, the body must chiefly depend to keep its temperature down to the normal point. Rubner calculates that a stout man, in summer, may thus lose 3,200 calories in a day, the equivalent in heat of the amount generated by an ordinary military ration. So long as this evaporation is free, the degree of solar heat does not matter and the body functions remain unaffected. Thus a temperature greatly exceeding $100^{\circ}$ is not oppressive in the very dry air of New Mexico and Arizona. Very different is the result in the moist atmosphere of the tropics: there the skin and lung evaporation is greatly impeded. 
Not only does a high degree of humidity interfere with evaporation but likewise with radiation from the body. It is true that, on the other hand, it favors conduction, since moist air is a better conductor of heat than dry air, but such conduction is a poor substitute for the greatly impaired radiation. This failure of heat dissipation results in an increase of body temperature which is further added to and aggravated by the more active metabolism thus produced.

The dangerous effect of this increased temperature, however, is probably mitigated by the depletion of internal organs caused by the rushing of blood to the skin to facilitate loss of heat by evaporation and radiation. Owing to the close correlation existing between the circulation of the skin and of the brain, the latter organ is especially affected, and to its depletion should be chiefly attributed, according to some physiologists, the dull feeling, listlessness and discomfort of a muggy summer day,

The physiological effects of moist heat are well shown by the experiments of Phalen at Los Banos, P. I.* Four able-bodied men were subjected for 4 hours to a temperature $92^{\circ}$ to $98^{\circ}$ in a room saturated with moisture but otherwise well ventilated. They were clothed in thin underwear and allowed to recline or walk as they preferred. The loss in weight averaged 3.25 pounds and the loss of strength (as measured by the ergometer) 38 per cent. In each case there was a notable rise in pulse, respiration and temperature, as well as a fall in blood pressure. Sweating was very profuse and the clothing was soon saturated. All the men felt tired and relaxed and had no appetite for the next meal, but denied the presence of headache or other disagreeable symptoms, and were in their normal condition the next day.

This observer also reports the result, on a baseball team, of hard practice for two hours at a temperature of about $93^{\circ}$, with relative humidity of approximately 8o, under a cloudless sky and in a moderate breeze. The average temperature of the men was $99.7^{\circ}$, the lowest being $99^{\circ}$ and the highest $100.4^{\circ}$. The blood pressure rose from I 20.7 to $129.5 \mathrm{~mm}$. This record hardly differs from what would be expected after a similar game played on a warm day in the United States, and shows the possibility of strenuous exercise even in the tropics if not too long continued.

* J. D. Phalen, Philippine J. of Sc., Dec., igro. 
Whatever may be the degree of atmospheric humidity in hot countries, relief is always afforded by agitation of the air. Any breeze or wind increases the evaporation and radiation from the body and thus reduces its temperature. Fortunately, it happens that tropical countries are nearly constantly swept by monsouns and trade-winds so that there are few where the white man, by using the means suggested by ordinary intelligence, cannot live in safety and comfort, so far as climatic conditions are concerned.

One of the effects of the greatly increased perspiration is a diminution of urine and of the digestive fluids. The urea is lessened, but this is mostly, if not entirely, from lessened food.

In spite of the more abundant perspiration, the skin evaporation seems unable to prevent a rise of body temperature in new-comers, amounting to half a degree or more; but gradually the system adjusts itself to its new conditions so that, in a year or two, the temperature is again normal.

Under the direct rays of the hot sun the skin becomes moist with perspiration, but should the exposure continue and the natural cooling processes of the body be interfered with, the sweat becomes checked and the skin dry, one of the first symptoms of heatstroke.

According to the observations of Wickline and other members of the Medical Corps, U. S. Army, made on IO4 American officers and enlisted men, in the Philippines (Mil. Surgeon, October, 1908), the composition of the blood undergoes notable changes. In 86 per cent. of all men examined, the hemoglobin was decreased from 94 per cent. at the first examination to 83 per cent. at the last, nearly 2 years later. The erythrocyte count, on the contrary, was increased up to an average of $5,640,000$. The most remarkable change was found to occur in the differential leukocyte count, consisting in the continuous decrease of polymorphonuclears, from 64.4 down to an average of 54 per cent., with a relative increase in the small lymphocytes and eosinophiles. This low percentage of the phagocytic polymorphonuclear elements, which also appears to be the normal condition of the natives of tropical countries, may account for their relative lack of resistance to certain infectious diseases, and gives point to Cabot's remark: "It would appear that the degree of health in persons not organically diseased, might perhaps prove to vary directly with the percentage of polymorphonuclear cells in the blood."

The number of respirations, in Wickline's observations, shows an 
increase, with an average of 19.6 per minute. The pulse rate is not reported, but it may be assumed that, if changed at ail, it is slightly increased in harmony with that of respiration.

Phalen's observations* corroborate those of Wickline.

Those of Chamberlain and Vedder, $\dagger$ based on a large number of men, yield somewhat different results. After 20 months of Philippine service, 700 American soldiers living near sea level, showed a red-cell count of 5,200,000 per c. m., a hemoglobin reading of 89.6 per cent., and a color index of 0.86 . Such red-cell count, Dr. Chamberlain concludes, " does not differ from the normal at present recognized for healthy young men in the temperate zone. The hemoglobin percentage and the color index are probably a little low, but not sufficiently so to indicate a definite anemia. The pallor not infrequently met with among apparently healthy persons in the tropics we believe to be due as a rule to superficial ischemia and not to a deficiency in the total quantity, or in any particular constituent, of the blood."

Chamberlain and his colleagues also found that the blood-pressure of Americans in the Philippines differs little if at all from the average at home. The body temperature, after 19 months' service in the Islands, averaged $98.74^{\circ}$, which is less than 0.2 over the generally recognized mean of $98.6^{\circ}$. The pulse rate for both races, white soldiers and Filipinos, averaged a few beats above the usual standard of 72 per minute.

The expansion or rarefaction of the air caused by the heat in the tropics, reduces the amount of oxygen contained in it to an extent of about 3 per cent. Since the hemoglobin of the blood is the oxygen-carrier-to the tissues, its decrease further reduces the amount of oxygen which becomes available for the system, and the capacity of the lungs to eliminate carbon. To make up to a great extent for this deficiency, nature, as stated above, increases the number of red blood-cells, together with the number of respirations, as it does in people residing at high altitudes. It has been proved that the higher the temperature the more active the respiration, that is to say, the greater the ventilation of the lungs and output of $\mathrm{CO}_{2}$. Regarding the increase of erythrocytes, however, Phalen inclines to the belief that it is only apparent, being due to excessive perspiration and

* Loc. cit.

$\dagger$ Philippine J. of Sc., Sec. B, vi, I9II. 
resulting concentration of the blood, rather than to an actual augmentation of cells.

Of IOI men examined by Wickline, it was found that, at the end of about 2 years, 82 had lost an average of 8 .I pounds, 4 showed no change, while 15 showed an average gain of 6 pounds. In 200 men examined by Chamberlain, the loss in weight during the first year was 3.2 pounds.

Some of the effects of tropical heat upon men and animals have been observed by Hans Aron at Manila, P. I. He found that monkeys exposed to the sun show a steady rise of temperature and die in about an hour, with hemorrhages of the brain and heart. Dogs and rabbits succumbed in a few hours with hyperemia and small hemorrhages of the brain and meninges. If care is taken to conduct away the excessive heat incre nent by brisk fanning, the body temperature remains approximately normal and the animals do not suffer. Exposure of the head alone is not fatal so long as the rest of the body is kept cool.

If the effect of sunlight in such fatal cases were to be attributed to the absorption of the ultraviolet, or actinic, rays, as has been contended, their influence, it is claimed, would not thus be completely neutralized by a current of air. Such deduction, however, is not convincing. It is very probable that the actinic rays make themselves felt on the body only at a high temperature, and that any artificial cooling of the skin prevents their action.

The temperature of the skin, in man, normally about $9 \mathrm{I}^{\circ}$, rises quickly upon exposure to the sun, to about $97^{\circ}$, at which point the sweat breaks out; there it stops or even declines if perspiration is free. In the hair of an uncovered head the temperature increases enormously, and as but little perspiration takes place in the scalp, no fall occurs. In the black dense hair of a native, it rose to II $3^{\circ}$ F. within 30 minutes, and to $122^{\circ}$ within an hour. The necessity of protecting the head from the solar rays is thus conclusively demonstrated. In the sun, perspiration is so great that a man lying down quietly, lost 280 grams in weight in an hour, without counting the moisture absorbed by the clothing. The pulse rate of a man sitting quietly, increased Io to 12 beats a minute, while the quantity of air respired also increased 23 per cent., namely, from $3 \mathbf{I} 7$ c. c. in the shade, to 390 in the sun.

The effect of a tropical climate upon the nervous system is notice- 
able in susceptible people, causing general depression and loss of vital energy, with tendency to neurasthenia and mental irritability. There is no evidence that, so far as Americans are concerned, blonds are oftener or more seriously affected than brunettes.

To sum up, it may be stated as an accepted fact that in our colonies, as in other tropical countries, from climatic conditions alone, irrespective of endemic disease, the endurance of the North American is put to a severer test, while he is generally incapable of the same mental and muscular exertion. Our views on this subject, however, have had to be somewhat modified and readjusted to the results obtained in the Panama Canal Zone, demonstrating to what an astonishing extent the so-called deteriorating climatic effects of the tropics are preventable. Gorgas reports that, after the elimination of yellow fever and malarial fever, and the enforcement of all reasonable sanitary measures in the Zone, the American employes have become hardy, rugged, active and energetic, with a mortality as low as that of the healthiest regions of the United States (see Chapter I).

Soldiers sent to the tropics should be picked men, in good physical condition and not less than 22 years old; younger men have but little power of endurance and are more susceptible to infectious diseases. They should arrive at the beginning of the dry and cold season, usually November or December, so that they may partly adapt themselves to their new surroundings before the advent of summer. The sanitary conditions under which our soldiers in the colonies are obliged to live have very much improved of late years, and their power of resistance to the depressing effects of the climate have correspondingly increased. The length of time they can serve in the tropics without detriment to body and mind cannot be definitely stated, depending as it does upon many variable factors, but there is no doubt that, under ordinary circumstances, they could remain at least 3 or 4 years with entire impunity. Statistics' show that the longer our men serve in the Philippines, up to ro or more years, the smaller is their ratio of non-effectives. A large proportion of the English troops in India remain from 5 to 8 years, but it must be noted that part of their time is spent at posts located in mountainous regions, and therefore in a subtropical if not temperate climate. Their statistics show that, within certain limits, the longer a soldier remains, the less susceptible he grows to his pathogenic surroundings, 
so that he is able to render better service the third and fourth years than the first and second; in other words, in healthy men of good habits a marked degree of physiological acclimation takes place. The War Department, in reducing the term of service of our soldiers in the Philippine Islands to two years, has doubtless been inspired by other considerations than the mere economic and sanitary aspects of the subject. However, under the best possible conditions, it is obvious that white troops, sent from the United States to the colonies, not only require very expensive care but will never acquire the natural resistance which is enjoyed by the natives. The latter, as the experience of all countries has shown, if well trained and well officered, will make excellent and reliable troops with much less effort and cost. Therefore, speaking from the viewpoint of hygiene, the easiest way to reduce the mortality rates for disease in our colonies would be to replace the white troops serving therein, as far as possible, by native troops.

Food. - The food suitable for warm countries has already been discussed (page 40r). There can be no doubt as to the principle that, to preserve his health and yield his greatest efficiency in a tropical climate, the soldier must reduce his daily ration; but this principle is very imperfectly understood and, in practice, carelessly observed. Eating is to a large extent a matter of habit, and men will consume the same quantity of food they have been accustomed to, regardless of climate, unless their attention is especially called to the danger of such indulgence.

Not only the system does not require so much food, but the amount of muscular exercise being necessarily reduced, less of it can be metabolized and excreted, so that, should the same quantity be ingested, the system gets clogged, while an unusual amount of physiological work is thrown upon the liver and kidneys in their efforts to get rid of all useless waste products. A condition of physiological hyperemia is brought about which is the first stage of the "tropical liver" ; this condition soon passes into one of static congestion with diminished functional activity. In the first stage, there is usually a copious flow of bile with perhaps bilious diarrhea, but in that of congestion there is impaired hepatic action and those digestive disturbances which result from lack of healthy bile. One step further and congestion may pass into actual inflammation, or hepatitis, with enlargement of the organ, fever, pain and tenderness on pressure. 
The reduction of food should be chiefly in fats and meats whose waste products throw most work upon the already strained eliminative organs. Nitrogenous food is, of course, everywhere necessary, but, in the tropics, it should be chiefly supplied by fish, poultry, eggs, cereals rich in gluten, and the pulses (beans, peas and lentils, indigenous and introduced); the bulk of the food should always consist of starchy cereals, fresh vegetables and fruits.

In India, sulphur fumes are used quite successfully to prevent the rapid decomposition of meats and render them more tender (see page 335 ).

Beverages. - Concerning beverages, it is well to bear in mind the French axiom that, in the tropics: "To seek pure water and shun alcoholic drinks is the beginning of wisdom." No water should be used which is not known to be wholesome, or has not been purified. More of it is necessary in warm than in temperate regions so as to provide for the increased perspiration. The best time to take it is between meals, and in the morning and evening. To drink much and often is a bad habit, disturbing the digestive functions and weakening the power of endurance. Water should be cooled with ice so as to be palatable and refreshing, but ice-cold water is liable to produce gastric and intestinal troubles and always dangerous in the tropics. In the absence of ice, water can be cooled by the usual native methods, namely, by letting it filter or ooze through a porous earthern jar or olla, the evaporation of the fluid on the surface abstracting the heat of the jar and contents; or a metal receptacle can be used, with covering of felt or flannel occasionally wetted, like the soldier's canteen. The process of evaporation and cooling is much more rapid if the receptacle be hung in a draft, overnight. Incidentally, the olla always clarifies the water and may also materially purify it if brushed inside and outside and sterilized once a week.

Alcoholic drinks, in the tropics, should be entirely discarded or else ased with the greatest discretion. They throw more work on the liver and kidneys already overtaxed, and lower still further the nervous energy already depressed. "Alcohol is â predisposing cause to all endemic diseases; on this point all authors in exotic pathology are unanimous; in epidemics, the intemperate are the first and surest victims" (Treille). There is no objection to tea and coffee; they will furnish all the stimulation that may be needed under most circumstances. To relieve fatigue and quench thirst; on a hot day, 
Englishmen, Japanese and Russians rely mostly on tea. Coffee and tea are made with boiled water and have therefore the merit of being sterilized drinks. For this purpose, tea will admit of a much higher degree of dilution than coffee (see Coffee and Tea).

\section{SOLAR RAYS.}

It will be useful to remember that the solar spectrum consists of a series of colors, ordinarily described as red, orange, yellow, green, blue, indigo and violet. These colors result from the dispersion of the rays of different wave-lengths, the wave-length of the extreme red at one end of the spectrum being about twice that of the extreme violet at the other end. Outside the visible spectrum, however, there is also an invisible part beyond the red, called the infra-red region with much greater wave-length, and another beyond the violet, called the ultra-violet with shortest wave-length. The spectrum has been divided into three parts formed respectively by the invisible heat rays, the luminous rays and the chemical or actinic rays. This division, however, is more or less arbitrary for all rays of the spectrum are heat rays and possess more or less chemical power. It is true, nevertheless, that chemical changes are most stimulated by the violet and ultra-violet rays, and least by the red and orange.

Of late years, it has been more clearly realized that the solar rays affect animals and plants in their threefold power: by heat, light and actinic or chemical action. These effects are probably never separated but may be combined in greatly varying proportion. That heat alone cannot produce all the symptoms of insolation is made evident by noting the effects of simple artificial heat in rolling-mills and the fire-rooms of ships where the temperature not infrequently rises to $200^{\circ} \mathrm{F}$. Such heat is borne, for short periods of time, without injury. On the other hand, active work in the open sunlight at a temperature exceeding $100^{\circ}$ is perilous for the white man; the colored man endures it better, although, on the other hand, he is less resistant to the effects of extreme artificial heat.

Of the nature of the chemical action of sun-rays upon man but little definite is known. It is apparently directly proportional to luminosity, but also greatly influenced by heat; in fact it is" seldom noticeable except when accompanied by a high degree of heat, so that only in warm countries does it become sufficiently marked to be injurious. On the summit of high mountains, solar radiation and 
luminosity are greater (see p. $5^{\mathrm{I}} \mathrm{7}$ ), and the intensity of chemical action is said to be much increased (Hann); but, provided the head be kept well protected, the cold air and perhaps also the active evaporation from the skin prevent any dangerous effect of insolation.

It is to the constant stimulus of sunlight that are attributed the quick fancy, vivacity of gesture and animated language of the people of southern countries. But light, like heat, is often excessive and must be guarded against. A strong light reflected from a white bright surface soon becomes painful and, if continued, may produce headache, blurred vision and vertigo; in other words, a lightstroke.

According to some writers, the stimulating effect of sunlight is due to its actinic rays; pleasant and helpful when moderate, but harmful, depressing and overwhelming when excessive. The well-known disinfecting effect of sunlight, that is, its destructive action upon pathogenic bacteria, the decomposition of the atmospheric carbon dioxid under its influence by plants containing chlorophyll, its effect in tanning, inflaming and blistering the human skin, in blackening silver salts in photographic films, as well as in the process of bleaching fabrics, are so many instances of chemical action. The strong disinfecting power of the ultra-violet rays is utilized in the purification of water (page 2S2). The decomposition of a solution of oxalic acid under the influence of uranyl acetate (as catalyser) only occurs through the action of the ultra-violet rays. Bacon found that this decomposition in Manila, in July, was from 5 to 20 times greater than in Chicago, in June.

Effects of actinic or chemical rays upon man. - The effects of the actinic rays upon the health and efficiency of man, especially of the white man, is a question upon which much light has recently been shed. The majority of the races of mankind have pigmented skins ranging through various combinations of brown, red and yellow. It has been naturally inferred that the purpose and function of this cutaneous pigment is to exclude the actinic rays of the sun. This inference suggested to the white man, in warm climates, to protect himself in a similar manner by lining his hat and coat with a fabric of the color that best excludes the objectionable rays. It is well known that white men are occasionally seen who are unable to remain exposed to the sun without acute headache and great discomfort until they have recourse to this simple expedient.

What color is most effective to that end? Which shade of human 
pigment should we approximate? The copper of the American Indian, the bronze of the Hindoo, the yellow of the Chinese or the black of the negro? It appears that the nearer we approach to the equator the darker becomes the pigment, and that black, therefore, should be preferred. It is known that red and orange keep out more of the actinic rays than any other color of the solar spectrum and. for that reason, are used by photographers in the windows of their dark rooms. In following out this indication, undue prominence has been given these two colors by tropical hygienists, while the claim of the plain black, which for obvious reasons cannot be used by photographers, has been overlooked. If small squares of silk, of exactly the same texture and thickness but of the different colors of the spectrum (as near as they can be approximated in dry-goods stores), with black and white added, are placed on very sensitive photographic bromide paper and exposed an instant to the light, it will be found (Fig. 231) that black excludes the chemical ray's more completely than any color, and that the colors exclude them in the following order: red, orange, green, blue, indigo, violet, yellow, white. In studying Fig. 23I, it is interesting to note the selvage edge which, in all the samples, is on the right side. This edge is not appreciably thicker than other parts of the fabric but more closely woven, therefore more opaque. The result is seen in the fact that this edge is white, or nearly so, in all the colors except yellow, violet and white. The effect of color, however, is obvious in the selvage of the black and blue squares where a white line becomes black, and in that of the yellow square where a reddish line becomes nearly white.

From these and similar observations, it is deduced that the nonactinic value of a textile fabric depends more or less upon its color, but more upon its opacity; that any fabric which excludes light also shuts out actinic rays, and that this result is most surely and easily. obtained, the thickness of the cloth remaining the same, with black than red or orange; black is also more easily procured and less conspicuous; therefore it should be the color of the fabric used to line the head-dress and part of the coat, or for the undershirt. Silk being light and a poor heat conductor may be particularly recommended as the best material for the purpose (see p. 424).

This question seemed of sufficient importance to the War Department to warrant the undertaking of experiments on a large scale to ascertain the real value of anti-actinic clothing. These experiments 
were continued for over one year, in the Philippines, and fully reported upon in I9Io by Phalen, of the Medical Corps. Five hundred men were supplied with orange-red underwear (shirt and drawers) and hat lining; 500 other men wearing ordinary white underwear were selected as controls. Both kinds of underwear were practically identical in material and weight. The men wearing the special clothing and the controls were taken from the same companies, the two groups being as nearly as possible equal in physique and assigned to the same duties. It was found that in the special group, as com-

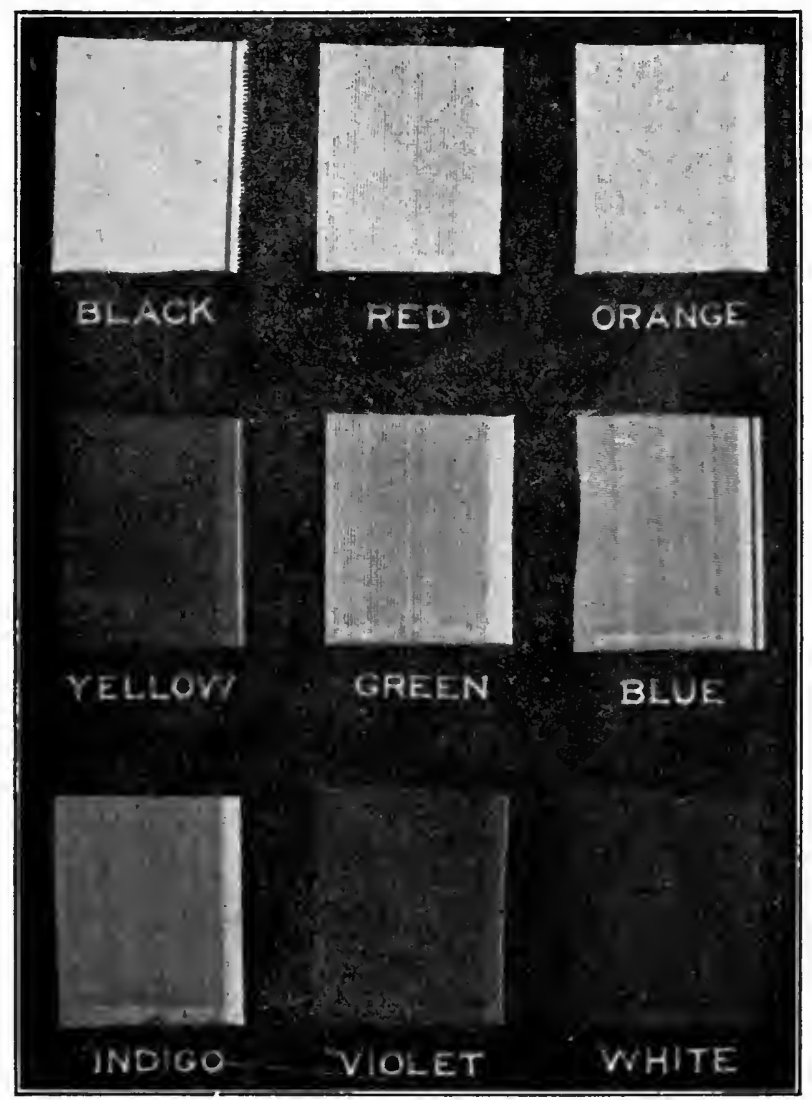

FIG. 23I.- Photograph showing the anti-actinic value of colors, including white and black. 
pared with the controls, the loss of weight was greater; that the rates of temperature, pulse and respiration were slightly higher; that the loss of blood pressure was greater; that the blood changes (increase of red-cells and loss of hemoglobin) were more pronounced. The general health condition, as estimated from the admissions to the sick report, was practically the same in both groups. The great majority of the wearers of colored under-garments, that is, 4 out of every 5, pronounced them distinctly less comfortable than white ones.

Although the differences noted above are but slight they justify the conclusion, when considered collectively, that colored undergarments are more absorptive of heat rays than white ones. This was directly proved by many experiments both on and off the body, showing that, when exposed to the sun, the temperature was greater beneath the orange-red material than beneath similar white material. Thus of two flasks covered, one with orange-red, and the other with white material, after one hour's exposure, the first showed a temperature of $48.2^{\circ} \mathrm{C}$. and the other $44^{\circ}$. In the case of a man wearing a composite shirt, half white and half orange-red, it was found, after Io minutes' exposure, that the thermometer beneath the white side registered $37.75^{\circ} \mathrm{C}$. and the one beneath the colored side $43^{\circ}$. The same results followed when both materials were overlaid with khaki cloth, although the difference was not so marked.

It must be conceded that orange-red underclothing protects the body from the actinic rays of the sun, at least from those rays that act upon photographic plates. This protection, however, does not only depend upon the color of the fabric but also upon its thickness and the tightness of its weave, that is, upon its opacity. It was found that the campaign hat is as opaque to chemical rays without a red lining as with it, while this lining added materially to the opacity and anti-actinic power of the khaki cap. The greater thickness and tighter weave of the American khaki, as compared with English khaki, render it more protective. The blue and olive-drab flannel shirts were shown to be not nearly so protective as had been expected.

We may conclude that, if underclothing of undoubted anti-actinic power has no favorable effect upon the vitality and efficiency of the white men wearing it, it is because the chemical rays of the sun, under ordinary circumstances, really play an unimportant part as a 
climatic factor and are, so far as the great majority of men are concerned, a negligible quantity. It is possible, however, that under a blazing sun and with inadequate protection, the actinic rays may acquire dangerous power; thus might some of the symptoms of fulminant insolation be accounted for. It is also known that, occasionally, men are found who are especially susceptible and need to be guarded against their influence. Thus of the 500 subjects of the experiment noted above, 7 preferred the colored underwear because it relieved them of " headache and dizziness" (in 4), of " headache" (in I), of "fever" (in I) and of "prickly heat" (in I). Of the few officers who also tried it, one stated that he had been entirely relieved from "a feeling of depression of spirits and irritation" which previously always followed exposure to the sun. For this small percentage of men, colored or black underwear and hat lining will always be desirable and should be provided.

That the skin pigment has some protective action against chemical rays must be conceded, but there is nothing to prove that this action possesses physiological importance; otherwise, how to explain the various colorings of this pigment in different races subjected to practically the same climatic influences, and why should people living in northern latitudes, like the Esquimos, North American Indians and Chinese, have so much of it? We also know that races greatly differing in kind and degree of pigmentation have lived together for centuries in the same regions (as in Egypt) without any tendency to an approximation of pigmentary hue. It seems more probable that pigmentation is one of the racial characteristics, acquired ages ago, and that it is no more the direct effect of the sun than is the shape of the nose, the thickness of the lips or the projection of the cheeks.

Some of the experiments reported by Phalen bear on this subject. Specimens of human skins of different hues, made as nearly as possible of uniform thickness, were tested with photographic plates to ascertain the extent of their opacity to the chemical rays. Comparing a very dark Filipino skin with a white one, there was, as expected, a notable difference, but only for short exposure; by increasing the exposure to one minute the effects on the photographic plates did not differ very greatly. A comparison of the effect produced through the light brown and white skins showed practically no difference. It was noted that the texture of the former was much softer than that of the latter. On a photograph made by short 
exposure through the darkest skin, and through the white skin reinforced by one layer of khaki cloth, it was observed that the latter fabric completely neutralized the difference in opacity existing between the skins. Another fact was shown, namely, that the pigment of the darkest skin does not by any means approximate the orangered of the experimental garments in excluding the chemical rays. The abundant blood circulating in the deeper layers of the skin, says Phalen, is probably much more efficient as a protection against the chemical ray than is the layer of pigment.

Black pigment seems to be of much greater use in mitigating the heat rays than in intercepting the chemical rays. As is well known, black absorbs more solar heat than any color, but also radiates most. Thus hot tea poured in a black pot will cool more rapidly in the shade than if put in a light-colored vessel. A dark-hued skin, therefore, loses heat more quickly than a white skin at night or whenever protected from the sun. But when exposed to the sun, another physiological process comes into play which again gives it the advantage, as shown by Aron in the Philippines. Being more quickly heated, the temperature at which the sweat starts is more promptly reached and, with the sweat, comes evaporation and cooling of the surface. The regulatory apparatus of the dark skin, with its abundant sweat glands, is more sensitive than that of the light skin and responds more promptly and successfully to the stimulus of heat; accordingly Aron found that, in the sun, the white skin is always slightly hotter than the brown skin. How much of this effect is due to color, to the innervation of the sweat glands, or the number and structure of the latter is not yet clearly determined. The white man is more likely to perspire profusely, with dripping sweat, a condition which weakens the body from loss of water without corresponding cooling effect, the comparatively thick sweat layer absorbing more heat from the air than from the skin in the process of evaporation. In the brown man, on the contrary, the perspiration, although very free, is spread in a thinner film over the skin, seldom in excess of what can be evaporated, while the evaporation is more completely at the expense of the body temperature.

An interesting question is the relative ability of blonds and brunettes to withstand tropical conditions. It has been contended by most writers that the latter were better fitted by nature to resist the detrimental influences of warm countries. This subject having been 
elaborately investigated by a medical board, its president, Major Chamberlain, reported that: "From a consideration of all the data it appears that, among American soldiers, blonds are quite as well able as brunettes to withstand the influences of the Philippine climate for a period of two years and probably for a period of five and onehalf years," the time covered by the investigations. Among the two groups there was the same relative amount of sickness, the same proportion invalided home and discharged, and the same influence on character and behavior.

The intense luminosity or glare of hot climates is often distressing to the eye, and should be mitigated so far as practicable; the brim of the hat or helmet, or the visor of the cap, should be wide enough to afford adequate protection. Colored glasses are often useful. Gould thinks that amber-colored glasses are best, admitting enough of the more luminous rays (yellow) and excluding those which are injurious to the retina.

\section{Sunstroke.}

Under this comprehensive poptlar name are included three separable conditions, although the first two are not always clearly differentiated: insolation, heat-stroke and heat exhaustion, and the soldier may suffer from one or another, or a combination of them.

Insolation (siriasis, sunstroke proper), the most dangerous of them, results from exposure to the direct rays of the sun at a high temperature. It has been ascribed to the action of the ultra-violet or actinic rays of sunlight but this contention has'not been confirmed by more recent investigations. Whatever influences these rays may exert, it seems pretty well established that siriasis is chiefly the result of a rapid and excessive increase of body temperature, and it appears probable that the immediate cause of such dangerous increase is the stoppage of perspiration brought about by a disturbance in the cutaneous innervation. It is not unlikely, however, that the actinic rays are responsible for this disturbance of innervation, as they are in the production of sunburn. Thus would be explained the fact that the degree of temperature which in sunlight causes sunstroke is harmless in the engine-room of a steamer.

-Insolation is always manifested by rapid and severe symptoms, such as intense headache, a quick, full pulse and hot, dry skin, suggesting meningeal congestion or inflammation; or, in grave cases, by 
sudden unconsciousness from paralysis of the heart or of respiration, the result of hemorrhages in the brain and meninges. It may be seen on the march but is more likely to occur during the stress of battle, under a blazing sun, when the head is imperfectly protected.

Heat-stroke, also called thermic fever, is of common occurrence in this country wherever great heat and a high degree of humidity are combined, as in the eastern and southern States. Intemperance, in eating as well as in drinking, fatigue, bad ventilation, malaria and all other depressing conditions are predisposing influences. Its etiology is still uncertain; heat is probably the chief factor, with more or less actinic effect. From the fact that direct exposure to the sun is not necessary for its production, and the peculiarities of its distribution and prevalence, Sambon and Manson are inclined to consider it a germ disease, a view not generally accepted. The symptoms are, in the first stage: fatigue, pains in the limbs, drowsiness, headache, vertigo, mental confusion, intolerance of light, contracted pupils, suffused eyes and face, nausea and vomiting, hot, dry skin (rarely moist) and quick pulse. They are soon succeeded by high fever and burning skin, the temperature rising to $108^{\circ}$ to $109^{\circ}$ F. (H.C. Wood), an exceedingly rapid pulse, later becoming irregular and intermittent, labored breathing, convulsions and complete unconsciousness. In mild cases, the symptoms may be limited to some febrile movement, disturbance of the digestive functions and torpidity of bowels. The treatment consists in taking the patient to a cool, shady, well-ventilated place, removing his clothing and reducing his temperature by cold baths. Hot baths have also been highly recommended. The administration of digitalis and strychnine will generally be indicated, and artificial respiration may be necessary.

It must be borne in mind that some of the pathological effects of sunstroke are more or less permanent, that the patient seldom completely recovers and always remains more susceptible to a subsequent attack; hence the saying, in some of the British colonies, that "once sunstruck always sunstruck." It seems an admitted fact that man does not become habituated to hot solar rays, so as to be immune to their effects, any more than he becomes habituated to the contact of fire. The native of hot countries, although endowed by nature with a dense and very black (non-actinic) head of hair, generally protects himself with ample hat or thick turban. The 
present custom or fashion of riding and indulging in all sorts of outdoor sports, bare-headed, during the hot summer days, is not approved by the best judgment of hygienists and may certainly result in serious harm. It is known to have produced alopecia.

Heat-exhaustion is a state of syncope or faintness resulting from high atmospheric temperature combined with fatigue or privation, and often induced by alcoholic excess. The patient is pale, with cold skin and subnormal temperature; the pulse is small and soft, and the breathing shallow; the pupils are generally dilated. There is seldom complete unconsciousness. He should be laid on his back in a cool, shady place, his clothing loosened and a little water dashed on his face and chest. Gentle stimulation is indicated; spirits of ammonia may be held to the nose or given by the mouth; whiskey, suitably diluted, may likewise be given by the mouth or else, in case of unconsciousness, injected into the rectum.

\section{CLOTHING.}

The general subject of clothing has already been considered, and so far as it relates to the tropics can be summed up in a few words.

For underclothing, flannel, even of light material, is seldom comfortable. It should preferably consist of a cotton-knit loose undershirt with short sleeves, jean or muslin drawers not coming much below the knees, and cotton socks. For those who can afford it, a gauze of wool and silk makes the ideal material for underwear. The so-called linen-meshed garments are also very satisfactory and preferred by many. Care must be taken that the material is never so thin and porous as to allow the body to become chilled in the evening.

When the coat is not worn, the olive-drab flannel shirt is substituted. The best head-gear is a well-constructed, black-lined helmet; but, for the field, the campaign hat is more serviceable, provided it is sufficiently thick, well ventilated and water-proof. With suitable head-gear it is best to keep the hair cut short.

A flannel abdominal band or apron (see page 440), worn at night, is often serviceable in the tropics, especially in the hot season. As one lies in bed, uncovered and perspiring, the abdomen, which is the most sensitive part of the body, will be chilled before any other part; the bowels lie so near the wall of the abdomen that their circulation is easily deranged by changes of temperature, and digestive 
or intestinal troubles may result in susceptible people. The abdominal band is never useful during the day, and seldom at night when the cold is so marked as to require one or two blankets over the sleeper.

\section{SHELTER.}

In the erection of quarters or barracks in the tropics, the objects to be aimed at, from the hygienic viewpoint, are to exclude heat and excessive light, and provide ample ventilation. Greater air space and movement should be provided than in northern climates, and this can be done chiefly by raising the ceiling to a height of I 3 or 14 feet and extending the windows downward to near the floor and upward to near the ceiling. The barracks, and as many of the other buildings as possible, should be so oriented as to face north, therefore with the long axis running east and west. This, however,

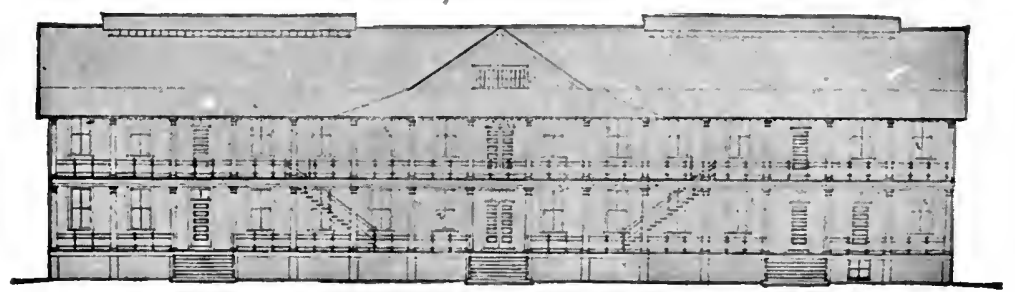

FIG. 232.-U. S. barrack in the tropics. (Honolulu.)

may be modified in accordance with prevailing breezes; thus to get the benefit of the N. E. trade-winds, the axis should preferably run N. W. to S. E. The barracks should be of two stories, each story with a broad veranda going all around and protected by Venetian blinds on the sunny sides. The upper story is to be used for dormitories, and above it should be a spacious, well-ventilated attic. The best building material is iron, brick and stone; a thick wall with a course of perforated bricks permitting a free air circulation is nearly ideal. The roof should be of tile or concrete, and the floors of cement, vitrified brick or tile. The color of the outside paint or kalsomining must not be white, which injuriously dazzles the eye, but a soft light tint of gray, yellow, pink or blue.

In India, according to Melville, the 2-story barrack has not been found very satisfactory. In its stead are preferred half-company 
bungalows with simple verandas, each bungalow consisting of two dormitories, 20 feet high, in which 1,800 cubic feet of air are allowed per man. Between these dormitories a mess-room and kitchen are provided.

The effect of water evaporation in cooling the air of habitations is seldom fully appreciated and utilized; thus the temperature of a room 70 feet long by 30 feet wide, sprayed with a gallon of water, will speedily fall from $80^{\circ}$ to $70^{\circ} \mathrm{F}$. Verandas should be freely sprinkled. The East Indian method of suspending mats in doorways on the windward side, and keeping them constantly wet is also very efficient, provided there is a breeze. The methods employed in cold-storage rooms, especially the ammonia process, are also applicable, on a smaller scale, to all other kinds of buildings. Where artificial ventilation is used, the air can be driven through an ice chamber before its distribution. Simpler economic devices

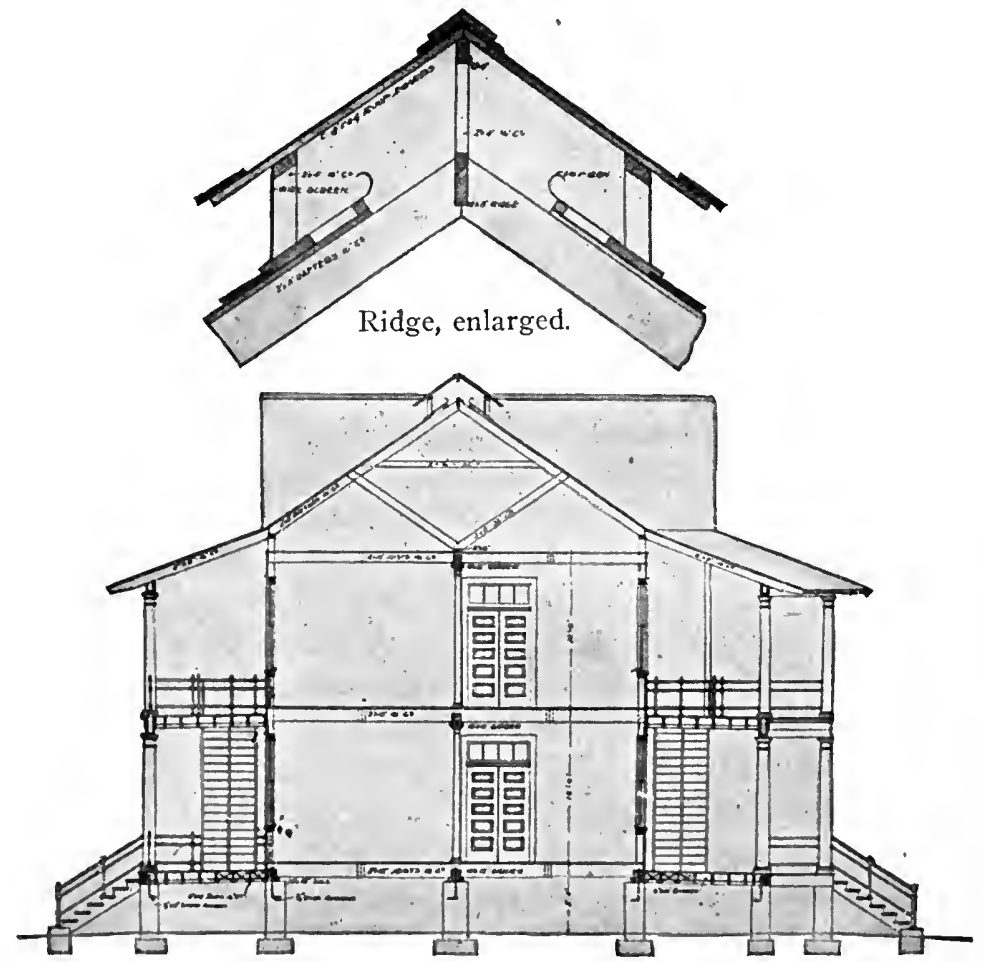

FIG. 233.-Cross section of Fig. 232 . 
have been recommended, such as that of Moore in which the air passes down a cylinder filled with cracked ice and salt, and that of Gates in which the air is purified and cooled by water spray.* Dr. Manning, of Washington, D. C., suspends horizontally a strip of cotton twill, three feet wide and as long as desired, in the room to be cooled, and keeps it saturated with water by means of a perforated pipe passed through a hem in the upper edge, the water being collected below in a trough hanging from the lower edge. With an electric fan playing upon one end of it, a wavy, serpentine motion can be imparted to the entire strip and the evaporation greatly increased. $\uparrow$

The mosquito being always a dangerous enemy in warm countries, every effort must be made to exclude it; in a malarial locality all quarters should be completely screened, and originally planned with a view to the possibility of this effective screening. But, under all circumstances, at least the kitchen and mess-room should be entirely protected by wire netting, so as to keep out not only mosquitoes but also flies and other insects.

In cantonments, I-story frame buildings well raised above the ground, with suitable verandas, large windows, ridge ventilation and tiled roof (or double roof of corrugated iron) are very satisfactory, but it will often be found advantageous to utilize native material and labor and erect structures of bamboo and thatch which can be made fairly comfortable and sanitary.

The use of tents, in warm climates, should be avoided as much as possible, as it is always difficult to keep them reasonably cool, dry and otherwise comfortable. Whenever used, each should be covered with its fly set at an interval of about a foot from the roof. In a camp of some duration, the tents should be shaded, whenever possible, with a roof of brush, straw or grass. An excellent method of cooling them is to dash water against the walls and over the roof, but not enough to close the pores of the canvas. The best tents for hot weather are the tropical hospital tent and the tropical wall tent described under Camps (page 620). For the sick, a black, dark blue or dark red lining, to exclude actinic rays, will add to the comfort of the hospital tent; if more light is desired this lining can be covered with pale yellow chintz. The conical tent is entirely unfitted

* See Bergey's Principles of Hygiene.

$\dagger J . A . M$. A., June 4, 1910 . 
for hot countries; it is more stable but, on account of the low roof and lack of fly, always several degrees warmer than the hospital tent.

\section{GENERAL DIRECTIONS.}

Physical exercise, in the tropics, is highly useful and should never be neglected, but must be performed at the proper time. Drills and all military exercises should not be permitted, as a rule, whenever the temperature exceeds $85^{\circ} \mathrm{F}$.; with a cooling breeze this limit might be raised to $90^{\circ}$; therefore the best time for them will be in the early morning before $8 o^{\circ}$ clock, or in the evening after 5 o'clock. All work not absolutely necessary should be suspended between I I A. M. and 4 P. Mr., the hours of siesta. During these hours it is not necessary that the men should sleep, but desirable that they should enjoy complete mental and physical relaxation. Marching should begin at dawn, or even before, and stop at or before 9 o'clock, to be resumed, if necessary, after $5 \mathrm{P}$. i. T. To break the usual night rest is always to be deprecated but, whenever optional, it is less exhausting and greatly preferable to do so than to march during the day under a torrid sun, provided time is given to make up sleep at the first opportunity. There is a time of day when the sun is exceedingly trying, that is when approaching the horizon, before setting; then its horizontal rays strike the face, eyes and temples, parts well protected at other times, with great actinic intensity. If the command is not yet in camp, and the direction of the march is westward, it will be wise, circumstances permitting, to halt until after sunset. The step should be an easy route step, in open order and with load as light as possible. The men should seldom drink on the march, except at the halts, after cooling off, and then moderately.

With proper care, even large bodies of troops can march in summer over extensive desert tracts, in tropical countries, without serious losses. Such is the march described by Dr. James McGregor, of an English army from India, which disembarked at Kosseir on the Red Sea, in June, I80 i by marching always at night it reached the banks of the lower Nile (over 400 miles) in a month, in " a very healthy state," in spite of the intense heat, dust and hot winds, and of uncomfortable garments with much neck gear and strapping.

In warm countries the loss of heat by radiation is generally very rapid after sunset, causing a sudden fall of temperature and chilling 
sensation. The same sudden change may take place after a rain. This is to be guarded against by buttoning th the shirt and blouse, adding or changing garments, especially by those susceptible to malarial or diarrheal diseases. The experienced soldier never parts with his blanket, however irksome it may be to carry during the day. The effect of altitude on the tropical climate is also very remarkable, a rise of 1,000 feet being equivalent to a change of $6^{\circ}$ to $10^{\circ}$ latitude further north, while the noctural temperature falls still in a greater ratio. Thus while the heat is intolerable in Santiago de Cuba, in June and July, Cristo, a few miles away, at an altitude of only 800 feet, is delightfully cool. This fact should be taken advantage of in locating barracks and hospitals ; even an elevation of 100 or 200 feet is decidedly advantageous.

The principle that soldiers in camp should never sleep on the ground applies especially to tropical countries. If cots are not available, the poncho or slicker should be interposed between the ground and the blanket or overcoat used as bedding; but still better, if time permits, a bedstead should be improvised. The mosquito-bar is never to be dispensed with, except by authority, when the complete absence of mosquitoes has been ascertained.

The cold bath in hot countries is certainly an excellent means to combat the heat and prevent the loss of nervous energy. The swim at the beach or the shower-bath at the barracks cannot be too highly recommended, provided it is short and no depressing effect is produced.

To observe the state of his bowels and regulate them as required should be one of the important hygienic cares of the soldier in the tropics. The waste products from excessive proteid alimentation, the sluggish digestion and rapidity of bacteria multiplication greatly increase the danger of auto-infection. Many cases of "undetermined " fever are doubtless the result of the reabsorption of toxic products from the intestinal canal. The heat (and solar actinic rays) so affect the nervous system as to often check the neristaltic motion of the bowels. with consequent constipation. This should be overcome by cold shower-baths, massage and vegetable and fruit diet. 


\section{TROPICAL DISEASES.}

(See also page 15. )

As has been stated before, constant high temperature and humidity are favorable to the multiplication of microbes, as well as that of insect life and vermin, and this explains the rapid decomposition and decay of organic matter in the tropics, with or without putrefactive odors. These microbes, however, are practically all saprophytes and harmless to man. As to pathogenic germs, the conditions under which they breed and are transmitted are now well understood, with the result that the prevalent infectious diseases which they produce are all preventable and can be stamped out by the application of well-known sanitary measures. Such diseases as leprosy, bubonic plague and cholera continue to lurk in some of our colonies after having disappeared from colder climates, more on account of the ignorance and indifference of the natives than because of more favorable conditions for the breeding of germs. There are, however, many insects, parasites and organisms only found in warm countries, so that the infections they generate, like yellow fever and amebic dysentery, do not easily spread in temperate latitudes; but these also are preventable.

There is no foundation for the belief that tropical climates are favorable to the development of mental and nervous diseases. The reports of the Surgeon General for the last decade show that insanity, neurasthenia and suicide have not been more common in the Philippine Islands than in the United States.

Among American soldiers the two classes of diseases by far the most prevalent in the Philippines are venereal disorders and malarial fevers (see page I7). In I9Io, the ratio of the former was nearly twice, and of the latter over six times that of troops in the United States. Venereal diseases in the Philippines are of a decidedly more contagious and virulent type. They have widely spread since the American intervention and many of the interior towns where they were formerly unknown have become infected.

There are also indications that much of the morbidity among our troops in tropical territory is due to excessive or improper diet. This is shown, in the first place, by the rates of diarrheal diseases which, in the Philippines, are more than double those in the United States: in the second place, by the high prevalence of "Furuncle and Phlegmon" which, during the period I908-I9IO, was one of 
the five diseases having the highest rates of admissions; and in the third place, by the rather large proportion of cases reported as " undetermined fevers," under such headings as simple continued fever, febricula, ephemeral fever. In $1909,25.46$ of admissions per $\mathrm{I}, \infty 00$ of strength were for this class of cases in the Philippines, against 2.15 in the United States; in 1910 , the ratios were 10.75 and 2.42 respectively. Owing to the well-known care with which diagnoses are made in all suspicious febrile cases, it seems probable that many of these undetermined cases are the result of auto-intoxication from excessive or inappropriate proteid alimentation.

Dysentery which, as a cause of death, is a negligible disease in the United States under normal conditions, assumes much greater importance in the Philippines; next to tuberculosis it had the highest ratio of deaths in I9IO. It is the tropical disease most to be apprehended by Americans.

Typhoid fever, in the Philippines, shows a much lower rate for the Filipino scouts and natives generally than for white troops; for the latter the admission rate exceeds that for troops serving in the United States, showing that the comparative immusity of the natives is not a matter of climate but rather of racial resistance, or of diet.

The so-called tropical anemia of the natives which has been generally attributed to depressive climatic influences, is now regarded as secondary to certain specific diseases or the result of several causes combined, such as malaria, uncinariasis and semi-starvation.

In conclusion it may be said that: Any soldier, in the tropics, who takes an intelligent interest in the sanitary measures enacted for his benefit and cheerfully complies therewith, who reasonably adapts his diet to the climate, keeps away from the hot sun when off duty, and shuns the saloon and brothel, has as good chances of health and longevity as in temperate climates. 


\section{CHAPTER LVIII.}

\section{SERVICE IN COLD CLIMATES.}

A temperature of $40^{\circ}$ to $45^{\circ} \mathrm{F}$. below zero is not uncommon in some of our interior northern States, while a still more intense cold is experienced in parts of Alaska. The coldest day recorded by Nansen in "Farthest North" while drifting, only a few degrees from the pole, was on January I5, when the temperature ranged from $-58^{\circ}$ to $-61.6^{\circ} \mathrm{F}$. At Cape Sheridan (1at. 82.30 $0^{\circ}$ ), the coldest month recorded in 1909 was February, with mean temperature of $-3 \mathrm{I} .7 \mathrm{I}^{\circ}$, and the lowest minimum $-52^{\circ}$, in March. The mean of February at Fort Conger (lat. 81.30 $0^{\circ}$ ), in $188 \mathrm{I}-1883$, was $-40.13^{\circ}$. For comparison, it may be mentioned that the mean temperature of February along our northern boundary line $\left(49^{\circ}\right.$ of lat.) seldom falls below zero. When Peary left Camp Columbia, March 13 , (lat. $83^{\circ}$ ) for his dash to the pole, the temperature was $-53^{\circ}$ and reached $-59^{\circ}$ in the evening. He notes that as there was no wind the cold was not unbearable. At the pole, on April 6 and 7 , the temperature ranged from $-\mathrm{II}^{\circ}$ to $-30^{\circ}$. The lowest temperature recorded by Amundsen in his winter quarters at the Bay of Whales, in the Antarctic Circle, was $-74^{\circ}$ F., on. August I3.

In northern latitudes, when the air is dry and perfectly still, the temperature falls to its lowest point, to rise again as soon as the air becomes disturbed by wind currents. Such calm cold, although intense, is much more bearable than a lesser cold with wind blowing, for the body becomes surrounded with a layer of air warmer than the atmosphere and loses less heat. It is the blizzard that constitutes the dangerous feature of the winter of our northwestern States. As explained in another place (page 514), the same degree of cold is much more bearable if dry than if damp; thus a temperature of $-10^{\circ}$ or $-15^{\circ}$ in Dakota is less trying and depressing than one of zero on the shores of the Great Lakes, causing a smaller loss of animal heat from the body.

That man can maintain himself in excellent health and lead an active life in climates with extreme winter cold has been amply demonstrated. It has also been shown that, with proper food and 
clothing, it is possible for troops to conduct an active campaign in winter snow and ice, with temperature often below zero. Several of our successful Indian campaigns were thus prosecuted without a single death from congelation or serious accident attributable to the cold. During the Russo-Japanese War, one of the bloodiest battles, lasting several days, was fought in January, with temperature from $8^{\circ}$ to $12^{\circ}$ below zero, a sharp wind blowing from the north during much of the time; there were many cases of frost-bite, but most of them could have been prevented by proper hand and foot covering.

Severe cold causes loss of body temperature by conduction and radiation, and reduces the force and frequency of the pulse. It constricts the cutaneous capillaries and greatly diminishes perspiration, while the urine is correspondingly increased. Should the exposure be prolonged and severe, the contracting superficial arterioles do not permit enough blood to reach the extremities which become blanched and frost-bitten. Frost-bite, in hands and feet, is generally accompanied by a sharp tingling pain, but may also manifest itself, especially when the result of intense dry cold, by a painless ivory-white spot on the cheek, nose or ear. In general refrigeration, after long exposure, the patient experiences difficulty in speaking; his sight grows dim and his faculties fail him; muscular exertion becomes difficult and he staggers like a drunken man; he is overcome by a sense of languor and an irresistible desire to go to sleep, a sleep which leads to coma and death.

To combat cold successfully, the system endeavors to produce more animal heat by an increased demand for food and by stimulating the digestive and assimilative functions, while the combustion of tissues and elimination of wastes are more rapid and complete. The food, in very cold climates, should be abundant and rich in fats (fat meats, bacon or pork, oil and butter). The Eskimo is said to eat 12 to 15 pounds of fat meat, raw or cooked, daily, when able to procure it, and to be very fond of raw blubber and walrus beef. The increased desire for rich food, in northern latitudes, may be safely indulged provided one performs active work, or takes brisk exercise; but, with a quiet or idle life, this desire should be restrained and regulated, otherwise accidents of auto-infection or disorders of nutrition are sure to result.

The conclusions reached by Peary as to the best diet for strenu- 
ous work in the Arctic Circle contain information of practical value to any military command operating in very cold latitudes. In his opinion, the only food essentials needed under such circumstances, no matter what the season, the temperature or the duration of the journey might be, are four: pemmican, tea, ship's biscuit and condensed miik. Pemmican, he writes, " may be regarded as the most concentrated and satisfying of all meat foods and is absolutely indispensable in protracted Arctic sledge journeys." (See composition, page 335.) In all his journeys towards the pole, the standard daily ration was: pemmican, I pound; ship's biscuit, I pound; condensed milk, 4 ounces; compressed tea, $1 / 2$ ounce; liquid fuel (alcohol or petroleum), 6 ounces; a total of 2 pounds Io $1 / 2$ ounces for ration and fuel. Of this, Peary says: "I believe that no other item of food, either for heat or muscle building, is needed."

Of the trip culminating in the discovery of the south pole, Amundsen writes: "For food we relied entirely on pemmican, biscuits, chocolate, powdered milk and, of course, dog meat. The dogs were fed on pemmican. In my opinion we had the best and most satisfying provisions possible." The pemmican contained oatmeal and vegetables as well as meat and fat. The biscuits consisted of oatmeal, powdered milk and sugar. The powdered milk " proved a splendid thing" as it packs and keeps well under all circumstances.

Experience has shown that alcoholic drinks, except in great moderation, are especially dangerous in cold countries, and that men are healthier and capable of greater endurance to cold and fatigue without them. This has led to the entire suppression of the daily dose of rum or whiskey which was formerly part of the ration of the crews of whaling vessels and other ships cruising in the northern seas. When Nansen sailed for the north pole, the only spirits on board his ship were a few bottles of cognac in the doctor's stores; he returned after a three years' strenuous cruise with every member of his expedition in the best of health. According to Pierson, of the Medical Corps, who spent severa! years in Alaska, alcohol, for anybody exposed to extreme cold, is almost suicidal, having a prompt and overpowering effect upon the nervous system. Amundsen, after describing the bill of fare of his men while wintering near the south pole, adds: "Every Saturday evening a glass of toddy and a cigar," thus showing it was not a common and regular indulgence. On sledge journeys, says he, "we all know from experience that 
spirits must be banished," because weight and space must be saved and he does not regard spirits as one of the necessary things.

The most suitable clothing is woolen undergarments and fur outer garments. Too much clothing is uncomfortable and unsafe while marching or exercising; the undergarments become dampened with perspiration and there is danger of chilling of the body surface from rapid evaporation at the next halt. The underwear should only be of meditum weight and thickness in post or camp, and when more warmth is needed it should be obtained chiefly by outer clothing which can be changed or removed as desired. The buffalo fur coat formerly worn by officers and men in winter expeditions is now replaced by a thick canvas overcoat lined with heavy blanket cloth. Buckskin suits are excellent, keeping out the cold wind and saving the body heat without impeding overmuch the aëration of the skin.

The extremities require special attention. In very cold weather leather shoes are of little use; they do not keep the feet warm, and are very slippery. But they are necessary when the ground is damp or muddy, as after a thaw; in such case they should be oiled or otherwise made waterproof. Wetting of the foot-gear renders the danger of frost-bite serious and constant. The socks should always be perfectly dry before being put on. According to Pierson, so long as the ground is dry and frozen there is nothing better than German socks and moccasins, the usual foot-gear of the Alaskan mail carriers. Likewise very suitable are the woolen stockings, felt shoes and Arctic overshoes provided by the Q. M. Corps. Care must be taken that the feet are not compressed, and that nothing interferes with the free circulation of blood in the toes. For the hands, mittens are better than gloves, and the fur mittens of muskrat skin lined with lamb's fleece, issued to our soldiers in very cold latitudes, can hardly be excelled. Mittens of tan buckskin are also provided when needed. They should be several sizes larger than the hands so as to permit the latter to slip in and out easily, or the wearing of the woolen knitted glove inside of them. The half-breed's way of wearing his mittens, tied to both ends of a cord passing around his neck, secures them against loss, and otherwise saves time and trouble, especially when the soldier is obliged to withdraw his hands to handle his gun.

For the head, the fur muskrat cap formerly issued to the men is 
a most excellent covering. It has been replaced by the less expensive canvas cap lined with olive-drab kersey, with visor to protect the face, and extended below into a cape to cover the neck and throat. A very useful and popular type of cap in cold countries is that with adjustable side pieces tied across the top in mild weather, but let down and tied under the chin in cold weather, thus protecting the ears, sides of the face, throat and chin. The hood of the Army overcoat would be improved, for service in high latitudes, by the addition of a fur edging which keeps out the cold without collecting frost from the moisture of the breath.

For bedding, the best device is the sleeping-bag of the Arctic explorer and Canadian voyageur, made of strong canvas lined with fur. However, inasmuch as no part can be removed so as to adjust it to variations of temperature, it is not desirable in changeable weather or in the proximity of a fire. (See page 468.) Fur robes are always useful.

In marching, certain precautions are required. If the face suffers from the cold it should be anointed with cold cream, vaseline or any kind of grease. Bright sunlight reflected from the snow produces a painful glare, sometimes strong enough to cause snow blindness, an affection which begins with pricking sensations in the eyeballs and lasts from a few hours to one or two weeks. To guard against this glare the Eskimos wejar wcoden spectacles with a slit cut through the eye pieces. Colored glasses are also useful. The best protection is afforded by goggles with amber glass which excludes the injurious glare without interfering with the acuity and range of vision. The metal parts of spectacles in contact with the skin should always be wrapped in wool. Inasmuch as glasses may become steamed and have to be frequently wiped, some writers recommend the use of a piece of thin, black crepe fastened to the front of the cap or hat and falling over the eyes like a veil.

The column should march in close order so that the men may shelter and protect one another. No. straggling must be permitted. There is always great danger that men exhausted by the march, mentally and physically benumbed by the cold, may drop out, if unobserved, yield to their intense desire to go to sleep and freeze to death; then "whoever stops goes to sleep, and whoever goes to sleep wakes no more." Such men must be urged, helped, shaken and kept awake at all hazards until shelter is available and rest possible. 
When undertaking long marches in very cold weather, a sufficient supply of tea and coffee should always be on hand, along with small portable spirit lamps or oil stoves. Alcoholic stimulants, when deemed necessary, may be used in small doses cautiously repeated.

Here may be mentioned the Japanese " pocket stove" which many Japanese soldiers carried on their persons in Manchuria during the Russo-Japanese War; it is semi-cylindrical in shape, one foot long and four inches in diameter; a slow burning powder is the fuel and a pleasant warmth is thus readily produced.

In frost-bite there is more or less congelation of tissue and arrest of circulation. Its intelligent treatment requires some knowledge of its pathology. As the blood of the exposed part freezes, the water, of which it mostly consists, expands (as when ice is formed) and stretches the constricted capillaries which are thus more or less jnjured. If the part is rapidly thawed out by the application of heat, the circulation is restored before the capillaries have time to recover enough of their tone and integrity to withstand the full force of the blood pressure. The result is continued paralysis of the blood-vessels, with stasis and possibly necrosis and gangrene. To prevent such serious danger, the circulation must be restored very slowly, so that the walls of the arterioles may recover nearly their normal state by the time the blood begins to flow. When an exposed part is in danger of becoming frozen, it is enough to rub it with the hand or the back of the mitten. If it be blanched and actually frosted, snow should be rubbed on it, gently but persistently until the blood returns to the skin. In case of a frost-bitten extremity, immersion in ice-cold water, with continuous frictions, must be kept up until the muscular tone of the arterioles is sufficiently restored to prevent stasis, that is to say, one or two hours after the return of the circulation. If upon removing the frost-bitten part from the water, it becomes blue, thus giving evidence of stasis, it should be put back again. Hot water, as hot as can be borne, is recommended by some Russian writers who claim excellent results from this mode of treatment, with much less discomfort to the patient. Although the blood is thus quickly thawed out, the capillaries are stimulated and kept constricted by the heat, checking the circulation until they have recovered their normal tone.

Cold countries, that is, those in which a freezing temperature per sists for long periods without thaw, are generally very healthy, 
Alaska (for whites) is the healthiest part of all the territory under the flag of the United States. Their prevalent diseases, namely, colds, tonsillitis, bronchitis, etc., are those which result from the usual tendency of people, in cold weather, to crowd together in overheated and ill-ventilated rooms, neglecting open-air exercise and the requirements of personal hygiene. As would be expected under such conditions, any epidemic breaking out is liable to spread rapidly. Pneumonia is very rare in Arctic regions. Tuberculosis among the Esquimos is frightfully prevalent (4 per cent.), showing how dangerous is the neglect of ordinary measures of prophylaxis even in the most favorable climates. A common disease in Alaska, according to Pierson, is the " chichoker" knee, a painful local inflammation of low grade with stiffening of the joint, due to exposure. It is prevented by wearing some form of knee protector. Peary notes the interesting phenomenon that outbreaks of grip among Arctic explorers have generally been coincident with epidemics of this disease in Europe and America.

In the case of general congelation, the patient must not be carried at once into a warm place, but kept for a while in a shed or fireless room; there the body is rubbed with dry towels and the extremities treated as in frost-bite; heart stimulants will probably be needful. 


\section{CH.APTER LIX.}

\section{DISINFECTION AND DISLNFECTANTS.}

(See also Insecticides.)

The term disinfectant, although popularly used in a more comprehensire sense, should be restricted to any substance which destroys the specific germs and toxins of infectious diseases. It is practically symonymous with germicide. Disinfection, therefore, consists in the destruction of pathogenic germs. Sterilization has a more extended sense and means the destruction of all micro-organisms. Antiscptics are substances which, without killing organisms, lower their vitality so as to arrest their power of propagation, thereby restraining or preventing the decomposition of organic matters. Deodorants merely oxidize the products of decomposition and thereby corsect or destroy offensive adors withont any direct effect upon the causal organisms.

The lines separating disinfectants, antiseptics and deodorants are not strictly drawn, most substances in either class sharing also, more or Irss. the properties of the other classes; thus disinfectants, when user? in small doses, lose their porrer to destroy germs and become simply antiseptics; for instance, formalin which is an efficient germicide in a strength of 5 per cent., is only an antiseptic and deodorant in a strength of I per 3.000 .

Disiniectants are conveniently divided into physical and chemical. The phrsical include air, sunlight and heat.

\section{AIR AND IIGHT.}

Fresh air and sunlight, especially on account of their availability at all times, have great ralue as disinfectants or aids to disinfection. Fresh air oxidizes the organic dust of bullings and thus removes much of the pabulum of bacteria: it acts much more efficiently under the influence of the solar rays. Direct sunlight, chiefly through the actinic effect of the ultra-riolet rays, has a strong restraining action on the growth of bacteria and kills most of them. According to T. Weinzirl.* it destroys all non-spore bearing bacteria in from 2 to ro

*J. Imf. Dis., May, 1907. 
minutes. The germs of plague, typhoid fever and cholera are particularly sensitive to it. This effect of light is materially increased by heat, and is most marked with clear, warm atmosphere. Diffuse light is much less efficient but also very useful. Germs floating in dry air become desiccated and, in this state, lose their power of propagation and often their vitality. There is considerable difference, however, in their resistance, those of tuberculosis and diphtheria, for instance, can endure desiccation for weeks, while those of plague, cholera and typhoid fever are killed in a few hours.

From the above remarks it follows that nature provides us with simple and useful means of disinfection and antisepsis which should not be neglected. These are the thorough ventilation and sunning of dwellings, the airing, shaking and sunning of clothing, bedding, carpets, hangings, etc. In garrisons, the bedding, clothing and much of the equipment of the men should be regularly aired and shaken once a week, even in the absence of sunlight.

The sterilizing influence of sunlight on the bacteria of drinking water is very remarkable. Thus, within a depth of 2 or 3 feet, the germs of typhoid fever and cholera are quickly oxidized. In clear water this destructive effect extends to a depth of 5 or 6 feet. It is advisable, therefore, for this and other reasons, to draw the water from near the surface of streams and ponds.

\section{HEAT.}

Heat may be used dry or moist.

Dry Heat. - This is hardly ever used for disinfection. It requires such a high temperature for its action that it is liable to scorch exposed articles, especially woolen materials; it acts very slowly, and has such a low power of penetration as to make it useless for the disinfection of bulky articles like mattresses and folded blankets.

Moist Heat. - This is used in the form of boiling water and steam. Boiling is a simple and efficient method of disinfection, especially for cotton and linen goods, as well as cuspidors, bed pans, urinals and a great variety of objects. In half an hour it destroys all bacteria. It is not applicable to woolen goods which shrink and lose their elasticity, nor to leather and rubber goods which become hard and brittle. It has also the objection of fixing and rendering indelible albuminous stains such as those from blood, pus and excreta; fabrics so stained should first be soaked and rubbed in cold 
water to which a little washing soda is added. For the disinfection of bright steel objects or cutting instruments, the addition of I per cent. of soda will prevent rusting and injury to the cutting edge.

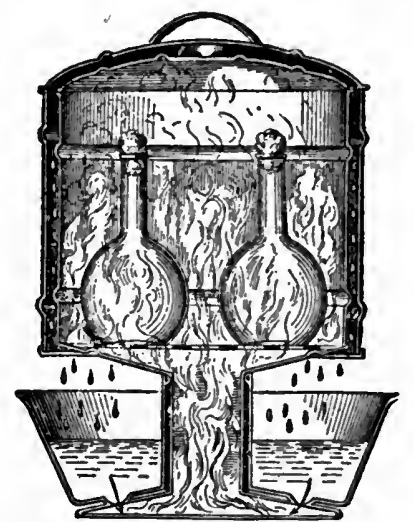

FIG. 234. - Section of Arnold steam sterilizer.

Steam. - All things considered, steam is the best and most useful of disinfectants, being cheap, reliable, quick, easily manipulated and applicable to a majority of the articles requiring disinfection. It is used saturated or superheated.

Saturated steam is steam as it streams or flows from boiling water under atmospheric pressure only. It is entirely reliable for many practical purposes, killing most bacteria in a few minutes. It has but little power of penetration and therefore can only be used for small, loose articles, or for the sterilization of dressings in operating rooms. It is successfully utilized in the Arnold sterilizer commonly issued to our post hospitals (Fig. 234); from the pan, the water passes slowly into the shallow boiler from which the steam rises into the sterilizing chamber; the water resulting from condensation drips back into the pan; as the heat is turned off, the hood and lid should be removed in order to let the steam escape and prevent the wetting of the sterilized articles which would result from its condensation.

Disinfection with streaming steam can be readily effected without the use of special apparatus, whenever a tin boiler is found to furnish the steam; sticks are laid across the top and the material to be disinfected placed over them; the whole should be covered with a sheet to retain the heat, and steamed for at least an hour. 


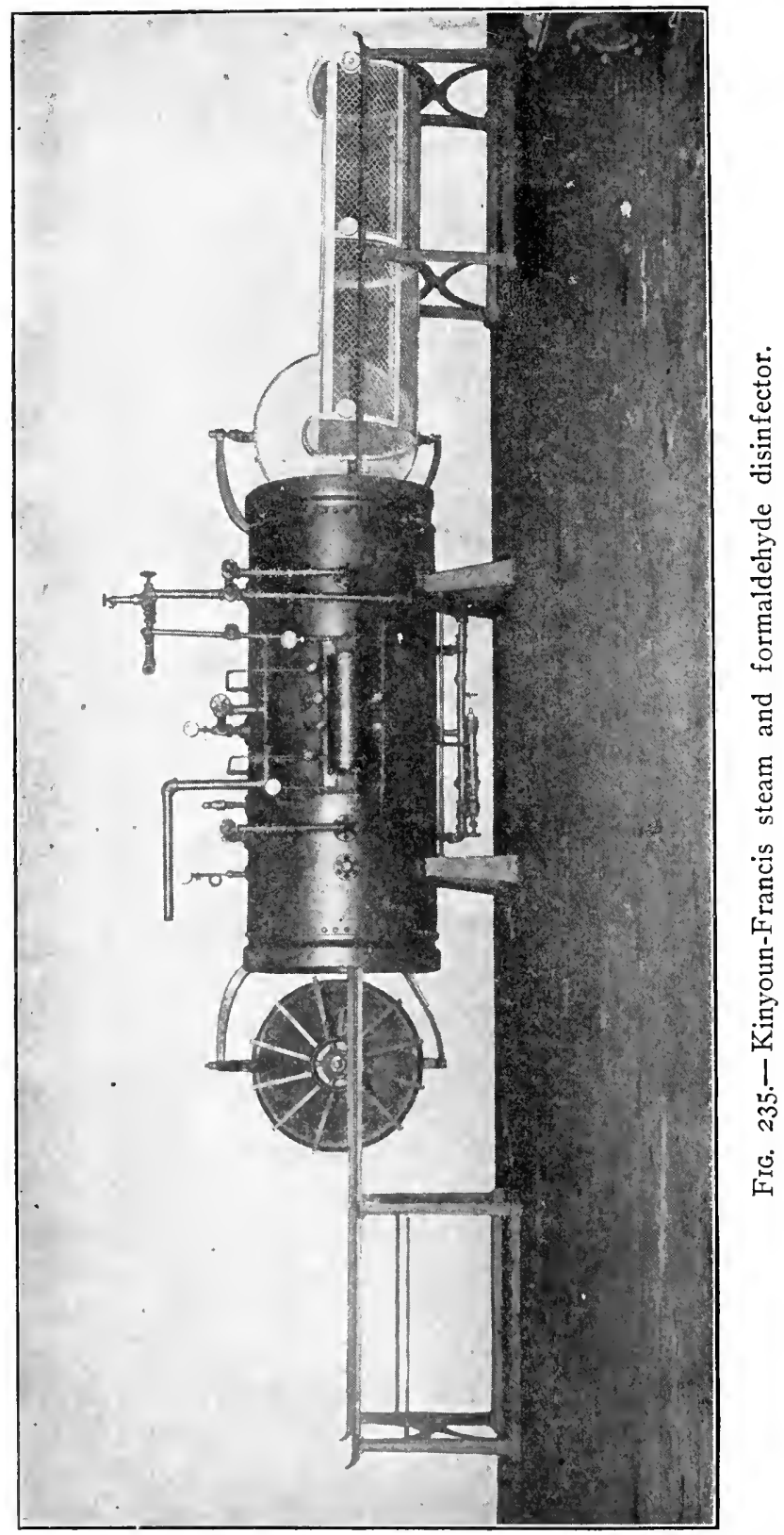


Superheated steam is steam under pressure, and so heated that it can be cooled without condensing, and even take up moisture from surrounding objects. It is the form commonly used for general disinfection. Superheated steam at $230^{\circ} \mathrm{F}$. will destroy not only all

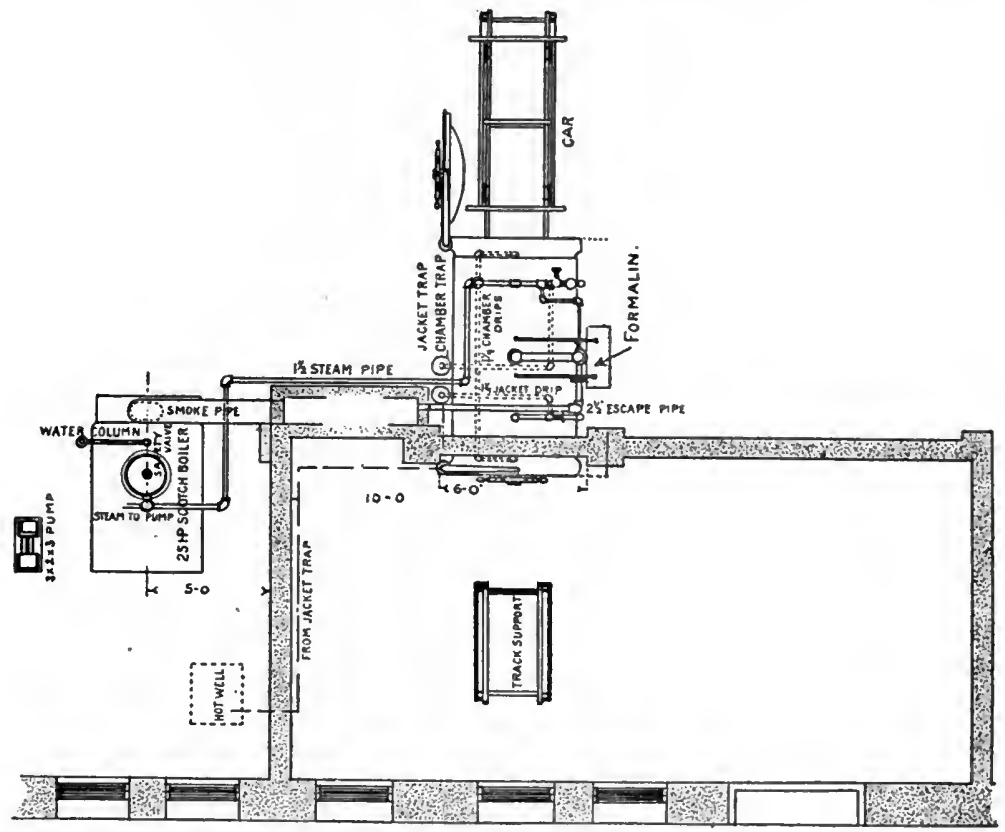

FIG. 236.- Ground plan of disinfecting plant, Fort Slocum, X. Y. (Kensington Engine Works Co.)

bacteria but the most resistant spores in a few minutes. It does not injure cotton and linen articles, nor most household effects, but is quite apt to shrink woolens and damage silk fabrics, while it ruins furs, leather, felt and rubber goods. As in the case of boiling water, spots of blood, pus or feces should be removed prior to disinfection.

Steam sterilizers of various types are found in all large hospitals and public institutions not only for the sterilization of infected articles whenever required, but also for the systematic sterilization of the clothing and effects of all inmates admitted therein, as a measure of prevention. For the use of permanent camps or cantonments, sterilizers mounted on wheels so as to be drawn to any required place, will often answer an excellent purpose. 


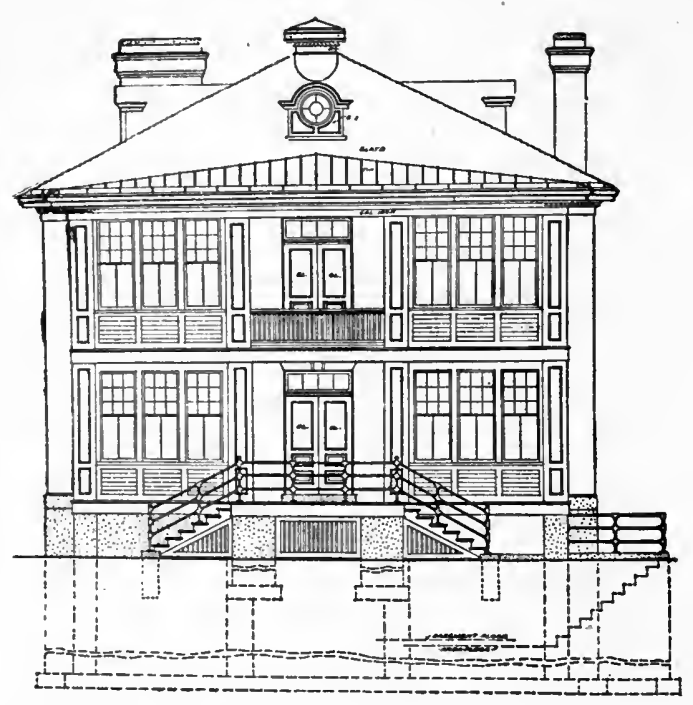

FIG. 237.- Pavilion for infectious diseases. U. S. Army hospitals. Front elevation.

The ordinary apparatus (Fig. 235) consists of a chamber of steel large enough to admit mattresses and other bulky packages, with an outer jacket of same metal, and an intervening space of two inches. At each end is a door made to fit air-tight. Inside and outside the chamber are rails upon which rolls a car for convenience of loading and unloading. To operate it, the steam is first let into the jacket so as to warm the chamber and thus prevent the condensation of the steam which would otherwise occur when first introduced. The goods are then placed in the car, rolled into the chamber and the doors closed and made steam-tight. The steam exhauster is now turned on until a vacuum of $I_{5}$ to 20 inches is obtained; this removes the air and moisture from the chamber and its contents. Air in the chamber would interfere with the penetrating action of the stean and retard the attainment of the required temperature. Steam is then admitted and a temperature of $230^{\circ}$ to $240^{\circ} \mathrm{F}$. maintained for about I 5 minutes, when another partial vacuum is made. A current of air is now drawn through the chamber, the outer door opened and the contents removed. As the result of the combined action of the vacuum and fresh air inlet, the contents are found to be completely dry after 3 or 4 minutes' exposure. 


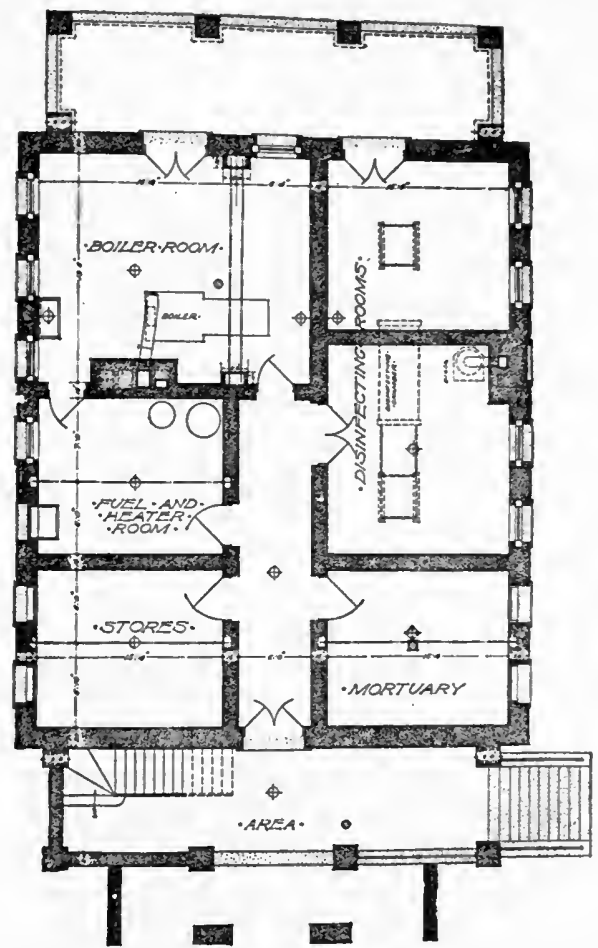

FIG. 238.-Pavilion for infectious diseases. U. S. Army hospitals. Basement plan.

It has been stated above that certain articles are injured by steam. They may be safely disinfected with formaldehyde under pressure, in a partial vacuum; for this purpose, steam chambers are generally provided with a formaldehyde attachment, used as described on page 735 .

The body of the apparatus should pass through a partition wall so that the ends of the chamber open into separate rooms, one room being used for the reception of infected articles and the other for their delivery after sterilization. (Fig. 236.)

Our larger military posts have a separate pavilion for infectious diseases (Figs. 237, 238, 239) which is supplied with a complete disinfecting outfit in the basement, generally consisting of the KinyounFrancis circular disinfecting chamber with the usual formaldehyde retort attachment. 


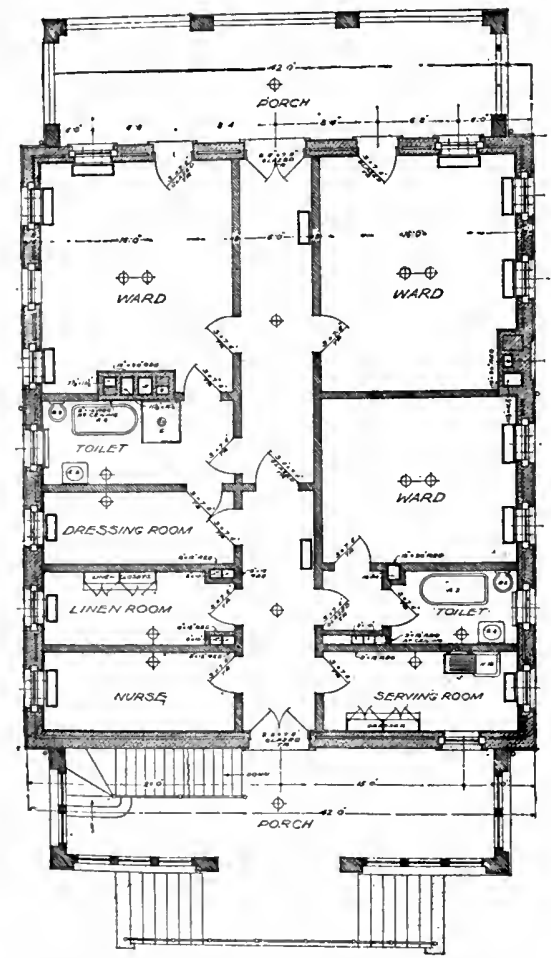

FIG. 239.-Pavilion for infectious diseases. U. S. Army hospitals. First floor plan.

In such a pavilion it is generally impossible to assign separate floors or parts of the building to different infections. It should consist of separate rooms, each completely isolated. Patients are admitted to any vacant rooms, as they come, according to convenience and regardless of contiguous cases. This plan, for a post, has been found to give the best results, requiring a smaller building, smaller personnel and insuring better care to the patients.

\section{CHEMICAL DISINFECTANTS.}

Chemical disinfectants are conveniently divided into those which are used in aqueous solution and those used under the form of gas. 


\section{GASEOUS DISINFECTANTS.}

Of the several substances belonging to this class, only few are of practical importance, namely, chlorin, sulphur dioxid and formaldehyde.

Chloris. - This gas has powerful disinfecting properties, but on account of its high specific gravity which prevents an even diffusion, its lack of penetrating power, bleaching effects on colors and destructive action on fabrics, is very seldom used to disinfect rooms, clothing or household effects. Its principal application is in the purification of water (page 283).

Sulpiur Dioxid, $\mathrm{SO}_{2}$. - This gas, produced by the burning of sulphur, has long been considered one of our most reliable disinfectants, probably on account of its pungent, irritating and persistent odor, and is still largely employed. The experiments of most bac-

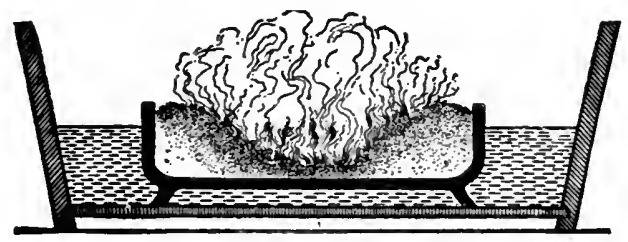

FIG. 240.- Pan for burning sulphur.

teriologists, however, have demonstrated that, although it has undoubted germicidal properties under certain favorable conditions, it is untrustworthy for general disinfection. Plenty of moisture is necessary for its action but, even with it, its power of penetration is always feeble, while its high specific gravity renders its even diffusion almost impossible. Furthermore, it bleaches fabrics or materials dyed with vegetable or anilin dyes, injures most metals, and as part of it becomes oxidized, the resulting sulphuric acid corrodes the fiber of linen and cotton goods, seriously weakening their tensile strength. Whenever used as a disinfectant, 5 pounds of sulphur shuuld be burned to each $\mathrm{I}, 000$ cubic feet of space to produce any marked effect. The sulphur, broken into small fragments, is placed in a broad and shallow iron pot which is itself set on bricks in a tub of water, to guard against the danger of fire, and is ignited after being freely wetted with alcohol. (Fig. 240.) The necessary moisture is automatically produced from the water in the tub. The room 
should previously be sealed tight in order to prevent leakage of the gas.

It is as a fumigant that sulphur dioxid finds its most useful application. Sulphur fumes are most effective in the destruction of rats, mice, roaches, bedbugs, flies and mosquitoes, and, for this purpose, act best in dry air, without added moisture. As an insecticide, 2 pounds of sulphur to each I,OOO cubic feet of space are enough.

Sulphur dioxid can also be used in the liquid form. The process consists simply in inverting the can containing it in a metal or earthen vessel, when volatilization takes place rapidly. It is free from any danger of fire and has also the advantage of liberating a large quantity of gas in a short time, but costs ten times as much as the method of burning sulphur. For disinfection, ten pounds of liquid sulphur are required for every $\mathrm{I}, 000$ cubic feet. The necessary moisture must be specially provided.

Wherever sulphur dioxid is used on a large scale, as at quarantine stations, special and highly efficient appliances have been devised, such as the "sulphur furnace" and the "Roberts' sulphur stove."

Formaldehyde, $\mathrm{CH}_{2} \mathrm{O}$. - A colorless, pungent gas, extremely irritating to the mucous membrane of the nose and eyes, and of the same specific gravity as air in which it diffuses evenly. It is a product of oxidation of wood alcohol and is soluble in water up to 40 per cent., forming the solution known in trade as formalin. If the latter be concentrated by heat, the gas is changed into a white crystalline solid by polymerization, called paraformaldehyde or simply paraform $\left(\mathrm{C}_{3} \mathrm{H}_{6} \mathrm{O}_{3}\right)$, consisting of 3 molecules of formaldehyde. The gas is changed in the same manner when generated in a cold room or at a low temperature. The readiness with which it is thus polymerized into an inert substance is one of the drawbacks to its use.

Formaldehyde is the strongest and most practically efficient 'gaseous disinfectant known. It is applicable to the disinfection of rooms, clothing, fabrics, effects and furniture, but cannot be depended upon for mattresses, upholstered furniture, and the like, which require deep penetration; for this purpose, steam is to be used. It has no injurious action on metallic substances, woolen goods, furs, rubber and leather articles, colors and paintings. It is also an excellent deodorant, as it readily combines with and destroys the foul-smelling products of decomposition. It fails to kill vermin 
and is of but little value even against mosquitoes, unless used in large amounts in tightly closed rooms. (See page 74I.)

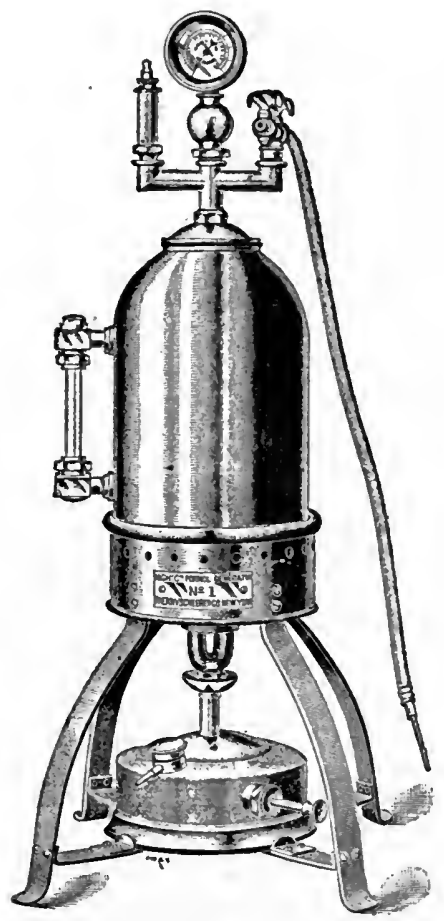

FIG. 24I.-Autoclave formaldehyde generator.

Methods of Generation and Use. - In all these methods (the third excepted), formaldehyde is generated from formalin and may be relied upon as a powerful surface disinfectant under certain conditions, namely, a sufficiently ligh temperature and degree of himidity. Below $60^{\circ}$ F., formaldehyde polymerizes into paraform and has but little value. In the absence of moisture it is practically inert as a germicide; for satisfactory results the relative humidity of the air of the room, before generating the gas, must be at least 60 per cent. In all these methods, about the same amount of watery vapor is given off, but, for disinfection purposes, this does not seem to answer as well as the natural humidity of the atmosphere (McClintic). Leakage must be prevented by closing all doors, windows, chimneys, ventilators and registers, as well as chinks and fissures, 11sing paper strips and paste very freely for the purpose: sometimes large paulins or ample sheets of strong paper are necessary. Upon the completion of the process, the best way to get rid of the gas is to open all the doors and windows and let it blow away. Ammonia may be sprinkled about the room to neutralize it, but the resulting substance (formamid) has such a persistent odor that this is now seldom resorted to.

Of the many methods of generating formaldehyde, the most practical and efficient are:

I. With Retort, without pressure. - Of this the Trenner Lee 
generator is a good type. It consists of a copper retort from which formalin can be readily and conveniently vaporized, either from outside or inside the room, without pressure and automatically.

2. With Autoclave, under pressure. - This requires a somewhat complicated apparatus, not free from danger, and only used from the outside. The gas is very quickly liberated under a pressure of about 3 atmospheres. To the formalin should be added 20 per cent. of calcium chloride or some other neutral salt to prevent polymerization and facilitate the evolution of the gas. (Fig. 24I.)

3. With Generating Lamp. - Of this the Kuhn generator is the best type. The gas is obtained by the oxidation of methyl or wood alcohol brought about by the action of incandescent platinized asbestos, and is therefore in its nascent or most active state. The pan containing the alcohol is surrounded by water which is slowly vaporized. Three pints of alcohol are required to disinfect 2,000 cubic feet of space. One of the disadvantages of this lamp is that the gas is generated very slowly so that it lacks the penetrating power of the quicker processes; but, on the other hand, it does not show the same tendency to polymerize into paraform.

(Figs. 242 and 243.)

4. Formalin-Permanganate Method. - This is based upon the fact that when formalin is poured upon potassium permanganate, a violent reaction takes place with strong ebullition of the mixture, rapid generation of formaldehyde and considerabie vaporization of water. The reaction is over in 5 minutes and leaves hardly any residue. The average yield of gas is $8 \mathrm{I}$ per cent. of the amount present in the solution. The best proportion of formalin and permanganate is two to one, namely, io onnces of the former to 5 of the latter for each $\mathrm{I}, \mathrm{OOO}$ cubic feet of space if the atmosphere is warm and tolerably humid, and double these quantities if it is dry and cold. On account of the frothing and sputtering which occur, the permanganate should be put in a tin vessel, Io inches wide at bottom and about I 8 inches high, preferably with sides flaring out toward the top. This vessel is placed in a shallow tub of water, or simply upon a piece of stont orown paper projecting a couple of feet all around it to catch the sputtering drops and protect the floor. Formaldehyde being inflammable care must be taken that there is no gas jet or flame of any kind in the room. To obtain the best bactericidal results the charge of permanganate in any one vessel should not exceed 200 grams.

For simplicity and rapidity this method is superior to any other. 
It liberates the gas almost instantaneously and in nearly as large quantities as the retort and autoclave methods which it has largely superseded. No advantage is gained by adding water to the formalin before mixing with the permanganate. For the best restlts the temperature of the room should not be less than $65^{\circ} \mathrm{F}$.

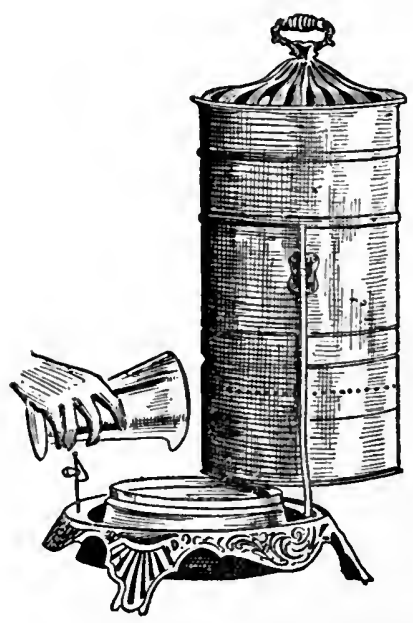

FIG. 242.- Kuhn formaldehyde generator. Filling. (Rosenau.)

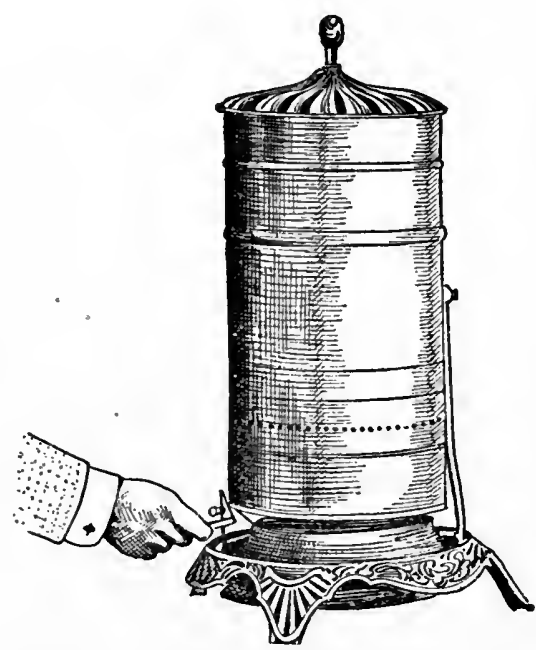

FIG, 243.- Kuhn formaldehyde generator. Lighting. (Rosenau.)

5. Sheet-spraying Method. - For each I,O0o cubic feet of space, a sheet 5 by 7 feet is hung up in the room to be disinfected, in a slanting position, at an angle of about $45^{\circ}$. It should be wrung out of water so as to be just damp to the touch when hung up. Three hundred c. c. (about IO ounces) of formalin are then uniformly sprayed upon it, so that the small discrete droplets will not run together. The room must be tightly sealed and kept closed at least 24 hours. This method is not applicable to rooms or apartments of a much greater capacity than 2,000 cubic feet, as the very slow evaporation from the sheets would not be equal to the loss from leakage. It is fairly efficient when long exposure is not objectionable and the weather is warm (at least $75^{\circ} \mathrm{F}$.) or the house artificially heated. In cold weather very little gas is liberated and it quickly polymerizes.

6. With Paraform. - Paraform or paraformaldehyde is a poly- 
meric condensation of formaldehyde gas. It is a white powder, soluble in warm water and burning readily with a blue flame, leaving no residue if pure. By heating, it becomes converted into its own weight of formaldehyde, but should not be allowed to burn as its combustion practically destroys all of the gas. Special lamps have been devised for the volatilization of paraform, but an ordinary pint tin cup will suffice to volatilize an ounce or two of paraform, provided the flame is not too strong. For each 1,000 cubic feet of space, 2 ounces of paraform should be used. On account of the simplicity of the process, requiring only a sufficient amount of an easily transportable powder, this method seems to be specially adapted to field conditions.

Candles of paraform, prepared by reliable firms, are now in the market and extensively used.

The so-called paraform-permanganate method has been found efficient and safe, and is now used by the New York Department of Health. For each I,O0o cubic feet of space the proportions are as follows: paraform 30 grams, potassium permanganate 75 grams, warm water 90 grams. The two chemicals are thoroughly mixed in a small, deep tin can (pint size), then the water is added and the mixture again thoroughly mixed. The evolution of gas is slow in starting but is practically complete in 5 to Io minutes, the amount generated running as high as 87 per cent. There is no sputtering and no danger of fire.

7. Munson's Method.* - The apparatus used in this method is described as " of simple, light, compact and inexpensive character, by which gaseous disinfection of the equipment of the soldier in the field may be accomplished in as short a time as 20 minutes, and the destruction of insects, vermin and parasites thereon, . . within half that time." The problem consisted in finding a simple, practical and effective method of gas disinfection for the field, and appears to have been solved in an ingenious and successful way, whereby formaldehyde is applied in as concentrated a form as possible.

The generator, of copper or brass, is 8 inches high, 6 inches in diameter and weighs 5 pounds. It consists of 4 parts, simple, solid and not liable to injury (Fig 244):

* The Military Surgeon, February, 1913. 
I. The outer container (A), a cylinder with solid bottom, and two perforations at opposite points near the top, into each of which is screwed a short tube. One of these tubes is provided with a turncock, the other is left permanently open to prevent any possibility of explosion. When the apparatus is used inside a container, the gas escapes through both openings; when used outside, the turncock is closed and the gas forced to pass through the other outlet.

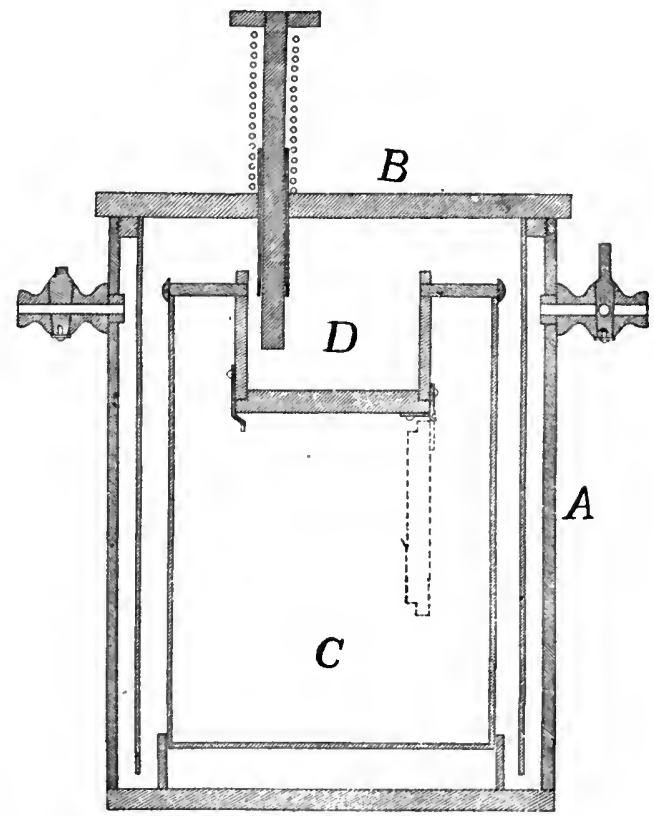

FIG. 244.- Section of Munson's disinfectant gas generator.

2. The cover and gas bell (B) consisting of a top or cap held in position with thumbscrews and perforated to receive a plunger; from the under surface projects a cylinder which reaches down within half an inch of the bottom of the container, and intended to prevent spattering and the escape of harmful chemicals.

3. The mixing can $(\mathrm{C})$, a simple cylinder resting in a frame which raises it about half inch above the bottom of the container to permit free movement of gas and prevent overheating. In the upper rim are grooves for the arms of the dosing box. 
4. The dosing box (D) is a small metal receptacle suspended within the mixing can, being supported by four arms which fit in the rim of the latter. The bottom is hinged in such manner that the plunger (projecting through the cap) when pressed down strikes against it and causes it to fall open.

In operating this apparatus, the dosing box is evenly filled with permanganate (6o c. c.), and formalin poured into the mixing can until the mark on its inside is reached ( 50 c. c.). The cover being clamped, the apparatus is put in the container, if to be operated inside, or connected with it by a rubber tube if to be operated from outside. The plunger is pressed down so as to drop the permanganate into the formalin, and the reaction started. The amount of gas thus generated will be sufficient for the usual disinfection of I,O00 cubic feet of space. The quantity of the chemicals may be reduced as desired.

It is well known that for disinfection purposes, the more confined is the space in which a gas is generated, and the more free from admixture with air, in other words, the greater its concentration, the more efficient is its action. In his method, Munson avails himself of these facts by using a collapsible and expansible container, one holding very little air in its collapsed state and becoming gradually distended by the pure gas to the degree desired. This concentration of the gas greatly increases its penetration and immensely shortens the factor of time exposure. Such a container, in the shape of an impermeable gas bag, is readily improvised with ordinary rubber sheeting. This, as furnished by the Medical Department, comes in rolls 54 inches wide. A strip 6 feet long folded on itself (rubber surface outside) makes a bag sufficiently capacious to hold the complete clothing, blankets and equipment of two soldiers, together with the disinfecting apparatus. The edges are pinned or stitched together and holes or cracks sealed with adhesive plaster. The soldier's poncho, or ordinary paper bag (especially if impermeable) may also be utilized.

A great advantage of this method, especially when the apparatus is placed within a collapsible container, is the conservation of heat, so that the hot gas does not polymerize, and carries more moisture.

This apparatus is also well fitted for use in rigid containers, such as trunk, box, closet or room. For rooms, a larger generator (IO $1 / 2$ inches) should be used. 
The conclusions reached from the results of many experiments are that with $100 \mathrm{c}$. c. of formalin in the generator and the use of an impermeable collapsible bag, the sterilization of bedding and clothing may be relied upon to be accomplished in 20 minutes, while insects (bedbugs, flies, ants, roaches, etc.) are practically always killed in 15 minutes.

8. With Steam Chamber and Dry Heat in Partial Vacuum. - This method requires a special apparatus, such as the Kinyoun-Francis disinfecting chamber with formaldehyde attachment, in which may be obtained a high percentage of formaldehyde, a temperature of $175^{\circ} \mathrm{F}$. and a vacu11m of $\mathrm{I}_{5}$ inches. The attachment consists of a copper retort (Fig. 24I) in which is poured a formalin mixture (consisting by volume, of formalin 5 parts, calcium chloride I part and water 2 parts) which is heated by means of a copper steam coil. 'It is very reliable, quick, not destructive, and gives good penetration although lacking the deep penetrating power of steam. It is applicable to baggage, clothing, household effects, and especially to articles liable to be damaged by steam. Letter mail is rendered safe by this process without puncturing the envelopes.

To operate the apparatus, the steam is first turned into the jacket and the chamber heated to $175^{\circ} \mathrm{F}$.; the steam ejector is then set to work until a vacuum of ${ }_{15}$ inches is produced; the formaldehyde is now forced in from its retort under a pressure of 3 atmospheres. At least three ounces of formalin should be used for every Ioo cubic feet. The size of the retort is such that if one-fifth filled, it will entirely saturate the chamber with gas, one liter of formalin generating about $I, 45^{\circ}$ liters of gas. An exposure of one hour is ample for thorough disinfection. At the completion of the process the formaldehyde may be neutralized by ammonia from a special retort; but, according to Rosenat, it is simpler and better to open both doors of the chamber and allow the gas to blow away.

Simple Spraying. - Spraying formalin upon walls, floors and furniture is always an excellent additional precaution just before or after the application of any of the methods of room disinfection. Formalin can also be successfully used to disinfect contents of trunks and boxes, but, for this purpose, it will not do to simply pour it into the corners; it should be carefully sprayed or sprinkled in small drops and distributed uniformly between the layers, using from 2 to 3 ounces per cubic foot (Rosenau). 
Formalin-phenol Method. - What may be designated by this name is the method described by W. B. McLaughlin who showed that if formaldehyde is mixed with vapor of carbolic acid its tendency to polymerization ceases, while its power of penetration is much increased. He uses a mixture of 75 parts of formalin and 25 parts of carbolic acid, 8 ounces of which, to each I,O00 cubic feet of space, are volatilized from a retort or sprayed on sheets. By this method, resistant strains of bacteria were killed through I2 layers of blankets, under conditions where formaldehyde alone would not penetrate 3 layers.

Ozone. - As gaseous disinfectant of rooms, ozone has not yet been proved practically useful, the amount produced and degree of purity varying greatly under different conditions. It has undoubted antiseptic power but only in such concentration (I 3 parts per million) as is by no means innocuous. According to Macfie its odor is detectible when present in such proportion as I volume in 2,500,000. As little as I per million is irritating to the respiratory tract, and an exposure of two hours to a concentration of 15 to 20 per million is not without danger to life.

Ozone is more or less successfully used for the sterilization of water. As an adjunct to ventilating systems it is claimed to be a useful deodorizer. It has also been found a valuable aid in the preservation of the contents of cold-storage rooms.

\section{DISINFECTING AGENTS IN SOLUTION OR POWDER.}

Bichloride of mercury or mercuric chloride, popularly known as corrosive sublimate, is the most powerful of all metallic salts as a disinfectant under favorable conditions. A solution of $I / I 000$ is generally stated to destroy ordinary bacilli, free from spores, in a few seconds. Experimenters differ rather widely in their views of its efficiency, but it is evident that the high reputation given it by former observers must be modified. It has three great disadvantages: it is virulently poisonous, corrodes metals, and forms with albumin an inert insoluble compound. It also acts as mordant, permanently staining clothing spotted with blood or pus. Its solution, when in contact with certain mineral or organic substances, is also liable to precipitate as calomel, sulphide or other insoluble compound. In the treatment of excretions, for instance, such as sputum or feces, a coagulum of albuminate is formed on the surface which 
protects the innermost bacilli from contact with the disinfectant. This may be prevented by the addition of 5 parts of acid (sulphuric, hydrochloric or tartaric) or Io parts of common salt for each part of sublimate. For use in infectious diseases (clothing, floors, walls, furniture, etc.) the strength of the solution is usually $1 /$ rooo. The solution should be made in an earthen or wooden vessel. The addition of 2 parts of common salt per $\mathrm{r}, 000$ parts of water renders the bichloride much more soluble. According to Harrington, a solution of I/I000O will promptly sterilize certain bacteria when their resistance has been lowered by desiccation, while a solution of $1 / 5000$ is ineffective against the common pathogenic bacteria in a moist condition. According to the same authority, even I/IOOO solution requires at least Io minutes to kill some of the commonest of the skin bacteria, so that it would be of advantage to abandon its use in surgery.

The tablets and solutions of this salt are generally colored with aniline blue or some other neutral dye to identify them and prevent accidents.

Mincral acids possess decided disinfecting powers and may be used, largely diluted, wherever their corrosive action is not objectionable. A solution of 0.02 per cent. of hydrochloric acid kills the cholera organism in 2 hours, and one of 0.07 per cent. the bacillus of typhoid fever in the same time, while a solution of 0.08 per cent. of sulphuric acid will sterilize foul sewage in 15 minutes.

Formalin, a 40 per cent. solution of formaldehyde already described, is extensively used, in variable degrees of dilution, as a most valuable disinfectant, antiseptic and deodorant. It is not corrosive; articles are not injured by it, and its action is not retarded by albuminous matter. A solution of I to 3,000 or 4,000 restrains the growth of all pathogenic bacteria, while a ro per cent. solution mixec' with fecal discharges renders them odorless at once, and completely sterile within an hour. Tubercle bacilli in sputum are killed by a 5 per cent. solution in 60 minutes. Its use for the destruction of flies is described on page I 55. Soiled linen and bed clothing can be disinfected by immersion in a 5 per cent. solution for one hour. The objection to formalin is the irritating gas given off from it, which precludes its use in the sick room. Occasionally, in susceptible people, its solution may cause a severe dermatitis, the mere presence of the vapor in the room producing, in such people, stinging formication over the whole surface of the body. 
Carbolic Acid (Phenol or Phenic Acid). - A product of the distillation of coal-tar. When chemically pure (phenol) it forms white crystals which liquefy by the addition of 8 per cent. of water. This "liquefied phenol" of the pharmacopœia dissolves in 12 parts of water, while the so-called pure acid of commerce, which contains more or less cresols, dissolves in 20 parts. A solution of 3 to 5 per cent. destroys all the ordinary pathogenic bacteria in sputum and excreta in a few hours. As it does not injure fabrics nor affect wood, leather or metal, its range of application is very great. Carbolic acid dissolved in alcohol is stronger than in aqueous solution, but loses its germicidal properties when dissolved in oil.

The crude acid, which is the form generally used for disinfection, contains variable amounts of cresols and other similar strongly bactericidal bodies, as well as inert tar oils. On account of its unreliable strength the amount used should be at least equal to the volume of excreta.

The cresols occur as impurities of carbolic acid and are generally combined under the trade name of "tricresol." They are about twice as powerful as carbolic acid and, in a I per cent. solution, promptly destroy the pus-producing organisms. They are extensively used under many preparations; being insoluble in water they are combined with various solvents or made into emulsions. Of the best-known preparations may be mentioned lysol and creolin. Lysol contains about 50 per cent. of cresols dissolved in potash soap; it is fully as strong as carbolic acid, miscible in water in all proportions, and extensively used in surgical practice. Creolin is a dark brown alkaline liquid containing about Io per cent. of cresols. These preparations are less poisonous and less irritating to the skin than carholic acid and quite as potent germicides.

The official Liquor cresolis compositus is similar to lysol. It can be extemporized by mixing equal weights of tricresol and official soft soap (J. R. Barber).

Chloride of lime or more properly chlorinated lime, is a white powder of somewhat unstable nature, obtained by the exposure of water-slaked lime to nascent chlorin gas. To be efficient it must contain at least 25 per cent. by weight of chlorin. As it readily undergoes decomposition, it should be kept in tight vessels in a cool and dry place. Its composition, although still uncertain, is generally considered to be a mixture of calcium chloride and calcium hypo- 
chlorite. It is an effective disinfectant in a solution of 5 per cent. or even less. A solution is best made by triturating the requisite amount in water to the consistency of cream and then diluting to the required volume. Chlorinated lime corrodes metals, but its action upon agate ware is very slight. It also bleaches and otherwise injures fabrics. It is useful for the disinfection of excreta and sputum, the mopping of floors and the washing of hands. It is commonly used as a deodorant on account of its great affinity for hydrogen, readily decomposing hydrogen sulphide, ammonia and other volatile odorous substances. It must be remembered, however, that its own odor is often quite objectionable.

Chlorinated lime is extensively used on the battlefields of Europe in the treatment of infected wounds, after proper neutralization with anhydrous sodium carbonate and sodium bicarbonate according to the method of Dakin. The proportion of these two salts varies according to the titration of the chlorinated lime. With a titration of 30 , the quantities of the three substances required to prepare Io liters of Dakin's solution would be $154 \mathrm{gms}$. of chlorinated lime, 77 of anhydrous sodium carbonate and 64 of sodium bicarbonate.*

Quicklime or calcium oxid is an excellent disinfectant well adapted to military needs. Treated with about one-half its weight of water, it evolves considerable heat and becomes "hydrated or "slaked." "Milk of lime" is made by thoroughly mixing slaked lime with from 4 to 8 parts of water. If more water is added, the familiar "white wash" is obtained. Lime dissolves in 700 parts of water, yielding a clear solution which is "lime water." When exposed to the air, quicklime is rapidly acted upon by carbon dioxid, and most of it becomes changed into carbonate which is practically inert; hence the necessity of always using freshly burned and slaked lime.

Lime has been proved to be a strong and reliable disinfectant, especially in the treatment of fecal discharges; it can be used as dry powder, after partial slaking, but fresh milk of lime, of 15 to 20 per cent. strength, is preferable. At least as much should be added as the bulk of matter to be disinfected, and thoroughly mixed with it; in this way complete sterilization may be expected in an hour or two. It is probable that lime of good quality is very nearly, if not quite, as good a disinfectant as the average chloride of lime, while it is

* Carrel's Method of Wound Sterilization. Colonel W. H. Arthur. The Military Surgeon, May, 1917. 
much cheaper and odorless. Whitewashing the walls of barracks, cellars, store-rooms and outbuildings is an excellent practice; for this purpose chloride of lime can often be advantageously mixed with the lime. The sprinkling of dry powdered lime on infected soil, or soil liable to be infected, as, for example, around the edges of latrines, along picket lines, or in the vicinity of kitchens, laundries and lavatories, is always advisable and often necessary.

The basic Fuchsins, soluble in water and alcohol, possess (according to E.S. May) a marked germicidal action. Especially great is the disinfectant power of rosanilin acetate (the most basic of them), exceeding that of phenol, with greater diffusibility and less toxicity.

Ferrous sulphate, also called sulphate of iron or copperas, occurring in green, efflorescent crystals, has been extensively used as a deodorant, especially for the removal of odors from privies and vaults. It is useless as a disinfectant and has very little claim to be considered even a deodorant.

\section{ROOM DISINFECTION.}

So long as a room is occupied by the patient, disinfection is impossible, but every effort should be made to prevent the dissemination of infectious material; to that end the rules to be observed are thus comprehensively formulated by Harrington:*

"In order to prevent or restrict the carriage of living organisms from the room, ingress should be denied to all whose presence is unnecessary; the wearing of other than cotton and linen dresses, that is, smooth-surfaced and washable, by the attendants should be interdicted; no food remainder should be taken away to be consumed by others; no used bed-linen or body-linen removed until after immersion in disinfectant solutions, and no discharges finally disposed of until after appropriate treatment. If it be necessary to use the broom, the dust should be kept down by the use of wet sawdust or tea leaves, which, with the gathered dirt and dust, should be treated with disinfectant and burned."

In any case of infectious sickness, all linen used in the room and liable to have been infected must be thrown into a tub, immersed for an hour in a disinfecting solution and then carried under cover or wrapped in a disinfected sheet to the laundry. The solution may be

* Manual of Practical Hygiene, Charles Harrington. I905. 
of corrosive sublimate ( $1 / 1000)$, carbolic acid (5/100), cresol $(3 / 100)$, or formalin $(4 / 100)$. Chlorinated lime is liable to injure fabrics and therefore not suitable for this purpose. All eating utensils should be well scalded after meal, each patient having his own set, not to be used by anybody else.

For the disinfection of roons, formaldehyde gas combines more of the requirements of the ideal disinfectant than any other so far tried, and is now almost exclusively used for the purpose. But all hygienists recognize that the complete destruction of bacteria in an infected room, by the application of any gas, is extremely difficult, generally impossible, and that the result, although undoubtedly useful, is nearly always imperfect. It is necessary therefore to have recourse to supplementary measures before and after the application of the gas. In the first place, if a steam sterilizer be available, mattresses, pillows and stuffed articles should be removed (wrapped in disinfected sheets) and treated in it. If the stuffing be of inexpensive material it is best to burn it, while the ticking can be disinfected and boiled. The contents of the room should be so arranged as to be easily reached by the gas on all sides: articles of furniture moved away from the walls, clothing and bedding suspended on lines, pockets turned inside out, and drawers of all bureaus. and cabinets left open. The careful closing of all openings and sealing of all cracks and fissures has already been dwelt upon.

After the fumigation, the carpets and hangings should be exposed to the sunlight and beaten; the floors must be scrubbed with a solution of chloride of lime (I/IOO) or of corrosive sublimate (I/2000), and the walls sprayed with the same or, still better, a solution of formalin $(5 / 100)$. Should the walls be covered with soiled and cracked paper, it is best to remove it so that they may be rekalsomined and new paper put on. All woodwork and articles of furniture not liable to be injured thereby should be washed with soft soap and hot water, followed, if deemed necessary, by formalin spray.

The experiments and observations of Chapin and others have lately considerably modified our views of the necessity of terminal disinfection, that is, the disinfection of rooms and their contents after the recovery of infectious cases. Attention has been more forcibly drawn to the already recognized facts that the danger is chiefly from infected persons and not from infected things; that pathogenic germs do not grow outside of the body and soon perish, 
or at least soon lose their virulence. Thus, pus-forming bacteria are often found in the air of operating rooms, but surgeons no longer sterilize the air. Extension of infection usually takes place by immediate or mediate contact with convalescents or "carriers." The belief in transmission by fomites or through aërial germs never had but the flimsiest basis in observation or experiment. There is little doubt that transmission may be effected by mediate contact, that is, the transfer of fresh infective material on inanimate objects. This is best combated by scrupulous cleanliness in the care of patients, the free use of soap and water, and frequent washing of the bed linen and clothing.

In practice, it has been found that the results of compulsory terminal disinfection in preventing the spread of infectious diseases are not better than where such disinfection is omitted. As a consequence of this new trend of medical opinion, much less importance is now attached to the fumigation of room and fomites, and much more to the scrubbing of floors and the mopping of walls and furniture.

Cleaning of the hands is frequently necessary in the nursing of infectious diseases. Under such conditions disinfectants injure the skin and should be used as little as possible. Bearing in mind that soap has decided germicidal properties, the process should always begin with soap and warm water, not in a basin but under a tap, in running water, followed or not by immersion in alcohol. When a stronger effect is desired the hands may be immersed in a 3 per cent. solution of formalin, carbolic acid, cresol or lysol.

DiSINFECTION IN THE FIELD. - Even when troops are so situated as to be deprived of the usual modern sanitary appliances, practical means of disinfection are never entirely wanting and should not be neglected. Exposure to sunlight and wind, as well as boiling, are generally possible and always efficient. Of chemical disinfectants for use in the field, light, compact, safely and easily transported, the best two appear to be mercury bichloride and paraform. Any tent which needs disinfection should be turned inside out, scrubbed with a bichloride solution and repitched on new ground, exposed to sun and wind. The original tent area is covered with straw and fired. Whenever clothing or other articles need disinfection, other than boiling, a formaldehyde chamber is readily improvised, a large packing box being utilized for the purpose, covered over with a tarpaulin 
or tent fly to prevent leakage, and the gas produced by the volatilization of paraform. The Munson method of using formaldehyde (see p. 732) is especially applicable to field conditions, provided the chemicals are at hand. Another useful disinfectant, lime, can also be procured in most situations, or at least wherever limestone is found in sufficient quantity.

For disinfection of ships, see Marine Hygiene.

Disinfection of Feces. - The fecal discharges in typhoid fever, dysentery and cholera, as often stated, contain most of the excreted infectious germs and therefore must be carefully treated. The amount of disinfectant should be at least equal to that of the discharges, thoroughly mixed with them and allowed to stand for about an hour before final disposal. Formalin $(5 / 100)$ is best for the purpose. Milk of lime is too bulky for water-closets but very useful in camps. Chloride of lime, carbolic acid and the cresols are also effective and may be used whenever the odor is not found objectionable. In typhoid fever, the urine should be disinfected with about a twentieth of its volume of formalin.

Disinfection of Sputum. - In tuberculosis and pnetumonia, special individual spit-cups should be used, partly filled with disinfectant and kept covered. A 5 per cent. solution of carbolic acid, or 4 per cent. of cresol or formalin, is best for the purpose. Paper spit-cups, which are afterwards thrown into the fire, are still better. The discharges from the mouth and nose in diphtheria, tonsillitis, whoopingcough and cerebrospinal meningitis should be received in rags and burned. 


\section{HYGIENE OF THE BATTLEFIELD.}

The soldier, in presence of the enemy, and with a battle impending, must bear in mind the value of certain precautions which, should he be wounded, will greatly tend to prevent a fatal issue and hasten his recovery.

I. Let him make sure that he has a first-aid packet, as required by the Field Service Regulations, that it is in good condition and that he knows how to apply it. This is of prime importance for he may thus be able to save his own life or that of a comrade. Inasmuch as a wound always stands in danger of infection from the clothing, the prompt application of an aseptic dressing gives the patient much better chances of escaping dangerous complications.

2. Let him fill his canteen with boiled water or as good water as can be procured, whenever the opportunity offers, for he does not know how long it may be before a new supply is available. If wounded, especially in case of free hemorrhage, he will be tormented with thirst. Should he escape injury, he may be able to quench the thirst of a less fortunate comrade.

3. Let him be as clean in body and clothing as circumstances permit, washing himself with a wet towel if a bath is impossible, and changing his underclothing. The value of this precaution is obvious in view of the preponderant part played by skin and clothing in the infection of wounds.

4. Let him see to it that his bowels have moved freely and that his bladder is empty. This is in order to mitigate the danger of urine and fecal extravasation in case of abdominal injury. For the same reason, his food should be nourishing in small bulk, and eaten in moderation.

5. If gravely injured, or exsanguined, it will generally be the better course for the soldier (in a war with a civilized enemy) to remain quietly recumbent where he fell, or at the nearest shelter, until rescued by the litter-bearers, either of his own side or of the enemy. Any great effort to walk may aggravate the injury and jeopardize life. 
There are prominent features, in modern warfare, of special interest to the military hygienist. The most striking is the enormous extension of the battle line, requiring the calling to arms or other military duties of all able-bodied men. In the Russo-Japanese War of 1904-I905 the battle line had already reached a length of 85 to 90 miles, but at this writing (1917) the western front of the belligerent armies in Europe is over 400 miles, while their eastern front is more than twice as long. Let this be compared with the battle line of Waterloo, 3 or 4 miles long, or that of Antietam and of Gettysburg, 4 or 5 miles long.

This tactical disposition of troops in thin, long-drawn-out lines, has the excellent effect, from the viewpoint of hygiene, of preventing large massing of troops, thus reducing to a minimum the risk of transmitting infectious diseases and making serious outbreaks practically impossible along the front. The danger will lie further in rear, in camps of concentration and instruction where the active efforts of medical officers in preventing the incidence and spread of disease will save many more lives and contribute much more efficiently to maintain the strength of the combatant units, than in tending the wounded on the battlefield.

Another amazing feature of war is the leading part taken by the aëroplane, the new war engine that carries death and destruction many miles in rear of the fire zone and ascertains the movements of the enemy so as to render surprises almost impossible. It is especially a serious menace to camps under canvas and will tend more and more to drive troops under ground or in otherwise inconspicuous shelters.

Also very remarkable is the protracted duration of campaigns; they have ceased to be "short and decisive." Battles consist of series of engagements covering days and weeks. Field intrenchments are used to an extent never known before, so that the soldier at the front, except when charging, is hidden in trenches and invisible to the enemy. Advances are slow and must be prepared by several days of terrific artillery fire. When of about equal strength, the belligerents may remain in their positions, confronting each other, for weeks, without any decisive movement forward or backward. Under these circumstances, underground works such as trenches, dugouts, galleries, magazines, dressing-stations, etc., soon assume much importance. 
This comparative immobility of the battle line should be taken advantage of to promote the comfort and health of the men. It will probably be possible then to supply fresh bread and needful components of the field ration, as well as to issue clothing. Medical officers will be able to bring up supplies, and to give serious cases suitable treatment before their transportation to the rear. In case of retreat they will probably also have time to take measures for a safe and orderly evacuation.

Season and weather may cause an occasional lull in the fighting but no serious interruption of a campaign. The Manchurian War of I904-5 and the present European War have shown the possibility of carrying on a winter campaign and fighting decisive battles with the temperature at about zero $F$. without many casualties from congelation and frost-bite. This had already been shown in some of our Indian campaigns but never on the same scale nor with the same convincing effect. With head and extremities suitably protected, a severe degree of cold, even below zero, does not appear to have an important inhibitive effect on the efficiency of the soldier.

Of all the great developments of the several branches of military science, the most momentous in its effects is that achieved by the artillery, in the increased number and power of pieces and the wonderful accuracy of their fire. The result is a much larger proportion of wounds from shrapnel and shell fragments than ever before - mostly extensive and lacerated wounds of great gravity and a decidedly higher rate of killed to the wounded.

Trench Hygiene. - Trench warfare, with all its accompaniments of underground shelters, constitutes a sanitary problem which always calls for the best judgment and greatest ingenuity of the medical officer. The dead and wounded may encumber the trenches, and their removal made impossible, sometimes for days, by the continuity of bursting shells, or if possible, very difficult by reason of the size of the stretcher and the many obstacles in the way of the bearers. The disposal of excreta, garbage and dirt in trenches and dugouts may present many difficulties and should receive constant medical supervision in order to avoid offensive and dangerous accumulations. It is in crowded dugouts that the transmission of disease by contact is most to be apprehended. Fortunately, cooking is seldom necessary in the front trenches, the food being brought from the rear through connecting galleries or boyaux, so that the usual kitchen wastes are avoided. 
In case of rain the discomfort of the men is greatly increased. If the drainage is bad, as often happens, they may be obliged to stand for hours in water and mud, a situation tending to produce what has been called "Trench Foot" (page I2I).

The disposal of excreta is generally by the bucket system or by the straddle trench. The bucket system is seldom satisfactory, as the receptacle is quite likely to be emptied into a near shell crater or other improper place ; in the absence of water its thorough cleaning and disinfection may be impossible. The straddle trench is effective provided it is frequently inspected. Box latrines, if suitable material be at hand, may also be used to advantage. Garbage and refuse should only be deposited at designated and marked places, and are carried out to be burned or buried whenever possible.

In trenches, soldiers, although often placed in an environment more or less polluted by the domestic wastes of generations, are unable to bathe and wash and, for a number of days, to change their clothing. The result is an inevitable accumulation of dirt upon the clothing and upon the skin, a dirt often rich in pathogenic and septic germs. The effect of such environment is that all wounds are infected, excepting perhaps a small proportion of those inflicted with the rifle. Always and severely infected are the large, torn wounds made by artillery projectiles and grenades. During the first part of the European War, tetanus and gas gangrene were frequent complications of wounds, but antitetanic serum and the surgeon's skill have largely overcome them.

Trench warfare offers some advantages from the surgeon's viewpoint. It facilitates first-aid treatment; the wounded can often be carried out of the danger zone without risk from the enemy's fire. It is also possible, in abdominal and other urgent wounds, to operate at once in a suitably equipped dugout, thus saving patients from the peril of long and rough transportation.

From what is known of the life of soldiers at the front, much of the time in trenches and ill-smelling dugouts, with depressing environment and exposed to all changes and extremes of weather, one would naturally infer that their health would undergo gradual deterioration. But it is pleasant to realize that the contrary is true. Official reports from the French, English. and German headquarters show that there has been a steady improvement in health, vigor and resistance to fatigue and disease in all men who have served at the 
front during the last 2 or 3 years. Alarming accounts have been circulated of a great increase of tuberculosis among soldiers as result of camp and trench life, but such reports are positively denied by competent observers. Sir William Osler, in. i916, stated that " of $1,000,000$ enlisted men between the ages of 18 and 40 , the proportion to develop tuberculosis is probably much smaller than if these men had remained in civil life."

Subsequent experience has confirmed his statement.

A. M. Fauntleroy, of the U. S. Navy, after careful observations behind the allied armies, reports that "tuberculosis of the lungs is of rather infrequent occurrence."

The 100,000 tuberculous discharged soldiers in France, at present, do not represent a higher percentage of that disease than could be expected in that country under modern normal conditions. According to Prof. Fishberg,* figures published by Rénon show clearly that tuberculosis has not increased in the population of France since the war began.

We may conclude that, in favor of the able-bodied soldier at the front, there are factors which make for strength and vitality, wellknown factors indeed but deserving to be again mentioned, namely:

A life in conformity with intelligent regulations, and free from all excesses; moderate (often scant) and wholesome diet; reduction or suppression of alcoholic drinks; free exposure to open air day and night; active exercise, including much hard work and many hardships.

After the Battle. - The duties of medical officers generally become more strenuous toward the close of the battle. Upon them rests the responsibility of collecting, transporting and treating the wounded, of recommending and superintending the proper disposal of the dead and of all that relates to the sanitation of the battlefield.

The search for, and collecting of the wounded and the dead may prove to be arduous work when the fighting has taken place through broken grounds, woods, brush and crops, and every available help should be secured. Trained dogs have proved invaluable for that purpose and are kept in most of the European armies ready for use, an example worthy of our imitation. Inasmuch as the searching must often be carried on at night, it is important to provide a

* Tuberculosis and War. Maurice Fishberg. Jour. A. M. A., June I6, 1917. 
suitable system of illumination. Experience has shown the superiority of acetylene lamps for the purpose, not powerful searchlights which project impenetrable shadows behind every bush and hillock, but small portable lights carried in the hand, at the waist or the hat band of the litter-bearer.

Disposal of the Dead. - The disposal of the dead is a serious question, whether viewed from the humanitarian or sanitary standpoint, and an important duty devolving upon the belligerent that remains in possession of the field. "The dead are collected by details from the line as soon as practicable after the battle and disposed of as the commander directs. No body is buried or otherwise disposed of without being identified and a proper record made." (F. S. R.) These details should be, so far as possible, from the regiments actually engaged in the battle, therefore having a knowledge of the battlefield and better able to find and identify the bodies. The natural claims of humanity demand this identification in civilized warfare whenever practicable; to those who die in its defense, the country owes the duty, at least, to preserve their names and mark the place of their burial. The Geneva Convention also requires that the enemy's dead should be collected, carefully examined and buried.

"Before a command enters upon a campaign, every member thereof is provided with an identification tag by which he can be identified if killed or wounded. Regimental and other commanders are charged with seeing that their commands are provided with these tags in ample time, and that they are properly worn. They are not removed from the dead, but left on the bodies when interred or otherwise dispesed of." (F. S.R.) The tag is worn under the shirt, suspended from a cord around the neck. Being of durable metal it will last as long as the skeleton of the body so that the remains can be identified if exhumed at any later date. Tags or other marks of identification, as well as valuables, found on dead bodies of the enemy are turned over to the commander of trains, who sends them to the provost marshal at the base.

Two general methods of disposal of the dead may be used, namely, burial and cremation. The latter method is seldom practicable on the battlefield nor is it generally desirable. For obvious reasons the preservation of remains is preferable; it permits their identification at any subseruent time, and their transfer to the family plot 
or national cemetery. But there are circumstances when cremation imposes itself ; for instance, in case of a siege, where space for burial is not available, or again when the soil is so rocky or so hard frozen that graves cannot be dug with means at hand; it is also the better method to dispose of the bodies of men dead from acutely infectious diseases, like plague, cholera, smallpox.

The bodies must be carefully examined to make sure that life is extinct. As probably several hours, perhaps a day or two, will have passed since death occurred, rigor mortis will have set in and perhaps signs of putrefaction will be present in most of the bodies and furnish infallible tests. In case of doubt and an early decision is necessary, the Icard fluorescin test may be applied. It consists in injecting into the veins or the muscular masses an aqueous solution of fluorescin, so as to show the persistence or the cessation of the circulation of the blood. "When an hour, or at most, two hours, after the injection, no characteristic coloration from the absorption of fluorescin is observed, such as intense yellowness of the skin and the mucosa, or green coloring of the eyes, it may be affirmed without doubt that death has taken place."

For the normal and prompt disintegration of buried bodies, the soil should be dry, porous and naturally well drained, in order to permit a free circulation of air and promote the necessary biological processes. The incipient putrefaction of the body attracts all kinds of necrophagous insects which lay their eggs on or in the soil; the larva penetrate downward to the body and therein undergo their various metamorphoses. Meanwhile, the saprophytic bacteria in the body multiply enormously. With free soil ventilation, insects and bacteria will rapidly bring about the oxidation and destruction of all the soft tissues. On the contrary, if the soil be clayey and damp, or marshy, their action is slow or arrested, and the tissues undergo transformation into adipocere, or corpse fat, which may remain unchanged an indefinite number of years.

When, for special reasons, it is desired to prevent the rapid decomposition of a buried body, it should be placed, completely clothed, in an air-tight box, and the latter covered with a layer of lime or other disinfectant. On the battlefield, however, the desideratum is to promote rapid oxidation and disintegration of the tissues with as little putrefaction as possible. Therefore the bodies should be stripped of clothing (at least of outer clothing), free aëration provided and disinfectants avoided. 
A good method is to dig a pit with sloping sides, 35 feet long, 7 wide at bottom, and 6 or 7 deep according to the nature of the subsoil. All around the bottom is a small trench draining into a well at the lower end, trench and well being filled with stones or brush. The bodies are laid across in 3 or 4 superposed rows, the rows separated by brush, straw or other available vegetable material. The clothes, if not otherwise needed, can be laid on the top row to secure additional aerration. The pit is then filled up with earth which is piled up above ground into a convex or ridged top capable of shedding rain. The greater the number of bodies buried together, the greater and more active will be the biological processes, and the more rapid and complete the disintegration of the bodies. Lime should not be used except in the trench and well at the bottom of the pit, and other places where it is desired to prevent odors. It would be well, if time permits, to dig a flue leading from the upper part of the end of the pit to a shallow hole, a few feet away, in which a fire is made; this fire aspirates and burns the gases from the pit.

In selecting sites for pits, care should be taken that they do not drain into a nearby water-course likely to be used as water-supply.

Concerning cremation, it must be remembered that human bodies, and all animal bodies, are not easily reduced to ashes. They require a hot fire kept up by a very active combustion. In most situations an improvised furnace of brick or stone, with good draft to windward, will answer the purpose provided the fuel is abundant. If at hand, kerosene or tar should be freely poured over the bodies and fuel.

The carcasses of horses and other dead animals must also be disposed of on the battlefield. They may be buried or cremated as seems best. If buried the general principles enunciated above should be observed. If cremated, a good plan is to eviscerate them and throw some of the fuel inside the body. 


\section{NAVAL AND MARINE HYGIENE.}

The application of the principles of hygiene to ships is, in many respects, very different from their application to troops on land and requires special consideration. The whole subject has been carefully investigated by the Medical Corps of the Navy and described in special text books. In this Manual it is only intended to advert to its most important features.

A war ship is comparable to a small active manufacturing town, with a crowded population of carpenters, blacksmiths, electricians, firemen, coal-heavers, machinists, gunners, etc., surrounded by complex mechanical devices and exposed to many dangers which menace life and limb.

A ship at sea is entirely thrown upon its own resources and must make the most of the special and inevitable conditions in which it is placed. These conditions are favorable and unfavorable. Sea air is free from micro-organisms and all obnoxious organic matter; it is healthy, invigorating and not liable to sudden or great changes of temperature; advantages from which the seaman benefits. The vital question of the disposal of excreta and wastes presents no difficulty since everything is thrown into the sea. The water, usually distilled, is therefore of unquestioned purity, while the quantity and quality of the food are also more under control and easily maintained to the proper standard than among troops on land. The men, while afloat, are constantly under military discipline and precluded from indulging in dangerous dissipation. Finally, it is easier on a ship to direct and supervise the application of all sanitary measures, as well as to detect and isolate the first cases of infectious diseases than in a camp or garrison.

The great and irremediable drawback of life aboard is restricted space, causing inevitable crowding, close intercourse and personal contact, together with more or less community of effects and clothing, all conditions most favorable to the transmission of communicable diseases. Ventilation of various degrees of excellence can be secured above, but is often very unsatisfactory below decks, 
especially in the depths where engineers, mechanics, firemen and stokers perform their habitual duties. Physical exercise, so necessary to all young men (especially walking and running, that easiest and best form of exercise), is quite restricted, of ten impossible, or else becomes an irksome task.

It is somewhat difficult to balance these advantages and disadvantages of life afloat and draw conclusions as to its effect upon human health. According to the mortuary records in the reports of the Surgeon General of the Navy for the years 1906 and 1907, only 73 men died of disease on board vessels out of a total disease mortality of 3 I8. Of the four diseases which caused the highest mortality in the Navy, viz., tuberculosis, pneumonia, typhoid fever, cerebrospinal meningitis, there were is deaths afloat and $9 \mathrm{I}$ ashore. For I9I I, the ratio per thousand of daily sick (disease and injury) in the force afloat, ranged from 8.62 to 10.46 , while that for yards and land stations was 12.27. Although the force afloat, during that year, was more than six times the strength of that in yards and stations, its mortality from disease was only one-fourth more, namely 25 and 20 deaths respectively. From these and other data it has been pretty conclusively shown that, while cruising, seamen have better chances of health (accidents disregarded) than while on shore duty, or than soldiers in garrison.

A comparison between the statistics of the Army and Navy for the year 1907 (an average normal year) is interesting. The ratio of deaths in the Army was 5.8I per thousand of strength, namely, 3.44 from disease and 2.37 from other causes; in the Navy, 5.67, namely, 3.49 from disease and 2.18 from other causes. The five diseases with the highest ratio of deaths are the same in both services, in the following order of mortality: For the Army, tuberculosis, pneumonia, cerebrospinal meningitis, typhoid fever and heart disease; for the Navy, pneumonia, typhoid fever, cerebrospinal meningitis, tuberculosis and heart disease. While the rate of discharges in the Army was 20.15 per thousand, it was 28.57 in the Navy, a notable difference, probably exerting a marked influence in lessening the mortality of the Navy. The most remarkable discrepancy, however, between the two services is in the ratio of admissions and readmissions (to hospital or treatment involving exemption from duty); being 1,218 per thousand in the Army and only 740 in the Navy, with a ratio of constantly non-effective of 46 
in the Army and only $3 I$ in the Navy. Evidently this discrepancy is to be explained chiefly by the different methods of accounting for the sick and wounded in the two services, especially in recording minor ailments; but, after making this allowance, it seems quite probable that the morbidity in the Navy is somewhat less than in the Army, and that this better showing is mainly due to the excellent health prevailing among the men afloat.

In the British Navy, the death rate for 1905 was 3.9, namely 2.74 for disease and I.I5 for injuries, with admission rate of 735 and discharge rate of 23,89 .

For the year I9II, the rate of deaths from disease in the United States Navy and Marine Corps was 2.II, as against 2.66 in the Army. This slight difference, however, appears to be more than offset by the much higher rate of discharges in the Navy ( I8.75) than in the Army (12.28), especially for tuberculosis (4.30 and 2.85 respectively), the disease causing the greatest number of discharges.

The mean death rate for the two years I9I4 and I9I5, from disease and injuries, was 4.42 in the Army and 4.33 in the Navy.

For the year I9II, the five most prevalent diseases in the Navy and Marine Corps, in order of admissions, were: venereal diseases, tonsillitis, skin diseases, influenza and bronchial affections; while the five which caused the greatest number of deaths, in order of mortality, were: tuberculosis, pneumonia, heart disease, typhoid fever and nephritis. During that year the diseases which gave the highest rate of death in the Army were tuberculosis, measles, pnet1monia, heart disease and typhoid fever. It is seen that, with occasional exceptions, the above diseases are practically the same in both services.

In the Navy we may expect higher rates of those infectious diseases specially favored by crowding, such as influenza, measles and mumps. The confinement of many men, particularly among the engine and fire-room forces, below decks, where they are deprived of sunshine and a free circulation of air, and the violent changes of temperature to which they are subjected, cause a higher rate of admission for tuberculosis in the Navy. Thus, in I9ro, it was shown that with an average admission rate for tuberculosis of 5.9 per I,000 for the entire Navy, it rose to 8 for engineers, 7.3 for electricians, 8.7 for messmen and I3.4 for the hospital corps.

From the discharge tables it appears that during the years I9ro 
and I9I I the proportion of mental diseases was likewise greater in the Navy. For this there is no satisfactory explanation.

One class of ear injuries much more frequent in the Navy than in the Army is that caused by the firing of heavy guns. To prevent this the usual practice, until lately, was for each man to place a pledget of cotton in each ear, loosely packed, and keep his mouth slightly opened so as to maintain full air pressure in the Eustachian tube. Many appliances have been tried, but none found entirely successful. The "Frank ear stopple" of soft rubber, and the "E1liot ear protector" have thus far proved the most satisfactory. The latter consists essentially of a perforated celluloid ball, of the proper size to fit the auditory canal, with wing to hold it in position. Another preparation, a mixture of molder's cla" and wool, has also been well spoken of in the British Navy.

Recruiting. - Recruiting in the Navy is governed by practically the same regulations as in the Army. To enlist, applicants, as a general rule, must be $2 \mathrm{I}$ and not over 35 years old, but these limits admit of exceptions; thus an ordinary seaman may enlist when 18 and not over 30 years old; an apprentice seaman when 17 and not over 25. May also enlist at 18 , landsmen (not for seaman branch), hospital apprentices, mess attendants and ship's cooks.

The standard of vision is higher than in the Army, although the necessity for this is not apparent. A minimum visual acuteness of $20 / 30$ is required in each eye for all applicants, but no man can be a gun pointer who has not a vision of $20 / 15$ in the right, or aiming eye (that is to say, who cannot read at 20 feet the line on the test card normally read at 15 feet), and a vision of $20 / 20$ in the other eye. The reason given for this high requirement in gun pointers is in order to eliminate certain ocular defects, such as minor degrees of astigmatism.

Owing to the great strain to which the sense of hearing is subjected by the firing of guns aboard, great care is taken in the examination of the ears to ascertain that the drums are sound and the Eustachian tubes patent.

Training Station. - On account of the crowding more or less unavoidable on a ship of war, it is most important that every possible measure be taken to prevent outbreaks of infectious disease aboard. The first and most effective measure to that end is to draw the recruits from a clean source of supply, that is to say, from " train- 
ing stations" and "receiving ships" carefully protected from pathogenic organisms. The receiving ship is an anachronism and should be discontinued. The training station, to fulfill its functions successfully, should consist of several separate departments through which the recruits are successively advanced: one for their reception and examination, another for their detention and further observation, and a third for those finally accepted and considered fit to go aboard. No man should be sent aboard, under normal conditions, who has not been in detention, under observation, for at least I 5 days. In these departments it is desirable that the men be quartered. in small isolated squad-rooms, in order the more easily to check the spread of any infection. Each recruit received on board must be carefully inspected. His medical history, so far as known, should always accompany him. Men who have been, or are suspected to have been, exposed to infection are mustered and examined, morning and evening, during the full incubation period of the disease.

\section{VENTILATION.}

On board ship the air space is a variable quantity and difficult to determine, depending upon the size of available compartments, the amount of stores, furniture and baggage they may contain, and the number of men to be berthed in each. This space is necessarily restricted, sometimes to an almost inconceivable degree, but it should never fall below 140 cubic feet, which is hardly any more than the area of the hammock multiplied by the height of the deck. English law requires that in forecastles each bunk should have at least 72 cubic feet entirely free from stores; but according to the best authorities this minimum should never be less than 100 feet. The sick bay on our modern ships is given all the space that is deemed practicable; thus, on the U. S. S. Maryland, it is to feet high, contains 35 folding metal beds in pairs, and each bed has an air space of 260 feet. The effect of such close confinement is, of course, mitigated by the free aëration, natural and artificial, of the compartments, and by the fact that watches are changed every four hours so that, except on troop-ships, few men sleep continuously the entire night in foul air.

The construction and equipment of troop-ships (transports) is of the highest importance for this country and has already received 
careful attention from the War Department, on account of the frequent changes of the regiments stationed in our distant colonies.

They should never have more than two decks, preferably only one, occupied by the berths of soldiers. Twenty square feet of floor space should be allowed as a minimum, and not more than two tiers of hammocks or bunks permitted. "Where bunks are used, the lower tier should be raised not less than 18 inches above the deck; with not less than three feet between the two tiers, and between the upper tier and the ceiling, so as to enable the occupants to sit upright. All bunks should be arranged so as to leave a passage-way at least 30 inches wide between each other and the side of the ship, to facilitate cleansing, allow ready communication and ventilation" (Munson). In dimensions, the bunks should be no less than 6 feet 3 inches by 2 feet. A high standard of cleanliness on board troopships is of the utmost importance. As a large proportion of the men will be the victims of sea-sickness for a few days, special arrangements must be made to catch vomited matters, for their prompt removal and the cleansing of soiled floors and furniture.

The ventilation of a ship is always a complicated problem, seldom successfully solved for various obvious reasons: the immersion of the larger part of its body which precludes openings, while many of those above the water-line have often to be kept closed; the special causes of pollution to which its atmosphere is exposed; its structural irregularity, involving many compartments of different shapes and sizes and used for a variety of purposes.

The air of a ship is contaminated by the breathing of its inmates, the combustion of fuel and illuminants, and by soiled clothing and bedding like that of barracks, but to a much higher degree on account of the greater crowding. There are, besides, other contributory causes of pollution more or less special to ships: the profuse perspiration of the men in the boiler- and engine-rooms, and other heated compartments : the charred organic dust of the air; the gases from coal bunkers, products of heat and moisture; the effluvia from heated oil and grease, from tar, paint, bilge water, vomited matters, water-closets, galleys, mess-rooms, and from the components of the store-rooms and cargo.

The bilge water is the drainage of the ship, to which, in wooden ships, is added more or less leakage from without; the putrefaction of the organic matters it contains, together with the sulphides derived from the sulphates of the sea-water, give it a most repulsive 
fetid odor. In modern iron ships it collects into the main drain which runs nearly the whole length of the ship, and seldom accumulates to any objectionable extent, the drain being frequently pumped out and flushed.

Next to the bilge, the fore- and after-peaks are the most insanitary parts of vessels and likewise require special attention.

The bedding should be thoroughly aired once a week when the weather permits, the blankets and mattress covers washed twice a year and the hammocks once a month.

The ventilation of ships, like that of buildings, is natural and artificial. Natural ventilation is effected through hatchways, skylights, stairways, hollow masts, port-holes, side-lights and other openings, as well as through revolving tubes with hood or cowl made to face the wind, and windsails or canvas cylinders used in the same manner. The principal outlets for foul air are the casings or jackets of the funnels, in which the heat causes a strong up-draft. In living spaces the air-ports are usually provided with pivoted sidelights by means of which the air can be scooped in or directed outward as desired. Inlets may become outlets with change of wind or temperature.

On troop-ships effective ventilation demands that every possible obstruction to the circulation and diffusion of air be removed; hence large chests and bulky articles should not be allowed in quarters. Bulkheads must be as few as possible and always partly opened or latticed. An ideal berth-deck on such ships would be one consisting of a single open compartment running the whole length of the vessel. Passage-ways, longitudinal and transverse, should be as straight as possible so as to favor the free movement of air.

Natural ventilation, however, although very valuable, is irregular, unreliable and, except for the upper decks, often insufficient.

In engine-rooms the air should be changed at least every two minutes; in dynamo-rooms, every minute; in all quarters and living space, every 12 minutes; in water-closets, wash-rooms, store-rooms, magazines, every eight minutes. The amount of air needed in engine- and boiler-rooms varies also with the speed of the ship and the draft of the fires. Only artificial or mechanical ventilation can always meet such requirements. Two systems are used; namely, propulsion, whereby the fresh air is driven in by electric fans or blowers, and extraction or exhaustion in which the foul air is drawn out. 
Until recently the exhaust system had been depended upon in battleships to a much greater extent than is approved by good hygiene; although generally satisfactory on the upper decks, it is quite in-

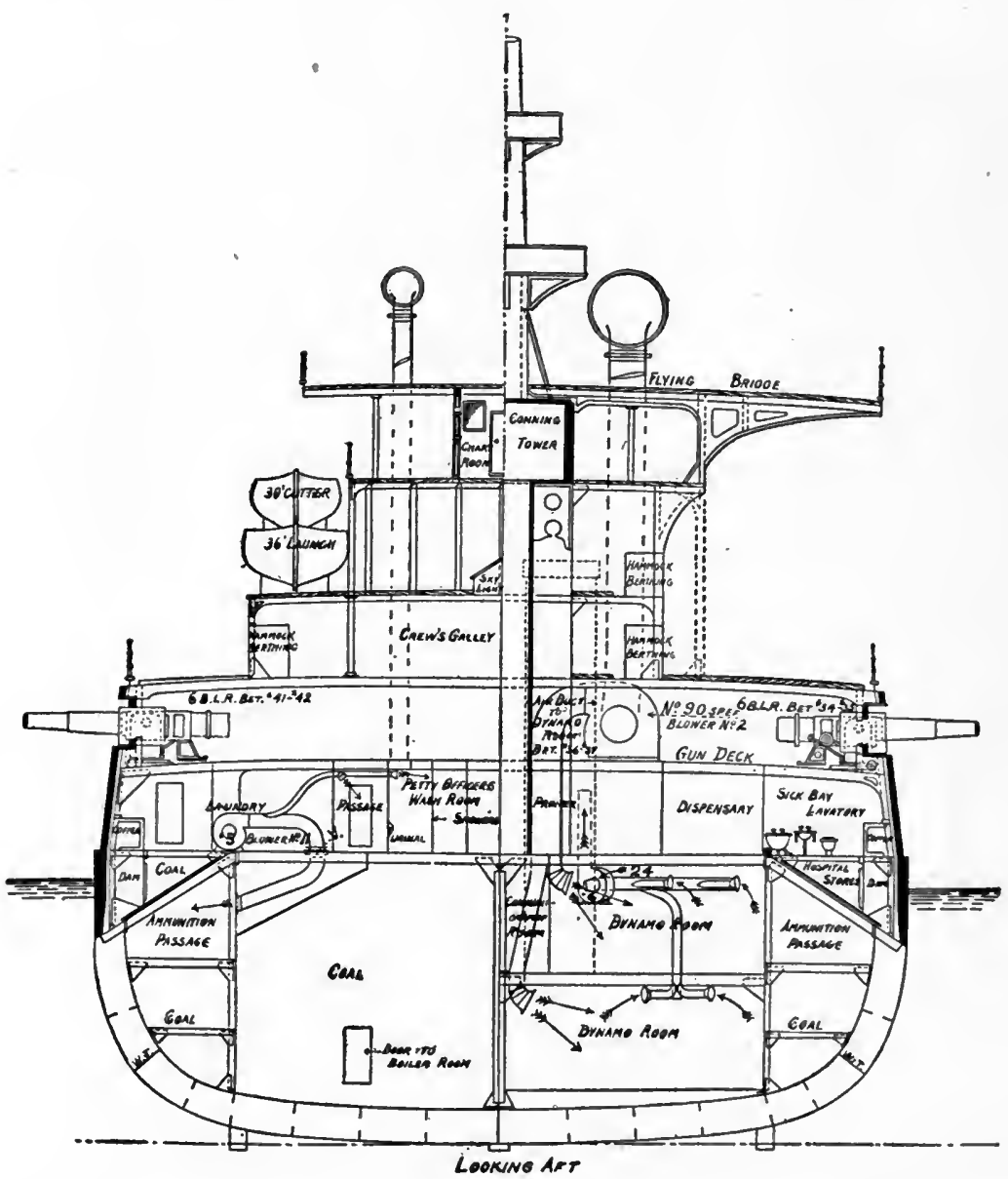

Fig. 245.- Cross section of ship shówing system of ventilation in our Navy, by propulsion and extraction.

adequate below the armored deck, especially within the citadel, for want of enough chance inlets to admit fresh air. It also frequently draws air from contiguous compartments, and may thus cause foul- 
ing of living quarters. It is indicated for water-closets, lavatories, pantries, store-rooms for volatile supplies, galleys, laundries and isolation rooms.

As a general rule, however, experience has shown that both systems are necessary for complete ventilation, and therefore a double set of ducts or trunks, one for supply and the other for exhaust. Each important compartment should have its own ventilating system, for the movement of air is much impeded by bends in the distributing ducts. The inlets and outlets should be as far apart as possible so that the fresh air may diffuse itself and fulfill its purpose before escaping. Thus in boiler- and engine-rooms the best method would seem to be to.propel the air in through or near the floor by many jets, and aspirate it through the ceiling by a number of outlets leading into an exhaust trunk opening above the upper deck.

Air ducts, on account of convenience of construction, are generally rectangular, although the circular form is more economical as regards friction. Wherever they pass through watertight bulkheads they are provided with valves which, in case of accident and flooding, close automatically.

In living spaces the fresh-air inlets should be so placed and trimmed or protected by baffles that the streams of air will be broken up and so distributed as to prevent drafts. In cold weather, the introduction of cold air in heated (or often superheated) rooms exposes the occupants to unpleasant and dangerous chilling drafts. It is desirable therefore that the incoming fresh air should be moderately warmed before its admission and diffusion. This can be done by placing the steam coils used to heat the compartments in small chambers through which the air is made to pass. This decided improvement in ventilation is being tested in our Navy, and it is hoped that it will be found of general applicability.

Temperature and Humidity. - The temperature and humidity of ships deserve mention. They are heated within by boilers, engines, steam pipes, chemical clianges in the cargo, galleys and ovens, and without by the sun. It is generally considered desirable to scatter the auxiliary engines (dynamos, windlasses, condensers, motors, etc.) in various parts of the ship so that in case of damage or accident some may be left in a serviceable condition. The compartments in which they are placed, as well as contiguous rooms, are thus made hot and damp, and often difficult to ventilate. The neces- 
sity of such dispersion is beginning to be questioned by naval engineers and, should they conclude to concentrate all these engines - within the strongest part of the citadel, where ventilation is generally very good, the comfort and hygiene of the ship will be much improved thereby.

Iron heats and cools rapidly; metal ships are exceedingly warm in summer and cold in winter. These extremes can be much diminished by inside wood sheathing, but this is liable to decay and to harbor vermin ; morover, on men of war, such combustible material is not permissible as it would considerably add to the danger of conflagration in an engagement. Solar heat is best excluded by awnings and curtains, often sprinkled with water, and by white paint on the outside. The awnings are much more effective if double, particularly if the inner canvas is dark-colored. While at anchor, in the tropics, the ship should have a line made fast to the stern so that its broadside may always be kept to the wind, and free ventilation by perflation secured. Besides thorough ventilation, no better method of cooling compartments on board ship has as yet been devised than the use of electric fans which stir the air and increase the cutaneous evaporation.

Dampness is quite marked in many parts of ships; it is the resultant of several causes: the high degree of relative humidity of the air, the water used for mopping, the breaking of the waves upon the decks, the leaks from steam pipes, the respiration of men crowded within very limited spaces. This moisture readily condenses upon the cool iron, especially at night, producing the phenomenon of "s'veating," or formation of droplets of water on metal surfaces, often dripping into the bunks and a cause of much annoyance. During the summer, only good ventilation has any marked effect upon it, while in winter it is removed by the steam heating. Some mitigation of this evil is obtained by using cork paint on all sweating surfaces, that is to say, ordinary paint sprinkled with granulated cork and covered with a smooth layer of zinc white. Asbestos cloth has also been recommended.

The old pernicious method of washing wooden decks by first flooding them with water is fortunately gradually disappearing. The washing of decks should be done with as little water as possible, wetting only small portions at a time and drying thoroughly, and preferably on sunny days, in order to prevent prolonged dampness. 
The lower decks, especially, should be mopped only when necessary to insure cleanliness. By sprinkling clean sand and sawdust over them, wooden decks can be preserved from much soiling. Simple mopping or scrubbing in places where required, together with holystoning and dry rubbing, will generally accomplish all that is necessary.

The use of linoleum, varnished over with shellac, as a deck covering and dressing now general in the Navy, renders the decks neat, clean and free from the dampness which formerly resulted from their frequent washing. This covering is particularly of sanitary value in the living and messing spaces below the main deck. But to $f$ ulfill its purpose and really improve the healthfulness of ships it requires constant attention. The shellac should be put on freely so that the linoleum does not become exposed, stained and cracked. The linoleum, when worn and torn, permits an unseen accumulation of filth and moisture underneath it, admirably suited to the proliferation of disease germs. When in that state it is worse than useless and had far better be removed.

If the cooling of the living spaces is always difficult in summer, their heating in winter is easily accomplished. Steam heating, through coils, is the method generally used. Hot water heating would give a more moderate and equable warmth, but is of more difficult and costly application. Heating must always be accompanied by suitable and adequate ventilation, preferably with air moderately warmed as already explained.

Personal Hygiene. - In the Navy, where men must inevitably live in close contact, personal hygiene is even more essential than in the Army. Body cleanliness has become one of the characteristics of our Navy personnel and is effectually maintained by force of public opinion aboard and the instrumentality of disciplinary measures. "On board ship the recruit who shows a disposition to eschew the bath is always an object of the crew's active attention and, if necessary, a scrubbing is administered to initiate the change of habit " (Rep. Surg. Gen., Igo8). Cleanliness of linen is as essential as that of the body, for not only. is soiled linen malodorous but even more liable to disseminate germs and parasites than the skin itself. Since the infection complicating gunshot wounds is the result of the dirt on skin and clothing, rather than on the missile, all authorities recommend that prior to an engagement, soldiers and sailors should 
bathe and put on clean underwear and outer garments; if this be advisable on land it is still more so aboard where the danger from shell wounds is much more serious.

Ample bathing facilities, in the form of shower-baths, are required on board ships, especially for the engineer's force whose perspiring bodies are often begrimed with dirt and oil. Salt- and soft-water baths should be provided. It has been reported that the use of softwater for this purpose is not as general as it might be in our Navy. Complaints have also been made that sometimes the salt water is so hot when it reaches the showers (having circulated through the distillers) that it renders them almost useless.

Swimming is for the sailor one of the most valuable of accomplishments, therefore one which he should be obliged to acquire, since it may be the means of saving his own life or that of his comrades. Swimming, besides cleaning the body, is a capital exercise and, were it for that reason alone, should be encouraged whenever practicable. It is necessary, however, to use some caution and ascertain that the water is reasonably pure. Most of the harbors frequented by our ships are more or less polluted with sewage from cities; in such places, therefore, the men should only be allowed to go swimming during floodtide. There is also danger of infection when swimming on sides of ships near where the flush from the heads (water-closets) is ejected.

The floors of water-closets should be of cemented concrete or tiling and made thoroughly impervious. Unless well constructed and kept in good repair, the jarring and vibration of the ship are quite likely to cause cracks and fissures in them, which become receptacles for urine and washings and the cause of offensive smells. The use of valves and vent pipes is necessary in water-closets to prevent "blowing" as the ship rolls, and the discharge of tainted air in the compartments. In the best form of closet the outlet of the pan is closed by two valves so arranged that only one opens at a time. In closets placed below the water line and where, therefore, discharge by gravity is impossible, the flushing is effected by a hand pump. The flush water should not be hot; if the heated water from the distillers is used, it may constitute a nuisance on account of the odorladen steam arising from the fixtures.

Considering the very offensive and repulsive odors emanating from anclean mouths and decayed teeth, especially in crowded spaces, oral 
hygiene must receive careful attention. The use of the toothbrush and tooth powder should be encouraged, if not insisted upon. Toothpicks should likewise be provided and kept in canteens; the common practice of using matches for toothpicks has been found to be responsible for a certain number of cases of inflammation and ulceration of the gums and other parts of the mouth. The employment of dentists in the Navy, as now provided by law, has filled an urgent need.

The ship's barber may be a potent agent in the transmission of various diseases from his hands, clothing, instruments and excretions, and must therefore be subjected to strict sanitary regulations. Some naval surgeons insist that every man on board should be required to shave himself and the barber's functions limited to hair cutting, but this is probably no more feasible than in the Army. Each person should at least have his own shaving implements and material, except soap which had better be used in liquid form or as paste (in tubes). Instruments used on different persons should be disinfected after each operation by immersion in alcohol and afterward in a strong solution of either formalin or cresol; clean towels must be supplied each customer. (See Personal Hygiene.)

Tattooing, formerly very prevalent in the Navy, and still too common, is a serious evil to be entirely suppressed, one which, in the opinion of the Surgeon General, calls for prompt and vigorous official action. It is well known that this practice, as dangerons as it is absurd, is a vehicle for the transmission of constitutional and cuticular disease; cases of syphilis and tuberculosis, even of death from blood-poisoning, having been caused by it.

The danger of the communication of diseases by the saliva, from the mouth-to-mouth use of common drinking cups, has long been realized in the Navy, and made the scuttle-butt cup an object of suspicion and apprehension. This difficulty has been removed by the introduction of the bubble fountain system (sce p. 25I) whereby. a man may drink without contact of the lips to the pipe, the flow being governed by a spring faucet, and the overflow passing into the expanded end of the surrounding drain. This device also saves water and time. On ships not provided with it the cup should be kept submerged in a solution of formalin ( $\mathrm{I} / 250 \mathrm{O})$.

On board ship, where the danger of communicating infectious diseases by contact is so great, nothing is better calculated to main- 
tain a high standard of health and cleanliness than individual insppection of the enlisted personnel at regular times, by a medical officer. For instance, once a month, each man, stripped, should pass before the surgeon and his genital organs and skin be rapidly examined. Thus are venereal and cutaneous diseases discovered and habits as to cleanliness ascertained. This examination will prompt the men to report venereal affections soon after they break out, which is in the interest of good hygiene, and induce the uncleanly to make more frequent use of the bath.

Food. - The Navy ration has been described in another chapter. It is in every way satisfactory as to quantity and quality, the commanding officer having ample authority to avail himself of local markets whenever desirable. In long cruises, however, fresh supplies may become scant and the diet somewhat monotonous, with deficiency of vegetable components and an excess of preserved meats. Most war ships and transports are provided with ice machines and cold storage so as to insure fresh meat and other perishable supplies for several weeks. The chief trouble in feeding the enlisted men of the Navy, as of the Army, is to secure the services of competent cooks in sufficient number, so that the components of the ration may be best utilized and always well prepared and served. Medical officers, in both services, cannot devote too much time and attention to the composition and preparation of the diet, under the various conditions of duty and climate. A consolidated mess has decided advantages over divided messes, but for its success, requires a competent and zealous commissary steward and the intelligent supervision of officers.

In order to prevent scurvy and other detrimental effects of a prolonged salt-meat diet, the Revised Statutes require that all vessels bound across the Atlantic and Pacific, or around Cape Horn or Cape of Good Hope, or engaged in whaling or sealing, shall carry a sufficient supply of lemon-juice or lime-juice, and vinegar.

Water-supply. - This is an easy question on board war ships, transports and steam liners, all supplied with evaporators and distillers, and where only distilled water is allowed for drinking and culinary purposes. It is to be remembered, however, that distilled water, although free from organisms and therefore incapable of transmitting infectious diseases, may have an unpleasant and repulsive odor and taste, and possibly cause intestinal irritation, when the raw water is pumped from shallow, polluted harbors. 
In ports where cholera, typhoid fever or dysentery prevail, or where the harbor is contaminated with much sewage or animal refuse, the use of harbor water on any of the decks should not be permitted.

In the absence of a distilling apparatus, no water should be taken on board without previous examination and approval by the ship surgeon, if practicable. The storage of such water is sometimes a matter of difficulty. Galvanized-iron tanks are best for the purpose; they should be coated with cement to prevent the action of the water upon the iron, and have large manholes for purposes of inspection and cleansing. When an emergency requires the use of hogsheads or casks, their interior should be thoroughly charred, so that the water may not become tainted by the decomposition of the substances it extracts from the wood. The amount needed for drinking, cooking, ablutions and washing of clothing should be calculated on the basis of four gallons per capita.

\section{DISINFECTION OF SHIPS.}

As soon as an infected vessel is brought alongside the quarantinestation pier, the sick (provided their condition admits of removal), together with their effects, are taken directly to the lazaretto without disinfection. The rooms thus vacated are immediately disinfected. Cabin passengers and, afterwards, steerage passengers and the personnel of the ship, are taken off by sections to the steam and formaldehyde disinfection chambers for treatment of their clothing and baggage, and then assigned to quarters at the station. Each set of rooms thus vacated is at once subjected to disinfection. Bedding and other articles needing the action of steam are also carried out.

The principal agents used in the disinfection of the vessel proper are sulphur dioxid, cyanid gas, bichloride of mercury and formaldehyde gas. For the hold of vessels, either of the first two is used, followed, in the case of wooden vessels, by washing with a solution of bichloride.

Sulphur dioxid is an efficient disinfectant so far as the hold and compartments of vessels are concerned. It is also invaluable for its power of destroying rodents and insects. It is generated by burning sulphur, 5 pounds per $\mathrm{r}, 000$ cubic feet of air space, or liberated from to pounds of liquid sulphur dioxid, sufficient moist- 
ure being supplied by steam or water; time of exposure 24 hours in metal ships and 48-72 hours in wooden ships. When the cargo cannot be removed, the sulphur should be generated from a special furnace to minimize the danger of fire. The chief objection to sulphur is the long exposure required on account of its lack of diffusiveness.

Cyanid gas (hydrocyanic acid) exceeds sulphur dioxid in efficiency as a fumigant because more toxic and more diffusive. With it the fumigation of loaded ships is effected in one hour, without injury to the most delicate fabrics, nor to a perishable cargo of fruits or vegetables, tea, coffee, tobacco, etc. It has been found that 5 ounces of potassium cyanid per I,OOO cubic feet are sufficient for the destruction of rats and insects, making cyanid gas the cheapest of fumigants.*

Compared to sulphur dioxid, cyanid gas has only one disadvantage, its greater danger to human life, but this is easily guarded against by proper care. (See Insecticides.)

In computing the capacity of the hold of a vessel to determine the quantity of fumigant required, it is assumed that the net tonnage of the vessel indicates the cubic capacity of her cargo-carrying space, and that ten net tons represent $\mathrm{I}, 000$ cubic feet of space.

In the case of all vessels treated for yellow fever or plague infection, fumigation must precede everything else in order to insure the destruction of mosquitoes, rats and other vermin. In wooden vessels it must also be used first, for water would seal many of the cracks and prevent effective gas penetration.

Before applying the bichloride solution there should be a thorough mechanical cleansing of all parts requiring it, with lye and a stiff brush. This solution (made up of I part of bichloride and 2 parts of hydrocloric acid or sodium chloride in I,000 parts of water) is thrown against all surfaces with hand pumps.

For application to polished woods, bright metals and other sur. faces injured by the bichloride, a 5 per cent. solution of formalin or carbolic acid should be preferred.

The living apartments, parlors, dining-rooms, cabins and forecastles may also be disinfected with sulphur or bichloride, but formaldehyde gas (as generated by the autoclave) is generally prefer-

* Public Health Report. Surgeon R.'H. Creel. Dec. 3, 1915. 
able. When sulphur is used its destructive action must be borne in mind and all metal surfaces and gilt decorations protected by a coating of vaseline.

Vessels are seldom so badly infected as to need disinfection throughout; the parts which require it, and the best method to effect it must first be determined. There is no reason, for instance, to disinfect the staterooms of the first cabin passengers because there is a case of smallpox in the steerage or in the quarters of the crew : nor to disinfect the hold of a vessel and break the cargo because of a case of smallpox. cholera or typhus fever in the cabins or steerage. Special measures are called for with each disease: thus, with cholera, typhoid fever and dysentery particular attention must be paid to the water, clothing and bedding; especial efforts should be directed against mosquitoes in yellow fever and malaria; against rats and vermin in plague; while in smallpox and other exanthematous diseases, the usual careful disinfection of living compartments is required.

As a rule, the cargo and stores do not need disinfection, excepting when consisting of rags or other absorbent articles, household goods or food products coming from infected localities. In the case of plague infection, sulphur or cyanid gas fumigations are necessary before and while unloading the cargo, in order to insure the destruction of all rats (as described under Quarantine). The ballast is liable to infection and its removal may be required.

\section{THE SICK BAY.}

The hospital or infirmary of the ship should be so located as to have as much light and air as possible. with a minimum of noise and motion. On war vessels it is generally on the berth deck. abaft of the forward turret. It should be borne in mind that the further aft it is. the less motion and noise will disturb it, and the less the liability to flooding in case of storm: the facilities for isolation will also be better and the danger of disseminating infection less. As a rule, the sick bay in our Navy combines most of the advantages which it is practicable to secure for the sick and wounded afloat. It is thus described by Surgeon General Rixey:

"Turning to the most modern type of ship, like the 'Louisiana' or 'Pennsylvania' or 'Connecticut,' we find a relatively commodious, admirably equipped emergency hospital, consisting of 
wards, isolation room, dispensary, bathroom, closet, and operating room, modern in all its appointments. All the indispensable requisites are provided and placed in a manner that is irreproachable for operation, dressing, and all medical care. In addition to modern aseptic furniture, instruments of precision, laboratory facilities, an elaborate surgical outfit and sterilizers, not to mention an abundant store of dressings and other material, there is good light and ventilation, hot and cold water, ice, etc., and, above all, attendance by trained nurses qualified to undertake all the duties of their calling" ("Keen's Surgery").

Unfortunately, the sick bay is rarely, if ever, protected by heavy armor, so that in war time, at the beginning of an engagement, it becomes untenable and must be abandoned. A naval battle is very different from a land fight, often more severe and destructive. Its issue is not decided by rifle fire, as upon land, but by heavy ordnance, so that wounds are mostly of a much more serious character. Combatants and non-combatants are equally exposed above the water-line; nor is there entire safety below it, the men thus located being in greater danger of mines and torpedoes.

Where to collect the wounded during an engagement, so that they may be relatively safe and not in the way of combatants while receiving appropriate treatment, is an important problem which, in the event of war, would have to be solved on each ship in accordance with its structural type. Such a place must be sought behind the heavy armored belt, preferably below the water-line; the spaces between the bunkers can be utilized. since coal affords relative protection. Accessibility is a great advantage, but does not matter so much as safety.

The great value of protected dressing-stations in saving lives without interfering with the fighting efficiency of the ship, has been recognized in our Navy. They are provided for in our new battleships, one forward and one aft, behind heavy armor and adjacent to hatches extending vertically through the decks so that the wounded may be rapidly passed down from the various deck levels. They are fitted with all necessary appliances for lighting, ventilation, steam connections for sterilizers, hot and cold water, water-closets, lockers, etc. In ordinary peace time this compartment is to be utilized for stores or other purposes. 


\section{HOSPITAL SHIP.}

The hospital ship, in peace as in war, is now recognized as a necessary component of a fleet. The Navy Regulations characterize it as follows:

"A hospital ship being assimilated to a naval hospital on shore, will be commanded by a naval medical officer not below the grade of a surgeon, detailed by the Navy Department. Such vessels will be manned by a merchant crew and officers and, in addition, a detail from the Hospital Corps of the Navy for carrying out the service to which the vessel is specially assigned."

"All hospital ships shall be distinguished by being painted white outside, with a horizontal band of green about a meter and a half in breadth. The boats shall be distinguished by similar painting. In accordance with the terms of the Geneva Convention, all hospital ships will fly the Geneva Cross flag at the main in lieu of the narrow pennant or coach whip."

"The neutrality of the hospital ship will at all times be preserved."

During an engagement, the hospital ship will seldom be able to render much service. Any attempt to do so must be clearly in pursuit of its humane mission and always at its own risk and peril. It may help the wounded and crews of sinking ships after they have surrendered, or when in such condition as to have ceased to be a factor in the fight. Most of its efficient work, however, will be performed at the close of the battle.

Besides the hospital ship, the needs of a fleet also include the service of several fast "medical transports" ready, during and after an engagement, to carry needed personnel and material to any of the ships and transfer the wounded to the hospital ship.

Hospital ships for the Army or Navy, whether constructed for the purpose or adapted from the merchant marine, should combine certain essential features, described as follows:

Hull : Of iron or steel.

Size: Not less than 4,000 tons.

Compartments: At least four good water-tight compartments.

Bilge keels.

Length: Not less than 350 feet.

Beam: Not less than 40 feet, with broad under-water lines to secure steadiness, even at the expense of speed. 
Steaming radius: 5,000 miles.

Free board: There should be as much free board as possible consistent with steadiness and safety.

Decks: At least four good unincumbered decks.

I. Superstructure deck.

2. Upper deck.

3. Main deck.

4. Lower deck.

5. Hold.

Disposition of Space.

Superstructure Deck. - Navigating officers with offices, staterooms, and mess-rooms, forward; senior medical officer's adjoining captain's.

Aft: Infectious ward; removable, mosquito-proof, double canvas top; closets and washrooms with separate plumbing; utensil closet, nurses' room, and medical attendants' room.

Extreme aft: Disinfector for infected material.

Boats: At least two steam launches specially equipped for transport of sick.

Junior medical officers have staterooms near sick officers' quarters, and medical officers mess in the wardroom of sick officers.

UpPer DEck, Forward. - Ward for sick officers: At least two suites of communicating rooms for officers of high rank, with bath and closet in one room, and standing bed,.stationary eating table and clothes locker in the other.

Other rooms: At least Io single rooms for officers of commissioned rank, except cadets. There should also be a mess-room with mess table, sideboard, library, easy chairs, pantry, and dumb-waiter. At the after end there should be common closets, baths, and washroom.

Aft: Medical ward, with standard bunks single or double banked, according to plan, with eating board, spit cups, and ditty-box attached. Head nurse's room. In rear of ward: Baths, closets, washroom, and examining room. Starboard side engine-room uptakes: Dispensary with Navy standard fittings complete, and adjoining stateroom for pharmacist. Port side:

I. Lounging- and smoking-room for officers.

2. Commissary's office.

3. Medical records and business office. 
MaIn Deck, Forward. - Medical convalescent ward arranged the same as the medical ward.

Aft: Surgical ward, with operating room, X-ray and electrotherapeutic room, and small dark and developing room on starboard side forward; recovery room adjoining. Aft, on port side of ward, pus operating room and dressing room, baths, closets, and washroom; on starboard side, head nurse's room, clinical bacteriological room and dentist's office, outfit complete, Navy or Army standard, with working library. Port side uptakes: Mess-rooms for convalescents, with pantries and warming room. On starboard uptakes, galleys, bakeshops, etc. Adjoining convalescent ward, forward; 'ward for non-commissioned officers, with adjoining messroom and pantries; closets and baths on port side connecting with same for convalescent medical ward. Aft of galleys: Mess-room for nurses, hospital stewards, etc. Pharmacist to mess with warrant officers.

Lower Deck, Forward Extreme. - Petty officers' mess-room, quarters, bath, closets; corresponding space on main deck above for crew.

Mid-deck Space. - Steam laundry, port side forward. Ice machines (two), capacity 3-4 tons, starboard side. Disinfector, drying room, freight elevator, cargo ports, cells for prisoners, and padded cells for insane. Dynamo room.

Aft: Crew space, with mess tables, Navy standard lockers, library, and master-at-arms' office and stateroom. Further aft, nurses, with mess tables, lockers, Navy standard pattern, books of instruction, lecture room, berths. Closats with washrooms extreme aft.

Hold, Forward. - Storerooms for vegetables and meats, pantries for officers' mess, storeroom for perishable goods, and dead-room, with Navy standard outfit.

Aft: Engineer stores, paymaster stores, medical stores for use on board ship and for distribution.

Extreme Aft: Two Army regimental hospitals each for 1,000 men, with wheeled litters, for use with landing parties.

Means of transportation for sick and wounded:

I. Wheeled carriage.

2. Stretchers, Stokes' and Army.

3. Cargo ports. 
4. Electric "Otis" light-running elevator at site of after cargo ports, running to all decks.

5. Davits and whip.

6. Steam launches.

7. Ladders.

The entire ship should be screened.

Medical and Hospital Corps Personnel for a Ship of 200 Beds. Medical: One medical officer in command of ship; four medical officers to attend the patients.

Hospital Corps:

Noncommissioned officers: Five (5).

Privates: Thirty-five (35).

Note.-In 1916, Congress having, for the first time, authorized the building of a hospital ship for the U. S. Navy, according to plans carefully prepared in the Surgeon General's Office, it is believed that, when completed, it will be a model of its kind and the best representative of all the advanced features and latest improvements to be expected in an up-to-date hospital ship. 


\section{CHAPTER LXII.}

\section{QUARANTINE.*}

By quarantine is meant the employment of such measures as are deemed necessary to avoid the introduction or transmission of disease from one country or locality to another.

The word itself is derived from the Italian quarantina, meaning forty, the number of days during which ships were formerly detained. The first maritime quarantine was instituted in 1403 at Venice, but measures to prevent the spread of plague had heen previously enforced by several Italian states.

All infectious diseases may be subjected to quarantine. For many years quarantinable diseases were limited to cholera, typhus, smallpox, and yellow fever. The statutes of New York now comprise as such, "yellow fever, measles, cholera, typhus, small-pox, scarlatina, diphtheria, relapsing fever, and any disease of a contagious, infectious, or pestilential character which shall be considered by the health officer dangerous to public health."

Under present United States regulations, the quarantinable diseases are cholera, typhus fever, plague, small-pox, yellow fever, and leprosy.

The period of detention imposed on ships and persons in quarantine for any disease should not exceed the usual period of incubation of said disease.

Quarantine may be maritime or inland; inland quarantine may be interstate, state, or municipal.

Inasmuch as maritime quarantine, in any harbor of the United States, is intended to protect not only the State in which said harbor is situated, but also the adjoining States and indirectly the entire country, and also for reasons of convenience, efficiency, uniformity and economy of administration, it is obvious that it should be entirely under federal control. Accordingly, the great majority of the maritime States have surrendered their quarantine functions to

\footnotetext{
* Based on laws and regulations of the United States as executed by the Public Health Service.
} 
the U.S. Government. At the present time (1917), there are only two ports (Baltimore and New York) whose quarantine stations are still directed by State authorities, but it is expected that they also will soon come under national control.

All maritime and interstate quarantine powers of the United States are vested in the Public Health Service, which is a branch of the Treasury Department, and directed by a Surgeon General.

Under existing law the Public Health Service coöperates with and aids State and municipal boards of health in the execution and enforcement of the rules and regulations of such boards to prevent the introduction of contagious or infectious diseases into the United States from foreign countries and into a State or Territory from another State or Territory; and for such ports and places within the United States as have no quarantine regulations under State or municipal authority, or adequate regulations to prevent the introduction of such diseases into the United States or into a State or Territory from another State or Territory, the Secretary of the Treasury shall, if he deems necessary, make such rules and regulations as may be required to that end. If the States and municipalities fail or refuse to execute and enforce these regulations the President is authorized to execute and enforce them and adopt such measures as in his judgment shall be necessary for the purpose.

The federal control of maritime quarantine does not affect the right of the seaboard States and municipalities to maintain their own quarantine, in addition, if they so desire.

Maritime quarantine is directed against the introduction of disease, chiefly from abroad, through the medium of vessels, their crews, passengers and cargoes. It begins at the port of departure. The United States regulations require that masters of vessels departing from any foreign port, or from any port in the colonies of the United States, for a port in the United States or its colonies, must obtain a bill of health signed by the proper consular or medical officer of the United States. Should such vessels call at intermediate ports they must procure at said ports a supplemental bill of health.

Are usually exempt from this requirement all vessels clearing from adjacent Canadian and Mexican ports unless quarantinable diseases prevail thereat.

Before issuing the bill of health the consular or medical officer will satisfy himself that the vessels, passengers, crews and cargo have complied with all the quarantine regulations of the United States. 
Quarantine stations must have adequate provisions for the boarding and inspection of vessels ; appliances for their mechanical cleansing and disinfection; a hospital for the treatment of contagious dis. eases, and another for the treatment of non-contagious diseases; barracks for the detention, in groups, of persons who have been exposed to contagion; also laboratory, steam laundry, crematory, etc. (see Disinfection of Ships).

Every vessel subject to quarantine inspection, entering a port of the United States or its colonies, shall fly a yellow flag at the foremasthead and be considered in quarantine until given free pratique.

\section{SPECIAL UNITED STATES QUARANTINE REGULATIONS.}

For Cholera. - For the purpose of these regulations, five days are considered as the period of incubation.*

The law provides that officers of the Public Health Service shall be stationed in cholera-infected ports, or in ports where emigrants from infected districts embark for America. They enforce the United States regulations providing for five days' observation in barracks before sailing, elimination of foodstuffs from baggage and disinfection of their effects. On arrival in an American port, the ship is again subject to a detention of five days. Inasmuch as the cholera bacillus may exist in the intestines of healthy persons and that the usual quarantine inspection is ineffective against such " carriers," a bacteriological examination of the stools of all steerage passengers coming from infected ports and places is also required before release. Such examination is likewise made of the stools of any passenger suffering from diarrhea.

"If cholera has appeared on board, remove all passengers from the vessel and all of the crew, save those necessary to care for her; place the sick in hospital. Carefully isolate those especially suspected, and segregate the remainder in small groups. No communication should be held between these groups. Those believed to be especially capable of conveying infection must not enter the place of detention until they are bathed and furnished with non-infected clothing; nor shall any material capable of conveying infection be taken into the place of detention, especially food and water."

* International Sanitary Convention of Paris, 1903. 
The water-supply of the vessel, if suspected of infection, must be disinfected, then discharged and the tanks thoroughly rinsed.

Living apartments and other portions of the vessel, as well as all baggage, effects and articles of cargo, which have been exposed to infection, must be disinfected.

Water ballast taken on at a cholera-infected port should be discharged at sea and the tanks disinfected.

For Plague. - For the purpose of these regulations, five days shall be considered as the period of incubation.*

At ports where plague prevails, every precaution must be taken to prevent the vessel becoming infected through the agency of rats, mice, flies, fleas, ants, or other animals. The vessel should not be at the dock, or anchor near any place where such animals may gain access to it. Access through the cables is prevented by tarring them and providing them with inverted cones. Communication between the vessel and shore must be reduced to the minimum absolutely necessary.

Careful observations should be made during voyages from plagueinfected ports to ascertain any marked sickness or increased mortality among the rats on shipboard. Experience has shown that an outbreak of plague in man is almost invariably preceded by an increased mortality among rats and mice.

Suspected vessels coming to quarantine shall be anchored at a stufficient distance from the shore or other vessels to prevent the escape of rats by swimming.

If any cases have occurred during the voyage, passengers and crew should be examined with special reference to the glandular regions. Doubtful cases, especially of the pneumonic type, may be subjected to bacteriological examination before the vessel is released.

The sick are sent to the hospital. The crew and passengers are removed and segregated into small groups and held under close observation for five days. Any person who has been directly exposed should be bathed and his belongings fumigated.

Nothing shall be thrown overboard from the vessel, not even deck sweepings. Such material will be burned in the furnaces of a steamer or in a place specially designated, but not in the galley.

As soon as practicable there shall be a thorough and simultaneous disinfection of all parts of the vessel with sulphur dioxid or cyanid

* International Sanitary Convention of Paris, 1903. 
gas for the destruction of rats and vermin. The killing of escaping rats should be provided for by a water guard in small boats. No person with abrasions or open sores must be employed in the landling of the vessel or her cargo. The rats will be subsequently gathered and burned, due precautions being taken not to touch them with bare hands. With sulphur dioxid the disinfection is fractional; after twelve hours' exposure, overnight, the upper layer of cargo is removed and placed on lighters exposed to the sun; this process of disinfection by night and removal of successive layers of cargo by day is to be continued until the hold is empty. With cyanid gas, fractional disinfection is seldom necessary.

For Yellow Fever. - For the purpose of these regulations, six days are considered as the period of incubation.*

While in an infected port, the vessel must lie at approved moorings in the open harbor and not approach the wharves; nor must the crew be allowed ashore. Every possible precaution should be taken to prevent the introduction of mosquitoes, and their breeding on board. Before the stowing of cargo or receiving of passengers the vessel should be carefully fumigated.

Inasmuch as the yellow-fever mosquito (Stegomyia) occurs naturally from the Gulf of Mexico to Virginia, it follows that measures of quarantine at southern ports need be much stricter than further north where the Stegomyia is not found, except when imported and then only for a short time.

If the vessel has properly complied with the quarantine regulations to be observed at foreign infected ports, and arrives at a northern port (north of the southern boundary of Maryland) without sickness, after 'six days from the time of departure, she may be admitted to pratique immediately after fumigation, without detention. If she arrives in less than six days she may be admitted to pratique without further detention than is necessary to complete the six days. If arriving after a longer voyage than twelve days, she may be subjected to the full period of detention, on the ground that a case of yellow fever might have occurred aboard and recovered, thus making her infection possible.

Vessels shall be held in quarantine if arriving at a port south of the southern boundary of Maryland in the season of close quarantine, May I to November I (directly or via a northern port), from

* Second Sanitary Convention of American Republics, Washington, 1905. 
a tropical American port, unless said port is known to be free from yellow fever.

If cases of yellow fever have occurred aboard, remove them to the hospital by screened ambulance or litter, and place them in a ward thoroughly protected against mosquitoes. Remove likewise and isolate all persons not required for the care of vessel; then destroy the mosquitoes on board by the simultaneous fumigation of all parts of the vessel by sulphur dioxid. If a fresh crew is available the vessel may now be released without further detention, reshipping the old crew left at the quarantine station on her next outward voyage.

Vessels need not be subject to quarantine when engaged in the fruit trade, provided they have fully complied with the special rules and regulations enacted for their guidance and benefit.

Passenger traffic without detention may also be allowed during the close quarantine season from infected ports to ports in the United States, even south of the southern boundary of Maryland, when all passengers and crew are immune to yellow fever, the vessel is "of iron and in first-class sanitary condition, and every precaution has been taken in the infected port to prevent the ingress of mosquitoes.

The disinfection of baggage for yellow fever is not required unless the quarantine officer has sufficient grounds to believe that it harbors mosquitoes.

For Small-pox. - For the purpose of these regulations, fourteen days are considered as the period of incubation.

On all vessels arriving with small-pox on board, or having had small-pox during the voyage, any of the passengers and crew who have been exposed to the infection must be vaccinated or detained in quarantine not less than fourteen days, unless they show satisfactory evidence of recent successful vaccination or of having had small-pox.

Such vessels need not be quarantined further than the removal of the sick, the disinfection of all compartments, baggage and objects that have been exposed to infection, and such vaccination of the personnel as required in the previous paragraph.

For Typhus Fever. - For the purpose of these regulations, twelve days are considered as the period of incubation.

Vessels in otherwise good sanitary condition, but having typhus fever on board which has been properly isolated, need not be quar- 
antined further than the removal of the sick, and fumigation of the compartments and their contents likely to be infested with vermin.

If the cases have not been isolated, or the disease has spread, the vessel will be quarantined, the sick removed, all persons who have been exposed examined and, so far as deemed necessary, their persons, clothing and effects treated for the destruction of vermin. Passengers upon whom lice are found will be detained to the end of the period of incubation, $i$. e., 12 days.

Living compartments and all other parts of the vessel, as well as baggage and effects likely to be infested, must be fumigated.

For Leprosy. - The period of incubation of this disease is still undetermined.

Vessels arriving at quarantine with leprosy on board shall not be granted pratique until the leper, with his baggage, has been removed to the quarantine station, and the living compartment or part of the vessel which has been exposed to infection properly disinfected.

No alien leper shall be landed. He will be detained at the quarantine station until again put aboard the vessel that brought him, for his return to the port of embarkation, as provided by law.

\section{INTERSTATE QUARANTINE.}

For the purpose of these regulations the quarantinable diseases are the same as in maritime quarantine, namely: cholera, plague, yellow fever, small-pox, typhus fever, and leprosy.

The general principles governing interstate quarantine are the same as those pertaining to maritime quarantine, but instead of dealing with ships as media of transportation we must deal with trains, steamboats, and wheeled vehicles.

The law requires that State and municipal health officers shall immediately notify the Public Health Service, by telegraph or letter, of the existence of any of the quarantinable diseases in their respective States or localities. All cases of quarantinable diseases or suspected of belonging to this class shall be at once reported by the physician in attendance to the proper authorities. These notifications are of extreme importance and must be strictly enforced so that suitable measures may be taken before the epidemic acquires headway and begins to spread.

Persons suffering from a quarantinable disease, or suspected to be so, shall be removed to a hospital or otherwise isolated until no 
longer capable of transmitting the disease. Those who have been exposed to the infection shall be isolated, under observation, for such a period of time as may be necessary to demonstrate their freedom from it.

The apartments occupied by persons suffering from quarantinable disease, and adjoining apartments when deemed infected, together with articles therein, shall be disinfected upon the termination of the disease.

No common carrier shall accept for transportation any person suffering with a quarantinable disease, nor any infected article of clothing, bedding or personal property. Bodies of persons who have died from any such disease shall not be transported save in hermetically-sealed coffins, and by the order of the State or local health officer.

In the case of yellow fever, a place shall not be considered entirely free from infection until after the expiration of eighteen days (nanely, twelve days for incubation in mosquito and six days for incubation in bitten patient).

In addition to the national quarantine, State or municipal authorities may make such additional regulations as they deem necessary for the preservation of the health of the people within their jurisdiction.

Under interstate and State quarantine are included sanitary cordon, camp of detention, railroad quarantine, disinfection stations, and inspection service.

The sanitary cordon consists of a line of guards, military or civil, thrown around a district or locality, either to protect the same from the surrounding country when infected, or to protect the surrounding country from an infected district or locality. Sometimes a double cordon is necessary, the outer one embracing the whole suspected territory, the inner investing more closely the well-defined infected locality. Sanitary cordons have been mostly used in Europe and Asia to guard against the spread of plague and cholera, and in this country against the spread of yellow fever.

A camp of detention is a place to which persons from infected points can go or are sent, to be kept under observation a sufficient length of time to demonstrate whether they are or are not infected; if infected they are removed to a special hospital; if not, they are released after disinfection of their clothing and baggage. 
Camps of detention should be established with due regard to site, water and drainage, and subjected to strict discipline. Every applicant must be examined by a physician before admission to ascertain the state of his health. His clothing and baggage are carefully inspected and, if need be, disinfected. All ingress and egress are allowed only through the established portal. The inmates are made as comfortable and cheerful as possible, all proper amusements and entertainments being encouraged and promoted. Each person, on leaving camp, is given a certificate that he has passed the required period of detention.

Such places should not be confounded with camps of refuge which are simply residence camps established to receive the non-immune population of an infected community and where it can remain in comparative safety until the epidemic is stamped out.

Railroad and steamboat quarantine consists in restricting all egress from an infected State or locality to a few stations on railroad and steamboat lines, and maintaining an efficient system of inspection at those stations. Inspectors, properly uniformed, board all outgoing trains and steamboats and demand of each passenger a certificate from a health officer showing where he has been during the previous five or six days, according to the disease to be guarded against. In case of doubt, passengers are sent to camps of detention.

Municipal quarantine includes not only. the measures necessary to prevent the introduction of disease into communities or its transmission from a city to neighboring cities and States, but also those required to prevent its spread within cities. It applies not only to the quarantinable diseases already described, but also to all other infectious diseases such as scarlet fever, measles, diphtheria and tuberculosis.

An important factor in municipal quarantine is the house-to-house inspection to ascertain the actual number of existing cases of disease. This naturally involves domiciliary quarantine or the exercise of restrictive measures against a particular house or part of a house; these are enforced by the stationing of guards to see that none but atthorized persons enter or leave the infected premises.

It is very important that as many of the sick as possible should be removed to special hospitals, thus increasing the facilities and efficiency of administration and diminishing the number of foci of infection. 
THE FOLLOWING ARTICLES OF THE SECOND INTERNATIONAL SANITARY CONVENTION OF THE AMERICAN REPUBLICS (WASHINGTON, D. C., 1905), BASED ON THE CONVENTION OF PARIS, 1903, HAVE SPECIAI INTEREST.

ARTICLE I. - Each government shall immediately notify other governments of the first appearance in its territory of authentic cases of plague, cholera, or yellow fever.

ARTícle II. - This notification is to be accompanied, or very promptly followed, by the following additional information:

I. The neighborhood where the disease has appeared.

2. The date of its appearance, its origin and its form.

3. The number of established cases, and the number of deaths.

4. For plague, the existence among rats or mice of plague or of an unusual mortality; for yellow fever, the existence of Stegomyia fasciata in the locality.

ARTICLE IV. - The notification and the information prescribed in Articles I and II are to be followed by further communications dispatched in a regular manner in order to keep the governments irformed of the progress of the epidemic.

These communications which are to be made at least once a week, and which are to be as complete as possible, should indjcate in detail the precautions taken to prevent the extension of the disease.

They should set forth: first, the prophylactic measures taken relative to sanitary or medical inspection, to isolation and disinfection; second, the measures taken relative to departing vessels to prevent the exportation of the disease, and, especially under the circumstances mentioned in paragraph 4 of Article II, the measures taken against rats and mosquitoes.

Article V. - The prompt and faithful execution of the preceding provisions is of the very first importance.

The notifications have only a real value if each government is warned in time of cases of plague, cholera and yellow fever, or of suspicious cases of those diseases occurring in its territory. It cannot then be too strongly recommended to the various governments to make obligatory the declaration of cases of plague, cholera or yellow fever, and the giving of information concerning an unusual mortality of rats and mice especially in ports. 
ARTICLE VI. - It is understood that neighboring countries reserve to themselves the right to make special arrangements with a view of organizing a service of direct information between the chiefs of administration upon the frontiers.

Article VII. - Information of a first case of plague, cholera or yellow fever does not justify, against a territorial area where it may appear, the application of quarantine measures.

Upon the occurrence of several non-imported cases of plague, or a non-imported case of yellow fever, or when cases of cholera form a focus, the area is to be declared infected.

Article VIII. - To limit the measures to the affected regions alone, governments should only apply them to persons and articles proceeding from the contaminated or infected areas.

* $* * \quad * \quad * * *$

But this restriction should only be accepted upon the formal condition that the government of the infected country shall take necessary measures, I, to prevent, unless previously disinfected, the exportation of articles coming from the contaminated area and, 2, to prevent the extension of the epidemic; and provided further, there be no doubt that the sanitary authorities of the infected country have faithfully complied with Article I.

When an area is infected, no restrictive measure is to be taken against departures from this area which have occurred five days at least before the beginning of the epidemic.

ArTICLE IX. - That an area should no longer be considered as infected, official proof must be furnished:

First, that there has been neither a death nor a new case of plague or cholera for five days after isolation, death, or cure of the last plague or cholera case. In the case of yellow fever the period shall be eighteen days, but each government may reserve the right to extend this period.

Second, that all the measures of disinfection have been applied; in the case of plague, that the precautions against rats, and in the case of yellow fever that the measures against mosquitoes, have been observed.

ARTICLE X. - The government of each country is obliged to immediately publish the measures which it believes necessary to take against departures either from a country or from an infected territorial area. 
Article XI. - There exists no merchandise which is of itself capable of transmitting plague, cholera, or yellow fever. It only becomes dangerous in case it is soiled by pestous or choleraic products, or, in the case of yellow fever, when such merchandise may harbor mosquitoes.

ARTICLE XII. - No merchandise or objects shall be subjected to disinfection on account of yellow fever, but in cases covered by the previous article the vehicle of transportation may be subject to fumigation to destroy the mosquitoes. In the case of cholera and plague, disinfection should only be applied to merchandise and objects which the local sanitary authority considers as infected.

Nevertheless, merchandise or objects may be subjected to disinfection, or prohibited entry, independently of all proof that they may or may not be infected.

* * * * * * * *

Article XIII. - In the case of cholera and plague there is no reason to forbid the transit through an infected district of merchandise and objects which are so packed that they are not exposed to infection in transit.

In like manner, when merchandise or objects are so transported that, in transit, they cannot come in contact with soiled objects, their transit across an infected territorial area should not be an obstacle to their entry into the country of destination.

ARTICLE XVI. - Letters and correspondence, printed matter, books, newspapers, business papers, etc. (postal parcels not included), are not to be submitted to any restriction or disinfection. In case of yellow fever, postal parcels are not to be subjected to any restriction or disinfection.

* * * * * * *

Article XIX. - Baggage. In the case of soiled linen, bed clothing, clothing and objects forming a part of baggage or furniture coming from a territorial area declared infected, disinfection is only to be practised in cases where the sanitary authority considers them as contaminated. There shall be no disinfection of baggage on account of yellow fever. 


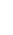




\section{BIBLIOGRAPHICAL LIST OF THE MORE RECENT AND USEFUL WORKS OF HYGIENE.}

\section{Aitken, Sir Wm.:}

\section{MILITARY HYGIENE.}

Growth of the recruit and young soldier. $12^{\circ}$, London I887.

Ashburn, P. M.:

The elements of military hygiene, especially arranged for officers and men of the line. $12^{\circ}$, New York, 1909.

Burot, F. \& Legrand, M. A.:

Les troupes coloniales; hygiène du soldat sous les tropiques. $8^{\circ}$, Paris, 1898.

Caldwell, R.:

Military hygiene. 2 ed. $8^{\circ}$, London \& New York, I9Io.

Davies, A. M.:

Handbook of hygiene. $16^{\circ}$, London, 1895.

Duncan, A.:

The prevention of disease in tropical and subtropical campaigns. $8^{\circ}$, London, $\mathrm{I} 888$.

Firth, R. H.:

Military hygiene; a manual of sanitation for soldiers. $8^{\circ}$, London, 1908.

Freeman, E. C.:

The sanitation of British troops in India. $12^{\circ}$, London, 1899.

Friedheim \& Richter:

Taschenbuch für den Felddienst des Sanitäts offiziers. $4^{\circ}$, Berlin, I899.

Havard, V.:

Manual of military hygiene. 3 ed., $8^{\circ}$, New York, 1917. Hoffmann, W. \& Schwiening, H.:

Hygiene der militärischen Unterkünfte; Hygiene des Dienstes. $8^{\circ}$, Berlin, 1911 .

Keefer, F. R.:

A text book of military hygiene and sanitation. Philadelphia, I9I4.

Kirchner, M.:

Lehrbuch der Militärgesundheitspflege. 2 Auf. der "Grundriss der Militärgesundheitspflege." $4^{\circ}$, Leipzig, 1910.

Laveran, A.:

Traité d'hygiène militaire. $8^{\circ}$, Paris, 1896.

Melville, C. H.:

Morache, G.:

Military hygiene and sanitation. $8^{\circ}$, London, I9I2.

Traité d'hygiène militaire. 2 ed., $8^{\circ}$, Paris, 1886. 


\section{Morrison \& Munson:}

Munson, E. L.:

A study in troop leading and management of the sanitary service. Fort Leavenworth, Kans., I9IO.

The theory and practice of military hygiene. $8^{\circ}$, New York, I90I.

The soldier's foot and the military shoe. Fort Leavenworth, I9I2.

Niehaus, W.:

Die Sanitäts ausrüstung des Heeres im Kriege. $8^{\circ}$, Berlin, I9I3.

Parkes, E. A.:

A manual of practical hygiene, prepared especially for use in the medical service of the army. 8 ed. edited by J. Lane Notter. $8^{\circ}$, London, I89I.

\section{Rouget \& Dopter:}

Hygiène militaire. $8^{\circ}$, Paris, 1906.

Tournade, A.: La pratique de l'hygiène en campagne. 1916.

Troussaint \& Schneider, G. E.:

Viry, C. M. J.:

Pages d'hygiène militaire pour les officiers. $8^{\circ}$, Paris.

Woodhull, A. A.:

Principes de $1^{\prime}$ hygiène militaire. $8^{\circ}$, Paris, 1896.

Military hygiene for officers of the line. $8^{\circ}$, New York, I909.

\section{NAVAL AND MARINE HYGIENE.}

\section{Duchateau, Jan et Plante:}

\section{Fonssagrives, J. B.:}

Hygiène navale. $8^{\circ}$, Paris, 1908.

Gatewood, J. D.:

Traité d'hygiène navale. 2 ed., $8^{\circ}$, Paris, 1877.

Germany:

Naval Hygiene. Phila., I909.

Kaiserliches Gesundheitsamt. Anleitung zur. Gesundheitspflege an Bord von Kauffahrteischiffen. 2 Auf., $12^{\circ}$, Berlin, 1899.

Holt, J.:

Marinesanitätsordnung. 3 vols., $8^{\circ}$, Berlin, 1893 .

An epitomized review of the principles and practice of Plumert, A.: maritime sanitation. $8^{\circ}$, New Orleans, I892.

Gesundheitspflege auf Kriegsschiffen. 2 Aufl, $8^{\circ}$, Wien \& Berlin, I900. 
Abbott, A. C.:

The hygiene of transmissible diseases; their causatior. modes of dissemination and methods of prevention. $8^{\circ}$, Phila., I 899 .

Abel, F.:

GENERAL HYGIENE.

Grundriss der Hygiene. $8^{\circ}$, Berlin, 1912.

Albrecht (\& al.):

Arnould, J.:

Handbuch der Hygiene. Io vol., Jena, 1893-190I.

Nouveaux éléments d'hygiène. Entiérement refondus

Bergey, D. H.: par E. Arnould. 5 ed., $8^{\circ}$, Paris, 1907.

Billings, J. S.:

The principles of hygiene. 4 ed., $8^{\circ}$, Phila., 1912.

Principles of ventilation and heating. New York, 1886.

Brouardel, P., Chantemesse, A. \& Mosny, E.:

Traité d'hygiène. Publié en 20 fascicules. $8^{\circ}$, Paris, I906-I9I3.

Carpenter, R. C.:

Heating and ventilation of buildings. 5 ed., New York, 1902.

Chapin, C. V.:

The sources and modes of infection. New York.

Chittenden, R. H.:

Physiological economy in nutrition. $8^{\circ}, 1904$.

Nutrition of man. $8^{\circ}, 1907$.

Cimmino, R.:

Appunti di igiene. $8^{\circ}$, Napoli, 1905.

Combastel, G.:

Eléments d'hygiène pratique a l'usage des écoles normales, des instituteurs et des familles. $12^{\circ}$, Paris, 1902.

Currier, C. G.:

Outlines of practical hygiene. 5 ed., $8^{\circ}$, New York, 1905.

Daniels, C. W. \& Wilkinson, E.:

Tropical medicine and hygiene. 3 vol., 8 , London \& New York, I9I0-I912.

De Giaxa, V.:

Compendio d'igiene. 4 ed., $8^{\circ}$, Milano.

Dieudonné, A.:

Doane, R. W.:

Bacterial food poisoning. New York, 1909.

Insects and disease. New York, I9ı. 
Drasche, A.:

Duchesne, L.:

Bibliothek der gesammten medicinischen Wissenschaften für praktische Aerzte and Specialaerzte. Hygiene. $8^{\circ}$, Wien \& Leipzig, 1899.

Egbert, S.:

Hygiéne générale et hygiène industrielle. $8^{\circ}$, Paris, 1896 .

A manual of hygiene and sanitation. $8^{\circ}, 5$ ed., Phila., I9r3.

Ferguson, R. B.:

Aids to the mathematics of hygiene. 4 ed., $16^{\circ}$, London, I909.

Fisher, B.:

Kurzgefasste Anleitung zu den wichtigeren hygienischen Untersuchungen. 2 Auf., $8^{\circ}$, Berlin, 1912.

Flügge, C.:

Grundriss der Hygiene für Studirende und praktische Aerzte. 4 Auf., $8^{\circ}$, Leipzig, 1897.

Folwell, A. P.:

Fuller, G. W.:

The designing, construction and maintenance of sewerage systems. 6 ed., $8^{\circ}$, New York.

Gärtner, A.:

Sewage disposal. New York, I9I2.

Leitfaden der Hygiene für Studirende, Aerzte, Architekten, Ingenieure und Verwaltungsbeamte. 5 Auf., $8^{\circ}$, Berlin, 1909.

Gerhard, W. P.:

The water supply, sewerage and plumbing of modern city buildings. New York, I9Io.

Guide to sanitary inspections. 4 ed., $12^{\circ}$, New York, 1909.

Glaister, J.:

Greene, A. M.:

Manual of hygiene, for students and nurses. 2 ed., $12^{\circ}$, Edinburgh, I905.

Guiraud, L.:

Elements of heating and ventilation. $8^{\circ}$, New York.

Manuel pratique d'hygiène a l'usage des médecins et des étudiants. $8^{\circ}$, Paris, 1904.

Harrington, C. \& Richardson, N. W.:

A manual of practical hygiene. 4 ed., $8^{\circ}$, Phila., I9Ir.

Hazen, A.:

Filtration of public water supplies. 3 ed., $8^{\circ}$, New York, I900. 
Heim, L.:

Hooker, A. H.:

Clean water. New York, I907.

Lehrbuch der Hygiene. $8^{\circ}$, Stuttgart, I903.

Chloride of lime in sanitation. $8^{\circ}$, New York.

Hough, T. \& Sedgwick, W. T.:

Elements of hygiene and sanitation, being part II of "The human mechanism; its physiology and hygiene and the sanitation of its surroundings."

Howard, L. O.: $8^{\circ}$; Boston, 1907 .

The house-fly, disease carrier. New York, I9II.

Kinnicut, Winslow \& Pratt:

Kober, G. M.:

Sewage disposal. $8^{\circ}$, New York, I9ı.

La Bonnardière:

Industrial and personal hygiene. Washington, I908.

Précis d'hygiène pratique générale et spéciale; première

Langlois, J. P.: partie, hygiène générale. $12^{\circ}$, Lyon, I901.

Leach, A. E.:

Précis d'hygiène publique et privée. 4 ed., $8^{\circ}$, Paris, I909.

Food inspection and analysis. 2 ed., large $8^{\circ}$, New York,

Lehmann, K. B.: 1909.

Die methoden der praktischen Hygiene. Lehrbuch zur hygienischen Untersuchung und Beurtheilung für Aerzte, Chemiker und Juristen. 2 Auf., $8^{\circ}$, Wiesbaden, I90I.

Le Play, A. \& Mesureur, A.:

Macaigne, M.:

Précis d'hygiène. $8^{\circ}$, Paris, I909.

Macfie, R. C.:

Précis d'hygiène. $8^{\circ}$, Paris, I9I I.

Mason, W. P.:

Air and health. New York.

Water supply. 3 ed., $8^{\circ}$, New York.

Examination of water. 4 ed., $12^{\circ}$, New York.

Notter, J. L. \& Firth, R. H.:

Hygiene. 6 ed., $8^{\circ}$, London, I905.

Pagliani, L.:

Trattato di igiene e de sanita publica colle applicazioni all'ingegneria e alla vigilanza sanitaria. $8^{\circ}$, Milano, 1905. 
Parkes, L. C.:

Pfeiffer, R. \& Proskauer, B.:

Hygiene and public health. 5 ed., $12^{\circ}$, London, 1897.

Encyklopädie der Hygiene. Lfrg. I-8, $8^{\circ}$, Leipzig, 1902-1903.

Plicque, A. F.:

Prausnitz, W.:

Précis populaire d'hygiène pratique. $12^{\circ}$, Paris, 1902.

Grundzüge der Hygiene unter Berücksichtigung der Gesetzgebung des deutschen Reichs und Oesterreichs. 8 Auf., $8^{\circ}$, Munchen, 1908 .

Proust, A.:

Reid, G.:

Traité d'hygiène. 3 éd., avec la collaboration de A. Netter et H. Bourges. $8^{\circ}$, Paris, 1904 .

Practical sanitation; a handbook for sanitary inspectors and others interested in sanitation. 8 ed., $8^{\circ}$, London, I90r.

Rideal, S.:

Disinfection and the preservation of food. $8^{\circ}$, London \& New York.

Rohe, G. H.:

Sewage and bacterial purification of sewage. London.

Text-book of hygiene. 3 ed., $8^{\circ}$, Phila., I899.

Rosenau, M. J.:

Rubner, M.:

Preventive medicine and hygiene. New York, I9I3.

Lehrbuch der Hygiene; systematische Darstellung der Hygiene und ihrer wichtigsten Untersuchungs methoden. 8 Auf., $8^{\circ}$, Wein, 1907.

Rubner, M., von Gruber, M. \& Ficker, M.:

Schoofs, F.:

Handbuch der Hygiene. 4 ed., Leipzig, I9II.

Traité d'hygiène pratique; méthodes de recherches. $8^{\circ}$, Paris, 1908.

Sedgwick, W. T.:

Principles of sanitary science and public health. Boston, 1902.

Shaw, W. N.:

Air currents and the laws of ventilation. Cambridge Univ. Press, 1907.

Simpson, W. J.:

The principles of hygiene as applied to tropical and sub-tropical climates. $8^{\circ}$, London \& New York, 1908. 
Smolensky, P.:

Traité d'hygiène. Procédés rapides de recherche des falsifications et alterations. Traduction du russe, avec annotations. $8^{\circ}$, Paris, 1904.

Smyth, A. W.:

A text-book of the principles of hygiene, based on physiology: For the use of school teachers. $8^{\circ}$,

Sternberg, G. M.: London, 1905.

Report on building of model houses. Washington, 1908.

Stevenson \& Murphy:

Treatise on hygiene. 3 vols., London.

Thoinot, L. H. \& Nordon:

Hygiène. $8^{\circ}$, Paris, I9I I.

Von Esmarch, E.:

Hygienisches Taschenbuch für medizinal und verwaltungsbeamte Aerzte, Techniker und Schulmänner. 3 Auf., $12^{\circ}$, Berlin, 1902.

Wanhill. C. F. \& Beveridge, W. W. O.:

The sanitary officer's handbook of practical hygiene. $8^{\circ}$, London, 1909.

Waring, G. E.:

How to drain a house.

Weill-Mantow, J.:

Hygiène individuelle et économie domestique. $12^{\circ}$; Paris, 1909.

Whipple, G. C.:

Williams, M.:

Microscopy of drinking water. Boston, 1905.

Wilson, G.:

Chemistry of cookery.

A handbook of hygiene and sanitary science. 8 ed., $12^{\circ}$, London, 1898. 


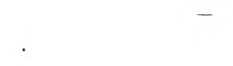




\section{INDEX}

Abdominal band, 440, 704

Acetone bodies, 3 I 7

Acetylene, as illuminant, 560 field outfit for, 56I

Acids, mineral, as disinfectants, 737 vegetable, as food, 3I 3

Actinic rays and their action, 695 effect of color on, 697 opacity on, 697 in relation to clothing, 425 in water purification, 282

Actinomycosis in cattle and man, 326 Administration in the field, 683

Aëroplane in war, 745

Age, as influencing sickness and mortality, 20

in relation to physical development, 183

of applicants for enlistment in U. S. Army, 183

in U. S. Navy, 755

Air, amount of, necessary, $53^{1}$ aqueous vapor in, 5 II

composition of, 509

contamination of, 5I9, 524

cooling of, in habitations, 706

as disinfectant, 719

passage of, through walls, 477,536

purification of, in nature, 529

sewer, 565

symptoms produced by pollution of, 524

principal causes of, 524

Air space necessary, in barracks, 53I in hospitals, 532

on board ships, 756

Airing of barracks, 492, 534

Albuminoids of meat, 307

Alcohol, food value of, 4I4

methylic or wood, $4 \mathrm{I} 7$

Alcoholic drinks, 4I4 in the tropics, 694

in cold climates, 714

Alcoholism, rates for, in Army and Navy, I3I

symptoms and effects of, 416

Altitude, effects of, 515

in the tropics, 709

Ammonia, in air, 511

in water, 267

permissible amount of, 268

Amœbæ, in relation to dysentery, 47
Ankylostomiasis, 108

cause and symptoms of, 108 prophylaixs of, III

Ankylostomum duodenale, 108

Anopheles mosquitoes, I68

Anthrax in man and cattle, I00, 32-

Antiseptic, definition of, 719

Anti-siphon trap, 572

Antitoxins, 27

Applicants for enlistment, 183 age limits of, in Army, 183 in Navy, 755

causes of rejection of, 192

chest measurement of, 189

height and weight requirements of, 187

method of examination of, 193 relation between height and weight of, 19I

special disqualifications of, I97

vision and hearing requirements of, 195, 197

in the Navy, 755

Arnold sterilizer, $72 \mathrm{I}$

Ascaris lumbricoides, 106, 107

Asphyxiating gases, I2I

Athlete's heart, 220

Athletic games and races, 229

Autogenesis, 26

Aviation, requirements for, 197

Bacillus, definition of, 23 aërogenes capsulatus, 14, 272 anthracis, 100

botulinus, 327

bulgaricus, 3 I 5,342

coli communis, $27 \mathrm{I}$

comma, 5I

diphtheria, 9 I

of Ducrey, I22

dysenteria, 46

enteritidis (Gärtner), 328

enteritidis sporogenes, 272

lactis aërogenes, 272

lepræ, I02

mallei, IOI

paratyphosus, 42

perfringes, 14

pestis, 86

prodigiosus, 263

proteus vulgaris, 328,369

pyocyaneus, I4 
subtilis, 369

tetani, 97

tuberculosis, 56

Bacon, qualities of, $322,387,402$ ration of, 383

Bacteria, action of, on proteins, 3I 5 on carbohydrates, 3 I 7

classification of, 23

denitrifying, 60I

effect of diet on intestinal, 369

in air, 521

in water, 270

in sewer, 565

in soil, 601

introduction of, in body, 27

nitrifying, 580 , $60 \mathrm{I}$

of putrefaction, 602

Bake-ovens, 375

Balantidium coli, 48

Ball trap, 568

Barber shop, hygiene of, 248 on board ships, 764

Barrack bag, 490

Barracks, arrangement of, 474

basement of, 483,485

bedding in, 490

building materials of, 477,483

care of, 492

ceilings of, 485,532

construction of, 477,483

distribution of rooms in, 485

floors of, 484, 493

foundations of, 480

furniture of, 489

improvised, 624

in the tropics, 705

lavatory in, 486

orientation of, 474,475

pavilion system, 474

plaster finish in, 480

portable, Ducker system of, 627

Doecker system of, 627

specifications for. 483

shape and size of, 474

Tollet system of, 474

walls of, 485

windows of, 482

Barthelemy's pack, 460

Basements, requirements of, 479,483

Bathing and washing, 246

Baths, cold and warm, 252 effects of, 252

shower and tub, 253

Turkish and Russian, 253

Battles, sanitary features of, 745 sanitary precautions before, 744

Battlefield, hygiene of, 744 disposal of the dead on, 748
Beans and peas, 357

Bedbug as disease transmitter, 143 destruction of, 495 Mexican, I44

Bedding, regulation, 490

Bedsack for the field, 490

Bedstead, regulation, 490

Beef, characteristics of good, 322 corned, 335

jerked, 335

measly, 325

Bell trap, 568

Belt, cartridge, 468

Benzin as insecticide, $18 \mathrm{I}$

Benzoic acid as preservative, 336

Beriberi, etiology of, II5 part played by rice in, II6

Berkefeld filter, 29I

Beverages, alcoholic, 4I4 proportion of alcohol in, $4 \mathrm{I} 6$ in hot countries, 694 in cold countries, $7 \mathrm{I} 4$ non-alcoholic, 409

Bibliography, 787

Bilge water, 757

Bilharziasis, I06

Bivouacs, 603

Blackwater fever, 72

Blanket, regulation, 468,490

Blanket-roll, 46i

Blood changes in tropics, 689

Boiling, as means of disinfection, 720

Borax, as food preservative, 336

Boston vent in water-closets, 575

Botulism, 327

Bowels, care of, 255 in the tropics, 709

Bread, characteristics of good, 354 composition of, 355

fermented and non-fermented, 353

field. 356

hard, 355

making of, 354

ration of, 383

soft, 355

Break-bone fever, 78

Breathing, in exercise, 213 through the nose, $2 \mathrm{I} 3$

Brick, as building material, 477

Brill's disease, 83

Bromidrosis of feet, 249

Bromin, as water purifier, 282

Broth as food, 33I

Bubble fountain, $25 \mathrm{I}$ in the Navy, 764

Building materials, 477 permeability of, 477 porosity of, 477 
Burial of bodies on battlefield, 749

Butter as food, 345

Buttermilk as food, 346

Cabbage as food, $36 \mathrm{r}$

Calories, definition of, 363

Camps, care of water-supply in, 632, 678

concentration of troops in, 583

disposal of excreta in, 636

of garbage and refuse in, 666

establishment of, 632

food and eating in, 677

form of, 629

improvements in installation of, 635

laundry and lavatory in, 666

personal hygiene in, 680

plan of infant.ry, 629

poisonous plants in, 606 animals in, 609

police of, $68 \mathrm{I}$

regimental hospital in, 631

sanitation of, regulations for, 684

site of, 603

duration of occupancy, 682

influence of vegetation upon, 606

reasons for changing, 682

soil of, 605

Campaign or service hat, 436

Campho-phenol as insecticide, 179

Candles as illuminants, $\mathbf{5 5 9}$

Canned meat, 333

Canteen and cup, 470

Caps, regulation, 435

for cold regions, 439

knitted, night, 439

Carbohydrates, amount necessary, 365 composition of, 3I I

in tropics, 403

kinds of, $3 \mathrm{Ir}$

nutritive functions of, 317

Carbolic acid as disinfectant, 738

Carbon bisulphide as insecticide, I8I

Carbon dioxid, how produced, 5 Io

as air constituent, 410

as index of air pollution, 526

effects of, $\mathbf{5 2 5}$

in alveolar air, 525

reduction of, in nature, $5 \mathrm{Ir}$

tests for, in air, 526

Carbon monoxid, danger from, 528

in coal gas, 528,560

in tobacco smoke, 528

tests for, 529

Carriers of infections, 25

Cartridge belt, 468
Cassava as food, 360

Cellars, requirements of, 479

Cellia mosquitoes, 173

Cellulose as food, 3 I 2

Cerebro-spinal meningitis, 90

Charcoal in filters, 304

Cheese as food, 346

Chest, effect of exercise upon, 212 how to expand, 2I3

in relation to height and weight, 187

method of measuring, I 89 mobility of, 190

Chicken-pox, 94

Chimneys, effects of, on ventilation, 539

Chittenden's experiments, 367

Chloride of lime, 738

Chlorine, in water, 267

as disinfectant, 727

as water sterilizer, 28,3

Chocolate, composition of, 4 I 2 nutritive value of, $4 \mathrm{I} 3$

Cholera, etiology of, 51 prophylax is of, 52 quarantine regulations in, 776

Cigar and cigarette smoking, 136

Cistern, for rain water, 258

Venetian, 306

Citric acid as water purifier, 289

Clams as food, 337

Clark's process for hard water, 265 soap test, 266

Climatic bubo, 103

Climatic variations, 686

Clonorchis endemicus, 106

Clothing, evaporation of, 423

fibers used for, 419

heat absorption of, $42 \mathrm{I}$

heat conductivity of, 422

heat radiation of, 422

hygroscopicity of, $42 \mathrm{r}$

in cold countries, $7 \mathrm{r} 5$

in relation to actinic rays, 425 , 697

in the tropics, 704

outer, 424

permeability of, 420

waterproofing of, 432

weight of materials, 410

Coal gas as illuminant, $\mathbf{5 5 9}$

Coat, regulation dress, 428 regulation service, 429

Cocaine habit, 138

Cockroaches as disease transmitters, 146

species of, 146

destruction of, 148 
Cocoa, composition of, 4 I2 effects of, $4 \mathrm{I} 3$

Coffee, composition of, 409 effects of, 409 value of, in field, 4 I I

Coindreau strap for shoe, 445

Cold, effects of on body, $7 \mathrm{I} 3$

Cold climates, 7 I 2 campaigning in, $712,716,746$ clothing in, 7 I 5

diet in, 713

diseases of, 717 stimulants in, 7I4

Cold storage, for fish, 337

for meat, 332

for oysters, 338

for poultry, 333

Colors and actinic rays, 697

Combustion, effects on air of, 523

Conduction of heat, 544

Congelation, prevention and treatment of, 7 I 7

Contact filter for sewage, 584

Contagious diseases, 26

Convection of heat, 544

Cooking, effects of, 328

of meat, modes of, 328

loss in, 330

in the field, $37 \mathrm{I}$

in foreign armies, 377

range, for the field, 372

outfit, individual, 372

Copper sulphate as water purifier, 288

Cork paint, on ships, 761

Corn or maize as food, 356

Cot, regulation, folding, 634

Cotton as material for clothing, 424

Cotton khaki, why discarded, 429

Cow pea as food, 357

Cremation of bodies on battlefield, 749, 75 I

Crematories for garbage and refuse: the rock-pile, for camps, 672 the grate, for garrison, 594 (see Incinerators)

Creolin, 738

Cresols as disinfectants, 738

Crowding, effects of, 524, 680

Crustaceans as food, 338 composition of, 339

Culex mosquitoes, 165

Cuspidors in barracks, 494

Cyanid gas, I80

see hydrocyanic acid, 180

Cycloleppteron mosquitoes, I7 I

Cysticercus bovis, 324

cellulosæ, 324
Dakin's solution, 739

Dampness, influence on health, 473, 598

in new buildings, 478

in cellars, 479

on ships, 761

Darnall, filter, 300

water purification by chlorin, 284

Datura or Jimson weed, as insecticide, I 78

Deaths in U. S. Army, 4

in U. S. Navy, 4

in United States, 5

Dengue, 78

Deodorant, definition of, 719

Desiccation as means of preservation, 335

as means of disinfection, 720

Dew point, 512

Diarrhea, causes of, 45 prevalence of, in camps, 44

Diet, the ideal, 366,370 (see Food)

Diphtheria, 9 I

Disconnecting trap, 568

Diseases, as influenced by age, 20

infectious, nature of, 23

in time of peace, 3

in war, II

miscellaneous, I20

most prevalent and fatal, 3

nutritional, I I4

of tropical countries, I5, 7 IO

of cold countries, $7 \mathrm{I} 7$

parasitic, I05

Diseases of cattle and hogs, 324

Diseases and wounds, losses from, compared, 8

Disease-transmitting animals, I4I

Disinfectant, air and light as, 7 I9 definition of, 7 I9

heat as, 720

steam as, $72 \mathrm{I}$

Disinfectants, chemical, gaseous, 727 in solution, 736

Disinfecting apparatus, steam, 724 steam and formaldehyde, 735

Disinfection, nature of, 7 I9 in the field, 742

of feces, 743

of hands, 742

of latrines, 648

of rooms, 740

of sewage effluent, 585

of ships, 766

of sputum, 74 :

of urine, 743 
Draft, nature and effects of, 543

Drain in house plumbing, 565 trapping of, 567

Drawers, regulation, 433

Dress coat, uniform, 428

Dress trousers, uniform, 428

Drinking cup, 25I

Drinks, see Beverages

Dry heat as disinfectant, 720

Dugout as winter quarters, 625

Dust, atmospheric, 517 microbial, 52I in air contamination, 520 in relation to heating, 554

Dysentery, etiology of, 45 types of, 46 prophylaxis of, 49

Ear, hygiene of, 248 protection of, 755

Earth, absorbent power of, 602

Earth-closet, 656 earth to be used in, 656

Eating and drinking, rules in, 406

Eggs as food, 347

Electricity as illuminant, 562

Emergency ration, 389 of the British army, 394 of foreign armies, 395

Entamœba coli, 47 histolytica, 47 tetragena, 47

Entamebic dysentery, 47

Equipment, requirements of, 453 distribution of, on body, 455 weight of, 453

U. S. Army, $46 \mathrm{I}$ composition and weight of, 462

for cavalry, $47 \mathrm{I}$

for hospital corps, 47I

Equitation as exercise, 226

Examination of applicants, 193

Excavating wagon for excreta, 655

Excreta, amount discharged, 564

as cause of infection, 636

composition of, 564

danger from, 636

disposal of, in camp, 636

by earth-closet, 656

by incineration, 658

by post holes, 653

by sink, 640

by sub-surface irrigation, 59 r

by trenches, 637

nature of, 564

Exercise, object of, 201

effect of, on heart, 210 on height, 216

on lungs; 212

on muscles, 209

on skin, 215

on temperature, 202

on weight, 216

fatigue from, 207

forms of, 206

in the tropics, 688,708

in cold countries, $7 \mathrm{I} 3$

lactic acid in, 203

overtraining in, 219

physiology of, 201

regulation of, 218

Exercises, special military, 225

the setting-up, 225

Extractives in meat, 310

Falk's winter quarters, 624

Fatigue, nature of, 207

Fatigue suit, canvas, 430

Fats, amount necessary, 365

composition of, 3II

digestibility of, 322

nutritive functions of, 316

reduction of, in tropics, 402

Feet, care of, 242, 249

deformity of, from shoes, 442

Fencing as exercise, 226

Ferrous sulphate as disinfectant, $\mathbf{7 4 0}$

Field baking, 375

cooking, 37 I

kitchen, 677

lavatory, 666

mess-shelter, 678

ovens, 375

range, 372

Field hospital, 632

Filariasis, I05

Filters, water, Berkefeld, 29I

Cincinnatus, 292

Darnall, 300

double-barrel, 305

granular, 304

improvised, 305

Japanese, 303

Jewell, gravity, 297

mechanical, 297

Pasteur-Chamberland, 29I

pressure, 298

rain water, 258

sand-bed, 294

Filtration of water, sand-bed, 293 mechanical, 297 air-wash system, 298 rake system, 298 nature of, 290

of sewage, 58I 
by contact filter, 584

by sand bed, 583

by sprinkling filter, 584

Fireless cooker, 374

Fireplace, heating by, 546

First-aid packet and pouch, 47I

Fish as food, 337

as larva destroyers, I76

Flannel, properties of, 423

Flask trap, 567

Fleas, as disease transmitters, I45

species of, I45

destruction of, 146

Flies, as disease transmitters, 148

in cholera, $5^{2}$

in dysentery, 49

in typhoid fever, 34

in tuberculosis, 57

breeding of, I5O

modes of conveying infections, I52

prevention and destruction of, I 54

species of, house-fly, I48

biting stable-fly, 156

common flies, 156

flesh or blow flies, 157

horse-flies, 157

phlebotomus flies, 158

simulium flies, I57

Floors, construction and materials

$$
\text { of, } 48 \mathrm{I}, 484
$$

dressing for, 482, 494

hygiene of, 493

Floor space, in barracks, 532

Floor trap, 568

Flour, wheat, 352

characteristics of good, 353

grades of, $35^{2}$

proteins of, 353

Flukes, in meat, 326

Fluorescin in testing wells, 262 as test for death, 749

Food, amount required, 365,370 decrease of, southward, $40 \mathrm{O}$ digestibility, 32I

dynamic action of, $40 \mathrm{OI}$

in the tropics, 400,693

in cold countries, 713

on board ships, 765

Foods, classification of, 307

animal, classification of, 318 composition of, 3I9, 339 cooking of, 328 excess of, 367

proximate principles of, 307 nutritive functions of, $3 \mathrm{I} 4$ nutritive value of, 363 nitrogenous constituents of, 307

vegetable, classification of, 349 composition of, 350

Foot, anatomical lines of, 44I how measured for shoes, 444 military type of, 444

Football as exercise, 23I

Foot-wear, importance of, 44I effects of bad, 442 in cold countries, 715 (see Shoes)

Foot-and-mouth disease, 327

Forbes water-sterilizer, 274 transportable sterilizer, 279

Forests, influence of, on climate, $5 I_{5}$

Formaldehyde as disinfectant, 728 generation of, by autoclave, 730 by formalin and permangan. ate, 730

by lamp, 730

by Munson's method, 732

by paraform, 73I

by paraform and permanganate, 732

by retort, 729

by sheet spraying, 73I

by simple spraying, 735

by steam chamber, 735

as insecticide, I8I

Formalin as disinfectant, 736,737

Fort Benjamin Harrison, sewage plant of, 587

Fort Ethan Allen, plan of, 476

Foundations of buildings, 480

Frambœsia (yaws), I03

Freezing, effect of, on organisms in water, 280

Frost-bite, nature and treatment of, $7 \mathrm{I} 3,7 \mathrm{I} 7$

Fruits as food, $36 \mathrm{I}$

used as vegetables, $36 \mathrm{r}$

Fuchsins as disinfectants, 740

Gangosa, I 26

Garbage, disposal of, 593 in camps, 667

Gas, coal, composition of, 559 products of combustion of, 560

Gas gangrene, 15

Gasolin as insecticide, $18 \mathrm{I}$

Gauntlets, uniform, 439

Gelatinoids, 3 IO

nutritive functions of, $3 \mathrm{I} 5$

Glanders, in man and horses, IOI

Gloves, uniform, 439

Gonorrhea (see Venereal diseases), I 22

Grease, clogging of pipes by, 569 
trap, 569

Green vegetables, 36I

Griffith water-sterilizer, 279

Grippe or influenza, 96

Ground air, 598

moisture, 598

temperature, 599

water, 605

Ground, danger of sleeping on, 635

Guarana as beverage, 413

Guard-house, care of, 493

Gymnasium, hygiene of, 227

Gymnastic exercises, 22I

Hallux valgus, 443

Hair, care of, 247,248

Hands, washing of, 256,742

Hard bread, 355

Hat, campaign or service, 436

Havelock, of helmet, 435

Haversack, 465

Head covering, 433

in cold countries, $7 \mathrm{I} 7$

Hearing, examination of, 197, 755

Heart, diseases of, 120 effect of exercise on, 210

effect of overstrain on. 220

examination of, 198

irritability of, 244

Heat, as disinfectant, 720

loss of, from body, 364

effects of dry, 545 moist, 688

effects of, on air dust, $5 \mathbf{5 4}$

of flame, 559

transmission of, 544

Heat-exhaustion, 704

Heat-stroke, 703

Heating, by hot-air furnace, 549

by hot water, 550

by open fire or grate, 546

by steam, $55 \mathrm{I}$

by stoves, 547

coils, location of, $\mathbf{5 5 2}$

systems of, 553

direct system of, 553

direct-indirect system of, 553

drying of air from, 545

indirect system of, $\mathbf{5 5 3}$

of ships, $760,76 \mathrm{I}$

use of, in ventilation, $\mathbf{5 4 6}$

Height, regulation, for soldiers, 187

Helmet, as head covering, 435

Hematuric fever, 72

Hemoglobinuric fever, 72

Henneberg, trinkwasser wagon, 278

Heroin, 139

Hila monster, 612
Hookworm, 108

old world, 108

new world, 109

Horse meat, 322

Hospital, air space required in, 532 construction and disposition of, 498

floors and walls of, 500, 502

field, 632

general, 503

temporary, 505

pavilions of, 506

regimental, 631

standard post, 498

brigade, 502, 503

in tropics, 503

Hospital ship, 770

Hospital tropical tent, 620

ward tent, 622

Hot air, heating by, 549

Hot water, heating by, 550

Humidifiers, 546

Humidity of air, $5 \mathrm{I} 3$

effect of, on body, $5^{\mathrm{I}} 3$

effect of, in cold weather, 514

effect of, in hot weather, 514

indoor, 545

of ships, 760

Humus, nature of, 597

Huts, log, 624 excavated or dugouts, 625

Hydric trap, 572

Hydrocarbons or fats, $31 \mathrm{I}, 322,402$ functions of, 316

Hydrocyanic acid as insecticide, 180 in disinfection of ships, 766

Hydrophobia, 99

Hygiene, nature and scope of, XV military, scope and aims of, XVIII of battlefield, 744

of toilet rooms, 576

Hygroscopicity of fabrics, 42I

Hymenolepis nana, 106

Ice, ration of, 386

Ice-cream, 344

Identification record, 200 tag, 462, 749

Illuminants, 559 relative cost of, 563 vitiation of air by, 559

Immunity, nature and kinds of, 28

Imhoff tank, 582

Incineration of excreta, 658 of garbage and refuse, 668

Incinerators for excreta, 658 the ambulant, 665

the Conley, 665 
the Harris, $66 \mathrm{r}$

the Jones, 660

the Lewis and Kitchen, 665

the McCall, $6_{5} 8$

the Q. M. Corps, 663

Incinerators for garbage and refuse, 668

the Caldwell, 672

company or kitchen, 668

the Guthrie, 671

rock-pile, 672

railroad-iron, 675

Infection, production of, 26

Infectious diseases, 23, 680 marked decrease of, I2

Infirmary, 63I

Influenza, 96

Inlets, fresh air, 54I

Insects as disease-transmitters, I43

in barracks, 494

Insecticides, 178

Insolation, 702

Inspection, physical, 127

in the Navy, 764

Interaction of food and work, 407

Intercepting trap, 568

Intestinal flora, how affected by diet, 369

Intestinal parasites, I06

Iodine as water purifier, 283

Irrigation in sewage disposal, $58 \mathrm{I}$

Irritable heart, 244

Itch or scabies, 112

camp, I12

dhobie, I12

Japanese field cooking stove, 378

pocket stove, 7I7

filter, 303

Jerusalem artichoke as food, 359

Jigger or sand flea, I46

Kala-azar, 103

Kephir as food, 346

Kidneys, overstrain of, 206, 221, 368

Kit, surplus, 465 field, 465

Kitchen, care of, 495

ambulant, 378

field, 677

improvised, in camp, 371

Kitchen pit, for liquid wastes, 667

Knapsack, 457

Kola nut, as stimulant, $4 \mathrm{I} 3$

Koumiss, 346

Lactic acid in muscle and blood, 203 action of, 204
Latrines, in camp, 63I, 636

danger from, 636

disinfection of, 648

earth-closet, 656

firing of, 649

pit (see Sinks), 640

police of, 648

post-hole, 653

sanitary trough, 653

spraying of, $65^{\circ}$

trench, 637

Latrine box, 643

the Havard, 643

the Lyster, 645

the standard, 645

the Straub, 645

Laundry in garrison, 2.54 in field, 666

Lavatory in garrison, 250 in field, 666

Leggins, uniform, $45^{\circ}$

Leishmaniasis, 103

Lentils as food, 357

Leprosy, 102

quarantine regulations in, 780

Lice as disease transmitters, 144 destruction of, 145 species of, 144

Light and lighting, 557 effects of, 557 location of, 558

Lime as disinfectant, 739

bisulphide as insecticide, 181

chloride (chlorinated), 283

as disinfectant, 738

as water purifier, 283

as sewage sterilizer, 586

in treatment of wounds, 739

hypochlorite, 283

milk of, 739

Liquid wastes, disposal of, 668,670 , 671

Liver, abscess of, 49

effect of food excess on, 40I, 693

effect of alcohol on, 416

Lizards, as poisonous animals, 6I2

Locliers for squad-rooms, 490

Lockjaw or tetanus, 97

Log huts, 624

Luminosity in flame, $\mathbf{5 5 9}$

Lysol, 738

Maize as food, 356

Malarial fevers, etiology of, 64 human cycle of parasites in, 65 mosquito cycle of parasites in, 67 parasites of, 64 
prevalence of, 63

prophylaxis of, 67, 7I

Malta fever, 80

Manchineel as poisonous plant, 608

Miansonia mosquitoes, 167

Manual of arms as exercise, 225

Manure, treatment of, 154,674

March, forced, 234

general remarks, 233

rate and lengtli of, 237

regulation of, 237

water drinking on the, 239

Marching, flexion step in, 235

in the tropics, 708

steps used in, 234

work done in, 244

Marine hygiene, 752

Marshes near camps, 604

Mate as beverage, 413

Mattress, regulation, 490

Measles, 94

German, 95

Meat, roasting or baking of, 329

boiling of, 329

broiling of, 329

canning of, 333

characteristics of good, 322

composition of, 3 I9

constituents of, 318

cooking of, 328

desiccation of, 335

digestibility of, $32 \mathrm{I}$

extracts, $33 \mathbf{I}$

extractives, 318

frying of, 330

loss by cooking, 330

parasites of, 324

poisoning by, 327

preservation of, $33 \mathrm{I}$

refrigeration of, 332

salting of, 335

stewing of, 330

Meat-can, 465

Nedical officer, status of, XIX duties and responsibilities of, $\mathrm{XX}$

Meningitis, cerebro-spinal, 90

Mercury, bichloride of, as disinfectant, 736

as insecticide, I8I

Merriam pack, 459

Mess, company and general, 486

Mess kit, individual, 462

Mess-room, care of, 495

Mess shelter, for camps, 678

Methylic alcohol, 417

Meyer line in foot, 442

Micro-organisms in air, 521, 522

Milk, as medium for bacteria, 340 composition of, 340

diseases transmitted by, 34I

dried or powdered, 344

examination of, 343

lactic bacilli in, 342

pasteurization of, 344

sterilization of, 343

toxic substances in, 34I

Milk of lime as disinfectant, 739

Mites as disease transmitters, I4I

Mittens, regulation, 439

Modern warfare, 745 prominent features in, 745

Moisture in heated air, 545

in walls of new building, 478, 479 in soil, 598

Mollusks as food, 337

composition of, 339

Morbidity and mortality in military service, I

in the Navy, 4

in time of peace, 3

in tropical countries, 15

in war, 6

Morphine habit, 138

Mortality, decrease of, XVI

from disease and wounds compared, 8

in U. S. Army, 4

in U. S. Navy, 4

influence of age on, 20

of length of service on, 20

of climates and stations on, 20 of race on, 18

Mortar and plaster, 478

Mosquitoes as disease transmitters, I 59

classification of, I58, $164^{\circ}$

description and breeding of, I59

destruction of, 68,174

eggs of, 160

mode of conveying infection, 160

screening against, 68, 68I

Mosquito bars, use of, $68,68 \mathrm{I}$

Mountain sickness, 515

Mouras vault, 589

Mouth, care of, 247

Mumps, 96

Muscles, effect of exercise on, 209

Mushrooms, useful and poisonous, 608

Mutton, characteristics of, 322

Myzomyia mosquitoes, I7 I

Myzorhynchus mosquitoes, 172

Native forces, use of, in tropics, 693 
Naval hygiene, 752 characteristics of, 752 statistics of, 753

Necator Americanus, 109

Night marches, 239, 708

Night urinals, 652

Nitrates in water, significance of, 269

Nitrites in water, significance of, 268

Nitrogen in nature, 510

Nitrogenous diet, excess of, 368

Non-effective rates, 5

Nucleo-protein, 308

Nuts as food, 362

Nyssorhyncus mosquitoes, I72

Oatmeal as food, 356

Ocean, influence of, on climate, 515

Oil, linseed, for floors, 482

Oil, mineral, for lighting, 559 for destruction of mosquitoes, 175 for latrine disinfection, 649

Oleomargarin as food, 346

Olive-drab cotton cloth, 429

Olive-drab flannel shirt, 430

Onions as food, $36 \mathrm{I}$

Outlets, ventilating, $54 \mathrm{I}$

Ovens, field, for baking, 375 improvised, 376

Overcoat, regulation, 43I for cold climates, 432

Overshirts, regulation, 433

Overshoes, regulation, $45^{\circ}$

Overstrain in exercise, 220

Overtraining in exercise, 219

Oxygen, for animals and plants, 509

Oxygenation of blood, effect of hot climates on, 690

effect of high altitudes on, 515

Oxyuris vermicularis, 106

Oysters as food, 337

Ozone, as air constituent, 5II

as disinfectant, 736

as water purifier, $28 \mathrm{I}$

effect of heat on, 554

Pack-carrier, 465

Pack, composition of, 462

Pack, Merriam, 459

Pain de guerre, French army, 355

Pain biscuité, French army, 356

Panama Canal Zone, I6 rates of diesase and death, I6 results obtained in, 692

Papataci fever, 8I

Papering, walls and ceilings, $48 \mathrm{I}$

Paragonomiasis, I06
Parasites, of circulatory system, I05 intestinal, I06 skin, $11 z$

Paratyphoid fever, 42

Pasteurization of milk, 343

Pavilion wards, 506

Peanuts as food, 362

Peas as food, 357

Pectin as food, 312

Pediculus, species of, 144 destruction of, 145

Pellagra, II4

Pemmican as food, 335, 7I4

Pemphigus contagiosus, II 3

Permanganate as water purifier, 288

Permeability of fabrics, 420 of soils, 597,598

Personal hygiene, 246 in the field, 680 on board ships, 762

Perspiration, in hot climates, 687

Petroleum, as illuminant, 559 to destroy bedbugs, 495 to destroy mosquitoes, I 75 to disinfect latrines, 649

Phagocytosis, 27

Physical training, 22I for officers, 224

Picket line, care of, 674

Pied forcé, 242

Pignet's index, 191

Pinole as food, 356

Plague, types of, 85 cause of, 86 diagnosis of, 88 mode of transmission, 86 prophylaxis, 88 quarantine regulations, 777

Plasmodium parasites, 64

Plaster and plastering, $478,479,480$

Plumbing fixtures, 565

Plumbing system, 565

Pneumonia, 62

Poisonous plants, 606

Poisonous animals, 609

Police, sanitary, of camps, $68 \mathrm{r}$

Poncho for foot soldiers, 468

Pork, characteristics of good, 322, 323 measly, 324

Portable, knock-down barracks, 627 hospitals, 627

Post, military, plan of, 476 disposition of buildings, 474 site of, 473

Post holes for excreta disposal, 653

Potassium permanganate as water purifier, 288

Potatoes as food, 358 
Preservatives, food, 336

Prismatic glass in windows, 559

Privy (see Latrines) the L. R. S. sanitary, $59 \mathrm{I}$

Proteins, amount necessary, 365, 370 bacterial decomposition of, 315 composition and nature of, 308 danger of excess of, 368 digestibility of, 32 I experiments regarding, 367 kinds of, 307 nutritive functions of, $3 \mathrm{I} 4$ reduction of, reasons for, 368 in the tropics, 402,693

Protozoa, 24

Proximate principles of food, 306

Psilosis or sprue, 50

Ptomains, 327

Pulses as food, 357

Purin bodies, 309

Puttees, 450

Pyrethrum as insecticide, 178

Pyrofume as insecticide, 179

Quarantine, definition and object, 774 interstate, 780

maritime, 775

municipal, 782

camp of detention in, $78 \mathrm{I}$

sanitary cordon in, $78 \mathrm{I}$

Quarantinable diseases, 774 special regulations for, $776-780$ (see Sanitary convention)

Rabies or hydrophobia, 99

Race, influence of, I8

Radiation of heat, 544

from body and clothing, 364, 422

Radiation, solar, 517, 695

Radiators, location of, 552

Range, cooking, for field, 372

Ratio of killed to wounded, I4

Ration, Army, 383

conclusions regarding the, 396

emergency, 389

field, 388

Filipino, 388

garrison, 383

composition of components, 310

value in calories, 384

in foreign armies, 391

British army, 393

Chinese army, 398

French army, 39I

German army, 393

Japanese army, 394

Russian army, 394 in the tropics, 400

conditions affecting, 400

reserve (haversack), 387

savings of, how expended, 398

trail, 390

travel, 386

U. S. Navy, 390

Recruiting, 182

(see Applicants for enlistment)

in the Navy, 755

Recurrent fever, 83

Red bug, I4I

Refrigeration, 332 (see Cold storage)

Refuse in camps, disposal of, 666

Relapsing fever, 83

Respiration, vitiation of air by, 519 in the tropics, 690

Rhus or sumach, 606

Rice as food, 357,405 in etiology of beriberi, II6 structure and composition of, II6

Ridge ventilation, 539

Ringworm, II 2 tropical, I 2

Rocky Mountain spotted fever, 84

Roofing of buildings, 483,485

Room disinfection, 740

Roots as food, 360

Rubeola or measles, 94

Rules in eating and drinking; 406

Running drill as exercise, 226

Russian ambulant kitchen, 378

Russian stove, 548

Saccharin, 312

Salts, mineral, as food, 314

Sanatorium for tuberculosis, 60

Sanitary cart, 668

Sanitary convention of American republics, 783

Sanitary inspector, 683

Sanitary rules in the field, 677

Sanitary service, regulation of, 683

Sanitary squads, 684

Sanitas trap, 57I

Scabies or itch, I4I cause of, I4I

Scarlet fever, 94

Schistosomum, species of, 106

Scorpions as venomous animals, 612

Screening against flies, 68I

Scurvy, II9

Seal of traps, 567 loss of, 569

Septic tank, $58 \mathrm{r}$ action of, $58 \mathrm{r}$ Imhoff, 582 
Service uniform, 429

Service at the front, 747

Service in cold climates, 7/2 (see Cold climates)

Service in warm climates, 686 (see Tropics)

Sewage, biological treatment of, 580 by irrigation, $58 \mathrm{I}$ by filtration, $58 \mathrm{I}, 583$ chemical treatment of, 579 composition of, 578 definition of, 564 discharge of, into the sea, 578 into lakes and rivers, 579 disinfection of effluent, 585 disposal of, 578 in garrison and camps, 586 domestic treatment of, 589 purification plant, 587 stability of effluent, 585

Sewer air, nature of, 565

Sewerage, definition of, 564 effects of, upon health, 565 requirements of, 564

Sewers, combined system of, 577 separate system of, 577

Ships, care of decks on, 761 causes of air pollution on, 757 conditions affecting hygiene of, 752

disinfection of, 766

humidity of, 760

personal hygiene on, 762

sick bay of, 768

temperature of, 760

ventilation of, 758

water-supply of, 765

Shirts, 433

olive-drab overshirt, 430

Shoes, breaking a new pair of, 445

care of, 448

effects of ill-fitting, 442

fitting of, 444

military, 444

requisites of, 447

shape of rational, 446

uniform, 449

Shower bath, in garrison, 253 in the field, 666

- Sick bay of ships, 768

Six-day fever, 8I

Sinks or pit latrines, box for, 643 construction of, 640

dimensions of, 640

location of, 640

objections to, $65 \mathrm{I}$

police and disinfection of, 648 use of paper in, 648
Siphonage in traps, cause of, 569 prevention of, 570

Skin, effects of washing the, 246 functions of, 246 parasites of, II 2 pigment of, 700 vitiation of air by, 520

Sleeping bag, 468, 7 I6

Sleeping sickness, I04

Slicker for mounted troops, 468

Smallpox or variola, 92 quarantine regulations in, 779

Smell, in air pollution, 524, 526

Smoking habit, I33

Snakes as poisonous animals, 6ro

Snake bite, treatment of, 6II

Snow blindness, 558, 716

Soap, use of, $247,250,742$

Socks, regulation, 452

Sodium bisulphate as water purifier, 288

Sodium hypochlorite as water purifier, 283

Soft drinks, $4 \mathrm{I} 3$

Soil, absorbent power of, 602 air in, 597

bacteria of, 601 constituents of, 597

danger of sleeping on, 635

effect of vegetation on, 600

moisture of, 598

permeability of, to water, 598

pollution of, 601

porosity of, 597

temperature of, 599

Soil pipe, in house plumbing, 565

Solanin in potatoes, 359

Solar rays, nature and effects of, 695

Solaro fabrics, 425

Soup, 33I

Soy bean as food, 358

Spiders as poisonous animals, 613

Spirillum, 24 of cholera, $5 \mathrm{I}$

Spirochrete (treponema) of syphilis, 122

Spittoons, use and care of, 494

Spring water, 260

Sprinkling filter for sewage, 584

Sprue or psilosis, 50

Sputum, danger of, 494 disinfection of, 743

Star ventilator, 539

Starches as food, 312

Stations, influence of, on health, 20

Steam as disinfectant, 721 saturated or flowing, 721 superheated, 723 
Steam disinfecting apparatus, 724

Steam, heating by, $55 \mathrm{I}$

Stegomyia mosquitoes, 166

Step, the flexion, 235

Steps, cadence and length of, 234

Sterilization, definition of, 7 I9

Stomoxys calcitrans, 156

Stove, heating by, 547 ventilation by, 547

Russian, 548

Japanese, 717

Straddle trench, 637 cover for, 639

Streptococcus fecalis, 14

Strongyloides stercoralis, 106

Sub-surface irrigation, 591

Sugar, cane, or saccharose, 312 grape, or glucose, 3 I I maltose, 3I I

milk, or lactose, 312

on the march, 240 value as food, 317

Sulphur dioxid, as disinfectant, 728 as insecticide, 179

as preservative, 335 method of generating, 728

Sunlight, as disinfectant, 7 I9 in the tropics, 695

Sunning of bedding, 720

Sunstroke, cause and effects of, 702

Surplus kit, 465

Suspenders, for soldiers, 426

Sweater, regulation, 431

Sweet potatoes as food, 360

Swimming as exercise, 227,763

Swimming pool, hygiene of, 228

Symptomatic anthrax, Ior

Syphilis, cause of, 122 diagnosis of, 125

prophylaxis of, 126

statistics of, I23

from tattooing, 764

Tag, identification, 462,749

Trenia saginata, 324 solium, 324

Tannier as food, 360

Taro as food, 360

Tattooing, danger from, 764

Tea, composition of, 4 IO preparation of, 4 IO value of, in the field, $4 \mathrm{II}$

Teeth, care of, 247

Tempelhof hospital, Berlin. 507

Temperature, of body, in tropics, 690 of air, indoor, 545 variations of, 686 of ships, 760
Tents, common, 616

conical, 617

cooling of, 707

ditching of, 633

flooring of, 615

general considerations, $6 \mathrm{I} 4$

Herbet, 623

hospital, tropical, 620

hospital ward, 622

pyramidal, 620

shelter, 6i5

shifting of, in camp, 682

spacing of, 629

storage, 623

Tollet, 623

tortoise, 623

ventilation of, 614

wall, large, 616 small, 617

Terrazo floors, $48 \mathrm{I}$

Tetanus or lockjaw, 97

Textile fibers, 419

Three-day fever, 8I

Ticks as disease transmitters, I4I species of, I42

Tinea circinata or ringworm, II2 imbricata or tropical ringworm, II 2

Tirotoxicon, 34I

Tobacco, use and effects of, 133 composition of, I 33

Toilet of the soldier, 247

Toilet paper, in camps, 648

Toilet room, hygiene of, 576

Tollet pavilion, 474

Tomatoes as food, 36r

Tonsillitis, 4, 555

Tooth-powders, 248

Toxins, 27

Training, physical, 22I for officers, 224

Training stations in Navy, 755

Transports, bunks on, 757 space on, 756 ventilation of, 757

Traps, back-pressure in, 570 anti-siphon, 572 common forms of, 567 depth of seal in, 567 grease, 569 loss of seal in, 569 non-siphoning, 57I object of, 567 sanitas, 571 siphonage of, 569 venting of, 570

Travel ration, 386

Trench foot, I2I, 747 
Trench hygiene, 746

Trenches as straddle sinks, 637

Treponema (or spirochæte), 24 pallidum of syphilis, I22 pertenue of yaws, I03

Trichina spiralis, 325

Trichuris trichiura or whipworm, 106

Tricresol as disinfectant, 738

Tropical diseases, I5, 7 IO

Tropical sore, I03

Tropics, barracks in, 705 cooling of, 706

beverages in, 694

blonds and brunettes in, 701

care of bowels in, 709

chilling of body in, 708

climatic features of, 687

clothing in, 704

diet for the, 402

effects of proteid diet in, 402

exercise in, 688, 708

fats in, 402

food in, 693

danger of excess of, 693

glare in, 557,702

head-gear in, 704

heat dissipation in. 687

influence of, on blood, 689 on nervous 'system, 691

on pulse, 690

on respiration, 689

on body temperature, 690

on weight, 691

length of service in, 692

marching in, 708

rarefaction of air in, 690

skin pigmentation in, 700

tents in, 707

ventilation of barracks in, 705 water cooling in, 706

Trough latrine, 653 advantages and objections, 655

Trousers, uniform, 428, 45 I

Trypanosomiasis, 104

Tse-tse fly, I04, I56

Tuberculosis, cause of, 56

in cattle, 326

mode of infection in, 57

prevalence of, 54

prophylaxis of, 58

sanatorium treatment of, 59

Tubers as food, 358

Typhoid bacillus, 30

in feces and urine, 3I

transmission of, 32

by flies, 34

by contact, 33

by clothing and tentage, 33 by water, 34

Typhoid fever, in the Army, 29

prophylaxis of, 38

statistics of, 36

susceptibility to, 37

vaccination against, 39

Typho-malarial fever, 30

Typhus fever, 8I

etiology of, 82

former prevalence of, II

quarantine regulations in, 779

Ultra-violet or actinic rays, 695 in water purification, 282

in illumination, 558

Underclothing, requirements of, 423 materials used for, 423

orange-red, 697

regulation, 433

Undershirts, regulation, 433

Uniform, requirements of, 426 color of the, 426

Uniforms in U. S. Army, 428

Uric acid, excretion of, 309

Urinals, in camps, 652 in garrison, 576

Urine, danger of, in typhoid fever, $3 \mathrm{I}$

disinfection of, 743

Vaccination, in smallpox, 92 in typhoid fever, 39

Vaillard-Desmaroux sterilizer, 278

Vapor as air constituent, 5II

Varicella, 94

Vai icocele in applicants, 199

Varicose veins in applicants, 200

Variola or smallpox, 92

Varioloid, 93

Vegetable acids, 3 I 3

Vegetable foods, classification of, 349 composition of, 350

Vegetables, green, $36 \mathrm{I}$ fruits used as, $36 \mathrm{I}$

Vegetation, effect of, on air, 5 II on camp site, 606

on mosquitoes, 606

on soil, 601

Venereal diseases, 122 in the tropics, 123, 710 prophylaxis of, I 26 rates in Army and Navy, I23 in foreign armies, 124

Venomous animals, 609

Ventilation, nature of, 519, 530 artificial, 539 advantages and objections, 540 
downward system, 540

upward system, $5+0$

effect of chimneys on, 539

effect of heat on, 534

effect of stoves on, 539

inlets for, 541

position and size of, $54 \mathrm{I}$

natural, 534

devices to promote, 537

forces governing, 534

on board ships, 756

outlets for, 54I

position and size, 54 I

ridge. 539

Ventilators, kinds of, 538

Venting of traps, 570

Vermin in barracks 494

Vision requirements, in Army, 195 in Navy, 775

Vitality, effect of service on, 747

Vclunteers, losses by disease in, I 82

VVainscoting in barracks, 490

Walls, dampness of, 478,479

permeability of, 477.536

plastering and finishing of, 480 , 485

thickness of, 477

Waring systen 1 of disposal, 59 I

Warm climates, 686

(see Tropics)

IVastes, disposal of, 593, 667

IVaste-pipes in house plumbing, 565

Water, ammonia in, 267

bacterial indicators in, 2\%2

bacterial examination of, 270

chemical examination of, 264

chlorine in, 267

distilled, 280

drinking of, on the march, 239 at meal, 406

in the tropics, 694

evaporation of, to cool air, 706

external qualities of, 263

hardness in, 265

lake, 260

metals in. 266

microscopic examination, 270

nitrates in, 260

nitrites in, 268

organic matter in, 266

pathogenic organisms in, $27 \mathrm{I}$

purification of, 273

by chemical means, $28 \mathrm{r}$

by cold, 280

by filtration, 290

by heat, 273

by sunlight, 260,720 quantity of, required, 257

rain, 258

river, 259 self-purification of, 259

spring, 260

sterilizers, objections to, 279

the Forbes, 274

the Griffith, 279

wagon, on the march, $2.4 \mathrm{I}$

wheeled-sterilizers, 277

Water bag, sterilizer, 286

Water-closets, fixtures, 572

hopper, 574

requirements of; $\mathbf{5 7 2}$

short-hopper, $\mathbf{5 7 4}$

siphon-jet, 574

wash-down, 574

wash-out, 574

Water-gas, as illuminant, 559

Waterproofing of clothing, 432 of shoes, 448

Water-supply, in camps, 632, 6,8 on board ships, 765

IVater-wagons ,24I, 277

Weight, as affected by exercise, 2I6 for applicants, I 88 in relation to height, 189 loss of, in the tropics, $69 \mathrm{I}$

Wells, construction of, 26I

location of, 262

pollution of, 26 I

tubular, 263

Welshach gas-burner, 560

Wheat, $35^{1}$

flour of, 352

Wheeled kitchen, 378

Wheeled water-sterilizers, 277

White duck suit, 430

Windows, construction of, 482 double, 483

French, 482 ventilating panels in, 537

Wood alcohol. 4I7

Wood work, disinfection of, 74I

Wool fiber, 419

Woolen fabrics, advantages of, 423 oljections to, 424

Wounds by modern projectiles, I3 infection of, $\mathrm{I} 4$

character of, 14

Yam as food, 360

Yaws, I03

Yellow fever. 73

etiology of, 73

historical appendix, 76

prophylaxis of, 74

quarantine regulation in, 778 


